COLUMBIA LIBRARIES OFFSITE

HX00008621 


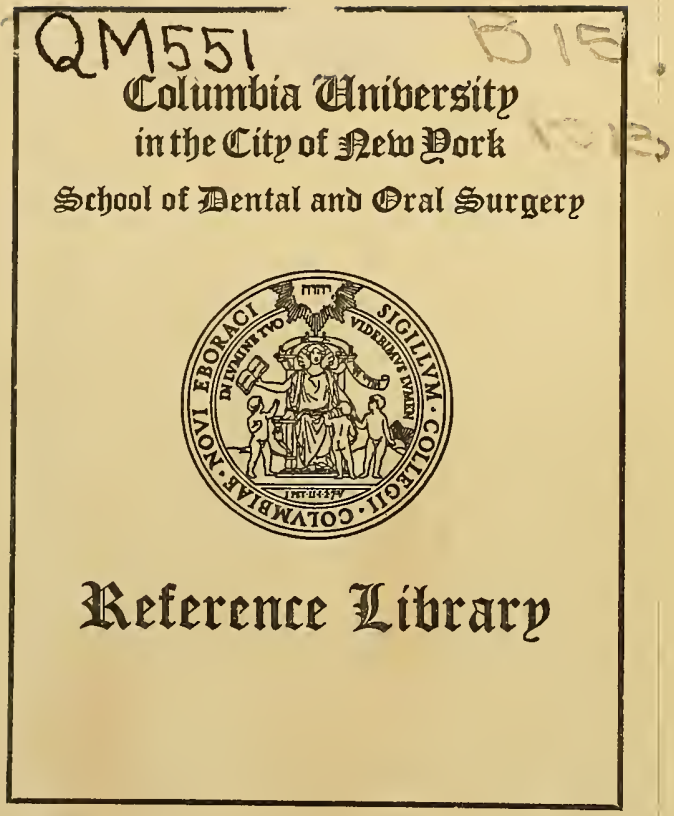




$$
\text { comfor }
$$





A

\section{TEXT-BOOK OF HISTOLOGY}

BY

FREDERICK R. BAILEY, A. M., M. D.

FOURTH REVISED EDITION

PROFUSELY ILLUSTRATED

NEW YORK

WILLIAM WOOD AND COMPANY

MDCCCCXIII 


$$
\begin{aligned}
& Q M 551 \\
& 315 \\
& 1913
\end{aligned}
$$

Copyright, I9I3

By IVILLIAM WOOD AND COMPANY 


\section{PREFACE TO THE FOURTH EDITION}

THE very gratifying approval which the previous editions of the Text-book received has made it seem unwise to attempt any change in the general plan and scope of the work as outlined in the preface to the first edition. The text has been thoroughly revised, some parts of it rewritten. Some figures have been replaced by new ones and a considerable number of new figures, have been added. For these the writer wishes to acknowledge his obligations. To Prof. H. von W. Schulte and to Mr. A. M. Miller the writer is indebted for many valuable criticisms and suggestions. The chapter on the nervous system which was rewritten by Dr. Oliver S. Strong for the third edition, has been revised by him for the present edition. For Dr. Strong's careful and painstaking work on this chapter, for his thoroughly original treatment of his subject, and for the original drawings and photographs in this chapter, the author wishes again to express his most grateful appreciation. Dr. Strong wishes to acknowledge his indebtedness to Dr. Adolf Meyer for many ideas and terms found valuable in the preparation of the chapter on the nervous system. 
Digitized by the Internet Archive in 2010 with funding from

Open Knowledge Commons (for the Medical Heritage Library project) 


\section{PREFACE TO THE FIRST EDITION}

THE primary aim of the writer in the preparation of these pages has been to give to the student of medicine a text-book of histology for use in connection with practical laboratory instruction, and especially to furnish to the instructor of histology a satisfactory manual for classroom teaching. With these objects in view, the text has been made as concise as possible consistent with clearness, and the writer has attempted to make the more essential elements stand out somewhat from the necessarily accompanying details.

It has been impossible to accomplish this without some sacrifice of uniformity of treatment and of logical sequence. This is especially noticeable in the chapter on the nervous system, which has been made much fuller and more "practical" than is usual. The author's reason for the method of treatment there adopted and for the considerable amount of anatomy which this chapter contains is the apparent success the method has met with in the teaching of this always difficult subject to students.

The chapter on general technic is intended to furnish the student with only the more essential laboratory methods. For special and more elaborate methods the student is referred to the special works on technic mentioned at the close of the chapter. The special technic given in connection with the different tissues and organs is in most cases such as can be conveniently used for the preparation of class sections.

The original illustrations are from drawings by Mr. A. M. Miller, to whom the writer is greatly indebted for his careful and accurate work. The uselessness of redrawing perfectly satisfactory illustrations has led the writer to borrow freely from various sources, each cut being duly accredited to the work from which it has been taken. For all of these the author wishes to express his appreciation and obligation. He is also deeply indebted to Dr. O. S. Strong for his careful review and criticism of the chapter on the nervous system and for his supervision of the drawing of Figs. 263 and 264 ; to Dr. G. C. Freeborn, his predecessor as Instructor of Histology at the College of Physicians and Surgeons, for many valuable suggestions; and to Dr. T. Mitchell Prudden for his careful and critical review of the author's copy. 



\section{CONTENTS}

\section{PART I.-HISTOLOGICAL TECHNIC}

\section{CHAPTER I}

General Technic.

PAGE.

General Considerations,. . . . . . . . . . . . . . . . . . . 3

Examination of Fresh Tissues, . . . . . . . . . . . . . . . . . 3

Dissociation of Tissue Elcments, . . . . . . . . . . . . . . . . 4

Teasing, . . . . . . . . . . . . . . . . . . . . . . . . . . . . 4

Maceration, . . . . . . . . . . . . . . . . . . . . . 4

Preparation of Sections, . . . . . . . . . . . . . . . 5

Fixation, . . . . . . . . . . . . . . 5

Hardening, . . . . . . . . . . . . . . . . . 8

Preserving, . . . . . . . . . . . . . . . . . . . . . . 9

Decalcifying, . . . . . . . . . . . . . . . . . 10

Embedding, . . . . . . . . . . . . . . . . . . . . . I I I

Celloidin Embedding, . . . . . . . . . . . . . . . . . . . . . II II

Paraffin Embedding, . . . . . . . . . . . . . . . . 13

Section Cutting, . . . . . . . . . . . . . . . . . . . I4

Celloidin Sections, . . . . . . . . . . . . . . . . . . . . . . 14

Paraffin Sections, . . . . . . . . . . . . . . . ${ }^{15}$

Frozen Sections, . . . . . . . . . . . . . . . I5

Staining, . . . . . . . . . . . . . . . I7

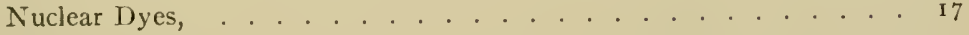

Plasma Dyes, . . . . . . . . . . . . . . . . . . . . . . . 19

Staining Sections, . . . . . . . . . . . . 20

Staining in Bulk, . . . . . . . . . . . . . . 2 $2 \mathrm{I}$

Mounting, . . . . . . . . . . . . . . . . . 22

Staining and Mounting Iaraffin Sections, . . . . . . . . . . . 23

Injection,. . . . . . . . . . . . . . . . . . . . 25

\section{CHAPTER II}

\section{Siectal Staining Methods.}

Silver Nitrate $\mathrm{N}$ ethod of Staining the Intercellular Substance,. . . . . . . 28

Chlorid of Goll for I) cmonstrating Connective-tissuc Cclls, . . . . . . . . 28

Weigert's Elastic Tissue Stain. . . . . . . . . . . . . . . . 28

Verhoeff's 1)ifferential lilastic Tissue Stain, . . . . . . . . . . . . 28

(iolgi's ('hrome-silver for stalining Secretory 'Jubules, . . . . . . . . . . 29

Mallory's l'hosphomolybdic Acir] Hacmatoxylin Stain for Connective 'Tissue, 29

Mallory's P'hosphotungstic Acid Ifrmatoxylin Stain for Connective Tissue, , 30 
Mallory's Aniline Blue Stain for Connective Tissue . . . . . . . . . . . 30 Maresh's Modification of Bielschowsky's Stain for the Finer Connective Tissue Fibrils,. . . . . . . . . . . . . . 3 I

Osmic Acid Stain for Fat, . . . . . . . . . . . . . . . . . . 3 I

Jenner's Blood Stain, . . . . . . . . . . . . . . . . . $3^{\text {I }}$

\section{CHAPTER III}

Special Neurological Staining Methods.

Weigert's Method of Staining Medullated Nerve Fibres, . . . . . . . . . 32

Weigert-Pal Method, . . . . . . . . . . . . . . . . . . . 33

Marchi's Method for Staining Degenerating Nerves, . . . . . . . . . . 34

Golgi Methods of Staining Nerve Tissue, . . . . . . . . . . . . . . . . 35

Slow Method, . . . . . . . . . . . . . . . . . . . . . 35

Rapid Method, . . . . . . . . . . . . . . . . . . . . . . . . . 35

Mixed Method, . . . . . . . . . . . . . . . . . . . . . . . . 35

Formalin Bichromate Method, . . . . . . . . . . . . . . . 36

Bichloride Method, . . . . . . . . . . . . . . . . . . . . . . 36

Golgi-Cox Method,. . . . . . . . . . . . . . . . . . . . 36

Cajal's Method, . . . . . . . . . . . . . . . . . . . . . . . . 37

Nissl's Method, . . . . . . . . . . . . . . . . . . . . . . . . . 38

General References on Technic, . . . . . . . . . . . . . . . . . . 39

\section{PART II.-THE CELL}

\section{CHAPTER I}

The Cell, . . . . . . . . . . . . . . . . . . . . . . . . . . . . . . . 43

General Structure, . . . . . . . . . . . . . . . . . . . . . 43

Structure of a Typical Cell, . . . . . . . . . . . . . . . . . . . . . . . 43

The Cell Body, . . . . . . . . . . . . . . . . . . . . . . . . . 44

The Cell Membrane, . . . . . . . . . . . . . . . . . . . . . . . 47

The Nucleus, . . . . . . . . . . . . . . . . . . . . . . . . . . 47

The Centrosome, . . . . . . . . . . . . . . . . . . . . . . 49

Vital Properties of Cells, . . . . . . . . . . . . . . . . . 50

Metabolism,. . . . . . . . . . . . . . . . . 50

Function, . . . . . . . . . . . . . . . . . 5 I

Irritability, . . . . . . . . . . . . . . . . . 5 I

Motion, . . . . . . . . . . . . . . . . . . 5 I

Amœboid, . . . . . . . . . . . . . . . . . . . 52

Protoplasmic, . . . . . . . . . . . . . . . . . 52

Ciliary, . . . . . . . . . . . . . . . . 52

Reproduction, . . . . . . . . . . . . . . . . . . 52

Direct Cell-division, . . . . . . . . . . . . . . . . . . . 53

Indirect Cell-division, . . . . . . . . . . . . . . . . . 53

Fertilization of the Ovum, . . . . . . . . . . . . . . 58

Technic, . . . . . . . . . . . . . . . 63

References for further study, . . . . . . . . . . . . . . . 64 


\section{PART III.-THE TISSUES}

\section{CHAPTER I}

HistogeNesis-Classification,

Tissues Derived from Ectoderm,

Tissues Derived from Entoderm,

Tissues Derived from Mesoderm,

\section{CHAPTER II}

Epithelium (Including Mesothelium and Endothelium), . . . . . . . . . 69

Histogenesis, . . . . . . . . . . . . . . . 69

General Characteristics, . . . . . . . . . . . 69

Classification, .. . . . . . . . . . . 70

Simple Epithelium, . . . . . . . . . . . . . . 7 I

Simple Squamous, . . . . . . . . . . . . . . . 7 I

Simple Columnar, . . . . . . . . . . . . . . . . . 7 I

Pseudostratified, . . . . . . . . . . . . . . . 73

Stratified Epithelium, . . . . . . . . . . . . . . . . . . . 73

Stratified Squamous, . . . . . . . . . . . . . . . . . . . . . . . 73

Stratified Columnar, . . . . . . . . . . . . . . . . . . 74

Transitional, . . . . . . . . . . . . . . . . . . 74

Modified Forms of Epithelium, . . . . . . . . . . . . . . . 75

Ciliated Epithelium, . . . . . . . . . . . . . . . . 75

Pigmented Epithelium, . . . . . . . . . . . . . . . 76

Glandular Epithelium, . . . . . . . . . . . . . . . . 77

Neuro-epithelium, . . . . . . . . . . . . . . . . . . 77

Mesothelium and Endothelium, . . . . . . . . . . . . 77

Technic, ................. . . 78

\section{CHAPTER III}

The Consective Tissues, . . . . . . . . . . . . . . . . 80

General Characteristics, . . . . . . . . . . . . . . . . 80

Classification, ................. . . 8 I

Development, . . . . . . . . . . . . . . . . 8 I

Connective Tissue, Proper . . . . . . . . . . . . . . . . . . 82

Embryonal Connective Tissue, . . . . . . . . . . . . . . 82

Fibrillar Connective Tissue, . . . . . . . . . . . . . . . 82

Connective-tissue Cells, ................ . . . 83

Intercellular Substance, . . . . . . . . . . . . . . . . 86

Areolar or Loose Connective Tissue, . . . . . . . . . . . . . . . . . . 87

Fat 'Tissue, . . . . . . . . . . . . . . . . 87

Formed Connective Tissue, . . . . . . . . . . . . . . . . . 9r

Jendons and ligaments, . . . . . . . . . . . . . . . . . . . . . . . 9I

Elastic Tissue, . . . . . . . . . . . . . . . . . . . . 92

Reticular Tissue,. . . . . . . . . . . . . . . . . . . . . . 94

Technic, . . . . . . . . . . . . . . . . . 95

Cartilage, . . . . . . . . . . . . . . . . . 97

Ilyaline, . . . . . . . . . . . . . . . . . . . 98 
Elastic,

Fibrous,

Technic,

Bone Tissue,

Technic,

THE BlOOD, . . . . . . . . . . . . . . . . . . . . . I03

Red Blood Cells, . . . . . . . . . . . . . . . . . . . . 103

White Blood Cells, . . . . . . . . . . . . . . . . . . . . I05

Blood Platelets, . . . . . . . . . . . . . . . . . . 108

Blood Dust, ..... . . . . . . . . . . . . . . . . . . то 108

Development, . . . . . . . . . . . . . . . . 108

Technic,................... . . I Iо

\section{CHAPTER V}

Muscle Trssue, . . . . . . . . . . . . . . . . . . . . . . . III

Involuntary Smooth Muscle, . . . . . . . . . . . . . . . . . III

Voluntary Striated Muscle, . . . . . . . . . . . . . . . . . . . . . III

Involuntary Striated Muscle (Heart Muscle), . . . . . . . . . . . . . II9

Development of Muscle Tissue, . . . . . . . . . . . . . . . . . . . I 22

Technic, . . . . . . . . . . . . . . . . . . . . . . . . . . . . . . I 24

\section{CHAPTER VI}

Nerve Tissue, . . . . . . . . . . . . . . . . . . . . . . I 26

The Neurone, . . . . . . . . . . . . . . . . . . . . . . . . . . . . . I 26

General Structure, . . . . . . . . . . . . . . . . . . I 26

The Cell Body, . . . . . . . . . . . . . . . . . . . . . . . . I26

The Nucleus, . . . . . . . . . . . . . . . . . . . . . . . . . . I 27

The Cytoplasm, . . . . . . . . . . . . . . . . . . . I28

Neurofibrils, . . . . . . . . . . . . . . . . . . . . I 28

Perifibrillar Substance, . . . . . . . . . . . . . . . . . . . . 128

Chromophilic Bodies, . . . . . . . . . . . . . . . . . . . I 29

The Protoplasmic Processes or Dendrites, . . . . . . . . . . . . . . I3 I

The Axone, . . . . . . . . . . . . . . . . . . . . . . . . . . . . . $\mathrm{I}_{3} 2$

Non-medullated Axones (Non-medullated Nerve Fibres), . . . . . . . 132

Medullated Axones (Medullated Nerve Fibres), . . . . . . . . . . . I33

Theories as to Physiology of the Neurone, . . . . . . . . . . . . . . . I37

Significance of Degenerative Changes in the Neurone, . . . . . . . . . . I39

Neuroglia, . . . . . . . . . . . . . . . . . . I42

Technic, . . . . . . . . . . . . . . . . . . . . . . I44

General References, . . . . . . . . . . . . . . . . . I45

\section{PART IV.-THE ORGANS}

\section{CHAPTER I}

The Circulatory System, . 
General Structure,

Capillaries,

Arteries,

Teins,

Technic,

The Heart, I6I

Technic: 163

Development of the Circulatory System,

The Lymph-vessel System, . . . . . . . . . . . . . . . . . . 65

Lymph Capillaries, . . . . . . . . . . . . . . 665

Lymph Spaces, . . . . . . . . . . . . . . . . . . . . . . . .

Development of Lymph-vessel System, . . . . . . . . . . . . . . . . . .

Technic, . . . . . . . . . . . . . . . . . . .

General References on Circulatory System,

\section{CHAPTER II}

LYMPHATIC ORGANS,

The Lymph Nodes,

Development,

Technic,

Hæmolymph Nodes, . . . . . . . . . . . . . 175

Technic, . . . . . . . . . . . . . . . . . . . . . . .

The Thymus, . . . . . . . . . . . . I77

Development, . . . . . . . . . . . . . . . . . . . . .

Technic, . . . . . . . . . . . . . . . . . . . . . .

The Tonsils, . . . . . . . . . . . . . . . . . . . 178

The Palatine Tonsils, . . . . . . . . . . . . . . . . . 180

The Lingual Tonsils, . . . . . . . . . . . . . . . . . . . . 180

The Pharyngeal Tonsils, . . . . . . . . . . . . . . . . . . . . . . I8o

Development, . . . . . . . . . . . . . . . . . . . . . . . .

Technic, . . . . . . . . . . . . . . . . . . . . . . . . . . . .

The Spleen, . . . . . . . . . . . . . . . . . . . 187

Technic, . . . . . . . . . . . . . . . . . . . . . . . . . . . . . 87

General References,

\section{CHAPTER III}

The Sketetal Systeyi . . . . is8

The Bones, ............ 192

Red Marrow, . . . 194

Red Marrow, . . . . . . . . . . . . . . . . 197

Yellow . Narrow, .

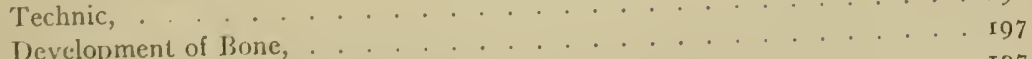

Development of Bone, . . . . . . . . . . . . . . . . . . . . . . . . . . .

Intramembranous Development, . . . . . . . . . . . . . 199

Intracartilaginous Development, . . . . . . . . . . . . . . . . 20 20I

Subperiostcal, . . . . . . . . . . . . . . . . . . . . . . 203

Growth of Bone, . . . . . . . . . . . . . . . . . . . . . . . 203

Technic, . . . . . . . . . . . . . . . . . 204

The Cartilages, 
Articulations,

Technic,

General References,

\section{CHAPTER IV}

The Muscular System.

A Voluntary Muscle, . . . . . . . . . . . . . . . . . . . . . . 207

Tendon Sheaths and Bursæ, . . . . . . . . . . . . . . 208

Growth of Muscle, . . . . . . . . . . . . . . . . . . . . . . 209

Technic, . . . . . . . . . . . . . . . . . . . 2 II

\section{CHAPTER V}

Glands and the General Structure of Mucous Membranes, . . . . . . 2 i2

Glands-General Structure and Classification, . . . . . . . . . . . 2I2

Duct Glands, . . . . . . . . . . . . . . . . . . . . $2 \mathrm{I} 5$

Tubular Glands, . . . . . . . . . . . . . . . . . . . 215

Aveolar Glands, . . . . . . . . . . . . . . . . . . . 217

Ductless Glands, . . . . . . . . . . . . . . . . $2 \mathrm{I} 7$

General Structure of Mucous Membranes, . . . . . . . . . . . 2 $2 \mathrm{I} 8$

\section{CHAPTER VI}

The Digestive System, . . . . . . . . . . . . . . . . . . 220

Anatomical Divisions, . . . . . . . . . . . . . . . . . . . 220

The Headgut, . . . . . . . . . . . . . . . . . . . . . . . . . 22 I

The Mouth, . . . . . . . . . . . . . . . . . . . . . 22 I

The Mucous Membrane of the Mouth, . . . . . . . . . . 22 I

Glands of the Oral Mucosa, . . . . . . . . . . . . . 221

Technic, . . . . . . . . . . . . . . . . . 223

The Tongue, . . . . . . . . . . . . . . . . 223

Technic, . . . . . . . . . . . . . . . 227

The Teeth, . . . . . . . . . . . . . . . . . . 227

Development of the Teeth, . . . . . . . . . . . . 238

Technic, . . . . . . . . . . . . . . . . . 242

The Pharynx, . . . . . . . . . . . . . . . . . . . . . . 242

Technic, . . . . . . . . . . . . . . . . . 243

The Foregut, . . . . . . . . . . . . . . . . . . . . . . . 243

The Esophagus, . . . . . . . . . . . . . . . . . . . . . . . 243

Technic, . . . . . . . . . . . . . . . . . . . . 245

General Structure of the Walls of the Gastro-intestinal Canal, . . . . 245

The Stomach, . . . . . . . . . . . . . . . . . . . . . . . . . . 247

Technic, . . . . . . . . . . . . . . . 254

The Midgut, . . . . . . . . . . . . . . . . . . . . 255

The Small Intestine, . . . . . . . . . . . . . . . . . . 255

Peyer's Patches, . . . . . . . . . . . . . . . . . . . . 260

The Endgut, . . . . . . . . . . . . . . . . . . . . . . . . 263

The Large Intestine, . . . . . . . . . . . . . . . . . . . . . . . 263

The Vermiform Appendix, . . . . . . . . . . . . . . . . . . . . 265

The Rectum, . . . . . . . . . . . . . . . . . . . . . . . . 266 
The Peritoneum, Mesentery, and Omentum,. . . . . . 267

Blond-ressels of the Stomach and Intestine, . . . . . . . . . . 268

Lymphatics of the Stomach and Intestine, . . . . . . . . 270

Nerves of the Stomach and Intestine, . . . . . . . . . . . 270

Secretion and the Absorption, . . . . . . . . . . . 27 I

Technic, . . . . . . . . . . . . . . . . . 275

The Larger Glands of the Digestive System, . . . . . . . . . . . . . . . 275

The Salivary Glands, . . . . . . . . . . . . . . . 276

The Parotid, . . . . . . . . . . . . . . . . . 277

The Sublingual, . . . . . . . . . . . . . . . . . . 277

The Submaxillary, . . . . . . . . . . . . . . . 278

Technic, . . . . . . . . . . . . $28 I$

The Pancreas, . . . . . . . . . . . . . . . . . $28 \mathbf{I}$

Technic, . . . . . . . . . . . . . 287

The Liver, . . . . . . . . . . . . . . . . . . . . . . . . 287

Excretory Ducts of the Liver, . . . . . . . . . . . . . . 289

The Gall-bladder, . . . . . . . . . . . . . . . . 295

Technic, . . . . . . . . . . . . . . . . 295

Development of the Digestive System, . . . . . . . . . . . . . . . . . 296

General References, . . . . . . . . . . . . . . . . . 298

\section{CHAPTER VII}

The Respiratory System, . . . . . . . . . . . . . . . . . . . . . . . 299

The Nares, . . . . . . . . . . . . . . . . . . . . . . . . . 299

The Larynx,. . . . . . . . . . . . . . . . . . . . . . . . 30I

The Trachea, . . . . . . . . . . . . . . . . . . . . $30 \mathrm{I}$

Technic, . . . . . . . . . . . . . . . . 304

The Bronchi, . . . . . . . . . . . . . . . . . . . . 304

The Lungs, . . . . . . . . . . . . . . . . . . . . . . 308

Development of the Respiratory System, . . . . . . . . . . . . . . . 3 I5

Technic, . . . . . . . . . . . . . . . . . . . 3 I7

General References, . . . . . . . . . . . . . . . . . . 3 I 7

\section{CHAPTER VIII}

THE URINARY SYSTEMT, . . . . . . . . . . . . . . . . . . . . . . . . . .

The Kidney, . . . . . . . . . . . . . . . . . . . . . . .

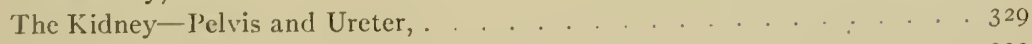

The Urinary Bladder, . . . . . . . . . . . . . . . . . . . . 330

Technic, . . . . . . . . . . . . . . . . . 33 I

General References, . . . . . . . . . . . . . . . . 332

CIIAPTER IX

TH: RERODLCTIV: SYSTEM, . . . . . . . . . . . . . . . . 333

Walc Organs, . . . . . . . . . . . . . . . . . . . 333

The Testis, . . . . . . . . . . . . . . . . . . . . . 333

The Seminal loucts, . . . . . . . . . . . . . . . . . . . . . 339

The Epididymis, . . . . . . . . . . . . . . . . . . 339

The Vas Jeferens, . . . . . . . . . . . . . . . . 340 
PAGE

The Seminal Vesicles and Ejaculatory Ducts, . . . . . . . . . . . 34I

Rudimentary Structures Connected with the Development of the Genital

System,

The Spermatozoön, . . . . . . . . . . . . . . . . . . . . 343

Development of the Spermatozoön, . . . . . . . . . . . . 344

Technic, . . . . . . . . . . . . . . 346

The Prostate Gland, . . . . . . . . . . . . . . . . . . . . 347

Cowper's Glands, . . . . . . . . . . . . . . . . . . . 348

Technic, . . . . . . . . . . . . . . . . 349

The Penis, . . . . . . . . . . . . . . . . . . . 349

The Urethra, . . . . . . . . . . . . . . . . . . . 35 I

Technic, . . . . . . . . . . . . . . . . 352

Female Organs, . . . . . . . . . . . . . . . . . . . 352

The Ovary, . . . . . . . . . . . . . . . . . . . $35^{2}$

The Graafian Follicle, . . . . . . . . . . . . . . . . . . . 354

The Corpus Luteum, . . . . . . . . . . . . . . . . . . . . . . 359

Rudimentary organs connected with the Ovary, . . . . . . . . . . 363

The Oviduct, . . . . . . . . . . . . . . . . . . . . 364

Technic, . . . . . . . . . . . . . . . 365

The Uterus, . . . . . . . . . . . . . . . . . . . . . . 366

The Mucosa of the Resting Uterus, . . . . . . . . . . . . . 367

The Mucosa of the Menstruating Uterus, . . . . . . . . . . . 368

The Mucosa of the Pregnant Uterus, . . . . . . . . . . . . . 370

The Placenta, . . . . . . . . . . . . . . 37 I

The Vagina, . . . . . . . . . . . . . . . . . . . . . . . . . . 375

Development of the Urinary and Reproductive Systems, . . . . . . . . . 376

Technic, . . . . . . . . . . . . . . . . . . . . . . 379

General References, . . . . . . . . . . . . . . . . . 379

\section{CHAPTER $\mathrm{X}$}

The Skin and tts Appendages, . . . . . . . . . . . . . . . . . . . . . 380

The Skin, . . . . . . . . . . . . . . . . . 380

Technic, . . . . . . . . . . . . . . . . . . . 384

The Nails, . . . . . . . . . . . . . . . . . . . . . 385

Technic, . . . . . . . . . . . . . . . . . . . . 387

The Hair, . . . . . . . . . . . . . . . . . . . . . . . . . . . . . 387

Technic, . . . . . . . . . . . . . . . . 393

Blood-vessels of Skin, . . . . . . . . . . . . . . . . . . . . 393

Technic, . . . . . . . . . . . . . . . . . . . . . . 395

Development of Skin, Nails, and Hair, . . . . . . . . . . . . . . . . 395

The Mammary Gland, . . . . . . . . . . . . . . . . . . . . . . . 395

Technic, . . . . . . . . . . . . . . . . . . $40 \mathrm{I}$

General References, .. . . . . . . . . . . . . . . . . . . $40 \mathrm{I}$

\section{CHAPTER XI}

The_Thyreom and Parathyreold, the Prtuitary Body, the Paraganglia and THE AdRENAL.

The Thyreoid, . . . . . . . . . . . . . . . . . . . . 402

Development, . . . . . . . . . . . . . . . 403 
The Parathyreoids,

Technic,

The Pituitary Body,

Paraganglia,

Carotid Glands,

Coccygeal Gland,

Adrenal Gland,

\section{CH.APTER XII}

The Neryous System,

416

Histological Development and General Structure, _. . . . 4 4 I6

Membranes of the Brain and Cord,

422

Technic,

The Peripheral Xerves, . . . . . . . . . . . . 424

Technic,

The Afferent Peripheral Neurones, . . . . . . . . . . . . . 426

The Cerebro-spinal Ganglia, _. . . . . . . . . . . 426

The Peripheral Processes of the Cercbro-spinal Ganglion Cells. . 429

The Central Processes of the Cerebro-spinal Ganglion Cells, . . 436

The Sympathetic Ganglia, . . . . . . . . . . . . . . . 436

Technic. . . . . . . . . . . . 4 441

The Efferent Peripheral Cerebro-spinal Neurones, . . . . . . . . . 44I

The Spinal Cord, . . . . . . . . . . . . . . 442

Origin of the Fibres which make up the White Matter of the Cord, . . 443

(I) The Spinal Ganglion Cell and the Origin of the Posterior Columns, . . . . . . . . . . . 443

(2) Cells Situated in Other Parts of the Central Nervous System which Contribute Axones to the White Columns of the Cord, . . 444

(3) Root Cells-Motor Cells of the Anterior Horn, . . . . . 444

(4) Column Cells, . . . . . . . . . . . . . . 444

(5) Cells of Golgi Type II, . . . . . . . . . . . . . 445

Technic, . . . . . . . . . . . . . 445

Practical Study, . . . . . . . . . . . . . . . . . 447

General Topography of the Cord, Cell Groupings, Arrangement of Fibres and Finer Structure, . . . . . . . . . . . . . . 448

Practical Study of Sections through Lumbar Enlargement, . . . 448

General Topography, . . . . . . . . . . 448

Cell Groupings, . . . . . . . . . . . . . . . 450

Arrangement of Fibres, . . . . . . . . . . . 452

Finer Structure, . . . . . . . . . . . . . . . 453

Blood-vessels, . . . . . . . . . . . . . . . . 454

Variations in Structure at Different Levels, . . . . . . . . 455

Practical Study, . . . . . . . . . . . . . . . 455

Section through the Twelfth Thoracic Segment, . . . . . 455

Section through the Mid-thoracic Region, . . . . . . 455

Section through the Cervical Enlargement, . . . . . 455

Fibre Tracts of the Corrl, . . . . . . . . . . . . . . . . 459

Ascending 'Tracts, . . . . . . . . . . . . $46 \mathrm{I}$

I. Long Ascending Arms of Dorsal Root Fibres, . . . . 46r 
II. Spino-thalamic Tract, PAGE

III. Dorsal Spino-cerebellar Tract, . . . . . . . . . . . . 463

IV. Ventral Spino-cerebellar Tract, . . . . . . . . . . 463

Descending Tracts, . . . . . . . . . . . . . . . . . 464

I. The Pyramidal Tracts, . . . . . . . . . . . . 464

II. The Colliculo-spinal Tract, . . . . . . . . . . . . 465

III. The Tract from the Nucleus of the Posterior or Medial Longitudinal Fasciculus, . . . . . . . . . . . . . 465

IV. The Rubro-spinal Tract, . . . . . . . . . . . . . . 466

V. The Deitero-spinal Tract, . . . . . . . . . . . . 466

VI. The Fasciculus of Thomas, . . . . . . . . . . . . . 467

VII. Helweg's Tract, . . . . . . . . . . . . . . . . . . 467

VIII. The Septo-marginal Tract, . . . . . . . . . . . . 467

IX. The Comma Tract of Schultze, . . . . . . . . . . . 467

Fundamental Columns or Ground Bundles, . . . . . . . . . . . 468

A Two-neurone Spinal Reflex Arc, . . . . . . . . . . . . . . . . 469

A Three-neurone Spinal Reflex Arc, . . . . . . . . . . . . . . 469

A Cerebellar Arc, . . . . . . . . . . . . . . . . . . . . 470

A Cerebral or Pallial Arc, . . . . . . . . . . . . . . . . . 471

Technic, . . . . . . . . . . . . . . . $47 \mathrm{r}$

The Brain, . . . . . . . . . . . . . . . . . . . . 473

General Structure, . . . . . . . . . . . . . . . . . . 473

Segmental Brain and Nerves, . . . . . . . . . . . . . . . . . 474

Suprasegmental Structures, . . . . . . . . . . . . . . . . . . 478

The Hindbrain or Rhombencephalon, . . . . . . . . . . . . . . . 479

The Medulla Oblongata or Bulb, . . . . . . . . . . . . . . . 479

The Pons, . . . . . . . . . . . . . . . . . . 482

The Cerebellum (also p. 507), . . . . . . . . . . . . 482

Technic, . . . . . . . . . . . . . 482

Practical Study, . . . . . . . . . . . . . . . . . . 483

I. Transverse Section of the Medulla through the Decussation of the Pyramidal Tracts (Motor Decussation), . . . . . . 483

2. Transverse Section of the Medulla through the Decussation of the Fillet or Lemniscus (Sensory Decussation), . . . . 485

3. Transverse Section of the Medulla through the Lower Part of the Inferior Olivary Nucleus, . . . . . . . . . . . . 487

4. Transverse Section of the Medulla through the Middle of the Olivary Nucleus, . . . . . . . . . . . . . . . . $40 \mathrm{I}$

5. Transverse Section of the Medulla through the Entrance of the Cochlear Root of Nerve VIII, . . . . . . . . . . . 49 I

6. Section through the Hindbrain at Level of Junction of Pons, and Cerebellum and Entrance of Vestibular Nerve, . . . 499

7. Transverse Section of the Hindbrain through the Roots of Nerves VI (Abducens) and VII (Facial), . . . . . . . 502

8. Transverse Section of the Hindbrain through the Roots of Nerve V (Trigeminus), . . . . . . . . . . . . . 504

The Cerebellum, . . . . . . . . . . . . . . . . . 507

The Cerebellar Cortex, . . . . . . . . . . . . . . 509

The Isthmus, . . . . . . . . . . . . . . . . . . . . . . . 5 55

Practical Study, . . . . . . . . . . . . . 515 
9. Transverse Section through the Isthmus at the Exit of Nerve IV (Trochlearis),

The Midbrain or Mesencephalon,

Practical Study,

I0. Transverse Section through Midbrain at Level of Anterior

Corpora Quadrigemina and Exit of Nerve III (Oculomotor), 5 I7

The Forebrain or Prosencephalon, . . . . . . . . . . . . . . 522

The Interbrain (Diencephalon or Thalamencephalon), . . . . . 522

Practical Study, . . . . . . . . . . . . 524

II. Transverse Section through the Junction of Midbrain and Thalamus, . . . . . . . . . . . 524

I2. Section through the Interbrain at the Level of the Optic

Chiasma,

The Endbrain or Telencephalon, . . . . . . . . . . . 532

The Rhinencephalon, . . . . . . . . . . . . . 532

The Corpus Striatum, . . . . . . . . . . . . . 532

The Pallium, . . . . . . . . . . . . . . 532

Practical Study, . . . . . . . . . . . . . . 535

I3. Transverse Section through the Cerebral Hemispheres, Corpora Striata and Thalamus, . . . . . . . . 535

The Cerebral Cortex, . . . . . . . . . . . . . 536

Technic, . . . . . . . . . . . . . . 543

The Pineal Body, . . . . . . . . . . . . . . . . . 544

Technic, . . . . . . . . . . . . . 547

Table of Cranial and Spinal Nerves, . . . . . . . . . . . . . 545

General Reference for Further Study, . . . . . . . . . . . . 547

\section{CHAPTER XIII}

The Organs of Special Sense, . . . . . . . . . . . . . . 548

The Organ of Vision, . . . . . . . . . . . . . . . . . . 548

The Eyeball, . . . . . . . . . . . . . . . . 548

The Cornea, . . . . . . . . . . . . . . . 548

The Chorioid, . . . . . . . . . . . . . . 550

The Ciliary Body, . . . . . . . . . . . . . 552

The Iris, . . . . . . . . . . . . . . . . . . 554

The Retina, . . . . . . . . . . . . . . . . 555

The Optic Nerve, . . . . . . . . . . . . . . . . . . . 559

The Relations of Optic Nerve to Retina and Brain, . . . . . . . 560

The Lens, . . . . . . . . . . . . . . . . . 564

The Lacrymal Apparatus, . . . . . . . . . . . . . . . . 567

The Eyelid, . . . . . . . . . . . . . . . . . . . 567

Development of the Eye, . . . . . . . . . . . . . . . 569

Technic, . . . . . . . . . . . . . 570

The Organ of Hearing, . . . . . . . . . . . . . . 572

The Extcrnal Ear, . . . . . . . . . . . . . . . 572

The Middle Ear, . . . . . . . . . . . . . . . . . . . . 573

The Internal Ear, . . . . . . . . . . . . . . . . . . 574

The Vestibule and Semicircular Canals, . . . . . . . . 575

The Saccule and Utricle, . . . . . . . . . . . . 575 


\section{CONTENTS}

The Semicircular Canals,. PAGE

The Cochlea, . . . . . . . . . . . . 576

Development of the Ear, . . . . . . . . . . . . . . . . . . . . 576

Technic, . . . . . . . . . . . . . 583

The Organ of Smell, . . . . . . . . . . . . . . . . 584

Technic, . . . . . . . . . . . . . . . 584

The Organ of Taste, . . . . . . . . . . . . . . . . . . . 586

Technic, . . . . . . . . . . . . . . . . . . 587

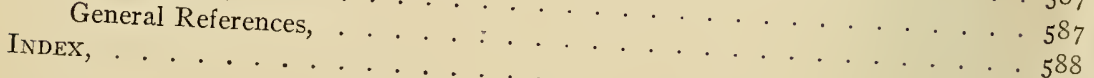

. 589 
PART I

HISTOLOGICAL TECHNIC 



\section{CHAP'TER I}

\section{GENERAL TECHNIC}

CERTAIN body fluids, e.g., blood, urine, etc., may be examined by simply placing them on a slide under a cover-glass. A few tissues, e.g., thin membranes, such as the omentum and the mesentery, may be examined fresh in some such inert medium as blood serum or normal salt solution ( 0.75 -per-cent. aqueous solution sodium chlorid). For such examination the tissue is immersed in the salt solution on a slide and covered with a cover-glass. Most tissues and organs, however, require much more elaborate preparation to render them suitable for microscopic examination. Tissues too dense and thick to be readily seen through with the microscope must be so treated as to make them transparent. This is accomplished either by pulling the tissue apart into fine shreds, teasing, or by cutting it into thin slices, section cutting. Some tissues admit of teasing in a fresh condition; others can be satisfactorily teased only after they have been subjected to the action of a chemical which breaks down the substance holding the tissue elements together, maceration. Fresh tissue can rarely be cut into sections sufficiently thin for microscopic examination. It must first be treated in such a manner as to preserve as nearly as possible the living tissue relations, fixation. If too soft for section cutting it must next be put through a process known as hardening. If, however, as in the case of bone, the tissue is too hard, it must be softened by dissolving out the mineral salts, decalcification. If very thin sections are to be cut, it is further necessary to impregnate the tissue with some fluid substance which will harden in the tissue and give to the mass a firm, even consistency. This is known as embedding.

Furthermore, most tissue elements have refractive indices which are so similar that their differentiation under the microscope is often extremely difficult. 'To overcome this difficulty, recourse is had to staining the tissue with dyes which have an affinity for certain only of the tissue elements, or which stain different elements with different degrees of intensity. This is known as differential or selective staining. 
The final step in the process is the mounting of the specimen, after which it is ready for microscopic study.

Only the more common procedures used in the preparation of tissues for microscopic study are described in this section. At the end of each section are given the technical methods most satisfactory for the demonstration of the tissues described in that section. For other methods the student is referred to special works upon microscopic technic.

\section{Dissociation of Tissue Elements}

This method of preparing tissues for microscopic study has only a limited application, most specimens being preferably fixed, and cut into thin sections. Certain of the structural features of such tissues as nerves, muscle, and epithelium, which have but little intercellular substance may be well demonstrated by dissociation.

This is accomplished by (I) teasing, or (2) maceration, or both.

(r) TEAsING.-This consists in pulling apart fresh or preserved tissues by means of teasing needles. Instructive specimens of such tissues as muscle and nerve may be obtained in this way.

(2) Maceration.-This is the subjecting of a tissue to the action of some chemical which breaks down the substance uniting the tissue elements, thus allowing them either to fall apart or to be more easily dissociated by teasing. The most commonly used macerating fluids are:

(a) Ranvier's Alcohol (33-per-cent., made by adding 35 c.c. of 96-per-cent. alcohol to 65 c.c. of water).--Bits of fresh tissue are placed in this fluid for from twenty-four to forty-eight hours. The cells may then be easily separated by shaking or by teasing. Ranvier's alcohol is an especially satisfactory macerating fluid for epithelia.

(b) Formaldehyde, in very dilute solutions (0.2- to o.4-per-cent. commercial formalin ${ }^{1}$ ).- Tissues should remain in the formaldehyde solution from twenty-four to forty-eight hours. This is also especially useful for dissociating epithelial cells.

(c) Sodium or Potassium Hydrate (30- to 35-per-cent. aqueous solution).-From twenty minutes to an hour is usually sufficient to cause the tissue elements to fall apart or to be readily pulled apart with teasing needles. If it is at any time desirable to stop the action of the

${ }^{1}$ Commercial formalin is a 40 -per-cent. solution of formaldehyde gas in water. 
caustic alkali, this may be accomplished by neutralizing with glacial acetic acid or by replacing the alkali with a 6o-per-cent. aqueous solution of potassium acetate. The specimens may then be preserved in the potassium-acetate solution, in glycerin, or in 50-per-cent. alcohol. This dissociating fluid is largely used for muscle cells and fibres.

(d) Nitric acid (1o- to 20-per-cent. aqueous solution).-This is especially useful for dissociating involuntary and voluntary muscle.

After any of the above procedures, the macerating fluid containing the tissue elements should be placed in a long tube, allowed to stand for a time and the fluid decanted. Water is then poured into the tube, the tissues allowed to settle and the water poured off, this being repeated until all trace of macerating fluid is removed. The tissue elements may then be preserved or mounted in glycerin or in glycerin jelly. It is frequently advisable to stain the tissues. For this purpose alum-carmin (p. I9) is especially satisfactory. (For details see technic I, p. I 24 and technic 2, p. I24). After staining and washing, the tissues may be preserved or mounted in glycerin, eosin glycerin, or glycerin jelly. It frequently happens that on examining dissociated tissue elements after mounting, the bits of tissue are still too large. This may be remedied by gently tapping on the cover-glass with a lead pencil.

\section{PREPARATION OF SECTIONS}

\section{Fixation}

Even tissues, the structure of which admits of their being examined in the fresh condition, as described on p. 3, soon undergo post-mortem changes if placed in an inert medium such as normal salt solution or blood serum. As a result they soon lose their characteristic appearance and disintegrate. A proper killing or fixation is therefore the first step in the preparation of most tissues for microscopic study, the object being to so preserve the tissues that they retain as nearly as possible, and more or less permanently, the same structure and relation which they had during life. Fixed tissue may be placed in water, salt solution, alcohol, etc, in fact may be subjected to many and varied manipulations which would destroy fresh tissues, without disturbing the relation of the tissue elements. Fixation of such a thin film of tissuc as a blood smear may be accomplished by 
heating. A blood smear or even small pieces of tissue may be fixed by exposure to certain chemical vapors as, e.g., the vapor of formalin or of osmic acid. Fixation is, however, usually accomplished by means of chemicals in solution, the solution being known as a fixing agent or fixative. The tissue is immersed in the fixative and allowed to remain there until fixation is complete. The pieces of tissue should be small, and large quantities of the fixative should be used. It may be necessary to change the fluid a number of times, in order to keep it up to the proper strength. The length of time required depends upon the character of the tissue and upon the fixative used. In general it should be only long enough to bring about the desired result, as prolonged immersion in the fixative may make the tissue brittle or may interfere with the subsequent treatment of the tissue, especially staining.

Organs and even bodies may be fixed in toto by injecting the fixative through an artery and allowing it to escape through the veins. After the injection, the whole specimen should be placed in a large quantity of the same fixative. This method fills the entire vascular system including the capillaries with the fixative, thus bringing the latter into very prompt and close contact with the tissue elements. The result is a very rapid and accurate fixation, which is especially valuable where it is necessary to preserve the topographic relations of various parts of an organ or a body.

A mercuric chlorid solution (p. 8) followed immediately by strong alcohol makes a very good injection fixative. Satisfactory fixation is largely dependent upon the freshness of the tissue when placed in the fixative. The following are the fixatives in most common use:

(1) Strong Alcohol (96-per-cent.).-This is a rapid fixative and should be used on small pieces of tissue. The time required is from six to twenty-four hours, though tissues may remain longer without injury. The alcohol should be changed after two or three hours. This fixative should not be used where fine histological detail is desired, since it causes some shrinkage. One advantage in its use is the fact that tissues are hardened and ready for embedding at the end of fixation.

(2) Dilute Alcohol (30-per-cent. to 8o-per-cent.).-This, as a rule, gives unsatisfactory results, causing much shrinkage of the tissue elements.

(3) Formalin (2-per-cent. to Io-per-cent. aqueous solution).Formalin is rapid in its action and probably has better penetrating 
qualities than any other fixative. For general purposes a ro-per-cent. solution ( $\mathrm{I}$ part commercial formalin to 9 parts water) should be used. This fixes in from six to twenty-four hours. The results after formalin are not always good, owing to the fact that it has little hardening power, and the subsequent action of alcohol is likely to cause some distortion of the tissues. It acts better when combined with other fixatives than when used alone. (See Orth's fluid.)

(4) Miiller's Fluid.

Potassium bichromate,

Sodium sulphate,

Water,

$$
\begin{array}{r}
2.5 \mathrm{gm} . \\
\text { I. } 0 \mathrm{gm} . \\
\text { I00. } 0 \text { c.c. }
\end{array}
$$

This fluid gives rery good results, but is extremely slow in its action, requiring from a week to several months. Fairly large pieces of tissue may be fixed, but in all cases large quantities of the fixative should be used and frequently renewed.

(5) Orth's Fluid.

Müller's fluid (double strength),

Formalin, 8-per-cent.,

Equal parts.

This is one of the best general fixatives. Its action is similar to that of Müller's fluid but much more rapid, fixation being accomplished in from twenty-four to forty-eight hours, though specimens may remain in the fluid several days without disadvantage. Fairly large pieces of tissue may be fixed with good results. The fixative should be changed after a few hours. Fixation with Orth's fluid gives an excellent basis for a hæmatoxylin-eosin stain (see (I), p. 20.) The fixative should always be freshly prepared. It is convenient to keep the 8-per-cent. formalin solution and the double-strength Müller's fluid in stock. Orth's fluid is then prepared by simply taking equal parts of each.

(6) Osmic Acid.-This, in a I-per-cent. aqueous solution, is a quick and excellent fixative of poor penetrating power. Very small pieces of tissue must therefore be used. They should remain in the fluid from twelve to twenty-four hours. Osmic acid stains fat and myelin black and is consequently useful in demonstrating their presence in tissues. Fixation should take place in the dark.

(7) Flemming's Fluid.

Chromic acid, I-per-cent. aqueous solution,

25 c.c.

Osmic acid, I-per-cent. aqueous solution,

IO C.C.

Glacial acetic acid, I-per-cent, aqueous solution, ro c.c.

Water,

55 c.c. 
Flemming's fluid is one of the best fixatives for nuclear structures, and is of especial value in demonstrating mitotic figures. Very small pieces of tissue should be placed in the fixative, where they remain for from twenty-four hours to three days. The solution should be freshly made as required, or a stock solution without the osmic acid may be kept, and the latter added at the time of using.

(8) Mercuric Chlorid.-This may be used either in saturated aqueous solution or in saturated solution in 0.75 -per-cent. salt solution. Fixation is complete in from twelve to twenty-four hours, and is usually very satisfactory.

A saturated solution of mercuric chlorid in 5-per-cent. aqueous solution of glacial acetic acid also gives good results.

(9) Zenker's Fluid.

Potassium bichromate,

Sodium sulphate,

Mercuric chlorid,

Glacial acetic acid,

Water,

$$
\begin{array}{r}
2.5 \mathrm{gm} . \\
\text { I. } 0 \mathrm{gm} . \\
5.0 \mathrm{gm} . \\
5.0 \mathrm{c.c} . \\
\text { I00. } 0 \text { c.c. }
\end{array}
$$

This fluid should be freshly made, or the salts may be kept in solution and the acetic acid added at time of using.

Zenker's fluid is a good general fixative, but usually causes some shrinkage of the tissue elements. Fixation requires from six to twenty-four hours. The most serious drawback to Zenker's fluid is the fact that the mercuric chlorid sometimes produces dark, irregular precipitates in the tissues. This may be remedied, however, by the use of iodine and iodid of potassium in the hardening process (see Hardening, p. 9).

(Io) Picric acid is an excellent fixative for cytoplasm. It may be used in: (a) Saturated aqueous solution; (b) saturated solution of picric acid in I-per-cent. aqueous solution of acetic acid; $(c)$ saturated solution of picric acid in 2-per-cent. aqueous solution of sulphuric acid.

\section{Hardening}

Most fixatives are also hardening agents if their action is prolonged. This is, however, often detrimental. It is, therefore, customary, after fixation is complete, to carry the specimens, with or without washing, through successively stronger grades of alcohol for the purpose of hardening the tissues. For general histological 
purposes the specimens may be transferred directly to 70-per-cent. or 8o-per-cent. alcohol, which should be changed once or twice. In the case of delicate tissues the first grade of alcohcl should be 40-per-cent. or 50-per-cent., the second 70-per-cent., and the third 8o-per-cent. The specimens should remain in each grade from twelve to twenty-four hours.

Washing the tissues after fixation is not a matter of indifference. In some cases water should be used, while in other cases water is liable to undo the action of the fixative, in which cases alcohol must be used for washing.

After fixation in alcohol no washing, of course, is necessary. Specimens fixed in strong alcohol are embedded immediately (see Embedding, p. II), or preserved (see Preserving, p. 9). After fixation in dilute alcohol the specimens are passed through the graded alcohols up to 8o-per-cent.

After fixation in formalin solutions the specimens are passed directly through the graded alcohols without washing in water. Specimens fixed in any solution containing picric acid should not be washed in water, but passed directly through the alcohols; and it is usually necessary to change each grade in order to wash out the picric acid.

Specimens fixed in osmic acid or any solution containing osmic acid should be washed in running water before being passed through the graded alcohols. After solutions containing potassium bichromate the specimens should be washed in water sufficiently to remove the excess of bichromate, though too prolonged washing secms to be detrimental. A precipitate forms in the alcohols, but this apparently does no harm.

After mercuric chlorid or Zenker fixation the washing may be done either in water or in alcohol. To avoid precipitates in the tissues add a small quantity of an iodin solution (equal parts tincture iodin and ro-per-cent. aqueous solution potassium iodid) to any of the grarles of alcohol. As the alcohol becomes clear more of the solution is added until the alcohol remains slightly tinged.

\section{Preserving}

Hardened tissues are usually preserved in 8o-per-cent. alcohol. Formalin in aqueous solutions of I-per-cent. to ro-per-cent. is also used as a preservative. In either case, when it is necessary to pre- 
serve the specimens for a considerable length of time (several months or longer), the tissues are likely to lose their staining qualities to a certain extent. Preserving the specimens in equal parts of strong alcohol, glycerin, and distilled water is successful as a partial remedy for this.

\section{Decalcifying}

Tissues containing lime salts, like bones and teeth, must have the lime salts dissolved out before sections can be cut. This process is known as decalcification.

Tissues to be decalcified must be first fixed and hardened. Fixation in Orth's fluid and hardening in graded alcohols give good results. After hardening, the tissue is washed in water and placed in one of the following decalcifying fluids. The quantity of fluid should always be large and the fluid frequently changed. The completion of decalcification can be determined by passing a needle through the specim en by cutting it with a scalpel. The time required varies with the size and hardness of the specimen and the decalcifying fluid used. When decalcification is complete, the specimen is washed in sufficient changes of water to remove all trace of acid. This may be quickly accomplished by the addition of a little ammonium hydrate to the water. The specimen is then carried through graded alcohols.

(I) Hydrochloric Acid.-This may be used in aqueous solutions of from 0.5-per-cent. to 5-per-cent. A very satisfactory decalcifying mixture is that known as Ebner's hydrochloric-salt solution. It consists of:
Sodium chlorid, saturated aqueous solution,
I part.
Water,
2 parts.
Hydrochloric acid, sufficient to make a from 2-per-cent. to 5- per-cent. solution.

The addition of the salt prevents swelling of the tissue. This fluid is slow in acting and should be changed every day.

(2) Nitric Acid.-This is less used than the preceding. The strength should be from I-per-cent to Io-per-cent. aqueous solution. Weak solutions (I-per-cent. to 2-per-cent.) will decalcify small fœtal bones in from three to twelve days. For larger bones stronger solutions and longer time are required. Transferring from the nitric solution to a 5-per-cent. alum solution for twenty-four hours before washing will prevent swelling of the tissue. Phloroglucin is some- 
times added to the nitric acid solution for the purpose of protecting delicate tissues or of allowing stronger solutions of the acid to be used. One gram of phloroglucin is dissolved in to c.c. of nitric acid. To this are added soo c.c. of Io-per-cent. aqueous solution of nitric acid. Small pieces of tissue decalcify in a few hours. If less rapid action is desired, reduce the amount of nitric acid without changing the percentage of phloroglucin.

(3) Small bones may be satisfactorily decalcified in Zenker's fluid (see Fixatives, page 8), or in the following:

Picric acid,

Chromic acid,

Glacial acetic acid,
I part.

I part.

5 parts.

\section{Embedding}

Most hardened tissues are still not firm enough to be cut into the thin sections suitable for microscopic study. In order to support the tissue elements and render them more firm for section cutting, recourse is had to embedding. This consists in impregnating the tissues with some substance which is liquid when the tissues are placed in it, but which can be made to solidify throughout the tissues. In this way the tissue elements are held firmly in place. The embedding substances most used are celloidin and paraffin.

\section{Celloidin Emibeding}

(i) Alcohol-ether Celloidin.-Two solutions should be made. Solution No. 2. Thick celloidin-a 5-per-cent. solution of celloidin in equal parts 96-per-cent. alcohol and ether.

Solution No. I. Thin celloidin-made by diluting solution No. 2 with an equal volume of equal parts of alcohol and ether.

The hardened tissues are placed successively in:

Strong alcohol (96-per-cent.) twelve to twenty-four hours, to dehydrate.

Equal parts alcohol and ether, twelve to twenty-four hours.

Thin celloidin, twenty-four hours to several days.

Thick celloidin, twenty-four hours or longer.

The exact time tissues should remain in the different grades of celloidin depends upon the character of the tissue, the size of the specimen, and the thinness of section desired. Many tissues may be advantageously left for weeks in thin celloidin. 
The specimen must now be "blocked" and the celloidin hardened. By blocking is meant fastening the specimen impregnated with celloidin to a block of wood or other suitable material which may be clamped in the microtome (see Section Cutting, p. I4). The specimen may be taken from the thick celloidin (considerable of the latter adhering to the specimen), quickly pressed upon a block of wood or vulcanized fibre, allowed to harden five to ten minutes in air, and then immersed in 8o-per-cent. alcohol. The alcohol gives an even hardening of the celloidin, attaching the specimen firmly to the block. Another method, and one by which very even-shaped blocks may be obtained, is to place the specimen from the thick celloidin into a little paper box (made by folding paper over a wooden block), slightly larger than the specimen, and covering with thick celloidin. The celloidin should dry slowly under a bell-jar for from two to twelve hours, according to the amount of celloidin, after which it should be immersed in 8o-per-cent. alcohol and the paper pulled off. Such a block may be cut into any desired shape. It is attached to the wooden or vulcanized block by dipping for a moment in thick celloidin, and then pressing firmly down upon the block. After five to ten minutes' drying in air it is transferred to 8o-per-cent. alcohol.

Old, hard, celloidin-embedded specimens are sometimes difficult to attach to blocks. This usually may be accomplished by first thoroughly drying the specimen and then dipping it in equal parts alcohol and ether for a few minutes. This softens the surface of the celloidin, after which the specimen is dipped in thick celloidin and blocked. Embedded or blocked specimens can be kept in 8o-percent. alcohol. After several months, however, the celloidin is likely to become too soft for good section cutting. In that case the specimens can be readily re-embedded by dissolving out the old celloidin with alcohol and ether and putting them again through the regular embedding process.

(2) Clove-oil Celcoidin.-A more rapid impregnation of the tissue may be obtained by means of what is known as clove-cil celloidin.

Celloidin,

Clove oil, $30 \mathrm{gm}$.

Ether,

Alcohol, absolute,

I00 c.c.

400 c.c.

20 c.c.

The celloidin is first placed in a jar and the clove oil and ether added. From two to four days are required for solution of the celloidin. 
During this time the jar should be shaken several times. After the celloidin is dissolved the absolute alcohol is added and the solution is ready for use.

The specimen must be thoroughly dehydrated, placed in alcohol and ether or pure ether for a few hours, and then transferred to the clove-oil celloidin. From six to twelve hours is sufficient to impregnate small pieces of tissue. The tissue is now taken from the celloidin, placed directly upon a wooden or vulcanized block, and immersed in chloroform. The celloidin hardens in about an hour, and is then ready for sectioning. The specimen is very firm, and very thin sections can be cut.

A disadvantage in clove-oil celloidin is that neither the blocks nor the sections can be kept permanently in alcohol, as can those embedded in alcohol-ether celloidin. They may, however, be kept for several weeks in pure chloroform.

\section{Paraffin Embedding}

For paraffin embedding a thermostat or paraffin oven is necessary in order that a constant temperature may be maintained. The temperature should be about $56^{\circ} \mathrm{C}$. Pure paraffin, the melting-point of which is from $50^{\circ}$ to $55^{\circ} \mathrm{C}$., is used. In very warm weather it may be necessary to add to this a little paraffin, the melting-point of which is $62^{\circ} \mathrm{C}$.

The hardened tissue is first put in 96 -per-cent. alcohol for from twelve to twenty-four hours, and then completely dehydrated by putting in absolute alcohol for the same length of time, or less for small specimens. It is then transferred to some solvent of paraffin. Some of the solvents used are xylol, oil of cedarwood, chloroform, and toluol. Of these the best are perhaps xylol and oil of cedarwood. The tissue should remain in either of these for several hours, or until the tissue becomes more or less transparent. It is then placed in melted paraffin, in the paraffin oven, for from one to three hours, according to the size and density of the specimen. This allows the tissues to become impregnated with the melted paraffin. The paraffin should be changed twice.

In case of very delicate tissues it is well to transfer them from the absolute alcohol to a mixture of equal parts absolute alcohol and xylol for a short time before putting them into the pure xylol. In the same way a mixture of equal parts xylol and paraffin may be used before putting the tissues into pure paraffin. 
For hardening the paraffin in and around the tissue a very convenient apparatus consists of a plate of glass and several L-shaped pieces of iron or lead. Two of these are placed on the glass plate in such a manner as to enclose a space of the desired size. Into this are placed the specimen and sufficient melted paraffin to cover it. Both glass and irons should be smeared with glycerin to prevent the paraffin from adhering, and should be as cold as possible, so that the paraffin may harden quickly. The same paper boxes described under celloidin embedding may also be used for paraffin. Another good method for small pieces of tissue is to place the specimen in paraffin in an ordinary watch-glass which has been coated with glycerin. Both paper-box and watch-glass specimens are immersed in cold water as soon as the surface of the paraffin has become hard. After the paraffin has hardened any excess may be cut away with a knife.

Paraffin-embedded specimens may be kept indefinitely in air. For section cutting, the block of paraffin is attached to a block of wood or of vulcanite or to the metallic block-holder of the microtome. This is done by heating the block-holder, pressing the paraffin block firmly upon it, and then dipping the whole into cold water.

\section{Section Cutting}

The older method of making free-hand sections with a razor has been almost completely superseded by the use of a cutting instrument known as the microtome. This consists essentially of a clamp for holding the specimen and a microtome knife or razor. The two are so arranged that when knife and specimen meet, a section of any desired thickness may be cut.

The technic of section cutting differs according to whether the specimen is embedded in celloidin or in paraffin.

In cutting celloidin sections the knife is so adjusted that it passes obliquely through the specimen, as much as possible of the cutting edge being used. The knife is kept flooded with 8o-per-cent. alcohol and the specimens are removed by means of a camel's-hair brush to a dish of 8o-per-cent. alcohol, where they may be kept for some time if desired. When celloidin sections tear or when very thin sections are desired it is often of advantage to paint the surface of the block, after cutting each section, with a coat of very thin celloidin.

Celloidin sections are usually not thinner than Io $\mu$, although 
under farorable conditions sections $5 \mu^{1}$ or even $3 \mu$ in thickness may be obtained.

In cutting paraffin sections the knife is kept dry and is passed not obliquely but straight through the specimen, only a small part of the cutting edge being used. An exception is made in the case of very large paraffin sections, where an oblique knife is used. Sections are removed from the knife by a dry or slightly moistened brush. If not desired for immediate use the sections may be conveniently kept for a short time on a piece of smooth paper. If sections curl they may be flattened by floating on warm 3o-per-cent. alcohol or on warm water.

Paraffin sections may be so cut that the edges of the sections adhere. Long series or "ribbons" of sections may thus be secured. This is of decided advantage when serial sections are desired. Failure of the secticns to cut evenly or to adhere in ribbons is usually due to the paraffin being too hard and brittle, which of course is due to its low temperature. If much section cutting is to be done, the operator will find himself amply repaid by having the room-temperature fairly high. In case paraffin of a melting-point of $50^{\circ}$ to $55^{\circ} \mathrm{C}$. is used, a room temperature of $73^{\circ}$ to $75^{\circ} \mathrm{F}$. will greatly facilitate the work. Where it is not possible to have a high room temperature, recourse may be had to coating the surface of the block with a paraffin of lower melting-point than that used for the embedding. A similar effect may be obtained by holding a heated metal plate or bar near the block until the paraffin is slightly softened. This process may be repeated as often as necessary.

In addition to the fact that ribbon series can be cut in paraffin, this embedding substance also has the advantage over celloidin that thinner sections can be obtained. On the other hand, celloidin embedding produces less shrinkage of the tissues than paraffin embedding with the accompanying heat.

Frozen Sections.-This method, while more used in pathology where rapid diagnosis is more often important, is still sometimes extremely useful to the histologist, especially as an easy and quick method of testing material. Perfectly fresh tissues may be used or tissues which have been previously fixed. Fixed tissues must have the fixative thoroughly removed before freezing. Of fixatives roper-cent. formalin is probably the best and the tissue should remain in

$1 \mu=$ micromillimeter or micron $={ }_{1}^{1}(60)$ of a millimeter $=$ microscopic unit of measure = abrut zastoo of an inch. 
the fixative from one to two hours. If time is important the tissue may be boiled for two or three minutes in the formalin solution.

Ether, rhigolene and carbon dioxid are the most common freezing agents. A special freezing microtome and knife may be used, or a freezing attachment may be used with the ordinary microtome. In ether freezing the ether is vaporized by means of compressed air and the vapor carried to the under side of a metallic plate upon which the block of tissue is placed. In carbon dioxid freezing, which has now largely superseded ether freezing on account of convenience and economy, the commercial cylinder of gas such as is used for charging soda fountains is used. As the liquid carbon dioxid and not the gas is required for freezing, the cylinder should be hung valve end down, with the valve about the level of the microtome. The carbon dioxid is carried to the metallic disc of the microtome by means of a stout rubber tube and it is advisable to screw a cap. with a small hole in it over the valve and to attach a longer handle to the valve leverin order to more perfectly control the flow. The piece of tissue to be frozen should not be over $5 \mathrm{~mm}$. thick and the freezing should be done slowly. A proper hardness of the tissue is important and is of course determined by the amount of carbon dioxid admitted.

As the frozen section is not supported by any embedding mass, special handling is required, the section being attached to a slide or cover-glass before staining. For this purpose either egg albumen or celloidin may be used.

Egg albumen,

Distilled water,

Saturated solution sodium salicylate (made

slightly alkaline with lithium carbonate)

50 c.c.

I 5 O c.c.

(or sufficient to completely dissolve the albumen).

Sections, from material previously fixed are transferred directly from the microtome to the albumen solution. Sections from fresh material are placed for three to five minutes in 5-to ro-per-cent. formalin solution before being transferred to the albumen solution.

From the albumen solution sections are floated on a slide or cover-glass, the excess of solution drained off and the section firmly blotted with several thicknesses of washed cheese-cloth. The slide or cover-glass is next immersed in alcohol or in equal parts alcohol and ether to coagulate the albumen, thus fixing the section to the glass. The section may now be stained and mounted in the usual way 
In the celloidin method the section is transferred from the microtome to water, floated upon a slide, blotted with filter paper, flooded with absolute alcohol and drained. Before the alcohol dries the slide is flooded with a very thin celloidin solution which is immediately drained off. The celloidin is then allowed to harden a moment and the slide immersed in water. The section is now fixed to the slide and may be stained and mounted as usual.

\section{Staining}

This is for the purpose of more readily distinguishing the different tissue elements from one another by their reactions to certain dyes.

Based upon their action upon the different tissue elements, stains may be classified as ( $\mathrm{x}$ ) nuclear dyes, which stain nuclear structures; and (2) plasma dyes, which stain the cell body or cytoplasm. Plasma dyes, also, as a rule, stain the intercellular tissue elements, and are therefore known as diffuse stains.

The dyes most frequently used for staining tissues are:

I. Nuclear dyes: (a) Hæmatoxylin and its active principle, hæmatein; (b) carmine and its active principle, carminic acid; $(c)$ basic aniline dyes.

II. Plasma dyes: (a) Eosin; (b) neutral carmine, (c) picric acid; (d) acid aniline dyes.

\section{Nuclear Dyes.- (a) HжMatoxylin.}

x. Gage's Hamatoxylin.

Ammonia or potash alum, Distilled water,

Boil for Io minutes to sterilize; cool and add the following solution:

Hæmatoxylin crystals,

O. I gm.

Alcohol 95-per-cent., IO. O c.C.

Four grams chloral hydrate are then added to the mixture to prevent growth of germs.

This dye may be used in full strength or diluted witl alum water. It stains in from two to five minutes.

2. Delafield's Hamatoxylin.

Hamatoxylin crystals,

I $\mathrm{gm}$.

Alcohol,

6 c.c.

Ammonia alum, saturated aqucous solution,

IOO C.C. 
The hæmatoxylin should be first dissolved in the alcohol and then added to the alum solution. The mixture should next be allowed to stand in the light for from seven to ten days to ripen. It is then filtered, and to the filtrate are added:

Glycerin, 25 c.c.

Wood naphtha, 25 c.c.

The mixture is again allowed to stand for from two to four days and filtered. It may be used full strength or diluted with equal parts of water. It stains in from two to five minutes.

3. Heidenhain's Hamatoxylin.

Hæmatoxylin crystals, I $\mathrm{gm}$.

Water, IOO c.c.

Sections are first placed for from four to eight hours in a 2.5-per-cent. aqueous solution of ammonium sulphate of iron. They are then washed in water and transferred to the hæmatoxylin solution until they are intensely blue or black (usually several hours). The sections are now washed in water and differentiated by again placing in the iron solution till they have a light grayish color. After this they are thoroughly washed in water.

4. Mayer's Hamalum.

Hæmatein,

Alcohol,

Ammonia alum, 5-per-cent. aqueous solution,
I gm.

50 c.c.

I,, 000 c.C.

The hæmatein is first dissolved in the alcohol, after which the alum is added. This dye does not require any ripening, and is thus available for immediate use. It is a rapid nuclear dye usually requiring not more than from three to five minutes.

5. A combination of Gage's and Mayer's formulæ makes a very satisfactory nuclear dye.

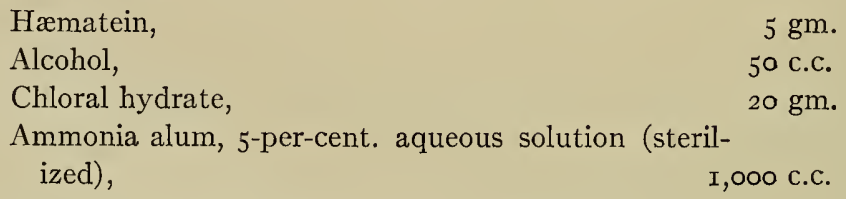

The hæmatein is first dissolved in the alcohol and then added with the chloral hydrate to the alum solution. This solution is used in full strength and stains in from three to five minutes. 
6. Weigert's Hamatoxylin.

Two stock solutions shculd be made up as follows:

A. I-per-cent. hæmatoxylin, in 96-per-cent. alcohol.

B. Hydrochloric acid (sp. gr. I.I 26),

Ferric chloride, 30-per-cent.,

Distilled water,

IO C.C.

40 c.c.

950 c.c.

For use, mix equal parts of $A$ and B. The mixture will keep two or three days. This is a rapid stain usually requiring not more than a minute. A more brilliant nuclear stain may be obtained by overstaining and then decolorizing. After the stain wash the sections in water and then decolcrize to the pioper degree in weakly acidulated rater. To stcp the action of the acid the sections should be dipped in water made slightly alkaline with ammonia. This is an excellent stain and gives brilliant results. It is especially good in cases where the material has become old and lost its affinity for ordinary hæmatoxylin stains.

(b) ALUMI-CARMINE.

Carmine,

$2 \mathrm{gm}$.

Alum,

Carbolic acid, .

$5 \mathrm{gm}$.

Water,

$2 \mathrm{gm}$.

I00 c.c.

The alum is first dissolved in the I00 c.c. of warm distilled water, after which the carmine is added. This mixture is then boiled for twenty minutes, allowed to cool, and filtered. The carbolic acid is then added. This is a slow-acting pure nuclear dye.

(c) BAsrc Aniline Dyes-gentian violet, methyl violet, methyl green, methyl blue, toluidin blue, fuchsin, thionin, safranin, etc.

These are best kept in stock in saturated alcoholic solutions. When desired to use, a few drops of the alcoholic solution are added to distilled water. No rule as to exact proportions can be given, as these depend upon the material, the fixation, and the intensity of stain desired.

II. Plasma Dyes.-(a) Eosin.

This is prepared as follows: Water-seluble eosin is dissolved in water to saturation. It is then precipitated by hydrechloric acid and the precipitate washed with water upon a filter until the filtrate is tinged with cosin. After drying, the precipitate is dissolved in strong alcohol, I gm. of cosin to $\mathrm{I}, 500$ c.c. of alcohcl, or watersoluble eosin may be kept in saturated aqueous solution containing 
a trace of thymol as a preservative. This may be diluted to any desired strength at time of using. Eosin is a rapid plasma stain.

(b) Neutral Carmine.

Carmine,

I gm.

Liquor ammonii caustici,

5 c.c.

Distilled water, $5 \circ$ c.c.

The last two ingredients are first mixed, and the carmine then added. This solution is allowed to remain in an open vessel for about three days, or until the odor of ammonia has disappeared, after which it is filtered.

(c) PICRIC ACID-used mainly as the plasma-staining element of such a staining mixture as picro-acid-fuchsin.

(d) Acid Aniline Dyes.-Of these, acid fuchsin, erythrosin, and orange $\mathrm{G}$ are most used. They may be prepared and kept in stock in the same manner as the basic aniline dyes (see above). Erythrosin is of especial value for sections which take the eosin stain poorly.

\section{Staining Sections}

It is often of advantage to stain the different tissue elements different colors. This may be accomplished either by staining successively with several dyes, or by a single staining with a mixture of dyes. The following are the methods in most common use:

(i) Staining Double with Hamatoxylin and Eosin.-Sections are first washed in water. They are then stained with hæmatoxylin (solutions I, 2, 4, 5, or 6, pp. I 7-I9) from one to five minutes. After being thoroughly washed in water, they are dehydrated in strong alcohol and transferred to the alcoholic eosin solution (page I9). Most sections stain in from two to five minutes. By this method nuclei are stained blue or purple, cell bodies and intercellular substances red.

Very often a more brilliant staining may be accomplished as follows: Overstain in hæmatoxylin and wash thoroughly in water; decolorize in water slightly acidulated (8 or ro drops of hydrochloric acid to Ioo c.c. of water) until only the nuclei retain the stain; wash in water which has been made slightly alkaline with ammonium hydrate; then stain with eosin as usual.

(2) Staining with Picro-Acid-fuchsin.

Acid-fuchsin, I-per-cent. aqueous solution,

Picric acid, saturated aqueous solution,

IOO C.C. 
This solution usually stains in from one to three minutes. Occasionally a longer staining is required. Cell bodies including muscle cells and fibres are stained yellow by the picric acid, connectivetissue fibres red by the fuchsin. After staining, the sectionsare washed in distilled water.

(3) Triple Staining with Hæamatoxylin and Picro-acidFUCHSIN.-This is the same as the preceding except that before staining with picro-acid-fuchsin, the sections are overstained in hæmatoxylin (solutions $\mathrm{I}, 2,4,5$, or 6 , pp. I7-rg). The usual purple of hæmatoxylin-stained nuclei is changed to brown by the action of the picric acid. Care should be taken that the sections do not remain too long in the picro-acid-fuchsin, or the hæmatoxylin may be completely removed. After staining, sections are washed in distilled water and transferred to 96-per-cent. alcohol.

If sections overstain with fuchsin, the staining solution may be diluted with water; if sections are understained with fuchsin, more fuchsin may be added. If the picric-acid stain is not sufficiently intense, the 96-per-cent. alcohol should be tinged with picric acid.

(4) Staining with Picro-Carmine.

Ammonium carminate,

Distilled water,

Picric acid, saturated aqueous solution,
I gm.

35 C.c.

I 5 C.C.

The ammonium carminate is first dissolved in the water, after which the saturated aqueous solution of picric acid is added with constant stirring. The mixture is then allowed to stand in an open vessel for two days, when it is filtered. This fluid stains nuclei and connective tissue red, cell protoplasm yellow.

\section{StAINING IN BULK}

By this is meant the staining of blocks of tissue before cutting into sections. The method is much less used than formerly. It is slower than section staining and more difficult to control. Blocks of the hardened tissue are transferred to the stain from water or alcohol according to the solvent of the stain. Alum-carmine and boraxcarmine are the most used general bulk stains.

(i) Alum-Carmine.

Carmine,

Ammonis alum, 4-per-cent. aqueous solution, 
After mixing the ingredients, the solution should be boiled fifteen minutes, and after cooling, enough sterile water added to replace that lost by evaporation. The time required for staining depends upon the size of the specimen. There is, however, little danger of overstaining. After washing out the excess of stain with water the specimen is dehydrated and embedded in the usual way.

(2) Borax-carmine, Alcoholic Solution.

Carmine, $3 \mathrm{gm}$.

Borax,

$4 \mathrm{gm}$.

Water, 93 c.c.

After mixing the above, add roo c.c. 7o-per-cent alcohol, allow the mixture to settle, then filter.

About twenty-four hours is required to stain blocks $0.5 \mathrm{~cm}$. in diameter. Larger blocks require longer staining.

\section{Mounting}

It is usually desirable to make permanent preparations or "mounts" of the stained specimens.

The most satisfactory media for mounting specimens are glycerin and Canada balsam.

(I) GLycerin.-Sections may be transferred to glycerin from either water or alcohol. In the case of double-stained specimenshæmatoxylin-eosin - the glycerin should be tinged with eosin, as the pure glycerin abstracts the eosin from the tissues. In many cases satisfactory eosin staining may be obtained by simply placing the hæmatoxylin-stained specimens in glycerin strongly tinged with eosin (eosin-glycerin). The specimen in a drop of glycerin is transferred to the glass mounting slide, the excess of glycerin removed with filter paper or with a pipette, and a cover-glass put on.

Glycerin mounts, as a general rule, are unsatisfactory. Furthermore, they must be cemented to exclude the air. This can be done by painting a ring of gold-size, or a thick solution of gum shellac in alcohol to which a little castor oil has been added, around and over the edge of the cover-glass. Both cover-glass and slide must be cleaned free from glycerin before the cement is applied. A camel'shair brush moistened with alcohol is the best means of removing the excess of glycerin.

Glycerin jelly is a more satisfactory mounting medium than pure glycerin. It can be purchased from firms dealing in microscopical 
supplies and needs merely the application of heat to make it fluid. Specimens can then be mounted in it in the usual manner, and after being allowed to cool, do not require cementing.

(2) BALSAM.-This is the most satisfactory general mounting medium. It has an advantage over glycerin in drying down perfectly hard and thus needing no cement, and in preserving colors more permanently. Its disadvantage is that its refractive index is so high that it sometimes obscures the finer details of structure, especially of unstained or slightly stained specimens.

Specially prepared Canada balsam is dissolved either in xylol or in oil of cedar, the solution being made of any desired consistence. Xylol-balsam dries much more quickly than does the oil-of-cedar balsam.

Preparatory to mounting in balsam, stained sections must be thoroughly dehydrated and then passed through some medium which is miscible with both alcohol and balsam. This medium, which at the same time renders the section transparent, is known as a clearing medium. For celloidin specimens the most satisfactory are:

(I) Oil of origanum Cretici.

(2) Carbol-xylol (xylol, Ioo c.c., carbolic-acid crystals, 22 gm.), followed by pure xylol.

(3) Xylol and cajeput oil, equal parts, followed by pure xylol. After clearing, the section is transferred by means of a sectionlifter to a glass mounting slide. In case oil of origanum is used, it is then blotted firmly with filter paper to remove the excess of oil. Care must be taken to have the filter paper several layers thick in order that the oil may be completely removed. The specimen should also be blotted firmly, giving the oil time to soak into the paper. These two precautions are necessary to prevent the section adhering to the paper instead of to the slide. After blotting, a drop of balsam is placed upon the centre of the specimen and a cover-glass put on.

When xylol is used, blotting is not necessary. Drain off the excess of oil, put a drop of balsam on the specimen and put on a cover-g'ass.

Paraffin Sections.--The technic of staining and mounting paraffin sections differs from that of celloidin sections. This is due mainly to the fact that while cellodin is transparent and may remain permanently in the specimen, paraffin is opaque and must be dissolved out before the section is fit for microscopic study. 
Bulk staining with carmine (page $2 \mathrm{I}$ ) is frequently used for specimens which are to be embedded in paraffin. Sections may be counterstained if desired.

The following are the steps to be followed in staining and mounting paraffin sections:

I. To attach sections to slide:

Place a drop of egg albumen (equal parts white of egg and glycerin to which a little carbolic acid may be added for preserving) on a slide, and spread it out thin with the finger.

Place a few drops of distilled water on the slide.

Float sections on the water.

Warm gently to allow sections to flatten-must not melt paraffin.

Pour off excess of water, holding the ends of the ribbons to prevent them floating off.

Stand slides on end a few hours to allow water to evaporate.

2. To remove paraffin:

Place slide in xylol three to five minutes.

3. To stain sections:

Place slide in fresh xylol three minutes.

Transfer to absolute alcohol.

Transfer to 9 c-per-cent. alcohol.

Transfer to 8o-per-cent. alcohol.

Transfer to 50-per-cent. alcohol. (May be omitted.)

Transfer to water.

Stain with an aqueous stain.

Wash in water.

Transfer to 50-per-cent. alcohol. (May be omitted.)

Transfer to 8o-per-cent. alcohol.

Transfer to 9o-per-cent. alcohol.

Transfer to absolute alcohol.

Transfer to xylol.

Transfer to fresh xylol.

Mount in xylol-balsam.

If an alcohol stain is used instead of an aqueous one, the carrying down and up through the graded alcohols is omitted.

If it is desired to stain double with eosin-hæmatoxylin (page 20) use the above technic in staining with hæmatoxylin; then the alcoholic eosin stain before final transfer to absolute alcohol. 


\section{Injection}

For the study of the distribution of the blood-vessels in tissues and organs, it is often necessary to make use of sections in which the blood-vessels have been injected with some transparent coloring matter. The injecting fluid most commonly used is a solution colored gelatin.

The gelatin solution is prepared by soaking I part gelatin in from 5 to ro parts water-the proportion depending upon the consistence desired-and when soft, melting on a water-bath.

Various dyes are used for coloring the gelatin, the most common being Prussian blue and carmine.

Prussian Blue gelatin is prepared by adding saturated aqueous solution Prussian blue to the gelatin solution, the proportions depending upon the depth of color desired. Both solutions should be at a temperature of $60^{\circ} \mathrm{C}$. After thoroughly mixing, the blue gelatin is filtered through cloth.

CARMINE GELATIN is prepared by first dissolving I gm. carmine in 30 c.c. distilled water. To this is added ammonia until the mixture becomes a dark cherry red. A ro-per-cent. aqueous solution of acetic acid is next added, drop by drop, with constant stirring until the mixture becomes neutral. The carmine and gelatin solutions both being at about $60^{\circ} \mathrm{C}$., are now mixed in the desired proportions. If the carmine injection mass is alkaline, it diffuses through the walls of the vessels; if acid, there is a precipitation of the carmine which may interfere with its free passage through the capillaries. If, however, the alkaline carmine and gelatin be first mixed, and the ro-per-cent. acetic acid solution be then added as directed above, the precipitated granules are so fine, even with an acid reaction, that they readily pass through the capillaries. The precipitation of the carmine in the shape of coarser granules is of advantage when it is desired to have an injection mass which will fill the arteries or veins only, without passing over into the capillaries.

The injecting apparalus consists of a vessel which contains the injection mass, and some means of keeping the latter under a constant but easily varied pressure. With the vessel is connected a tube ending in a cannula, through which the injection is made.

A very simple apparatus consists of a shelf which can be raised and lowered, and upon which the vessel stands. The tube connecting with the cannula may be attached to a faucet in the vessel or to 
a bent glass tube which passes into the top of the vessel and acts on the principle of a siphon.

In a somewhat more elaborate apparatus the injection mass is placed in a closed vessel, and this is connected with a second vessel containing air compressed by means of an air pump.

Accurate regulation of the pressure may be obtained by connecting the injection vessel with a manometer.

If the injection is to occupy considerable time, a hot-water bath in which the gelatin may be kept at an even temperature is also necessary.

Whole animals or separate organs may be injected. For injecting a whole animal, the animal, which is usually a small one such as a guinea-pig, rat, mouse, or frog, is chloroformed, the tip of the heart is cut away and a cannula is inserted through the heart into the aorta. This is first connected with a tube leading to a bottle containing warm normal saline solution. Pressure is obtained in the same manner as above described for the injection mass. By this means the entire arterial and venous systems are thorcughly washed out until the return flow from the vena cava is perfectly clear. The cannula is next connected with the tube from the vessel containing the injection mass, the pressure being only sufficient to keep the liquid flowing. When the injection mass flows easily and freely from the vena cava, the vessel is tied and the pressure is increased slightly and continued until the color of the injection mass shows clearly in the superficial capillaries. The aorta is now tied and the animal immersed in cold water to solidify the gelatin. After the gelatin becomes hard, the desired organs are removed and fixed and hardened in the usual way. Sections of injected material are usually cut rather thick, that the vessels may be traced the greater distance.

Better results are frequently obtained by injecting separate organs. This is accomplished by injecting through the main artery of the organ (e.g., the lungs through the pulmonary, the kidney through the renal). The injection is best done with the organ in situ, although it may be accomplished after the organ has been removed. The method is the same as given above for injecting an animal in toto.

The so-called double injection by means of which an attempt is made to fill the arteries with an injection mass of one color (red), while the veins are filled with an injection mass of another color (blue), often gives pretty, but usually inaccurate pictures, it being, as a rule, impossible to confine each injection mass to one system. 
Double injection is accomplished by first washing out the vessels with normal saline and then connecting the artery with the red gelatin, the rein with the blue gelatin, and injecting both at the same time, the pressure driving the saline out of the vessels into the tissues. The difficulty is that either the arterial injection carries over into the veins, or the venous injection carries over into the arteries. A somewhat more accurate method is first to inject the veins with an injection mass in which the coloring matter is in the form of granules too large to pass through the capillaries, and then to inject the arteries and capillaries in the usual manner. This method is especially useful in demonstrating the vessels of the kidney, liver, and gastrointestinal canal. 


\section{CHAPTER II}

\section{SPECIAL STAINING METHODS}

OF these only the more common will be described.

(i) Silver-nitrate Method of Staining Intercellular SubSTANCE.-After first washing, the tissue, e.g., omentum or cornea, is placed in a from 0.2- to I-per-cent. solution of silver nitrate, or better, protargol, where it is kept in the dark for a half-hour or more according to the thickness and density of the tissue. The specimen is then washed in water, transferred to 40-per-cent. alcohol and placed in the direct sunlight until it assumes a light brown color. It is then placed in fresh 8o-per-cent. alcohol for preservation.

(2) CHLORID OF GOLD in I-per-cent. aqueous solution is used in the same manner for demonstrating connective-tissue cells and their finer processes.

(3) Weigert's Elastic-tissue Stain.-This is prepared as follows:

Fuchsin,
Resorcin,
Water,

$$
\begin{aligned}
& 2 \mathrm{gm} . \\
& 4 \mathrm{gm} . \\
& 200 \mathrm{c.c} \text {. }
\end{aligned}
$$

These are boiled for five minutes, during which 25 c.c. of liquor ferri sesquichlorati are stirred in. The result is a precipitate which should be filtered out after the liquid has become cool. After drying, 200 c.c. of 95-per-cent. alcohol are added to the filtrate and boiled until the latter dissolves. Lastly, 4 c.c. of hydric chlorid are added to the solution. Sections should remain in the stain thirty minutes, after which they are washed in alcohol until the stain ceases to be given off.

(4) Verhoeff's Differential Elastic Tissue Stain.Hæmatoxylin crystals (Grubler), Absolute alcohol,

○. 5 gm.

25.00 C.C.

Dissolve by heating, then add 5-per-cent. aqueous solution ammonium hydrate, I drop. Allow to stand 5 minutes or longer, then add Lugol's solution (iodin 2 parts, potassium, iodid 4 parts), 22.00 c.C.

Filter, let stand in corked bottle 24 hours. 
In using this stain add to each cubic centimeter required of the above solution I drop of a 7 -per-cent. solution of ferric chlorid in abșolute alcohol.

Sections are carried from alcohol into the staining fluid where they remain one to three hours. The stain should be kept covered to prevent evaporation. Sections are next washed in water one or two minutes and examined under the microscope. If further differentiation is desirable, place a few seconds in I-per-cent. aqueous solution ferric chlorid. Wash in water. Counterstain in 0.2-per-cent. eosin, dehydrate in alcohol, clear and mount in balsam.

Elastic tissue is stained black, nuclei take hæmatoxylin, while other connective tissues, myelin and neuroglia stain with eosin. Fixation by Zenker's fluid gives perhaps the best results although the stain may be used after other fixations. Water in the staining fluid causes precipitates. It is therefore important to see that all dishes used are perfectly dry or washed with alcohol before using. Too much ferric chlorid gives precipitates and overstaining, too little results in failure of the elastic fibres to stain.

(5) Golgi's Chrome-silver Method for Demonstrating SeCretory Tubules.-Small pieces of perfectly fresh tissue, e.g., liver, are placed in the following:

Potassium bichromate, 4-per-cent. aqueous solution, 4 vols.

Osmic acid, I-per-cent. aqueous solution, I vol.

After three days they are transferred without washing to a 0.75 -percent. aqueous solution of silver nitrate, which should be changed as soon as a precipitate forms. The specimens remain in the second silver solution from two to three days, after which they are rapidly dehydrated, embedded in celloidin, and cut into rather thick sections.

(6) Mallory's Phosphomolybdic Acid Hematoxylin Stain for Connective Tissue.- Thin sections are placed for from two to ten minutes in a ro-per-cent. aqueous solution of phosphomolybdic acid. They are then washed in distilled water and transferred to:

$\begin{array}{lr}\text { Phosphomolyldic acid, ro-per-cent. aqueous solution, } 100.0 \mathrm{c.c} . \\ \text { Distilled water, } & 200.0 \mathrm{c.c} . \\ \text { Hamatoxylin crystals, } & \text { I.75 gm. } \\ \text { Carbolic-acid crystals, } & 5.00 \mathrm{gm} .\end{array}$

The phosphomolybdic acid and water are first mixed, after which the hamatoxylin and carbolic acid are added.

After staining from ten to twenty minutes the sections are washed 
in distilled water, placed for five minutes in 50-per-cent. alcohol, then in strong alcohol, cleared in xylol and mounted in xylol-balsam. This stain works best after Zenker's fluid fixation.

(7) Mallory's Phosphotungstic Acid Hæmatoxylin Stain for Connective Tissue.

Hæmatoxylin (or hæmatein ammonium), $\quad$ ○.I gm. Water, 8o. c.c.

Phosphotungstic acid (Merck), Io-per-cent. aqueous solution, 2o. c.c.

The hæmatoxylin should be dissolved in a little water with the aid of heat and, when cool, added to the rest of the solution. Allow to ripen for several weeks or months. Or, if it is desired for immediate use, the solution can be ripened by the addition of ro c.c. of a I/4-per-cent. solution of potassium permanganate.

Tissues should be fixed in Zenker's fluid (p. 8).

I. Treat sections with iodin solution to remove mercury precipitate, 5 to ro minutes (p. 9.)

2. Several changes 95-per-cent. alcohol.

3. Water.

4. One-fourth-per-cent. aqueous solution potassium permanganate, 5 to ro minutes.

5. Wash in water.

6. Five-per-cent. aqueous solution oxalic acid, 5 to to minutes.

7. Wash thoroughly in water.

8. Phosphotungstic acid_hæmatoxylin solution, twelve to twentyfour hours.

9. Dip for a few seconds in 95-per-cent. alcohol.

Io. Clear in carbol-xylol and xylol and mount in xylol-damar.

(8) Mallory's Aniline Blue Stain for Connective TissuE.-Tissues should be fixed in Zenker's fluid (p. 8).

I. Stain thin sections in a 0.2 -per-cent. aqueous solution of acid fuchsin for five to ten minutes. (This step may be omitted.)

2. Stain in the following solution for twenty minutes:

Aniline blue (soluble in water),

Orange G.,

Phosphomolybdic acid, I-per-cent. aqueous solution,

3. Decolorize in several changes of 95-per-cent. alcohol.

4. Clear in carbol-xylol and xylol and mount in xylol-damar. 
(9) Maresh's Modification of Bielschowskys' Stain for the Finer Connective Tissue Fibrils.

I. Very thin paraffin sections are fixed to the slide and placed for twelve to twenty-four hours in a 2-per-cent. silver nitrate solution.

2. For fifteen to thirty minutes in freshly prepared alkaline silver solution (20 c.c. of 2 -per-cent. silver nitrate solution, to which have been added 3 drops of 40-per-cent. caustic soda, and the precipitate redissolved by adding ammonia drop by drop while stirring).

3. Rinsed quickly in distilled water and placed in 20-per-cent. formalin for three minutes or until black.

4. Washed in distilled water and transferred for ten minutes to acid gold bath (Io c.c. distilled water to which have been added 2 or 3 drops of I-per-cent. gold chlorid and 2 or 3 drops acid).

5. Placed one-half to one minute in 5-per-cent. hyposulphite of soda to remove all unreduced silver.

6. Washed in distilled water, dehydrated, cleared in xylol and mounted in balsam.

(io) Osmic-Acid Stain for Fat.-For this purpose osmic acid is used in a I-per-cent. aqueous solution. The method is especially useful for demonstrating developing fat, fatty secretions (mammary gland), and fat absorption (small intestine). Very small bits of the tissue are placed in the osmic-acid solution for from twelve to twentyfour hours. They are then hardened in graded alcohols, embedded in celloidin, and the sections mounted in glycerin.

(i I) Jenner's Blood Stain.

Water-soluble eosin (Grübler), I-per-cent. aqueous solution, IOO C.C.

Methylene blue-pure (Grübler), I-per-cent. aqueous solution, IOO c.c.

Mix, and after standing 24 hours, filter. The filtrate is dried at $65^{\circ} \mathrm{C}$., washed, again dried and powdered.

To make the staining solution, $0.5 \mathrm{gm}$. of the powder is dissolved in roo c.c. pure methyl alcohol. Blood smears stain in from two to five minutes. They are then washed in water, dried, and mounted in balsam.

This solution acts as a fixative as well as a stain. 


\section{CHAPTER III}

\section{SPECIAL NEUROLOGICAL STAINING METHODS}

\section{Weigert's Method of Staining Medullated Nerve Fibres}

IN preparing material for the Weigert method, two points are to be kept in mind: Ist, proper fixation and preservation of the myelin sheaths; $2 \mathrm{~d}$, treatment (mordanting) with a reagent which enters into combination with the myelin, the result being that the myelin sheaths stain specifically with hæmatoxylin. Formalin fulfils the first requirement, the bichromates the first and second. Consequently the material may be fixed and hardened in bichromate, and, if not to be used immediately, is best kept in formalin to avoid overhardening. Or the material may be fixed and kept in formalin and impregnated with the bichromate before using, the latter being done before dehydrating in alcohol. Further mordanting, which is usually done, especially when the material has been kept for some time in formalin or alcohol, is for the purpose of intensifying the stain.

Material is fixed in one of the following fluids:

(a) Müller's fluid (page 7).

(b) Potassium bichromate, 5-per-cent. aqueous solution.

(c) Formalin, Io-per-cent. aqueous solution.

(d) Formalin, I volume; potassium bichromate, 5-per-cent. aqueous solution, 9 volumes.

In Müller's fluid or in plain potassium-bichromate solution a hardening of two days to four weeks is required; in formalin or formalin-bichromate from a week to ten days is sufficient. All material is better kept until used in 5-per-cent. to Io-per-cent. formalin solution than in alcohol. The specimens are then hardened in graded alcohols, embedded in celloidin, and sections cut in the usual way. Material fixed in formalin should be placed for several days in the following:

Chrome alum,

I gm.

Potassium bichromate,

$3 \mathrm{gm}$.

Water, roo c.c.

before hardening in alcohol. 
Sections from material fixed in any of the chrome-salt solutions are placed for from twelve to twenty-four hours in a saturated aqueous solution of neutral cupric acetate diluted with an equal volume of water. The cupric acetate forms some combination with the tissues which intensifies their staining qualities, thus acting like the chrome salts as a mordant.

After mordanting, the sections are washed in water and transferred to the following staining fluid:

Hæmatoxylin crystals,

Alcohol, 95-per-cent.,

Lithium carbonate-saturated aqueous solution,

Water,
I gm.

IO c.c.

I c.c.

90 c.c.

This solution must either be freshly made before using, the hæmatoxylin being dissolved first in the alcohol, or the hæmatoxylin may be kept in ro-per-cent. alcoholic solution, the lithium carbonate in saturated aqueous solution, and the staining fluid made from these as needed.

Sections remain in the hæmatoxylin solution from two to twentyfour hours, the longer time being required for staining the finer fibres of the cerebral and cerebellar cortices. They are then washed in water and decolorized in the following:

Potassium ferricyanid,

Sodium biborate,

Water,
$2.5 \mathrm{gm}$.

$2.0 \mathrm{gm}$.

300.0 c.c.

While in the decolorizer, sections should be gently shaken or moved about with a glass rod to insure equal decolorization. In the decolorizer the sections lose the uniform black which they had on removal from the hxmatoxylin. They remain in the decolorizing fluid until the gray matter becomes a light gray or yellow color, in sharp contrast with the white matter which remains dark. Sections are then washed in several waters to remove all traces of decolorizer, and dehydrated in alcohol.

Weigert-Pat, Method.-In this modification of the Weigert method, material hardened in formalin should be further hardened in potassium bichromate 5-per-cent. for two weeks, or in copper bichromate 3-per-cent. for about a week, after which it may be cut and stained without further mordanting. Sections from material hardened in the other above-mentioned ways are mordanted in a $3^{-}$to $5^{-}$ per-cent. aqueous solution of potassium bichromate instead of in the 
copper-acetate solution. After rinsing in water the sections are stained in hæmatoxylin as in the ordinary Weigert method. The lithium carbonate may, however, be omitted. They are then washed and transferred to a 0.25 -per-cent. solution of potassium permanganate, where they remain from one-half to two minutes, after which they are again washed and placed in the following:

Oxalic acid,

I gm.

Potassium sulphite,

I gm.

Water,

200 c.c.

In this solution differentiation takes place, the medullary sheaths remaining dark, while the color is entirely removed from the rest of the tissue. If the section is still too dark, it may again be carried through the permanganate and oxalic-acid solutions, rinsing in water between changes, until sufficiently decolorized.

All formalin-fixed material is best stained by the Weigert-Pal method. An intensification of the stain, especially of the very fine fibres, may sometimes be obtained by placing the sections for a minute in a 0.5 -per-cent. aqueous solution of osmic acid before decolorizing.

\section{Marchi's Method for' Staining Degenerating Nerves}

Small pieces of tissue are fixed and hardened for from seven to ten days in Müller's fluid. Thin slices of the tissue are then transferred to a solution of one part I-per-cent. osmic acid and two parts Müller's fluid, in which they remain from two to seven days. After embedding and sectioning in the usual manner, sections are mounted, usually without further staining, in xylol-balsam. The treatment with Müller's fluid so affects the normal medullary sheaths that they will not take the osmic-acid stain, but appear yellowish-brown, while the degenerating sheaths (probably fatty) stain black. The result is a positive picture of stained degenerating fibres in contrast with the stained normal and unstained degenerated fibres as seen after Weigert staining. Another advantage of the Marchi method is that, as the picture is a positive one, an early or slight degeneration may be recognized which would escape notice in material stained by Weigert's method; on the other hand, in a long-standing degeneration when the medullary sheaths have completely disappeared and their places have been taken by connective tissue, there being no degenerated myelin. remaining, the Marchi method is inapplicable. 
Busch's modification of the Marchi method gives sharp pictures and has the advantage of allowing formaldehyd fixation and hardening. Tissues thus treated are placed for from five to seven days in a solution of one part osmic acid, three parts iodate of sodium, and 300 parts water. They are then embedded, cut, and mounted as usual.

\section{Golgi Methods of Staining Nerve Tissue}

The Golgi methods in most common use at present are the following:

(I) Golgi Silver Methods.-(a) Slow Method.-Blocks of tissue are placed for several months in a 3 -per-cent. aqueous solution of potassium bichromate. Small pieces of the tissue are then transferred immediately to a 0.75 -per-cent. aqueous solution of silver nitrate. This is changed several times or until no more precipitate is formed. In the last silver solution they remain for from one to three days. The only method of determining whether the tissue has been sufficiently long in the bichromate is to try at intervals small bits of the tissue in the silver solution until a satisfactory result is secured. Sections should usually be from 50 to $80 \mu$ thick and are mounted in balsam without a cover-glass.

(b) Rapid Method.-Small pieces of tissue, 2 to $4 \mathrm{~mm}$. thick, are placed in the following solution for from two to six days, the time depending upon the age and character of the tissue, the temperature at which fixation is carried on, and the elements which it is desired to impregnate:

Osmic acid, I-per-cent. aqueous solution, I part.

Potassium bichromate, 3.5-per-cent. aqueous solution, 4 parts.

As a rule, the longer the hardening the fewer are the elements stained, but these few are clearer. The tissue is next transferred to silver nitrate as in the slow method. Pieces of tissue should be tried each day until a satisfactory result is obtained. The pieces may be kept in silver nitrate some time, but not in alcohol, and are better cut without embedding, the pieces being simply washed in 95-per-cent. alcohol several hours, then gummed to the block with celloidin, cut in 95-per-cent. alcohol, and mounted as in the slow method.

(c) Mixed Melhod.-Specimens are placed in the bichromate solution for about four days, then from one to three days in the osmium-bichromate mixture (see Rapid Method), after which they are transferred to the silver solution (see Slow Method). 
(d) Formalin-bichromate Method.-Tissues are placed for from two to six days in the following solution:

Formalin,

Potassium bichromate, 3-per-cent. aqueous solution, Io to 20 parts.

90 to 80 parts.

Subsequent treatment with silver is the same as in the previously described method. The results resemble those of the slow method. The specimens may be kept in strong alcohol. The method is satisfactory only for the adult cerebrum and cerebellum.

(2) Golgr Bichlorid Method.-Material, which need not be cut into small pieces, remains for several months in the potassiumbichromate solution (see Slow Silver Method), after which it is transferred to a 0.25 -per-cent. to I-per-cent. aqueous solution of mercuric chlorid for from four to twelve months or longer, the solution being changed as often as discolored. The degree of impregnation must be determined by frequently testing the material, but is usually indicated by the appearance of small white spots on the surface of the tissue.

A modification of the bichlorid method, known as the Cox-Golgi Method, often gives good results. The following fixing solution is used:

Potassium bichromate, 5-per-cent. aqueous solution,

Mercuric chlorid, 5-per-cent. aqueous solution,

Distilled water,

After mixing the above, add

Potassium chromate, 5-per-cent. aqueous solution,
20 parts.

20 parts.

40 parts.

Tissues remain in this fluid for from two to five months.

In the Golgi silver methods the result of the treatment, first with bichromate and then with silver nitrate, is that a precipitate is formed in the tissue, a chromate or some other silver salt, which in favorable cases is largely confined to certain of the nerve cells and their processes. It must be remembered that only a few of the cells and processes are stained, these often only partially, and that other irregular precipitations are usually present. In the mercury methods, the bichromate of potassium and the bichlorid of mercury may be used combined in the same solution. There are other modifications of the Golgi methods, in which similar precipitates of other metallic salts are secured. 
Golgi specimens should be dehydrated and embedded as rapidly as possible. This is especially true of specimens treated by the rapid and the mixed methods. Those treated by the slow silver method and by the bichlorid method are more permanent, and more time may be taken with their dehydration. Thick sections should be cut ( 75 to $100 \mu$ ) and mounted in xylol-balsam. After the rapid method, it is safer to mount without a cover-glass; after the slow method, specimens may be mounted with or without a cover. The balsam should be hard, and melted at the time of using. (See Mounting, page 22.)

\section{Cajal's Methods for Staining the Neurofibrils in the NERVE-CELLS}

In these methods, besides the neurofibrils, the cell processes and especially the axis cylinders are often beautifully displayed, the stain giving a picture in this respect much more general than that of the Golgi methods, but much more specific, and sharper than that of the ordinary stains.

The methods consist mainly of two steps: (I) The staining of the tissue in a solution of silver nitrate; (2) the further reduction of the silver stain with a weak photographic developer. Three methods, or variations, are here given:

(I) Pieces about $0.5 \mathrm{~cm}$. thick are placed in a liberal quantity of from I-per-cent. or I.5-per-cent. (new-born or embryonic mammalian material) to 5-per-cent. (adult material) solution of silver nitrate and kept at a temperature of $32^{\circ}$ to $40^{\circ} \mathrm{C}$. for two to five days. When properly stained (shown by a yellowish or light brown coloration of freshly cut surfaces) the pieces are very briefly rinsed in distilled water and placed in: pyrogallol (or hydroquinone) I gm., distilled water roo c.c., formalin 5 to ro c.c., for twenty-four hours or more. They are then washed a few minutes in water and transferred to 95-per-cent. alcohol, which is changed when discolored, and where they may often be kept for some time without injury. They may then be embedded in celloidin or paraffin and sections cut, usually $15^{-2} 5 \%$ in thickness. Different depths of the blocks of tissue usually vary in stain, the most favorable being intermediate between the surface and centre of the block. Celloidin sections usually keep well in 95-per-cent. alcohol. They may be cleared in carbol-xylol, rinsed in xylol, and mounted in xylol-balsam or xylol-damar in the usual way. In delicate objects (study of pathological changes 
in neurofibrils) it may be best to abbreviate the dehydration, and block and cut without infiltration with celloidin.

(2) Pieces are first placed in 95-per-cent. alcohol or in absolute alcohol $\left(32^{\circ}\right.$ to $40^{\circ} \mathrm{C}$.) for twenty-four hours. For neurofibrils it is better to add from 0.25 c.c. to I c.c. of ammonium hydrate to each Io c.c. of the alcohol. They are then treated with silver nitrate I-percent. or I.5-per-cent., as in (I). This method gives better pictures of the cell processes and axis cylinders and a better fixation of the cells.

(3) Pieces are first placed in distilled water roo c.c., formalin 20 c.c., ammonia I c.c., for twenty-four hours at $32^{\circ}-40^{\circ} \mathrm{C}$., washed in water twelve to twenty-four hours, and then treated with silver nitrate I-per-cent. or I.5-per-cent., as in ( $\mathrm{I}$ ). This method gives pictures of the terminations of nerve fibres on the periphery of nerve cells and their dendrites (end-feet or end-buttons of Auerbach).

In general it is best to avoid, in the above methods, any excessive exposure to the light while the pieces are in the silver bath (especially when the pieces are very small), though they may be brought into the light for examination and while being transferred to the reducing fluid.

\section{NissL's Method}

This method is useful for studying the internal structure of the nerve cell. It depends upon a rapid fixation of the tissue, its subsequent staining with an aniline dye, and final decolorization in alcohol.

The aniline dye most commonly used is methylene blue. There are many variations and modifications of Nissl's method. The following is simple and gives uniformly good results:

Specimens are first fixed in mercuric-chlorid solution (page 8), in formalin (ro-per-cent. aqueous solution), or in absolute alcohol, and embedded in celloidin.

Thin sections are stained in a I-per-cent. aqueous solution of pure methylene blue (Grübler). The sections are gently warmed in the solution until steam begins to be given off. They are then washed in water and differentiated in strong alcohol. The degree of decolorization which gives the best results can be learned only by practice. Several alcohols must be used, and the last alcohol must be perfectly free from methylene blue. The sections are cleared in 
equal parts xylol and cajeput oil and mounted in xylol-balsam. A contrast stain may be obtained by having a little eosin or erythrosin in the last alcohol.

\section{General References for Further Study of Technic.}

Freeborn: Histological Technic. Reference Handbook of Medical Sciences, vol. iv.

Lee: The Microtomist's Vade-mecum.

Mallory and Wright: Pathological Technic. 

PART II

THE CELL 



\section{CHAPTER I}

\section{THE CELL}

IN the simplest forms of animal life the entire body consists of a little albuminous structure, the essential peculiarity of which is that it possesses properties which we recognize as characteristic of living organisms. This albuminous material basis of life is known as protoplasm, while the structure itself is known as a cell. Within the cell is usually found a specially formed part, the mucleus. Peripherally cells are limited by a modified surface cytoplasm or a more or less distinct cell wall or cell membrane.

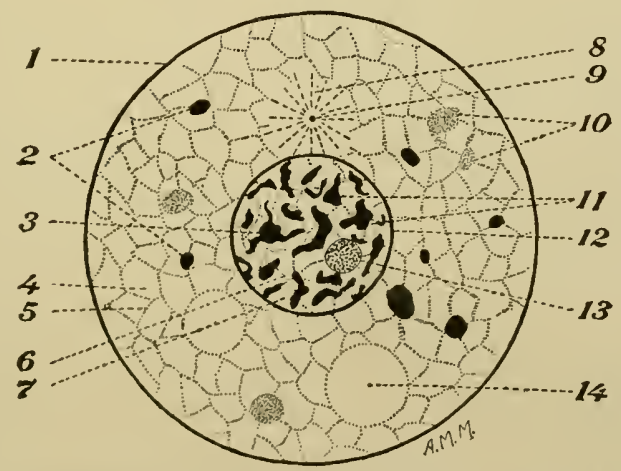

FIG. I.-Diagram of a typical cell. I, Cell membrane. 2, Metaplasm granules. 3, Karyosome or net-knob. 4, Hyaloplasm. 5, Spongioplasm. 6, Linin network. 7, Nucleoplasm. 8, Attraction-sphere. 9, Centrosome. Io, Plastids. I I, Chromatin network. I2, Nuclear membrane. I3, Nucleolus. I4, Vacuole.

An actively multiplying cell contains a minute structure associated with the reproductive function and known as the centrosome.

A typical cell thus consists of the following structures (Fig. I): (I) The cell body; (2) the cell membrane; (3) the nucleus; (4) the centrosome. Of these only the cell body with its modified surface cytoplasm or cell membrane is present in all cells. A few cells contain, in their fully developed condition, no nuclei. In many mature cells it is impossible to distinguish a centrosome.

All plants and animals consist of cells and their derivatives, and if an attempt be made to resolve any of the more complex living struc- 
tures into its component elements, it is found that the smallest possible subdivision still compatible with life is the cell. The cell may therefore be considered as the histological element or unit of structure.

I. The Cell Body.-This consists of a viscid, colorless, semi-fluid substance, belonging to the general class of albumens. It is of complex chemical composition containing the elements carbon, hydrogen, oxygen and nitrogen in quite constant proportions, and smaller

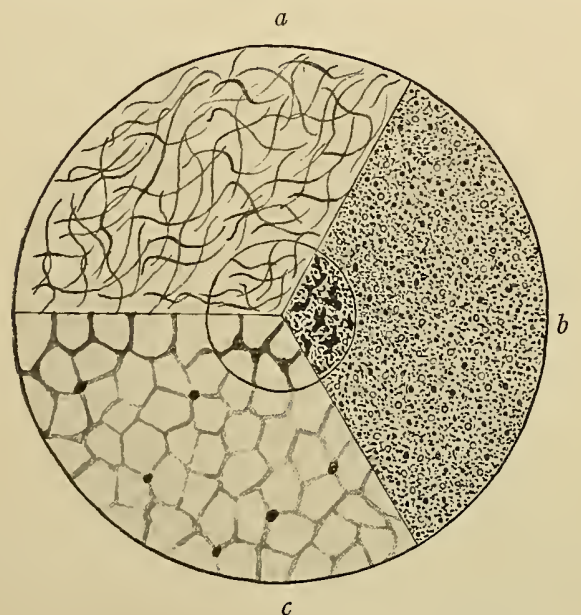

Fig. 2.-Diagram Illustrating Theories of Protoplasmic Structure. $a$, Fibrillar theory; $b$, granule theory; c, "foam" theory. (The general structure of cell body and nucleus corresponds.) variable quantities of phosphorus, sulphur, iron and other substances. It contains a peculiar nitrogenous proteid, plastin. Structurally it can be differentiated into a formed element, spongioplasm, and a homogeneous element, hyaloplasm. Distributed along the spongioplastic network are minute granules, microsomes. The exact relations which these elements bear to one another and to the cell as a whole have been the subject of much investigation and speculation. The earlier cytologists concerned themselves with the question as to whether protoplasm was homogeneous (i.e., a mere solution or at most a mixture of various substances) or had a definite structure. The theory of a structureless protoplasm having been long since abandoned, the question as to the character of the protoplasmic structure still remains unanswered.

Altmann's granule theory considers protoplsm as composed of fine granules embedded in a gelatinous intergranular substance. Altmann believed these granules the ultimate vital elements, and for this reason gave them the name of bioblasts (Fig. 2, b).

According to Bütschli, protoplasm is a foam or emulsion, the microscopic appearance of which can be simulated by artificial emulsions. He ascribes the appearance of a reticulum to the fact that each little foam space forms a complete cavity filled with fluid, the cut walls of these spaces giving a reticular appearance on section (Fig. 2, $c$ and Fig. 3). 
Other investigators consider protoplasm as made up of ( $\mathrm{I}$ ) a fibrillar element, either in the form of a network of anastomosing fibrils (cytoreticulum) or of a feltwork of independent fibrils (filar mass or miton), and (2) a fluid or semi-fluid substance which fills in the meshes of the reticulum or separates the fibrils (interfilar mass or paramiton) (Fig. 2, a).

That the question as to the ultimate structure of protoplasm still remains unanswered is dependent mainly upon the extreme technical difficulties which have confronted the cytologist. Living protoplasm has a homogeneous glossy appearance, showing even under the highest magnification rarely more than a granular structure. It is usually only after death of the cell and the use of chemical fixatives and stains, that the so-called "structure" of protoplasm becomes visib!e. How closely the picture presented by such chemically treated protoplasm corresponds to the structure of living protoplasm is as yet undetermined. It is quite possible that the structure even of undifferentiated protoplasm or protoplasm proper, such as is found in early embryonal cells, is not entirely uniform. The protoplasm of the more highly specialized cells certainly differs markedly in structure and somewhat in chemical composition in different cells. It even differs in the same cell under different functional conditions. These differences are apparently due to special development of the protoplasm for its peculiar functions. Thus, for example, in the muscle cell and the nerve cell most of what in the embryonal cell was undifferentiated protoplasm has become differentiated into contractile or conductile fibers. The nucleus and a small amount of undifferentiated or less differentiated protoplasm remain and are probably largely active in maintaining the nutrition of the cell. Such changes in the protoplasm are of course permanent. Temporary or periodic changes in the structure of protoplasm are seen in such cells as the secreting cells of the pancreas or of the salivary glands where marked
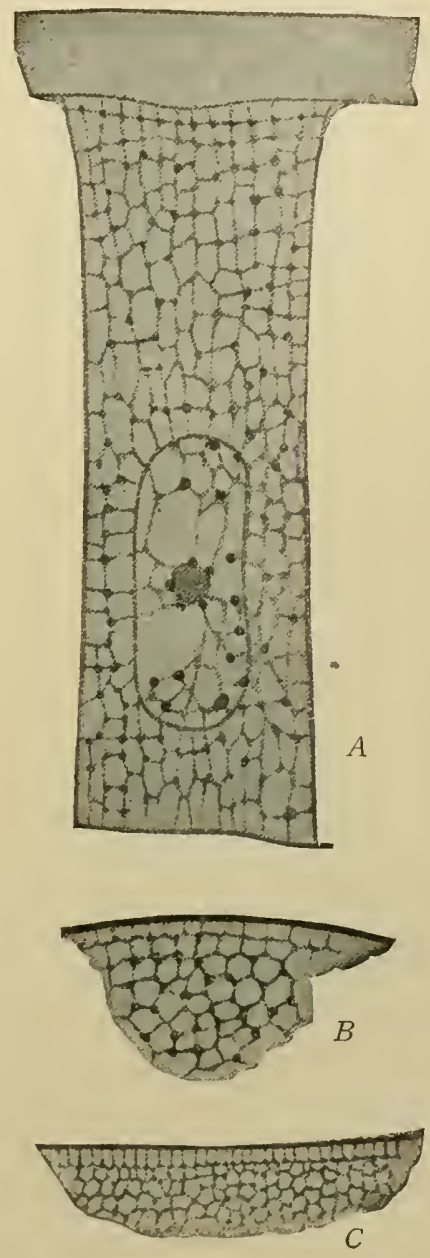

FIG. 3.-Foam or emulsion structure of protoplasm according to Butschli (Butschli). A, Epidermal cell of the carthworm. $B$, Peripheral cytoplasm of sea urchin's egg. $C$, Artificial emulsion of olive oil, sodium chlorid and water.

differences in the protoplasm occur, dependent upon whether the cell is in a resting, or actively secreting condition.

Protoplasm is thus probably best considered as the material basis 
of cell activity, i.e., of life, rather than as a substance having fixed and definite chemical or morphological characteristics.

It is convenient to use the term protoplasm to mean the entire substance of the cell, karyoplasm to designate the protoplasm of the nucleus, and cytoplasm the protoplasm of the cell body exclusive of the nucleus.

Peculiar bodies known as plastids (Fig. I) are of frequent occurrence in vegetable cells, and are also found in some animal cells. They are apparently to be regarded as a differentiation of the cytoplasm, but possess a remarkable degree of independence, being capable of subdivision and in some cases of existence outside of the cell.

In addition to the granules which are apparently an integral part of the protoplasmic structure, other granules and various cell "inclusions" occur, to which the term metaplasm (paraplasm, deutoplasm) granules, has been applied (Fig. I). Some of these are intimately associated with the cell activities and represent either food substances in process of being built up into the protoplasm of the cell or waste products of cell metabolism. Others, such e.g., as the glycogen

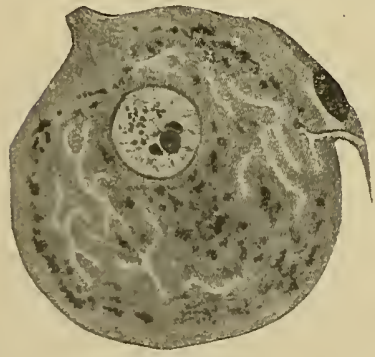

FIG. 4.--Intracellular canals (trophospongium) of a ganglion cell (E. Holmgren). granules of the liver cell or the mucous granules of the mucous cell, are specific secretion products. Still others are fat droplets, pigment granules, and various excrementitious substances.

When the protoplasm of a cell can be differentiated into a central granular area and a peripheral clear area, the former is known as endoplasm, the latter as exoplasm. When the exoplasm forms a distinct limiting layer, but blends imperceptibly with the rest of the protoplasm, it is known as

the crusta.

In some cells minute channels or canals are present in the cytoplasm (Fig. 4). These channels may contain branching processes from other cells, forming what is known as a trophospongium. Some intracellular canals are apparently secretory in character and may communicate with fine intercellular secretory channels. In this way the secretion of such cells as the serous cells forming the demilunes of mucous tubules (p. 222), or of the parietal cells of the stomach glands (Fig. I 55), is carried to the lumen. 
2. The Cell Membrane (Fig. I).-In most vegetable cells the cell membrane is the most conspicuous part of the cell and was responsible for the name "cell" which the seventeenth-century botanists, overlooking the importance of the enclosed protoplasm, gave to the little spaces or cavities of which they thought plants composed. A distinct cell membrane is present in but few animal cells. An exception is seen in the fat cell where a distinct membrane exists. In most animal cells no membrane has as yet been demonstrated and this has led to a persistent denial of the existence of such a membrane. It is nevertheless probable that most if not all animal cells have some modification, however slight, of the periphery of their protoplasm which serves to retain the protoplasm within definite bounds, to protect it as it were and at the same time permit osmosis. When a membrane surrounds the cell, it is known as the pellicula; when cells lie upon the surface, and only the free surface of the cells is covered by a membrane, it is known as the cuticula.

3. The Nucleus (Fig. I).-This is a vesicular body embedded in the cytoplasm. The typical nucleus, like the typical cell, is spheroidal, but the shape of the nucleus varies for different cells and corresponds somewhat to the shape of the cell body, e.g., the rod-shaped nucleus of the elongated smooth muscle cell. It may also be modified by intracellular pressure as, e.g., in the mucous cell and in the fat cell.

The position of the nucleus is usually near the center of the cell. It may, however, be eccentric. Such eccentricity may be due to pressure of cell contents as, e.g., in the mucous cell and in the fat cell. Considered by earlier cytologists an unessential part of the cell, the nucleus is now known to be most intimately associated with cellular activities. It is not only essential to the carrying on of the ordinary metabolic processes of the cell, but is an active agent in the phenomena of mitosis, which in most cases determine cell reproduction.

As a rule, each cell contains a single nucleus. Some cells contain two nuclei (quite common in the liver cell, rare in the ovum and in the nerve cell). A few cells contain many nuclei, e.g., the multinuclear "giant" cells of the spleen, bone-marrow, and certain tumors. Some cells, such as the human red blood cell and the respiratory epitheliun, are, in their mature condition, non-nucleated. All non-nucleated cells, however, contained nuclei in the earlier stages of their development. Non-nucleated cells, while capable of performing certain functions, are wholly incapable of proliferation. 'The non-nucleated 
condition must, therefore, be regarded as not only a condition of maturity, but of actual senility, at least so far as reproductive powers are concerned.

In some of the lowest forms of animal life, the nuclear material instead of being grouped to form a definite body or nucleus, is more or less evenly distributed as granules through the cytoplasm.

Chemically the nucleus is extremely complex, being composed of the proteids nuclein, paranuclein, linin, paralinin, and amphipyrenin.

Morphologically also the nucleus is complex, much of the apparent structural differentiation being determined by the staining reactions of the different elements when treated with certain aniline dyes. The nuclear structures and their relations to the chemical constituents of the nucleus are as follows:

(a) The muclear membrane (amphipyrenin). This forms a limiting membrane separating the nucleus from the cell protoplasm. It is doubtful whether the nuclear membrane is different either chemically or morphologically from the nucleoreticulum. It is wanting in some nuclei. When present it appears from its staining reactions to be structurally continuous with, and chemically identical with, in some cases, the linin, in others, the chromatin of the intranuclear network. It may be complete, or fenestrated allowing free communication between the cytoplasm and the nuclear contents.

(b) The intranuclear network, or nucleoreticulum, consists of a chromatic element (nuclein or chromatin) and of an achromatic element (linin). The linin constitutes the groundwork of the reticulum along which the chromatin granules are distributed. At nodal points of the network there are often considerable accumulations of chromatin. These nodal points, at first thought to be nucleoli, are now known as false mucleoli, or karyosomes. Instead of a distinct network there may be disconnected threads or simply granules of chromatin. Chromatin is the most characteristic of the chemical constituents of the nucleus, the only one which contains phosphoric acid, and also, apparently, the only nuclear substance which is always transmitted from parent to daughter cell in cell-division. Fine granules have been described as occurring in the linin, differentiated from chromatin by the fact that they are most susceptible to acid dyes, while chromatin takes basic dyes.

(c) The nucleolus or plasmosome (paranuclein, pyrenin) is a small spherical body within the nucleus. Not infrequently there are several nucleoli. Similar cells vary as to the number of nucleoli they 
contain. The same cell may vary as to the number of its nucleoli under varying functional conditions. The nucleolus regularly disappears during mitosis, and during functional activity in some gland cells. It stains intensely with basic dyes. Its function is unknown.

(d) Karyoplasm (nuclcoplasm, nuclear fuid, nuclcar sap). This is the fluid or semi-fluid material which fills in the meshes of the nucleoreticulum.

While the nucleus is a perfectly distinct structure capable in some animal and in some vegetable cells of moving about more or less actively in the cytoplasm, and is usually separated by a membrane from the rest of the cell, a marked similarity exists between the structure of nucleoplasm and cytoplasm. This similarity is emphasized by the absence in some resting cells of any nuclear membrane, by the apparent direct continuity in some cases of nucleoreticulum and cytoreticulum, and by the continuity of karyoplasm and cytoplasm in all cells during cell-division.

4. The centrosome (Fig. 5) is a small spheroidal body found sometimes in the nucleus, or more commonly in the cytoplasm near the nucleus. In actively dividing cells the centrosome is frequently double, this being apparently in preparation for the suc-

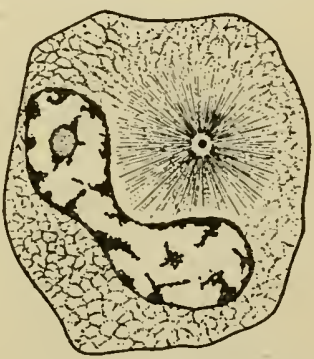

Fig. 5.- Spermatogonium from frog (Hermann). Single centrosome at center of attraction sphere or aster. Nucleus contains a plasmosome. ceeding cell-division. In some cases the centrosome is triple or even multiple. It was first found in the ovum and described as peculiar to that cell. It is now believed to occur in most, if not in all, animal cells. It usually consists of (I) a minute central granule or granulesthe centriole, which stains intensely with iron-hæmatoxylin, and outside of which is (2) a clear zone, the attraction sphere. From this centre, radiations extend outward into the cytoplasm. There is much confusion of terms in connection with the centrosome, the term centrosome being by some applied to the entire structure including the radiating fibrils, by others to the central granule only, by still others to the central granule plus the surrounding clear area. By some the radiations are believed to be composed of a different substance than the general cytoplasm, which is designated archoplasm. The main significance of the centrosome is in connection with cell-division, under which head it will be further considered (p. 53). 


\section{Vital Properties of Cells}

It has already been noted that the essential peculiarity of the cell is that it possesses certain properties which are characteristic of life. By this is meant that a cell is able: $\mathrm{I}$. To nourish itself and to grow-metabolism. 2. To do its own particular work in the body economy-specialfunction. 3. To respond to stimulation-irritability. 4. To move-motion. 5. To produce other cells-reproduction.

As would be expected, these properties, existing as they do in living cells, cannot always be sharply separated but frequently overlap. Thus, e.g., in the muscle cell "motion" equals "special function."

In the simplest forms of animal life, where a single cell constitutes the entire individual, all of these functions are performed by the one cell. In all higher, that is, multicellular animals, there are not only many cells but many kinds of cells, and this morphological differentiation corresponds to a physiological differentiation, each group of cells developing along certain well-defined lines for the performance of its own special function.

I. Metabolism.-This term is used to designate those cellular activities which have to do with the nutrition of the cell. A cell is able (I) to take up from without substances suitable for its nutrition and to transform these into its own peculiar structure, and (2) to dispose of the waste products of intracellular activities. The former is known as constructive melabolism or anabolism, the latter as destructive metabolism or katabolism.

It is possible, for example, to watch an amœeba send out projections (pseudopodia) (see p. 52) around an adjacent bit of food material. These projections coalescing finally completely enclose the food material within the body of the amœba where it is acted upon by the protoplasm in such a way (digested) as to completely lose its identity and to finally become an integral part of the cytoplasm.

There is normally maintained within the cell a state of equilibrium between this constructive and destructive metabolism, between the intake of food on the one hand and the outflow of material products or of energy on the other. Stated as an equation, intake $=$ outgo. Any marked + or - on one side of the equation without corresponding + or - on the other side must disturb this equilibrium and must result in physical changes within the cell. Thus any marked + in intake without corresponding + in outgo 
must result in growth of the cell, while any + in outgo without corresponding + in intake must tend toward diminution in bulk of cell and final destruction.

2. Special Function.-This is the special work which it is the part of the cell to perform. It varies greatly for different cells. Some cells, as, e.g., the surface cells of the skin, appear to act mainly as protection for more delicate underlying structures. Other cells-gland cells-in addition to maintaining their own nutrition, produce specific substances (secretions), which are of great importance to the body as a whole. Still other cells, e.g., nerve cells and muscle cells, have the power to store up their food substances in such a way as to make them available in the form of energy. This appears to be accomplished by the building up within the cell of highly complex and, consequently, unstable molecular combinations. By reduction of these unstable combinations, molecules of greater stability and less complexity are formed. This results in the transformation of potential into kinetic energy, and the expenditure of this energy is expressed in function.

3. Irritability is that property which enables a cell to respond to external stimuli. Cells vary in respect to their irritability, the most markedly irritable cells in higher animals being those of the neuromuscular mechanism. Stimulation may be mechanical, electrical,

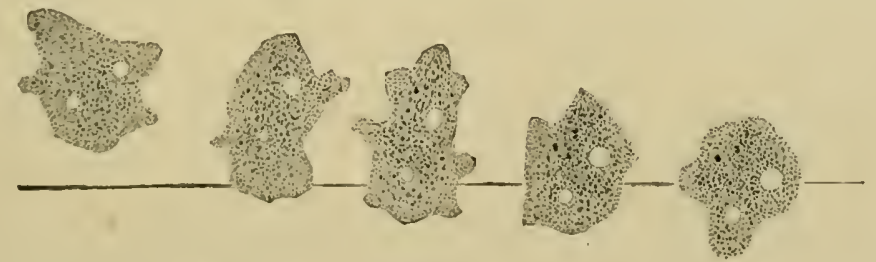

FIG. 6.-Amœboid Movement. Successive changes in shape and position of fresh-water amoba.

thermal, chemical, etc. The response of the cell to certain forms of chemical stimulation is known as chemotaxis. Some substances attract cells (positive chemotaxis); others repel cells (negative chemotaxis). Stimuli other than chemical possess similar properties, as indicated by the terms thermotaxis, galvanotaxis, etc. Some cells are so specialized as to react only to certain kinds of stimulation, e.g., the retinal cells only to light stimuli.

4. Motion.-This is dependent wholly upon the protoplasm of the cell, and is exhibited in several somewhat different forms. 
(a) Amoboid Movement. This consists in the pushing outward by the cell of processes (pseudopodia). These may be retracted or may draw the cell after them. In this way the cell may change both its shape and position (Fig. 6). White blood cells exhibit to a marked degree the power of amœboid movement.

(b) Protoplasmic Movement. This occurs wholly within the limits of the cell, changing neither its shape nor position. It occurs in both plant and animal cells, and consists of a sort of circulation or "streaming" of the protoplasm. It is evidenced by the movement of minute granules present in the protoplasm, by changes in the position of the nucleus, etc.

(c) Ciliary Movement. This is the whipping motion possessed by little hair-like processes called cilia, which project from the surfaces of some cells.

Certain cells which are specialized for the particular purpose of motion as, e.g., muscle cells, possess such powers of contraction that they are able to move not only themselves but other parts with which

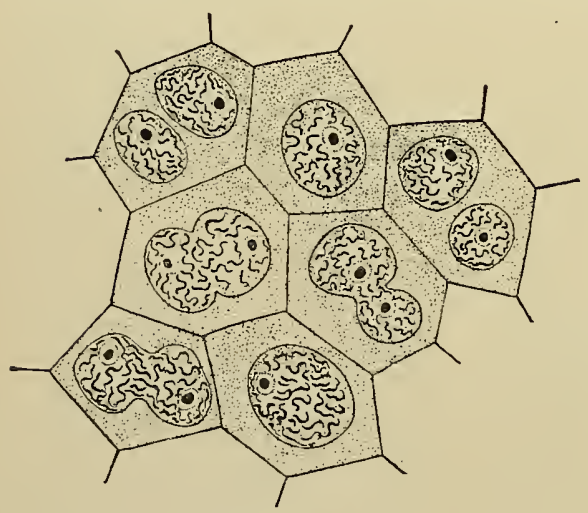

FIG. 7.-Epithelial Cells from Ovary of Cockroach, Showing Nuciei Dividing Amitotically. (Wheeler.) they are connected. This power of contractility is dependent upon the spongioplasm, the hyaloplasm playing a more passive rôle. In muscle cells the highly developed contractile powers appear to be due to the excessive development and peculiar arrangement of the spongioplasm.

5. Reproduction. - The overthrow of the long-held biological fallacy of spontaneous generation was soon followed by the downfall of a similar theory regarding the origin of cells. We now know that all cells are derived from cells, and that the vast number and complex of cells which together form the adult human body are all derived from a single primitive cell, the ovum.

Reproduction of cells takes place in two ways, by direct cell division or amitosis, and by indirect cell division or mitosis. In both amitosis and mitosis the division of the cell body is preceded by division of the nucleus. 
DrRect Cell-Division-Amitosis (Figs. 7 and 8). - In this form of cell-division the nucleus divides into two daughter nuclei without any apparent preliminary changes in its structure. The division of the nucleus may or may not be followed by division of the cell body, in the latter case resulting in the formation of polynuclear cells. This form of cell-division is uncommon in higher animals where Flemming considers it a degenerative phenomenon rather than a normal method of cell-increase. It is a common method of celldivision in the protozoa.

INDIRECT CELL-DIVISION-Mitosis (Figs. 9, Io).- In this form of celldivision also, the nucleus divides into two daughter nuclei, and the cell into two daughter cells, but only after they have passed through certain characteristic and complicated changes. These changes occur as a continuous process, but it is convenient for clearness of description to arbitrarily

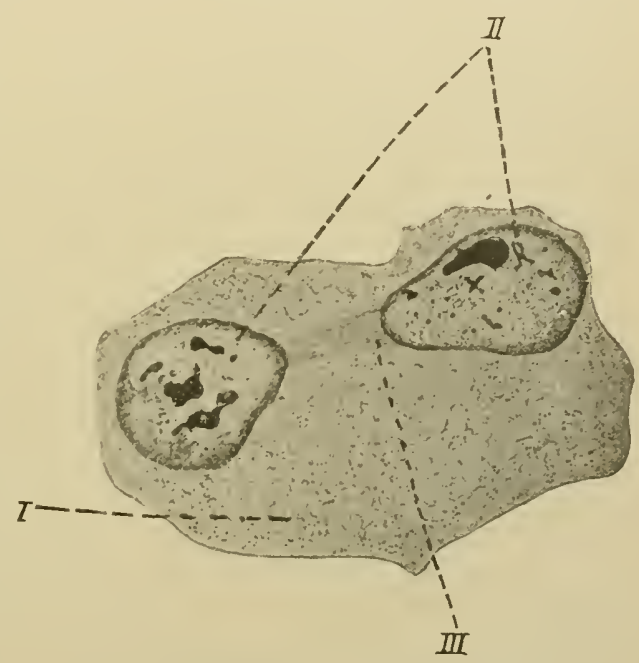

FIG. 8.-Epithelial cell from bladder showing amitotic division of its nucleus. (Nemileff.) I, Cytoplasm; $I I$, two daughter nuclei; $I I I$, fibrils uniting daughter nuclei. divide them into stages or phases. Thus we recognize in mitosis: (a) the prophase; $(b)$ the metaphase; (c) the anaphase; (d) the lelophase. The prophase is the stage of preparation on the part of the nucleus for division; the metaphase, the actual scparation of the nuclear elements; the anaphase, the formation of the two daughter nuclei; the telophase, the reconstruction of the two daughter resting nuclei and the division of the cytoplasm.

(a) The Prophase (Fig. 9, B, C, D) is marked by the following changes:

I. The centrosome, if single, divides into two daughter centrosomes. In most actively dividing cells, however, the centrosome is at this stage already double (Fig. 9, A) having divided as early, frequently, as the anaphase of the preceding mitosis.

The two daughler centrosomes, each surrounded by its attraction 
sphere, now move apart but remain connected by fibrils, probably derived from the linin (Fig. 9, B). These fibrils form the central or achromatic spindle. Two other sets of fibrils radiate from each centrosome - one, known as the polar rays, passes out toward the periphery of the cell; the other, known as the mantle fibres, extends from the centrosome to the chromosomes (Fig. 9, C). The two centrosomes with their fibres constitute the amphiaster.
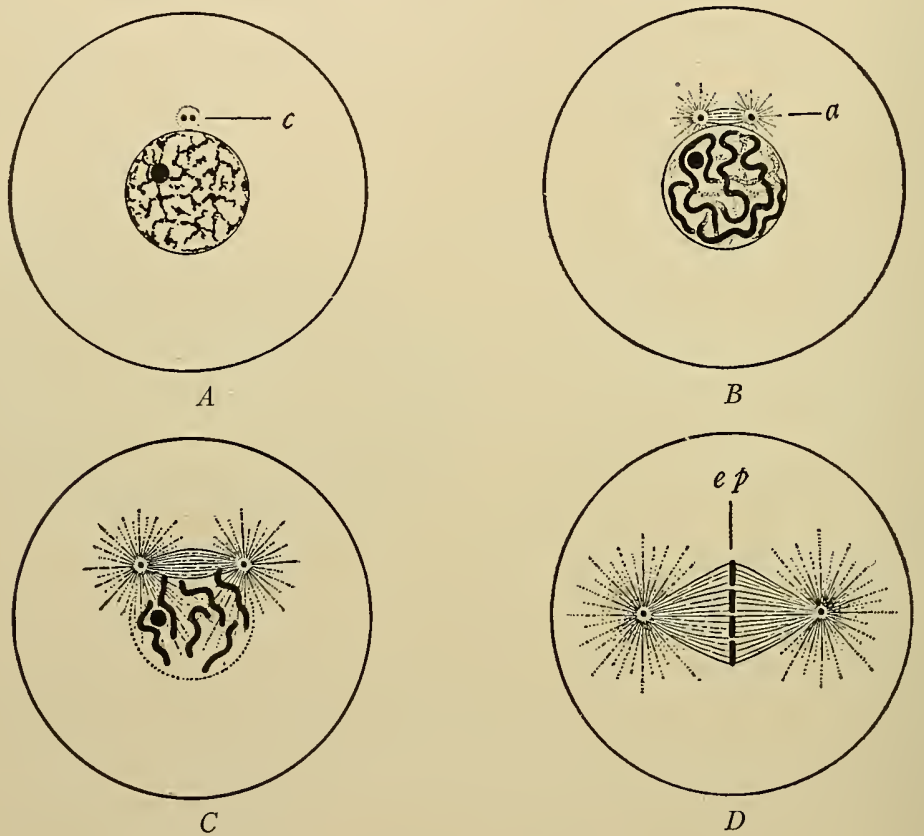

Fig. 9.-Diagrams of Successive Phases of Mitosis.

$A$, Resting cell, with reticular nucleus and true nucleolus; $c$, attraction sphere with two centrosomes.

$B$, Early prophase. Chromatin forming continuous thread-the spireme; nucleolus still present; $a$, amphiaster; the two centrosomes connected by fibrils of achromatic spindle.

$C$, Later prophase. Segmentation of spireme to form the chromosomes; achromatic spindle connecting centrosomes; polar rays; mantle fibres; fading of nuclear membrane.

$D$, End of prophase. Monaster-mitotic figure complete; $e p$, chromosomes arranged around equator of nucleus; fibrils of achromatic spindle connecting centrosomes; mantle fibres passing from centrosomes to chromosomes. (E. B. Wilson, "The Cell," The Macmillan Co.)

2. During or immediately following the formation of the amphiaster, important changes take place in the nucleus. It increases in size and loses the reticular appearance of the resting nucleus, its chromatic elements becoming arranged in a long spireme-thread or in several shorter threads, the closed skein_or closed spireme. This next 
becomes thicker and more loosely arranged, thus forming the open spireme. That some chemical as well as morphological change has taken place in the transformation of the reticulum of the resting nucleus into the spireme is shown by the marked increase in staining intensity, the spireme taking a much darker stain than the reticulum. Late in the prophase the nucleolus and nuclear membrane disappear. The cytoplasm and karyoplasm then become continuous and both spireme and amphiaster lie free in the general cell protoplasm (Fig. $9, C)$.

3. The spireme next breaks up into a number of segmentschromosomes (Fig. 9, C). These are usually rod-shaped at first, later they may become U's or V's or may even become spheroidal. The chromosomes now arrange themselves regularly around the equator of the nucleus, their closed ends being directed centrally.

The details of the transformation of the reticulum into chromosomes vary. In some cases a single spireme-thread is formed. In others the spireme-thread first splits longitudinally into two threads before segmenting into chromosomes. Again the spireme-thread may show segmentation into chromosomes from the beginning. In still other cases the chromosomes apparently form directly from the reticulum without the intervention of the spireme stage. It is most important to note that while the number of chromosomes varies for different species of plants and animals, it is fixed and characteristic for a given species. Thus in Ascaris megalocephala (much used for study on account of its small number of chromosomes) the number is 4 , in the mouse 24 , in man, estimated by some as 16 , by others $24 .{ }^{1}$ This means that whenever mitosis occurs in Ascaris, the spireme-thread invariably segments into 4 chromosomes. Chromosomes and amphiaster now constitute the mitotic figure which at this stage is known as the monaster, its formation marking the end of the prophase.

(b) Metaphase (Fig. Io, E). This marks the beginning of actual division of the nucleus. Each chromosome splits longitudinally (longitudinal cleavage) into two daughter chromosomes, each contain-

${ }^{1}$ Guyer (Biol. Bul. Marinc Biol. Lab. Wood's Hole, Mass., Vol. XIX) describes twenty-two chromosomes in human spermatogonia, of which two are "accessory". Apparently"half the resulting spermatirls contain ten chromosomes, the other half twelve, two of which are accessory. In Syromastes Wilson found an identical condition and it was later determined that the somatic number of chromosomes for Syromastes was twenty-two for the male and twenty-four for the femalc. Guyer concludes that the probable somatic number for the human male is twenty-two, for the female twenty-four. 
ing exactly one-half the chromatin of the parent chromosome. Uand V-shaped chromosomes always begin to split at the apex, from which point the separation extends to the open ends.

(c) Anaphase (Fig. Io, $F, G$ ).-An equal number of daughter chromosomes now travels along the fibrils of the achromatic spindleapparently under the influence of the mantle fibres-toward each daughter centrosome around which they become grouped. In this
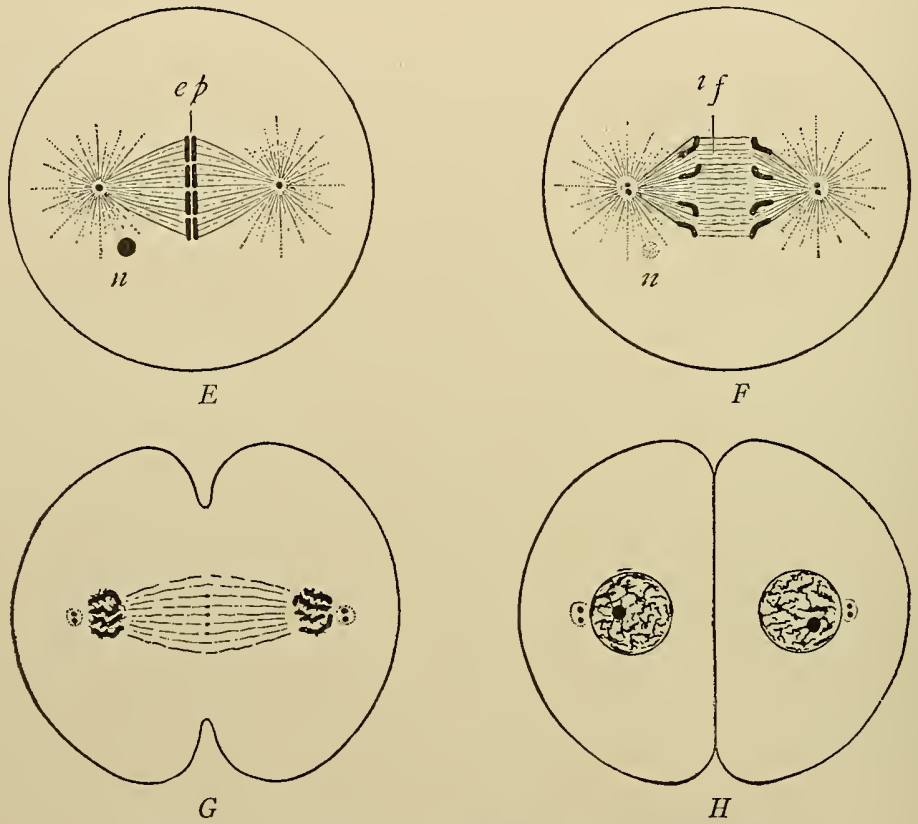

FIG. Io.-Diagrams of Successive Phases of Mitosis.

$E$, Metaphase. Longitudinal cleavage; splitting of chromosomes to form daughter chromosomes, $e p ; n$, cast-off nucleolus.

$F$, Anaphase. Daughter chromosomes passing along fibrils of acromatic spindle toward centrosomes; division of centrosomes; if, interzonal fibres or central spindle.

$G$, Late anaphase. Formation of diaster; beginning division of cell body.

$H$, Telophase. Reappearance of nuclear membrane and nucleolus; two complete daughter cells, each containing a resting nucleus. (E. B. Wilson, "The Cell," The Macmillan Co.)

way are formed two daughter stars, the mitutic figure being known at this stage as the diaster (Fig. Io, $G$ ). These daughter stars are at. first connected by the fibrils of the achromatic spindle. In this. stage may also occur beginning division of the cell body. In actively dividing cells each centrosome frequently undergoes division at this. stage, resulting in four centrosomes to the cell.

(d) Telophase (Fig. Io, $H$ ).--This is marked by division of the 
cell protoplasm and consists of a cycle of changes, by means of which each group of daughter chromosomes is transformed into the chromatin network of a resting nucleus. These changes are the same as those described in the prophase, but occur in the reverse order, the chromosomes uniting to form the spireme, and the spireme becoming transformed into the nuclear network. The result is the formation of two daughter cells. The nuclear membrane reappears, as does also the nucleolus. Each daughter cell is thus provided with a resting nucleus. The fact that the number of chromosomes which enter into the formation of the chromatic reticulum of the nucleus of each daughter cell is the same as the number into which the spireme of the parent cell divided, has suggested the hypothesis that the chromosomes maintain their identity even during the resting stage.

The time required for the mitotic process is usually from one-half to three-quarters of an hour. Exceptionally it is prolonged to several hours.

The rôle which each part of the cell plays in the vital activities of the cell, and the physiological correlation of these various parts, have been but partially determined. Experiments upon some of the protozoa show that if the cell be divided into two parts, one part containing the nucleus, the other part nonnucleated, the behavior of the two parts is very different. The part containing the nucleus soon again becomes a complete cell with all the properties which the cell originally possessed. The non-nucleated part responds somewhat to stimulation, is capable of some movement, makes some feeble attempts at digestion, but is incapable of secretion, of reconstructing the complete cell, of reproduction, and soon dies. Even when a small part of the nucleus remains in the cut-off piece of cytoplasm reconstruction may take place. On the other hand a nucleus completely deprived of its cytoplasm is incapable of reconstructing the cell. In Infusoria each cell has two nuclei, a macro-nucleus and a micronucleus, the former connected with nutrition, the latter with reproduction. These facts together with the behavior of the nucleus during the ingestion of food, during secretion, and even during motion, warrant the belief that while in all probability most of the actual work of the cell takes place in the cytoplasm, the nucleus exerts a more or less controlling influence over all cell activities.

The parts which the several cell structures play in mitosis have been the subjects of much study and are as yet not fully determined.

As to the behavior of the chromatic portion of the mitotic figure little doubt exists. It originates in the chromatic portion of the nuclear reticulum of the parent cell and its destination is the chromatic portion of the reticulum of the daughter cells.

'The rôle of the centrosome in mitosis is not so clear. It has been called the "dynamic centre" of the cell because in most cases it appears to be the active agent in initiating and probably further directing the mitotic process. The 
origin of the astral fibres is not always the same. In Infusoria the centrosome is found within the nucleus and both amphiaster and chromosomes are of nuclear origin. In some of the higher plants the amphiaster is derived wholly from the spongioplasm. In the egg cells of Echinoderm, part of the amphiaster (central spindle) is of nuclear, the remainder (asters) of cytoplasmic origin. That the centrosome is not always the active factor in mitosis is shown by the fact that in the higher plants no centrosome can be demonstrated during any stage of mitosis, and also that in some cases the chromosomes divide without previous division of the centrosome. Between mitotic periods the centrosome with or without its aster may remain as an integral part of the resting cell. It may, on the other hand, entirely disappear during the resting stage.

Branca calls attention to the fact that the centrosome is an organ of no "constancy; permanence, or specificity," that (I) in certain cells it is impossible to demonstrate a centrosome at any time; (2) that when a centrosome has been demonstrated for a certain type of cell, it cannot always be found even in that type of cell; (3) that certain parts of the centrosome, the rays, are cytoplasmic, while another part, the centriole, reacts like nuclear material (chromatin). He concludes that the centrosome is a portion of the protoplasm "temporarily differentiated for a specific function; not unlike the basal filaments which appear temporarily in gland cells when they become active, both being functional forms of protoplasm which can succeed each other as the cell changes the character of its function."

It is through the above-described process of cell-division that new cells are produced to replace those worn out as a result of their labors or destroyed by injury. It is through the same process that the vast number of cells which make up the adult body are developed from one original cell-the ovum. Such powers of evolution are not, however, inherent in the ovum itself, but, in sexual reproduction, are acquired only after its union with germinal elements from the male. This union of male and female germinal elements is known as fertilization of the ovum.

\section{Fertilization of the Ovum}

Prior to and in preparation for fertilization, both male and female cells must pass through certain changes. These are known as maturation of the spermatozoön on the male side (p. 344) and of the ovum on the female (p. 357).

The spermatozoön (Fig. II) is developed from a cell of the seminiferous tubule of the testis. The nucleus of this cell so divides its chromosomes that each spermatozoön contains just one-half the number of chromosomes characteristic of cells of the species. These are contained in the head of the spermatozoön, which thus represents the 
mucleus of the male sexual cell, the middle piece probably containing the centrosome, the tail piece the remains of the protoplasm.

The nucleus of the ovum or germinal vesicle also passes through a series of changes by which it loses one-half its chromosomes. The germinal vesicle or nucleus of the ovum first undergoes mitotic division with the usual longitudinal cleavage of its chromosomes and the formation of two daughter muclei. One of these and its centrosome are extruded from the cell as the first polar body. The remaining nucleus and centrosome again divide mitotically, only in this second division, instead of the usual longitudinal cleavage of chromosomes, by which each daughter nucleus is provided with the same number of chromosomes as the mother nucleus, the chromosomes simply separate, one-half going to each daughter nucleus. One of the daughter nuclei and its centrosome are now extruded as the second polar body. The polar bodies ultimately disappear, as does also the centrosome remaining within the egg. This leaves in the now matured ovum a single nucleus, which is known as the female pronucleus, and which contains one-half the number of chromosomes characteristic of cells of the species.

During this process in some animals-in others after its ccmpletion-the spermatozoön enters the ovum. The head of the spermatozoön becomes the male promucleus, the middle piece becomes a centrosome, while the tail is, in some instances at least, left behind as the spermatozoön enters the egg. The chromatin of the male next becomes

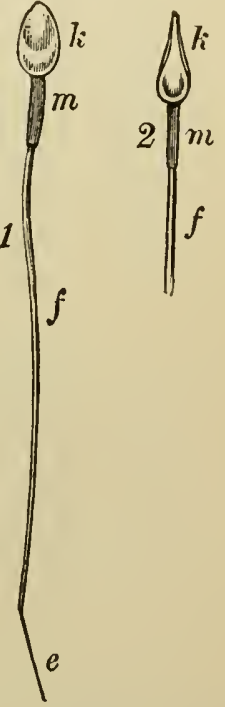

FIG. II.-Human Spermatozoa. (After Retzius.) I, Head seen on flat; 2 , head seen on edge; $k$, head; $m$, body; $f$, tail; $e$, end piece. arranged as chromosomes. Male and female pronuclei now lose their limiting membranes and approach each other, their chromosomes intermingling. As each pronucleus contained one-half the number, the monaster thus formed contains the full number of chromosomes characteristic of the species. Meanwhile the male centrosome, formed from the body of the spermatozoön, divides into two daughter centrosomes. These with their radiating fibrils have the same arrangement relative to the monaster of mingled male and female chromosomes, already described under mitosis. By longitudinal cleavage of these chromosomes, as in ordinary mitosis, two sets of daughter chromosomes are formed. Each set 
passes along the filaments of the achromatic spindle to its centrosome. Thus is formed the diaster. By continuation of the mitotic process two new nuclei are formed, each nucleus containing the number of chromosomes characteristic of the species, and each being
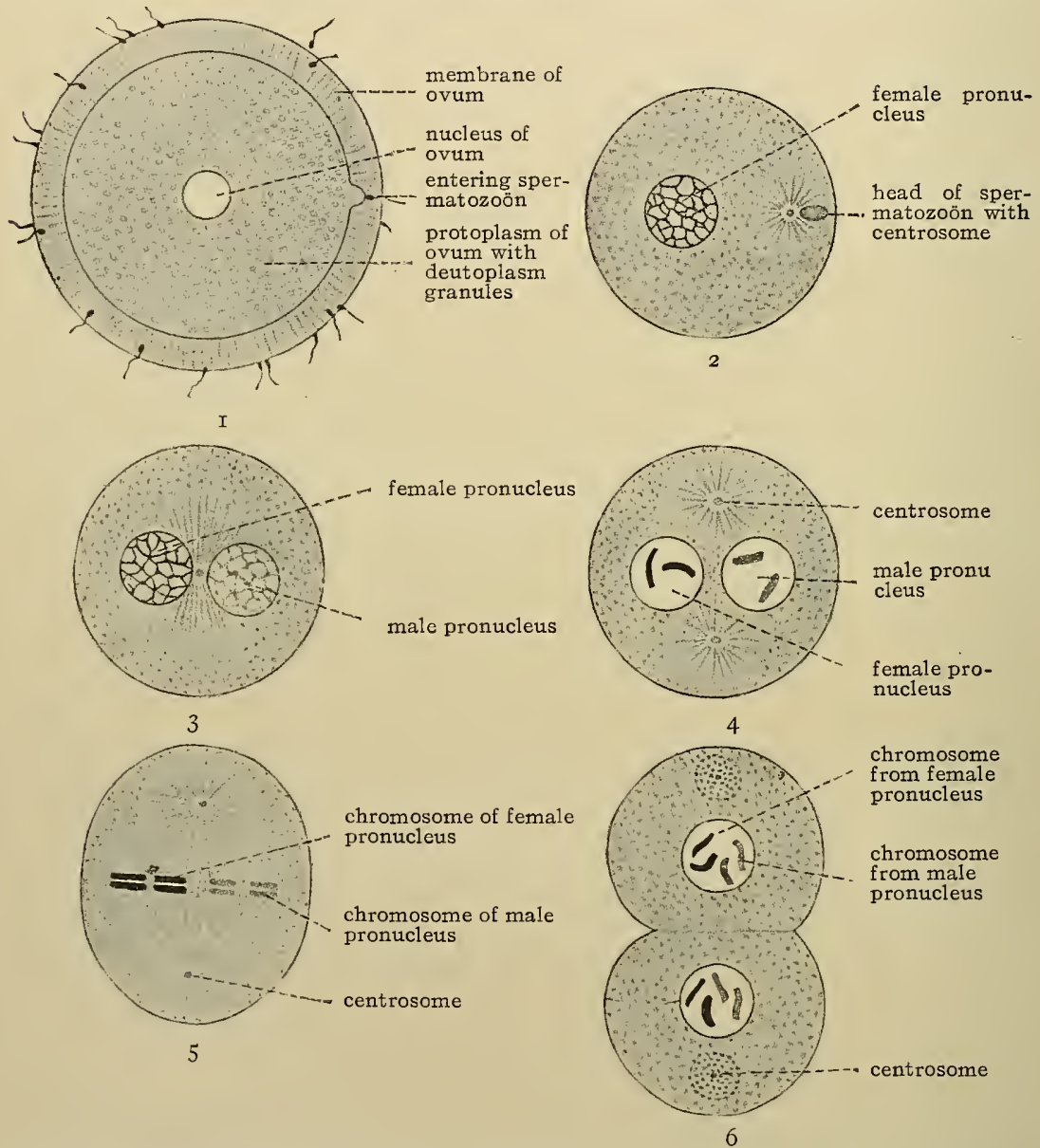

FIG. I2-Diagram of Fertilization of the Ovum. (The somatic number of chromosomes being four.) (From Bohm and von Davidoff, after Boveri.

I, Ovum surrounded by spermatozoa, only one of which is in the act of penetration. Toward the latter the protoplasm of the ovum sends out a process; 2 , Head of spermatozoön has entered ovum, its body becoming the male centrosome, its tail having disappeared; 3, The head of spermatozoön has become the male pronucleus. Male and female pronuclei approach each other. Between them is the (male) centrosome; 4 , The spiremes of male and female pronuclei have each formed two chromosomes. The centrosome has divided; 5, Male and female chromosomes have mingled and by longitudinal cleavage (see Mitosis, p. 53) have become eight. These become arranged in the equatorial plane of the ovum. Mantle fibres extend from centrosomes to chromosomes; 6, Division of the ovum; two daughter cells, each containing a daughter nucleus. Each daughter nucleus contains four chromosomes, two derived from each pronucleus. 
made up equally of male and female chromosome elements. Thus occurs the first division of the fertilized ovum into two daughter cells. By similar mitotic processes these two cells become four, the

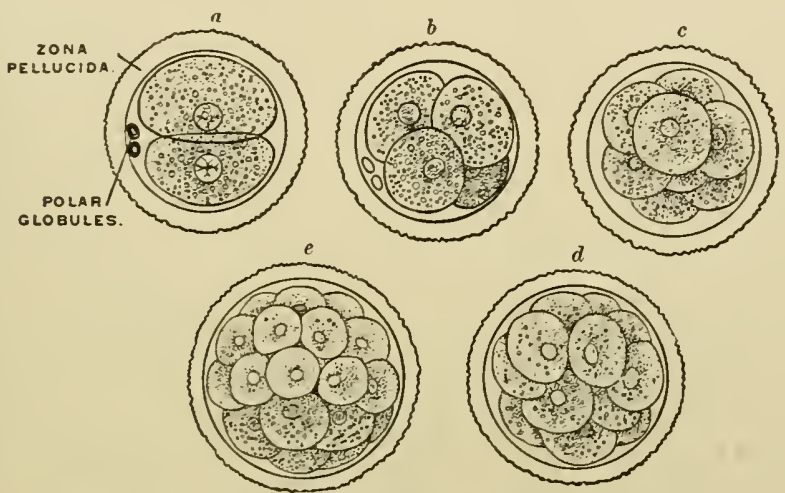

A
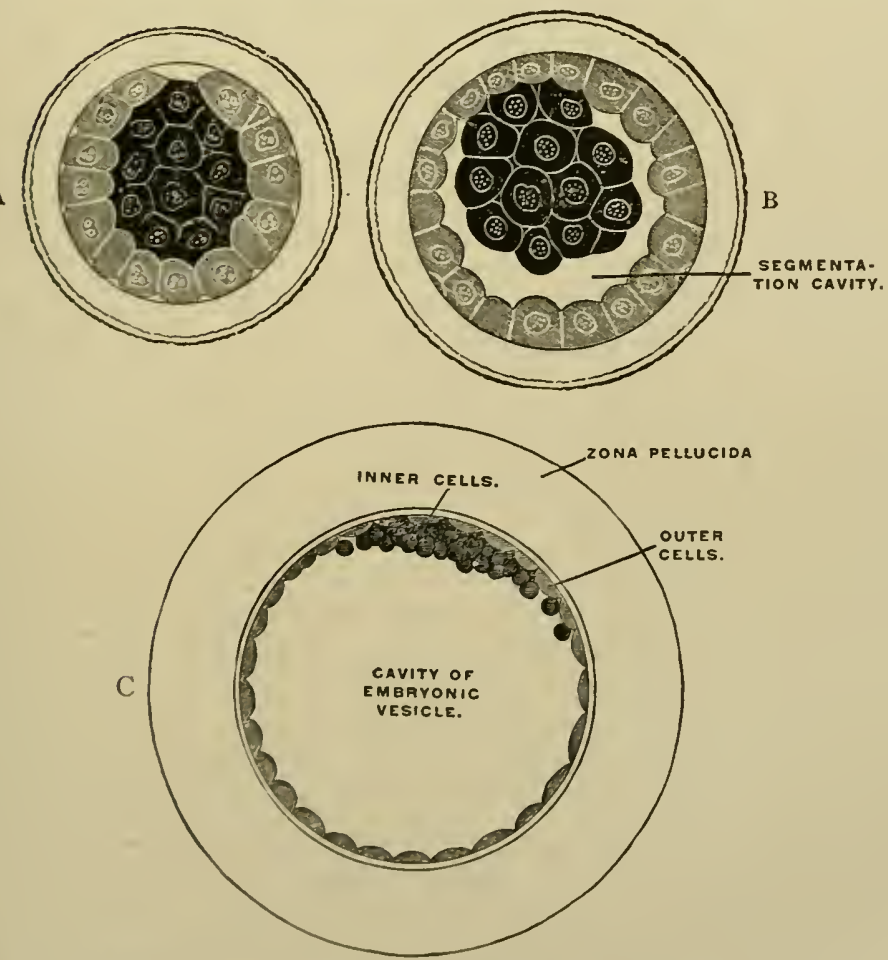

FIG. 13.- Segmentation of the Ovum. (From Gerrish, after van Beneden.)

$a$, Two-cell stage resulting from first division of fertilized ovum; $b$, four-cell stage; $c, d$, $e$, later stages. $A$, Differentiation into inner and outer cells; $B$, Formation of segmentation cavity; $C$, Embryonic vesicle, showing two primary germ laycrs. Outer cells, ectroderm; inner cells, cntoderm. 
four cells become eight, etc. This is known as segmentation of the оvит.

The earlier generations of these cells are morphologically alike and are known as blastomeres. Soon, however, these cells become spread out and at the same time differentiated into two primary germ layers. The outer of these is known as the ectoderm or epiblast, the inner as the entoderm or hypoblast. Between these two layers and derived from them a third layer is formed, the mesoderm or mesoblast. These three layers constitute the blastoderm.

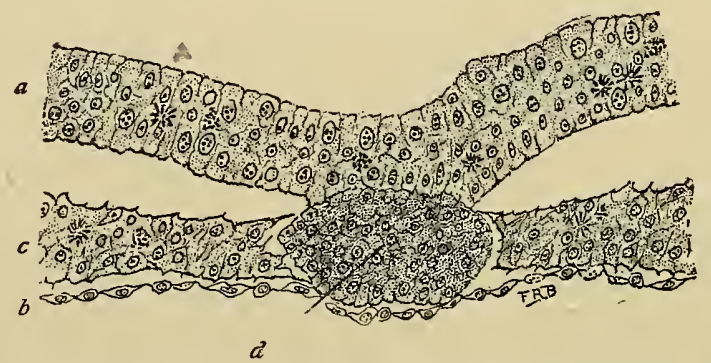

Fig. I4.-The Two Primary Germ Layers; from transverse section through primitive groove of a chick of 27 hours' incubation. $a$, Ectoderm (outer germ layer); $b$, entoderm (inner germ layer); $c$, mesoderm (middle germ layer); $d$, anlage of notochord.

As to what determines and controls fertilization, comparatively little is known. As in ordinary mitosis, the origin of the centrosome is obscure. In some forms, at least, the centrosome of the spermatid enters into the formation of the middle piece of the spermatozoön. The male centrosome thus enters the ovum. It is also known that in some eggs the egg-centrosome disappears soon after the extrusion of the second polar body, and that the centrosome of the fertilized egg develops in close relation to the middle piece of the spermatozoön. These facts point to the male centrosome as the centrosome of fertilization.

The ovum and spermatozoön are apparently brought together by a definite attraction on the part of the ovum toward the spermatozoön. The nature of this attraction is unknown. It is possibly chemical, and is exerted only between ova and spermatozoa of the same species. This has been proved for lower forms by mixing ova of one species and spermatozoa from several species in an inert medium when only spermatozoa of the same species will attach themselves to the ova. That the attractive force lies in the cytoplasm is shown by the fact that small pieces of the egg protoplasm free from nuclear elements will exert sufficient powers to cause spermatozoa to enter them.

As to the point of entrance of the spermatozoön, some eggs may be entered at any point, others are permeable at but one point.

One spermatozoön only is required for fertilization, and when this spermatozoön has entered, the egg apparently loses its power of attracting spermatozoa, or else develops some actual defense against further entrance of spermatozoa. 


\section{TECHNIC}

I. Fresh cells may be studied by gently scraping the surface of the tongue, transferring the mucus thus obtained to a glass slide and covering with a coverglass.

2. Red blood cells from the frog are prepared as follows: After killing the frog the heart is opened and the blood allowed to drop into a tube containing Hayem's fluid (sodium chlorid I gm., sodium sulphate $5 \mathrm{gm}$., mercuric chlorid 0.5 gm., distilled water Ico c.c.). After shaking, the cells are allowed to settle for from twelve to twenty-four hours. The fixative is then replaced by water, the tube again shaken, the cells allowed to settle, and the water is replaced with So per-cent. alcohol tinged with iodin. After from twelve to twenty-four hours the alcohol is decanted and the tube partly filled with alum-carmine solution (page I9). About twenty-four hours usually suffices for staining the nuclei. The alum-carmine is then poured off and the cells well shaken in water. After settling, the water is replaced by glycerin, to which a small amount of picric acid has been added. In this the cells may be permanently preserved. The nuclei are stained red by the carmine, the cytoplasm yellow by the picric acid.

3. Surface cells from the mucous membrane of the bladder. The bladder is removed from a recently killed animal, pinned out mucous membrane side up on a piece of cork and floated, specimen side down, on equal parts Müller's fluid and Ranvier's alcohol (technic, 4, p. 7, and $a$, p. 4) for from twenty-four to forty-eight hours. The specimen is then washed in water and the cells removed by gently scraping the surface. These may then be stained and preserved in the same manner as the preceding. Cells from the different layers should be studied; also the appearance of the large surface cells seen on flat and on edge, showing pitting of under surface by cells beneath.

4. Amœboid movement may be studied by watching fresh-water amœbæ or white blood cells. A drop of water containing amœbæ is placed on a slide, covered, and a brush moistened with oil is passed around the cover to prevent evaporation. The activity of the amœbæ may be increased by slightly raising the temperature. An apparatus known as the warm stage is convenient for demonstrating amœboid movement. A drop of blood, human, or better from one of the cold-blooded animals, may be used for the study of amœeboid movement in the white blood cells. It should be placed on a slide, covered, and immediately examined on the warm stage.

5. Ciliary movement is conveniently studied by removing a small piece of the gill of an oyster or mussel, teasing it gently in a drop of normal salt solution and covering. The cilia being very long, their motion may be easily studied, especially after it has become slow from loss of vitality.

6. Mitosis. The salamander tadpole and the newt are classical subjects for the study of cell-division. The female salamander is usually full of embryo tadpoles in January and February. The embryos are removed and fixed in Flemming's fluid (technic 7, p. 7), after which they may be preserved in equal parts of alcohol, glycerin, and water. Mitotic figures may be found in almost any of the tissues. Pieces of epidermis from the end of the tail, the parictal peritoneum, and bits of the gills are especially satisfactory. If the newt's tail 
is used, it should be fixed in the same manner, embedded in paraffin and cut into thin sections. These are stained with Heidenhain's hæmatoxylin, technic 3, p. I8.

Certain vegetable tissues, such as the end roots of a young, rapidly growing onion or magnolia buds, are excellent for the study of mitosis. The technic is the same as for animal tissues.

\section{General References for Further Study of the Cell}

Branca, A.: Histologie, Paris, I906.

Conklin, E. G.: Karyokinesis and Cytokinesis. Jour. Acad. Nat. Sci. of Phila., vol. xii, I902.

Harper, E. H.: The Fertilization and Early Development of the Pigeon's Egg. Amer. Jour. of Anat., vol. iii, No. 4, I904.

Hertwig, O.: Die Zelle und die Gewebe, 1898.

Hertwig, R.: Eirife, Befruchtung u. Furchungsprozess. In Hertwig's Handbuch d. vergleich. u. experiment. Entwickelungslehre der Wirbeltiere, Bd. I, Teil I, I903.

Lillie, F. R.: A Contribution toward an Experimental Analysis of the Karyokinetic Figure. Science, New Series, vol. xxvii, I9o8.

McMurrich: The Development of the Human body.

Minot: Human Embryology. A Laboratory Text-book of Embryology.

Sobotta, J.: Die Befruchtung u. Furchung des Eies der Maus. Arch. f. mik. Anat., Bd. xlv, I895.

Wilson, E. B.: The Cell in Development and Inberitance, $2 \mathrm{~d}$ ed., 1900. 


\section{PART III \\ THE TISSUES}





\section{CHAPTER I}

\section{HISTOGENESIS-CLASSIFICATION}

Ectoderir, mesoderm, and entoderm (see page 62) are known as the primary layers of the blastoderm. They differ from one another not only in position, but also in the structural characteristics of their cells. The separation of the blastomeres into these three layers represents the first morphological differentiation of the cells of the developing embryo. By further and constantly increasing differentiation are developed from these three primary layers all tissues and organs, each layer giving rise to its own special group of tissues. The tissue derivations from the primary layers of the blastoderm are as follows:

Ectoderm.-(I) Epithelium of skin and its appendages-hair, nails, sweat, sebaceous and mammary glands, including smooth muscle of sweat glands.

(2) Epithelium of mouth and anus, of glands opening into mouth, and enamel of teeth.

(3) Epithelium of nose and of glands and cavities connected with nose.

(4) Epithelium of external auditory canal and of membranous labyrinth.

(5) Epithelium of anterior surface of cornea, of conjunctiva, of crystalline lens, of lacrymal gland, and of lacrymal canal.

(6) Epithelium of male urethra, except prostatic portion.

(7) Epithelium of medulla of suprarenals, of pineal bodies, of pituitary body and of a few other small ductless glands.

(8) Entire nervous system, including retina and its forward extension over iris, also muscle tissue of sphincter and dilator pupillæ.

Entoderm.-(I) Epithelium of digestive tract (excepting mouth and its glands and anus,) and of glands connected with digestive tract including liver, gall bladder and pancreas.

(2) Epithelium of respiratory tract and of its glands.

(3) Epithelium of blarlder except the trigonum, of female urethra, of vaginal vestibule and glands of Bartholin, of prostatic portion of male urethra, prostatic glands and glands of Cowper. 
(4) Epithelium of tympanum and of Eustachian tube.

(5) Epithelium of thyreoid and parathyreoid, reticulum of thymus, and Hassall's corpuscles.

Mesoderm.-(I) All the connective or supporting tissues except neuroglia.

(2) Lymphatic organs with the exception of Hassall's corpuscles and reticulum of the thymus.

(3) Blood cells and bone-marrow.

(4) Striated, cardiac and smooth muscle (with the possible exception of smooth muscle of sweat glands and of iris).

(5) Endothelium lining blood-vessels and lymphatics.

(6) Mesothelium lining serous membranes-pleura, pericardium, and peritoneum.

(7) Epithelium of genito-urinary system with the exception of the urethra and a large part of the bladder.

(8) Epithelium of the suprarenal cortex.

In all but the lowest forms of animal life the body consists of an orderly arrangement of many kinds of cells. From the cells is developed a substance which lies outside the cells and is known as intercellular substance. This may be small in amount, just sufficient to unite the cells, as in epithelium, or it may so predominate as to determine the character of the tissue, as in some forms of connective tissue. It does not always completely separate the cells which may be connected across the intercellular substance by extensions of their protoplasm, as in the "intercellular bridges" of epithelium or the anastomosing processes of connective-tissue cells. Less commonly cells are united in such a multinuclear continuum that their boundaries are almost or wholly lost. Such a structure is known as a syncytium. The association of a particular type of cell with a particular type of intercellular substance is known as a tissue. The character of a tissue depends upon the character of its cells, of its intercellular substance, and their relations to each other. Further differentiation of cells and intercellular substance within a particular tissue gives rise to various sub-groups of the tissue. The association of tissues to form a definite structure for the performance of a particular function is known as an organ. The physiological association of organs constitutes a system. The fact that chemical changes take place in intercellular substance as well as in cells has led to the suggestion that the intercellular substance is endowed with the same 
vital properties as the cell. The consensus of opinion is, however, that the intercellular substance is derived originally from the cell, is replenished by the cell, is dependent upon the cell for its nourishment, and is incapable of activity or existence apart from the cell.

A scientific classification of the tissues is at present impossible.

The foregoing list of tissue derivations shows how unsatisfactory is any attempt at classification on the basis of histogenesis, many tissues which are morphologically similar being derived from two or even all three of the blastodermic layers.

The following is the usual classification of adult tissues: (I) Epithelial tissues; (2) connective tissues; (3) blood; (4) muscle tissue; (5) nerve tissue.

Of these, epithelium and connective tissue may be regarded as the more elementary tissues, being common to both plants and animals. Blood is sometimes classified among the connective tissues. Muscle and nerve are the most highly specialized tissues and are peculiar to animals. While the individuality of the tissues classified is recognized, the physiological necessities of nutrition, innervation, etc., scarcely permit the existence of any one tissue alone by itself. Thus blood and blood vessels permeate almost all tissues except epithelium, while the latter is everywhere traversed by elements of nerve tissue. 


\section{CHAPTER II}

\section{EPITHELIUM (INCLUDING MESOTHELIUM AND ENDOTHELIUM)}

General Characteristics.-Epithelium is derived from all three germ layers. It consists almost wholly of cells. The intercellular substance is merely sufficient to attach the cells to one another and is, consequently, known as cement substance. A characteristic of this cement substance is its reaction to silver nitrate (Figs. I5, 26, 27). In some instances the protoplasm of adjacent epithelial cells is seen to be even more closely associated, the intervening cement substance being bridged over by delicate processes of protoplasm which pass from one cell to another and are known as "intercellular bridges" (see Fig. 19, p. 74). It seems probable that the minute spaces between the processes serve as channels for the passage of food (lymph) to the cells. Reference to Figs. I 55 and 187 shows that a precipitation of silver also occurs in some tubules filled with glandular secretions. It is possible that the so-called intercellular cement may be of the nature of an intercellular lymph. The surface cells of epithelium are united by continuous cement substance in which there are apparently no spaces. In this way escape of lymph is prevented.

Epithelial cells vary in size and shape, the element of pressure being a frequent determining factor. They are often extremely elastic, allowing great changes in shape and relation. Thus the epithelium of the collapsed bladder is thick and the cells columnar, but as the bladder fills, the cells become flatter until in complete distention the epithelium is thin and consists of flattened cells. Their protoplasm may be clear, finely or coarsely granular, or pigmented. Each .cell usually contains a single well-defined nucleus. Two or more nuclei are sometimes present. Some epithelial cells are, when fully matured, non-nucleated, e.g., respiratory epithelium of lung.

When epithelium rests upon connective tissue, it is usually separated from the latter by a thin, apparently homogeneous membrane known as the basal membrane or membrana propria. Authorities 
differ as to whether this membrane is of connective-tissue or of epithelial origin.

Surface epithelial cells frequently have thickened free borders or culicula, which unite to form a continuous membrane, the culicular membrane. Striations extend from the cytoplasm into the cuticulæ. A still greater specialization of the surface of the cell is seen in the ciliated cell. In this cell fine hair-like projections-cilia-extend from the surface of the cell.

Some epithelial cells show important changes dependent upon their functional activities. An example of this is seen in the mucous cell in which there is a transformation of the greater part of the cytoplasm into, or its replacement by, mucus.

Epithelia are devoid, as a rule, of both blood- and lymph-vessels. An exception to this is the stria vascularis of the cochlea. Nerves, on the other hand, are abundant.

Classification.-Epithelia may be classified according to shape and arrangement of cells as follows:

(I) Simple Epithelium.-(a) Squamous; (b) columnar.

(2) Stratified Epithelium.-(a) Squamous; (b) columnar.

(3) Mesothelium and Endothelium.

Specializations of the above-mentioned types are known as: (a) Ciliated epithelium; (b) pigmented epithelium: (c) glandular epithelium; (d) neuro-epithelium.

Pigment may occur in any type of epithelium. Cilia are found only in the simple columnar and stratified columnar forms.

\section{Simple Epithelium}

In simple epithelium the cells are arranged in a single layer.

(a) Simple squamous epithelium consists of flat scale-like cells which are united by an extremely small amount of intercellular substance. The edges of the cells are smooth or serrated. Seen on flat, they present the appearance of a mosaic. Seen on edge, the cells appear fusiform, being thickest at the center, where the nucleus is situated, and thinning out toward the periphery. Simple squamous epithelium has but a limited distribution in man. It occurs in the lungs as non-nucleated respiratory epithelium, in Bowman's capsule of the renal corpuscle, in the descending arm of Henle's loop of the uriniferous tubule, in the retina in the form of pigmented cells, and on the posterior surface of the anterior lens capsule.

(b) Simple columnar epilhelium consists of a single layer of elon- 
gated cells. The bases of the cells are usually separated from the underlying connective tissue by a basement membrane. The nucleus is, as a rule, in the deeper part of the cell, near the basement membrane. Many of these cells have prominent thickened free

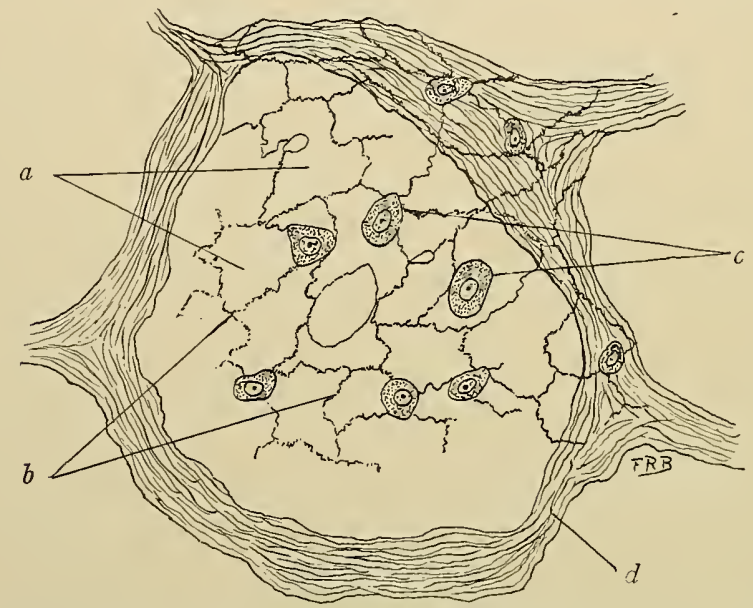

FIG. I5.-From Section of Cat's Lung, stained with silver nitrate, showing outlines of the Simple Squamous Epithelium Lining the Air Vesicle. a, Two epithelial cells; $b$, the wavy stained intercellular substance; $c$, fœetal cells; $d$, connective tissue.

borders or cuticulæ. This form of epithelium is often ciliated. The height of the cell varies greatly, there being all gradations from high columnar to low cuboidal. Simple columnar epithelium lines the

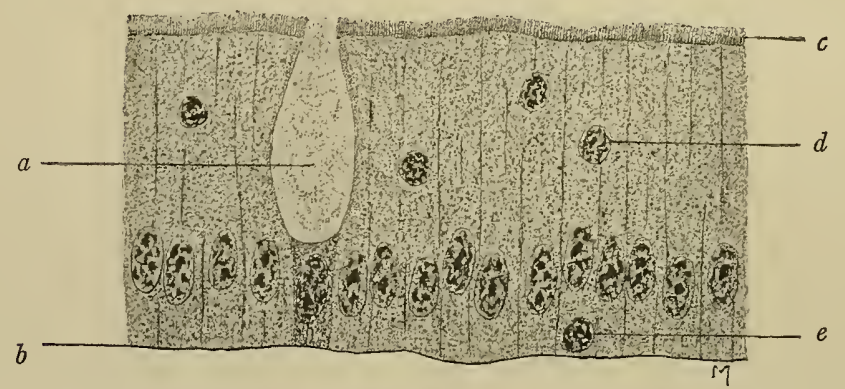

FIg. I6.-Simple Columnar Epithelium from the Human Small Intestine. $a$, Mucous (goblet) cell; $b$, basement membrane; $c$, thickened free border (cuticula); $d$, leucocyte among the epithelial cells; $e$, replacing cell.

gastro-intestinal canal, the uriniferous tubule (excepting the descending arm of Henle's loop), simple tubular glands, the ducts of some compound tubular glands, the smaller bronchi, the membranous and penile portions of the male urethra, and the gall-bladder. 
In simple columnar epithelium, in addition to the single row of epithelial cells, there are found lying near the basement membrane, between the bases of the epithelial cells, small, spherical, or irregular cells, which frequently show mitosis and which are known as replacing cells. They appear to develop into columnar epithelial cells as they are needed to replace older cells. The so-called pseudo-strati-

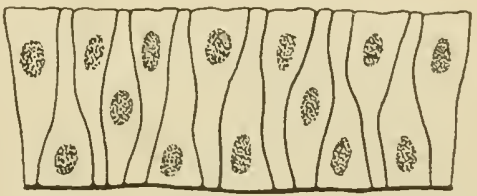

Fig. 17.-Diagram of Pseudostratified Epithelium. showing Nuclei situated at Different Levels. fied epithelium is a form of simple columnar epithelium, in which, from crowding of the cells, the nuclei have come to lie at different levels, thus giving the appearance of stratification (Fig. I7).

\section{Stratified Epithelium}

In stratified epithelium the cells are arranged in more than one layer.

(a) Stratified squamous epithelium is developed from simple epithelium by the growth of new cells between the old cells and the underlying connective tissue. It consists of several layers of cells

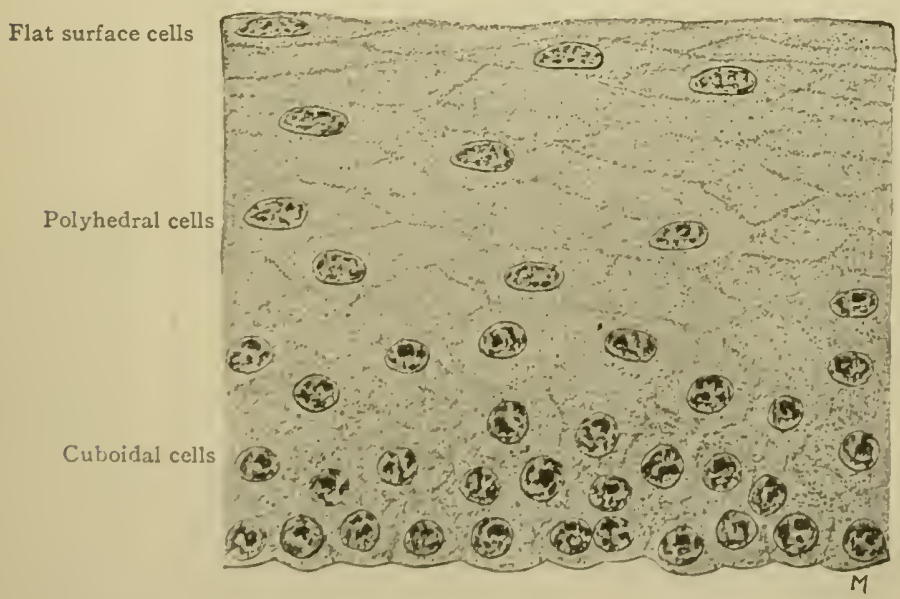

FiG. 18.-Stratified Squamous Epithelium from Cat's Esoplhagus.

which vary greatly in size and shape. 'The surface cells are large and flat. Iseneath these are several layers of polyhedral cells, with often very distinct protoplasmic intercellular connections ("intercellular bridges," see also p. 74). The deepest cells are columnar or cuboi- 
dal. It is thus seen that in stratified squamous epithelium only the surface cells are squamous. This form of epithelium rests upon a

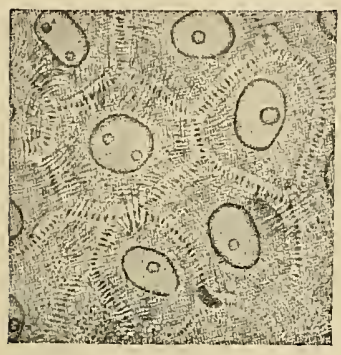

FIg. r9.-Epithelial Cells from the Stratum Spinosum of the Human Epidermis showing "Intercellular Bridges." $\times 700$. (Szymonowicz.) cuticula. Some epithelia of this type are ciliated. Stratified columnar epithelium is found in the larynx, nose, palpebral con-

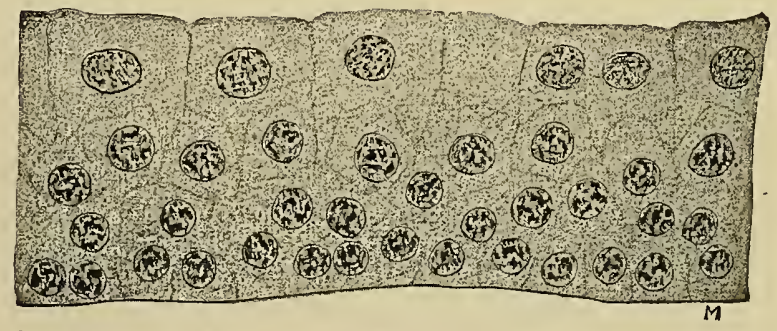

FIG. 20.-Transitional Epithelium from the Human Bladder.

junctiva, largest of the gland ducts, the vas deferens, and part of the male urethra.

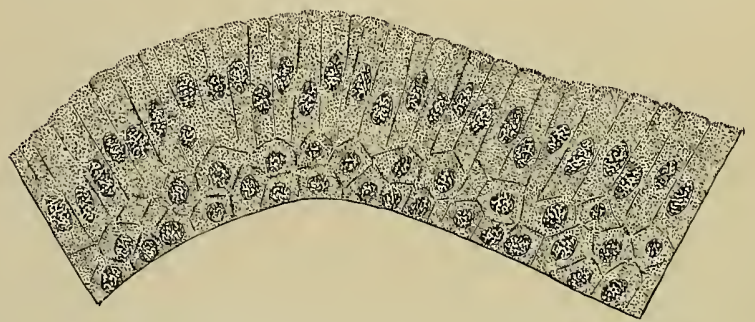

FIG. 21.-Stratified Columnar Epithelium from the Human Male Urethra. $\times_{400}$.

Stratified epithelium composed of only from three to six layers of cells is sometimes designated "transitional epithelium." This 
type of epithelium usually rests upon a basement membrane free from papillæ. The surface cells are large and frequently contain two or three nuclei. Their free surfaces are flat, while their under surfaces show depressions due to pressure from underlying cells. The deeper

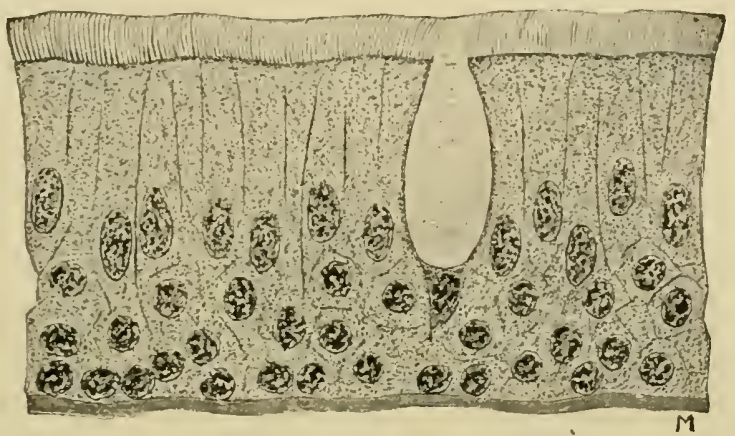

FIG. 22.--Stratified Columnar Ciliated Epithelium from the Human Trachea. A mucous (goblet) cell also is present.

cells are polygonal or irregularly cuboidal. This form of epithelium lines the bladder, ureter, pelvis of the kidney, and prostatic portion of male urethra.

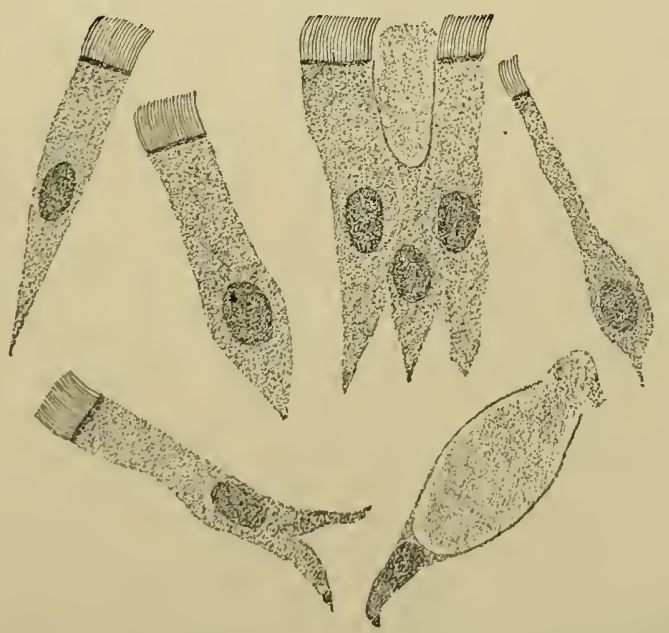

FIG. 23.-Isolated Ciliated Cells and Goblet Cells from Dog's Trachea. $\times 700$.

\section{Modified Forms of Epithelium}

(a) Cilialed Epithelium.--In this form of epithelium, fine hair-like processes-cilia-extend from the surface of the cell. These cilia vary from twelve to twenty-five for each cell and may be short as in 
the trachea or long as in the epididymis. There is usually a welldefined cuticula from which the cilia appear to spring. According to Apáthy, the cilia extend through the cuticula, giving to the latter a striated appearance (Fig. 24). Just beneath the cuticula each cilium shows a swelling-the basal granule. Lenhossék considers these

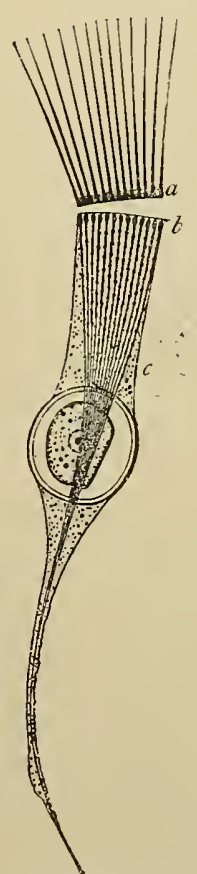

FIG. 24. granules centrosomes. The intracellular extensions of the cilia converge toward the nucleus, and are continuous with the reticular or fibrillar structure of the cell body. The motion of cilia is wave-like, the wave always passing in the same direction. Various explanations of ciliary motion have been given. It has been suggested that it is due to the contractile powers of the spongioplasm, also that it is due to changes in surface tension of the film of protoplasm which covers each cilium.

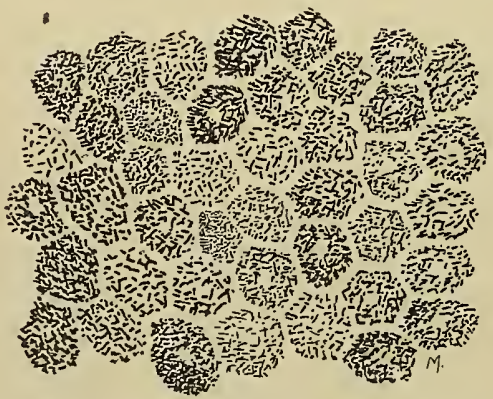

FIG. 25.

FIG. 24.-Ciliated Epithelial Cell from Intestine of Mollusk (Engelmann), showing $a$, cuticula, $b$, basal granules, and $c$, intracellular extensions of cilia.

FIG. 25.-Pigmented Epithelial Cells from the Human Retina $\left(\times_{350}\right)$, showing different degrees of pigmentation. The clear spots in the centres of the cells represent the unstained nuclei.

Cilia are confined to the surface cells of simple columnar and stratified columnar epithelium.

Simple columnar ciliated epithelium occurs in the smaller bronchi, uterus, Fallopian tubes and central canal of the spinal cord.

Stratified columnar ciliated epithelium occurs in large bronchi, trachea, larynx, nose, Eustachian tube, vas deferens and epididymis.

(b) Pigmented epithelium consists of cells the cytoplasm of which contains brown or black pigment. It is usually present in the form. of spherical or rod-like granules. Examples of it are seen in the pigmented epithelium of the retina and in the pigmented cells of the deeper layers of the epidermis in colored races (Fig. 25). 
(c) Glandular Epithelium.-This forms the essential or secreting element of glands and is mostly of the simple cylindrical variety. The different kinds of glands and their epithelia will be described among the organs.

(d) Neuro-epilhelium.-This is a highly specialized form of epithelium which occurs in connection with the end organs of nerves, under which heading it will be described.

\section{Mesothelium and Endothelium}

While recognizing the present tendency toward considering those tissues formerly classified as endothelium, as simple squamous epithelium, the correctness of the newer classification still remains sub judice and, so long as this is the case, we prefer to retain the certainly

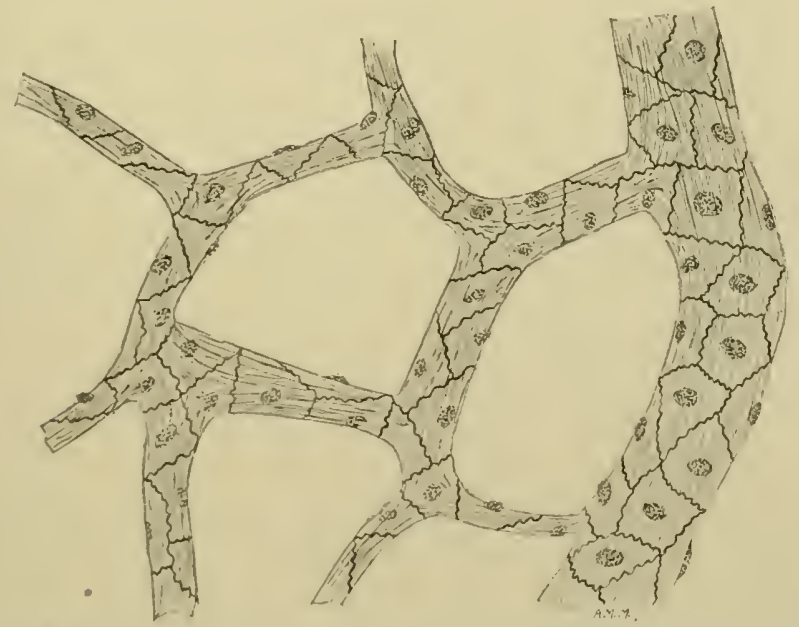

FIG. 26.-Mesothelium from Omentum of Dog Treated according to Technic 7 , p. 79. $\times 350$. Black wavy lines indicate the intercellular cement substance. The mesothelial cells cover the strands of connective tissue, the fibres of the latter being visible through the transparent cell bodies.

much more convenient classification of Minot, which coincides with his subdivision of the mesoblast. According to this classification for those tissues which resemble epithelium in structure and which are derived from the sublayer of the mesoderm which lie designates the mesenchyme, the term endothelium is retained. The term mesolhelium is used for those tissues which resemble epithelium but are derived from a subdivision of the mesoderm which he designates the mesothelial layer. 
Mesothelium and endothelium are similar in structure. Each consists of thin flattened cells with clear or slightly granular protoplasm and bulging oval or spherical nuclei. The edges of the cells are usually wavy or serrated. The cells are united by an extremely small amount of intercellular "cement" substance, which is usually indistinguishable except by the use of a special technic.

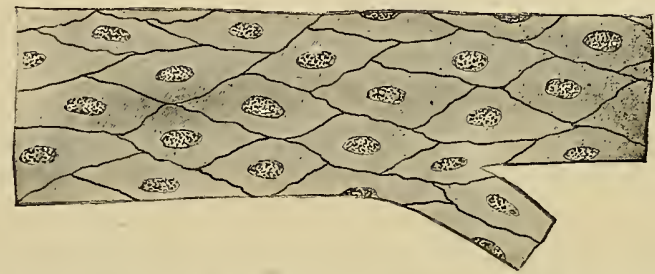

Fig. 27. -The Endothelium of a Small Blood-vessel. Silver nitrate stain. $\quad \times 350$.

Endothelium forms the walls of the blood and lymph capillaries and lines the entire blood-vessel and lymph-vessel systems.

Mesothelium lines the body cavities - the pleura, the pericardium and the peritoneum.

Recent researches make it seem probable that the surface cells of serous membranes are modified connective-tissue cells rather than epithelium.

\section{TECHNIC}

I. Simple Squamous Epithelium.-That of the lung may be demonstrated by injecting with silver solution (technic I, p. 28) through a bronchus and then immersing the tissue in the same solution. The lungs of young kittens furnish especially satisfactory material.

2. Simple Columnar Epithelium.-A piece of small intestine, human or animal, is pinned out flat on cork and fixed in formalin-Müller's fluid (technic 5, p. 7). Sections are cut perpendicular to the surface, stained with hæmatoxylin and eosin (technic r, p. 20) and mounted in glycerin tinged with eosin (p. 22). Little processes known as villi project from the inner surface of the intestine. These are covered by a single layer of columnar epithelial cells. The cuticulæ and cuticular membrane are usually well shown. Among the simple cylindrical cells are seen large clear or slightly blue-stained cells. These are known from their secretion as mucous cells, from their shape as goblet cells, and are classed as modified epithelium of the glandular type. These should be studied in their various stages of secretion, from the cell in which only a small amount of mucus is present near the outer margin, to the cell whose protoplasm is almost wholly replaced by mucus. Some cells will be found in which the surface has ruptured and the mucus can be seen pouring out of the cell. 
3. Stratified Squamous Epithelium.--The cornea furnishes good material for the study of stratified squamous epithelium. An eye is removed from a freshly killed animal and the cornea cut out and fixed in formalin-Müller's fluid. Sections are cut perpendicular to the surface, and treated as in the preceding. The cells are laid down in from six to eight layers. The œsophagus may be used instead of the cornea, its mucous membrane being lined by a somewhat thicker epithelium.

4. Transitional Epithelium.-This is conveniently studied in the mucous membrane of the bladder. Technic same as 2, above.

5. Stratified Columnar Epithelium.-A portion of trachea from a recently killed animal is treated according to the same technic. The surface cells are ciliated so that this specimen also serves to demonstrate that type of modified epithelium. Isolated cells or clumps of cells may be obtained from the trachea in the manner described in technic 3, p. 63 .

6. Pigmented Epithelium. - Fix a freshly removed eye in formalin-Müller's fluid (p. 7). After hardening, cut transversely and remove the vitreous and retina. The pigmented cells remain attached to the inner surface of the chorioid, and may be removed by gently scraping. They may be preserved and mounted in glycerin.

7. Mesothelium.-Part of the omentum of a recently killed animal is removed and washed in water, care being taken not to injure the tissue in handling. The water is then replaced by a I to 500 aqueous solution of silver nitrate. After half an hour the specimen is removed from the silver, washed in water, transferred to 8o-per-cent. alcohol and placed in the sunlight until it becomes light brown in color. It is then preserved in fresh 8o-per-cent. alcohol. The nuclei may be stained with hæmatoxylin (stain 5, p. I8). The specimen should be mounted in glycerin. Wavy black lines indicate the intercellular cement substance. The nuclei of the mesothelial cells are stained blue, those of the underlying connective-tissue cells a paler blue. It must be borne in mind in studying this specimen that the strands or trabeculæ of the omentum are not composed of mesothelium, but of fibrous connective tissue, and that the flat mesothelial cells merely lie upon the surface of the connective-tissue strands.

8. Endothelium may be demonstrated by removing the bladder from a recently killed frog, distending it with air and subjecting it to the same technic. By this means the intercellular substance of the endothelium of the blood-vessels of the bladder wall is stained and the outlines of the cells are thus shown. 


\section{CHAPTER III}

\section{THE CONNECTIVE TISSUES}

Under this head are classified Connective Tissue Proper, Cartilage, and Bone.

General Characteristics. - The most prominent characteristic of the connective tissues is the predominance of the intercellular substance. In this respect the connective tissues differ markedly from epithelium. Moreover it is the intercellular substance and not the cells which determines the physical character of the tissue. Thus, for example, the hardness of bone and teeth, the firmness and elasticity of cartilage, the toughness of tendon, the softness of subcutaneous connective tissue, are all due to the character and arrangement of the intercellular elements. In most forms of supportive tissue the cellular elements are very similar and in no way determine the physical character of the tissue.

The rôle of the connective tissues is mainly passive, and the cells, instead of playing the most active part, as in epithelial, muscle and nerve tissues, serve mainly for the maintenance of the nutrition of the more important intercellular substance. The latter thus predominates in function as well as in quantity, its character according with the specific function of the tissue. Thus, where strength and preservation of form are essential, are found connective tissues with such intercellular substance as occurs in bone; where softness and flexibility are required, such loosely arranged intercellular elements as are present in areolar tissue.

Most of the connective tissues are extremely vascular, differing also in this respect from epithelium. An exception to this rule is hyalin cartilage.

All of the connective tissues have a common origin in the more loosely arranged portion of the mesoderm which is known as the mesenchyme.

A very close relation exists between the different forms of connective tissue as evidenced by a marked tendency and ability of one form to be closely united to, or to be transformed into, or to be 
replaced by, another form. This may be progressive in line of development or retrogressive in line of degeneration. For example the close physical union which exists between tendon and bone or between cartilage and bone or tendon: or the manner in which the forms of bone are first laid down in connective tissue, which is replaced by bone, or by cartilage followed by bone. Again in the callus which occurs in the uniting of fractures, there is first fibrous tissue, then bone, or there may be an intervening cartilaginous stage, or repair may fail of completion, leaving permanent connective tissue or cartilage.

The correlation of the different forms of connective tissue is also shown by the fact that in different species the same anatomical structure is sometimes formed by different members of the group. Thus in some birds the leg tendons are formed of bone, while in certain fish the skeletal system is cartilaginous. In Mammals the sclera of the eye is fibrous connective tissue. In Batrachians the same structure is cartilage, in Birds, bone.

\section{Classification:-}

Connective Tissue $\left\{\begin{array}{c}\begin{array}{c}\text { Connective Tissue } \\ \text { Proper }\end{array} \\ \text { Cartilage }\left\{\begin{array}{l}\text { Hyalin } \\ \text { Fibrous } \\ \text { Elastic }\end{array}\right. \\ \text { Bone }\end{array}\right.$
(a) loose or areolar (fat tissue, pig- mented tissue)
(b) tend o n-aponeu- rosis

The mesenchyme consists at first wholly of small spheroidal or ovoid cells These cells proliferate and elaborate a substance which separates the cells from one another and is the primitive ground or intercellular substance. As the cells separate they assume irregular stellate branching forms and anastomose to form a network or syncytium. Such a tissue is known as mucous or embryonal connective tissue (Fig. 28), is found in the adult only in the umbilical cord and possibly in the vitreous of the eyc, but in the embryo is widely distributed. With the exceptions noted it represents but a stage in the development of the more specialized forms of connective tissue.

Portions of this embryonal connective tissue develop fibres in the ground substance. Some of these fibres are known as white or fibrillated fibres or, because of their chemical constitution, as collagenous fibres. Others are known as yellow or elastic fibres and consist of clastin. This differentiation of the ground substance gives rise to connective-tissue proper. At other points in the mesenchyme, cartilaginous material appears in the ground substance. These points are numerous and widely separated and each represents a chondrification center or point of development of cartilage. At some points in the con- 
nective tissue or in the cartilage there are depositions of lime salts in the ground substance-calcification. This is followed by the formation of true boneossification.

Regarding the development of the connective-tissue fibrils, there are two theories: (I) According to one, they are developed directly from the protoplasm of the connective-tissue cells. The cells increase in length, and fine granules appear, which arrange themselves in rows in the cytoplasm; these granules unite to form fibrils. Such cells are known as fibroblasts, and their fibrils are the forerunners of the intercellular fibrils of connective tissue. A modification of this theory derives the fibrils from the peripheral portion of the cell-the exoplasm. (2) According to the other theory the fibrils are developed from the matrix, minute granules first becoming arranged in rows and later uniting to form fibrils.

Regarded as opposing theories, there is in reality but little antagonism between them. There is no doubt as to the intercellular matrix being a product of the cell. Whichever theory, therefore, is accepted, the entire intercellular substance, fibres and ground substance are ultimate derivatives of the cell. Recent studies, especially those of Mall, are confirmatory of the second of the theories given above. He maintains that the connective-tissue fibrils, both white and elastic, are derivatives of an active intercellular matrix, which latter is a direct product of the cell.

Two similar theories exist as to the development of elastic fibres, a cellular theory and an extracellular theory. According to some advocates of the cellular theory, the elastic fibres are derived from the exoplasm; according to others, from the cytoplasm immediately surrounding the nucleus. Recent researches favor the extracellular theory. Mall describes extremely minute fibrils in the ground substance, which later develop irto elastic fibres.

\section{CONNECTIVE TISSUE PROPER}

(Mucous Tissue-Gelatinous Tissue)

\section{EMBRYONAL CONNECTIVE TISSUE}

This is the least differentiated of the connective tissues and has been already partly described (p. 8I). It occurs only in the fœtus. It consists of irregular stellate, branching cells which anastomose, and are irregulary scattered through an apparently structureless ground substance rich in mucin (Fig. 28).

\section{FIBRILLAR CONNECTIVE TISSUE}

Fibrillar connective tissue, also known as white fibrous tissue or connective tissue proper, consists of cells and fibres lying in a basement or ground substance. The elements of fibrillar tissue may be classified as follows: 
Cells

I. Fixed

(a) Ordinary connective-tissues cells.

(b) Plasma cells.

(c) Mast cells.

(d) Clasmatocytes

2. Wandering.

Intercellular substance $\left\{\begin{array}{l}\text { (a) Fibres }\left\{\begin{array}{l}\text { white or fibrillated, } \\ \text { yellow or elastic. }\end{array}\right. \\ \text { (b) Ground or basement substance. }\end{array}\right.$

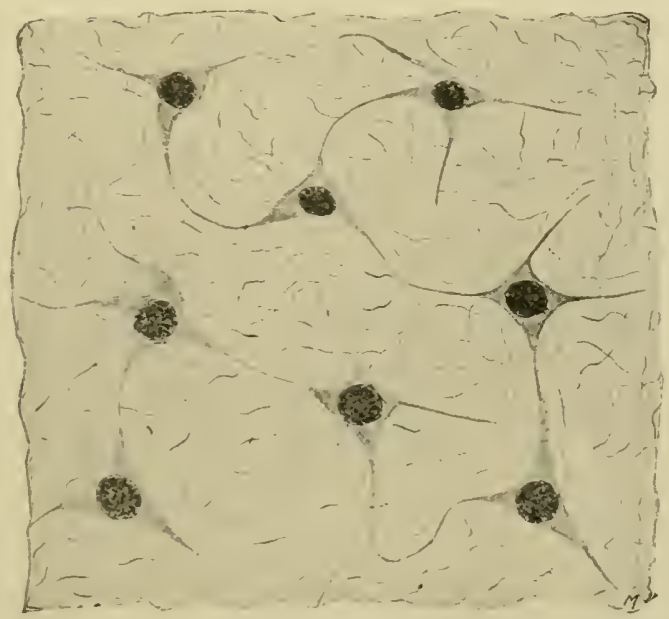

FIG. 28.-Mucous Connective Tissue from Umbilical Cord of Eight-inch Fœtal Pig. $\times 600$. At this stage the ground substance shows some fibrillation.

Connective-tissue Cells.-(a) The ordinary fixed connectivetissue cell is often the only connective-tissue cell seen in ordinary sections. It is an irregular shaped cell, often quite flattened with clear or slightly granular cytoplasm and an oval nucleus. (Figs.29 and 30.) In looscly arranged tissue when the cells are well separated, the cell is usually stellate with many branches, which anastomose with branches of neighboring cells. In a dense tissue such as the cornea, these cells apparently lie in little cell spaces or lacunx from which minute channels (canaliculi) extend in all directions to unite with canaliculi from adjoining spaces (Fig. 3I). Delicate cell processes extend into the canaliculi and there anastomose with processes from other cells thus forming a sort of syncytium (Fig. 32). Owing to the extreme sensitiveness of the 


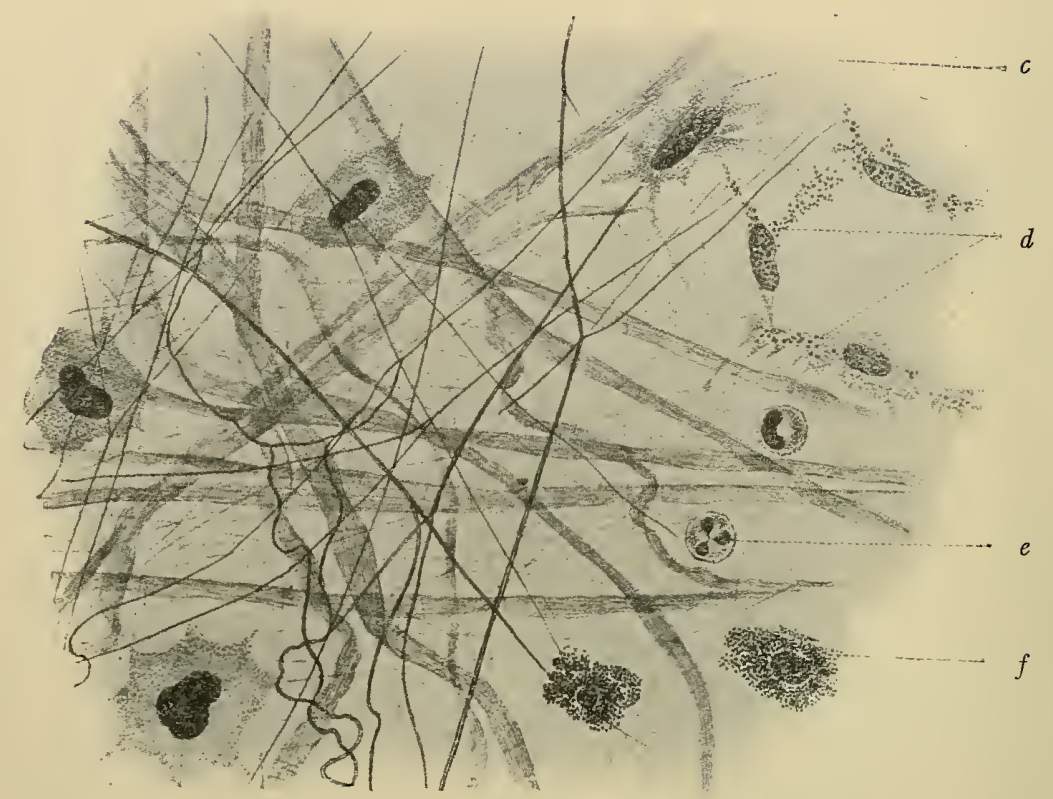

FIG. 29.-Areolar Connective Tissue (Rauber-Kopsch).

$a$, White fibre. $b$, Elastic fibre. $c$, Fixed connective tissue cell. $d$, Clasmocyte. $e$, Leucocyte (wandering cell). $f$, Mast cell.

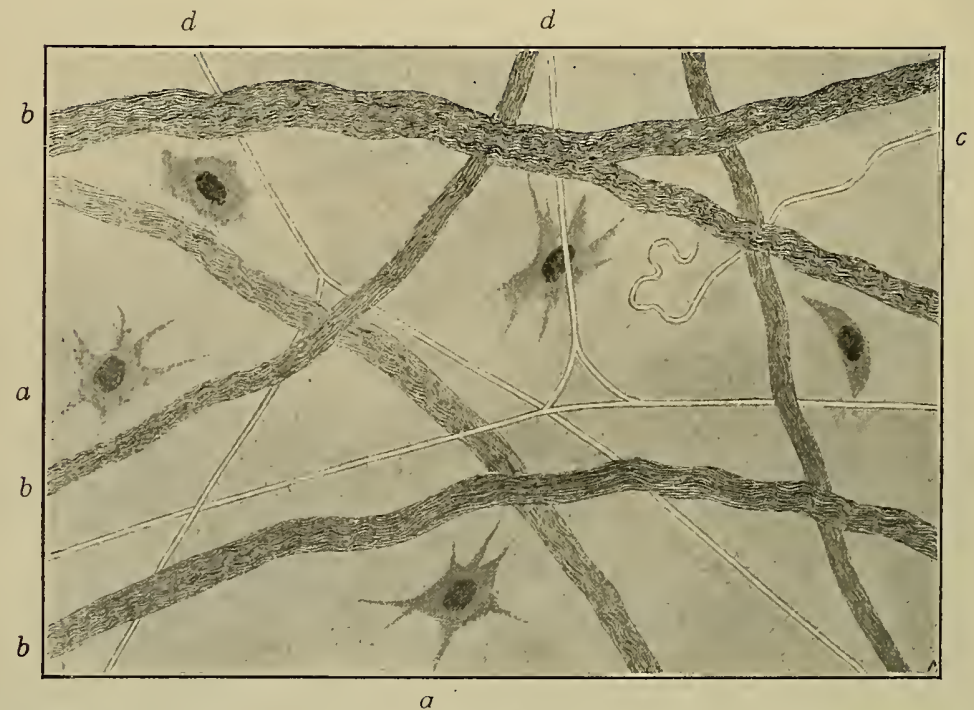

FIG. 30.-Fibrillar Connective Tissue (Areolar Type) from Subcutaneous Tissue of Rabbit (technic 2, p. 96). $\times 500 . \quad a$, Fixed connective-tissue cell; $b$, fibrillated fibres; $c$ elastic fibre with curled broken end; $d$, elastic fibres showing Y-shaped branching. 
protoplasm of the connective-tissue cell to most fixatives, its usual appearance is that of a minute amount of cytoplasm shrunken down around a nucleus. Somewhat similar cells with more coarsely granular or vacuolated cytoplașm have been designated "clasmocytes" (Ranvier). By some these are believed to be of leucocyteorigin, by others to be an earlier stage in the development of the fixed connective-tissue cell.

(b) Plasma Cells.-These cells occur mainly near the smaller blood-vessels. Their protoplasm is finely granular and stains with

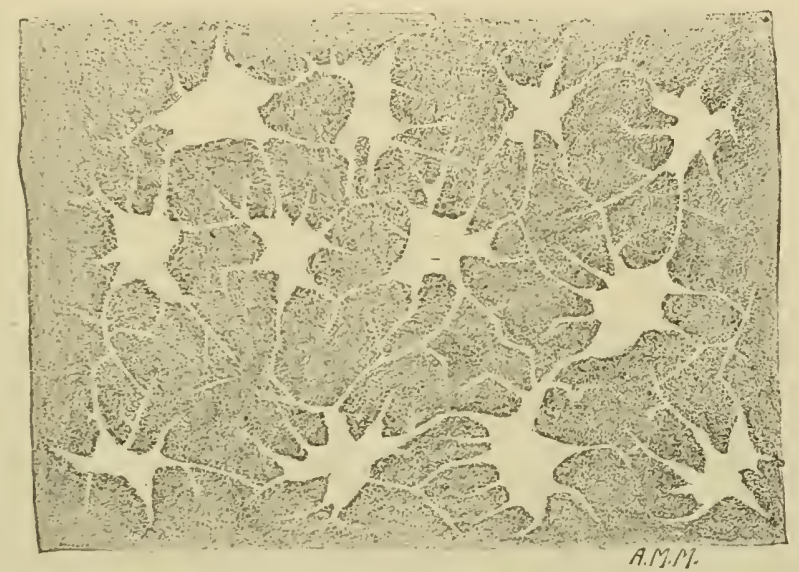

FIG. 3r. - Section of Human Cornea cut Tangential to Surface. $\times_{350}$. (Technic 9, p. 97.) Connective-tissue Cell Spaces (Lacunæ) and Anastomosing Canaliculi, white; whole Intercellular Substance (Ground Substance and Fibres), dark.

basic aniline dyes. They frequently contain vacuoles. Their nuclei are small, spherical and usually excentric. Small plasma cells are about the size of leucocytes, which they closely resemble. Large plasma cells are larger than leucocytes and richer in protoplasm. Some consider them as derived from leucocytes, others as a modified form of the ordinary fixed connective-tissue cell.

(c) Mast cells are spherical or irregular-shaped cells, found like the preceding in the neighborhood of the blood-vessels. Their protoplasm contains coarse granules which stain intensely with basic aniline dyes (Fig. 29). They are believed by some investigators to be connected with the formation of fat; by others to represent a stage in the development of the fixed connective-tissue cell.

(d) Clasmatocytes. - These are large, mostly spindle shaped cells with granular protoplasm. 
Connective-tissue cells may be pigmented (Fig. 33). In such cells the cytoplasm is more or less filled with brown or black pigment granules. In man pigmented connective-tissue cells occur in the skin, chorioid and iris.

The so-called wandering cells (Fig. 29) are not properly a part of

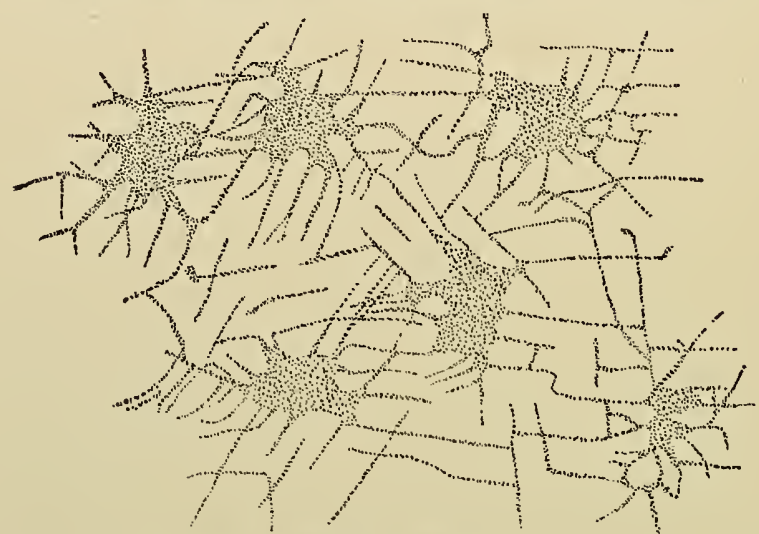

FIg. 32. -Section of Human Cornea cut Tangential to Surface. $\times 35 \circ$. (Technic 8, p. 97.) Connective-tıssue Cells with Anastomosing Processes, stained; Intercellular Substance: (Ground Substance and Fibres), unstained.

connective tissue, being merely amœboid white blood cells (see page I07) which have passed out from the vessel into the tissues. They are not peculiar to connective tissue, being found in other tissues, e.g., in epithelium.

The Intercellular Substance.-(a) Fibres. White or fibrillated

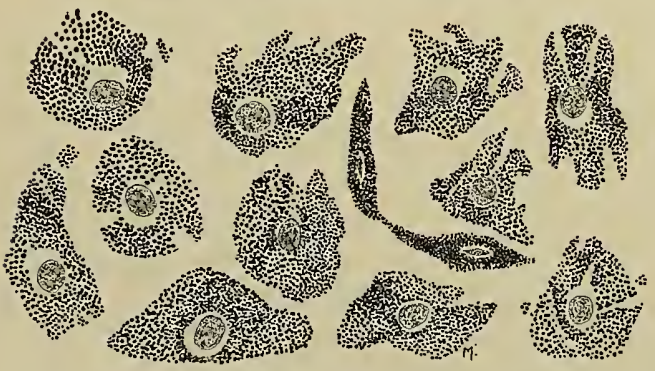

FIG. 33.-Pigmented Connective-tissue Cells from Chorioid Coat of Human Eye. $\times 35 \circ$. (Technic 7, p. 97.) fibres are bundles of extremely fine fibrillæ $\left(0.5^{\mu}\right.$ in diameter) (Fig. 30). The fibrillæ lie parallel to one another and are united by a small amount of cement substance. The fibrillæ do not branch. The fibre bundles, on the other hand, branch dichotomously and anastomose. White fibres, on boiling, yield gelatin.

Yellow or elastic fibres are apparently homogeneous, highly refractive fibres, varying in diameter from I to Ior (Fig. 30). They branch and anastomose, forming networks. The smaller fibres are 
round on cross section, the larger flattened or hexagonal (Figs. 43 and 44). Their elasticity is easily demonstrated in teased specimens by curling of the broken ends of the fibres (Fig. 30). On boiling they yield elastin. Although, when subjected to the usual technic, elastic fibres appear homogeneous, they are probably composed of a thin sheath or membrane, enclosing the more granular elastin. The latter stains intensely with magenta, the sheath remaining unstained.

In addition to the white fibres and elastic fibres above described, so-called "reticular" fibres are frequently present in fibrillated connective tissue. (See p. 82.)

(b) Basement or ground substance occurs in extremely minute amounts between the individual fibrillæ of the white fibres, where it acts as a cement substance. The same material also forms the basement or ground substance in which the connective-tissue cells and fibres lie (Fig. 3I). Difficulty in seeing this ground substance is due to its transparency. It may be demonstrated by staining with silver nitrate. (See technic 9, p. 97.)

Much variation exists in regard to the proportions of the different elements. This gives rise to variations in the physical characteristics of the tissue. When fibres predominate over cells and ground substance, the tissue is dense and hard and is known as dense fibrous tissue. The terms fine connective tissue and coarse connective tissue designate the character of the fibres. When many cells are present, the tissue is softer and is known as cellular connective tissue.

According to the arrangement of the white fibres, fibrous connective tissue is subdivided into areola or loose connective tissue and formed connective tissue.

\section{Areolar or Loose Connective Tissue}

In this the fibres are irregular, running in all directions and interlacing, leaving between them meshes or areole (Fig. 30).

Subcutaneous connective tissue is a typical example of areolar tissuc. Both white and elastic fibres are present, although the former predominate. Areolar tissue varies greatly as regards the relative number of cells and fibres and the closeness with which the different elements are packed. It thus varies greatly in density.

Fat Tissue.-Adipose tissue or fat tissue is a form of areolar tissuc in which some of the cells have become changed into fat cells. Fat tissue is peculiar among the connective tissues in that the cells and not the intcrcellular substance make up the bulk and determine the 
character of the tissue. The adult fat cell is surrounded by a distinct cell membrane, and almost the entire cell is occupied by a single droplet of fat (Figs. 35 and 36). The nucleus, flattened and surrounded by a small amount of cytoplasm, is usually found pressed against the cell wall (Fig. 36). This appearance of a distinct cell membrane enclosing the spherical fat droplet, with the nucleus and cytoplasm pressed into a cresent-shaped mass at one side, has given rise to the term "signet-ring cell." Fat cells which occur singly,

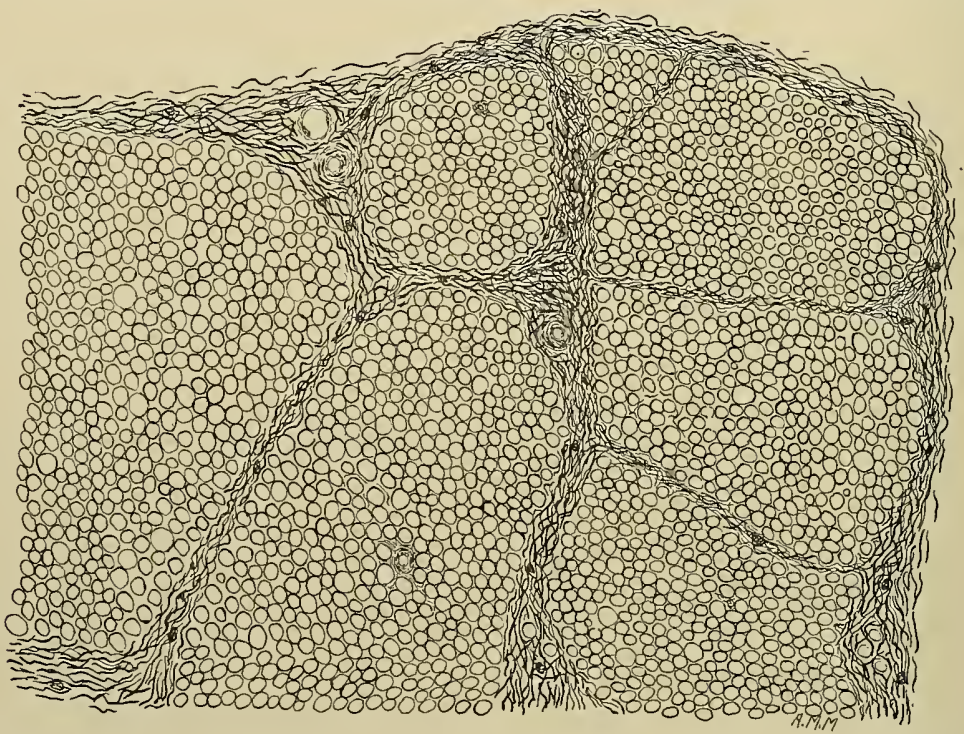

FIg. 34--Fat Tissue from Human Subcutaneous Tissue (Cliild) to show Lobulation. $\times 25$. (Technic $x$, p. 95.)

or in small groups, or in the developing fat of young animals are spherical (Fig. 35). In large masses of adult fat, the closely packed cells are subjected to pressure and are polyhedral (Fig. 36). Fat cells are usually arranged in groups or lobules, each lobule being separated from its neighbors by fibrillar connective tissue (Fig. 34).

The appearance which adult fat presents can be understood only by reference to its histogenesis. Fat cells are developed directly from embryonic connective-tissue cells. In the human embryo they are first distinguishable as fat cells about the thirteenth week. The connective-tissue cells which are to become fat cells gather in groups in the meshes of the capillary network which marks the ending of a small artery. Each group is destined to become an adult fat lobule (Fig. 37). 
a. $b$

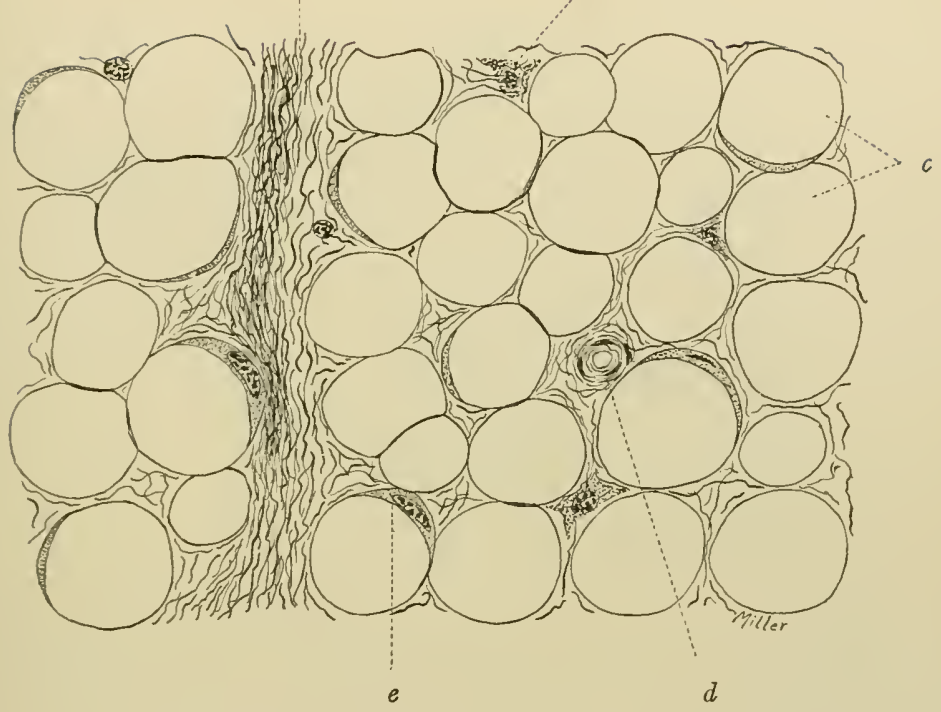

FIG. 35.-Young Fat from Human Subcutaneous Tissue. (Child.) XI75. (Technic I, p. 95.) $a$, Interlobular connective tissue; $b$, fixed connective-tissue cell; $c$, fat cells; $d$, artery; $e$, nucleus of fat cell and remains of cytoplasm ("signet ring").

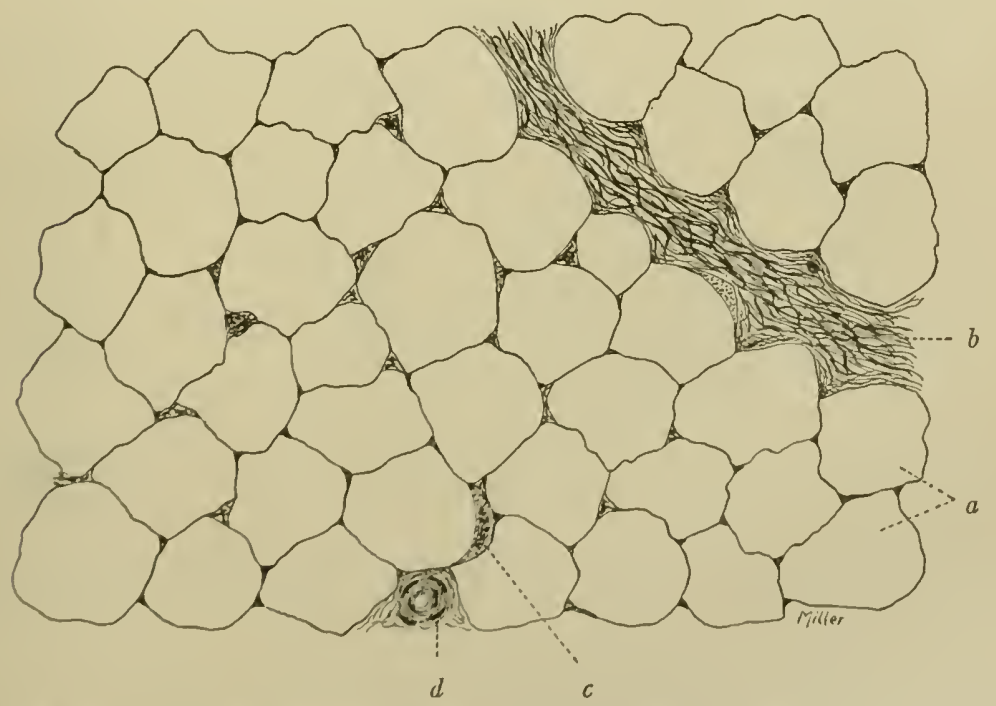

Fis: 30.-Adult Fat Tissue from Human Sulscutancous Tissue. $X_{175}$. (Technic I, p. 95.) $a$, lat cclls; $b$, interlobular connective tissue; $c$, nucleus of fat cell and remains of cytroplasm ("signet ring"); $d$, artery. 
Fat first appears as minute droplets in the cytoplasm of the embryonic connective-tissue cell (Fig. 38). These small droplets in-

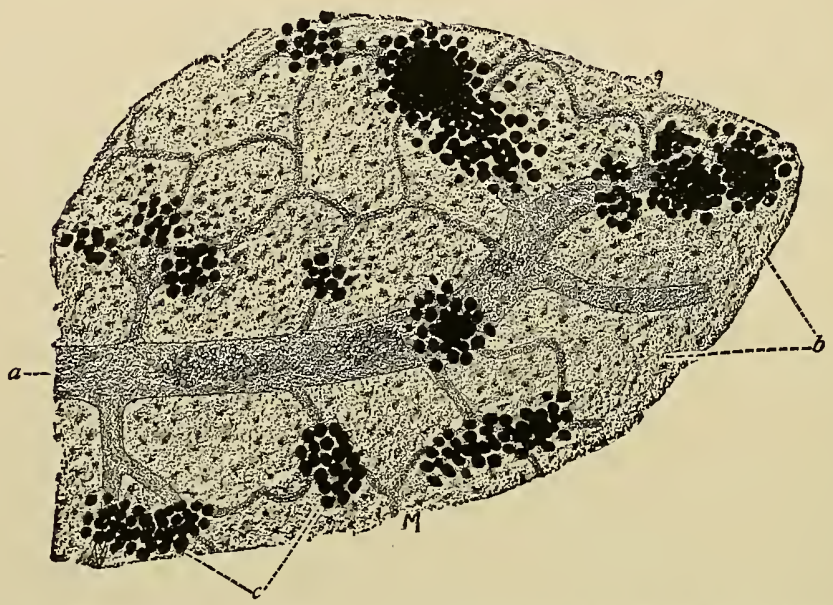

FIG. 37--Developing Fat Tissue from Subcutaneous Tissue of Five-inch Fœtal Pig. $\times 75$. (Technic 2, p. 96.) $a$, Arteriole breaking up into capillary network; $b$, embryonal connective tissue; $c$, embryonal fat lobule developing around blood-vessels.
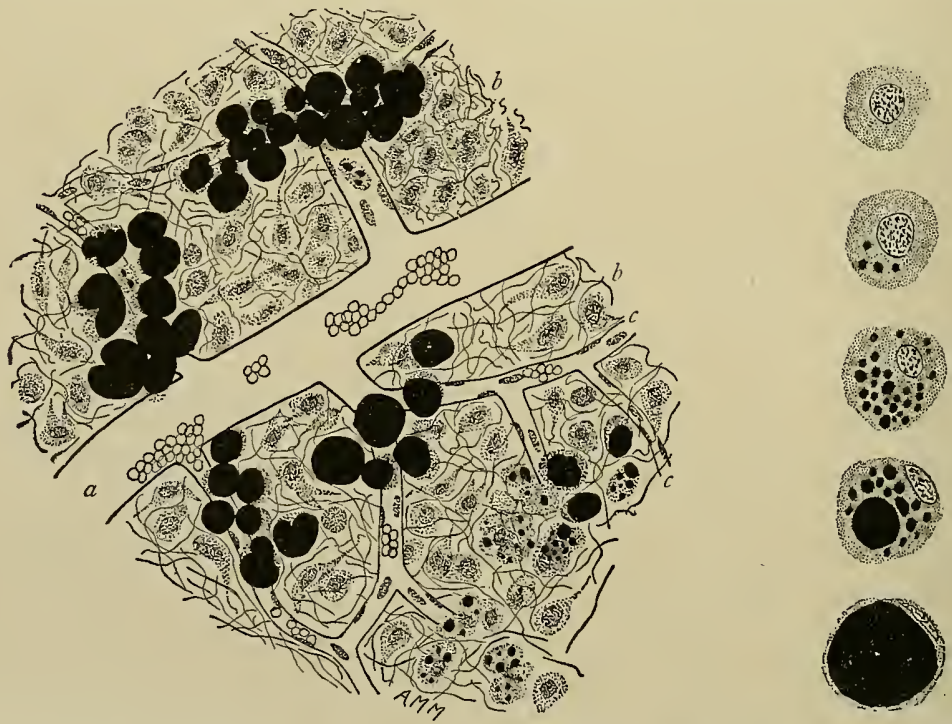

Fig. 38.-Developing Fat Tissue from Subcutaneous Tissue of Five-inch Fœtal Pig. (Technic 2, p. 96.) $a$, Arteriole breaking up into capillary network; $b$, embryonal connective tissue, embryonal cells from which fat cells are developing; $c$, capillaries. Fat droplets stained black. At the right are five individual cells showing stages of development from an embryonal cell to an adult fat cell.

crease in number and finally coalesce to form a single larger droplet. This increases in size and ultimately almost wholly replaces the cyto- 
plasm. In this way the nucleus and remaining cytoplasm are pressed to one side and come to occupy the inconspicuous position which they have in adult fat.

The blood supply of fat is rich and the adult lobule maintains its embryonic vascular relations, in that the vascular supply of each lobule is complete and independent. One artery runs to each lobule, where it breaks up into an intralobular capillary network, which in turn gives rise to the intralobular veins, usually two in number.

Fat is thus seen to be a connective tissue in which some of the cells have undergone specialization. There still remain, however, embryonal connective-tissue cells which are not destined to become fat cells, but which develop into cells and fibres of ordinary fibrous connective tissue. A few of these remain among the fat cells to become the delicate intralobular connective tissue seen in adult fat. The majority are, however, pushed to one side by the developing lobules, where they form the interlobular septa.

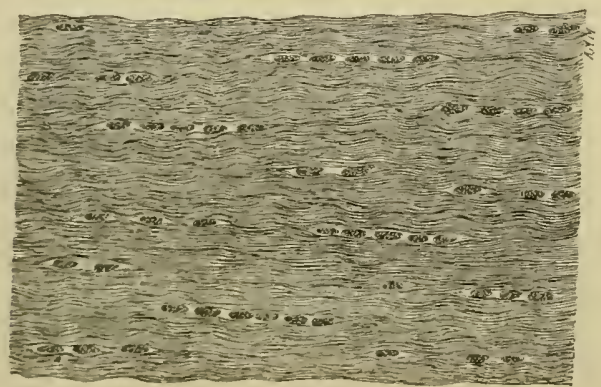

FIG. 39.

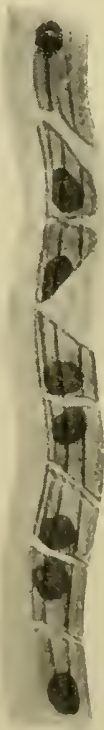

FIG. 40.

FIG. 39.-Longitudinal Section of Tendon from Frog's Gastrocnemius. $\times_{25}$. The nuclei of the flattened cells are seen lying in rows between the connective-tissue fibres.

FIG. 40.- Teased tendon fibres with cells lying on their surface. $\times_{400}$. (Ranvier.)

Formed Connective Tissue.-In formed connective tissue the tissue elements instead of being disposed irregularly as in areolar tissue, are arranged with some regularity or order, thus giving the tissue more or less definite form.

Tendons and ligaments are examples of formed connective tissue in which the fibres all run in approximately the same direction (Fig. 39). Elastic fibres are absent or present in very small numbers. The predominance of the white fibres and their parallel arrangement result in great strength with almost no extensibility. While 
the individual fibrils do not branch, groups of fibrils pass from one bundle to another. There is little ground substance and cells are comparatively few. The latter are, however, so characteristic as to have received the name of tendon cells (Fig. 4I). They areirregularly rectangular cells, have rather more breadth than length and are arranged in characteristic rows between the fibre bundles. The cell margins are contiguous and the usually excentric nuclei tend to lie in adjacent sides of two cells, thus giving the cells the appearance of being arranged in pairs (Fig. 40). Thin plate-like extensions of the

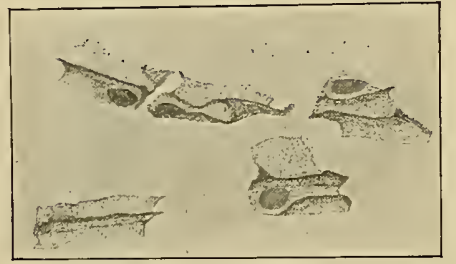

FIG. 4I.

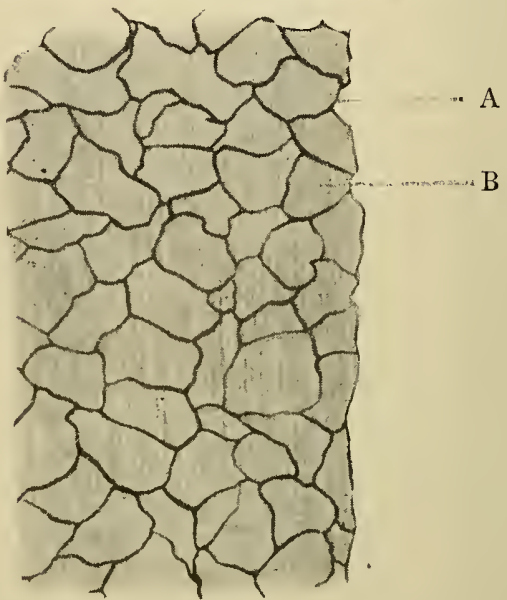

FIG. 42 .

FIG. 4I.-Tendon Cells from the Tail of a Rat. Stained in methylene-blue (intra vitam). (Bohm-Davidoff.)

FIg. 42.-Pavement Endothelium of Tendon of Rat. $A$, intercellular substance impregnated with silver nitrate; $B$, tendon fibres. $\times \operatorname{Ir}_{5}$. (Branca.)

cell (Fig. 4I) pass into the ground substance between the fibre bundles and when the cell is seen on flat, the greater thickness of the cell through the extensions gives the optical effect of dark lines in the cell body.

Aponeuroses.-In thin aponeurotic tissue the fibres are disposed in two planes, the fibres of one plane running approximately at right angles to those of the other plane. The cells resemble tendon cells and lie upon the fibre bundles. In thicker aponeuroses the fibres are arranged in planes but their disposition is more irregular.

\section{Elastic Tissue}

Elastic fibres occurring in fibrous connective tissue have been described. When the elastic fibres predominate the tissue is known as elastic tissue. Almost pure elastic tissue is found in the ligamentum nuchæ of quadrupeds. In man it occurs mainly in the ligamenta subflava, in some of the laryngeal ligaments, in the walls 
of the trachea and bronchi and of arteries. In the ligamentum nuchæe the fibres are coarse and arranged in bundles separated from one another by white fibrous tissue containing connective-

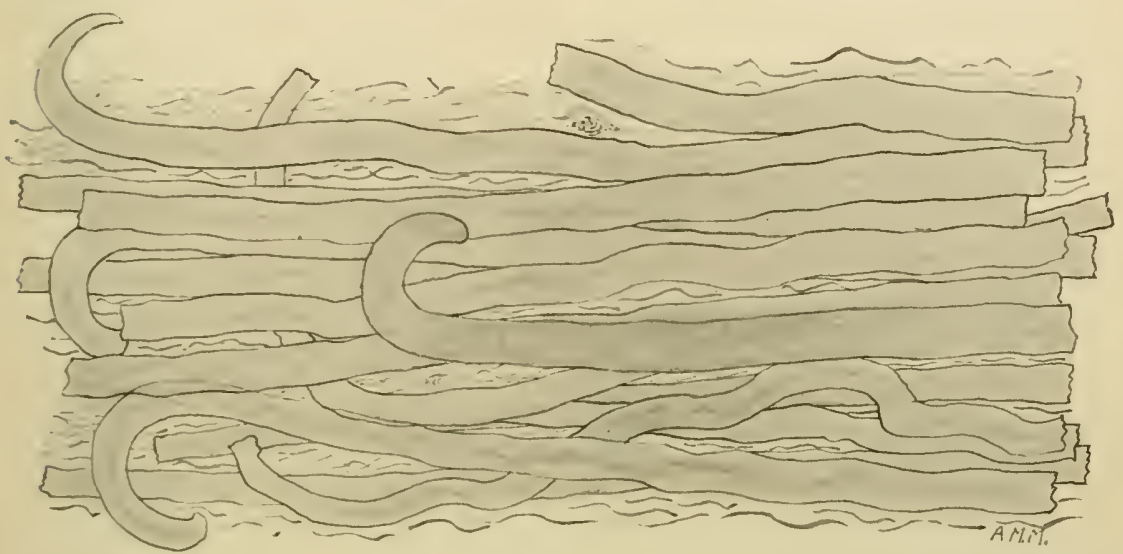

FIG. 43.-Coarse Elastic Fibres from Ligamentum Nuchæ. $\times_{500 .}$ Teased specimen. (Technic ro, p. 97.)

tissue cells. This white fibrous tissue also penetrates the bundles and separates the individual elastic fibres. A few white fibres and connective-tissue cells are also present (Figs. 43 and 44 ).

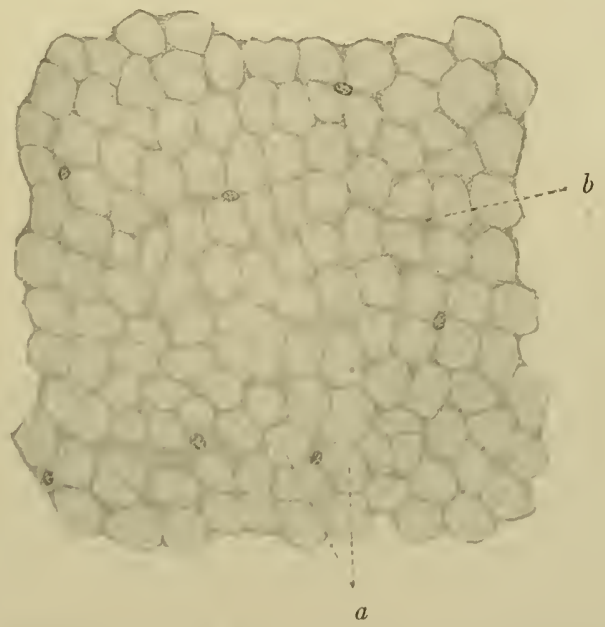

FIG. 44.-Cross Section of Coarse Elastic Fibres from Ligamentum Nuchac. $\times 500$. (Technic 10, p. 97.) $a$, Elastic fibres; $b$, white fibrous tissue and cement substance. The nuclci are the nuclei of fixed connective-Lissue cells.

Elastic tissue may be arranged as thin membranes, as $e . g$. , in the walls of blood-vessels. 'These membranes are usually described as composed of a dense mass of flat, ribbon-like elastic fibres, which 
interlace in such a manner as to leave openings in the membrane. Hence the term "fenestrated membrane." They have been recently described as consisting of a central layer composed of elastin, staining with magenta, and on either side a thin, transparent sheath unstained by magenta. This is seen to correspond to Mall's description of the structure of the elastic fibre. Only the middle of these layers is fenestrated.

\section{Reticular Tissue}

Reticular connective tissue is a form of fibrillar connective tissue. It consists of extremely delicate fibrils with no ground

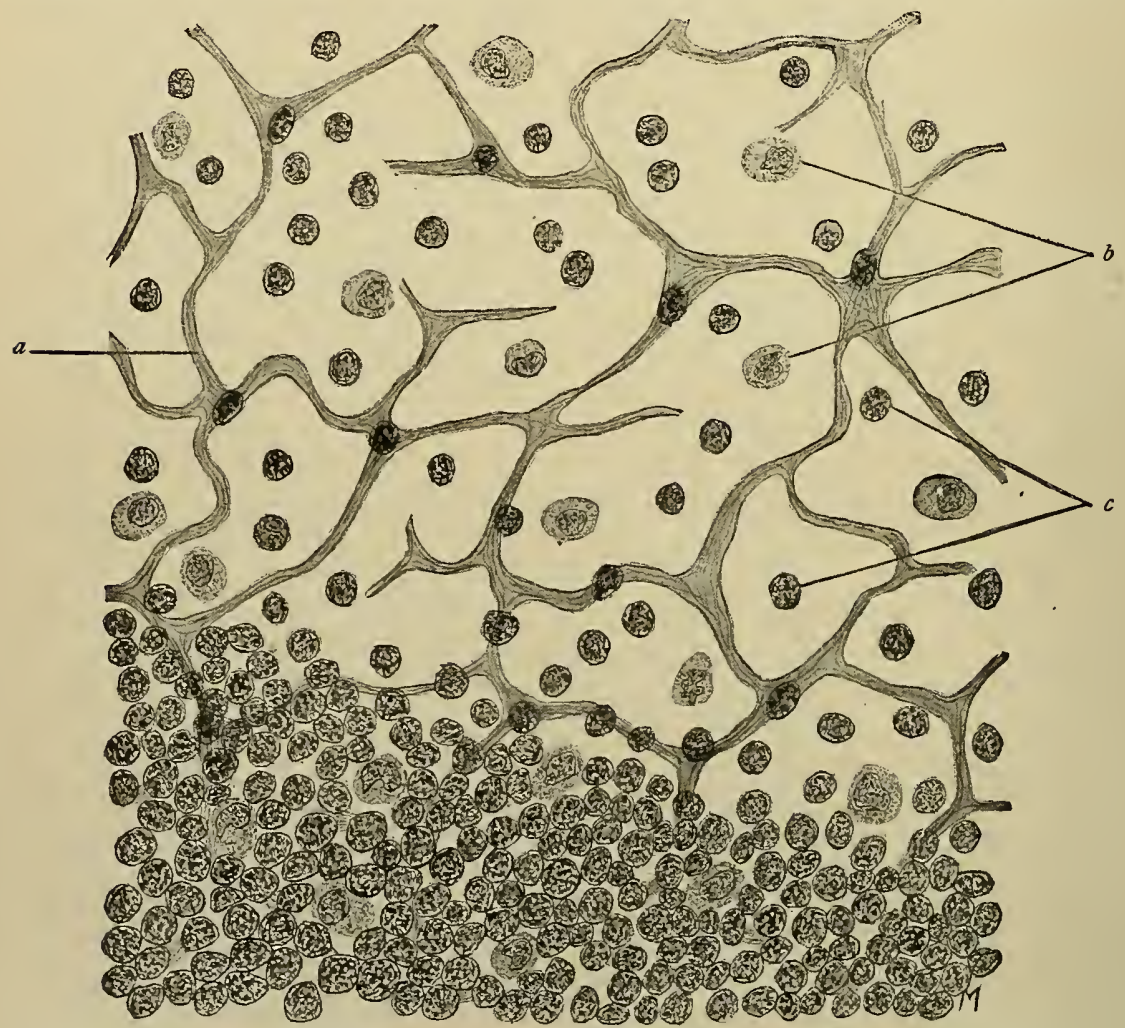

Fig. 45.-Reticular Tissue from a Human Lymph Node. (Technic, below.) a, Reticular connective tissue, in the meshes of which are suspended $b$, leucocytes, and $c$, lymphocytes. The reticular connective tissue is present also in the more dense lymphatic tissue seen in the lower part of the figure, but is not visible on account of the closely packed cells.

substance. The fibrils are grouped in larger or smaller bundles which form a network or feltwork enclosing spaces, thus constitut- 
ing a reticulum. The fibrils present much the same microscopic appearance as the white fibres of areolar tissue. Also in certain organs, e.g., the lymph nodes, the direct continuity of the frbres of the coarser fibrous tissue of the trabeculaæ with the fibrils of the reticular tissue can be easily demonstrated. In some locations, e.g., in the lymph nodes (Fig. 45) the cells of the reticular tissue lie upon the surface of the fibrils and so completely invest them that the fibrillar character of the tissue cannot be seen until the overlying cells have been removed. This led to the description of the reticulum of lymphatic tissue as composed wholly of anastomosing cells.

Reticular tissue has been described as yielding on boiling, a substance called by some elastin by others reticulin instead of gelatin which results from boiling fibrous tissue. Other recent studies upon the chemistry of reticular tissue are not however in accord with this view, reticular tissue bring found to yield gelatin on boiling. Both tissues resist pancreatic digestion, and the question as to the structural and chemical relations of the two tissues remains at present unsettled.

Reticular connective tissue forms the framework of adenoid tissue and of bone-marrow. It is also present in large amounts in the spleen and in the mucous membrane of the gastro-intestinal tract, lung, liver, kidney, and other organs where it forms the finer part of the framework, supporting the capillaries, and often apparently serving as a basement membrane for the gland cells.

\section{TECHNIC}

I. Arcolar Tissue, to show White and Elastic Fibres.-Remove a bit of the subcutaneous tissue, as free from fat as possible, from a recently killed animal. Place it upon a mounting slide and with teasing needles quickly spread it out in a thin layer. During this manipulation the specimen should be kept moist by breathing on it. Put a drop of sodium chlorid solution upon the specimen and cover.

As the specimen is unstained, a small diaphragm should be used for the microscopic examination.

The white fibres are straight or wavy, are crossed in all directions, and are longitudinally striated. The elastic fibres have been stretched and show as sharp lines with curled ends where the fibres are broken.

Place a drop of hydric acetate, I-per-cent. alqueous solution, at one side of the cover and a bit of filter patper at the other side. The filter paper absorbs the salt solution, which is replaced by the hydric acetate. The latter causes the white fibres to swell and become indistinct while the clastic fibres show more plainly. 
2. Areolar Tissue, to show Cells and Elastic Fibres.-Prepare second specimen of areolar tissue in the same manner as the preceding. Instead of mounting in salt solution, allow it to become perfectly dry, then stain in the following solution:

Gentian violet, saturated aqueous solution, Water,

Wash thoroughly, dry, and mount in balsam.
40 c.c.

60 c.c.

The nuclei of the fixed connective-tissue cells are stained violet. Their delicate cell bodies show as an irregular haze around the nuclei. Both nuclei and cell bodies appear cut in all directions by the stretched elastic fibres. Wandering cells (leucocytes) may usually be seen. Plasma cells are frequently not demonstrable, and mast cells are only occasionally present. The elastic fibres are stained violet. The white fibres are almost unstained.

While these methods are most satisfactory for bringing out the different connective-tissue elements, they are misleading to the student in that they show a picture of connective tissue after special preparation, rather than as it usually appears in sections. For contrast the student should study carefully the connective tissue as it appears in sections through the skin, the mucous membranes and other organs.

3. Formed Connective Tissue.-Fibrous tissue arranged in the form of a network may be seen in the specimen of omentum (technic 7, p. 79).

4. Densely formed connective tissue may be studied in tendon. Cut through the skin of the tail of a recently killed mouse about half an inch from the tip and break the tail at this point. By pulling on the end of the tail this portion may now be separated from the rest of the tail, carrying with it long delicate tendon fibrils, which have been pulled out of their sheaths. These should be immediately examined in salt solution, using the high power and a small diaphragm. The fibrils are seen arranged in parallel bundles.

5. Place a drop of hydric acetate (2-per-cent. aqueous solution) at one side of the cover-glass, absorbing the salt solution from the opposite side by means of filter paper. The fibres swell and become almost invisible, while rows of connective-tissue cells (tendon cells) can now be seen. The cells may be stained by allowing a drop of hæmatoxylin or of carmine solution to run under the cover. After the cells are sufficiently stained, the excess of stain is removed by washing, and the specimen mounted in glycerin.

6. Fix a small piece of any good-sized tendon in formalin-Müller's fluid (page 7). After a week, harden in alcohol, embed in celloidin, and make longitudinal and transverse sections. Stain strongly with hæmatoxylin, followed by picroacid-fuchsin (page 20). Mount in balsam.

7. Pigmented connective-tissue cells are most conveniently obtained from the chorioid coat of the eye. Fix an eye in formalin-Müller's fluid (see page 7), cut in half, remove chorioid and retina and pick off the dark shreds which cling to the outer surface of the chorioid and inner surface of the sclera. These may be transferred directly to glycerin, in which they are mounted, or the bits of tissue may be first stained with hæmatoxylin (page I 7). In addition to the pig- 
mented cells should be noted the ordinary fixed connective-tissue cells which lie among them. Only the nuclei of these cells can be seen.

S. Connective-tissue cells to show anastomosing processes.-Stain a cornea with gold chlorid (see page 28). Sections are made tangential to the convex surface and are mounted in glycerin.

9. Connective-tissue cell spaces (lacunæ) and their anastomosing canaliculi may be demonstrated by staining a cornea with silver nitrate (see page 28). The silver stains the ground substance of the cornea, leaving the lacunæ and canaliculi unstained. The relation which this picture bears to the preceding should be borne in mind (see Figs. $3 \mathrm{I}$ and 32 ).

ro. Coarse elastic fibres may be obtained from the ligamentum nuchæ, which consists almost wholly of elastic tissue. A piece of the ligament is fixed in saturated aqueous solution of picric acid and hardened in alcohol. A bit of this tissue is teased apart on a glass slide in a drop of pure glycerin, in which it is also mounted. Before putting into glycerin, the specimen may be stained with picroacid-fuchsin. This intensifies the yellow of the elastic fibres and brings out in red the fibrillar connective tissue. Pieces of the ligament fixed and hardened in the same manner may be embedded in celloidin and cut into longitudinal and transverse sections. These stained with picro-acid-fuchsin show well the relation of the coarse elastic fibres (yellow) to the more delicate fibrous tissues (red).

Ir. Fat Tissue.-Human subcutaneous fat as fresh as possible is fixed in formalin-Müller's fluid (technic 5, p. 7), hardened in alcohol and embedded in celloidin. Sections are stained with hæmatoxylin and picro-acid-fuchsin (technic 3, p. 2I). The alcohol and ether of the celloidin remove the fat from the fat cells, leaving only the cell membranes. The fat gives the celloidin a milky appearance. Such celloidin does not cut well. The celloidin should, therefore, be changed until it ceases to turn white. The sections are cleared in oil of origanum or carbol-xylol, and mounted in balsam. The fibrillar tissue is stained red by the fuchsin, and the protoplasm of the fat cell yellow by the picric acid.

I2. Developing Fat Tissue.-Remove bits of tissue from the axilla or groin of a five-inch fœtal pig, or other fœtus of about the same development. Fix twenty-four hours in a r-per-cent. aqueous solution of osmic acid (technic ro, p. 3I), wash thoroughly and mount in glycerin. A part of the tissue mounted should be thoroughly teased, the rest gently pulled apart. The teased portion will show the fat cells in various stages of development. The unteased part will usually show brownish blood-vessels and the grouping of fat cells around them, to form embryonic fat lobules. Note the developing connective tissuc between the groups of fat cells. It is from this that the arcolar tissue, which envelops and separates the lobules of adult fat, is developed.

I3. Reticular Tissue.-Fix a lymph node in formalin-Müller's fluid (technic 5 , p. 7), and stain very thin sections with hrmatoxylin and picro-acid-fuchsin (technic 3, p. 2I). In the lymph sinuses of the medulla the reticulum can usually be plainly seen.

\section{Cartilage}

Cartilage is a form of connective tissue in which the ground substance is firm and dense and determines the physical character of the 
tissue. On boiling it yields chondrin. Cartilage cells are differentiated connective-tissue cells. While varying greatly in shape they are most frequently spherical or oval. Each cell lies in a cell space or lacuna, which it completely fills. The intercellular substance immediately surrounding a lacuna is frequently arranged concentrically, forming a sort of capsule. Fine canaliculi connecting the lacunæ are present in some of the lower animals and have been described in human cartilage. They can be demonstrated, however, in human cartilage, only by special methods, and probably represent artefacts.

Cartilage contains no blood-vessels, and in human cartilage no lymph channels have been positively demonstrated.

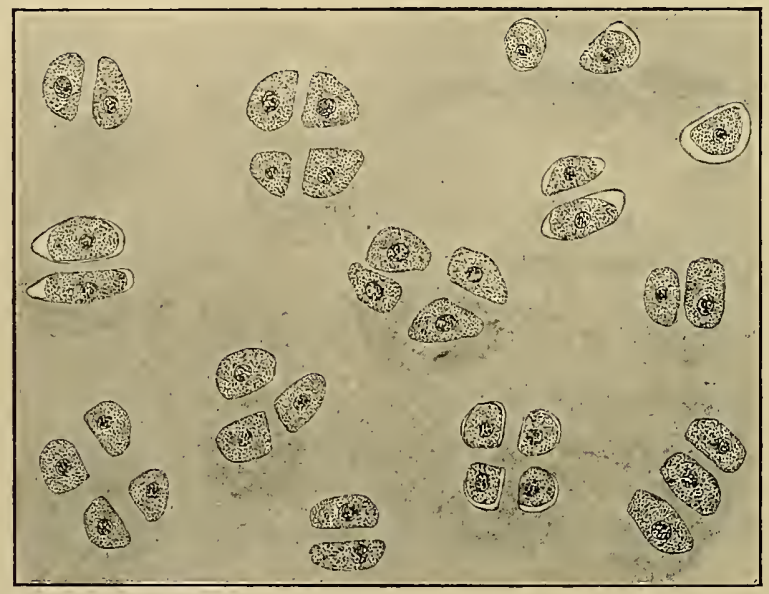

FIg. 46.-Hyaline Cartilage from Head of Frog's Femur. $\times_{35}$. $\quad$ (Technic I, p. roo.) Groups of cartilage cells in apparently homogeneous matrix.

Cartilage is subdivided according to the character of its intercellular substance into three varieties: (I) Hyaline, (2) elastic, (3) fibrous.

I. Hyaline Cartilage (Fig. 46).-The cells occur singly or in groups of two or multiples of two. An entire group of cells frequently lies in one lucuna surrounded by a single capsule. Such a group of cells has developed within its capsule from a single parent cell. In other cases delicate hyaline partitions separate the cells of a group. The cells are spherical or oval, with flattening of adjacent sides. The nucleus is centrally placed, and has a distinct intranuclear network and membrane. The cytoplasm is finely granular, and may contain drop- 
lets of fat, of glycogen, or of both. Toward the perichondrium the arrangement of the cells in groups is less distinct. Here the cells are fusiform and parallel to the surface.

The intercellular matrix, when subjected to the usual technic, appears homogeneous. By the use of special methods, such, e.g., as artificial digestion, this apparently structureless matrix has been shown to be made up of bundles of fibres, quite similar to those found in fibrous connective tissue.

Hyaline cartilage forms the articular cartilages of joints, the costal cartilages, and the cartilages of the nose, trachea, and bronchi. In the embryo a young type of hyaline cartilage, known as embryonal cartilage, forms the matrix in which most of the bones are developed.

\section{Elastic cartilage (Fig. 47)} resembles hyaline, but differs from the latter in that its hyaline matrix contains a large number of elastic fibres. These vary in size, many being extremely fine. The elastic fibres branch and run in all directions, forming a dense network of interlacing and anastomosing fibres.

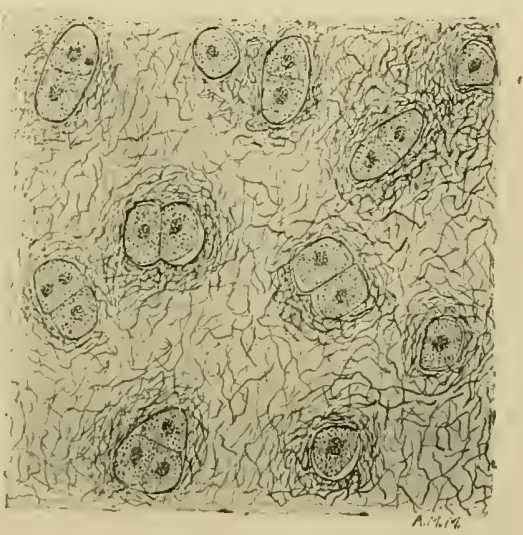

FIG. 47.-Elastic Cartilage from Dog's Ear. $\times 350$. ('Technic 2, p. I00.) Groups of cartilage cells in fibro-elastic matrix.

Elastic cartilage occurs in the external ear, the Eustachian tube, the epiglottis, and in some of the laryngeal cartilages.

3. Fibrous cartilage (Fig. 48) is composed mainly of fibrillar connective tissue. The fibres may have a parallel arrangement, or may run in all directions. Cells are few, and are usually arranged in rows of from two to six, lying in elongated cell spaces between the fibre bundles.

Fibrous cartilage occurs in the inferior maxillary and sternoclavicular articulations, in the symphysis pubis, and in the intervertebral discs.

Cartilage, except where it forms articular surfaces, is covered by a membrane, the perichondrium. This is composed of fibrillar connective tissue, and blends without distinct demarcation with the superficial layers of the cartilage.

Like the other connective tissues, cartilage develops from meso- 
derm. It is at first wholly cellular. Each cell forms a capsule around itself, and by blending of these capsules are formed the first elements of the intercellular matrix. This increases in quantity and assumes the structural characteristics of one of the forms of cartilage. The white fibres of fibro-cartilage and the yellow fibres of elastic cartilage develop in the same manner as in fibrillar and elastic tissue.

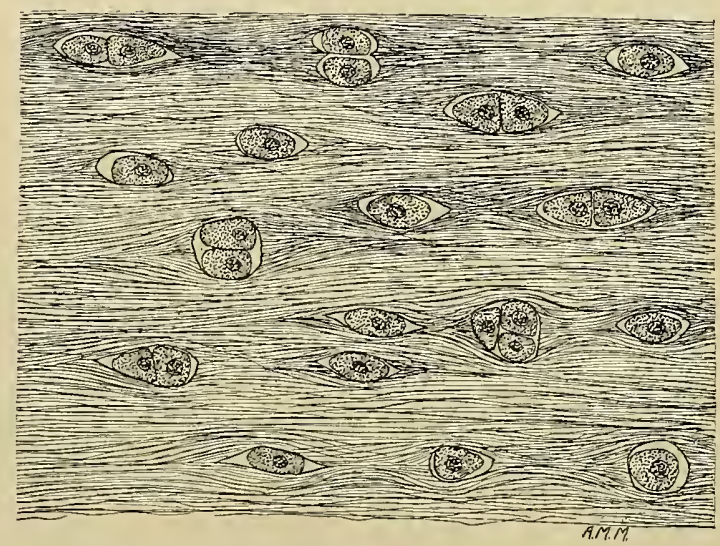

FIG. 48.-Fibrous Cartilage from Dog's Intervertebral Disc. $\times 35 \circ . \quad$ (Technic 3 , p. roo.) Groups of cartilage cells in matrix of fibrillar connective tissue.

\section{TECHNIC}

(I) Hyaline Cartilage.- Remove a frog's femur and immediately immerse the head in saturated aqueous solution of picric acid. Cut sections tangential to the rounded head, keeping knife and bone wet with the picric acid solution. As bone must be cut, a special razor kept for the purpose should be used. Cut sections as thin as possible. The first sections consist wholly of cartilage. As bone is reached, the cartilage is confined to a ring around the bone. Mount in the picric-acid solution, cementing the cover-glass immediately.

(2) Elastic Cartilage.-Remove a piece of cartilage from the ear and fix in formalin-Müller's fluid (technic 5, p. 7). Stain sections strongly with hæmatoxylin, followed by picro-acid-fuchsin (technic 3, p. 2I). Clear in carbol-xylol and mount in balsam. The capsules around the cartilage cells are thick and, as they usually retain some hæmatoxylin, can be readily seen. Note also the flattened cartilage cells near the surface, and the perichondrium.

(3) Fibro-cartilage.-Fix pieces of an intervertebral disc in formalin-Müller's fluid. Sections are stained either with hæmatoxylin-eosin or with hæmatoxylin-picro-acid-fuchsin and mounted in balsam.

\section{Bone tissue}

Bone is a form of connective tissue in which the matrix is rendered hard by the deposition in it of inorganic matter, chiefly the 
phosphate and the carbonate of calcium. These salts are not merely deposited in the matrix, but are intimately associated and combined with its histological structure. The intimacy of this association of the organic and inorganic constituents of bone is shown by the fact that, though the salts compose two-thirds of bone by weight, it is impossible to distinguish them by the highest magnification. Furthermore, if either the lime salts are dissolved out by means of acids (decalcification) or the organic matter removed by heating (calcination), the histological structure of the bone still remains.

Like the other connective

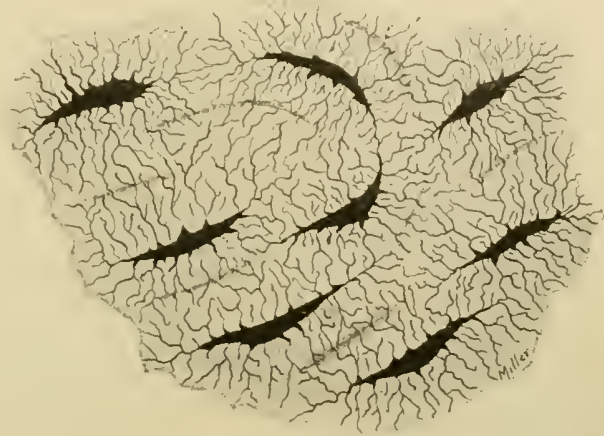

Fig. 49.-Bone Tissue showing Lacunæ and Canaliculi. $\times 700 . \quad($ Technic I, p. I02.) tissues, bone consists morphologically of cells and intercellular substance.

Bone cells or bone corpuscles lie in distinct cell spaces or lacunce. From the lacunæ pass off in all directions minute canals-canaliculiwhich anastomose with canaliculi of neighboring lacunæ (Fig. 49). At the surface of bone these canaliculi open into the periosteal lymph-

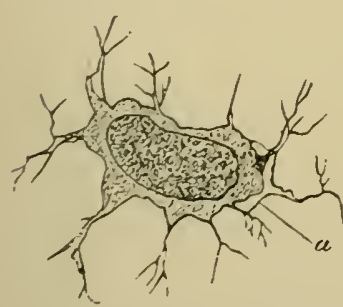

Fig. 50.-Bone Cell and Lacuna. (Joseph.) At $a$ the cell body has shrunken, allowing the outline of the lacuna to be seen. atics. A complete system of canals is thus formed, which traverse the bone and serve for the passage of nutritive fluids. The bone cells themselves (Fig. 50) are flat, ovoid, nucleated cells, with numcrous fine processes, which extend in all directions into the canaliculi. In young developing bones the processes of adjacent cells anastomose. In adult bone the processes extend but a short distance into the canaliculi, and probably do not anastomose.

The basement substance or matrix has a fibrous structure, closely resembling that of fibrillar connective tissue, and it is in this fibrillar matrix that the lime salts are deposited. The fibrils are held together by cement substance into bundles. In most bone the bundles are fine and arranged in layers or lamella. Less commonly the fibre bundles are coarser and have an irregular arrangement. 


\section{TECHNIC}

(I) For the study of the minute structure of bone a section of undecalcified or hard bone is required. Part of the shaft of one of the long bones is soaked for several days in water and all the soft parts are removed. It is then placed in equal parts alcohol and ether to remove all traces of fat and thoroughly dried (the handle of a tooth or nail brush frequently furnishes good material and is already dried). Thin longitudinal and transverse sections are now cut out with a bone saw. One surface is next ground smooth, first on a glass plate, using emery and water, then on a hone. The specimen is now fastened polished side down on a block of wood or glass by means of sealing wax, and the other side polished smooth in the same manner as the first, the bone being ground as thin as possible. The sealing wax is removed by soaking in alcohol and the specimen looked at with the low power. If not thin enough, it is gently rubbed on a fine hone. It is then soaked in equal parts alcohol and ether, dried thoroughly, and mounted in hard balsam. This is accomplished by placing a small bit of hard balsam on a slide, melting, pushing a bit of the bone into the hot balsam, covering and cooling as quickly as possible. The object of the hard balsam and quick cooling is to prevent the balsam running into the lacunæ and canaliculi and obscuring them by its transparency. The air imprisoned in the lacunæ and canaliculi causes them to appear black when viewed by reflected light.

(2) The structure of the bone cell is best studied in sections of decalcified bone which has first been carefully fixed. (See technic I, p. I 96.) 


\section{CHAPTER IV}

\section{THE BLOOD}

BLOOD is best considered as a tissue, the intercellular substance of which is fluid. This fluidity of the intercellular substance allows the formed elements or cells to move about freely, so that there is not the same definite and fixed relation between cells and intercellular substance as in other tissues. There are about 5 litres of blood in the adult body, blood thus constituting about one-thirteenth of the entire body weight.

The fluid intercellular substance or plasma is slightly alkaline in reaction. It consists of serum albumen, globulin, fibrinogen and inorganic salts, chiefly the chlorid, carbonate, bicarbonate and phosphate of soda. The reaction of blood is distinctly alkaline, due mainly to the phosphate of soda. Its specific gravity is about 1.030 , while that of the whole blood is about I.06o. The bulk of the plasma is about equal to that of the red and the white cells.

The formed elements of the blood are: (I) Red blood cells (red blood corpuscles, erythrocytes); (2) white blood cells (colorless corpuscles-leucocytes); (3) blood platelets (thrombocytes); (4) blood dust

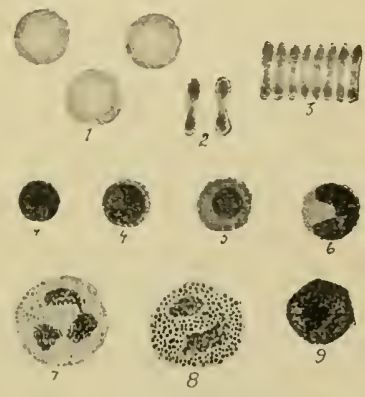

Fig. 5I.-Cells from Human Blood. $\times 600$. (Technic 2, p. I Iо.) I, Red blood cell seen on flat; 2 ,red blood cell seen on edge; 3 , red blood cells forming rouleaux; 4, 4, small and large lymphocytes; 5, mononuclear leucocyte; 6, transitional leucocyte; 7 , polymorphonuclear leucocyte, containing neutrophile granules; 8, polynuclear leucocyte, containing eosinophile granules; 9, mononuclear leucocyte, containing basophile granules. (hrematokonia).

I. Red blood cells (erythrocytes) (Fig. 5 I, I, 2, 3) are in man non-nucleated circular discs. ${ }^{1}$ Their average diameter is about $7 \cdot 5 "$, their thickness $2 "$ at the thin centre. A few red blood cells of a diameter of 8 " to $8.5 \%$ (macrocytes) and about the same number of red cells only about one-half the usual diameter (microcytes)

Isome olservers describe the red! blood cell as bell- or rup-shaped. (Lewis: Jour. Ifed. Rescarch, X. S., wol. v, s0o.4; Rardasch: Anat. Anz. xxviii, 1000. Weidenreich:

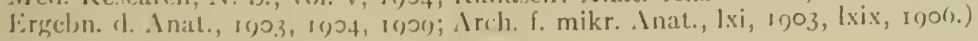


are usually present. They are biconcave, with rounded edges. Seen on the flat, the difference in thickness between centre and periphery is evidenced by the difference in refraction (Fig. 5I, I). Seen on edge, the shape resembles that of a dumb-bell (Fig. 5I, 2). Singly or in small numbers, red blood cells have a pale straw color, due to the presence of hæmoglobin. Redness is apparent only when the cells are seen in large numbers. If fresh blood be allowed to stand for a moment, the red cells are seen to adhere to one another by their flat surfaces, forming rows or rouleaux (Fig. 5I, 3).

Subjected to the usual technic, the red blood cell appears homogeneous. By the use of special methods, this apparently homogeneous substance can be separated into $(a)$ a color-bearing proteidhamoglobin, and $(b)$ a stroma, the latter representing the protoplasm of the cell. Peripherally the stroma probably forms an extremely delicate cell membrane, although the presence of any membrane whatever is denied by some. It is the hæmoglobin which gives color to the corpuscles. Hæmoglobin is a complex proteid, which can be resolved into a globulin and a pigment, hamatin. It is held in solution or in suspension in the stroma.

The red blood cells are soft and elastic, and are easily twisted to accommodate themselves to the smallest capillaries.

The red blood cell is extremely susceptible to changes in the plasma. Thus even slight evaporation of the plasma results in osmosis between the now denser surrounding fluid and the contents of the cell. This causes fluid to leave the cell, with the result that the latter becomes spherojdal and irregularly shrunken, with minute knob-like projections from its surface. This is known as crenation of the red cell. The addition of water to blood, thus decreasing the specific gravity of the plasma, has the opposite effect, resulting in swelling of the cell. It also causes solution of the hæmoglobin, which leaves the cell, the latter then appearing colorless, with a faint circular outline-the membrane of the cell. This separation of the hæmoglobin from the corpuscle is also caused by freezing and thawing, by heat $\left(60^{\circ} \mathrm{C}\right.$.), by the addition of dilute acids, ether, or chloroform.

Dilute alkaline solutions and bile first cause the red corpuscles to swell and become spherical, and then to dissolve. This is known as hamolysis, and may also be effected by mixing the blood of one species with that of another. Dilute acetic acid causes swelling and fading of the red cells, with the formation of prismatic crystals of hæmoglobin. 
The red blood cells number from 4,500,000 to 5,000,000 per cubic millimeter of blood.

2. White blood cells (leucocytes) (Fig. 5I, 4 to 9 inclusive) are colorless nucleated structures which have a generally spherical shape, but which are able to change their shape on account of their powers of amœboid movement. They have a diameter of from 5 to $10 \mu$, and are much less numerous than the red cells, the proportion being about one white cell to five hundred red cells, or about 10,000 to the cubic millimeter. This proportion is, however, subject to wide variation.

Leucocytes may be classified as follows: (a) Lymphocytes; (b) mononuclear leucocytes; $(c)$ transitional leucocytes; $(d)$ polymorphonuclear or polynuclear İeucocytes.

(a) Lymphocytes (Fig. 5I, 4).-These vary in diameter from 5 to $8 \mu$, and are sometimes subdivided into small lymphocytes and large lymphocytes. The nucleus is spherical, stains deeply, and almost completely fills the cell, the cytoplasm being confined to a narrow zone around the nucleus. Lymphocytes constitute about 20-per-cent. of the white blood cells.

(b) Mononuclear Leucocytes (Fig. 51, 5 and 9) are of about the same size as large lymphocytes. The nucleus, however, stains more faintly and is smaller, while the cytoplasm is greater in amount. From 2-per-cent. to 4-per-cent. of the white cells are mononuclear leucocytes.

(c) Transitional Leucocytes (Fig. $5 \mathrm{I}, 6$ ) occur in about the same numbers as the preceding, and are of about the same size., There is relatively more cytoplasm, and the nucleus, instead of being spherical, is crescentic or horseshoe or irregular in shape. These cells represent a transitional stage between the mononuclear and the polymorphonuclear and polynuclear varieties.

(d) Polymorphonuclear and polynuclear leucocytes (Fig. $5 \mathrm{I}, 7,8)$ constitute about 70 -per-cent. of the white blood cells. Their size is about the same as that of the mononuclear form, but they are somewhat more irregular in shape. The appearance of the nucleus is characteristic. In the polymorphonuclear form the nucleus consists of several round, oval, or irregular nuclear masses connected with one another by cords of nuclear substance. These cords are frequently so delicate as to be distinguished with difficulty. The polynuclear form is derived from the polymorphonuclear by breaking 
down of the connecting cords, leaving several separate nuclei or nuclear segments.

Granules in small numbers may be present in the protoplasm of any of the leucocytes, but the protoplasm of about 70 per cent. of all leucocytes is so distinctly and regularly granular that by some authors a primary division into granular leucocytes and non-granular leucocytes is made. Under this classification, lymphocytes and some mononuclear leucocytes are placed in the non-granular group, while transitional leucocytes, polymorphonuclear leucocytes and some mononuclear leucocytes, are placed in the granular group. Aniline dyes may be divided into acid, basic and neutral, according to whether the coloring matter is an acid, a base, or a combination of an acid and a base, and the granules of the granular leucocytes react in a definite manner to these dyes, thus allowing the following classification:

$$
\text { Granular leucocytes } \begin{cases}\text { I } & \text { neutrophile. } \\ 2 & \text { acidophile (eosinophile). } \\ 3 & \text { basophile. }\end{cases}
$$

As the neutrophile granules are fine and the eosinophile granules coarse, a classification of leucocytes into finely granular and coarsely granular is sometimes made."

I. Neutrophile Leucocytes. - These are the most numerous of all leucocytes, making up about 68 per cent. Their protoplasm is thickly studded with very fine granules which stain violet with a mixture' of eosin (acid) and toluidin blue (basic). Most neutrophiles are polymorphonuclear, a few are transitional. They have a wide distribution, being found not only in the blood itself, but in the spleen and lymph nodes and as wandering cells in various tissues and organs.

2. Acidophile Leucocytesl or, because the most common acid dye used is eosin, eosinophile. The granules in these cells are coarse and sharply defined. They stain strongly with acid dyes. Eosinophiles are mainly polymorphonuclear, more rarely they are transitional. They make up from I per cent. to 4 per cent. of all leucocytes. In certain pathological conditions their number is greatly increased.

3. Basophile Leucocytes.-The granules in these cells are rather coarse and irregular in shape and are distributed unevenly through the cytoplasm. They stain strongly with basic dyes. They are 
present in small numbers (Ehrlich, 0.2 per cent. to 0.5 per cent.) in normal blood, or they may not be demonstrable. Ehrlich identifies them with the "mast cells" which are found in various tissues and organs, especially in areolar connective tissue, but this identity has been questioned.

Upon the basis of the foregoing description the following classification of leucocytes on the basis of granulation may be made.

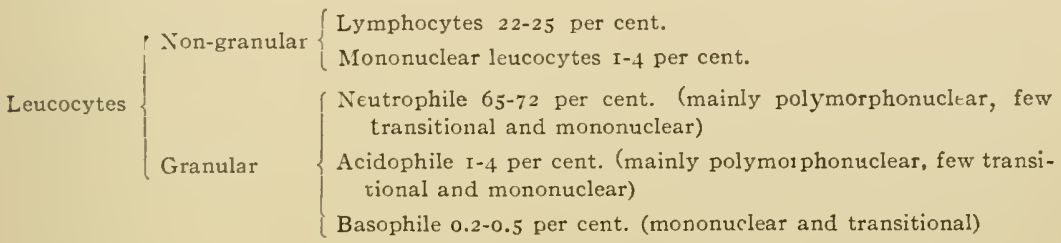

The function of the red cells is primarily the carrying of oxygen from the lungs to the tissues and of carbonic acid from the tissues to the lungs. This oxygen-carrying ability is dependent upon the hæmoglobin and is directly proportionate to the number of red cells and to the richness of the individual cells in hæmoglobin. In the capillaries of the tissues and again in the capillaries of the lung the hæmoglobin is undergoing constant change. The hæmoglobin of arterial blood is known as oxyhæmoglobin, of venous blood as reduced hæmoglobin. The difference is readily demonstrable by the spectroscope. ${ }^{1}$

That leucocytes possess in a marked degree the power of amœboid movement has been noted (p. 52). On account of this motility leucocytes are able (I) to leave the blood-vessels (diapedesis) and move about freely in the tissues (wandering cells), (2) to surround and take up substances from without (phagocytosis).

Diapedesis.-This power is directly dependent upon motility and while possessed by all leucocytes is most markedly characteristic of the polymorphonuclear forms. (For details of amoboid movement see p. 52.)

Phagocylosis.-Phagocytic powers are not possessed equally by all leucocytes, but are confined largely to the mononuclear and polymorphonuclear forms. Such cells can take up foreign substances, bacteria, degeneration products, etc., carry them to other

'Of interest in this connertion is the fact that in poisoning by illuminating gas, a very definite and stalse combination of the carbon dioxid with the hamoghobin is formerl (rarloxyhaemoglobin). It is this subetance which determines the darker red (s) los of the blowe in this condition and is apprarently the rause of cleath. 
parts of the body or entirely outside the body (salivary corpuscles), or apparently absorb or digest them. (See also p. 50.) ${ }^{1}$

3. Blood platelets (thrombocytes) are minute round or oval bodies from $2 \mu$ to $4 \mu$ in diameter. They are colorless and vary in shape. In fixed preparations they often appear stellate. Their number has been variously estimated. The average is probably about 200,000 to 300,000 per cubic millimeter of blood. In the blood stream they are separate, but show a marked tendency to agglutination directly the blood is drawn. Some comparatively recent observations tend toward considering the platelets as true cells. Thus they have been described as amœboid, as having the same chemical composition as cells, and as containing either granules of chromatin or distinct nuclear structures. The so-called thrombocytes of Ovipara are larger than those of man and are unquestionably nucleated cells. Their appearance is, however, wholly unlike human thrombocytes and the identity of the two forms is doubtful.

Various functions have been ascribed to the thrombocytes. It is fairly established that they have something to do with the formation of fibrin and the coagulation of blood.

4. The blood dust (hæmatokonia) occurs in the form of small refractive granules.

In the blood of the lower mammals and in herbivorous animals small droplets of fat derived from the chyle are found. They are known as elementary granules and are not present in normal human blood.

\section{Development of the Blood.}

At an early stage of embryonic development certain mesodermic cells of the area vasculosa, which surrounds the embryo, become arranged in groups known as blood islands. It is from these "islands" that both blood and blood-vessels develop. The peripheral cells arrange themselves as the primitive vessel walls, within which the central cells soon become free as the first blood corpuscles. In this way vascular channels are formed, inside of which are developing blood cells. This division of the mesoblastic cells of the blood islands into (I) endothelial cells of the vessel walls and (2) progenitors of the blood cells or primitive blood cells, is quite generally accepted. From this point, however, opinions

${ }^{1}$ It is to be noted that phagocytosis is not confined to leucocytes but has been observed in other cells, e.g., fixed connective-tissue cells including endothelium. It is also to be noted that phagocytic cells are not equally phagocytic to all substances. In other words phagocytes apparently have some powers of selection. Thus if two kinds of bacteria be presented to them, they may take up only one kind, or one kind much more readily than the other. 
diverge, the two main theories of blood-cell formation being known as the polyphyletic and the monophyletic theories.

According to the polyphyletic theory, after the original division of the mesoblast cells of the blood islands into vessel wall cells (endothelium) and blood cells (primary blood cells), the latter go on to the development of erythroblasts and these to the development of erythrocytes. Leucocytes develop later in connective tissue, in the liver, spleen and bone marrow, while lymphocytes have their origin in the germ centers of the lymphoid organs. There are thus three separate sources of blood cells: (I) Red blood cells, originally from erythroblasts of the blood islands; in adult life from the erythroblasts of the bone marrow and possibly of spleen; (2) leucocytes, first in connective tissue, liver, spleen and marrow; in adult life in the marrow; (3) lymphocytes, in the lymphoid organs. According to the polyphyletic theory these three types are genetically independent and remain so throughout life, the cells of each type undergoing mitotic division to produce new cells of the same type only.

According to the monophyletic theory the blood-cell-forming elements of the blood islands fall into two groups as regards their future development: (I) cells which give rise to primary erythroblasts, these to secondary erythroblasts and these again to erythrocytes, thus completing the line of red blood cells, and (2) cells which remain undifferentiated, not only in the embryo but throughout life, and retain the capability of differentiating into erythroblasts or into white blood cells, either leucocytes or lymphocytes. According to Weidenreich ${ }^{1}$ and other supporters of this theory, red and white blood cells stand in very close genetic relation. He claims that an undifferentiated mother cell exists which is capable of differentiating either in the direction of the red cell or of the white cell; that this mother cell exists not only in the embryo but throughout life and is constantly giving birth to cells which are to develop into red blood cells or white blood cells to replace those being constantly used up and cast off. Morphologically the mother cell is apparently identical with the lymphocyte. In postembryonic life, this mother cell is found in marrow in the germ center of lymphoid organs and possibly in connective tissue. On the other hand a once differentiated cell remains a differentiated cell, and while able to divide mitotically and thus give rise to cells of its own kind, it is never capable of producing cells of any other kind.

Two views are held in regard to the manner in which the embryonic nucleated red cell gets rid of its nuclcus in becoming the non-nucleated red cell of the adult. According to one the nuclcus is absorbed within the cell and gradually secreted; according to the other the nucleus, as a whole, is extruded.

In early embryonic life especially active proliferation of red cells occurs in the blood-vessels of the liver. This has led to the considering of the liver as a blood-forming organ. The liver cells themselves, however, take no actual part in the formation of blood cells, the blind pouch-like venous capillaries of the liver, with their slow-moving blood currents, merely furnishing a peculiarly suitable place for cellular proliferation. Before birth the splenic pulp and bone marrow become blood-forming organs. In the adult the bone marrow is probably, under normal conditions, the main if not the sole seat of red-cell formation.

${ }^{1}$ Ant. Rec., vol. iv., Sept., Igro. 
During fotal life the number of nucleated red cells constantly diminishes, while the number of non-nucleated red cells increases. At birth there are usually but few nucleated red cells in the general circulation, although even in the adult they are always found in the red bone-marrow.

The origin of the blood platelets is not known. They have been described as originating in the extruded nuclei of the red cells, as disintegrating leucocyte products, as red cells in process of development, as red cells in process of disintegration, as albuminoid precipitates, as a specific blood cell. According to Wright ${ }^{1}$, they are derived from the megakaryocytes of bone marrow and other blood-forming organs.

\section{TECHNIC}

(I) Fresh Blood-- Prick a finger with a sterile needle. Touch the drop of blood to the centre of the slide and cover quickly. For immediate examination of fresh blood no further preparation is necessary. Evaporation may be prevented by cementing, or by smearing a rim of vaseline around the cover-glass.

(2) Blood Smears.-From the same or a second prick take up a drop of blood along the edge of a mounting slide. Quickly place the edge against the surface of a second slide and draw the edge across the surface in such a manner as to leave a thin film or smear of blood. Allow the smear to become perfectly dry and stain by technic Ir, p. 3r. By this method the acidophile granules are stained red, basophile granules purple, and neutrophile granules a reddish-violet.

Good results may also be obtained by fixing the dried smear for half an hour in equal parts alcohol and ether and staining first in a strong alcoholic solution of eosin, then in a rather weak aqueous solution of methylene blue.

1 Jour. Morph., xxi, I9Iо. 


\section{CHAPTER V}

\section{MUSCLE TISSUE}

While protoplasm in general possesses the property of contractility, it is in muscle tissue that this property reaches its highest development. Moreover, in muscle this contractility is along definite

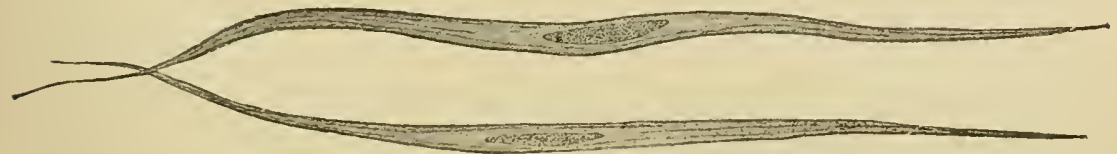

Fig. 52.-Isolated Smooth Muscle Cells from Human Small Intestine. $\times_{40}$. (Technic I, p. I24.) Rod-shaped nucleus surrounded by area of finely granular protoplasm; longitudinal striations of cytoplasm.

directions, and is capable of causing motion, not only in the cell itself, but in structures outside the cell.

Muscle may be classified as: (I) Involuntary smooth muscle; (2) voluntary striated muscle; (3) involuntary striated muscle or heart muscle.

Involuntary Smooth Muscle.-This is the simplest form of muscle tissue and consists of long spindle-shaped cells (Fig.

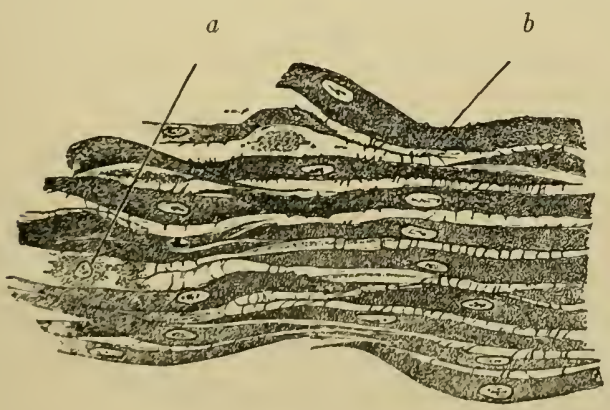

$A$

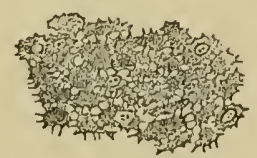

$B$

Fig. 53. - Ipparent Intercellular Bridges of Smooth Mluscle. A, From longitudinal section of intestinc of guincat-pig; B, from transverse section of intestinc of rabbit. $\quad X+20$. $a$, Nerve cell; $b$, end of muscle cell. (Stöhr.)

52) which are prismatic on transverse section (Fig. 53). 'The length of the smooth muscle cell varies usually from 30 to 200 ", its width from 3 to $8, \mu{ }^{1}$ except in the pregnant uterus where the cells frequently attain a much greater size. Less commonly the smooth

1 In the walls of very small blorel-vesects smooth muscle cells 15 to 20 th are found. (Apathy). 
muscle cell is flattened, and, in tissues rich in elastic fibres, e.g., the media of some arteries, especially the aorta, is quite irregular in shape. The central thickest part of each cell contains an elliptical or long rod-shaped nucleus. The

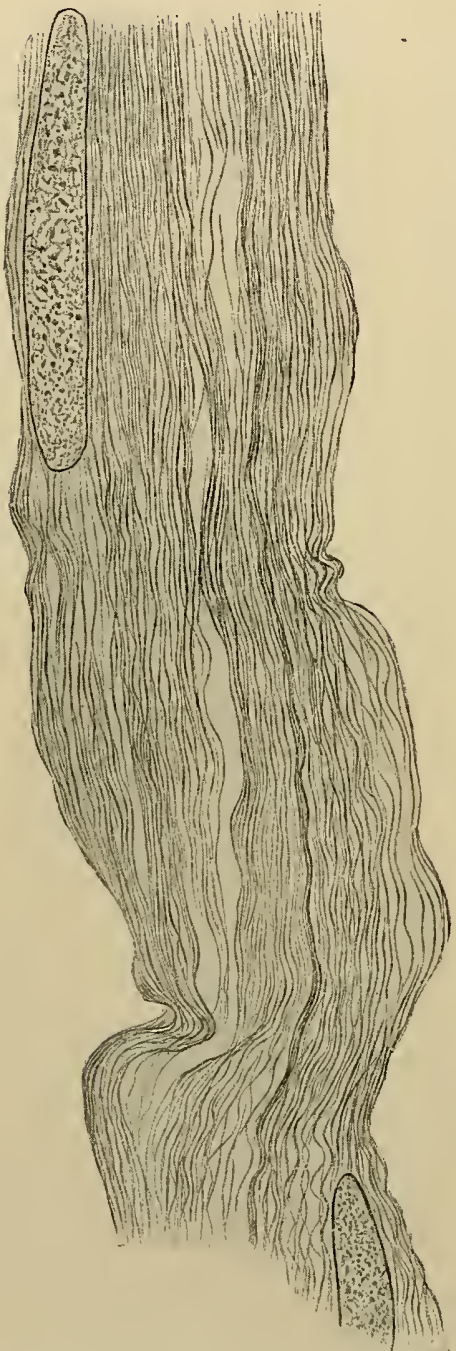

FIG. 54.-Preparation of Smooth Muscle Cells to show Fibrillar Structure. From intestine of Triton. $\times 2300$. (Heidenhain.) nucleus has a rather coarse intranuclear network and one or more nucleoli. In some cells a centrosome has been demonstrated. It lies outside and usually just to one side of the nucleus. The irregular, wavy and twisted nuclei often seen are probably due to contractions of the cytoplasm. The nucleus is surrounded by an area of finely granular cytoplasm, most abundant at the poles of the nucleus where it frequently forms a little pointed cap. The rest of the cytoplasm shows delicate longitudinal striations, which probably represent a longitudinal arrangement of the spongioplasm. These fibrils are extremely fine, are frequently present in small numbers, are not arranged in bundles, are apparently homogeneous and anisotropic, and are often very difficult of demonstration. The fibrils lie in a less differentiated cytoplasm. The smooth muscle cell has no such distinct envelop as the sarcolemma of striated muscle. The outer cytoplasm is, however, modified to form a delicate cell membrane or at least a modified surface layer.

The cells are united by a small amount of intercellular "cement" substance which reacts to silver nitrate. Intercellular "bridges" similar to those connecting epithelial cells have been described (Fig. 53), but are regarded by many observers as artefacts. By others fine 
intercellular fibrils are described as continuous from cell to cell, thus forming a syncytium. Between and surrounding the smooth muscle cells is a network of delicate connective-tissue fibrils (Fig. 55).

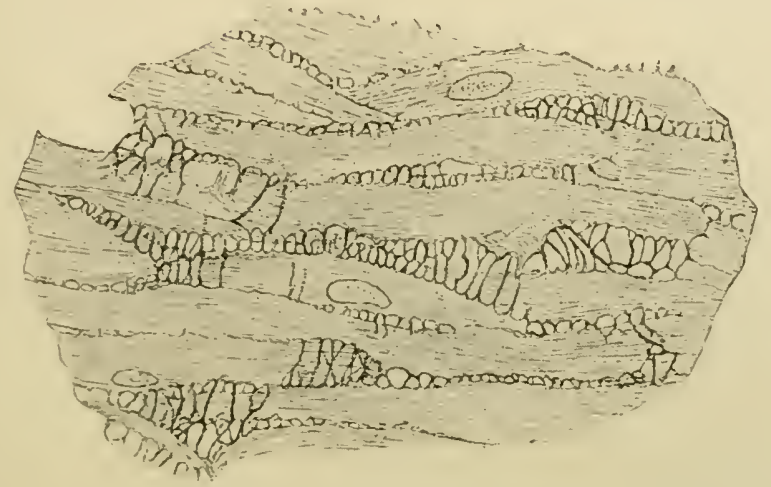

FIG. 55--Longitudinal Section of the Musculature of Cat's Intestine to show Intercellular Connective Tissue. (Bohemann.)

These are not apparent in sections which have been subjected to the usual technic, but can be demonstrated either by digesting the smooth muscle cells by means of trypsin, thus bringing out the undigestible collagenous fibrils, or by special staining methods (p. I 24).

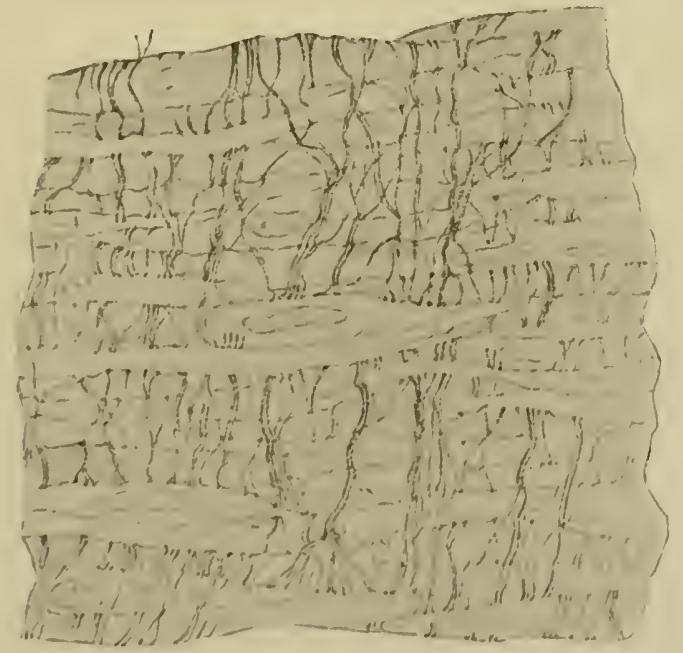

Fic. 56.- Filastic Fibres in the Smooth Muscle of Intestine of Cat. (IIolmgren.)

Smooth muscle cells may be arranged in layers of considerable thickness, the cells having a definite direction, as in the so-called "musculature" of the intestine (Fig. 56). In such masses of smooth. 
muscle the cells are separated into groups or bundles by connective tissue. Smooth muscle cells may be arranged in a sort of network, the cells crossing and interlacing in all directions, as in the wall of the frog's bladder. Again, they may be scattered in small groups or singly among connective-tissue elements, as in the villi of the small intestine.

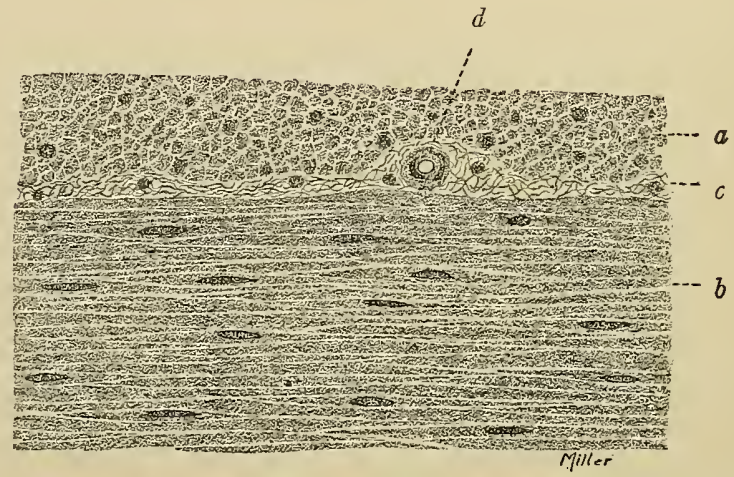

FIg. 57.-Smooth Muscle from Longitudinal Section of Cat's Small Intestine, showing Portions of Inner Circular and Outer Longitudinal Muscle Coats with Intervening Connective Tissue. $\times 350$. (Technic 3 , p. I24.) $a$, Transversely cut cells of inner circular layer; in comparatively few has the plane of section passed through the nucleus; $b$, longitudinally cut cells of outer longitudinal layer. In many of the cells the plane of section has not passed through the nucleus; $c$, intermuscular septum (connective tissue); $d$, small artery.

Voluntary Striated Muscle.-This consists of cylindrical fibres from $5 \circ$ to $\mathrm{I} 30 \mathrm{~mm}$. in length and from Io to $100 \mu$ in diameter. Generally speaking, the fibres of greater diameter are found in the larger animals. There is apparently no relation between the size of the muscle and either the length or diameter of its component fibres. There is also no relation of length to diameter, within the same muscle, fibres of many different sizes intermingling.

Each muscle fibre consists of $(a)$ a delicate sheath, the sarcolemma, enclosing $(b)$ the muscle substance proper, in which lie $(c)$ the muscle nuclei.

The sarcolemma is a clear, apparently structureless, very elastic membrane, which adheres so closely to the underlying muscle substance as to be indistinguishable in most preparations. In teased specimens it may frequently be seen at the torn ends of the fibres (Fig. 58). In staining reaction it differs from both white and elastic tissue and is apparently to be considered a distinct cell membrane.

The muscle substance consists of ergastoplasm (fibrilla) and 
sarcoplasm (interfibrillar substance) and shows two sets of striations (Fig. 59), longitudinal striations and cross striations. The longitudinal striations are due to parallel running ultimate fibrilla, which lie in and are more or less separated from one another by the sarcoplasm. Each fibrilla when examined, unstained, by reflected light is seen to be composed of alternating light and dark segments. As like segments lie in the same transverse plane, the whole muscle fibre appears composed of alternate light and dark bands (Figs. 59 and 6o), and this distinction is maintained even in stained specimens, as the light bands stain little if any, with most staining agents, while the dark bands react strongly to stains. The light band is composed of a singly refracting (isotropic) substance, the dark band of a doubly refracting (anisotropic) substance. Through the middle of the light band runs a fine line (Krause's line), while an even finer line (Hensen's line) can sometimes be seen running through the middle lighter portion of the dark band. Both Krause's and Hensen's lines cross the intervening sarcoplasm as well as the fibrilla and extend to the sarcolemma, thus completely crossing the fibre. As all of these structures run through the entire thickness of the fibre, they in reality constitute discs of muscle substance (Fig. 60). By means of certain chemicals these discs may be separated, the separation taking place along the lines of Krause. Each "muscle disc" thus consists of that portion of a fibre included between two adjacent lines of Krause and is composed of a central dark disc, and on either side onc-half of each arljacent light disc. A muscle filore is thus seen to be divisible

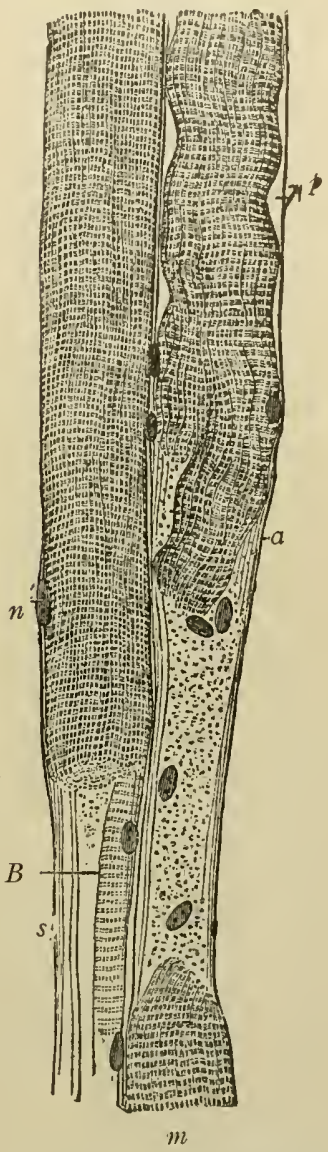

FIG. 58.-Semidiagrammatic Drawing of Parts of two Muscle "Fibres which have been broken, showing the relations between Muscle Substance Proper and Sarcolemma (Ranvier.) $m$, $a$, Retracted ends of muscle substance, between which is seen the sarcolemma with several adherent muscle nuclei; $B$, thin layer of muscle substance which has adhered to the sarcolemmil; $n$, muscle nucleus; $s$, satroslemma; $p$, sprace between sarcolemma and muscle substance. longitudinally into ultimale fibrilla, transversely into muscle discs. 
What is known as the sarcous element of Bowman is that portion of a single fibrilla which is included in a single disc, i.e., between two adjacent lines of Krause (Fig. 60).

The sarcoplasm is not evenly distributed throughout the fibre. On cross section irregular trabeculæ of sarcoplasm are seen extending in from the sarcolemma (Fig. 6r). These separate the fibrillæ into bundles, the muscle columns of Kölliker. A transverse section

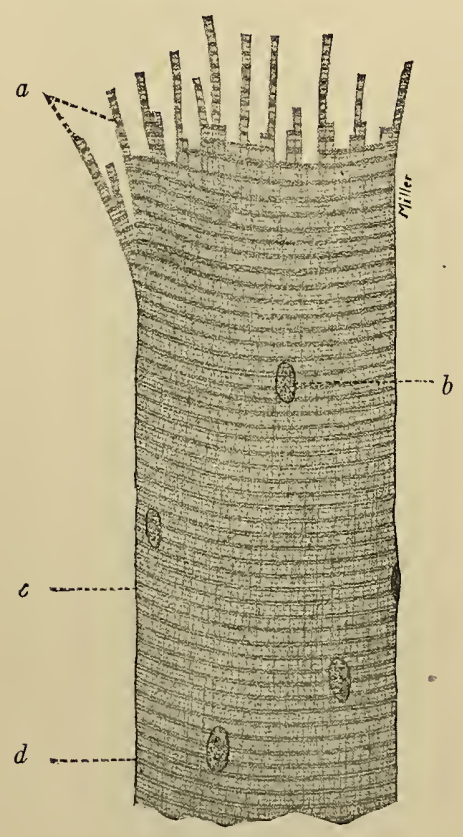

FIG. 59 .

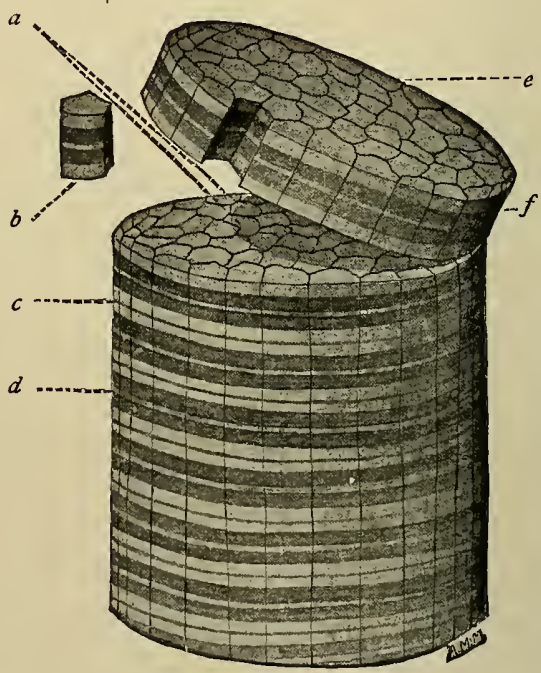

FIG. 60.

FIG. 59.--Portion of Striated Voluntary Muscle Fibre. $\times_{350}$. (Technic 4, p. I25.) The fibre is seen to be marked transversely by alternate light and dark bands. Through the centre of the light band is a delicate dark line (Krause's line); through the centre of the dark band a fine light indicates Hensen's line. The black line outlining the fibre represents the sarcolemma. $a$, Fibrillæ; $b$, muscle nucleus; $c$, Krause's line; $d$, Hensen's line.

FIG. 60.-Diagram of Structure of a Muscle Column of Kolliker. The appearance presented by the cross-cut muscle column=Cohnheim's field. $a$, Muscle fibrillæ; $b$, sarcous element; $c$, Krause's line; $d$, Hensen's line; $e$, Cohnheim's field; $f$, muscle disc.

of one of these columns presents the appearance of a network of sarcoplasm and of interfibrillar cement substance enclosing the fibrillæ. This appearance is known as Cohnheim's field (Figs. 60 and $6 r$ ).

Many of the details of structure of striated muscle have been determined by studies upon the muscles of lower animals. These details are extremely complicated and the numerous terms used to designate the same structure very con- 
fusing. The following is the scheme of structure and the nomenclature according to Heidenhain (Fig. 62).

The older terms muscle cell for the smooth muscle cell, and muscle fibre for the analogous multinuclear element of striated muscle are retained, also Apathy's myofibril for the ultimate fibrilla, Kölliker's muscle column for the smallest bundles of fibrils, and sarcoplasm for interfibrillar substance.

The muscle fibre is subdivided into a series of segments by transverse discs which completely cross the fibre, involving both fibrillæ and sarcoplasm and are attached to the sarcolemma. This disc (ground membrane-membrane of Krause,) Heidenhain designates the telophragma (Z). That part of a fibre which lies between two telophragmata is an inokomma. The middle of each inokomma is crossed by a disc (Hensen's membrane) which also involves both fibrillæ

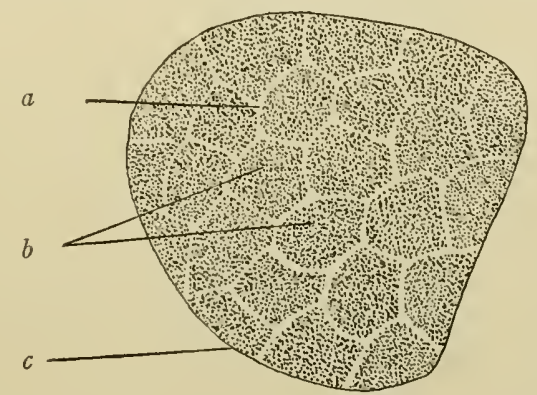

FIG. 6I.-Semidiagrammatic Drawing of Transverse Section of a Voluntary Muscle Fibre, showing Sarcolemma; sarcoplasm separating fibrils into bundles, each bundle constituting a muscle column of Kölliker and the appearance of its cross-cut end being Cohnheim's field. $a$, Sarcoplasm; $b$, Cohnheim's fields; $c$, sarcolemma.

and sarcoplasm and is attached to the sarcolemma. This membrane having apparently the same structure as the telophragma is designated the mesophragma (M). The mid-portion of the inokomma consists of anisotropic substance $(\mathrm{Q})$, the ends of isotropic $(\mathrm{J})$. That portion of $\mathrm{Q}$ which lies on either side of $\mathrm{M}$ is lighter than the rest of $\mathrm{Q}$ and is designated $\mathrm{Qh}(\mathrm{h}=\mathrm{hell}=$ clear $)$. That portion of $\mathrm{J}$ which lies close on either side of $\mathrm{Z}$ is darker than the rest of $\mathrm{J}$ and is designated $\mathrm{Jd}(\mathrm{d}=\mathrm{dich} t=$ thick$)$. In the sarcoplasm between the fibrillæ are granules; those in $\mathrm{J}$ are arranged in a regular row and are known as $\mathrm{J}$-granules, those in $\mathrm{Q}$ are more irregularly placed and are known as Q-granules. In Fig. 62 are also shown the "cross-fibre-nets" which are brought out by metallic impregnation and which possibly represent intracellular canals.

Two varieties of striated voluntary muscle fibres are distinguished, while fibres and red fibres. 'The difference between the two is due to the amount of sarcoplasm - the red fibres being rich in sarcoplasm, the white fibres poor. Red fibres contract less rapidly than white, but are less easily fatigued. In man white fibres are in the large majority, red fibres never occurring alone, but mingled with white fibres in some of the more active muscles, such as those of respiration 
and mastication. In some of the lower animals are found muscles made up wholly of red fibres.

Muscle fibres ending within the substance of a muscle have pointed extremities. Where muscle fibres join tendon, the fibre ends

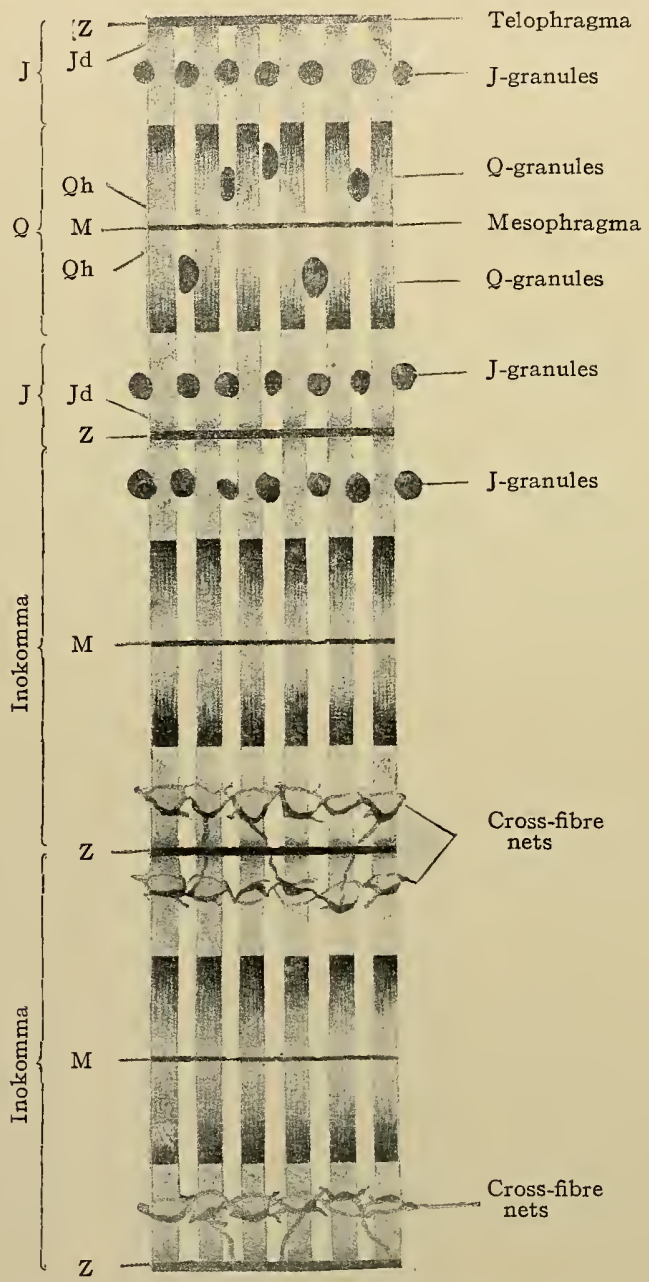

FIG. 62.-Scheme of Structure of Striated Voluntary Muscle Fibre with Nomenclature of Heidenhain. (Heidenhain.)

in a rounded or blunt extremity, the sarcolemma being continuous with the tendon fibres (Figs. 63 and 64).

Muscle fibres are usually unbranched. In some muscles-e.g., those of the tongue and of the eye-anastomosing branches occur. 
When muscle fibres end in mucous membranes - e.g., the muscle fibres of the tongue - their terminations are often branched.

Muscle fibres are multinuclear, some of the large fibres containing hundreds or even thousands of nuclei. ${ }^{1}$ In the white fibres the nuclei are situated at the periphery just beneath the sarcolemma. In red fibres they are centrally placed.

\section{Involuntary Striated Muscle (Heart Mus-} cle).-This, as its name implies, is a striated muscle not under control of the will. It occurs only in the cardiac musculature. Like voluntary muscle, heart muscle is striated both longitudinally and transversely. Like smooth muscle, on the other hand, it is composed of units which at least resemble cells and have long been called heart muscle cells. Heart muscle also resembles smooth muscle in that there is usually but one nucleus to a cell and this nucleus is centrally placed instead of lying at the periphery as in voluntary muscle. Also the sarcolemma if present at all is extremely delicate and is probably, as in smooth muscle, only a modification of the surface sarcoplasm. The amcunt of sarcoplasm throughout the cell is large. Around the nucleus is an area of sarcoplasm free from fibrillæ. This area often extends some distance toward the ends of the cell.

Heart muscle fibres are not the parallelrunning unbranched fibres of voluntary muscle, but, while having a generally parallel arrangement, give off side branches which anastomose, making it impossible to trace a single fibre for any great distance. These side branches have the same structure as the main fibre except that they are for the most part of smaller diameter and are nonnucleated.

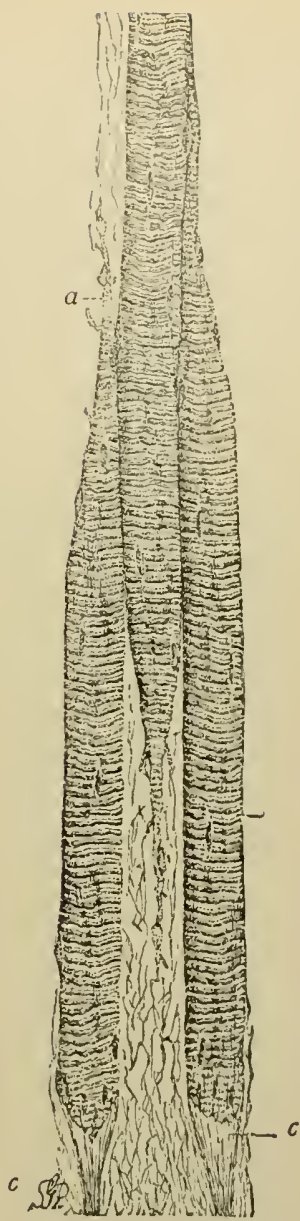

FIG. 63.-Semidiagrammatic Illustration of Endings of Muscle Fibres within a Muscle and in Tendon. (Gage.) $a$, Tapering end of fibre terminating within the muscle; the lower end of the central fibre shows the same method of termination; $c, c$, each fibre terminates above in pointed intramuscular ending, below in blunt ending connected with tendon.

${ }^{1}$ Stöhr calls attention to the fact that such a structure may be considered either a multinuclear cell or a syncytium and that there is really no true distinction between the two. 
The striations of heart muscle are less distinct than are those of voluntary muscle, but apparently represent very similar structures. The longitudinal striations indicate fibrille lying in the sarcoplasm. From the central mass of sarcoplasm which surrounds the nucleus, strands radiate toward the periphery. These strands, anastomosing, separate the fibrillæ into columns, the muscle columns of Kölliker. In cross section these present the appearance described under voluntary muscle as Cohnheim's fields. The disposition of the sarcoplasm,

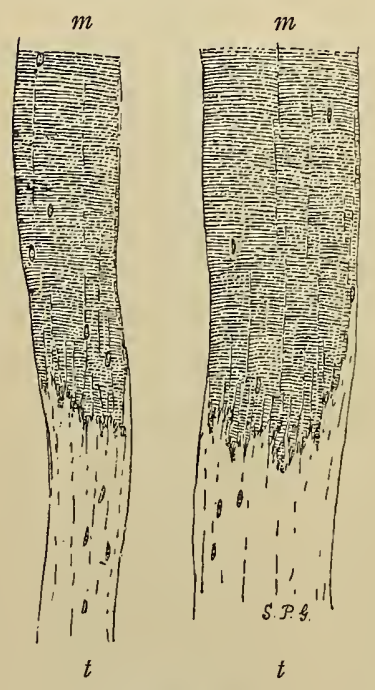

FIG. 64 .

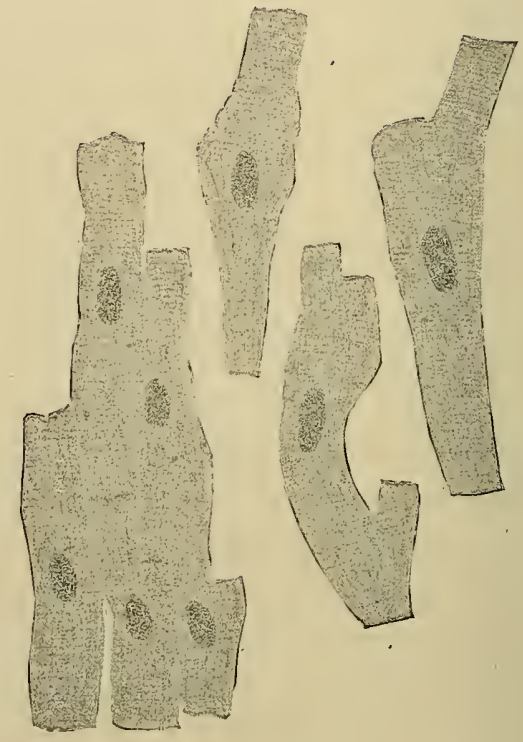

FIG. 65 .

FIG. 64.-Two Muscle Fibres from Upper End of Human Sartorius, to show connection of muscle and tendon. $\times 350$. (Gage.) $m$, Muscle fibres; $t$, tendon fibres.

Frg. 65.-Muscle Cells from the Human Heart (technic 6, p. I25), showing lateral branches and lines of union between cells. $\times 500$.

extending outward from the region of the nucleus like the spokes of a wheel, gives to the cross section a characteristic radiate appearance (Fig: 66), not seen in cross sections of voluntary muscle. The transverse markings represent, as in voluntary muscle, alternate light and dark discs. Through the middle of the light disc can be seen the membrane of Krause. McCallum describes Krause's membrane as crossing not only the fibrillæ, but also the sarcoplasm, as in the voluntary muscle fibre. The sarcoplasm he describes as further subdivided by membranes, which are transversely continuous with Krause's membranes, into minute discs. The centre 
of the cell around the nucleus is wholly composed of these little discs of sarcoplasm.

Peculiar to heart muscle are what appear in longitudinal sections to be dark lines which cross transversely both main fibres and side branches. These are known as intercallated discs and divide the muscle fibre into irregular, short, thick cylindrical segments. A disc may pass straight across a fibre, or it may cross it in a series of steps, or it may extend only part way across the fibre. It always touches at some point one of the ground membranes ${ }^{1}$ and sometimes

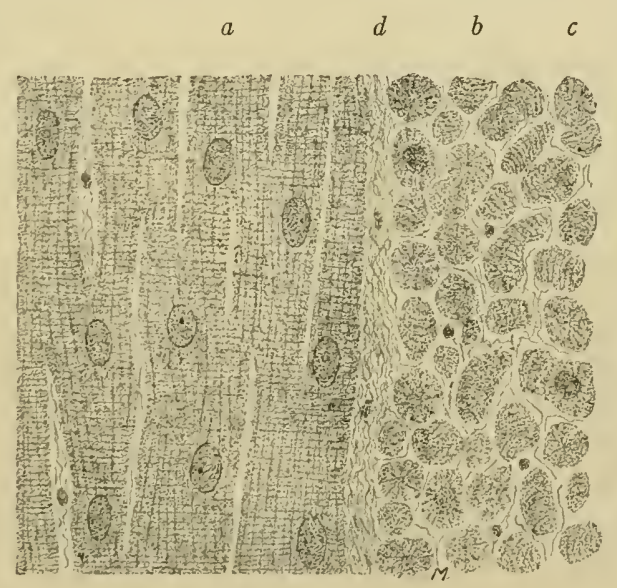

FIG. 66.-Section of Heart Muscle. $\times 35$. (Technic 7, p. 125.) $a$, Cells cut longitudinally; $b$, cells cut transversely (only three nuclei have been included in the plane of section); $c$, cells cut obliquely; $d$, connective-tissue septum.

entirely fills the space between two ground membranes which lie unusually close together. Special technic has demonstrated the fact that the fibrillæ do not stop at the disc but are continuous through it, although they show in passage some modification of structure.

The one question which has been most discussed in regard to the structure of heart muscle and which remains still unanswered is whether heart muscle is cellular or a syncytium. The solution of the problem is of course dependent upon the determination of the significance of the intercallated discs--whether or not they represent true cell boundaries. The segments into which the heart muscle fibre is divided by the discs have long been described as cells. The fact that the nuclei are usually placed about midway between two discs; that the discs show the same staining reaction as intercellular substance when subjected to the action of silver nitrate, and the ease with which heart muscle may be

\footnotetext{
${ }^{1}$ Some authoritics deny this.
} 
separated into "cells," especially in young animals and in lower vertebrates, by use of those chemicals usually used to break down intercellular cement; have all been used as arguments in favor of a cellular structure. On the other hand, the fact that it has not been proved that the segments bounded by the discs correspond to the original myoblasts; that in the later stages of development of heart muscle the type of nuclear division is usually amitotic, a type frequently unaccompanied by division of the cytoplasm; that the fibrillæ pass uninterrupted through the discs; that some discs only partially cross a fibre; that some segments contain more than one nucleus while others are non-nucleated; all favor a syncytial interpretation. How open the question still remains is shown by the fact that Heidenhain regards the "cells" as "growth segments"; that Marceau considers them non-contractile "supports" for the fibrillæ; that Jordan from his work on humming-birds concludes that they are a coarser development of the anisotropic bands; while Schaffer looks upon them as post-mortem contraction artefacts.

\section{Development of Muscle Tissue}

Smooth Muscle.-In the higher animals, muscle tissue with the exception of that connected with the sweat, lacrymal and mammary glands, which is of
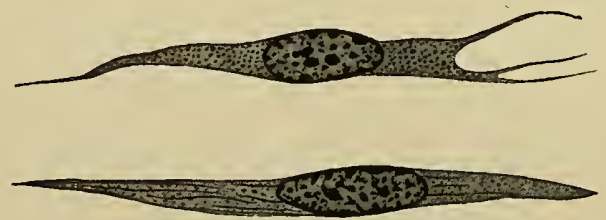

Fig. 67.-Myoblasts in Different Stages of Development. (Godlewski.)

The upper cell represents a myoblast with granular cytoplasm (from sheep embryo of I3 mm.); the middle, a myoblast with fibrils in process of formation (from guinea-pig embryo of Io $\mathrm{mm}$.); the lower, a myoblast with still further differentiated, segmented fibrils (from a rabbit embryo of $8.5 \mathrm{~mm}$.).

ectodermic origin, is derived wholly from mesoderm. The cells (myoblasts) which are to become smooth muscle cells develop in the general mesenchymal tissue among cells which are to become connective-tissue cells and with which they are at first apparently identical. In becoming a smooth muscle cell the myoblast changes its shape, becoming greatly elongated, its nucleus at the same time becoming oval or rod shaped. Such cells anastomose freely. During these changes in shape, delicate longitudinal fibrils appear in the cytoplasm. Just how these fibrils originate is not known, but they probably represent a specialization and rearrangement of the spongioplasm.

Striated Voluntary Muscle.-This develops from the mesoblastic somites. Each somite early divides into an outer part, sclerotome or cutis plate, and an inner part, the myotome or muscle plate. Cells of the myotome soon show changes 
which distinguish them as myoblasts or muscle-forming cells. These cells, which are at first spherical, become elongated and spindle shaped. The nucleus is at this stage centrally placed and the spongioplasm is in the form of a reticulum.

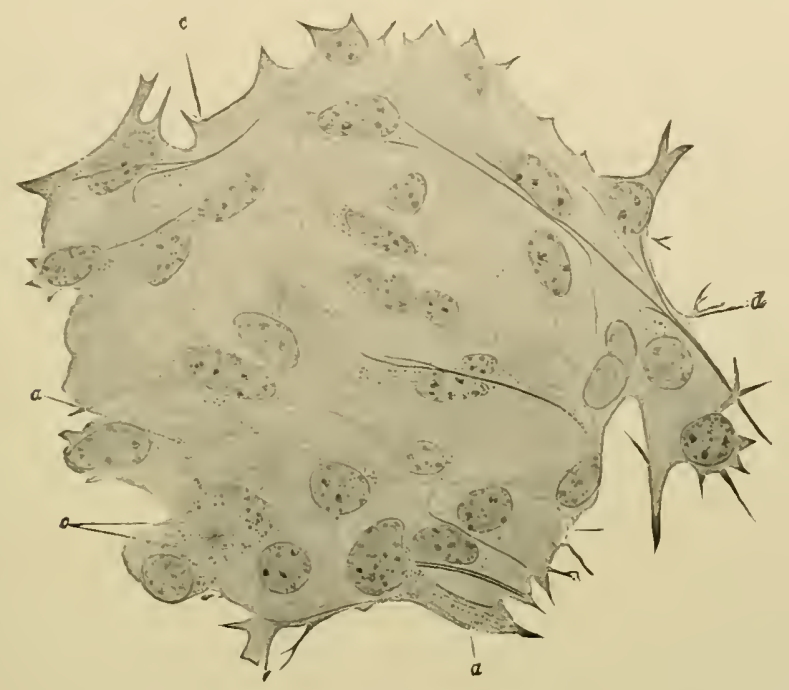

FIg. 68.-From a Section of Developing Heart Muscle from a Rabbit Embryo of 9 mm. (Godlewski.)

$a$, Cell body with granules arranged in series; $b$, cell body with centrosome and attraction sphere; $c$, branching fibril; $d$, fibrils extending through several cells.

Fibrillar arrangement of the spongioplasm first appears around the periphery where granules form in the cytoplasm and become arranged in rows lengthwise of the cell. The central portion of the cell is at this stage still occupied by retic-

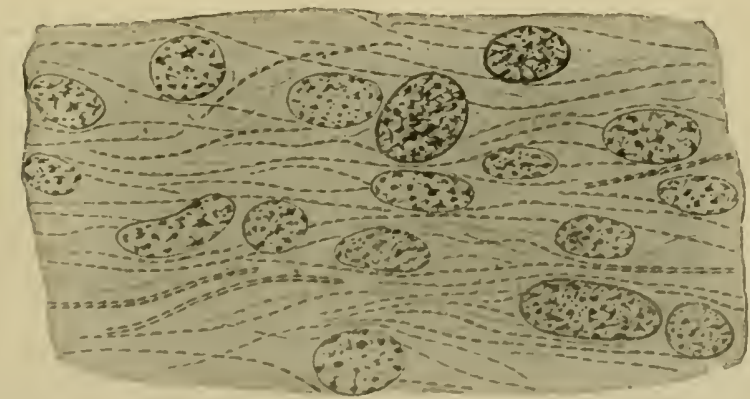

FIG. 69.-From a Section of I)eveloping II eart Muscle in a Rabbit Embryo of ro mm. (Godlcwski.)

The fibrils are segmenterl, indicating the beginning of the cross striation charactistic of heart muscle.

ular spongioplasm and the nucleus. These granules next unite to form delicate fibrils. New fibrils form both by longitudinal arrangement of more granules and by longiturlinal splitting of fibrils already formed, until they finally fill 
the entire cell. After the union of the granules to form fibrils the latter are apparently homogeneous, but later differentiate into the alternate light and the dark substances which determine the cross striations characteristic of striated muscle. Just how this differentiation takes place is not known. During this process of fibrillation the nucleus has been undergoing mitotic division without corresponding division of the cytoplasm. In white fibres these nuclei migrate to the surface and come to lie just beneath the sarcolemma. The sarcoplasm in which the fibrils lie probably represents the remains of the undifferentiated protoplasm (hyaloplasm).

Some authorities deny the origin of the muscle fibre from a single cell, describing it as derived from a number of myoblasts which unite to form a fibre.

Heart muscle develops from mesenchyme. The myoblasts are at first small, spheroidal, and closely arranged. With the appearance of intercellular substance the cells become separated and irregular in shape, and anastomose to form a syncytium. A little later the cells become arranged in parallel columns and cross markings, the "intercallated discs" appear, dividing the syncytium into the so-called "heart-muscle cells." Whether these coincide with the original myoblasts is not known. As in voluntary muscle, fibrils first develop in the periphery of the cell apparently by union of granules which arrange themselves in lines lengthwise of the cell. The fibrils increase in number and invade the entire cell except the small area of undifferentiated protoplasm which remains around the nucleus. Later the fibrils show the alternate light and dark markings or cross striations. As with voluntary muscle the way in which these cross markings develop is not known.

Attention has already been called (p. $5^{2}$ ) to the spongioplasm as the contractile element of protoplasm. It is to be noted that in the development of muscle no new element appears, the contractile fibrillce representing nothing more than a specialization of the already contractile spongioplasm.

\section{TECHNIC}

(I) Isolated Smooth Muscle Cells.-Place small pieces of the muscular coat of the intestine in o.I-per-cent. aqueous solution of potassium bichromate, or in 30-per-cent. alcohol for forty-eight hours. Small bits of the tissue are teased thoroughly and mounted in glycerin. Nuclei may be demonstrated by first washing the tissue and then staining for twelve hours in alum carmine (p. xg). This is poured off, the tissue again washed in water and preserved in eosin-glycerin, which gives a pink color to the cytoplasm.

(2) Potassium hydrate in 40-per-cent. aqueous solution is also recommended as a dissociater of smooth muscle cells. Pieces of the muscular coat of the intestine are placed in this solution for five minutes, then transferred to a saturated aqueous solution of potassium acetate containing I-per-cent. hydric acetate for ten minutes. Replace the acetate solution by water, shake thoroughly, allow to settle, pour off water, and add alum-carmine solution (p. Ig). After twelve hours' staining, wash and transfer to eosin-glycerin.

(3) Sections of Smooth Muscle.-Fix small pieces of intestine in formalinMüller's (technic 5, p. 7) or in Zenker's fluid (technic 9, p. 8). Thin transverse 
or longitudinal sections are stained with hæmatoxylin-eosin (technic I, p. 20), and mounted in balsam. As the two muscular coats of the intestine run at right angles to each other, both longitudinally and transversely cut muscle may be studied in the same section.

(4) Striated Voluntary Muscle Fibres.-One of the long muscles removed from a recently killed animal is kept in a condition of forced extension while a Iper-cent. aqueous solution of osmic acid is injected into its substance at various points by means of a hypodermic syringe. Fixation is accomplished in from three to five minutes. The parts browned by the osmic acid are then cut out and placed in pure glycerin, in which they are teased and mounted.

(5) Sections of Striated Voluntary Muscle-Fix a portion of a tongue in formalin-Müller's fluid or in Zenker's fluid (p. 8 ). Thin sections are stained with hæmatoxylin-picro-acid-fuchsin (technic 3, p. 21) and mounted in balsam. As the muscle fibres of the tongue run in all directions, fibres cut transversely, longitudinally, and obliquely may be studied in the same section. The sarcolemma, the pointed endings of the fibres, and the relation of the fibres to the connective tissue can also be seen.

(6) Isolated heart-muscle cells may be obtained in the same manner as smooth muscle cells. (See technic I, p. I24.)

(7) Sections of Heart Muscle.-These are prepared according to technic 3 (above). By including the heart wall and a papillary muscle in the same section, both longitudinally and transversely cut cells are secured. The stain may be either hæmatoxylin-eosin (technic I, p. 20), or hæmatoxylin-picro-acid-fuchsin (technic 3, p. 2I). 


\section{CHAPTER VI}

\section{NERVE TISSUE}

\section{The Neurone}

In most of the cells thus far described the protoplasm has been confined to the immediate vicinity of the nucleus. In the smooth muscle cell was seen an extension of protoplasm to a considerable distance from the nuclear region, while in the connective-tissue cells of the cornea the protoplasmic extensions took the form of distinct processes. Processes, often extending long distances from the cell body proper, constitute one of the most striking features of nervecell structure. Some of these processes are known as nerve fibres; and nerve tissue was long described as consisting of two elements, nerve cells and nerve fibres. With the establishment of the unity of the nerve cell and the nerve fibre, the nerve cell with its processes was recognized as the single structural unit of nerve tissue. This unit of structure is known as a neurone. The neurone may thus be defined as a nerve cell with all of its processes.

In the embryo the neurone is developed from one of the ectodermic cells which ccnstitute the wall of the primitive neural canal. This embryonic nerve cell, or neuroblast, is entirely devoid of processes. Soon, however, frcm one end of the cell a process begins to grow out. This proress is known as the axone (axis-cylinder process, neuraxone, neurite). Other prccesses appear, alro as outgrowths of the cell body; these are known as protoplasmic processes or dendrites.

Each adult neurone thus consists of a cell body, and passing off from this cell body two kinds of processes, the axis-cylinder process and the dendritic processes (Fig. 70).

An important exception to this rule is presented by the cells of the cerebrospinal ganglia (Fig. 292). Here the typical neurone has two processes, a peripheral process, and a central process which enters the central nervous system. Both processes have a typical axis-cylinder structure. Some authorities have interpreted the peripheral process as a modified dendrite.

I. The Cell Body.-Like most other cells, the nerve cell body consists of a mass of protoplasm surrounding a nucleus (Fig. 7I). 
Nerve cell bodies vary in size from very small cell bodies, such as those found in the granule layers of the cerebellum and of the olfactory lobe, to the large bodies of the Purkinje cells of the cerebellum

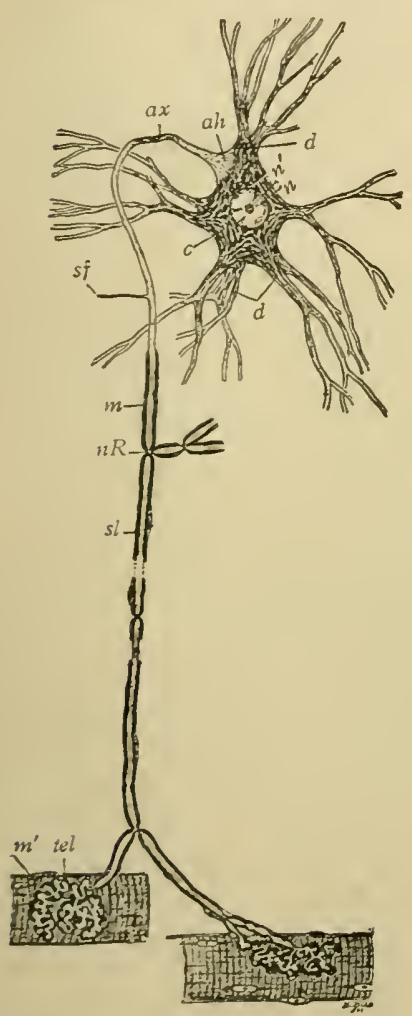

FIG. 70.

and of the motor cells of the ventral horns of the cord, which are among the largest in the body. There is as much variation in shape as in size, and some of the shapes are characteristic of the regions in which the cells are situated. Thus, many of the bodies of the cells

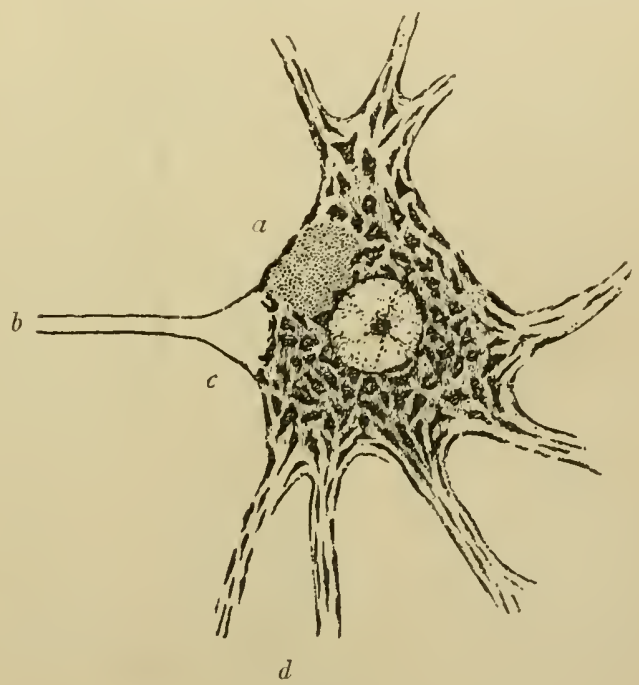

FIG. 7 I.

FIG. 70.-Scheme of Peripheral Motor Neurone. (Barker.) The cell body, protoplasmic processes, axone, collaterals, and terminal arborizations in muscle are all seen to be parts of a single cell and together constitute the neurone. c, Cytoplasm of cell body containing chromophilic bodies, neurofibrils, and perifibrillar substance; $n$, nucleus; $n^{\prime}$, nuclcolus; $d$, dendrites; $a h$, axone hill free from chromophilic bodies; $a x$, axone; sf, branch (collateral); $m$, medullary sheath; $n R$, node of Ranvier where branch is given off; $s l$, neurilemma (probably not present in central nervous system); $m^{\prime}$, striated muscle fil)re; tel, motor end plate.

FIg. 7 I. - Large Motor Nerve Cell from Ventral IIorn of Spinal Cord of Ox, showing Chromophilic Borlies. (From Barker, after von Lenhossék.) $a$, Pigment; $b$, axone; $c$, axone hill; $d$, dendrites.

of the spinal ganglia are spheroidal; of most of the cells of the cortex cerebri, pyramidal; of the cells of Purkinje, pyriform; of the cells of the ventral horns of the cord, irregularly stellate. According to the number of processes given off, nerve cells are often referred to as unipolar, bipolar, or mullipolar.

The NuCi.fus of the nerve cell (Fig. $7 \mathrm{I}$ ) differs in no essential 
from the typical nuclear structure. It consists of (I) a nuclear membrane, (2) an intranuclear network of linin and chromatin, (3) an achromatic nucleoplasm, and (4) a nucleolus.

The CyTOPLASM of the nerve cell consists of at least two distinct elements: (I) Neurofibrils, and (2) perifibrillar substance. In most nerve cells a third element is present, (3) chromophilic bodies.

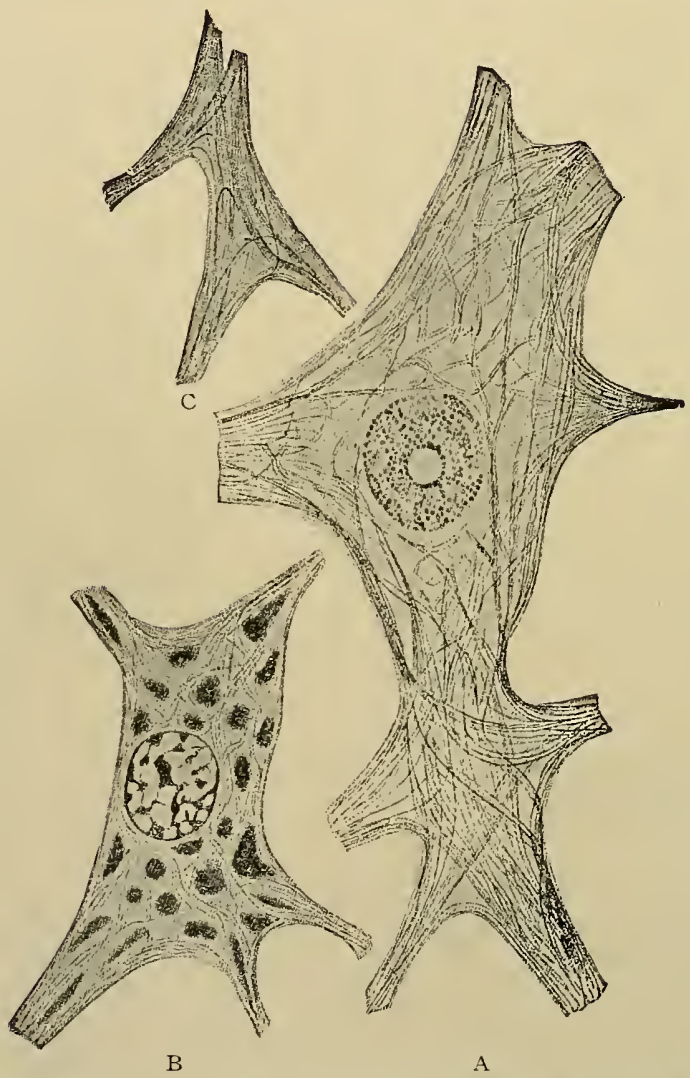

FIG. 72.-Ganglion Cells, Stained by Bethe's Method, showing Neurofibrils. A Anterior horn cell (human); B, cell from facial nucleus of rabbit; C, dendrite of human anterior horn cell showing arrangement of neurofibrils. (Bethe.) In B the chromophilic bodies are shown. In this picture the neurofibrils are shown as not anastomosing.

(1) The neurofibrils are extremely delicate fibrils which are continuous throughout the cell body and all of its processes. Within the body of the cell they cross and interlace and probably anastomose (Figs. 72 and 73 ).

(2) The perifibrillar substance (Fig. 72) is a fluid or semi-fluid substance which both in the cell body and in the processes surrounds 
and separates the neurofibrils. It is believed by some to be like the fibrils, continuous throughout the cell body and processes, by others to be interrupted at certain points in the axone (see p. I37).

(3) The chromophilic bodies (Fig. 7I) are granules or groups of granules which occur in the cytoplasm of all of the larger and of many of the smaller nerve cells. They are best demonstrated by means of a special technic known as the method of Nissl (page 38).

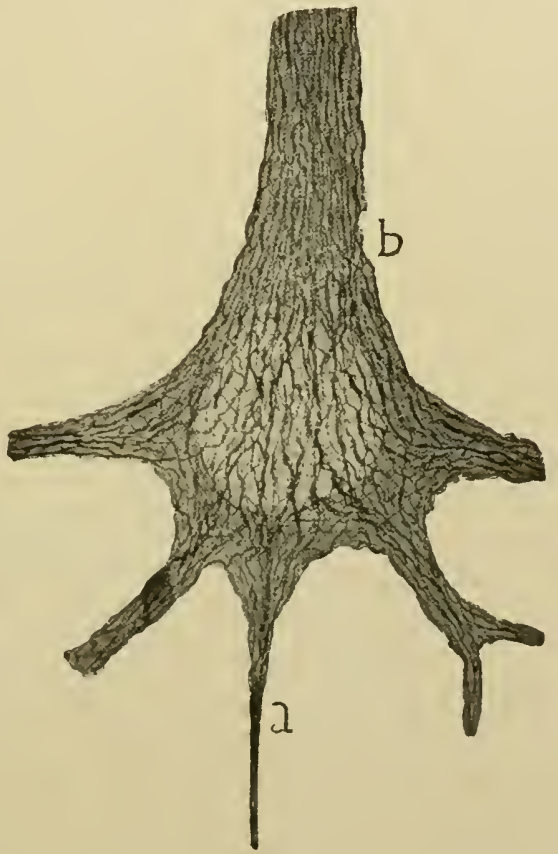

FIG. 73.-Body of Large Pyramidal Cell from Cortex of Cat. Silver Method of Cajal. Shows nucleus pale and arrangement of neurofibrils within the cell; $a$, axone; $b$, main or apical dendrite. (Cajal). In this picture the neurofibrils are shown as anastomosing.

When subjected to this technic, nerve cells present two very different types of reaction. In certain small cells the amount of cytoplasm is extremely small and only the nuclei stain with the Nissl method. Such cells are found in the granule layers of the cerebellum, olfactory lole, and retina. They are known as caryochrome cells, and apparently consist wholly of neurofibrils and perifibrillar substance. ()ther cells react, both as to their nuclei and as to their cell bodies, to the Nissl stain. 'These cells are known as somalochrome cells. Taking as an example of this latter type of cell one of the motor cells of the ventral horn of the cord and subjecting 
it to the Nissl technic, we note that the cytoplasm is composed of two distinct elements: (a) a clear, unstained ground substance, and, scattered through this, (b) deep-blue-staining masses, the chromophilic bodies (Fig. $7 \mathrm{I}$ ). These bodies are granular in character and

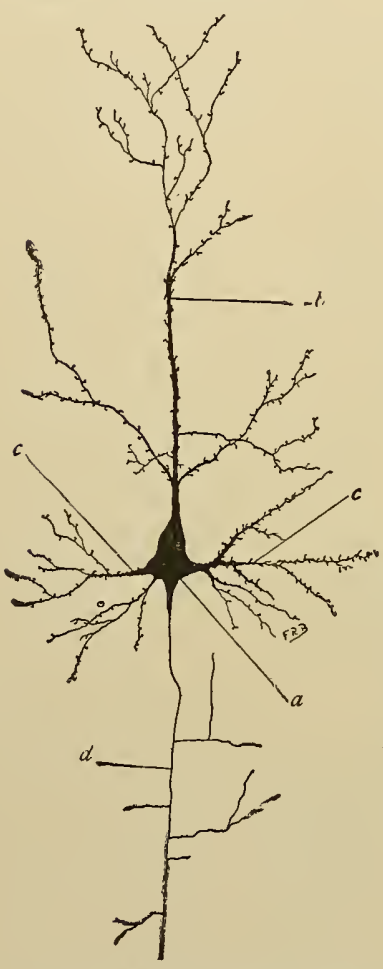

FIG. 74.-Pyramidal Cell from Human Cerebral Cortex. (Golgi bichlorid method. See 2, p. 36.) Golgi cell type I. $a$, Cell body; $b$, main or apical dendrite showing gemmules; $c$, lateral dendrites showing gemmules; $d$, axone with collaterals. Only part of axone is included in drawing. differ in shape, size, and arrangement. They may be large or small, regular or irregular in shape, may be arranged in rows or in an irregular manner, may be close together, almost filling the cell body, or quite separated from one another. Presenting these variations in different types of cells, the appearance of the chromophilic bodies in a particular type of cell remains constant, and has thus been used by Nissl as a basis of classification. ${ }^{1}$

It is important to note in studying the nerve cell by this method that somatochrome cells of the same type frequently show marked variations in staining intensity. This appears to depend upon the size and closeness of arrangement of the chromophilic bodies, and this again seems dependent upon changes in the cytoplasm connected with functional activity.

In cells stained by Nissl's method the cytoplasm between the chromophilic bodies remains unstained and apparently structureless, and it is this part of the cytoplasm that corresponds to the neurofibrils and a part at least of the perifibrillar substance.

The relation which the appearance of the Nissl-stained cell bears to the structure of the living protoplasm is still undetermined. According to some investigators the Nissl bodies exist as such in the living cell. Others believe that they are not present in the living cell, but represent precipitates due either to postmortem changes or to the action of fixatives. The significance: of the Nissl picture from the standpoint of pathology lies in the fact that when subjected to a given technic, a particular type of nerve cell always pre-

1 For this classification, the significance of which is somewhat doubtful, the reader is referred to Barker, "The Nervous System and Its Constituent Neurones," p. I 2 I. 
sents the same appearance ("equivalent picture"), and that this appearance furnishes a norm for comparison with cells showing pathological changes, and which have been subjected to the same technic.

Many nerve cells contain more or less brownish or yellowish pigment (Fig. 7I). This pigment, which is a lipochrome, is not present in the cells of the new-born, but appears in increasing amounts with age. Its significance is not known. Another black pigment known as melanin is present in certain cells in the central nervous system (e.g., the substantia nigra). This pigment is said to increase in amount until adolescence.

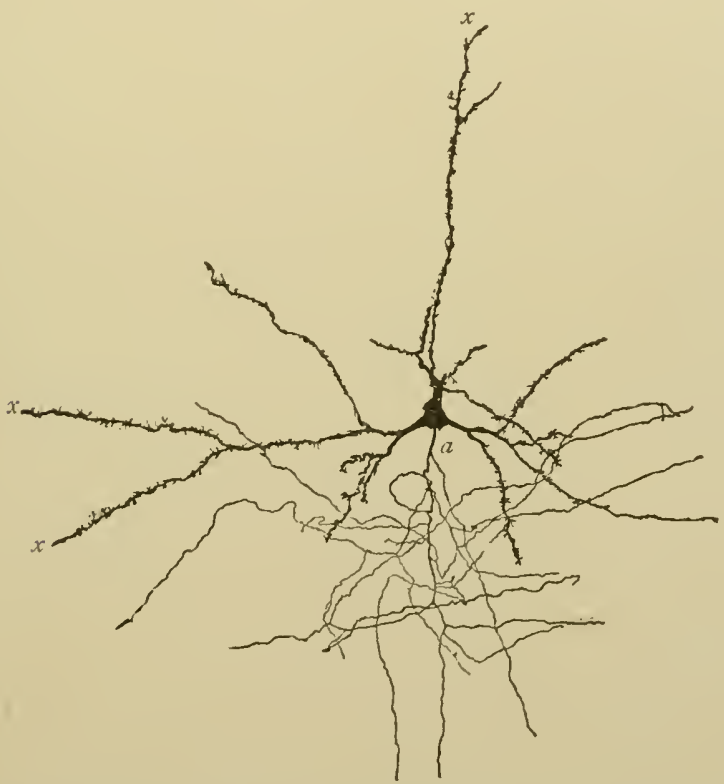

Fig. 75--Golgi Cell Type II. from Cerebral Cortex of Cat. (Kölliker.) x, Coarse protoplasmic processes with gemmules easily distinguishable from the more delicate, smoother axone, $a$. The latter is seen breaking up into a rich plexus of terminal fibres near its cell of origin, practically the entire neurone being included in the drawing.

In addition to its characteristic structure, the nerve cell may contain many elements found in other cells (p. 43). Golgi, Holmgren, Cajal, and others have also demonstrated a network of canals within the nerve cell similar to that found in other cells (p. 46, Fig. 4).

II. The Protoplasmic Processes or Dendrites.-These have a structure similar to that of the cell body, consisting of neurofibrils, perifibrillar substance, and, in somatochrome cells, chromophilic bodies (Figs. 7 I and 72). Dendrites branch dichotomously, become 
rapidly smaller, and usually end at no great distance from the cell body (Figs. 74 and 75).

III. The Axone.- This differs from the cell body and dendrites in that it contains no chromophilic bodies (Fig. 7 I), consisting wholly of neurofibrils and perifibrillar substance. Not only is it entirely achromatic itself, but it always takes origin from an area of the cell body, the axone hill (Fig. $7 \mathrm{I}$ ), which is also free from chromophilic bodies. It is as a rule single, and while usually arising from the body of the cell may be given off from one of the larger protoplasmic trunks. Some few cells have more than one axone, and nerve cells without axones have been described. In Golgi preparations the axone is distinguished by its straighter course, more uniform diameter, and smoother outline (Fig. 74). It sends off few branches (collaterals), and these approximately at right angles. Both axone and collaterals usually end in terminal arborizations. In most cells the axone extends a long distance from the cell body. Such cells are known as Golgi cell type I or long axone neurones (Fig. 74). In others the axone branches and ends in the gray matter in the vicinity of its cell of origin-Golgi cell type II or short axone neurones (Fig. 75).

As they leave the cell body the neurofibrils of the axone converge to a very narrow portion of the axone, where the perifibrillar substance is much reduced in amount, or according to some, entirely interrupted. Beyond this the fibrils become more separated and the perifibrillar substance more abundant.

Some axones pass from their cells of origin to their terminations as "naked" axones, i.e., uncovered by any sheath. Other axones are enclosed by a thin membrane, the neurilemma or sheath of Schwann. Still others are surrounded by a sheath of considerable thickness known as the medullary or myelin sheath.

Depending upon the presence or absence of a medullary sheath, axones may thus be divided into two main groups-medullated axones and non-medullated axones.

I. Non-Medullated Axones (non-medullated nerve fibres) (Fig. 76). These are subdivided into non-medullated axones without a neurilemma and non-medullated axones with a neurilemma.

(a) Non-medullated axones without a neurilemma are merely naked axones. Present in large numbers in the embryo, they are in the adult confined to the gray matter and to the beginnings and endings of sheathed axones, all of the latter being uncovered for a 
short distance after leaving the nerve cell body, and also just before reaching their terminations.

(b) Ton-medullated axones with a neurilemma-fibres of Remak (Fig. 76). In these the axone is surrounded by a delicate homogeneous, nucleated sheath, the neurilemma or sheath of Schwann (see p. I35). These axones are described by some writers as having

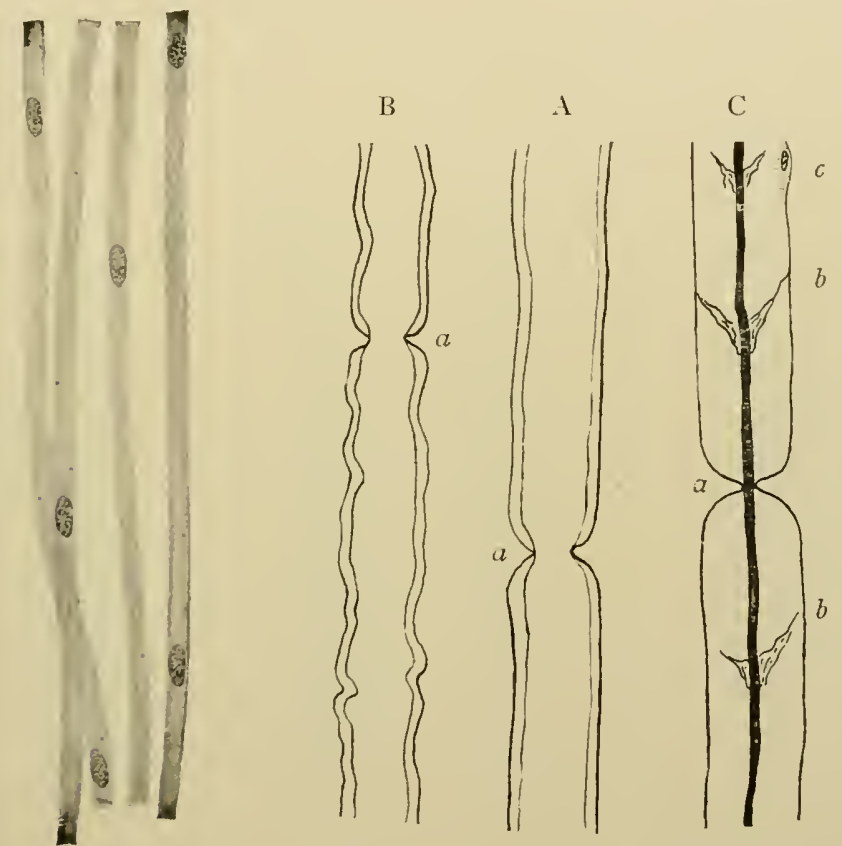

Fig. 77 .

Fig. 76.-Non-medullated Nerve Fibres with Neurilemma, only the nuclei of which can be seen. $\times_{300 .}$

FIG. 77. - A, Fresh nerve fibre from sciatic nerve of rabbit teased apart in normal salt solution, showing broad unshrunken axone and comparatively thin medullary sheath. $B$, Showing crenation of medullary sheath which occurs soon after placing fibres in salt solution. $C$, Same after fixation and staining with picro-acid-fuchsin, showing shrunken axone and broad medullary space. The latter usually contains irregular clumps of myelin. a, Node of Ranvier; $b$, incisures of Schmidt; $c$, nucleus of neurilemma.

no true neurilemma, but merely a discontinuous covering of flat connective-tissue cells, which wrap around the axone and correspond to the endoneurium of the nerve trunk (see page 426). The majority of the axones of the cells of the sympathetic ganglia fall under this category.

2. MadulaAtED Axones (medullated or myelinated nerve fibres). -These, like the non-medullated, are subdivided according to the 
presence or absence of a neurilemma into medullated axones with a neurilemma and medullated axones without a neurilemma.

(a) Medullated axones with a neurilemma constitute the bulk of the fibres of the cerebro-spinal nerves. Each fibre consists of (I) an axone or axis-cylinder, (2) a medullary sheath, and (3) a neurilemma.

(I) The axone is composed of neurofibrils continuous with those of the cell body, and like them lying in a perifibrillar substance or neuroplasm (Fig. 82). In the fresh condition the axone is broad,

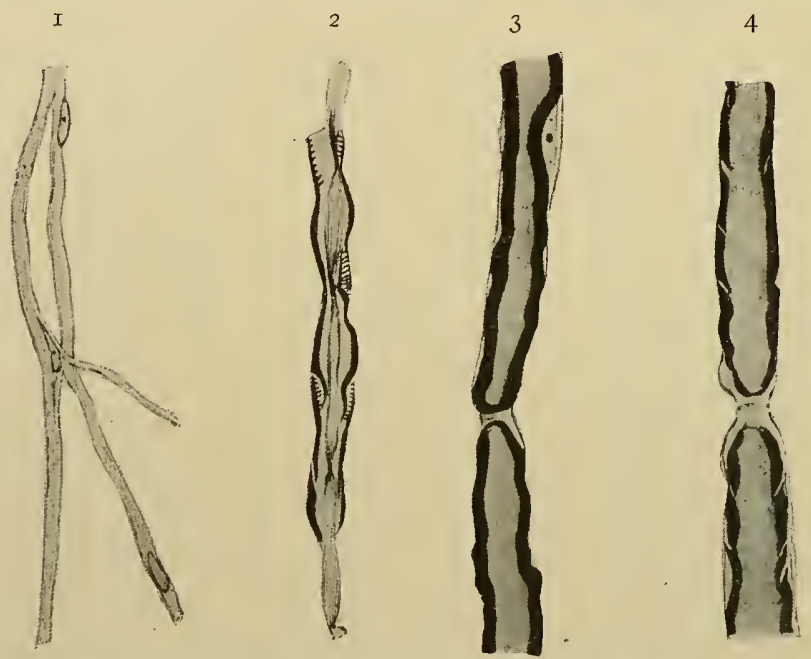

Fig. 78,-1, Fibres of Remak; 2, nerve fibre from central nervous system (medullated but without sheath of Schwann); 3 and 4 , nerve fibres from the sciatic of frog, showing nodes of Ranvier, and in 4 the incisures of Schmidt.

and shows faint longitudinal striations corresponding to the neurofibrils, or appears homogeneous (Fig. 77,A). Fixatives usually cause the axone to shrink down to a thin axial thread, whence its older name of axis-cylinder (Fig. 77,C). A delicate membrane has been described by some as enveloping the axone. It is known as the axolemma or periaxial sheath (Fig. 80).

(2) The medullary sheath (Figs. 77 and 82) is a thick sheath largely composed of a semi-fluid substance resembling fat and known as myelin. In the fresh state the myelin has a glistening homogeneous appearance. It is not continuous, but is divided at intervals of from 80 to $6 \mathrm{co} \mu$ by constrictions, the nodes or constrictions of Ranvier. That portion of a fibre included between two nodes is known as an internode (Fig. 80). The length of the internode is 
usually proportionate to the size of the fibre, the smaller fibres having the shorter internodes. In fresh specimens the medullary sheath of an internode appears continuous (Fig. $77, A$ ), but in fixed specimens it is broken up into irregular segments, Schmidt-Lantermann segmonts, by clefts which pass from neurilemma to the axolemma or axone, and are known as the clefts or incisures of SchmidtLantermann (Fig. 77,C). On boiling medullated nerve fibres in alcohol and ether a fine network is brought out in the medullary sheath, the neurokeratin network. Owing to the resistance of neurokeratin to the action of trypsin, it has been considered as possibly similar in composition to horn.

(3) The neurilemma or sheath of Schwann (Figs. 82, B, and 8I) is a delicate structureless membrane which encloses the myelin. At the nodes of Ranvier the neurilemma dips into the constriction and comes in contact with the axone or axolemma. Silver nitrate staining shows a black transverse line in the neurilemma at the node of Ranvier. This line represents the boundary between two neurilemma cells. Against the inner surface of the neurilemma, usually about midway between two nodes, is an oval-shaped nucleus, the nucleus of the neurilemma (Figs. 76, $C$, and $8 \mathrm{I}$ ). Each nucleus is surrounded by an area of granular protoplasm, and makes a little depression in the myelin and a slight bulging of the neurilemma (Fig. 77, C). According to most observers no neurilemma is present in the central nervous system. An important exception is Cajal, who describes the medullated fibre of the central nervous system as having a neurilemma.

In addition to the above-described sheaths, most medullater fibres of peripheral nerves have,

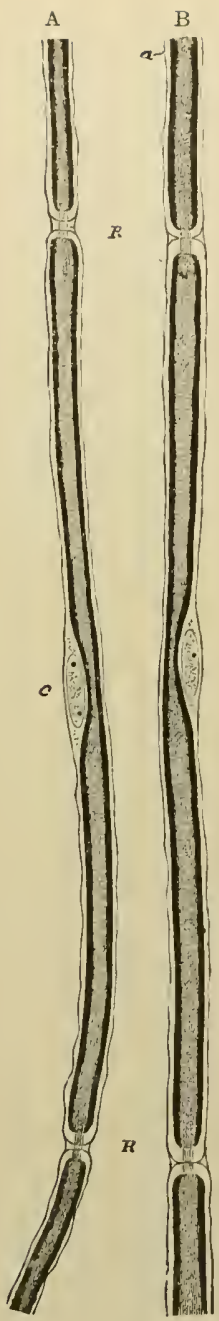

Fri. 79.-1)iagrammatic Representation of Portions of Two Medullated Nerve Fibres, its scen in Longitudinal Section, stained with Osmic Acid. (Length of internode is proportionately shortencll.) $R, R$, Nodes of Ranvier, with axis cylinder passing through; $a$, ncurilemma; $c$, nucleus surrominderl by protoplasm, lying at abrut the midflle of the internode between the neurilemma and the medlullary sheath. (From drawing t)y J. E. Neale in Quain's Anatomy.) 
outside the neurilemma, a nucleated sheath of connective-tissue origin, known as the sheath of Henle (Fig. 8I).

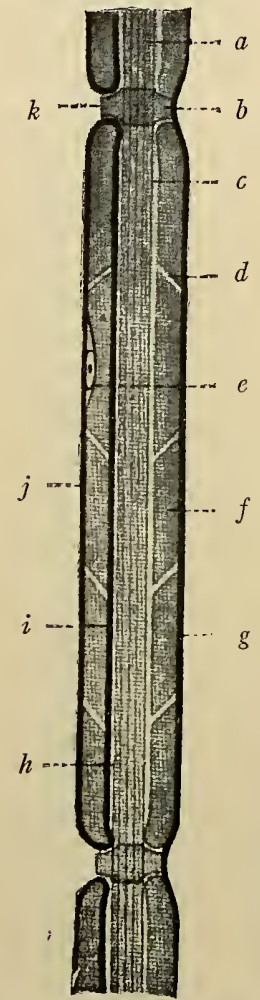

FIG. 80.

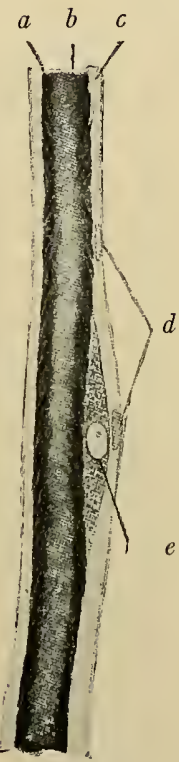

FIG. 8I.
FIG. 80.--Diagram of Structure of a Medullated Nerve Fibre of a Peripheral Nerve showing two different views (one on each side) as to relations of neurilemma and axolemma and their behavior at the nodes of Ranvier. (Szymonowicz.) a, Neurofibrils; $b$, cement substance; $c$, axone; $d$, incisure of Schmidt; $e$, nucleus of neurilemma; $f$, medullary sheath; $g$, sheath of Schwann; $h$, axone; $i$, axolemma; $j$, sheath of Schwann; $k$, node of Ranvier.

FIg. 8r.-Piece of Medullated Nerve Fibre from Human Radial Nerve. $\times 400$. Osmic-acid fixation and stain. (Szymonowicz.) $a, \mathrm{Me}-$ dullary sheath; $b$, axone; $c$, sheath of Henle; $d$, nuclei of Henle's sheath; $e$, nucleus of neurilemma.

Two views as to the relation of the axolemma to the neurilemma are illustrated in Fig. 8o. According to one the neurilemma is continuous, merely dipping into the nodes of Ranvier, where it touches the axolemma or the axone. According to the second both neurilemma and axolemma are interrupted at the node, but unite with each other there to enclose completely the medullary substance of the internode.

According to the views illustrated in Fig. 82, that part of the axone which lies between two nodes is enveloped by a cell, or by several cells forming a syncytium. The outer homogeneous membrane there pictured would thus be of the nature of a cell membrane or cuticle, and would correspond to the neurilemma. The trabeculæ (protoplasmic strands and neurokeratin network, of which some of the larger strands would represent the incisures of Schmidt) would correspond to the spongioplasm, and just along the outer side of the axone would constitute the axolemma. The myelin would thus be enclosed within the cylindrical neurilemma cell which surrounds the axone.

Recent experiments of Bethe and others tend to prove an interruption of the perifibrillar substance at the node of Ranvier. They consider the axone at the node as probably crossed by a sieve-like plate, through the holes of which the fibrils pass, but which completely interrupts the perifibrillar substance. The accuracy of these observations has been disputed.

Medullated nerve fibres vary greatly in size. The finer fibres have a diameter of from 2 to $4 \mu$, those of medium size from 4 to ro $\mu$, the largest from to to $20 \mu$. They have few branches, and these are always given off at the nodes of Ranvier. (b) Medullated axones without a neurilemma are the medullated 
nerve fibres of the central nervous system as described by most observers. Cajal, as already mentioned (p. I35), describes these fibres as having a neurilemma. Their structure is similar to the above-described structure of a medullated nerve fibre with a neurilemma, except for the absence of the latter sheath.

As to the physiological significance of the structural elements of the neurone, we have little absolute knowledge but certain fairly wellgrounded theories.

That portion of the neurone which surrounds the nucleus-the cell body-is, as already stated, the genetic centre of the neurone, the nucleus as in other cells being probably concerned in the general cell metabolism. From the behavior of the processes when cut off from the cell body it is evident that the latter is the trophic or nutritive centre of the neurone.

It is probable that in most or perhaps all neurones the usual direction of conduction along the axone is cellulifugal, i.e., from cell body to terminal arborization. The dendrites and cell body would receive and probably integrate the various nerve impulses received by the neurone. In other words, the dendrites and cell body receive, the axone transmits and the axone branches and terminal arborizations distribute. This general direction of conduction indicates a certain polarity of the neurone. While the cerebro-spinal ganglion cell is obviously polar-

FIG. 82.-Scheme of Structure of Medullated Peripheral Nerve Fibre of a Fish (Nemileff). A, Cross section; $B$, longitudinal section; on left, fibre is shown as stained intra vitam with methylene blue; on right, myelin is shown black as in osmic acid staining, with the incisures of Schmidt indicated; $s z$, cells of sheath of Schwann; $n$, their nuclei; ss, sheath of Schwann; $s p$, processes of the cells of sheath of Schwann or the myclin sheath network; te, larger trabecula of protoplasmic framework of medullary sheath arrangerl obliquely tr) axis-cylinder and forming the so-caller "funnels"; $l c o$, clear streaks in fibres treated with osmic acid, corresponding to le, incisures of Schmidt; mo, myclin blackenerl with osmic acirl; ax, axiscylinder; $p a$, periaxial space around itxis-cylinder; $g s$, "cragulum sheath," granules probably representing cragulated fluid in periaxial space; $p f$, peripheral, non-fibrillar, part of axis-cylinder; $f$, ncurofibrils of axis-cylinder; $r$, ring-like thickening of Schwann's sheath at norle of Kanvicr; $n$, cavity in $r$.

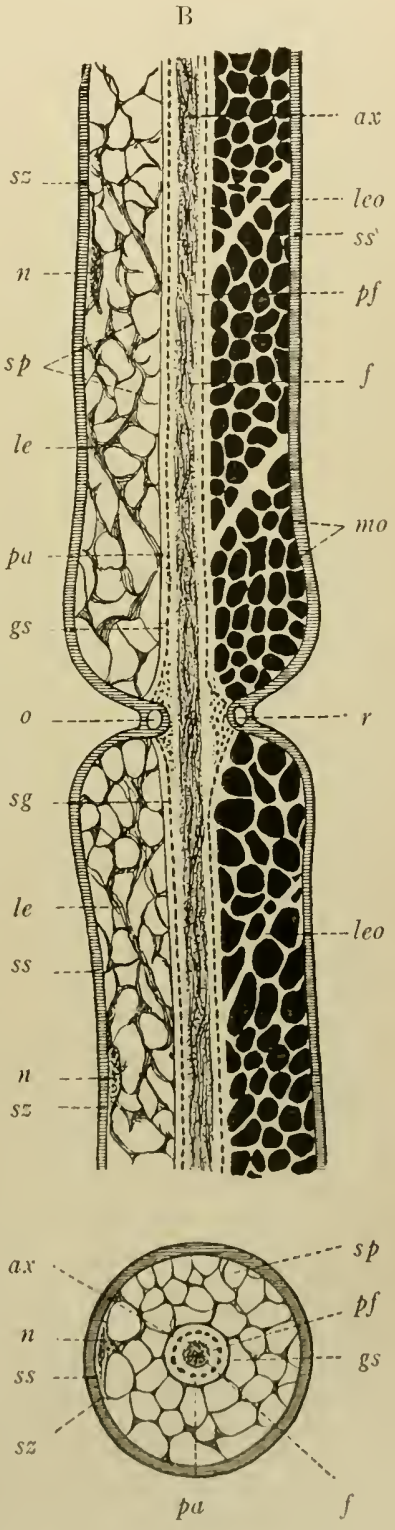

1 
ized, yet if its peripheral process be regarded as an axone, the latter would evidently constitute an exception to conduction along the axone being cellulifugal. There are cases (e.g., unipolar cerebro-spinal ganglion cell) where the nervous impulse may apparently pass from one process to another without traversing the body of the cell.

Regarding the chromophilic substance, certain facts, such for example as the entire absence of chromophilic bodies in many nerve cells, which nevertheless undoubtedly functionate; the absence of these bodies in all axones; the diminution of the chromatic substance during functional activity; its much greater diminution if activity be carried to the point of exhaustion; these together with its behavior under certain pathological conditions, all favor the theory that the stainable substance of Nissl is not the active nerve element of the cell, but is rather of the nature of a nutritive element.

There thus remains to be considered as possible factors in the transmission of the nervous impulse the neurofibrils and the perifibrillar substance. While a few investigators are inclined to magnify the importance of the latter, the majority agree in considering the neurofibrils as the principal conducting mechanism of the neurone. The already referred to observations of Bethe regarding the interruption of the perifibrillar substance at the constricted portion of the axone and at the nodes of Ranvier, would, if true, be obviously in favor of this view. It is also obvious that if the neurofibrils are the sole conducting substance and if they do not anastomose within the body of the vertebrate neurone, we would be driven to conclude there must be an extracellular anastomosis. The probability, however, is rather against both of these premises. The neurofibrils are probably a differentiation of the spongioplasm, while the perifibrillar substance and chromophilic bodies are specializations of the hyaloplasm.

As to the manner in which neurones are connected, there are two main theories, the contact theory and the continuity theory.

According to the contact theory each neurone is a distinct and separate entity. Association between neurones is by contact or contiguity of the terminals of the axone of one neurone with the cell body or dendrites of another neurone, and never by continuity of their protoplasm. This theory, which is known as the "neurone theory" and which received general acceptance as a result of the work of Golgi, His, Forel, Cajal, and others, has been recently called in questioin by such prominent neurologists as Apáthy, Bethe, Held, and Nissl, on the ground that in some cases the neurofibrils are continuous throughout a series of neurones. The point of contact between two neurones is called a synapsis (Fig. 83), and the conception that there is some kind of interruption or discontinuity in neural circuits involving more than one neurone has proved useful to physiologists. It affords an explanation of certain differences between conduction through a circuit of two or more neurones and conduction through a nerve fibre alone. For example, an impulse takes longer to traverse the circuit than to traverse a nerve fibre of equal length. Also a stimulus may pass in either direction along a nerve fibre, but cannot be "reversed" along a circuit. Based upon the contact theory is the so-called "retraction hypothesis," which held that a neurone being associated with other neurones only by contact was able to retract its terminals, thus breaking the association and throwing itself, as it were, out of circuit. 
According to the continuity theory, while the perifibrillar substance is interrupted as above described, the neurofibrils, are continuous. According to this theory the neurofibrils, which form a plexus or network within the cell body and dendrites, are connected with a pericellular network-the Golgi net - which closely invests the cell body and its dendrites. Externally the Golgi net is further connected with the neurofibrils of the axones and collaterals of other nerve cells. This connection is either direct, or, as some believe, through another general (diffuse) extracellular netzork. The neurofibrils are thus, accord-

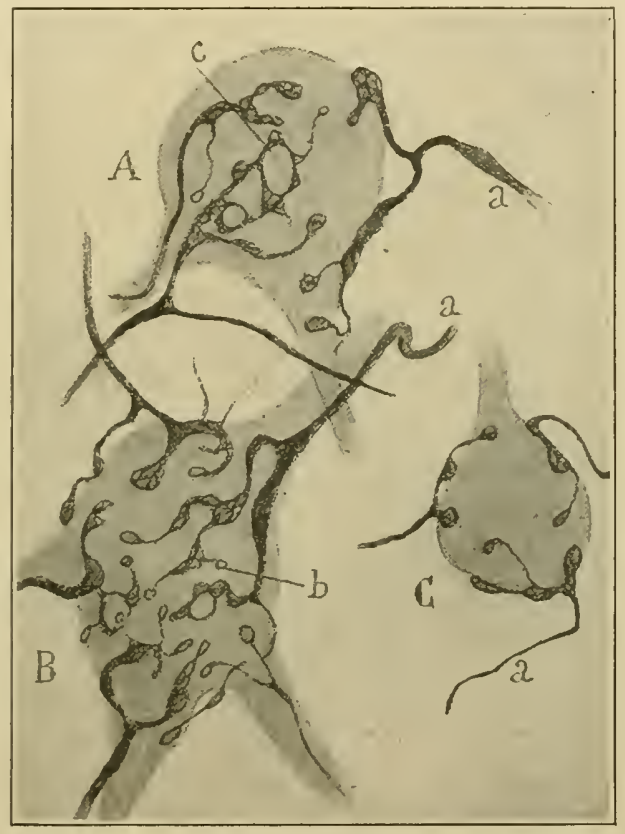

Fig. 83.-A, $B, C$, Three cells of the Ventral Cochlear Nucleus of Rabbit, showing terminals of fibres of the cochlear nerve and their relations to the cell bodies (Cajal). $a, a, a$, Fibres of the cochlear nerve, which break up into terminal arborizations upon the cells; $b, c$, terminal rings. The points of contact between the terminals of the axone of one neurone and the cell body and dendrites of the other neurone constitute a "synapsis."

ing to this theory, continuous and form two or possibly three continuous networks: (a) an intracellular network, (b) a pericellular network (Golgi), and (c) a more diffuse extracellular network, lying between the cells. The existence of (c) is extremely doubtful and it seems probable that the Golgi network is either non-nervous or an artefact. Thus the main point at issue is whether the neurofilbrils, in such pericellular terminals ats are illustrated in Fig. 83, are continuous with the neurofibrils within the cell enveloped or are separate from the latter.

The individuality of the neurone and the interdependence of its various parts are strikingly shown by its behavior when injured. That degenerative changes, which progress to complete disappearance of the nerve structures, take place in 
the distal part of a nerve when that nerve is cut across has long been accepted as one of the fundamental laws of neuropathology (law of Wallerian degeneration). In terms of the neurone concept this would mean that an axone cut off from its cell of origin degenerates and disappears and this behavior of the axone would accord with the already stated fact that the cell body is the trophic center of the neurone. The degenerative changes in the axone are best shown in their earlier stages by an osmic acid (p. 7) or by a Marchi (p. 34) stain. Later, when the degenerated fibres have been largely replaced by connective tissue, the Weigert method is most satisfactory, especially in the central nervous system. In this case, of course, the degeneration is indicated by an absence of stain, while in the Marchi method a positive picture of the degenerating myelin sheaths is

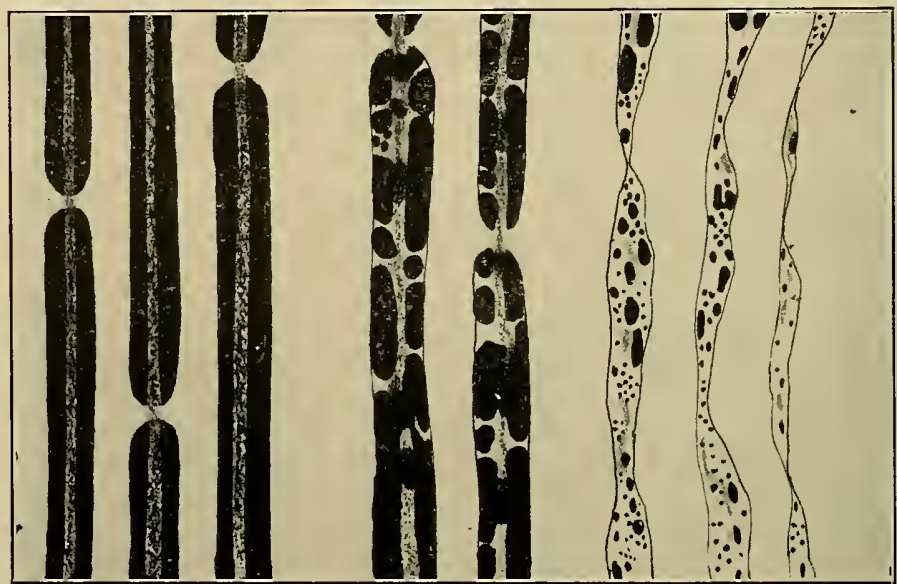

A

$B$

$C$

Fig. 84.- $A$, Normal Nerve Fibres from Sciatic Nerve of Rabbit, osmic acid fixation and stain; each fibre shows node of Ranvier. $B$, Two fibres from distal part of rabbit's sciatic five days after cutting the nerve; shows segmentation of myelin; $C$, three fibres from distal part of rabbit's sciatic three weeks after cutting nerve; most of the myelin has been absorbed and only traces of the axones remain.

seen. When the nerve is cut the first changes affect the cut ends and seem to be of a traumatic character such as formation of bulbous enlargements of the ends of the axones, increase and separation of neurofibrils and formation of sprouts. This phase is soon passed over in the distal stump, while in the central it may pass over into a beginning regeneration of the nerve fibres. In the distal portion a degeneration of the nerve fibres throughout their length and including their terminal arborization now takes place. All parts of the nerve fibre are affected. In the medullary sheath the changes consist in segmentation of the sheath, breaking up of the segments into granules and finally complete absorption (Fig. 84). While undergoing these physical changes chemical changes are also taking place in the myelin which result in its breaking down into simpler fatty substances which give the fat reaction to the Marchi stain. At the same time the neurofibrils become irregular and granular and the axis-cylinders finally disappear. 
The neurilemma cells of the peripheral nerve fibres are peculiar in that they do not degenerate; instead of this their protoplasm increases, and their nuclei proliferate. These cells are apparently concerned in the disintegration and absorp-. tion of the myelin. They also form protoplasmic bands which play an important part in the regeneration of the nerve (see below). The same rule holds good for dendrites as for axones as far as it has been possible to determine, namely, that cut off from their cells of origin they undergo complete degeneration.

At the time the law of Wallerian degeneration was established it was believed that the central portion of the nerve and the cell bodies remained intact after division of the nerve. More recently the method of Marchi (for nerve fibres) and

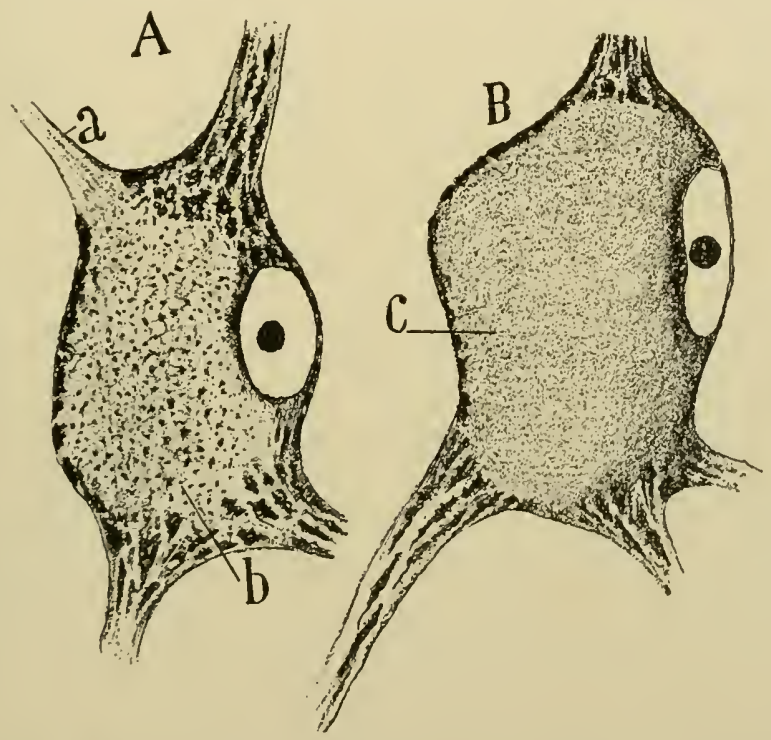

Fig. 85.-Two Motor Cells from Ventral Horn of Dorsal Cord of Rabbit; fifteen days after cutting major sacro-sciatic nerve. $A$, Cell in which the chromophilic bodies appear disintegrated and nucleus eccentric; $B$, cell showing more advanced chromatolysis, the chromophilic sulsstance being present only in the dendrites and around the nucleus in the form of a homogeneous mass; nucleus causes bulging of surface of cell.

the methorl of Nissl (for neurone bodies) have shown marked degenerative changes in the parts proximal to the lesion. The extent and rapidity of these changes are dependent mainly upon three factors ( $\mathrm{J}$ ) the type of neurone-some neurones being apparently more resistant than others to injury; (2) the character of the injury-e.g., tearing the nerve causing the greatest reaction; cutting the nerve, a reaction of less intensity; pinching the nerve, the least degree of reaction; and (3) the location of the injury, an injury near the cell body causing more effect centrally than one at a rlistance. In other words, the effect depends upon the percentage of the neurone cut off. If the injury is very near the cell borly the latter may ultimately disappear, the proximal portion of the nerve fitse which remains altacher to it undergoing a final degencration similar to that of the distal severed portion. In the cell body there is an initial turgescence, 
followed by disintegration and disappearance of the chromophilic bodies beginning near the centre of the cell. and a displacement of the nucleus toward the periphery. This reaction on the part of the cell body to injury to its axone"central chromatolysis" and "nuclear eccentricity"--is sufficiently characteristic to have led to the designation "axonal degeneration" (Fig. 85).

If the neurone body survive the injury regeneration takes place. This consists in a reappearance of the chromophilic substance, beginning near the nucleus, a return of the nucleus to its normal position, and a slow subsidence of the turgescence. While this is going on the axones of the central stump grow across the scar to the protoplasmic bands beyond formed by the neurilemma cells of the peripheral stump, and find their way along or in them to their former terminations. According to some authorities the neurilemma cells can do more than this and form, in young animals at least, new nerve fibres which, however, do not persist unless they form connections with the central stump. The outgrowth (centrogenetic) theory seems more probable than the latter (autogenetic) theory.

$A$

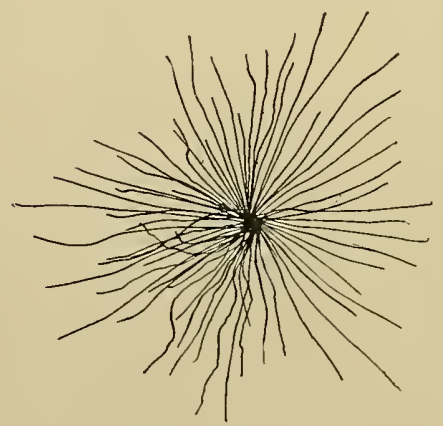

$B$

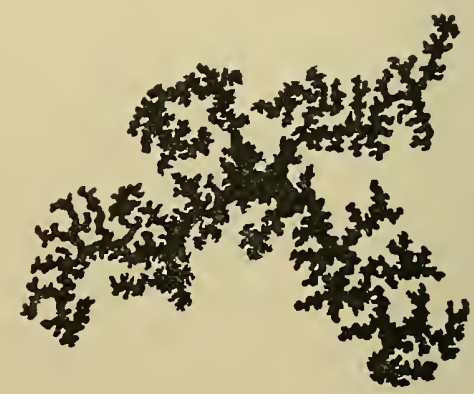

Frg. 86.-A, Neuroglia Cell-Spider Type-Human Cerebrum. B, Neuroglia Cell -Mossy Type-Human Cerebrum.

The rapidity of the above degenerative and regenerative changes varies according to the metabolic activity of the animal, i.e., they are much more rapid in warm-blooded than in cold-blooded animals, and in summer than during hibernation. They are also influenced by the age of the animal.

The importance of these degenerations from the standpoint of anatomy lies in the fact that, by using such methods as Nissl, Weigert, and Marchi, one is enabled to trace the connections between cell bodies and nerve fibres throughout the nervous system.

\section{Neuroglia}

This is a peculiar form of connective tissue found only in the nervous system. Unlike the other connective tissues, neuroglia is of ectodermic origin, being developed from the ectodermic cells which line the embryonic neural canal. These cells, at first morphologically identical, soon differentiate into neuroblasts or future 
neurones, and spongioblasts or future neuroglia cells, the latter most probably being in the form of a syncytium. Later this syncytium differentiates fibres, the neuroglia fibres, which, according to Weigert and others, may be entirely separate from the cells (Fig. 87 ), but more probably lie wholly or at least partly within them. In the syncytium there are also to be distinguished an endoplasm of granular protoplasm and a clear ectoplasm which may perhaps be regarded as a matrix. (See also Fig. 3I3.) The structure of neuroglia would thus be analogous to that of fibrous connective tissue, i.e., composed

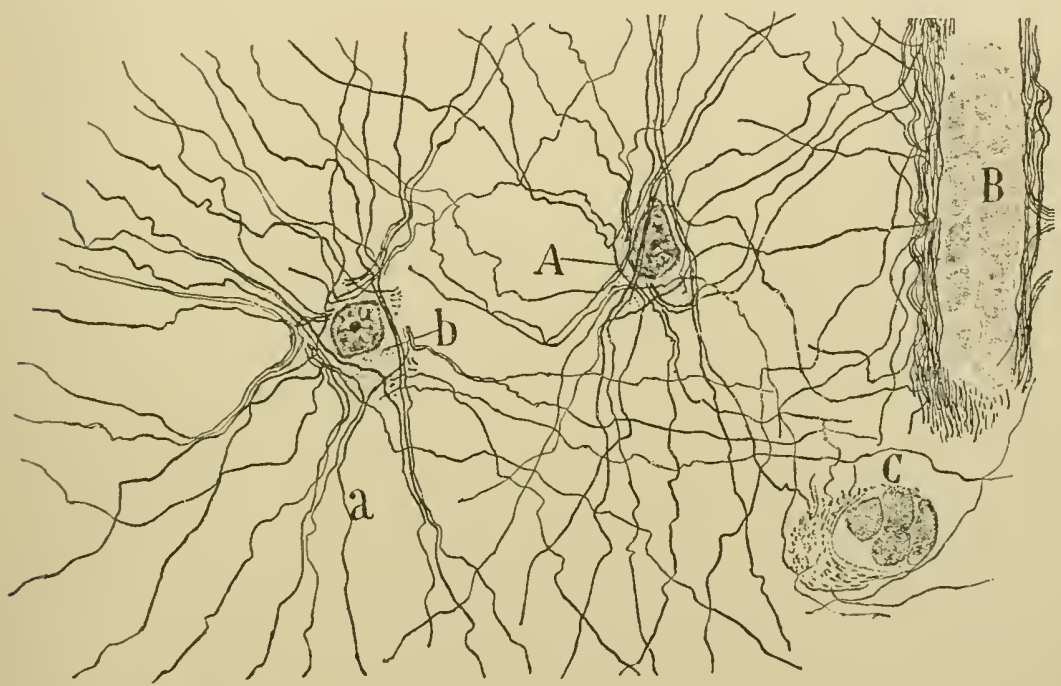

Fig. 87.-Neuroglia Cells and Fibres from the White Matter of the Human Cerebellum stained by Weigert's neuroglia stain. $A$, Neuroglia cell; $B$, blood-vessel cut longitudinally, and $C$, blood-vessel cut transversely, showing enveloping neuroglia fibres; $a$, neuroglia fibres; $b$, cytoplasm of neuroglia cell. (Cajal.)

of cells, the neuroglia cells, a fibrillar substance, the neuroglia fibres, and a ground substance. The Golgi method apparently reveals a great variety of neuroglia cells which may be divided into cells with straight radiating unbranched processes, spider cells, and rough thick branching cells, mossy cells. It seems probable that in the former both cells and fibres are stained while in the latter only portions of the cells or syncytium, this method not differentiating between cells and fibres.

An increased activity of the neuroglia, due it may be to some pathological cause, is indicated by increase in the endoplasm, by proliferation of the nuclei, and by some cells detaching themsclves 
from the syncytium and becoming amœboid. Such amoboid cells may convey various products of degeneration to the vicinity of the perivascular lymph spaces. There may also result a temporary increase in the glial fibres (gliosis).

According to good authorities a continuous glial membrane forms the outer boundary of the ectodermal elements of the central nervous system, i.e., the nerve tissue and glia. This glial membrane is of course in apposition with the mesodermic connective tissue which forms the pia or inner covering of the brain and cord and also with the connective tissue of the adventitia of the blood-vessels which penetrate the central nervous system. The glial membrane is formed by superficial flattened cells and processes of other glial cells which come into contact with the connective tissues. The lining of the cavity of the neural tube is formed by the ependymal cells which are a form of neuroglia cells. These cells are columnar epithelial cells each with a process penetrating a variable distance into the wall of the neural tube and joining the general neuroglia syncytium. Their nuclei lie near the central cavity of the tube and they contain glia fibres. The inner lining of the chorioid plexuses of the brain is a single layer of cuboidal epithelial cells which have probably a secretory function and contribute to the production of the cerebro-spinal fluid. It is probable that the ependyma cells also have a secretory activity.

It is probable that the neurilemma cells also originate from the neural tube. If this is the case it is evident that they may be regarded as a special form of neuroglia cells. It has already been seen that in pathological changes they behave similarly to the neuroglia cells in the central nervous system.

\section{TECHNIC}

(I) Pieces of the cerebral cortex are stained by one of the Golgi methods. If the rapid or mixed silver method is used, sections must be mounted in hard balsam without a cover; if the slow silver or the bichlorid method is used, the sections may be covered. Sections are cut from 75 to $100 \mu$ in thickness, cleared in carbol-xylol or oil of origanum and mounted in balsam. This section shows only the external morphology of the neurone. It is also to be used for studying the different varieties of neuroglia cells as demonstrated by Golgi's method (see page I43).

(2) Thin transverse slices from one of the enlargements of the spinal cord are fixed in absolute alcohol. Thin sections ( 5 to Io $\mu$ ) are stained by Nissl's method (page $3^{8}$ ) and mounted in balsam. This section is for the purpose of 
studying the internal structure of the nerve cell and processes as demonstrated by the method of Nissl.

(3) Medullated Nerve Fibres (fresh).-Place a small piece of one of the sciatic or lumbar nerves of a recently killed frog in a drop of salt solution and tease longitudinally. Cover and examine as quickly as possible. Note the diameter of the axone and of the medullary sheath and the appearance of the nodes of Ranvier. An occasional neurilemma nucleus can be distinguished.

(4) Medullated nerve fibres--fibres from the cauda equina (this material has the advantage of being comparatively free from fibrous connective tissue)-are fixed in formalin-Müller's fluid (technic 5, p. 7), and hardened in alcohol. Small strands are stained twenty minutes in strong picro-acid-fuchsin solution (technic 2, p. 20), washed thoroughly in strong alcohol, cleared in oil of origanum, thoroughly teased longitudinally and mounted in balsam.

\section{General References for Further Study of Tissues}

Barker: The Nervous System.

Bethe: Allgemeine Anatomie und Physiologie des Nervensystem.

Cabot: A Guide to the Clinical Examination of the Blood for Diagnostic Purposes.

Ewing: Clinical Pathology of the Blood.

Hertwig: Die Zelle und die Gewebe.

Kölliker: Handbuch der Gewebelehre.

Prenant, Bouin et Maillard: Traité d'Histologie.

Ranvier: Traité Technique d'Histologie.

Van Gehuchten: Le Système nerveux de l'homme.

Wood: Laboratory Guide to Clinical Pathology. 



\section{PART IV}

THE ORGANS 

A tissue such as any one of those described in the preceding chapters scarcely exists in the pure state, that is alone by itself, in the adult body. Two or more tissues are always associated and such an association of two or more tissues for the purpose of performing a definite function constitutes an organ.

In practically all cases one of the tissues is connective tissue, the main function of which is to form a supportive framework for the more active specific tissue of the organ. In many cases this connective tissue forms a definite covering or capsule. From the capsule strands of connective tissue frequently extend down into the organ which branch and form its connective tissue framework. Sometimes the subdivision of the organ by connective tissue is quite regular macroscopic subdivisions being marked off by coarse connective tissue septa, and these again subdivided by finer septa. In such case the former are known as lobes, the smaller as lobules.

But while an organ has been defined as consisting of two or more tissues and while in general one of these tissues is connective tissue framework and the other the specific functioning tissue of the organ, such a simple combination of two tissues does not actually exist, for all organs are supplied with blood and lymph which are distributed to them and through them by the blood and lymph vessels. There are thus carried into the organs not only the blood and lymph themselves, but also the tissues which compose the walls of the blood and lymph vessels. Alsc every organ has its nerve supply; thus nerve tissue is distributed through all organs.

Many organs are hollow tubes and the study is the study of the structure of the walls of the tube, such, $e . g$. , are the stomach and intestines, the heart and the blood-vessels, the trachea and bronchi. Other organs not so apparently tubular still show their tubular structure on closer analysis, such, e.g., are the lungs and the duct glands.

As an organ has a specific function and as this function is performed mainly by the specific cclls of the organ, these cells show variations in structure dependent upon whether the organ is at work or at rest.

Many ardult organs are so complex that it is possible to understand them only by reference to their development from more simple structures. 
From the foregoing it follows that in studying an organ there are to be considered primarily:

(r) The specific tissue of the organ.

(2) The connective tissue framework.

(3) The blood supply.

(4) The nerve supply.

(5) Function.

(6) Development. 


\section{CHAPTER I \\ THE CIRCULATORY SYSTEM}

THE circulatory apparatus consists of two systems of tubular structures, the blood-vessel system and the lymph-vessel system, which serve, respectively, for the transmission of blood and lymph.

\section{THE BLOOD-VESSEL SYSTEM}

This consists of $(a)$ a central propelling organ, the heart; $(b)$ a series of efferent tubules-the arteries-which by branching constantly increase in number and decrease in calibre, and which serve to carry the blood from the heart to the tissues; $(c)$ minute anastomosing tubules-the capillaries - into which the arteries empty and through the walls of which the interchange of elements between the blood and the other tissues takes place; $(d)$ a system of converging tubules - the veins-which receive the blood from the capillaries, decrease in number and increase in size as they approach the heart, and serve for the return of the blood to that organ.

The entire system-heart, arteries, veins, capillaries-has a common and continuous lining, which consists of a single layer of endothelial cells. Of the capillaries this single layer of cells forms the entire wall. In the heart, arteries, and veins, the endothelium serves simply as the lining for walls of muscle and connective tissue.

\section{Capillaries}

It is convenient to describe these first on account of their simplicity of structure. A capillary is a small vessel from 4.5 to $16 \mu$ in diameter. Its wall consists of a single layer of endothelial cells. The cells are somewhat elongated in the long axis of the vessel. The smaller the calibre of the capillary the more elongated are the cells. Two endothelial cells suffice to complete the circumference of the smaller capillaries, while the larger requires three or four. The protoplasm of the cells is clear or fincly granular. The nuclei are oval, with their long axes in the long axis of the vessel. In fixed material the 
nuclei bulge into the lumen. In the living condition the lining of the capillary is probably nearly smooth. According to some investigators a delicate cuticle limits the cytoplasm on the side toward the lumen. The edges of the cells are serrated and are united by a small amount of intercellular substance (p. 77), which can be demonstrated by the silver nitrate stain. ${ }^{1}$ In certain capillaries-those of the early embryo, of the kidney glomeruli, of the chorioid coat of the eye, of the liver - no cell boundaries can be made out. In these capillaries the endothelium appears to be of the nature of a syncytium (p. 68). Capillaries branch without diminution in calibre, and these branches

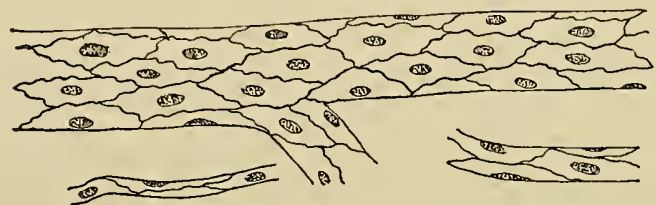

FIG. 88.-Large and Small Capillaries. Silver-nitrate and hæmatoxylin stain (technic 7, p. 79), to show outlines of endothelial cells and their nuclei.

anastomose to form capillary networks, the meshes of which differ in size and shape in different tissues and organs (Figs. 88, 89, 90). The largest meshed capillary networks are found in the serous membranes and in the muscles, while the smallest are found in the glands, as, e.g., the liver. As to calibre, the largest are found in the liver, the smallest in muscles.

In such thin membranous parts as the web of the frog's foot, or the wall of the frog's bladder, the blood may be observed as it flows through the arteries, capillaries, and veins. The current is seen to be fastest in the arteries, and faster in the centre of the vessel than at its periphery. It is slower in the veins and slowest in the capillaries. In the case of the frog's bladder, the mere exposure to the air acts as a sufficient irritant to cause slight inflammatory changes, and the leucocytes may be seen adhering to the walls of the capillaries and passing through them into the tissues. The capillary, both from the thinness of its wall and from the slowness with which the blood passes through it, is peculiarly adapted for the interchange of material between the blood and the tissues, and it is probable that it is in the capillary that all such interchange takes place.

${ }^{1}$ Some authors describe delicate protoplasmic anastomoses between the cells and describe an irregular precipitation of the silver nitrate corresponding to the spaces between the anastomosing threads. These spaces are interpreted as intercellular channels through which the leucocytes and plasma can pass. Intracellular spaces reacting to silver nitrate and supposed to have a similar function have also been described. 


\section{Arteries}

The wall of an artery consists of three coats:

(I) An inner coat, the intima.

(2) The middle coat, the media.

(3) An outer coat, the adventitia.

The intima consists of a single layer of endothelial cells, continuous with and similar to that forming the walls of the capillaries, or,

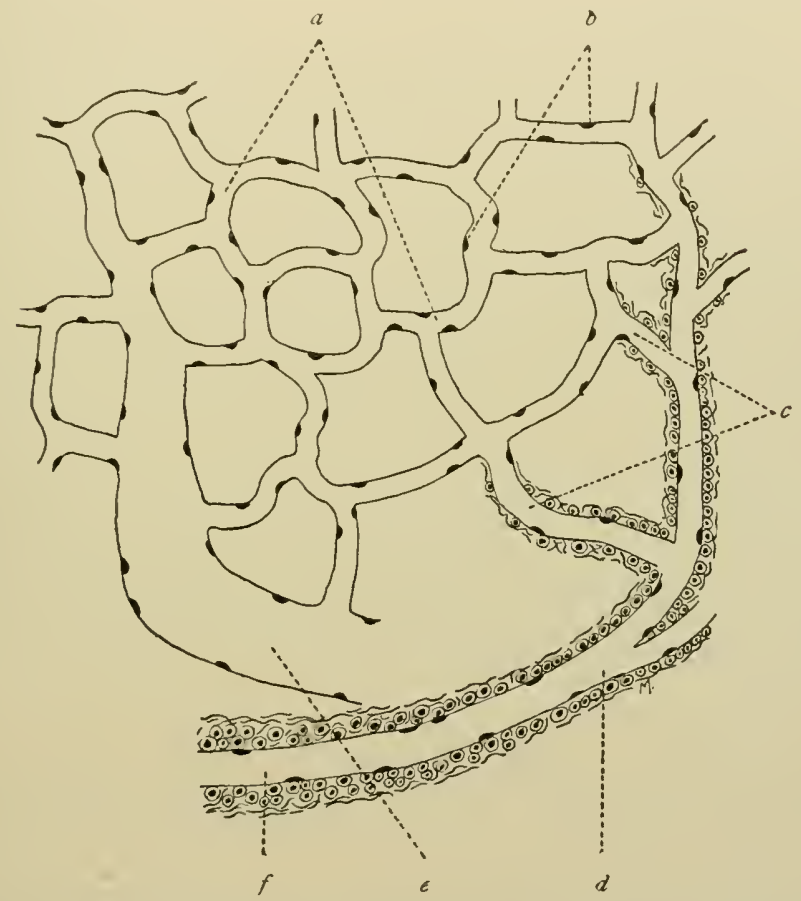

FIG. 89.-T)iagram of Capillaries and Small Artery showing their structure and relations. $a$, Capillaries; $b$, nuclei of capillary endothelium; $c$, precapillary arteries; $d$, arteriole; $c$, large capillary; $f$, small artery.

in arteries of considerable size, of this layer plus more or less connective tissue. The middle coat consists mainly of smooth muscle, the outer of connective tissue.

The structure of these three coats varies according to the size of the artery, and while the transition between them is never abrupt, it is convenient, for purposes of description, to distinguish (a) small arterics, (b) medium sized arteries, and (c) large arteries.

Small Arteries.-P'assing from a capillary to an artery, the first. change is the addition of a thin sheath of connective tissue, the fibres 
of which are disposed longitudinally, around the outside of the endothelial tube. A little farther back isolated smooth muscle cells, circularly arranged, begin to appear between the endothelium and the connective tissue. Such an artery is known as a precapillary artery. The next transition is the completion of the muscular coat, the muscle cells now forming a continuous layer. Such an artery, consisting of three distinct coats, the middle coat composed of a single continuous layer of smooth muscle cells, is known as an arteriole (Fig. 89, $d$; Fig. 90, b).

Medium-sized Arteries.-This group comprises all the named arteries of the body with the exception of the aorta and the pulmo-

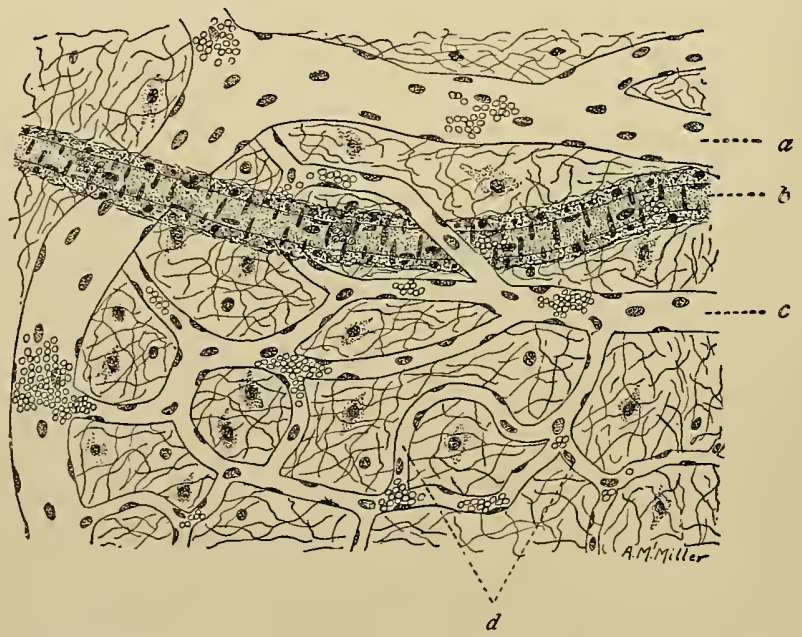

FIG. 90.-Capillary Network from Human Pia Mater, showing also an arteriole in "optical section" and a small vein. $\times 350$. (Technic I, p. I60.) a, Vein; $b$, arteriole; $c$, large capillary; $d$, small capillaries.

nary. Their walls are formed of the same three coats found in the arteriole, but the structure of these coats is more elaborate.

r. The INTIMA consists of three layers (Fig. 9r).

(a) An inner endothelial layer already described.

(b) A middle layer, the intermediary layer of the intima. This is composed of delicate white and elastic fibrils which run longitudinally, and connective-tissue cells.

(c) An outer layer, the elastic layer of the intima, or membrana elastica interna - a thin fenestrated membrane of elastic tissue. This membrane is intimately connected with the media and marks the boundary between the latter and the intima. In the smallest of 
the medium-sized arteries the intermediary layer is often wanting, the endothelial cells resting directly upon the elastic membrane. Owing to the extensive amount of elastic tissue in their walls, there is a postmortem contraction of arteries which results in the intima being thrown up into folds. For this reason the elastic membrane presents, in transverse sections of an artery, the appearance of a wary band (Fig. 9I).

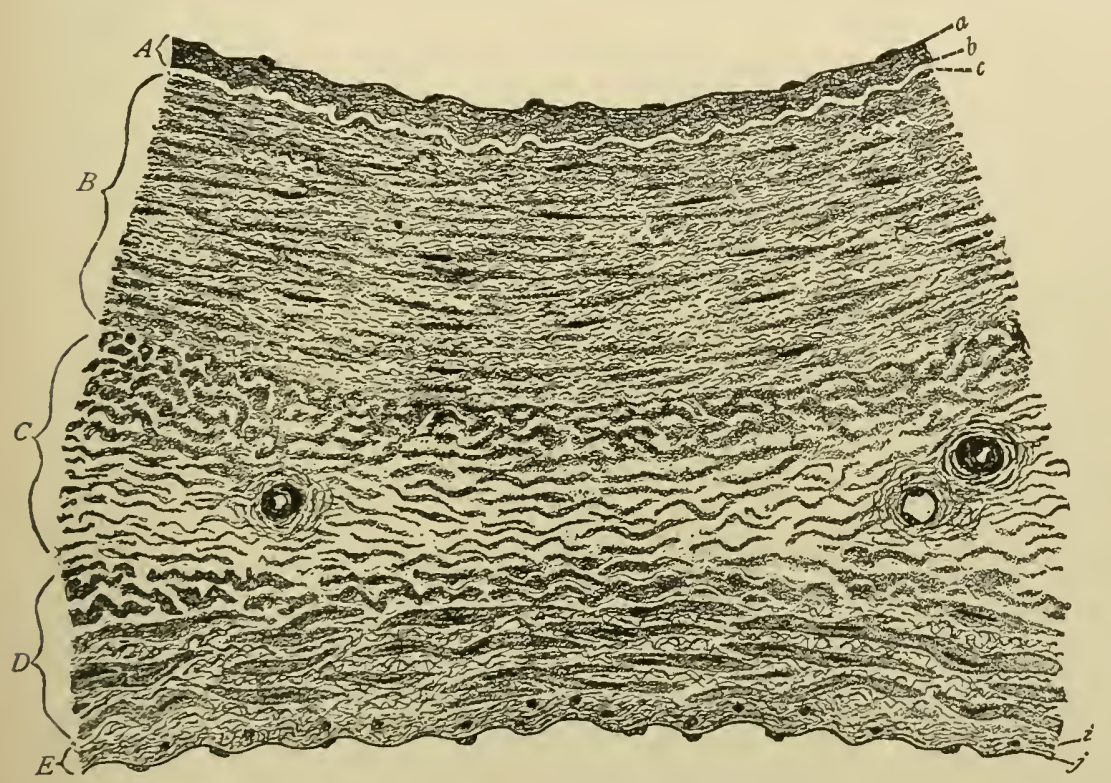

FIG. 91.-From Cross-section through Walls of Medium-sized Artery and its Accompanying Vein. $\times 75$. (Technic 3 , p. I6o.) $A$, Intima of artery; $a$, its endothelial layer; $b$, its intermediary layer; $c$, its elastic layer; $B$, media of artery; $C$, adventitia, the upper part belonging to the artery, the lower to the vein; within the adventitia are seen the vasa vasorum; $D$, media of vein; $E$, intima of vein; $i$, its intermediary layer; $j$, its endothelial layer.

2. The MEDIA is a thick coat of circularly disposed smooth muscle cells (Fig. 9I, B). Its thickness depends largely upon the size of the vessel, though varying somewhat for different vessels of the same size. A small amount of fibrillar connective tissue supports the muscle cells. Elastic tissue is present in the media, the amount being usually proportionate to the size of the vessel. In the smaller of the mediumsized arteries, the clastic tissue is disposed as delicate fibrils among the muscle cells. In larger arteries many coarse fibres are interminglerd with the fine fibrils. When much elastic tissue is present the muscle cells are separated into more or less well-defined groups. In such large arteries as the subclavian and the carotid, elastic tissue 
occurs not only as fibrils but also as circularly disposed plates or fenestrated membranes.

3. The adventitia (Fig. 9I, C) is composed of loose fibrous connective tissue with some elastic fibres. Occasionally there are scattered smooth muscle cells. Both smooth muscle cells and elastic fibres are arranged longitudinally. The adventitia does not form a definitely outlined coat like the media or intima, but blends

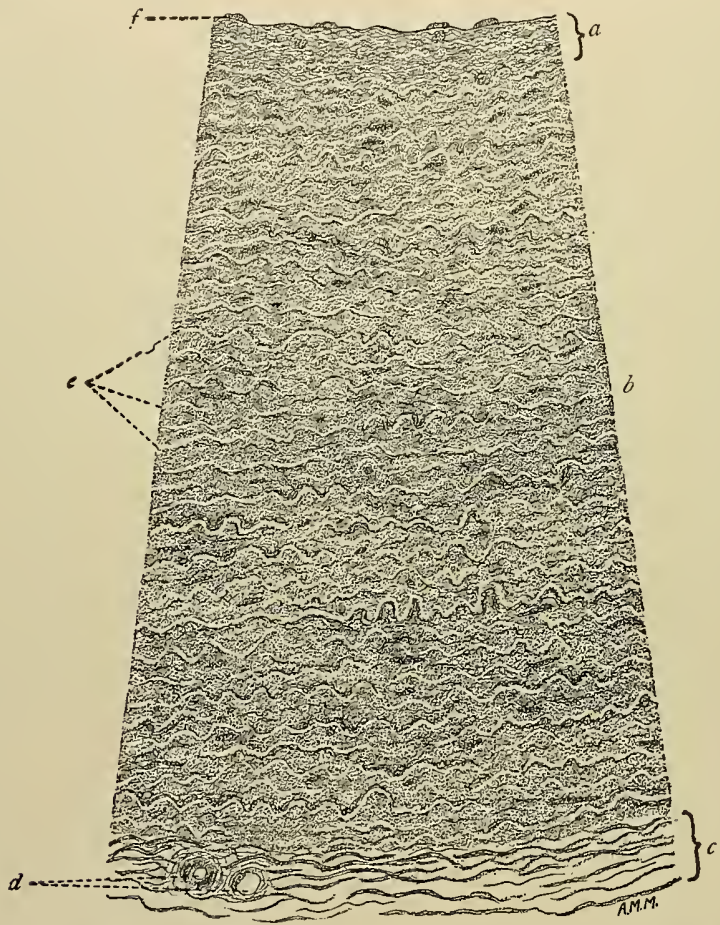

Fig. 92.-From Transverse Section of Dog's Aorta. $\times 60$. (Technic 4, p. I6o.) $a$, Intima; $b$, media; $c$, adventitia; $d$, vasa vasorum; $e$, elastic tissue; $f$, endothelium.

externally with the tissues surrounding the artery and serves to attach the artery to these tissues. In some of the larger arteries the elastic tissue of the adventitia forms an especially well-defined layer at the outer margin of the media. This is known as the membrana elastica externa. In general, it may be said that the thickness of the adventitia and the amount of elastic tissue present are directly proportionate to the size of the artery.

Large arteries like the aorta (Fig. 92) have the same three coats as small and medium-sized arteries. The layers are not, however, so distinct. This is due mainly to the excessive amount of elastic 
tissue in the media (Fig. 93), which makes indistinct the boundaries between intima and media, and between media and adventitia. The walls of the aorta are thin in proportion to the size of the ressel, increased strength being obtained by the decided increase in the amount of elastic tissue. Of the intima, the endothelial cells are short and polygonal; the intermediary layer similar to that of a medium-sized artery; the elastic layer less distinct and often broken up into several

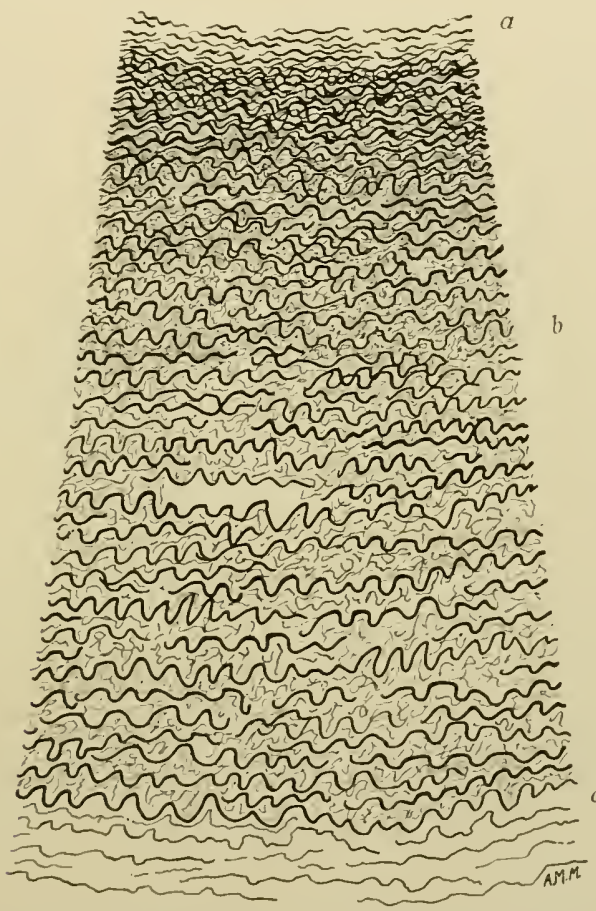

Frc. 93.-- From Transverse Section of Dog's Aorta, to show Vilastic Tissue $\times 60$. ('Technic 7 , p. r6o.) Elastic tissue stained black. $a$, Intima; $b$, media; $c$, adventitia.

thin layers. 'The media consists mainly of elastic tissue arranged in circular plates or fenestrated membranes. Between the elastictissue plates are groups of smooth muscle cells and some fibrillated connective tissuc. The adventitia resembles that of the medium-sized artery. 'There is no external elastic mombranc.

Certain arteries have structural peculiarities. The arteries of the brain and cord are thin-walled in proportion to their calibre, the inner clastic membrane is especially well defined, and there are few elastic fibres in the media. In the renal, creliac, mesenteric, and external iliac arteries there is little or no connective 
tissue separating the endothelium from the media. In the subclavian, the media contains longitudinal muscle cells. Longitudinally running muscle cells occur in the adventitia of the umbilical arteries, in the iliac, splenic, renal, superior mesenteric and dorsalis penis. The radial, femoral and cœliac arteries have comparatively little elastic tissue, while in the common iliac, carotid, and axillary the elastic tissue is in excess of the muscular.

\section{Veins}

The walls of veins resemble those of arteries. There are the same three coats, intima, media, and adventitia, and the same elements enter into the structure of each coat (Fig. 9I). Venous walls are not, however, so thick as those of arteries of the same calibre, and the coats are not so distinctly differentiated from one another. The transition from capillary through the precapillary vein to the small vein is similar to that described under arteries (page I54). Unlike the artery, the thickness of the wall of a vein and its structure are not directly proportionate to the size of the vessel, but depend also upon other factors such as the position of the vein and the support given to its walls by surrounding structures.

Of the INTIMA the endothelial layer and the intermediary layer are similar to those of the artery. The elastic layer is not always present, is never so distinct, and is not wavy as in the artery (Fig. 9I). The result is a lack of demarcation between intima and media, the connective tissue of the intermediary layer of the intima merging with the mixed muscle and connective tissue of the media. Projecting at intervals from the inner surface of the wall of some veins are valves. These are derived entirely from intima and consist of loose fibrous and elastic tissue covered by a single layer of endothelium. Valves are especially large and strong in the larger veins of the lower limbs. They are absent in the veins of the brain and cord and their membranes, in the veins of bones, in the umbilical vein, and in most of the visceral veins with the exception of some branches of the portal.

The MEDIA of veins is thin as compared with that of arteries of the same size. It consists of fibrous and elastic tissue and smooth muscle cells. The amount of muscle is comparatively small and the cells are arranged in groups through the connective tissue.

The ADVENTITIA is well developed in proportion to the media. It consists of mixed fibrous and elastic tissue and usually contains along its inner margin small bundles of longitudinally disposed smooth muscle cells. 
The media is thickest in the veins of the lower extremities, especially the popliteal, and in the reins of the skin. In the veins of the head and abdomen the media is very thin, while in the subclavian and superior vena cava and in the reins of bones, of the pia mater, dura mater, and retina, there is an almost entire absence of media.

Arteries are as a rule empty after death, while veins contain blood. The absence of much elastic tissue in the walls of the veins prevents any such extensive post-mortem contraction as occurs in the arteries. Veins tend to collapse after death, but are usually prevented from doing so by the presence of blood in them.

In the iliac and femoral veins, longitudinally disposed muscle occurs in the inner part of the media. The umbilical vein, like the corresponding artery, has three distinct muscular coats. Longitudinal muscle fibres are present in the adventitia of the superior vena cava (hepatic and abdominal portion) and of the portal and hepatic veins. In the upper portion of the inferior vena cava, in the superior vena cava, the jugular, innominate, and subclavian, there is little muscle tissue in any of the coats, while in the veins of the brain and its membranes, the retina, the placenta and the bones, no muscle is present.

Vasa Vasorum.-Medium and large arteries and veins are supplied with small nutrient vessels - vasa vasorum. These vessels run in the adventitia, small branches penetrating the media (Figs. 9I and 92).

Lymph channels are found on the outer surface of many bloodvessels. Some of the smaller vessels are surrounded by spaces lined by endothelium-perivascular lymph spaces. These communicate with the general lymphatic system.

Nerves.-The walls of the blood-vessels are supplied with both medullated and non-medullated fibres. The latter are axones of sympathetic neurones. As these nerves control the calibre of the vessels they are known as vasomotor nerves. They form plexuses in the adventitia, from which are given off branches which penetrate the media and terminate on the muscle cells. The medullated fibres are the peripheral arms of spinal or cranial ganglion cells. The larger fibres run in the connective tissue outside the adventitia. From these are given off branches which enter the media, divide repeatedly, lose their medullary sheaths, and terminate mainly in the media, although some fibres have been traced to their terminations in the intima. 


\section{TECHNIC}

(I) Capillaries, Arterioles, Small Arteries, and Veins.-Fix an entire brain, or slices about an inch thick from its surface, in formalin-Müller's fluid for twentyfour hours (technic 5, p. 7). Remove the pia mater, especially the thinner parts which lie in the sulci between the convolutions, and harden in graded alcohols. Select a thin piece, stain with hæmatoxylin (lightly) and eosin (strongly) (technic I, p. 20), and mount in balsam or in eosin-glycerin. The veins, having thin walls and being usually well filled with blood, appear distinct and red from the eosin-stained red cells. The arteries, having thicker walls, in which are many hæmoglobin-stained nuclei, have a rather purple color. Between the larger vessels can be seen a network of anastomosing capillaries with their thin walls and bulging nuclei. Some are filled with blood cells; others are empty with their collapsed walls in apposition. Note the appearance of an arteriole, first focusing on its upper surface, then focusing down through the vessel. In this way what is known as an "optical section" is obtained, the artery appearing as if cut longitudinally. Trace the transition from arteriole to precapillary artery and the breaking up of the latter into the capillary network. Similarly follow the convergence of capillaries to form a small vein.

(2) Instructive pictures of the relations of arteries, capillaries, and veins in living tissues may be obtained by curarizing a frog, distending the bladder with normal saline introduced through a small catheter or cannula, opening the abdomen and drawing out the bladder, which can then be arranged upon the stage of the microscope. The passage of the blood from the arteries through the capillary network and into the veins is beautifully demonstrated.

(3) For studying the structure of the walls of a medium-sized artery and vein remove a portion of the radial artery, or other artery of similar size, and its accompanying vein, together with some of the surrounding tissues. Suspend the vessels, with a small weight attached, in formalin-Müller's fluid (technic 5, p. 7). Sections should be cut transversely, stained with hæmatoxylin-eosin (technic I, p. 20), or with hæmatoxylin-picro-acid fuchsin (technic 3, p. 2I), and mounted in balsam. The vessels of the adventitia-vasa vasorum-are convenient for studying the structure of arterioles and small veins.

(4) Fix a piece of aorta in formalin-Müller's fluid, care being taken not to touch the delicate endothelial lining. Stain transverse sections with hæmatoxylin-eosin or with hæmatoxylin-picro-acid fuchsin and mount in balsam.

(5) The outlines of the lining endothelial cells may be demonstrated as follows: Kill a small animal, cut the aorta, insert a glass cannula and, under low pressure, thoroughly wash out the entire vascular system with distilled water. Follow the water by a one-per-cent. aqueous solution of silver nitrate. Remove some of the smaller vessels, split longitudinally, mount in glycerin, and expose to the direct sunlight. After the specimen has turned brown examine with the low power. The outlines of the cells should appear brown or black.

(6) The endothelium of the smaller vessels and capillaries may also be demonstrated in the specimen described under technic 8, p. 79 .

(7) The elastic tissue of the blood-vessels is best demonstrated by means of Weigert's elastic-tissue stain. Prepare sections of medium-sized vessels and of the aorta, as above described (3), and stain as in technic 3, p. 28. 


\section{The Heart}

The heart is a part of the blood-vessel system especially differentiated for the purpose of propelling the blood through the vessels.

The main mass of the heart wall consists of a special form of muscle tissue already described as heart muscle (page IIg). This constitutes the myocardium. On its inner and outer sides the myocardium is covered by connective-tissue membranes lined, respectively, with endothelium and mesothelium and known as the endocardium and epicardium.

The MYOCARDIUM varies in thickness in different parts of the heart, being thickest in the left ventricle, thinnest in the auricles. A ring of dense connective tissue, the auriculo-ventricular ring, completely separates the muscle of the auricles from that of the ventricles, except in the median septum where the auricular and ventricular muscle is continuous. The auricular muscle consists of an outer coat common to both auricles, the fibres of which have a transverse or somewhat oblique direction, and of an inner coat, independent for each auricle, the fibres of which are longitudinally disposed. Bundles of these fibres stand out as ridges along the inside of the auricles. They are more strongly developed in the right than in the left auricle. Between the two coats bundles of muscle fibres are frequently found which run in various directions.

The disposition of the muscle tissue of the ventricles is much more complicated. It is usually described as composed of several layers, the fibres of which run in different directions. The meaning of these fibre layers becomes apparent when we study the arrangement of the fibres in embryonic hearts in which the connective tissue has been broken down by maceration. Thus dissected, the muscle of the ventricles is seen to consist mainly of two sets of fibres, a superficial set and a deep set. These run at approximately right angles to each other. Both sets of fibres begin at the auriculo-ventricular rings. The superficial fibres wind around both ventricles in a spiral manner, becoming constantly deeper, to terminate in the papillary muscles of the opposite ventricle. The deeper fibres pass from the auriculo-ventricular ring around the ventricle of the same side, through the interventricular septum and terminate in the papillary muscles of the opposite ventricle.

The ENDOCARDIUM covers the inner surface of the myocardium and forms the scrous lining of all the chambers of the heart. At. 
the arterial and venous orifices it is seen to be continuous with and similar in structure to the intima of the vessels. It consists of two layers: (a) an inner composed of a single layer of endothelial cells, corresponding to the endothelial lining of the blood-vessels; and (b) an outer composed of mixed fibrous and elastic tissue and smooth muscle cells. Externally the cndocardium is closely attached to the myocardium.

Strong fibrous rings (annuli fibrosi), composed of mixed fibrous and elastic tissue, surround the openings between auricles and ventricles. Similar but more delicate rings encircle the openings from the heart into the blood-vessels.

The heart valves are attached at their bases to the annuli fibrosi. They are folds of the endocardium, and like the latter consist of fibrous and elastic tissue continuous with that of the rings and covered by a layer of endothelium.

The EPICARDIUM is the visceral layer of the pericardium. It is a serous membrane like the endocardium, which it resembles in structure. It consists of a layer of mixed fibrous and elastic tissue covered over by a single layer of mesothelial cells. Beneath the epicardium there is usually more or less fat.

Blood-vessels.-Blood for the nutrition of the heart is supplied through the coronary arteries. The larger branches run in the connective tissue which separates the bundles of muscle fibres. From these, smaller branches pass in among the individual fibres, where they break up into a rich capillary network with elongated meshes. From the myocardium, capillaries penetrate the connective tissue of the epicardium and endocardium. The auriculo-ventricular valves are supplied with blood-vessels, while in the semilunar valves. blood-vessels are wanting.

Lymphatics.- Lymph channels traverse the epicardium and endocardium and enter the valves. Within the myocardium minute lymph vessels have been demonstrated between the muscle fibres and accompanying the blood-vessels.

Nerves.-These are derived from both cerebro-spinal (vagus) and sympathetic systems (cervical ganglia) and consist of both medullated and non-medullated fibres. Sympathetic ganglion cells are distributed in groups throughout the myocardium, the largest, lying in the epicardium near the base of the heart, being known as the cardiac ganglion or ganglion of Wrisberg. Among these cells the nerve fibres form plexuses from which both motor and sensory 
terminals are given off to the muscle. (For nerve endings in heart muscle see page 440.)

\section{TECHNIC}

(I) The Heart.-Cut pieces through the entire thickness of the wall of one of the ventricles, care being taken not to touch either the serous surface or the lining endothelium. Fix in formalin-Müller's fluid (technic 5, p. 7). Cut transverse and longitudinal sections; stain with hæmatoxylin-eosin (technic $x$, p. 20) and mount in balsam.

(2) Treat the entire heart of a small animal (e.g., guinea-pig or frog) in the same manner as the preceding, making transverse sections through both ventricles.

(3) An entire heart, human or animal, may be fixed in the distended condition by filling with formalin-Müller's fluid under low pressure and then tying off the vessels. The entire heart thus distended is placed in a large quantity of the same fixative.

\section{Developient of the Circulatory System}

The blood-vessels and the heart begin their development separately and afterward become united. Both are derived from mesoderm, but while the heart develops within the embryo, the earliest blood-vessels and blood are developed from extra-embryonic mesoderm. The earliest vessels to be formed are the capillaries. These make their appearance in the mesodermic tissue near the periphery of the area vasculosa which surrounds the developing embryo. Here groups of cells known as "blood islands" differentiate from the rest of the mesodermic cells, appearing in the chick by the end of the first day of incubation. The superficial cells of these islands become flattened to form the endothelium, which is at this stage apparently a syncytium through which the nuclei are scattered and showing no evidences of cell boundaries. This endothelial syncytium surrounds the remaining more central cells, from which blood cells are developed. These represent the earliest blood-vessels. The channels, which are at first unconnected, anastomose and give rise to a network of channels which are the carliest capillaries. These develop rapidly in the area vasculosa and some of them increase in size to bccome arteries and veins, the smooth muscle and connective tissue of their walls being differentiated from the surrounding mesoderm. These grow toward, and finally into, the embryo where they unite with the heart. In granulation tissue, and in new growths in general, both normal and pathological, new bloorl-vessels apparently develop as off-shoots from other vessels. These are at first solid extensions of enrlothelium which become hollowed out. In regard to the origin of the later vessels in the extraembryonic area, and those within the embryo, there are two views: (I) that they represent outgrowths from the original capillaries; (2) that they arise in situ in the same manner as the carliest capillaries and unite secondarily to form networks. The weight of evidence at present favors the latter view. The entire vascular system is at first represented by a network 
of capillaries. As development proceeds some of the channels enlarge to form the arteries and veins.

The heart first appears as an endothelial tube, the primitive endocardium, at a very early age of embryonic life, in the chick embryo during the first day of incubation. It apparently begins its rhythmic contraction before any contractile fibrils can be distinguished in its walls and before any connection with blood-vessels has been established. The origin of the cardiac endothelium is not definitely known. It is believed by some to be of entodermic origin, by others of mesodermic, by still others to be partly derived from each of these layers. Around this endothelial tube, but separated from it by a space, there develops from mesoderm an entirely distinct muscular tube, the primitiv

Cœlom

Parietal mesoderm

Ectoderm

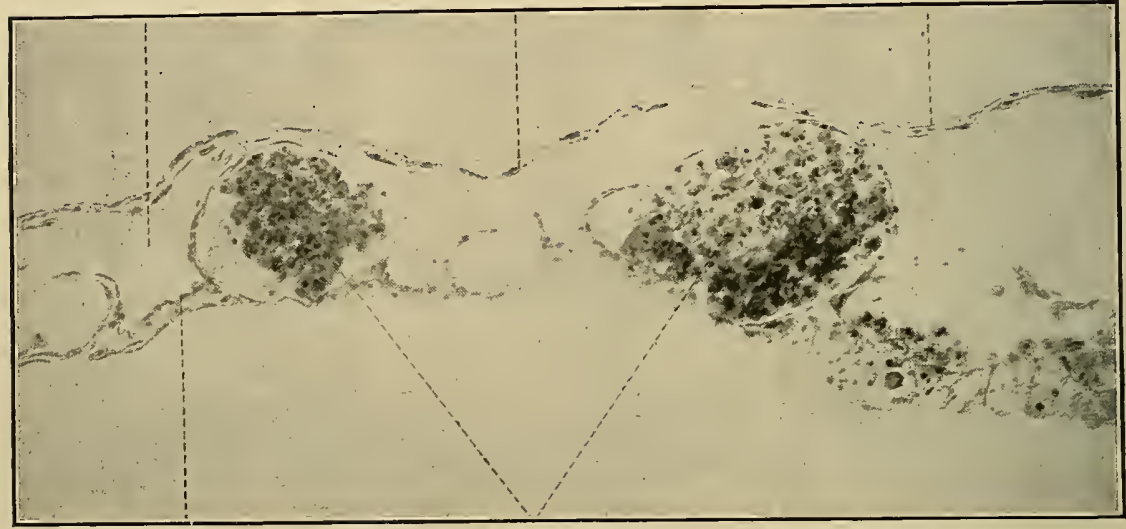

Visceral mesoderm

Blood islands

FIG. 94.-Section of Blastoderm of Chick of 42 Hours' Incubation. PhotographThe cells of the blood islands are difierentiated into nucleated red blood cells (erythro. blasts) and the endothelium of the vessels.

myocardium. These two tubes are at first united only in places by bands of connective tissue. Later they unite so that the inner tube, the endocardiume becomes a lining for the outer tube, the myocardium. The union of the two heart tubes occurs very early. The foregoing description applies to the chick and to those mammals thus far studied. In the earliest human embryos ( 2 to $3 \mathrm{~mm}$.) the heart is already a single, slightly coiled tube connected at its cephalic end with the ventral aorta and caudally with the omphalo-mesenteric veins. The epicardium, as the visceral layer of the pericardium, has a separate origin, being constricted off from that portion of the mesoderm which lines the primary body cavity.

\section{THE LYMPH-VESSEL SYSTEM}

The larger lymph vessels are similar in structure to veins. Their walls are, however, thinner than those of veins of the same calibre and they contain more valves. These are folds of the intima, and 
always occur in pairs. They are arranged with considerable regularity and in small vessels where the intima forms the entire wall there is a distinct bulging just above the valve which gives the vessel a quite characteristic appearance. They are capable of great distention, and when empty collapse so that their thin walls are in appositicn.

The largest of the lymph vessels, the thoracic duct, has three welldefined coats: an intima consisting of the usual lining endothelium resting upon a subendothelial layer of delicate fibro-elastic tissue, the outermost elastic fibres having a longitudinal arrangement; a fairly thick media of circularly disposed smooth muscle cells which occur in groups separated by connective tissue containing a few elastic fibres and an adventitia of longitudinally running connective tissue containing elastic fibres and strengthened by bundles of longitudinal smooth muscle.

Lymph capillaries resemble blood capillaries in that their walls are composed of a single layer of endothelial cells. The cells are rather larger and more irregular than in blood capillaries, the capillaries themselves are larger, and, instead of being of uniform diameter throughout, vary greatly in calibre within short distances. In certain tissues dense networks of these lymph capillaries are found. Cleft-like lymph spaces-perivascular lymph spaces-partially surround the walls of the smaller blood-vessels.

Lymph spaces without endothelial or other apparent lining also occur. Examples of these are the pericellular lymph spaces found in various tissues and the canaliculi of the cornea and of bone (pages 83 and Ior).

Similar in character to lymph spaces are the body cavities, peritoneal, pleural, and pericardial, with their linings of serous membranes. These cavities first appear in the embryo as a cleft in the mesoderm -lhe calom, body cavity, or pleuroperiloneal cleft. This cleft is lined with mesothelium beneath which the stroma is formed. 'These membranes not only line the cavities, but are reflected over most of the viscera of the abdomen and thorax. They consist of a stroma of mixed fibrous and elastic tissue, covered on its inner side by a layer of mesolhelium, the two being separated by a homogeneous basement membrane. The stroma contains numerous lymphatics. These have been described as communicating with the free surfaces by means of openings-slomata. Recent observations, however, would seem to indicate that these stomata are artefacts. 
That the lymph-vessels form a definite and closed system of channels and are not in direct communication with the lymph spaces has been clearly demonstrated.

The origin of the lymphatic vessels is not clear. According to some investigators they originate as evaginations from the vascular system, starting as four buds, two from the veins of the neck, and two from the veins in the inguinal region. The two anterior buds appear first (pig embryo of $\mathbf{1} 4.5 \mathrm{~mm}$.) and are in communication with the anterior cardinal vein. A little later the posterior buds connected with the posterior cardinals appear. From these buds the entire lymphatic plexus develops by a process of evagination.

According to other investigators, the lymphatic vessels first appear as minute spaces in the mesenchyme of the axilla and groin (during second month in human embryo). These spaces enlarge and by sending off branches give rise to the network of lymphatic vessels. According to this veiw, the connection between lymphatic vessels and veins is secondary.

\section{TECHNIC}

(I) Remove a portion of the central tendon of a rabbit's diaphragm. Rub the pleural surface gently with the finger or with a brush to remove the mesothelium. Rinse in distilled water and treat with silver nitrate as in technic 7, p. 79 . Mount in glycerin. If the silver impregnation is successful, the networks of coarser and finer lymphatics can be seen as well as the outlines of the endothelium of their walls. If care has been taken not to touch the peritoneal surface, the peritoneal mesothelium and the stomata are frequently seen.

(2) The Thoracic Duct.-Remove a portion of the thoracic duct, fix in formalin-Müller's fluid (technic 5, p. 7), and stain sections with hæmatoxylin-eosin (technic I, p. 20).

\section{General References for Further Study of the Circulatory System}

Kölliker: Handbuch der Gewebelehre des Menschen, vol. iii.

Stöhr: Text-book of Histology.

Schäfer: Histology and Microscopic Anatomy, in Quain's Elements of Anatomy, tenth edition. 


\section{CHAPTER II \\ LYMPHATIC ORGANS}

\section{Lymphatic Tissue ${ }^{1}$}

So-CALLED lymphatic tissue consists of reticular connective tissue and a special type of cells, lymphoid cells, which fill in the meshes of the reticulum. Lymphoid cells are small spheroidal cells, each having a single nucleus which almost completely fills the cell. Lymphatic tissue may be diffuse or circumscribed. In diffuse lymphatic tissue the cells are not closely packed and there is no distinct demarcation between the lymphatic and the surrounding tissues. An example of diffuse lymphatic tissue is seen in the stroma of the mucous membrane of the gastro-intestinal canal. In circumscribed lymphatic tissue the cells are very closely packed, often completely obscuring the reticulum. There is also a quite distinct demarcation between the lymphatic and the surrounding tissues. Such a circumscribed mass of lymphatic tissue is known as a lymph nodule.

\section{The Lymph Nodes}

Lymph nodes are small bodies, usually oval or bean-shaped, which are distributed along the course of the lymph vessels. In some regions they are arranged in series forming "chains" of lymph nodes as, e.g., the axillary and inguinal.

Each lymph node is surrounded by a capsule of connective tissue which sends trabecula or sepla into the organ. The capsule and septa constitute the connective-lissue framework of the node, and serve as a support for the lymphatic tissue (Fig. 95).

The capsule is composed of fibrous connective tissue. Toward the surface of the capsule the fibres are loosely arranged and there are comparatively few elastic fibres. This outer layer of the capsule

${ }^{1}$ In the preceding crlitions "lymphatic tissue" was placed according to name rather than structure among the tissues. As it consists of a conneclive tissic framework supporting a special type of cell which has a specific function, it is more properly classified as an organ. On account of the peculiar structure and wide distribution of the "tissue" and on account of convenience and long usage, the term "lymphatic tissue" is still retained. 
blends with the surrounding tissues and serves, like the fibres of the arterial adventitia, to attach the organ to them. The inner layer of the capsule consists of a more dense connective tissue, is richer in elastic fibres, and contains some smooth muscle cells. At one point, known as the hilum (Fig. 95), there is a depression where the connective tissue of the capsule extends deep into the substance of the node. This serves as the point of entrance for the main arteries and nerves, and of exit for the veins and efferent lymph vessels.

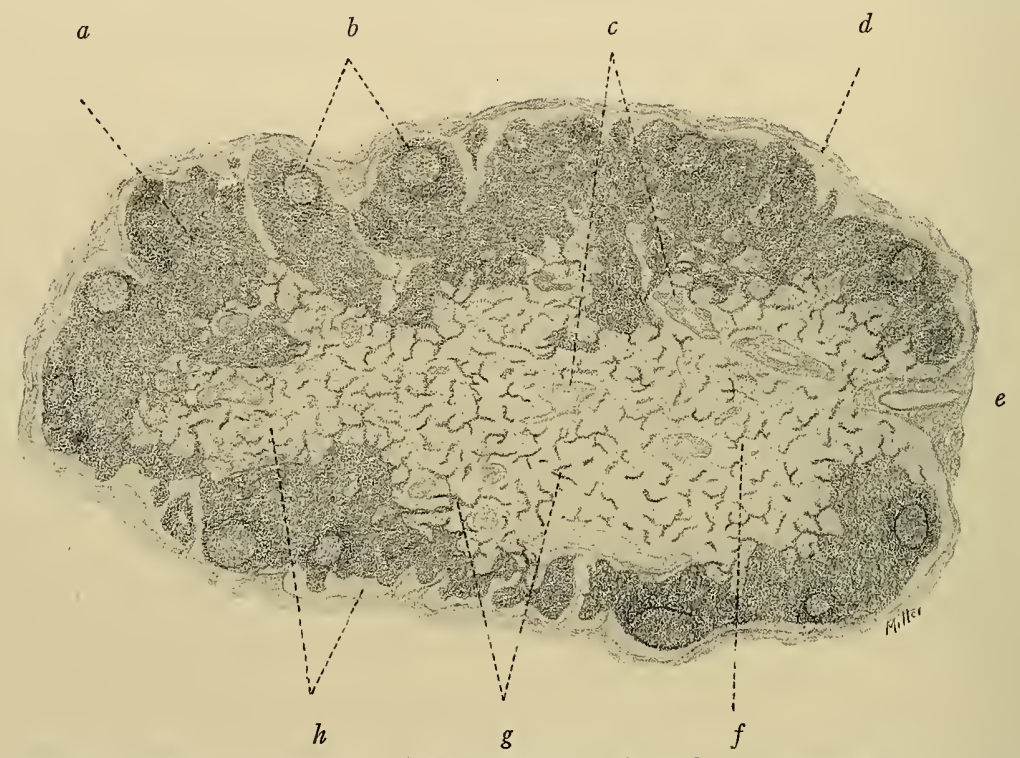

Fig. 95.--Section through Entire Human Lymph Node, including Hilum. $\times \times 5$. (Technic I, p. x 72.) Dark zone, cortex; light central area, medulla. $a$, Lymph nodule of cortex; $b$, germinal centres; $c$, trabeculæ containing blood-vessels; $d$, capsule; $e$, hilum; $f$, lymph sinus of medulla; $g$, lymph cords of medulla; $h$, lymph sinuses of medulla and cortex.

The connective-tissue septa, which extend from the capsule into the interior of the node incompletely divide it into irregular intercommunicating compartments. In the peripheral portion of the node these compartments are somewhat spheroidal or pear-shaped. Toward the centre of the node the septa branch and anastomose freely, with the result that the compartments are here narrower, more irregular, and less well defined.

Within the compartments formed by the capsule and the septa is the lymphatic tissue. Near the capsule where the compartments are large and spheroidal or pear-shaped, the lymphatic tissue is arranged in masses which correspond in shape to the compartments. 
These are known as lymph nodules (Fig. 95). In the centre of each nodule is usually an area in which the cells are larger, are not so closely packed, and show marked mitosis. As it is here that active proliferation of lymphoid cells takes place, this area is known as the germinal centre (Figs. 95 and 96). Immediately surrounding the germinal centre is a zone in which the lymphoid cells are more closely packed than elsewhere in the nodule (Fig. 96). This is apparently due to the active production of new cells at the germinal centre and the consequent pushing outward of the surrounding cells. In stained sections the centre of the nodule is thus lightly stained, while immediately surrounding this light area is the darkest

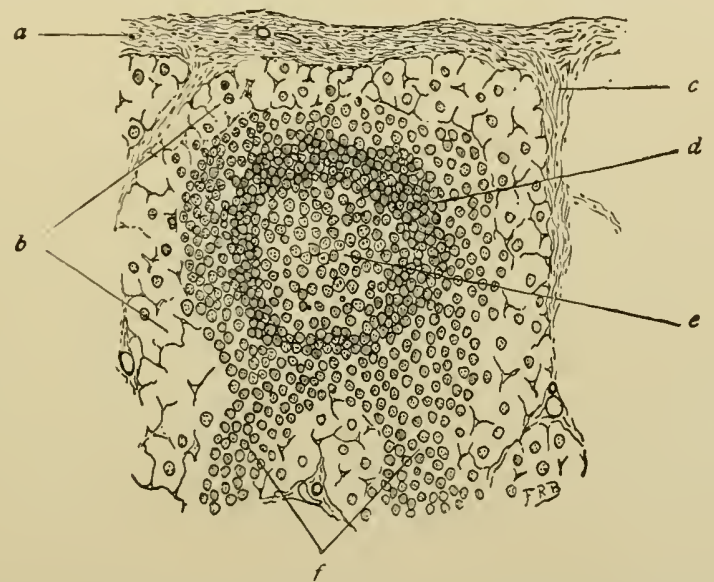

FIG. 96.-Section through Cortex and Portion of Medulla of Human Lymph Node (Technic 2, p. I 72.) $a$, Capsule; $b$, lymph sinus; $c$, trabecula; $d$, closely packed cells at outer border of lymph nodule; $e$, germinal centre; $f$, lymph cords in medulla.

portion of the nodule (Fig. 96). From the inner sides of the nodules strands of lymphoid tissue extend into the center of the node. These are known as lymph cords, and anastomose freely. The regular arrangement of the lymph nodules and trabecula in the peripheral portion of the node contrasts strongly with their irregular arrangement in the centre. This determines a division of the nodule into two zones, an outer peripheral zone or cortex and a central zone or medulla. In both cortex and medulla the lymphoid tissue is always separated from the capsule or from the septa by a distinct space - the lymph sinus - which is bridged over by reticular tissue containing comparatively few lymphoid cells (Fig. 96). These sinuses form a continuous system of anastomosing channels throughout the node. 
The regular arrangement of trabeculæ, and lymph nodules with sinuses between, which is characteristic of the cortex, makes this part of the organ easily understood. To appreciate the structure of the medulla it must be borne in mind that all of these cortical structures extend down into the medulla, the trabeculæ as anastomosing networks of connective tissue, the lymph nodules as cord-like structures which divide and anastomose, the sinuses as more or less clear channels which always separate the connective tissue from the lymph cords. These parts - trabecula, sinus, lymph cord-all anastomosing freely and most irregularly in the medulla, always maintain the same relation to one another as in the cortex, namely, that sinus is always interposed between lymph tissue and trabecula.

The reticular connective tissue (page 94), which forms a part of the lymphatic tissue proper, is continuous with the fibrous connective-tissue framework of the organ in such a manner that it is impossible to determine any demarcation between the two tissues. In the lymph nodules, and wherever the lymphoid cells are densely packed, the underlying reticular network is almost completely obscured. Crossing the sinuses, especially those of the medulla, and in specimens in which the cells have been largely washed out or removed by maceration, the reticular structure is well shown.

The lymphoid tissue proper, as represented by the lymph nodules and anastomosing lymph cords, is thus, as it were, suspended in the meshes of a reticulum which is swung from the capsule and trabeculæ. As both nodules and cords are everywhere separated from capsule and trabeculæ by the sinuses, and as these latter serve for the passage of lymph through the node, it is seen that the lymphatic tissue of the node is broken up in such a manner as to be bathed on all sides by the circulating lymph.

In addition to the definitely formed lymph nodes and the welldefined collections of lymph nodules, such as those of the tonsil or of Peyer's patches, small nodules or groups of lymphoid cells have a wide distribution throughout the various organs. While many of these collections of lymphatic tissue are inconspicuous, still the aggregate of lymph tissue thus distributed is by no means inconsiderable. The most important will be described in connection with the organs in which they occur.

Blood-vessels.-Those which enter the hilum carry the main blood supply to the organ. Most of the arteries pass directly into the lymphatic tissue, where they break up into dense capillary net- 
works. Some of the arteries, instead of passing directly to the lymphatic tissue, follow the septa, supplying these and the capsule, and also sending branches to the surrounding lymphatic tissue. A few small ressels enter the capsule along the convexity of the organ and are distributed to the capsule and to the larger septa.

Lymphatics. - The afferent lymph vessels enter the node on its convex surface opposite the hilum, penetrate the capsule, and pour their lymph into the cortical sinuses. The lymph passes through the sinuses of both cortex and medulla, and is collected by the efferent

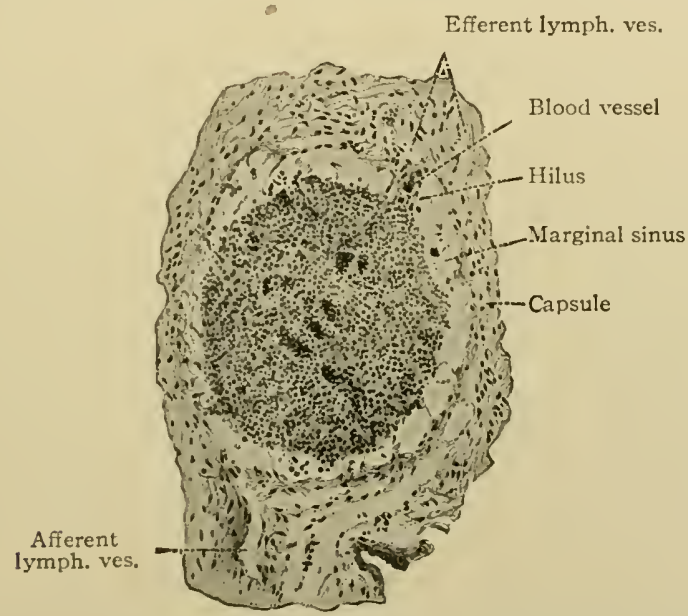

FIG. 97.-From a Section through the Axilla of a Human Embryo of $125 \mathrm{~mm}$. (4-5 months), showing an Early Stage of a Lymph Gland. (Kling.)

lymph vessels which leave the organ at the hilum. Within the node the lymph comes in contact with the superficial cells of the nodules and of the lymph cords. These cells are constantly passing out into the lymph stream so that the lymph leaves the node much richer in cellular elements.

Nerves are not abundant. Both medullated and non-medullated fibres occur. Their exact modes of termination are not known.

Development.-The first indications of lymph node formation are found in the axilla and groin (pig embryo of about $30 \mathrm{~mm}$.; human toward end of third month) in the connective tissue in which the lymphatic vessels are best developed (p. I66). Here groups of more closely packed cells appear. As the tissue is richly vascular it secms impossible to determine whether the closely packed cells originate within the blood-vessels or develop from fixed connective tissue cells. Each group of cells is the anlage of a lymph node (Fig. 97). 'The immediately surrounding connective tissue forms the capsule, while the lymph channels just 
beneath form the subcapsular or marginal sinus (Fig. 98). The point of main connection with outside blood-vessels becomes the hilum. As the lymph node grows outward, parts of the capsule remain within to form trabeculæ while the lymph channels within the nodule apparently develop as ingrowths from the marginal sinus (Figs. 97 and 98).

\section{TECHNIC}

(I) Remove several lymph nodes from one of the lower animals (ox, cat, dog, rabbit), fix in formalin-Müller's fluid (technic 5, p. 7), and harden in alcohol. Cut thin sections through the hilum, stain with hæmatoxylin-eosin (technic I, p. 20), or with hæmatoxylin-picro-acid-fuchsin (technic 3, p. 2I), and mount in balsam.

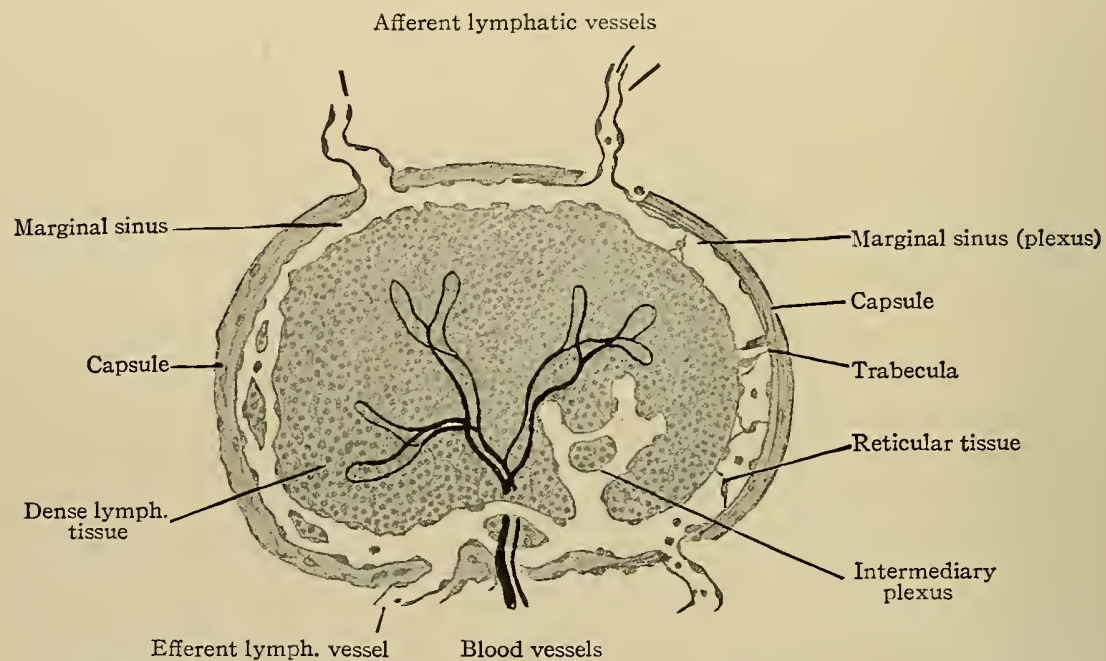

FIG. 98.-Diagram lllustrating a Stage (Later Than Fig. 97) in the Development of a Lymph Gland. (Stöhr.)

(2) Expose a chain of lymph nodules (e.g., the cervical or inguinal of a recently killed dog or cat). Insert a small cannula or needle into the uppermost node and inject formalin-Müller's fluid until the node becomes tense. By now slightly increasing the pressure the fluid may be made to pass into the second node, and so through the entire chain. The nodes are then carefully dissected out and placed for twenty-four hours in formalin-Müller's fluid, then hardened in alcohol. Sections are cut through the hilum, stained with hæmatoxylin-eosin or with hæmatoxylin-picro-acid-fuchsin and mounted in balsam. Near the centre of the chain are usually found nodes in which the lymph sinuses are properly distended. The most proximal nodes are apt to be overdistended, but for this very reason are often excellent for the study of the reticular tissue from which most of the cells have been washed out, especially in the medulla.

(3) Human lymph nodes may be treated by either of the above methods. 
Owing to the coalescence of their cortical nodules their structure is not so easily demonstrated as that of the lymph nodes of lower animals.

\section{Hæmolymph Nodes}

These are lymphoid structures which closely resemble ordinary lymph nodes, but with the essential difference that their sinuses are blood sinuses instead of lymph sinuses.

Each node is surrounded by a capsule of varying thickness, composed of fibro-elastic tissue and smooth muscle cells. From the cap-

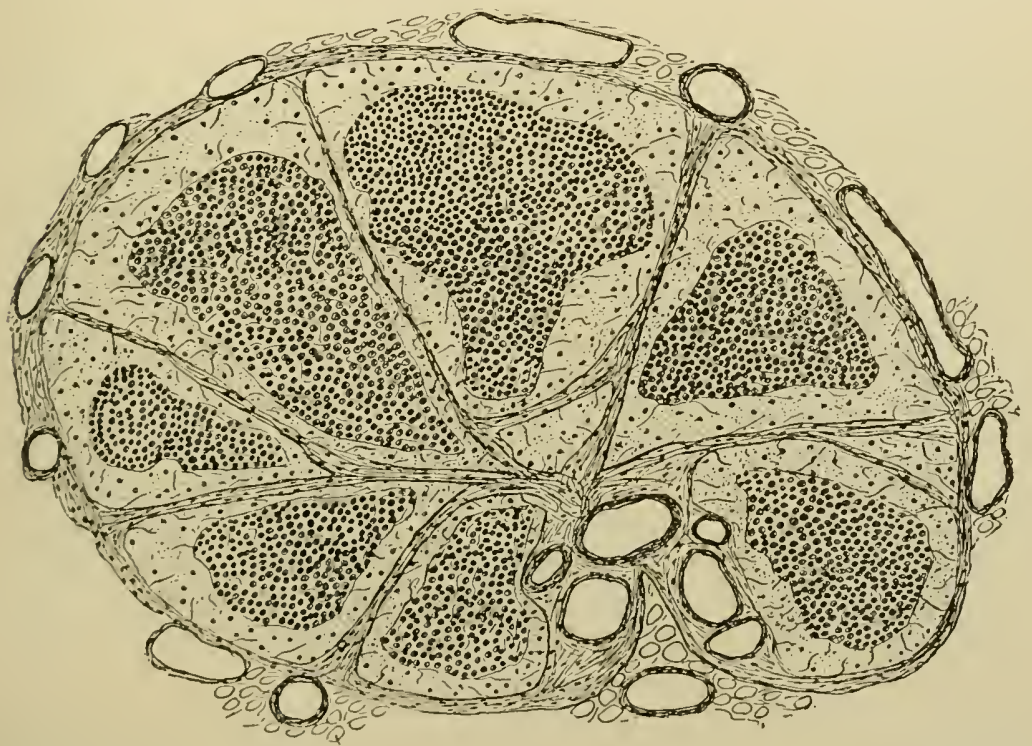

Frg. 99.- Section through I Iuman Hæmolymph Node, including Hilum, showing capsule, trabeculæ, sinuses filled with blood, and lymph nodules. (Warthin.)

sule trabeculoe of the same structure pass down into the node, forming its framework (Fig. 99). Beneath the capsule is a blood simus, which may be broad or narrow, and usually completely surrounds the node. Less commonly the sinus is interrupted by lymphoid tissue extending out to the capsule. From the peripheral sinus branches extend into the interior of the node, separating the lymphoid tissue into cords or islands. The relative proportion of sinuses and lymphoid tissue varies greatly, some nodes being composed almost wholly of sinuses, while in others the lymphoid tissue predominates. 'There is usually a fairly distinct hilum. In many glands no differentiation into corlex and medulla can be made. Where there are a distinct medulla 
and cortex the peripheral lymphoid tissue is arranged in nodules as in the ordinary lymph node. Reticular connective tissue crosses the sinuses and supports the cells of the lymph nodules and cords (Fig. I00).

The cellular character of the lymphoid tissue has led to the subdivision of hæmolymph nodes into splenolymph nodes and marrowlymph nodes. In the splenolymph node the lymphoid tissue resembles that of the ordinary lymph node of the spleen. In the marrowlymph node, which is the much less common form, the lymphoid

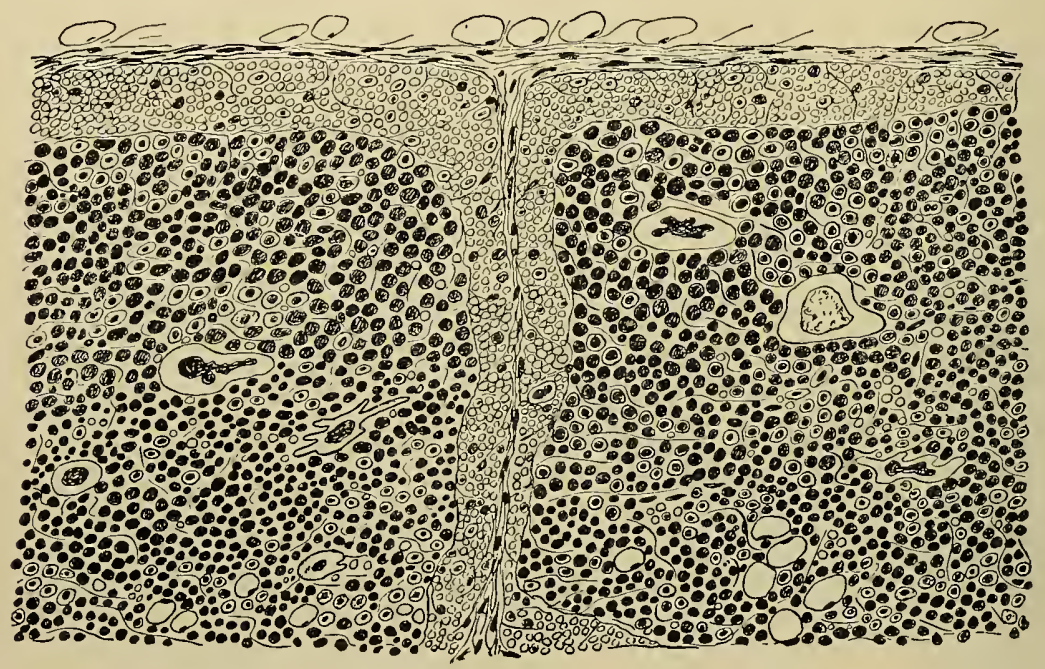

Fig. I00.-Section through Superficial Portion of Human Hæmolymph Node (Marrowlymph Node). (Warthin.) Capsule, trabeculæ, and parts of two adjacent nodules; sinuses filled with blood; among the lymph cells are large multinuclear cells resembling those of marrow, nucleated red blood cells, etc.

tissue resembles red marrow. There are no distinct nodules, and there is a quite characteristic distribution of small groups of fat cells. The most numerous cells are eosinophiles and mast cells (see page I06). Polynuclear leucocytes and large leucocytes with a single lobulated nucleus are less numerous. The very large multinuclear cells of red marrow are also found, but usually in small numbers.

Large phagocytes containing blood pigment and disintegrating red blood cells are found in both forms of hæmolymph nodes, but are most numerous in the splenolymph type. In nodes which have a brownish color when fresh, these phagocytes frequently almost completely fill the sinuses. 
Further classification of hæmolymph nodes has been attempted, but is unsatisfactory, owing to the large number of transitional forms. Thus many nodes are transitional in structure betwen the hæmolymph node and the ordinary lymph node, between the splenolymph node and the marrowlymph node, and between the splenolymph node and the spleen.

Under normal conditions the hæmolymph nodes appear to be concerned mainly in the destruction of red blood cells; possibly also in the formation of leucocytes. Under certain pathological conditions they probably become centres for the formation of red blood cells.

Blood-vessels.-An artery or arteries enter the node at the hilum, and break up within the node into small branches, which communicate with the sinuses where the blood comes into intimate association with the lymphoid tissue. From the sinuses the blood passes into veins, which leave the organ either at the hilum or at some other point on the periphery. The course which the blood takes in passing through the hæmolymph node is thus apparently similar to that taken by the lymph in passing through the ordinary lymph node.

The relation of the hæmolymph node to the lymphatic system is not known, and like ignorance exists as to its innervation.

The development of the hæmolymph nodes is probably much the same as that of the lymph nodes, except for the relation of the latter to the lymphatic vessels, the sinuses of the hæmolymph nodes being developed from blood-vessels.

\section{TECHNIC}

Same as for lymph nodes (technic I, p. I72). The nodes are found in greatest numbers in the prevertebial tissue, and are often difficult to recognize. Fixing the tissues in 5-per-cent. formalin aids in their recognition as it darkens the nodes while bleaching the rest of the tissues.

\section{The Thymus}

The thymus is an organ of foctal and early extra-uterine life; reaching in man its grcatest development at the end of the second year. After this age it undergoes a slow retrograde change into fat and connective tissue, until by the twentieth year scarcely a vestige of glandular tissue remains. The fully developed thymus presents the following structure. The entire gland is surrounderl by a rather delicate and loose connective-tissue capsule which attaches it to the surrounding tissues. From the capsule septa extend down into the 
organ. These branch and subdivide the gland into lobes, and these into larger and smaller lobules.

From the perilobular connective tissue, septa extend in to the lobule, incompletely separating it into a number of chambers. Each lobule consists of a cortical portion and a medullary portion. The cortex consists of nodules of compact lymphatic tissue, composed of reticular tissue and lymphoid cells, similar to those found in the lymph node. These occupy the chambers formed by the connective-tissue septa. The medulla consists of the same elements only more loosely arranged,

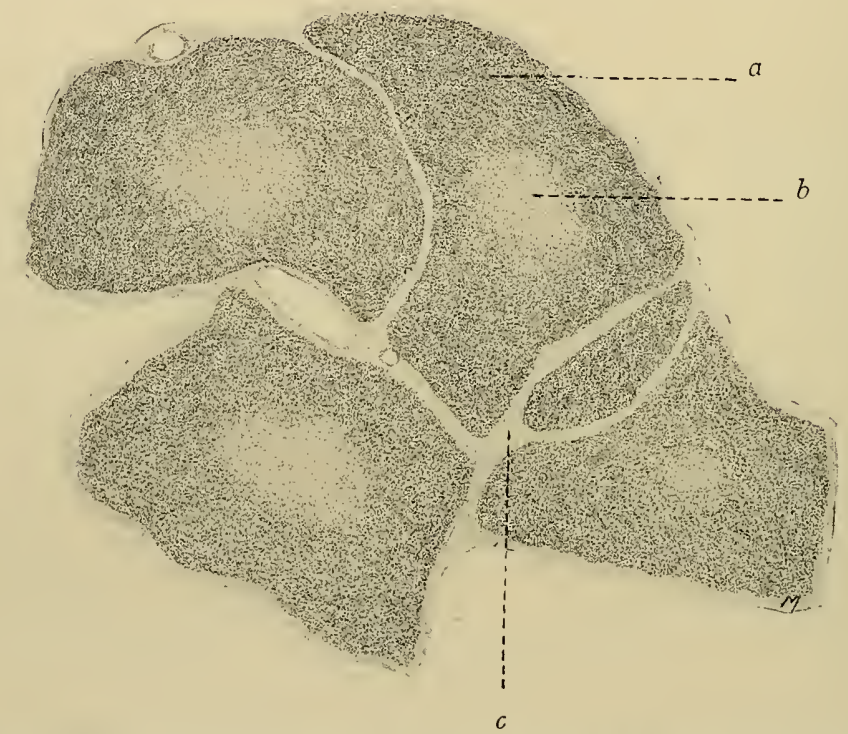

FIG. ror.-From Section of Human Thymus, showing parts of five lobules and interlobular septa. $\times 20$. (Technic, page 178 .) $a$, Cortex; $b$, medulla; $c$, interlobular septum.

the cells being much less closely packed, thus forming a more diffuse lymphatic tissue. There are also in the medulla no connective-tissue septa. In some lobules the more dense cortical substance completely encloses the medulla. It is common, however, for the medullary tissue to extend to the surface of a lobule at one or more points and to be there continuous with the medullary substance of an adjacent lobule. These interlobular connecting strands of medullary substance are known as medullary cords. In the medulla are found a number of spherical or oval bodies composed of concentrically arranged epithelial cells. These are known as Hassall's corpuscles (Fig. IO2), and represent the only remains of the original glandular epithelium. 
They are characteristic of the thymus. The central cells of the corpuscles are usually spherical and contain nuclei, while the peripheral cells are flat and non-nucleated. As the entire corpuscle takes a bright red stain with eosin-hæmatoxylin, the corpuscles stand out sharply from the surrounding bluish or pinkish lymphatic tissue. With low magnifications they are apt to be mistaken for bloodvessels.

Unlike the other lymphatic organs, the lymph nodules of the thymus contain no germinal centres. Mitosis can, however, usually be seen in the lynphoid cells. No definite lymph sinuses have been demonstrated. $\mathrm{Nu}$ cleated red blood cells occur in the thymus. The thymus must therefore be considered one of

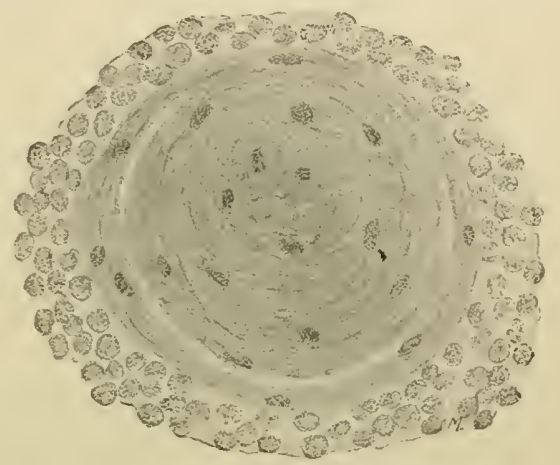

Fig. 102.- Hassall's Corpuscle and Small Portion of Surrounding Tissue. $\times 600$. (See Technic below.) the sources of lymphoid cells and of red blood cells.

Blood-vessels. - The larger arteries run in the connective-tissue septa. From these, smaller intralobular branches are given off, which break up into capillary networks in the cortex and medulla. The capillaries pass over into veins. These converge to form larger veins, which accompany the arteries.

Of the lymphatics of the thymus little is known. They appear to originate in indefinite sinuses within the lymphoid tissue, whence they pass to the septa where they accompany the blood-vessels.

Nerves.-These are distributed mainly to the walls of the bloodvessels. A few fine fibres, terminating freely in the lymphatic tissue of the cortex and of the medulla, have been described.

The thymus originates in the entoderm in the region of the third branchial groove, first as two hollow evaginations of the endothelium of the pharyngeal cavity, which later become solid cords, and then separate entircly from the pharynx. The thymus thus begins its fotal existence as a typical epithelial gland. Into this epithelial structure mesodermic cells grow and differentiate into lymphatic tissue. This almost completely replaces the epithelial tissue, only rudiments of which remain as Hassall's corpuscles.

Stöhr denies the mesodermic invasion of the thymus and consequently the lymphatic character of the gland. He considers the specific cells of the thymus as modified epithelial cells which have become "deceptively like lymphoid cells." This explanation has not, however, been generally accepted. 


\section{TECHNIC}

Fix the thymus of a new-born infant in formalin-Müller's fluid (technic 5, p. 7), and harden in alcohol. Stain sections with hæmatoxylin-eosin (technic I, p. 20), or with hæmatoxylin-picro-acid-fuchsin (technic 3, p. 21), and mount in balsam.

\section{The Tonsils}

The Palatine Tonsils or True Tonsils.-These are lymphatic organs, essentially similar in structure to those already described.

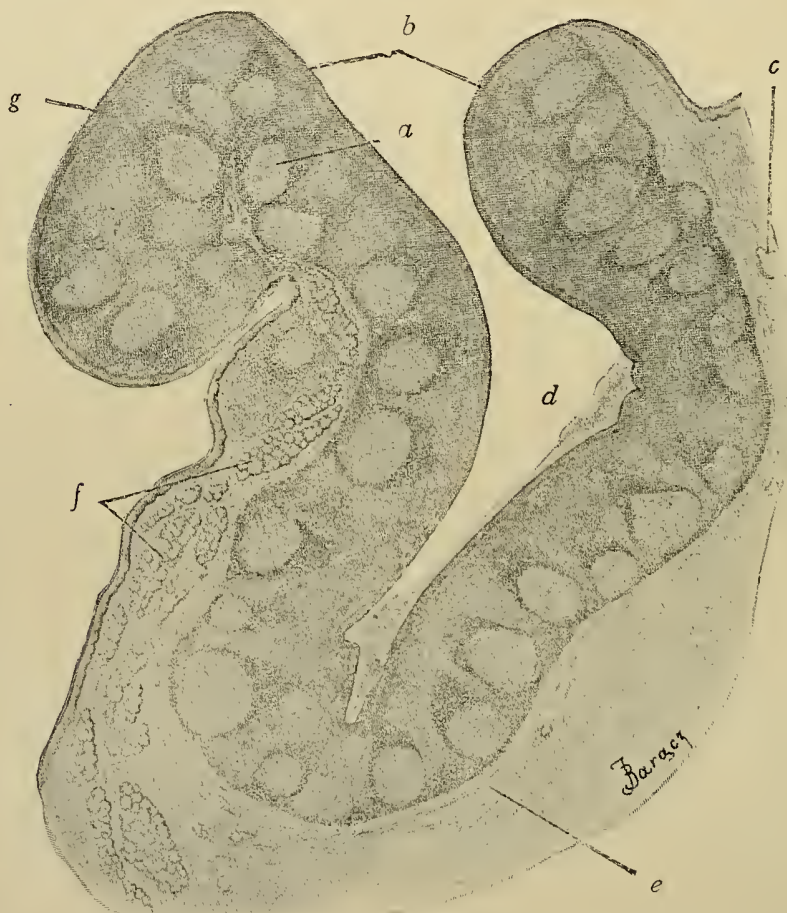

FIG. I03.- Vertical Section of Dog's Tonsil through Crypt. $\times$ I5. (Szymonowicz.) $a$, Lymph nodule; $b$, epithelium of crypt; $c$, blood-vessel; $d$, crypt; $e$, connective-tissue capsule; $f$, mucous glands; $g$, epithelium of pharynx.

The usual fibrous capsule is present only over the attached surface, where it is firmly adherent on the one side to the tonsillar tissue and on the other to the surrounding structures from which it separates the tonsils. From the capsule, connective-tissue trabecule extend into the substance of the organ and branch to form its framework. The free surface of the tonsil is covered by a reflection of the 
stratified squamous epithelium of the pharynx (Fig. 103). This epithelium presents the same structure as elsewhere in the pharynx, flat surface cells, beneath which are irregular cells, while the deepest cells are more or less distinctly columnar. The latter rest upon a papillated stroma from which they are separated by the basement membrane. At several places on the surface of the tonsil deep indentations or pockets occur. These are from ten to twenty in number, are known as the crypls of the tonsil (Fig. I03), and are lined throughout by a continuation of the surface epithelium which becomes thinner as the deeper part of the crypt is reached. Passing off from the bottoms and sides of the main or primary crypts are frequently several secondary crypts, also lined with the same type of epithelium.

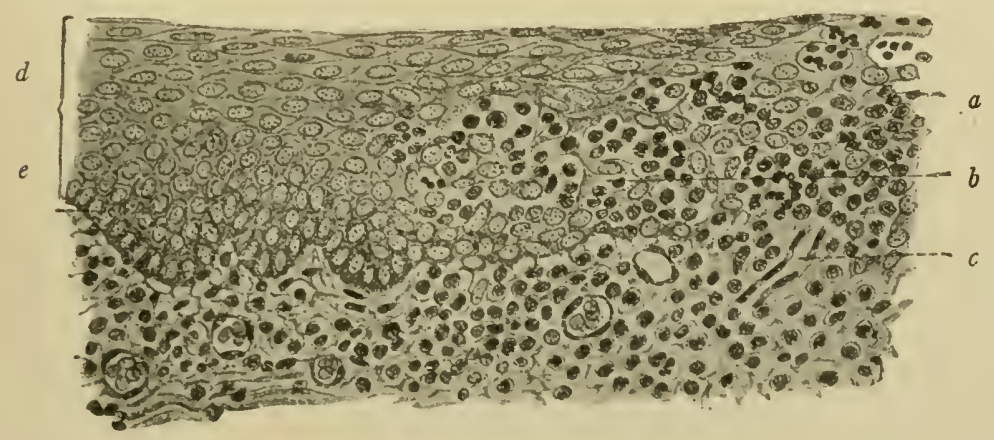

FIG. I04.-Vertical Section through Wall of Crypt in Dog's Tonsil, showing lymphoid infiltration of epithelium. $\times I_{50}$. (Böhm and von Davidoff.) $a$, Leucocytes in epithelium; $b$, space in epithelium filled with leucocytes and changed epithelial cells; $c$, bloodvessel; $d$, epithelium beyond area of infiltration; $e$, basal layer of cells.

The stroma consists of diffuse lymphatic tissue in which are found nodules of compact lymphatic tissue similar to those in the lymph node. Each nodule has a germ centre, where active mitosis is going on, and a surrounding zone of more densely packed cells. The nodules may have a fairly definite arrangement, forming a single layer beneath the epithelium of the crypts or may be arranged quite irregularly, several nodules uniting to form masses of dense lymphatic tissue. At various points on the surface of the tonsil, and especially in the crypts, occurs what is known as lymphoid infiltration of the epithelium (Fig. 104). 'This consists in an invasion of the epithelium. by the underlying lymphoid cells. It varies from the presence of only a few lymphoid cells scattered among the epithelium, to an almost 
complete replacement of epithelial by lymphoid tissue. In this way the latter reaches the surface and lymphoid cells are discharged upon the surface of the tonsil and into the crypts. These cells probably form the bulk of the so-called salivary corpuscles. In the connective tissue adjacent to the tonsil are numerous mucous glands, the ducts of which empty into the tonsillar crypts.

The Lingual Tonsils-Folliculi Linguales.-These are small lymphatic organs situated on the dorsum and sides of the back part of the tongue between the circumvallate papillæ and the epiglottis. They are similar in structure to the true tonsils. Each lingual tonsil has usually one rather wide-mouthed deep crypt (the foramen cacum lingua) which may be branched and which is lined with a continuation of the surface stratified squamous epithelium. Into these crypts frequently open the ducts of some of the mucous glands of the tongue.

The Pharyngeal Tonsils.- These are lymphatic structures which lie in the naso-pharynx. They resemble the lingual tonsils, except that they are, as a rule, not so sharply circumscribed. Hypertrophy of the pharyngeal tonsils, with consequent obstruction of the nasal openings, is common especially in children, constituting what are known as adenoids.

The blood-vessels have a distribution similar to those of the lymph nodes, but enter the organ along its entire attached side and not at a definite hilum.

Of the lymphatics of the tonsil little is known.

The nerves which are branches of the glosso-pharyngeal and of the spheno-palatine ganglion also enter the organ along its attached side.

The palatine tonsils make their first appearance during the third month of intra-uterine life as hollow evaginations of entoderm which grow down into the underlying mesenchyme, in the region of the second inner branchial groove. The earliest of the tonsillar lymphoid cells are apparently white blood cells which have migrated from the vessels of the stroma of the mucosa and have infiltrated the surrounding connective tissue. Further development of the tonsil is by proliferation of these cells. The crypts are at first solid ingrowths of surface epithelium. These later become hollowed out.

The lingual and pharyngeal tonsils begin their development during the later months of intra-uterine life. In the pharyngeal tonsils, definite nodules appear about the time of birth or during the first or second year. In the lingual tonsils the nodules are not fully formed until the fifth or sixth year. 


\section{TECHNIC}

Normal human tonsils are so rare, owing to the frequency of inflammation of the organ, that it is best to make use of tonsils from one of the lower animals (dog, cat, or rabbit). Treat as in technic I, p. I72, care being taken that sections pass longitudinally through one of the crypts.

\section{The Spleen}

The spleen is a lymphatic organ and might be quite properly classed as a hæmolymph node. Its peculiar structure appears to depend largely upon the arrangement of its blood-vessels.

Except where attached, the spleen is covered by a serous membrane, the peritoneum (page 267 ). Beneath this is a capsule of fibrous tissue containing numerous elastic fibres and some smooth muscle

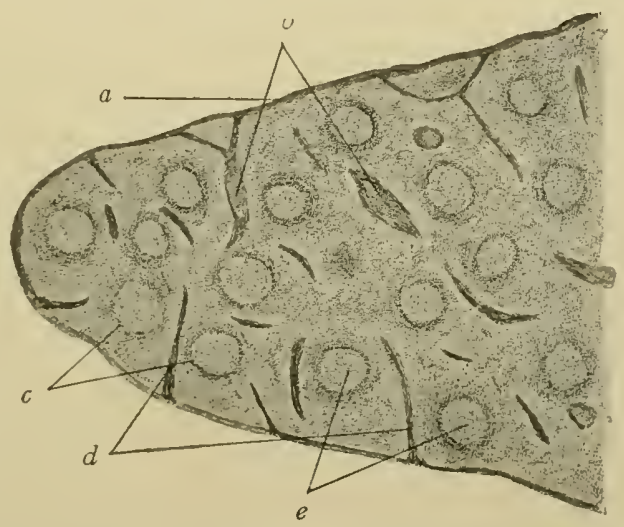

Fig. 105.-Section through Portion of Cat's Spleen, to show general topography. $\times$ I5. (Technic I, p. I87.) $a$, Capsule; $b$, septa containing blood-vessels; $c$, germinal centres; $d$, septa; $e$, lymph nodules.

cells. From the capsule strong connective-tissue septa, similar to the capsule in structure, extend into the interior of the organ. These branch and unite with one another to form very incomplete anastomosing chambers. The capsule and septa form, as in the lymph node, the connective-tissue framework of the organ (Fig. I05).

The chambers incompletely bounded by the connective-tissue septa are filled in with tissue resembling lymphatic tissue, composed of reticular connective tissue, lymphoid cells, and other varieties of cells described on p. I84. This tissue constitutes the substantia propria of the organ and is everywhere traversed by thin-walled vascular channels, the tissue and vascular channels together con- 
stituting the splenic pulp (Fig. I06). Compact lymphatic tissue occurs in the spleen as spherical, oval, or cylindrical aggregations of closely packed lymphoid cells. These are known as Malpighian bodies or splenic corpuscles (Figs. 105 and 106) and are distributed throughout the splenic pulp. Each splenic corpuscle contains one or more small arteries. These usually run near the periphery of the corpuscle; more rarely they lie at the centre. Except for its relation to the blood-vessels, the splenic corpuscle is quite similar in structure to a lymph nodule. It consists of lymphoid cells so closely packed as completely to obscure the underlying reticulum. In a

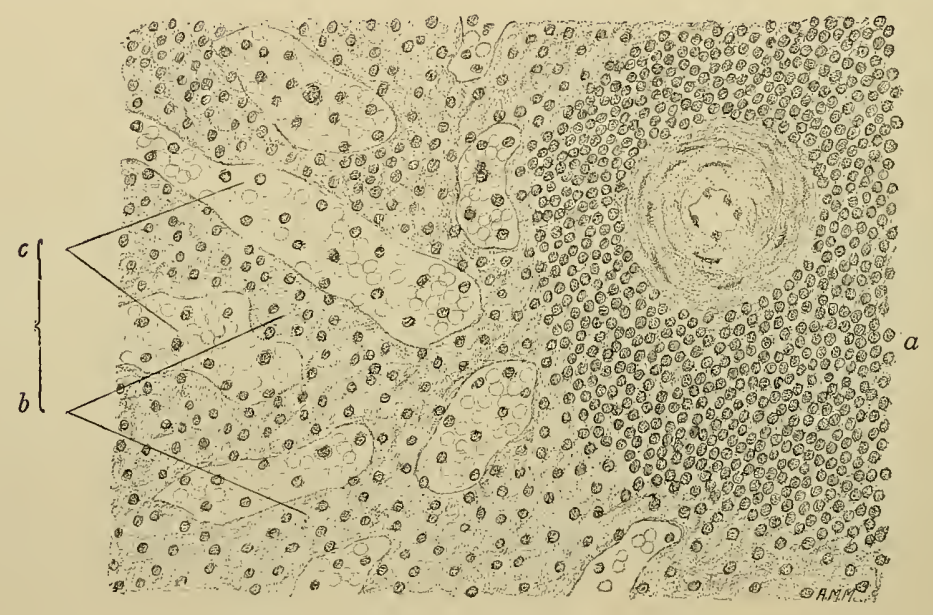

Fig. I06.--Section of Human Spleen, including portion of Malpighian'body with its artery and adjacent splenic pulp. $\times_{300}$. (Technic 2, p. I 87.) a, Malpighian body; $b$, pulp cords, $c$, cavernous veins; $b$ and $c$ together constituting the splenic pulp.

child's spleen the centre of each corpuscle shows a distinct germinal centre (see page $\mathrm{r} 69$ ). In the adult human spleen germ centres are rarely seen. The blood-vessels of the spleen have a very characteristic arrangement, which must be described before considering further the minute structure of the organ.

The arteries enter the spleen at the hilum and divide, the branches following the connective-tissue septa. The arteries are at first accompanied by branches of the splenic veins. Soon, however, the arteries leave the veins and the septa, and pursue an entirely separate course through the splenic pulp. Here the adventitia of the smaller arteries assumes the character of reticular tissue and becomes infiltrated with lymphoid cells. In certain animals, as e.g., the guinea- 
pig, this infiltration is continuous, forming long cord-like masses of compact lymphoid tissue. In man, the adventitia is infiltrated only at points along the course of an artery. This may take the form of elongated collections of lymphoid cells-the so-called spindlesor of distinct lymph nodules, the already mentioned splenic corpuscles. Although usually eccentrically situated with reference to the nodules, these arteries are known as conlral arteries. They give rise to a few capillaries in the spindles, to a larger number in the nodules. Be-

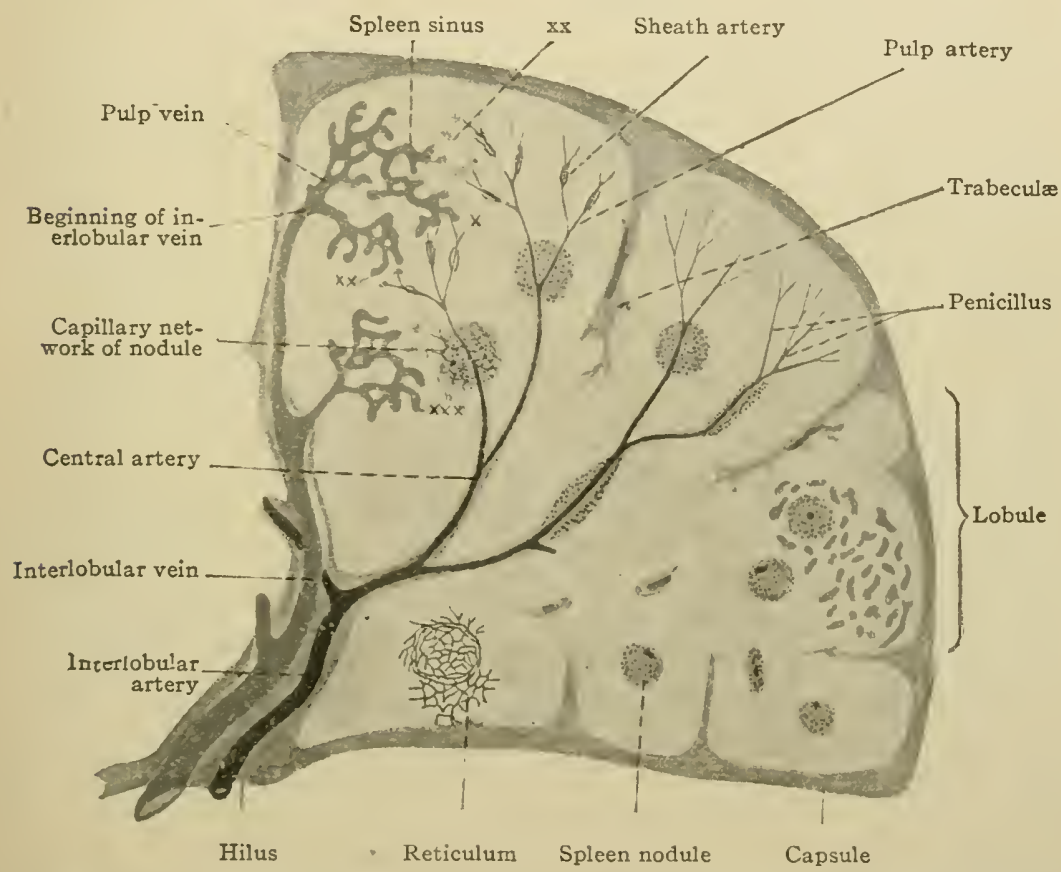

FIG. 107.-Scheme of Human Spleen. $x$, Opening of arterial capillaries into spleen sinus; $x x$, interruption of closed blood course at ends of arterial capillaries, at margin of nodule, $x x x$. For sake of clearness, sinus is placed too far from margin of nodule. (Stöhr.)

yond the latter the arteries divide into thick sheathed terminal arteries-pulp arlcries - which do not anastomose, but lie close together like the bristles of a brush or penicillus. The pulp arteries break up into unusually thick walled arterial capillaries which still retain an adventitia, the so-called shealhed arleries. These are of remarkably uniform diameter-6- $8 \mathrm{cc}-$ - and empty into broader spaces from ro to $40 \%$ in diameter-the splecn, sinuses or ampullawhich in turn empty into the cuvernous reins of the splenic pulp (Fig. Io6). 
The Splenic Pulp.--The anastomosing cavernous veins break up the diffuse lymphatic tissue of the spleen into a series of anastomosing cords similar to those found in the medulla of the lymph node. These are known as pulp cords (Fig. I06), and with the cavernous veins constitute, as already mentioned, the splenic pulp. The pulp cords consist of a delicate framework of reticular connective
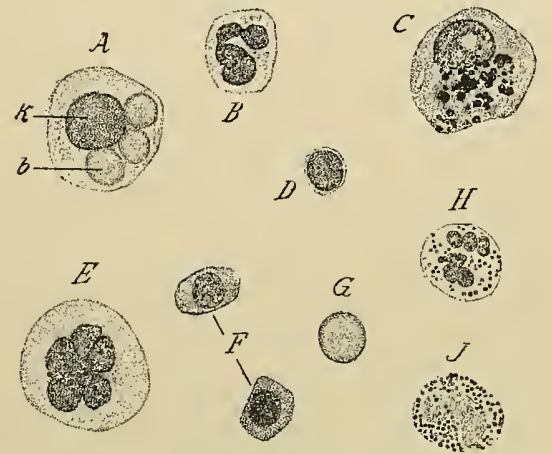

Fig. ro8.--Isolated Spleen Cells. $\times 700$ (Kölliker.) $A$, Cell containing red blood cells; $b$, blood cell; $k$, nucleus; $B$, leucocyte with polymorphous nucleus; $C$, "spleen" cell with pigment granules; $D$, lymphocyte; $E$, large cell with lobulated nucleus (megalocyte); $F$, nucleated red blood cells; $G$, red blood cell; $H$, multinuclear leucocyte; $J$, cell containing eosinophile granules.

destruction of red blood cells.

(4) Multinuclear cells. These are most common in young animals. Each cell contains a single large lobulated nucleus, or more frequently several nuclei. These cells resemble the osteoclasts of developing bone and the multinuclear cells of bone-marrow.

In macerated splenic tissue or in smears from the spleen, there are found, in addition to the above varieties of cells, long spindleshaped cells with bulging nuclei. These come from the walls of the cavernous veins.

Two views have been held regarding the vascular channels of the pulp. According to one, these channels have complete walls, the blood-vessel system of the splenic pulp being a closed system as in other organs. According to the other, the cavernous veins or spleen sinuses into which the arterial capillaries. open, have fenestrated walls-open system. These fenestra are of sufficient size to allow fluid and formed elements of the blood to pass out freely into the pulp. cords and elements of the pulp cords to pass freely into the vessels. From these open-walled sinuses the veins proper take origin. The smaller veins, even those within the trabeculæ, have only endothelial walls. These uniting, form veins. 
which enter the septa and ultimately converge to form the splenic veins which leave the organ at the hilum.

Mollier ${ }^{1}$ describes the walls of the spleen pulp sinuses as having the following structure. (I) A protoplasmic endothelial syncytium, with scattered oval nuclei (their long diameter lying in the long diameter of the vessel) and no evidence of cell boundaries. This syncytium is arranged as a network, the meshes of which are sometimes quite irregular, at other times quite regular and

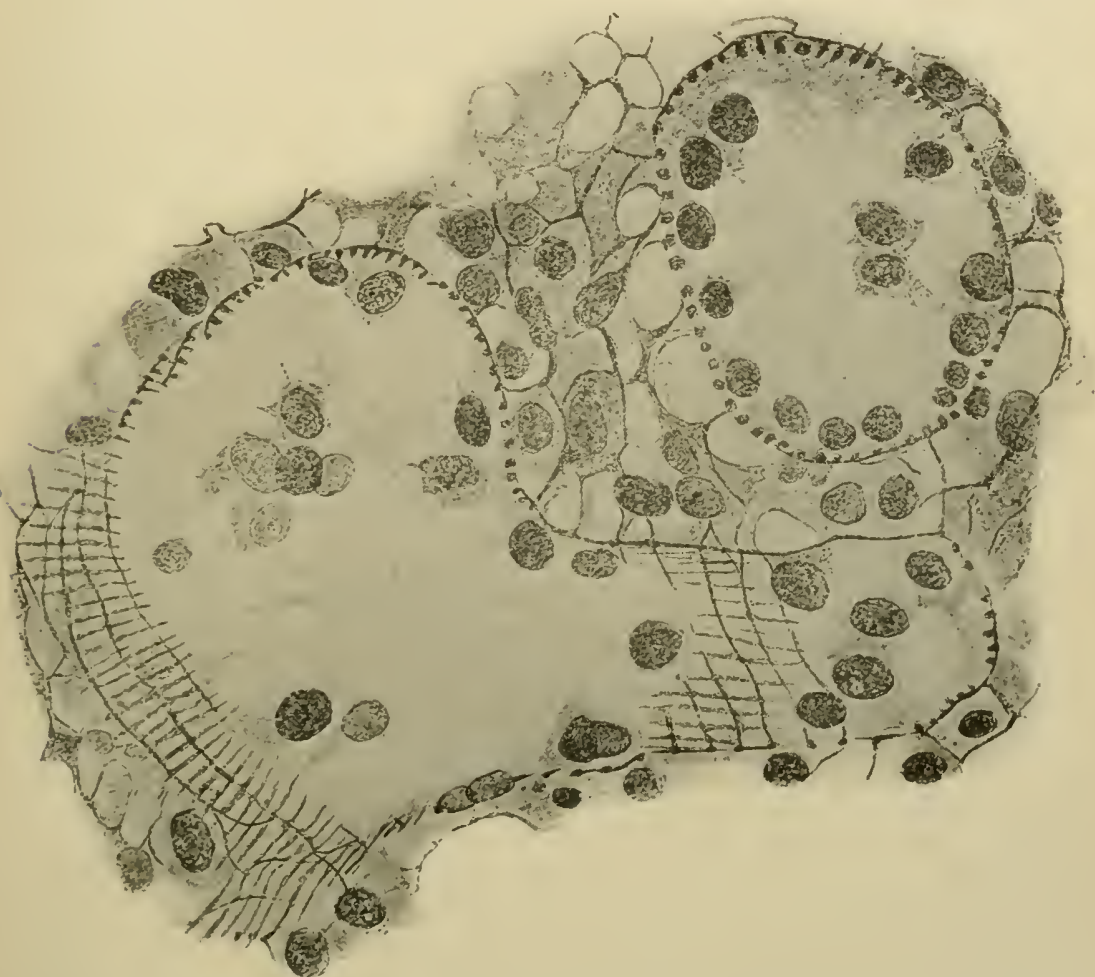

FIG. I09.-Section of Monkey's Spleen. Two pulp sinuses are shown and between them some of the reticular tissue of the pulp. The sinus in the upper right corner is cut transversely so that the longitudinal fibres of its wall are cut across and appear as a row of dots. The prominent dark nuclei belong to the endothelium. The other sinus is cut obliquely and a branch from it is seen partly in longitudinal section. It shows well the disposition of both longitudinal and circular fibres. (Mollier.)

rectangular, with the long diameter of the mesh rumning in the long diameter of the vessel. This protoplasmic syncytium lines the sinus. (2) Outside the endothelial syncytium are closely-placed longitudinal fibres which lie upon the lengthwise-running strands of the protoplasmic reticulum (Fig. 109). (3) Outside the lengthwise-running strands transverse fibres or ring fibres which at rather longer intervals encircle the tube (Fig. 110). Both longitudinal and ring fil)res are connective tissue (reticular), and the reticulum which they form is

${ }^{1}$ Arch. f. mikr. Anat., Bcl. lxxvi, I910-Ir. 
everywhere continuous with the reticulum of the pulp cords and apparently identical with it, except that to form walls, the reticulum is flattened out and usually has a more regular arrangement. The strands of the fibre reticulum lie upon the strands of the protoplasmic reticulum in such a way that the meshes correspond. A thin structureless fenestrated membrane lying just outside the endothelial syncytium has been described. The sinus walls probably possess a certain elasticity or contractility and undoubtedly change their diameter to accord with varying functional conditions. It follows that the meshes of the

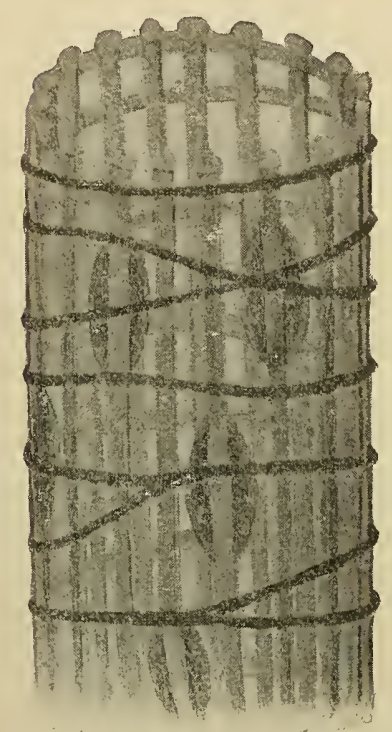

Fig. I I o.-Diagram of a Sinus of the Splenic Pulp, showing Relations of Longitudinal Fibres, Circular Fibres and Endothelial Nuclei. (Mollier.) reticulum may be at one time widely open (congested spleen), at another partly open, at still another entirely closed.

The main functions of the adult spleen in health appear to be the production of leucocytes and the destruction of red blood cells. It is possible that to a limited degree the spleen produces red blood cells. During fœtal life the spleen is actively engaged in the development of both red and white cells. Also in adult life a severe secondary anæmia or a pernicious anæmia may stimulate the spleen to resume production of red and white cells. The spleen can be removed without apparently seriously interfering with the body functions. Enlargement of lymph nodes and increased blood-forming activity of bone marrow as a result, have been described.

The ultimate origin of the spleen in man has not been definitely determined. The anlage of the spleen can be seen in embryos of five weeks as a thickening of the mesenchyme in the left dorsal mesogastrium and it was believed that the organ developed wholly from this mesenchyme. The mesothelium covering the thickened mesenchyme also shows thickening, and demarcation between the two layers becomes almost entirely lost. Recent investigations have made it seem probable that the spleen develops in part at least from these mesothelial cells which grow down into the mesenchyme. In this case it is probable that mesenchyme gives rise to capsule, trabeculæ, and reticular connective tissue-the connective tissue framework of the organ-while mesothelium is responsible for some at least of the various cellular elements of the splenic pulp.

Lymphatics are not numerous. In certain of the lower animals large lymph vessels occur in the capsule and septa. These are not well developed in man. Lymph vessels are present in the connective tissue of the hilum. They probably do not occur in the splenic pulp or in the splenic corpuscles. 
Nerves.- These are mainly non-medullated, although a few medullated fibres are present. Among the latter are dendrites of sensory neurones whose cell bodies are situated in the spinal ganglia. They supply the connective tissue of the capsule, septa, and blood-vessels. The non-medullated fibres-axones of sympathetic neurones-accompany the arteries, around which they form plexuses. From these plexuses terminals pass to the muscle cells of the arteries, to the septa, to the capsule, and possibly also to the splenic pulp. The exact manner in which both medullated and non-medullated fibres terminate is as yet undetermined.

\section{TECHNIC}

(I) The spleen of a cat is more satisfactory for topography than the human spleen, as it is smaller, contains more connective tissue and its Malpighian bodies are more evenly distributed and more circumscribed. Fix in formalin-Müller's fluid (technic 5, p. 7), and harden in alcohol. Cut sections through the entire spleen. Stain with hæmatoxylin-eosin (technic I, p. 20), or with hæmatoxylinpicro-acid-fuchsin (technic 3, p. 2I).

(2) Human Spleen.--Small pieces are treated as in technic (I).

(3) Human Spleen (Congested).-Congested human spleens are usually easy to obtain from autopsies. Treat as in technic (I). The cavernous veins being distended with blood, the relations of the veins to the pulp cords are more easily seen than in the uncongested spleen. The contrasts are especially sharp in sections stained with hæmatoxylin-picro-acid-fuchsin.

(4) The cells of the spleen may be studied along the torn edges or in the thinner parts of any of the spleen sections. Or a smear may be made in a manner similar to that described in technic (page 110 ), by drawing the end of a slide across a freshly cut spleen surface and then smearing the tissue thus obtained across the surface of a second slide. Dry, fix in equal parts alcohol and ether (one-half hour), stain with hæmatoxylin-eosin and mount in balsam. Or the cut surface of the spleen may be scraped with a knife, the scrapings transferred to Zenker's fluid, hardened in alcohol, stained with alum-carmine (pages I9 and 63 ) and mounted in cosin-glycerin.

\section{General References for Further Study}

Kölliker: Handbuch der Gewebclehre des Menschen, vol. iii.

Szymonowicz and MacCallum: Histology and Microscopic Anatomy.

Warthin: Hacmolymph Glands (with bibliography). Reference Ilandbook of the Merlical Sciences, vol. iv.

Mall: Lobule of the Spleen. Bul. Johns Hopkins IIospital, vol. ix.-Architecture and Jilood-vessels of the Dog's Spleen. Zeit. f. Morph. u. $\Lambda$ nth., Bd. ii.

Oppel: Ueber Gitterfasern der menschlichen Leber und Mlilz. Anat. Anz., 6 Jahrg., S. 165. 


\section{CHAPTER III \\ THE SKELETAL SYSTEM}

ThE skeletal system consists of a series of bones and cartilages which are united by special structures to form the supporting framework of the body. Under this head are considered: (I) bones, (2) marrow, (3) cartilages, (4) articulations.

\section{The Bones}

A bone considered as an organ consists of bone tissue laid down in a definite and regular manner. If a longitudinal section be made through the head and shaft of a long bone, the head of the bone and also part of the shaft are seen to be composed of anastomosing bony

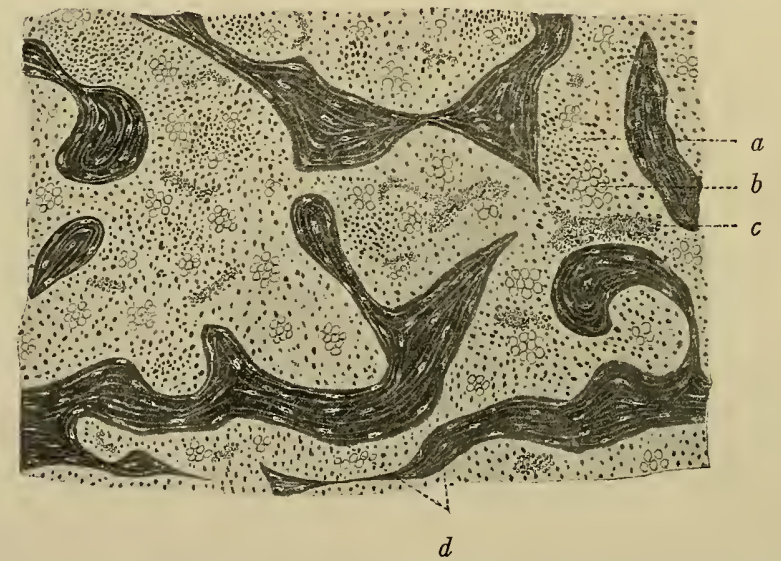

FIG. III.-Section of Spongy Bone. $\times 75 . \quad$ (Technic 3, p. I97.) $a$, Marrow space; $b$, group of fat cells; $c$, blood-vessel; $d$, trabeculæ of bone.

trabeculæ enclosing cavities. This is known as cancellous or spongy bone. The shaft of the bone consists of a large central cavity surrounded by spongy bone, which, however, passes over on its 'outer side into a layer of bone of great density and known as hard or compact bone. Spongy bone forms the ends and lines the marrow cavities of the long bones, and occurs also in the interior of short bones and flat 
bones. Compact bone forms the bulk of the shafts of the long bones and the outer layers of the flat and short bones.

In compact bone the layers or lamellix of bone tissue have a definite arrangement into systems, the disposition of which is largely dependent upon the shape of the bone and upon the distribution of its blood-vessels.

In spongy bone (Fig. I I I) there is no arrangement of the bone tissue into systems. The trabeculæ consist wholly of bony tissue laid down

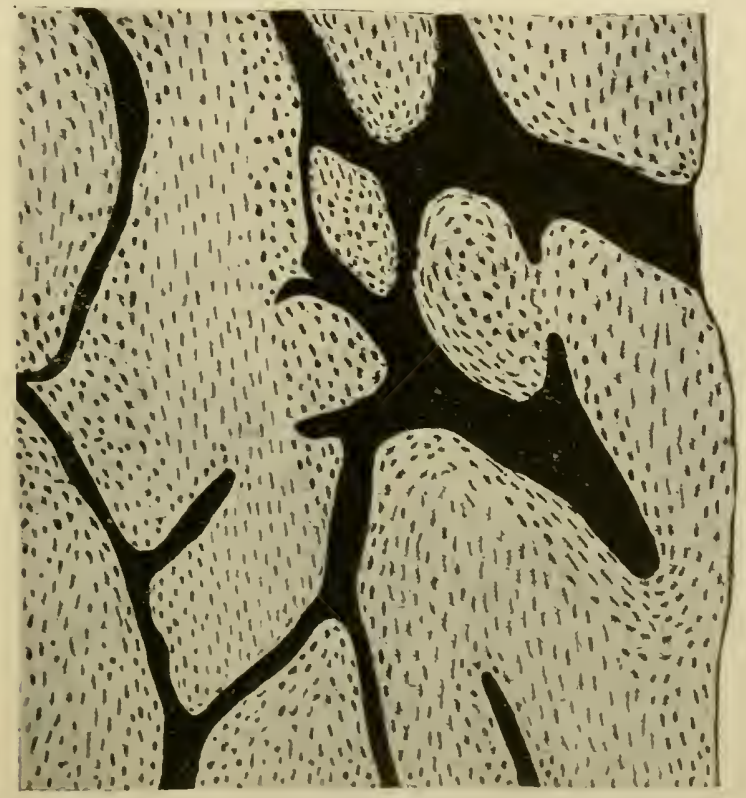

FIG. I I 2.-Longitudinal Section of Hard (Undecalcified) Bone: Shaft of Human Ulna. Xgo. (Szymonowicz.) Haversian canals, lacune, and canaliculi in black.

in lamellæ. These trabeculæ anastomose and enclose spaces which contain marrow and which serve for the passage of blood-vessels, lymphatics, and nerves.

On examining a longitudinal section of compact bone (Fig. II 2 ) there are seen running through it irregular channels, the general direction of which is parallel to the long axis of the bone. These channels anastomose by means of lateral branches, and form a complete system of intercommunicating tubes. They are known as IIaversian canals, contain marrow elements, and serve for the transmission of blood-vessels, lymphatics, and nerves. They anastomose not only with one another, but are in communication with the sur- 
face of the bone and with the central marrow cavity. Between the Haversian canals most of the lamellæ run parallel to the canals.

In a cross section through the shaft of a long bone (Fig. I I3), three distinct systems of lamellæ are seen. These are known as Haversian lamella, interstitial lamelle, and circumferential lamella.

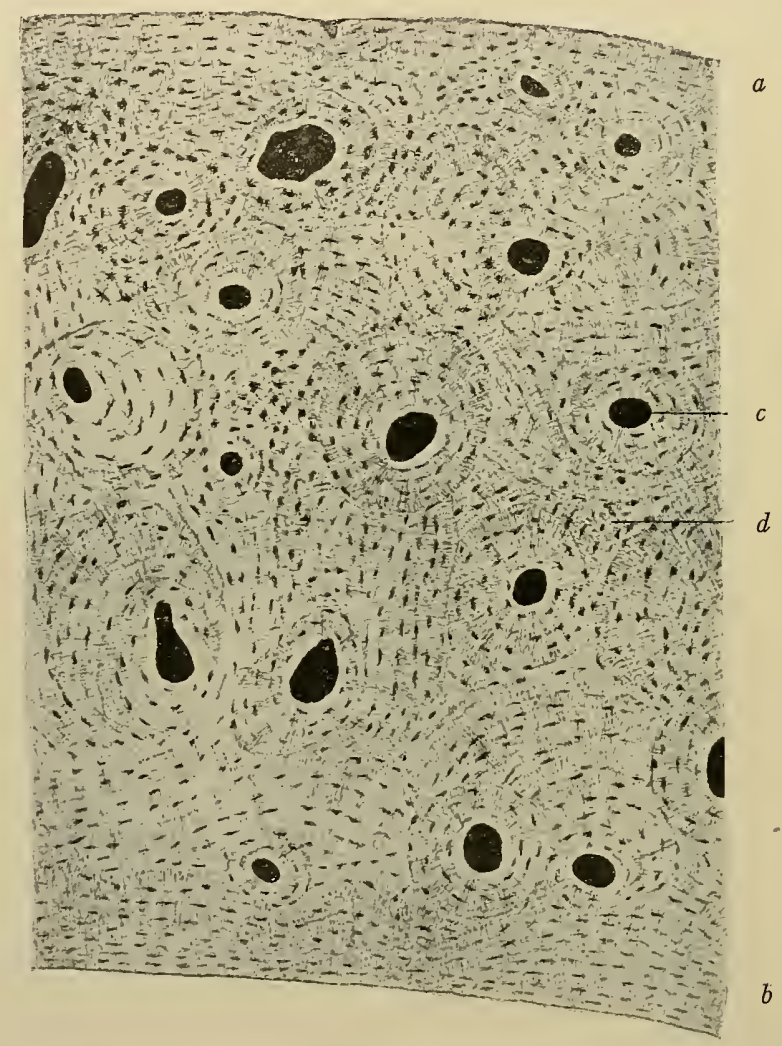

FIG. I 13.-Cross-section of Hard (Undecalcified) Bone from Human Metatarsus. $\times 90$. (Szymonowicz.) Haversian canals, lacunæ, and canaliculi in black. $a$, Outer circumferential lamellæ; $b$, inner circumferential lamellæ; $c$, Haversian lamellæ; $d$, interstitial lamellæ.

(I) HaversianLamellæ (Fig. I I 4).--These are arranged in a concentric manner around the Haversian canals. Between the lamellæ, their long axes corresponding to the long axes of the Haversian canals, are the lacunce with their inclosed bone cells (page ror). The lacunæ of adjacent lamellæ are usually arranged alternately. In a section of ordinary thickness the lacunæ are not nearly so numerous as the lamellæ, and are seen only between some of the lamellæ. The 
lacunæ of a Haversian system communicate with one another and with their Haversian canal by means of the canaliculi. In Haversian systems the fibres of the matrix (see page Ior) run in some lamellæ parallel to the canal, in others concentrically. Adjacent fibres thus frequently cross at right angles.

(2) Interstitial (Intermediate or Ground) Lamelle (Figs. II 3 and II4).-These are irregular short lamellæ, which occupy the spaces left between adjacent Haversian systems.

(3) Circumferential LaMELLE (Fig. I I3).-These are parallel lamellæ which run in the long axis of the bone, just beneath the periosteum and at the outer edge of the central marrow cavity. Occasionally circumferential lamellæ are absent, the Haversian systems abutting directly upon periosteum.

Channels for the passage of blood-vessels from the periosteum to the Haversian canals pierce the circumferential lamellæ. They are known as Volkmann's canals, and are not surrouncied by concentric lamella as are the Haversian lamella, but are mere channels through the

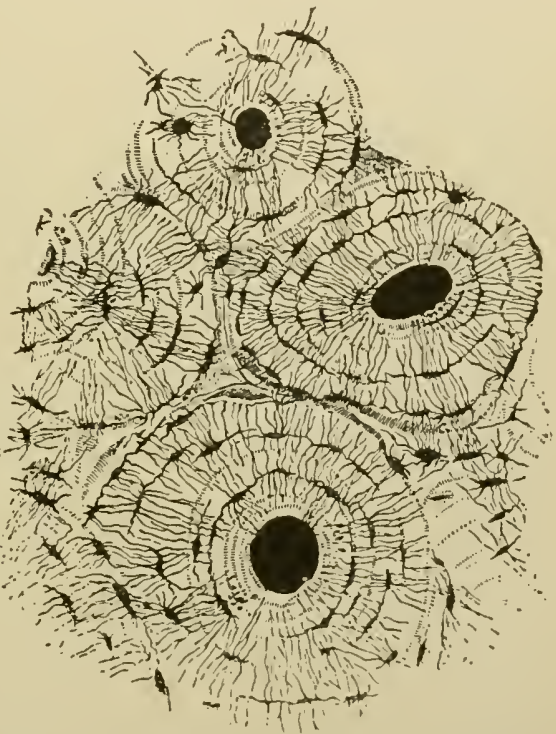

FIG. 1 I 4.-Transverse Section of Compact Bone from Shaft of Humerus. $\times 150$ and slightly reduced. (Sharpey.) (Technic I, p. I 96.) Three Haversian canals with their concentric lamellæ and lacune; canaliculi connecting lacunie with each other and with Haversian canal. Between the IIaversian systems of lamellæ are seen the interstitial lamellic.

bone. Similar canals pass from the inner Haversian canals into the marrow cavity.

The Periosteum.-This is a fibrous connective-tissue membrane which covers the surfaces of bones except where they articulate. It is firmly adherent to the superficial layers of the bone and consists of two layers. The outer layer is composed of coarse fibrillated fibres and contains the larger blood-vessels. The inner layer consists of fine white fibres and delicate elastic fibres which support the smaller blood-vessels.

From the periosteum distinct bundles of white fibres, with often 
some elastic fibres, pierce the outer layers of the bone. These are known as the perforating fibres of Sharpey. When tendons and ligaments are attached to bone, their fibres are prolonged through the periosteum into the bone as perforating fibres.

\section{Bone Marrow}

Bone marrow is a soft tissue which occupies the medullary and Haversian canals of the long bones and fills the spaces between the trabeculæ of spongy bone. It consists of a delicate reticular connective tissue, in the meshes of which lie various kinds of cells. Because of its function as a blood-forming organ it contains all varieties of blood cells as well as certain other cells. It is convenient to divide marrow cells primarily into (I) blood cells, adult and developmental forms, and (2) other cells.

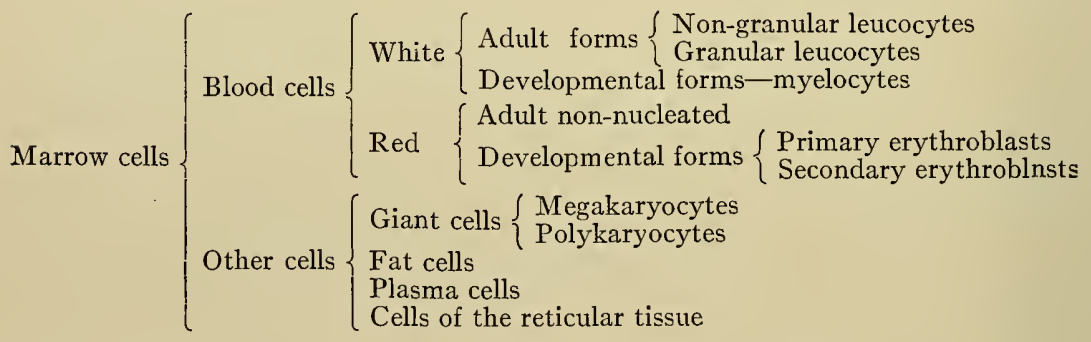

The following description omits the adult forms of blood cells for which the student is referred to p. Io3.

(I) Myelocytes.-These resemble the mononuclear and some of the transitional forms of leucocytes. The nucleus is large and may be lobulated. It contains a comparatively small amount of chromatin and therefore stains faintly. The cytoplasm is finely granular and stains with neutrophile dyes. Myelocytes are not present in normal blood, but occur in large numbers in leukæmia. It is from the myelocytes that some and possibly all leucocytes, which are of bonemarrow origin, are derived.

(2) Nucleated Red Blood Cells.-These are divisible into primary erythroblasts and secondary erythroblasts or normoblasts. The former represents an earlier, the latter a later stage in the evolution of the non-nucleated adult red blood cell.

The primary erythroblast, the younger of the two, has a wellformed nucleus with a distinct intranuclear network. The proto- 
plasm contains but little hæmoglobin. In the secondary erythroblast the intranuclear network has disappeared and the protoplasm has become richer in hæmoglobin. The secondary erythroblast is converted into the adult red blood cell either by extrusion of its nucleus, or by the disintegration of the nucleus within the cell body.

(3) Giant Cells.-(a) Megakaryocytes. These are from 25-30, in diameter and are distinctly amobcid. They have an abundant granular protoplasm which is usually acidephile, more rarely basophile. Peripherally the protoplasm is comparatively free from granules so that a clear exoplasm and a granular endoplasm may be distinguished. The nucleus varies greatly both in shape and size. It is usually single; may be spheroidal, but is more commonly lobulated with the lobules arranged in a circle or like the letter C. There are many nucleoli and centrosomes. The latter may be clumped or distributed through the protoplasm. When the nucleus is circular or C-shaped the centrosomes lie in the centre. Both mitosis and amitosis have been described in these cells. The origin and function of the megakaryocytes is unknown. Wright describes them (p. I Io) as giving rise to the blood platelets, Stöhr as probably associated with the formation of leucocytes. They sometimes apparently entirely lose their protoplasm, thus giving rise to free giant nuclei.

(b) Polykaryocytes (myeloplaxes-osteoclasts). These are even larger than the megakaryocytes, having sometimes a diameter of гоo $\mu$. They are flat with a thickness of only 6-1o $\mu$ and are rather scarce in adult bone. In developing bone they are numerous and lie near the bone or cartilage. Their protoplasm is granular and frequently contains fat droplets. They are multinuclear ( 5 to 40 ) and contain many centrosomes arranged in pairs, the number of pairs apparently corresponding to the number of nuclei. The nuclei may be clumped near the centre of the cell or may be arranged in a ring or skein or irregularly. In development of bone these cells apparently have to do with its absorption. It is of interest to note that they are also found at the roots of milk teeth which are being absorbed, and Billroth describes them as apparently causing the absorption of ivory pegs which had been driven into bone.

Fat cells (p. 88), plasma cells (p. 85), mast cells (p. 85) and cells of the reticular connective tissue (p. 94) are found in marrow in varying numbers.

While all marrow contains the elements described, their proportions vary greatly. Dependent upon the amount of fat present are 
distinguished two main varieties of marrow, red marrow and yellow marrow.

Red marrow is found in all bones of embryos and of young animals, also in the vertebræ, sternum, ribs, cranial bones, and epiphyses of long bones in the adult. In the diaphyses of adult long bones the marrow is of the yellow variety. The difference in color between red marrow and yellow marrow is due to the much greater propor-

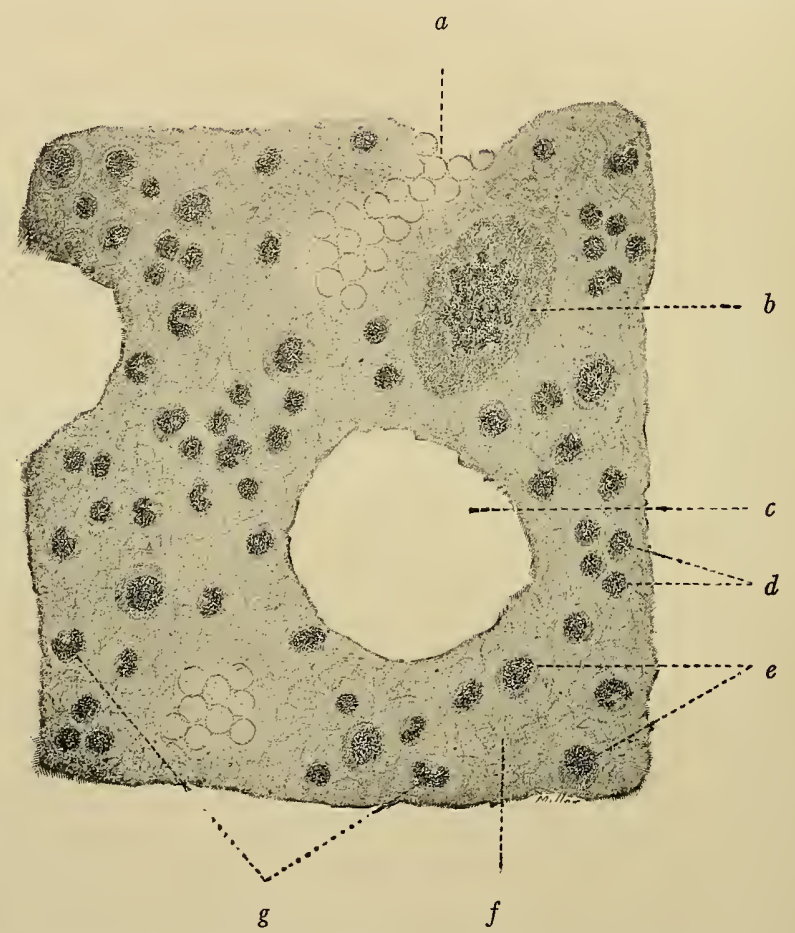

FIG. I I5.-Section of Red Bone-marrow from Rabbit's Femur. $\times 700 . \quad$ (Technic 4, p. I 97.) $a$, Red blood cells; $b$, myeloplax; $c$, fat space; $d$, nucleated red blood cells; $e$, myelocytes; $f$, reticular connective tissue; $g$, leucocytes.

tion of fat in the latter, yellow marrow being developed from the red by an almost complete replacement of its other elements by fat cells.

Red marrow is of especial interest as a blood-forming tissue, being in the healthy adult the main if not the sole source of red blood cells, and one of the sources from which the leucocytes are derived. (See also p. 109.) At the same time it is quite probable that it functionates as a place where blood cells are destroyed. It is also active in the development of bone. 
Yellow marrow (Fig. II6) consists almost wholly of fat cells, which have gradually replaced the ether marrow elements. Under certain conditions the yellow marrow of the bones of the old or greatly emaciated undergoes changes due for the most part to the absorption of its fat. Such marrow becomes reddish and assumes a somewhat gelatinous appearance. It is known as "gelatinous

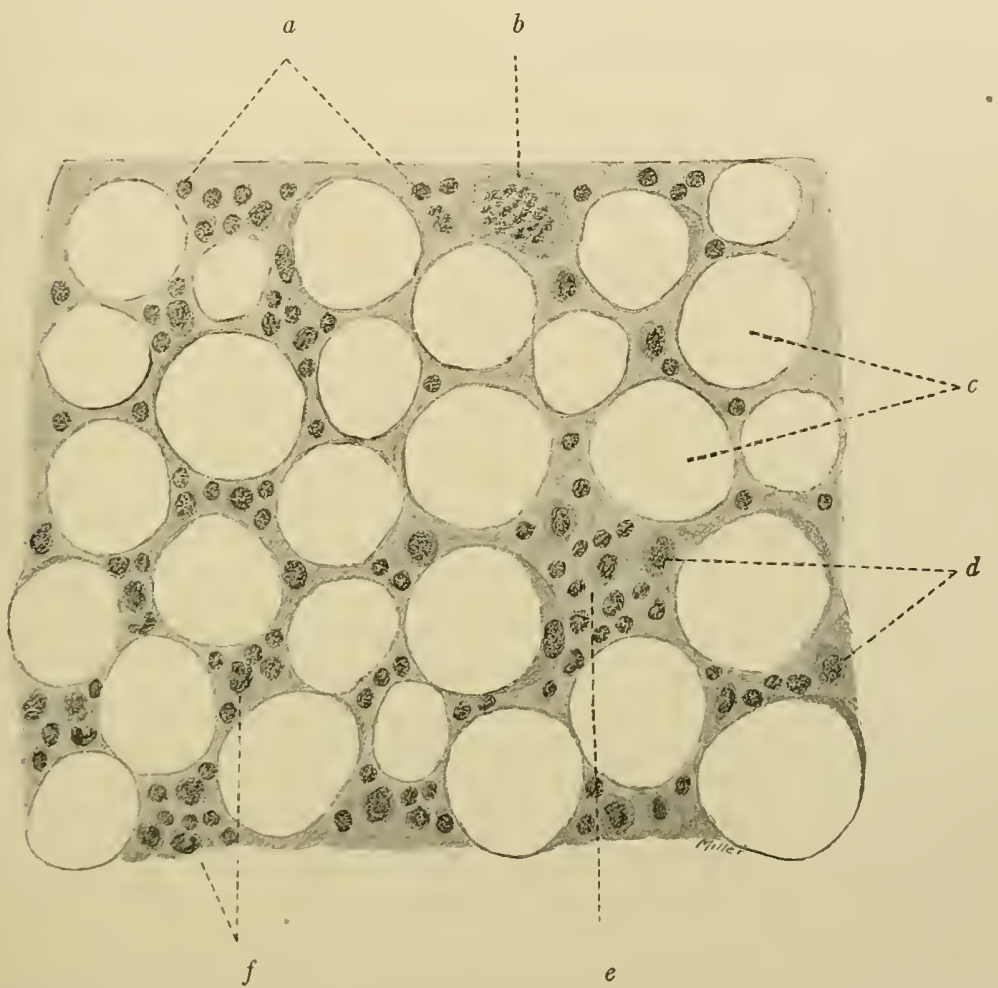

Fig. i I6.-Yellow Marrow from Rabbit's Femur. $\times_{560 .}$ (Technic 4, p. 197.) $a$, nucleated red blood cells; $b$, myeloplax; $c$, fat cells; $d$, myelocytes; $e$, reticular connective tissue; $f$, leucocytes.

marrow." Under certain conditions, e.g., fracture of shaft of long bone, yellow marrow may assume the character of red marrow and take an active part in the process of repair. It also serves as a storage place for fat.

The large marrow cavities, such as those of the shafts of the long bones, are lined by a layer of fibrous connective tissue, the endosteum.

Blood-vessels. - The blood-vessels of bone pass into it from the periosteum. Near the centre of the shaft of a long bone a canal passes obliquely through the compact bone. This is known as the 
mutrient canal and its external opening as the mutrient foramen. This canal serves for the passage of the nutrient vessels-usually one artery and two veins-to and from the medullary cavity. In its passage through the compact bone the nutrient artery gives off branches to, and the veins receive branches from, the vessels of the Haversian canals.

Each of the flat and of the short bones has one or more nutrient canals for the transmission of the nutrient vessels.

- In addition to the nutrient canals the surface of the bone is everywhere pierced by the already mentioned (page I9I) Volkmann's canals, which serve for the transmission. of the smaller vessels. In compact bone these vessels give rise to a network of branches which run in the Haversian canals. In spongy bone the network lies in the marrow spaces. Branches from these vessels pass to the marrow cavity, and there break up into a capillary network, which anastomoses freely with the capillaries of the branches of the nutrient artery.

The capillaries of marrow empty into wide veins without valves, the walls of which consist of a single layer of endothelium. So thin are these walls that the veins of marrow were long described as passing over into open or incompletely walled spaces in which the blood came into direct contact with the marrow elements. These veins empty into larger veins, which are also valveless. Some of these converge to form the vein or veins which accompany the nutrient artery; others communicate with the veins of the Haversian canals.

Lymphatics with distinct walls are present in the outer layer of the periosteum. Cleft-like lymph capillaries lined with endothelium accompany the blood-vessels in Volkmann's and in the Haversian canals. The lacune and canaticuli constitute a complete system of lymph channels which communicate with the lymphatics of the periosteum, of Volkmann's and the Haversian canals, and of the bone-mårrow.

Nerves,-Both medullated and non-medullated nerves accompany the vessels from the periosteum through Volkmann's canals, into the Haversian canals and marrow cavities. Pacinian bodies (page 433) occur in the periosteum. Of nerve endings in osseous tissue and in marrow little definite is known.

\section{TECHNIC}

(I) Decalcified Bone--Fix a small piece of the shaft of one of the long bones -human or animal-in formalin-Müller's fluid (technic 5, p. 7) and decalcify 
in hydrochloric or nitric acid solution (page ro). After decalcifying, wash until all traces of acid are removed, in normal saline solution to which a little ammonia has been added. Dehydrate, and embed in celloidin. Transverse and longitudinal sections are made through the shaft, including periosteum and edge of marrow cavity. Stain with hæmatoxylin-eosin (technic I, p. 20) and mount in eosin-gylcerin.

(2) Hard Bone.-Transverse and longitudinal sections of undecalcified bone may be prepared as in technic I, p. 102.

(3) Spongy Bone.--This may be studied in the sections of decalcified bone, technic (I), where it is found near the marrow cavity. Or spongy bone from the head of one of the long bones or from the centre of a short bone may be prepared as in technic (2).

(4) Red Marrow.-Split longitudinally the femur of a child or young animal, and carefully remove the cylinder of marrow. Fix in formalin-Müller's fluid and harden in graded alcohols. Cut sections as thin as possible, stain with hæmatoxylin-eosin, and mount in balsam.

(5) Marrow: fresh specimen.--By means of forceps or a vice, squeeze out a drop of marrow from a young bone, place on the centre of a mounting slide, cover and examine it immediately.

(6) Place a similar drop of marrow on a cover-glass and cover with a second cover-glass. Press the covers gently together, slide apart and fix the specimen by immersion for five minutes in saturated aqueous solution of mercuric chlorid. Wash thoroughly, stain with hæmatoxylin-eosin, and mount in balsam.

\section{Development of Bone}

The forms of bones are first laid down either in cartilage or in embryonic connective tissue. The bones of the trunk, extremities, and parts of the bones of the base of the skull develop in a matrix of cartilage. This is known as intracartilaginous or endochondral ossification. The flat bones, those of the vault of the cranium and most of the bones of the face, are developed in a matrix of fibrillar connective tissuc-intramembranous ossification. A form of bone development, similar in character to intramembranous, occurs in connection with both intramembranous ossification and intracartilaginous ossification. This consists in the formation of bone just beneath the perichondrium-subperichondrial ossification-or, as with the development of bone perichondrium becomes periosteum-subperiosteal ossification.

There are thus three forms of bone development to be considered: (I) Intramembranous, (2) intracartiliginous, and (3) subperiosteal.

I. Intramembranous Development (Fig. II 7).-In intramembranous ossification the matrix in which the bone is developed is conncetive tissue. The process of bone formation begins at one or more points in this matrix. These are known as ossification centres. Here some of the bundles of white fibres become calcificd, i.e., become impregnated with lime salts. There is thus first established a centre or centres of calcification. Between the bundles of calcified fibres the connective tissue is rich in cells and vascular, and from its future rôle in bone formation is known as osteogenetic tissue (lïg. 117). Along the surfaces 
of the calcified fibres certain of the osteogenetic cells arrange themselves in a single layer (Figs. II 7 and II8). These are now known as osteoblasts or "bone formers." Under the influence of these osteoblasts a thin plate of bone is formed between themselves and the calcified fibres. This plate of bone at first contains no cells, but as the lamella of bone grows in thickness, the layer of osteoblasts becomes completely enclosed by bone. The osteoblasts are thus transformed into bone cells (Fig. II8), the spaces in which they lie becoming bone lacunce. The bone cell is thus seen to be derived from the embryonic connective-tissue cell, the osteoblast being an intermediate stage in its development. In this way

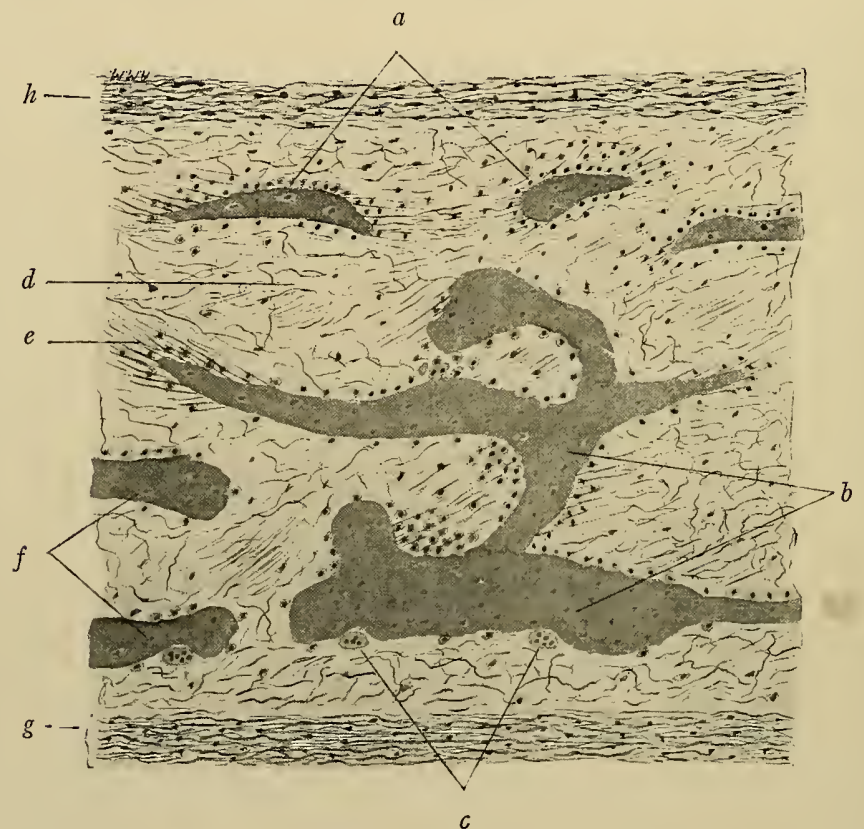

FIG. I 7 7.- Intramembranous Bone Development. Vertical section through parietal bone of human fœtus. XI6o. (Technic I, p. 203.) $a$, Osteoblasts; $b$, bone trabeculæ; $c$, osteoclasts lying in Howship's lacunæ; $d$, internal periosteum; $e$, bone cells; $f$, calcified fibres; $g$, osteogenetic tissue; $h$, external periosteum (pericranium.)

irregular anastomosing trabeculæ of bone are formed enclosing spaces (Fig. II7). The bony trabeculæ at first contain remains of calcified connectivetissue fibres, while the spaces, which are known as primary marrow spaces, contain blood-vessels, osteogenetic tissue, and developing marrow. The osteoblasts ultimately disappear and the spaces are then occupied by blood-vessels and marrow. The connective-tissue membrane has now been transformed into cancellous or spongy bone (Fig. III).

The bone thus formed is covered on its outer surface by a layer of connective tissue, a part of the membrane in which the bone was formed, but which from its position is now known as the periosteum, or, in the case of the cranial bones, as the peri- or epicranium (Fig. 117 ). 
In this form of bone development, occurring as it does in the bones of the skull, provision must be made for increase in the size of the cranial cavity to accommodate the growing brain. This is accomplished in the following manner: Along the surface of the bone, directed toward the brain, large multinuclear cells-osteoclasis or "bone breakers"-make their appearance (Fig. I I8). The origin of these cells is not clear. Similar cells are conspicuous elements of adult, marrow. They have been variously described as derived from leucocytes from osteoblasts, or directly from the connective-tissue cells. A recent theory holds that they are derived by a process of budding from the endothelial cells, which form the walls of the capillaries. These osteoclasts apparently possess the power of breaking down bone. They are found mainly along its inner surface, and can be seen lying in little depressions-Howship's lacunce (Fig. Ir8)-

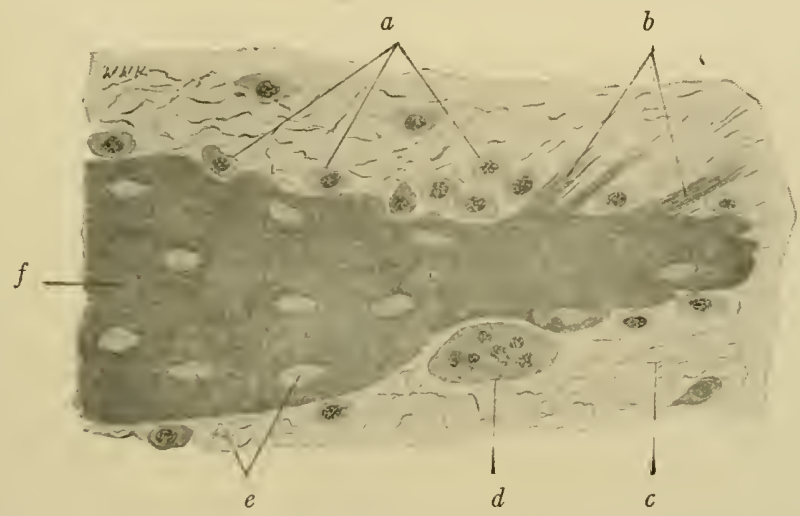

Frg. I I 8.- Intramembranous Bone Development. Vertical section through parietal bone of human foetus. $\times 350$. (Technic I, p. 203.) $a$, Osteoblasts; $b$, calcified fibres; $c$, osteogenetic tissue; $d$, osteoclast lying in Howship's lacuna; $c$, bone lacunæ; $f$, bone.

which they have hollowed out in the bonc. Between the outer surface of the bone and the pcricranium is a layer of osteogenetic tissue, the innermost cells of which are arranged as osleoblasts along the outermost osseous lamellix. Here they are constantly adding new bone beneath the pericranium. This new bone is laid down, not in flat, cvenly disposed layers, but in the form of anastomosing trabeculx enclosing marrow spaces.

It is thus seen that subperiostcal bone, like intramembranous, is at first of the spongy variety, and that with the development of the cranium the original intramembranous bone is entirely absorbed, together with much of the subperiosteal.

2. Intracartilaginous Development.-In this form of ossification an cmbryonal type of hyaline cartilage precedes the formation of bone, the cartilage corresponding more or less closely in shape to the future bone (Fig. I i 9 ). Covering the surface of the cartilage is a membrane of fibrillar connective tissue, the perichondrium or primary periostrum.

In most of the long bones the earliest changes take place within the cartilage at about the centre of the shaft (lig. rig). Here the cartilage cells increase in 
size and in number in such a way that several enlarged cartilage cells come to lie in a single enlarged cell space, and the cartilage assumes the character of hyaline cartilage. The cell groups next arrange themselves in rows or columns, which at first extend outward in a radial manner from a common centre, but later lie in the long axis of the bone. During these changes in the cells there is

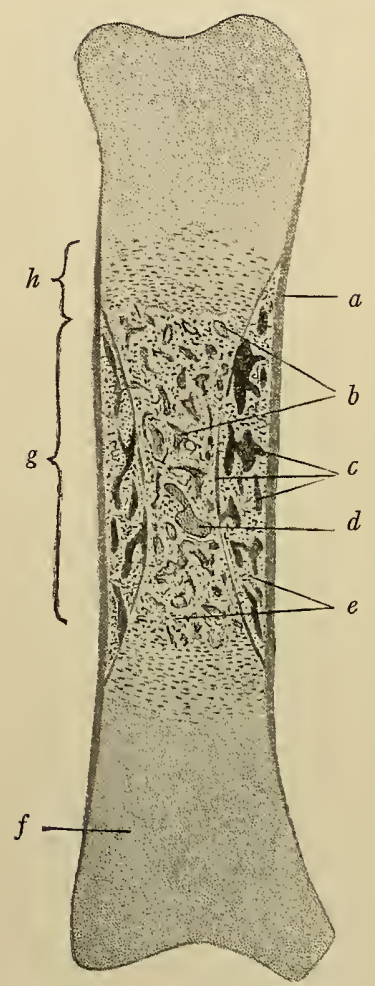

FIG. I I9.--Intracartilaginous Bone Development. Longitudinal section of one of the bones of embryo sheep's foot, showing ossification centre. $\times$ 2o. (Technic 2, p. 203.) $a$, Periosteum; $b$, bloodvessels; $c$, subperiosteal bone; $d$, intracartilaginous bone; $e$, osteogenetic tissue; $f$, cartilage; $g$, ossification centre; $h$, calcification zone.

they proceed to deposit a thin layer of bone between themselves and the cartilage (Fig. 122). As this increases in thickness some of the osteoblasts are enclosed within the newly formed bone to become bone cells, while the remains of the cartilage diminishes in amount and finally disappears. The calcification centre

${ }^{1}$ The term "periosteum" is admissible from the fact that the first bone actually formed is beneath the perichondrium, which thus becomes converted into periosteum an increase in the intercellular matrix and a deposit there of calcium salts. In this way the cartilage becomes calcified, the area involved being known as the calcification centre. Further growth of cartilage at the calcification centre now ceases and, as growth of cartilage at the ends of the bone continues, the central portion of the shaft appears constricted. The changes up to this point seem to be preparatory to actual bone formation.

Ossification proper begins by blood-vessels from the periosteum ${ }^{1}$ pushing their way into the calcified cartilage at the calcification centre, carrying with them some of the osteogenetic tissue from beneath the periosteum. These blood-vessels with their accompanying osteogenetic tissue are known as periosteal buds (Fig. I20). Osteoblasts now develop from the osteogenetic tissue and appear to dissolve the calcified cartilage from in front of the advancing vessels. In this way the septa between the cartilage cell spaces are broken down, the cartilage cells disappear, and a central cavity is formed-the primary marrow cavity. From the region of the primary marrow cavity blood-vessels and osteogenetic tissue push in both directions toward the ends of the cartilage which is to be replaced by bone. These break down the transverse septa between the cell spaces, while many of the longitudinal septa at first remain to form the walls of long anastomosing channels, the primary marrow spaces (Fig. I2I). As in intramembranous bone, these contain blood-vessels, embryonal marrow, and osteoblasts, all of which are derived from the osteogenetic tissue brought in from the periosteum by the periosteal buds. The osteoblasts next arrange themselves in a single layer along the remains of the calcified cartilage, where 
has now become the ossification centre, and its anastomosing osseous trabeculæ, with their enclosed spaces containing osteogenetic tissue and marrow, constitute primary spongy bone.

At either end of the ossification centre the cartilage presents a special structure. Nearest the centre the cell spaces are enlarged, flattened, arranged in rows and contain shrunken cells. Some of the walls break down and irregular spaces are formed. The ground substance is calcified. Passing away from the ossification centre, the cell spaces become less flattened, still arranged in rows,

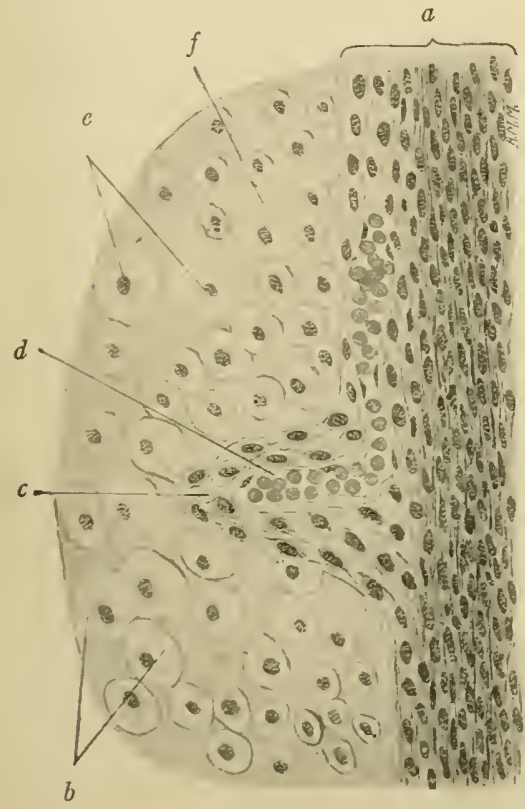

FIG. I 20.

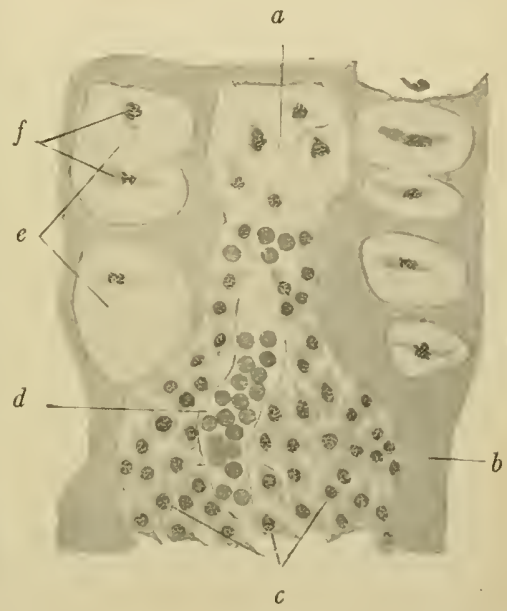

FIG. I2I.

FIG. I 20.-Intracartilaginous Bone Development. $\times 350$. Showing osteogenetic tissuc pushing its way into the cartilage (periosteal bud) at the ossification centre. $a$, Periosteum; $b$, cartilage cell spaces; $c$, periosteal bud; $d$, blood-vessel; $e$, cartilage cells; $f$, cartilage matrix.

FIG. 121.- Intracartilaginous Bone Development. Same specimen as Fig. 119 $\left(\times_{350}\right)$, showing osteogenetic tissue pushing its way into the cartilage and breaking it up into trabecula; also formation of primary marrow spaces and disintegration of cartilage cells. $a$, Disintegrating cartilage cells; $b$, cartilage trabecula; $c$, osteogenetic tissuc in primary marrow space; $d$, blood-vessels; $e$, cell spaces; $f$, cartilage cclls.

the contained cells larger, and there is a lesser degree of calcification. This area passes over into an arca of hyaline cartilage which blends without distinct demarcation with the ordinary embryonal cartilage of the rest of the shaft. The area of calcified cartilage at either end of the ossification centre is known as the calcification zone and everywhere precedes the formation of true bone (Fig. II0).

3. Subperiosteal or subperichondrial development (Fig. II9) has alrcady been largely described in connection with intramembranous ossification, and 
differs in no important respect from the latter. It always accompanies one of the other forms of ossification. Bone appears beneath the perichondrium somewhat earlier than within the underlying cartilage. Beneath the perichondrium is a layer of richly cellular osteogenetic tissue. The cells of this tissue nearest the cartilage become osteoblasts and arrange themselves in a single layer along its surface. Under their influence bone is laid down on the surface of the cartilage in the same manner as in intramembranous ossification.

Intracartilaginous and subperiosteal bone can be easily differentiated by the presence of cartilaginous remains in the former and their absence in the latter.

All bone is at first of the spongy variety. When this is to be converted into compact bone, there is first absorption of bone by osteoclasts, with increase in size of the marrow spaces and reduction of their walls to thin plates. These spaces are now known as Haversian spaces.

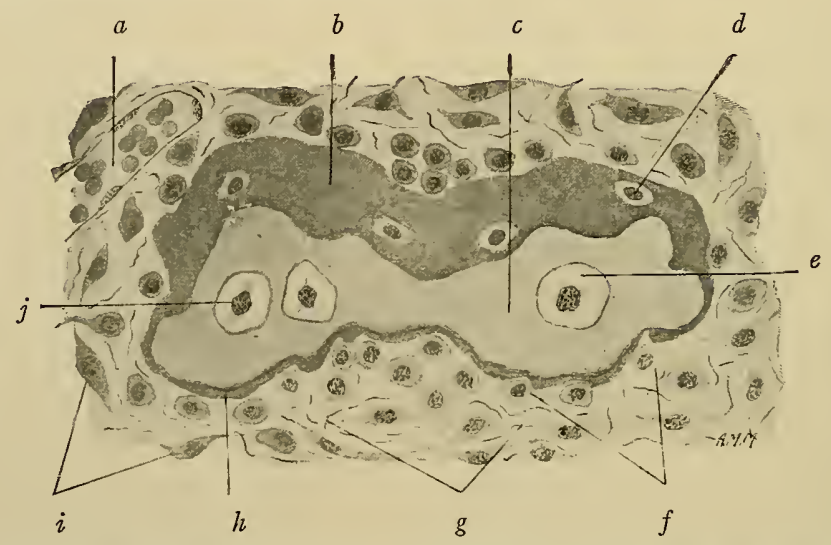

FIG. I22,- Intracartilaginous Bone Development. Same specimen as Fig. II9. $(\times 35 \mathrm{c})$, showing bone being deposited around one of the trabeculæ of cartilage. $a$, Blood-vessel; $b$, bone; $c$, cartilage remains; $d$, bone cell; $e$, cartilage cell space; $f$, osteoblasts; $g$, osteogenetic tissue; $h$, lamella of bone; $i$, connective-tissue cells; $j$, cartilage cell.

Within these new bone is deposited. This is done by osteoblasts which lay down layer within layer of bone until the Haversian space is reduced to a mere channel, the Haversian canal. In this way are formed the Haversian canals and the Haversian systems of lamella. Some of the interstitial lamellæ are the remains of the spongy bone which was not quite removed in the enlargement of the primary marrow spaces to form the Haversian spaces; other interstitial lamellæ appear to be early formed Haversian lamellæ which have been more or less replaced by Haversian lamellæ formed later.

While these varieties of ossification have been described, we would emphasize the essential unity of the process. The likeness between intramembranous and subperiosteal ossification has been already noted. The differences observed in intracartilaginous ossification are more apparent than real. In intracartilaginous ossification the bone is developed in cartilage but not from cartilage. As in intramembranous and in subperiosteal ossification, intracartilaginous bone is developed from osteogenetic tissue. This osteogenetic tissue is a differentiation of embryonal connective tissue, in this case carried into the cartilage from the 
periosteum in the periosteal buds. In intramembranous ossification the bone is developed within and directly from the embryonal connective tissue of which the membrane is composed. In intracartilaginous ossification there is the same embryonal connective-tissue membrane, but within this membrane the form of the bone is first laid down in embryonal cartilage. Surrounding the cartilage there remains the embryonal connective tissue of the membrane, now perichondrium. It is from tissue which grows into the cartilage from this membraneembryonal connective tissue-that the bone, although developed in cartilage, is formed.

Marrow develops from the mesenchymal tissue which enters the cartilage anlage in the periosteal buds, at the beginning of ossification.

\section{Growth of Bone}

The growth of intramembranous bone by the formation of successive layers beneath the periosteum has been already described (page Ig 8 ).

Intracartilaginous bones grow both in diameter and in length.

Growth in diameter is accomplished by the constant deposition of new layers of bone beneath the periosteum. During this process, absorption of bone from within by means of osteoclasts leads to the formation of the marrow cavity. The hard bone of the shaft of a long bone is entirely of subperiosteal origin, the intracartilaginous bone being completely absorbed.

Growth in length takes place in the following manner: Some time after the beginning of ossification in the shaft or diaphysis, independent ossification centres appear in the ends of the bone (epiphyses). So long as bone is growing, the epiphyses and diaphysis remain distinct. Between them lies a zone of growing cartilage, the epiphyseal or intermediate cartilage. Increase in length of the bone takes place by a constant extension of ossification into this cartilage from the ossification centres of the epiphyses and diaphysis. After the bone ceases to grow in length, the epiphyses and diaphysis become firmly united.

\section{TECHNIC}

(I) Developing Bone-Intramembranous.--Small pieces are removed from near the edge of the parietal bone of a new-born child or animal. These pieces should include the entire thickness of bone with the attached scalp and dura mater. Treat as in technic I, p. 196, except that the sections which are cut perpendicular to the surface of the bone should be stained with hematoxylin-picroacid-fuchsin (technic 3, p. 2 I) and mounted in balsam.

(2) Developing Bone-Intracartilaginous and Subperiostcal.-Remove the forearms and legs of a human or animal embryo by cutting througl the elbow, and knec-joints. (Fotal pigs from five to six inches long are very satisfactory.) Treat as in technic (I). Block so that the two long bones will lic in such a plane that both will be cut at the same time. Cut thin longitudinal sections through the ossification centres, stain with hematoxylin-picro-icid-fuchsin, and mount in balsam. Cut away the endls of one or two of the embedded bones, leaving only the ossification centres. Block so as to cut transverse sections through the ossification centre. Stain and mount as the preceding. 
In the picro-acid-fuchsin stained sections of developing bone the cartilage is stained blue; cells, including red blood cells, yellow; connective tissue from pale pink to red, according to density; bone a deep red.

\section{The Cartilages}

The costal cartilages are hyaline. They are covered by a closely adherent connective-tissue membrane, the perichondrium. Where cartilage joins bone there is a firm union between the two tissues and the perichondrium becomes continuous with the periosteum.

The articular cartilages are described below under articulations.

The other skeletal cartilages, such as those of the larynx, trachea, bronchi, and of the organs of special sense, are more conveniently considered with the organs in which they occur.

\section{Articulations}

Joints are immovable (synarthrosis) or movable (diarthrosis). In synarthrosis union may be cartilaginous (synchondrosis), or by means of fibrous connective tissue (syndesmosis).

SyNCHONDROSIS. - The cartilage is usually of the fibrous form except near the edge of the bone, where it is hyaline. The intervertebral discs consist of a ring of fibro-cartilage surrounding a central gelatinous substance, the nucleus pulposus, the latter representing the remains of the notochord.

Syndesmosis.- Union is by means of ligaments. These may consist wholly of fibrous tissue, the fibres and cells being arranged much as in tendon, or mainly of coarse elastic fibres separated by loose fibrous tissue. In such syndesmoses as the sutures of the cranial bones, the union is by means of short fibrous ligaments between the adjacent serrated edges.

DiARTHRosis.- - In diarthrosis must be considered (a) the articular cartilages, $(b)$ the glenoid ligaments and interarticular cartilages, (c) the joint capsule.

(a) Articular cartilages cover the ends of the bones. They are of the hyaline variety, ${ }^{1}$ being the remains of the original cartilaginous matrix in which the bones are formed. Next to the bone is a narrow strip of cartilage in which the matrix is calcified. This is

${ }^{1}$ In the acromio-clavicular, sterno-clavicular, costo-vertebral, and maxillary articulations the cartilage is of the fibrous form. The same is true of the cartilage covering the head of the ulna, while the surface of the radius, which enters into the wrist-joint, is covered not by cartilage, but by dense fibrous tissue. 
separated from the remaining uncalcified portion of the cartilage by a narrow so-called "striated" zone. The most superficial of the cartilage cells are arranged in rows parallel to the surface; in the midregion the grouping of cells is largely in twos and fours as in ordinary hyaline cartilage (page 98 ); while in the deepest zone of the uncalcified cartilage the cells are arranged in rows perpendicular to the surface.

(b) The glenoid ligaments and interarticular cartilages conform more to the structure of dense fibrous tissue than to that of cartilage.

(c) The joint capsule consists of two layers, an outer layer of dense fibrous tissue intimately blended with the ligamentous structures of the joint and known as the stratum fibrosum, and an inner layer, the stratum synoviale or synovial membrane, which forms the lining of the joint cavity. The outer part of the stratum synoviale consists of areolar tissue with its loosely arranged white and elastic fibres interlacing in all directions and scattered connective-tissue cells and fat cells. Nearer the free surface of the membrane the fibres run parallel to the surface and the cellular elements are more abundant. The cells are scattered among the fibres and are stellate branching cells like those usually found in fibrous connective tissue. On the free surface, however, the cells lie close together, in places forming a single surface layer, in other places being disposed in three or four layers. Formerly described as endothelium, they are now generally considered connective-tissue cells or "mesenchymal epithelium."

From the free surfaces of synovial membranes, processes (synovial villi-Haversian fringes) project into the joint cavity. Some of these are non-vascular and consist mainly of stellate cells similar to those of the synovial membrane. Others have a distinct core of fibrous tissue containing blood-vessels and covered with stellate connective-tissue cells. From the primary villi small secondary nonvascular villi are frequently given off.

\section{TECHNIC}

(1) Joint Capsule and Articular Cartilage.-Remove one of the small joints - human or animal - cutting the bones through about one-half inch back from the joint. Treat as in technic I, p. 196, making longitudinal sections through the entirc joint.

(2) Synovial Villi.-Remove a piece of the capsular ligament from near the borrler of the patella and cut out a bit of the velvety tissue which lines its inner surface. Examine fresh in a drop of normal salt solution. Fix a second piece 
of the ligament in formalin-Müller's fluid (technic 5, p. 7), make sections perpendicular to the surface, stain with hæmatoxylin-eosin (technic r, p. 20), and mount in balsam.

\section{General References for Further Study}

Braunca: Précis d'Histologie.

Kölliker: Handbuch der Gewebelehre, vol. i.

Stöhr: Text-book of Histology.

Schäfer: Histology and Microscopical Anatomy, in Quain's Elements of Anatomy. 


\section{CHAPTER IV.}

\section{THE MUSCULAR SYSTEM ${ }^{1}$}

THE voluntary muscular system consists of a number of organsthe muscles - and of certain accessory structures - the tendons, tendon sheaths, and burse.

A VoluntaRy MUSCLE consists of striated muscle fibres arranged in bundles or fascicles and supported by connective tissue.

The entire muscle is enclosed by a rather firm connective-tissue sheath or capsule - the epimysium (Fig. I23). This sends trabeculæ

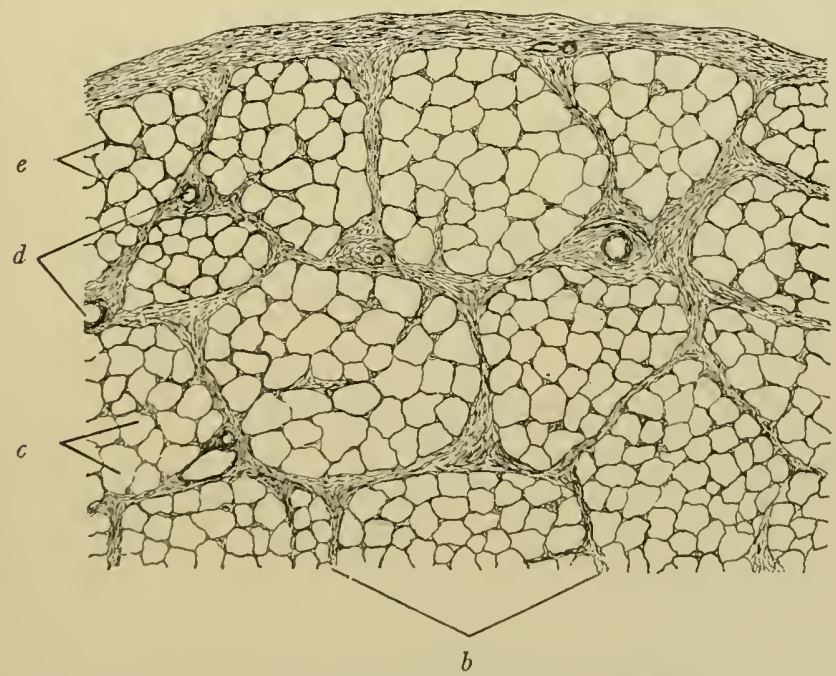

Fig. I 23. - From a Transverse Section of a Small Human Muscle, showing relations of muscle fibres to connective tissue. $a$, Epimysium; $b$, perimysium; $c$, muscle fibres; $d$, arteries; $e$, endomysium.

of more loosely arranged connective tissue into the substance of the muscle. These divide the muscle fibres into bundles or fascicles. Around each fascicle the connective tissue forms a more or less definite

'Iefinite arrangements of smooth muscle, such as are founcl in the stomach and intestines, also the muscle of the heart, are properly a part of the muscular system. They are, however, best considered under tissues and in connection with the organs in which they occur. 
envelope, the perifascicular sheath or perimysium. From the latter delicate strands of connective tissue pass into the fascicles between the individual muscle fibres. This constitutes the intrafascicular connective tissue or endomysium, which everywhere completely separates the fibres from one another so that the sarcolemma of one fibre never comes in contact with the sarcolemma of any other fibre. It should be noted that these terms indicate merely location; epi-,

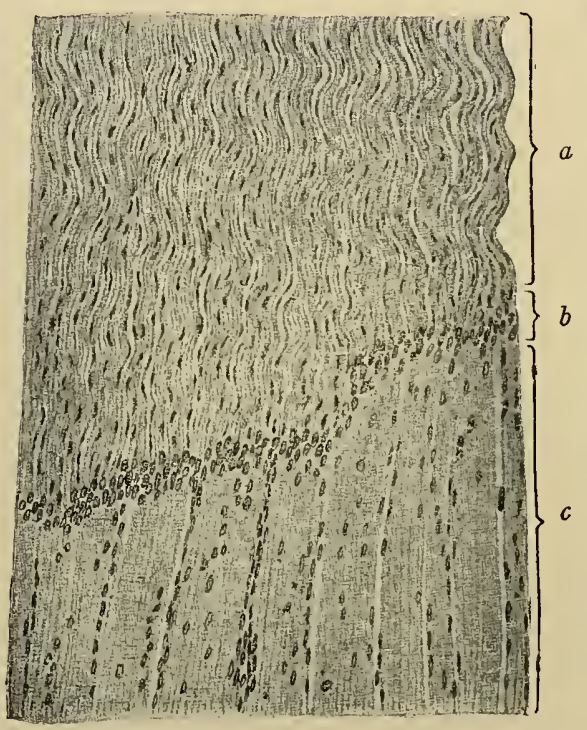

FIg. I 24.-From a Longitudinal Section through Junction of Muscle and Tendon. $\times$ I5०. (Böhm and Davidoff.) $a$, Tendon; $b$, line of union showing increase in number of muscle nuclei; $c$, muscle.

peri-, and endo-mysium all being connective tissue grading from coarse to fine, as it passes from without inward. The structure of the muscle as an organ is thus seen to conform to the structure of other organs, in that it is surrounded by a connectivetissue capsule, which sends septa into the organ, dividing it into a number of compartments and serving for the support of the essential tissue of the organ, the muscle fibres or parenchyma.

The structure of tendon has been described (see page 9I).

Tendon sheaths and bursa are similar in structure, consisting of mixed white and elastic fibres. Their free surfaces are usually lined by flattened connective tissue-cells.

At the junction of muscle and tendon, the muscle fibre with its sarcolemma ends in a rounded or blunt extremity (Fig. 63, p. II9). Here the fibrils of the tendon fibres are in part cemented to the sarcolemma, and in part are continuous with the fibres of the endo- and peri-mysium. This has been for a long time the accepted idea of the transition from muscle to tendon, and is perhaps more nearly correct where the insertion is oblique. It has been more recently proved, however, that for some muscles at least, and especially where muscle and tendon join end to end a much more intimate union of elements occurs. This is shown in Figs. I25 and I 26 from Stöhr. 
In Fig. I 25 the individual muscle fibrillæ are seen passing over into tendon fibrillæ with no line of demarcation. In Fig. I 26 a similar continuity is shown except that groups of muscle fibrillæ are seen to be continuous with groups of tendon fibrillæ. Both sarcoplasm, which appears somewhat augmented at this point, and sarcolemmæ, extend between the tendon bundles beyond the line of cross muscle striations. Along the line of union of muscle and tendon the muscle nuclei are more numerous than elsewhere (Fig. I24, b), and it has been suggested that there is here a zone of indifferent or formative

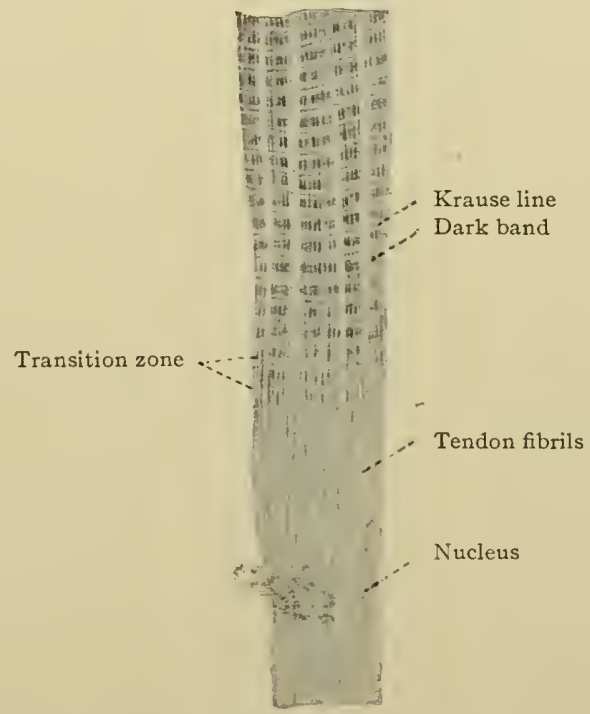

FIG. 125.-Longitudinal Section through Muscle-tendon Junction: Human Intercostal. $\times 750$. Only part of a fibre is shown, without sarcolemma. (Stöhr.)

tissue which is capable of developing on the one hand into muscle, on the other into the connective tissue of tendon.

Growti of Muscle takes place mainly at the ends of the fibres where the nuclei are most numerous. In addition to the growth incident to increase in size of the individual or of the particular muscle, there is a constant wearing out of muscle fibres and their replacement by new fibres. 'This is accomplished as follows: The muscle fibre first breaks up into a number of segments (sarcostyles), some of which contain nuclei while others are non-nucleated. The sarcostyles next divide into smaller fragments, and finally completely disintegrate. This is followed by a process of absorption and complete disappearance of the fibre. From the free sarcoplasm new 
muscle fibres are formed. In the early stages of their development these are known as myoblasts. The latter develop into muscle fibres in the same manner as described under the histogenesis of muscle (p. I22).

Blood-vessels. - The larger arteries of muscle run in the perimysium, their general direction being parallel to the muscle bundles. From these, small branches are given off at right angles. These in

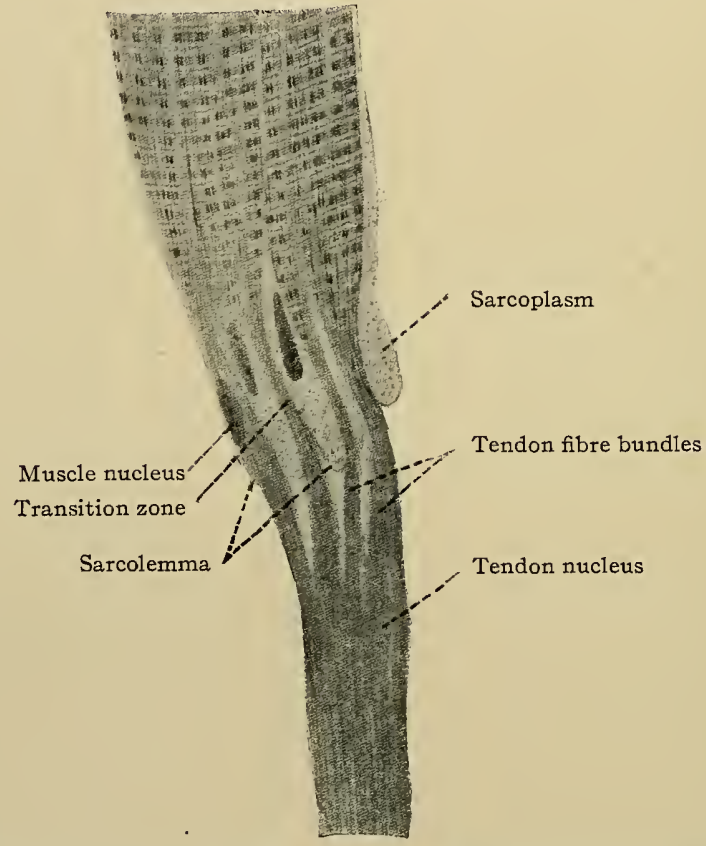

FIG. I 26.-Junction of Muscle and Tendon showing Continuity of Fibrils and Extension of Sarcolemma beyond the Limits of Cross Striations. Rectus abdominis of frog. $\times 75$ o. (Stöhr.)

turn give rise to an anastomosing capillary network with elongated meshes, which surrounds the individual muscle fibres on all sides. From these capillaries, veins arise which follow the arteries. Even the smallest branches of these veins are supplied with valves.

In tendons blood-vessels are few. They run mainly in the connective tissue which surrounds the fibre bundles. Tendon sheaths and bursæ, on the other hand, are well supplied with blood-vessels.

The lymphatics of muscle are not numerous. They accompany the blood-vessels. In tendon definite lymph vessels are found only on the surface. 
Nerves.-The terminations of nerves in muscle and tendon are described under nerve endings (page 434).

\section{TECHNIC}

(I) A Mruscle.-Select a small muscle, human or animal, and, attaching a weight to the lower end to keep it stretched, fix in formalin-Müller's fluid (technic 5, p. 7), and harden in alcohol. Stain transverse sections with hæmatoxylinpicro-acid-fuchsin (technic 3, p. 2I) and mount in balsam.

(2) Junction of Muscle and Tendon.-Any muscle-tendon junction may be selected. Fix in formalin-Müller's fluid, keeping stretched by means of a weight attached to the lower end. Cut longitudinal sections through the muscletendon junction, stain with hæmatoxylin-picro-acid-fuchsin, and mount in balsam. The gastrocnemius of a frog is convenient on account of its small size, and because by bending the knee over and tying there, the muscle can be easily put on the stretch and kept in that condition during fixation. Place the entire preparation in the fixative removing the muscle-tendon from the bone after fixation. 


\section{CHAPTER V.}

\section{GLANDS}

\section{General Structure and Classification}

Attention was called in describing the functional activities of cells (page $5 \mathrm{I}$ ) to the fact that certain cells possess the power of not only carrying on the nutritive functions necessary to maintain their own existence, but also of elaborating certain products either necessary for the general body functions (secretions) or for the body to eliminate as waste (excretions). Such cells are known as gland cells or glandular epithelium, and an aggregation of these cells to form a definite structure for the purpose of carrying on secretion or excretion is known as a gland.

A gland may consist of a single cell, as, e.g., the mucous or goblet cell on the free surface of a mucous membrane or the unicellular glands of invertebrates. Such a cell undergoes certain changes which result in the production within itself of a substance which is to be used outside the cell. The appearance which this cell presents depends upon the stage of secretion. It is thus possible to differentiate between a "resting" and an "active" cell or between an "empty" and a "loaded" cell. The mucous secreting cell of the intestine is one of the simple columnar cells which constitute the epithelium of the mucous membrane. It is distinguishable as a mucous or goblet cell only after secretion begins. The resting cell is granular and takes a rather dark cytoplasmic stain. As the cell becomes active, part of the cytoplasm is transformed into, or is replaced by, a clear substance which does not stain like cytoplasm, but reacts to hæmatoxylin. The mucus collects first in the free end of the cell, and gradually increases in amount until the entire cell is filled, with the exception of a small area at the base, where a little unchanged protoplasm surrounds a flattened nucleus. The cell at this stage is much larger than in the resting state, and finally ruptures on the free surface and pours out its secretion. Opinions differ as to the further behavior of this cell. According to some, its life history is now ended, and its place is taken by other cells which pass through the same process. Others 
believe that in most cases the cell is reconstructed from the nucleus and unchanged cytoplasm, and again passes through the process of secretion. In stratified epithelium secretion may begin while the cell is still deeply situated, but is completed only as the cell reaches the surface, where its mucus is to be discharged.

In describing the vital properties of cells (p. 50) attention was called to the fact that all cells take up from the surrounding blood and lymph substances required for their own nutrition, and give off waste products (excretions). In such sense all cells secrete and excrete. What distinguishes the gland cell is that in addition to carrying on its own metabolism (p. 51) it manufactures a specific substance not for its own use but to be extruded from the cell, and used elsewhere in the body (secretion, e.g., gastric juice), or discarded (excretion, e.g., urine). Such a cell takes up from blood or lymph the substances required, more or less completely assimilates them, and finally transforms them into its specific secretion.

Certain changes, other than those described as seen in the mucous secreting cell, may occur in the protoplasm of actively secreting gland cells. In many cells there appears at the onset of secretion a modification of the cytoplasm which has been designated ergastoplasm (Fig. I 27). In some cells the ergastoplasm takes the form of slender threads

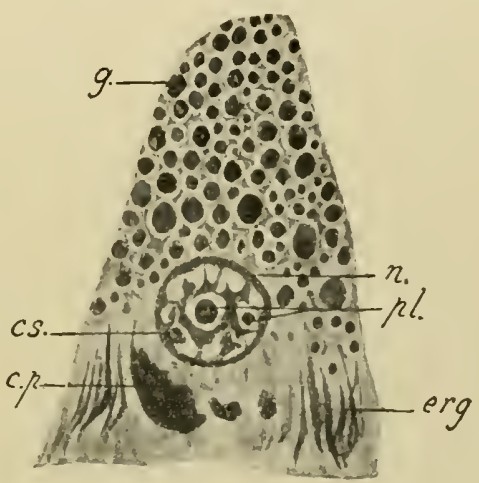

FIG. I 27--Gland Cell from Pancreas of Salamander; $n$, Nucleus; $c s$, caryosomes; $p l$, plasmosomes; $c p$, paranucleus; erg, ergastoplasm filaments; $g$, secretory granules. $\times 500$. (Prenant.) near the base of the cell, basal filaments, in others of minute rods. Other form shave been described as mitochondria, cytosomes, pseudochromosomes, etc.

Many gland cells have intracellular secretory canals (see p. 46).

In some gland cells a body known as the paramucleus appears at the beginning of secretion. It is a rather large, usually irregular mass, differing somewhat in staining qualities from both nucleus and ergastoplasm. Its relation to either of these structures, to the cytoplasm, or to the secretion is not known.

The function of the nucleus in secretion is apparently of grcat importance. Non-nuclcated portions of cells are probably not able to claborate any true secretion. When (sce below) the entire cell enters the secretion as in the mammary gland, the entire nucleus of course becomes a part of the secretion. In any event the onset of secretion is apt to be evidenced in the nucleus by enlargement and irregularity, in some cases by the giving off of nuclear material to the cytoplasm, in others by amitotic division. According to some observers both ergastoplasm and paranucleus are of nuclear origin.

In connection with the mucous cell (p. 212 ) it was noted that according to some authorities the cell dies in secreting, while others believe that the cell 
reconstructs itself and can again secrete. In certain glands, e.g., the mammary and sebaceous, the cast off cells themselves form the secretion. More commonly the cell merely gives off its secretion, the remainder of the cell recovering and again going through the same process.

Most glands are composed of more than one cell, usually of a large number of cells, and these cells, instead of lying directly upon the surface, line more or less extensive invaginations into which they pour their secretions.

In the simplest form of glandular invagination all the cells lining the lumen are secreting cells. In more highly developed glands only the deeper cells secrete, the remainder of the gland serving merely to carry the secretion to the surface. This latter part is then known as the excretory duct, in contradistinction to the deeper secreting portion. In both the duct portion and secreting portion of a gland the epithelium usually rests upon a more or less definite basement membrane or membrana propria (page 70). Beneath the basement membrane, separating and supporting the glandular elements, is the connective tissue of the gland. This varies greatly in structure and quantity in different glands.

When the secreting portion of the gland is a tubule, the lumen of which is of fairly uniform diameter, the gland is known as a tubular gland. When the lumen of the secreting portion is dilated in the form of a sac or alveolus, the gland is known as a saccular or alveolar gland. Intermediate forms have been described as tubulo-alveolar glands.

A gland may consist of a single tubule or saccule, or of a single system of ducts leading to terminal tubules or saccules-simple gland. A gland may consist of a number of more or less elaborate duct systems with their terminal tubules or saccules-compound gland. A few glands, e.g., the thyreoid and thymus, have no ducts, and are known as ductless glands.

All compound glands are surrounded by conneciive tissue which forms a more or less definite capsule. From the capsule connectivetissue septa or trabecula extend into the gland. The broadest septa usually divide the gland into a number of macroscopic compartments or lobes. Smaller septa from the capsule and from the interlobar septa divide the lobes into smaller compartments usually microscopic in size-the lobules. A lobule is not only a definite portion of the gland separated from the rest of the gland by connective tissue, but represents a definite grouping of tubules or alveoli with reference to 
one or more terminal ducts. The glandular (epithelial) tissue is known as the parenchyma of the gland, in contradistinction to the connective or interstitial tissue.

The relations of the glandular tissue proper to the connective tissue are best understood by reference to development. All glands, simple and compound, originate as simple evaginations from a surface lined with epithelium. The epithelial evagination grows down into the underlying connective tissue. In a compound gland this invagination tubule becomes the main excretory duct. As the tubule grows, it divides and subdivides to form the larger and smaller ducts and finally the secreting tubules or alveoli. During the development of the gland tubules, the connective tissue is also developing, but is being largely replaced by the more rapidly growing tubules. The gland tubules do not develop irregularly, but in definite groups, each group being dependent upon the tubule (duct) from which it originates. Thus the invagination tubule (main excretory duct) gives rise to a few large branches (lobar ducts), each one of which gives off the subdivisions which constitute a lobe. From each lobar duct there arise within the lobe a large number of smaller branches (lobular ducts) each one of which gives rise to the subdivisions included in a lobule. As the lobe groups and lobule groups of tubules develop, the largest strands of connective tissue are left between adjacent lobes (interlobar connective tissue), smaller strands between lobules (interlobular connective tissue), and the finest connective tissue between the tubules or alveoli within the lobule (intralobular connective tissue).

Glands may thus be classified as follows:

A. Duct glands or glands of external secretion.

I. Tubular glands.

(a) Simple tubular

$$
\left\{\begin{array}{l}
\text { straight. } \\
\text { coiled. } \\
\text { branched. }
\end{array}\right.
$$

(b) Compound tubular.

2. Alveolar or saccular glands.

(a) Simple alveolar.

(b) Compound alveolar, saccular or racemose.

B. Ductless glands or glands of internal secretion.

\section{Duct Glands}

I. Tubular Glands.-(a) Simple tubular Glands are simple tubules which open on the surface, their lining epithelium being continuous with the surface epithelium. All the cells may be secreting cells or only the more deeply situated. In the latter case the upper portion of the tubule serves merely as a duct. In the more highly developed of the simple tubular glands we distinguish a mouth, open- 
ing upon the surface, a neck, usually somewhat constricted, and a fundus, or deep secreting portion of the gland.

Simple tubular glands are divided according to the behavior of the fundus, into (I) straight, (2) coiled, and (3) branched.

(I) A straight tubular gland is one in which the entire tubule runs a straight unbranched course, e.g., the glands of the large intestine (Fig. I 28, I).

(2) A coiled tubular gland is one in which the deeper portion of the tubule is coiled or convoluted, e.g., the sudoriferous glands of the skin (Fig. I 28, 2).
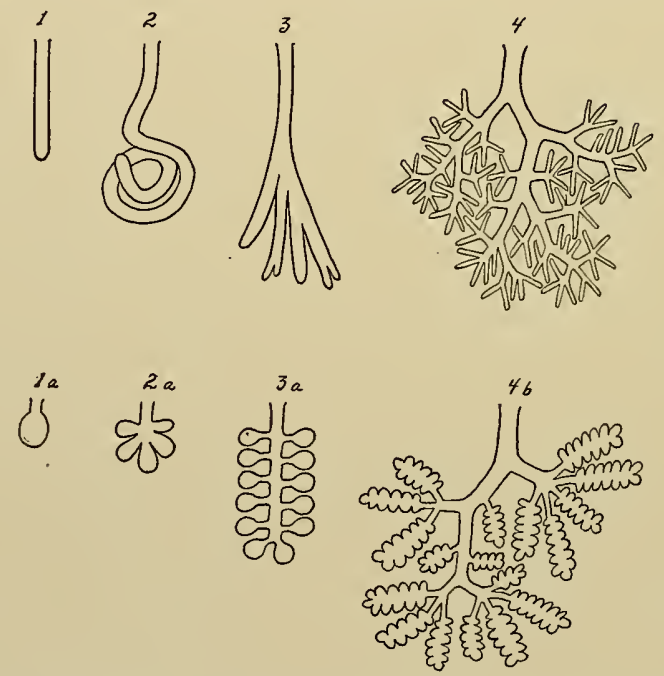

FIG. I 28.-Diagram Illustrating Different Forms of Glands. Upper row, tubular glands; 1,2 , and 3 , simple tubular glands; 4, compound tubular gland. Lower row, alveolar glands; г $a, 2 a$, and $3 a$, simple alveolar glands; $4 a$, compound alveolar gland. For description of $1 a, 2 a$, and $3 a$, see simple alveolar glands in text.

(3) A forked or branched tubular gland is a simple tubular gland in which the deeper portion of the tubule branches, the several branches being lined with secreting cells and opening into a superficial portion, which serves as a duct. Examples of slightly forked glands are seen in the cardiac end of the stomach, and in the uterus. Other tubular glands show much more extensive branching, the main duct giving rise to a number of secondary ducts, from which are given off the terminal tubules. The mucous glands of the mouth, osophagus, trachea, and bronchi are examples of these more elaborate simple tubular glands (Fig. I 28, 3). 
(b) Compound tubular glands consist of a number, often of a large number, of distinct ducl syslems. These open into a common or main excrelory duct. The smaller ducts end in terminal tubules. Many of the largest glands of the body are of this type, e.g., the salivary glands, liver, kidney, and testis (Fig. I 28,4 ).

In certain compound tubular glands, as, e.g., the liver, extensive anastomoses of the terminal tubules occur. These are sometimes called reticular glands.

2. Alveolar Glands.- (a) Simple Alveolar Glands.--The simplest form of alveolar gland consists of a single sac connected with the surface by a constricted portion, the neck, the whole being shaped like a flask (Fig. I28, I $a$ ). Such glands are found in the skin of certain amphibians; they do not occur in man. Simple alveolar glands, in which there are several saccules (Fig. I 28, 2 a), are represented by the smaller sebaceous glands. Simple branched alveolar glands, in which a common duct gives rise to a number of saccules (Fig. $128,3 a$ ), are seen in the larger sebaceous glands, and in the Meibomian glands.

(b) Compound Alveolar Glands.-These resemble the compound tubular glands in general structure, consisting of a large number of duct systems, all emptying into a common excretory duct. The main duct of each system repeatedly branches, and the small terminal ducts, instead of ending in tubules of uniform lumen, as in a tubular gland, end in sac-like dilatations, the alveoli or acini (Fig. I 28, 4 a). The best example of a compound alveolar gland is the mammary gland, although the lung is constructed on the principle of a compound alveolar gland.

\section{Ductless Glands}

Certain structures remain to be considered which are properly classified as glands, but in which during development the excretory duct has disappeared. Such glands are known as ductless glands.

The ovary is a ductless gland, the specific secretion of which, the ovum, is under normal conditions taken up by the oviduct and carried to the uterus. This is known as a dehiscent gland.

Other ductless glands, such as the thyreoid, hypophysis and adrenal, are known as glands of internal secretion, their specific secretions passing directly into the blood or lymph systems.

A few glands, e.g., the liver and pancreas, have both an internal secretion, and an external secretion. 


\section{General Structure of Mucous Membranes}

The alimentary tract, the respiratory tubules, parts of the genitourinary system, and some of the organs of special sense are lined by mucous membranes. While differing as to details in different organs, the general structure of all mucous membranes is similar. The essential parts are (I) surface epithelium, (2) basement membrane, and (3) stroma or tunica propria. The epithelium may be simple columnar, as in the gastro-intestinal canal; ciliated, as in the bronchi; stratified squamous, as in the œsophagus, etc. The epithelium rests upon a basement membrane or membrana propria which, like the same membrane in glands, is described by some as a product of the epithelium, by others as a modification of the underlying connective tissue. Beneath the basement membrane is a connective-tissue stroma, or tunica propria. This usually consists of loosely arranged fibrous tissue with some elastic fibres. It may contain smooth muscle cells and lymphoid tissue.

In addition to the three layers above described there is frequently a fourth layer between the stroma and the underlying connective tissue. This consists of one or more layers of smooth muscle, and is known as the muscularis mucose.

A mucous membrane usually rests upon a layer of connective tissue rich in blood-vessels, lymphatics, and nerves-the submucosa. 


\section{CHAPTER VI}

\section{THE DIGESTIVE SYSTEM}

THE digestive system consists of the alimentary tract and certain associated structures such as glands, teeth, etc.

The alimentary tract -is a tube extending from lips to anus. Different parts of the tube present modifications both as to calibre and as to structure of wall.

The embryological subdivision of the canal into headgut, foregut, midgut, and endgut admits of further subdivision upon an anatomical basis as follows:

I. Headgut: (a) Mouth, including the tongue and teeth.

(b) Pharynx.

II. Foregut: (a) Esophagus.

(b) Stomach.

III. Midgut: Small intestine.

IV. Endgut: (a) Large intestine.

(b) Rectum.

The entire canal is lined by mucous membrane, the modifications of which constitute the most essential difference in structure of its several subdivisions.

Bencath the mucosa is usually more or less connective tissue, which in a large portion of the canal forms a definite submucosa.

Muscular tissue is present beneath the submucosa throughout the greater part of the canal. In most regions it forms a definite, continuous, muscular tunic.

The upper and lower ends of the tube-mouth, pharynx, osophagus, and rectum-are quite firmly attached by fibrous tisue to the surrounding structures. The remainder of the tube is less firmly attached, lying coiled in the abdominal cavity, its surface covered, except along its attached border, by a serous membrane, the visceral peritoneum. 


\section{THE HEADGUT}

\section{The Mouth}

The Mucous Membrane of the Mouth.-This consists of stratified squamous epithelium. lying upon a connective-tissue stroma or tunica propria. The latter is thrown up into papille, which do not, however, appear upon the free surface of the epithelium. The submucosa is a firm connective-tissue layer with few elastic fibres. The thickness of the epithelium, the character of the stroma, and the height of the papillæ vary in different parts of the mouth. There is no muscularis mucosæ.

At the junction of the skin and mucous membrane (red margin of the lips) the epithelial layer is much thickened, the stroma is thinned, and the papillæ are very high. At this point the stratum corneum of the skin passes over into the softer nucleated epithelium of the mouth, while the stratum lucidum and stratum granulosum of the skin terminate (see skin, page 383 ).

The mucous membrane of the gums has prominent, long, slender papillæ, the summits of which are covered by a very thin layer of epithelium. This nearness of the vascular stroma to the surface accounts for the ease with which the gums bleed. That portion of the gums which extends over the teeth is devoid of papillæ. The submucosa of the gums is firmly attached to the underlying periosteum.

The mucous membrane lining the cheeks has low, small papillæ, and the submucosa is closely adherent to the muscular fibres of the buccinator.

Covering the hard palate, the mucous membrane is thin and the short papillæ are obliquely placed, their apices being directed anteriorly. The submucosa is firmly attached to the periosteum.

Over the soft palate the papillæ of the mucous membrane are low or even absent. They are somewhat higher on the uvula, the posterior surface of which shows a transitional condition of its epithelium, areas of stratified squamous alternating with areas of stratified columnar ciliated epithelium. Throughout the mucous membrane of the soft palate, uvula, and fauces, the stroma and submucosa contain diffuse lymphatic tissue. In some places the lymphoid cells are so closely placed as to form distinct nodules.

Glands of THE ORAL Mucosa. ${ }^{1}$-Distributed throughout the

${ }^{1}$ For description of the larger salivary glands see page 276 . 
oral mucosa are small branched tubular glands. Only in those parts of the mucous membrane which are closely attached to underlying bone, as on the gums and hard palate, are mucous glands few or entirely absent. While the deeper portions of the glands are in the submucosa, some of the tubules usually lie in the stroma of the mucous membrane.

The ducts open upon the surface and are lined with a continuation of the surface stratified squamous epithelium as far as the first bifurcation. Here the epithelium becomes stratified columnar, and this, as the smaller branches are approached, passes over into the simple columnar type. Not infrequently ducts of small secondary glands empty into the main duct during its passage through the mucosa.

According to the character of their secretions, the oral glands are divided into:

(a) Mucous glands, which secrete a mucin-containing fluid (mucus);

(b) Serous glands, which secrete a serous (albuminous) fluid;

(c) Mixed glands, the secretion of which is partly mucous and partly serous.

Morphologically, also, a similar distinction can be made in regard to the glandular epithelium which lines the terminal tubules, the tubules of mucous glands being lined with "mucous" cells, those of serous glands with "serous cells," while of the mixed glands the cells of some tubules are mucous, of others serous. In certain tubules both mucous and serous cells occur. The appearance which these cells present depends largely upon their secretory condition at the time of death.

Serous cells when resting have a slightly granular protoplasm, which in the fresh condition is highly refractive, giving the cells a transparent appearance. With the beginning of secretion the granules increase in number and the cells become darker. Stained with hamatoxylin-eosin, serous tubules have a purplish color. The nuclei are spherical or oval, and are situated between the centre and base of the cell (Fig. I8I, p. 279).

Mucous cells are in the quiescent state rather small cuboidal or pyramidal cells, with cloudy cytoplasm and nuclei situated at the base of the cell. When active the mucous cells are much larger, with clear cytoplasm and with nuclei flattened against the basement membranc. 'The protoplasm of the fresh unstained mucous cell is less highly refractive than that of the serous cell. It consequently 
appears darker and less transparent. Mucous tubules are larger and more irregular in shape than serous tubules, and when stained with hæmatoxylin-eosin either remain almost wholly unstained or take a pale blue hæmatoxylin stain (Fig. I8I, p. 279). Many mucous tubules have in addition to the mucous cells a peculiar, of ten crescentic-shaped group of cells on one side of the tubule, between the mucous cells and the basement membrane. These cells are granular and stain very much like serous cells with hæmatoxylineosin, thus resembling the latter in appearance. On account of the shape of the groups, they are known as the crescents of Gianuzzi or demilunes of Heidenhain (Fig. I8I, p. 279). The cells of the crescents are connected with the lumen by means of secretory canals, which pass between the mucous cells and end in branches within the protoplasm of the crescent cells. It is quite possible that some of the crescents are not serous cells but mucous cells in the non-active condition which have been pushed away from the lumen by the more active cells. Such cell groups are not connected with the lumen of the gland by intercellular secretory canals.

Peculiar irregular branching cells have been described, extending from the basement membrane in between the mucous cells. They are known as "basket" cells and are supposed to be supportive in character.

The cells of both mucous and serous tubules rest upon a membrana propria, outside of which, separating the tubules, is a cellular connective-tissue stroma.

Of the small glands of the mouth, a group near the root of the tongue are of the mucous variety, some "lingual" glands in the region of the circumvallate papillæ are serous, while the remainder are of the mixed type.

Blood-vessels. - The larger vessels run mainly in the submucosa. The arteries of the submucosa give off one group of branches to the tunica propria, where they break up into a dense subepithelial capillary network, sending capillary loops into the papillæ. A second group of arterial branches pass to the submucosa, where they give rise to capillary networks among the tubules of the mucous glands. From the capillaries veins arise which accompany the arteries.

Lymphatics.- The larger lymph vessels lie in the submucosa. These send smaller branches into the tunica propria, where they open into small lymph capillaries and spaces.

Nerves.-Medullated nerve fibres form plexuses in the submucosa 
and deeper parts of the mucosa. From these plexuses, branches are given off which lose their medullary sheaths and form a second plexus of non-medullated fibres just beneath the epithelium. From this subepithelial plexus, branches pass in between the epithelial cells to terminate in end brushes or in tactile corpuscles. The nerves belong to the cerebro-spinal system, and are dendrites of sensory ganglion cells. Axones of sympathetic neurones are also present in the oral mucosa, destined mainly for the muscle-tissue of the blood-vessels.

\section{TECHNIC}

(I) The superficial cells of the oral mucous membrane may be prepared for examination as in technic 1 , page 63 .

(2) For the study of the mucous membrane of different parts of the mouth, fix small pieces in formalin-Müller's fluid (technic 5 , p. 7), cut sections perpendicular to the surface, stain with hæmatoxylin-eosin (technic I, p. 20), and mount in balsam.

(3) Small mucous and serous glands of the mouth may be studied in the preceding sections.

\section{The Tongue}

The tongue is composed mainly of striated muscle fibres, supported by connective tissue and covered by a mucous membrane.

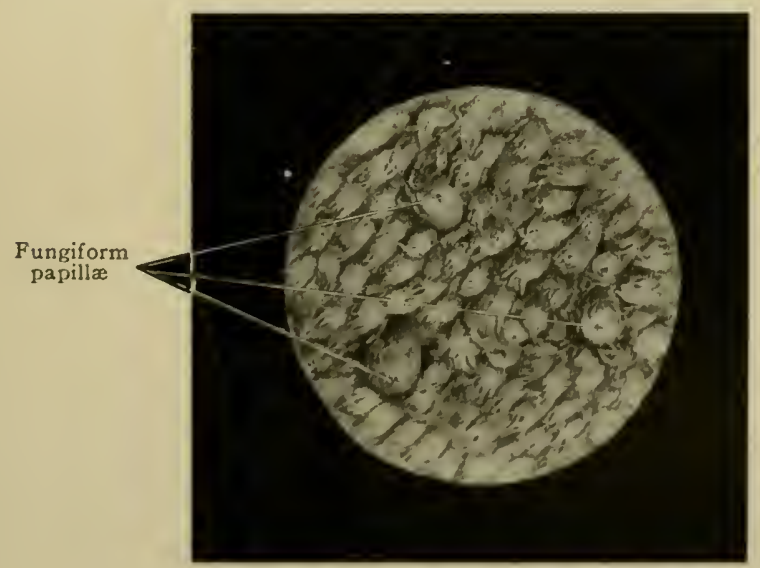

Fig. I 29.-Surface View of Tongue showing filiform papillic and three fungiform papilla (Spaltehol\%).

While the bundles of fibres interlace in all directions, three fairly distinct planes can be differentiated. 
(I) Vertical and somewhat radiating fibres-hyoglossus, genioglossus, and vertical fibres of the lingualis.

(2) Transverse fibres - transverse fibres of the lingualis.

(3) Longitudinal fibres - the styloglossus and longitudinal (superior and inferior) fibres of the lingualis.

The connective tissue which supports the muscle fibres and separates them into bundles contains mucous glands and fat. A strong band of connective tissue, the septum lingue, extends lengthwise through the middle of the tongue, dividing it into right and left halves.

The submucosa of the tongue is not well developed, the stroma of the mucosa resting directly upon the underlying muscle.

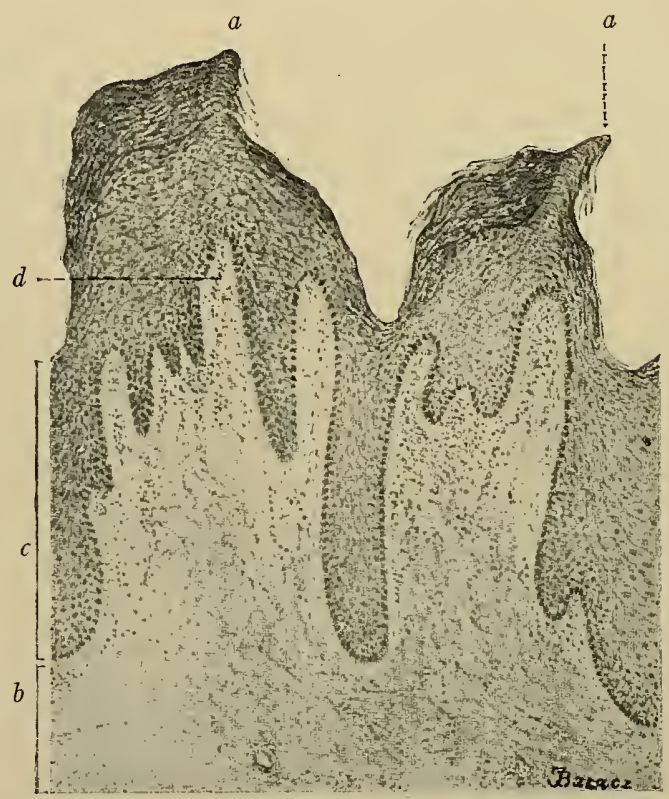

Fig. I30.--Vertical Section through Two Filiform Papillæ from Human Tongue. $\times 80$. (Szymonowicz.) $a$, Horny epithelium; $b$, stroma; $c$, epithelium; $d$, secondary papilla.

The mucous membrane of the tongue resembles that of the mouth, but dffers from the latter in that in addition to the low papillæ, such ais are found in the oral mucosa, the upper surface of the tongue is studded with numerous and much larger papillæ or villi. These project from the surface and give to the tongue its characteristic roughness. Three forms of papillæ are distinguished:-Filiform, fungiform, and circumvallate. 
(I) Filiform Papilla (Fig. I30).-These are the most numerous and are distributed over the entire dorsum of the organ. Each consists of a central core of connective tissue containing elastic fibres, which is long and slender, and is covered by stratified squamous epithelium. From the summit of each papilla are given off several secondary papilla. The epithelium covering the papillæ is hornified and often extends from the surface as a long thread-like projectionhence the name, filiform.

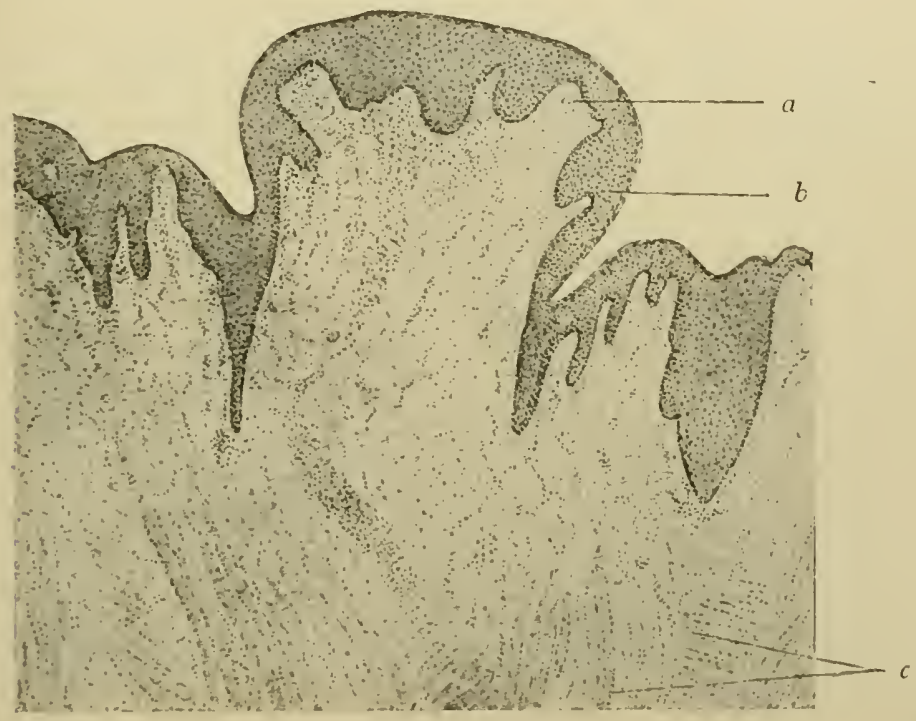

FIG. I3I.-Vertical Section through Fungiform Papilla of Human Tongue. $X_{45}$. (Szymonowicz.) $a$, Secondary papilla; $b$, epithelium; $c$, muscle fibres.

(2) Fungrarm Papille (Fig. I3I).-Scattered irregularly over the entire dorsum among the filiform papillæ, but fewer in number, are larger papille of somewhat different structure known as fungiform papilla. Their summits are rounded instearl of pointed and their bases are narrowed. Secondary papillie are given off not only from the summit, but from the sides of the papilla. The epithelial covering is comparatively thin and is not hornified. The connectivetissue corc of these papillac contains but few clastic fibres.

(3) The Circumbaldate Papilde (Fig. 132).-These are from nine to fifteen in number, and are grouped on the posterior surface of the dorsum of the tonguc. 'They resemble the fungiform papillae, but are much larger. Jiach lies rather deep in the mucous membrane, 
surrounded by a groove or trench and wall (whence the name circumvallate). The wall is somewhat lower than the papilla, thus allowing the latter to project slightly above the surface. Secondary papillæ are confined to the upper surface of the papilla, the sides being free from secondary papillæ. The surface of the papilla and the borders of the groove and wall are covered by stratified squamous epithelium. Lying in the epithelium of the side wall and sometimes of the opposite trench wall are oval bodies, the so-called taste buds, which serve as

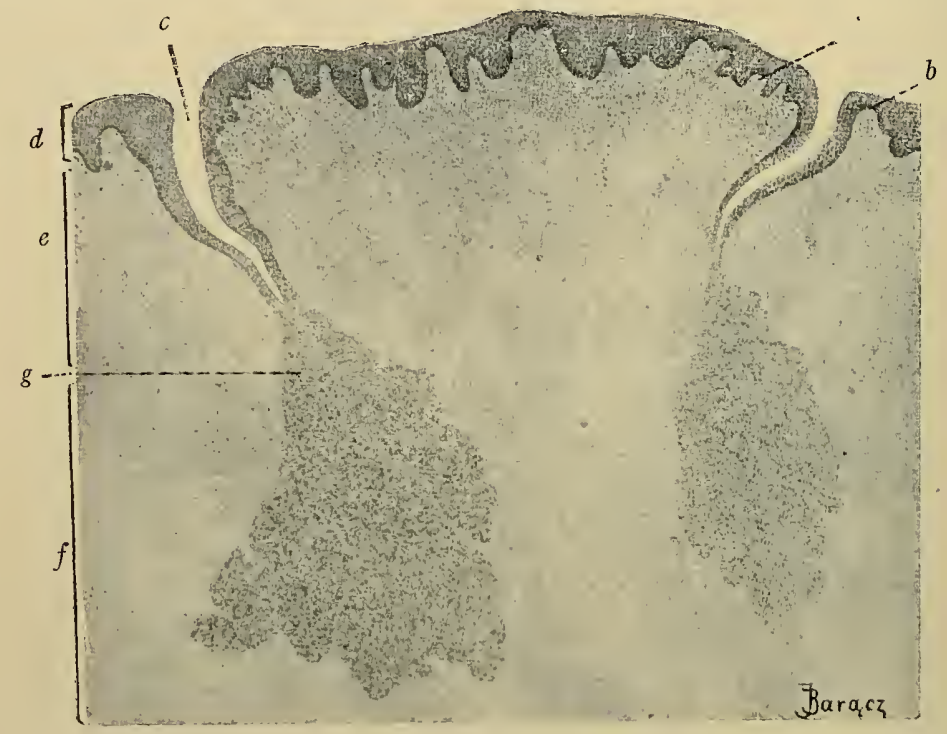

FIG. I 32.--Vertical Section through a Circumvallate Papilla of Human Tongue. $\times 37$. (Szymonowicz.) $a$, Secondary papilla; $b$, wall; $c$, trench; $d$, epithelium of tongue; $e$, stroma; $f$, submucosa; $g$, Ebner's glands.

organs for the nerves of taste (see nervous system). Into the trench surrounding the circumvallate papilla open the ducts of serous glands (Ebner's glands).

The lymph follicles of the tongue have been already described (page r80) under the head of the lingual tonsils.

For glands of the tongue see page 222 .

The larger blood-vessels run in the connective-tissue septa. These give off smaller branches, which break up into capillary networks surrounding the muscle fibres and forming a plexus just beneath the epithelium. From the latter are given off capillaries to the papillæ. The capillaries converge to form veins, which in general follow the course of the arteries. 
Fine lymph spaces occur in the papillæ and open into a plexus of small lymph capillaries just beneath the papillæ. These communicate with a deeper plexus of larger lymphatics, which increase in size and number as they pass backward and form an especially dense lymphatic network at the root of the tongue in the region of the lingual tonsils.

Nerves.-Sympathetic fibres pass mainly to the smooth muscle of the blood-vessels and to the glands. Medullated motor nerve fibres supply the lingual muscles. Medullated sensory nerves include those of the special sense of taste as well as those of ordinary sensation. They end freely among the epithelial cells or in connection with special end-organs - the tasle buds mainly in the circumvallate papillæ, and the end-bulbs of Krause in the fungiform papillæ.

\section{TECHNIC}

Remove pieces of the dorsum of the tongue, selecting parts that will include the different forms of papillæ and cutting well into the underlying muscular tissue. Treat as in technic 2, p. 223 , or sections may be stained with hæmatoxylin-picro-acid-fuchsin (technic 3, p. 2 I).

In sections from the back part of the tongue good examples of mucous and serous glands are usually found.

In small sections of the tongue the muscle fibres are seen arranged in bundles, surrounded by connective tissue and interlacing in all directions. For the study of the arrangement of the different planes of muscle, complete transverse sections should be made at intervals through the entire tongue. The muscle and connective-tissue relations are best brought out by the hæmatoxylin-picro-acidfuchsin stain.

\section{The Teeth}

A tooth is a hard bone-like structure, part of which projects above the surface of the jaw as the crown, while the deeper portion, the root or fang, is buried in a socket of the alveolar margin (Fig. 133). The junction of the root and crown is known as the neck.

A tooth consists of a soft central core, the pulp cavity, surrounded by dentine (Figs. I 33 and I35). The latter constitutes the main bulk of the tooth. The exposerl portion of the dentine is covered by a thin layer of extremely hard substance, the enamel (Fig. I33, I), while the alverlar portion of the dentine is covered with cementum (Fig. 133, 3). Of these the dentine and cementum are of connective-tissue origin, the enamel of epithelial. 
The pulp cavity occupies the central axis of the tooth (Figs. I33 and $\mathrm{I}_{35}$ ). In the root it is known as the root canal. At the apex of the root it communicates with the underlying tissue by means of one or more minute apical foramina, through which blood-vessels and nerves enter the pulp cavity.

The dental pulp consists of loose connective tissue approaching the embryonal in type, composed of many fusiform and stellate cells and

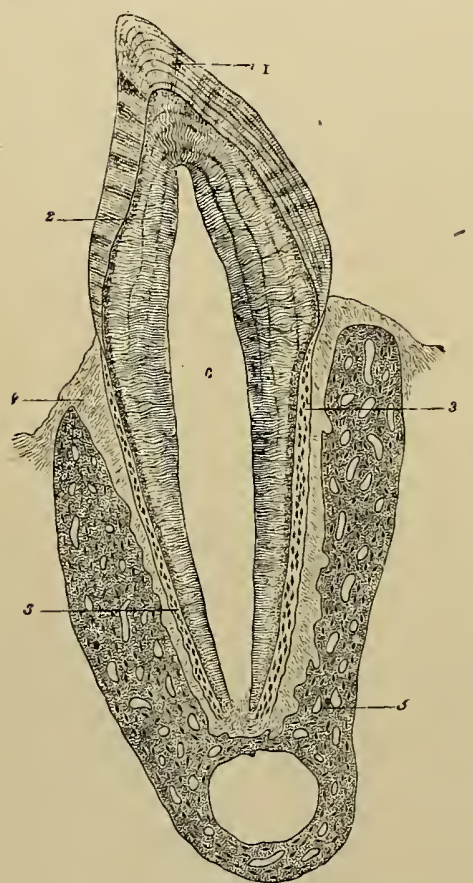

FIg. I33.-Vertical Section of Tooth in situ. $\times \mathrm{I}_{5}$. (Waldeyer.) c, Pulp cavity, the letter being at about the junction of crown and root; I, enamel showing radial and longitudinal markings; 2, dentine showing dental canals; 3 , cementum (containing bone corpuscles); 4, peridental membrane; 5 , bone of lower jaw. delicate white fibrils. There are apparently no elastic fibres. A few smooth muscle cells have been described. The pulp is richly supplied with blood-vessels and nerves which are found only in this part of the tooth. Along the dentinal surface of the pulp the connective-tissue cells are arranged as a single layer of columnar cells, the odontoblasts. These cells are closely allied to osteoblasts. Their nuclei lie toward their inner ends. Each cell sends out an inner process, which is usually single and passes into the dental pulp, several lateral processes which interlace with and probably anastomose with similar processes from other cells, and one or more outer fibrelike processes which enter the dentine, where they form the dentinal fibres. These frequently extend entirely through the dentine. Just beneath the layer of odontoblasts, the connective-tissue cells are much fewer in number than in the rest of the dental pulp. Appearing in sections as a clear band, it is known as the layer of Weil. Immediately internal to the layer of Weil, the cells are more closely arranged than elsewhere in the pulp.

Dentine (Figs. 135 and $136, D$ ) is somewhat harder than bone which it resembles in structure. According to von Bibra its chemical composition is: 


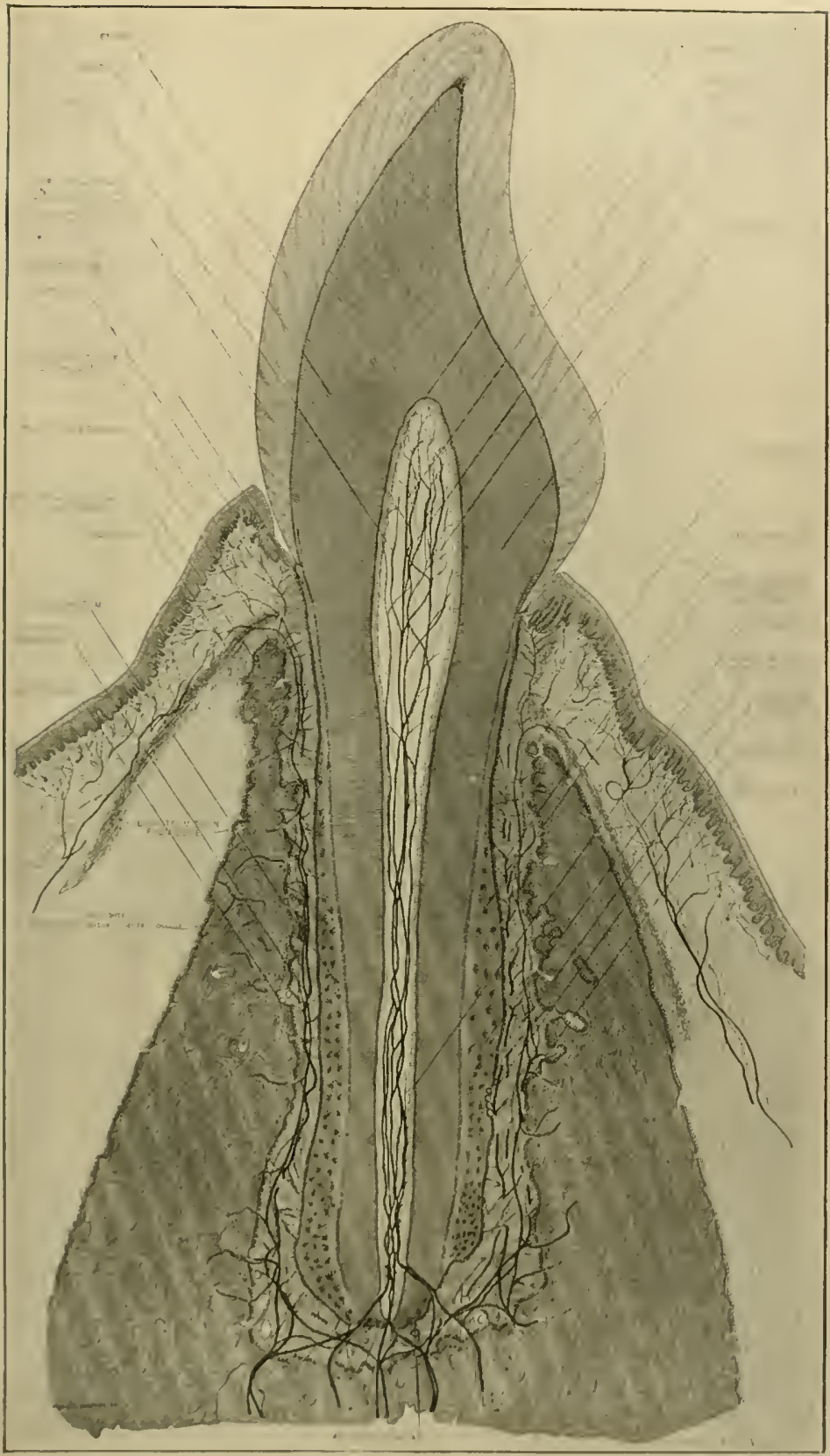

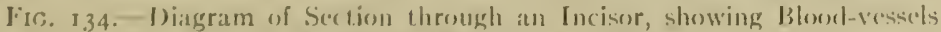
and Periclental Mcmoranc. (Noyes.) 
Organic matter, 28.01

Calcium phosphate and fluorid, 66.72

Calcium carbonate,

Magnesium phosphate,

I. 18

Other salts,

0.73

Dentine constitutes the bulk of the tooth and is peculiar in that it contains canaliculi, dentinal canals (Figs. 135 and $136, D k$ ), but no lacunæ or bone cells. The latter are represented by the odonto-

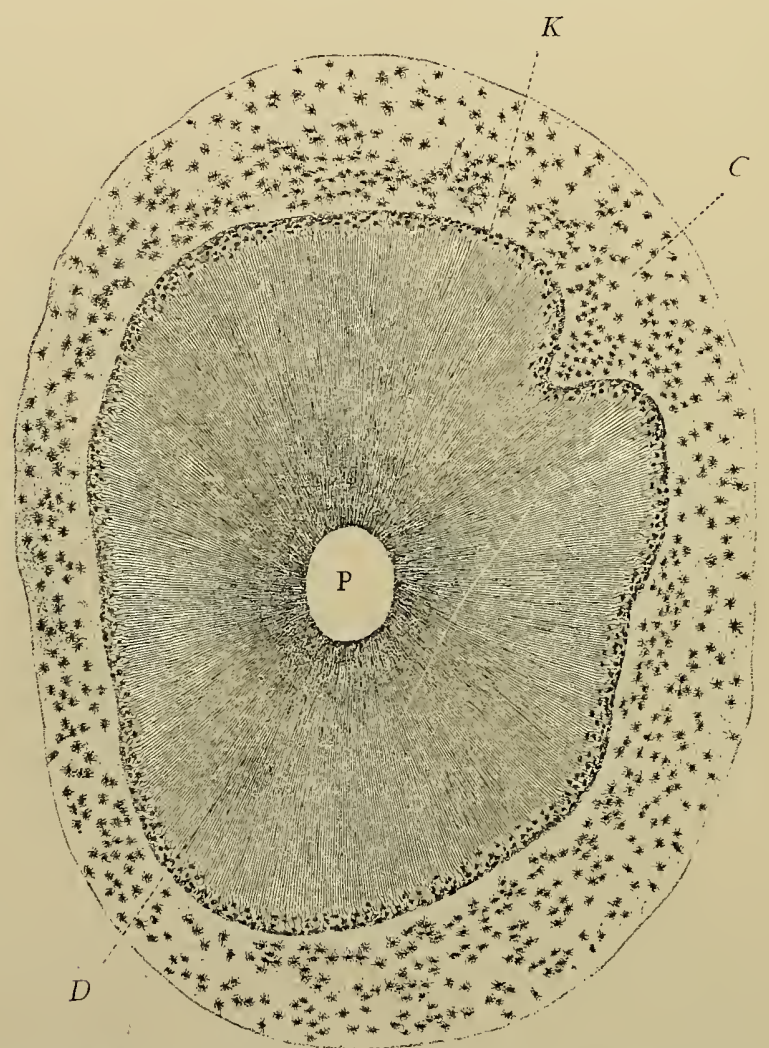

FIG. I35--Cross Section through Root of Human Canine Tooth $\left(\times_{25}\right)$ (Sobotta), showing relations of pulp cavity, dentine, and cementum. $P$, Pulp cavity; $D$, dentine; $C$, cementum; $K$, Tomes' granular layer.

blasts of the pulp, which, as already noted, lie at the inner side of the dentine, into the canaliculi of which they send the dentinal fibres. Dentine is non-vascular. The dentinal canals begin at the dental pulp, into which they open and where they have a calibre of 2 to $5 \%$. They pass outward radially, to the limit of the dentine, and, while taking different directions in different parts of the dentine, are essen- 
tially parallel. In their passage through the dentine the dentinal canals describe two series of curves, known as primary and secondary curves. The former take the form of an elongated $\mathrm{S}$ from pulp to enamel or cementum, the secondary curves are twistings of the canals (drawn out corkscrew). These twistings are very fine as many as 200 turns having been described in the length of a canal. In their passage through the dentine the main canals gradually grow smaller until their diameter is from 0.5 to $x \mu$. They give off minute side branches from 0.3 to $0.6 \mu$ in diameter, which leave the main tubules

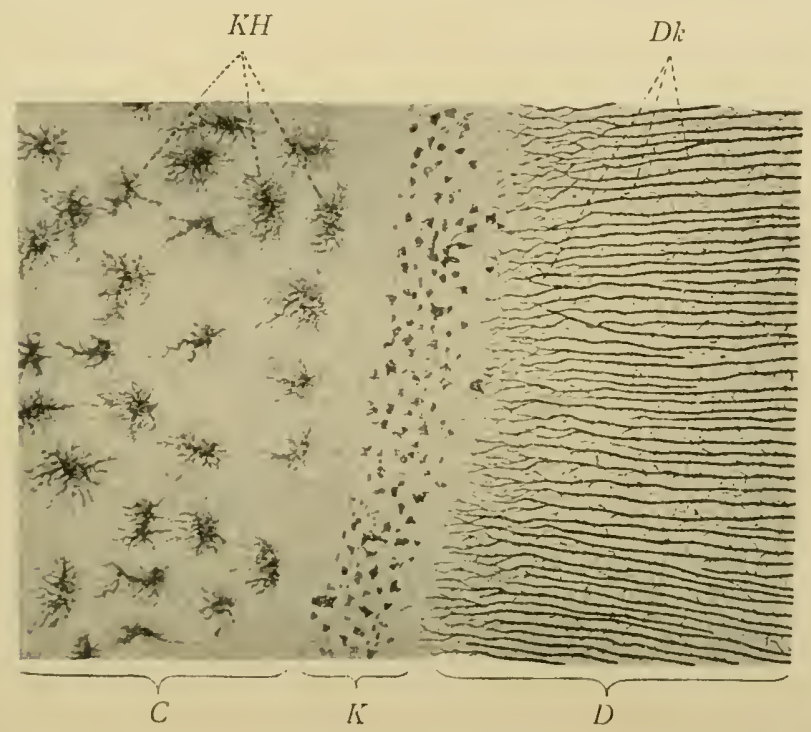

Fig. I36.-From Longitudinal Section through Root of Human Molar Tooth (X200) (Sobotta), showing junction of dentine and cementum. C, Cementum; $D$, dentine; $K$, Tomes' granular layer; $D k$, dental canals: $K I I$, lacunx of cementum.

at almost right angles, but soon turn slightly outward. 'They anastomose with similar branches from other canals. This anastomosis takes place not only between branches of adjacent canals, but also between branches of canals some distance apart. The main canals terminate either in blind extremities, or form loops by anastomosing with neighboring tubules. Some of the tubules have quite extensive terminal branchings, other tubules have only two or three end branches. A few tubules run slightly beyond the limits of the dentine into the enamel. The arrangement of the dentinal canals and their branches differs in different parts of the tooth. In the crown there are few large branches and the main canals show distinct primary and secondary curves, most of them ending blindly in brush-like branchings 
just under the enamel, but some continuing over into the enamel for from Io to $4.0 \mu$, where they lie in the cement between the prisms. In the root the canals have an almost straight direction (without primary curves). Large branches are more numerous and the main canals have a somewhat more irregular arrangement. They probably do not pass over into the cementum, but end at the granular layer. The dentine immediately around a dentinal canal is more dense and harder than elsewhere and forms a sort of sheath for the canalNeumann's dental sheath. Between the dentinal canals is a calcified ground substance, in which are connective-tissue fibres running in a direction parallel to the surface of the pulp, this corresponding, as in bone, to the deposition of the dentine in successive layers. In a longitudinal section of the dentine of the crown, lines are seen running parallel to the surface of the pulp. They are known as the lines of Schreger and are probably due to irregularities in deposition of the dentine.

Spaces which probably represent incomplete calcification of the dentine occur in the peripheral portion of the dentine of the crown. These are known as interglobular spaces (Fig. I37, Jg). They are filled with a substance resembling uncalcified dentine. The interglobular spaces do not interrupt the dental canals which pass through them with no break in their continuity.

In the outer part of the dentine of the root are similar spaces which are smaller and more closely placed. These form the so-called Tomes' granular layer (Fig. $136, K$ ). In the root of the tooth this layer is quite thick, separating the cementum from the dentine. Its spaces or lacunæ send off tiny canaliculi which run in all directions and anastomose with one another, with the dentinal tubules, and with the lacunæ of the cementum. This layer, with its small closely placed spaces and fine irregularly running canaliculi, contrasts sharply on the one side (Fig. I36) with the dentine and its straight parallel tubules; and on the other side, with the cementum and its large and more widely separated lacunæ. Over the crown of the tooth this layer is much thinner and as the apex is approached, becomes lost completely, the dental tubules extending to, and some of them entering slightly, (see above) the enamel. ${ }^{1}$

${ }^{1}$ Distinction is sometimes made between primary and secondary dentine. Primary dentine is that dentine normally formed during the development of a tooth and corresponds to the description given, later under some stimulus, e.g., excessive wear, formation of dentine again takes place, in which case the canals are often much more irregularly arranged; this is known as secondary dentine. 
The ENAMEL covers the exposed part or crown of the tooth and is the hardest substance in the body. It is thickest over the crown and gradually decreases in thickness along the sides of the tooth until it reaches the neck where it stops. It contains little more than a trace of organic substance, its chemical composition being, according to von Bibra:

Organic matter,

Calcium phosphate and fluorid,

$3 \cdot 59$

Calcium carbonate,

89.82

Magnesium phosphate,

$4 \cdot 37$

Other salts,

I. 34

0.88

It consists of long six-sided prisms 3 to $6 \mu$ in diameter-enamel fibres or enamel prisms (Fig. I37, Sp) - which take a slightly wavy course through the entire thickness of the enamel. The prisms are attached to one another by a small amount of cement substance, and are grouped into bundles, the prisms of each bundle being parallel, but the bundles themselves frequently crossing one another at acute angles. In the human adult the prisms are homogeneous; in the embryo they show a longitudinal fibrillation. Rather indistinct parallel lines (the lines of Retzius) cross the enamel prisms. They probably represent the deposition in layers of the lime salts, although they are considered by some as artefacts. The enamel is covered by an apparently structureless membrane, the cuticula dentis.

The cementum (Fig. $136, C$ ) covers the dentine of the root in a manner similar to that in which the enamel covers the dentine of the crown (Fig. I33, I and 3). It forms a thin layer at the neck, but increases in thickness as the deeper part of the root is reached. Cementum is bone tissue. It contains lacuno and bonecells. These vary much more in size and shape than do those of bone, are very irregularly distributed and may be absent from considerable areas. From the lacunx radiate canaliculi, but there is no distinct lamellation and no Haversian systems or blood-vessels, excepting in the large teeth of the larger mammalia, and in the teeth of the aged, where they may be present. Channels, similar to Volkmann's canals in bone, not surrounded by concentric lamellac, but serving for the passage of bloodvessels, are quite frequent in the thicker portions of the cementum. The ground substance of the cementum is continuous with that of the dentine and many canaliculi of the former open into the interglobular spaces of the latter. Nany uncalcified Sharpey's fibres penetrate the cementum. 
Tre Peridental Membrane.--Surrounding the root of the tooth and filling in the space between it and the wall of the alveolus is a layer of connective tissue which is known as the peridental membrane (Fig. I34). It attaches the tooth to the alveolus, attaches the teeth to each other, supports the free margin of the gum and holds it to the tooth, and serves for the transmission of vessels and nerves. It consists of dense white fibrous tissue with few or no elastic fibres. In general it resembles periosteum and has been described as a reflection of the alveolar periosteum upon the root of the tooth. The fibres fall into two classes, long fibres which pass from the cementum to the coarser connective

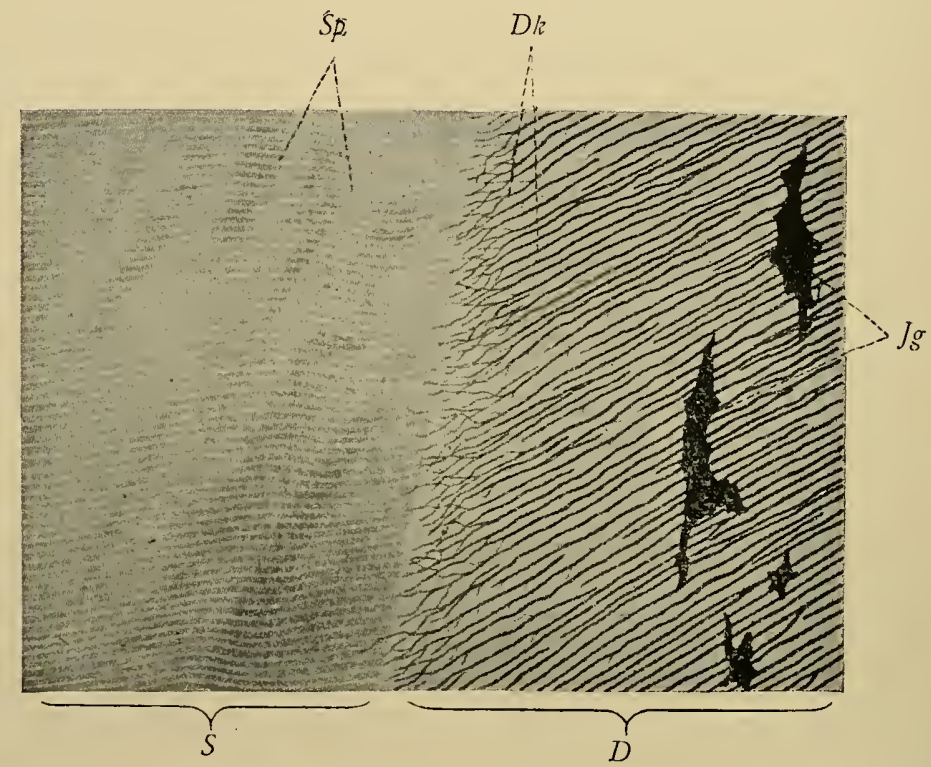

FIG. I37.-From Longitudinal Section of Crown of Human Premolar $\left(X_{200}\right)$ (Sobotta), showing junction of enamel and dentine. $S$, Enamel; $D$, dentine; $S p$, enamel prisms; $D k$, dental canals; $J g$, interglobular spaces. A few dentinal fibres are seen passing beyond the limits of the dentine into the enamel. The oblique dark bands in the enamel are the lines of Retzius.

tissue of the gum, to the alveolar wall or to the cementum of an adjacent tooth, and short fibres which fill in the interstices between the long fibres. Many of the long fibres are continued as calcified fibres into the cementum on the one hand and into the bone of the alveolus on the other. They are analogous to Sharpey's fibres of bone (p. 192). As they enter the cementum or bone, the fibres are grouped in bundles but in the central portion of the membrane these bundles break up and their fibres interlace in all directions. The long fibres differ in direction in different parts of the membrane. Those which spring from the cementum near its junction with enamel, pass out at right angles and then turn sharply toward the free margin of the gum which they support. Passing toward the apex, the next fibres instead of bending toward the free margin of the gum pass out at right angles and blend with the gum connective tissue. All fibres 
to the gums are more developed on the lingual than on the labial side. Between the teeth, fibres of this level pass from the cementum of one tooth to that of the next adjacent, here also supporting the free margin of the gum. Still further apically the fibres are grouped in coarser bundles to form the so-called dental ligament, and pass over or into the edge of the alveolus; between the teeth into the edge of the septum or into the cementum of the adjacent tooth. From the dental ligement for about one-third the depth of the root, rather coarse bundles of fibres pass from cementum almost straight to alveolar wall. For most of the remaining two-thirds, th. direction of the fibres is away from the apex, the bundles tending to break up and to radiate to a more extended insertion into the alveolar bone. The apical fibres also radiate to their insertion into bone.

Among the connective-tissue fibres are found fixed connective-tissue cells, groups or cords of cells which are apparently epithelial, and, during development and sometimes sparingly later, osteoblasts, osteoclasts, and cementoblasts.

The fixed connective-tissue cells are described on p. 83 .

The epithelioid cells are mostly arranged in cords which anastomose to form a network. The cells themselves are granular and have oval or round nuclei rich in chromatin. In some places their arrangement resembles tubules, but whether they have a glandular character is not known.

Osteoblasts and osteoclasts are the same as in developing bone (p. 198). The latter are present wherever absorption is taking place. They are especially numerous during the absorption of the roots of the milk teeth to make way for the permanent set.

Cementoblasts are cells which lie against the cementum and are analogous to the osteoblasts, from which however they differ morphologically. They are flattenerl and their protoplasm fills in the spaces between the ends of the fibres so that the cell bodies show the indentations of the fibres lying against them. From the cementoblasts processes extend into the cementum in much the same manner as processes of bone cells extend into their canaliculi.

Blood-vessels. - The arteries which supply the tooth and peridental membrane, enter the apical portion of the latter from the adjacent bone of the alveolus (Fig. 134). On entering the membrane the ressels divide into two main sets one of which passes through the foramina of the apex to supply the dental pulp, while the other passes along the outside of the tooth in the peridental membrane which it supplies (Fig. I34). The ressels in the membrane anastomose with vessels from the bone of the alveolar wall and at the margin of the alveolar cavity with vessels from the gums. From the capillary network veins follow the arteries back to the apical portion of the membrane and into the bone at the bottom of the alveolus. The arteries to the pulp run mainly through its centre giving off branches which form a capillary network which is especially rich at the periphery of the pulp) (Fig. 138). From these capillaries arise veins which pass 
back through the apical foramina into the peridental membrane where they unite with veins from the membrane and pass into the bone of the alveolus. Enamel, cementum, and dentine are non-vascular. A marked feature of the pulp vessels is the thinness of their walls, ves-

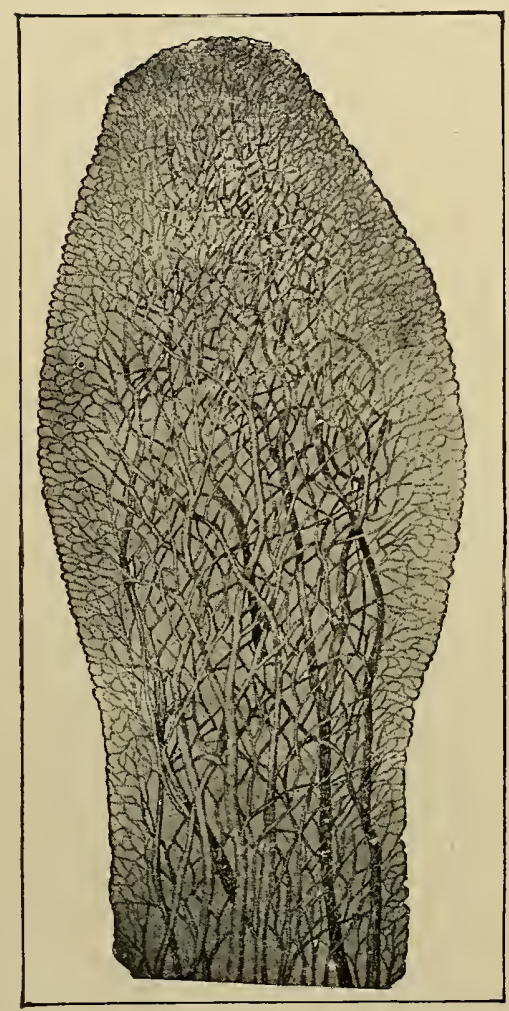

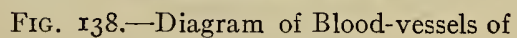
Pulp. (Stowell). sels of much greater calibre than are usually classed as capillaries having walls of capillary thinness.

Lymph Vessels.- Most authorities have denied the existence of definite lymph channels in the pulp. Schweitzer on the other hand describes an arborization of small lymph vessels in the pulp of the crown, converging to a few larger lymph vessels in the root pulp and accompanying the bloodvessels through the foramina of the apex.

Nerves.-The distribution of nerves to the tooth follows quite closely that of the blood-vessels. Bundles of medullated fibres from. the bone at the bottom of the alveolus enter the peridental membrane just beneath the apex where they divide into two main sets, one of which follows the vessels of the membrane while the other passes with the vessels through the apical foramina to the dental pulp. The membrane surrounding the tooth also receives nerves from the bone of the side wall of the alveolus. The branches to the pulp pass up through its center, giving off branches which are mostly non-medullated and which radiate toward the periphery where they form a plexus in the layer of Weil just beneath the odontoblasts. From this plexus branches are given off which pass in between the odontoblasts, some terminating there while others end between the odontoblasts and the dentine. ${ }^{1}$

${ }^{1}$ Noyes calls attention to the extreme sensitiveness of dentine in spite of the fact that no nerve fibres have been demonstrated in its canals, and believes that sensation is carried to the nerve fibres through the processes (dentinal fibres) and cell bodies of the odontoblasts. 


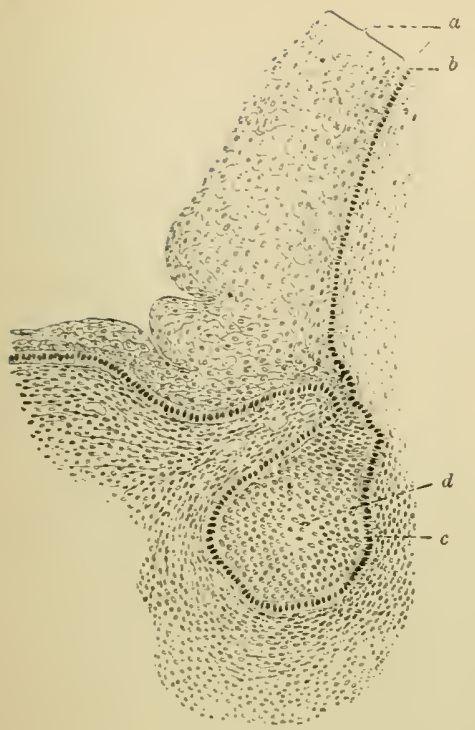

FIG. I39.

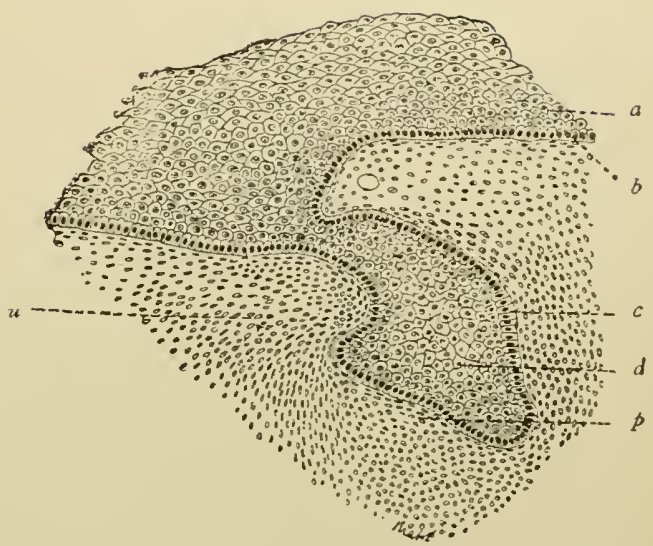

FIG. I 40 .

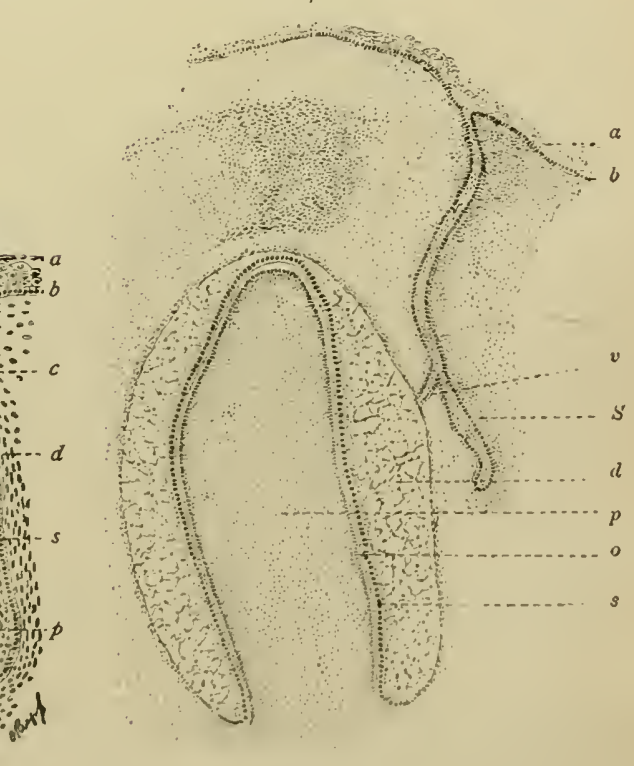

FIG. 142.

I.IC. I 4 I.

Fics. 139, 140, 141, 1 42.-Four Stages in the Develop)ment of a Tooth (from lower jaw of sheep embryo). (Bohm-Davidoff.) Fig. 139, Beginning of enamel organ showing connection with epithelium of mouth; Fig. I 40 , Later stage showing same with first trace of papilla; Fig. I 1 I, Later stage showing papilla well formed, the differentiation of the enamel pulp and of the inner and outer enamel cells can be secn; odontoljlist appearing along periphery of the papilla; Fig. 142 , shows also beginning enamel organ of permanent tooth; Figs. I39, 140, 141, X110, Fig. 142, X40. $a$, Fipithelium of mouth; $b$, its basal layer; $c$, superficial cells of enamel organ; $d$, enamel pulp); $p$, (lental papillat; $s$, enamel cells; $o$, odontoblasts; $S$, cnamel organ of permanent tooth just beginning to differentiate; $v$, remains of enamel ledge of milk tooth; $u$, surrounding connective tissue. 
Development.--The enamel of the teeth is of ectodermic origin, the remainder of mesodermic. The earliest indication of tooth formation occurs about the seventh week of intra-uterine life (embryos $\mathrm{x}_{2}$ to $\mathrm{I} 5 \mathrm{~mm}$.). It consists in a dipping down of the epithelium covering the edge of the jaw into the underlying connective tissue (mesoderm) where it forms the dental shelf, or common dental germ. Soon after the formation of the dental shelf, a groove appears along the margin of the jaw where the ingrowth of epithelium occurred. This is known as the dental groove. The epithelium of the dental shelf is at first of uniform thickness. Soon, however, at intervals along the outer side of the dental shelf, the cells of the shelf undergo proliferation and form thickenings, ten in

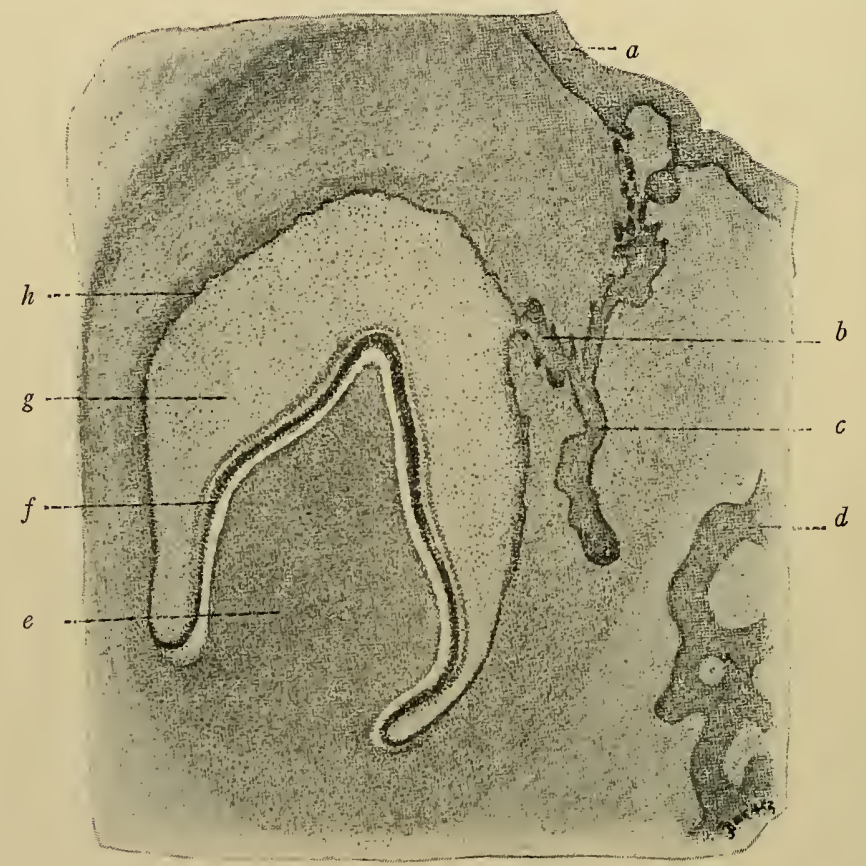

FIG. 143.- Developing Tooth from Three-and-one-half-months' Human Embryo. $\times 65$. (Szymonowicz.) $a$, Epithelium of gums; $b$, neck of enamel organ; $c$, dental germ of permanent tooth; $d$, bone of lower jaw; $e$, dental papilla; $f$, inner enamel cells; $g$, enamel pulp; $h$, outer enamel cells.

the upper and ten in the lower jaw, each one corresponding to the position of a future milk tooth. These are known as special dental germs, and remain for some time connected with one another and with the surface epithelium by means of the rest of the dental ridge.

Into the side of each special dental germ there occurs about the end of the third month (embryos of about $40 \mathrm{~mm}$.) an invagination of the underlying connective tissue. In the upper jaw the invagination takes place on the upper and inner side, in the lower jaw on the lower and inner side, of each dental germ.

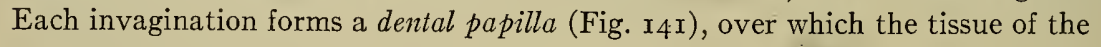


special dental germ forms a sort of cap, the latter being known from its subsequent function as the cnamcl organ, the papilla itself giving rise to the pulp and dentine. The dental germs are at this stage connected with each other by remaining portions of the dental shelf, and with the surface epithelium by remains of the original invagination. The next step is the almost complete separation of the special dental germs and ridge from the surface epithelium (Fig. I +2 ), and the formation around each special dental germ of a vacsular membrane, the denlal sac. The attenuated strand of epithelial cells, which

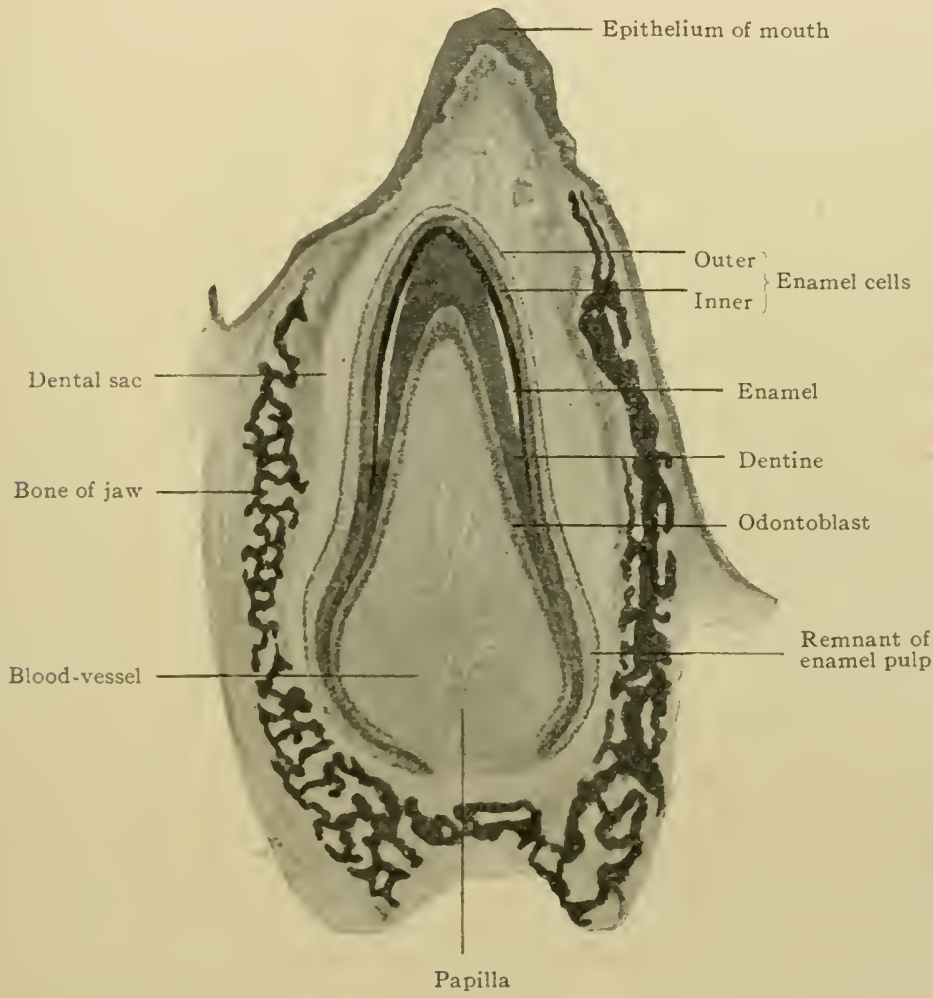

Fig. I44-- Jongitudinal Section of a Developing Tooth of a New-born Puppy: (Bonnett.) Late Stage.

still maintains a connection between the dental germs and the epithelium of the gums, is known as the neck of the cmonel orgen and it isfrom this that an extension soon occurs to the inner sirle of the dental germs of the milk teeth, to form the denlal germs of the permanent leeth (Fig. 143, c). Into the latter, connective-tissue papillae extend as in the case of the nilk teeth. There are thus present as early as the fifth month of fotal existence the germs of all milk and of some permanent teeth.

The liNAsEr, is formed by the enamol organ. At the stage represented in lig. 145, it consists of three layers: (I) The ouler cnamel cells, somewhat flat- 
tened; (2) the inner enamel cells, high columnar epithelium; (3) a layer of enamel pulp, situated between the other layers, and consisting of stellate anastomosing cells with considerable intercellular substance (Figs. I45 and I46). A membrane, the cuticular membrane, is first laid down between the inner enamel cells and the dentine. Each of the inner enamel cells now sends out a process, Tomes' process, from its inner end. The processes are separated by a considerable amount of cement, and are the beginnings of the enamel prisms. Calcification now takes place both in the prisms and in the cement substance, beginning in the ends nearest

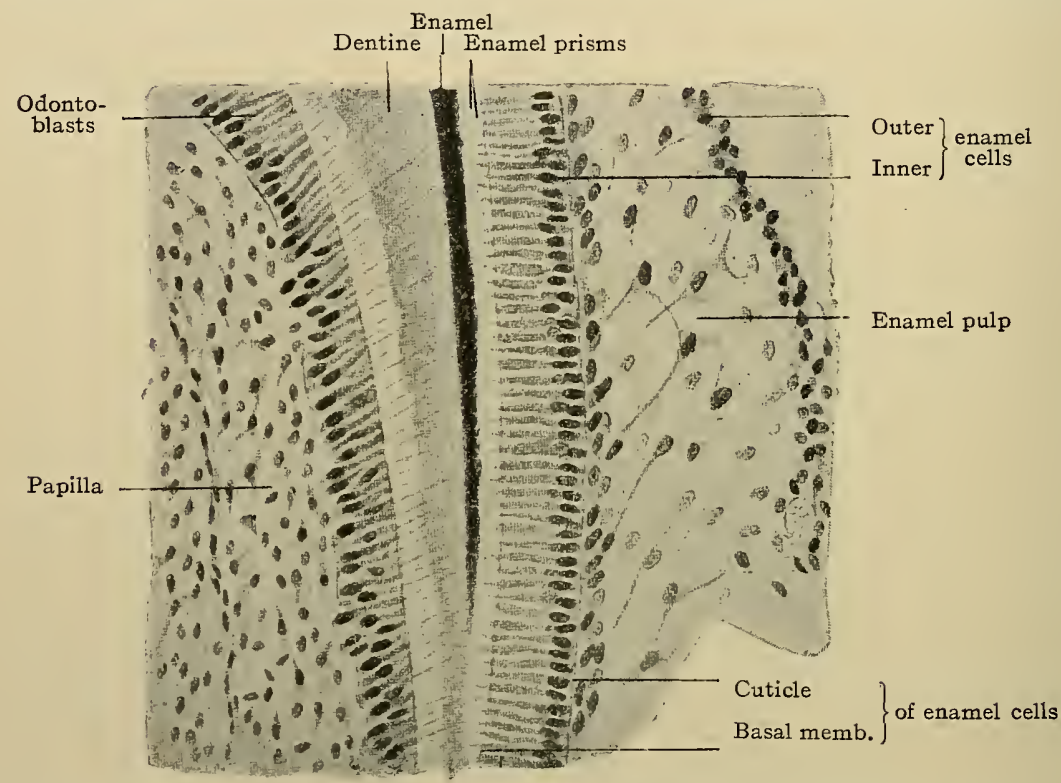

FIG. I45.-Section through Border of a Developing Tooth of a New-born Puppy.

(Bonnett.)

the papilla. As this proceeds outward, the prisms become much elongated and the cement substance reduced in amount. Further growth in thickness of enamel occurs by lengthening of the enamel prisms. During the formation of the enamel, the enamel pulp and the external enamel cells disappear.

The formation of enamel in the milk teeth begins about the end of the fourth month and continues until the eruption of the teeth. The extent of the enamel organ is considerably greater than that of the enamel, the former covering the entire tooth, both root and crown, with the exception of the base of the papilla where the latter is connected with the underlying connective tissue. As the tooth develops, the enamel organ disappears over the root, remaining to form the enamel only over that part of the tooth which is to be subsequently exposed. The function of the enamel pulp is not known. It disappears as the tooth grows. It has been suggested that it may furnish nutrition or serve as an avenue through which nutrition reaches the non-vascular enamel organ. It may serve as an area of least resistance through which the tooth grows. 
The Dextine is the first of the dental tissues to become hard. Both dentine and pulp develop, as noted on p. $23 \mathrm{~S}$, from the mesoderm of the dental papilla. When the latter is first formed it is of the same structure as the surrounding mesoderm with which it is continuous, except that it is somewhat more dense; later it assumes more the character of embryonal connective tissue, blood-vessels and nerves growing into it from the underlying connective tissue. The most peripheral cells of the pulp, those lying nearest the enamel organ, differentiate from the rest of the pulp to form a single layer of colummar or pyramidal cellsthe odontoblasts. The outer end of each cell is broad, while the inner end which contains the nucleus narrows to a point from which a delicate process extends into the pulp and probably anastomoses with other cell processes. These cells are analogous to the osteoblasts of developing bone and like them appear to determine the deposition of lime salts. The lime salts are first laid down in a membrane-like structure-the membrana preformativa-which the odontoblasts apparently form between themselves and the enamel. From this membrane the transformation of the connective tissue into dentine progresses inward toward the pulp, additional dentine continuing to be laid down in layers, each new layer internal to the preceding. In this way the dental papilla is reduced in size to form the pulp cavity. In the outer part of the dentine spaces remain in which no lime salts are deposited. These are the interglobular spaces. As calcification proceeds the odontoblasts do not become enclosed within the dentine as do the

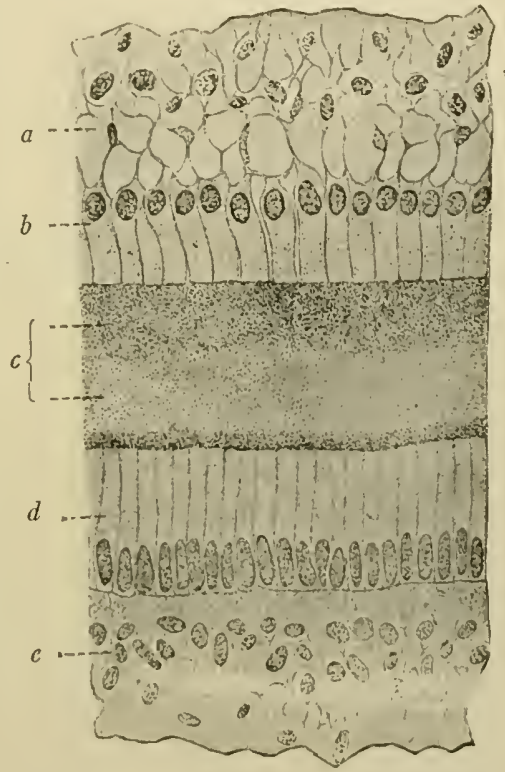

Fig. I 46.-From Cross-section through a Developing Tooth. $\times 720$. (Böhm and von Davidoff.) Note close relationship between odontoblasts and tissue of dental pulp. $a$, Dental pulp; $b$, odontoblasts; $c$, dentine; $d$, inner enamel cells; $e$, enamel pulp. osteoblasts within bone. They leave merely long slender processes, the dental fibres, lying in uninute channels through the dentine, dental canals, while the bodies of the cells form a single layer along the inner margin of the dentine. There are thus no lacunie and no cells within the dentine. 'This relation of odontol,asts to dentine, and probably the original odontoblasts, persist, not only throughout embryonic lut through adult life.

While the tooth lies within the gum, the somewhat condensed connective tissue which surrounds it constitutes the dental sac.

As the germs of the milk teeth develop, the dental shelf broadens by extending inward toward the tongue. Along this inner margin appear the germs of the permanent teeth, the develepment of the various teeth structures from the germs leeing idcentical with the process described in the case of the milk teeth. 
Twenty of the permanent teeth correspond in position to the twenty milk teeth, while twelve new molar germs, six in the upper and six in the lower jaw, represent the true molars of the adult. The first permanent tooth germ to appear is that of the first molar, about the beginning of the fifth month (embryos of about $\mathrm{I} 80 \mathrm{~mm}$.). It lies just behind the second molar of the milk dentition. The germs of the incisors and canines appear about the end of the sixth month, those of the premolars, which replace the milk molars, in the beginning of the eighth month. The germs of the second and third (wisdom teeth) molars do not appear until after birth, those- of the former at about six months, of the latter during the fifth year.

The CEMENTUM is developed by ossification of that part of the dental sac which covers the root, its development being similar to subperiosteal bone formation (p. 20I), without the formation of Haversian systems.

\section{TECHNIC}

(I) Teeth are extremely difficult organs from which to obtain satisfactory material for study. Sections of hard (undecalcified) and of decalcified teeth may be prepared in the same manner as sections of bone-technics $1, p .196 ; 2$, p. I97. The decalcified tooth should include if possible the alveolar margin of the jaw, so that in longitudinal sections the mode of implantation and the relation of the tooth to the surrounding structures can be seen.

(2) For the study of developing teeth, embryo pigs, sheep, cats, dogs, etc., are suitable. For the early stages fœtal pigs should be five to six inches long; for the intermediate, ten to twelve inches. The later stages are best obtained from a small new-born animal, e.g., kitten or small pup. The jaw-preferably the lower-or pieces of the jaw are fixed in formalin-Müller's fluid (technic 5, p. 7), hardened in alcohol, and decalcified (page ro). Subsequent treatment is the same as for developing bone (technic $x$, p. 203).

\section{The Pharynx}

The wall of the pharynx consists of three coats-mucous, muscular, and fibrous.

I. The mucous membrane has a surface epithelium and an underlying stroma.

The EPITHELIUM is stratified squamous except in the region of the posterior nares, where it is stratified columnar ciliated, continuous with the similar epithelium of the nasal mucosa.

The sTROMA, or tunica propria, consists of mixed fibrous and elastic tissue infiltrated with lymphoid cells. In certain regions these cells form distinct lymph nodules (see pharyngeal tonsils, page I80). Beneath the stratified squamous epithelium the stroma is thrown up into numerous low papillc. These are absent in regions 
covered by ciliated cells. Bounding the stroma externally is a strongly developed layer of longitudinal elastic fibres, the elastic limiting layer, which separates the stroma from the muscular coat and sends stout bands in between the muscle bundles of the latter.

2. The muscular coat lies beneath the elastic layer and is formed of very irregularly arranged muscle fibres belonging to the constrictor muscles of the pharynx.

3. The fibrous coat consists of a dense network of mixed fibrous and elastic tissue. It has no distinct external limit, and binds the pharynx to the surrounding structures.

The distribution of blood-vessels, lymphatics, and nerves is similar to that in the oral mucosa.

Small, branched, tubular, mucous glands are present in the stroma, and extend down into the intermuscular connective tissue. They are most numerous near the opening of the Eustachian tube.

\section{TECHNIC}

For the study of the structure of the walls of the pharynx, material should be prepared as in technic 2, p. 223.

\section{THE FOREGUT}

\section{The Esophagus}

The walls of the œesophagus are continuous with those of the pharynx and closely resemble the latter in structure. They consist of four layers, which from within outward are mucous, submucous, muscular, and fibrous (Fig. I47).

I. The mucous membrane resembles that of the pharynx except that beneath the stroma is a well-developed muscularis mucosa composed of smooth muscle cells arranged longitudinally. The muscularis mucosx forms a complete coat only in the lower part of the oesophagus. The epithelium is stratified squamous and rests upon a papillated stromá.

2. The submucosa is composed of loosely arranged fibrous and clastic tissue. It contains mucous glands, the larger blood-vessels, lymphatics, and nerves.

3. The Muscular Coat.-In the ujper portion of the oesophagus this coat is composed of striated muscle fibres; in the middle portion, of mixed striated and smooth muscle. In the lower portion there are 
two distinct layers of smooth muscle, an inner circular and an outer longitudinal. The latter is not continuous.

4. The fibrous coat consists of bundles of white fibrous tissue with many elastic fibres. It serves to connect the osophagus with the surrounding structures.

Two kinds of glands occur in the œesophagus.

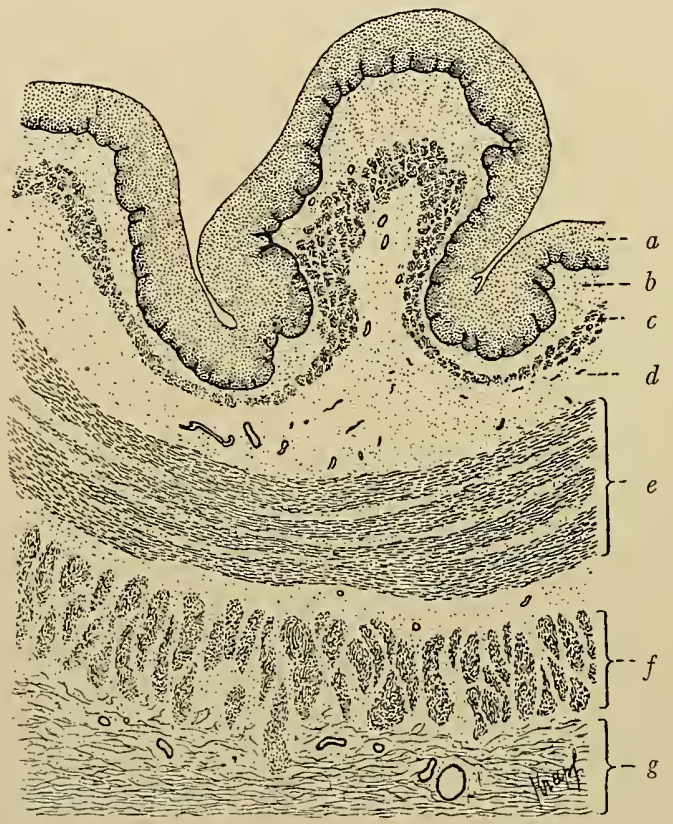

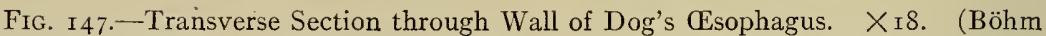
and von Davidoff.) $a$, Epithelium; $b$, stroma; $c$, muscularis mucosæ; $d$, submucosa; $e$, circular muscle layer; $f$, longitudinal muscle layer; $g$, fibrous layer.

(I) Mucous Glands.--These are of the same structure as those of the tongue, but much smaller. They lie in the submucosa and are distributed throughout the entire œsophagus, though most numerous in its upper third. The ducts pass obliquely downward on their way to the surface. Just before entering the muscularis mucosæ the duct widens out to form a sort of ampulla. Beyond this it again becomes narrow and enters the epithelium in the depression between two adjacent papillæ. A small lymph nodule is usually attached to the duct as it passes through the tunica propria.

(2) Simple Branched Tubular Glands.-These resemble the glands of the cardiac end of the stomach, but branch much more 
profusely. Some contain both chief and acid cells, others only chief cells (see stomach, page 249). They lie in the tunica propria, and are for the most part confined to a narrow zone at the lower end of the œsophagus and to the level of the fifth tracheal ring. Scattered groups also occur in other regions.

The distribution of blood-vessels, lymphatics, and nerves in the œsophagus is similar to their distribution in the mouth (p.222). Between the two muscular coats the nerve fibres form plexuses in which are many sympathetic nerve cells. These plexuses are analogous to the plexuses of Meissner in the stomach and intestine.

\section{TECHNIC}

Remove a portion of the wall of the œesophagus, wash carefully in normal salt solution, and pin out, mucous-membrane side up, on a piece of cork. Fix in formalin-Müller's fluid and harden in alcohol (technic 5, p. 7). Transverse or longitudinal sections should be cut through the entire thickness of the wall. If the details of the muscular coat are to be studied, sections from at least three different levels should be taken: one near the upper end, one at about the middle, and the other in the lower third. Stain with hæmatoxylin-eosin or hæmatoxylin-picro-acid-fuchsin (technic I, p. 20; 3, p. 2I) and mount in balsam.

General Structure of the Walls of the Gastro-Intestinal CANAL

The walls of the stomach and intestines are made up of four coats (Fig. 148). These from the lumen outward are mucous, submucous, muscular, and serous.

I. The mucous membrane (Fig. I48) consists of surface epithelium, glands, stroma, and muscularis mucosx. The surface epithelium is simple columnar and rests upon a distinct basement membrane. The arraligement of the glands and the nature of the gland cells differ in different parts of the tract. The stroma is a loosely arranged, richly cellular connective tissue, which in many places is so infiltrated with lymphoid cells as to constitute diffuse lymphatic tissue. In other places it contains circumscribed masses of lymplatic tissue, lymph nodules. The amount of stroma depends upon the closeness with which the glands are packed. The musculuris mucose consists of smooth muscle cells, which have a generally longitudinal arrangement. Where, however, the muscularis mucosa is thick there are usually two distinct layers-an inner circular and an outer longitudi- 
nal. Folds of considerable extent occur in the mucous membrane. Those of the stomach are known as ruge, and are not constant, depending upon the degree of distention of the organ. Those of the small intestine are much more definite, and are known as valvulce conniventes.

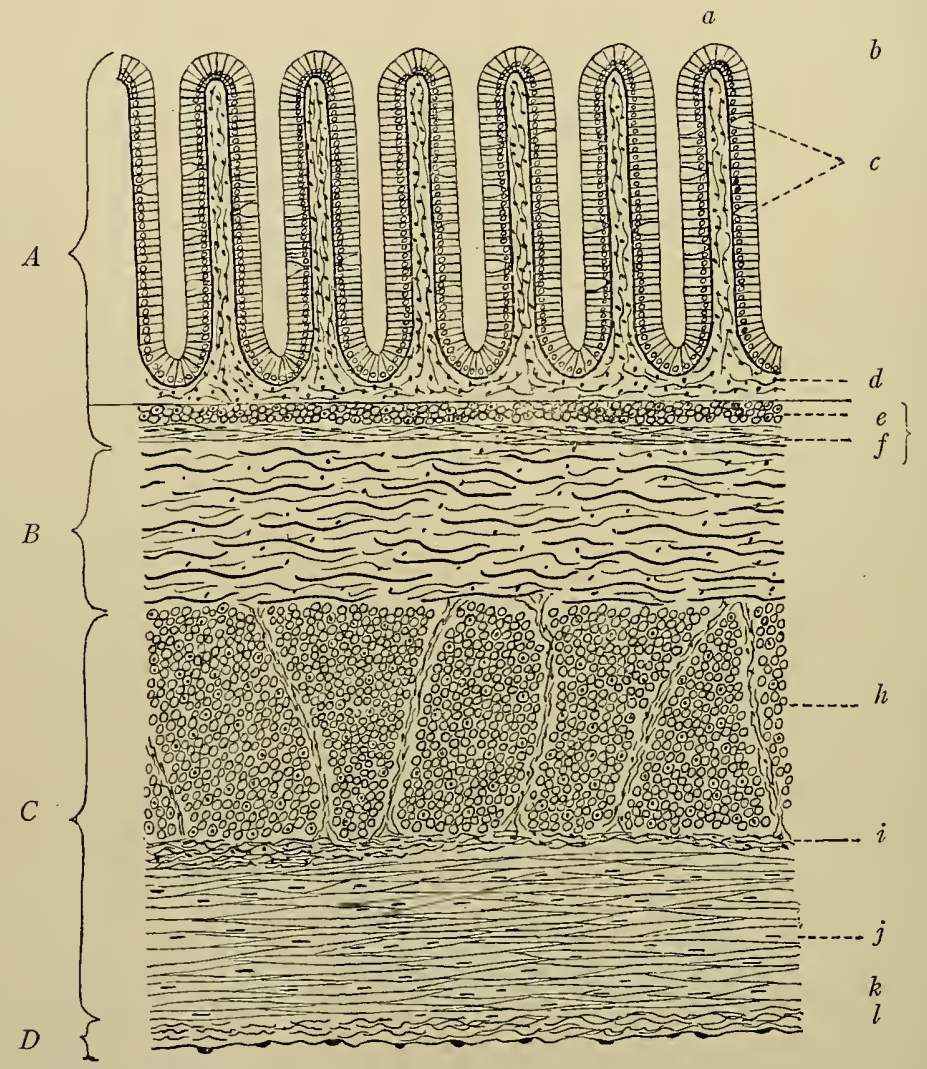

FIG. I48.-Diagram of Structure of Wall of Gastro-intestinal Canal. A, Mucous membrane; $a$, glands; $b$, epithelium; $c$, goblet cells; $d$, stroma; $e$, inner circular; $f$, outer longitudinal layers of $g$, muscularis mucosæ. $B$, Submucosa. $C$, Muscular coat; $h$, its inner circular layer; $j$, its outer longitudinal layer; $i$, intermuscular connective-tissue septum. $D$, serous coat; $k$, its connective-tissue layer; $l$, its endothelial layer.

2. The submucosa (Fig. I48) is a loose connective-tissue stucture. It contains the larger blood-vessels, lymphatics, and nerves.

3. The muscular coat (Fig. I48) consists of two layers of smooth muscle, which in the intestine are sharply differentiated into an inner circular and an outer longitudinal. In the stomach the direction of the layers of the muscular coat is less definite. A narrow layer of 
connective tissue separates the two layers of muscle. From this, septa extend in to the muscle tissue, separating it into bundles.

4. The serous coat (Fig. I48) is the visceral layer of the peritoneum. It consists of a thin layer of connective tissue covered by a single layer of mesothelium. Along the attachment of the mesentery the serous coat is wanting.

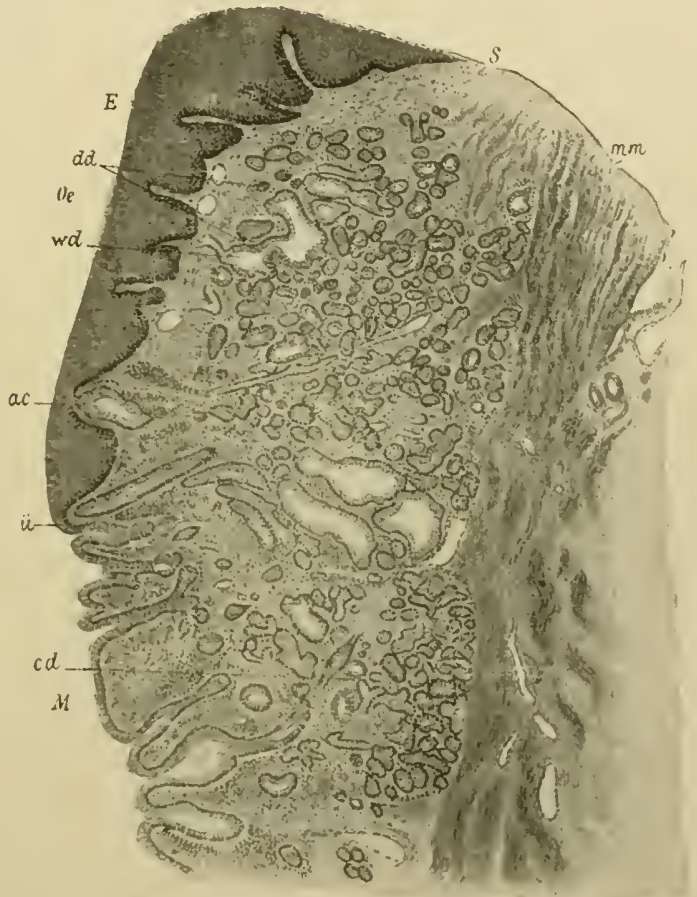

Fig. I49.-Section through Junction of Esophagus and Stomach of Man. XI2I. (Schäfer.) Oe, Esophagus; iI, stom:tch; $c d$, carcliac glands; $w$, dilated ducts of cardiac glands; $S$, stroma; $E$, stratified squamous epithelium of oesophagus; $m m$, muscularis mucosæ; $c d$, irregularly cut tubules of cardiac glands; $d d$, cardiac glands in lower end of the cesophagus; $i i$, limit of stratified cesophageal epithelium.

The subdivisions of the gastro-intestinal canal differ from one another mainly in regard to the structure of their mucous membranes, and especially in regard to the structure of the glands of the mucous membrane and submucosa.

\section{The Stomach}

At the junction of (xesophatgus and stomach there is atn abrupt transition from the stratified squamous ejpithelium of the former with 
its smooth surface to the simple columnar epithelium of the latter with its elevations and depressions. In the deeper structures the line of demarcation is not so abrupt, the muscularis mucosæ of the œsophagus being continuous with that of the stomach, and glands of the stomach type extending up under the stratified epithelium of the œsophagus (Fig. I49).

I. The mucous membrane of the stomach is folded into ridges or ruge, the height and number of which depend, as already noted, upon

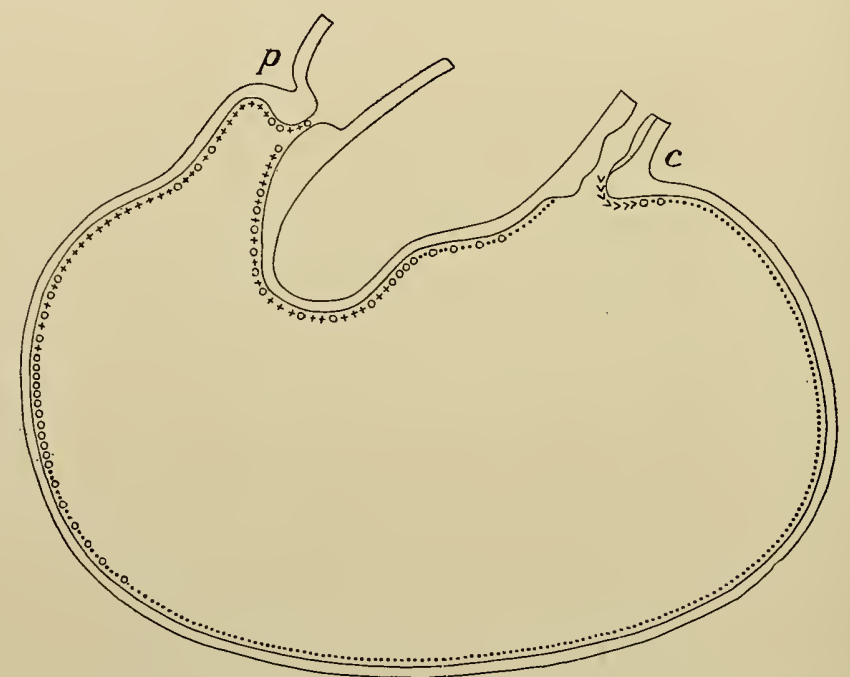

FIG. I 50.- Outline Diagram of Stomach to showLocation of Different Kinds of Glands. $c$, Cardia; $p$, pylorus; vvvvvv, cardiac glands; ......., fundus glands; +++++ , pyloric glands; $\infty 0^{\circ}, 000$, intestinal type glands (of Lieberkuhn). (Jouvenal.)

the degree of distention of the organ. The rugæ are most prominent in the collapsed organ, almost absent when the organ is fully distended. In addition to the rugæ the entire mucous membrane is studded with minute depressions barely visible to the naked eye, the so-called gastric pits (Fig. I52, $M g$ ). These mark the openings of the gastric glands. In the fundus they are comparatively shallow, extending through about one-fifth the thickness of the mucosa; in the pylorus the pits are much deeper, extending through half or more of the thickness of the mucous membrane (compare Figs. I 52 and I 56 ).

The Epithelium.-This is of the simple columnar type, covers the entire surface of the gastric mucosa and extends down into the pits. (Fig. I 52). The cells are of the high, clear, mucous type (Fig. I53, $M$ and $M^{\prime}$ ). The end of the cell toward the lumen is clear, usually 
consists mostly of mucus, and consequently stains lightly. There is no such distinct cuticle as in the intestine. The basal end of the cell contains the spheroidal, oval, or sometimes flattened nucleus, is granular, and takes a darker stain. The amount of mucus in the cell depends upon its functional condition. The cells rest upon a distinct basement membrane.

The Gastric Glands.-Extending from the bottoms of the pits, their epithelium continuous with that of the pits themselves, are the gastric glands. These are of two main kinds, fundus or peptic glands,

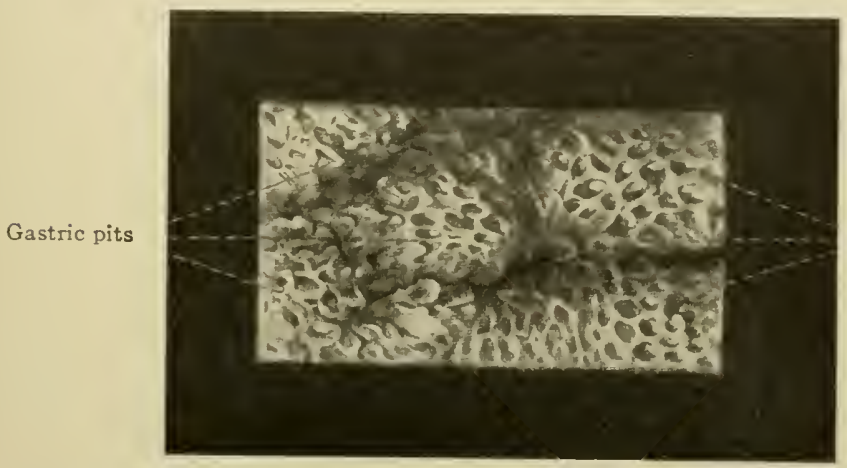

Gastric pits

FIG. I5I.-Surface View of Mucous Membrane of Stomach showing gastric pits (Spalteholz).

distributed through the greater part of the gastric mucosa, and $p y$ loric glands, confined to the immediate region of the pylorus.

The fundus glands (Fig. 152) are simple, sometimes branched, tubular glands, of which from three to seven open into each gastric pit. They extend through the entire thickness of the stroma, to the muscularis mucosæ.

Each gland consists of (I) a mouth opening into the pit; (2) a constricted portion, the neck; (3) the body or main portion of the tubule; and (4) a slightly dilated and bent blind extremity, the fundus (Fig. I 52). The mouth marks the transition from the higher epithelium of the pit to the low cuboidal of the neck (Fig. I53, h). In the body and fundus of the gramel two types of cells are found: (a) chief cells (central, peptic, or adelomorphous), and (b) parictal cells (acirl, oxyntic, or delomorphous).

The chief cells (Figs. I 53, 154) are the more numerous. They are of the low columnar type, of ten pyramidal with apjeces directed toward the lumen. Their bases rest either on the basement membrane or 
against the parietal cells. The appearance which these cells present depends upon their functional condition (p. 272). They usually appear clear and granular and take a light stain.

The parietal cells (Fig. I53,) are oval or polygonal in shape, and

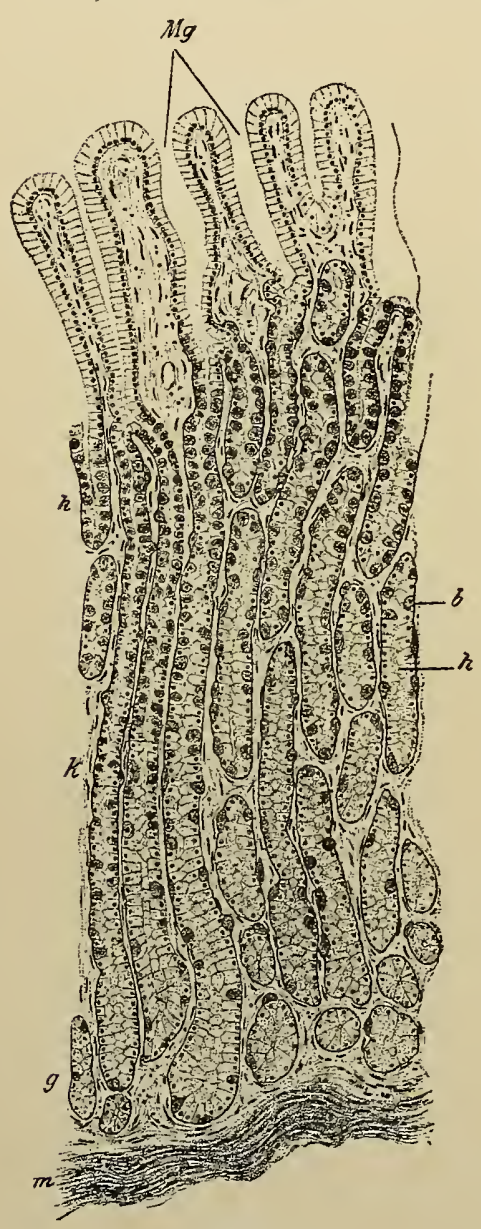

FIG. I 52 . lie here and there against the basement membrane. The nucleus is spherical, somewhat larger than that of the chief cell, and is usually situated at the center of the cell. There may be two nuclei. The protoplasm is finely granular and in the fresh unstained condition appears clearer than that of the chief cells. The parietal cells stain intensely with the aniline dyes with the result that in

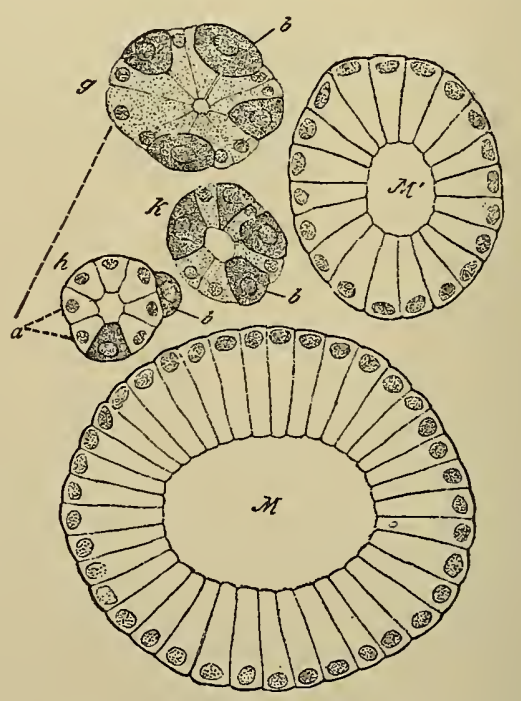

Fig. I53.

Fig I52.-Vertical Section through the Mucous Membrane of the Fundus of the Stomach. $\times 85$. (Kölliker.) $M g$, Gastric pits; $h$, neck; $k$, body; $g$, fundus of peptic glands; $h$, chief cells; $b$, parietal cells; $m$, muscularis mucosæ.

FIG. I53.-Cross-sections at Various Levels of Peptic Glands of Stomach. $X_{400}$. (Kölliker.) $M$, Section through gastric pit near surface; $M^{\prime}$, section through gastric pit near bottom; $h$, mouth of gland; $k$, neck; $g$, body near fundus; $a$, chief cells; $b$, parietal cells. :

stained specimens the two kinds of cells are in marked contrast, the parietal cells being much darker than the chief cells. Although lying against the basement membrane and frequently pushing it out so as 
to form little protuberances beyond the even line of the gland tubule, the parietal cells always maintain a connection with the lumen. This is accomplished by means of little clefts between the chief cells, intercellular secretory tubules, which extend down to the parietal cells. $\mathrm{By}$ means of the method of Golgi may be demonstrated not only the intercellular secretory tubules, but also the fact that upon reaching the cells these are continuous with a network of minute spaces within the cell-the intracellular secretory tubules (Figs. I 54 and I55). Parietal

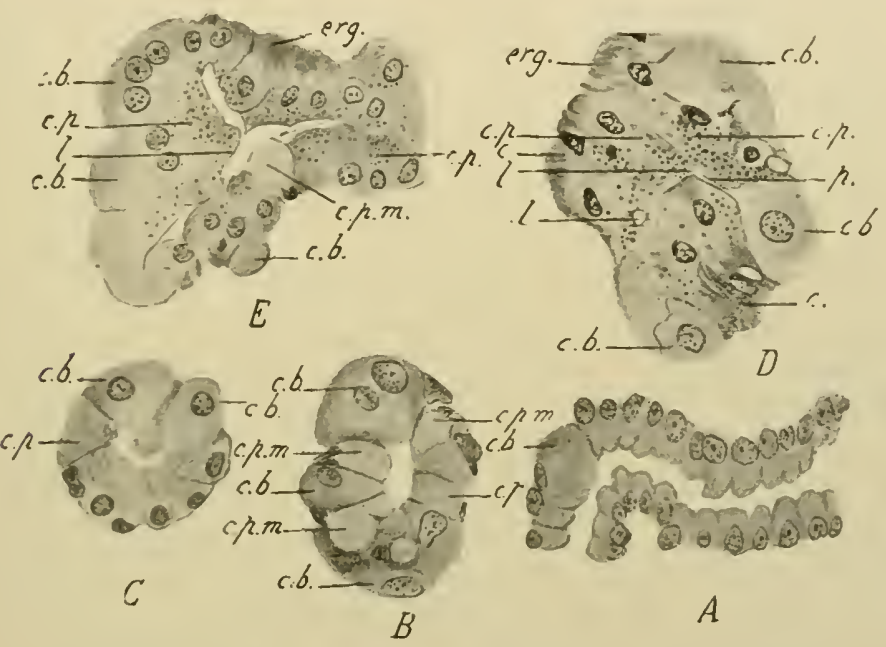

Fic. I 54.- Sections through Different Parts of a Fundus Gland of Human Stomach.

1 , mouth of gland; cells resemble surface cells except that they are shorter.

$B$, neck of gland; $c p$, principal cells; $c p m$, mucous cells; $c b$, parietal cells, one containing two nuclei.

$C$, midrlle portion of gland; $c p$, chief cells; $c b$, parietal cells.

$D$, deeper part of gland; $c p$, chief cells containing secretory granules, and at their bases ergastoplasm filaments $(\mathrm{erg})$ : at $\sigma$ are shown intracellular secretory canals penetrating base of cell; $c b$, parietal cells, one of which shows canaliculus leading to lumen of gland ( $\mathrm{r}$ ), the latter being cut twice in section owing to its irregular course.

$E$, blind end of gland; $c p$, chief cells with secretion granules and ergastoplasm filaments (erg); $c b$, parietal cells, one containing three nuclei; $c$ pm, mucous cells; $l$, lumen of gland. X250. (I'renant.)

cells are not distributed uniformly throughout the gland, but are most numerous in the body, where they frequently almost obscure the chief cells. In the fundus of the gland parietal cells are less numerous. For this reason and because of the wider lumen of the funclus, transverse and longitudinal sections of this part of the tubule are most satisfactory for the study of the relations of the two kinds of cclls (Figs. $5^{2}$ and 15.3 ). Mitosis is most active at the junction of 
the neck and body of the tubule which has consequently been designated the "growing point" of the tubule.

Lying near the basement membrane among the bases of the columnar epithelial cells are small spherical or irregular cells with dark nuclei. These are young epithelial cells which from their function are known as "replacing cells" (see page 73).

The PYLORIC GLANDS (Figs. I56 and ${ }_{5} 57$ ) are simple branched tubular glands, several of which open into each of the deep pyloric pits.

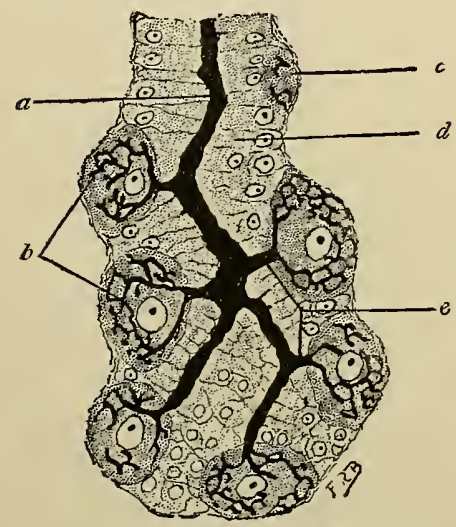

FIG. I 55--Longitudinal Section of Fundus of Gland from Pyloric End of Dog's Stomach. (Golgi method. See 5, p. 29.) a, Lumen of gland; $b$, intracellular canals in parietal cells; $c$, cut-off portion of parietal cell; $d$, chief cells; $e$, intercellular canals leading from lumen of gland to canals in parietal cells.
The glands, though short, are quite tortuous, so that in sections the tubules are seen cut mainly transversely or obliquely. In most of the pyloric glands but one type of cell is found. These resemble the chief cells of the fundus, but present a more uniform appearance, probably due to the absence of parietal cells. As in the fundus, "replacing cells" lie between the bases of the columnar epithelial cells. Parietal cells are not always entirely absent, but occur here and there in the pyloric tubules, especially near the fundus.

The transition from fundus to pylorus is not abrupt, but is marked by a "transitional border zone," in which fundus and pyloric glands are intermingled, and in which are also found single glands which resemble in structure both cardiac and pyloric.

In the transition zone between œsophagus and stomach are found glands which resemble those found in the lower end of the osophagus (p. 244). Their cells are clear resembling those of the pyloric glands. Some of the tubules have parietal cells. They have been designated cardiac glands and pass over by gradual transition into the fundus glands. There are also found in the stomach glands which are apparently identical in structure with those of the intestine (p. 260). Most of these resemble the glands of Lieberkühn and are distributed in small groups mainly in the pylorus and lesser curvature. Glands resembling Brunner's glands have also been described.

The STROMA (Figs. I 52 and I 56 ) -or TUNICA PROPRIA, in which the glands are embedded, consists of mixed fibrillar and reticular connective 
tissue infiltrated with lymphoid cells. In the fundus of the stomach the glands are so closely packed that the stroma is reduced to thin strands, which pass up between the glands and also separate them from the muscularis mucosæ. In the pylorus the glands are more widely separated and the stroma is correspondingly greater in amount. In both fundus and pylorus thicker strands of stroma surround a num-

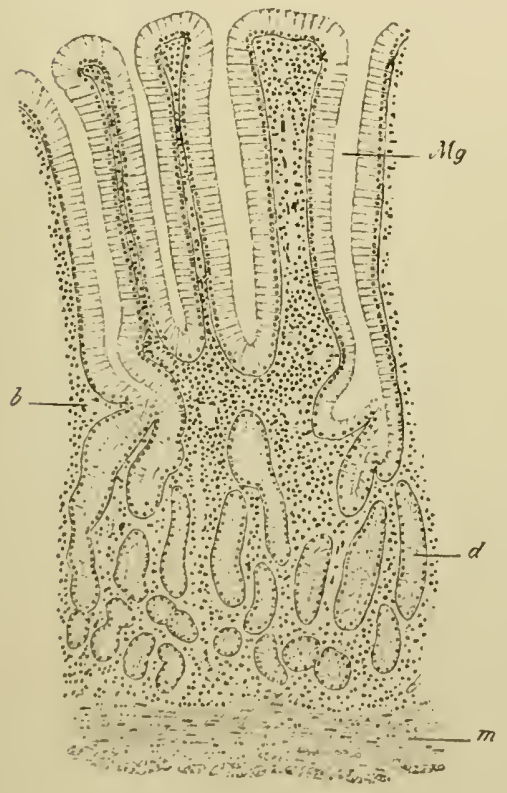

IFIG. I 56 .

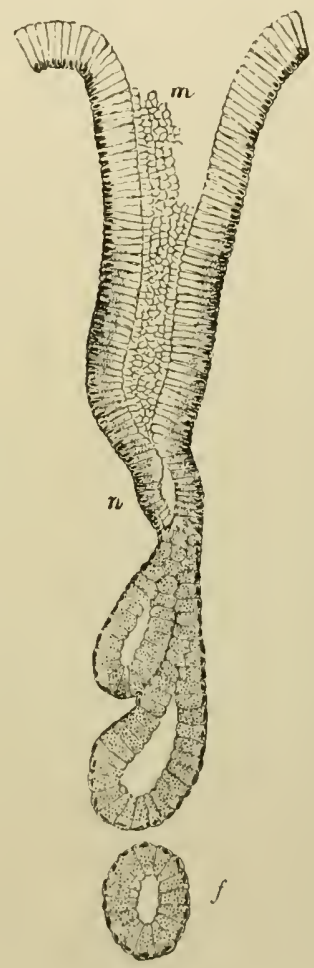

FIG. 157 .

FIG. I 56.-Vertical Section through Mucous Membrane of Pyloric End of Stomach. $\times 85$. (Kölliker.) $M g$, Gastric pit; $b$, blood-vessel in stroma; $d$, longitudinal section of bordy of gland; $m$, muscularis mucosæ.

FIG. I 57.- I'yloric Gland from Vertical Section through Wall of Dog's Stomach. (F),stein.) $m$, Gastric pit in which are seen some transversely cut cells; $n$, neck of gland; $f$, fundus cut transwersely.

ber of gland tubules, thus separating them into more or less welldefined groups. In addition to the diffuse lymphatic tissue of the stroma, closely packed aggregations of lymphoid cells are found in the shape of distinct nodules, known as "solilary follicles." 'These occur throughout the entire gastric mucosa, but are most numerous in the pylorus. 'The nodules are usually egg-shaperl, their apices 
lying just beneath the epithelium, their bases resting upon the muscularis mucosæ. Less commonly they lie partly in the submucosa. Over the nodules the epithelium is more or less infiltrated with migratory leucocytes. Most of the nodules contain germinal centres around which the lymphoid cells are more closely packed than elsewhere (see page I69).

The MUSCULARIS MUCOS 2 (Figs. I 52 and $156, m$ ) may consist of a single layer of smooth muscle with cells arranged longitudinally or obliquely, or there may be two distinct layers, an inner circular and an outer longitudinal. From the muscularis mucosæ single cells and groups of cells extend into the stroma between the gland tubules.

2. The submucosa consists of connective tissue, loosely arranged, many elastic fibres, and some fat. It contains the larger bloodvessels and nerves, including the plexus of Meissner (p. 27 I).

3. The muscular coat is usually described as consisting of three layers, an inner oblique, a middle circular, and an outer longitudinal.

In the fundus the muscle bundles run in various directions, so that the division of the muscular coat into layers having definite directions can be made out only in the pylorus. Here the inner and middle layers are thickened to form the sphincter pylori. In the connective tissue which separates the groups of muscle cells are collections of sympathetic nerve cells and fibres, which while much less distinct, are analogous to Auerbach's plexus of the intestine.

4. The serous coat consists of a layer of loosely arranged connective tissue covered by a single layer of mesothelium.

\section{TECHNIC}

(I) Remove a human stomach (not more than two or three hours after death) or that of a recently killed dog. Open along the lesser curvature, and carefully remove the excess of mucus by washing with normal saline. Cut pieces through the entire thickness of the wall, one from the fundus and one from the pylorus; pin out, mucous membrane side up, on pieces of cork, fix in formalin-Müller's fluid (technic 5, p. 7) or in Zerker's fluid (technic 9, p. 8), and harden in alcohol. Sections are cut as thin as possible, care being taken that the plane is such that the glands are cut longitudinally, stained with hæmatoxylin-eosin (technic I, p. 20), and mounted in balsam.

(2) Instead of removing pieces of stomach and pinning them out on cork, as suggested in the preceding technic, the entire stomach may be filled with the fixative, the ends being tied, and then placed in a large quantity of the fixing fluid. After fixation, pieces are removed and hardened in graded alcohols. If this method is used, great care must be taken not to overdistend the organ, only 
very moderate distention being desirable. Further treatment is the same as in the preceding technic (I).

(3) For comparison of resting with active gastric cells, preparations should be made from the stomach of an animal that has been for from twenty-four to fortyeight hours without food, and from a stomach during active digestion. Fix in Zenker's fluid as in technic (I), above. Examine unstained sections and sections stained with hamatoxylin-eosin.

(4) Sections through the junction of œsophagus and stomach and through the junction of stomach and duodenum furnish instructive pictures. They should be prepared as in technic ( $\mathrm{I}$ ).

(5) For the study of the distribution of the blood-vessels sections of an injected stomach should be made. This is best accomplished by selecting a small animal, such as a rat or guinea-pig, and injecting in toto through the ascending aorta, or by injecting only the hind part of the animal through the abdominal aorta. Technic, p. 25.

\section{THE MIDGUT}

\section{The Small Intestine}

On passing from stomach to small intestine the ruga of the former disappear, but are replaced by much more definite foldings of the mucosa, the valinla connivenles (Fig. I 59). These folds involve the entire thickness of the mucous membrane and part of the submucosa. They are in general parallel to one another, and pass in a circular or oblique manner, partly around the lumen of the gut. The entire surface of the intestine, including the valvula, is studded with minute projections just visible to the naked eye, and known as villi (Figs. I6o and r6r). These involve only the epithelium and stroma, although they also contain some muscular elements derived from the muscularis mucosx. The villi differ in shape in the different parts of the small intestine, being leaf-shaped in the duodenum, rounded in the jejunum, club-shaped in the ileum. The valvula conniventes and the villi are characteristic of the small intestine. It is important to note that while the pits of the stomach are depressions in the mucous membrane, the intestinal villi are definite projeclions aboie its general surface (Fig. ${ }_{5} 8$ ).

The wall of the intestine consists of the same four coats described as constituting the wall of the stomach, mucosa, submucosa, musculuris, and serosa.

1. The mucosa, as in the stomach, is composed of a lining cpilhelium, stroma, glands, and muscularis mucose. ()f these, the epithelium, 
the stroma, and cells from the muscularis mucosæ, are concerned in the formation of the villi.

The VILLUs consists of a central core-a fold of the stroma-of mixed fibrous and reticular tissue infiltrated with lymphoid cells, and of a covering epithelium.

The epithelium is disposed in a single layer, separated from the underlying connective tissue by a basement membrane. It consists of two apparently quite different kinds of cells, columnar cells and goblet or mucous cells. The former are the more numerous and are peculiar

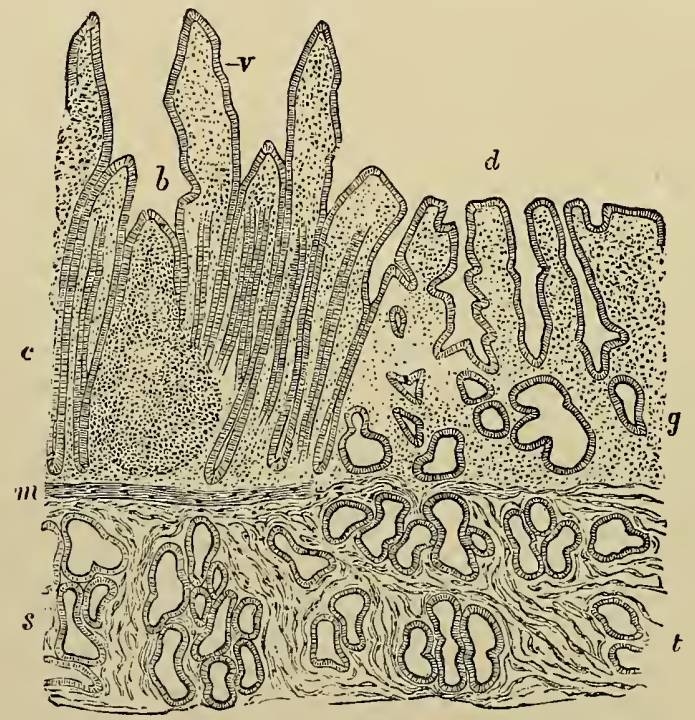

FIG. I 58.- Section through Junction of Pylorus and Duodenum. (Klein.) V, Villi of duodenum; $d$, stomach, showing gastric pits; $b$, apex of a solitary lymph nodule; $c$, crypt of Lieberkühn; $s$, secreting tubules of Brunner's glands; $g$, pyloric glands; $t$, tubules of Brunner's glands in submucosa of stomach; $m$, muscularis mucosæ.

to the intestine, while the mucous cells are identical with those found in other mucous membranes. The columnar cells are quite elastic and while generally long and narrow, vary as to length and breadth as they adapt themselves to movements of the intestine. The peculiarity of these cells is a striated free border, their contiguous free borders uniting to form the so-called cuticular membrane (Fig. I63, c). By means of special technic these striations can be resolved in to delicate parallel lying rods or fibrils. They have been interpreted by some as marking channels through the cuticle, by others as a fibrillar arrangement of the protoplasm. In some forms tiny swell- 
ings at the proximal ends of the fibrils, resembling the basal granules of ciliated cells (p. 76 ) have led to the suggestion that the fibrils are allied to cilia. The nuclei of these cells are ovoid and usually lie near the middle or in the base of the cell. Scattered among the columnar cells are the mucous or goblet cells (Figs. $16_{2}$ and $16_{3}, b$ ). These vary in appearance according to the amount of secretion which they contain. A cell at the beginning of secretion contains only small amount of mucus near its free border. As secretion increases the

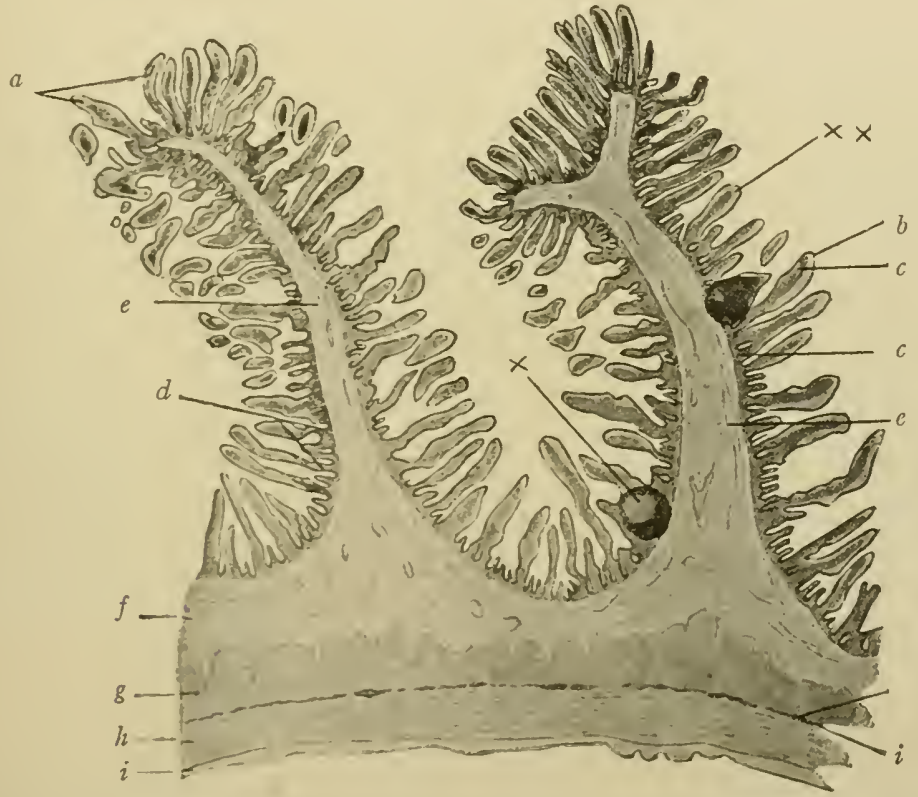

Frg. I 59.-Vertical Longitudinal Section of Human Jejunum $\left(X_{16}\right)$ (Stöhr), including two valvulæ conniventes. $a$, Villi, in many of which the stroma has shrunken away from the epithelium leaving a clear space, $X \times$. Lying free in the lumen of the gut are seen sections of villi cut in various directions. $b$, Epithelium; $c$, stroma; $d$, crypts of Lieberkühn; $X$, solitary lymph nodule with germinal centre; $e$, tissue of submucosa forming centre of one of the valvula conniventes; $f$, submucosa; $g$, inner circular layer of muscle; $h$, outer longitudinal layer of muscle; $i$, Auerbach's plexus; $j$, scrous coat.

mucus gradually replaces the cytoplasm until the latter is represented only by a crescentic mass containing a flattened nucleus and pressed against the basement membrane. The cell now discharges its mucus upon the free surface. The goblet cells possess no thickened border, appearing, when seen from the surface, as openings surrounded on all sides by the cuticulie of the adjacent columnar cells. Opinions differ as to the relation of the mucous to the columnar cell. Some authorities consider them two different and independent cells. The 
majority, however, look upon the mucous cell as specialization of the columnar cell. Again opinions differ as to whether the mucous cell, having discharged its secretion, dies or re-secretes, or returns to the

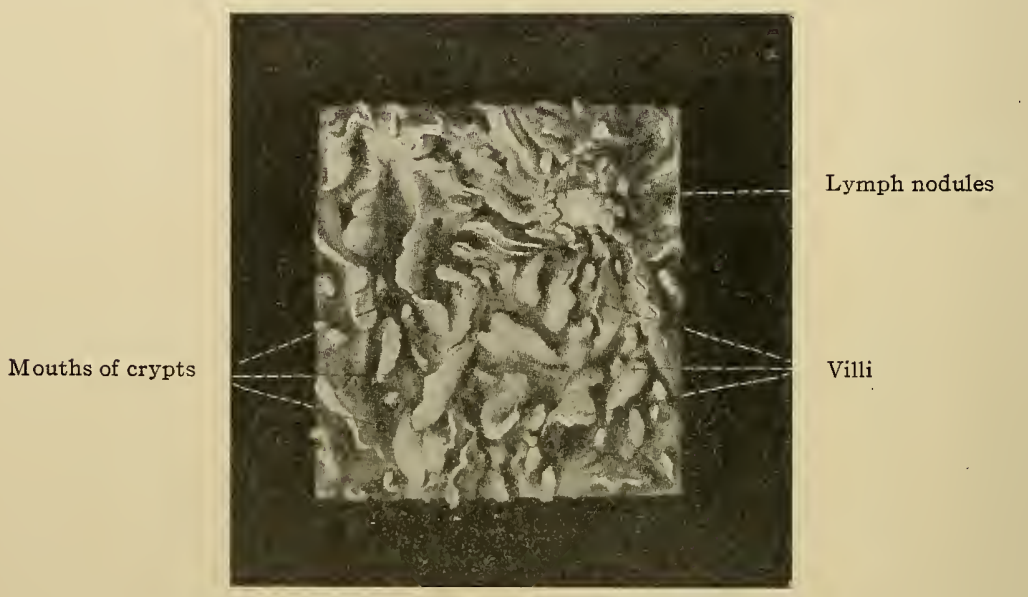

FIG. I60.-Surface View of Small Intestine near upper end, showing villi and one solitary lymph nodule. $X_{\mathrm{I} 2}$. (Spalteholz.)

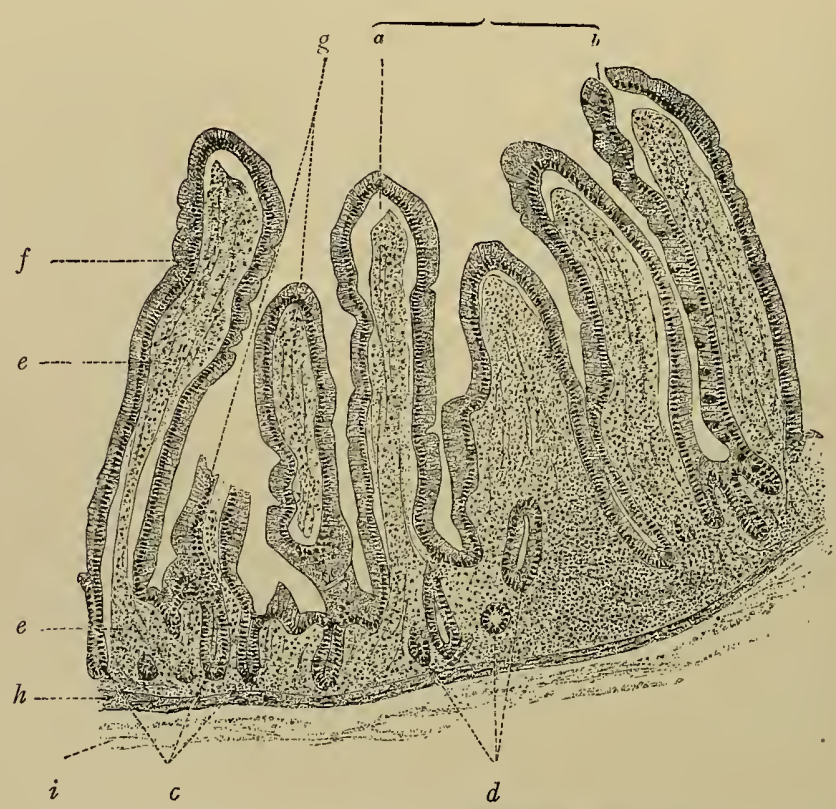

FIG. I6I.-Vertical Section through Mucous Membrane of Human Jejunum. $\times 80$. (Stöhr.) $a$ and $b$, Artifacts due to shrinkage; $c$, intestinal crypts (Lieberkühn); $d$, obique and transverse sections of crypts; $e$, stroma; $f$, epithelium; $g$, tangentially cut villi; $h$, muscularis mucosæ; $i$,submucosa. 
condition of the columnar cell. Small spherical cells with deeply staining nuclei are found in varying numbers among and sometimes within the epithelial cells. ${ }^{1}$ These are so-called wandering cells, migratory leucocytes, from the underlying stroma (Figs. $162, h$, and $\left.{ }^{1} 6_{3}, l\right)$. Other cells with dark-staining nuclei, "replacing cells," are found between the bases of the columnar cells (pages 73 and 252).

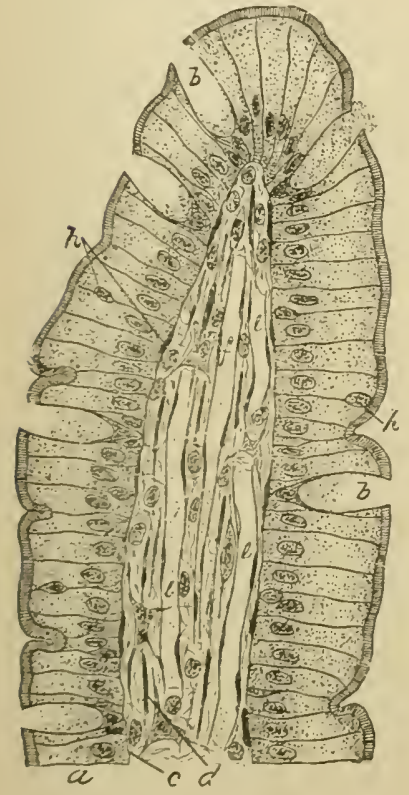

FIG. I62.

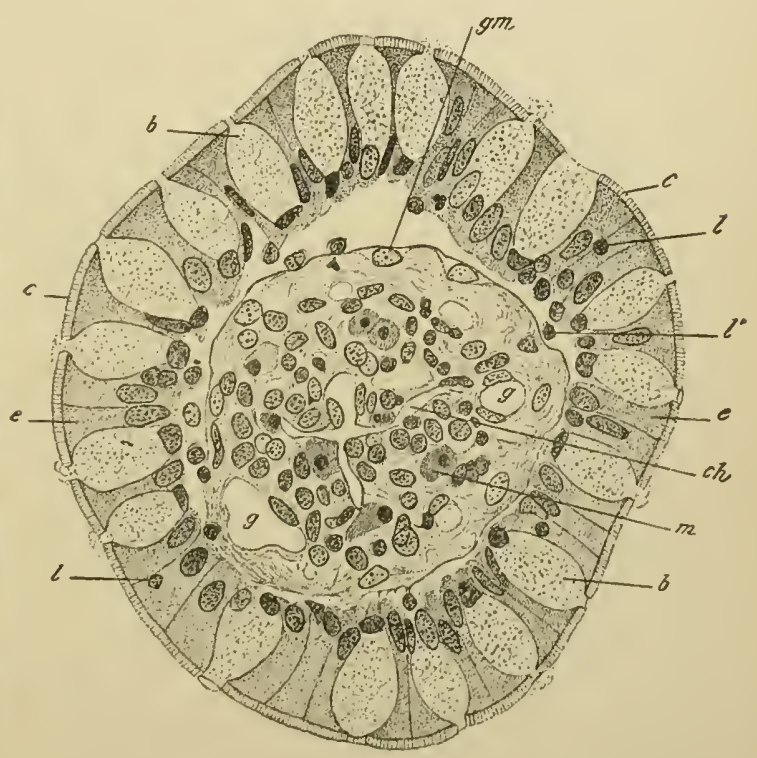

FIG. 163 .

FIG. 162.-Longitudinal Section of Villus from Small Intestinc of Dog. (Piersol.) $a$, Columnar epithelium; $b$, goblet cells; $h$, leucocytes; $c$, basement membrane; $d$, core of villus; $e$, blood-vessels; $f$, lacteal.

FIG. 163.-Cross-section of a Villus of Human Small Intestinc. $\times, 530 . \quad$ (Kölliker.) The stroma of the villus has shrunken away from the epithelium. $b$, Goblet cell; $c$, cuticula showing striations; $e$, columnar epithelial cell; gm, basement membrane with nuclei; $l$, leucocyte in epithelium; $l^{\prime}$, leucocyte just beneath epithelium; $m$ large leucocyte in stroma; ch, central chyle vessel; $g$, blood-vessel.

In addition to the connective-tissue and lymphoid cells, which constitute the main bulk of the villus core (Figs. 162 and ${ }^{6} 6_{3}$ ), isolated smooth muscle cells deriverl from the muscularis mucos $x$ occur, running in the long axis of the villus. A single lymph or chyle vessel (Fig. $\mathrm{x} 62, f ; 163, c h$ ) with distinct endothelial walls traverses the centre of cach villus, ending at its tip in a slightly dilated blind extremity. As it is usually scen colliujsed, it appears as two closely

1 J avideff consirlers these cells leucocyles in process of formation. He descriloes some of the columnar cells as having two nuclei, one of which remains within the cell, while the other is extruded with a little cytoplasm as a leucocyte. 
approximated rows of flat cells with bulging nuclei. The capillaries of the villus lie for the most part away from the chyle vessel, just beneath the basement membrane (Fig. I62, $e$; $16_{3}, g$ ).

From the depths of the depressions between the villi, simple tubular glands-glands or crypts of Lieberkühn (Figs. I6I and I64)--extend down through the stroma as far as the muscularis mucosæ. These crypts are lined with an epithelium similar to and

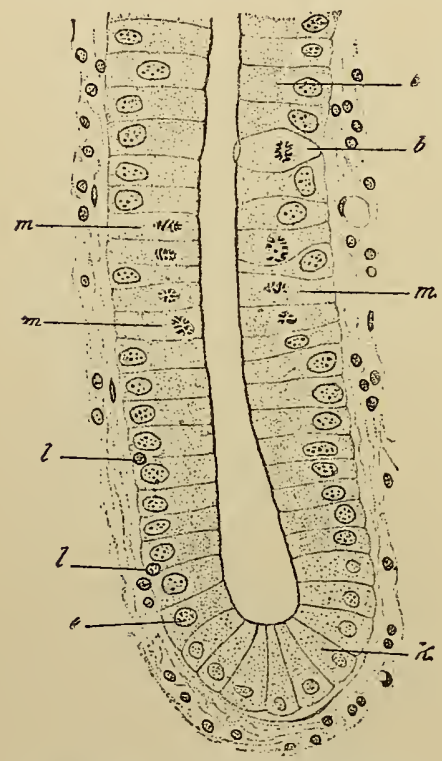

FIG. I64.-Longitudinal Section of Fundus of Crypt of Lieberkühn. $\times 530$. (Kölliker.) b, Goblet cell showing mitosis; $e$, epithelial cell; $k$, cell of Paneth; $l$, leucocyte in epithelium; $m$, mitosis in epithelial cell. Surrounding the crypt is seen the stroma of the mucous membrane.

are found in the stomach (see page 253 ).

Peyer's Patches (agminated follicles) (Fig. I65).-These are groups of lymph nodules found mainly in the ileum, especially near its junction with the jejunum. They always occur on the side of the gut opposite the attachment of the mesentery. Each patch consists of from ten to seventy nodules, so arranged that the entire patch has a generally oval shape, its long diameter lying lengthwise of the intestine. The nodules of which a patch is composed lie side by side. Their apices are directed toward the lumen and project 


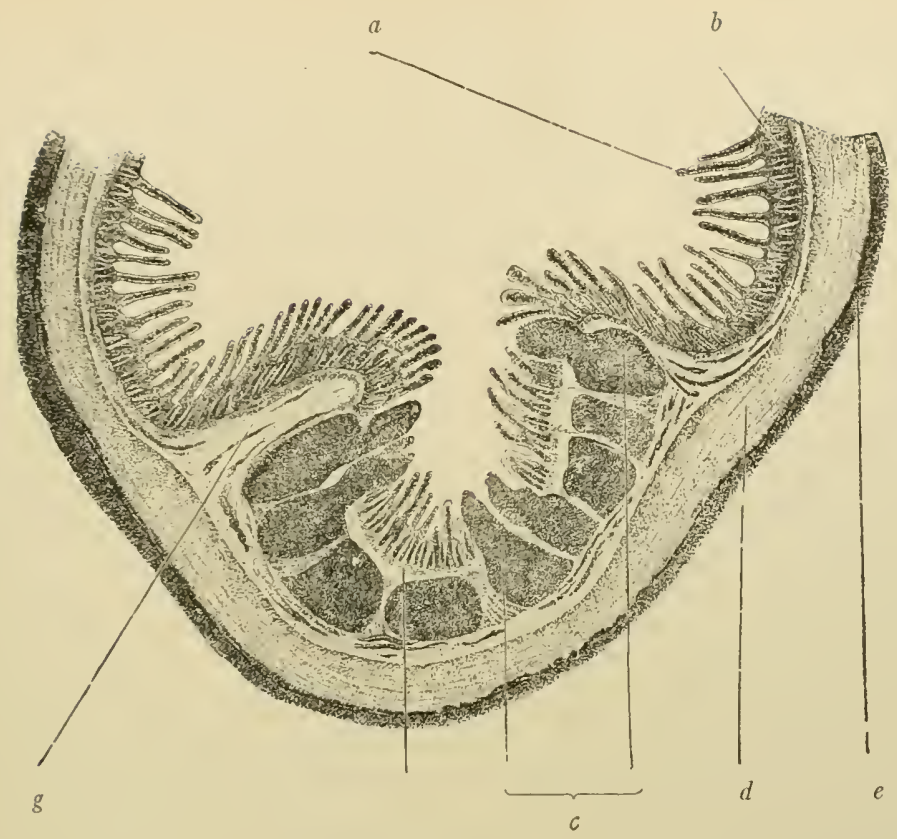

Fig. 165.- Transverse Section of Cat's Small Intestine through a l'eyer's Patch. (Stöhr.) $a$, Villi; $b$, crypts; $c$, longitudinal muscle layer; $d$, circular muscle layer; $e$, lymph nodules; $f$, muscularis mucos $x ; g$, submucosa.

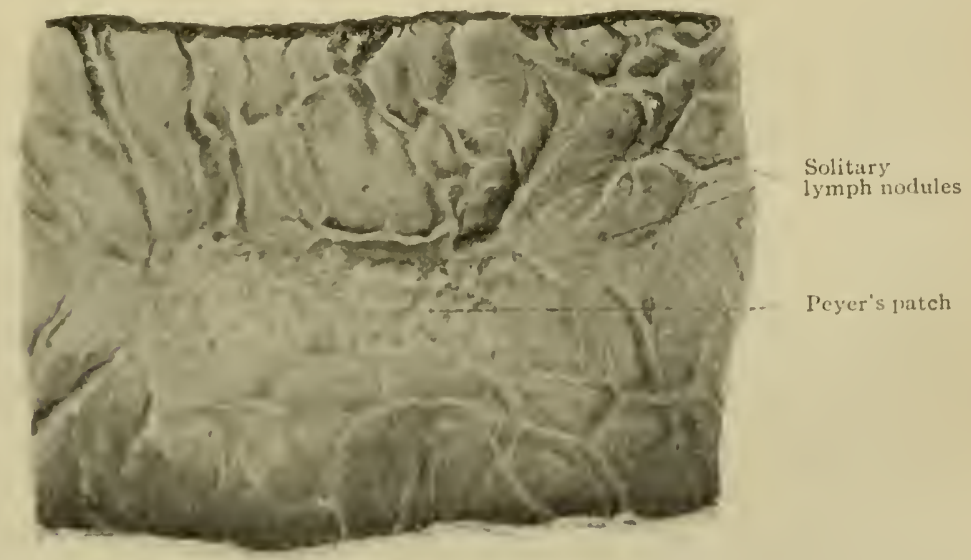

Fig. I 60 , Surface View of Mucous Vembrane of Simall Intestine (Ileum), showing Peyer's patelo (Spalteholz). 
almost through the mucosa, being uncovered by villi, a single layer of columnar epithelium alone separating their surfaces from the lumen of the gut. The bases of the nodules are not confined to the stroma, but usually spread out in the submucosa. The relation of the patch to the stroma and submucosa can be best appreciated by following the course of the muscularis mucosæ. This is seen to stop

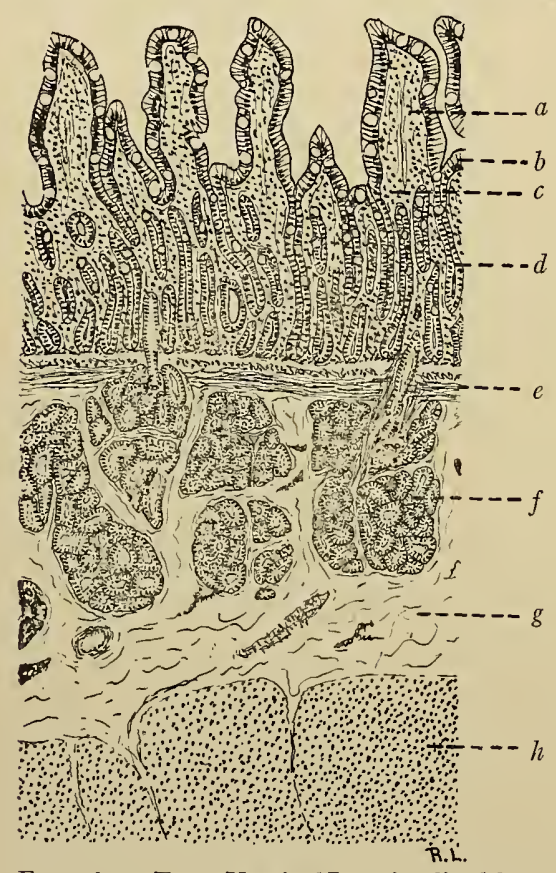

FIG. I67.-From Vertical Longitudinal Section of Cat's Duodenum to show Brunner's Glands. (Larrabee.) $a$, Villus; $b$, epithelium; $c$, stroma; $d$, glands; $e$, muscularis mucosæ; $f$, Brunner's glands; $g$, submucosa; $h$, circular muscle layer.

abruptly at the circumference of the patch, appearing throughout the patch as isolated groups of smooth muscle cells. The nodules rarely remain distinct, but are confluent with the exception of their apices and bases. It should be noted that both solitary nodules and Peyer's patches are structures of the mucosa, and that their presence in the submucosa is secondary.

THE MUSCULARIS MUCOSA (Figs. I6I and I67) consists of an inner circular and an outer longitudinal layer of smooth muscle.

2. The submucosa (Figs, I 59, I6 1. I67) consists, as in the stomach, of loosely arranged connective tissue and contains the larger blood-vessels. It is free from glands except in the duodenum, where it contains the glands of Brunner (Fig. I67). These are branched tubular glands lined with a granular columnar epithelium similar to that of the pyloric glands. The ducts are also lined with simple columnar epithelium. They pass through the muscularis mucosæ and empty either into a crypt of Lieberkühn or on the surface between the villi. Brunner's glands frequently occur in the pylorus, and it is not uncommon for the pyloric glands to extend downward somewhat into the duodenum. Meissner's plexus of nerve fibers, mingled with groups of sympathetic ganglion cells, lies in the sub-mucosa (see page $27 \mathrm{I}$ ). 
3. The muscular coat (Figs. I59 and 167 ) consists of two welldefined layers of smooth muscle, an inner circular and an outer longitudinal. Connective-tissue septa divide the muscle cells into groups or bundles, while between the two layers of muscle is a connective-tissue septum which varies greatly in thickness at different places and contains a plexus of nerve fibres and sympathetic ganglion cells known as the plexus of Auerbach (see page 270).

4. The serous coat consists as in the stomach of loose connective tissue covered by a single layer of mesothelium.

\section{THE ENDGUT}

\section{The Large Intestine}

The wall of the large intestine consists of the same four coats which have been described as constituting the walls of the stomach and small intestine, mucous, submucous, muscular, and serous.

I. The mucous membrane has a comparatively smooth surface, there being neither pits as in the stomach nor villi as in the small intestine (Fig. 168). The glands are of the simple tubular variety, are considerably longer than those of the small intestine, are almost straight, and extend through the entire thickness of the stroma. Owing to the closeness with which the gland tubules are packed, the amount of stroma is usually small. The surface cells (Fig. I68, a) are very high and narrow, with small, deeply placed nuclei, and are not usually intermingled with goblet cells. Passing from the surface down into the glands, the cells become somewhat lower and goblet cells become numerous (Fig. I69, $a$ and $d$ ). Both superficial and deep cells rest upon a basement membrane similar to that in the small intestine. The stroma also, though less in amount, is similar in structure to the stroma of the small intestine.

The MUSCULARIS MUCOSA (Fig. I 69, c) consists of an inner circular and an outer longitudinal layer of smooth muscle.

2. The submucosa (Fig. I68, e) consists of loosely arranged connective tissue. It contains large blood-vessels and the nerve plexus of Mcissner (sec page 271). Solitary lymph follicles occur throughout the mucous membranc of the large intestine. While properly considered as structures of the stroma from which they originate, the follicles lie mainly in the submucosit. (For details of structure see page 169.$)$ 
3. Of the muscularis (Fig. I68) the inner circular layer only is complete, the muscle tissue of the external longitudinal coat being arranged mainly as three strong, flat, longitudinal bands, the lineæ coli. Between these bands the longitudinal muscular coat is either very thin or entirely absent. In the connective tissue, lying to the

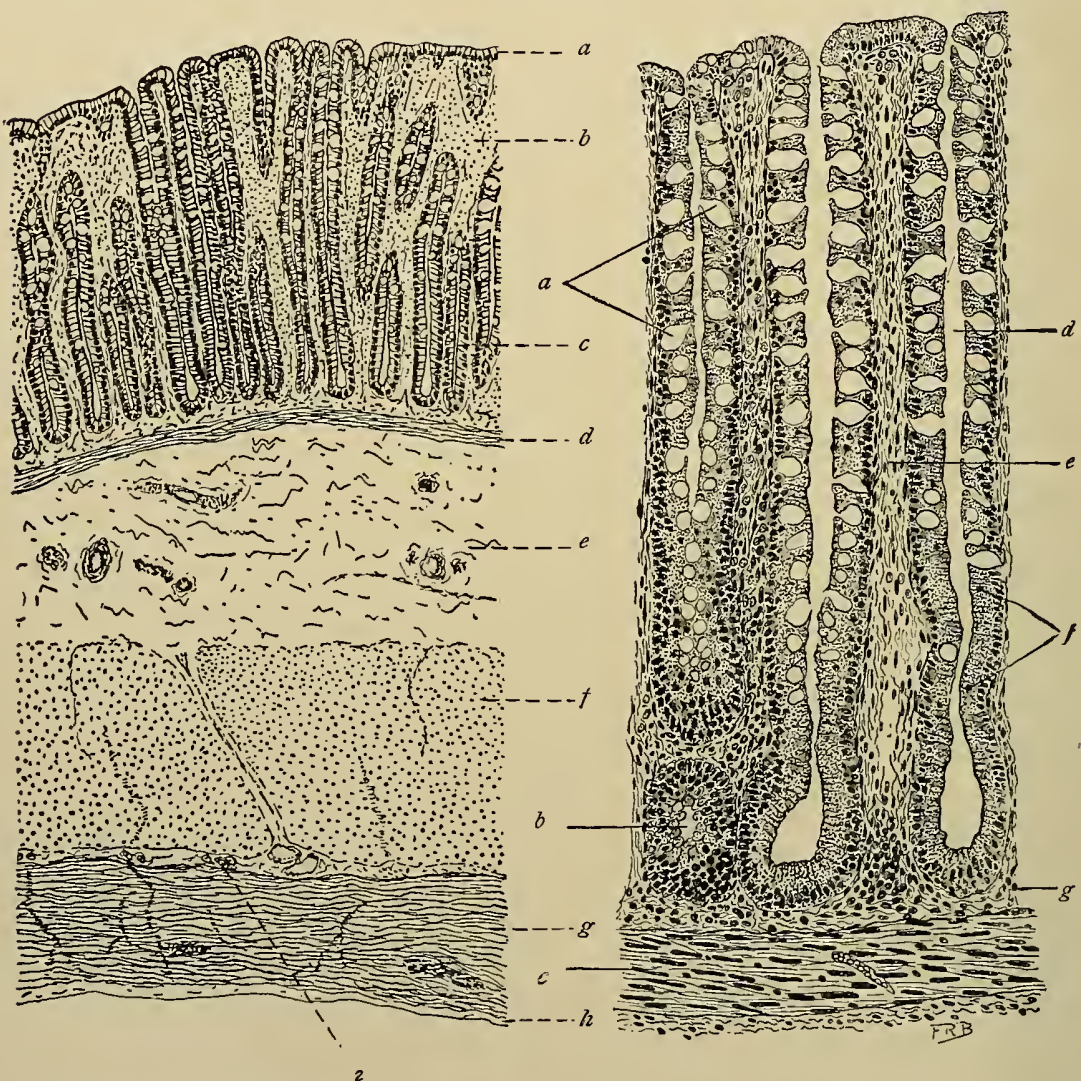

Fig. 168.

Fig. 169.

FIG. I68.- From Vertical Longitudinal Section of Cat's Large Intestine. (Larrabee.) a, Epithelium; b, stroma; c, fundus of gland; d, muscularis mucosæ; e, submucosa;f, circular muscle layer; $g$, longitudinal muscle layer; $h$, serous coat; $i$, Auerbach's plexus.

Fig. I69.-From Vertical Longitudinal Section of the Mucous Membrane of the Human Large Intestine. (Technic I, p. 275.) $a$, Mucous (goblet) cells; $b$, fundus of a gland cut obliquely; $c$, muscularis mucosæ; $d$, lumen of a gland cut longitudinally; $e$, stroma between the glands; $f$, leucocytes in the epithelium; $g$, stroma between fundi of glands and muscularis mucosæ.

outer side of the circular muscle coat, is the nerve plexus of Auerbach. (For details see page 270.)

4. The serous coat consists, as in the stomach and small intestine, of loose connective tissue covered by a single layer of mesothelium. 


\section{The Vermiform ApPendix}

The vermiform appendix is a diverticulum from the large intestine. Its walls are continuous with those of the latter, and closely resemble them in general structure. There are the same four coats, mucous, submucous, muscular, and serous.

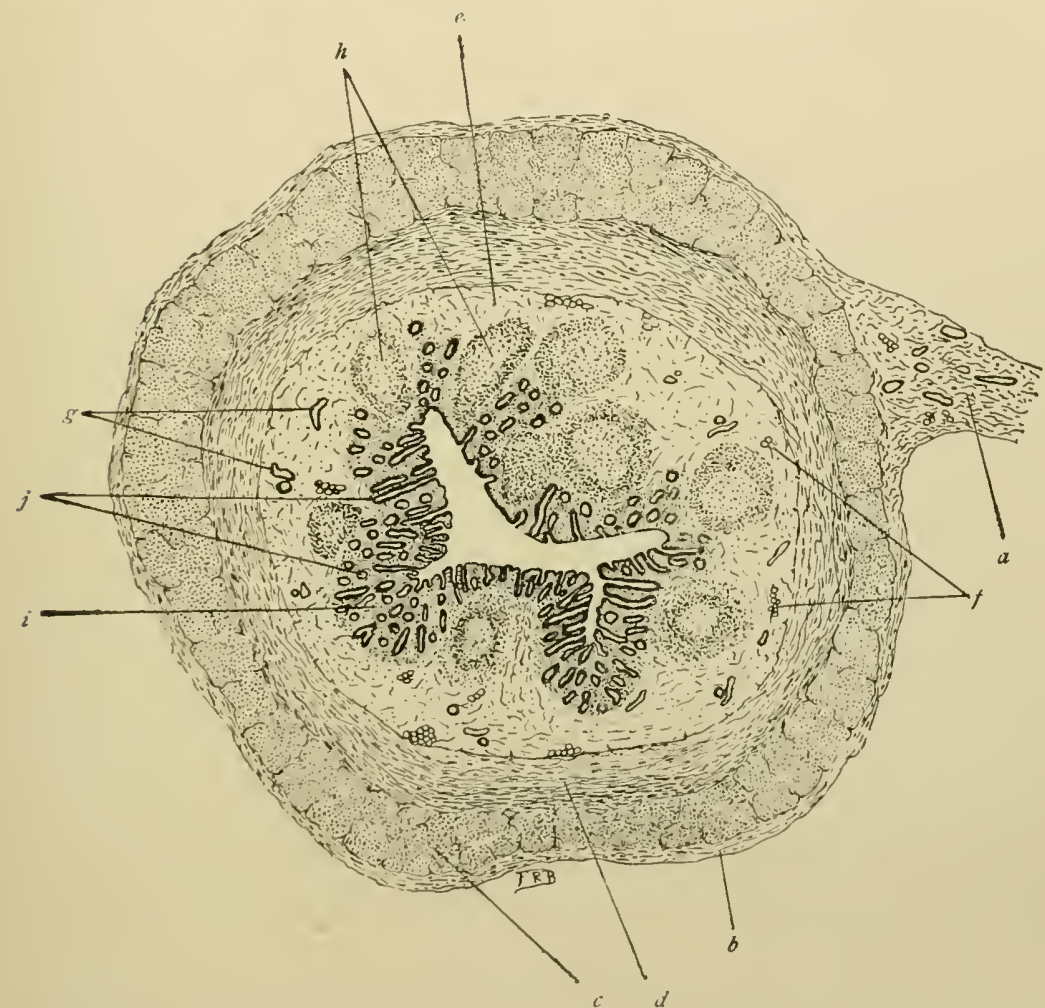

FIg. I 70.-Transverse Section of Human Vermiform Appendix. (Technic 2, p. 275.) $a$, Mesoappendix; $b$, serous membrane (serosa); $c$, outer longitudinal muscle layer; $d$, inner circular muscle layer; $e$, submucosa; $f$, groups of fat cells in submucosa; $g$, bloodvessels in submucosa; $h$, lymph nodules; $i$, stroma; $j$, glands opening into lumen and cut in various planes; muscularis mucose not present.

r. The mucous membrane (Fig. I70) consists of epithelium, glands, strema, and muscularis mucosie. The epilhelium resembles that of the large intestinc. The glands vary in number, but are usually much less closely packed than in the large intestine. They are most numerous in the appendices of infants and children. The gland tubules (Fig. I 70, $j$ ) are usually rudimentary, but in most cases have the same structure as the intestinal glands, and are evidently 
functional as they contain mucous cells in all stages of secretion. In consequence of the wider separation of the tubules the stroma is more abundant than in the large intestine, but has the same structure. The muscularis mucosa is usually fairly distinct as a thin circularly disposed band of smooth muscle cells just beneath the stroma. It is not always present. In some cases the mucosa as such is practically absent, being replaced by fibrous tissue. This condition is especially common after middle age, and may or may not be associated with obliteration of the lumen.

2. The submucosa (Fig. I $70, e$ ) is similar to that of the intestine.

3. The muscular coat varies greatly, both as to thickness and as to the amount of admixture of fibrous tissue. The inner circular layer (Fig. I $70, d$ ) is usually thick and well developed. The outer longitudinal layer (Fig. I $70, c$ ) differs from that of the large intestine in having no arrangement into linex, the muscle tissue forming a continuous layer. Less commonly a more or less marked tendency to an arrangement of the cells of the longitudinal coat into bundles, between which the outer coat is thin or wanting, is observed.

4. The serosa has the usual structure of peritoneum.

The lymph nodules (Fig. I $70, h$ ) constitute the most conspicuous feature of the appendix. They lie mainly in the submucosa. In children and young adults the nodules are oval or spherical; in later life somewhat flattened. The nodules may be entirely distinct, or may be arranged as in a Peyer's patch with distinct apices and bases, but with their central portions confluent. The muscularis mucosæ either passes through the superficial portions of the nodules, or, where they are separated from the lumen, passes over them.

The distribution of blood-vessels, lymphatics, and nerves is similar to that in the large intestine.

\section{The Rectum}

I. The mucous membrane of the rectum has a structure similar to that of the large intestine. The glands are longer and the mucosa is consequently somewhat thicker. In the lower part of the rectum definite longitudinal foldings of the mucosa occur, the so-called columne rectales. A change in the character of the mucous membrane begins at the upper end of the columnæ rectales. Here the simple columnar èpithelium of the gut passes over into a stratified squamous epithelium, beneath which is a papillated stroma. The glands con- 
tinue for a short distance beyond the change in the epithelium, but soon completely disappear. At the anus there is a transition from mucous membrane to skin similar to that described as occurring at the margin of the lips (page 220 ).

2. The submucosa is similar in structure to that of the large intestine.

The muscularis of the rectum differs from that of the large intestine in that the longitudinal layer is continuous and thick.

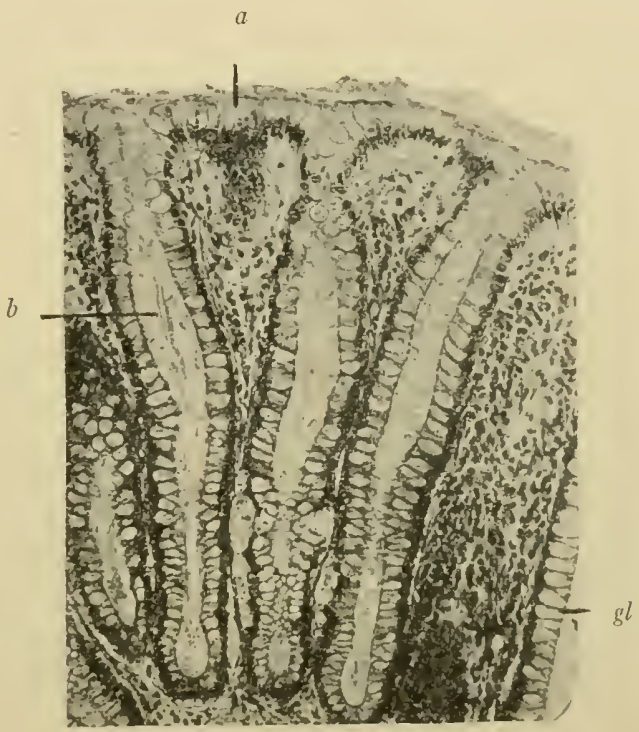

FIG. x 71.-Mucous Membrane of Human Rectum. a, Superficial epithelium, composed almost wholly of mucous cells, the columnar cells lying between them being so compressed as to appear as thin dark lines; gl, lumen of gland of Licberkülın. $\quad \times 60$. (Prenant.)

The serous coat is absent in the lower part of the rectum, being replaced by a fibrous connective-tissue layer, which connects the rectum with the surrounding structures.

\section{The Peritoneum, Mesentery, and Omentum}

The periloneum (sec also p. I65) is a serous membrane which lines the walls of the abdomen (parictal peritoneum) and is reflected over the contained viscera (visceral jeritoneum). It consists of two layers, a connective-tissue stroma and mesothelium. The stroma consists of lonsely arrangerl connective-tissue bundles, which inter- 
lace in a plane parallel to the surface. There are numerous elastic fibres, especially in the deeper layer of the parietal peritoneum. There are comparatively few connective-tissue cells. The mesothelium consists of a single layer of flat polygonal cells with bulging nuclei. The cells have irregular wavy outlines, which are easily demonstrated with silver nitrate (Fig. 26). The shape of the cells varies considerably according to the direction in which the tissues are stretched. Over some parts, e.g., the liver and intestine, the peritoneum or serosa is thin and very closely attached. In places where the peritoneum is freely movable, a considerable amount of loose connective tissue, rich in elastic fibres and containing varying numbers of fat cells, connects the peritoneum with the underlying tissue. This is known as the "subserous tissue." The peritoneum is well supplied with blood-vessels and lymphatics. The former give rise to a rich capillary network.

The mesentery is a sheet of loosely arranged connective tissue covered with peritoneum. It is reflected from the post-abdominal wall to the viscera, and serves to carry to these organs their bloodvessels, lymphatics, and nerves. In the case of the stomach, duodenum, and large intestine, the mesentery is comparatively short, and the organs are therefore quite firmly fixed to the abdominal wall. In the case of the small intestine the mesentery is long and the intestine, therefore, freely movable. The mesentery is richly supplied with lymph nodes and there is usually a considerable amount of fat. From the mesentery, the peritoneum passes over to and envelops the viscera.

The omentum (Fig. 26) is a sheet of tissue which passes from the liver to the lesser curvature of the stomach (gastro-hepatic omentum) to which it is attached, and from the greater curvature of the stomach to the transverse colon (greater omentum). It is similar in structure to the mesentery, and contains usually much fat and many lymph nodes. Its connective-tissue bundles are arranged in networks, the strands and meshes of which vary greatly in size and shape. The strands are covered by a single layer of mesothelium.

\section{Blood-Vessels of the Stomach and Intestines}

The arteries reach the gastro-intestinal canal through the mesentery and pass through the muscular coats to the submucosa, where they form an extensive plexus of large vessels (Heller's plexus) 
(Fig. I72,c). Within the muscular coats the main arteries give off small branches to the muscle tissue. From the plexus of the submucosa two main sets of ressels arise, one passing outward to supply the muscular coats, the other inward to supply the mucous membrane (Fig. I72). Of the former the larger vessels pass directly to the intermuscular septum, where they form a plexus from which branches are given off to the two muscular tunics. A few small branches from the

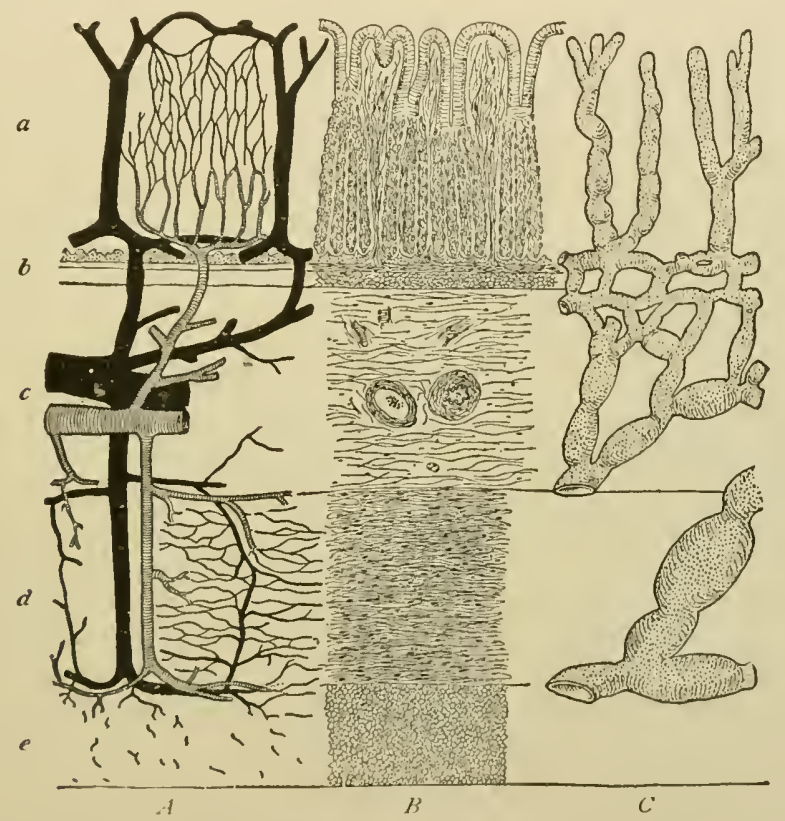

FIG. I 72,-Scheme of Blood-vessels and Lymphatics of Stomach. $\times 70 . \quad$ (Szymonowicz, after Mall.) $a$, Mucous membrane; $b$, muscularis mucosa; $c$, submucosa; $d$, inner circular muscle layer; $e$, outer longitudinal muscle layer; $A$, blood-vessels; $B$, structure of coats; $C$, lymphatics.

larger recurrent vessels also supply the inner muscular layer. Of the branches of the submucosa plexus which pass to the mucous membrane, the shorter supply the muscularis mucosa, while the longer branches pierce the latter to form a capillary plexus among the glands of the stroma. From the capillaries small veins take origin which pierce the muscularis mucosa and form a close-meshed venous plexus in the sulmucosa (Fig. I 72). These in turn give rise to larger veins, which accompany the arteries into the mesentery.

In the small intestine the distribution of the blood-vessels is modified by the presence of the villi (Fig. 174). Each villus reccives one small artery, or, in the case of the larger villi, two or three small 
arteries. The artery passes through the long axis of the villus close under the epithelium to its summit, giving off a network of fine capillaries, which for the most part lie just beneath the epithelium.

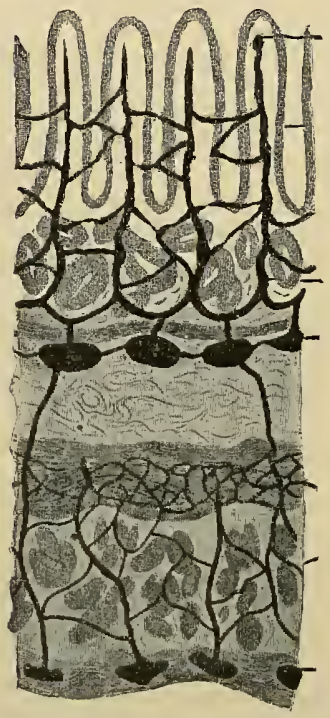

FIG. I73.-Diagram of wall of Stomach to show General Disposition of Lymphatics $a$, Terminal lymph channel in tissues separating glands; $b$, superficial lymphatic plexus in connective tissue surrounding bases of glands; $c$, submucous lymphatic plexus; $d$, intermuscular lymphatic plexus; $e$, subperitoneal lymphatic plexus. (Cuneo.)

From these, one or two small veins arise which lie on the opposite side of the villus from the artery.

\section{Lymphatics of the Stomach AND INTESTINE}

Small lymph or chyle capillaries begin as blind canals in the stroma of the mucous membrane among the tubular glands (Fig. I72). In the small intestine a lymph (chyle) capillary occupies the centre of the long axis of each villus, ending in a blind extremity beneath the epithelium of its summit (Fig. I74). These vessels unite to form a narrowmeshed plexus of lymph capillaries in the deeper part of the stroma, lying parallel to the muscularis mucosæ. Vessels from this plexus pass through the muscularis mucosæ and form a wider meshed plexus of larger lymph vessels in the submucosa. A third lymphatic plexus lies in the connective tissue which separates the two layers of muscle. From the plexus in the submucosa, branches pass through the inner muscular layer, receive vessels from the intermuscular plexus, and then pierce the outer muscular layer to pass into the mesentery in company with the arteries and veins.

\section{NeRVES OF tHe STOMACH AND INTESTINES}

The nerves which supply the stomach and intestines are mainly non-medullated sympathetic fibres. They reach the intestinal walls through the mesentery. In the connective tissue between the two layers of muscle, these fibres are associated with groups of sympathetic ganglion cells to form the plexus myentericus or plexus of Auerbach. The dendrites of the ganglion cells interlace, forming a 
large part of the plexus. The axones are grouped together in small bundles of non-medullated fibres, which pass into the muscular coats, where they form intricate plexuses, from which are given off clubshaped terminals to the smooth muscle cells. From Auerbach's plexus fibres pass to the submucosa, where they form a similar but

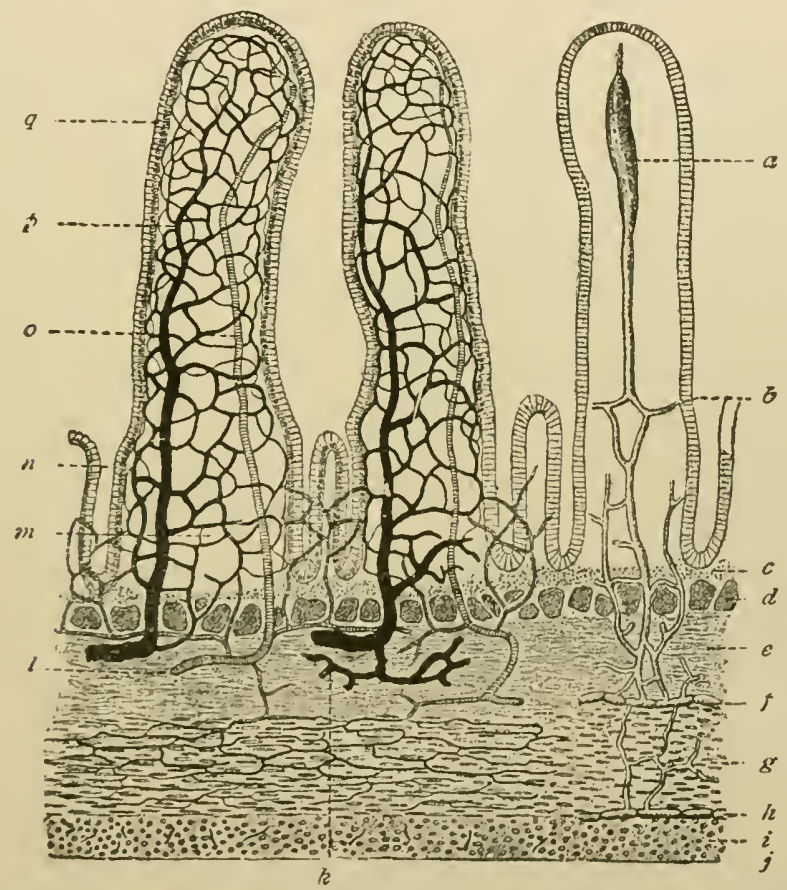

FIG. 174.- Scheme of Blood-vessels and Lymphatics of Iuman Smallintestine. (From Böhm and von Davidoff, after Mall.) $a$, Central lacteal of villus; $b$, lacteal; $c$, stroma; $d$, muscularis mucosa; $e$, submucosa; $f$, plexus of lymph vessels; $g$, circular muscle layer; $h$,plexus of lymph vessels; $i$, longitudinal muscle layer; $j$, serous coat; $k$, vein; $l$, artery; $m$, base of villus; $n$, crypt; $o$, artery of villus; $p$, vein of villus; $q$, epithelium.

finer-meshed, more delicate plexus, also associated with groups of sympathetic ganglion cells, the plexus of Meissner. Both fibres and cells are smaller than those of Auerbach's plexus. From Meissner's plexus delicate fibrils pass to their terminations in submucosa, muscularis mucosie, and mucous membrane.

\section{SECRETION ANI ABSORPTION}

The secretory activities of epithedial cells have already been mentioned (page 212). 'The epithelium of the gastro-intestinal tract must be considered as having two main functions: (I) The secretion 
of substances necessary to digestion; and (2) the absorption of the products of digestion.

(I) Secretion.-The production of mucus takes place in the mucous or goblet cell, which is present throughout the gastroenteric mucosa and, as already mentioned, probably represents a differentiation of the ordinary columnar epithelial cell. The chief

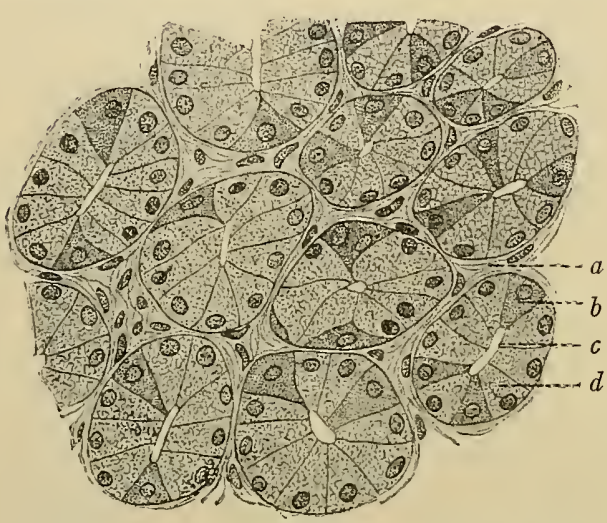

FIG. I75.-Section through Glands of Fundus of Human Stomach in Condition of Hunger. $\times 500$. (Böhm and von Davidoff.) $a$, Stroma; $b$, parietal cell; $c$, lumen; $d$, chief cell. cells, "peptic cells," of the stomach glands are comparatively clear during fasting, with development of ergastoplasm in their basal ends. With the onset of digestion these cells increase in size and become generally cloudy from development of granules with reduction of the ergastoplasm. These granules represent a pre-ferment or pepsinogen and with their discharge into the lumen of the gland, the cells again become smaller, clearer, and assume the resting condition. Bensley and Theohara have recently very clearly demonstrated the secretory chain in the chief cells from ergastoplasm or basal filaments through pepsinogen granules to pepsin. While some authorities still contend that a minor pepsin-forming rôle is played by the pyloric glands, the above facts together with the fact that activity of the chief cells (Fig. I76) is coincident with an increase in the pepsin found in the stomach, and that the amount of pepsinogen in the gastric mucosa is proportionate to the number of granules in the chief cells, (Langley and Sewell) may be accepted as proving these cells the main if not the sole producers of pepsin, and the granules as some stage in the elaboration of the ferment. As their name of "acid cells" would indicate, the parietal cells have been considered the source of the hydrochloric acid of the stomach. While doubt still exists as to the function of these cells, recent investigations make it probable that they secrete substances which as chlorides are transformed into hydrochloric acid by the action of the carbonic acid of the blood. According to some authorities other cells assist in the production of the stomach acid. The cells of Brunner's glands undergo changes 
during digestion, which are somewhat similar to those described as occurring in the chief cells of the stomach glands. By some they are believed to be concerned in the production of pepsin, by others to play a special rôle in the secretion of one or more of the intestinal ferments. The only function of the surface epithelium and of that of the intestinal crypts which has yet been determined is the secretion of mucus. The cells of Paneth are typical gland cells containing secretion granules and probably produce a specific secretion. (See also page 260.) Whether this secretion is the so-called "inverse

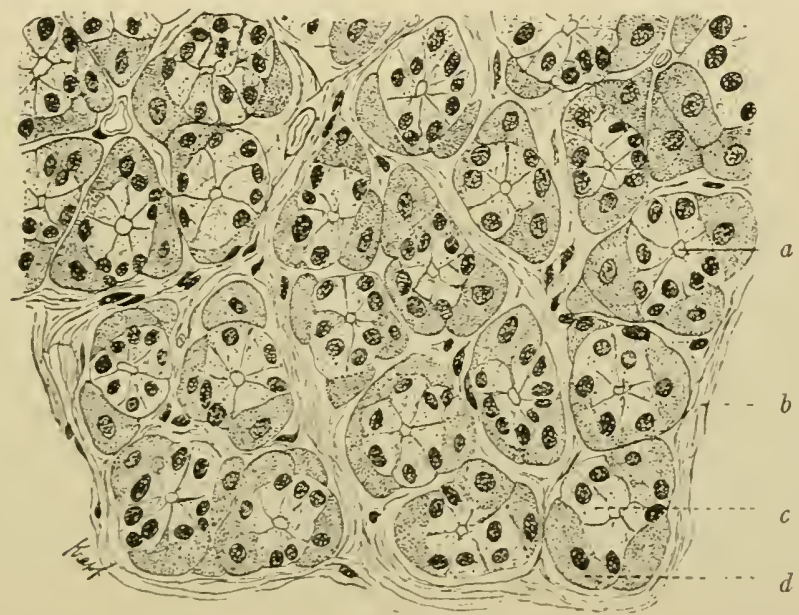

Fig. r76.-Section through Glands of Fundus of Human Stomach during Digestion. $\times 5$ oo. (Böhm and von Davidoff.) $a$, Lumen; $b$, stroma; $c$, chief cell; $d$, parietal cell.

ferment" or "invertin" which changes cane sugar into glucose and levulose or one of the other intestinal ferments has not been determined. It has been known that pancreatic fluid is inactive toward albuminoids unless mixed with intestinal secretions. This is due according to Pavlow to a special ferment "enterokinase." According to some this is a secretion of the epithelium, to others it is elaborated by eosinophile leucocytes which pass through the intestinal wall, and become a part of the intestinal secretion.

(2) ABsorption of Fat.-While various other products of digestion are absorbed by the intestine, the absorption of fat is the one most easily olsserved and the one of most interest from the histological standpoint because the passage of the droplets can be seen under the microscope. After feeding fat, fatty aciols, or soaps, fat globules are 
found to have penetrated the intestinal mucosa, and may be seen in (a) the epithelial cells, $(b)$ the leucocytes, and $(c)$ the lacteals of the villi (Fig. I77). Fat globules are never seen in the thickened free borders of the cells. Hence it seems probable that the fat before passing through this part of the cell becomes split up into glycerin and

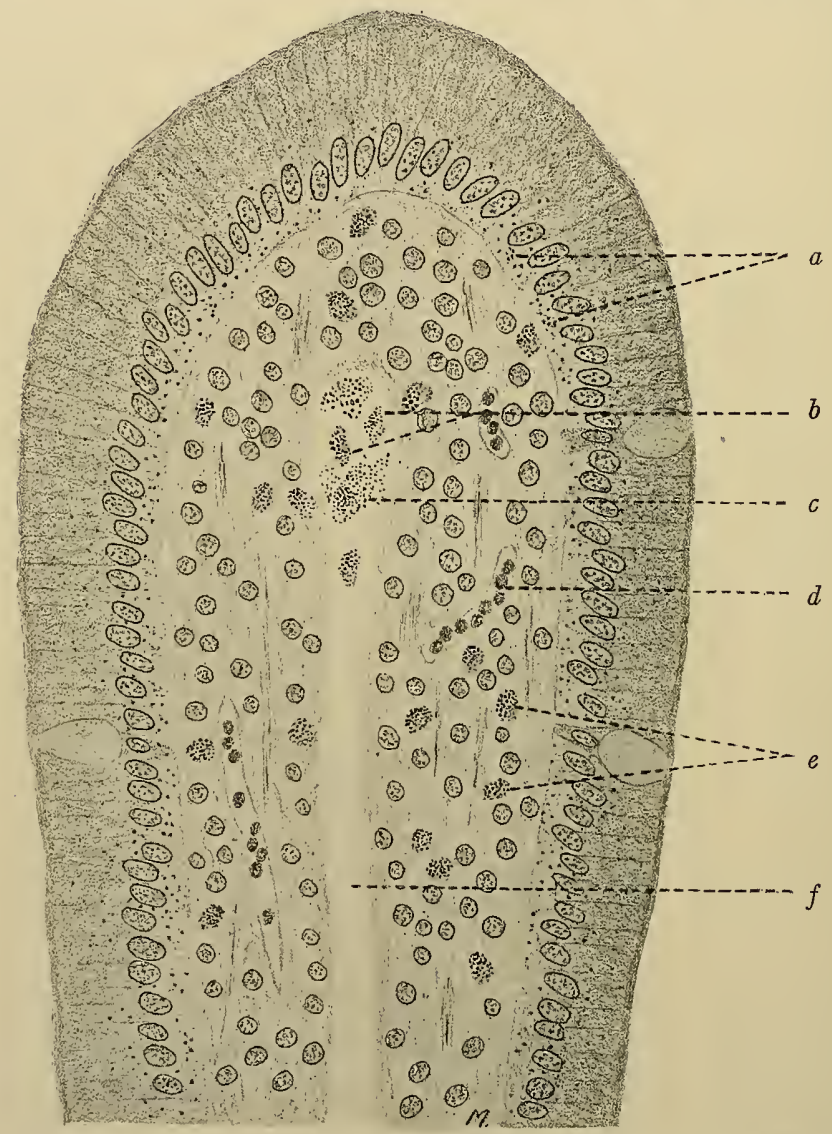

FIG. I77.-Fat Absorption. Longitudinal section of villus of cat's small intestine, three hours after feeding. $\times_{350}$. Osmic acid. $a$, Fat droplets in epithelial cells; $b$, fat droplets in leucocytes in stroma; $c$, fat droplets in leucocytes within lacteal; $d$, fat droplets free in lacteal; $e$, capillary containing blood cells; $f$, central lacteal of villus.

fatty acids which are united again to form fat within the protoplasm of the cell. Leucocytes containing fat globules are seen throughout the stroma. Within the lacteals are found fat-containing leucocytes and free fat droplets of various size. It would thus seem probable that the process of fat absorption consists in: (I) The passage of glycerin and fatty acids through the cell borders; (2) their reunion in 
the cell to form fat; (3) the transierence of these fat globules to leucocytes; which (4) carry them to the lacteals. In the lacteals the fat is probably set free by disintegration of the leucocytes. Other fat droplets-perhaps the majority-pass from the epithelial cells into the lacteal without the aid of leucocytes, possibly assisted by contractions of the smooth muscle fibres.

\section{TECHNIC}

(I) The technic for the small and large intestines and rectum is the same as for the stomach. Accurate fixation of the villi is difficult, there being usually some shrinkage of the connective tissue of the core away from the epithelium.

A longitudinal section should be made through the junction of small and large intestine, showing the transition from the villus-covered surface of the former to the comparatively smooth surface of the latter.

To show Brunner's glands a section of the duodenum is required.

To show the varying shapes of the villi in the different regions, sections should also be made of the jejunum and ileum.

Solitary follicles may usually be seen in any of the above sections.

A small Peyer's patch, together with the entire thickness of the intestinal wall, should be removed, treated as above, stained with hrmatoxylin-eosin (technic I, P. 20), or with hæmatoxylin-picro-acid-fuchsin (technic 3, p. 2I), and mounted in balsam.

(2) A vermiform appendix, as fresh as possible, should be cut transversely into small pieces, fixed in formalin-Müller's fluid (technic 5, p. 7), and hardened in alcohol. Thin transverse sections are made through the entire wall, stained with hæmatoxylin-eosin or hæmatoxylin-picro-acid-fuchsin, and mounted in balsam.

(3) Fat Absorption.-For the purpose of studying the process by which fat passes from the lumen of the gut into the chyle vessels, an animal should be killed at the height of fat absorption. A frog fed with fat bacon and killed two days later, a dog fed with fat meat, or a cat with cream and killed after from four to eight hours, furnishes good material. Usually if the preparation is to be successful, the lumen of the intestine will be found to contain emulsified fat and the lacteals of the mesentery are seen distended with chyle. Extremely thin slices of the mucous membrane of the small intestine are fixed in I-per-cent. osmic acid or in osmium bichromate solution (5-per-cent. aqueous solution potassium bichromate and 2-per-cent. arjucous solution osmic acirl-equal parts) for twelve to twenty-four hours, after which they are passed rather quickly through graded alcrhols. Sections should be thin and mounted, either unstainerl or after a slight cosin stain, in glycerin.

(4) The blower-vessels of the stomatch are best studied in injected specimens. (Sece page 25.)

\section{The Larger Glands of the Digestive System}

The smaller tubular glands which form a part of the mucous membrance and submucosia of the alimentary tract have been already de- 
scribed. Certain larger glandular structures, the development of which is similar to that of the smaller tubules but which come to lie wholly without the alimentary tract, connected with it only by their main excretory ducts, and which are yet functionally an important part of the digestive system, remain to be considered.

These are:
I. The salivary glands
(a) The parotid.
(b) The sublingual.
(c) The submaxillary.

2. The pancreas.

3. The liver.

\section{The Salivary Glands}

The salivary glands are all compound tubular glands. In man the parotid is serous; the sublingual and submaxillary, mixed serous and mucous (page 22I). Only the general structure of these glands is here described, the minute structure of mucous and serous glands having been described on page $22 \mathrm{I}$.

Each gland consists of gland tissue proper and of a supporting connective-tissue framework. The framework consists of a connective-tissue capsule which encloses the gland, but blends externally with and attaches the gland to the surrounding structures. From the capsule trabecula pass into the gland, subdividing it into lobes and lobules. The gland tissue proper consists of systems of excretory ducts opening into secretory tubules, all being lined with one or more layers of epithelial cells. Each gland has one main excretory duct. This divides into branches-interlobar ducts-which run to the lobes in the connective tissue which separates them. The interlobar ducts give rise to branches which, as they pass to the lobules in the interlobular connective tissue, are known as interlobular ducts. From the latter, branches enter the lobules - intralobular ducts - and split up into terminal secreting tubules which constitute the bulk of the lobule. As the smaller intralobular ducts are lined with cells of a secretory type, and probably take part in the elaboration of the secretion of the gland, they have been called salivary or secreting tubules. These, in the submaxillary and parotid glands, open into tubules which have a narrow lumen and are lined with low or flat epithelium; lying between the secreting tubules and the terminal tubules, they are known as intercalated or intermediate tubules. From 
the interlobular connective tissue delicate extensions pass into the lobules, separating the gland tubules. The glandular tissue is known as the parenchyma of the gland in contradistinction to the connective or interstitial tissue.

The parotid gland in man, dog, cat, and rabbit is a purely serous gland. Its duct system is complex. The main excretory duct (Stenoni) is lined by two layers of columnar epithelium resting upon a distinct basement membrane. The main duct divides into numerous branches, which in turn give rise to the secreting or salivary tubules. These are continuous with the long narrow intermediate tubules, from

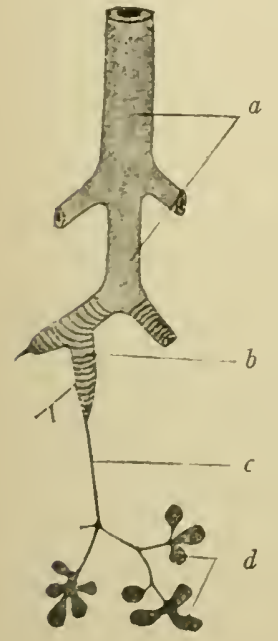

1

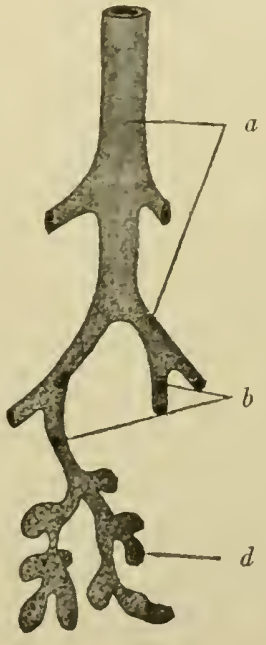

$B$

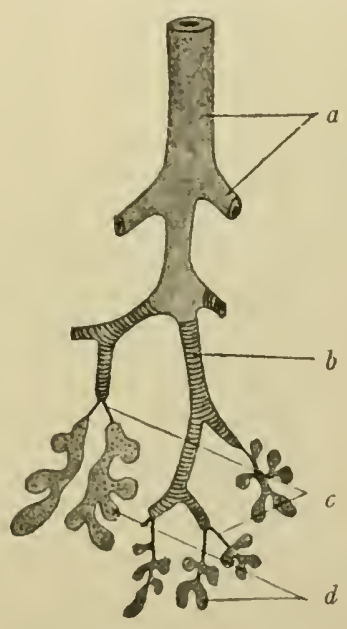

C

FIG. I78.-Diagrams to illustrate the Structure of the Salivary Glands. (Stöhr.) $A$, Parotid; $B$, sublingual; $C$, submaxillary. $a$, Excretory duct; $b$, secreting tubule; $c$, intermediate tubule; $d$, terminal tubule.

each of which are given off a number of short terminal tubules (Figs. I78, $A$ and 180). The two-layered epithelium of the main duct becomes reduced in the smaller ducts to a single layer of columnar cells. The salivary tubules are lined with high columnar epithelium, the bases of the cells showing distinct longitudinal striations. In the intermediate tubule the epithelium is flat, sometimes spindle-shaped. The terminal tubules are lined with scrous cells (page 22I). The connective tissue usually contains a considerable number of fat cells.

The sublingual gland is a mixed gland in man, dogr, cat, and rab)it. The duct system is less complex than in the parotid. The main duct (Bartholini) sends off branches which are continuous with 
tubules, showing a few secretory mucous cells. These open directly into the terminal tubules which are convoluted and vary greatly in

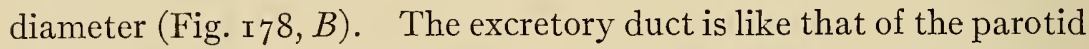
gland, lined with a two-layered columnar epithelium resting upon a basement membrane. In the smaller ducts the epithelium is reduced to a single layer of columnar cells. There are no intermediate tubules. The terminal tubules are lined with both serous and mucous cells (page 22I). The crescents of Gianuzzi (page 222) are numerous

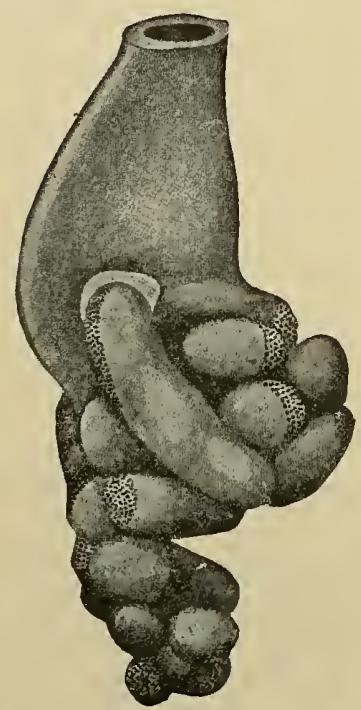

FIG. I 79.-Reconstructed Model of Small Muco-serous Gland. Dotted parts corresponding to the "crescents". (Maziarski). and large. The connective tissue of the gland contains many lymphoid cells (Fig. I 8I).

Near the sublingual gland is a group of some 5 to 20 simple tubular glands. Their terminal tubules are lined almost wholly with mucous cells. This group of tubules has been designated the "sublingualis minor."

The submaxillary gland is also a mixed gland in man, dog, cat, and rabbit. In complexity of its duct system it stands between the parotid and the sublingual (Fig. I78). The main duct (Wharton's) has not only a two-layered epithelial lining resting upon a basement membrane, but is distinguished by a richly cellular stroma and a thin layer of longitudinally disposed smooth muscle. Branches of the main duct open into long secreting tubules which communicate with the terminal tubules by means of short narrow intermediate tubules (Fig. I78, C). The secretory tubules are lined as in the parotid with columnar cells whose bases are longitudinally striated. These cells usually contain more or less yellow pigment. The intermediate tubules have a low cuboidal or flat epithelium. Most of the end tubules contain serous cells only (page 22I). The crescents of the mucous tubules (page 222) are less numerous and smaller than those in the sublingual, consisting as a rule of only from one to three cells (Fig. I82).

Blood-vessels.-The larger arteries run in the connective-tissue septa with the ducts, giving off branches which accompany the divisions of the ducts to the lobules, where they break up into capillary networks among the tubules. These give rise to veins which accompany the arteries. 


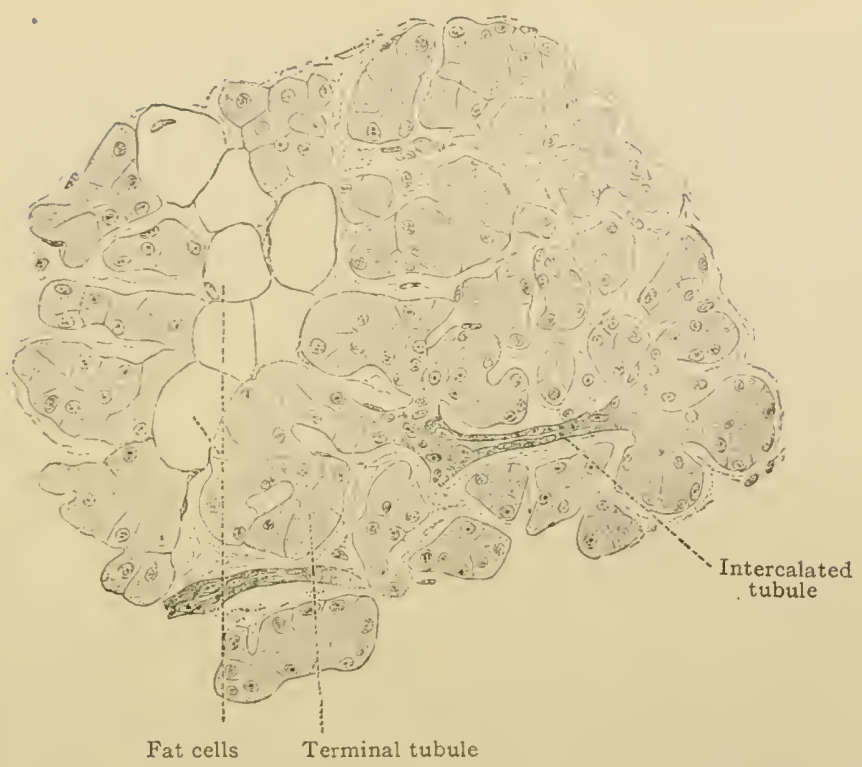

FIG. I80,-Section of Human Parotid Gland. $\times 252$. (Stöhr.) The narrow lumina of the terminal tubules do not show in this figure.

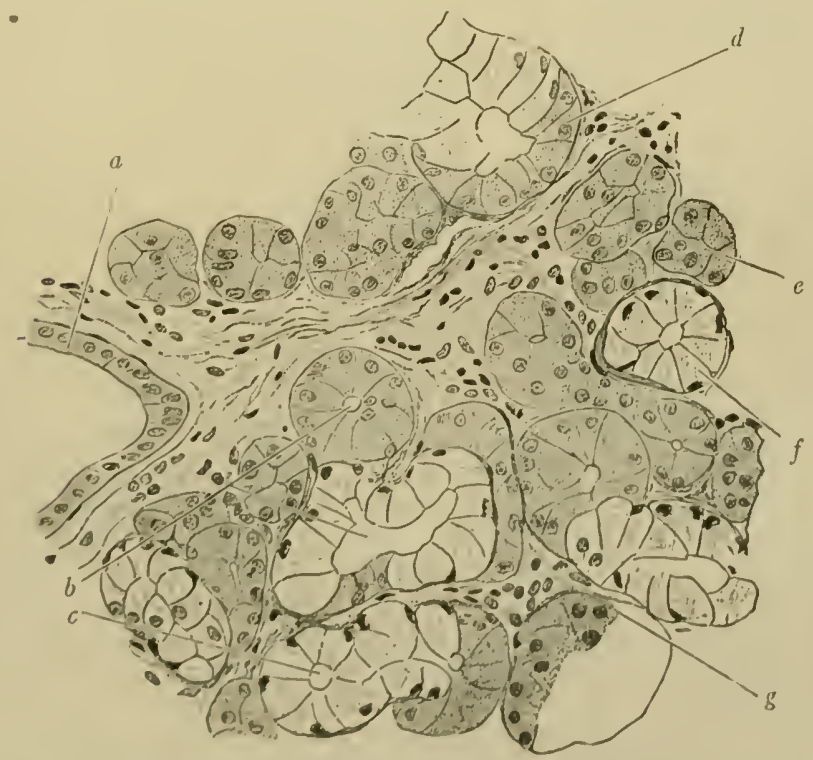

Frc. 38r.-Section of Iluman Sublingual (;land. $\times 252$, (Stölır.) a, Excretory rluct; $b$, lumina of serous and mucous tubules; $c$, mucous tubule; $d$, rlemilune; $e$, serous tubule; $f$, cross section mucous tubule; $g$, interstitial connective tissuc. 
The lymphatics begin as minute capillaries in the connective tissue separating the terminal tubules. These empty into larger lymph vessels which accompany the arteries in the septa.

The nerves of the salivary glands are derived from both cerebrospinal and sympathetic systems, and consist of both medullated and non-medullated fibres. The medullated fibres are afferent, probably the dendrites of cells located in the geniculate ganglion. Small bundles of these fibres accompany the ducts. Single fibres leave the

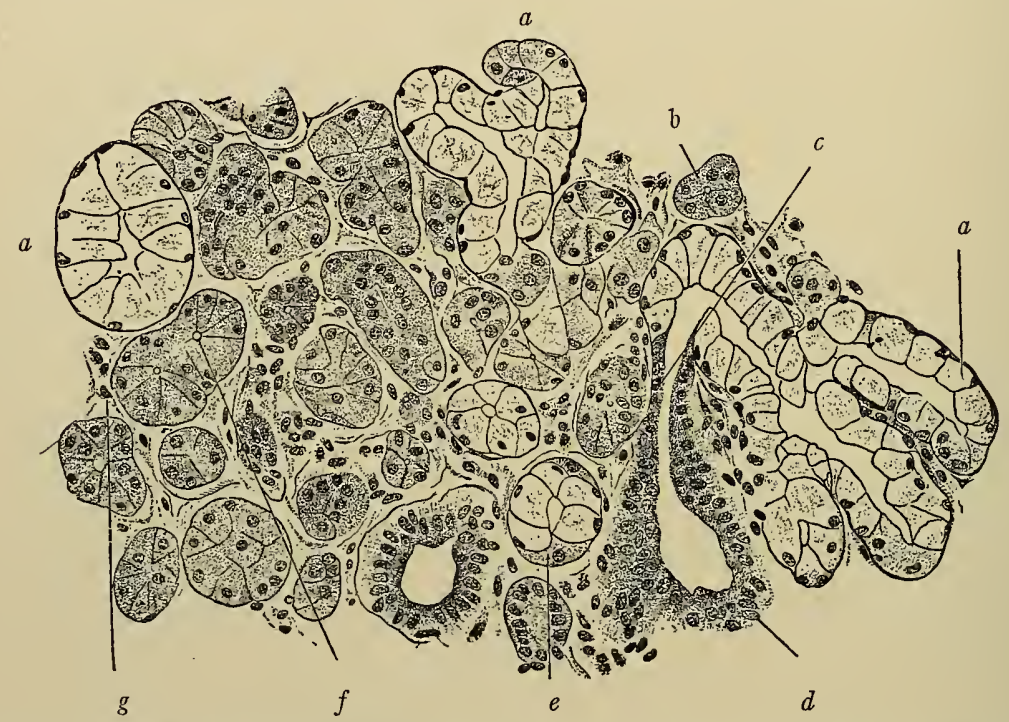

FIG. I82.- Section of Human Submaxillary Gland. $\times_{252}$. (Stöhr.) $a$, Mucous tubule; $b$, serous tubule; $c$, intermediate tubule; $d$, "secretory" tubule; $e$, demilune; $f$, lumen; $g$, interstitial connective tissue.

bundles, lose their medullary sheaths, and form a non-medullated subepithelial plexus, from which delicate fibrils pass to end freely among the epithelial cells. Efferent impulses reach the gland through the sympathetic. The fibres are axones of cells situated in small peripheral ganglia; the cells sending axones to the submaxillary lying upon the main excretory duct and some of its larger branches; those sending axones to the sublingual being situated in a small ganglion-the sublingual-lying in the triangular area bounded by the chorda tympani, the lingual nerve, and Wharton's duct; those supplying the parotid probably being in the otic ganglion. Axones from these cells enter the glands with the excretory duct and follow its branchings to the terminal tubules, where they form plexuses 
beneath the epithelium. From these, terminals pass to the secreting cells. It is probable that the salivary glands also receive sympathetic fibres from cells of the superior cervical ganglia.

\section{TECHNIC}

(I) The salivary glands should be fixed in Flemming's fluid (technic 7, p. 7), or in formalin-Müller's fluid (technic 5, p. 7). Sections are cut as thin as possible, stained with hæmatoxylin-eosin (technic I, p. 20), and mounted in balsam.

(2) For the study of the secretory activities of the gland cells, glands from a fasting animal should first be examined and then compared with those of a gland the secretion of which has been stimulated by the subcutaneous injection of pilocarpine. Fix in Flemming's or in Zenker's fluid (technic 9, p. S). Examine some sections unstained and mounted in glycerin, others stained with hæmatoxylin-eosin and mounted in balsam.

(3) The finer intercellular and intracellular secretory tubules are demonstrated by Golgi's method. Small pieces of absolutely fresh gland are placed for three days in osmium-bichromate solution (3-per-cent. potassium bichromate solution, 4 volumes; I-per-cent. osmic acid, I volume), and then transferred without washing to a 0.75 -per-cent. aqueous solution of silver nitrate. Here they remain for from two to four days, the solution being frequently changed. The processes of dehydrating and embedding should be rapidly done, and sections mounted in glycerin, or, after clearing in xylol, in hard balsam.

\section{Pancreas}

The pancreas is a compound tubular gland. While in general similar to the salivary glands, it has a somewhat more complicated structure. A connective-tissue capsule surrounds the gland and gives off trabeculæ which pass into the organ and divide it into lobules.

In some of the lower animals, as for example the cat, these lobules are well defined, being completely separated from one another by connective tissue. In this respect they resemble the lobules of the pig's liver. A number of these primary lobules are grouped together and surrounded by connective tissue, which is considerably broader and looser in structure than that separating the primary lobules. These constitute a lobule group or secondary lobulc.

In the human pancreas the division into lobules and lobule groups is much less distinct, although it can usually be made out. This is duc to the incompleteness of the connective-tissue septa, the human pancreas in this respect resembling the human liver. Rarely the human pancreas is distinctly lobulated.

The gland has a main excretory duct, the pancreatic duct or duct of Wirsung. In many cases there is also a secondary cxcrelory duct, 
the accessory pancreatic duct or duct of Santorini. Both open into the duodenum. The main duct extends almost the entire length of the gland, giving off short lateral branches, one of which enters the centre of each lobule group. Here it splits up into branches which pass to the primary lobules. From these intralobular ducts are given

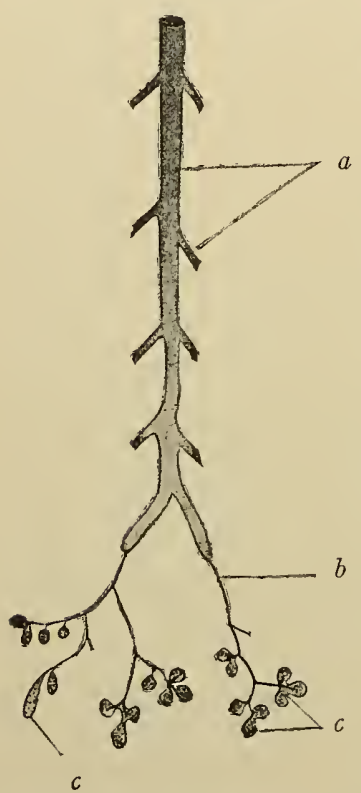

FIG. I83.-Diagram to illustrate Structure of Pancreas. (Stöhr.) a, Excretory duct; $b$, intermediate tubule; $c, c$, terminal tubules. off long, narrow, intermediate tubules, which in turn give rise to the terminal secreting tubules (Fig. I83).

The excretory ducts are lined with a simple high columnar epithelium which rests upon a basement membrane. Outside of this is a connective-tissue coat, the thickness of which is directly proportionate to the size of the duct. In the pancreatic duct goblet cells are present, and the accompanying connective tissue of the main duct and of its larger branches contains small mucous glands. As the ducts decrease in size, the epithelium becomes lower until the intermediate tubule is reached where it becomes flat.

The terminal tubules themselves are most of them very short, frequently almost spherical. This and the fact that several terminal tubules are given off from the end of each intermediate tubule have led to the description of these tubules as alveoli, and of the pancreas as a tubulo-alveolar gland, although there is no dilatation of the lumen. The terminal tubules are lined with an irregularly conical epithelium resting upon a basement membrane (Figs. I84 and 185 ). The appearance of these cells depends upon their functional condition. Each cell consists of a central zone bordering the lumen, which contains numerous granules known as zymogen granules, and of a peripheral zone next to the basement membrane, which is homogeneous and contains the nucleus (Fig. 185). The zymogen granules are quite large granules and as they are highly refractive stand out distinctly even in the fresh, unstained condition and under low magnification. The relative size of these zones depends upon whether the cell is in the active or resting state (compare Fig. 186, $A$ and $B$ ). During rest (fasting) the two zones are of about equal size. During the early 
stages of activity (intestinal digestion) the granules largely disappear and the clear zone occupies almost the entire cell. During the height of digestion the granules are increased in number to such an extent

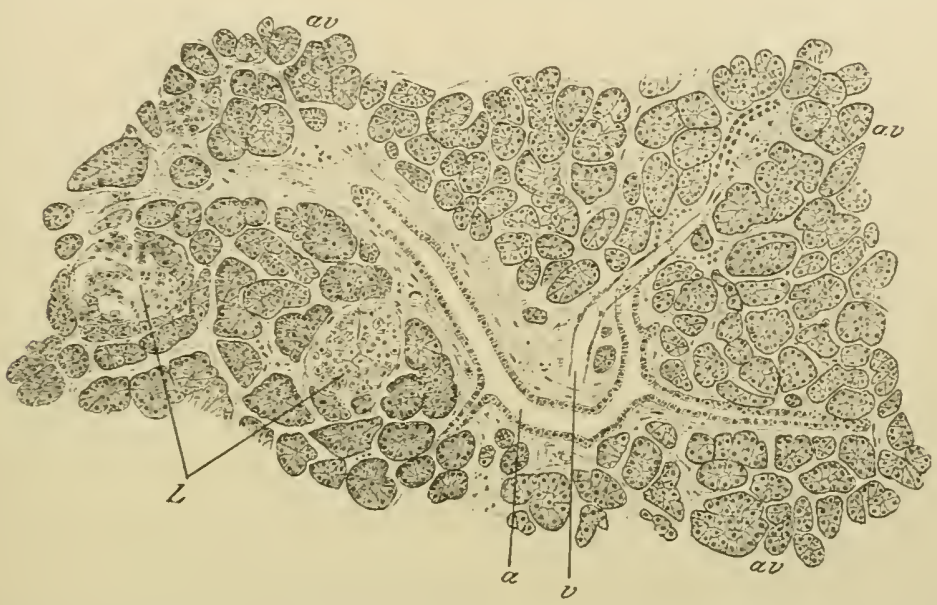

FIG. r84.-Section of Human Pancreas. XIr2. (Kölliker.) av, Alveoli; $a$, interlobular duct surrounded by interlobular connective tissue; $L$, islands of Langerhans; $v$, small vein.

that they almost fill the cell, while after prolonged secretion they are again almost absent. The cell now returns to the resting state in which the two zones are about equal. The increase and disappearance of the granules are marked by the appearance of the fluid secretion of the gland in the lumen. It would thus seem probable that the zymogen granules are the intracellular representatives of the secretion of the gland.

In sections of the gland there are seen within the lumina of many of the secreting tubules onc or more small cells of which little but the nucleus can usually le made

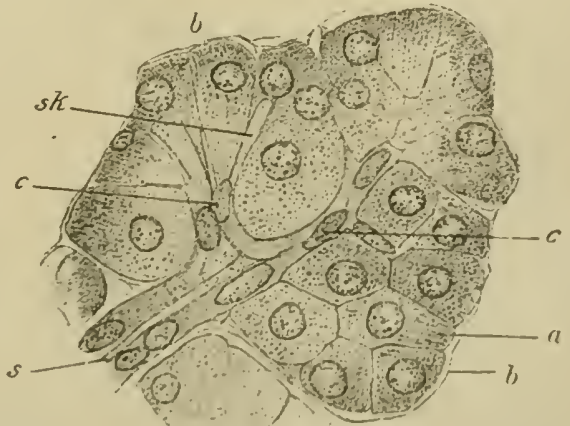

FIg. 185.-From Section of Ifuman Pancreas. $\times$ 700. (Kölliker.) $a$, Gland cell; $b$, basement membrane; $s$, intermerliate tubule; $c$, centroacinar cells; sk, intracellular secretory tubule. out. These cells lie in contact with the secreting cells, and resemble the flat cells which line the intermediate tubule. They are known as the centro-acinar (centro-tubular) cells of Langerhans (Fig. I85, c). 
Their significance is not definitely known. Langerhans believed that they were derived from the intermediate tubule, the epithelium of which, instead of directly joining that of the terminal

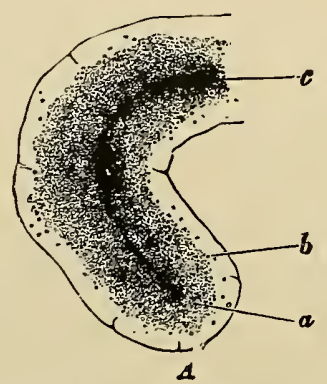

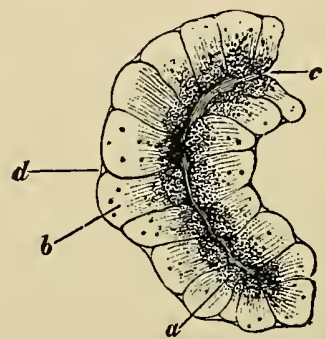

B.

FIG. I86.-Sections of Alveoli from Rabbit's Pancreas. (Foster, after Kühne and Lea.) $A$, Resting alveolus, the inner zone $(a)$, containing zymogen granules, occupying a little more of the cell than the outer clear zone $(b) ; c$, indistinct lumen. $B$, Active alveolus, granules coarser, fewer, and confined to inner ends of the cell $(a)$, the outer clear zone $(b)$ being much larger; outlines of cells and of lumen much more distinct.

tubule as in the submaxillary gland, was continued over into the lumen of the terminal tubule (Fig. 185). This interpretation has been quite generally accepted.

Cells which differ from the secreting cells are frequently found

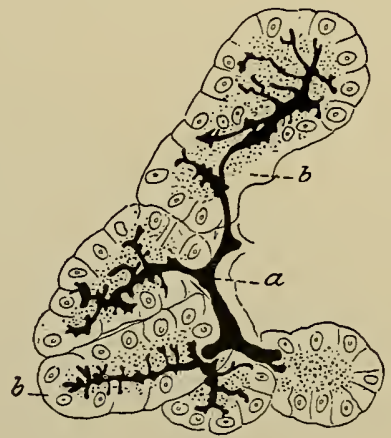

A

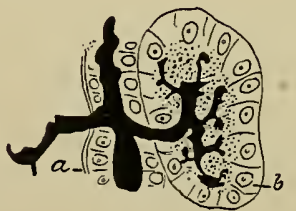

B

FIG. I87.-Sections through Alveoli of Human Pancreas-Golgi Method--(Dogiel), to show intracellular secretory tubules. $a$, Intermediate tubule giving off several terminal tubules, from which pass off minute intracellular secretory tubules; $b$, gland cells lining terminal tubules.

wedged in between the latter. They extend from the lumen to the basement membrane and are probably sustentacular.

Passing from the lumen of the terminal tubule, sometimes between the centro-tubular cells, directly into the cytoplasm of the secreting 
cells are minute intracellular secretory tubules. These are demonstrable only by special methods (Golgi) (Fig. IS7).

The pancreas also contains peculiar groups of cells, the cell-islands of Langerhans, having a diameter from 200 to $300 \mu$ (Figs. I84, I88, and I89). The "island" cells differ quite markedly both in arrangement and structure from those which line the terminal tubules (Fig. I88). They contain no zymogen granules. They are arranged in anastomosing cords or strands which are separated from one another by capillaries. There are no ducts and the method of Golgi shows no inter- or intra-cellular secretory tubules. Their protoplasm is un-

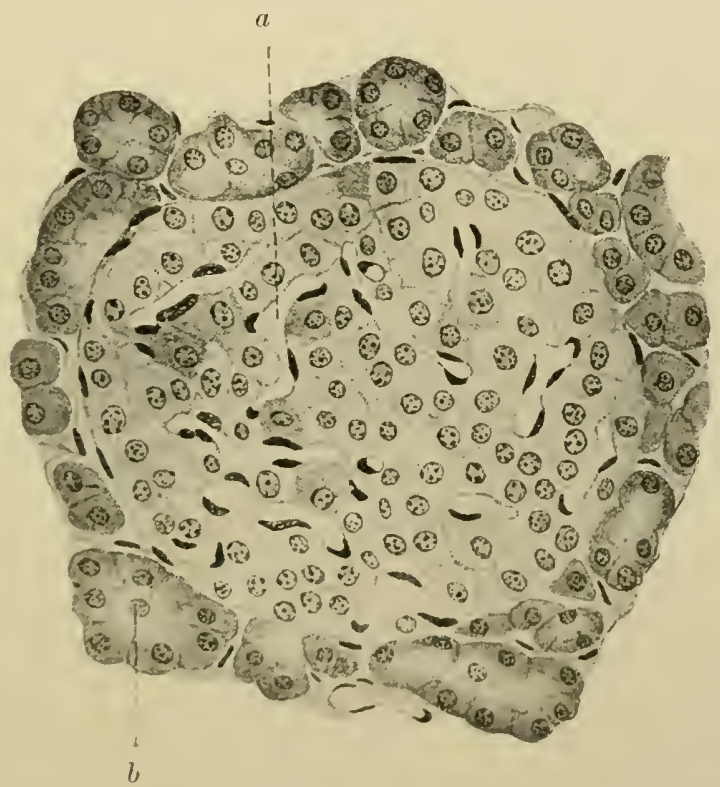

FIG. 188.- Island of Langerhans and few surrounding Pancreatic'Tubules. (Böhm and von Davidoff.) $a$, Capillary; $b$, tubule.

stained by basic dyes, but stains homogeneously with acid dyes. Their nuclei vary greatly in size, some, especially where the cells are closely packed, being small, others being large and vesicular. Some of the islands are quite sharply outlined by delicate fibrils of connective tissue containing a few elastic fibres (Fig. 188). Others blend with the surrounding tissues.

The origin, structure, and function of these islands have been subjects of much controversy. for some time they were considered of lymphoid origin. They are now believerl to lee epithelial cells having at developmental history similar to the cells lining the secreting tubules. Each cell-islind consists of, 
in addition to the cells, a tuft or glomerulus of broad tortuous anastomosing capillaries, which arise from the network of capillaries surrounding the secreting tubules. The close relation of cells and capillaries and the absence of any ducts have led to the hypothesis that these cells furnish a secretion-internal secretion - which passes directly into the blood-vessels.

In a recent publication Opie reviews previous work upon the histology of the pancreas and adds the results of his own careful researches. He concludes that the cell-islands of Langerhans are definite structures "formed in embryological life," that "they possess an anatomical identity as definite as the glomeruli of the kidney or the Malpighian body of the spleen, and that they subserve some

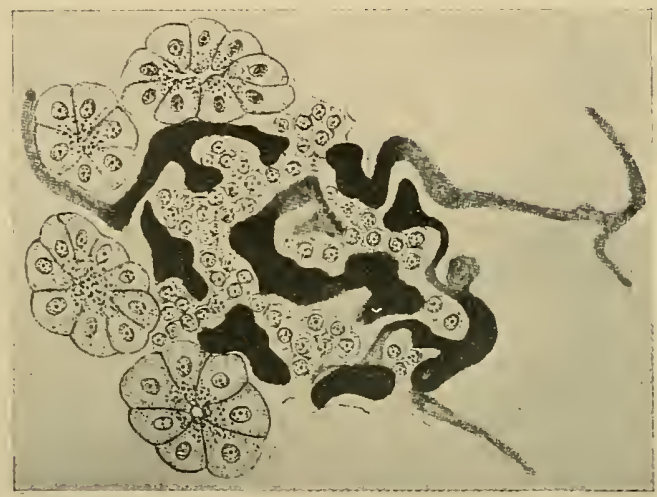

FIg. I 89.-From Section of Pancreas, the blood-vessels of which had been injected (Kühne and Lea), showing island of Langerhans with injected blood-vessels, surrounded by sections of tubules. Zymogen granules are distinct in inner ends of cells.

special function." He calls attention to the similarity which Schäfer noted between these cell-islands and such small ductless structures as the carotid and coccygeal glands and the parathyreoid bodies. From his study of the pancreas in diabetes, Opie concludes that the islands of Langerhans are concerned in carbohydrate metabolism.

Blood-vessels.- The arteries enter the pancreas with the main duct and break up into smaller arteries which accompany the smaller ducts. These end in a capillary network among the secreting tubules. From this, venous radicles arise which converge to form larger veins. These pass out of the gland in company with the arteries.

Lymphatics.-Of the lymphatics little is known.

Nerves.- The nerves are almost wholly from the sympathetic system, and are non-medullated. Some of them are axones of cells in sympathetic ganglia, outside the pancreas; others, of cells situated in small ganglia within the substance of the gland. They pass to plexuses among the secreting tubules, to which and to the walls of the vessels they send delicate terminal fibrils. 


\section{TECHNIC}

(I) The general technic for the pancreas is the same as for the salivary glands (page $28 \mathrm{I}$ ).

(2) Zymogen granules may be demonstrated by fixation in formalin-Müller's fluid (technic 5, p. 7), and staining with picro-acid-fuchsin (technic 2, p. 20), or with Heidenhain's iron hæmatoxylin (technic 3, p. IS).

(3) The arrangement of the blood-vessels in the islands of Langerhans may be studied in specimens in which the vascular system has been injected (page 25).

\section{The Liver}

The liver is a compound tubular gland, the secreting tubules of which anastomose. There are thus, strictly speaking, no "terminal tubules" in the liver, the lumina and walls of neighboring tubules anastomosing without any distinct line of demarcation.

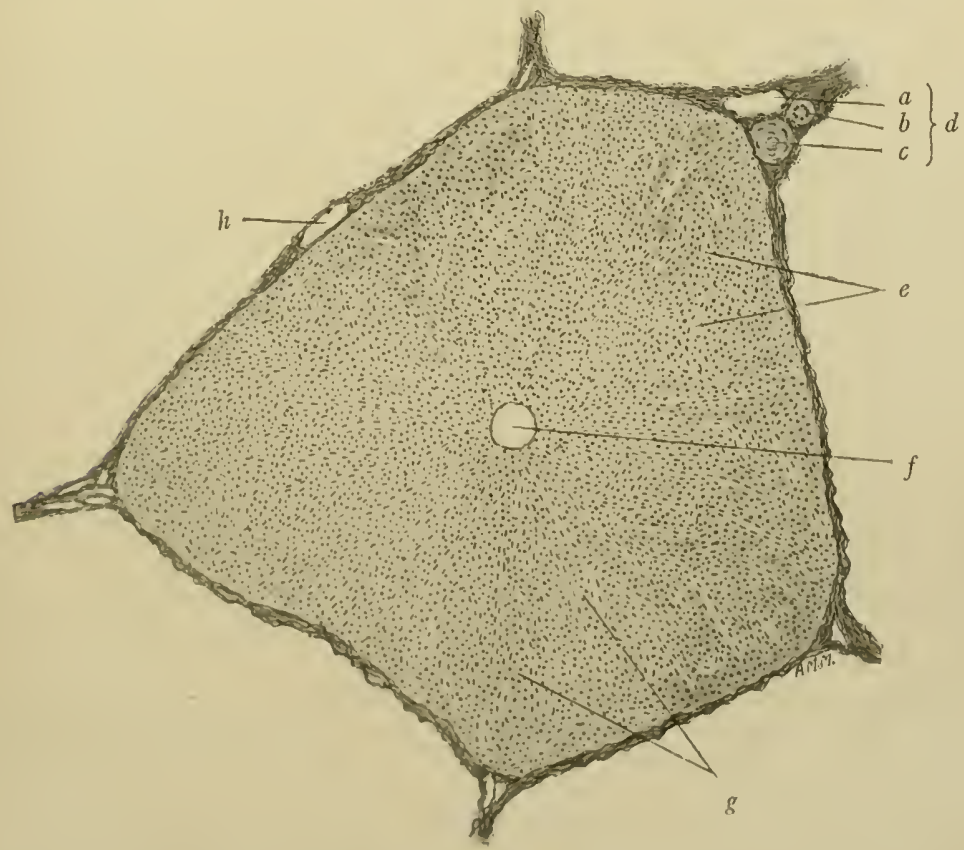

FIG. I90.-Section of Lobule of P'ig's Liver $\times 60$ (Lechnic $r$, p. 205), showing lobule completely surrounded by connective tissue. $a$, Portal vein; $b$, lile duct; $c$, hepatic artery; $d$, portal canal; $e$, capillaries; $f$, central vein; $g$, cords of liver cells; $h$, hepattic vein.

The liver is surrounded by a connective-tissue capsule, the capsule of Glisson. At the hilum this capsule extends deep into the substance of the liver, giving off broat connective-tissue sepla, which divide the organ into lobes. From the capsule and from these interlobar septa, 
trabeculæ pass into the lobes, subdividing them into lobules. In some animals, as for example the pig, each lobule is completely invested by connective tissue (Fig. I9o). In man, only islands of connective tissue are found, usually at points where three or more lobules meet (Fig. I9I). The lobules are cylindrical or irregularly polyhedral in shape, about $\mathrm{I} \mathrm{mm}$. in breadth and $2 \mathrm{~mm}$. in length. Excepting just beneath the capsule, where they are frequently arranged with

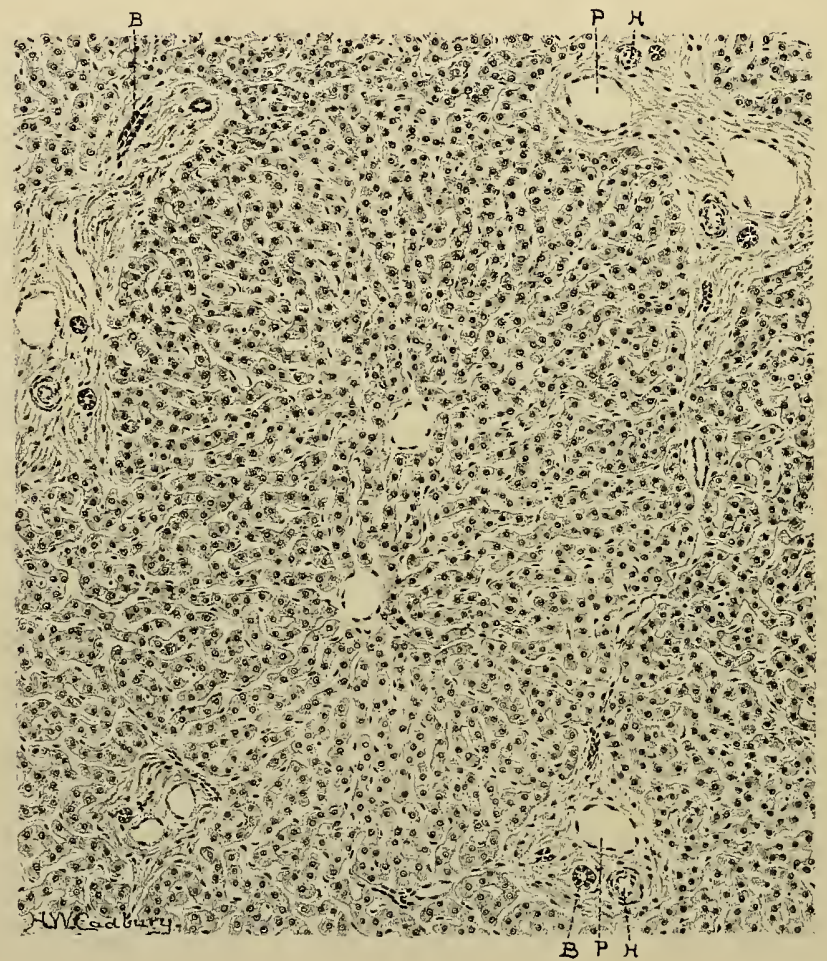

FIG. I9I.-Section of Human Liver. $\times 8$ o. (Hendrickson.) $P$, Portal vein; $H$, hepatic artery; $B$, bile duct. $P, H, B$ constitute the portal canal and lie in the connective tissue between the lobules.

their apices toward the surface, the liver lobules have an irregular arrangement.

The lobule (Fig. I90) which may be considered the anatomic unit of structure of the liver, consists of secreting tubules arranged in a definite manner relatively to the blood-vessels. The blood-vessels of the liver must therefore be first considered.

The BLOOD SUPPLY of the liver is peculiar in that in addition to the ordinary arterial supply and venous return, which all organs possess, 
the liver receives venous blood in large quantities through the portal vein. There are thus two afferent ressels, the hepatic artery and the portal sein, the former carrying arterial blood, the latter venous blood from the intestine. Both vessels enter the liver at the hilum and divide into large interlobar branches, which follow the connectivetissue septa between the lobes. From these are given off intcrlobular branches, which run in the smaller connective-tissue septa between the lobules. From the interlobular branches of the portal rein arise veins which are still interlobular and encircle the lobules. These send off short branches which pass to the surface of the lobule, where they break up into a rich intralobular capillary network. These intralobular capillaries all converge toward the center of the lobule, where they empty into the cenlral vein (Fig. I9o). The central reins are the smallest radicles of the hepatic veins, which are the efferent vessels of the liver. Each central vein begins at the apex of the lobule as a small vessel little larger than a capillary. As it passes through the centre of the long axis of the lobule the central vein constantly receives capillaries from all sides, and, increasing in size, leaves the lobule at

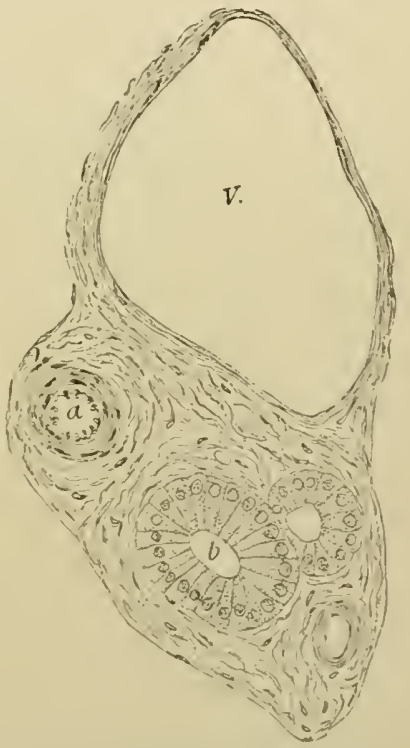

Frg. 192.- Portal Canal. $\times 315$. (Klein and Smith.) $a$, Hepatic artery; $V$, portal vein; $b$, bile duct. its base. Here it unites with the central veins of other lobules to form the sublobular vein which is a branch of the hepatic (Fig. I98).

The hepatic artery accompanies the portal vein, following the branchings of the latter through the interlobar and interlobular connective tissue, where its fincr twigs break up into capillary netzorks. Some of these capillaries empty into the smaller branches of the portal rein; others enter the lobules and anastomose with the intralobular portal capillaries.

The MAIN EXCRETORY DCCT - he patic duct-leaves the liver at the hilum near the entrance of the portal vein and hepatic artery. Within the liver the duct divides and subdivides, giving off interlobar, and these in turn interlobular branches. 'These ramify in the connective tissuc, where they always accompany the branches of the portal vein 
and hepatic artery. These three structures - the hepatic artery, the portal vein, and the bile duct, which always occur together in the connective tissue which marks the point of separation of three or more lobules-together constitute the portal canal (Fig. I92). From the interlobular ducts short branches pass to the surfaces of the lobules. From these are given off extremely narrow tubules, which enter the lobule as intralobular secreting tubules.

The walls of the ducts consist of a single layer of epithelial cells resting upon a basement membrane and surrounded by connective

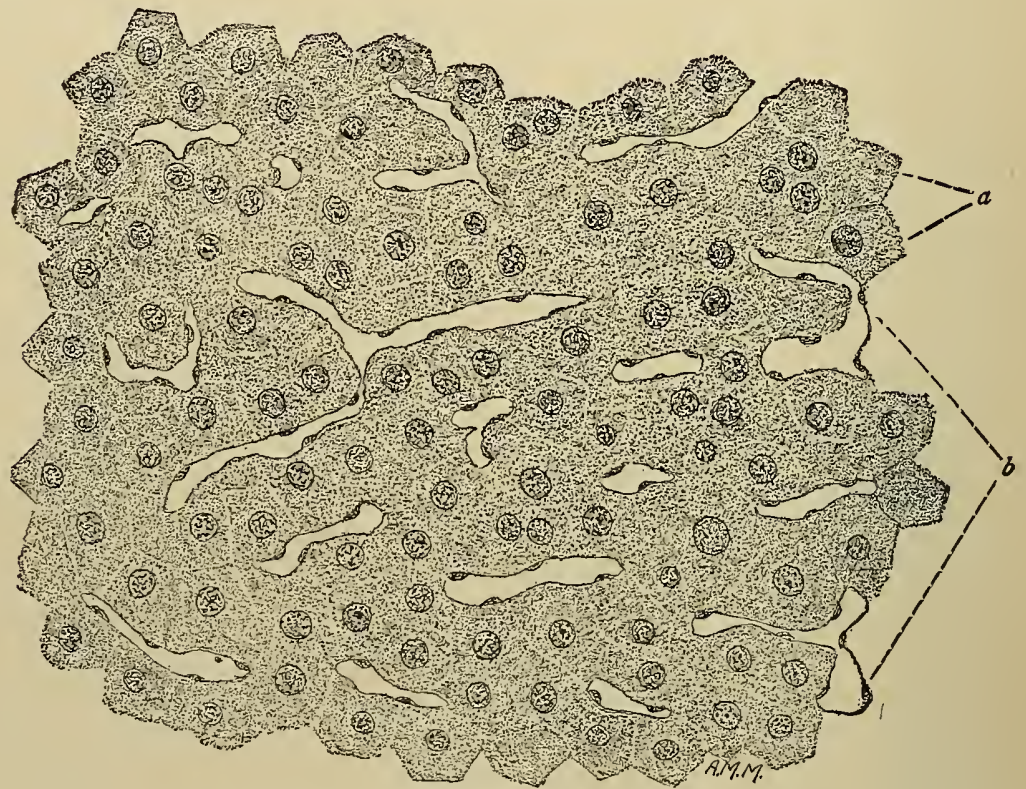

Fig. I93.-Part of Lobule of Human Liver, showing capillaries and anastomosing cords of liver cells. $\times 35^{\circ}$. $a$, Liver cells; $b$, capillaries.

tissue (Fig. 192). The height of the epithelium and the amount of connective tissue are directly proportionate to the size of the duct. In the largest ducts there are usually a few scattered smooth muscle cells.' The walls of the secreting tubules are formed by the liver cells.

The LIVER CELLS (Fig. I93) are irregularly polyhedral in shape. They have a granular protoplasm which frequently contains glycogen, pigment granules, and droplets of fat and bile. Each cell contains one or more spherical nuclei. Like other gland cells, the granularity of the protoplasm depends upon its functional condition. Within the cells are minute irregular canals, some of which can be 
injected through the blood-ressels, while others are apparently continuous with the secreting tubules (Fig. 195. $A$ and $B$ ).

The capillaries of the portal vein, as they anastomose and converge from the periphery to the centre of the lobule, form long-meshed

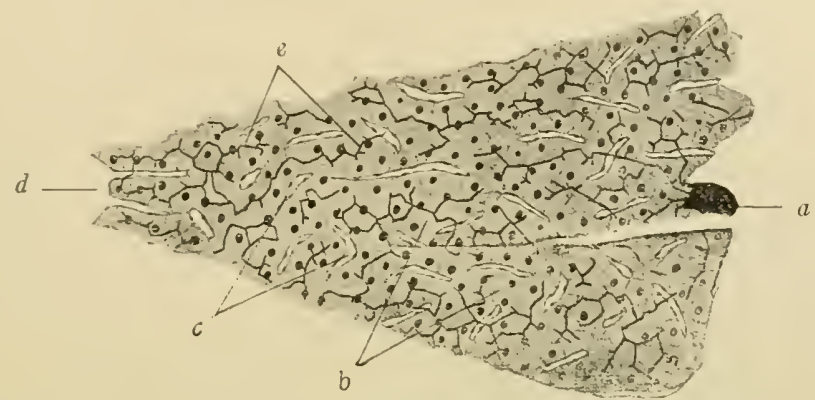

Fig. I 94.-Part of Lobule of Human Liver, Golgi Method (technic 3, p. 295), to show relations of bile duct to intralobular secretory tubules and of the latter to the liver cells. $\quad a$, Bile duct; $b$, cords of liver cells; $c$, blood capillaries; $d$, central vein; $e$, secretory tubules.

capillary networks. In the meshes of this network lie the anastomosing secreting tubules. On account of the shape of the capillary network, the liver cells, which form the walls of these tubules, are

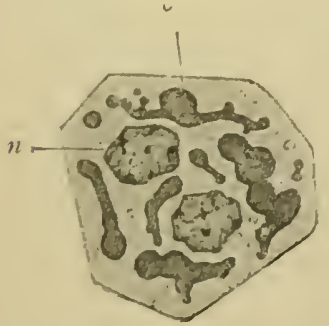

$A$

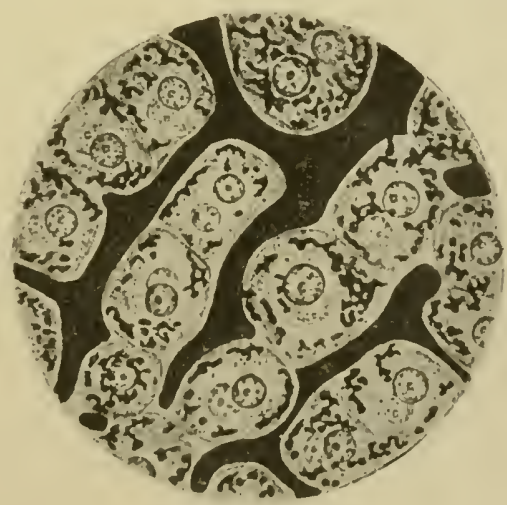

B

FIG. I95:- 1, Cell from human liver showing intracellulatr canals ('Srowicz); $c_{\text {, }}$ intraccellular canal; $n$, nucleus. B, From section of rabbit's liver injected inrouglh portal vein, showing intracellular canals (continuous with intercellular biond capillaries). ('sehäfer.)

arranged in anastomosing rows or cords, known as hepalic corls or cords of lizer cells (Jig. I 9.3).

The secreting tubules (Fig. 194) are extremely minute channels, the walls of which are the liver cells. A secretory lubule alway's runs 
between two contiguous liver cells, in each of which a groove is formed. The blood capillaries, on the other hand, are found at the corners where three or more liver cells come in contact. It thus results that bile tubules and blood capillaries rarely lie in contact, but are regularly separated by part of a liver cell. Exceptions to this rule sometimes occur. While most of the secretory tubules anastomose, some of them end blindly either between the liver cells or, in some instances, after extending a short distance within the cell protoplasm (Fig. 195 , $A)$. At the surface of the lobule there is a modification of some of

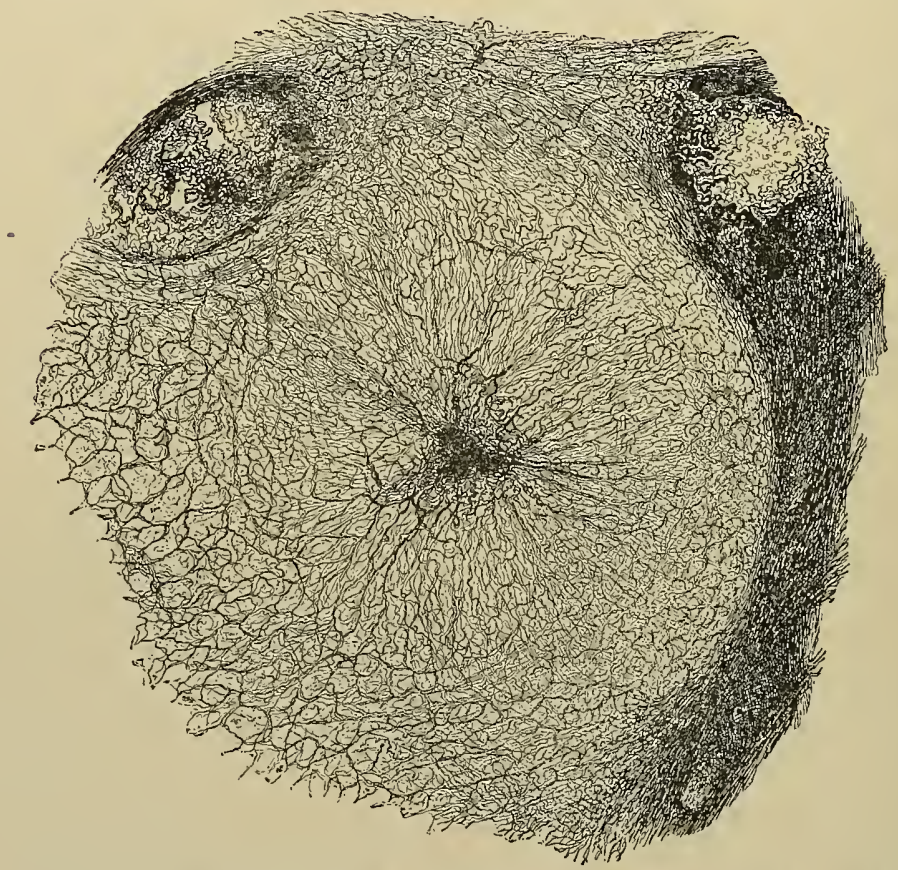

Frg. I96.-Liver Lobule, to show Connective-tissue Framework. (Mall.)

the liver cells to a low cuboidal type, and these become continuous with the lining cells of the smallest bile ducts, the secretory tubule being continuous with the duct lumen.

Special methods of technic have demonstrated a connective-tissue framework within the lobule. This consists of a reticulum of extremely delicate fibrils which envelop the capillary blood-vessels, and of a smaller number of coarser fibres which radiate from the region of the central vein-radiate fibres (Fig. I96).

Special technical methods also show the presence of stellate cells-cells of Kupffer-within the lobule. These are interpreted 
by Kupfier as belonging to the endothelium of the intralobular capillaries.

Comparing the liver with other compound tubular glands, it is seen to present certain marked peculiarities which distinguish it and which make its structure as a compound tubular gland difficult to understand. The most important of these are the following: (Figs. I97 and I98).

The extremely small amounl of connective tissue; in the human liver not enough interlobular connective tissue to outline the lobules,

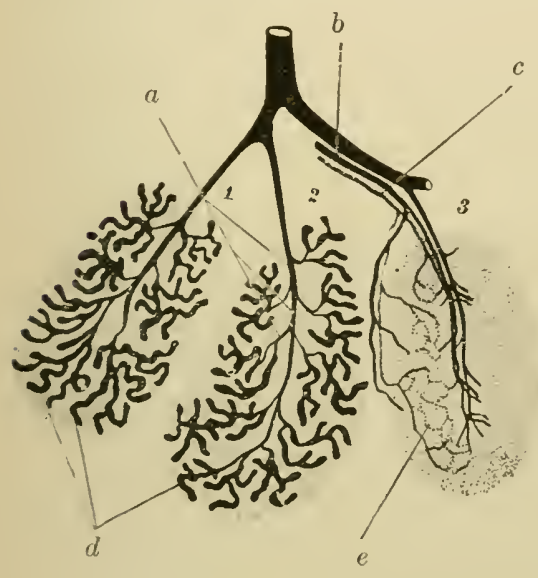

Frg. 197 .

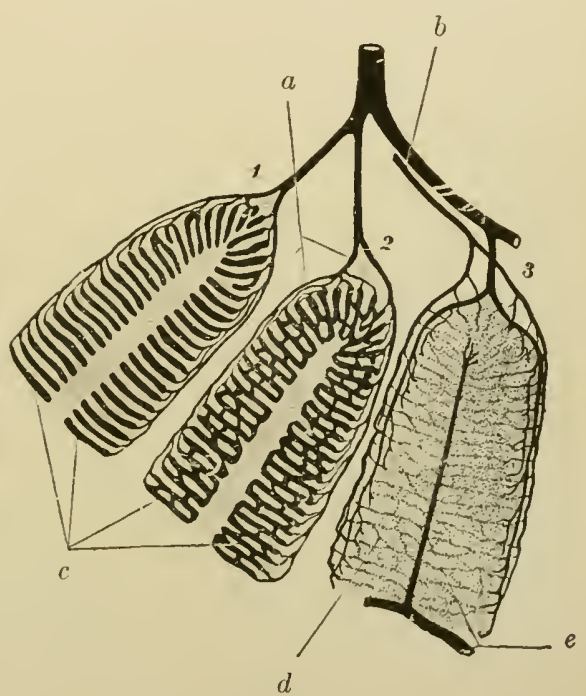

Fig. Ig8.

FIG. 197--Scheme of an Ordinary Compound Tubular Gland. In lobule 3 only the ramifications of the excretory duct, without endpieces, are shown. (Stöhr.) $a$, Branches of excretory duct; $b$, artery; $c$, vein; $d$, terminal tubules; $c$, capillaries.

Fig. I98. - Scheme of Liver. In lobule 1 , only the direction of the endpieces is shown; in lobule 2 only their branching; in 3 only the excretory ducts. (Stöhr.) $a$, Branches of excretory duct; $b$, portal vein; $c$, terminal tubules (hepatic cords); $d$, capillaries; $c$, vein (central and sublobular).

while intralobular connective tissue demonstrable by ordinary staining methods is wholly absent. There is thus no connective tissue seen separating the cells of one tubule from those of another as, for example, in such a gland as the submaxillary. The result is that cells of neighboring tubules lie sirle by side, and back to back as it were, with no intervening conneclive tissue.

The fact that unlike the tubules of olher glands, the liver lubule consists of only two rows of cells, between which lies the lumen. The latter is thus never in touch with more thatn two cells. 
The end-to-end anastomosis of the secreting tubules, there being no true terminal tubules; anastomosis of neighboring tubules by means of side branches; the arrangement of the bile capillaries in such a manner that a single liver cell abuts upon more than one capillary.

The more intimate relation of the liver cell to the blood capillaries. Thus most gland cells have one side on the lumen, one side only in contact with a capillary blood-vessel, the remaining sides being in contact with other cells of the same tubule. A liver cell, on the other hand, may and usually does come in contact with several blood capillaries.

The arrangement of both blood-vessels and tubules within the lobule. In the submaxillary, for example, the terminal tubules are convoluted and run in all directions. In the liver the terminal tubules are straight and run in a definite direction from the periphery of the lobule toward the centre. Again, while in other glands both intralobular arteries and ducts are distributed outward from the centre of the lobule, and the blood is returned through veins which pass to the periphery of the lobule, in the liver the interlobular ducts pass to the periphery of the lobule and give off secreting tubules which pass in toward the centre of the lobule. The afferent vessels also (portal veins) take the blood to the periphery of the lobule and distribute it to a capillary network which converges to an efferent vessel (hepatic vein) at the centre of the lobule. The veins are also peculiar in that they do not follow the arteries in leaving the liver but pursue an entirely independent course.

Blood-vessels. - These have.been already described.

Lymph vessels form a network in the liver capsule. These communicate with deep lymphatics in the substance of the organ. The latter accompany the portal vein and follow the ramifications of its capillaries within the lobule as far as the central vein.

The nerves of the liver are mainly non-medullated axones of sympathetic neurones. The nerves accompany the blood-vessels and bile ducts, around which they form plexuses. These plexuses give off fibrils which end on the blood-vessels, bile ducts, and liver cells.

Three main ducts, all parts of a single excretory duct system, are concerned in the transportation of the bile to the intestine, the hepatic, the cystic, and the common. Their walls consist of a mucous membrane, a submucosa, and a layer of smooth muscle. The mucosa is composed of a simple columnar epithelium resting upon a basement membrane 
and a stroma which contains smooth muscle cells and small mucous glands. The submucosa is a thin layer of connective tissue. Hendrickson describes the muscular coat as consisting of three layers, an inner circular, a middle longitudinal, and an external oblique. At the entrance of the common bile duct into the intestine, and at the junction of the duct of Wirsung with the common duct, there are thickenings of the circular fibres to form sphincters. In the cystic duct occur folds of the mucosa-the Heisterian valve-into which the muscularis extends.

\section{The Gall-Bladder}

The wall of the gall-bladder consists of three coats - mucous, muscular, and serous.

The mucous membrane is thrown up into small folds or ruga, which anastomose and give the mucous surface a reticular appearance. The epithelium is of the simple columnar variety with nuclei situated at the basal ends of the cells. A few mucous glands are usually found in the stroma.

The muscular coat consists of bundles of smooth muscle cells which are disposed in a very irregular manner, and are separated by considerable fibrous tissue. A richly vascular layer just beneath the stroma is almost free from muscle and corresponds to a submucosa. It frequently contains small lymph nodules.

The serous coat is a reflection of the peritoneum.

\section{TECHNIC}

(I) Before taking up the study of the human liver, the liver from one of the lower animals in which each lobule is completely surrounded by connective tissue should be studied. Fix small pieces of pig's liver in formalin-Mlüller's fluid (technic 5.p 7). Cut sections near and parallel to the surface. Stain with hematoxylin-picro-acid-fuchsin (technic 3, p. 21) and mount in balsam. In the pig's liver the lobules are completely outlined by connective tissue and the yellow picric-acid-stained lobules are in sharp contrast with the red fuchsin-stained connective tissue.

(2) For the sturly of the human liver treat small pieces of perfectly fresh tissue in the same manner as the preceding, but stain with hematoxylin-cosin (technic 1, p. 20).

(3) The secretory tubules and smaller bile ducts maly be denonstrated by terhnic, 5, p. 2\%). A lighte eosin stain l,rings out the liver cells.

(4) For the sturly of the blond-vessels of the liver, inject the vesseds through the inferior vena cavat or portal vein. If the vena cava is used, it is convenient to 
inject from the heart directly through the right auricle into the vena cava. Sections should be rather thick and may be stained with eosin, or even lightly with hæmatoxylin-eosin (technic I, p. 20), and mounted in balsam.

(5) For demonstrating the intralobular connective tissue, Oppel recommends fixing fresh tissue in alcohol, placing for twenty-four hours in a 0.5 -per-cent. aqueous solution of yellow chromate of potassium, washing in very dilute silver nitrate solution (a few drops of 0.75 -per-cent. solution to 50 c.c. of water) and then transferring to 0.75 -per-cent. silver nitrate solution, where it remains for twenty-four hours. Embed quickly in celloidin. The best tissue is usually found near the surfaces of the blocks. A similar result is obtained by fixing fresh $\mathrm{H}_{\mathrm{i}}$ tissue in 0.5 -per-cent. chromic-acid solution for three days, then transferring to 0.5 -per-cent. silver nitrate solution for two days.

\section{Development of the Digestive System}

In the development of the digestive system all the layers of the blastoderm are involved. Mesoderm and entoderm are, however, the layers most concerned, as the ectoderm is used only in the formation of the oral and anal orifices. The primitive alimentary canal is formed by two folds which grow out from the ventral surface of the embryo and unite to form a canal, in a manner that is quite similar to the formation of the neural canal. In this way the primitive gut is lined with cells which previously formed the ventral surface of the embryo, i.e., entoderm. A portion of the mesoderm accompanies the entoderm in the formation of the folds. This is known as the visceral layer of the mesoderm. The primary gut is thus a closed sac or tube. It is connected with the umbilical vesicle, but has no connection with the exterior. These connections are formed later by oral and anal invaginations of ectoderm which extend inward and open up into the ends of the hitherto imperforate gut. The ends of the alimentary tract, including the oral cavity and all of the glands and other structures connected with it, are of ectodermic origin. The epithelial lining of the gut and the parenchyma of all glands connected with it are derived from entoderm. The muscle, the connective tissue, and the mesothelium of the serosa are developed from mesoderm.

The mesodermic elements show little variation throughout the gut, the peculiarities of the several anatomical divisions of the latter being dependent mainly on special differentiation of the entoderm (epithelium). Beneath the entodermic cells is a narrow layer of loosely arranged tissue which later separates into stroma, muscularis mucosæ, and submucosa. Outside of this a broader mesodermic band of firmer structure represents the future muscularis.

The stomach first appears as a spindle-shaped dilatation about the end of the first month. Its entodermic cells, which had consisted of a single layer, increase in number and arrange themselves in short cylindrical groups. These are the first traces of tubular glands. They increase in length and extend downward into the mesodermic tissue. For a time the cells lining the peptic glands are all apparently alike, but at about the fourth month the differentiation into chief cells and parietal cells takes place.

In the intestines a proliferation of the epithelium and of the underlying 
stroma results in the formation of villi. These appear about the tenth week, in both small and large intestines. In the former they increase in size, while in the latter they atrophy and ultimately disappear. The simple tubular glands of the intestines develop in a manner similar to those of the stomach.

The mesothelium of the serosa is derived from the mesodermic cells of the primitive body cavity.

The development of the larger glands, connected with the digestive tract, takes place in a manner similar to the formation of the simple tubular glands. All originate in extensions downward of entodermic cords into the underlying mesodermic tissue. From the lower ends of these cords, branches extend in all directions to form the complex systems of tubules found in the compound glands.

The salivary glands being developed from the oral cavity, originate in similar invaginations of ectodermic tissue.

The pancreas originates as three separate evaginations from the entoderm of the future duodenum. Of these, one atrophies, while the other two unite to develop the adult pancreas. The two evaginations account for the two pancreatic ducts. The evaginations take place into the underlying mesenchyme, which develops the connective-tissue framework of the organ. The growths are at first solid cords of cells which later become hollowed out to form tubules and differentiate chief and centro-acinar cells, the former early showing zymogen granules. Increase in size of the gland is accomplished by continuous budding and extension of tubules. In some of the tubules the epithelial cells become darker and arranged as a wall around a mass of developing blood cells. These structures are known as primary islands. Neither their function nor subsequent history is known. Later in development some of the gland tubules lose their lumina, the zymogen granules disappear from their cells and the whole becomes transformed into a secondury island or island of Langerhans of the adult pancreas. According to Lageusse a transformation of islands into tubules also occurs, and this process of transformation of tubules into islands and islands into tubules persists throughout life. Claude on the other hand derives both primitive islands and islands of Langerhans from the mesenchyme. Recent observations tend to support the former of these views.

The liver originates as a downgrowth of the entoderm of the ventral wall of the future duodenum into the mesoderm of the transverse septum. At its cephalic end the downgrowth is solid and gives rise to the liver; at the caudal end it is hollow and gives rise to the gall-bladeler. As the evagination increases in size it becomes almost completely separated from the intestine, the slender connection which does remain lecoming the ductus choledochus. Between the latter and the liver anlage a slender connection also remains, the cystic duct. The mescnchyme surrounding these structures develops their connective-tissue framework. As the liver outgrowths develop they come into reliztion with the omphalomesenteric veins in such a manner that the vessels are broken up into an anastomosing network of smaller vessels, while the liver celis develop into anastomosing hepatic cylinders. 'These resemble the tubules of other grlands in that the walls are formerl l,y many eclls. The manner in which these are transformerl inte the slenrler hepatic: cords of the adult liver is not known. 'The 
change begins to take place about the time of birth in man. The breaking up of the larger vessels into smaller by ingrowth of the cords of liver cells gives rise to the so-called sinusoidal circulation.

\section{General References for Further Study}

Oppel: Løhrbuch der vergleichenden mikroskopischen Anatomie.

Kölliker: Handbuch der Gewebelehre des Menschen.

Opie: The Pancreas.

Stöhr: Salivary Glands, in Text-book of Histology. 


\section{CHAPTER VII}

\section{THE RESPIRATORY SYSTEM}

THE respiratory apparatus consists of a system of passages--nares, larynx, trachea, and bronchi. which serve for the transmission of air to and from the essential organ of respiration, the lungs.

\section{The Nares}

The nares, or nasal passages, are divided into vestibular, respiratory, and olfactory regions, the differentiation depending mainly upon the structure of their mucous membranes.

The veSTIBULAR REGION marks the transition between skin and mucous membrane (page 220). Its epithelium is of the stratified squamous variety and rests upon a basement membrane, which is thrown into folds by papillæ of the underlying stroma. The latter is richly cellular, and contains sebaceous glands (page 39I) and the follicles of the nasal hairs.

The RESPIRATORY REGION is much larger than both the vestibular and olfactory regions. Its epithelium is of the stratified columnar variety. The cells of the surface layer are ciliated and are interspersed with goblet cells. The stroma is distinguished by its thickness ( 3 to $5 \mathrm{~mm}$. over the inferior turbinates) and by the presence of networks of such large veins that the tissue closely resembles erectile tissue. It contains considerable diffuse lymphoid tissue and here and there small lymph nodules. In the stroma are small simple tubular glands lined with both serous and mucous cells. There is no submucosa, the stroma being connected directly with the periosteum and perichondrium of the nasal bones and cartilages.

The mucrous membrane of the accessory nasal sinuses is similar in structure to that of the respiratory region of the nares, but is thinner and contains fewer glands.

The offartoky REcion can be distinguished with the naked eye by its browni-h-yellow color, in contrast with the reddish tint of the surrounding respiratory mueosit. The epithelium is of the stratifiel 
columnar type, and is considerably thicker than that of the respiratory region. The surface cells are of two kinds: (I) sustentacular cells, and (2) olfactory cells.

(I) The sustentacular cells are the more numerous. Each cell consists of three parts: (a) A superficial portion, which is broad and cylindrical, and contains pigment, and granules arranged in longitudinal rows. The cells have well-marked, striated, thickened free borders, which unite to form the so-called membrana limitans olfactoria. (b) A middle portion which contains an oval nucleus. As the nuclei of these cells all lie in the same plane, they form a distinct narrow band, which is known as the zone of oval nuclei. (c) A thin filamentous process which extends from the nuclear portion down between the cells of the deeper layers. This process is irregular and pitted by pressure of surrounding cells. It usually forks and apparently anastomoses with processes of other cells to form a sort of protoplasmic reticulum.

(2) The olfactory cells lie between the sustentacular cells. Their nuclei are spherical, lie at different levels, and are most of them more deeply placed than those of the sustentacular cells. They thus form a broad band, the zone of round nuclei. From the nuclear portion of the cell a delicate process extends to the surface, where it ends in several minute hair-like processes. From the opposite pole of the cell a longer process extends centrally as a centripetal nerve fibre. The olfactory cell is thus seen to be of the nature of a ganglion cell (see also page 426 ).

Between the nuclear parts of the olfactory cells and the basement membrane are the basal cells. These are small nucleated elements, the irregular branching protoplasm of which anastomoses with that of neighboring basal cells and of the sustentacular cells to form the peculiar protoplasmic reticulum already mentioned.

The basement membrane is not well developed.

The stroma consists of loosely arranged white fibres, delicate elastic fibres, and connective-tissue cells. Embedded in the stroma are large numbers of simple branched tubular glands, the glands of Bowman. Each tubule consists of a duct, a body, and a fundus. The secreting cells are large and irregular and contain a yellowish pigment, which with that of the sustentacular cells is responsible for the peculiar color of the olfactory mucosa. These glands were long described as serous, but are now believed to be mucous in character. They frequently extend beyond the limits of the olfactory region. 


\section{The Larynx}

The larynx consists essentially of a group of cartilages united by strong fibrous bands and lined by mucous membrane.

The epithelium covering the true vocal cords, the laryngeal surface of the epiglottis, and the anterior surface of the arytenoid cartilages is of the stratified squamous variety with underlying papillæ. With these exceptions the mucous membrane of the larynx is lined with stratified columnar ciliated epithelium similar to that of the respiratory portion of the nares. Numerous goblet cells are usually present, and the epithelium rests upon a broad basement membrane. On the posterior surface of the epiglottis many taste buds (see Fig. 297 and page ${ }_{5} 87$ ) are embedded in the epithelium.

The stroma is especially rich in elastic fibres. The true vocal cords consist almost wholly of longitudinal elastic fibres covered by stratified squamous epithelium. Lymphoid cells are present in varying numbers. In some places they are so numerous that the tissue assumes the character of diffuse lymphoid tissue. Distinct nodules sometimes occur.

Owing to the absence of a muscularis mucosæ the stroma passes over with no distinct line of demarcation into the submucosa. This is a more loosely arranged, less cellular connective tissue, and contains simple tubular glands lined with both serous and mucous cells.

Externally the submucosa merges into a layer of more dense fibrous tissue which connects it with the laryngeal cartilages and with the surrounding structures. Immediately surrounding the cartilages the connective tissue forms an extremely dense layer, the perichondrium.

Of the cartilages of the larynx, the epiglottis, the middle part of the thyreoid, the apex and vocal process of the arytenoid, the cartilages of Santorini and of Wrisburg are of the yellow elastic variety. The main body of the arytenoid, the rest of the thyreoid and the cricoid cartilages are hyaline. After the twentieth year, more or less ossification is usually found in the cricoid and thyreoid cartilages.

\section{The Trachea}

The walls of the trachea consist of three layers-mucosa, submucosa, anrl filorosa (Fig. r99).

The mucosa is continuous with that of the larynx, which it closely resembles in structure. It consists of a stratified columnar ciliated 
epithelium, with numerous goblet cells, resting upon a broad basement membrane, and of a stroma of mixed fibrous and elastic tissue containing many lymphoid cells.

The submucosa is not distinctly marked off from the stroma on account of the absence of a muscularis mucosæ. It is distinguished from the stroma by its looser, less cellular structure, by its numerous large blood-vessels, and by the presence of glands. These are of the simple branched tubular variety and are lined with both serous and

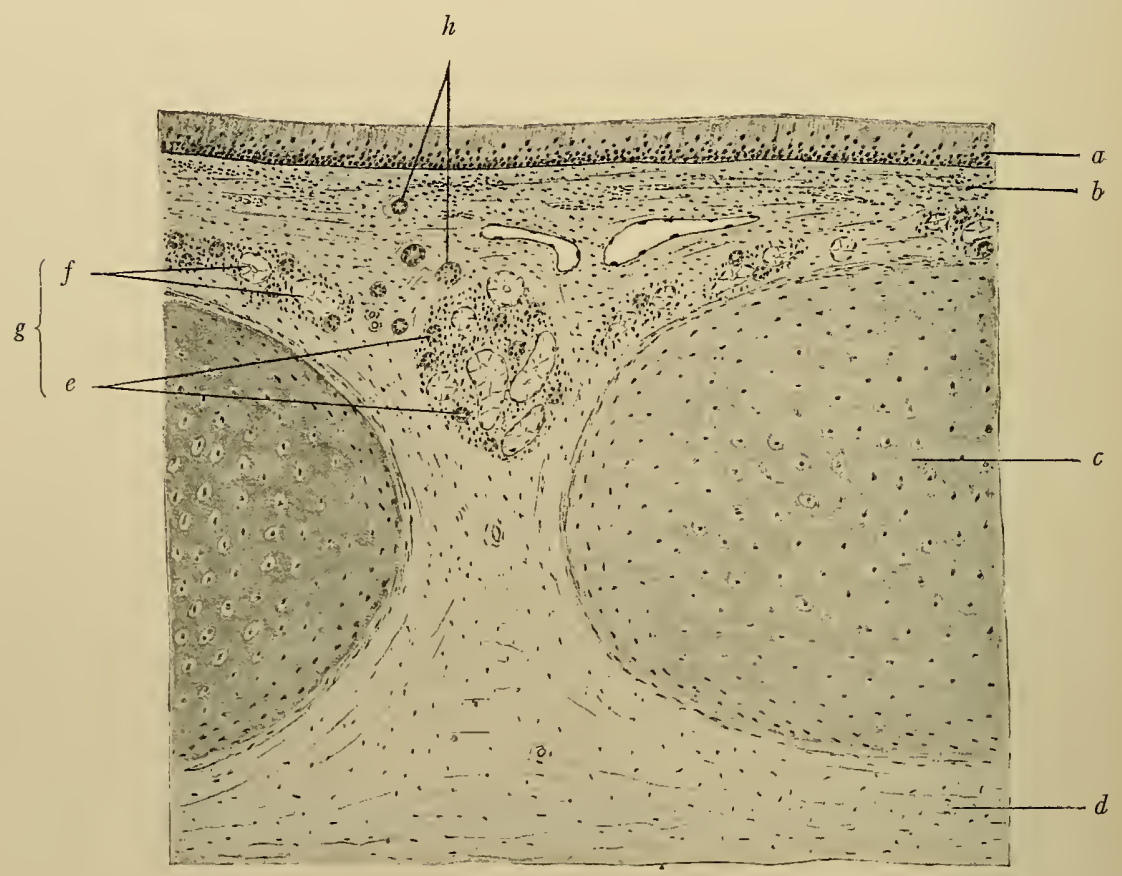

FIG. I99.-From Longitudinal Section of Human Trachea. $\times_{40}$. (Technic $3, p$ 304.) $a$, Epithelium; $b$, stroma; $c$, cartilage; $d$, fibrous coat; $e$, serous tubules; $f$, mucous tubules; $g$, glands in submucosa; $h$, ducts.

mucous cells. Some of the mucous tubules have well-marked.crescents of Gianuzzi. The glands are most numerous between the ends of the cartilaginous rings, where they frequently penetrate the muscle and extend into the fibrosa.

The fibrosa is composed of coarse, rather loosely woven connective-tissue fibres embedded in which are the tracheal cartilages. These are incomplete rings of hyaline cartilage shaped like the letter $\mathrm{C}$ (Fig. 200). They are from sixteen to twenty in number and encircle about four-fifths of the tube, being open posteriorly. The openings 
between the ends of the cartilaginous rings are bridged over by a thickened continuation of the fibrous coat, strengthened by a layer of smooth muscle (Fig. $200, \mathrm{~m}$ ). The bundles of muscle cells run mainly in a transverse direction, and extend across the intervals between adjacent rings as well as between their open ends. There

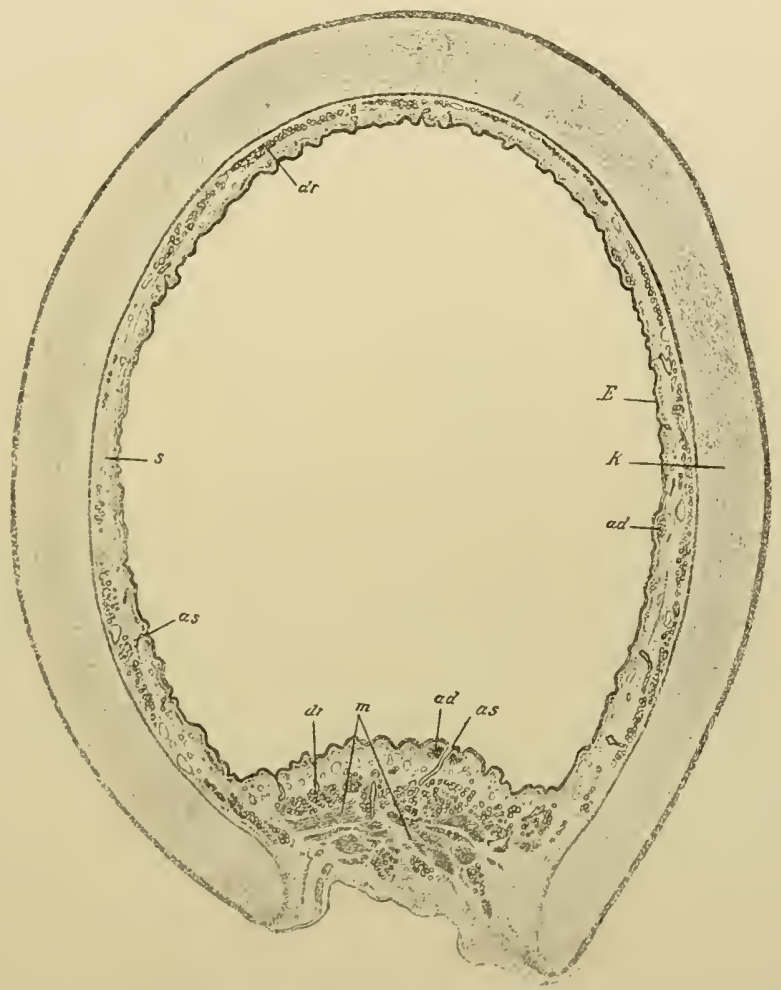

Fig. 200.-Transwerse Section of Human Trachea through One of the Cartilage Rings. $\times 8$. (Kölliker.) $E$, Epithelium of $(s)$ mucous membrane; $d r$, glands; as, gland duct; $a d$, arlenoid tissue; $K$, cartilage; $m$, smooth muscle cut longitudinally, extending across between ends of cartilage ring.

are frequently a few bundles of obliquely disposed cells and, most external, some few that run longitudinally.

Outside the fibrous coat proper is a looser, more irregular connective tissue, which serves to attach the trachea to the surrounding structures.

Blood-vessels, lymphatics, and nerves have a similar distribution in larynx and trachea. The larger vessels pass directly to the submucosa. From these, smaller branches pass to the different coats, where they break up into capillary networks. 
Lymphatics form plexuses in the submucosa and mucosa, the most superficial lying just beneath the subepithelial capillary plexus.

The nerves of the larynx and trachea are derived from both cerebro-spinal and sympathetic systems. The cerebro-spinal nerves are afferent, the dendrites of spinal ganglion cells. They form a subepithelial plexus from which are given off fibrils which pass into the epithelium and terminate freely among the epithelial cells. Other afferent fibres of cerebro-spinal nerves pass to the muscular coat of the trachea. Sympathetic nerve fibres form plexuses which are interspersed with minute groups of ganglion cells. Axones from these ganglion cells have been traced to the smooth muscle cells of the trachea. Sympathetic axones also pass to the glands of the trachea and larynx. On the under surface of the epiglottis small taste buds are found.

\section{TECHNIC}

(I) For the study of the details of structure of the walls of the nares and larynx, fix small pieces of perfectly fresh material from different regions in formalin-Müller's fluid (technic 5, p. 7), harden in alcohol, stain sections with hæmatoxylin-eosin (technic I, p. 20), and mount in balsam.

(2) The general relations of the parts can be studied by removing the larynx, upper part of the trachea, and corresponding portion of the œsophagus of an animal or of a new-born child, fixing and hardening as above, and cutting longitudinal sections through the entire specimen.

(3) Trachea.-Remove a portion of the trachea and treat as in technic (I). Both longitudinal and transverse sections should be made; the longitudinal including at least two of the cartilaginous rings; the transverse being through one of the rings.

\section{The Bronchi}

The primary bronchi and their largest branches have essentially the same structure as the trachea except that the cartilaginous rings are not as complete. Bronchi branch at acute angles and also give off small side branches.

As they decrease in calibre, the following changes take place in their walls (Figs. 20I to 204).

(I) The epithelium gradually becomes thinner. In a bronchus of medium size (Fig. 20I) it has become reduced to three layers of cells, which Kölliker describes as an outer "basal" layer, a middle "replacing" layer, and a surface layer of ciliated and goblet cells. In the smaller bronchi (Figs. 203, 204) the epithelium is reduced to a 
single layer of ciliated cells. These are at first high, but become gradually lower as the bronchi become smaller, until in the terminal branches the epithelium is simple cuboidal and non-ciliated. Among the ciliated cells are varying numbers of mucous or goblet cells.

(2) The stroma decreases in thickness as the bronchi become smaller. It consists of loosely arranged white and elastic fibres. This layer with the epithelium is folded longitudinally (Fig. 204). There is considerable diffuse lymphatic tissue, and in some places small nodules occur, over which there may be lymphoid infiltration

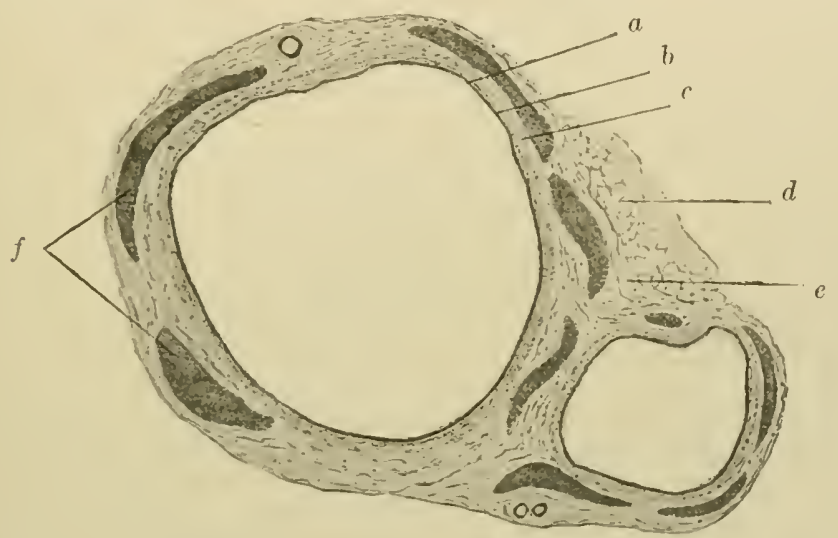

FIG. 201.-Transverse Section through two Medium-size Bronchi of the Human Lung. $\times \mathrm{r}_{5}$. (Technic 2, p. 317) In the fibrous coat are seen the bronchial arteries and veins. $a$, Epithelium; $b$, stroma; $c$, muscularis mucosæ; $d$, lung tissue; $e$, fibrous coat; $f$, plates of cartilage.

of the epithelium (see Tonsil, page I79). Near the root of the lung many small lymph nodules are found, which show different degrees of pigmentation.

(3) With decrease in thickness of the epithelium and of the stroma, the thickness of the mucosa is maintained by the appearance of a layer of smooth muscle. In the larger bronchi this is a continuous layer of circularly disposed smooth muscle, and lies just external (1) the stroma, forming a muscularis mucosic (Fig. 202). It reaches its greatest thickness relative to the size of the bronchus in the bronchi of medium size. As the bronchi become smaller it becomes thinner, then discontinuous, and in the smallest bronchi consists of only a few scattered muscle cells. These continue into the walls of the alveolar ducts, but are absent beyond this point.

(4) The submucosit decreases in thickness with decrease in the 
calibre of the bronchi. It consists of loosely arranged connective tissue. Mixed glands (Fig. 202) are present until a diameter of about. I $\mathrm{mm}$. is reached, when they disappear. They lie in the submucosa and frequently extend through between the cartilage plates into the fibrous coat. The ducts pass through the muscular coat and open into pit-like depressions lined with a continuation of the surface ciliated epithelium.

(5) The cartilages, which in the trachea and primary bronchi form nearly complete rings, become gradually smaller, and finally break up into short disconnected plates (Figs. 20I and 202). They

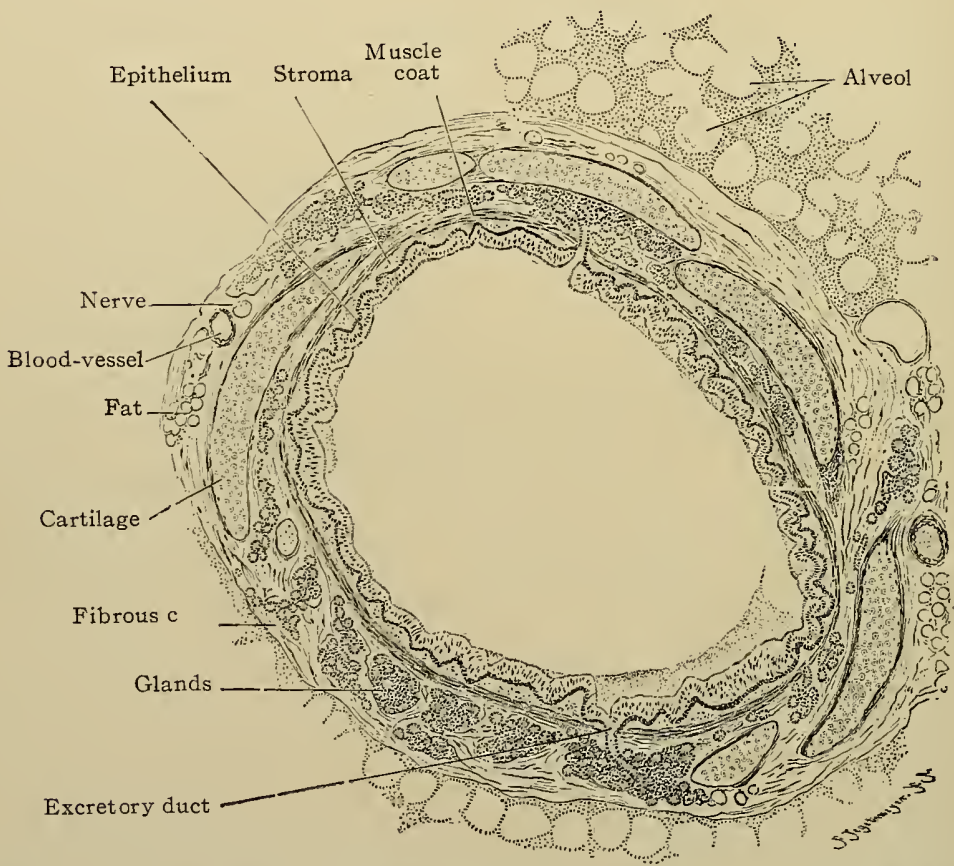

Fig. 202.-Cross Section of Human Bronchus (of a child) of $2 \mathrm{~mm}$. diameter. $\times 30$. (Stöhr.)

are frequently fibrocartilage rather than hyaline. These plates decrease in size and number, and are absent after a diameter of $\mathrm{I} \mathrm{mm}$. is reached. Cartilage and mucous glands thus disappear at about the same time, although it is common for glands to extend over into smaller bronchi than do the cartilage plates.

The bronchi down to a diameter of from I.5 to I mm. are interlobular, and belong to the duct system down to a diameter of about 
$0.5 \mathrm{~mm}$. From the small interlobular bronchi are given off the terminal bronchi. These are respiratory in character and are described with the lungs.

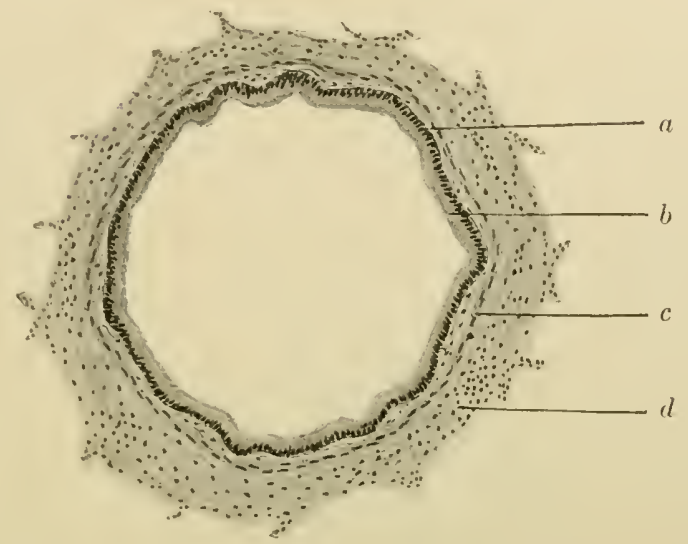

Fic. 203.-Transverse Section of Small Bronchus from Human Lung. Xir5. (Technic 2 , p. 3 I 7 .) $a$, Stroma; $b$, epithelium; $c$, muscularis mucosa; $d$, fibrous coat.

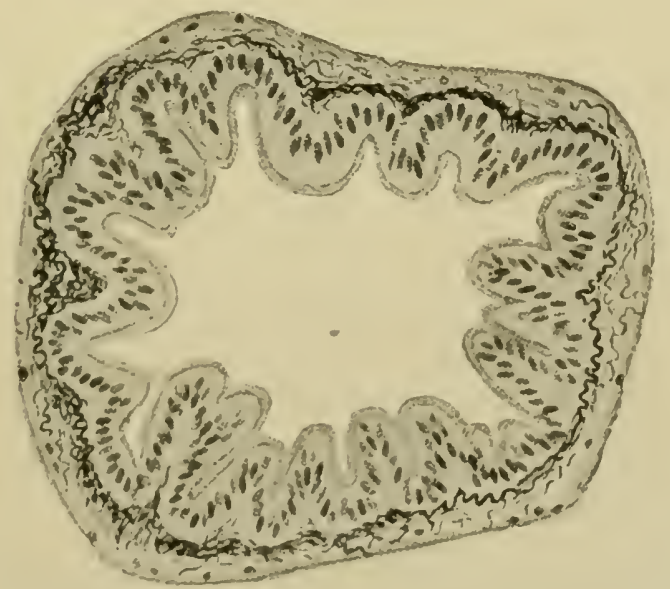

Fig. 204.-Transverse Section through small Bronchus of IIuman Lung. (Soljotta.) Simple columnar ciliated epithelium; no cartilage; no glands; mucosa folded longitudinally; elastic tissue stained with II eigert's elastic tissue stain.

In studying the bronchi it is convenient to arbitrarily divide them into large, medium-sized, and small bronchi.

Large bronchi have essentially the same structure ats the trathea except for somewhat thinner walls.

Medium-sized bronchi (Fig. 201) have an epithelium about three layers deep, disconnected plates of carrilitge, a continuous layer of smooth muscle disposed circularly as a muscularis mucosar and tubular glands. 
Small bronchi have a single layer of ciliated epithelium, a thinner muscular coat, no glands, and no cartilage. (Figs. 203, 204.)

\section{The Lungs}

The lung is built upon the plan of a compound alveolar gland, the trachea and bronchial ramifications corresponding to duct systems, the air vesicles to gland alveoli.

The surface of the lung is covered by a serous membrane-the pulmonary pleura - which forms its capsule, and which at the root of the lung, or hilum, is reflected upon the inner surface of the chest

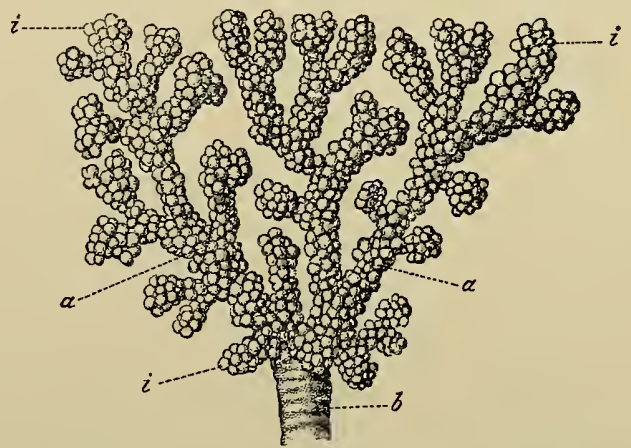

FIG. 205.-From Lung of an Ape. The bronchi and their dependent ducts and alveoli have been filled with quicksilver. $X_{5_{5}}$. (Kölliker, after Schulze.) $b$, Terminal bronchus; $a$, alveolar duct; $i$, alveoli.

wall as the parietal pleura. It consists of fibrillar connective tissue containing fine elastic fibres which are more numerous in the visceral than in the parietal layer. From the capsule broad connectivetissue septa pass into the organ, dividing it into lobes. From the capsule and interlobar septa are given off smaller septa, which subdivide the lobes into lobules.

The human pulmonary lobule (Figs. 206 and 208) is the anatomic unit of lung structure. Each lobule is complete in itself, having its own bronchial system, its own vascular system, and being more or less completely separated from its neighbors by connective tissue. In the young and in some lower animals the lobule is quite plainly outlined by connective tissue, but in the human adult the amount of connective tissue is extremely small, and the lobules, especially the more central, difficult of definition. The lobules are best observed at the surface of the lung where their bases which lie against the pleura can be seen with the naked eye. This is especially true in the aged 
where carbon deposits in the interlobular connective tissue assist in outlining the lobules. The base of the peripheral lobule is four-to eightsided and from I to $1.5 \mathrm{~cm}$. in diameter. It is pyramidal in shape, narrowing to an apex about I to $1.5 \mathrm{~cm}$. from the base. In the interior of the lung the lobules are not pyramidal but irregularly polyhedral, the apex being, however, distinguishable by the entrance of the lobular bronchus.

The apex of each lobule is the point of entrance of the lobular bronchus (branch of inter- or sublobular) (Figs. 205, and 208, bl) which is about $0.5 \mathrm{~mm}$. in diameter, and of the lobular branch of the

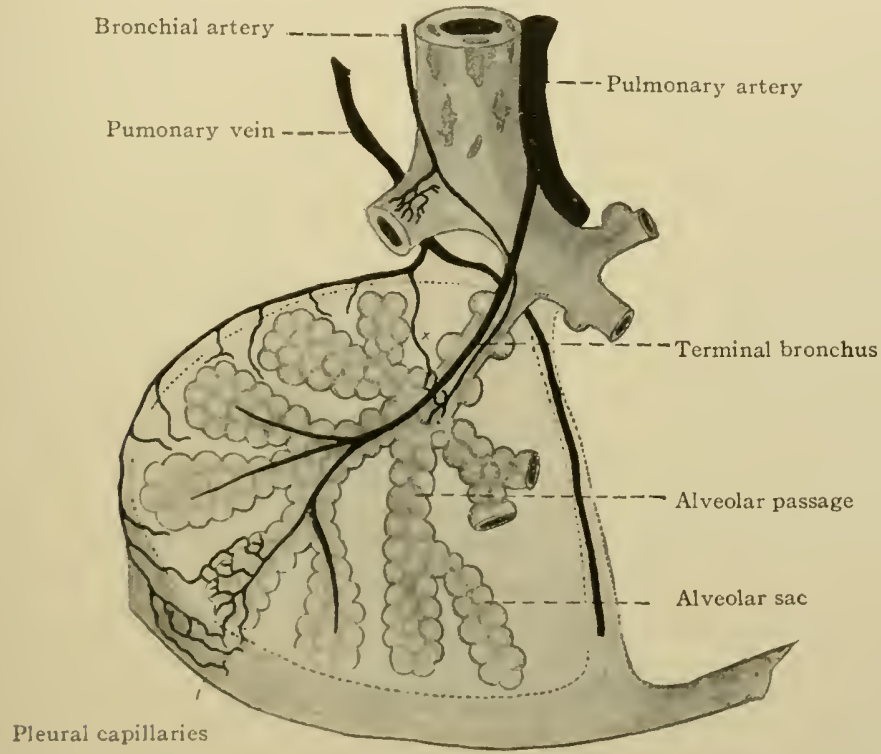

FiG. 206.-Scheme of a I'ulmonary Lobule and its Blood Supply. (Stöhr.) The two main branches of the pulmonary vein are seen lying in the interlobular connective tissue.

pulmonary artery. Accompanied by the artery (Fig. 206) the bronchus passes through the central axis of the lobule, giving off collateral branches (Fig. 205, $a, b$ ), to about the midllle of the tobule, where it divides into two branches (Fig. 206). These branches and also the collaterals branch dichotomously giving rise to from 50 to roo terminal or alveolar bronchi ("terminal", as being the last subdivision of the bronchial tree which preserves its identity as a bronchus; "alveolar", as it gives off especially toward its distal end, some alveoli). From each terminal bronchus open from three to six narrow 
passages-alveolar passages or alveolar ducts-from which are given off the alveoli-air vesicles or air cells. The somewhat dilated distal end of the alveolar passage is sometimes designated the alveolar sac or infundibulum. Laguesse divides the lobule schematically into three parts a proximal part containing the intralobular bronchus and collaterals, a middle part in which the main division of the

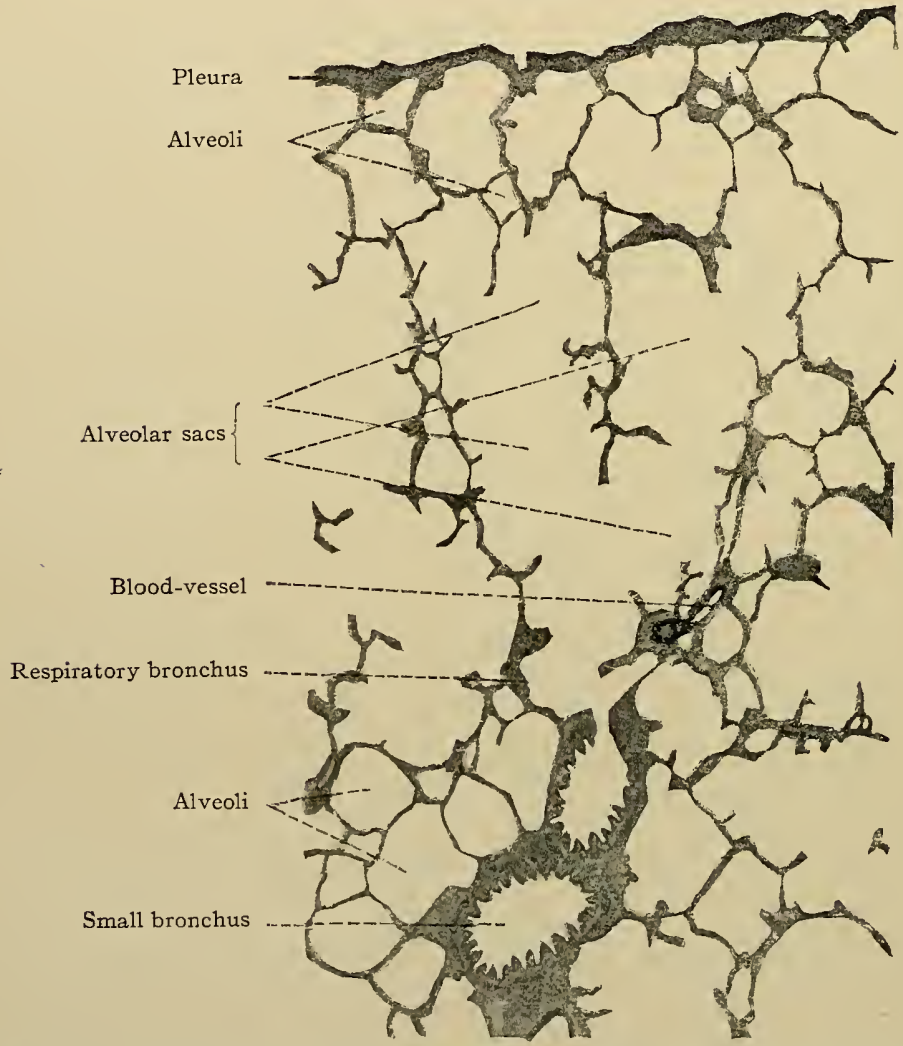

Frg. 207.--Section of Cat's Lung (Szymonowicz), surface lobule; respiratory bronchus opening into alveolar duct from which are given off two alveolar sacs.

lobular bronchus occurs, and a third part containing the alveolar bronchi and alveoli.

The terminal bronchus. The proximal portion of the terminal bronchus is lined by a simple columnar ciliated epithelium, resting upon a basement membrane. Beneath this is a richly elastic stroma containing bundles of circularly disposed smooth muscle cells. The epithelium becomes gradually lower and non-ciliated, and near 
the distal end of the terminal bronchus there appear small groups or islands of flat, non-nucleated epithelial cells-respiratory epithelium F. The alieolar passage. Here the cuboidal epithelium is almost completely replaced by the respiratory. Beneath the epithelium the walls have a structure similar to those of the distal end of the terminal

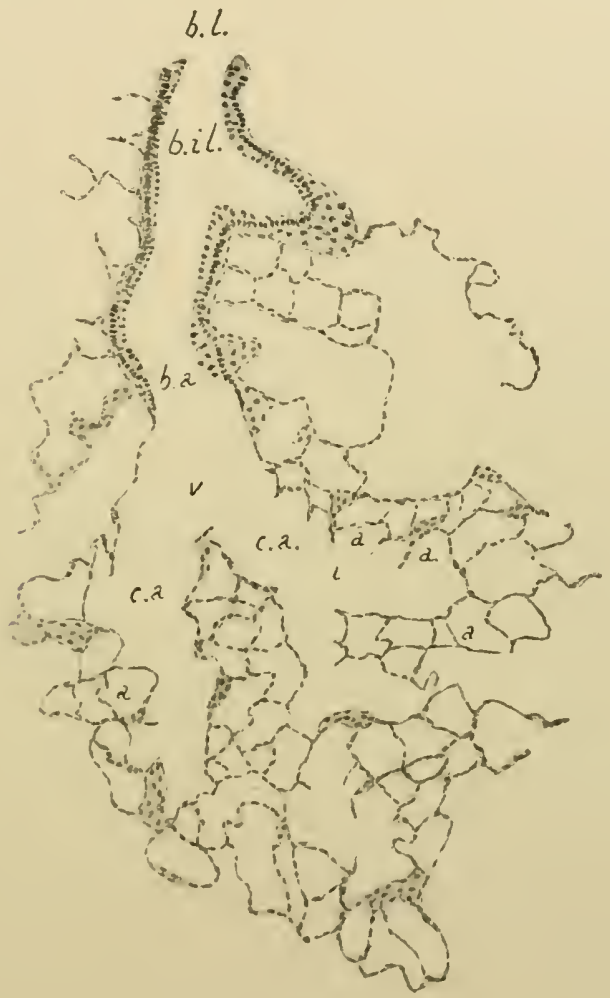

Fig. 208.- Section of Lung of Rat to show Arrangement of Bronchial Ramifications and of Alveoli within a Single Iobule. bl, Lobular or sublobular bronchus; bil, intralobular bronchus; $b a$, terminal bronchus; $v$, dilatation sometimes called vestibule; $c a$, alveolar canal; $i$, portion sometimes called infundibulum; $d$, alveoli, some of which are so cut as to show their openings into the infundibulum and alveolar canals, etc., while others appear closed. $\times 60$. (I'renant.)

bronchus, consisting of elclicate fibro-elastic tissuc with scattered smooth muscle cells. The basement membrane is extremely thin.

The alicolus. The epithelium of the alveolus consists of two kinds of cells, respiratory cells and so)-called "fatal" cells (see Develop)ment, jage 315 ).

The respirutory cells (Fig. 200) are some of them large, flat, nonnucleated plates, while others are much smaller, non-nucleated ele- 
ments. The absence of nuclei and the extremely small amount of intercellular substance render these cells quite invisible in sections stained by the more common methods. The cell boundaries are best demonstrated by means of silver nitrate (technic I, p. 78).

The "fotal" cells are granular, nucleated cells which are scattered among the respiratory cells. Their position appears to be less superficial than that of the respiratory cells, the fotal cells lying in the meshes of the capillary network, the respiratory cells covering the capillaries. In the embryonic lung and in the lungs of a still-born

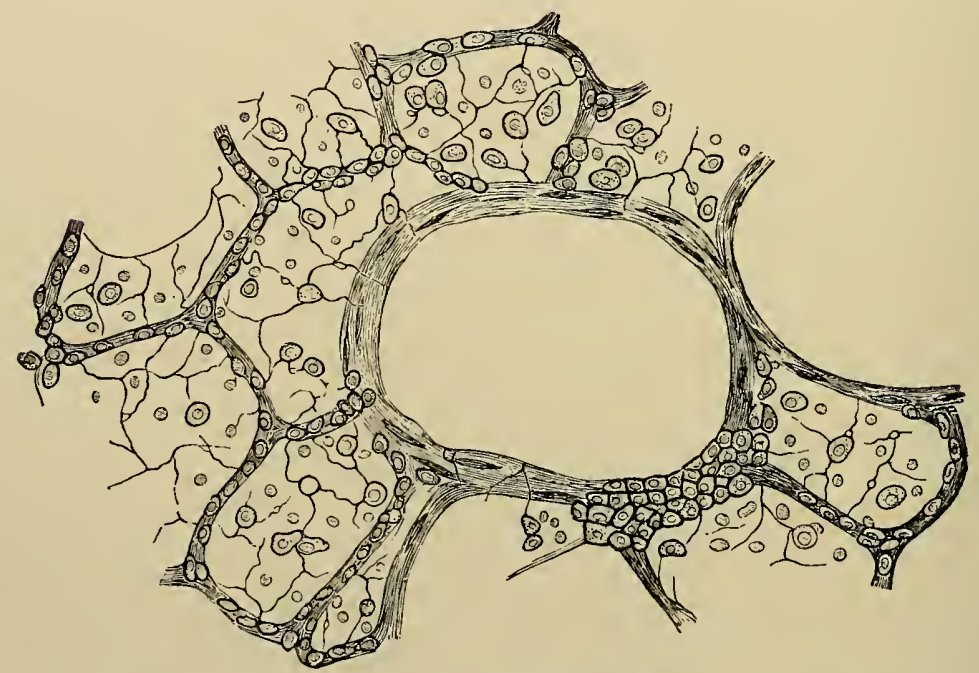

FIG. 209.--From Section of Cat's Lung Stained with Silver Nitrate. (Klein.) (Technic I, p. 78.) Small bronchus surrounded by alveoli, in which are seen both flat cells (respiratory epithelium) and cuboidal cells (fœtal cells).

child the air passages and alveoli contain only this type of cells, the small flat plates apparently resulting from a flattening out of the cuboidal cells due to pressure from inspiration, and the large flat plates to union of a number of small plates. Delicate elastic fibrils support the respiratory and fotal cells. Around the opening of the alveolus the elastic fibres are more numerous, forming a more or less definite ring. The disposition of elastic tissue in the wall of the alveoli is undoubtedly of importance in determining the contraction and expansion of the alveoli under varying conditions of pressure. It has been estimated that on forced inspiration an alveolus can expand to three times its resting capacity. Each alveolus communicates not only with its alveolar passage, or alveolar bronchus, by means of a 
broad opening, but alveoli are connected with one another by minute openings in their walls.

The interalicolar connective tissue, while extremely small in amount, serves to separate the alveoli from one another. Somewhat thicker connective tissue separates the alveoli of one alveolar passage from those of another. Still stronger connective-tissue bands as already noted separate adjacent lobules.

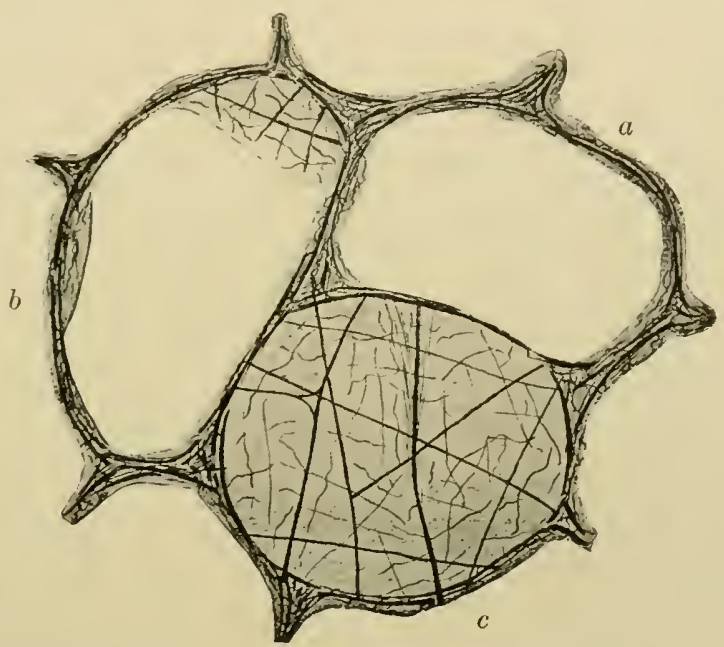

FIG. 2 Ic.- Section Through Three Alveoli of Human Lung. $\times 235$. Weigert's elastic-tissue stain (technic 3, p. 28) to show arrangement of elastic tissue. a, Alveolus cut through side walls only; $b$, alveolus cut through side walls and portion of bottom or top; $c$, alveolus in which either the bottom or top is included in section.

Blood-vessels. - Two systems of vessels distribute blood to the lungs. One, the bronchial system, carries blood for the nutrition of the lung tissue. The other, the much larger pulmonary system, carries blood for the respiratory function (Fig. 206).

The bronchial artery and the pulmonary artery enter the lung at its hilum. Within the lung the vessels branch, following the branchings of the bronchi, which they accompany (Fig. 206). The pulmonary vessels are much the larger and run in the connective tissue outside the bronchial walls. The bronchial vessels lie within the fibrous coat of the bronchus. A section of a bronchus thus usually shows the large pulmonary vessels, one on either side of the bronchus, and two or more small bronchial vessels in the walls of the bronchus (Fig. 20I).

'The pulmonary lobule forms a distinct "blood-vascular unit." A branch of the pulmonary artery enters the apex of each lobule close 
to the lobular bronchus, and almost immediately breaks up into branches, one of which passes to each alveolar passage (Fig. 206). From these are given off minute terminal arterioles which pass to the central sides of the alveolar passages and alveoli, where they give rise to a rich capillary network. This capillary network is extremely close-meshed, and invests the alveoli on all sides (Fig. 2 I I). Similar networks invest the walls of the respiratory bronchi, the alveolar ducts, and their.alveoli. All of these capillary networks freely anastomose.

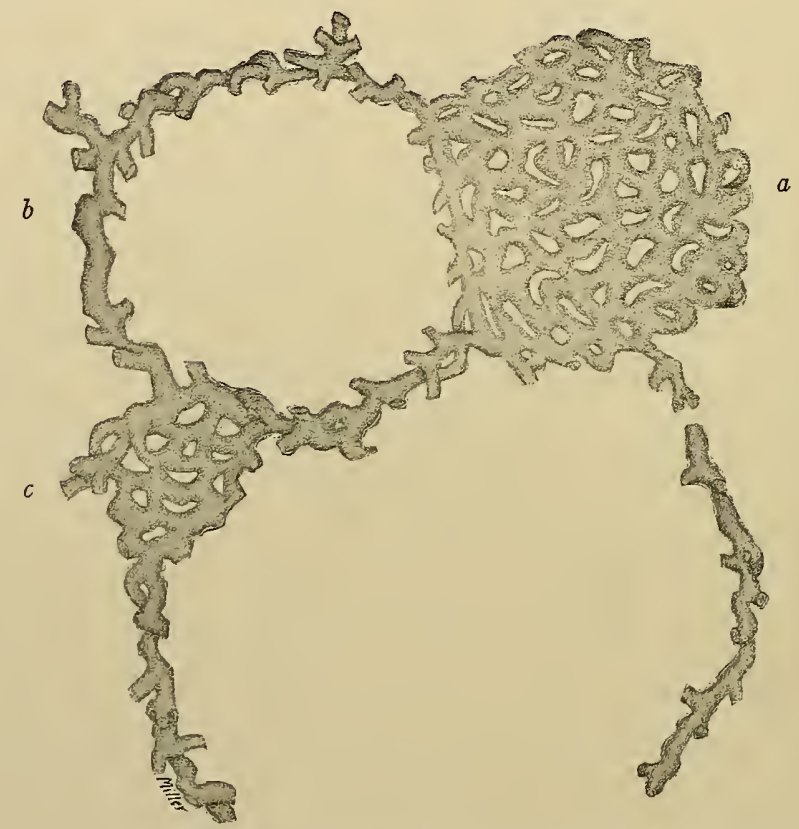

FIG. 2II.-Parts of Four Alveoli from Section of Injected Human Lung. $\times 200$. (Technic 5, p. $3^{\text {I } 7 .) ~ a, ~ W a l l ~ o f ~ a l v e o l u s ~ s e e n ~ o n ~ f l a t ; ~} c$, same, but only small part of alveolar wall in plane of section; $b$, alveoli in which plane of section includes only side walls; alveolar wall seen on edge.

There are thus interposed between the blood in the capillaries and the air in the alveoli only three extremely thin layers: (I) The thin endothelium of the capillary wall; (2) the single layer of flat respiratory epithelial plates; and (3) the delicate basement membrane upon which the respiratory epithelium rests together with an extremely small amount of fibrous and elastic tissues (see diagram, Fig. 2 г 2.)

The veins begin as small radicles, one from the base of each alveolus (Fig. 206). These empty into small veins at the periphery of the lobule. These veins at first run in the interlobular connective tissue 
away from the artery and bronchus. Later they empty into the large pulmonary trunks which accompany the bronchi.

The bronchial arteries break up into capillary networks in the walls of the bronchi, supplying them as far as their respiratory divisions, beyond which point the capillaries belong to the pulmonary system. The bronchial arteries supply the walls of the bronchi, the bronchial lymph nodes, the walls of the pulmonary vessels, and the pulmonary pleura. Of the bronchial capillaries some empty into the bronchial veins, others into the pulmonary veins.

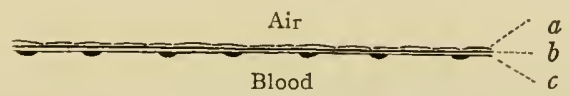

FIG. 2I 2.- Diagram of Tissues Interposed Between Blood and Air in Alveolus. $a$, Respiratory epithelium; $b$, fibro-elastic tissue; $c$, endothelium of capillary. As $b$ does not form a continuous membrane, the capillary wall is in many places in direct apposition with the respiratory epithelium, so that only two layers, $a$ and $b$, are interposed between blood and air.

Lymphatics.--The lymphatics of the lung begin as small lymph spaces in the interalveolar connective tissue. These communicate with larger lymph channels in the interlobular septa. Some of these empty into the deep pulmonary lymphatics, which follow the pulmonary vessels to the lymph glands at the root of the lung. Others empty into the superficial pulmonary lymphatics, which form an extensive subpleural plexus connected with small subpleural lymph nodes, whence by means of several larger vessels the lymph is carried to the lymph nodes at the hilum.

Nerves.-Bundles of medullated and non-medullated fibres accompany the bronchial arteries and veins. Small sympathetic ganglia are distributed along these nerves. The fibres form plexuses in the fibrous layer of the bronchi, from which terminals pass to the muscle of the bronchi and of the vessel walls and to the mucosa. Free endings upon the epithelium of bronchi, air passages, and alveoli have been described.

\section{Developalent of the Respikatory System}

The epithelium of the respiratory system develops from entoderm, the connective-tissue elements from mesoderm. The first differentiation of respiratory system appears as a dipping down of the entorlerm of the floor of the primitive pharynx (some investigators describe two original evaginations, one for each lung). The tubule thus formed divides into a larger and longer right branch, which subdivides into three branches corresponding to the three lobes of the future right lung, (Fig. $213, \mathrm{~b}, \mathrm{~b}, \mathrm{~b}$ ) and a smaller and shorter left branch, which subdivides into two branches corresponding to the two lobes of the future left 
lung. By repeated subdivisions of these tubules the entire bronchial system is formed. Up to this point (about six months in human fœtus) the development is that of a compound alveolar gland, (Fig. 213). The last to develop are the respiratory divisions of the bronchi with their alveolar passages and alveoli. The appearance of the alveoli is wholly characteristic of lung (Fig. 213, vpd.) The epithelium of the alveoli is at first entirely of the fotal-cell type, the large flat respiratory plates appearing only late in fœtal life. Just how and when

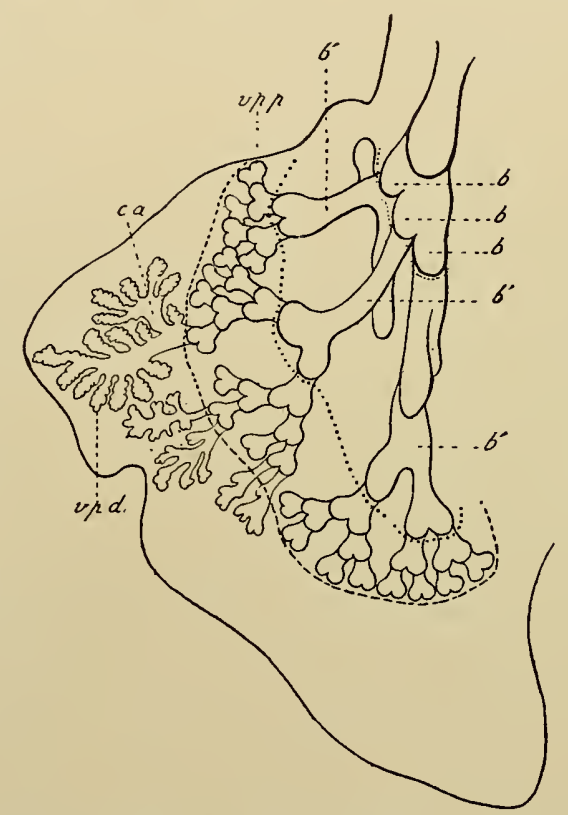

FIG. 2 I3.-Scheme of Development of Lung (Right). $\quad b, b, b$, thethree primary bronchial buds; $b^{\prime}, b^{\prime}, b^{\prime}$, collateral branches and secondary buds, terminating in $v p p$, the primary vesicles; $v p d$, pulmonary vesicles proper or alveoli; $c a$, alveolar canals. The broken line - - - - limits the stage of the three primary lung buds; between this line and the line.... only the three primary buds, their collaterals and secondary buds; between the line ...... and line - the stage of dichotomous division and of termination in primary vesicles. Up to this point the development is that of a compound alveolar gland. From this line to the surface represents the final period of development, which is peculiar to the lung and results in the formation of the pulmonary alveoli. (Prenant.)

the flattening of the epithelial cells takes place is not definitely known. The accepted theory has been that the cells become flattened rather suddenly at birth as a result of the first inspiration. Some authors describe a gradual thinning of the cells from the sixth fœtal month on. Bikfalir describes a gradual thinning which is completed rather rapidly on inspiration. The fœtal and respiratory cells of the adult lung have the same embryonic origin. During the early stages of lung development the mesodermic tissue predominates, but with the rapid growth of the tubules the proportion of the two changes until in the adult lung the mesodermic tissue becomes restricted to the inconspicuous pulmonary framework and the blood-vessels. 


\section{TECHNIC}

(I) The technic for the largest bronchi is the same as for the trachea (technic 3, p. 30.4). The medium size and small bronchi are studied in sections of the lung.

(2) Lung and Bronchi--Carefully remove the lungs and trachea (human, dog, or cat) and tie into the trachea a cannula to which a funnel is attached. Distend the lungs moderately (pressure of two to four inches) by pouring in formalin-Müller's fluid (technic 5, p. 7), and then immerse the whole in the same fixative for twenty-four hours. Cut into small blocks, using a very sharp razor so as not to squeeze the tissue, harden in alcohol, stain thin sections with hæmatoxylin-eosin (technic I, p. 20), and mount in balsam or in eosin-glycerin. The larger bronchi are found in sections near the root of the lung. The arrangement of the pulmonary lobules is best seen in sections near and horizontal to the surface. Sections perpendicular to and including the surface show the pulmonary pleura.

(3) Respiratory Epithelium (technic I, p. 78).

(4) Elastic Tissue of the Lung (technic 3, p. 28).

(5) Blood-vessels.-For the study of the blood-vessels, especially of the capillary networks of the alveoli, sections of injected lung should be made. A fresh lung is injected (page 25) with blue gelatin, through the pulmonary artery. It is then hardened in alcohol, embedded in celloidin, and thick sections are stained with eosin and mounted in balsam.

\section{General References for Further Study}

Miller, W. S.: Das Lungenläppchen, seine Blut- und Lymphgefässe.

Councilman: The Lobule of the Lung and its Relations to the Lymphatics.

Kölliker: Handbuch der Gewebelchre des Menschen. 


\section{CHAPTER VIII}

\section{THE URINARY SYSTEM}

\section{The Kidney}

THE kidney is a compound tubular gland. It is enclosed by a firm connective-tissue capsule, the inner layer of which contains smooth muscle cells. In many of the lower animals and in the human

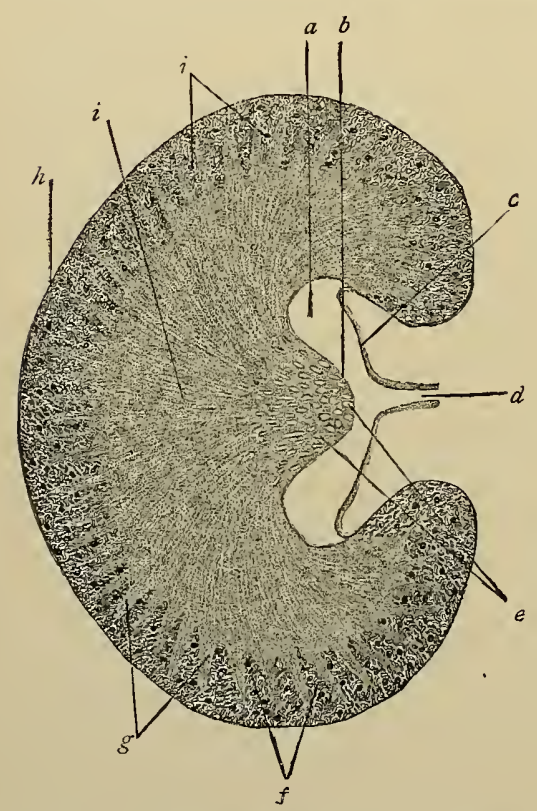

FIG. 2I4.-Longitudinal Section Through Kidney of Guinea-pig, including hilum and beginning of ureter. $\times 5$. (Technic I, p. 33I.) a, Pelvis; $b$, papilla; $c$, wall of pelvis; $d$, ureter; $e$, ducts of Bellini; $f$, cortical pyramids; $g$, medullary rays; $h$, cortex; $i$, medulla; $j$, renal corpuscles. fœetus septa extend from the capsule into the gland, dividing it into a number of lobes or renculi. In some animals, e.g., the guineapig and rabbit, the entire kidney consists of a single lobe (Fig. 2 I4). In the adult human kidney the division into lobes is not complete, the peripheral parts of the different lobes blending. Rarely the fotal division into lobes persists in adult life, such a kidney being known as a "lobulated kidney."

On the mesially directed side of the kidney is a depression known as the hilum (Fig. 2r4). This serves as the point of entrance of the renal artery and of exit for the renal vein and ureter.

On section, a division of the organ into two zones is apparent to the naked eye (Figs. $2 \mathrm{r} 4$ and 215). The outer zone or cortex has a granular appearance, while the inner zone or medulla shows radial striations. This difference in appearance between cortex and medulla is mainly due, as will be seen subsequently, to the fact that in the cortex the kidney tubules are convoluted, while in the medulla they run in parallel radial lines 
alternating with straight blood-vessels. The medullary portion of the kidney projects into the pelvis, or upper expanded beginning of the ureter (Figs. 214 and 2I 5) in the form of papilla. The number

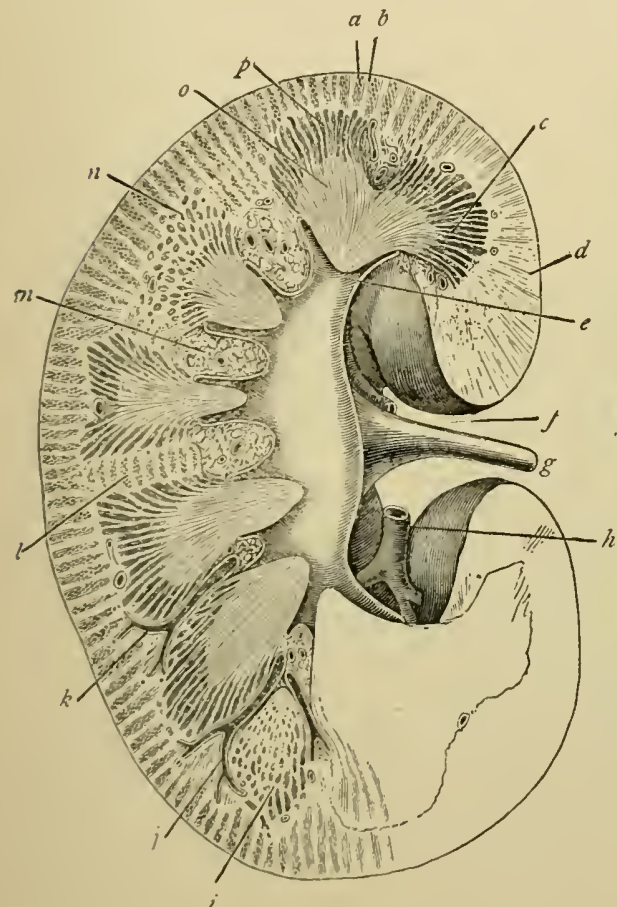

FIG. 2I5.

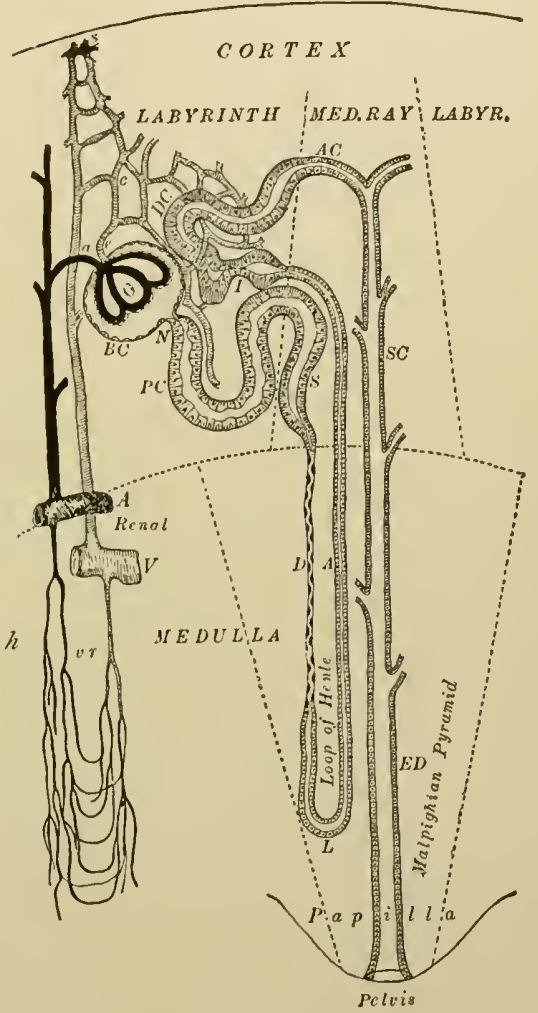

FIG. 216.

FIG. 2 15.-Longitudinal Section of Kidney Through Hilum. a, Cortical pyramid; $b$, medullary ray; $c$, medulla; $d$, cortex; $e$, renal calyx; $f$, hilum; $g$, ureter; $h$, renal artery; $i$, obliquely cut tubules of medulla; $j$ and $k$, renal arches; $l$, column of Bertini; $m$, connective tissue and fat surrounding renal vessels; $n$, medulla cut obliquely; $o$, papilla; p, medullary pyramid. (Merkel-Henle.)

FIG. 216.- Scheme of Uriniferous Tubule and of the Blood-vessels of the Kidney showing their relation to each other and to the different parts of the kidney. G, Glomerulus; $B C$, Bowman's capsule; $N$, neck; $P C$, proximal convoluted tubule; $S$, spiral tubule; $I$, descending arm of Henle's loop; $L$, Henle's loop; $A$, ascending arm of Henle's loop; $I, D C$, distal convoluterl tubule; $A C$, archerl tubule; $S C$, straight collecting tubule; $E D$, duct of Bellini; $A$, arcuate artery, and $V$, arcuate vein, giving off interlobular vessels to cortex and vasa recta to merlulla; $a$, afferent vessel of glomerulus; $e$, efferent vessel of glomerulus; $c$, capillary network in cortical labyrinth; $s$, stellate veins; $v r$, vasa recta and capillary network of medulla. (I'earsol.)

of papillac varies from ten to fifteen, corresponding to the number of lobes in the foetal kidney. 'The pyramidal segment of medulla, the ajex of which is a papilla-in other words, the medullary 
portion of a fotal lobe-is known as a medullary or Malpighian pyramid. The extensions downward of cortical substance between the Malpighian pyramids constitute the columns of Bertini or septa renis. Radiating lines-medullary rays or pyramids of Ferreinextend outward from the base of each Malpighian pyramid into the cortex (Fig. 2I5). As the rays extend outward in groups they outline pyramidal cortical areas. These are known as the cortical pyramids or cortical labyrinths.

The secreting portion of the kidney is composed of a large number of long tortuous tubules, the uriniferous tubules.

Each URINIFEROUS TUBULE begins in an expansion known as Bowman's capsule (Figs. 2 I6, $B C$, and $2 \mathrm{I} 7,3,4,5$ ). This encloses a
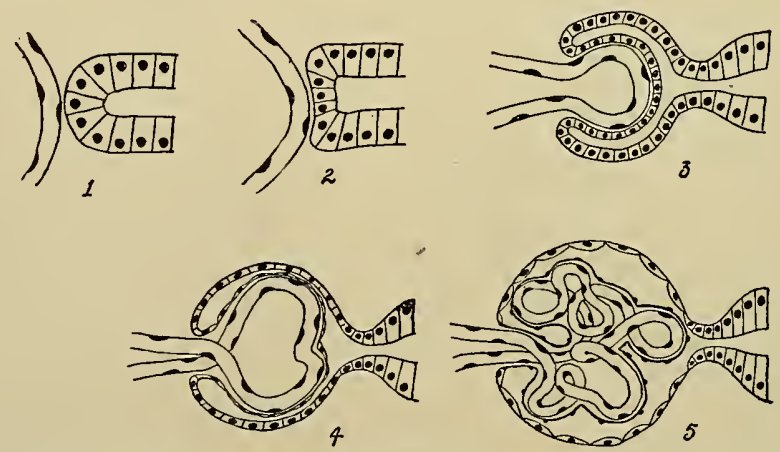

FIG. 217.-Diagrams Illustrating Successive Stages in Development of the Renal Corpuscle, $\mathrm{I}$ and 2, Approach of blood-vessel and blind end of tubule; 3 , invagination of tubule by blood-vessels; 4 and 5 , later stages, showing development of glomerulus and of the two-layered capsule of the renal corpuscle, the outer layer being the capsule of Bowman continuous with the epithelium of the first convoluted tubule.

tuft of blood capillaries, the glomerulus. Bowman's capsule and the glomerulus together constitute the Malpighian body or renal corpuscle (Fig. 218). As it leaves the Malpighian body the uriniferous tubule becomes constricted to form the neck (Figs. 2 I6, $N, 2$ I 7 , and 2 I $8, b$ ). It next broadens out into a greatly convoluted portion, the first convoluted tubule (Fig. 216, PC, and Fig. 2 I9). The Ma!pighian body, the neck, and the first convoluted tubule are situated in the cortical pyramid (Fig. 216). The tubule next takes a quite straight course downward into the medulla-descending arm of Henle's loop (Fig. 2 I 6 , $D$ ) - turns sharply upon itself-Henle's loop (Fig. 2 I6, L) -and passes again toward the surface-ascending arm of Henle's loop (Fig. 2I6, A) - through the medulla and medullary ray. Leaving the medullary ray, it enters the same cortical pyramid from which it took origin to become the second convoluted tubule (Fig. $2 \mathrm{I} 6, D C$ ). This tubule is in 
close proximity to the Malpighian body from which it started, lying, however, on the side of the afferent and efferent blood-vessels, i.e., on the side opposite its point of origin. The second convoluted tubule passes into the arched tubule $(A C)$ which enters a medullary ray and continues straight down through the medullary ray and medulla as the straight or collecting tubule $(S C)$. During its course the collecting tubule receives other arched tubules. As it descends it becomes broader, enters the papilla, where it is known as the duct of Bellini $(E D)$, and opens on the surface of the papilla into the kidney pelvis. About twenty ducts of Bellini open upon the surface of each papilla, their openings being known as the foramina papillaria.

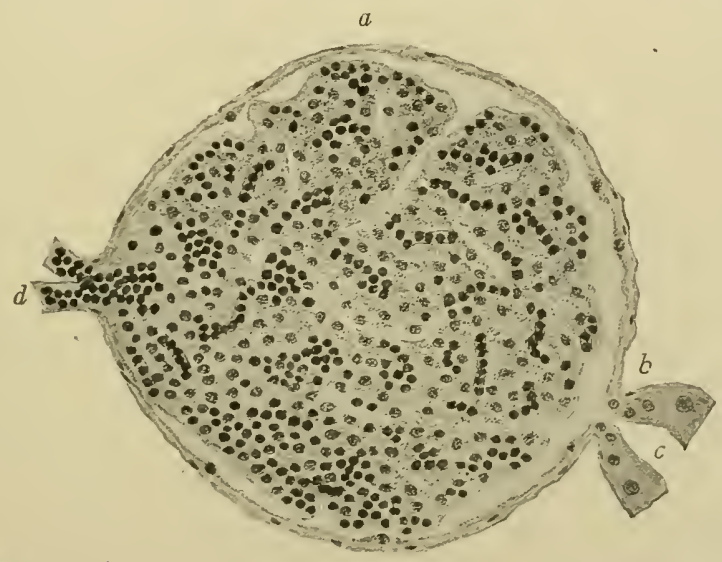

FIG. 218.-Malpighian Body from Human Kidney. $\times 280 . \quad$ (Technic 2, p. 33I.) $a$, Bowman's capsule; $b$, neck; $c$, first convoluted tubule; $d$, afferent and efferent vessels.

Each tubule consists of a delicate homogeneous membrana propria upon which rests a single layer of epithelial cells. The shape and structure of the epithelium differ in different portions of the tubule.

I. The Malpighian body is spheroidal, and has a diameter of from I 20 to $200 \%$. The structure of the Malpighian body can be best understood by reference to its development (Fig. 2I7). During the development of the uriniferous tubules and of the blood-ressels of the kidney, the growing end of a vessel meets the growing end of a tubule in such a way that there is an invagination of the tubule by the bloodvessel (see Fig. 217). The result is that the end of the vessel which develops a tuft-like network of capillaries - the glomerulus - comes to lie within the expanded end of the tubule, which thus forms a twolayered capsule for the glomerulus. One layer of the capsule closely invests the tuft of capillaries, dipping down into it and separating the 
groups of capillaries (see p. 326). This layer by modification of the original epithelium of the tubule is finally composed of a single layer of flat epithelial cells with projecting nuclei. The outer layer of the capsule lies against the delicate connective tissue which surrounds the Malpighian body. This layer consists of a similar though slightly higher epithelium and is known as Bowman's capsule. Between the glomerular layer of the capsule and Bowman's capsule proper is a space which represents the beginning of the lumen of the uriniferous tubule (Fig. 218), the epithelium of Bowman's capsule being directly continuous with that of the neck of the tubule.

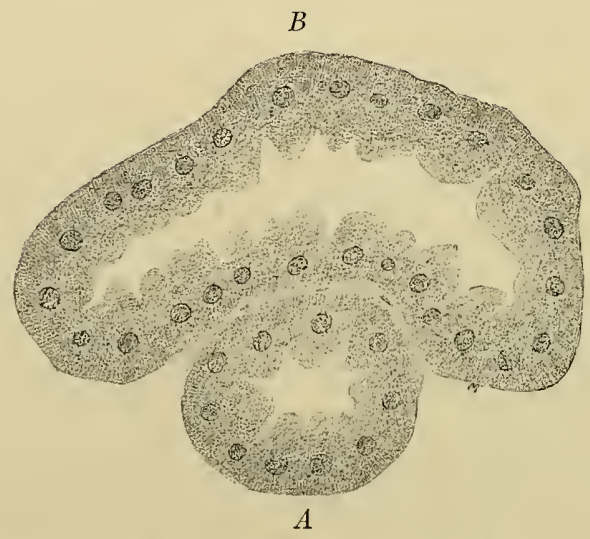

FIG. 219.- Proximal Convoluted Tubules of Human Kidney. $\times_{350}$. (Technic 2, p. 33.) $A$, Cross-section; $B$, oblique section.

2. The Neck.-This is short and narrow, and is lined by a few cuboidal epithelial cells. Toward its glomerular end the epithelium is transitional between the flat epithelium of Bowman's capsule and the cuboidal epithelium of the neck proper. At its other end the epithelium of the neck becomes larger and more irregular as it passes over into that lining the next division of the tubule (Fig. 2 I8).

3. The first convoluted tubule (Fig. 219) measures from 40 to $70 \mu$ in diameter. It is lined by irregularly cuboidal or pyramidal epithelium, with very indistinct demarcation between the cells. The cytoplasm is granular, and the granules are arranged in rows, giving the cell a striated appearance. This is especially marked at the basal end of the cell where the nucleus is situated. A zone of fine striations along the free surface frequently presents somewhat the appearance of cilia.

4. The descending arm of Henle's loop is narrow (Fig. 220, I), ro to $\mathrm{I}_{5} \mu$ in diameter. It is lined by a simple flat epithelium. The 
part of the cell which contains the nucleus bulges into the lumen, and as the nuclei of opposite sides of the tubule usually alternate, the lumen is apt to present a wavy appearance in longitudinal sections.

5. Henle's Loop.- The epithelium here changes from the flat of the descending arm to the cuboidal of the ascending arm. 'The exact point where the transition occurs varies. It may take place during the turn of the loop, or in either the ascending or descending arm.

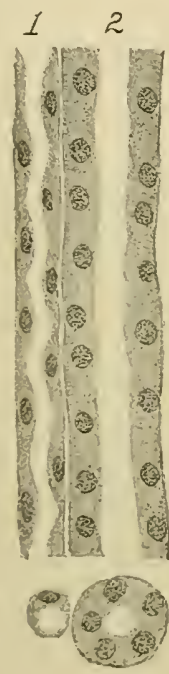

3

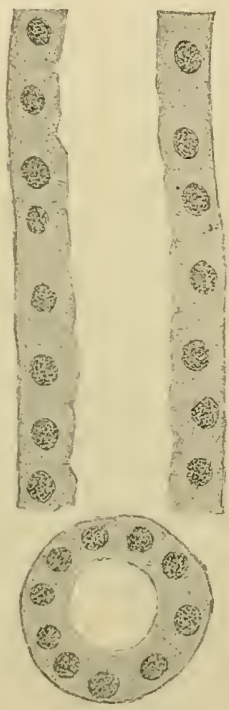

4

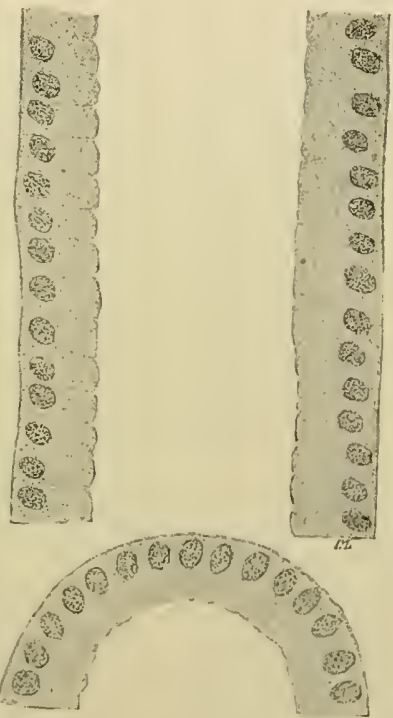

FIG. 220.-Tubules of Human Kidney. $\times 560$. From longitudinal section. (Technic 2, P. 33x.) I, Descending arm of Henle's loop; 2, ascending arm of Henle's loop; 3 , collecting tubule; 4 , duct of Bellini. Beneath the longitudinal sections are seen cross sections of the same tubules.

6. The ascending arm of Henle's loop (Fig. 220, 2) is broader than the descending, measuring from 20 to $30 \%$ in diameter. Its epithelium is cuboidal with granular striated protoplasm. The cells thus resemble those of the convoluted tubule, but are smaller, more regular, and less granular.

7. The second convoluled tubule has a diameter of 40 to $5 \%$ ". It is much less tortuous than the first convoluted tubule. Its epithelium is similar to that lining the first convoluted tubule except that it is slightly lower and less rlistinctly striated.

8. 'The arched lubule has a somewhat narrower lumen (about 25 ) than the second convoluted. It is lined with a low cuboidal epithelium with only slightly granular cytoplasm. 
9. The straight or collecting tubule (Fig. 220,3) has at its commencement at the apex of a medullary ray a diameter of from 40 to $50 \mu$. As it descends it receives other arched tubules, and increases in diameter until in the ducts of Bellini (Fig. 220,4) of the papilla it has a diameter of from 200 to $300 \mu$ and a widely open lumen. The epithelium is at first low and gradually increases in height. In the

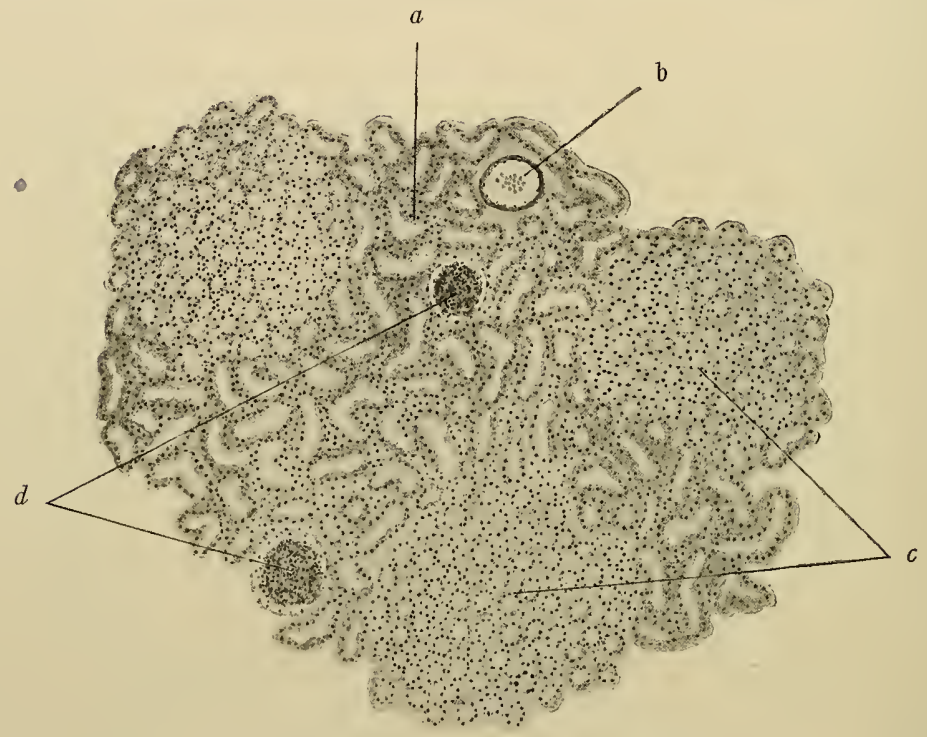

FIG. 221,-Cross Section Through Cortex of Human Kidney. $\times 60 . \quad$ (Technic 2, p. 33 I.) $a$, Convoluted tubules of cortical pyramid; $b$, interlobular artery; $c$, medullary rays; $d$, Malpighian bodies.

ducts of Bellini it is of the high columnar type. The cytoplasm of these cells contains comparatively few granules, thus appearing transparent in contrast with the granular cytoplasm of the ascending arms of Henle's loops and of the convoluted tubules.

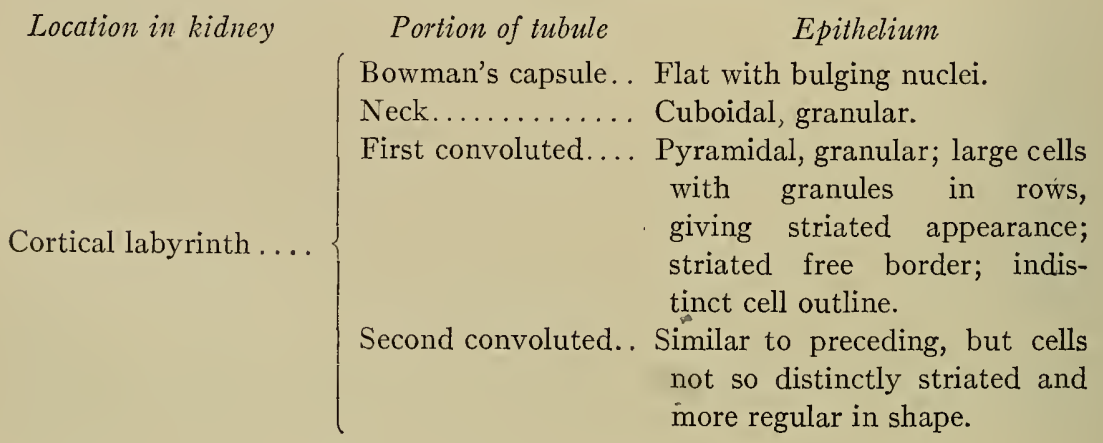


Location in kidney

Medullary ray in cortex

Medulla

Papilla
Portion of tubule

Epithelium

Arched (passing from Rather clear cuboidal cells.

labyrinth to ray)

Part of ascending Cuboidal, granular, regular.

arm of Henle's

loop

Collecting tubule... Cuboidal or columnar, clear; varying in height with diameter of tubule.

Descending arm, Clear flat cells with bulging Henle's loop nuclei.

Henle's loop........ Usually like descending, rarely like ascending arm.

Part of ascending Cuboidal, granular. arm Henle's loop

Collecting tubule... Cuboidal or columnar, clear; varying in height with diameter of tubule.

Ducts of Bellini.... Clear, cuboidal or columnar cells according to diameter of tubule.

The epithelium of the uriniferous tubule rests upon an apparently structureless basement membrane. Rühle describes the basement membrane as consisting of delicate longitudinal and circular connective-tissue fibrils. He regards the fibrils as merely a more regular arrangement of the interstitial connective tissue. According to Rühle the epithelium simply rests upon the basement membrane, being in no way connected with it. In the cortex the tubules are closely packed and the amount of interstitial connective tissue is extremely small. In the medulla the connective tissue is more abundant.

Of the function of the different parts of the uriniferous tubule our knowledge is extremely limited. The water of the urine is secreted in the Malpighian body, some specific action of the cells covering the glomerulus, allowing the water, normally free from albumen, to pass from the capillaries in to the lumen of the tubule. The urinary solids are secreted mainly or wholly by the cells of the convoluted tubule and of the ascending arm of Henle's loop.

Blood-vessels (cliagram, Fig. 223).-The blood supply to the kidney is rich and the blood-vessels come into intimate relations with the tubules. The renal arlery enters the kidney at the hilum, and 
immediately splits up into a number of branches-the interlobar arteries (Fig. 223, g). These give off twigs to the calyces and to the capsule, then without further branching pass between the papillæ through the medulla to the junction of medulla and cortex. Here they bend sharply at right angles and following the boundary line between cortex and medulla, form a series of arches, the arterice arciformes or arcuate arteries (Fig. 223,d). From the arcuate arteries two sets of vessels arise, one supplying the cortex, the other the medulla (Figs. 2 I 6 and 223).

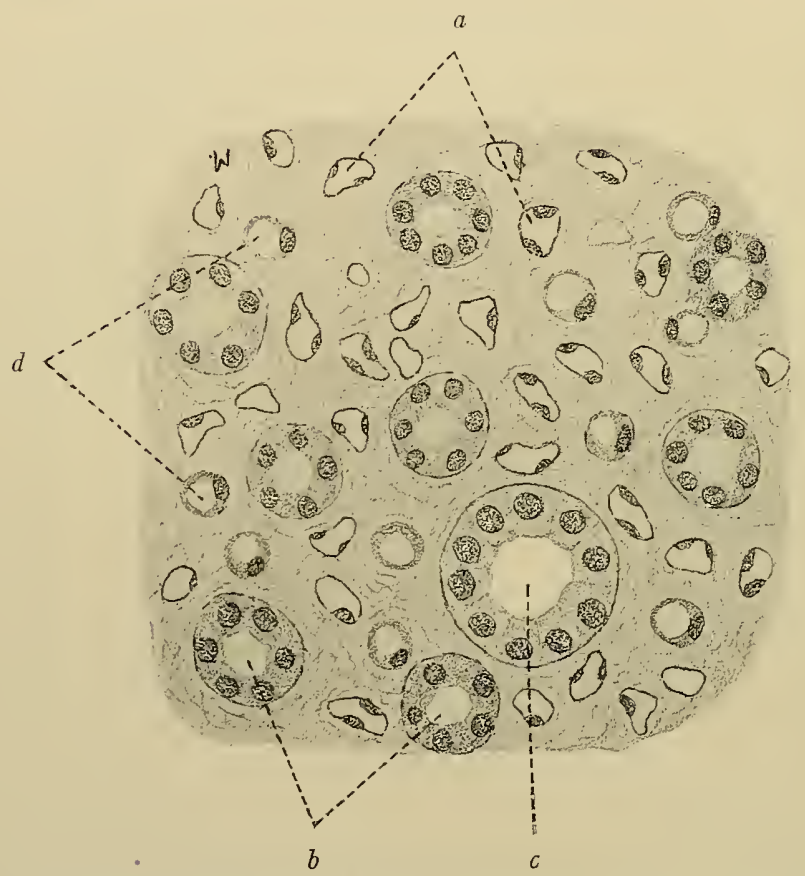

FIG. $222-$ Cross Section through Medulla of Human Kidney. $\times 465 . \quad$ (Technic 2 , p. 33 r.) $a$, Capillaries; $b$, collecting tubule; $c$, ascending arms of Henle's loops; $d$, descending arms of Henle's loops.

The arteries to the cortex spring from the outer (Fig. 223, $b$ ) sides of the arterial arches, and as interlobular arteries pursue a quite straight course through the cortical pyramids toward the surface, about midway between adjacent medullary rays. From each interlobular artery are given off numerous short lateral branches, each one of which passes to a Malpighian body. Entering a Malpighian body as its afferent vessel, the artery breaks up into a number of small arterioles, which in turn give rise to the groups of capillaries which form the 
glomerulus. Each group of glomerular capillaries arising from a single arteriole is separated from its neighbors by a rather larger amount of connective tissue than that which separates the individual capillaries. This gives to the glomerulus its lobular appearance. From the smaller glomerular capillaries the blood passes into some-

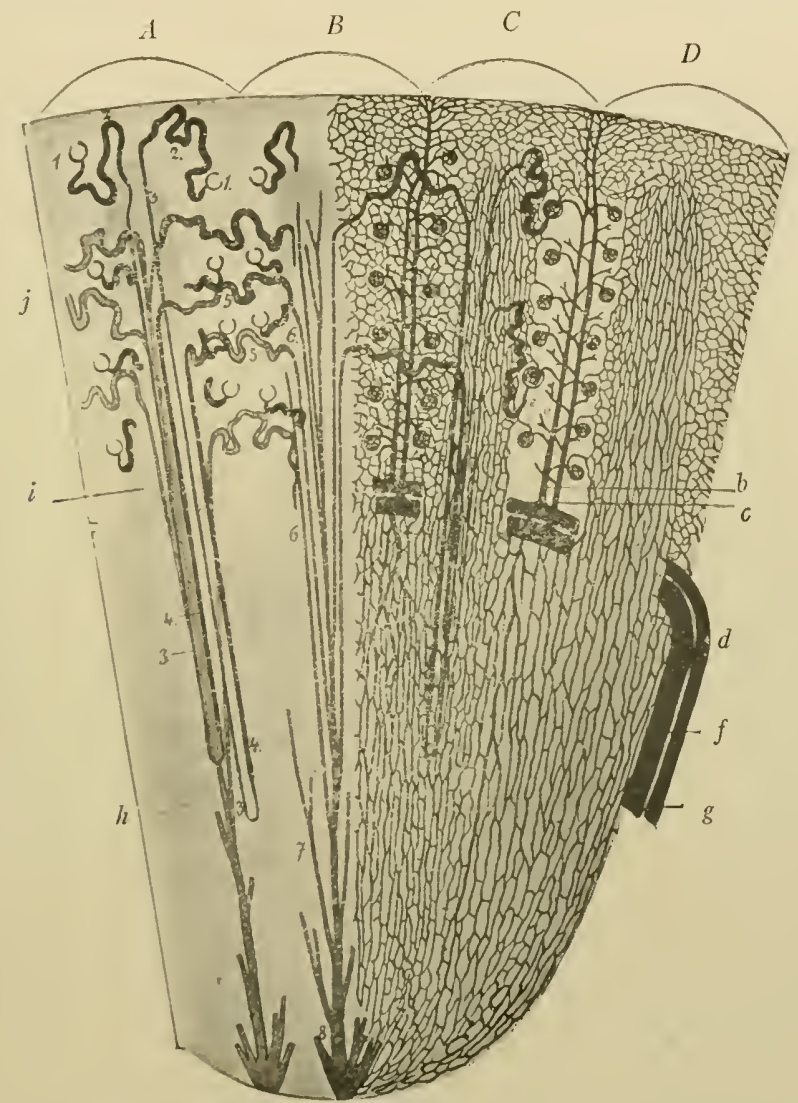

FIG. 223.-Diagram to Illustrate (left) the Course of the Uriniferous Tubule; (right) the Course of the Renal Vessels. (Szymonowicz.) $A, B, C$, $D$, each represents a kidney lobule limited laterally by the interlobular vessels; $a$, afferent vessel; $e$, efferent vessel of glomerulus; I, Bowman's capsule; 2, first convoluted tubule; 3 , descending arm of Henle's loop); 4, ascending arm of IIenle's loop; 5, second convoluted tubule; 6 and 7 , collecting tubules; 8 , duct of Bellini; $b$, interlobular artery; $c$, interlobular vein; $d$, renal arch (arcuate artery above and arcuate vein below); in their transverse course these vessels lic along the boundary line between cortex and medulla; $f$, interlobar vein; $g$, interlobar artery; $h$, merlulla; $i$, medullary ray; $j$, cortex.

what larger capillaries, which unite to form the efferent vessel of the glomerulus. As afferent and efferent vessels lie side by side, the glomerulus has the appearance of being suspended from this point (Figs. 216,218). The entire vascular system of the glomerulus is arlerial. 
After leaving the glomerulus, the efferent vessel breaks up into a second system of capillaries, which form a dense network among the tubules of the cortical pyramids and of the medullary rays. The mesh corresponds to the shape of the tubules, being irregular in the pyramids, long and narrow in the rays. In these capillaries the blood gradually becomes venous and passes into the interlobular veins (Fig. $223, c)$. These accompany the interlobular arteries to the boundary between cortex and medulla, where they enter the arcuate veins, which accompany the arcuate arteries (Fig. 223, $d$ ).

The main arteries to the medulla arise from the inner concave sides of the arterial arches. They pass straight down among the tubules of the medulla and are known as arteria recte. Branching, they give rise to a long-meshed capillary network which surrounds the tubules. This capillary network is also supplied by (I) efferent vessels from the more deeply situated glomeruli (false arteriæ rectæ) and (2) by medullary branches from the interlobular arteries. The veins of the medulla arise from the capillary network and follow the arteries back to the junction of medulla and cortex, where they empty into the arcuate veins (Fig. 223, $d$ ).

In addition to the distribution just described, some of the interlobular arteries extend to the surface of the kidney, where they enter the capsule and form a network of capillaries which anastomose with capillaries of the suprarenal, recurrent, and phrenic arteries. A further collateral circulation is established by branches of the abovenamed arteries penetrating the kidney and forming capillary networks within the cortex, even supplying some of the more superficial glomeruli. The most superficial of the small veins which enter the interlobular are arranged in radial groups, having the interlobular veins as their centres. These lie just beneath the capsule, and are known as the stellate veins of Verheyn. In addition to capillary anastomoses, direct communication between arteries and veins of both cortex and medulla, by means of trunks of considerable size, has been described.

The lymph vessels of the kidney are arranged in two systems, a superficial system which ramifies in the capsule, and a deep system which accompanies the arteries to the parenchyma of the organ. Little is known of the relation of the lymphatics to the kidney tubules.

Nerves.-These are derived from both cerebro-spinal and sympathetic systems. The medullated fibres appear to pass mainly to the walls of the blood-vessels which supply the kidney capsule. 
Plexuses of fine non-medullated fibres (sympathetic) accompany the arteries to the glomeruli. Delicate terminals have been described as passing from these plexuses, piercing the basement membrane and ending freely between the epithelial cells of the tubules.

\section{The Kidney-Pelvis and Ureter}

The kidney-pelvis, with its subdivisions the calyces, and the ureter constitute the main cxcretory duct of the kidney. Their walls consist of three coats: an inner mucous, a middle muscular, and an outer fibrous.

The mucosa is lined by epithelium of the transitional type. There are from four to eight layers of cells, the cell outlines are usually well defined, and the surface cells instead of being distinctly squamous are only slightly flattened. Less commonly large flat plate-like cells, each containing several nuclei, are present. The cells rest upon a basement membrane, beneath which is a stroma of delicate fibroelastic tissue rich in cells. Diffuse lymphatic tissue frequently occurs in the stroma, especially of the pelvis. Occasionally the lymphatic tissue takes the form of small nodules. Mucous glands in small numbers are found in the stroma of the pelvis and upper part of the ureter. There is no distinct submucosa, although the outer part of the stroma is sometimes referred to as such.

The muscularis consists of an inner longitudinal and an outer circular layer. In the lower part of the ureter a discontinuous outer longitudinal layer is added.

The fibrosa consists of loosely arranged connective tissue and contains many large blood-vessels. It is not sharply limited externally, but blends with the connective tissue of surrounding structures, and serves to attach the ureter to the latter.

The larger blood-vessels run in the fibrous coat. From these, branches pierce the muscular layer, give rise to a capillary network among the muscle cells, and then pass to the mucosa, in the stroma of which they break up into a rich network of capillaries. The veins follow the arteries.

The lymphatics follow the blood-ressels, being especially numerous in the stroma of the mucosa.

Nerves. - Plexuses of both medullated and non-medullated fibres occur in the walls of the ureter and pelvis. The non-merlullated fibres pass mainly to the cells of the muscularis. Medullated fibres 
enter the mucosa where they lose their medullary sheaths. Terminals of these fibres have been traced to the lining epithelium.

\section{The Urinary Bladder}

The walls of the bladder are similar in structure to those of the ureter.

The mucous membrane is thrown up into folds or is comparatively smooth, according to the degree of distention of the organ. The epithelium is of the same general type - transitional epithelium

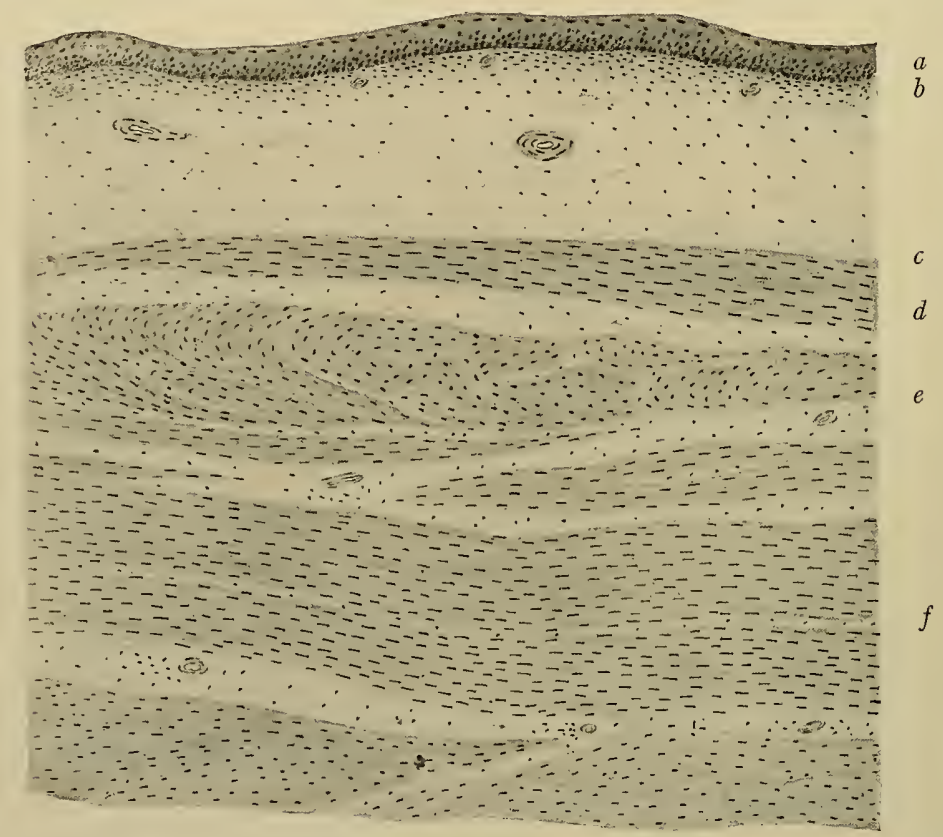

Fig. 224.-Vertical Section through Wall of moderately distended Human Bladder. $\times 60$. (Technic 5, p. 33I.) $a$, Epithelium, $b$, stroma, of mucous membrane; $c$, submucosa; $d$, inner muscle layer; $e$, middle muscle layer; $f$, outer muscle layer.

(see page 74)-as that of the ureter. The number of layers of cells and the shapes of the cells depend largely upon whether the bladder is full or empty. In the collapsed organ the superficial cells are cuboidal or even columnar, their under surfaces being marked by pit-like depressions caused by pressure of underlying cells. Beneath the superficial cells are several layers of polygonal cells, while upon the basement membrane is the usual single layer of small cuboidal cells. In the moderately distended bladder the superficial cells 
become flatter and the entire epithelium thinner (Fig. 224). In the distended organ there is still further flattening of the superficial cells and thinning of the entire epithelium. The stroma consists of fine loosely arranged connective tissue containing many lymphoid cells and sometimes small lymph nodules. It merges without distinct demarcation into the less cellular submucosa (Fig. 224, c).

The three muscular layers of the lower part of the ureter are continued on to the bladder, where the muscle bundles of the different layers interlace and anastomose, but can be still indistinctly differentiated into an inner longitudinal, a middle circular, and an outer longitudinal layer (Fig. 224, $d, e, f$ ).

The fibrous layer is similar to that of the ureter, and attaches the organ to the surrounding structures.

The blood-and lymph-vessels have a distribution similar to those of the ureter.

Nerves.-Sensory medullated fibres pierce the muscularis, branch repeatedly in the stroma, lose their medullary sheaths, and terminate among the cells of the lining epithelium. Sympathetic fibres form plexuses in the fibrous coat, where they are interspersed with numerous small groups of ganglion cells. Axones of these sympathetic neurones penetrate the muscularis. Here they form plexuses, from which are giren off terminals to the individual muscle cells.

For development of urinary system see page 376 .

\section{TECHNIC}

(I) Fix the simple kidney of a rabbit or guinea-pig in formalin-Müller's fluid (technic 5, p. 7). Make sections through the entire organ including the papilla and pelvis, stain with hæmatoxylin-cosin (technic I, p. 20), and mount in balsam. This section is for the study of the general topography of the kidney.

(2) Fix small pieces from the different parts of a human kidney in formalinMüller's fluid or in Zenker's fluid. Thin sections should be made, some cutting the tubules longitudinally, others transversely, stained with hxmatoxylin-eosin and mounted in balsam.

(3) Blood-vessels. - For the purpose of demonstrating blood-vessels of the kirlncy the method of double injection is useful (page 26).

(4) Ureter.-Cut transversely into short segments, fix in formalin-Müller's fluid (technic 5, p. 7), and stain transverse sections with hamatoxylin-eosin (technic I, p. 20), or with hxmatoxylin-picro-acirl-furhsin (technic 3, p. 2I). Mount in balsam.

(5) Blarder (technic 1, p. 254, or technic 2, p. 25.). By the latter method any rlesired degree of clistention may be obtained. 


\section{General References for Further Study}

Kölliker: Handbuch der Gewebelehre, vol. iii.

Gegenbauer: Lehrbuch der Anatomie des Menschen, vol. ii.

Henle: Handbuch der Anatomie des Menschen, vol. ii.

Johnston: A Reconstruction of a Glomerulus of the Human Kidney. Johns Hopkins Hosp. Bul., vol. xi., r 900 .

Müller: Ueber die Ausscheidung des Methylenblau durch die Nieren. Deutsches Archiv f. klin. Med., Bd. 63, I899. 
CHAPTER IX

\section{THE REPRODUCTIVE SYSTEM}

\section{MALE ORGANS}

\section{The Testis}

THE testes are compound tubular glands. Each testis is enclosed in a dense connective-tissue capsule, the tunica albuginea (Fig. 225, a). Outside the latter is a closed serous sac, the tunica vaginalis, the visceral layer of which is attached to the tunica albuginea, while the parietal layer lines the inner surface of the scrotum. Posteriorly the serous sac is wanting, the testis lying behind and outside of the tunica vaginalis. As the latter is derived from the peritoneum, being brought down with and invaginated by the testes in their descent to the scrotum, it is lined by mesothelial cells. To the inner side of the tunica albuginea is a layer of loose connective tissue rich in blood-vessels, the tunica rasculosa. Posteriorly the tunica albuginea is greatly thickened to form the corpus II ighmori, or mediastinum testis, from which strong connective-tissue septa radiate (Figs. 225, $m$ and $226, b$ ). These septa pass completely through the organ and blend with the tunica albuginea at various points. In this way the interior of the testis is subdi-

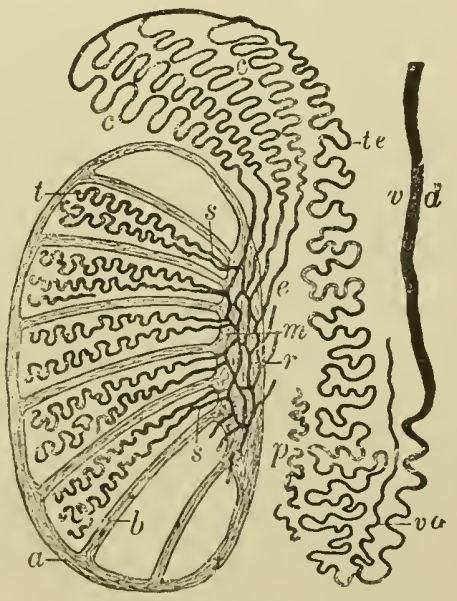

FIG. 225.-Diagram illustrating the Course and Relations of the Seminiferous Tubules and their Excretory Ducts. (Piersol.) a, Tunica albuginea; $b$, connective-tissue septum between lobules; $m$, mediastinum; $t$, convoluted portion of seminiferous tubule; $s$, straight tubule; $r$, rete testis; $c$, vasa efferentia; $c$, tubules of head of epididymis; te, vas epididymis; $v d$, vas deferens; $v a$, vas aberrans; $p$, paradidymis. vided into a number of pyramidal chambers or lobules, with bases directed toward the periphery and apices at the mediastinum (Figs. 225 and 226).

Bchind the testis and outside of its tunica albuginea is an elongated body-the cpididymis (Figs. 22 5, c and 226, c), consisting of convoluted 
tubules continuous with those of the mediastinum. The epididymis is divided into three parts: an expanded upper extremity, the head or globus major (Figs. 225 and 226, c); a middle piece, the body (Fig. 226, $d)$; and a slightly expanded lower extremity, the tail or globus minor. From the last named passes off the main excretory duct of the testis, the vas deferens (Fig. 225, vd). All of the tubules of the epididymis

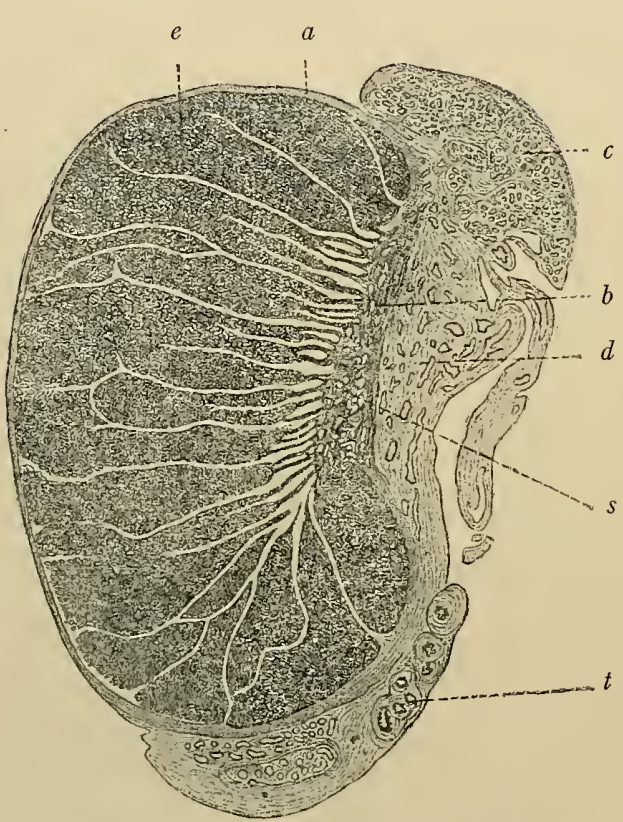

FIG. 226.--Longitudinal Section through Human Testis and Epididymis. $\times_{2}$. (Böhm and von Davidoff.) The light strands are connectivetissue septa. $a$, Tunica albuginea; $b$, mediastinum and rete testis; $c$, head of epididymis; $d$, body of epididymis; $e$, lobule; $s$, straight tubules; $t$, vas epididymis.

are continuous on the one hand with the tubules of the testicle, and on the other with the vas deferens. They thus constitute a portion of the complex system of excretory ducts of the testicle.

\section{The seminiferous} tubule may be divided with reference to structure and location into three parts. (I) A much convoluted part, the convoluted tubule, which begins at the base and occupies the greater portion of a lobule of the testis (Fig. 229, a). As they approach the apex of a lobule several of these convoluted tubules unite to form (2) the straight tubule (Fig. 225, s, 229). This passes through the apex of the lobule to the mediastinum, where it unites with other straight tubules to form (3) the irregular network of tubules of the mediastinum, the rete testis (Fig. $229^{\circ}, \mathrm{c}$ ).

I. The Convoluted Tubule.-This, which may be considered the most important secreting portion of the lobule, since it is here that the spermatozoa are formed, has a diameter of from I 50 to $250 \mu$. The tubules begin, some blindly, others by anastomoses with neighboring tubules, near the periphery of the lobule, and pursue a tortuous course toward its apex (Fig. 229, a).

The wall of the convoluted tubule (Fig. 227) consists of three 
layers: (a) An outer layer composed of several rows of flattened connective-tissue cells which closely invest the tubule; $(b)$ a thin basement membrane; and $(c)$ a lining epithelium. The epithelium consists of two kinds of cells, the so-called supporting or sustentacular cells and the glandular cells proper, the spermatogenic cells.

The sustentacular cells, or columns of Sertoli, are irregular, high, epithelial structures, whose bases rest upon the basement membrane,

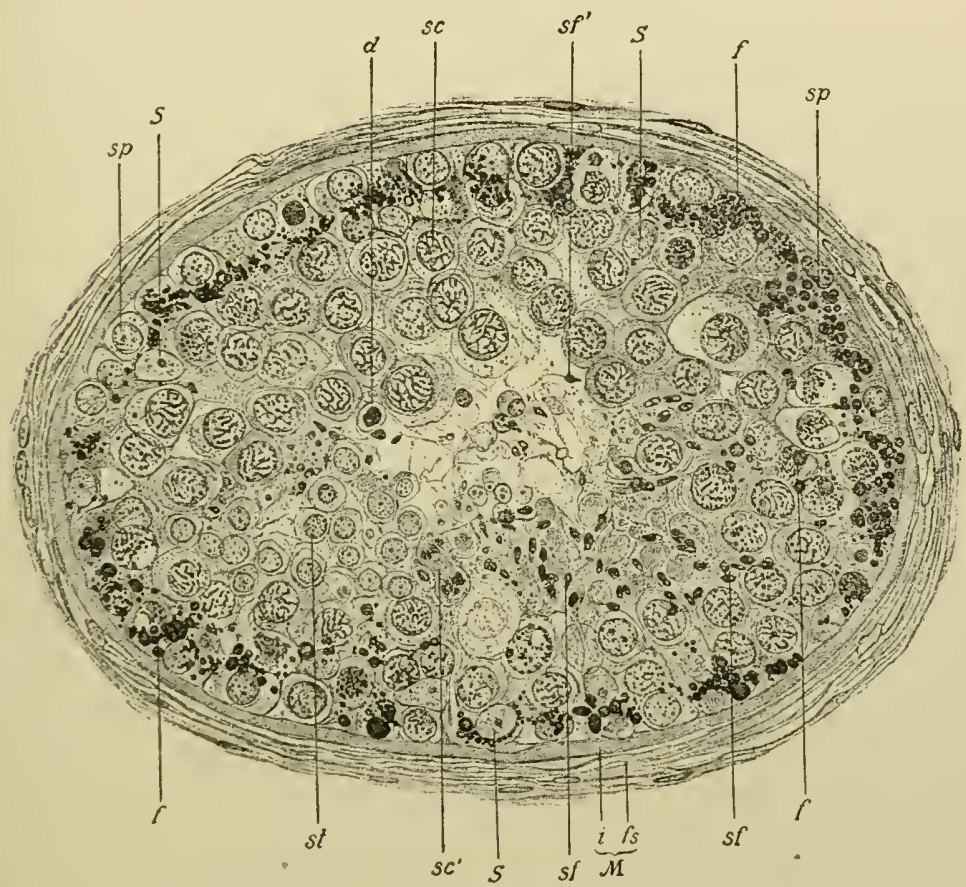

FIG. 227--Cross Section of Convoluted Portion of Human Seminiferous" Tubule. X480. (Kölliker.) II, Basement membrane; $i$, its inner homogeneous layer; $f s$, its outer fibrous layer; $s$, nucleus of Sertoli cell; $s p$, spermatogone; $s c$, spermatocyte; $s c^{\prime}$, spermatrocyte showing mitosis; $s f$, nearly mature spermatozoön; $s f^{\prime}$, spermatozoön free in lumen of tulsule; $d$, degenerating nucleus in lumen; $f$, fat droplets stained by $y_{i}$ osmic acirl.

and which extend through or nearly through the entire epithelium (Fig. 228,s). Their sides show marked irregularities and depressions, due to the pressure of surrounding spermatogenic cells. Their nuclei are clear, being poor in chromatin and their protoplasm contains brownish fat droplets. The cells of Sertoli have long been considered as sustentacular in character. It has recently been suggested that these cells are derived from the spermatogenic cells, but that, instead of developing into spermatozoa, they undergo retrograde changes, 
their protoplasm mingling with the intercellular substance, their nuclei becoming lost and the cells finally disappearing. According to this theory the tuft-like arrangement of the spermatozoa about the ends of the Sertoli cells is due to pressure by surrounding spermatogenic cells (Figs. 228, $h$ and $230, l$ ).

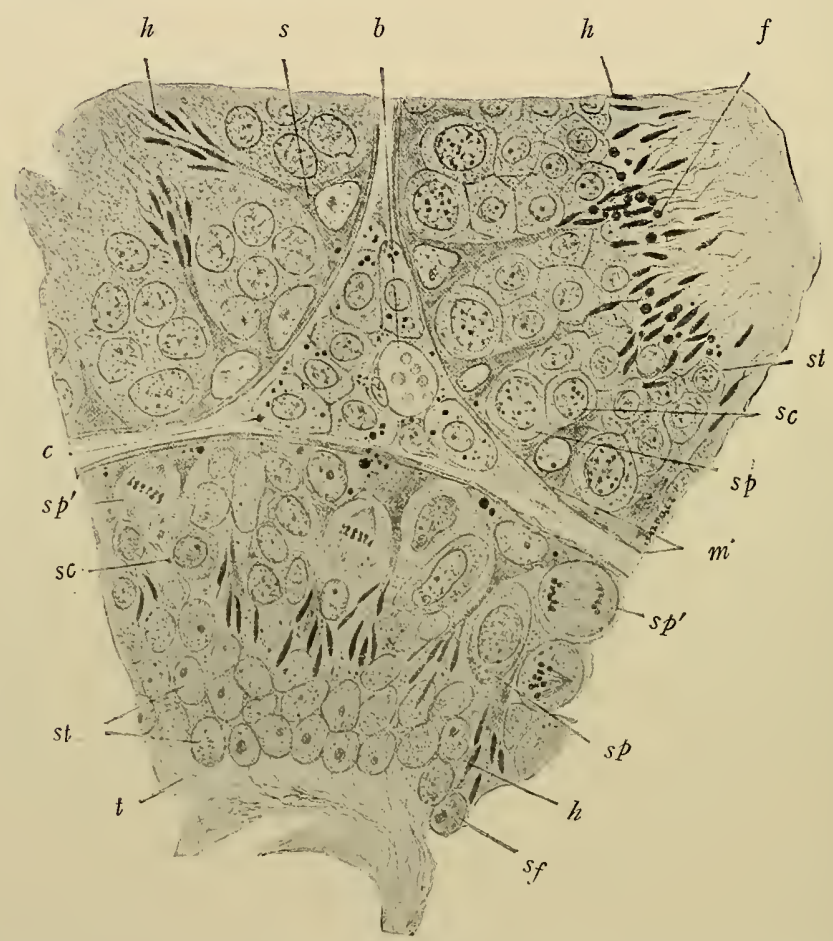

FIG. 228.-Parts of Transverse Section of three Seminiferous Tubules from Testis of White Mouse. $\times 600$. (Szymonowicz.) $s$, Sertoli cell with nucleus; $s p$, spermatogone, resting state; $s p^{\prime}$, spermatogone in mitosis; $s c$, spermatocyte; $s t$, spermatid; $s f$, spermatid developing into spermatozoön; $h$, head of spermatozoön; $t$, tails of developing spermatozoa; $b$, blood-vessel; $c$, interstitial cell; $m$, basal membrane; $f$, fat droplets.

The appearance which the spermatogenic cells present depends upon the functional condition of the tubule. In the resting state the epithelium consists of several layers of spherical cells containing nuclei which stain with varying degrees of intensity. In the active state several distinct layers of spermatogenic cells can be differentiated. These from without inward are as follows:

(I) Spermatogones (Figs. 227 and 228, sp).-These are small cuboidal cells which lie against the basement membrane. Their nuclei are spherical and rich in chromatin. By mitotic division of the spermatogones are formed the cells of the second layer, the spermatocytes. 
(2) Spermatocytes (Figs. 227 and $228, s c$ ).-These are larger spherical cells with abundant cytoplasm and large vesicular nuclei showing various stages of mitosis. They form from two to four layers to the inner side of the spermatogones, and are sometimes differentiated into spermatocytes of the first order and spermatocytes of the second order. By mitotic division of the innermost spermatocytes are formed the spermatids.

(3) The spermatids (Figs. 227 and 228 , st) are small round cells which line the

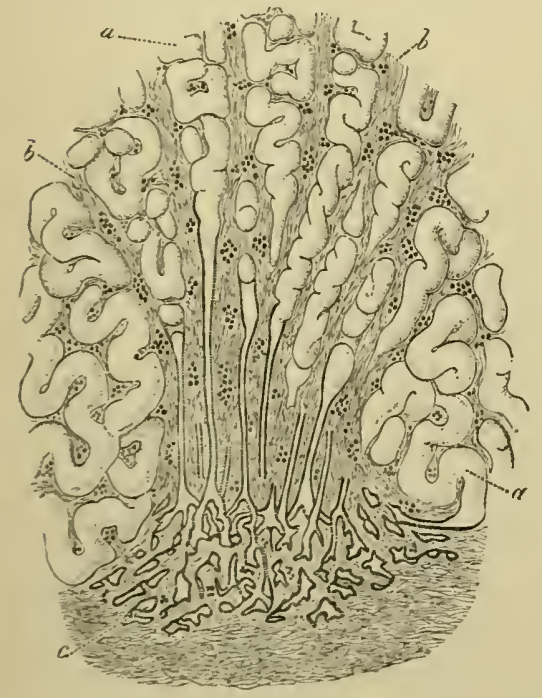

FIG. 229 .

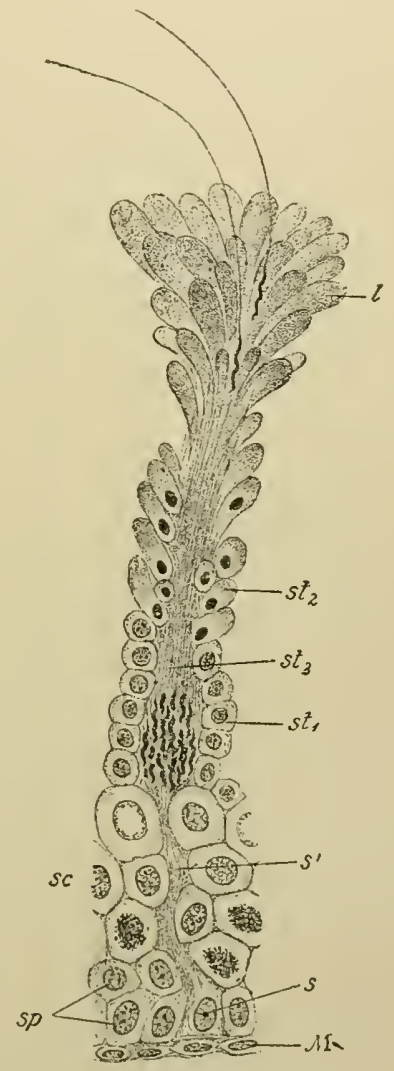

Fig. 230.

Fig. 220.- Passage of Convoluted Part of Seminiferous Tubules into Straight Tubules and of these into the Rete Testis (Milhalkowicz.) a, Convoluted part of tubule; $b$, fibrous stroma continued from the mediastinum testis; $c$, rete testis.

I'Ir. 230.-Spermatoblast with some Adjacent Sperm Cells, from Testis of Sparrow. (I'rom Kölliker, after Eitzold.) II, Basement membrane; $s$, nucleus of Sertoli cell; sp, spermatogones; $s c$, spermatocyte; $s t_{1}$ and $s t_{2}$, spermatids lying along the surface of the Sertoli cell, $s^{\prime}$ and $s t_{3}$; at $s t_{3}$ are seen the nearly mature spermatozoa; $l$, tuft-like arrangement of bodies of spermatids around free end of Sertoli cell, with two mature ș

Jumen of the seminiferous tubule. They are the direct progenitors of the spermatozoa. (For details of spermatogenesis see page 344.)

In the actively secreting testicle spermatozoa are frequently found either free in the lumen of the tubule or with their heads among the superficial cells and their tails extending out into the lumen (Figs. $227,5 f^{1}$ and 230). There are also found in the lumen many small 
cells with dark nuclei. These are spermatids which have become free and which degenerate without forming spermatozoa.

Separating and supporting the convoluted tubules is a small amount of interstitial connective tissue in which are the blood-vessels and nerves. Among the usual connective-tissue elements are found

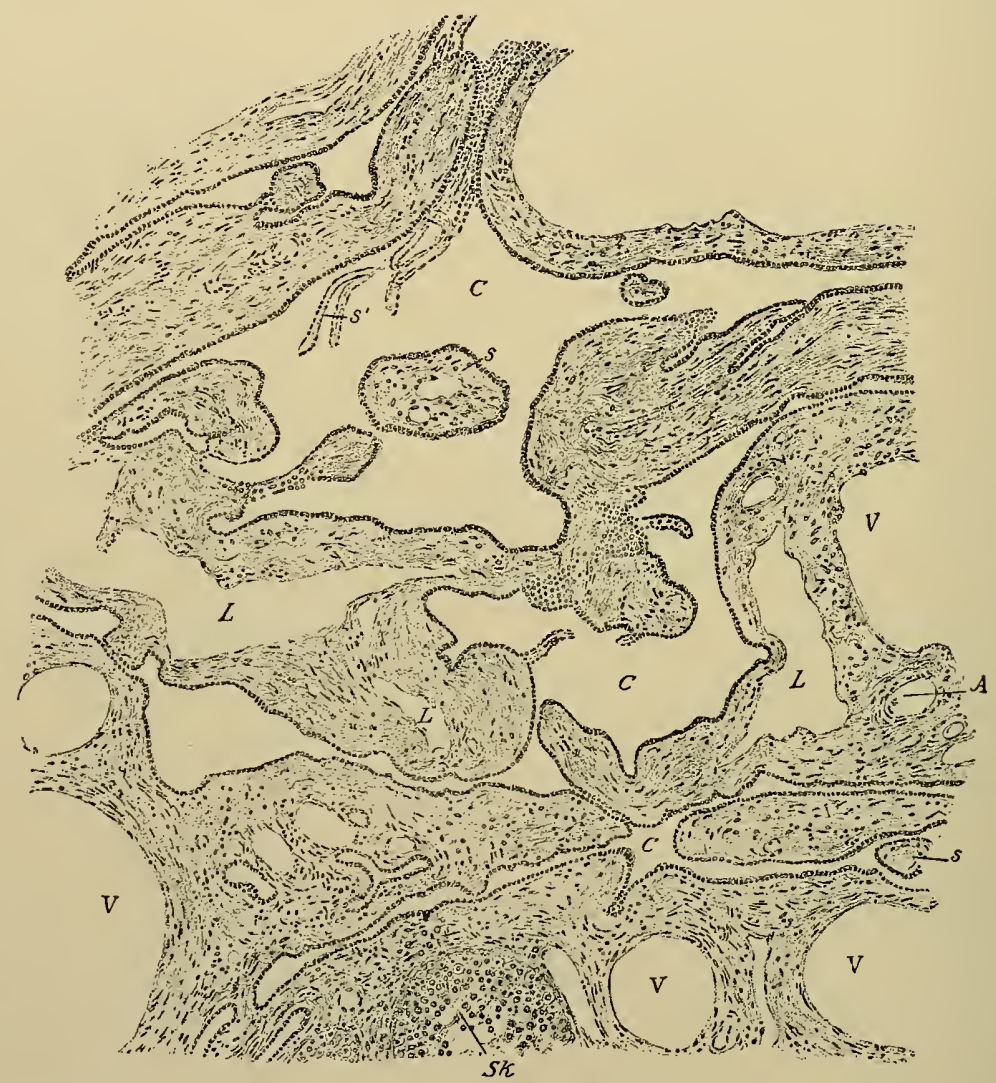

Fig. 23I.-From Section through Human Mediastinum and Rete Testis. $X_{96}$. (Kölliker.) $A$, Artery; $V$, vein; $L$, lymph space; $C$, canals of rete testis; $s$, cords of tissue projecting into the lumina of the tubules and so cut transversely or obliquely; $S k$, section of convoluted portion of seminiferous tubule.

groups of rather large spherical cells with large nuclei-interstitial cells. They are believed to represent remains of the Wolffian body (Fig. 228, c).

2. The Straight Tubule.-With the termination of the convoluted portion, the spermatogenic tissue of the gland ends, the remainder of the tubule constituting a complex system of excretory ducts. The straight tubule is much narrower than the convoluted, 
having a diameter of from 20 to $40 \%$. It is lined by a single layer of cuboidal cells resting upon a thin basement membrane. At the apex of the lobule the straight tubules become continuous with the tubules of the rete testis.

3. The Tubules of the Rete Testis.-These are irregular canals which vary greatly in shape and size. They are lined with a single layer of low cuboidal or flat epithelial cells (Fig. 23 I, C).

The SeminalDucts. - While

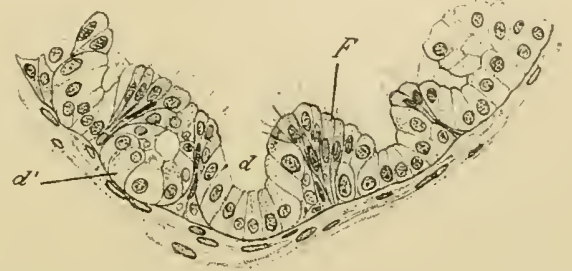

Fic. 232.- Part of a Cross Section through a Vas Efferens of the Human Epididymis. $\times$ I 40. (Kölliker.) $F$, Jligh columnar ciliated epithelium; $d$, lower non-ciliated epithelium, presenting appearance of a gland; $d^{\prime}$, the same cut obliquely.

the already described straight

tubules and the tubules of the rete testis must be regarded as part of the complex excretory duct system of the testis, there are certain structures which are wholly outside the testis proper, which serve to transmit the secretion of the testis, and are known as the seminal ducts. On leaving the testis these ducts form the epididymis, after

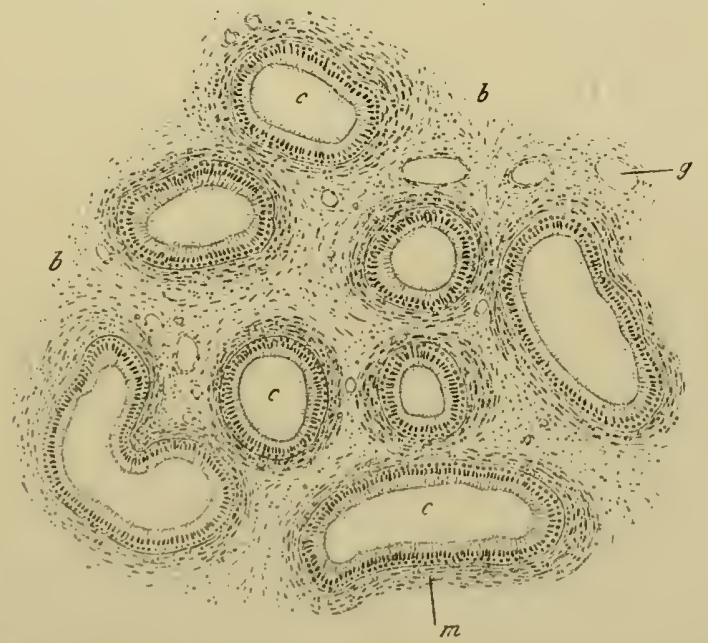

Firs. 23.3.-From Cross Section through Head of Epirlidymis. $\times 35 . \quad$ (Kïlliker.) $b$, Interstitial connective tissue; $c$ sections through tubules of epididy mis, showing twolayererl columnar epithelium; g, blosol-vessel.

which they converge to form the main excretory duct of the testis, the vas deferens.

Tin: Lipninyms.- From the tubules of the rete testis arise from eight to fifteen tubules, the vasa efferentia, or efferent ducts of the 
testis (Fig. 225, c). Each vas efferens pursues a tortuous course, is separated from its fellows by connective tissue, and forms one of the lobules of the head of the epididymis. The epithelium of the rasa efferentia consists of two kinds of cells, high columnar ciliated cells (Fig. 232, F), and, interspersed among these, lom cuboidal nonciliated cells (Fig. 232, d). Occasionally some of the high cells are free from cilia and some of the cuboidal cells may bear cilia. The cuboidal cells lie in groups between groups of the higher cells, often

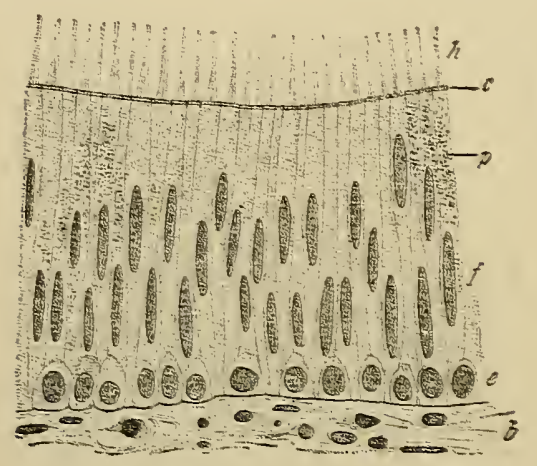

FIG. 234.-Tertical Section through WTall of Tubule of Epididymis. $\times>00$. (Kölliker.) (Fig. 233 more highly magnified.) $b$, Connective-tissue and smooth muscle cells; c, basal layer of epithelial cells; $f$, high columnar epithelial cells; $p$, pigment granules in columnar cells; $c$. cuticula; h, cilia. giving the appearance of cryptlike depressions. These have been referred to as intraepithelial glands. They do not, however, invaginate the underlying tissues. The epithelium rests upon a basement membrane, beneath which are several layers of circularly disposed smooth muscle cells.

The vasa efferentia converge to form the sas cpididymis (Fig. 233). Here the epithelium is of the stratified variety, there being two or three roms of cells. The sur face cells are narrow, high. and ciliated, and their nuclei are placed at different lerels (Fig. 234). The cilia are long and each cell has only a fem cilia. The deeper cells are irregular in shape. The basement membrane and muscular layers are the same as in the rasa efferentia. As the vas deferens is approached the muscular coat becomes thickened, and is sometimes strengthened by the addition of scattered bundles of longitudinally disposed cells.

The TAs Deferexs.- The walls of the ras deferens consist of four coats-mucosa, submucosa, muscularis, and fibrosa (Fig. 235).

The mucosa is folded longitudinally, and is composed of a stroma and a lining epithelium. The epithelium is of the stratified columnar type with two or three rows of cells, being similar to that lining the vas epididymis. The extent to which the epithelium is ciliated varies greatly. In some cases the entire vas is ciliated, in others only the upper portion, in still others no cilia are present beyond the epididymis. The epithelium rests upon a basement membrane beneath 
which is a fibro-elastic cellular stroma. The stroma merges without distinct demarcation into the more vascular submucosa.

The muscularis consists of two strongly developed layers of smooth muscle, an inner circular and an outer longitudinal (Fig. 235), which together constitute about seren-eighths of the wall of the ras. At the beginning of the vas deferens a third layer of muscle is added composed of longitudinal bundles, and situated between the inmer circular layer and the submucosa.

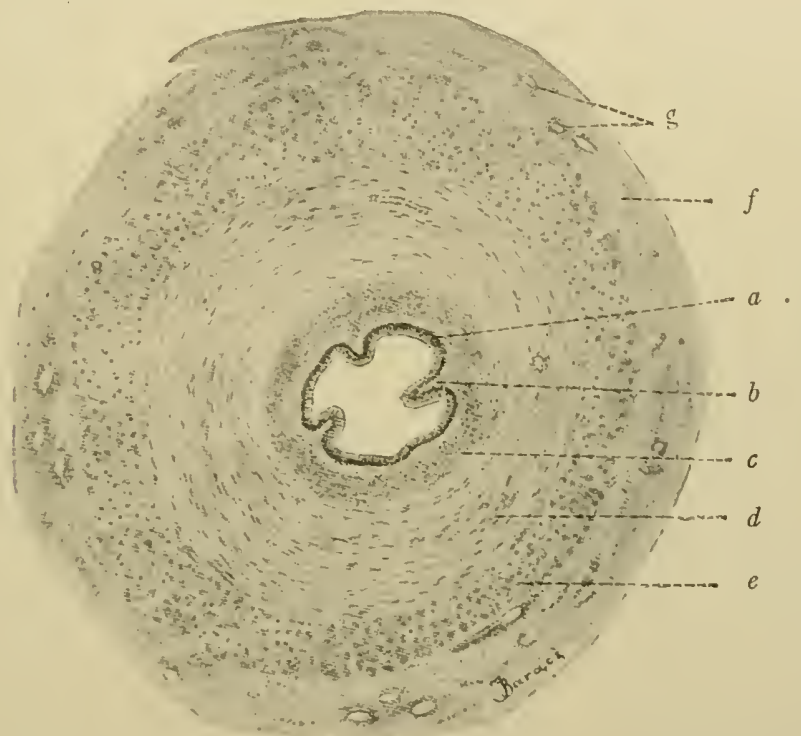

Fig. 235.-Cross Section of Human Vas Deferens. $X_{37}$. (Szymonowicz.) $a$, Epithelium; $b$, stroma; $c$, submucosa; $d$, inner circular muscle layer; $e$, outer longitudinal muscle layer; $f$, fibrous layer; $g$, blood-vessels.

The fibrosa consists of fibrous tissue containing many elastic fibres.

Near its termination the vas dilates to form the ampulla, the walls of which present essentially the same structure as those of the vas. The lining epithelium is, however, frequently markedly pigmented and the mucosa contains branched tubular glands.

The Seminal Vesicles and Ejaculatory Ducts.-The seminal resicles. The walls of the seminal vesicles are similar in structure to those of the ampulla. The epithelium is pseudo-stratified with two or three rows of nuclei and contains a yellow pigment. When the vesicles are distended the epithelium flattens out and the nuclei lie more in one plane, thus giving the appearance of an ordinary simple 
columnar epithelium. Beneath the epithelium is a thin stroma, outside of which is an inner circular and an outer longitudinal layer of smooth muscle, both layers being much less developed than in the vas. The seminal vesicles are to be regarded as accessory genital glands.

The ejaculatory ducts are lined with a single layer of columnar cells. The muscularis is the same as in the ampulla except that the inner circular layer is thinner. In the prostatic portion of the duct the muscularis is indistinct, merging with the muscle tissue of the gland. The ducts empty either directly into the urethra or into the urethra through the vesicula prostatica.

Rudimentary Structures Connected with the Development of the Genital System.-Connected with the testicle and its ducts are remains of certain fœtal structures. These are:

(I) The paradidymis, or organ of Giraldes, situated between the vessels of the spermatic cord near the testis. It consists of several blind tubules lined with simple columnar ciliated epithelium.

(2) The ductus aberrans Halleri, found in the epididymis. It is lined with simple columnar ciliated epithelium and opens into the vas epididymis. Instead of a single ductus aberrans, several ducts may be present.

(3) The appendix testis (stalked hydatid or hydatid of Morgagni), in the upper part of the globus major. It consists of a vascular connective tissue surrounding a cavity lined with simple columnar ciliated epithelium.

(4) The appendix epididymidis, a vascular structure, not always present, lying near the appendix testis. It resembles the latter in structure.

The paradidymis and ductus aberrans Halleri probably represent remains of the embryonal mesonephros. The appendix testis and the appendix epididymidis are believed by some to be derived from the primitive kidney, by others from the embryonal duct of Müller.

Blood-vessels. - Branches of the spermatic artery ramify in the mediastinum and in the tunica vasculosa. These send branches into the septa of the testicle, which give rise to a capillary network among the convoluted tubules. From the capillaries arise veins which accompany the arteries.

Lymph capillaries begin as clefts in the tunica albuginea and in the connective tissue surrounding the seminiferous tubules. These connect with the more definite lymph vessels of the mediastinum and of the spermatic cord.

Nerves.-Non-medullated nerve fibres form plexuses around the blood-ressels. From these, fibres pass to plexuses among the seminiferous tubules. Their exact method of termination in connection with the epithelium has not been determined. In the epididymis 
are found small sympathetic ganglia. The walls of the vasa efferentia, vas epididymis, and vas deferens contain plexuses of non-medullated nerve fibres, which give off terminals to the smooth muscle cells and to the mucosa.

The Spermatozoa.-The spermatozoa are the specific secretion of the testicle. Human spermatozoa are long, slender flagellate bodies, from 50 to $70 \%$ in length, and are suspended in the semen, which is a secretion of the accessory sexual glands. The general shape is that of a tadpole; and by means of an undulatory motion of the tail, the spermatozoön is capable of swimming about freely in a suitable medium. It has been estimated that the human spermatozoa average about sixty thousand per cubic millimetre of semen.

The human spermatozoön consists of (I) a head, (2) a middle piece or body, and (3) a tail or flagellum (Fig. 236).

The head, from 3 to $5 \mu$ long and about half that in breadth, is oval in shape when seen on flat, pearshaped when seen on edge. It consists mainly of chromatin derived from the nucleus of the parent cell. Enveloping the nuclear material of the head is a thin layer or delicate membrane of cytoplasm, the gulea capilis. The

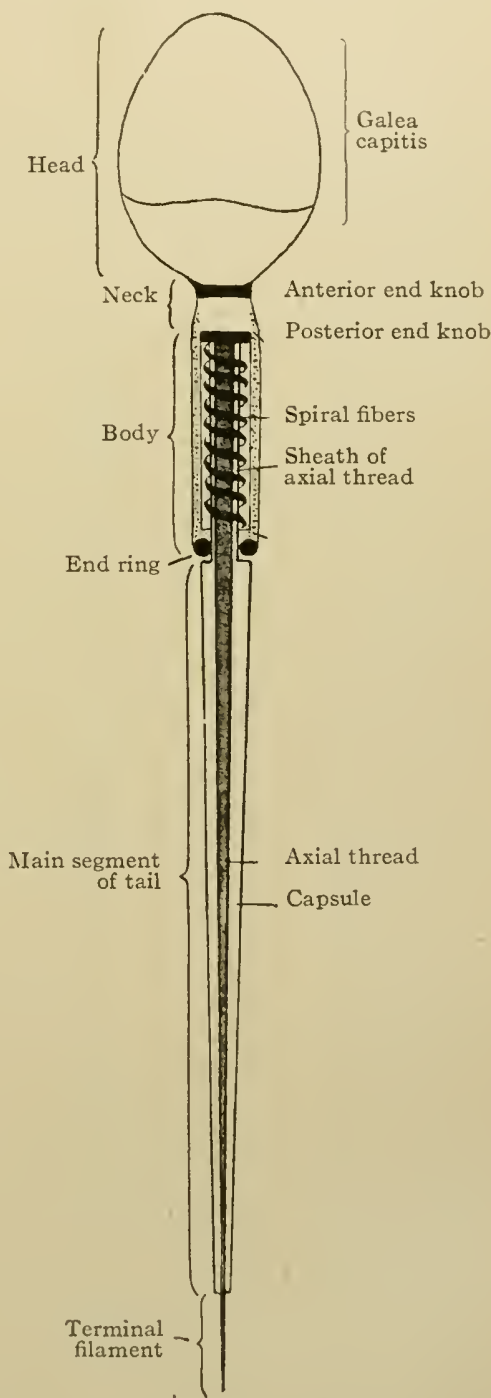

FIG. 236.-Diagram of a IIuman Spermatozoön. (Meves, Bonnet.) front of the head ends in a sharp edge, the apical body or acrosome. In some lower forms the acrosome is much more highly developed than in man and extends forward as a pointed or barbed spear, the perforutorium. The acrosome is differentiated from the nuclear 
portion of the head by taking an acid dye, the chromatin, of course, taking a basic stain.

The body is cylindrical, about the same length as the head, and consists of a fibrillated central core, the axial thread, surrounded by a protoplasmic capsule. A short clear portion, the neck, unites the head and body. Just behind the head the axial thread presents a bulbous thickening, the anterior end knob, which fits into a depression in the head. At the junction of neck and body are one or several posterior end knobs to which the axial thread is attached. The latter leaves the body through a perforated ring, the end ring or end disc. Delicate fibrils-spiral fibres-run spirally around the body portion of the axial thread.

The tail consists of a main segment, from 40 to $60 \%$ in length, and a terminal segment having a length of from 5 to ro $\mu$. The main segment has a central fibrillated axial thread which is continuous with the axial thread of the body. This is enclosed in a thin cytoplasmic membrane or capsule continuous with the capsule of the body. This membrane, inconspicuous and apparently structureless in man, is remarkably developed in some lower forms, e.g., the membrana undulata of birds. The terminal segment consists of the axial thread alone. The motility of the spermatozoön depends entirely upon the flagellate movements of the tail. In many of the lower animals the spermatozoön has a much more complicated structure.

Of the above-described parts of the spermatozoön only the head and tail can usually be differentiated, except by the use of special methods and very high-power objectives.

Development of the Spermatozoa.-As already noted in describing the testicle, the spermatozoa are developed from the epithelial cells of the seminiferous tubules. The most peripheral of the tubule cells, the spermatogones (Fig. 227, sp and Fig. 228, sp) are small round cells with nuclei rich in chromatin. By mitosis the spermatogone gives rise to two daughter cells, one of which remains at the periphery as a spermatogone, while the other takes up a more central position as a spermatocyte (Fig. 228, sc and Fig. 230, sc). The latter are rather large spherical cells, whose nuclei show very distinct chromatin networks. By mitotic division of the spermatocytes of the innermost row are formed the spermatids (Fig. 228, st and Fig. 230, st). These are small spherical cells, which line the lumen of the tubule and are the direct progenitors of the spermalozoa. In the transformation of spermatocyte into spermatid an extremely important change takes place in the nucleus. This consists in a reduction of its chromosomes to one-half the number specific for the species (page 58). The transformation of the spermatid into the spermatozoön differs somewhat in different animals and the details of the process must be regarded as not yet definitely determined. The 
nucleus of the spermatid first becomes oval in shape, and its chromosomes become condensed into a small homogeneous mass, which forms the head of the spermatozoön. During their transformation into the heads of the spermatozoa, the nuclei of the spermatids arrange themselves in tufts against the inner ends of the cells of Sertoli. This compound structure, consisting of a Sertoli cell and of a group of developing spermatozoa attached to its central end, is known as a spermatoblast (Fig. 230). The body or middle piece of the spermatozoön is described by most investigators as derived from the centrosome, while the tail is a derivative of the cytoplasm.

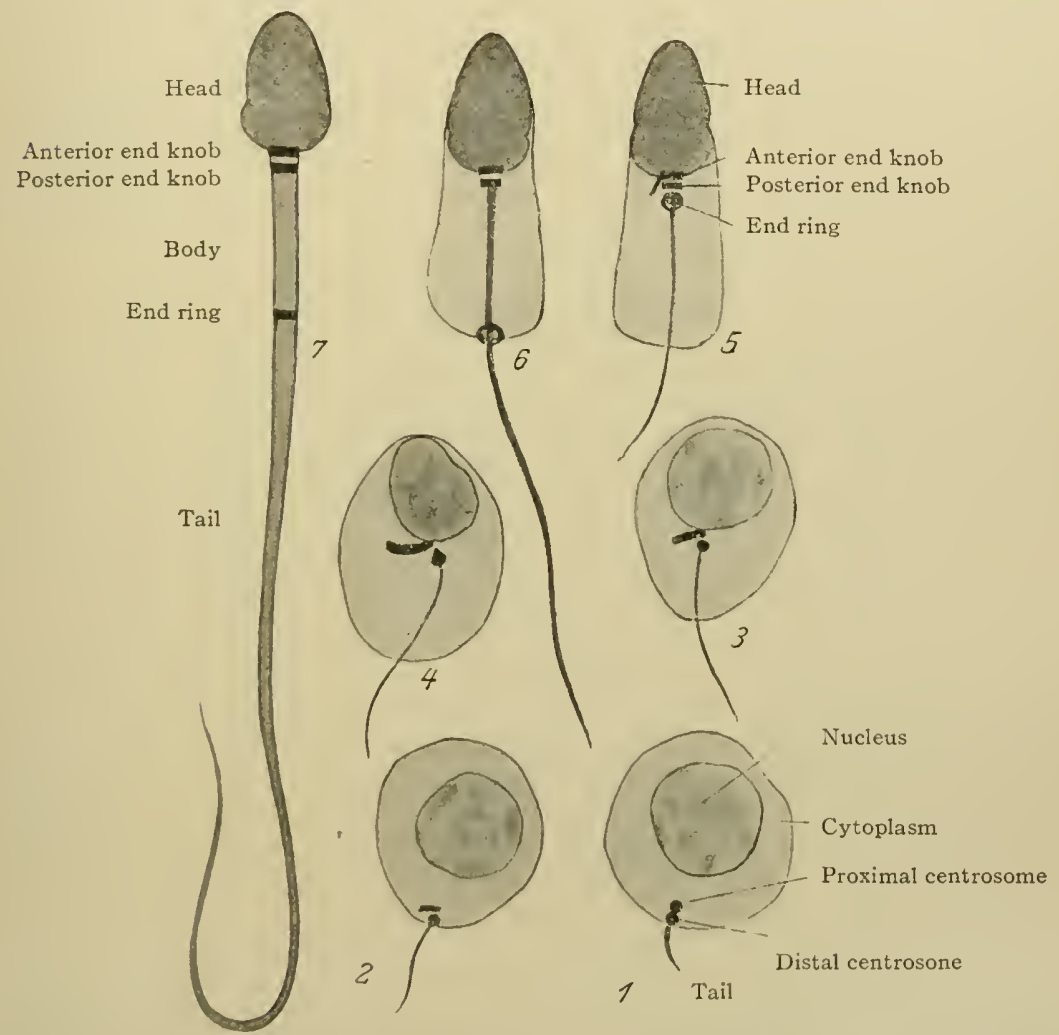

Fis. 237.-Transformation of a Spermatid into a Spermatozoön (human). Schematic (Meves, Bonnet.)

The details of the transformation of the spermatid into the spermatozoön are illustrated in Fig. 237. The centrosome either divisles completely, forming two centrosomes, or incompletely, forming a (lumb-bell-shaped Jorly. The nuclear material becomes very compact and passes to one end of the cell, forming the bulk of the head. Both centrosomes help to form the body. 'They first become disc-shaped. 'The one lying nearer the centre of the ecll becomes attached to the posterior end of the head as the anterior end-knob, the other takes a position a 
little more posteriorly as the posterior end-knob and from it grows out the axial filament. Some of this centrosome passes to the posterior limits of the body and there becomes the end ring. As the two parts of this centrosome separate the delicate cytoplasm between them forms the spiral fibres. During these changes the axial filament has been growing and projects beyond the limits of the cell. Most of the cytoplasm of the spermatid is not used in the formation of the spermatozoön, but is cast off and degenerates. A small amount is used for the sheath of the body, and for the galea capitis. The sheath of the main part of the filament appears to develop from the filament itself.

The significance of the different parts of the spermatozoön has been brought out in describing its development. From this it is seen that the spermatozoön, like the mature ovum, is a true sexual element with one-half the somatic number of chromosomes. The head and body, containing the chromatin and the centrosomes, are the parts of the spermatozoön essential to fertilization. The acrosome is an accessory which in some forms at least aids the spermatozoön in attaching itself to and in entering the ovum. The tail is an accessory structure which provides motion, enabling the spermatozoön to move about freely in the semen and in the fluids of the female generative tract. Considering their minuteness, their speed is considerable, having been estimated at from 1.5 to $3.5 \mathrm{~mm}$. per minute; enough to allow them to ascend through uterus and oviduct against the adverse action of the cilia. When in a favorable environment, such as the fluids of the female generative tract, the spermatozoön is capable of living for some time after leaving the testicle. Living spermatozoa have been found in the uterus or tubes three and a half weeks after coitus.

\section{TECHNIC}

(I) For the study of the general topography of the testis, remove the testis of a new-born child, make a deep incision through the tunica albuginea in order to allow the fixative to penetrate quickly, and fix in formalin-Müller's fluid (technic 5, p. 7). Antero-posterior longitudinal sections through the entire organ and including the epididymis should be stained with hæmatoxylin-picro-acid-fuchsin (technic 3, p. 2I) or with hæmatoxylin-eosin (technic I, p. 20) and mounted in balsam.

(2) The testis of a young adult is removed as soon after death as possible, is cut into thin transverse slices, which include the epididymis, and is fixed in formalin-Müller's or in Zenker's fluid (technic 9, p. 8). Select a slice which includes the head of the epididymis, cut away the anterior half or two-thirds of the testis proper in order to reduce the size of the block, and, after the usual hardening and embedding, cut thin sections through the remaining posterior portion of the testis, the mediastinum, and epididymis. Stain with hæmatoxylin-eosin (technic I, p. 20) and mount in balsam.

(3) For the study of spermatogenesis fix a mouse's testis in chrome-aceticosmic mixture (technic 7, p. 7). Harden in alcohol and mount thin unstained sections in balsam or in glycerin.

(4) Spermatozoa.-Human spermatozoa may be examined fresh in warm normal saline solution or fixed in saturated aqueous solution of picric acid and 
mounted in glycerin. Mammalian spermatozoa may be obtained from the vagina after intercourse, or by incision into the head of the epididy mis. Technic same as for human.

(5) A portion of the vas deferens is usually removed with the testis and may be subjected to technic (2) above. Transverse sections are stained with hæmatoxy-lin-eosin and mounted in balsam.

\section{The Prostate Gland}

The prostate is described by some as a compound tubular, by others as a compound alveolar gland. It is perhaps best regarded as a collection of simple branched tubular glands with dilated terminal tubules. These number from forty to fifty, and their ducts con-

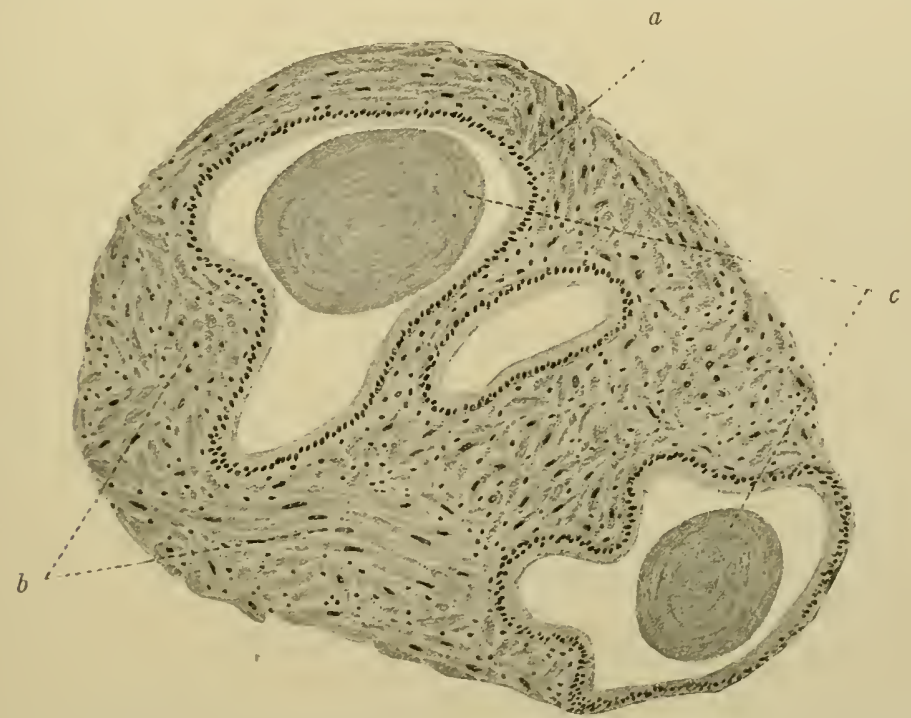

FIG. 238.- Section of Human Prostate. XI50. (Technic I, p. 349.) a, Epithelium of tubule; $b$, interstitial connective tissue; $c$, corpora amylacea.

verge to form about twenty main ducts, which open into the urethra. The gland is surrounded by a cupsule of fibro-clastic tissue and smooth muscle cells, the muscle cells predominating. From the capsule broad trabeculce of the same structure as the capsule pass into the gland. The amount of connective tissue is large. It is less in the prostate of the young than of the old. 'The hypertrophied prostate of age is due mainly to an increase in the connective-tissue elements. The tubules have wide lumina and are lined with simple cuboidal epithelium of the serous type, resting upon a delicate basement mem- 
brane (Fig. 238). Less commonly the epithelium is pseudo-stratified. The ducts are lined with simple columnar epithelium until near their terminations where they are lined with transitional epithelium similar to that lining the urethra. Peculiar concentrically laminated bodies, crescentic corpuscles, or corpora amylacea, are frequently present in the terminal tubules (Fig. 238, c). They are more numerous after middle life. Through the prostate runs the prostatic portion of the urethra.

Within the prostate is found the vesicula prostatica (utriculus prostaticus-uterus masculinus). It represents the remains of a fœtal structure, the Miillerian duct and consists of a blind sac with folded mucous membrane lined with a two-rowed ciliated epithelium which dips down to form short tubular glands. The prostatic secretion is serous.

The blood-vessels of the prostate ramify in the capsule and trabeculæ. The small arteries give rise to a capillary network which surrounds the gland tubules. From these arise small veins, which accompany the arteries in the septa and unite to form venous plexuses in the capsule.

The lymphatics begin as blind clefts in the trabeculæ and follow the general course of the blood-vessels.

Nerves.-Small groups of sympathetic ganglion cells are found in the larger trabeculæ and beneath the capsule. Axones of these cells pass to the smooth muscle of the trabeculæ and of the walls of the blood-vessels. Their mode of termination is not known. Timofeew describes afferent medullated fibres ending within capsular structures of flat nucleated cells. Two kinds of fibres pass to each capsule: one a large medullated fibre which loses its sheath and gives rise within the capsule to several flat fibres with serrated edges, the other small medullated fibres which lose their sheaths and split up into small varicose fibrils which form a network around the terminals of the large fibre.

\section{Cowper's Glands}

The bulbo-urethral glands, or glands of Cowper, are small, compound tubular glands. Both tubules and ducts are irregular in diameter so that some of them have more the character of alveoli than of tubules. They are lined with mucous cells. The smaller ducts are lined with simple cuboidal epithelium. They unite to form two main 
excretory ducts which open into the urethra and are lined with stratified columnar epithelium consisting of two or three layers of cells. In the main duct, as well as in its branches, smooth muscle occurs.

\section{TECHNIC}

(I) Fix small pieces of the prostate of a young man in formalin-Müller's fluid (technic 5, p. 7). Stain sections with hæmatoxylin-eosin (technic I, p. 20) and mount in balsam.

(2) The prostate of an old man should be treated with the same technic and compared with the above.

(3) Cowper's glands. Same technic as prostate (I).

\section{The Penis}

The penis consists largely of three long cylindrical bodies, the corpus spongiosum and the two corpora cavernosa. The latter lie side by side, dorsally, while the corpus spongiosum occupies a medial ventral position (Fig. 239). All three are enclosed in a common connective-tissue capsule which is loosely attached to the overlying skin. In addition each corpus has its own special capsule or tunica albuginea, about a millimetre in thickness, and composed of dense connective tissue containing many elastic fibres.

The corpus spongiosum and corpora cavernosa have essentially the same structure, being composed of so-called erectile tissue (Fig. 240). This consists of thick trabecula of inter.

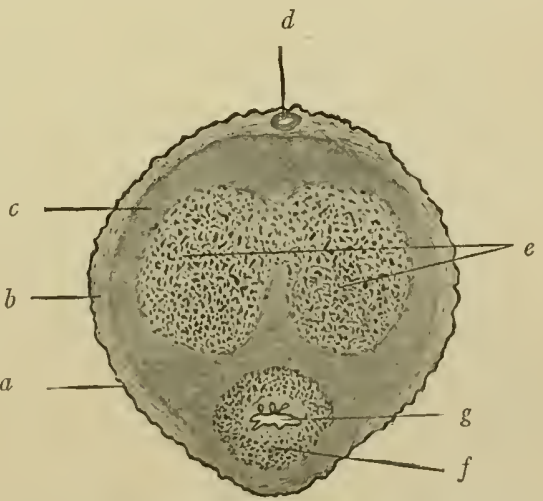

Fig, 239.-Transverse Section through Human Penis. $a$, Skin; $b$, subcutaneous tissue; $c$, fibrous tunic; $d$, dorsal vein; $e$, corpora cavernosa; $f$, corpus spongiosum; $g$, urethra.

\section{mingled fibro-elastic tissue and}

bundles of smooth muscle cells, which anastomose to form a coarse meshed network, the spaces of which are lined with endothelium. The spaces are known as cavernous sinuses, and communicate with one another, and with the blood-vessels of the penis. In the flaccid condition of the organ these sinuses are empty and their sides are in apposition. In erection these sinuses become filled with venous blood. 
The arteries have thick muscular walls and run in the septa. A few of them open directly into the venous sinuses. Most of them give rise to a superficial capillary network beneath the tunica albuginea. From this capillary plexus the blood passes into a plexus of broader venous channels in the periphery of the erectile tissue, and these in turn communicate with the cavernous sinuses. The usual direct anastomoses between arterial and venous capillaries also occur. The blood may therefore pass either through the usual course--arteries, capillaries, veins-or, under certain conditions, may pass through the cavernous sinuses. This determines the flaccid or the erect condition of the organ. The veins arise partly from the capillaries and

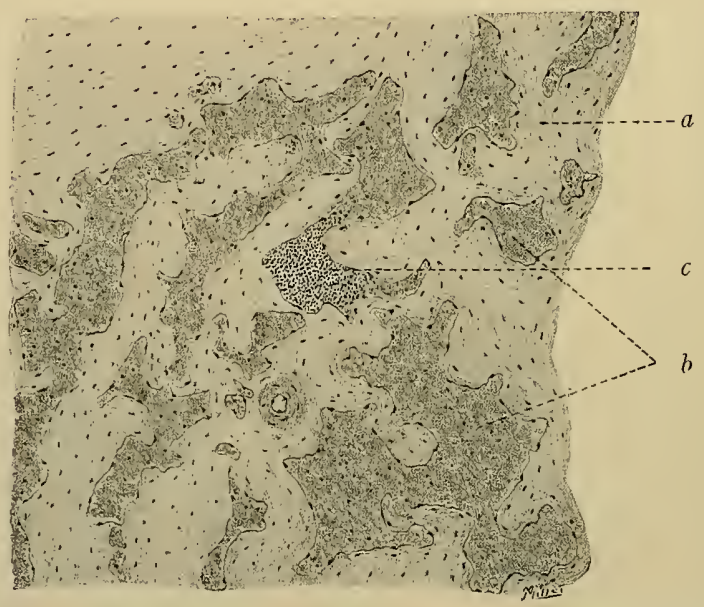

FIG, 240.-Erectile Tissue of Corpus Spongiosum of Human Penis. $\times 60$. $a$, Trabeculæ of connective tissue and smooth muscle; $b$, cavernous sinuses; $c$, groups of leucocytes in sinus.

partly from the cavernous sinuses. They pass through the tunica albuginea and empty into the dorsal vein of the penis (Fig. 239). In the corpus spongiosum there is probably no direct opening of arteries into sinuses. Both trabeculæ and sinuses are also smaller.

Of the lymphatics of the penis little definite is known.

The nerve endings, according to Dogiel, consist of: (a) free sensory endings, $(b)$ deeply situated genital corpuscles, $(c)$ Pacinian corpuscles and Krause's end-bulbs in the more superficial connective tissue, and $(d)$ Meissner's corpuscles in the papillæ. (For details see pages 432 and 433.)

The glans penis consists of erectile tissue similar in structure to 
that of the corpus cavernosum, except that the venous spaces are smaller and more regular. The mucous membrane is very closely attached to the fibrous sheath of the underlying erectile tissue. A few small sebaceous glands, unconnected with hairs - the glands of Tysonare found in the mucous membrane of the base of the glans penis.

The prepuce is a fold of skin which overlies the glans penis. Its inner surface is lined with mucous membrane.

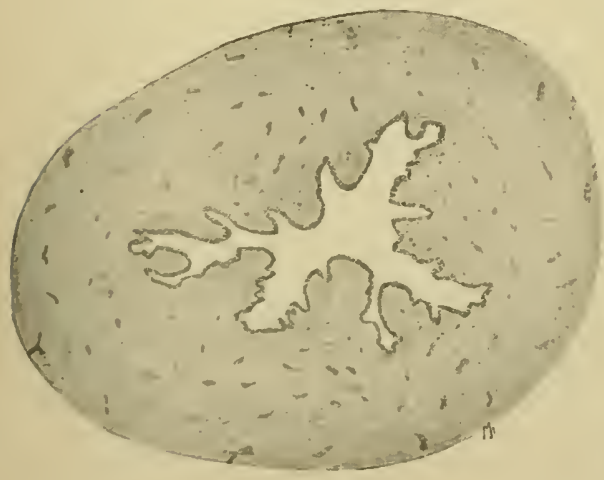

Fig. $24 \mathrm{I}$.

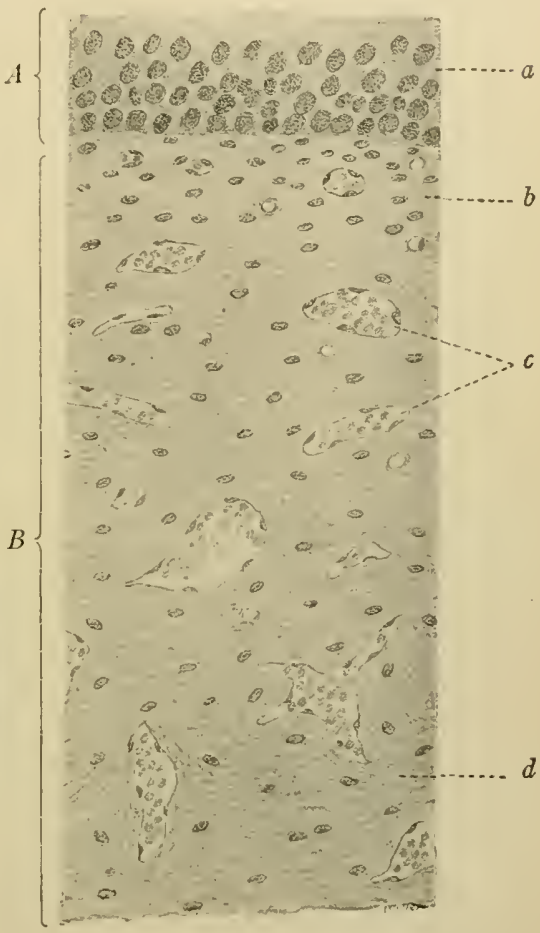

FIG. 242.

FIG. 24I.-From Transverse Section of Urethra and Corpus Spongiosum, including Mucous Membrane and part of Submucosa. $\times{ }_{15}$. The dark spots represent the cavernous veins.

Fig. 242.-Vertical Section through Portion of Wall of Human Male Urethra. $\times 350 . \quad A$, Mucous membrane; $B$, submucosa; $a$, epithelium; $b$, stroma; $c$, cavernous veins; $d$, connective tissue of submucosa.

\section{The Urethra ${ }^{1}$}

The MALE URETHRA is divided into three parts-prostatic, mcmbranous, and penile. The wall of the urethra consists of three coatsmucous, submucous, and muscular. The structure of the wall differs in the different parts of the urethra.

The mucous membrane (Fig. 242) consists of epithelium and stroma. The epithelium of the prostatic part is stratified squamous

The female urethra, while not so distinctly divisible into sections, presents essentially the same structure as the male urethra. The epithelium begins at the blarkler as siratified squamous of the transitional type, changes to at two-layered stratified or pseurlestratified, and finally passes over into stratified spluamous near the urethral opening. Clands of Liture are present, but are fewer than in the male. 
(transitional), resembling that of the bladder. In the membranous part it is stratified columnar or pseudostratified. In the penile portion it is pseudostratified up to the fossa navicularis, where it changes to stratified squamous. The epithelium rests upon a basement membrane, beneath which is a thin stroma rich in elastic fibres and having papillæ which are especially prominent in the terminal dilated portion of the urethra, the fossa navicularis. The stroma merges without distinct demarcation into the submucosa.

The submucosa consists of connective tissue and, in the penile portion, of more or less longitudinally disposed smooth muscle. It contains a dense network of veins - cavernous veins - which give it the character of erectile tissue (Fig. 242).

The muscular coat is thickest in the prostatic and membranous portions. Here it consists of a thin inner longitudinal and a thicker outer circular layer. A definite muscular wall ceases at the beginning of the penile portion, although circularly disposed smooth muscle cells are found in the outer part of the submucosa of the penile urethra.

Throughout the mucosa of the entire urethra, but most numerous in the penile portion, are simple branched tubular mucous glands, the glands of Littré. They are lined with columnar epithelium and the longer extend into the submucosa.

\section{TECHNIC}

(I) For the study of the general topography of the penis, remove the skin from the organ and cut into transverse slices about $0.5 \mathrm{~cm}$. in thickness. Fix in formalin-Müller's fluid (technic 5, p. 7), cut rather thick sections across the entire penis, stain with hæmatoxylin-picro-acid-fuchsin (technic 3, p. 2I) or with hæmatoxylin-eosin (technic I, p. 20) and mount in balsam.

(2) For the study of the structure of the penile portion of the urethra and of the erectile tissue of the corpus spongiosum, cut away the corpora cavernosa, leaving only the corpus spongiosum and contained urethra, and treat as above. Sections should be thin and stained with hæmatoxylin-eosin.

(3) The same technic is to be used for the membranous and prostatic portions of the urethra.

\section{FEMALE ORGANS}

\section{The Ovary}

The ovary is classed as one of the ductless glands. Its specific secretion is the ovum. The ovary has no duct system which is 
directly continuous with its structure. In place of this it is provided with what may be considered to be a highly specialized disconnected excretory duct - the oviduct or Fallopian tube-which serves for the transmission of its secretion to the uterus.

On one side the ovary is attached by a broad base, the hilum, to the broad ligament. Elsewhere the surface of the ovary is covered by a modified peritoneum. At the hilum the tissues of the broad ligament pass into the ovary and spread out there to form the ovarian

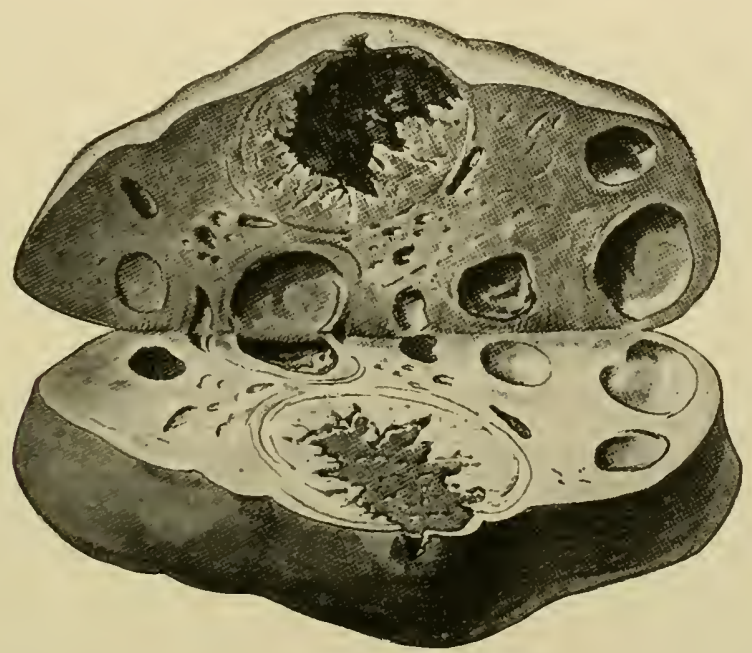

FIG. 243.- Ovary opened by Longitudinal Incision. Ovum has Escaped through Tear in Surface. Cavity of follicle filled with blood clot (corpus hæmorrhagicum) and irregular projections composed of lutein cells. (Kollmann's Atlas.)

stroma. This consists of fibrous connective tissue rich in elastic fibres and containing many smooth muscle cells. In the deeper central portion of the organ, stroma alone is found. Here it contains many large blood-vessels, and constitutes the medulla or zona vasculosa of the ovary (Fig. 244, 2). From the medulla the stroma radiates toward the surface of the ovary and becomes interspersed with glandular elements forming the ovarian corlex (Fig. 244, 3, 3'). At the surface of the ovary, just beneath the peritoneum, the stroma forms a rather dense layer of fibrous tissue, the lunica albuginea. At the margin of the peritoneal surface of the ovary the connective tissue of the peritoneum becomes continuous with the stroma of the ovary, while the flat mesothelium of the general peritoneum is replaced by a single layer of cuboidal cells, which covers the surface of the 
ovary and is known from its function as the germinal epithelium (Fig. 245, he). The parenchyma or secreting portion of the ovary consists of peculiar glandular elements, the Graafian follicles.

The structure of the Graafian follicle can be best appreciated by studying its development. The follicles originate from the germinal epithelium during fœtal life. At this time the germinal epithelium is proliferating, and certain of its cells differentiate into larger

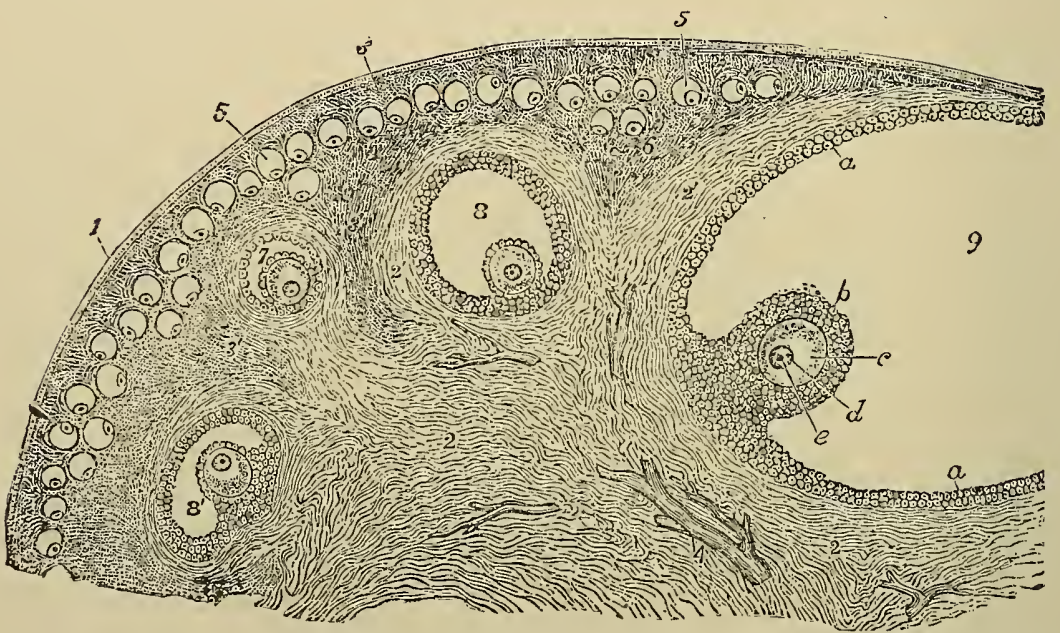

FIG. 244.- Semidiagrammatic Drawing of Part of Cortex and Medulla of Cat's Ovary. (From Schrön, in Quain's "Anatomy.") I, Germinal epithelium, beneath which is 3 , the tunica albuginea; 2, medulla, containing large blood-vessels, $4 ; 2,2^{\prime}$, fibrous stroma, arranged around mature Graafian follicle as its theca folliculi; $3^{\prime}$, stroma of cortex; 5 , small (primitive) Graafian follicles near surface; 6, same deeper in cortex; 7, later stage of Graafian follicle, beginning of cavity; 8 and $8^{\prime}$, still later stages in development of follicle; 9 , mature follicle; $a$, stratum granulosum; $b$, germ hill; $c$, ovum; $d$, nucleus (germinal vesicle); $e$, nucleolus (germinal spot).

spherical cells-primitive ova (Fig. $245, o p$ ). The primitive ova pass down into the stroma accompanied by a considerable number of the undifferentiated cells of the germinal epithelium. A cord-like mass of cells is thus formed, extending from the surface into the stroma. This is known as Pflïger's egg cord (Fig. 245). Each cord usually contains several ova. In some cases the differentiation of the ova cells does not occur upon the surface but in the cords after they have extended down from the surface. The connection of the cord with the surface epithelium is next broken so that each cord becomes. completely surrounded by stroma. It is now known as an egg nest (Fig. 245). During this process, proliferation of the epithelial cells of the cords and nests has been going on, and each ovum surrounded. 
by a layer of epithelial cells becomes separated from its neighbors (Fig. $245, f p$ ). This central orum surrounded by a single layer of epithelial cells (follicular cells) is the primitive Graafian follicle (Fig. 245, fp, Fig. 246, and Fig. 247, a). Rarely a follicle may contain more than one ovum, of which, however, only one goes on to maturity, the others degenerating. The follicle increases in size, mainly on account of proliferation of the follicular cells, which soon form several layers instead of a single layer, but also partly on account of growth of

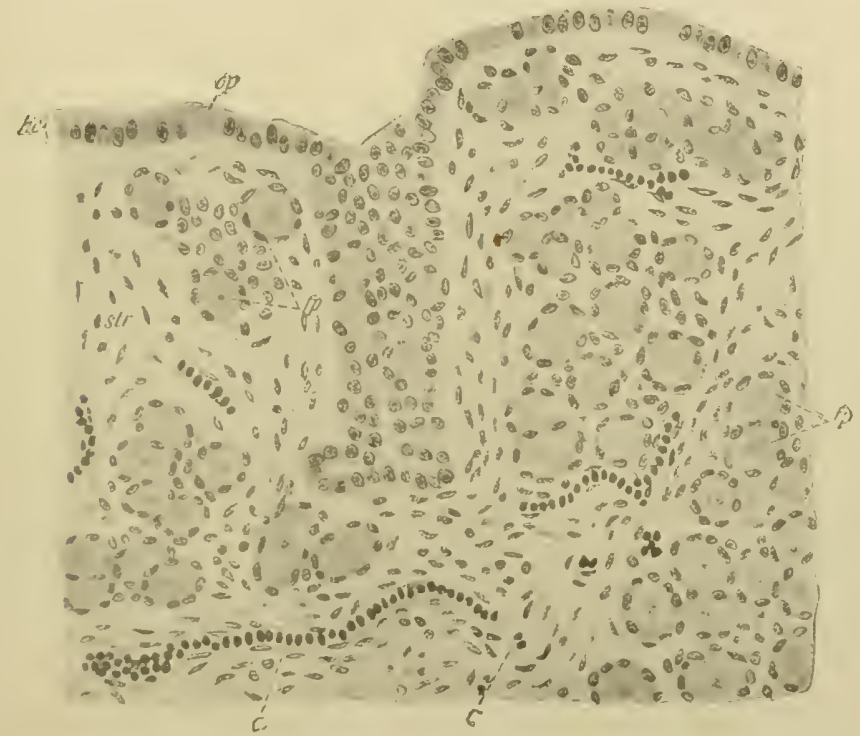

FIg. 245.-From Transverse Section of Ovary of New-born Child. $\times_{28} 8$ (Sobotta). Shows primitive ova in germinal epithelium; Pflüger's egg cords and nests of cells; $c$, capillaries; he, germinal epithelium; str, stroma; $f p$, primitive follicles; $o p$, primitive ova.

the ovum itself (Fig. 247). The latter now leaves the centre of the follicle and takes up an eccentric position. At the same time a cavity (or several small cavities which later unite) appears near the centre of the follicle (Fig. 247, $e$ and Fig. 244, 7). This is filled with fluid which seems to be in part a secretion of the follicular cells, in part a result of their disintegration. The cavity is known as the follicular cavily or antrum, the fluirl as the liquor folliculi. Lining the follicular Cavity are several rows of follicular cells with granular protoplasmthe stratum granulosum. With increase in the liquor folliculi the ovum becomes still further pressed to one side of the follicle, where, surrounderl by an accumulation of follicular cells, it forms a distinct projection into the cavity (Fig. 249, and Fig. 244,8 and 9). This 
is known as the germ hill (discus proligerus-cumulus ovigerus). The cells of the germ hill nearest the ovum become columnar and arranged in a regular single layer around the ovum - the corona radiata (Fig. 250). The ovarian stroma immediately surrounding the Graafian follicle becomes somewhat modified to form a sheath for the follicle-

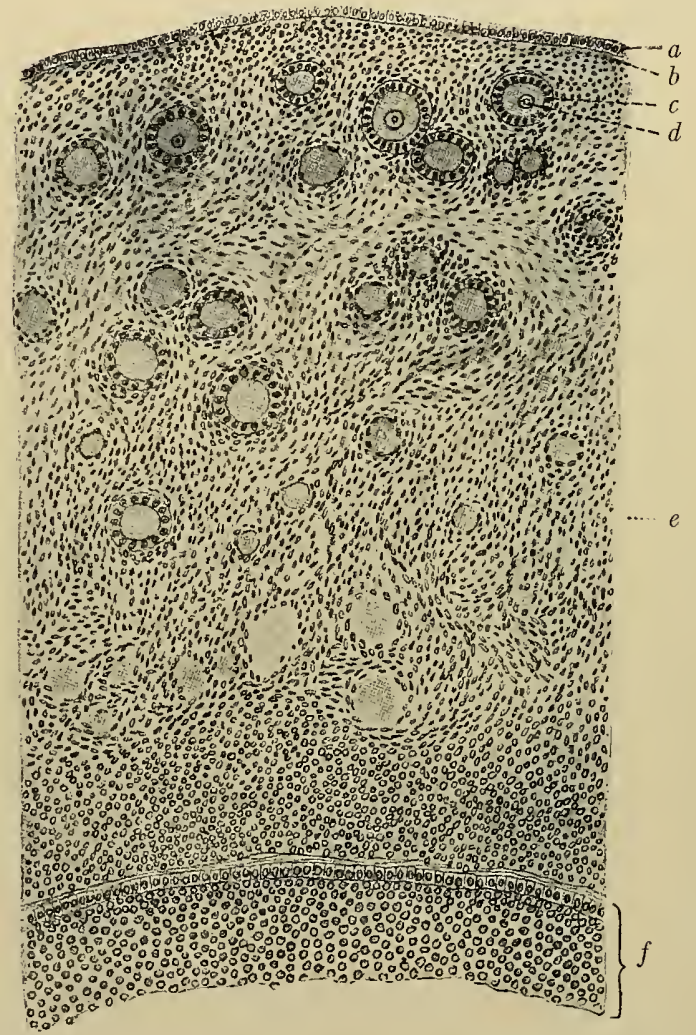

FIg. 246.- Vertical Section through Cortex of Ovary of Young Girl. $\times$ I9o. (Böhm and von Davidoff.) $a$, Germinal epithelium; $b$, tunica albuginea; $c$, follicular epithelium; $d$, ovum; $e$, primitive Graafian follicles in ovarian cortex; $f$, granular layer of large Graafian follicle.

the theca folliculi (Fig. 248). This consists of two layers, an outer more dense fibrous layer, the tunica fibrosa, and an inner more cellular and vascular, the tunica vasculosa. Between the theca folliculi and the stratum granulosum is an apparently structureless basement membrane.

While these changes are taking place in the follicle, the ovum is also undergoing development. The ovum of the primitive follicle 
is a spherical cell, having a diameter of from 40 to $70 "$ and the structure of a typical cell. The mucleus or germinal vesicle (so called on account of the part it takes in reproduction) is about half the diameter of the cell. and is spherical and centrally placed (Fig. 247). It is surrounded by a double-contoured nuclear membrane, and contains a distinct chromatic network and nucleolus or germinal spot. The cytoplasm is quite easily differentiated into a spongioplasm network and a homogeneous hyaloplasm. Such ova are present in all active ovaries, i.e. during the childbearing period, but are especially numerous in the ovary of the infant and child (Fig. 246).

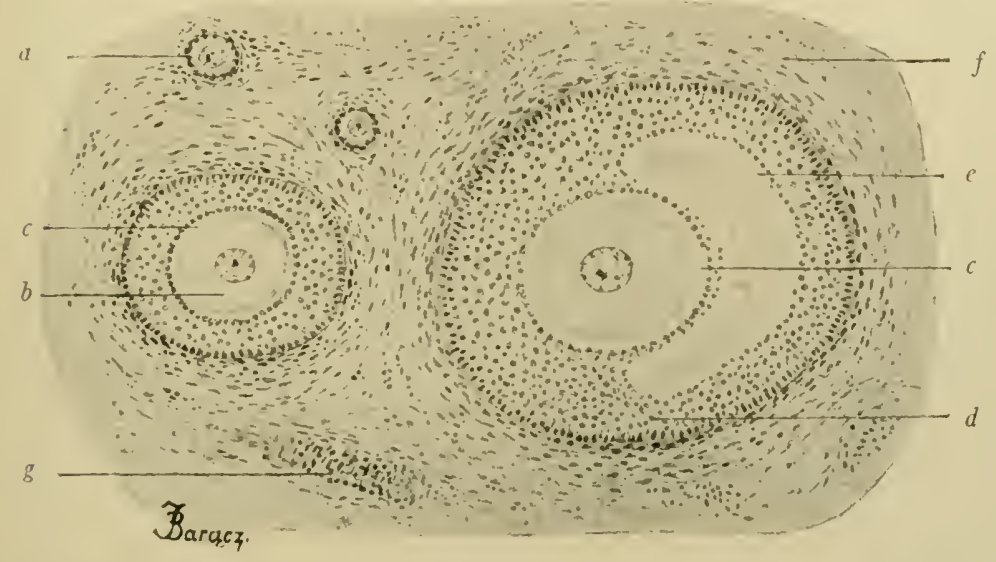

Fig. 247.- From Section through Cortex of Ape's Ovary. X X 50. (Szymonowicz.) $a$, l'rimitive follicle; $b$, ovum, with nucleus and nucleolus; $c$, zona pellucida; $d$, follicular epithelium; $e$, follicular cavity; $f$, ovarian stroma; $g$, blood-ressel in stroma.

With the development of the follicle the ovum increases in size and becomes surrounded by a clear membrane, the zona pellucida, believed by some to be a cuticular formation deposited by the egg cell, by others to be a product of the surrounding follicular cells. Minute canals extend into the zona pellucida from its outer surface. These contain processes of the cells of the corona radiata. A narrow cleft, the perivitelline space, has been described as separating the ovum from the zona pellucida. During the growth of the ovum its cytoplasm becomes coarsely granular from the development of yolk or deutoplatsm granules (Fig. 250). Immediately surrounding the nucleus, and just beneath the zona pellucida, the egg protoplasm is fairly free from yolk granules.

The further maturation of the ovum, which is neessary before the 
egg cell is in condition to be fertilized, consists in changes in the chromatic elements of the nucleus, which result in the extrusion of the polar bodies, and apparently have as their main object the reduction in number of chromosomes to one-half the number characteristic of the species. This process has been described (page $5^{8}$ ). In many of the lower animals maturation of the ovum is completed outside the ovary. In man and the higher animals the entire process takes place within the ovary, the second polar body being extruded just before the escape of the ovum from its follicle.

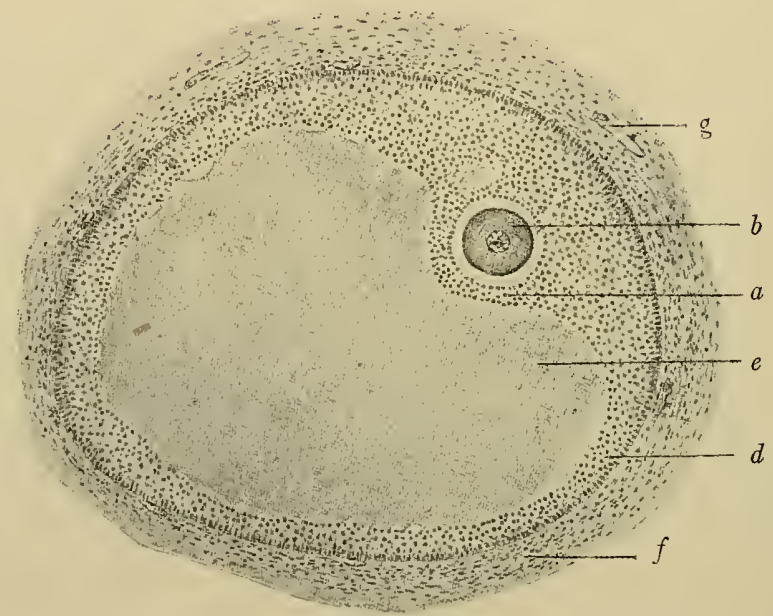

Fig. 248. - Section through Graafian Follicle of Ape's Ovary. $\times_{90} . \quad$ (Szymonowicz.) Later stage of development than Fig. 228. $a$, Germ hill; $b$, ovum with clear zona pellucida, germinal vesicle, and germinal spot; $d$, follicular epithelium (membrana granulosa); $e$, follicular cavity; $f$, theca folliculi; $g$, blood-vessel.

The youngest of the Graafian follicles are found just under the tunica albuginea near the germinal epithelium, from which they originate (Fig. 244, 5). As the follicle matures it passes deeper into the cortex. With complete maturity the follicle usually assumes macroscopic proportions -8 to $\mathrm{I} 2 \mathrm{~mm}$.- and often occupies the entire thickness of the cortex, its theca at one point touching the tunica albuginea. Thinning of the follicular wall nearest the surface of the ovary next takes place (Fig. 25I), while at the same time an increase in the liquor folliculi determines increased intrafollicular pressure. This results in rupture of the Graafian follicle and the discharge of its ovum, together with the liquor folliculi and some of the follicular cells. 
An escape of blood into the follicle from the torn ressels of the theca always accompanies the discharge of the ovum. The follicle again becomes a closed cavity, while the contained blood clot becomes organized by the ingrowth of vessels from the theca, to form the corpus hamorrhagicum (Fig. 252), which represents the earliest stage in the development of the corpus luteum.

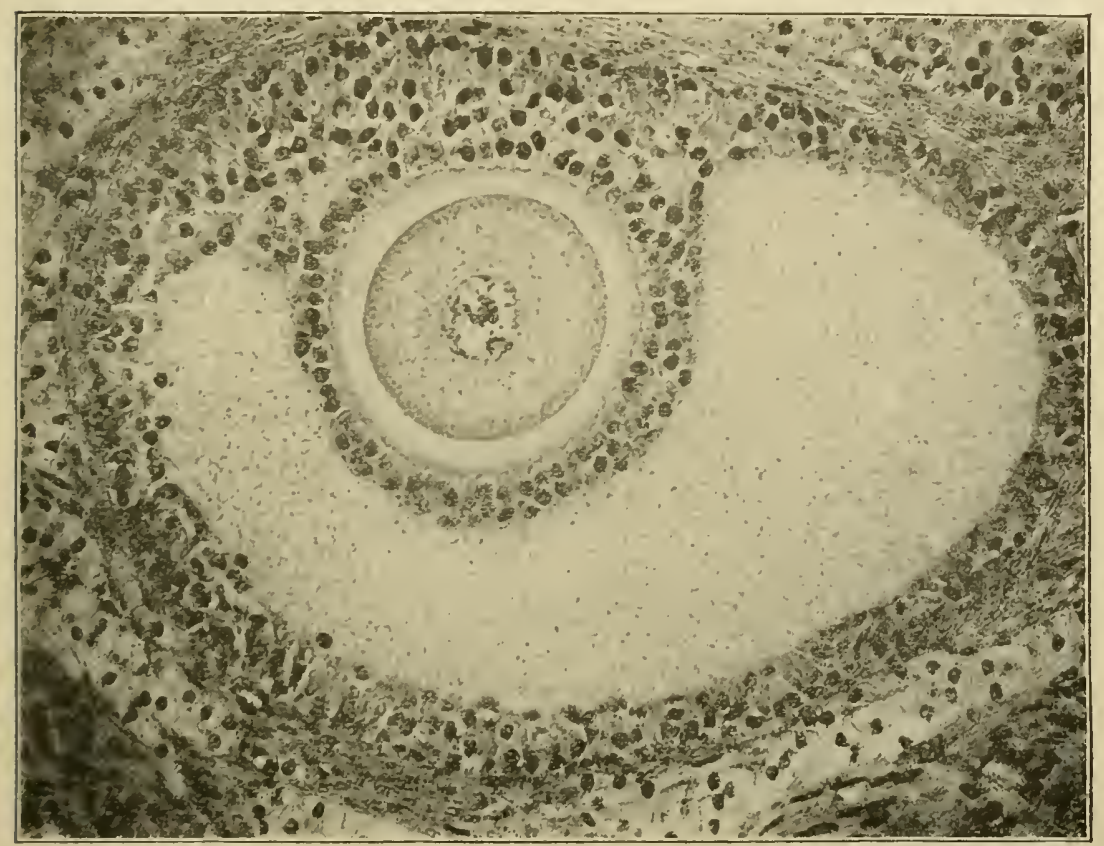

Fig. 249.-Graafian-Follicle and Contained Ovum of Cat; directly reproduced from a photograph of a preparation by Dahlgren. $\times 235$. (From "The Cell in Develropment and Inheritance," I'rof. E. B. Wilson; The Macmillan Company, publishers.) The ovum is seen lying in the Graafian follicle within the germ hill, the cells of the latter immediatcly surrounding the ovum forming the corona radiata. The clear zone within the corona is the zona pellucida, within which are the egg protoplasm, nucleus, and nucleolus. Iincircling the follicle is the connective tissue of the theca folliculi.

The corpus luteum (Fig. 253), which replaces the corpus hæmorrhagicum, consists of large yellow cells-lutein cells-and of connective tissuc. 'The latter with its blood-vessels is derived from the inner layer of the theca. The origin of the lutein cells is not clear. They are described by some as derived from the connective-tissue cells of the theca; by others as the result of proliferation of the cells of the stratum granulosum. The cells have a yellow color from the presence of fatty (lutcin) granules in their protoplasm, and it is to 
these granules that the characteristic yellow color of the corpus luteum is due. A definite cellular structure with a supporting connective-tissue framework thus replaces the corpus hæmorrhagicum, remains of which are usually present in the shape of orange-colored. crystals of hæmatoidin. By degeneration and subsequent absorption of its tissues the corpus luteum becomes gradually reduced in size,

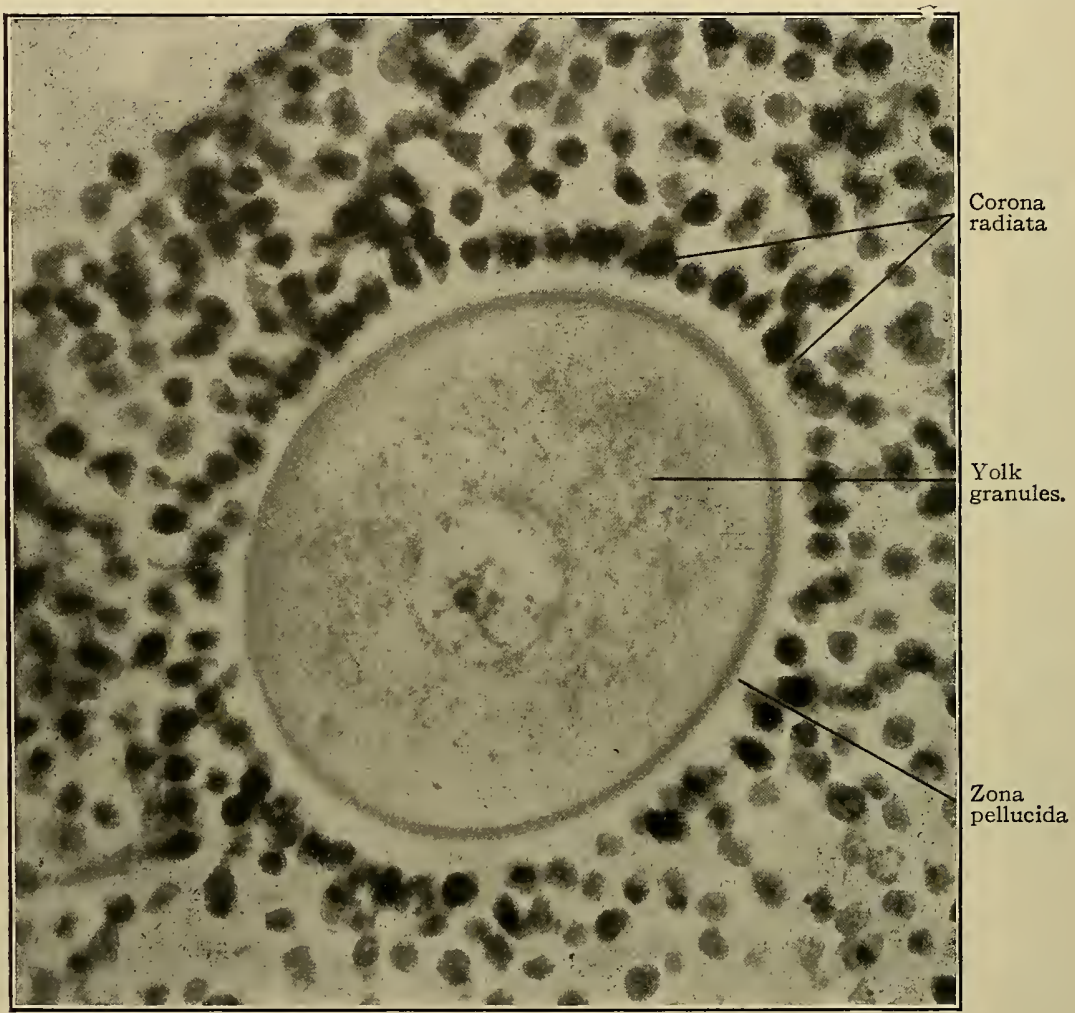

FIG. 250.-From a Section of a Human Ovum. Section taken from the ovary of a I 2 year old girl. The ovum lies in a large mature Graafian follicle and is surrounded by the cells of the "germ hill" (the inner edge of which is shown in the upper left-hand corner of the figure). Photograph. (Bailey and Miller.)

loses its yellow color, and is then known as the corpus albicans. This also is mostly absorbed, being finally represented merely by a small area of fibrous tissue.

Corpora lutea are divided into true corpora lutea (corpora lutea vera or corpora lutea of pregnancy) and false corpora lutea (corpora lutea spuria). The former replace follicles whose ova have undergone fertilization, the latter, follicles whose ova have not been fer- 


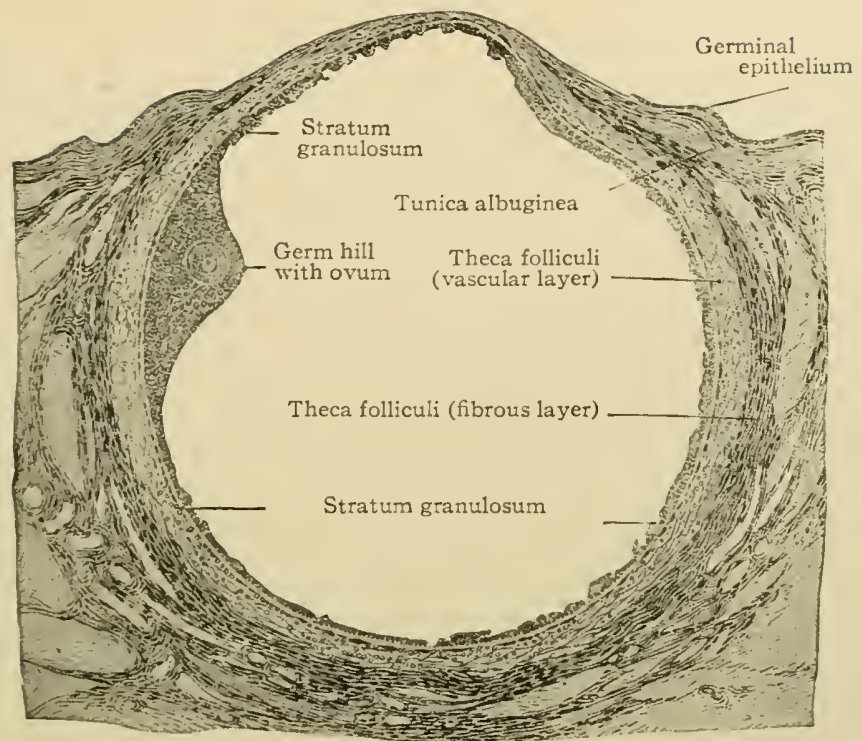

Fig. 25I.-From Section of Human Ovary, showing mature Graafian follicle ready to rupture. (Kollmann's Atlas.)

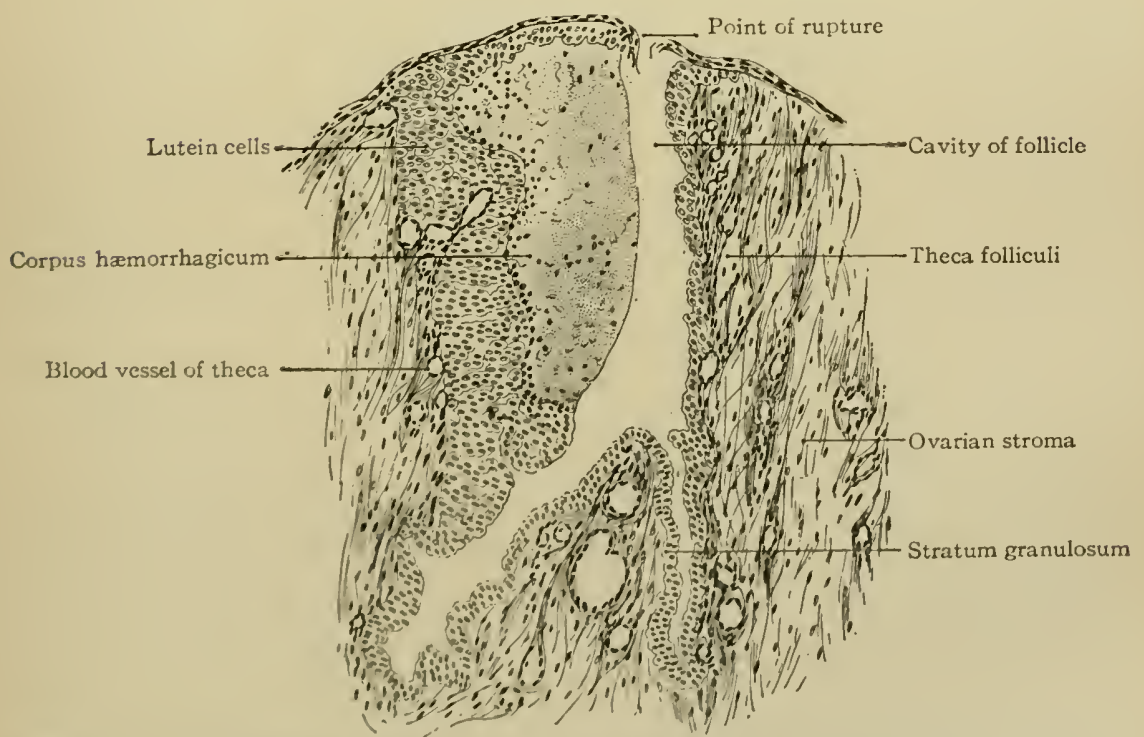

Fis. 252, From Section of Human Ovatry, showing carly stage in formation of Corpus l.uteum. (Kollmann's Itlas.) 
tilized. The structure of both is similar, but the true corpus luteum is larger, and both it and its corpus albicans are slower in passing through their retrogressive changes, thus remaining much longer in the ovary.

While the function of the corpus luteum is not known, the recent experiments of Fraenkel seem to be confirmatory of the theory advanced by Born, that the corpus luteum is a gland having an inter-

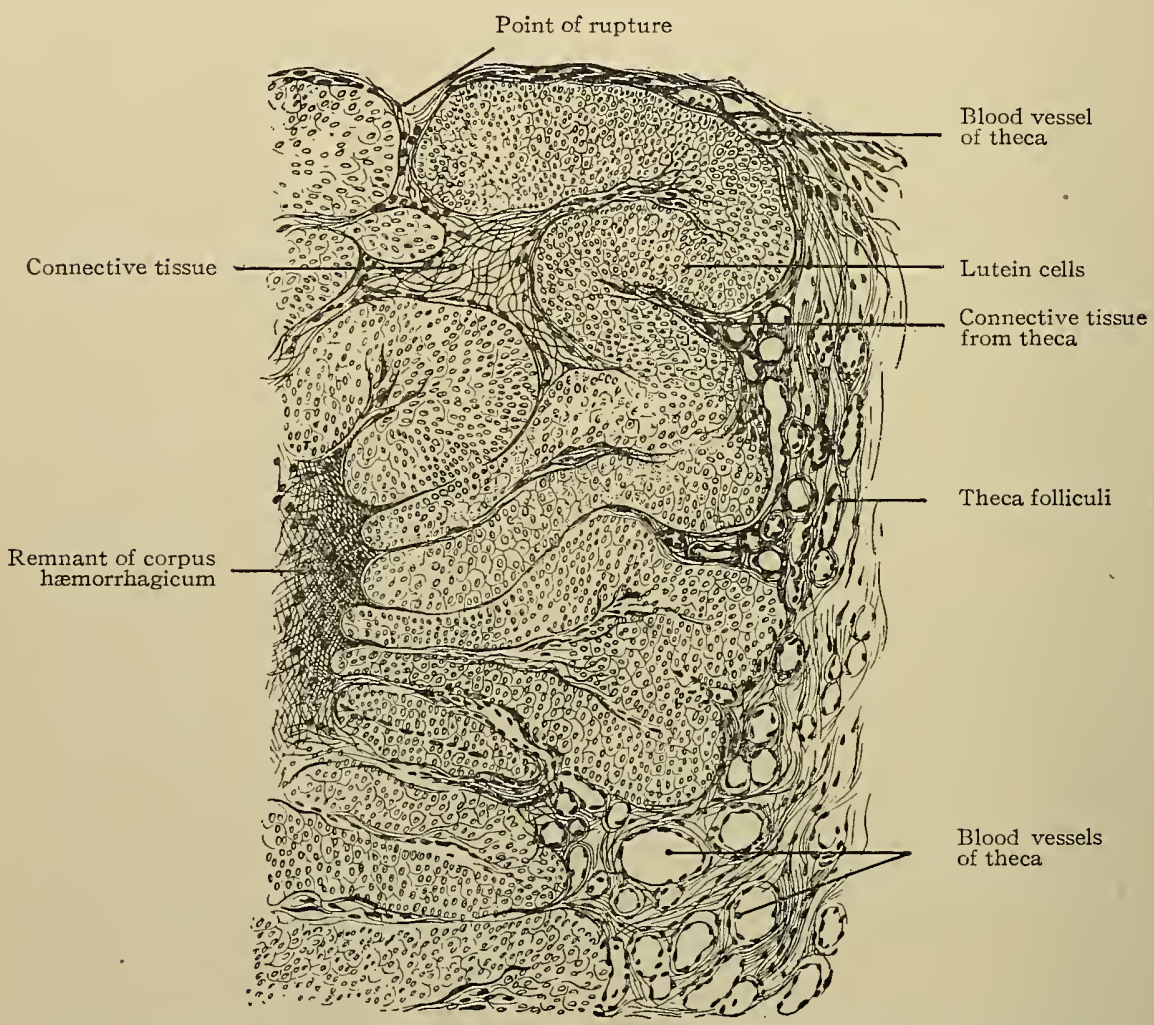

FIG. 253.-From Section of Human Ovary, showing later stage of Corpus Luteum than Fig. 252. (Kollmann's Atlas.)

nal secretion, which appears to have some influence upon the attachment of the fecundated ovum to the uterus and upon its nutrition during the first few weeks of its development. According to Fraenkel the corpus luteum is a periodically rejuvenated ovarian gland, which gives to the uterus a cyclic nutritional impulse, which prepares it for the implantation of the ovum or favors menstruation whenever the ovum is not fertilized. 
Of the large number of ova-estimated at seventy-two thousand in the human ovaries-only comparatively few, according to Henle about four hundred, reach maturity. The majority undergo, together with their follicles, retrogressive changes known as atresia of the follicle. The nucleus of the ovum, as well as the nuclei of the follicular cells, passes through a series of chromatolytic changes, or in some cases apparently simply atrophies. The cell bodies undergo fatty or albuminous degeneration and the cells become reduced to a homogeneous mass, which is finally absorbed, leaving in its place a connective-tissue scar, probably the remains of the theca folliculi.

Blood-vessels. - The arteries, branches of the ovarian and uterine, enter the ovary at the hilum and ramify in the medulla. From these are given off branches which pass to the cortex and end in a capillary network in the tunica albuginea. In the outer layer of the theca folliculi the capillaries form a wide-meshed network, which gives rise to a fine-meshed network of capillaries in the inner layer of the theca. From the capillaries veins arise which form a plexus in the medulla and leave the ovary at the hilum.

Lymphatics.-These begin as small lymph spaces in the cortex, which communicate with more definite lymph vessels in the medulla, the latter leaving the organ at the hilum.

Nerves.-Medullated and non-medullated fibres enter the ovary at the hilum and follow the course taken by the blood-vessels. Many of the fibres end in the vessel walls; others form plexuses around the follicle and end in the theca folliculi. Some describe fibres as passing through the theca and ending in the follicular epithelium. Others claim that nerve fibres do not enter the follicle proper. Groups of sympathetic ganglion cells occur in the medulla near the hilum.

As is the case with the testicle, certain rudimentary organs, the remains of fotal structures, are found connected with the ovary.

The paroöphoron consists of a number of cords or tubules of epithelial cells, sometimes ciliated, sometimes non-ciliated. It is found in the medulla, or, more commonly, in the connective tissue of the hilum.

'The epoöphoron is a similar structure found in the folds of the broarl ligament. Its tubules open into a rluct known as Gärtner's duct. In man this duct ends blindly. In some of the lower animals it opens into the vagina. Both paroöphoron and epoöphoron are 
remains of the embryonal mesonephros, the former of its posterior segment, the latter of its middle segment.

\section{The Oviduct}

The oviduct or Fallopian tube is the excretory duct of the ovary, serving for the transmission of the discharged ovum from ovary to uterus. Although there is no sharp demarcation between them, it is convenient to divide the tube into three segments: (I) The isthmus, beginning at the uterus and extending about one-third the

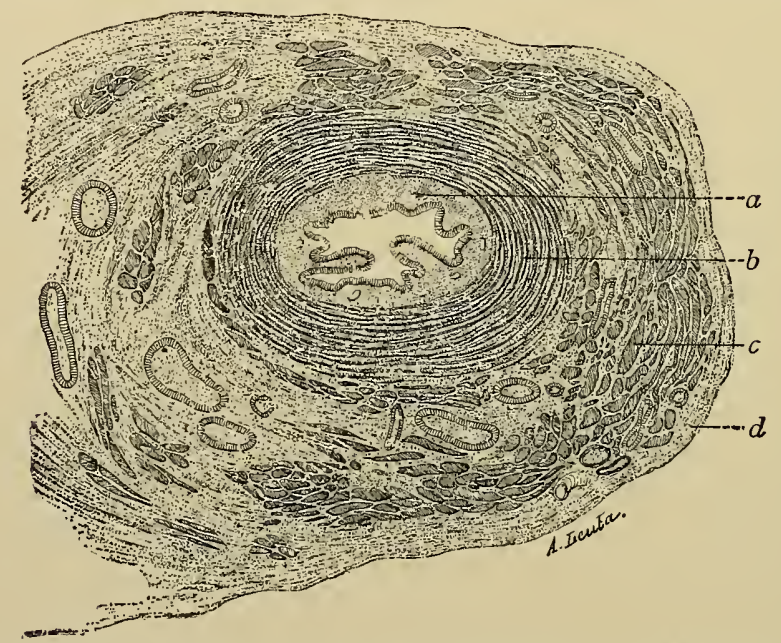

FIG. 254.-Cross Section of Oviduct near Uterine End. $a$, Mucous membrane; $b$, circular muscle coat; $c$, longitudinal muscle coat; $d$, connective tissue of serous coat. (Orthmann.)

length of the tube; (2) the ampulla, about twice the diameter of the isthmus, and occupying somewhat more than the middle third; and (3) the fimbriated or ovarian extremity.

The walls of the oviduct consist of three coats: (I) Mucous, (2) muscular, and (3) serous (Figs. 254 and 255).

The mucous membrane presents numerous longitudinal foldings. In the embryo four of these folds can usually be distinguished, and these are known as primary folds. In the adult many secondary folds have developed upon the primary, especially in the ampulla and fimbriated extremity where the folds are high and complicated (Fig. 255). The epithelium lining the tube is of the simple columnar ciliated type, and completely covers the foldings of the mucous mem- 
brane. The ciliary motion is toward the uterus. The stroma consists of a cellular connective tissue, quite compact in structure in the isthmus, where the folds are low, more loosely arranged in the high folds of the ampulla and fimbriated extremity.

The muscular coat consists of an inner circular and an outer longitudinal layer. The latter is a comparatively thin layer in the isthmus, consists of discontinuous groups of muscle cells in the ampulla, and in the fimbriated extremity is frequently absent.

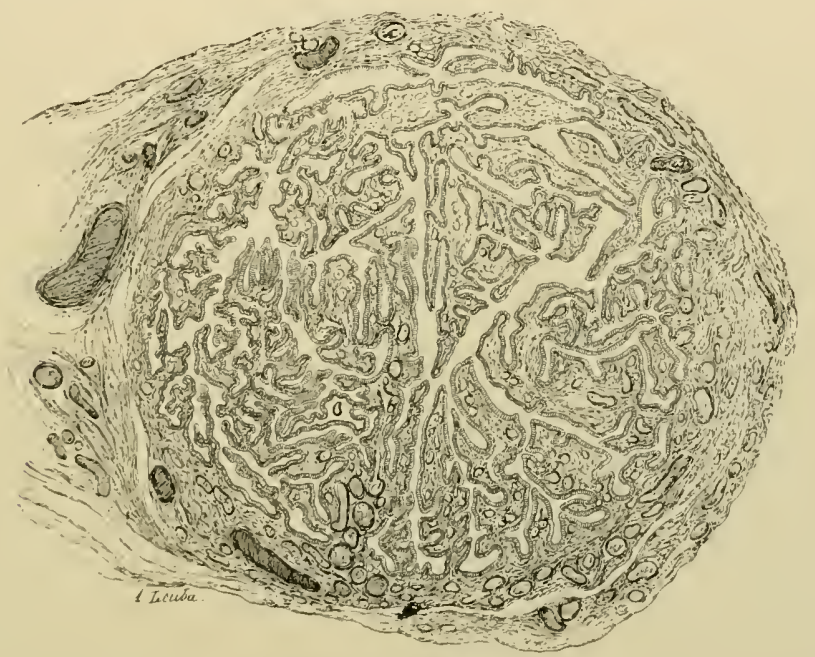

FIG. 255.-Cross Section of Oviduct near Fimbriated Extremity, showing complicated foldings of mucous membrane. (Orthmann.)

The serous coat has the usual structure of peritoneum.

The larger blood-vessels run in the stroma along the bases of the folds. They send off branches which give rise to a dense capillary network in the stroma.

Of the lymphatics of the tube little is known.

The nerves form a rich plexus in the stroma, from which branches pass to the blood-vessels and muscular tissue of the walls of the tube and internally as far as the epithelial lining.

\section{TECHNIC}

(1) Child's Ovary.-Remove the ovary of a new-born child, being careful not to touch the surface epithelium, fix in '/enker's fluid (technic 9, p. 8), and harden in alcohol. Cut sections of the entire organ through the hilum. Stain with hamatoxylin-cosin (technic $\mathrm{I}, \mathrm{p}, 20$ ) and mount in balsam.

(2) For the purpose of sturlying the Gratafian follicle in the different stages of 
its development remove an ovary from an adult cat or dog and treat as above. Technic (I). These sections also, as a rule, are satisfactory for the study of the corpus luteum.

(3) The adult human ovary is little used for histological purposes on account of the few follicles it usually contains and its proneness to pathological changes.

Its study is, however, so extremely important, especially

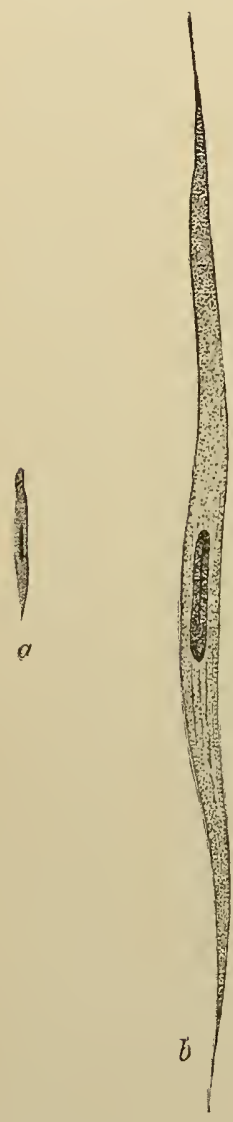

FIG. 25 6.--Muscle cells from $(a)$ nonpregnant uterus; $b$, pregnant uterus; drawn to same scale. (Sellheim.) with reference to the pathology of the ovary, that if possible a normal human ovary should be obtained from a young subject for purposes of comparison with the above. Technic (I).

(4) For studying the egg cords of Pflüger and their relation to the germ epithelium, ovaries of the human fotus, and of very young cats, dogs, and rabbits are satisfactory. Technic (I).

(5) Sections of the fimbriated end of the oviduct are usually found in the sections of ovary. For the study of other parts of the tube, cut out thin pieces from different regions, fix in formalin-Müller's fluid, stain transverse sections with hæmatoxylin-eosin, and mount in balsam.

\section{The Uterus}

The wall of the uterus consists of three coats which from without inward are serous, muscular, and mucous.

The serous coat is a reflection of the peritoneum, and has the usual structure of a serous membrane.

The muscularis consists of bundles of smooth muscle cells separated by connective tissue. The muscle has a general arrangement into three layers, an inner, a middle, and an outer, which are distinct in the cervix, but not well defined in the body and fundus.

The inner layer-stratum submucosum-is. mainly longitudinal, although some obliquely running bundles are usually present.

The middle layer-called from the large venous channels which it contains, the stratum vasculare - is the thickest of the three layers, forming the main bulk of the muscular wall. It consists mainly of circularly disposed muscle bundles.

The outer layer-stratum supravasculare-is thin and consists partly of circular bundles, partly of longitudinal. The latter predominate and form a fairly distinct layer just beneath the serosa. 
The muscle cells of the uterus are long spindle-shaped elements, some having pointed, others blunt, branched, or frayed ends. In the virgin uterus they have a length of from 40 to 6o". During pregnancy the muscular tissue of the uterus is greatly increased. This is due partly to increase in the number, partly to increase in the size of the muscle cells. At term the muscle cells frequently have a length of from 250 to $600 \%$. (Fig. 256.)

The mucous membrane. As the mucosa presents marked variation in structure, dependent upon the functional condition of the organ, it is necessary to describe:

I. The mucosa of the resting uterus.

2. The mucosa of the menstruating uterus.

3. The mucosa of the pregnant uterus.

\section{The Mucosa of the Rest- ING UTERUS}

This is from I to $2 \mathrm{~mm}$. thick, and consists of a stroma, glands, and a lining epithelium (Fig. 257). The stroma resembles embryonal connective tissue, consisting of fine fibrils and long, irregular branching cells which form a sort of network, the meshes of which are filled in with lymphoid cells and leucocytes. The epithelium is of the

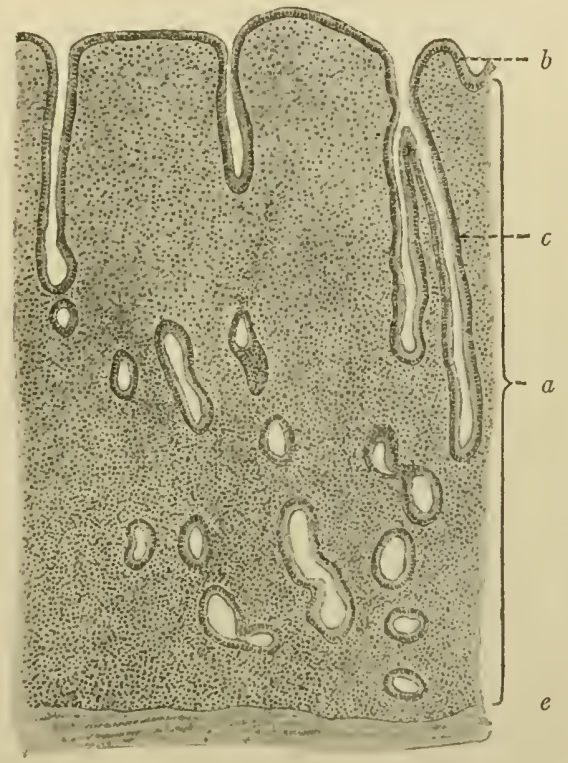

FIG. 257.-From Uterus of Young IVoman. (Böhm and von Davidoff; preparation by Dr. J. Amann.) $\times_{34}$. a, Mlucous membrane; $b$, surface cpithelitum; $c$, gland; $e$, muscle. simple high columnar ciliated variety, the ciliary motion being toward the cervix. $\Lambda$ basement membrane separates the epithelium from the underlying stroma. The glands are simple forked tubules lined by a single layer of columnar ciliated cells resting upon a basement membrane and continuous with the surface cells. The glands extend completely through the stroma. Near the surface they run a comparatively straight course. Deeper in the stroma their course is more tortuous, while the fundus is frequently turned at right angles to the rest of the tubule.

In the cervix the stroma is firmer and less cellular, and the mu- 
cous membrane is thicker and presents numerous folds - the plice palmate. The epithelium is higher than in the body of the organ. In addition to glands like those found in the body of the uterus, the cervical mucosa contains peculiar short, sac-like invaginations, lined with a continuation of the surface epithelium, which secrete a glairy mucus. Closure of the mouths of some of these sacs frequently

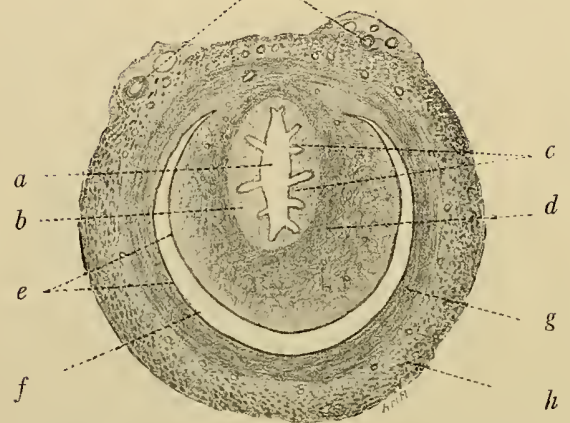

FIG. 258.-From Section of Dog's Cervix. $\times 4$. (Technic 2, p. 379.) a, Cervical canal; $b$, mucosa; $c$, folds of mucosa (plicæ palmatæ); $d$, muscle layers of cervix; $e$, epithelium of vagina and vaginal surface of cervix; $f$, vaginal epithelium; $g$, vaginal mucosa; $h$, submucosa and muscularis of vagina; $i$, bloodvessels.

squamous epithelium with underlying papillæ similar to and continuous with that of the vagina.

Near the external os the epithelium changes over into the stratified squamous epithelium with underlying papillæ, similar to that of the external surface of the cervix.

\section{The Mucosa of the Menstruating Uterus}

This consists of the same structural elements as the mucosa of the resting uterus: stroma, glands, and lining epithelium. These, however, undergo certain changes which may be conveniently divided into three stages:

(a) The stage of preparation.

(b) The stage of menstruation proper.

(c) The stage of reparation.

(a) The Stage of Preparation.-This begins several days before the actual flow of blood, and is marked by an intense hyperæmia determining a swelling and growth of the entire mucosa. 
The blood-ressels, especially the capillaries and veins, become greatly distended, thus contributing largely to the increase in thickness of the mucosa. There are also proliferation of the connective-tissue cells, an increase in the number of leucocytes, and a growth of the uterine glands. The surface at the same time becomes irregular, the glands opening into deep pits or depressions, and the glands themselves become more tortuous and their lumina more widely open. The mucous membrane has now reached a thickness of about 6 $\mathrm{mm}$., and is known as the decidua menstrualis (Fig. 259).

(b) The Stage of MenStruation Proper.--This is marked by the escape of blood from the engorged vessels and the appearance of the external phenomena of menstruation. The blood escapes partly by rupture of the vessel walls, partly by diapedesis. The hemorrhage is at first subepithelial, but the epithelium soon gives way and the blood escapes into the cavity of the uterus. Much difference of opinion exists as to the amount of epithelial destruction during menstruation, some claiming that the entire epithelium is de-

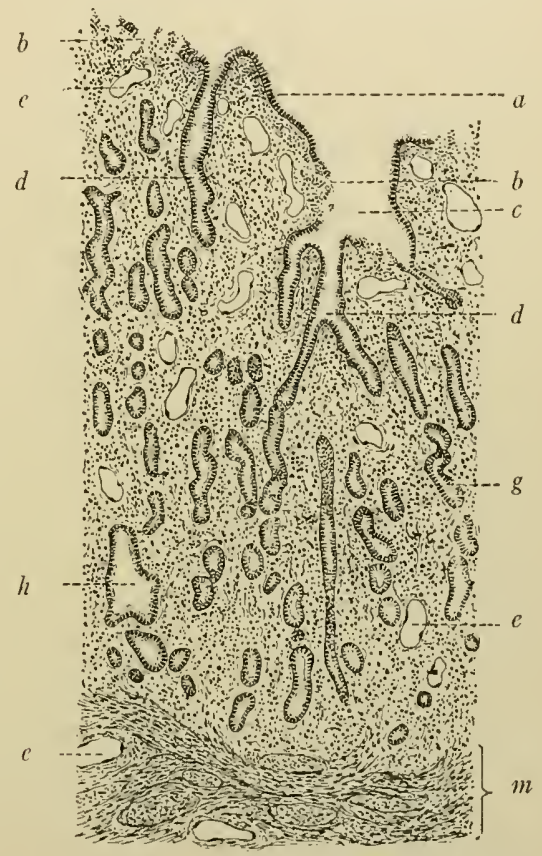

FIG. 250.-Section through Mucous Membrane of Virgin Uterus during First Day of Menstruation. $\times 30$. (Schaper.) $a$, Surface epithelium; $b$, disintegrating surface; c, pit-like depression in mucous membrane; $d$, excretory duct; $c$, bloodvessels; $g$, gland tubule; $h$, dilated gland tubule; $m$, muscularis.

stroyed with each menstrual period, others that the epithelium remains almost intact. Complete destruction of the epithelium is hardly compatible with the restoration of the epithelium which always follows menstruation. While there is undoubtedly destruction of most or all of the surface epithelium and of the glands to some consirlerable dejeth, the deeper portions of the glands always remain to take part in the succeeding regenerative phenomena.

(c) THE STAGE of REPARAfIon.- After from three to five days the bleeding from the uterine mucosa ceases and the return to the resting condition begins. 'This is marked by disappearance of the 
congestion, by decrease in thickness of the mucosa and in the size of the glands, and by restoration of the surface epithelium.

\section{The Mucosa of the Pregnant Uterus.}

This is known as the decidua graviditatis, and presents changes in structure somewhat similar to those which occur during menstruation, but more extensive. It is divided into three parts:

(a) The decidua serotina or decidua basalis - that part of the mucosa to which the ovum is attached.

(b) The decidua reflexa or decidua capsularis-that part of the mucosa which surrounds the ovum.

(c) The decidua vera-which consist of all the remaining mucosa.

The development of the decidua vera resembles the changes which take place in the mucosa during menstruation. There is the same thickening of the mucosa, and this thickening is due to the same factor, i.e., distention of the blood-vessels and proliferation of the tissue elements. These changes are, however, much more extensive than during menstruation. The superficial part of the stroma between the mouths of the glands becomes quite dense and firm, forming the compact layer. The deeper part of the stroma contains numerous cavities, which are the lumina of the now widely distended and tortuous glands. This is known as the spongy layer.

Within the stroma, especially of the compact layer, develop the so-called decidual cells. These are peculiar typical cells derived from connective tissue. They are of large size (30 to $100 \mu)$, vary greatly in shape, and in the later months of pregnancy have a rather characteristic brown color, which they impart to the superficial layers of the decidua vera. They are mostly mononuclear, although polynuclear forms occur.

During the latter half of pregnancy there is a gradual thinning of the decidua vera, due apparently to pressure. The necks of the glands in the compact layer disappear, and the gland lumina in the spongy layer are changed into elongated spaces, which lie parallel to the muscular layer.

The decidua reflexa and decidua serotina have at first the same structure as the decidua vera. The decidua reflexa undergoes hyaline degeneration during the early part of pregnancy, and by the end of gestation has either completely disappeared (Minot) or has fused with the decidua vera (Leopold). 
The decidua serotina undergoes changes connected with the development of the placenta.

\section{The Placenta ${ }^{1}$}

The placenta consists of two parts, one of which is of maternal origin-placenta uterina - the other of fœtal origin-the placenta fortalis. As it is in the placenta that the interchange takes place between the maternal and the fœtal blood, the relations between the maternal and fœtal parts of the placenta are extremely intimate. This relation consists essentially in the growing out from the foctal placenta of finger-like projections - villi-which penetrate the maternal placenta, the latter being especially modified for their reception.

The Placenta Fetalis.-This is a differentiated portion of the chorion. On the surface directed toward the foetus the chorion after the third month is covered by a delicate fœtal membrane, the placental portion of the amnion. This consists of a surface epithelium, resting upon a layer of embryonal connective tissue which attaches it to the chorion. The chorion consists of $(a)$ a compact layer-the membrana chorii-composed at first of embryonal, later of fibrous, connective tissue, and containing the main branches of the umbilical vessels and $(b)$ an inner villous layer, which gives rise to finger-like projections which extend down from the fœetal into the maternal placenta and serve to connect the two.

The chorionic villi first appear as short projections composed entirely of epithelium. Each of these primary villi branches dichotomously, giving rise to a number of secondary villi. As they develop, the central portion of the original solid epithelial structure is replaced by connective tissue. Septa of connective tissue from the maternal placenta pass down among the villi and separate them into groups or cotyledons. The main or primary villi run a quitestraight course from the chorion into the maternal placental tissue, apparently serving to secure firm union between the two. They are thus known as roots of attachment or fastening villi ( Fig. 260). The secondary villi are given off laterally from the primary villi, end freely in the spaces between the latter-intervillous spaces (Fig. 260) and are known as free, terminal or floating villi (lïg. 260).

The chorionic villus thus consists of a central core of connective

1 for many facts ats to the structure of the placentat, the writer is indebted to the excellent chapter on the subject adrled by Prof. Alfred Schaper to the fifth edition of Stishr's "lextlorek of Ilistologgy." 


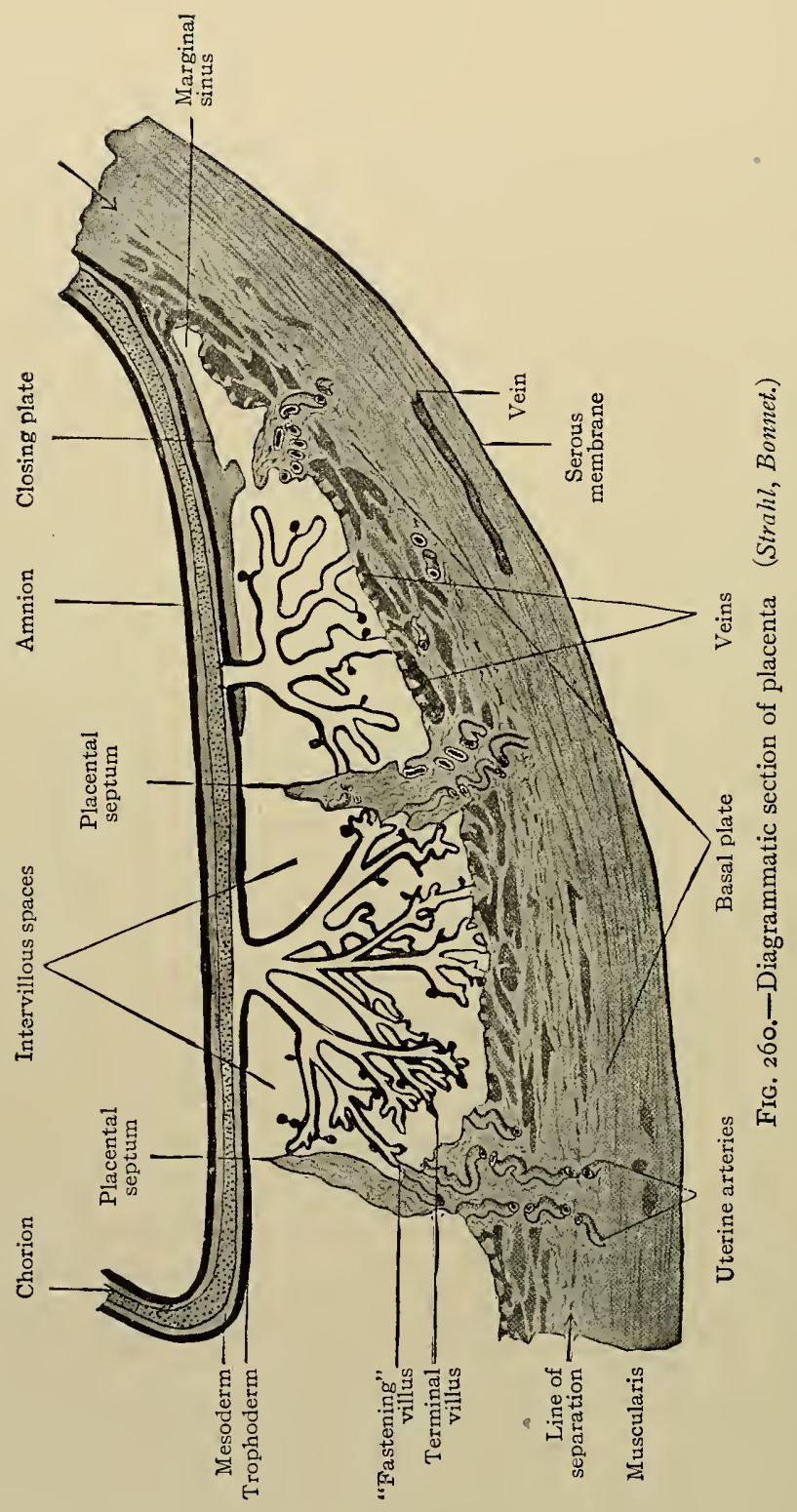


tissue covered by a layer of epithelium. The connective tissue is of the mucous type and serves for the transmission of numerous bloodvessels. In the villi of early pregnancy the epithelium consists of an inner layer of distinctly outlined cells and an outer layer of fused cell bodies-a syncytium (Fig. 262, A, a)-containing small scattered nuclei. The villi of the later months of pregnancy have no definite epithelial covering, but are surrounded by a delicate homogeneous membrane, probably the remains of the syncytium. At various points on the surface of the villus are groups of nuclei. These stain

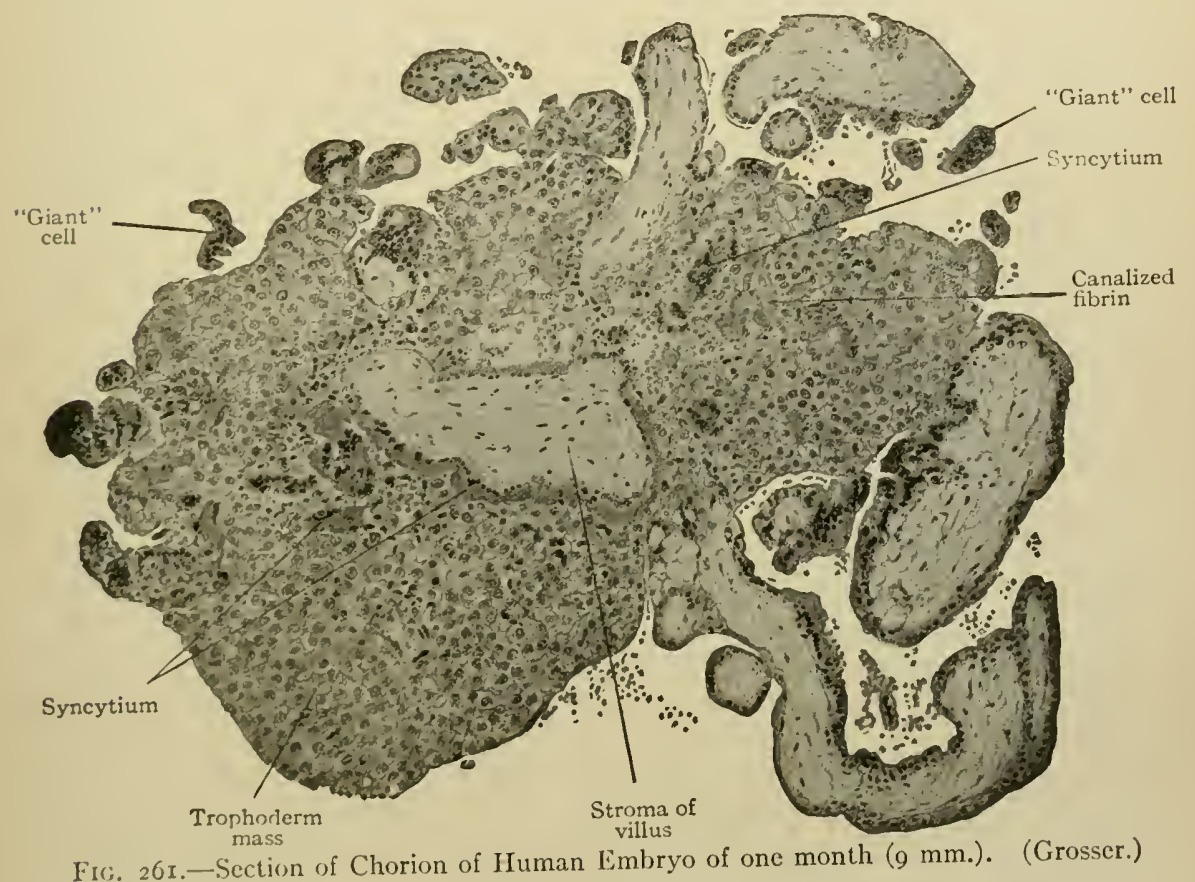

intensely, are surrounded by a homogencous protoplasm, and form knob-like projections above the general surface of the villus. They are known as cell patches, or more properly as nuclear groups (Fig. $262, c)$, and represent remains of the nuclei of the epithelium of the younger villus. Between the nuclear groups the villus is covered only by a thin homogeneous membrane. Small villi usually resemble more closely in structure the younger villus, being frequently covered by a nucleated syncytium. Portions of the syncytium, especially of older villi, sometimes become changed into a peculiar hyaline substance containing numerous channels. This is known as canalized 
fibrin, and may form dense layers upon the surface of the chorion. (Fig. 26r.)

The Placenta Uterina.-This develops from the decidua serotina. The latter becomes much thinner than the rest of the decidua (decidua vera), but still shows a division into a deeper spongy portion containing gland tubules, and a superficial compact portion in which are large numbers of decidual cells. From the superficial portion

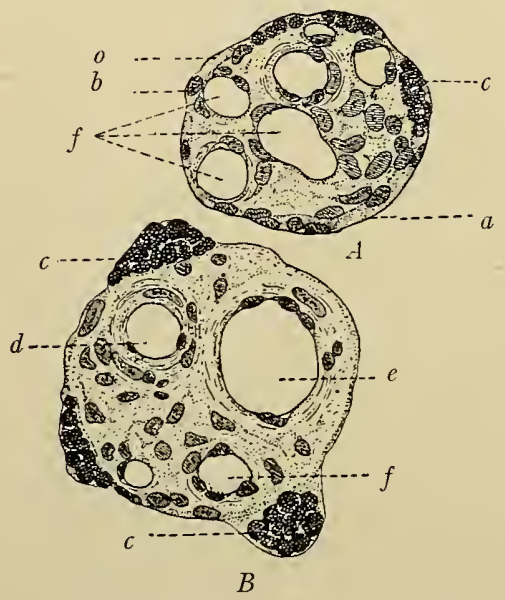

FIG. 262.-Cross Sections of Human Chorionic Villi at End of Pregnancy. $\times 250$. (Schaper.) $A$, Small villus; $B$, larger villus. $a$, Protoplasmic coat (syncytium); $b$, epithelial nucleus; $c$, nuclear groups; $d$, small artery; $e$, small vein; $f$, capillaries. from vessels of the uterine muscularis enter the serotina. In the very tortuous course which these vessels take through the serotina (Fig. 260) their walls lose their muscular and connective-tissue elements and become reduced to epithelial tubes. These branch in the placental septa and finally open into the intervillous spaces along the edges of the cotyledons. The veins take origin from these spaces near the centres of the cotyledons. The maternal blood thus passes through the intervillous spaces from periphery to centre, and in its course comes into direct contact with the freely terminating chorionic villi. It is to be noted that the blood-vessel systems of the mother and of the fœtus are both closed systems, and that consequently there is no direct admixture of maternal and fotal blood. Interchange of materials must therefore always take place through the capillary walls and through the walls of the chorionic villi. (Fig. 260.) 
Blood-vessels. - The arteries enter the uterus from the broad ligament and pass to the stratum vasculare of the muscularis, where they undergo extensive ramification. From the arteries of the stratum rasculare branches pass to the mucosa and give rise to capillary networks, which surround the glands and are especially dense just beneath the surface epithelium. From these capillaries the blood passes into a plexus of veins in the deeper portion of the mucosa, and these in turn empty into the venous plexuses of the stratum vasculare. Thence the veins accompany the arteries, leaving the uterus through the broad ligament.

Lymphatics.- These begin as minute spaces in the stroma and empty into the more definite lymph channels of the muscularis, which are especially well developed in the stratum vasculare. These in turn communicate with the larger lymph vessels in the subserous connective tissue.

Nerves.-Both medullated and non-medullated nerve fibres occur in the uterus. The latter are associated with minute sympathetic ganglia and supply the muscular tissue. The medullated fibres form plexuses in the mucosa, from which are given off fine fibres which terminate freely between the cells of the surface epithelium and of the uterine glands.

\section{The Vagina}

The wall of the vagina consists of four coats, which from without inward are fibrous, muscular, submucous, and mucous.

The fibrous coat consists of dense connective tissue with many coarse elastic fibres. It serves to connect the vagina with the surrounding structures.

The muscular coat is indistinctly divided into an outer longitudinal and an inner circular layer. The latter is usually not well developed and may be absent.

The submucosa is a layer of loose connective tissue, especially rich in elastic fibres and blood-vessels. Numerous large venous channels give to the submucosa the character of erectile tissue.

The mucous membrane consists of a papillated connective-tissue stroma of mixed fibrous and clastic tissue. The stroma usually contains diff use ly mphoid tissue and more rarely solitary nodules. Covering the stroma is a stratified squamous epithelium, the surface cells of which are extremely thin. The surface of the mucosa is not smooth, 
but is folded transversely, forming the so-called ruga. Most authorities agree that glands are wanting in the vagina, the mucus found there being derived from the glands of the cervix.

Blood-vessels.-The larger blood-vessels run in the submucosa, giving off branches which break up into capillary networks in the submucosa, muscularis, and stroma. The vascular networks have a general direction parallel to the surface. The capillaries empty into veins which form a plexus of broad venous channels in the muscularis.

The Lymphatics.-These follow in general the distribution of the blood-vessels.

Nerves.-Nerve fibres from both cerebro-spinal and sympathetic systems are found in the vagina. Medullated (sensory) fibres, the dendrites of spinal ganglion cells, form plexuses in the mucosa, from which are given off delicate non-medullated terminals to the epithelial cells. Non-medullated sympathetic fibres supply the muscularis and the muscle of the vessel walls. Along these nerves are small sympathetic ganglia.

In the vestibule the epithelium gradually takes on the structure of epidermis. Here are located small mucous glands--glandule vestibulares minores-especially numerous around the clitoris and opening of the urethra. Larger mucous glands-glandule vestibulares majores, or glands of Bartholin-analogous to Cowper's glands in the male, are also found in the walls of the vestibule.

The clitoris consists mainly of erectile tissue similar to that of the corpora cavernosa of the penis. It is covered with a thin epithelium with underlying papillæ, and is richly supplied with nerves having highly specialized terminations.

\section{Development of the Urinary and Reproductive Systems}

The development of the genito-urinary system is complicated by the appearance, and disappearance for the most part, of two sets of urinary organs, and the final formation of the permanent set. The three sets, in the order of their appearance, are the pronephroi, mesonephroi, and metanephroi. The first two sets, which are present only in the embryo in the higher animals, are the representatives of organs that function in the adult in the lower vertebrates. They are also intimately concerned in the development of the efferent duct system of the male reproductive organs in higher animals. The metanephroi, generally known as the kidneys, are the functional urinary organs in the majority of reptiles and in all birds and mammals.

The pronephroi are represented in the human embryo of 3 to $5 \mathrm{~mm}$. by one or two small, condensed masses of mesoderm just lateral to the primitive segments 
on each side in the cervical region. These masses, which are probably derived from the mesothelial lining of the body cavity, may or may not become hollow, but do not connect with the pronephric duct, and soon disappear. The pronephric duct appears about the same time as a derivative of the mesoderm just lateral to the primitive segments. It extends from the cervical region to the caudal region of the embryo where it bends mesially and opens into the gut. These ducts persist and become the ducts of the mesonephroi.

The mesonephroi begin to develop almost as soon as the pronephroi and just caudal to them. Condensations appear in the mesodermal tissue lateral to the primitive segments and become more or less tortuous. Lamina appear in these condensations, thus forming tubules which then connect at one end with the pronephric duct (now the mesonephric or Wolffian duct). The tubules develop progressively from before backward and finally form a series extending from the cervical region to the pelvic region of the embryo. At the distal end of each tubule a glomerulus, containing branches from the aorta, develops. The tubules increase in length and number and come to form a pair of large structures which project into the dorsal part of the body cavity. These are often spoken of as the IIolffin bodies. They reach the height of their development during the fifth or sixth week.

During the period of their existence in the embryo in higher animals, the mesonephroi functionate as urinary organs, not only through the agency of the glomeruli but also by means of the epithelium of the tubules themselves, among which numerous branches of the posterior cardinal veins ramify.

From the sixth week on, and coincident with the development of the metanephroi or kidneys, the mesonephroi atrophy, leaving finally only certain parts which differ in the two sexes. In the male some of the tubules in the cephalic portion persist as the vasa efferentia, while a few in the caudal portion remain as the paradidymis and vasa aberrantia; the duct persists as the vas epididymidis, vas deferens, and ejaculatory duct. In the female the mesonephric tubules disappear for the most part, only a few remaining to form the epoopphoron and paroöphoron, while the duct persists in part as Gärtner's canal.

Each kidney begins in embryos of 5 to $6 \mathrm{~mm}$. as a hollow bud on the dorsal side of each mesonephric duct near its opening into the gut. This bud grows dorsally, and then turns cranially in the mesoderm between the vertebral column and the mesonephros. The proximal portion remains more slender as the ureter, while the distal end becomes dilaterl to form the primitive renal pelvis. From this dilated end a number of secondary evaginations grow out to form the papillary ducts and straight collecting tubules.

The mesodermal tissue surrounding the outgrowths from the primitive renal pelvis becomes condensed in places and gives rise to the convoluted portions of the uriniferous tubules and to IIenle's loops. $\Lambda$ glomerulus develops in connection with the distal end of eatch convoluted tubule. The portion of each tubule derived from the mesorlermal tissue unites secondarily at the arched (junctional) tubule with the portion derived from the renal pelvis. Thus the kidney tubules are derived in part directly from undifferentiated mesoderm (convoluted tubules and Henle's loogs) and in part from an outgrowth from the mesonephric duct (straight collecting tubules and papillary ducts). 
During fœtal life the kidneys are distinctly lobulated, but after birth the surface becomes quite smooth.

The genital gland on each side appears on the mesial surface of the mesonephros as a thickening of a narrow band of the mesothelial lining of the body cavity. The cells in the band become differentiated into two kinds-small cuboidal cells which stain rather intensely, and larger spherical cells with clearer cytoplasm and vesicular nuclei. The latter are the sex cells which are destined to give rise to the ova in the female or the spermatozoa in the male. The whole thickened band is known as the germinal epithelium.

The cells of the germinal epithelium increase in number by mitosis and soon become differentiated into two layers-a superficial layer which retains its epithelial character and contains the sex cells, and a deeper layer which is destined to give rise in part to the stroma of the genital gland. The elevation caused by the increased number of cells projects into the body cavity as the genital ridge. From the superficial layer containing the sex cells a number of plugs or columns of cells grow down into the deeper layer carrying some of the sex cells with them. Thus far (up to about the fifth week) the changes are common to both sexes, and only later does the sex differentiation occur.

After about the fifth week certain changes occur in the genital ridge which differ accordingly as the ridge is to become an ovary or a testicle. In the case of the ovary a layer of loose connective tissue grows in between the surface epithelium and the cell columns or plugs mentioned above. The cell columns are thus pushed farther from the surface and constitute the medullary cords. These ultimately disappear. The surface epithelium again sends plugs of cells (Pflüger's egg cords) down into the underlying tissue. These cords are made up for the most part of epithelial cells which give rise to the follicular cells, but contain also a considerable number of sex cells (primitive ova). The egg cords then become broken up into smaller masses each of which contains a single primitive ovum (rarely more) and constitutes a primitive Graafian follicle. The sex cell grows in size and becomes the primary oöcyte (and finally the mature ovum), while the epithelial cells around it give rise to the stratum granulosum and germ hill of the mature Graafian follicle. During these processes the stroma also increases in amount, while the original germinal epithelium becomes reduced to a single layer of cuboidal cells. The formation of egg cords is usually completed before birth. (See also p. 354.)

In the case of the testicle a layer of dense connective tissue, the tunica albuginea, develops between the germinal epithelium and the sex cords. The epithelium becomes reduced to a single layer of flat cells. The sex cords which first grew into the underlying tissue and which contain the sex cells, are destined to give rise to the convoluted seminiferous tubules. This phase of development differs from that in the ovary inasmuch as in the latter case the first formed sex cords (medullary cords) disappear, Pflüger's egg cords being formed later and having no homologue in the testicle. The sex cords of the testicle become more and more convoluted and the sex cells (spermatogonia) proliferate rapidly. Beginning after birth and continuing up to the time of puberty, lumina appear in the sex cords and they thus give rise to the convoluted seminiferous tubules. The supporting cells (of Sertoli) are probably derived from the undifferentiated epithelial cells of the sex cords. (See also p. 335.) 


\section{TECHNIC}

(I) A human uterus - if possible from a young adult-or, if this cannot be obtained, the uterus of a cat or dog, is cut transversely into slices about $\mathrm{I} \mathrm{cm}$. thick and fixed in Zenker's fluid (technic 9, p. 8) or in formalin-Müller's fluid (technic 5, p. 7). For topography these slices are cut in half through the middle of the uterine cavity and sections made through the entire half organ. These are stained with hæmatoxylin-picro-acid-fuchsin (technic 3, p. 2I) and mounted in balsam. For details of the mucous membrane, cut away most of the muscle from around the half slice, being careful not to touch the mucous surface; make thin sections, stain with hæmatoxylin-eosin (technic I, p. 20), and mount in balsam.

(2) Sections of the cervix may be prepared in the same manner as the preceding.

(3) Placental tissue may be cut into small cubes and treated with the same technic (I).

(4) If a human or animal uterus with the placenta in situ is obtainable it should be cut into thin slices and fixed in formalin-Müller's fluid. The blocks of tissue should be so arranged that sections include the utero-placental junction. They may be stained with hæmatoxylin-eosin or with hæmatoxylin-picroacid-fuchsin (see above).

(5) Treat pieces of the human vagina according to technic I, p. 254 .

\section{General References for Further Study}

Ballowitz: Weitere Beobachtungen über den feineren Bau der SaugethierSpermatozoen. Zeit. f. wiss. Zool., Bd., lii, I891.

Hertwig: Lehrbuch der Entwickelungsgeschichte des Menschen und der Wirbelthiere, Jena, I896.

Kölliker: Handbuch der Gewebelehre des Menschen.

Nagel: Das menschliche Ei. Arch. mik. Anat., Bd. xxxi, I888.

Ruckert: Zur Eireifung der Copepoden. Anat. Hefte, I. Abth., Bd. iv, 1894 .

Schaper: Chapter on the Placenta in Stöhr's Text-buok of Histology, 5th ed.

Sobotta: Ueber die Bildung des Corpus luteum bei der Maus. Arch. f. mik. Anat., Bd. xlvii, 1896.-Ueber die Bildung des Corpus luteum beim Kaninchen. Anat. Hefte, I. Abth., Bd. viii, I897. 


\section{CHAPTER X}

\section{THE SKIN AND ITS APPENDAGES}

\section{The Skin}

THE skin or cutis consists of two parts: (I) The derma, corium, or true skin, and covering this, (2) the epidermis or cuticle. The derma is a connective-tissue derivative of the mesoderm, the epidermis an epithelial derivative of the ectoderm.

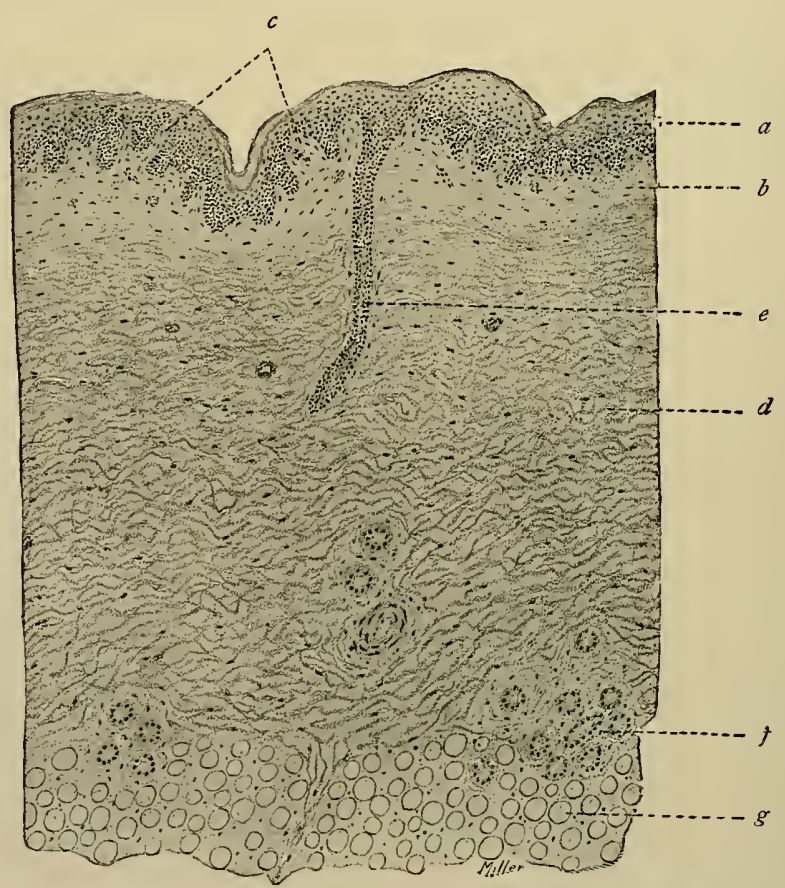

FIG. 263.-Vertical Section of Thin Skin, Human. $\quad \times 60 . \quad$ (Technic 2, p. 385.)" $a$, Epidermis; $b$, pars papillaris of derma; $c$, papillæ; $d$, pars reticularis of derma; $e$, duct of sweat gland; $f$, sweat gland; $g$, subcutaneous fat.

The Derma.-This is divided into two layers which blend without distinct demarcation. The deeper is known as the pars reticularis, the more superficial as the pars papillaris (Fig. 263).

The pars reticularis is made up of rather coarse, loosely arranged 
white and elastic fibres with connective-tissue cells in varying numbers. The fibres run for the most part parallel to the surface of the skin.

The pars papillaris is similar in structure to the preceding, but both white and elastic fibres are finer and more closely arranged. Externally this layer is marked by minute folds which are visible to the naked eye, and can be seen intersecting one another and enclosing small irregular areas of skin. In the thick skin of the palms and soles these furrows are close together and parallel, while between them are long corresponding ridges. In addition to the furrows and

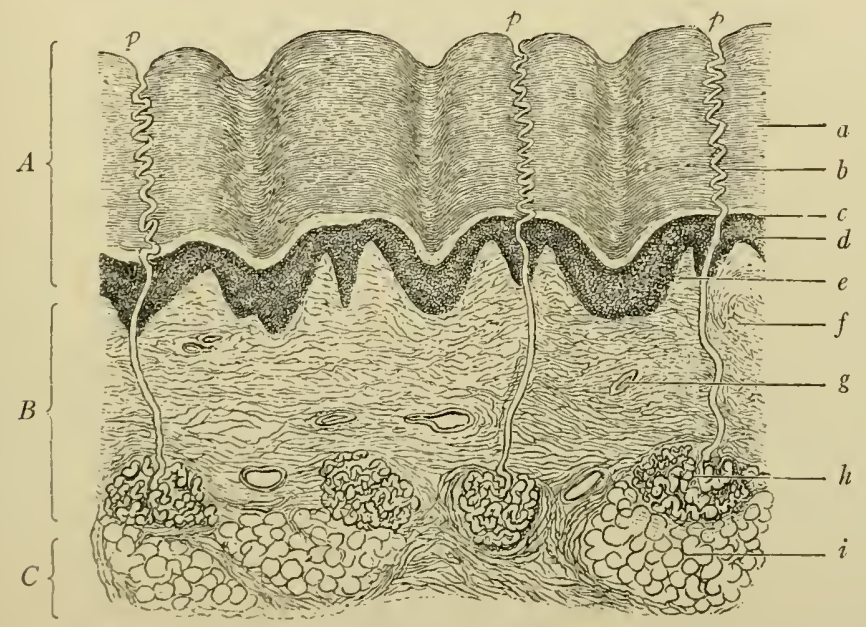

FIG. 264.-Thick Vertical Section through Skin of Finger Tip. (Merkel-Henle.) $A$, Epidermis; $B$, derma; $C$; subcutis. $a$, Stratum corneum; $b$, duct of sweat gland; $c$, stratum lucidum; $d$, stratum germinativum; $e$, papilla of derma; $f$, derma; $g$, bloodvessel; $h$, sweat gland; $i$, fat lobule; $p$, sweat pore.

ridges the entire surface of the corium is beset with minute papilla. These vary in structure, some ending in a single point-simple papilla-others in several points-compound papille; some containing blood-ressels-vascular papilla; others containing special nerve terminations-nerve papille (Fig. 265).

Smooth muscle cells occur in the corium in connection with the sweat glands. In the skin of the scrotum-tunica dartos - and of the nipple, the smooth muscle cells are arranged in a network parallel to the surface. In the face and neck striated muscle fibres penetrate the corium.

Bencath the corium is the subculaneous lissue. 'This consists of 
vertically disposed bands of connective tissue - the retinacula cutiswhich serve to unite the corium to the underlying structures and enclose fat lobules. In some parts of the body this subcutaneous fat forms a thick layer-the panniculus adiposus.

The EPIDERMis.-This is composed of stratified squamous epithelium. In the comparatively thin skin of the general body surface the epidermis is divided into two sublayers: (I) One lying just above the papillary layer of the derma, and known as the stratum

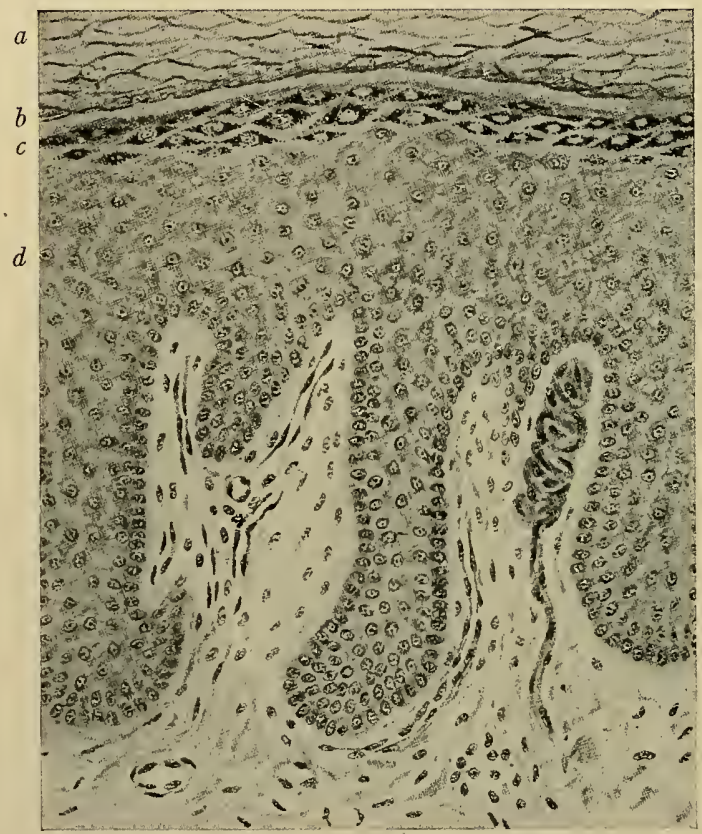

FIG. 265.-From Vertical Section through Skin of Human Finger Tip. $\times 200$. (Schäfer.) $a$, Stratum corneum; $b$, stratum lucidum; $c$, stratum granulosum; $d$, stratum germinativum. To the left a vascular papilla; to the right a nerve papillastcontaining tactile corpuscle.

germinativum (stratum mucosum-stratum Malpighii); (2) the other constituting the superficial layer of the skin - the horny layer or stratum corneum. In the thick skin of the palms and soles two additional layers are developed; (3) the stratum granulosum; and (4) the stratum lucidum (Fig. 264).

(I) The stratum germinatioum consists of several layers of cells, The deepest cells are columnar and form a single layer (stratum cylindricum), which rests upon a basement membrane separating it 
from the derma. The membrane and cells follow the elevations and depressions caused by the papillæ. The rest of the stratum germinativum consists of large polygonal cells. These cells have welldeveloped intercellular bridges, which appear as spines projecting from the surfaces of the cells. For this reason the cells are sometimes called "prickle" cells, and the layer, the "stratum spinosum." The spines cross minute spaces between the cells, which are believed to communicate with the lymph spaces of the derma (Fig. 266,c). The cells of the stratum germinativum are usually in a state of active mitosis.

(2) The stratum granulosum is well developed only where the skin is thick. It consists of from one to three layers of flattened polygonal cells. The protoplasm of these cells contains deeply staining granules-keratohyaline granules - which probably represent a stage in the formation of the horny substance-keratin-of the corneum cells. The nuclei of these cells always show degenerative changes, and there is reason for believing that this karyolysis is closely associated with the formation of the keratohyaline granules (Fig. 266, b).

(3) The stratum lucidum is also best developed where the skin is thickest. It consists of two or three layers of flat clear cells, the outlines of which are frequently so indistinct that the layer appears homogeneous. The

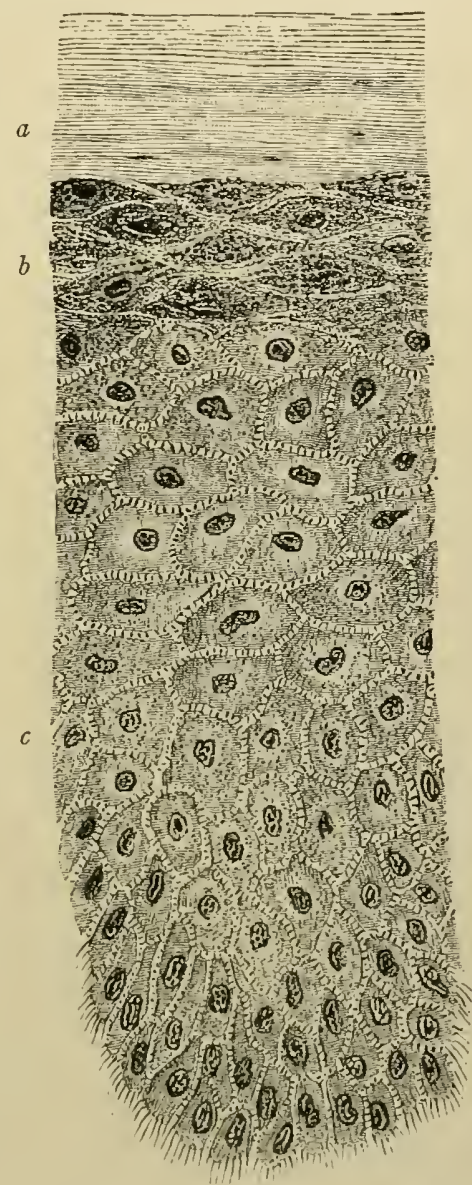

FIs: 266.-From Vertical Section through 'Thick Skin. (MerkelHenle.) $a$, Stratum luciclum; $b$, stratum uranulosum; $c$, stratum germinitivum, showing intercellular bridges. transparency of the cells is due to the presence of a substance known as elcidin, and derived from the keratohyaline granules of the stratum granulosum (Fig. 266, a).

(4) The stratum corneum varies grcatly in thickness, reaching its greatest development in the skin of the palms and soles. The cells 
are flattened and horny, especially near the surface. Some appear homogeneous, others have a lamellated appearance. They contain pareleidin, a derivative of the eleidin of the stratum lucidum. Nuclei are lost, but in many of the cells can be seen the spaces which the nuclei once occupied. Constant desquamation of these cells goes on, cells from the deeper layers taking their place.

The color of the skin in the white races is due to pigmentation of the deeper layers of the epidermis. In certain parts of the body pigmentation of the connective-tissue cells of the derma also occurs. In the dark races all cells of the epidermis are pigmented, although there is less pigment in the surface cells than in the cells more deeply situated.

Two kinds of glands occur in the skin-sebaceous glands and sweat glands.

Sebaceous Glands.- - These are usually associated with the hair follicles, and will be described in that connection. Sebaceous glands unconnected with hair occur along the margin of the lips, in the glans and prepuce of the penis, and in the labia minora.

SwEAT Glands (glandula sudoriparæ.)-These are found throughout the entire skin with the exception of the margin of the lips, the inner surface of the prepuce, and the glans penis. They are simple coiled tubular glands. The coiled portion of the gland usually lies in the subcutis, although it may lie wholly or partly in the deeper portion of the pars reticularis. The excretory duct runs a quite straight course through the derma, and enters the epidermis in one of the depressions between the papillæ. In the epidermis the duct takes a spiral course to the surface, where it opens into a minute pit just visible to the naked eye - the sweat pore (Fig. 264, p.). The coiled portion of the gland is lined with a simple cuboidal epithelium, having a granular protoplasm. In the smaller glands the epithelium rests directly upon the basement membrane. In the larger glands a longitudinal layer of smooth muscle cells separates the glandular epithelium from the basement membrane. The walls of the ducts consist of two or three layers of cuboidal epithelial cells, resting upon a delicate basement membrane, outside of which are longitudinally disposed connective-tissue fibres. On reaching the horny layer the epithelial wall of the duct ceases, the duct consisting of a mere channel through the epithelium (Fig. 264).

\section{TECHNIC}

(I) Fix the volar half of a finger-tip in formalin-Müller's fluid (technic 5, p. 7) or in absolute alcohol. Curling may be prevented by pinning to a piece of 
cork. Sections are cut transversely to the ridges, stained with hæmatoxylin-picroacid-fuchsin (technic 3, p. 2r), and mounted in balsam. Thick sections should be cut for the study of the coil glands with their ducts; thin sections for cellular details of the layers.

(2) Prepare in the same manner and for contrast with the preceding, sections of thin skin from almost any part of the body.

(3) Prepare a piece of negro skin in the same manner and note the position of the pigment.

\section{The Nails}

The nails are modified epidermis. Each nail consists of: $(a)$ a body, the attached uncovered portion of the nail; $(b)$ a free edge, the anterior unattached extension of the body; $(c)$ the nail root, the posterior part of the nail which lies under the skin (Fig. 267).

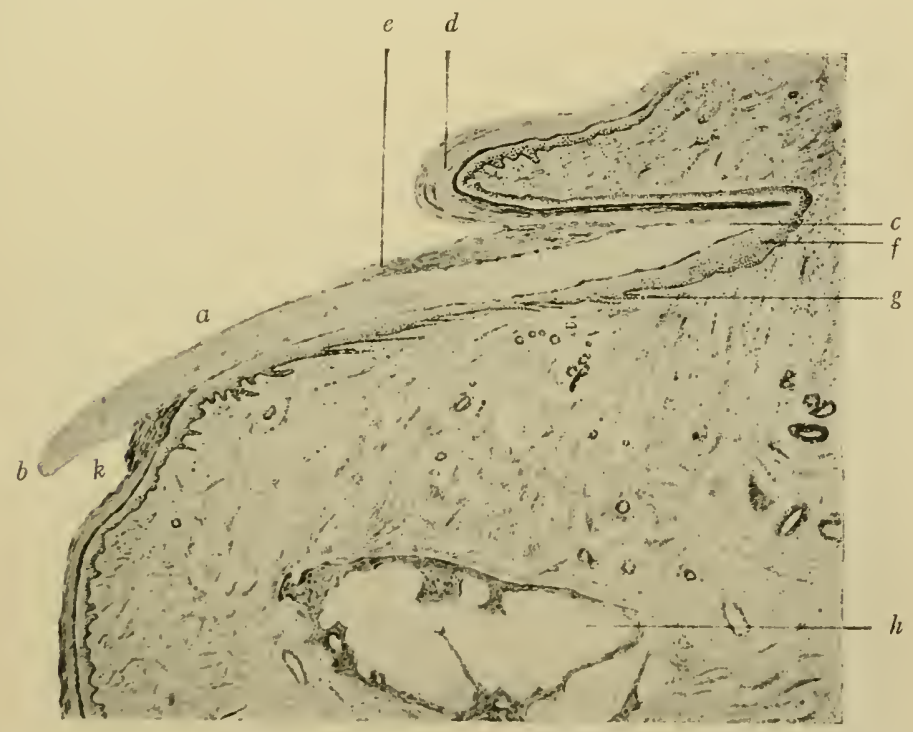

FIc. 267.-I ongitudinal Section through Root of IIuman Nail and Nail Bed. $X_{\text {Io. }}$ (Schaper.) $a$, Body of nail; $b$, free edge; $c$, root of nail; $d$, epidermis; $c$, eponychium; $f$, stratum germinativum of nail; $g$, folds in derma of nail bed; $h$, bone of finger; $k$, hyponychium.

The nail lies upon a specially modified portion of the corium, the nail bed, which beneath the nail root and somewhat forward of the root is known as the matrix. The nail bed is bounded on either side by folds of skin, the nail wall, while between the nail wall and the nail bed is a furrow, the nail groove (Fig. 268).

The nail bed consists of corium. Its connective-tissue fibres are arranged partly horizontal to the long axis of the nail, partly in a 
vertical plane extending from the periosteum to the nail. Papillæ are not present, but in their place are minute longitudinal ridges, which begin at the matrix and, increasing in height as they pass for-

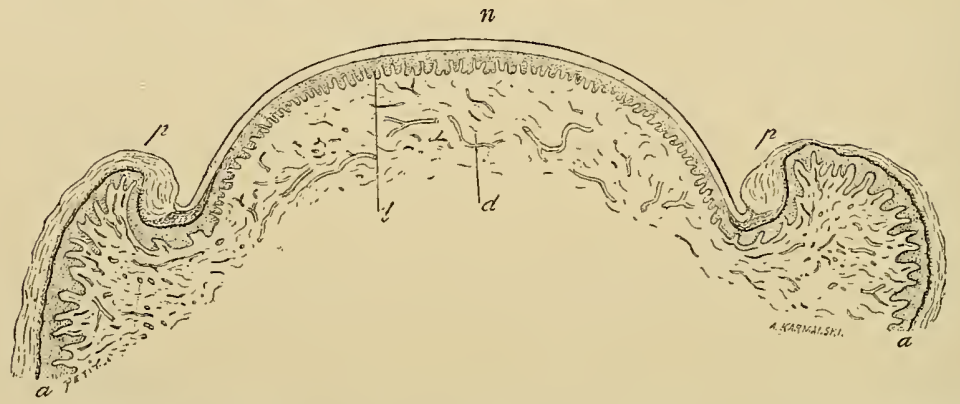

FIG. 268.-Transverse Section of Nail and Nail Bed. (Rannie.) $n$, Nail; $a$, epidermis; ${ }_{\varepsilon} p$, nail wall, to inner side of which is the nail groove; $l$, folds of derma; $d$, nailbed.

ward, terminate abruptly at the end of the nail bed, beyond which are the usual papillæ of the derma.

The nail itself consists of two parts ${ }^{1}$-an outer harder part or

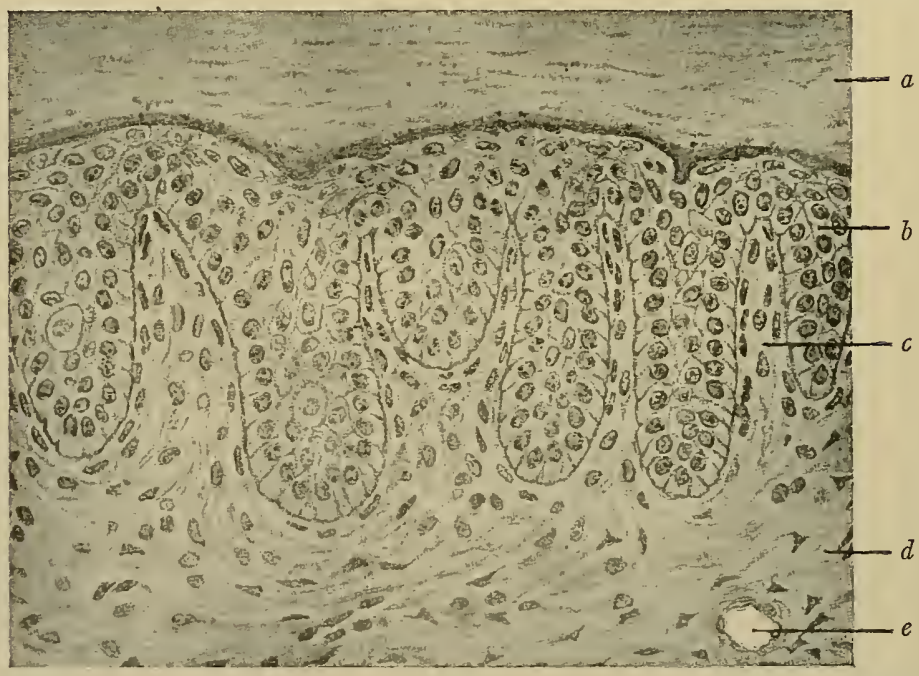

FIG. 269.-Vertical Transverse Section through Nail Body. $\times_{2} 80 . \quad$ (Szymonowicz.) $a$, Nail; $b$, stratum germinativum; $c$, ridge of nail bed; $d$, derma; $e$, blood-vessel.

true nail, and an under softer part. The outer portion is hard and horny, is developed from the stratum lucidum, and consists of several layers of clear, flat, nucleated cells. These layers overlap in such a

${ }^{1}$ Another division of nail and nail bed considers the nail as composed of the hard part only, the soft stratum germinativum being considered a part of the nail bed. 
manner that each layer extends a little farther forward than the layer above. The under softer portion of the nail corresponds to the stratum germinativum of the skin and, like the latter, consists of polygonal "prickle" cells and a stratum cylindricum resting upon a basement membrane. In the matrix where the process of nail formation is going on, this layer is thicker than elsewhere and is white and opaque from the presence of keratohyalin. The convex anterior margin of this area can be seen with the naked eye and is known as the Iunula.

At the junction of nail and skin, in the nail groove, the stratum corneum extends somewhat over the nail as its eponychium. A similar extension of the stratum corneum occurs on the under surface of the nail where the nail becomes free from the nail bed. This is known as the hyponychium (Fig. 267).

Growth of nail takes place by a transformation of the cells of the matrix into true nail cells. In this process the outer hard layer is pushed forward over the stratum germinativum, the latter remaining always in the same position.

\section{TECHNIC}

(I) Remove two or more distal phalanges from the fingers of a new-born child and fix in absolute alcohol or in formalin-Müller's fluid (technic 5, p. 7). After fixing, the bone should be carefully removed. Both longitudinal and transverse sections are made, stained with hæmatoxylin-picro-acid-fuchsin (technic 3, p. 21), and mounted in balsam. In cutting the sections it is usually best so to place the block that the knife passes through volar surface first, through nail last.

(2) The cellular elements of nail do not show well in sections. For demonstrating the nail cells, boil a piece of nail in concentrated potash lye or warm it in strong sulphuric acid, scrape off cells from the softened surface, and mount in glycerin.

\section{The Hair}

The hair, like the nail, is a development of the epidermis. The hair itself consists of a shaft, that portion of the hair which projects above the skin, and a root, that portion embedded within the skin. At its lower end the root presents a knob-like expansion, the hair bulb, in the under surface of which is a cup-like depression, which receives an extension of corium. This is known as the papilla. Enclosing the hair root is the hair follicle.

THE HAIR.-This is composed of epithelial cells arranged in three layers, which from within outward are medulla, cortex, and cuticle (Fig. 27I).

(I) The medulla occupies the central axis of the hair. It is absent 
in small hairs, and in the large hairs does not extend throughout their entire length. It is from 16 to $20 \mu$ in diameter, and consists of from two to four layers of polygonal or cuboidal cells with finely granular, usually pigmented protoplasm and rudimentary nuclei.

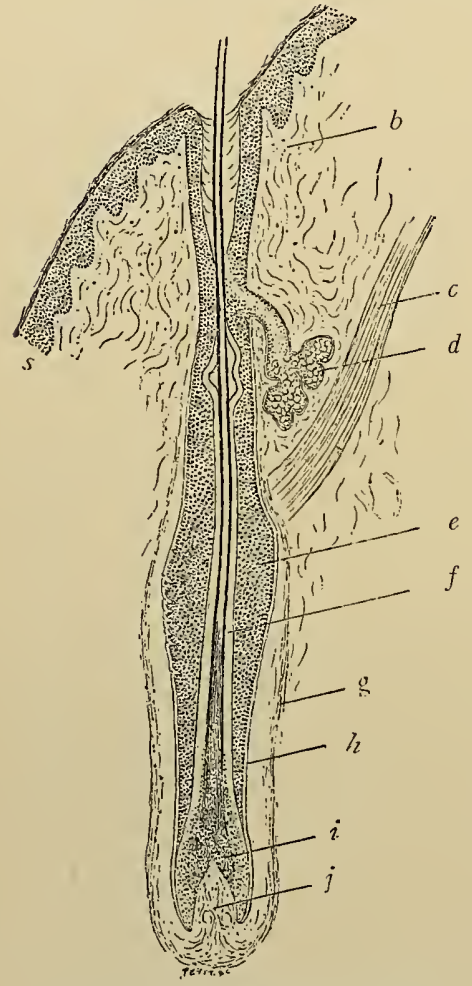

FiG. 270.-Longitudinal Section of Hair and its Follicle from Vertical Section of Scalp. (Ranvier.) a, Shaft of hair; $b$, derma; $c$, arrector pili muscle; $d$, sebaceous gland; $e$, outer root sheath; $f$, inner root sheath; $g$, connective-ti sule follicle; $h$, vitreous membrane; $i$, hair bulb; $j$, papilla; s, epidermis.

(2) The cortex makes up the main bulk of the hair and consists of several layers of long spindle-shaped cells, the protoplasm of which shows distinct longitudinal striations, while the nuclei appear atrophied. As these striations give the hair the appearance of being composed of fibrillæ, the term "cortical fibres" has been applied to them. In colored hair, pigment granules and pigment in solution are found in and between the cells of this layer. This pigment determines the color of the hair. In the root the cortical cells are less flattened than in the shaft.

(3) The cuticle has a thickness of about $\mathrm{I} \mu$, and consists of clear scalelike, non-nucleated epithelial cells. These overlap one another like shingles on a roof, giving to the surface of the hair a serrated appearance (Fig. 27I).

The HaIr Follicle.-This is also a modification of the skin. In the formation of the follicles of the finer (lanugo) hairs the epidermis alone is concerned. The follicles of the larger hairs contain both epidermal and dermal elements. The latter form the connective-tissue follicle, while the epidermis forms the root sheaths.

(I) The root sheath consists of two sub-layers-the inner root sheath and the outer root sheath (Figs. 272, 273 and 274).

(a) The inner root sheath consists of three layers, which from within outward are the cuticle of the root sheath, Huxley's layer, and Henle's layer. 
The cuticle of the root sheath lies against the cuticle of the hair and is similar to the latter in structure. It consists of thin scale-like overlapping cells, nucleated in the deeper parts of the sheath, nonnucleated nearer the surface (Figs. 272, 273 and 274, c).

Huxley's layer lies immediately outside the cuticle of the root sheath, constituting the middle layer of the inner root sheath. It consists of about two rows of elongated cells with slightly granular protoplasm containing eleidin. In the deeper portion of the root these cells contain nuclei. Nearer the surface the nuclei are rudimentary or absent (Figs. 272, 273 and 274, d).

Henle's layer is a single row of clear flat cells. In the bulb these cells may contain nuclei; elsewhere they are nonnucleated (Fig. 274,e).

(b) The outer root sheath is derived from the stratum germinativum to which it corresponds in structure, Next to the vitreous membrane is a single layer of columnar cells (stratum cylindricum). Inside of this are several layers of "prickle" cells (Figs. 272, 273 and $274, f)$.

(2) The connective-tissue follicle consists of three layers-an inner vitreous membrane, a middle vascular layer, and

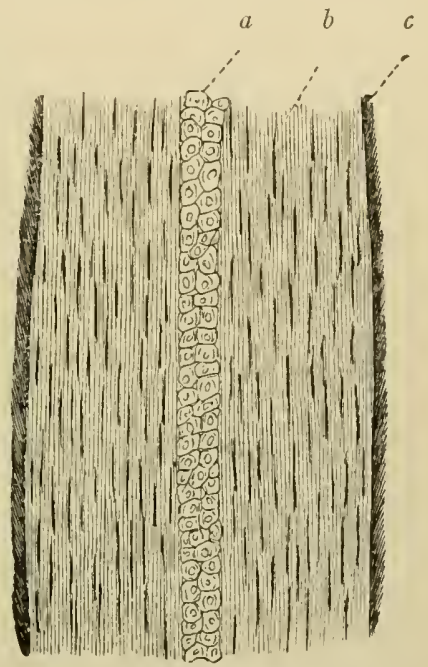

FIG. 271.-Longitudinal Section of Hair. $\times 35 \mathrm{c}$. (Kölliker.) $a$, Medulla; $b$, cortex; $c$, cuticle. an outer fibrous layer.

(a) The vitreous or hyaline membrane is a thin homogeneous structure of the nature of an elastic membrane. It lies next to the outer root sheath and corresponds to the basement membrane of the derma (Figs. 272, 273 and $274, g$ ).

(b) The middle or vascular layer is composed of fine connectivetissue-fibres, the general arrangement of which is circular. Cellular elements are quite abundant, while elastic fibres are, as a rule, alsent. As its name would indicate, this layer is especially rich in bloodvessels (Figs. 272, 273 and 274, i).

(c) 'The outer or fibrous layer consists of rather coarse, loosely woven bundles of white fibres, which run mainly in a longitudinal direction. Among these are elastic fibres and a few connective-tissue cells. 
In the deeper portion of the root, some little distance above the bulb, all the layers of the hair and its follicle can be distinctly seen. The differentiation of the layers becomes less marked as one passes in either direction. At about the level of the entrance of the ducts of the sebaceous glands (see p. 39I) the inner root sheath disappears, and the outer root sheath passes over into the stratum germinativum of the skin, while between the outer root sheath (now stratum germi-

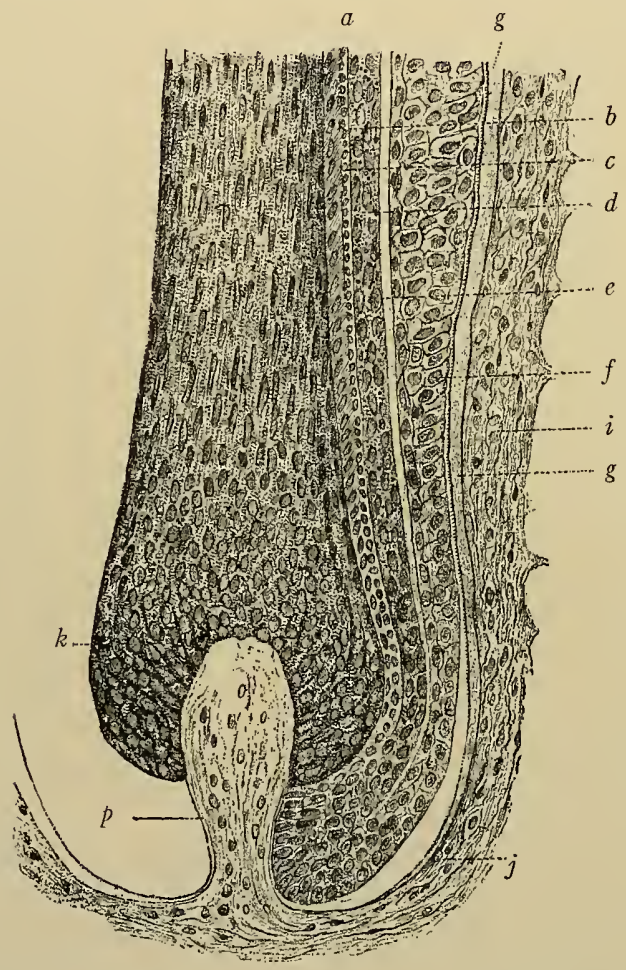

FIG. 272.-Longitudinal Section of Lower End of Root of Hair, including Papilla. (Kölliker.) $a$, Root of hair; $b$, cuticle of hair; $c$, cuticle of root sheath; $d$, Huxley's layer of inner root sheath; $e$, Henle's layer of inner root sheath; $f$, outer root sheath; $g$, vitreous membrane; $j$, connective-tissue follicle; $k$, bulb of hair; $p$, papilla.

nativum) and the hair are interposed the outer layers of the skin, stratum granulosum and stratum lucidum when present, and stratum corneum. All of these are continuous with the same layers of the skin. In the region of the bulb the outer root sheath first becomes thinner, then disappears, while the layers of the inner root sheath retain their identity until the neck of the papilla is reached, at which point the different layers coalesce. 
The arrector pili muscle (Fig. 270, c) is a narrow band, or bands, of smooth muscle connected with the hair follicle. It arises from the outer layer of the derma on the side toward which the hair slants, and is inserted into the wall of the follicle at the junction of its middle and lower thirds, the sebaceous gland being usually included between the muscle and the hair (see below). The contraction of the muscle thus tends to straighten the hair and to compress the gland.

The sebaceous glands are with few exceptions connected with the hair follicles. They are simple or branched alveolar glands. The

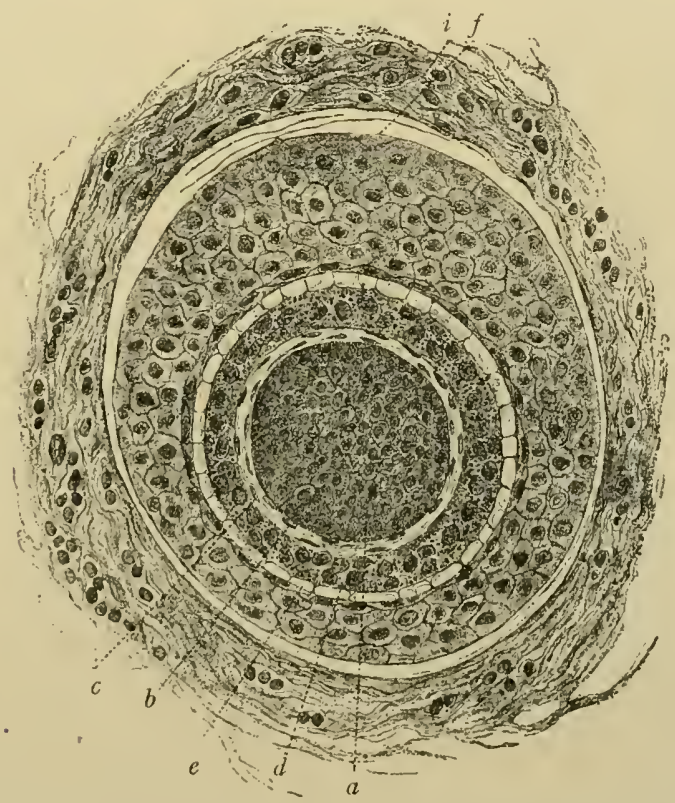

FIG. 273.-Transverse Section through Root of Hair and Hair Follicle. (Kölliker.) $a$, Hair; $b$, hair cuticle; $c$, cuticle of root sheath; $d$, Huxley's layer; $e$, Henle's layer; $f$, outer root sheath; $i$, connective-tissue follicle.

size of the gland bears no relation to the size of the hair, the largest glands being frequently connected with the smallest hairs. The glands are spherical or oval in shape and each gland is enclosed by a connective-tissue capsule derived from the follicle or from the derma. Beneath the capsule is a basement membrane continuous with the vitreous membrane of the follicle. The wide excretory duct empties in to the upper third of the follicle and is lined with stratified squamous epithelium continuous with the outer root sheath and stratum germinativum. The lower end of the duct opens into several simple or 
branched alveoli, at the mouths of which the epithelium becomes reduced to a single layer of cuboidal cells. In the alveoli themselves the cells are spheroidal or polyhedral, and usually fill the entire alveolus. These cells, like those lining the duct, are derivatives of

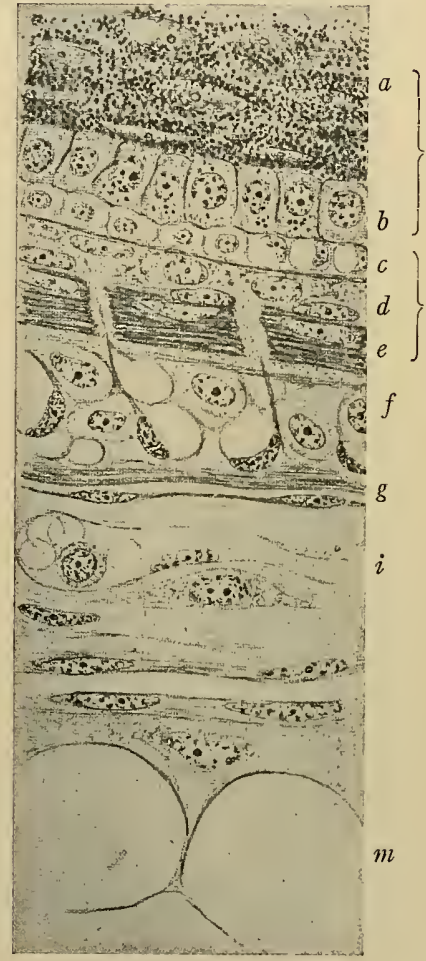

FIG. 274.-From Longitudinal Section through Hair and Hair Follicle. Enlarged to 800 diameters. (Schäfer.) $A$, Hair. $a$, Cortex of hair; $b$, cuticle of hair. $B$, Inner sheath. $c$, Cuticle of root sheath; $d$, Huxley's layer; $e$, Henle's layer; $f$, outer root sheath; $g$, vitreous membrane; $i$, connective-tissue follicle; $m$, fat cells.

the outer root sheath. The secretion of the gland-an oily substance called sebum-appears to be the direct product of disintegration of the alveolar ${ }^{A}$ cells, which can usually be seen in all stages of the process of transformation of the cell into the secretion of the $B$ gland. The most peripheral cells show the least secretory changes, containing a few small fat droplets. The central cells and those in the lumen of the duct show the most marked changes, their protoplasm being almost wholly converted into fat, their nuclei shrunken or disintegrated or lost. In the middle zone are cells showing intermediary stages in the process.

Shedding of hair occurs in most mammalia at regularly recurring periods. In man there is a constant death and replacement of hair. In a hair about to be shed, the bulb becomes cornified and splits up into a number of fibres. The hair next becomes detached from the papilla and from the root sheath and is cast off, the empty root sheaths collapsing and forming a cord of cells between the papilla and lower end of the shedding hair. If the dead hair is to be replaced by a new one, there sooner or later occurs a proliferation of the cells of the outer root sheath in the region of the old papilla. From this "hair germ" the new hair is formed in a manner similar to embryonal hair formation, the new hair growing upward under or to one side of the dead hair, which it finally replaces.

As to the manner in which growth of hair takes place, two views are held. According to one of these, the hair, cuticle, and inner root sheath are replenished by proliferation of the epithelial cells surrounding the papilla. These parts thus grow from below toward the surface. The oldest cells of the outer rootsheath, on the other hand, lie against the vitreous membrane, so that growth of 
this sheath takes place from without inward. According to the second view, the various parts of the hair and its follicle are direct derivatives of the different layers of the skin, and their growth takes place by a continuous process of invagination. Thus the most peripheral cells of the outer root-sheath-stratum cylindricum-pass over the papilla and turn upward to form the medulla of the hair; the deeper cells-stratum spinosum-of the outer root-sheath become continuous with the cortex of the hair; the stratum lucidum, with the sheath of Henle, which turns up on the hair as its cuticle; Huxley's layer, with the cuticle of the inner root-sheath. According to this view growth of hair is accomplished by continuous growth downward from the surface, and turning up into the hair, of these layers.

\section{TECHNIC}

Pin out small pieces of human scalp on cork and fix in absolute alcohol or in formalin-MI üller's fluid (technic 5, p. 7). From one block cut sections perpendicular to the surface of the scalp and in the long axes of the hair and follicles. From a second block cut sections at right angles to the hair follicles, i.e., not quite parallel to the surface of the scalp but a little obliquely. By this means not only are transverse sections secured, but if the block be sufficiently long the follicles are cut through at all levels. Sections are stained with hæmatoxylinpicro-acid-fuchsin (technic 3, p. 2I) and mounted in balsam.

Blood-vessels of the skin. From the larger arteries in the subcutaneous tissue branches penetrate the pars reticularis of the derma, where they anastomose to form cutaneous networks. The latter give off branches, which pass to the papillary layer of the derma and there form a second series of networks, the subpapillary, just beneath the papillæ. From the cutaneous networks arise two sets of capillaries, one supplying the fat lubules, the other supplying the region of the sweat glands. From the subpapillary networks are given off small arteries which break up into capillary networks for the supply of the papillæ, scbaceous glands, and hair follicles. The return blood from these capillaries first enters a horizontal plexus of veins just under the papillax. 'This communicates with a second plexus just beneath the first. Small veins from this second plexus pass alongside the arteries of the decper part of the corium, where they form a third plexus with larger, more irregular meshes. Into this plexus pass most of the veins from the fat lobules and sweat glands, although one or two small veins from the sweat glands usually follow the duct and empty into the subpapillary plexus. The blood next passes into a fourth plexus in the subcutaneous tissue, from which arise veins of considerable size. These accompany the arteries. 
Small arteries from the plexuses of the skin and subcutis pass to the hair follicle. The larger arterioles run longitudinally in the outer layer of the follicle. From these are given off branches which form a rich plexus of small arterioles and capillaries in the vascular layer of the follicle. Capillaries from this plexus also pass to the sebaceous glands, the arrectores pilorum muscles, and the papillæ.

The lymphatics of the skin. These begin as clefts in the papillæ, which open into a horizontal network of lymph capillaries in the pars papillaris. This communicates with a network of larger lymph capillaries with wider meshes in the subcutaneous tissue. The latter also receives lymph capillaries from plexuses which surround the sebaceous glands, the sweat glands, and the hair follicles.

The nerves of the skin. These are mainly sensory. Efferent sympathetic axones supply the smooth muscle of the walls of the bloodvessels, the arrectores pilorum, and secretory fibres to the sweat glands. The medullated sensory nerves are peripheralprocesses of spinal ganglion cells. The larger trunks lie in the subcutis, giving off branches which pass to the corium, where they form a rich subpapillary plexus of both medullated and non-medullated fibres. From the subcutaneous nerve trunks and from the subpapillary plexus are given off fibres which terminate in more or less elaborate special nerve endings (see page 430). Their location is as follows: (I) In the subcutis: Vater-Pacinian corpuscles, the corpuscles of Ruffini, and the GolgiMazzoni corpuscles of the finger-tip. The first two forms are most numerous in the palms and soles.

(2) In the derma: Tactile corpuscles of Meissner and Wagner. These are found in the papillæ, especially of the finger tip, palm, and sole. Krause's end bulbs-usually in the derma just beneath the papillæ, more rarely in the papillæ themselves. (3) In the epithelium: Free nerve endings among the epithelial cells.

Branches of the cutaneous nerves supply the hair follicles. As a rule but one nerve passes to each follicle, entering it just below the entrance of the duct of the sebaceous gland. As it enters the follicle the nerve fibre loses its medullary sheath and divides into two branches, which further subdivide to form a ring-like plexus of fine fibres encircling the follicle. From this ring, small varicose fibrils run for a short distance up the follicle, terminating mainly in slight expansions on the vitreous membrane. 


\section{TECHNIC}

For the study of the blood-vessels of the skin inject (technic, p. 25) the entire hand or foot of a new-born child. Examine rather thick sections either mounted unstained or stained only with cosin.

\section{Development of the Skin, Nails, and Hair}

The epidermis is, as already noted, of ectodermic origin. It consists at first of a single layer of cuboidal cells. This soon differentiates into two layers-an outer, the future stratun corneum, and an inner, the future stratum germinativum. The stratum granulosum and stratum lucidum are special developments of the stratum germinativum. The corium is of mesoblastic origin. It is at first smooth, the papillæ being a secondary development.

The nail first appears as a thickening of the stratum lucidum. This spreads until the future nail bed is completely covered. During development the stratum corneum extends completely over the nail as its eponychium. During the ninth month (intra-uterine) the nail begins to grow forward free from its bed and the eponychium disappears, except as already noted.

The hair also develops from ectoderm. It first appears about the end of the third fœtal month as a small local thickening of the epidermis. This thickening is due mainly to proliferation of the cells of the stratum mucosum, and soon pushes its way down into the underlying corium, forming a long slender cord of cells-the hair germ. Differentiation of the surrounding connective tissue of the corium forms the follicle wall, while an invagination of this connective tissue into the lower end of the hair germ forms the papilla. The cells of the hair germ now differentiate into two layers: a central core the middle portion of which forms the hair, while the peripheral portion forms the inner root sheath; and an outer layer which becomes the outer root sheath. The sublayers are formed from these by subsequent differentiation. The hair when first formed lies wholly beneath the surface of the skin. As the hair reaches the surface its pointed extremity pierces the surface epithelium to become the hair shaft (Fig. 275).

The sebaccous gland develops as an outgrowth from the outer root sheath. This is a flask-shaped and at first solid mass of cells, which later differentiate to form the ducts and alveoli of the gland.

The sweat glands first appear as solid ingrowths of the stratum germinativum into the underlying corium. 'The lower end of the ingrowth becomes thickened and convoluted to form the coiled portion of the gland, and somewhat later the central portion becomes channelled out to form the lumen. 'The muscle tissue of the sweat glands, which lies between the epithelium and the basement membrane, is the only muscle of the body derived from the ectoderm.

\section{The Mammary Gland}

The mammary gland is a compound alveolar gland. It consists of from fifteen to twenty lobes, each of which is subdivided into lobules. The gland is surrounded by a layer of connective tissue 


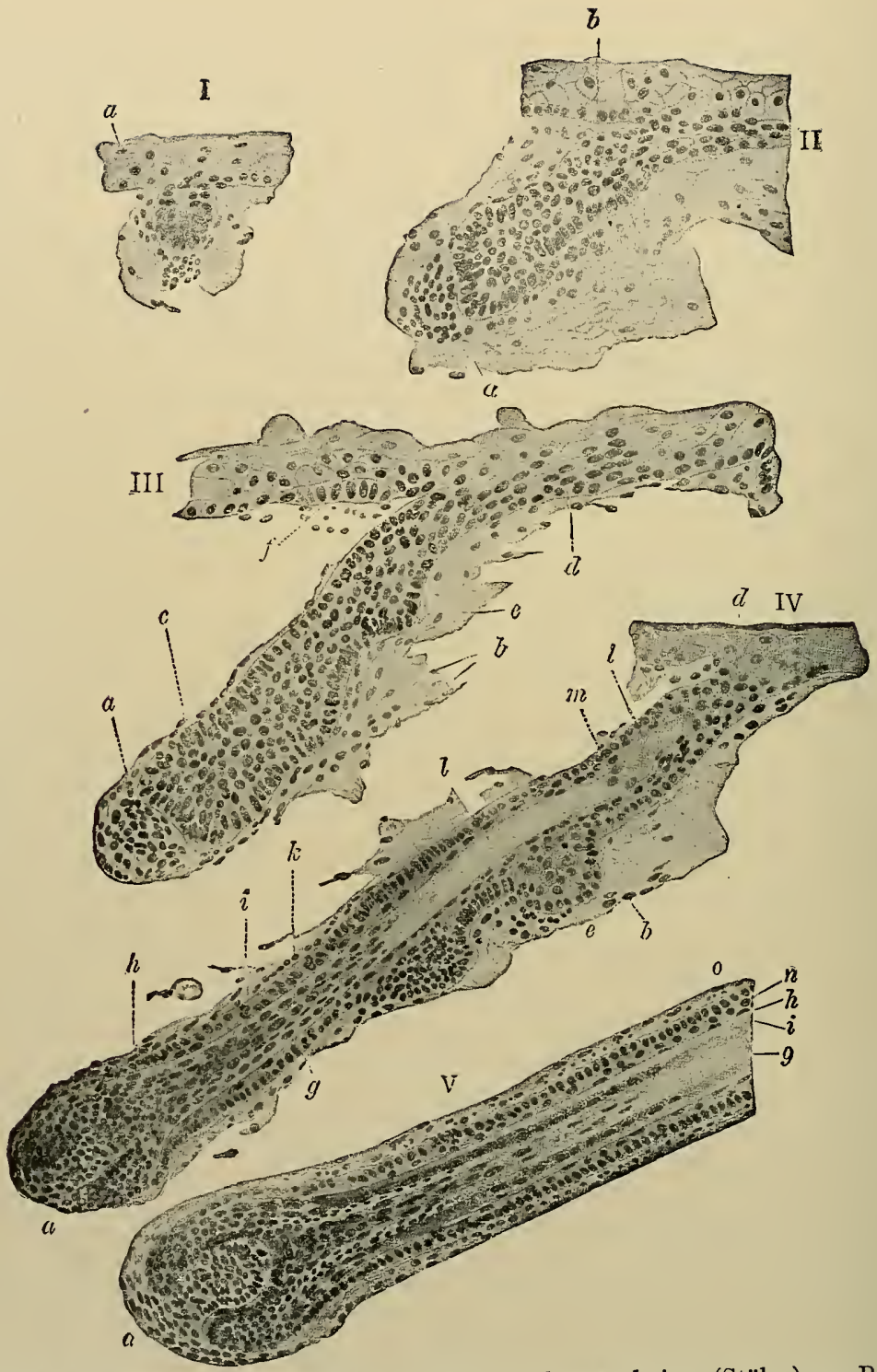

FIG. 275--Five stages in the development of a human hair. (Stöhr.) a, Papilla; $b$, arrector pili muscle; $c$, beginning of hair shaft; $d$, point where hair shaft grows through epidermis; $e$, anlage of sebaceous gland; $f$, hair germ; $g$, hair shaft; $h$, Henle's layer; $i$, Huxley's layer; $k$, cuticle of root sheath; $l$, inner root sheat; $m$, outer root sheath in tangential section; $n$, outer root sheath; $o$, connective-tissue follicle. 
containing more or less fat. From this periglandular connective tissue broad septa extend into the gland, separating the lobes (interlobar septa). From the latter finer connective-tissue bands pass in between the lobules (interlobular septa). From the interlobular septa strands of connective tissue extend into the lobule where they act as support for the glandular structures proper. An excretory duct passes to each lobe where it divides into a number of smaller ducts (lobular ducts), one of which runs to each lobule. Within the latter

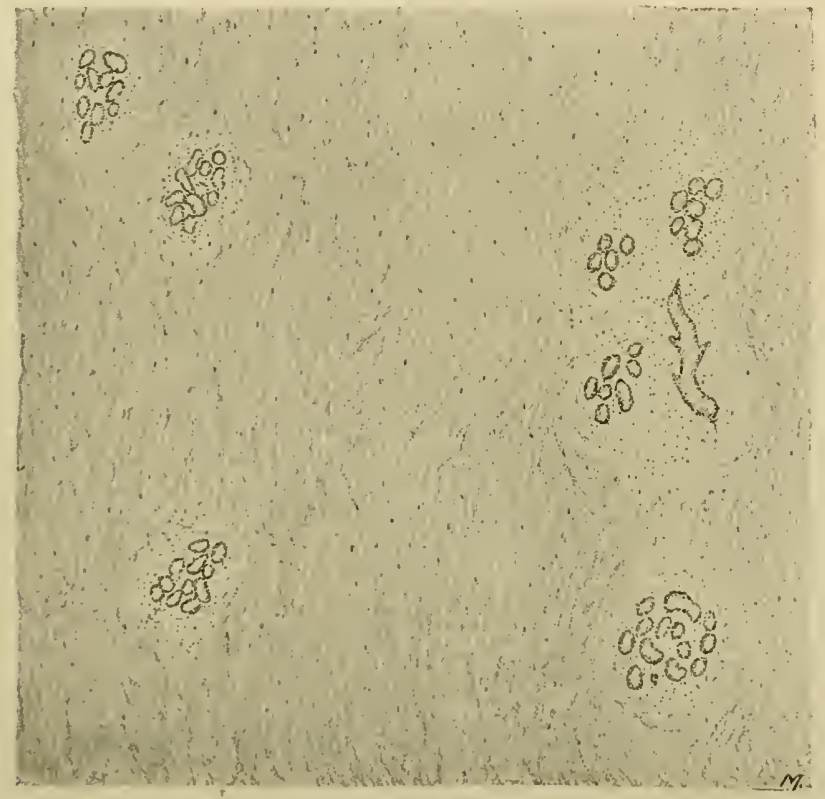

FIG. 276.-From Section of Human Inactive Mammary Gland. $\times 25$. (Technic I, p. 4or.) Gland composed almost wholly of connective tissue; few scattered groups of tubules.

the lobular duct breaks up into a number of terminal ducts, which in turn open into groups of alveoli. The fifteen to twenty main excretory ducts pass through the nipple and open on its surface. At the base of the nipple each main duct presents a sac-like dilatation, the ampulla, which appears to act as a reservoir for the storage of the milk.

Until puberty the gland continues to develop alike in both sexes, but after about the twelf th year the male gland undergoes retrogressive changes, while the female gland continues its development.

THE INACTIVE MAMMARY GLAND, by which is meant the female 
gland up to the advent of the first pregnancy and between periods of lactation, consists mainly of connective tissue and a few scattered groups of excretory ducts (Fig. 276). Around the ends of some of the ducts are small groups of collapsed alveoli. Both ducts and alveoli are lined with a low columnar, often rather flat epithelium. In some cases the flat cells are two or three layers thick, forming a thin stratified squamous epithelium. The relative amount of fat and connective tissue varies greatly, some inactive mammæ consisting almost wholly of fat tissue.

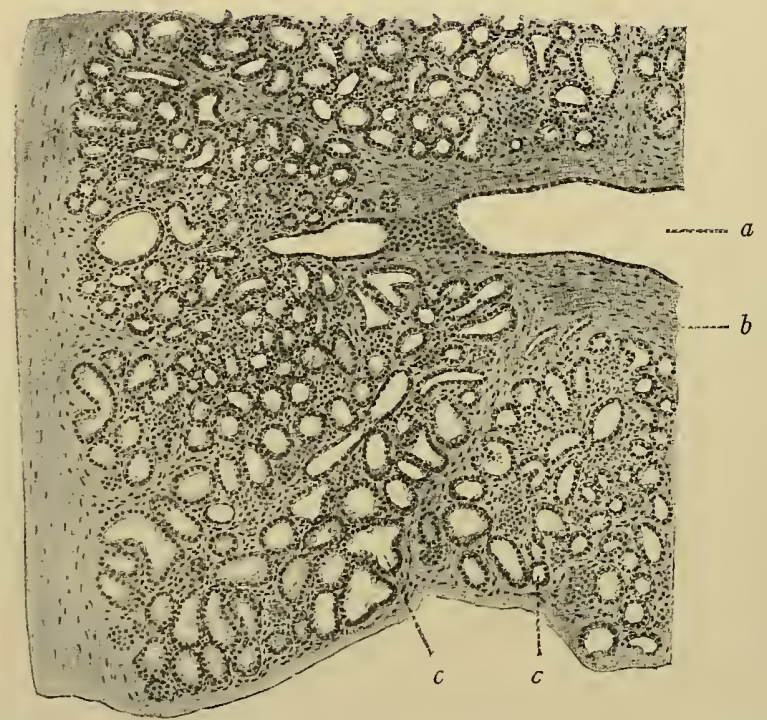

FIG. 277.-From Section of Human Mammary Gland during Lactation. $\times 50$.

(Stöhr.) $a$, Branch of excretory duct; $b$, interlobular connective tissue; $c$, alveoli.

The Active Mammary Gland.-Throughout pregnancy the gland undergoes extensive developmental changes and becomes functional at about the time of birth of the child. The microscopic appearance of the active gland differs greatly from that of the inactive (Fig. 277). There is a marked reduction in the connective tissue of the gland, its place being taken by newly developed ducts and alveoli. The alveoli are spheroidal, oval, or irregular in shape, and vary considerably in size. The alveoli are lined by a single layer of low columnar or cuboidal epithelial cells which rest upon a homogeneous basement membrane. The appearance of the cells differs according to their secretory conditions. The resting cell is cuboidal and its 
protoplasm granular. With the onset of secretion the cell elongates, and a number of minute fat droplets appear. These unite to form one or two large globules of fat in the free end of the cell. The fat is next discharged into the lumen of the alveolus, and regeneration of the cell takes place from the unchanged basal portion (Fig. 279). As to the number of times a cell is able to go through this process of secretion and repair before it must be replaced by a new cell, nothing definite is known. Active secretion does not as a rule take place in all the alveoli of a lobule at the same time. Each lobule

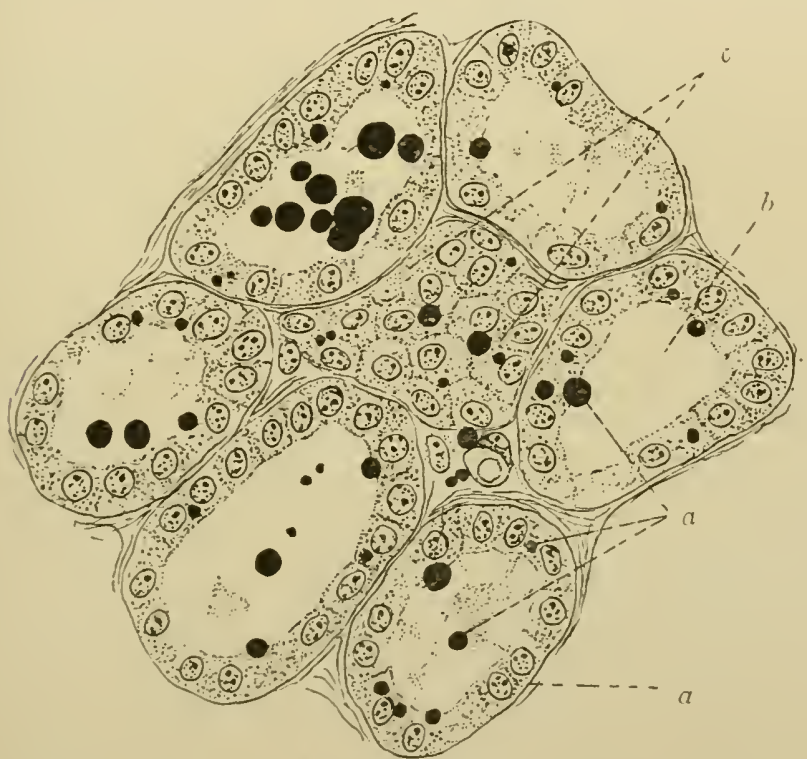

FIG. 278.-From Section, of Mammary Gland of Guinea-pig during Lactation. $\times$ 500. (Osmic acid.) (Szymonowicz.) a, Basement membrane; $b$, lumen of alveolus; $c$, tangential section of alveolus; $d$, fat globules.

thus contains both active and inactive alveoli. The smallest ducts are lined with a low columnar or cuboidal epithelium. This increases in height with increase in the diameter of the duct until in the largest ducts the epithelium is of the high columnar type.

The secretion of the gland is milk. 'This consists microscopically of a clear fluid or plasma in which are suspended the milk globules. The latter are droplets of fat from 3 to $5 /$ in diameter, each enclosed in a thin albuminous membrane which prevents the droplets from coalescing. Cells, probably leucocytes, containing fat droplets may also be present. In the secretion of the gland during the later months of pregnancy, and also for a few days following the 
birth of the child, a relatively large number of large fat-containing leucocytes - colostrum corpuscles - are found.

Blood-vessels. - These enter the gland, branch and ramify in the interlobar and interlobular connective tissue, and finally terminate in capillary networks among the alveoli and ducts. From the capillaries arise veins which accompany the arteries.

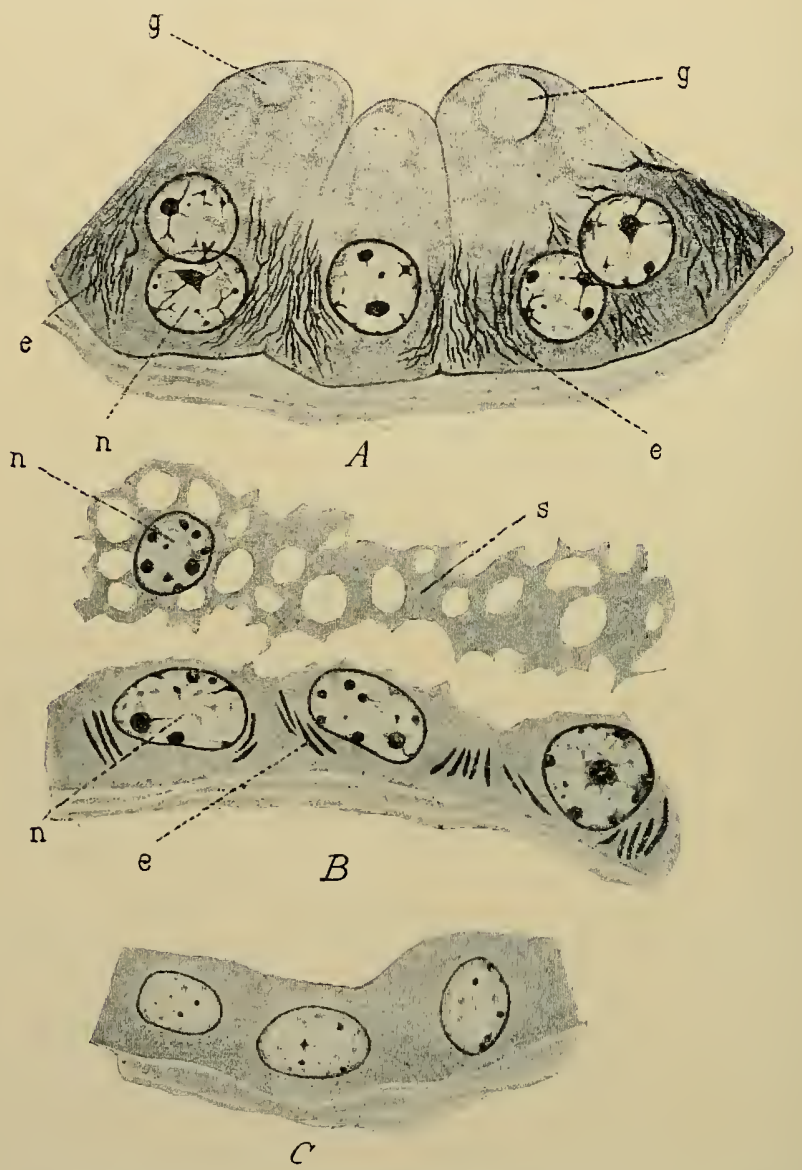

FIG. 279.- $A$, Mammary gland cells-secreting stage; $B$, same-excreting stage; the secretion having separated from the cells; $C$, resting stage; $n=$ nucleus. $l=$ ergastoplasm filaments. (Simon.)

Lymphatics.-Lymph capillaries form networks among the alveoli and terminal ducts. The lymph capillaries empty into larger lymphatics in the connective tissue. These in turn communicate with several lymph vessels which convey the lymph to the axillary glands.

Nerves.-Both cerebro-spinal and sympathetic nerves supply the 
gland, the larger trunks following the interlobar and interlobular connective-tissue septa. The nerve terminals break up into plexuses which surround the alvecli just cutside their basement membranes. From these plexuses delicate fibrils have been described passing through the basement membrane and ending between the secreting cells.

Detelopment.-The development of the mammary gland is quite similar to the development of the sebaceuus glands. The gland first appears as a dipping down of solid cord-like masses of cells from the stratum mucosum. The alveoli remain rudimentary until the advent of pregnancy. After lactation the alveoli atrophy, being replaced by connective tissue, and the gland returns to the resting state. After the menopause a permanent atrophy of the gland begins, fat and connective tissue ultimately almost wholly replacing the glandular elements.

\section{TECHNIC}

(I) Fix thin slices of an inactive mammary gland in formalin-Müller's fluid (technic 5, p. 7). Stain sections with hæmatoxylin-eosin (technic I, p. 20), and mount in balsam.

(2) Prepare sections of an active mammary gland, as in preceding technic (I).

(3) Fix very thin small pieces of an active gland in one per cent. aqueous solution of osmic acid. After twenty-four hours wash in water and harden in graded alcohols. Thin sections may be mounted unstained, or after slight eosin stain, in glycerin.

\section{General References for Further Study}

Kölliker: Handbuch der Gewebelehre des Menschen.

McMurrick: Development of the Human body.

Ranvier: Traité Technique d'Histologie.

Schäfer: Essentials of Histology.

Spalteholz: Die Vertheilung der Blutgefässe in der Haut. Arch. Anat. u. Phys., Anat. Abth., 1893 . 


\section{CHAPTER XI}

\section{THE THYREOID AND PARATHYREOID, THE PITUITARY BODY, THE PARAGANGLIA AND THE ADRENAL.}

\section{The Thyreoid}

The thyreoid (Fig. 280) is a ductless structure built upon the general principle of a compound alveolar gland. There are usually two lateral lobes connected by a narrow band of glandular tissue, the "isthmus." Each lobe is surrounded by a connective-tissue capsule, from which septa pass into the lobe, subdividing it into

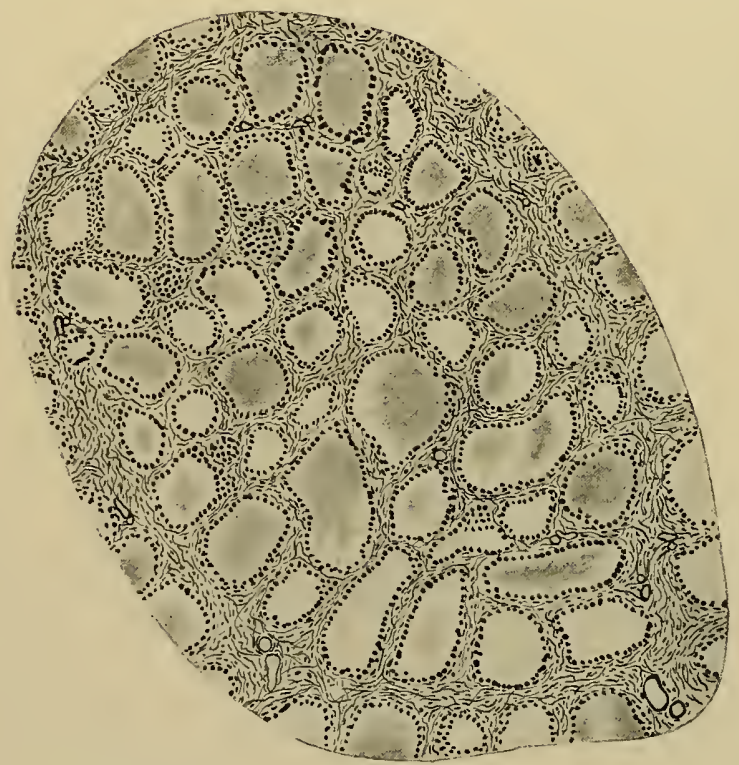

FIG. 280.--Section of Human Thyreoid. Most of the alveoli contain colloid.

lobules. From the perilobular connective tissue finer strands extend into the lobules, separating the alveoli. The latter are spherical, oval, or irregular in shape. They vary greatly in diameter (40 to $\mathrm{r} 2 \mathrm{O} \mu)$ and are as a rule non-communicating. At birth most of the alveoli are empty, but soon become more or less filled with a peculiar substance known as "colloid." The alveoli are lined with a single 
or double layer of cuboidal epithelial cells. Two types of cells are recognized.

One of these is actively secreting colloid and is known as a secreting or colloid cell. The other contains no colloid and is known as a resting or reserie cell. It is probable that their names indicate the relation of these cells to each other and that the reserve cell ultimately becomes a colloid-secreting cell.

The colloid cell appears in some cases simply to pour out its colloid secretion into the lumen, after which it may assume the character of a resting cell; in other cases the cell appears to be completely transformed into colloid, its place being taken by proliferation of the resting cells. In certain alveoli which are much distended with colloid the lining epithelium is flattened.

That the thyreoid exerts a decided influence upon general body metabolism is shown by the symptoms resulting from congenital absence of the gland (congenital myxœedema or cretinism) and by the effects of complete removal, the latter giving rise to a train of symptoms known as the "cachexia strumipriva."

The blood supply of the thyreoid is extremely rich, the vessels branching and anastomosing in the connective tissue and forming dense capillary networks around the alveoli.

Lymphatics accompany the blood-vessels in the connective tissue.

Nerves are mainly non-medullated fibres which form plexuses around the blood-vessels and in the connective tissue surrounding the alveoli. Terminals to the secreting cells end in club-like dilatations against the bases or between the epithelial cells. A few afferent medullated fibres are found in the plexuses surrounding the blood-vessels.

DEVELOPMENT. - The thyrcoid originates as a diverticulum from the entoderm of the primitive pharynx. It first appears in embryos of 3 to $5 \mathrm{~mm}$. and grows ventrally into the mesoderm of the ventral wall of the neck. Here it forms a mass which lies transversely across the neck. It is composed of solid cords of cells which become hollow to form the alveoli of the gland. At first the gland is connected with the surface by the thyreo-glossal duct. This either disappears entirely or is representerl in the adult by such rudimentary structures as the so-called prehyoid, suprahyoirl, and accessory thyreoirl glands. The gland at first consists of solicl cords of cells. Ingrowth of connective tissue divides these into groups or lolules, and at the sitme time breaks up the long tubules into short segments. Jilatation of the alveoli occurs with the formation of collorid. 


\section{The Parathyreoids}

These are small ductless glands which usually lie upon the posterior surface of the lateral lobes of the thyreoid. There are commonly two pairs, a superior and an inferior, on each side. The number is, however, subject to variation. Each gland is from 6 to $8 \mathrm{~mm}$. long, about half that in breadth, and $2 \mathrm{~mm}$. thick. Small groups of cells having the structure of the parathyreoids have been found below the thyreoid and within the thyreoid and thymus.

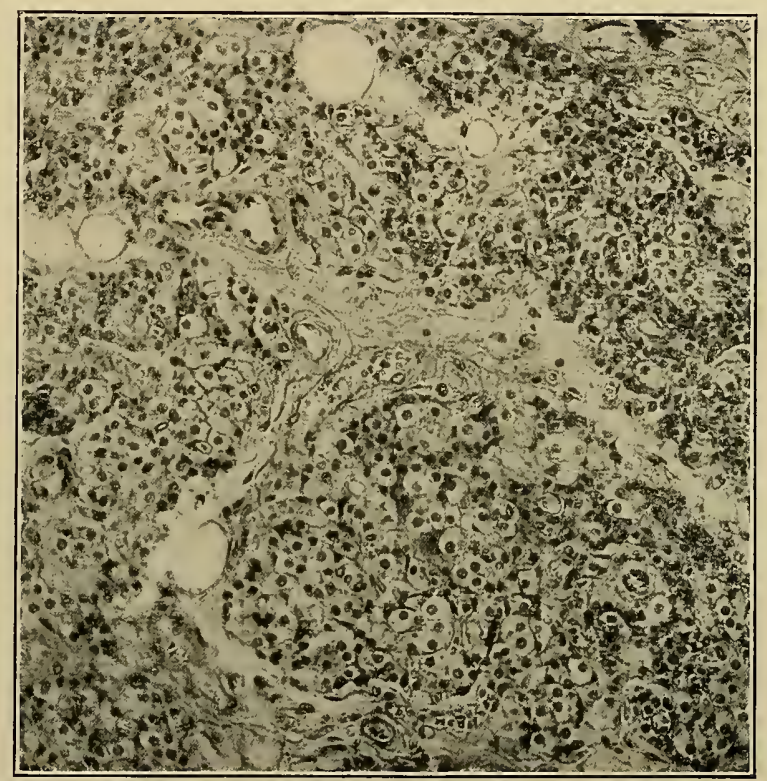

FIG. 28I.-Section of Human Parathyreoid; showing mainly "clear," "principal," or "chief" cells. (Pool.)

The parathyreoid is surrounded by a thin connective-tissue capsule which sends a variable amount of connective tissue into the gland as septa. When the amount is considerable the gland shows a subdivision into lobules. The stroma consists largely of reticular tissue and is very vascular. The number and arrangement of the cells vary. The gland may be almost wholly cellular with very little connective tissue, the groups of cells may be widely separated by interstitial tissue, or there may be any intermediate condition. The cells are arranged in irregular groups or cords (Figs. 28I, 282) sometimes around tubules, sometimes having a distinctly alveolar structure (Fig. 
283 ); in the latter case colloid may be present in the lumen. Colloid has also been found between, and colloid and glycogen within, the cells.

The cells themselves are spheroidal, cuboidal or pyramidal, with basal nuclei. The appearance of the cells varies sufficiently to have caused two or three types to be distinguished. All, however, probably represent different functional conditions of the same cell.

(I) Chief or clear cells (Fig. 28I). These are the more numerous. Their bodies are small and clear, and the cytoplasm does not stain readily. The nuclei

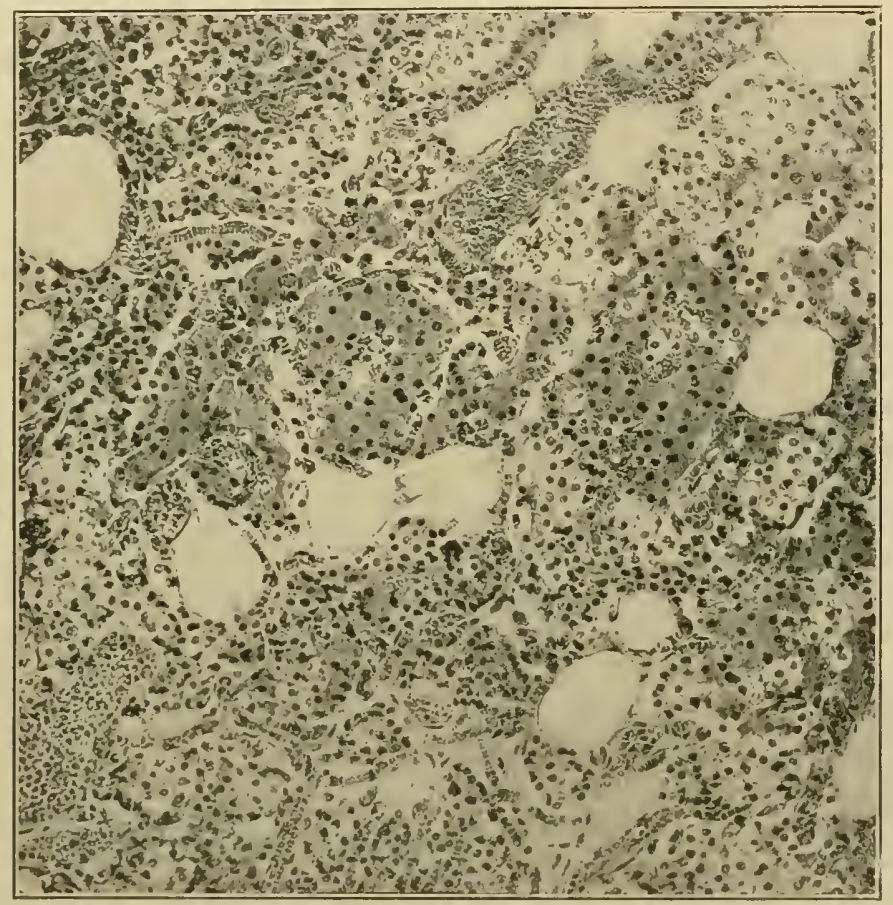

FIG. 282.-Section of Human Parathyrcoid showing groups of oxyphile cells. (Pool.)

are large in proportion to the cell and are clear and vesicular with loosely arranged pale chromatin network. (2) Oxyphile cells (Fig. 282). These are larger, their cytoplasm is more granular and takes a strong cosin stain. The nucleus is small, its chromatin network closely arranged and takes a dark stain. Compact groups of these cells occur especially just beneath the capsule. They are also found throughout the gland, arranged as cords, as single cells, or as small groujs among the clear cells. Intermediate types have been de- 
scribed. It is probable that the clear cells represent the resting, the granular cells the active secreting condition of the same cell.

The parathyreoids originate as epithelial evaginations from the third and fourth branchial grooves, and develop wholly independently of the thyreoid.

The powerful influence which these minute organs exert is shown both clinically and experimentally. Fatal tetany, resulting from the earlier operations for complete removal of the thyreoids, has been shown by animal experimentation to be due not to the removal of the thyreoids, but to coincident removal of the parathyreoids, the removal of the latter only, in animals, giving the same results. ${ }^{1}$

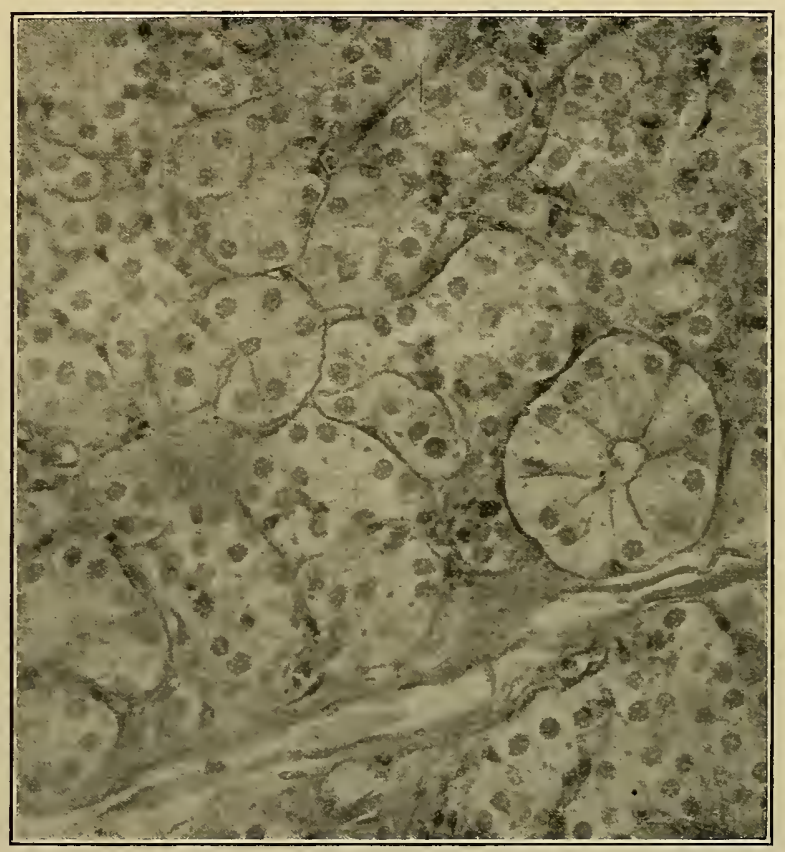

Fig. 283.-Section of Human Parathyreoid, showing lumina indicating tubular or tubulo-alveolar structure. (Pool.)

\section{TECHNIC}

The thyreoid and parathyreoid glands are best fixed in formalin-Müller's fluid. Sections may be stained with hæmatoxylin-eosin or hæmatoxylin-picro-acidfuchsin and mounted in balsam.

\section{General Reference for Further Study}

Pool: Tetany Parathyreopriva. Annals of Surgery, October, I907.

1 For much of the description of the parathyreoids and for the photograph the writer is indebted to Dr. Eugene H. Pool. 


\section{The Pituitary Body}

The Pituitary Body or Hypophysis Cerebri consists of two main lobes which are totally different both in structure and in origin. They are separated by a cleft, the interglandular cleft, and a narrow strip of the posterior lobe bordering the cleft presents a different structure from other parts of the gland. This has been designated the pars intermedia (Fig. 284).

The Anterior Lobe (pars anterior).--This is the larger of the two lobes and is distinctly glandular in character. It is of ectodermic origin, developing as a diverticulum from the primitive oral cavity. Its mode of development is that of a compound tubular gland, the single primary diverticulum undergoing repeated division to form the terminal tubules. The original diverticulum ultimately atrophies and disappears, leaving the gland entirely unconnected with the surface. The gland is enclosed in a connective-tissue capsule from which trabeculæ pass into the organ forming its framework. The gland cells are arranged in slightly convoluted tubules and rest upon a basement membrane. Between the tubules is a vascular connective tissue. Some of the gland cells are small cuboidal cells with nuclei at their bases and a clear or finely granular cytoplasm (chief cells). Others, somewhat less numerous than the preceding, are larger and polygonal with centrally placed nuclei, and cytoplasm containing coarse basophile granules (chromophile cells). Cells with distinctly eosinophile granules may also be present. There has been much controversy as to whether these cells are fundamentally different or merely represent different secretory conditions or stages. The large variation in relative number of the different forms, and the occurrence of cells which apparently represent intermediate stages, render it probable that all should be considered as merely different functional conditions of the one type of cell.

As in all ductless glands, the blood supply is rich, the vessels being sinusoidal in character, and the relations of capillaries to gland cells is extremely intimate, dense networks of capillaries surrounding the alveoli on all sides. In some places the cells are so placed around a capillary as to resemble the relation of gland cells to the lumen.

The Posterior Lobe (pars posterior, pars nervosa).-This like the anterior lobe is surrounded by a connective-tissue capsule which sends trabecule into its substance. In the human adult the lobe consists mainly of ncuroglia with a few scattered cells, which probably 
represent rudimentary ganglion cells and a few nerve fibres. In the human embryo, and in many adult lower animals, the nervous elements are much more prominent and more definitely arranged. Thus Berkley describes the posterior lobe of the pituitary body of the dog as consisting of three distinct zones: (I) An outer zone of three or four layers of cells resembling ependymal cells, connective-tissue

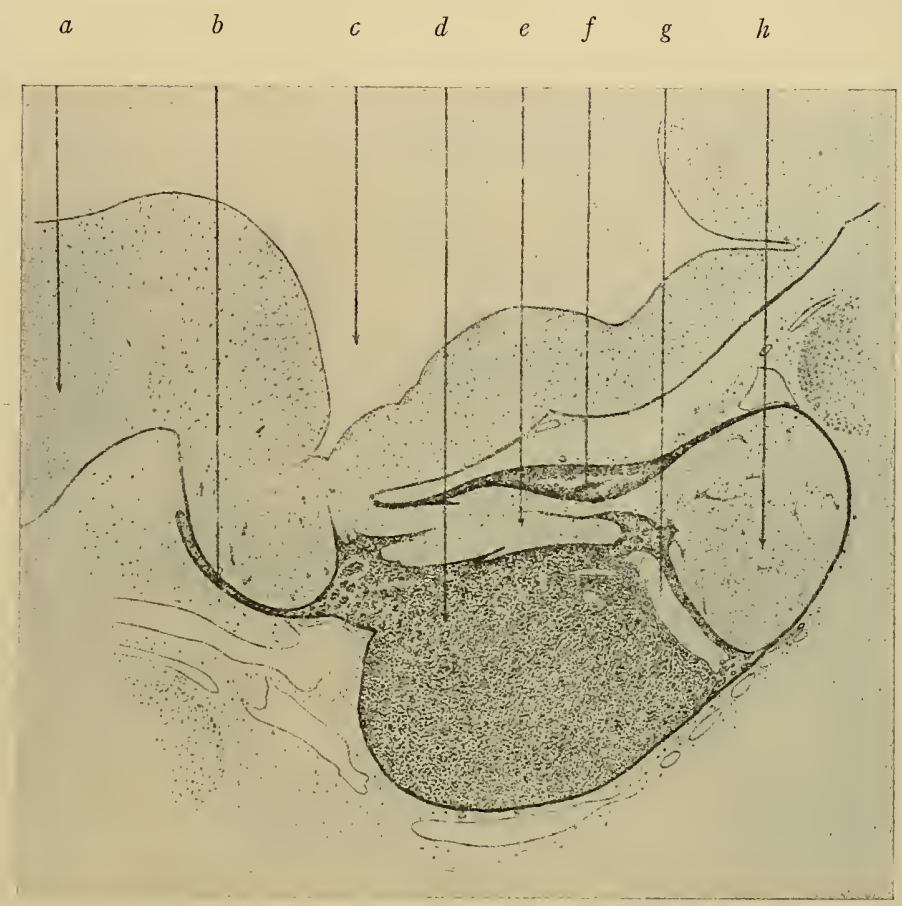

FIG. 284.-Mesial Sagittal Section through Pituitary Body of Five Months Human fotus. (Herring.) $a$, Optic chiasma; $b$, anterior extension of pituitary body; $c$; third ventricle (infundibulum); $d$, pars anterior; $e$, neck or isthmus of pars nervosa connecting it with bottom of infundibulum; $f$, epithelium continuous with pars intermedia surrounding neck; $g$, interglandular cleft; $h$, pars nervosa or posterior.

septa from the capsule separating the cells into irregular groups.

A middle zone of glandular epithelium, some of the cells of which are arranged as rather indefinite alveoli which may contain colloid. This is termed the pars intermedia. (3) An inner layer of nerve cells and neuroglia cells. These react to the Golgi stain, the nerve cells having axones and dendrites. Most of the axones appeared to pass in the direction of the infundibulum, but could not be traced into the latter. The posterior lobe is of ectodermic origin, developing as a 
diverticulum from the floor of the third ventricle. The remains of the diverticulum constitute the infundibulum.

The so-called middle lobe or pars intermedia is a thin layer of tissue which covers the anterior aspect of the posterior lobe thus lying between it and the interglandular cleft. It develops with the anterior lobe and like it consists of a connective-tissue framework and epithelial cells. It is less vascular, however, and its cells are smaller and less distinctly granular. The cells lining the cleft are columnar, thus contrasting with the flat cells of the opposite wall. Characteristic of the pars intermedia are small cyst-like structures which contain colloid, presenting an appearance not wholly unlike thyreoid, although chemically the colloid of the two glands is not identical. Lining these tiny cysts is a cuboidal epithelium.

The nature of the secretion, its function, and its relation to the various kinds of cells have been subjects of much controversy. The granules within the cells undoubtedly represent an intracellular stage of the secretion. According to some investigators each type of cell contributes its own special secretion to the general secretion of the gland. According to most authorities the different types of cells represent merely different stages in the elaboration of the secretion. Some consider the colloid a special secretion, others derive it from the cell granules and consider it as possibly representing a final stage, or "normal degenerative condition of the secretion."

Pregnancy apparently induces increased activity of the gland. This is of interest in connection with the clinical use of the extract of pituitary body for the purpose of inducing contractions in an atonic uterus during parturition or for the control of post-partum bleeding. Pathological conditions of the pituitary, more particularly tumors or hyperplasia of the anterior lobe, are apparently associated with a clinical condition known as acromegaly in which there is marked connective-tissue hypertrophy, especially of certain bones. The connection between acromegaly and lesions of the pituitary body would seem to be somewhat similar to that between myxodema and lesions of the thyreoid. Removal of the thyreoid results in enlargement of the pituitary body, especially in increase of colloid. Increase of colloid has also been reported after removal of the pancreas.

\section{The Paraganglia}

Under the hearl of Paraganglia are grouped certain small ductless glands and small groups of cells which are closely associated both anatomically and embryologically with the sympathetic system. They include the carotid, the cocrygeal, and tympanic glands, the parasympathetic organ of Zuckerkandl, and the medulla of the adrenals. The most marked characteristic of these organs aside from their 
close relation to the sympathetic, is that their cells take a yellowish brown stain when placed in solutions of chromic acid or chrome salts, retaining the color even after prolonged washing in water. For this reason the cells are sometimes referred to as chromaffin cells and the organs as chromaffin organs. ${ }^{1}$

While each organ has its own peculiar structure, all agree in certain general features. (I) The cells are polyhedral and have a general arrangement into cord-like structures rather than into lobules; (2) they are all ductless glands; (3) the cells lie in very close relation

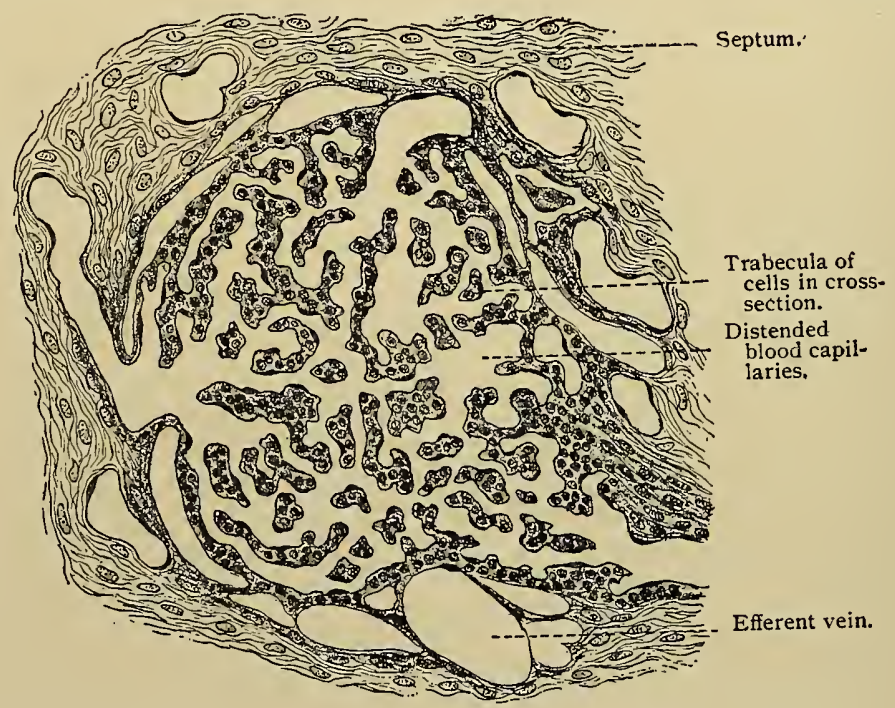

FIG. 285. -Section of human carotid gland. $\times{ }_{1} 60$. (Schaper.)

to rich capillary networks of large vessels, some of which resemble sinusoids and have been described as having incomplete walls, the gland cells thus being in direct contact with the blood stream; (4) unstained the cells show a clear, highly refractive cytoplasm, but contain secretion granules which stain yellowish brown with chromic acid and chrome salts, red with safranine, and black with osmic acid; (5) all, as far as has been determined, produce an internal secretion which passes directly into the blood and which acts as a regulator of vascular tone.

The Carotid Glands.-These are two small ductless glands, each about the size of a rice grain, which lie one on either side of the

${ }^{1}$ Cells containing chromaffin granules have also been described as occurring in sympathetic ganglia, and by Rose as present in many different organs, e.g., the ovary, testicle, blood-vessel walls, etc. 
bifurcation of the carotid artery. They are composed of a vascular connective tissue supporting spheroidal groups of large polyhedral epithelial cells with poorly marked boundaries and closely associated with tufts of capillaries. These capillaries are of large diameter and thin walled, and have been described as sinusoidal in character. The amount of connective tissue and the blood-vessels increase with age at the expense of the epithelial elements. This probably led to

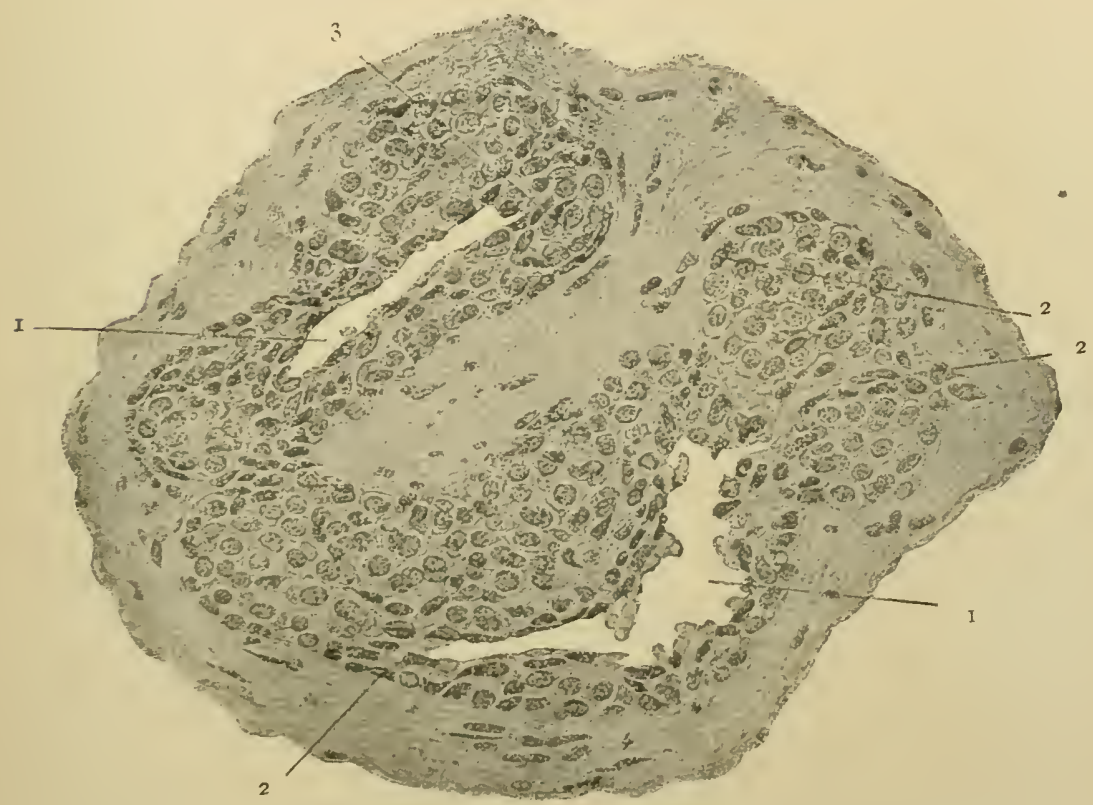

FIG, 286,-Section through coccygeal gland. (Walker.) r. Blood space; 2. epithelium; 3. connective tissue.

the earlier description of the gland as a vascular or glomerular structure. The gland cells themselves contain chromaffin granules (p. 4ro) and secrete a blood-pressure-raising substance apparently identical with, or similar in nature to, the secretion of the adrenal (p. 4 I 2).

The Coccygeal Gland.-This is a small ductless gland which lies just in front of the apex of the coccyx. It is similar in structure to the preceding but has its cell groups more irregularly arranged (Fig. 286). 'There is the same general arrangement of gland cells, the same relation of gland cells to the connective-tissue framework and to large sinusoidal blood-vessels, the same vascular and con- 
nective tissue changes with age, the same reaction of its cells to chrome salts, and the same or a similar secretion.

The tympanic gland and the organ of Zuckerkandl are small collections of chromaffin cells, the former lying on Jacobson's nerve in

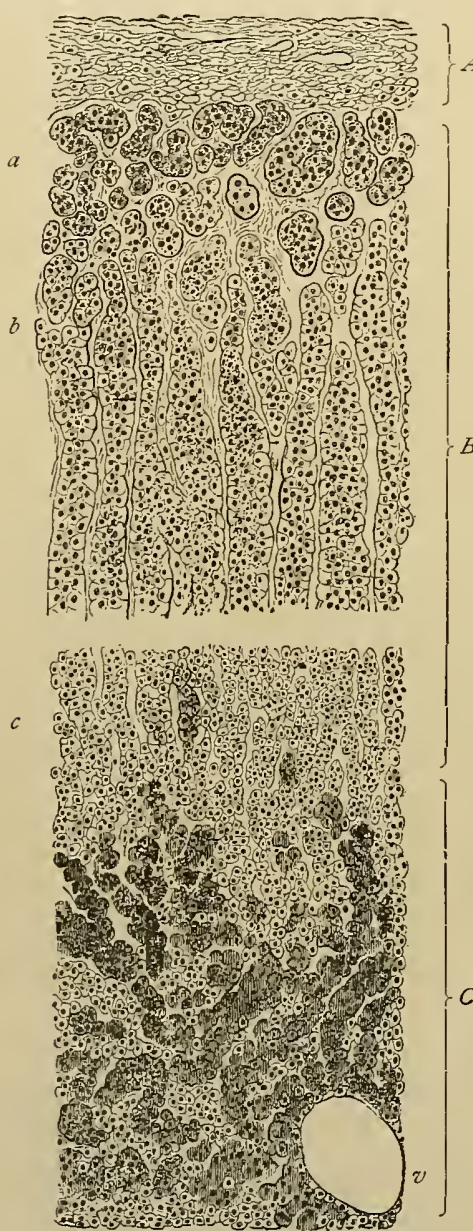

Fig. 287.-Vertical Section of Adrenal. (Merkel-Henle.) $A$, Capsule; $B$, cortex; $C$, medulla; $a$, glomerular zone; $b$, fascicular zone; $c$, reticular zone; $v$, vein in medulla. the tympanic canal, the latter lying in the retroperitoneal tissue at about the level of the bifurcation of the abdominal aorta.

\section{The Adrenal}

The adrenal is a ductless gland situated on the upper and anterior surface of the kidney. It is surrounded by a capsule and consists of an outer zone or cortex and a central portion or medulla. The cortex and medulla are sharply differentiated both in general appearance and in histological structure. The former is of rather firm consistency, its cells are arranged in rows with the bloodvessels between them, giving the zone a striated appearance. Its cells contain fat droplets and peculiar granules known as lipoid granules which give the cortex a yellowish tint. In contrast the medulla is soft, vascular, has a dark reddish appearance, and its cells contain granules known as chromaffin or pheochrome granules.

The CAPsule (Fig. 287, $A$ ) is composed of fibrous connective tissue and smooth muscle. In the outer part of the capsule the connective tissue is loosely arranged and merges with the surrounding fatty areolar tissue. The inner layer of the capsule is more dense and forms a firm investment for the underlying glandular tissue. From the capsule trabeculæ extend into the organ forming its framework and outlining compartments, 
which contain the glandular epithelium. This connective tissue is reticular in character.

The CORTEx (Fig $287, B$ ) is subdivided into three layers or zones: (a) A narrow, superficial layer, the glomerular zone; (b) a broad middle layer, the fascicular zone; and $(c)$ a narrow deep layer, the reticular zone. The names of the layers are indicative of the shape of the connective-tissue-enclosed compartments and of the contained groups of gland cells. In the glomerular zone (Fig. 287, a) the high, irregularly columnar epithelium is arranged in spherical or oval groups. The protoplasm of the cells is granular, contains fat droplets, and their nuclei are rich in chromatin. In the fascicular zone (Fig. $287, b$ ) polyhedral cells are arranged in long columns or fascicles. The cytoplasm is granular and usually contains many large fat droplets. The nuclei contains less chromatin than those in the glomerular zone. The appearance which the protoplasm of the cells richest in fat presents has led to their being called "spongioblasts." In the reticular zone (Fig. $287, c$ ) similar though somewhat more darkly staining cells, containing small fat droplets or sometimes no fat droplets, form a coarse reticulum of irregular anastomosing cords.

This division of the cortex into zones is more distinct in some of the lower animals. It does not indicate any fundamental structural or functional differences. The cells in the different zones show only minor variations in structure, the characteristic appearance of the zone depending upon the arrangement of the cells and the shape of the connective-tissue compartments. According to Gottschau the glomerular zone is the zone in which active formation of new cells takes place, these cells gradually passing toward the medulla, according to some, finally completing their history as medullary cells. Others terminate the life history of these cells with the deepest layer of the cortex. The pigment formation in the cells of the reticular zone is considered by some a degenerative, by others a secretory process. Still other investigators look upon the deeper cortical layers as the site of most active cell proliferation and an indifferent cell here situated as giving rise on the one hand to pigment cells and on the other to fat-producing cells. If the deeper cells are the older, the deeper zone would contain the more mature and probably the more functionally active cells, and it is here that are found cells crowded with fat droplets or with pigment granules. The former begin, according to some investigators, in the supcrficial cell as droplets of orrlinary fat, which becomes changed into lecithin-an easily brokendown, acid, phosphorus-containing fat with which many of the cells of the deeper layers are fillerl. As already noted, this pigment is regarded by some as the last stage in fat formation, by others as an entirely independent secretion. This interrelation of cortical and medullary cells is not, however, in accord with the findings of embryology or of comparative anatomy (p) 415). 
The MEDULLA (Fig. 287,C) consists of spherical and oval groups and cords of polygonal cells. After alcohol or formalin fixation these cells take a paler stain than those of the cortex. After fixation in solutions containing chromic acid or chrome salts the cells of the medulla assume a peculiar characteristic deep brown color, which cannot be removed by washing in water and which is due to chromaffin granules which they contain (p. 4I I). The chromaffin content varies in different animals and with age. Thus while in the adult human the chromaffin reaction is strong, little or no reaction is present in the fœetal adrenal. The secretion of the medullary part of the adrenal is known as adrenalin, apparently the mature condition of the intra-cellular chromaffin granules. As already noted (p. 410) it is probably an active agent in the regulation of arterial tension.

Scattered in irregular groups among the chromaffin cells are many sympathetic ganglion cells.

Blood-vessels.-The arteries supplying the suprarenal first form a poorly defined plexus in the capsule. From this are given off three sets of vessels-one to the capsule, one to the cortex, and one to the medulla. The first set breaks up into a network of capillaries, which supply the capsule. The vessels to the cortex break up into capillary networks, the shape of the mesh corresponding to the arrangement of the connective tissue in the different zones. The vessels to the medulla pass directly through the cortex without branching and form dense capillary networks among the groups of medullary cells. The relations of the capillaries to these glands cells are extremely intimate, especially in the reticular zone and medulla, where the cells in many cases immediately surround the capillaries in much the same manner as the glandular cells of a tubular gland surround their lumina. From the capillaries of both cortex and medulla small veins arise. These unite to form larger veins which empty into one or two main veins situated in the center of the medulla.

Lymphatics.-These follow in general the course of the bloodvessels. The exact distribution of the suprarenal lymph system has not been as yet satisfactorily determined.

Nerves.-The nerve supply of the suprarenal is so rich and the nerve elements of the gland are so abundant as to have led to its classification by some among the organs of the nervous system. Both medullated and non-medullated fibres-but chiefly the latter -form plexuses in the capsule, where they are associated with groups 
of sympathetic ganglion cells. From the capsular plexuses fine fibres pass into the cortex, where they form networks around the groups of cortical cells. The nerve terminals of the cortex apparently do nct penetrate the groups of cells. Bundles of nerve fibres, larger and more numerous than those to the cortex, pass through the cortex to the medulla. Here they form unusually dense plexuses of fibres, which not only surround the groups of cells, but penetrate the groups and surround the individual cells. Associated with the plexuses of the medulla, less commonly of the cortex, are numerous conspicuous groups of sympathetic ganglion cells.

DevelopMent.-The cortex and medulla have entirely different developmental histories. In the lower vertebrates (fishes) the two parts of the gland continue separate throughout life. In the ascending mammalian scale, the two parts become more and more closely united until in mammals they form a single organ. The cortex develops from mesoderm, first appearing in embryos of about five to six mm. At about the level of the cephalic third of the mesonephros the mesothelium sends outgrowths into the mesenchyme. These outgrowths soon lose their connection with the main mass of mesothelium and constitute the anlage of the suprarenal cortex. The medulla has an entirely independent origin, being derived from ectoderm, as part of the peripheral sympathetic nervous system. The cells of some of the sympathetic ganglia differentiate into sympathoblasts and phoochromoblasts, which give rise to the sympathetic cells and chromaffin cells respectively. These cells soon separate from their ganglia of origin and come to lie first near, then within, the developing cortex, thus forming the medulla.

\section{General References for Further Study}

Flint: The Blood-vessels, Angiogenesis, Organogenesis, Reticulum and Histology of the Adrenal. Contributions to the Science of Medicine, Johns Hopkins Press, 1900.

Pfaundler: zur Anatomie der Nebennierc. Anzeiger Akad. Wien, 29, I892.

Nagel: Ueber die Entwickelung des Urogenitalsystem des Menschen. Arch. f. Mik. Anat., Bd. xxxiv.

Stohr: Lehrbuch der Histologie B. I $5^{\text {th }}$ Ed.

Prenant: Traite d'Histologie. 


\section{CHAPTER XII \\ THE NERVOUS SYSTEM}

THE nervous mechanism in man consists of two distinct though associated systems, the cerebro-spinal nervous system and the sympathetic nervous system. Each of these systems is composed of a central portion which is its center of nervous activity, and of a peripheral portion which serves to place the center in connection with the organs which it controls. In the cerebro-spinal system the central portion is known as the central nervous system and consists of the cerebro-spinal axis, or brain and spinal cord. The peripheral portion is formed by the cranial and spinal nerves. The central portion of the sympathetic system consists of a series of ganglia from which the sympathetic nerves take origin. These latter constitute its peripheral portion. The whole sympathetic system is usually, however, included under the peripheral nervous system as opposed to the brain and spinal cord or central nervous system.

\section{HISTOLOGICAL DEVELOPMENT AND GENERAL STRUCTURE}

The beginning differentiation of the nervous system appears very early in embryonic life. It is first indicated by a longitudinal median thickening of the outer embryonic layer or ectoderm, to form the neural plate. The sides of the plate become elevated to form the neural folds, leaving between them the neural groove. By the dorsal union of these folds the neural groove is converted into the neural tube. The lumen of the neural tube corresponds to the central canal of the cord and the ventricles of the brain in the adult, and from the ectodermic cells which form the walls of this tube practically the entire nervous system is developed. The caudal portion of the tube is of nearly uniform diameter-the spinal cord. At the cephalic end, the neural plate, even before its closure, is wider and forms, when closed, an expanded portion of the tube, the brain. In their further development, the walls of the brain form three expansions-the three brain "vesicles" known as the forebrain (prosencephalon), midbrain (mes- 
encephalon), and hindbrain (rhombencephalon). In the forebrain two main divisions are usually distinguished, the endbrain (telencephalon) and the interbrain (diencephalon). The basal part of the endbrain forms the corpora striata and rhinencephalon, while the dorsal part expands into the pallium (cerebral hemispheres). In the interbrain may be distinguished a dorsal part, the epithalamus; a middle (largest) part, the thalamus; and a ventral expansion, the hypothalamus. In the midbrain the basal part becomes the tegmentum, and the dorsal part expands into the corpora quadrigemina or inferior and superior colliculi. The narrower part, connecting midbrain and hindbrain, is the isthmus. The basal part of the hindbrain forms the medulla oblongata and part of the dorsal wall expands into the cerebellum. In the later development of the brain, the pallium, and with it parts of the cerebellum, becomes enormously enlarged and structures are formed constituting connections between the pallium and the rest of the brain. The most massive of these are the pes pedunculi, added ventrally to the thalamus and midbrain; the pons Varolii, added ventrally to part of the midbrain, isthmus and part of the hindbrain; and the pyramids, added ventrally to the medulla. The basal part of the mid-and hindbrain, thus covered ventrally by the pes and pons, is the tegmentum. Other portions of the dorsal walls of the forebrain and hindbrain form thin-walled expansions which, together with vascular mesodermic coverings, are the chorioid plexuses of the lateral, third, and fourth ventricles. The cavities of the cerebral hemispheres are the lateral ventricles; the cavity of the interbrain is the third ventricle; that of the midbrain is the iter or aquaductus Sylvii; that of the hindbrain is the fourth ventricle.

'The wall of the neural tube is at first composed of a single layer of epithelial cells. By proliferation of these cells the epithelium soon becomes many-layered, and forms a syncytium-the myelospongium of His-although some of the original epithelial cells appear to extend through the entire thickness of the wall.

Some of the syncytial cells which extend through the entire thickness of the wall of the neural tube (spongioblasts of His) increase in length as the wall increases in thickness. The inner ends of these cells form the lining of the tube, while other parts of the cells between the lumen and the surface 1 end to collajese, forming cord-like structures. The outer ends of the cells, on the other hand, become perforaterl and unite to form a thick network-the marginal veil of His. ()f these cells, some retain this position, with nuclei near the lumen, 
in the adult and are known as ependymal cells; others move away from the central canal and become neuroglia cells.

Still other of the cells of the neural tube are destined to become neurones, and as such are known as neuroblasts. From the neuroblast a neurofibrillated process grows out-the future axone. Dendrites which at this stage are absent develop later in a similar manner, i.e., by extensions of the cell protoplasm. ${ }^{1}$ The neuroblasts soon leave their original position near the lumen and pass outward along the spaces between the elongated ependymal cells, but their bodies do not usually penetrate the marginal veil. Some may, however, pass through and even leave the neural tube along with the efferent roots. The axones of many neuroblasts located in the ventral part of the neural tube pierce the marginal veil and leave the neural tube as the efferent root fibres. These nerve cells together with many of the sympathetic neurones (see below) are the efferent peripheral neurones. Their axones pass to sympathetic ganglia or to various structures (muscles, glandular epithelia) the activities of which they affect. Such structures may be collectively termed effectors. The axones of other neuroblasts do not completely pierce the marginal veil, but are directed upward and downward within it, thus forming fibres connecting various parts of the central nervous system. Axones of still other neuroblasts, especially in suprasegmental structures (see p. 420), are directed toward the lumen. All such neurones whose axones do not leave the neural tube may be termed intermediate or central neurones, as distinguished from the peripheral neurones: intermediate, because they serve as intermediate links connecting, within the central nervous system, the terminations of the efferent peripheral neurones (see below) with the bodies of the efferent peripheral neurones; central, because they are confined to the central nervous system. Later becoming medullated, their axones constitute the great majority of the fibres of the white matter of the central nervous system.

During the closure of the neural groove, groups of cells from the crest of each neural fold become separated from the rest of the developing nervous system. Some of these cells form the cerebro-spinal ganglia, while, according to most authorities, others migrate still further from the neural tube and form the various sympathetic ganglia. Some cells (neuroblasts) develop into the nerve cells of the cerebro-

${ }^{1}$ According to other views, other cells may participate in the formation of the axone, and the dendrites may anastomose with other neuroblasts (see Chap. VI)., 
spinal and sympathetic ganglia. Others form, according to some authorities, enveloping cells, such as the capsule cells around the ganglion cell bodies and the neurilemma cells around the peripheral nerve fibres. These latter would thus correspond to the neuroglia cells of the central nervous system. The majority of the neuroblasts of the cerebro-spinal ganglia develop two processes: peripheral processes (afferent nerie fibres) to various structures (receptors) which receive stimuli; and central processes, forming the afferent roots and passing into the central nervous system where they usually bifurcate and form longitudinal ascending and descending arms. These cells are at first bipolar, later the cell body withdraws from the two processes and thus assumes the adult unipolar condition (see page 427). Other processes may also appear. Many of the neuroblasts of the sympathetic ganglia develop dendrites and axones while others form branching cells in which the two kinds of processes cannot be easily distinguished. Sympathetic cells are also derived from cells which migrate from the neural tube along the efferent root (p. 4I8).

The cerebro-spinal ganglionic neurones, together with some sympathetic neurones, certain neurones in the olfactory mucous membrane, retina, and possibly midbrain roof, constitute the afferent peripheral neurones of the entire nervous system. Most of the sympathetic neurones are efferent. The peripheral processes of the afferent peripheral neurones, the axones of the efferent peripheral neurones after they have emerged from the central nervous system, and the axones of the sympathetic ganglion cells, together with all their sheaths and connective-tissue investments, form the peripheral nerves. The bodies of the afferent peripheral neurones and sympathetic neurones form groups known as ganglia. The peripheral neurones are arranged segmentally, as shown by the series of ganglia and nerves.

The sympathetic neurones and those cerebro-spinal neurones which innervate sympathetic ganglia, viscera, glands, blood-vessels, and smooth and heart muscle are peripheral visccral or splanchnic ncurones. Those cranial nerve neurones which innervate the striated voluntary muscles of jaw, ear, face, pharynx, and larynx (branchiomotor) are usually also classed as splanchnic. The remainder of the cercbro-spinal perip heral neurones are peripheral somatic neurones.

From the foregoing it will be seen that all the neurones of the nervous system fall into two categories: I. Peripheral neurones, a 
part of whose processes, at least, lie outside the central nervous system, forming the peripheral nerves. II. Central or intermediate neurones. These lie entirely within the central nervous system. The peripheral neurones may be classified as follows: A. Afferent. peripheral (all the neurone bodies located outside the central nervous system, except possibly some in midbrain roof). The afferent peripheral neurones are (I) the cerebro-spinal ganglion cells, which may be (a) somatic, (b) splanchnic; (2) cells in olfactory membrane, retina, and possibly midbrain; (3) some sympathetic neurones (splanchnic). B. Efferent peripheral neurones. These are (I) cerebrospinal (neurone bodies located in ventral part of central nervous system), (a) somatic (to voluntary striated muscles except $(c)$ ), (b) splanchnic (sending axones to sympathetic ganglia), (c) splanchnic to voluntary striated branchiomotor muscles (p. 4rg); (2) sympathetic (splanchnic, bodies in sympathetic ganglia, sending axones to smooth muscle, heart muscle and glands).

In the central nervous system the bodies and dendrites of the neurones are usually aggregated within certain localities which, on account of their appearance when examined in fresh condition, are collectively termed the gray matter (substantia grisea). The gray matter also contains, of course, the beginnings and endings of the nerve fibres. The parts of the nervous system where the bodies and dendrites are absent and which are composed (exclusive of the neuroglia, blood-vessels, etc.) of the medullated nerve fibers are collectively termed the white matter (substantia alba).

The central nervous system may be divided into a segmental part and certain suprasegmental parts. The former is in more immediate relation with the peripheral (segmental) nerves. It comprises the spinal cord and basal part of the brain (segmental brain). It contains all the bodies of the efferent cerebro-spinal neurones and practically all the terminations of the central processes (afferent root fibres) of the afferent peripheral neurones. In it the gray matter is internal, as a rule, and the white matter external. The suprasegmental parts comprise the expanded portions of the dorsal wall of the neural tube already mentioned, namely the pallium, corpora quadrigemina, and cerebellum. These constitute the highest coördinating centers of the nervous system. Here the gray matter is external, constituting a cortex, and the white matter is internal.

The grouping of peripheral neurones into ganglia and nerves has already been mentioned. In the central nervous system more or 
less definite groups or systems of neurones having certain definite connections may also be distinguished. The axones of such a system constitute a tract or, when aggregated into a definite bundle, a fasciculus. The groups of neurone bodies are termed nuclei. The group or collection of bodies whose axones form a certain tract is the mucleus of origin of that tract. The same nucleus may receive the terminations of some other tract and is then the terminal mucleus of that tract. A given neurone system serves as a path for the conduction of some particular kind of nerve impulse. A conduction path, however, is often composed of several neurone systems linked together, thus forming a series of relays.

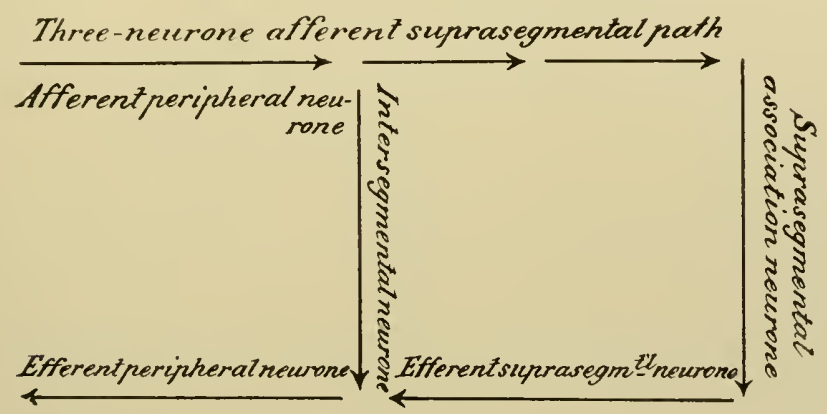

F1G. 288.--Diagram illustrating an arc transversing only the segmental, and an arc transversing the suprasegmental part of the nervous system.

All reactions performed by the nervous system must ultimately take effect upon some part of the body or effector and are usually initiated by changes in sume receptor. Such a circuit from receptor to effector may be termed a neural arc (Fig. 288), and will involve afferent peripheral neurones, efferent peripheral neurones and usually intermediate neurones. The complexity of such arcs depends largely upon the number and character of intermediate neurones intercalated in the arc between the afferent and efferent peripheral neurones. Such ares may obviously traverse centrally only the cord or segmental brain or may also traverse one or more of the suprasegmental parts of the nervous system. Intermediate neurones which link together different parts of the same segment may be termed intrasegmental neurones. Intermediate neurones which link together different segments of the cord and segmental brain may be termed intersegmental neurones. ()ther intermediate neurones form conduction paths to and from suprascgmental parts (afferent and efferent supro- 
segmental neurones) and still others are suprasegmental associative neurones, confined to suprasegmental structures (Fig. 2S8).

\section{MEMBRANES OF THE BRAIN AND CORD}

The brain and cord are enclosed by two connective-tissue membranes, the dura mater and the pia mater, a part of the latter being often referred to as a separate membrane, the arachnoid (Fig. 289).

The dura mater is the outer of the two membranes and consists of dense fibrous tissue. The cerebral dura serves both as an investing membrane for the brain and as periosteum for the inner surfaces of the cranial bones. It consists of two layers: (a) An inner layer of closely packed fibro-elastic tissue containing many connectivetissue cells, and lined on its brain surface with a single layer of flat cells; and (b) an outer layer, which forms the periosteum and is similar in structure to the inner layer, but much richer in blood-vessels and nerves. Between the two layers are large venous sinuses. The spinal dura corresponds to the inner layer of the cerebral dura, which it resembles in structure, the vertebræ having their own separate periosteum. The outer surface of the spinal dura is covered with a single layer of flat cells, and is separated from the periosteum by the epidural space, which contains anastomosing venous channels lying in an areolar tissue rich in fat. The spinal dura is said to contain lymphatics which open on both of its surfaces. Beneath the spinal dura, between it and the arachnoid, is the subdural space, a narrow cleft containing a fluid probably of the nature of lymph. It is stated that this space communicates by lymph clefts with the lymph spaces in the sheaths of nerves and also communicates with the deep lymphatic vessels and glands of the neck and groin. It has no direct communication with the subarachnoid space (see below).

The pia mater closely invests the brain and cord, extending into the sulci and sending prolongations into the ventricles. It consists of fibro-elastic tissue arranged in irregular lamellæ, forming a spongy tissue, the cavities of which contain more or less fluid. The outer lamellæ are the most compact, and are covered on the dural surface by a single layer of flat cells. It is this external layer of the pia which is frequently described as a separate membrane, the arachnoid. The space beneath the arachnoid is the subarachnoid space and contains cerebrospinal fluid. There is direct communication between these cavities and the subarachnoid space in the roof of the fourth 
ventricle. The subarachnoid space communicates with the lymphatic spaces within the nerve sheaths. It is also stated that it communicates with the lymph spaces contained within the vascular connective tissue (media and adventitia) of the blood-vessels which penetrate the central nervous system. Whether there is normally a peri-

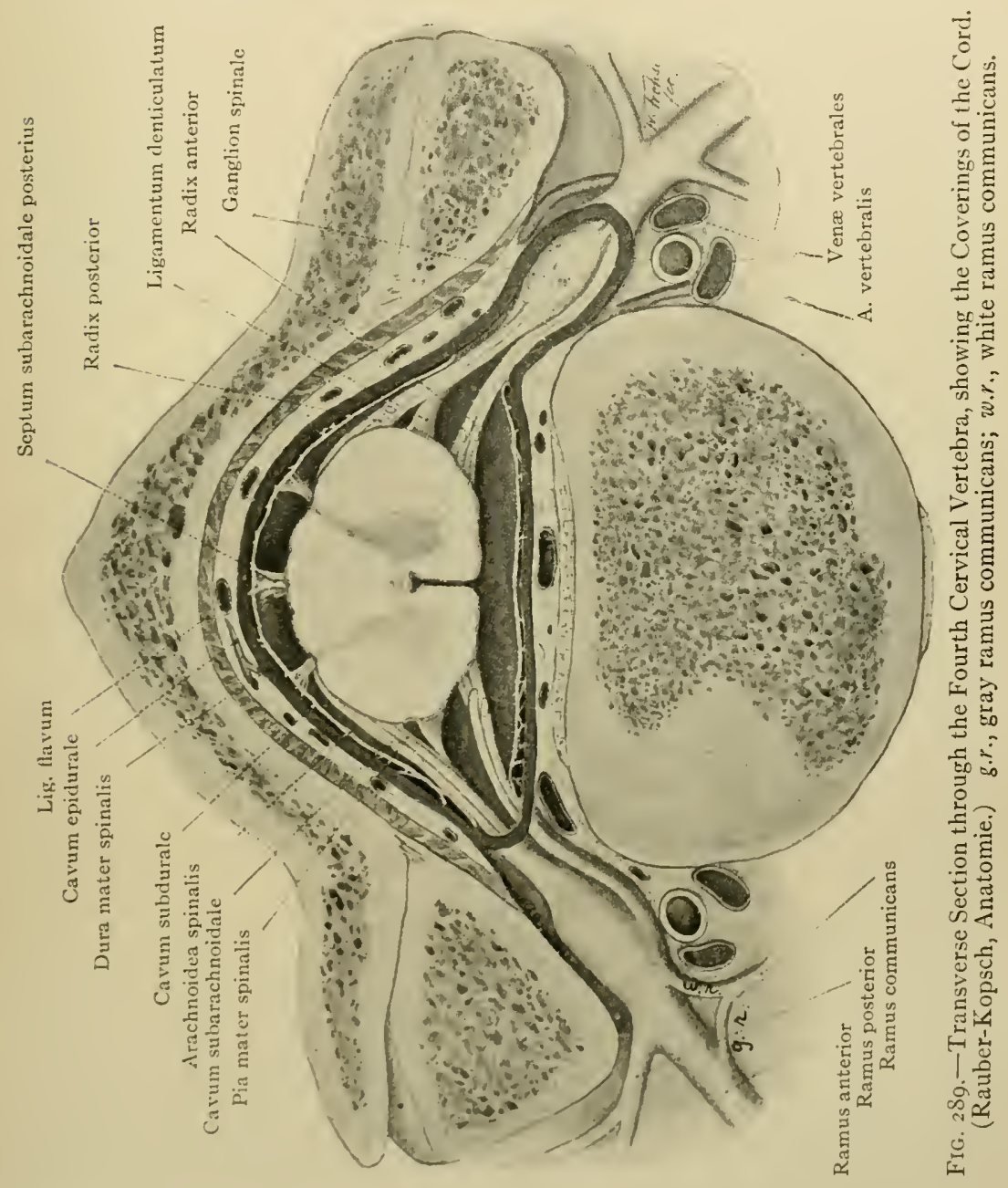

vascular lymph space between adventitia and glial membrane is doubtful and the existence has also been denied, by good authorities, of lymph spaces within the glia, the glial membrane forming in normal conditions a continuous structure. The inner lamella of the pia are more loosely arranged, are more cellular and more vascular. Espe- 
cially conspicuous are large, irregular cells with delicate bodies and large distinct nuclei. They lie upon the connective-tissue bundles partially lining the spaces.

The Pacchionian bodies are peculiar outgrowths from the outer layer of the pia mater cerebralis, which are most numerous along the longitudinal fissure. They are composed of fibrous tissue, and frequently contain fat cells and calcareous deposits.

Blood-vessels. - The spinal dura and the inner layer of the cerebral dura are poor in blood-vessels. The outer layer of the cerebral dura, forming as it does the periosteum of the cranial bones, is rich in blood-vessels which pass into and supply the bones. The pia is very vascular, especially its inner layers, from which vessels pass into the brain and cord.

\section{TECHNIC}

For the study of the structure of the membranes of the brain and spinal cord, fix pieces of the cord with its membranes, and of the surface of the brain with membranes attached, in formalin-Müller's fluid (technic 5, p. 7) and stain sections with hæmatoxylin-eosin (technic I, p. 20).

\section{THE PERIPHERAL NERVES}

The peripheral nerves are divided into spinal nerves and cranial nerves, the former being connected with the cord, the latter with the brain. Each spinal nerve consists of two parts-a motor or efferent part and a sensory or afferent part. Of the cranial nerves some are purely efferent, others purely afferent, while still others consist, like the spinal nerves, of both efferent and afferent fibres. The efferent fibres of the spinal nerves are axones of cell bodies situated in the anterior horns of the cord (see p. 45I, and Figs. 308 and $3 \mathrm{I} 8$ ) and axones of sympathetic ganglion cells. The former leave the cord as separate bundles, which join to form the motor or efferent root. The afferent fibres are peripheral processes of cell bodies situated in the spinal ganglia (p. 429 and Figs. 308, 292). These leave the ganglion and join with the fibres of the motor root to form the mixed spinal nerve (Fig. 308, f). The connection of the ganglion with the cord is by means of the central processes of the spinal ganglion cells, which enter the cord as the posterior root. Among the afferent fibres of the posterior root are also found, in some animals, a few efferent fibres (Fig. 308, c), processes of cells in the cord. Some fibres from the spinal ganglion and from the efferent roots form the white ramus 
communicans to the sympathetic ganglia. Fibres from the sympathetic ganglia form the gray ramus communicans to the mixed spinal nerve (Figs. 289 and 302). For further details regarding cranial nerves see pp. $474,+75$.

The peripheral nerve consists of nerve fibres supported by connective tissue (Fig. 290). Enclosing the entire nerve is a sheath of dense connective tissue, the epincurium. This sends septa into the nerve which divide the fibres into a number of bundles or fascicles. Surrounding each fascicle the tissue forms a fairly distinct sheath, the

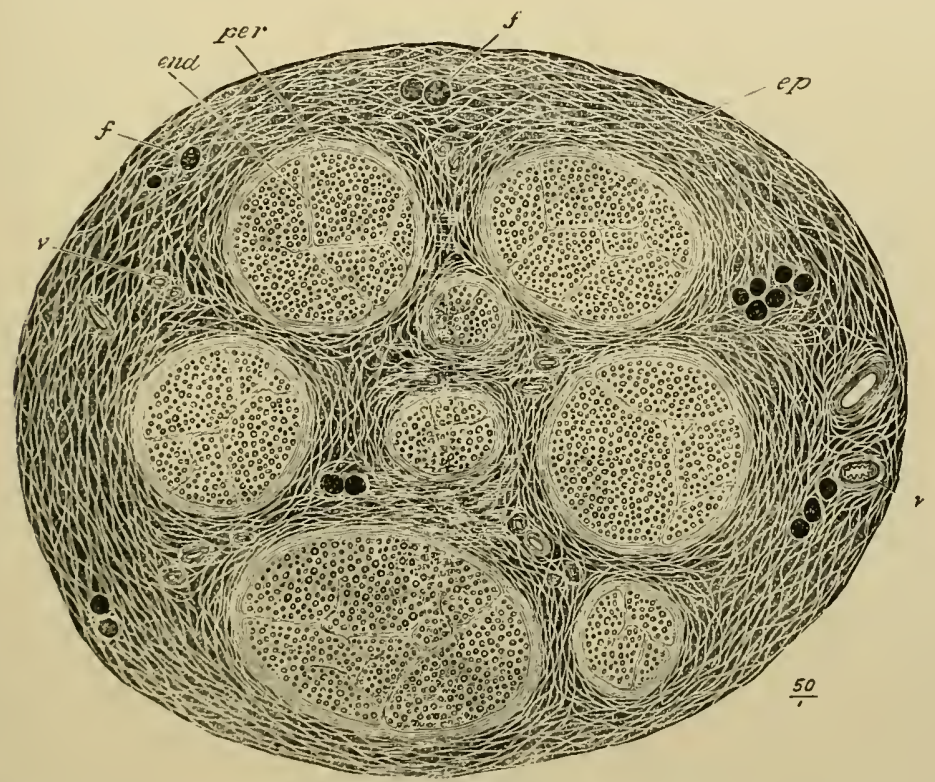

Fig. 290.-From Transverse Section of Human Nerve Trunk. (Osmic acid fixation.) (Quain.) ep, Nerve sheath or epineurium surrounding the entire nerve and containing blood-vessels $(v)$ and small groups of fat cells $(f)$; per, perifascicular sheath or perineurium surrounding each bundle or fascicle of nerve fibres; $e n d$, interior of fascicle showing supporting connective tissue, the endoncurium.

perifascicular sheath or perineurium, which is covered with a layer of flat cells. From the perineurium, delicate strands of connective tissue pass in to the fascicle, separating the individual nerve fibres. This constitutes the intrafascicular conneclive tissue or endoneurium. In the connective-tissue layers of the perineurium are blood-vessels, and lymph spaces lined with endothelium, which communicate with lymph channels within the fascicle. When nerves branch, the connective tissue sheaths follow the branchings. When the nerve becomes reduced to a single fithe, the perineural and endoneural 
connective tissue still remaining constitutes the sheath of Henle. The space between the neurilemma and sheath of Henle has been described as a lymph space communicating with the rest of the lymphatic system of the nerve. On emerging from the central nervous system, the root fibres receive an investment of connective tissue (endoneurium and perineurium) as they pass through the pia mater. This is reinforced by additional connective tissue (epineurium,) as the nerve passes through the dura mater. For description of medullated and non-medullated nerve fibres see Chap. VI.

\section{TECHNIC}

Fix a medium-sized nerve, such as the human radial or ulnar, by suspending it, with a weight attached to the lower end, in formalin-Müller's fluid (technic 5, p. 7). Stain transverse sections in hæmatoxylin-picro-acid-fuchsin (technic 3, p. 21), or hæmatoxylin-eosin (technic r, p. 20), and mount in balsam. Pieces of nerve should also be fixed in osmic acid imbedded, cut, and mounted without further staining. Pieces of fresh nerve may be tested and examined without staining.

\section{THE AFFERENT PERIPHERAL NEURONES}

These comprise, as already stated, the bodies and processes of the cerebro-spinal ganglion cells or cerebro-spinal ganglionic neurones, probably some of the sympathetic ganglion cells, certain cells of the retina and certain cells of the olfactory mucous membrane. The two last named are described in connection with the organs of special sense.

\section{The Cerebro-spinal Ganglia}

A cerebro-spinal ganglion contains the bodies of the cerebro-spinal ganglionic neurones whose processes form the afferent root of the cerebro-spinal nerve. It lies in the dorsal root, a dorsal root being that part of the nerve which lies between the exit of the dorsal root fibres from the cord and their junction with the ventral root. Each ganglion is surrounded by a connective-tissue capsule which is continuous with the perineurium and epineurium of the peripheral nerves. (Fig. 289.) From this capsule connective-tissue trabeculæ extend into the ganglion, forming a connective-tissue framework. Within the ganglion the nerve cells are separated into irregular groups by strands of connective tissue and by bundles of nerve fibres. Each ganglion cell contains a centrally located nucleus and a dis- 
tinct nucleolus, and is surrounded by a capsule of flat, concentrically arranged cells which are probably derived from the neural plate (p. 4I8) and are often called amplicytes or satellite cells (Fig. 293). Stained by Nissl's method the cytoplasm is seen to contain rather small. finely granular chromophilic bodies, which show a tendency to concentric arrangement around the nucleus. Pigmentation is common, the granules usually forming a group in the vicinity of the point of origin of the main process of the cell. The majority of the

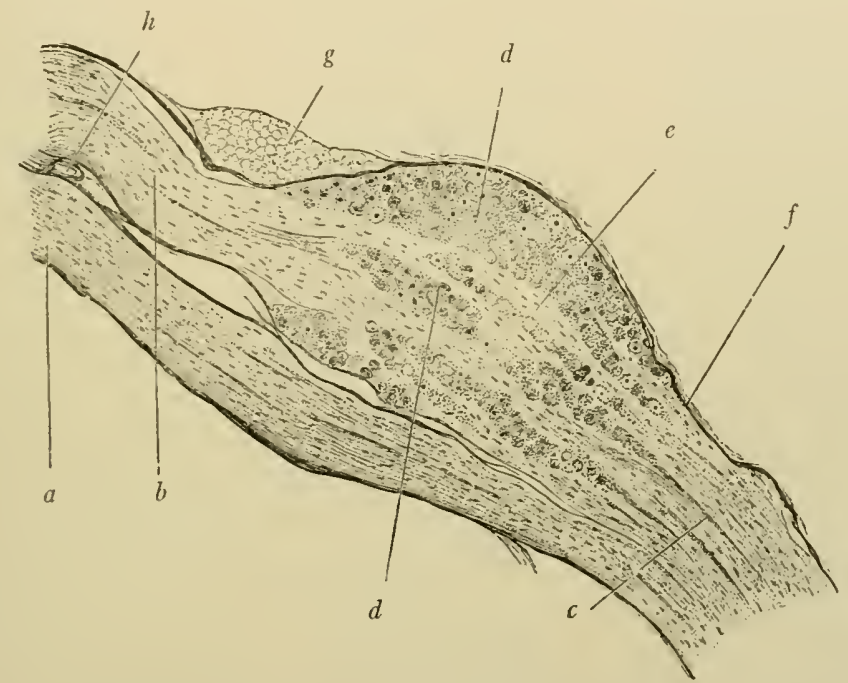

FIG. 29I.-Longitudinal Section through a Spinal Ganglion. $\times 20$. (Stöhr.) $a$, Ventral nerve root; $b$, dorsal nerve root; $c$, mixed spinal nerve; $d$, groups of ganglion cells; $e$, nerve fibres; $f$, perineurium; $g$, fat; $h$, blood-vessel.

cells of the spinal ganglia have one principal process which, at some distance from the cell body, divides into a peripheral branch which becomes an afferent fibre of the peripheral nerve and a central branch which enters the cord as a dorsal root fibre (p. 420). These cells are usually called unipolar cells. The principal process usually becomes medullated soon after emerging from the cell capsule (Fig. 292).

Among these cells a number of forms have been distinguished ly Dogiel: (a) Cells with only a principal process. This process may pass almost directly from the capsule, but often takes several turns around the cell body, forming a "glomcrulus," lefore emerging from the capsule (Fig. 292, I and Fig. 293, 1). This form is usually represented as the typical cercloro-spinal ganglion cell, but constitutes only a minority of these cells. (b) The principal process gives off collaterals. These lic within the capsule or are given off outside the carpsule and 
terminate in other parts of the ganglion and its covering, either in terminal arborizations or in terminal enlargements or bulbs. The collaterals may branch. Some of the terminations may be around the capsules of other cells. There may be one or several collaterals, sometimes a number of very short intracapsular collaterals. This last variety of cell may have more than one main process. (Fig. 292, 2 and 3, Fig. 293, B). (c) Cells with split processes. Here the main process divides into a number of fibres which reunite; this may be repeated. The splitting may be intracapsular or extracapsular. A variation of this is where there are two to six processes from the cell which form a complicated intracapsular network, finally uniting to form the single main process (Fig. 292, 4 and 5 and Fig. 293, C and $D$ ). (d) Cells with a number of dendrite-like processes

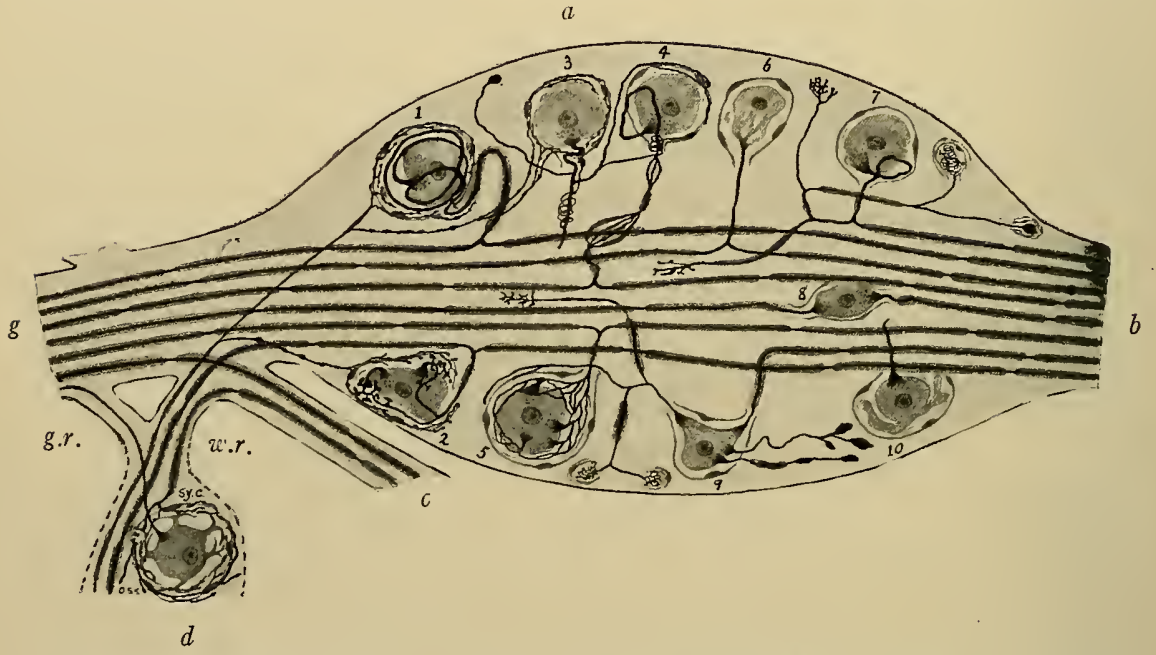

F1G. 292.- Various Types of Cells and Nerve Terminations found in a Spinal Ganglion. Schematized from Dogiel. g.r., gray ramus communicans; sy.c., sympathetic cell; w.r., white ramus communicans. $a$. Spinal ganglion; $b$, dorsal or afferent root; $c$, ventral or efferent root; $d$, sympathetic ganglion; $g$, spinal nerve. For further explanation see text (pp. 427-429).

which divide, forming an intracapsular network which finally fuses into the main process (Fig. 292,6). (e) Cells whose principal process divides as usual, but the peripheral branch terminates by arborizations or bulbs in the ganglion and in its covering, or in the neighborhood of the dorsal root (Fig. 292, 7). (f) Bipolar cells (Fig. 292, 8). (g) Multipolar cells with a number of intracapsular dendrites and a main process (Fig. 292, ro; Fig. 293, $E$ and $F$ ). (h) Cells with a principal process which probably enters. the dorsal root and a number of processes which may be dendrite-like in character, but also become medullated in places, and which branch and terminate in arborizations or bulbs in various parts of the ganglion. These latter apparently collectively represent the peripheral process which here ends in the ganglion (Fig. 292, 9)

The various endings in the ganglion of collaterals and other processes of ganglion cells often have capsules and resemble the terminations in receptors in 
other parts of the body (corpuscles of Pacini, end bulbs, etc.) and, together with their envelopes, may represent the receptors of the ganglion itself and of the connected nerves.

Sympathetic fibres enter the ganglion and form a plexus within it from which fibres pass and terminate within the capsules of the various ganglion cells.

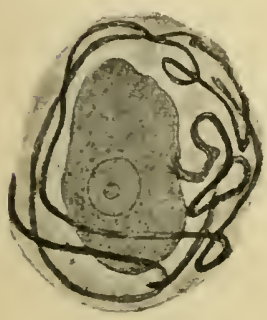

A

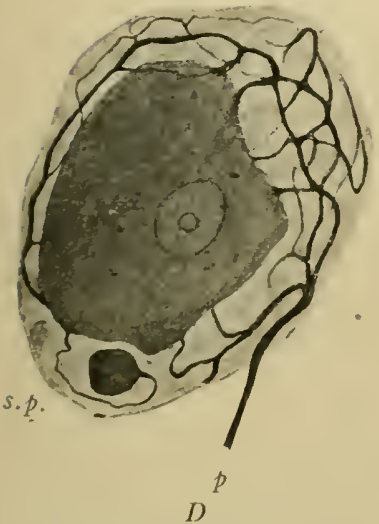

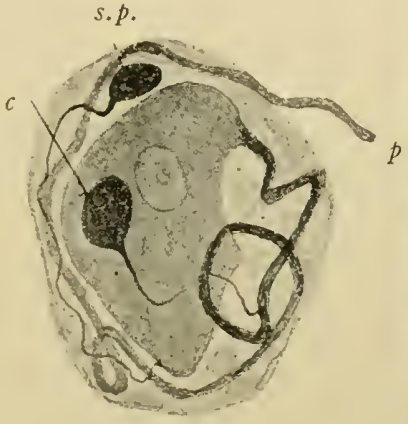

$B$

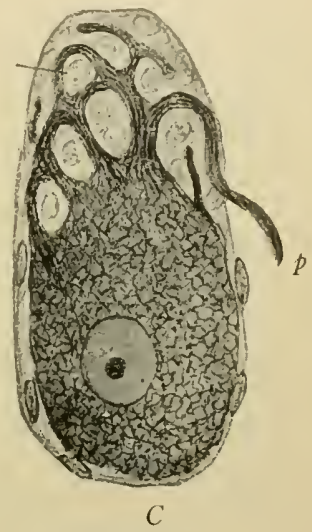

a.f.

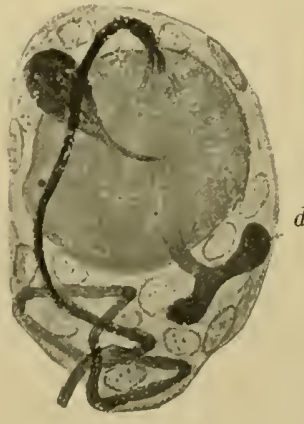

E

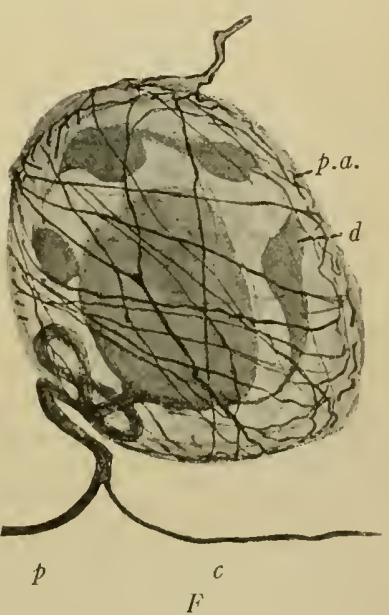

F

Fig. 293.-Cereloro-spinal Ganglion Cells and their Capsules. (Cajal.) A (adult man), Unipolar cell with single process forming a glomerulus; $B$ (man), cell with short process endling in intracapsular bulb and main process giving off in tracapsular collateral; $C$ (dog), "fenestrated" cell with several processes uniting to form main process; $D$ (ass), more complicated form of the same; $l:$ (man), cell with short bulbous dendrites; $F$ (man), (cill with bulbrous dendrites and enveloped with pericellular arborizations ( $p$.a.) of fibres (a.f.) terminating around cell; $c$, collateral; $d$, dendrite; $p$, principal process; s.p., short process. (('ajal's silver slatin.)

'THE JERIPHERA, PROCESSES OF THE CEREBRO-SPINAL GANGLION (ELJS are the afferent fibres of the cerebro-spinal nerves (1). 424). The morles of termination of these peripheral processes in receplors 
(p. 4I9) are extremely varied and complicated. These peripheral terminations are always free, in the sense that, while possibly sometimes penetrating cells, they probably never become directly continuous with their protoplasm.

In the skin, and in those mucous membranes which are covered with squamous epithelium, the nerve fibres lose their medullary sheaths in the subepithelial tissue, and, penetrating the epithelial layer, the axis-cylinder splits up into minute fibrils (terminal arborization) which pass in between the cells and terminate there, often in little knob-like swellings (Fig. 294). Such terminal

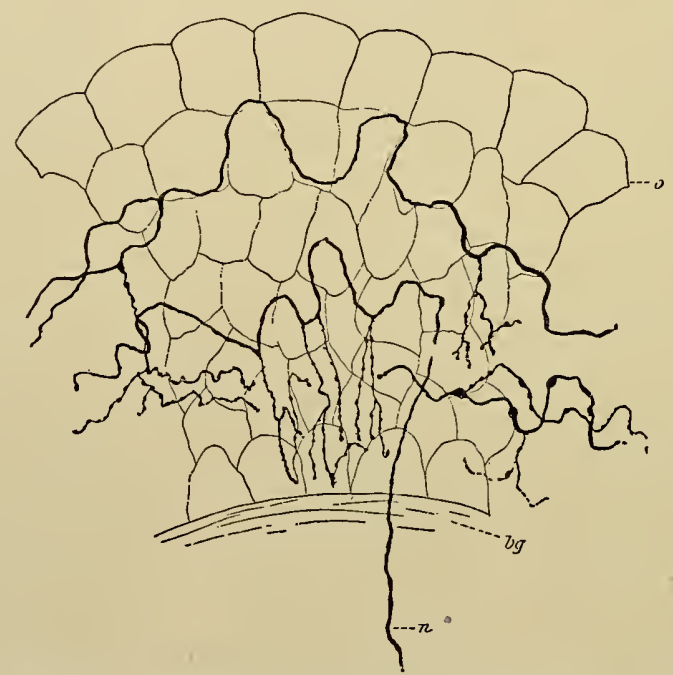

Fig. 294.-Free Endings of Afferent Nerve Fibres in Epithelium of Rabbit's Bladder. (Retzius.) $o$, Surface epithelium of bladder; $b g$, subepithelial connective tissue; $n$, nerve fibre entering epithelium where it breaks up into numerous terminals among the epithelial cells.

fibrils do not penetrate among the squamous cells. Similar "diffuse" endings are found in serous membranes and in simple epithelia, also in connective tissue. In the case of glandular epithelia such endings may, in part at least, be terminations of efferent (secretory) fibres from sympathetic ganglion cells (p. 439). Diffuse endings have also been described on endothelial surfaces such as endocardium and in smooth muscle. The latter are to be distinguished from the regular motor endings described below. An important form of diffuse ending is that found encircling and ending in the outer root sheaths of hair follicles (Fig. 295). Nerve endings are abundant in the pulp of teeth. There has been some dispute 
as to whether they penetrate the dentine. In addition to such comparatively simple nerve endings, there are also found in the skin and mucous membranes, especially where sensation is most acute, much more elaborate terminations. These may be classified as (I) tactile cells, (2) tactile corpuscles, and (3) end bulbs.

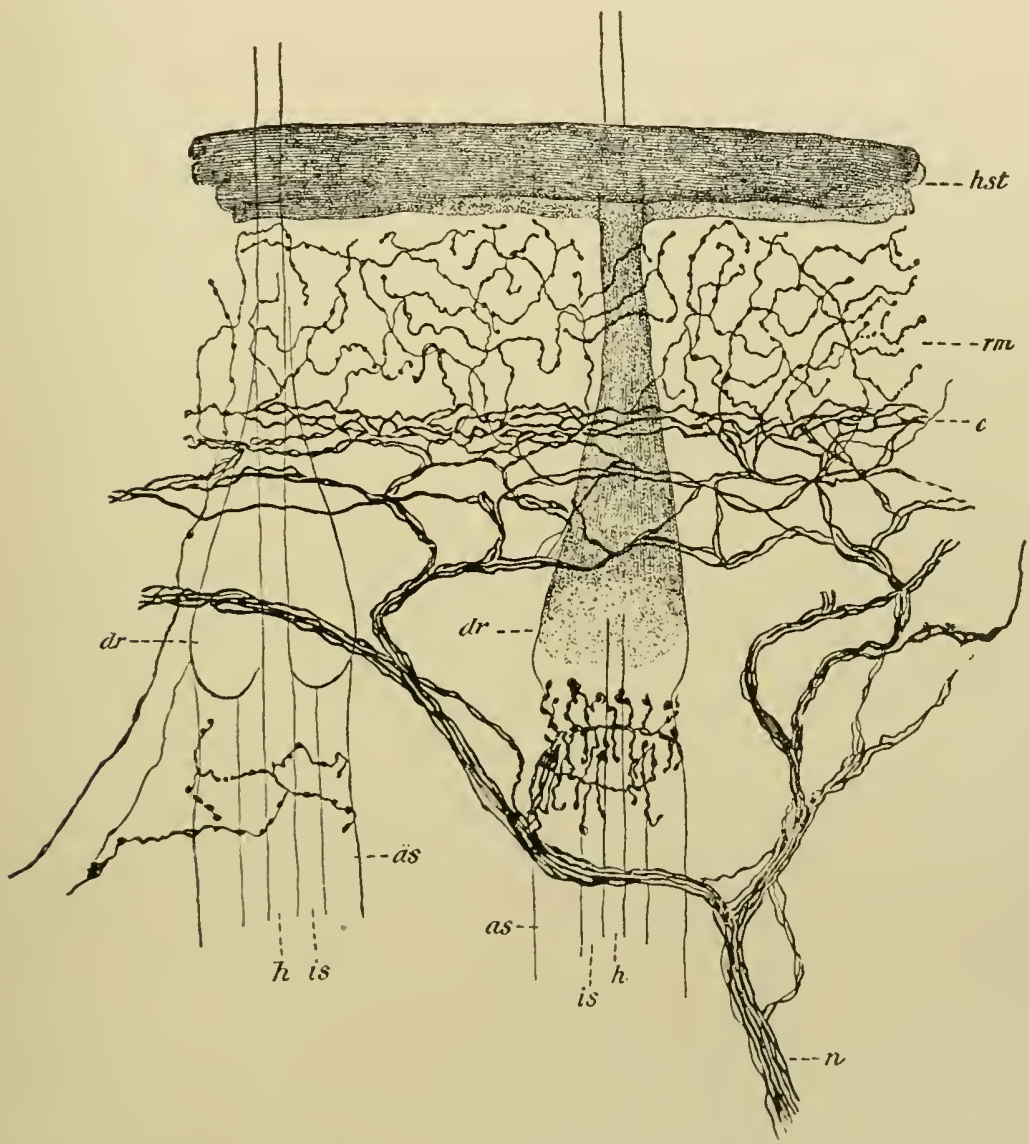

Fur. 295.-Nerves and Nerve Endings in the Skin and Hair Follicles. (After G. Retzius.) As, Outer root sheath; $c$, most superficial nerve-fibre plexus in the cutis: $d r$, schaceous glands; $h$, the hair itself; $h s t$, stratum corneum; is, inner root sheath of hair; $n$, cutaneous nerve; $r m$, stratum germinativum Malpighii.

A simple tactile cell is a single epithelial cell, the centrally directed end of which is in contact with a leaf-like expansion of the nerve terminal, the tactile meniscus. In the corpuscles of Grandry, found in the skin of birds, and in Merkel's corpuscles, which occur in mammalian skin, several epithclial cells are grouped together to 
receive the nerve terminations. These are known as compound tactile cells, the axis cylinder ending in a flat tactile disc or discs between the cells.

Of the tactile corpuscles (Fig. 296) those of Meissner, which occur

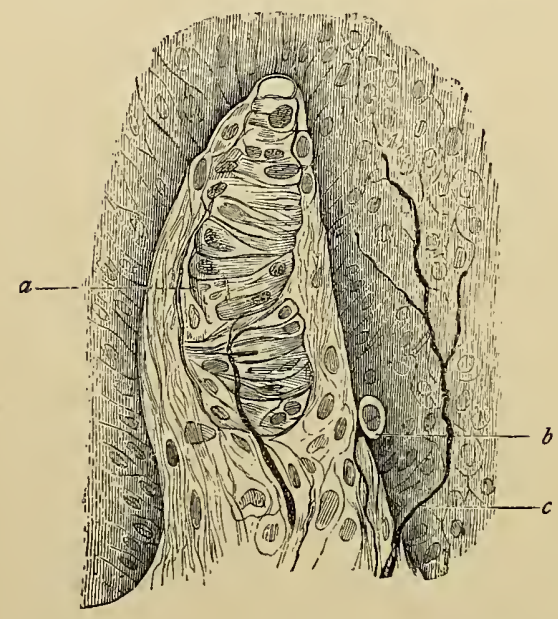

FIG. 296.

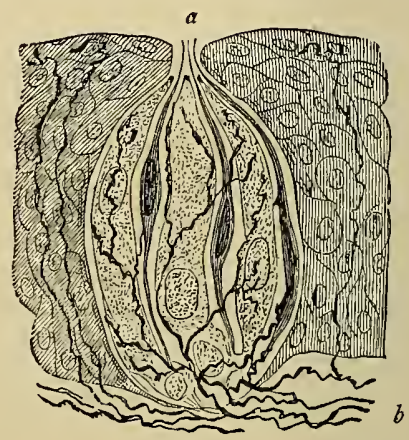

FIG. 297 .

FIG. 296.-Tactile Corpuscles of Meissner, tactile cell and free nerve ending. (Merkel-Henle.) a, Corpuscle proper, outside of which is seen in the connective-tissue capsule, $b$, fibre ending on tactile cell; $c$, fibre ending freely among epithelial cells.

FIG. 297.-Taste Bud from Circumvallate Papilla of Tongue. (Merkel-Henle.) a, Taste pore; $b$, nerve fibres entering taste bud and ending upon neuro-epithelial cells. On either side fibres ending freely among epithelial cells.

in the skin of the fingers and toes, are the best examples. These corpuscles lie in the papillæ of the derma. They are oval bodies, surrounded by a connective-tissue capsule and composed of flattened cells. From one to four medullated nerve fibres are distributed to

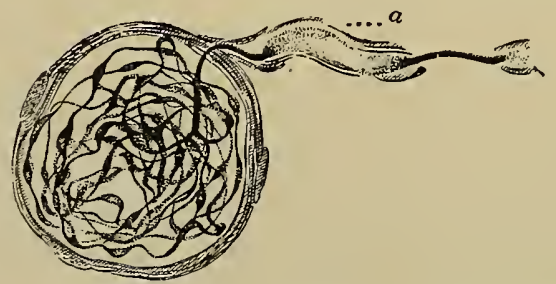

FIG. 298.-End Bulb from Conjunctiva. (Dogiel.) a, Medullated nerve fibre, axone of which passes over into dense end skein.

each corpuscle. As a fibre approaches a corpuscle, its connectivetissue sheath becomes continuous with the fibrous capsule, the medullary sheath disappears, and the fibrillæ of the terminal aborization pass in a spiral manner in and out among the epithelial cells. 
These terminal fibrils usually end in a flattened expansion consisting of neurofibrils and perifibrillar substance. Tactile corpuscles are also found on the volar surface of the forearm, eyelids, lips and tip of the tongue.

Of the so-called end bulbs, the simplest, which are found in the mucous membrane of the mouth and conjunctiva, consist of a central core formed by the usually more or less expanded end of the usually branched axis cylinder, surrounded by a mass of finely granular, nucleated protoplasm-the inner bulb-the whole enclosed in a capsule of flattened connective-tissue cells continuous with the sheath of Henle. Other end bulbs may be compound. End bulbs are found also in the mucous membrane of the tongue, epiglottis, nasal cavities, lower end of rectum, peritoneum, serous membranes, tendons, ligaments, connective tissue of nerve trunks, synovial membranes of certain joints and external genitals, especially the glans penis and clitoris. ${ }^{1}$

The Pacinian bodies (Fig. 299) are laminated, elliptical structures which differ from the more simple end bulbs already described, mainly in the greater development of the perineural capsule. The capsule is formed by a large number of concentric lamellæe, each lamella consisting of connective-tissue fibres lined by a single layer of flat connective-tissue cells. The

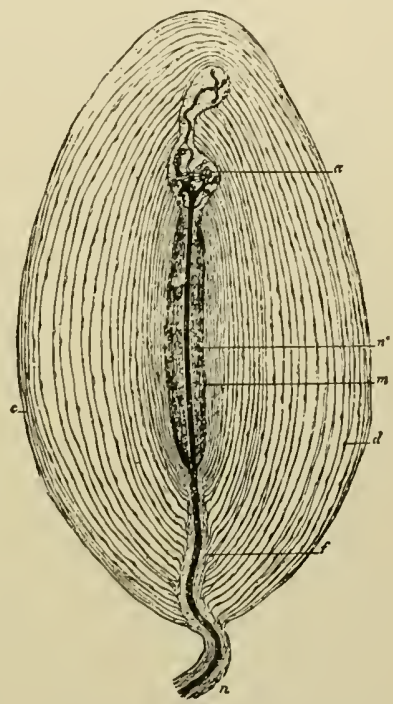

FIG. 299.-Pacinian Body from Mesentery of Cat. (Ranvier.) $c$, Lamina of capsule; $d$, epithelioid cells lying between laminæ of capsule; $n$, nerve fibre, consisting of axis cylinder surrounded by Henle's sheath, entering I'acinian body; $f$, perineural sheath; $m$, inner bulb; $n$, terminal fibre which breaks up at $a$ into an irregular bulbous terminal arborization. lamella are separated from one another by a clear fluid or semifluid substance.

As in the simpler end bulbs, there is a cylindrical mass of protoplasm within the capsule known as the inner bulb. Extending lengthwise through the centre of the inner bulb and often ending in a knol-like extremity is the axis cylinder. Arteries in the vicinity of the corpuscle break up into capillary networks surrounding the corpuscle.

1 This description of the clistribution of the various recoptors is principally taken from the excellent account in Schäfer's "T'ext-Brook of Microscopic Anatomy." $\Lambda$ n admiralile and still more detailerl account is to he found in "The Nervous System," by liarker. 
From this network capillaries enter the corpuscle, usually near the nerve fibre, and penetrate among the lamellæ, even reaching the distal end of the corpuscle. They do not enter the inner bulb. The Pacinian bodies are found in the subcutaneous fat, especially of the hand and foot, in the parietal peritoneum, mesentery, penis, clitoris, urethra, nipple, mammary gland, in the vicinity of tendons, ligaments and joints.

In voluntary muscle afferent nerves terminate in Pacinian corpuscles, in end bulbs, and in complicated end organs called muscle spindles, or neuromuscular bundles. The muscle spindle (Fig. 300) is an elongated cylindrical structure within which are muscle fibres, connective tissue, blood-vessels, and medullated nerves. The whole is enclosed in a connective-tissue sheath which is pierced at various points by nerve fibres. A single spindle contains several muscle fibres and nerve fibres. The muscle fibres differ from ordinary fibres, being fine and containing more nuclei and sarcoplasm in the middle. The muscle spindles are more numerous in the limb muscles than in those of the trunk, and in the distal than the proximal part of the limb. There are few in eye muscles and in some muscles they have not been detected. According to Ruffini, there are three modes of ultimate terminations of the nerve fibres within the spindles: one in which the end fibrils form a series of rings which encircle the individual muscle fibre, termed annular terminations; a second in which the nerve fibrils wrap around the muscle fibres in a spiral manner-spiral terminations; a third in which the terminations take the form of delicate expansions on the muscle fibre-arborescent terminations.

At the junction of muscle and tendon are found the elaborate afferent terminal structures, known as the muscle-tendon organs of Golgi (Fig. 30I). This is a spindle-shaped body composed of several tendon bundles. Into this there enter one or several nerve fibres which break up into complicated terminal arborizations upon the tendon bundles.

Other forms of nerve endings enclosed within connective-tissue sheaths are corpuscles of Ruffini found in the subcutaneous tissue of the finger and corpuscles of Golgi-Mazzoni found on the surfaces of tendons and also in the subcutaneous tissue of the fingers and in other parts of the skin.

It is evident from the above that the nerve terminations are only stimulated through the intermediation of surrounding cells which may form quite elab- 


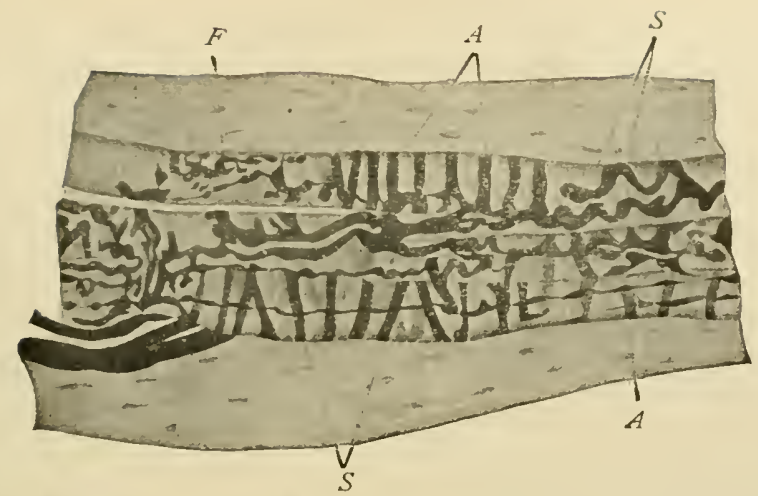

FIG. 300.-Middle Third of Muscle Spindle from Striated Voluntary Muscle of Cat. (From Barker, after Ruffini.) $A$, annular terminations; $S$, spiral terminations; $F$, arborescent terminations.

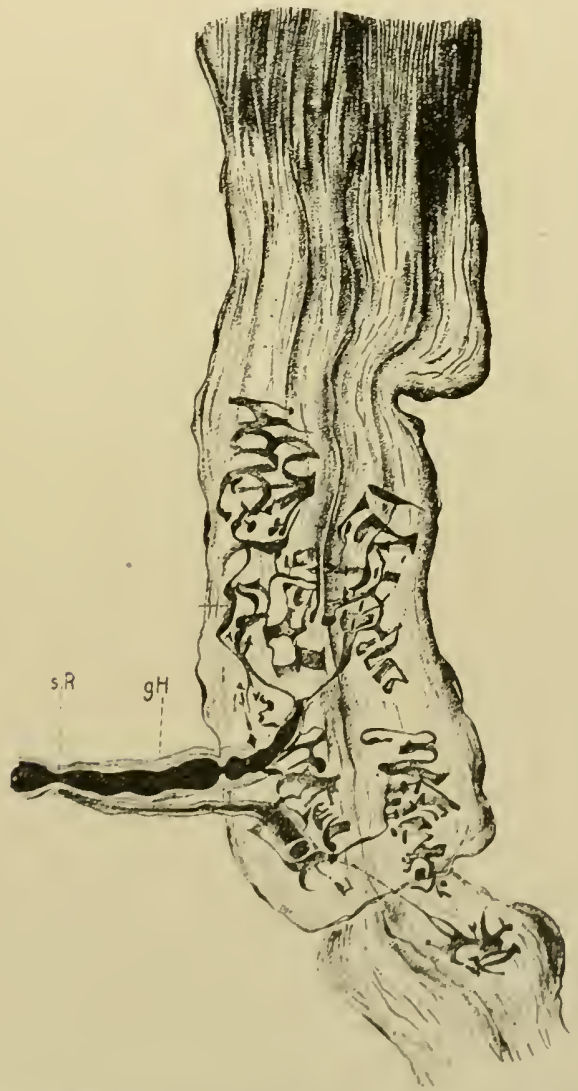

Jirs. 301 - Tenden of Muscle of liye of ()x. (ciacrio.) Two muscle-tendon organs of forlgi, each showing ring like and lirush like endlings. gh, sheath of Henle; sr, norle of Kanvicr. 
orate structures. These cells constitute the receptors and probably render the various nerve terminations they envelop more or less inaccessible to all but one particular kind of stimulus. The receptors described above are scattered throughout head and body (general or common senses) as distinguished from those which are concentrated into the organs of the special senses (smell, sight, hearing, taste) present only in the head (pp. 474, 475 and Chap. XIII). The various stimuli received by them may give rise to sensations of light pressure or touch (tactile cells and tactile corpuscles, terminations in hair sheaths), temperature and pain (diffuse terminations in epithelium and connective tissue?), muscle-tendon sense of movement and position (muscle spindles and possibly end bulbs, etc., in muscles, tendon organs of Golgi). These may be roughly grouped into general cutaneous or superficial sensation and deep sensation (muscle-tendon and other bodily sensations). All receptors, both of general and special senses, may be classified (Sherrington) as those receiving stimuli from the external world (exteroceptors, of superficial sensation, smell, sight, and hearing), those concerned with visceral reactions (intero-ceptors, including taste) and those giving information of bodily changes (proprio-ceptors, deep sensation).

\section{The CENTRAL PROCESSES OF THE CEREBRO-SPINAL GANGLION} CELIS enter the central nervous system as the fibres of the afferent root, the entire bundle of afferent root fibres of a single nerve consisting of all the central processes of the corresponding ganglion. Having entered the central nervous system, the central processes divide into ascending and descending arms, as already mentioned (p. 4I9). In the spinal cord the ascending arms are longer than the descending arms. In the brain the descending is usually the longer. These arms give off collaterals. Both collaterals and also the terminals of the arms enter the gray matter of the cord and segmental brain and terminate around various cell bodies and dendrites (terminal nuclei).

\section{THE SYMPATHETIC GANGLIA}

The sympathetic system of the neck and trunk consists of (I) a series of vertebral or chain ganglia, lying ventro-lateral to the vertebræ, connected with the cord by white rami communicantes and connected with each other by longitudinal cords, (2) of gangliated prevertebral plexuses connected with the vertebral ganglia and also connected with (3) ill-defined peripheral or terminal ganglia in the walls of the viscera (e.g., plexuses of Auerbach and Meissner). The sympathetic ganglia of the head are the ciliary, sphenopalatine, otic, and submaxillary.

The peripheral processes of certain spinal ganglion cells pass via 
the white rami communicantes to the vertebral ganglia and thence to visceral receptors. Axones of splanchnic efferent spinal neurones (preganglionic fibres) pass from the spinal cord via the ventral roots and white rami communicantes to terminate in various sympathetic ganglia. The axones of the cells of these ganglia (postganglionic fibres) complete the path by passing to the splanchnic effectors (heart muscle, smooth muscle or gland). Thus while the somatic efferent peripheral path consists of only one neurone, whose body is in the ventral gray of the cord or brain, and whose axone passes without interruption to the striated voluntary muscle, the splanchnic efferent peripheral path (except the branchiomotor) consists of two neurones. The first neurone body is in the gray of the cord or brain, the second neurone body is in a sympathetic ganglion. The preganglionic fibres emerging in the seventh cervical to the third lumbar spinal roots end either in the vertebral or prevertebral (cœliac, superior mesenteric, inferior mesenteric) ganglia. The vertebral ganglia send part of their axones to the peripheral nerves (grayrami communicantes), thence passing to superficial splanchnic effectors (smooth muscle of hairs and of superficial blood-vessels and glands of skin). Other axones may pass to the head. The axones of the prevertebral ganglia pass to the glands and smooth muscle of the viscera (Fig. 302). The other segments of the cord do not send out white rami communicantes except the second, third and fourth sacral. These fibres do not communicate with vertebral ganglia but terminate in prevertebral (pelvic) ganglia, which in turn send axones to the lower colon, rectum, bladder and genitals. In the head, preganglionic fibres emerge with the third nerve and pass to the ciliary ganglion, thence as postganglionic fibres to the ciliary and sphincter iridis muscles of the eye; others emerge with the seventh and pass to the sphenopalatine and submaxillary ganglia; others with the ninth and pass to the otic ganglion. Many of the axones of these ganglia pass to the salivary gland, others may be vasomotor. The vagus nerve sends preganglionic fibres to most of the viscera. These fibres probably terminate in the peripheral sympathetic ganglia. There is thus a cranial (III, VII, IX and X nerves), thoraco-lumbar and sacral outflow of preganglionic filores. Most visceral structures appear to receive a double innervation, one from the thoraco-lumbar and one from either the cranial or sacral system. By some writers the term sympathetic has been restricted to the thoraco-lumbar, the two others being termed autonomic. 


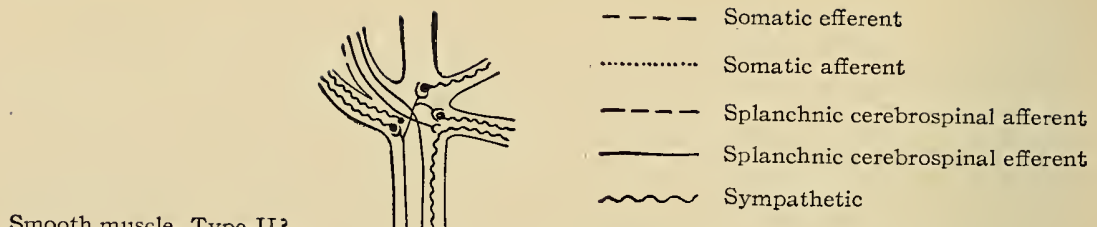

Smooth muscle Type II?

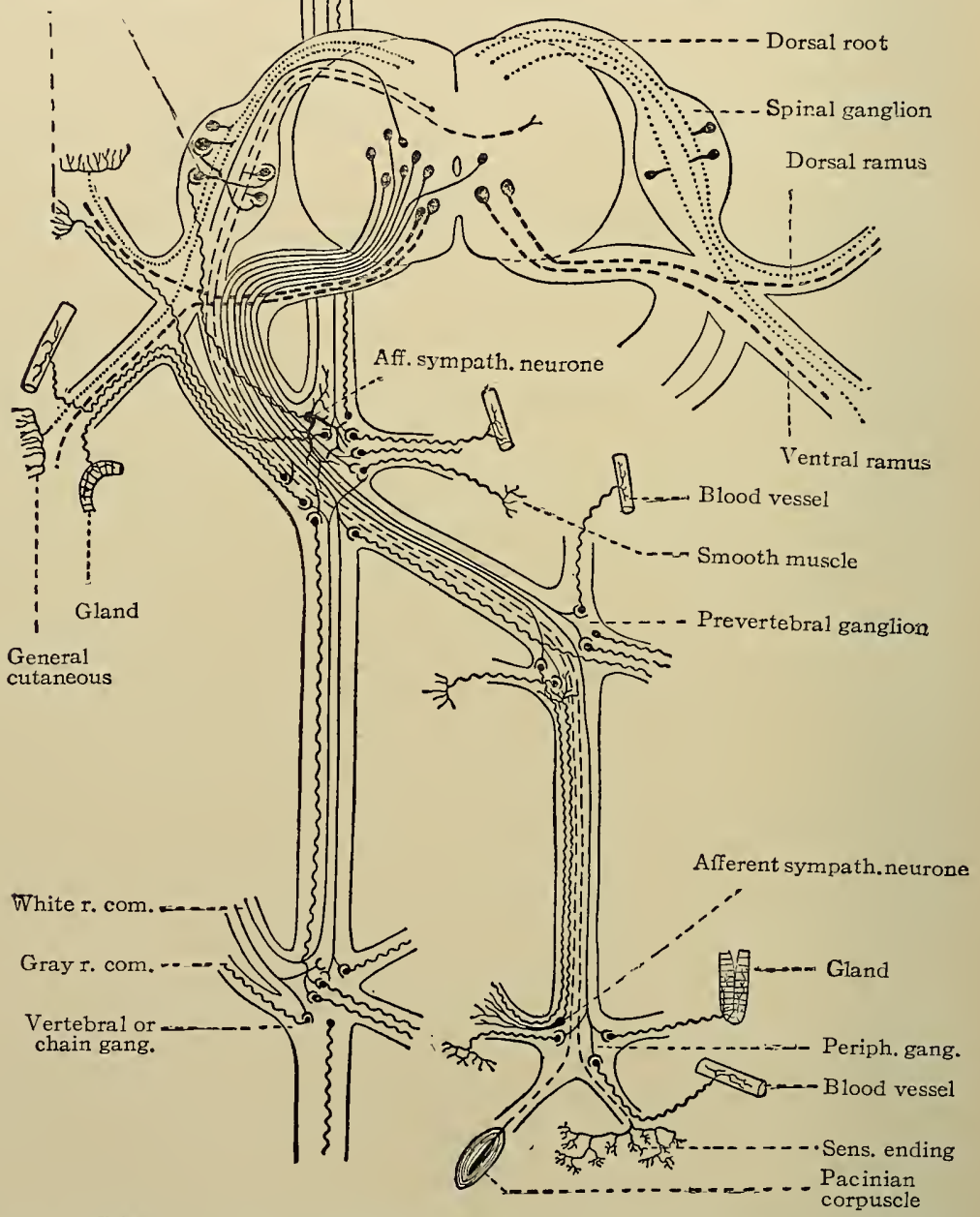

FIG. 302.-Diagram of the Sympathetic System and the Arrangement of its Neurones in a Mammal. On the left are shown the typical elements of a trunk segment including the sympathetic system. On the right are shown only the somatic afferent and efferent neurones of the spinal nerve. Of the sympathetic system are shown the white and gray rami, three ganglia of the chain, one prevertebral ganglion and one peripheral ganglion. The symbols used are explained in the figure. In most respects the diagram follows one of Huber's figures. (Johnston.)

It will be noted that the sympathetic outflow from any particular cord segment may ultimately reach other segments of the body. 
Some of the sympathetic cells are probably afferent but the majority are efferent. The fibres of the white rami communicantes are mostly fine and medullated. The axones of the sympathetic cells are fine and non-medullated or thinly medullated. For further details see Fig. 302.

The larger ganglia resemble the spinal ganglia in having a connective-tissue capsule and framework. The cells are smaller and often densely pigmenter. Each cell is surrounded by a capsule of cells

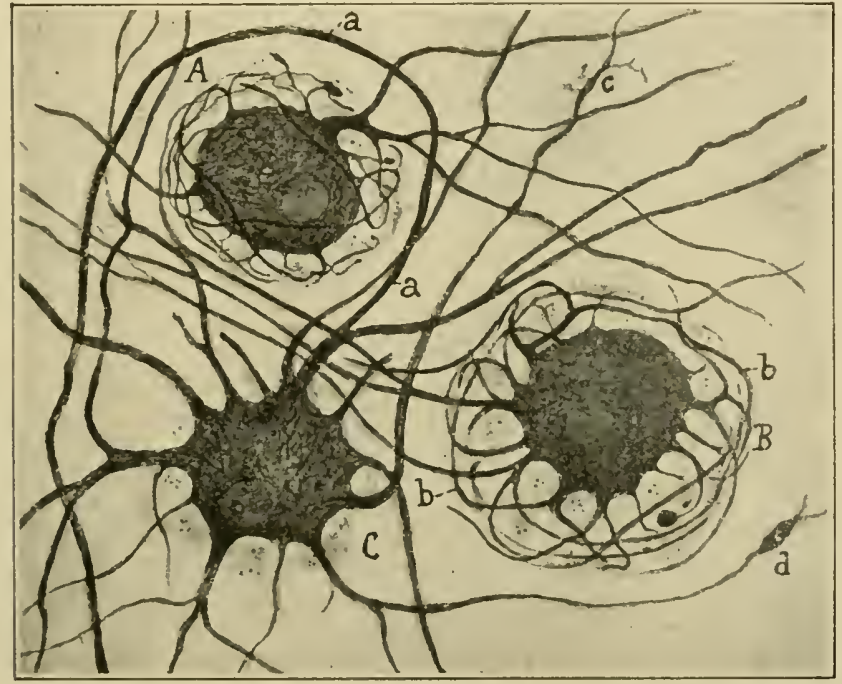

FIG. 303.- Sympathetic Nerve Cells (woman of 36 years). (Cajal.) $A$ and $B$, cells whose dendrites $(b)$ form a pericellular plexus. $C$, cell with long dendrites. $a$, axone; $c$ and $d$, terminal part of a dendrite. The capsular cells are faintly indicated. (Cajal's silver stain.)

similar to those surrounding the spinal ganglion cells. Often two or three cells and their interlocked dendrites are enclosed within a common capsule (Fig. 304, A).

The typical sympathetic nerve cell is a multipolar cell with short branching dendrites confined to the capsule of the cell and of ten interlocker with the dendrites of adjacent cells, forming glomeruli. Sometimes a dendrite will pass some distance from the cell, arborize, and interlock with a similar dendritic arborization of another cell. Another form of sympathetic cell has long slender dendrites often indistinguishable from axones. These cells are more frequent in the 
peripheral ganglia. Some of these cells have been considered to be afferent sympathetic cells. (Figs. 303 and 304.)

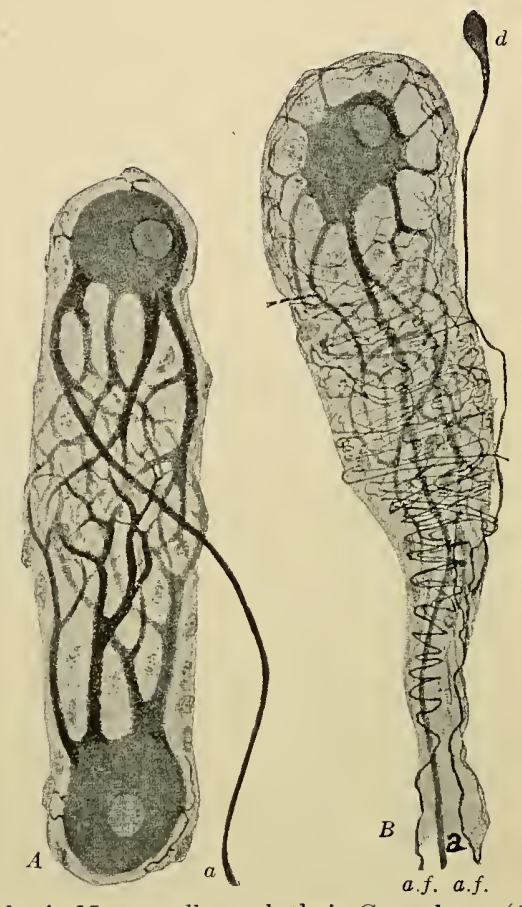

FIG. 304.-Sympathetic Nerve-cells and their Capsules. (Cajal.) $A$, Two-celled glomerulus; $B$, cell surrounded with the pericellular terminal arborizations of two fibres (a.f.) passing to the cell; $a$, axones; $d$, fibre, probably dendritic, with bulbous termination. (Cajal's silver stain.)

The sympathetic cells receive fibres which form arborizations around and within their capsules and also around the long dendrites.

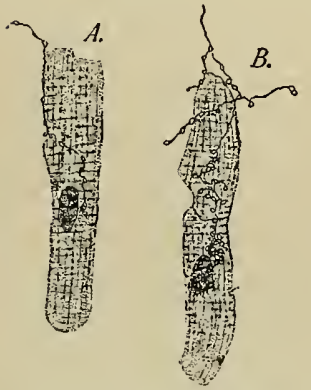

FIG. 305.-Nerve Endings on Heart Muscle Cells. (From Barker, after Huber and De Witt.)
Many of these are terminations of the visceral cerebro-spinal efferent neurones, $i e$. , the preganglionic fibers (Figs 302 and $304, B$ ).

As already noted, the axones of the efferent sympathetic cells terminate in heart muscle (cardio-motor), in smooth muscle of viscera (viscero-motor), of blood-vessels (vaso-motor) and of hairs (pilomotor), and in glandular epithelia (secretory). In heart muscle (Fig. 205) and in smooth muscle (Fig. 306) the nerves of the sympathetic system end in fine feltworks of fibres, which are in relation with 
the muscle cells. Satisfactory differentiation between efferent terminals and afferent terminals in heart and in smooth muscle has not yet been made.

In organs whose parenchyma is made up of glandular epithelium, the sympathetic nerves terminate mainly in free endings which lie in the cement substance between the cells, thus coming in contact with, though not penetrating, the epithelial cells.

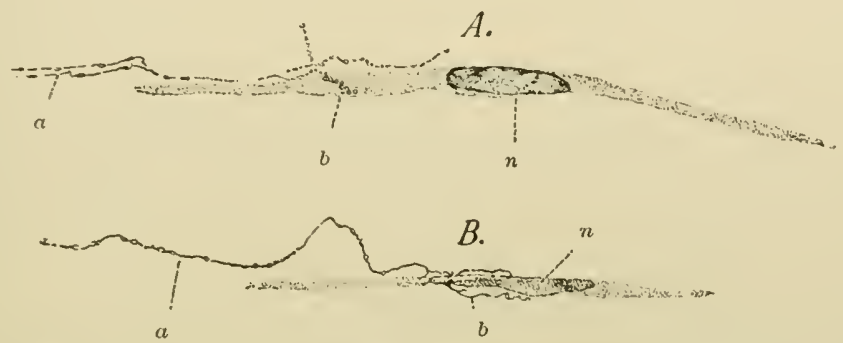

FIG. 306.- Nerve Endings on Smooth Muscle Cells. (From Barker, after Huber and De Witt.) $a$, Axis cylinder; $b$, its termination; $n$, nucleus of muscle cell.

\section{TECHNIC}

(I) Fix spinal and sympathetic ganglia in formalin-Müller's fluid (technic 5 , p. 7). Stain sections with hæmatoxylin-eosin (technic I, p. 20), or with hæmatoxylin-picro-acid-fuchsin (technic 3, p. 2I).

(2) Fix spinal and sympathetic ganglia in absolute alcohol or in ro-per-cent. formalin, and stain sections by Nissl's method (technic, p. 38).

(3) See also technic I, p. 446.

(4) Ganglia should also be prepared by Cajal's silver method, using the alcohol fixation (p. 37).

\section{EFFERENT PERIPHERAL CEREBRO-SPINAL NEURONES}

It has been seen that the bodies of these neurones lie in the ventral part of the neural tube where they form a part of the ventral gray column in the cord and the "motor" nuclei of cranial nerves in the segmental brain. The axones of these cell bodies emerge as the efferent roots and usually either pass (via the white ramus communicans, in spinal nerves) to various sympathetic ganglia to terminate there, or proceed as the efferent fil)res of the peripheral nerves to terminate in the striated voluntary muscles of the body and head. In the spinal nerves these fibres pass beyond the spinal ganglia and then join the afferent fibres; in some cranial nerves they pass out with the afferent fibres. On their way to the muscles the motor axones may bifurcate several times, thus allowing one neurone to innervate 
more than one muscle fibre. In the perimysium the nerve fibres undergo further branching, after which the fibres lose their medullary sheaths and pass to the individual muscle fibres. Here each fibre breaks up into several club-like terminals. These terminals are imbedded in a nucleated protoplasmic mass on the outside of the muscle fibre and probably composed of sarcoplasm. It is stated that the neurilemma of the nerve fibre is continous with the sarcolemma and that a continuation of the sheath of Henle covers the

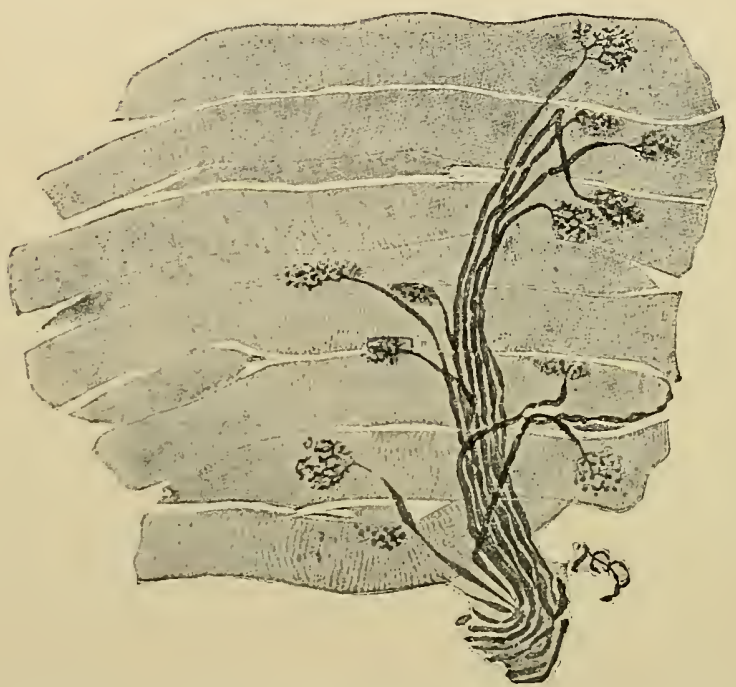

FIG. 307.- Motor nerve-endings in abdominal muscles of a rat. Gold preparation. Xi 7o. (Szymonowicz.)

whole structure which is known as the motor end plate. As a rule each muscle fibre is supplied with a single end plate, though in large fibres there may be several. (Fig. 307.)

\section{THE SPINAL CORD}

The spinal cord encased in its membranes lies loosely in the vertebral canal, extending from the upper border of the first cervical vertebra to the middle or lower border of the first lumbar vertebra. It is cylindrical in shape and continuous above with the medulla oblongata, while below it terminates in a slender cord, the filum terminale. At two levels, one in its cervical and one in its lumbar region, the diameter of the cord is considerably increased, These are known, respectively, as the cervical and lumbar enlargements. The spinal nerve roots leave the cord at regular intervals, thus indicating 
a division of the cord into segments, each segment extending above and below its nerve roots one-half the distance to the next adjacent roots. There are $3 \mathrm{I}$ segments corresponding to the 3 I spinal nerves; 8 cervical, I 2 thoracic, 5 lumbar, 5 sacral, and I coccygeal.

\section{Origin of the Fibres which Make up the White Matter of the Cord}

It has already been observed that the white matter of the cord is composed mainly of medullated nerve fibres, most of which run in a longitudinal direction. From the study of the neurone it follows that each of these fibres must be the axone of some nerve cell. The bodies of these cells, the medullated axones of which form the white matter of the cord are situated as follows:

A. Cells outside the spinal cord. (Extrinsic cells.)

B. Cells situated in the gray matter of the cord. (Intrinsic cells.)
(I) Cells outside the central nervous system (spinal ganglion cells).

(2) Cells in other parts of the central nervous system (the brain).

(3) Root cells, such as those of the anterior horn, whose axones form the ventral root (efferent peripheral neurones). These pass out directly, and thus do not become longitudinal column fibres.

(4) Intermediate neurones, whose axones enter into formation of the fibre columns of the cord (column cells.)

(5) Cells of Golgi, type II, the axones of which ramify in the gray matter. (These cells do not give rise to fibres of the white matter, but are conveniently mentioned here among the other cord cells.)

\section{(i) The Spinal Ganglion Cell and the Origin of the Posterior Columns}

It has already been seen that the central processes of these cells enter the cord as dorsal root fibres and split into ascending and descending longitudinal arms composing the greater part of the dorsal funiculus and the zone of Lissauer. They are described more in detail later (pp. $46 \mathrm{I}$ and ${ }_{4} 62$ ).

(2) Cells Situated in Other Parts of the Central Nervous System which Contribute Axones to the White Columns OF THE CORD.

These cells are situated in the motor areas of the cortex of the 
pallium, in the midbrain, cerebellum(?), and various parts of the segmental brain. The axones of these cells pass down the cord, forming the descending fibre tracts of the cord (p. 464). Collaterals and terminals of these fibres enter the gray matter of the cord to terminate there.

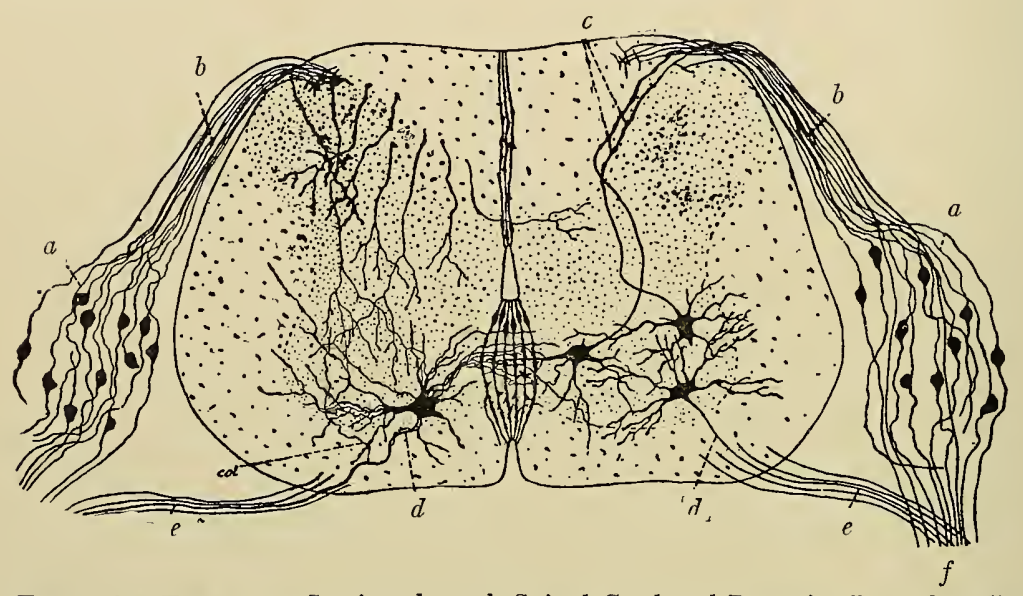

FIG. 308.- - Transverse Section through Spinal Cord and Posterior Root Ganglia of an Embryo Chick. (Van Gehuchten.) a, Spinal ganglion, its bipolar cells sending their peripheral processes outward to become fibres of the mixed spinal nerve $(f)$, their central processes into the dorsal columns of the cord as the dorsal root fibres $(b)$; within the posterior columns these fibres can be seen bifurcating and sending collaterals into the gray matter of the posterior columns, one collateral passing to the gray matter of the opposite side. The few efferent fibres of the dorsal root $(c)$ are disproportionately conspicuous. The large multipolar cells of the ventral horns are seen sending their axones $(d)$ out of the cord as the ventral root fibres $(e)$ which join the peripheral processes of the spinal ganglion cells to form the mixed spinal nerve $(f) ; c$ col, collateral from axone of ventral horn cell. Dendrites of the anterior horn cells are seen crossing the median line in the anterior commissure. About the centre of the cord is seen the central canal; dorsal and ventral to the latter some ependymal cells stretching from the canal to the periphery of the cord. (Golgi Method.)

\section{(3) Root Cells-Motor Cells of the Anterior Horn}

The course of the axones of these cells has been described (p. 44r). The bodies are described on pp. $45^{\mathrm{I}}$ and $45^{2}$.

\section{(4) Column Cells}

These are cells which lie in the gray matter of the cord and send their axones into the white columns or funiculi (see p. 450) where they either bifurcate or turn up or down, becoming longitudinal fibres. Some of the cells send their axones into the white matter of the same side of the cord. These are known as tautomeric cells (Fig. 309, 3). Others send their axones as fibres of the anterior commissure to the white matter of the opposite side of the cord- 
heteromeric cells (Fig. 309, I and 2). The axones of a few cross in the dorsal white commissure. In a few others the axone divides, one branch going to the white matter of the same side, the other to the white matter of the opposite side-hecateromeric cells (Fig. 309, 4).

The axones of many of these cells are short, constituting the short fibre tracts (fundamental columns-ground bundles) of the cord (see

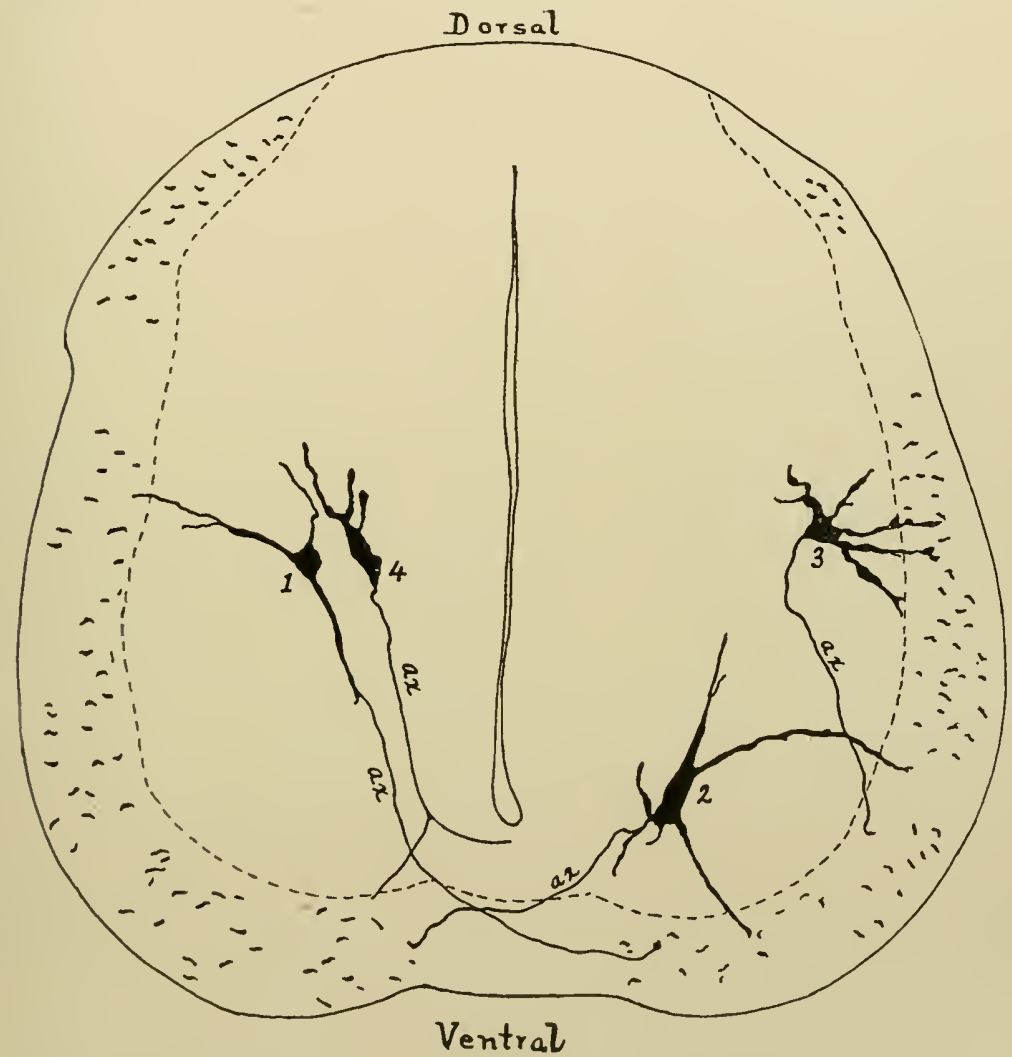

Fis. 309.-Transverse Section through Spinal Cord of Embryo Chick of Seven Days Incubation (Golgi Preparation). The boundary between the future gray and white matter is indicated by the dotted line. I and 2, Heteromeric column cells whose axones $(a x)$ pass through the anterior commissure to the opprosite ventral white columns; 3 , tautomeric column cells whose axone (ax) passes to the ventro-lateral white column on the same side; 4 , hecateromeric column cell whose axone $(a x)$ bifurcates, one branch entering the ventral white column of the same side and the other branch passing through the anterior commissure to the opposite side. $\Lambda$ number of longitudinil fibres in the white columns are shown, cut transversely or obliquely.

page 468; others form long ascending fibre tracts, passing up through the cord to the brain (see page 46r. 'Terminals and collateral branches of these longitudinal axones, especially of the short ones, are constantly re-entering the grily matter to end in arborizations around the nerve cells (Fig. 310 ). 


\section{(5) Cells of Golgi Type II}

The axones of these cells do not leave the gray matter, but

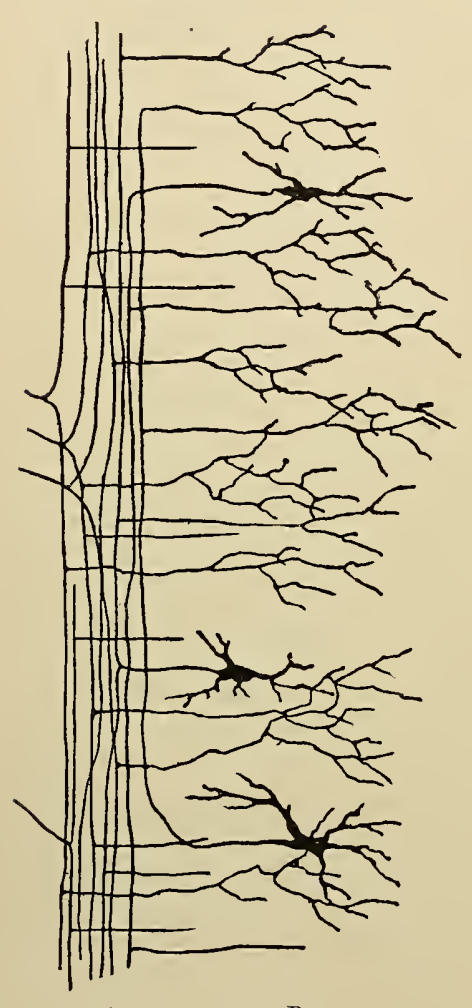

A

$B$

FIG. 310.-From Longitudinal Section of Spinal Cord of Embryo Chick. (Cajal.) $A$, White column of cord; $B$, gray matter. The cells of the gray matter (column cells) are seen sending their axones into the white matter, where they bifurcate, their ascending and descending arms becoming fibres of the white column. The dendrites of these cells are seen ramifying in the gray matter. To the left are seen fibres (posterior root fibres) entering the white matter and bifurcating, the ascending and descending arms becoming fibres of the white column. From the latter are seen fibres (collaterals and terminals) passing into the gray matter and ending in arborizations (Golgi Method). divide rapidly and terminate in the gray matter near their cells of origin, some crossing to terminate in the gray matter of the opposite side.

\section{TECHNIC}

(I) For the purpose of studying the spinal ganglion cell with its processes and their relations to the peripheral nerves and to the cord, the most satisfactory material is the embryo chick of six days' incubation, treated by the rapid silver method of Golgi (technic b, p. 35). Rather thick $\left(75^{\mu}\right)$ transverse and longitudinal sections are made and mounted in balsam without a cover-glass. Owing to the uncertainty of the Golgi reaction, several attempts are frequently necessary before good sections are obtained.

(2) The root cells of the anterior horn with their axones passing out of the cord and joining the peripheral processes of the spinal ganglion cells to form the spinal nerves, can usually be seen in the transverse sections of the six-day embryo chick cord prepared as above, technic (I).

(3) For studying the column cells of the cord, embryo chicks of from five to six days' incubation should be treated as in technic (I). Owing to the already mentioned uncertainty of the Golgi reaction, it is usually necessary to make a large number of sections, mounting only those which are satisfactorily impregnated. It is rare for a single section to show all types of cells. Some sections contain tautomeric cells, some contain heteromeric, some both, while in very few will the hecateromeric type be found.

Sections containing fewest impregnated cells frequently show collaterals to best advantage. These are seen as a fringe of fine transverse fibres crossing the boundary line between gray matter and white matter. 
(4) The silver method of Cajal, using the alcohol fixation (technic 2, p. 38), may also be advantageously used to display many of the above details of structure in embryo chicks. It is also capricious.

Whole neurones with long axones obviously cannot be directly demonstrated in single sections. To demonstrate these serial sections and indirect methods (degeneration, etc.) are used (see Fibre Tracts of Cord).

\section{PRACTICAL STUDY}

Transverse Section of Six-day Chick Embryo (Technic I, p. 446).--Using a lower-power objective, first locate the cord and determine the outlines of gray matter and white matter. Observe the spinal ganglia lying one on either side of the cord (Fig. 30S, $a$ ). At least one of the ganglia will probably show one or more bipolar cells, sending one process toward the periphery, the other toward the spinal cord. Note that the peripheral process is joined, beyond the ganglion, by fibres which come from the ventral region of the cord (fibres of the anterior root). In some specimens the latter can be traced to their origin in the cells of the anterior horn (Fig. 308, d). The union of the peripheral processes of the spinal ganglion cells and the anterior horn fibres is seen to make up the mixed spinal nerve (Fig. 308, $f$ ). Observe the central processes of the spinal ganglion cells entering the dorsal column of the cord and bifurcating (Fig. 308, b). As these branches pass up and down the cord, only a short portion of each can be seen in a transverse section. Note the fibres (collaterals) passing from the white matter into the gray matter. The fact that they are finer than the longitudinal fibres in the white matter shows that they are branches of the latter. Even in transverse section the point at which they leave the latter may occasionally be made out. Note in some of the sections a little round mass just ventral and to the inner side of the spinal ganglion, in which nerve cells may be seen, and some fibres passing into or out of it. This represents the beginning of the sympathetic system with its chain of ganglia. Note the relation which this bears to the spinal cord and spinal ganglia.

In the same or other transverse sections study the column cells of the cord, carefully distinguishing between the dendrites and axone. This is not always easy', but the axone can usually be distinguished as being more slender, with smoother outline and more uniform size throughout its course. Axones may come off from dendrites as well as from the cell bodies. At least one tautomeric and one heteromeric column cell shoulcl be found and studied (Fig. 309). Study also the collaterals if they are stainerl. Remember that only a few of the elements present are stained in Golgi preparations and that there are apt to be present irregular silver precipitates without any significance. Cappillaries often appear as a coarse brown-staincel meshwork.

Study also any spongiolblists that maty be stainerl. Those with nuclei near the eentral canal give at fair representation of the ependymat cells of the adult cord, except the cell does not usually in the latter extend entirely through the wall of the neural tube.

Longitudinal Section of Six-day Chick Embryo (Technic 1, p. 446).-Using a low-power objective locate gray matter and white matter and identify plane 
of section relative to transverse section above described. Note in the white matter longitudinally-running fibres from which branches pass off into the gray matter (Fig. 310). The longitudinal fibres of the posterior columns are the ascending and descending branches of the central processes of the spinal ganglion cells, and the branches passing into the gray matter are their collaterals and terminals. If the section happens to include the entering fibres of a posterior root, these can be seen branching in the posterior columns into ascending and descending arms (Fig. 3 Io). The longitudinal fibres of the lateral and anterior columns are axones of column cells and of cells situated in higher centres (see pages 444 and 445 ). These also send collaterals and terminals into the gray matter.

\section{General Topography of the Cord, Cell Groupings, Arrangement of Fibres and Finer Structure}

The further description of the cord is best combined with the practical study of sections of the cord, taking sections through the lumbar enlargement as a type.

\section{PRACTICAL STUDY OF SECTIONS THROUGH LUMBAR ENLARGEMENT}

General Topography (Figs. 3II and 3I2). -The general features of the sections can be best seen with the naked eye or with a low-power dissecting lens.

Note the shape and size of the cord, and that it is surrounded by a thin membrane, the pia mater spinalis; the deep anterior median fissure, into which the pia mater extends; the posterior median septum consisting principally of neuroglia, over which the pia mater passes without entering; and the postero-lateral grooves or sulci at the entrance of the posterior root fibres. The gray matter is seen in the central part of the section (stained more lightly in the Weigert preparation, on account of the presence of numerous unstained cell bodies and dendrites) and arranged somewhat in the form of the letter $\mathrm{H}$. Dorsally the gray matter extends almost to the surface of the cord as the dorsal gray columns (posterior horns or cornua). The ventral gray columns (anterior horns) are, on the other hand, short and broad, and do not approach the surface of the cord. Surrounding the gray matter is the white matter (stained deep blue in the Weigert preparation). This is divided by the posterior horn into two parts, one lying between the horn and the posterior median septum, the posterior funiculus (dorsal white column); the other comprising the remainder of the white matter, the antero-lateral funiculus (antero-lateral white column). This latter is again divided by the anterior horn and anterior nerve roots into a lateral funiculus (lateral white column) and an anterior funiculus (ventral white column). In the concavity between the anterior and posterior horns some processes of the gray matter extend out into the white matter where they interlace with the longitudinally running-fibres of the latter to form the reticular process (not well marked in the lumbar cord).

For the study of further details the low-power objective should be used. 
GRAY MATter.-In the cross portion of the $\mathrm{H}$ is seen the central canal, usually obliterated in the adult and represented only by a group of epithelial cells. The central canal divides the gray matter connecting the two sides of the cord into a central gray commissure and a dorsal gray commissure. Inmediately surrounding the epithelial cells is a light granular area composed mainly. of neuroglia and known as the central gelatinous substancc. Toward the surface of the

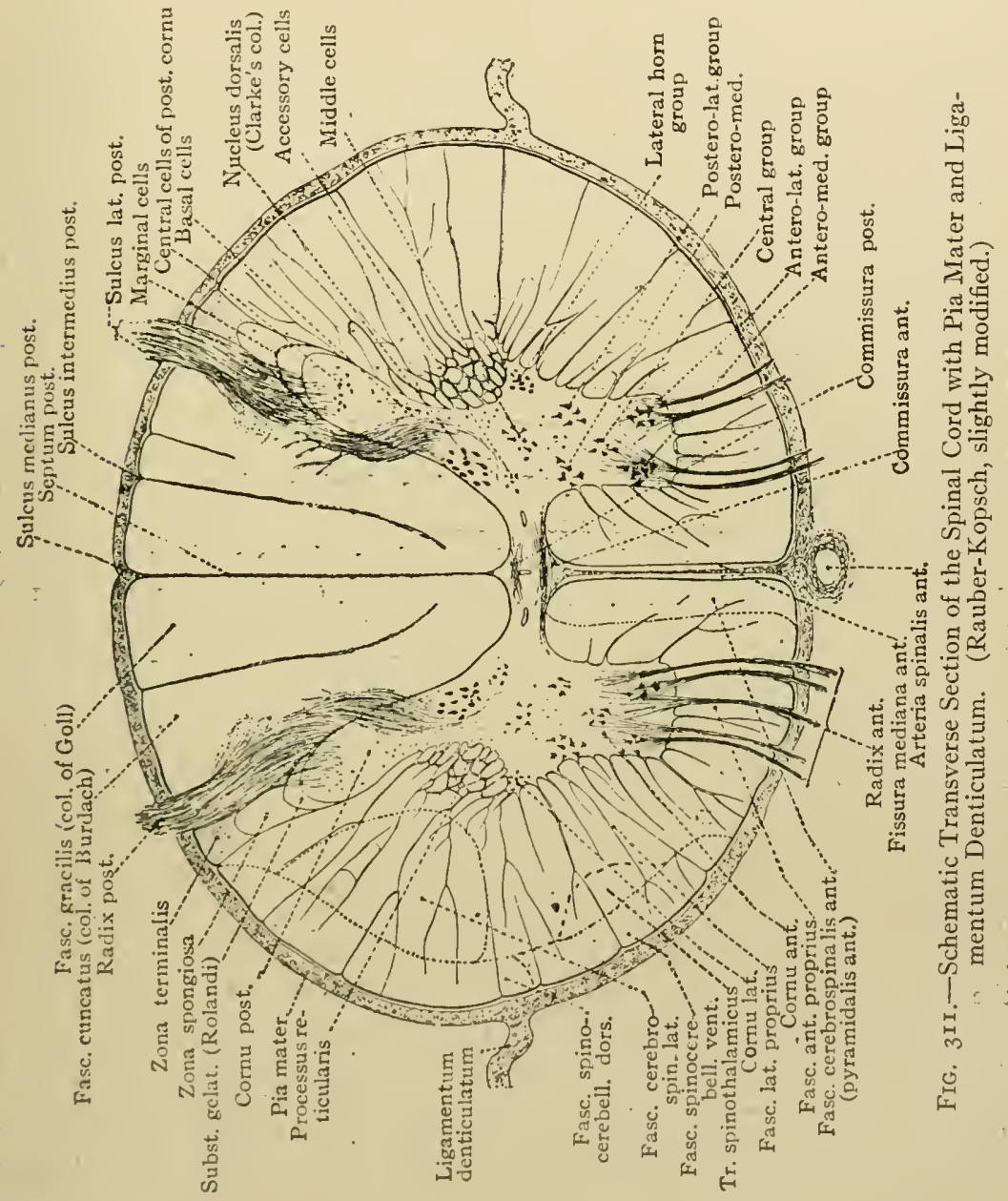

cord the posterior horn expands into a head or caput, external to which is an arca similar in general appearance to that surrounding the central canal, the gelatinous substance of Rolando. 'The head is connected with the base of the dorsal horn by a narrower neck or cervix. External to (he gelatinous substance of Rolindo is a thin zone containing a plexus of fine medullated fibres (W'eigert stain) known as the marginal zone or zona spongiosa, and external to this, occupying the space 
between it and the periphery, is a zone composed of fine longitudinal medullated fibres rather sparsely arranged and therefore staining more lightly with the Weigert method. This is the zona terminalis or zone of Lissauer. It belongs obviously to the white matter of the cord (see page 452). The portion of the gray matter connecting the dorsal and ventral horns may be termed the intermediate or middle gray. Note the well-defined groups of large nerve cells in the anterior horns and the fibres passing out from the anterior horns to the surface of the cord, ventral (anterior) nerve roots. (Figs. $3 \mathrm{II}$ and $3 \mathrm{I} 2$.)

White Matter.-Note the general appearance of the white matter and the disposition of the supporting strands of neuroglia tissue (light in the Weigert, usually darker in other stains). The neuroglia is seen to form a fairly thick layer just beneath the pia mater from which trabeculæ pass in among the fibres, the broadest strand forming the posterior median septum. If the section has been cut through a dorsal (posterior) nerve root, a strong bundle of dorsal root fibres can be seen entering the white matter of the cord along the dorsal and mesial side of the posterior horn. Just ventral to the anterior gray commissure is a bundle of transversely-running medullated fibres-the anterior white commissure. (Fig. 3I 2.) It is composed of the axones of various heteromeric column cells and of decussating terminals of various fibres. In the dorsal part of the dorsal gray commissure are also a few fine transversely running medullated nerve fibres - the dorsal white commissure. It consists of collaterals of fibres in dorsal funiculi and axones of heteromeric column cells.

Cell Groupings.-The positions and groupings of the various cell bodies should now be studied. (Nissl, Hæmatoxylin-Eosin, Cajal, Figs. 3I I and 3I2.) (For convenience, their general arrangement throughout the cord is here given; also the course of their axones, though this is usually only seen in Golgi preparations.)

(A) Cells of the Dorsal Horn.-(a) Marginal cells arranged tangentially to the border of the gelatinous substance of Rolando. (b) Cells in the gelatinous substance of Rolando, arranged radially. The Golgi method indicates that the axones of (a) and (b) principally enter the adjoining lateral column. (c) Large stellate cells in the apex of the caput, most of the axones of which go to the lateral columns, but some cross in the ventral white commissure. (d) A central group in the central part of the dorsal cornu, some of the axones of which may cross in the ventral commissure. (e) Basal cells in the base of the horn and in the processus reticularis, the axones of which usually go to the lateral column but may cross. (f) Dorsal (thoracic) nucleus or cells of Clarke's column in the mesial part of the base of the dorsal horn. These are tautomeric cells, the axones of which from the dorsal spino-cerebellar tract (see page $46_{3}$ ). Clarke's column is mainly confined to the dorsal or thoracic cord, but is also present in the first lumbar segment.

(B) Cells of the Intermediate Gray.-(a) Middle nucleus whose cells may send their axones across in the ventral commissure or uncrossed to the lateral column; (b) various small cells including the accessory nucleus near Clarke's column; (c) intermedio-lateral group which in parts of the cord forms a projection of the gray known as the lateral horn. These are probably root cells whose axones pass as preganglionic fibres into the sympathetic system. This nucleus. 
is more conspicuous in the thoracic cord, but extends from the seventh or eighth cervical to about the third lumbar segment and is also present in the sacral cord (especially the third segment). (pp. 456,457 and 458 .)

From the above it is seen that all the cells of the dorsal and intermediate gray, except the intermedio-lateral group, are column cells, either tautomeric, heteromeric or hecateromeric.

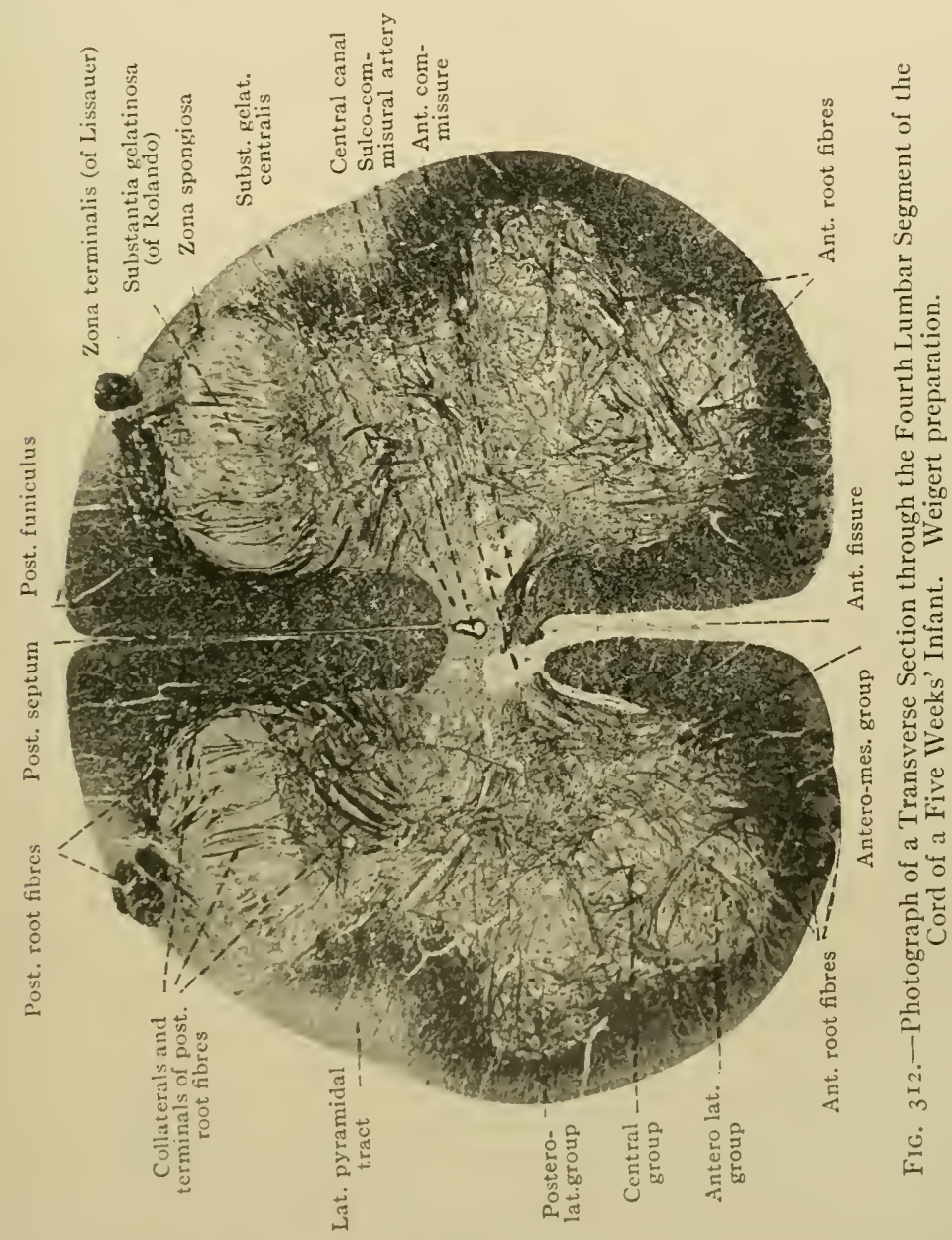

(C) Celis of the Vhitrat. Horn.-These fall into two calegories: (i) Column Cells. These may be tautomeric, sending axones to the adjoining white matter or heteromeric, the axones of which cross in the ventral commissure to the white matter of the opposite side. Among the latter may lec a well-marked group) in the dorso-mesial part of the horn (commissural nucleus). Hecateromeric cells may be present. (2) Rool cells. Two main divisions may be distinguished: (a) Mesial group, present throughout the cord above the fifth sicral segment 
(Bruce). This group probably innervates the striated voluntary (somatic) muscles of the trunk. The mesial group is in part of the cord subdivided into a ventro-mesial and dorso-mesial group, the latter being present in the first, sixth and seventh cervical, thoracic (except first), and first lumbar segments. (b) Lateral group, present in the cervical, first thoracic, and lumbo-sacral regions. This group innervates the muscles of the extremities and exhibits the following subdivisions: An antero-lateral $\left(\mathrm{C}_{4}\right.$ to $\mathrm{C} 8, \mathrm{~L}_{2}$ to $\left.\mathrm{S}_{2}\right)$, a postero-lateral $\left(\mathrm{C}_{4}\right.$ to $\mathrm{C} 8, \mathrm{~L}_{2}$ to $\left.\mathrm{S}_{3}\right)$ and a post-postero-lateral (C8 to $\mathrm{Thr}, \mathrm{Sr}$ to $\mathrm{S}_{3}$ ). There is also a central group $\left(\mathrm{L}_{2}\right.$ to $\left.\mathrm{S}_{2}\right)$ and a small anterior group ( $\mathrm{Lr}_{\mathrm{I}}$ to $\left.\mathrm{L}_{4}\right)$. The exact muscle groups innervated by these cell groups, respectively, have not yet been definitely determined. Other special cell groups are the phrenic group $\left(\mathrm{C}_{4}\right)$, centrally located, cilio-spinal and other cells (C8 to Th2, to sympathetic ganglia which send fibres to dilator pupillæ and blood-vessels of head), and the spinal accessory ( $\mathrm{C}_{\mathrm{I}}$ to $\mathrm{C} 6$ ). The latter is located laterally and innervates the sternomastoid and trapezius muscles. In the lumbo-sacral cord below the fourth lumbar there is also a medio-ventral splanchnic group which together with the lateral horn group of the sacral cord furnishes the preganglionic fibres emerging from the sacral cord.

For the determination of the destination of the axones of the efferent root cells the method of studying the changes in the cell body (Nissl stain) in definite lesions of the peripheral fibres (axonal degeneration, see Chapter VI) is used.

Arrangement of Fibres (Fig. 3 I 2). -With the low-and high-power objectives the course of the transverse (i.e., longitudinally cut) nerve fibres should be carefully studied in Weigert and Cajal preparations. These fibres pass from gray to white matter or vice versa, and are in general (a) root fibres entering or leaving the cord; (b) either axones of column cells in the gray passing out into the white there to become longitudinal fibers by turning or splitting, or they are the collaterals and terminals of the fibres of the white matter entering the gray to terminate there.

The arrangement of these fibres should be carefully studied in all parts of the section (Weigert and Cajal), taking one field at a time. In the dorsal part of the cord, the dorsal roots can be seen entering. From their lateral portion fine fibres detach themselves and enter the zone of Lissauer, the fibres of which are largely composed of their short ascending and descending arms. Most of the fibres of the root pass along the dorsal and mesial side of the dorsal horn, forming the zone of entry of the dorsal roots. By bifurcating (not visible in the preparation) they become the majority of the longitudinal fibres of the dorsal funiculus. From the entering root fibres and fibres of the dorsal funiculus, bundles of fine fibres (collaterals and terminals) pass radially through the gelatinous substance of Rolando or sweep around its mesial side and enter the gray. Some of these terminate in the gelatinous substance of Rolando (Golgi preparations), some form part of the dense plexus of fibres in the caput and terminate there, others can be traced to the intermediate gray and, in some cases, some ("direct reflex collaterals") can be traced to the ventral horn. It will be noted that not many come from the mesial part of the dorsal funiculus. Collaterals from the zone of Lissauer enter the gelatinous substance of Rolando. In the middle part of the cord there is a similar interchange of fibres between 
the plexus in the gray and the adjoining white matter. In the ventral part of the cord a similar interchange takes place, but here besides these fine fibres are seen the coarse fibres of the ventral roots gathered from various parts of the ventral horn to form bundles which leave the ventral side of the horn, pass through the white matter and emerge as the ventral root fibres. The larger bundles of fibres in the ventral horn separate the cell groups, but between individual cells are seen numerous fine medullated fibres (principally terminals of fibres from the white funiculi). Trace as far as possible the course of the fibres of the ventral and dorsal white commissures.

Finer Structures. ${ }^{1}$ - Study with the high power the general histological structure of the gray and white matter. In the gray matter note (Cajal, Nissl, H.-E.), besides the nerve cells and their processes, the neuroglia nuclei. Note also the structure and size of the medullated nerve fibres (Weigert). In the white matter

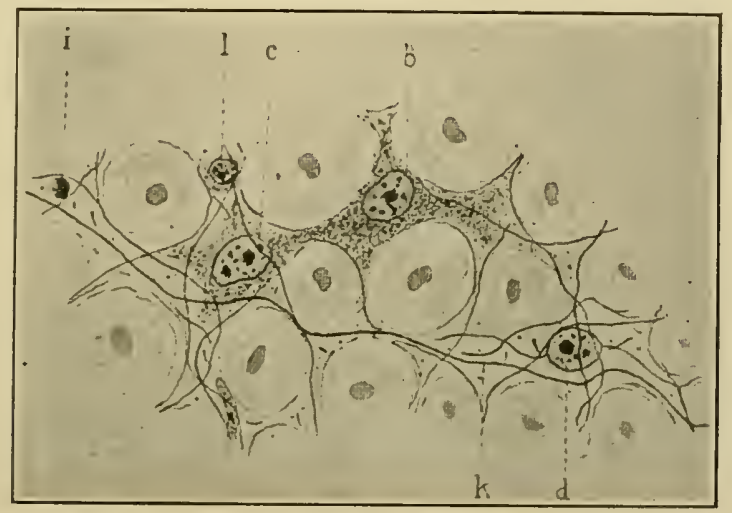

FIG. 313.-Firom Transverse Section of Elephant's Cord. (Hardesty.) Benda's Neurcglia Stain. $b, c, d$ and $i$, Four types of ncuroglia cells; $k$, neuroglia fibre passing through several neuroglia cells; $l$, leucocyte.

note the appearance of the cross-cut medullated nerve fibres in Weigert, Cajal and H.-E. preparations. With the neuroglia stains study carefully the neurogliu cells and neuroglia fibres, including the neuroglia zone forming the margin of the cord. (Fig. 313.) Note also the pia mater and the connective-tissue septa (usually perivascular) entering the cord from the pia accompanied usually by a denser aggregation of glia fibres. Note carefully the number of neuroglia nuclei in some small field. Increase in neuroglia is characteristic of many pathological conditions. Study the ependyma. (Weigert, Nissl, Cajal and glia stains).

Study the internal structure of the nerve-cells of various sizes present, especially the amount and arrangement of the chromophilic substance (Nissl). The smallest nerve cells of the ecord have a limited amount of chromophilic substance, often either in the form of perinucleatr capss or small bodies near the periphery

1 It may sometimes be advantagesus to have the high power sturly of the general histological structure of the gray and white matter of the cord precele the sturly of the architectural arrangements of the cord here placed first. 
of the cell. In the medium cells more chromophilic bodies are present. The cells of Clarke's column have a considerable number of chromophilic bodies arranged near the periphery of the cell. The root cells are richest in chromophilic substance (for further details see Chap. VI). In general it seems that the intermediate neurones belonging to efferent paths (i.e., acting on peripheral motor neurones) have the definite, clear-cut. coarse chromophilic granules characteristic of the peripheral motor neurones; while the neurones forming parts of afferent paths have the fine, indefinitely grouped granules characteristic of the cerebro-spinal ganglionic neurones (Jacobsohn and Malone).

Blood-vessels (Fig. 3 I 4.- -) Study the arrangement and structure of the bloodvessels of the cord and pia. There are three principal longitudinal arteries, the anterior spinal artery given off from the vertebral arteries near their union

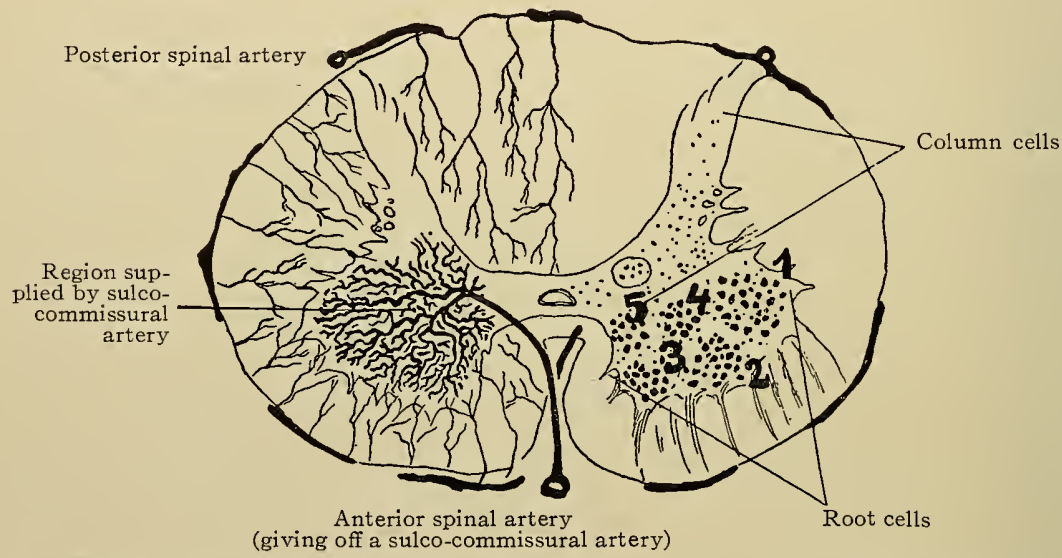

FIG. 3I4.--Schematic transverse section of Cord, Showing General Distribution of Blood-vessels (left) and Nerve-cells (right) (Bing). Root-cells; I, postero-lateral group; 2, antero-lateral group; 3 , antero-medial group; 4, central group; 5, postero-medial group. The broken black lines on the surface of the cord are portions of the vascular network in the pia mater.

into the basilar artery, and two posterior spinal arteries, given off also from the vertebral arteries. These arteries are reënforced by small arteries passing to the cord along the dorsal and ventral roots and form an arterial network in the pia mater. From the network terminal (i.e., non-anastomosing) branches enter the cord, supplying all parts except the ventral horn and column of Clarke. The latter are supplied by branches from the anterior spinal artery which pass dorsally in the ventral sulcus (sulco-commissural arteries) and enter the cord alternately to right and left. They then break up into a rich capillary network in the ventral horn, supplying also a branch to the column of Clarke. The veins of the cord also form a plexus in the pia mater. Larger posterior median and anterior median veins can be distinguished. Portions of the above vessels can be seen, cut in various planes in the pia and in the cord. The general appearance and structure of the blood-vessels, including capillaries, should be noted in the various methods of staining. 


\section{Variations in Structure at Different Levels}

While the general structure above described obtains throughout the cord, the size and shape of the cord, the size and shape of the gray matter, and the relative proportion of gray matter and white matter, vary in different parts of the cord, which must therefore be separately considered. These variations are due to: (I) Variations in the size of the nerves entering and leaving, which cause corresponding variations in the gray matter which receives the afferent fibres and contains the cells of origin of the efferent fibres. Thus the larger nerves of the extremities cause the increase in size of the gray matter of the cervical and lumbo-sacral cord with which they are connected, and also an increase in the dorsal funiculi. (2) A gradual increase in the white matter of the cord, as higher levels are reached, due to an increase in the number of long ascending and descending fibres to and from the brain.

\section{PRACTICAL STUDY}

Section through the Twelfth Thoracic Segment (Fig. 316).-Note that the cord is smaller than in the lumbar enlargement and somewhat flattened dorsoventrally; that the amount of gray matter and white matter is diminished; that both anterior and posterior horns are more slender, the anterior horn containing comparatively few cells. At the inner side and base of the posterior horn may be scen the group of cells known as Clarke's column (p. 450). Medullated fibres can be seen passing from the dorsal funiculus into Clarke's column, where they interlace among the nerve cells. These fibres are collaterals of the dorsal root fibres terminating in the nucleus. From the nucleus coarser fibres can be seen gathering at its ventral side and thence passing outward to the periphery of the cord where they bend upward forming the beginning of the dorsal spino-cerebellar tract (see p. 463).

Section through the Mid-thoracic Region (Fig. 316).-Compare with the lumbar sections. Note the change in shape and size; that the cord is more nearly round and smaller; that while the rerluction in size affects both gray matter and white matter it is the former that shows the greater decrease. The horns are even more slender than in the twelfth thoracic section, and the anterior horn contains still fewer cells. Clarke's column is present, but not so large.

Section through the Cervical Enlargement (Fig. 315).-Note the markerl increase in size of the corl, which affects both gray matter and white matter. Dependling upon the exact level at which the section is taken, the cord may be nearly round or flattened dorso-ventrally. The posterior horns remain stender while the anterior are much broarler than the posterior horns. The reticular process is more prominent than in any of the previous sections. As in the lumbar cord, the cell groujes of the anterior horn are numerous and well defined. A more or less 


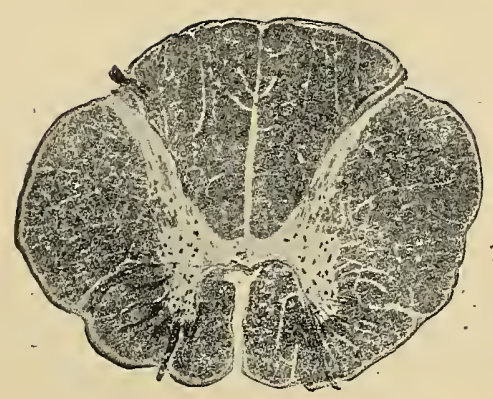

C. II

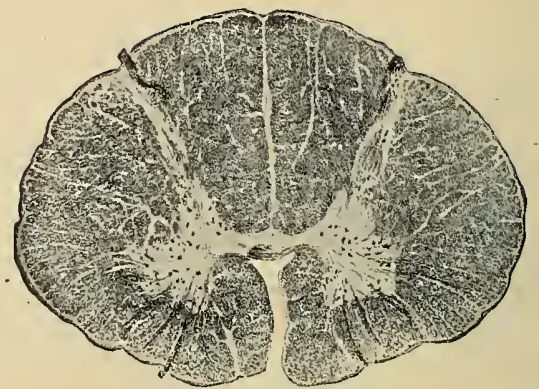

C. III

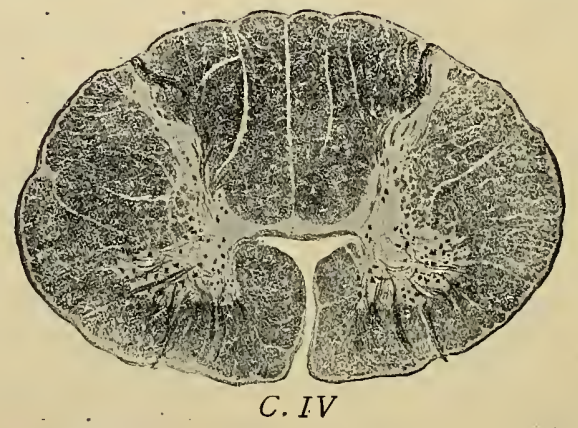

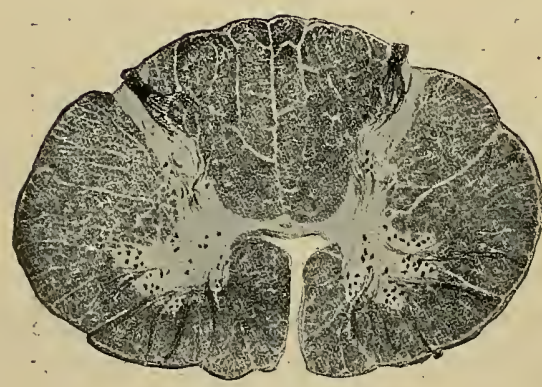

C. $V$

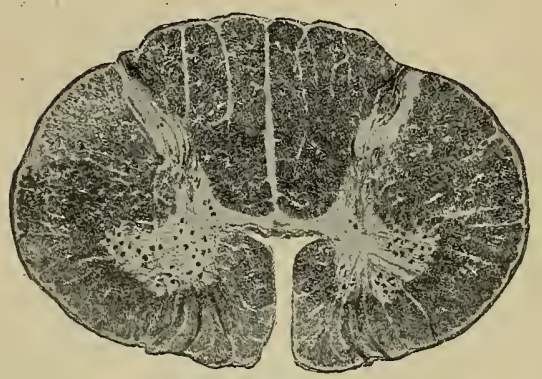

C. VII

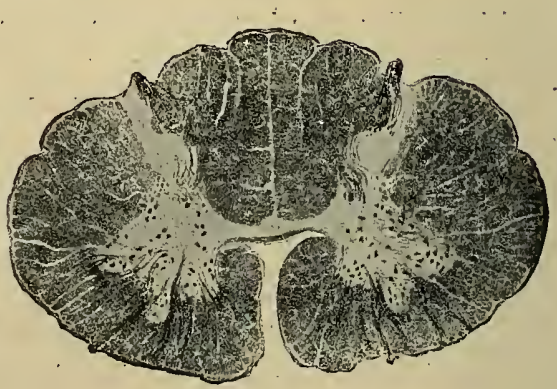

C. VI.

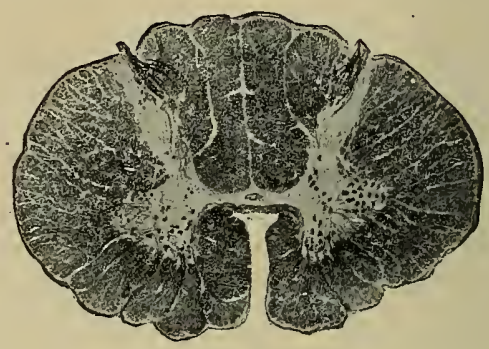

C. VIII

FIg. 3I5--Transverse Sections through the Cervical (II-VIII) Segments of the Cord. Weigert preparations. (Rauber-Kopsch.) 


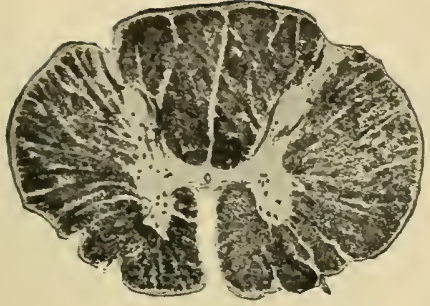

Th. I

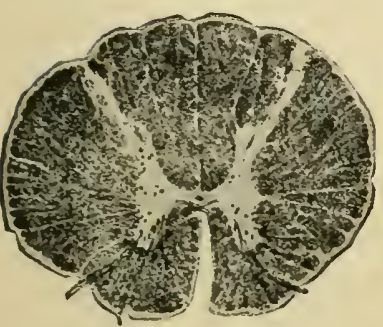

Th. III

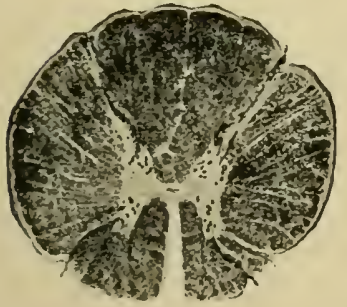

Th. VI

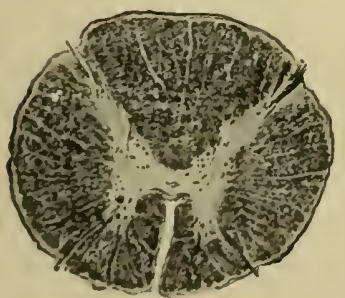

Th. IX

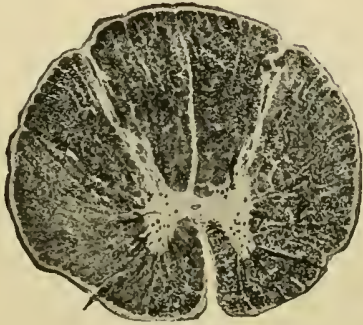

Th. IV

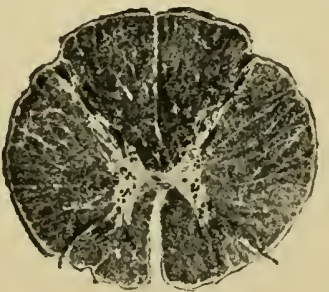

Th. VII

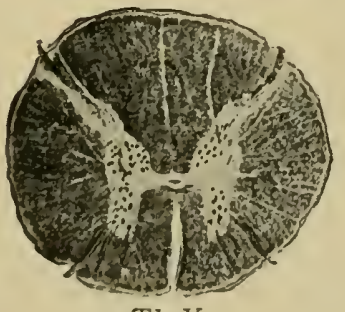

Th.X

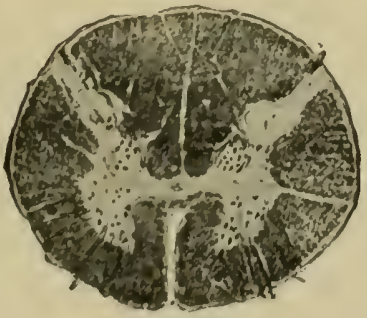

Th. XII

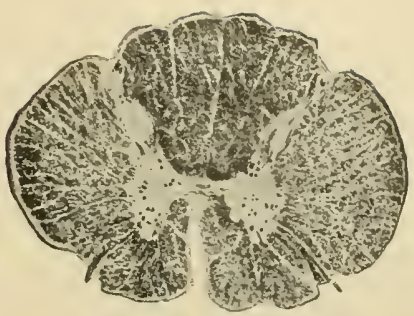

Th. II
Th. $V$

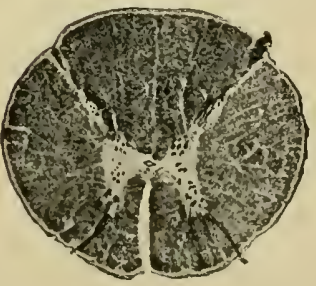

Th. VIII

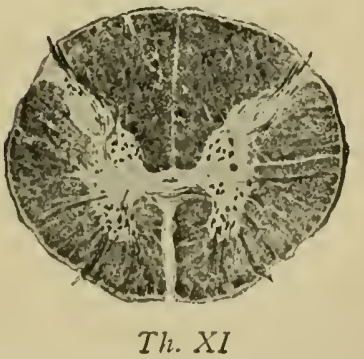

Th. XI 


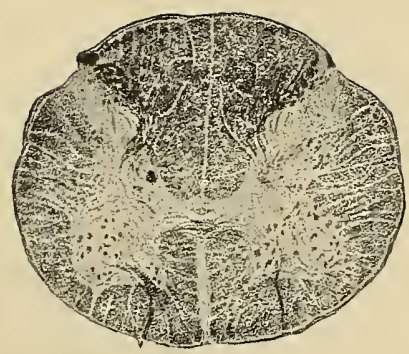

L. I

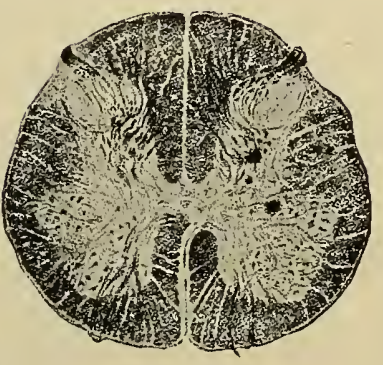

L. III

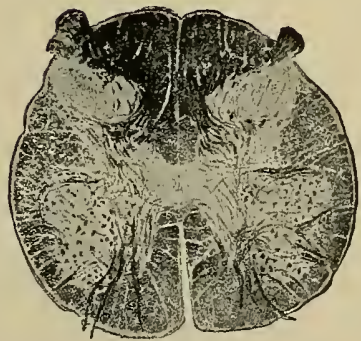

L. $V$

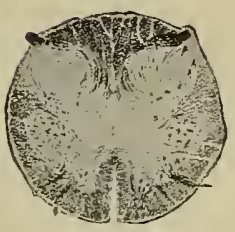

S. II

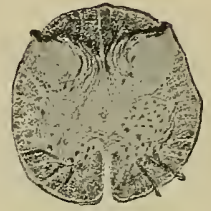

S. III

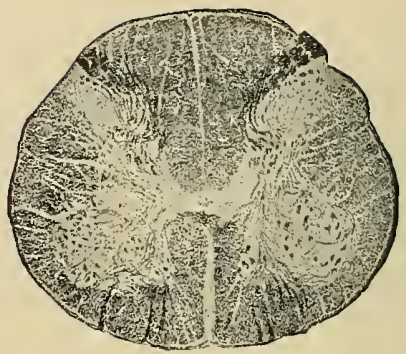

L. $I I$

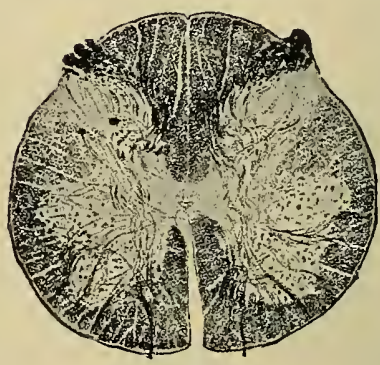

L. IV

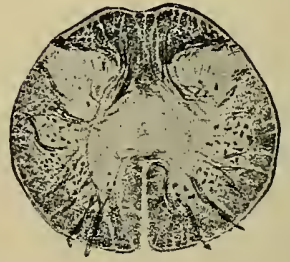

S. $I$

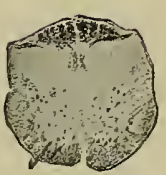

S. IV

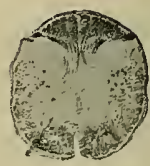

S. $V$

FIG. 3I 7.- Transverse Sections through the Lumbar (I-V) and Sacral (I-V)Segments of the Cord. Weigert preparations. Rauber-Kopsch.) 
definite septum divides the posterior column into an inner part, the column of Goll, and an outer part, the column of Burdach.

For further variations and differences between the segments of the cord, compare Figs. 315,316 and 317 .

\section{Fibre Tracts of the Cord}

The determination of the fibre tracts of the cord has been accomplished principally by two methods: (I) The myelogenetic method, which is based upon the fact that the fibres of different systems acquire their myelin sheaths at different periods of embryonic development. Thus by examining cords from embryos of various ages and young specimens it is possible, using a myclin stain (e.g., Weigert), to distinguish different tracts by the presence or absence of myelinization of their fibres. (2) The method of secondary or axonal degeneration, based upon the fact that a fibre separated from its cell undergoes degenerative changes and ultimately disappears and that the cell body also usually shows certain changes (see page 140). The fibres distal to the injury can be distinguished during active degeneration by applying the Marchi stain (page 34). After their disappearance, however, a negative picture is obtained by staining the surrounding normal fibres (Weigert). The changes in the cell bodies whose axones are injured are distinguished by applying the Nissl stain (p. 38). Thus if the cord is cut at some particular level, at any level above the cut all fibres present which originate from cells below the cut will show degeneration ("ascending" degeneration), while the cell bodies of the cut fibres will show the axonal degeneration changes. On the other hand, at any level below the cut, fibres which originate from cells above the cut will show degeneration ("descending" degeneration), while the cell bodies of these fibres, located above the cut, will exhibit axonal degeneration. The indication this gives as to the direction of conduction is evident when it is remembered that the impulse passes from neurone body along the axone to its termination. (3) Alrophy (von Gurlden's method). 'This method is based upon the fact that extirpation of some part of the nervous system in a young animal is followed by an atrophy of parts in intimate relation therewith. This method only demonstrates grosser changes than the preceding, but on the other hand whole conduction paths involving more than one neurone relay maly show changes. Other methods are the methorl of comparative analomy, i.e., study of the simpler nervous 


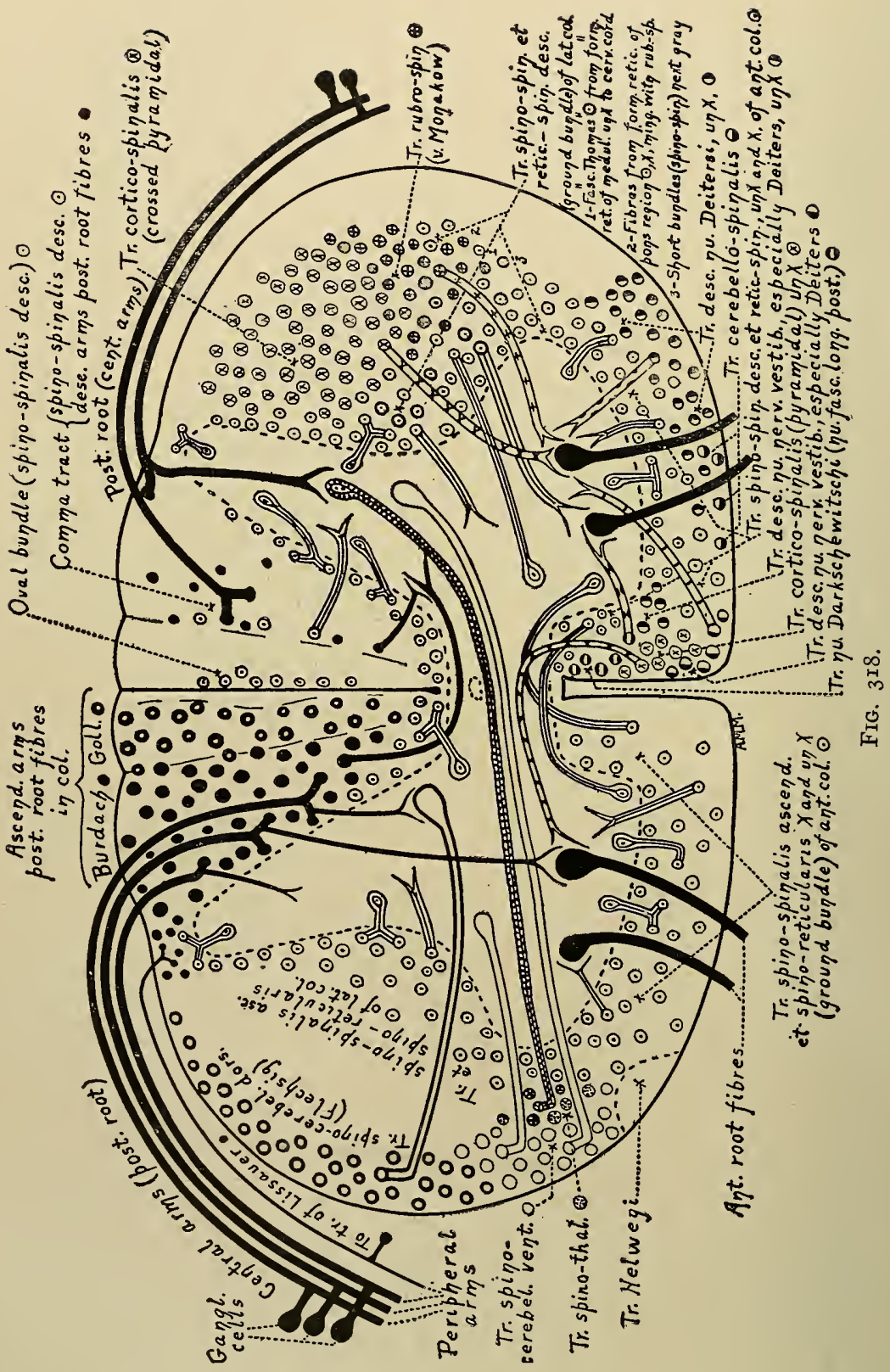


systems of lower forms and the correlated development or absence of related parts of the nervous system, and the method of physiology, i.e., study of the physiological effects of stimulation or extirpation of various portions of the nervous system thereby indirectly demonstrating anatomical pathways.

\section{ASCENDING Tracts.}

It will be recalled that various groups or systems of neurones (nuclei and tracts) are linked together to form conduction paths (p. 42I). In general an afferent conduction path consists of (I) a primary system (afferent ganglionic) whose central processes (afferent root fibres) end in its terminal nucleus; (2) a secondary system whose bodies constitute the terminal nucleus of (I) and whose axones form a second ary tract and end in a secondary terminal nucleus; (3) the conduction path may continue through tertiary nuclei and tracts, etc.

\section{A. Tracts forming parts of afferent pallial paths.}

I. Long Ascending Arms of Dorsal Root Fibres (Posterior Funiculi).-The origin of these tracts-central processes of the cells of the spinal ganglia-has been described (page 436). The distribution of the posterior root fibres to the gray matter of the cord was noted in connection with the study of the lumbar enlargement section (page 452). The general arrangement of these fibres in the dorsal funiculi remains to be noted.

Each successive dorsal root sends its fibres into the cord next to the dorsal horn and therefore lateral to the ascending fibres from the next root below. Thus the fibres of the lower roots as they ascend the cord are gradually pushed toward the median line until they finally occupy that part of the posterior column lying near the posterior septum. The separation of the posterior column by a connective-tissue septum into the column of Groll and the column of Burdach occurs

Frc. 318.-Diagram of the Tracts of the Cord (Cervical Region). Ascending tracts are shown on the left side, and descending tracts on the right. It will be noticed that the tracts of the cord are roughly divisible into three concentric zones: ( 1 ) $\perp$ zone occupying most of the posterior columns and the peripheral part of the lateral columns. This zone comprises the principal long ascending tracts (beginnings of afferent suprasegmental paths). (2) The secrnd zone lies immediately within the first in the lateral columns and also occupies the peripheral part of the antcrior columns. It comprises the principal long descending tracts from various parts of the brain (terminal portions of efferent suprasegmental paths). (3) The third zone borders the gray matter and includes the ground or fundamental bundles of the cord (chiefly spinal intersegmental fibres).

In the figure, for mu, Darkschewitschi read neucteus of merlial longitudinal fasciculus. 
only in the cervical cord (Fig. 3 I I and 3I5). Here the most median fibres, i.e., those lying in the column of Goll, are the longest fibres of the posterior columns, having come from the lower spinal ganglia (lower thoracic, lumbar and sacral), while the column of Burdach (Fig. 3II) consists of short and medium length fibres (upper thoracic and cervical dorsal root fibres). The fibres of Goll's column end in the nucleus funiculi gracilis or nucleus of the column of Goll in the medulla (see p. 485 and Fig. 326). Those fibres of Burdach's which do not terminate in the spinal cord terminate in the medulla in the nucleus funiculi cuneati or nucleus of the column of Burdach (p. 485 and Fig. 326). The nucleus gracilis and nucleus cuneatuswhich will be seen in sections of the medulla (Fig. 326) - thus serve as terminal nuclei for the afferent root fibres in the columns of Goll and those of the columns of Burdach which do not terminate within the cord. Inasmuch as many of the ascending arms are short, it is evident that only a fraction of the dorsal root fibres are represented at the higher levels. Those long arms which reach the medulla constitute the beginning of one of the principal afferent cerebral or pallial pathways. The axones of the neurones, whose bodies are the nuclei of Goll and Burdach, cross and form the tract known as the medial fillet (or lemniscus), composing the second system of this path. The fillet terminates in the thalamus and the path is completed by a third system of thalamo-cortical neurones to the cortex pallii, (probably principally to the post-central area). This path is, in brief, as follows: spinal ganglionic (long ascending arms of dorsal roots) + fillet + thalamo-cortical path, decussating in the medulla (Fig. 322).

II. The Spino-thalamic Tract.-This arises from heteromeric cells lying probably principally in the dorsal horn (groups $c$ and $d$, p. 450). Their axones cross in the ventral commissure and reach the opposite lateral funiculus where they ascend in a position mesial to the ventral spino-cerebellar tract (see below). This tract terminates in the thalamus whence the path is completed by thalamo-cortical neurones. The path is thus: spinal ganglion (short arms and collaterals of dorsal roots) + spinothalamic + thalamo-cortical neurones, three systems of neurones. Its decussation takes place in the cord at about the level of entry of the dorsal roots involved. Associated with this system may be some fibres to the superior colliculus (spino-collicular tract). (Figs. 318, 319 and 323).

$B$. Tracts forming part of paths to the cerebellum. 
III. The Dorsal Spino-cerebellar Tract (Tract of Flechsig, Direct or Uncrossed Cerebellar Tract).--This tract lies along the dorsal-lateral periphery of the cord, being bounded internally by the crossed pyramidal tract (Fig. 3II and Fig. 3I8). The fibres of the direct cerebellar tract are the axones of the cells of Clarke's column (tautomeric column cells) (Figs. 3I8, 319 and 323). These axones cross the intervening gray matter and white matter of the same side and turn upward as the direct cerebellar tract. In the medulla they form part of the restiform body or inferior cerebellar peduncle and pass to the cerebellum. Here they enter the gray matter of the vermis of the same or opposite side, ending in ramifications among the nerve cells. Some fibres either end in, or send off collaterals to, the cerebellar nuclei. The tract first appears in the upper lumbar cord, and increases in size until the upper limit of Clarke's column has been reached (page 450).

As already noted above, some fibres of the posterior root, or their collaterals, end in the column of Clarke. This path is composed then of two systems of neurones, spinal ganglion cells and Clarke's column cells, and is

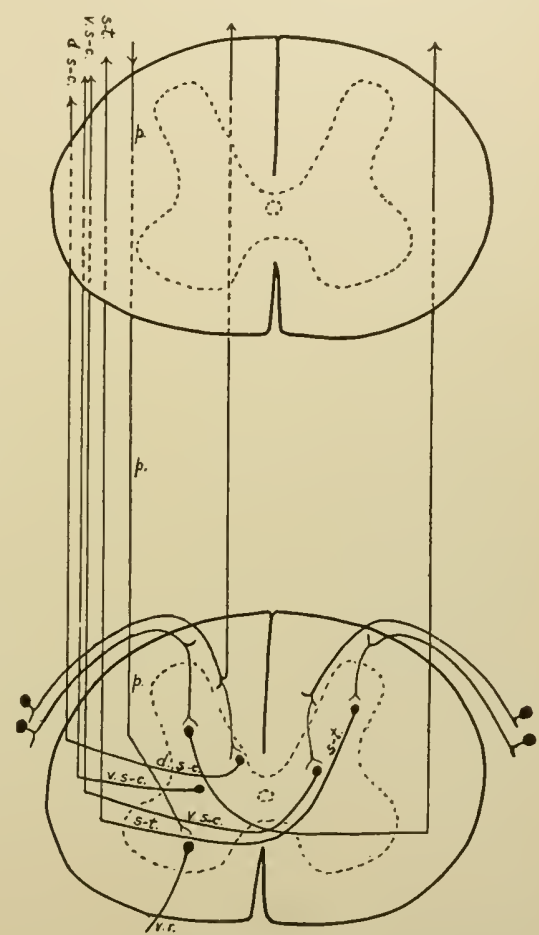

FIG. 319.-Diagram showing Beginnings of Principal Long Ascending Tracts of Cord and Termination of Lateral Pyramidal Tract. Each group of neurones is represented by one or two neurones. d.s-c., Dorsal spino-cerebellar tract; $p$, lateral pyramidal tract; $s-t$., spino-thalamic tract; v.s-c., ventral spino-cerebellar tract; v.r., ventral root. uncrossed (with the exception that some fibres are interrupted in the nucleus lateralis of the medulla and that some of the filores from the nucleus lateralis cross). (Fig. 337.)

IV. The Ventral Spino-cerebellar Tract.-This tract lies along the periphery of the cord, extending from the anterior limit of the direct cerebellar to about the exit of the ventral roots (Fig. 3 I a and Fig. 3 18). It is probably formed by axones whose cell bodies are scattered 
through the intermediate gray matter, possibly group $a$ (p. $45^{\circ}$ and Fig. 3II). Some fibres come from tautomeric, others from heteromeric cells, the axones of the latter crossing in the ventral commissure. The tract first appears in the upper lumbar cord and naturally increases in size as it passes upward. The fibres of this tract also end in the vermis of the cerebellum. They reach their destination in the cerebellum by a different route, ascending considerably farther than the dorsal spino-cerebellar fibres and then turning back along the outer side of the superior cerebellar peduncle to the vermis. This path is thus also a two-neurone path (spinal ganglion cells and spino-cerebellar neurones) and is partly crossed and partly uncrossed. The ventral spino-cerebellar and spino-thalamic tracts are sometimes referred to as Gower's tract. (Figs. 323 and 337.)

It seems probable that muscle-tendon sense passes up the cord by tract I (uncrossed in the cord), while pain and temperature pass up by the spinothalamic tract (crossed). The path pursued by touch is more doubtful but it may pass up partly by tract $I$ and partly by ascending arms of varying lengths which end in the cord around heteromeric column cells (thus partly uncrossed and partly crossed in the cord). This path may join the fillet in the medulla. It would seem probable that the cerebellar tracts convey stimuli from muscletendon receptors. Ascending paths may also be formed by successive relays of shorter tracts in the ground bundles of the cord and the reticular formation of the brain. This has been especially claimed for the pain pathway. The afferent visceral path to the pallium is not known.

\section{Descending Tracts}

I. The Pyramidal Tracts (Tractus Cortico-spinalis, Cerebrospinalis or Pallio-spinalis).-The cell bodies of the neurones whose axones make up this system are situated in the cerebral cortex anterior to the fissure of Rolando (precentral area, Fig. 357). Their axones converge in the corona radiata and pass downward through the internal capsule, pes pedunculi, pons, and medulla, sending off fibres to the motor nuclei of the cranial nerves. In the medulla the tracts come to the surface as the anterior pyramids. At the junction of medulla and cord occurs what is known as the pyramidal decussation. Here (a) most of the fibres of each tract cross to the opposite dorso-lateral region of the cord and continue downward as the crossed or lateral pyramidal tract. This lies in the dorsal part of the lateral column (Figs. 3 I I and 3I8). It extends to the lowermost part of the cord. In the cervical and dorsal region it is separated from the surface of the cord by the direct cerebellar tract. In 
the lumbar region the latter tract is no longer present and the crossed pyramidal tract comes to the surface. (b) The minority of the fibres of the anterior pyramids, instead of decussating, remain on the same side to pass down the cord along the anterior median fissure as the direct or anterior pyramidal tract, occupying a small oval area adjacent to the anterior sulcus (Fig. 3I8). It does not usually extend below the middle or lower dorsal region of the cord. As the pyramidal tracts descend they decrease in size from loss of fibres, which continually leave them to terminate in the ventral horns, The fibres of the crossed tract terminate mainly in the horn of thor same side, while most of the fibres of the direct tract probably cros through the anterior commissure to the opposite side of the corcts These tracts are thus mainly crossed tract;, as the great majorit ${ }_{\text {ar }}$, of their fibres cross to the opposite side of the cord. There $\operatorname{arc}{ }_{a}$ however, some homolateral (uncrossed) fibres in the lateral pyramidal tract. The tracts are apt to differ in size on the two sides of the cord, owing to the fact that the proportion of fibres which decussate is not constant. The axones terminate in arborizations around the motor cells of the ventral horns. The pyramidal tracts or palliospinal system together with the spinal efferent peripheral neurone system constitutes the pallio-spino-peripheral efferent conduction path. According to some authorities the pyramidal fibres terminate around cells in the intermediate gray matter whose axones, which form a part of the ground bundles, in turn terminate around the efferent root cells. Short axone (Golgi type II) cells might also of course be intercalated in this connection. (Figs. 322 and 323.)

The pyramidal tracts convey to the cord the impulses which result in voluntary movements, especially, probably, individual movements of parts of the limbs (foot, hand, finger, etc.).

II. The Colliculo-spinal Tract (Tecto-spinal Tract) orginates in the colliculi of the midbrain roof, decussates and descends to the cord, where it lies near the ventral sulcus. Its presence in the cord has been disputed. (Fig. 350.)

\section{The Tract from the Nucleus of the Posterior or Medial} Longitudinal Fasciculus.- This nucleus is located in the reticular formation of the tegmentum of the midbrain cephalad to the nucleus of nerve III. ${ }^{1}$ The tract originating from it forms in the brain a part

${ }^{1}$ The fitses in question have been variously stated to originate from the nucleus of Darkschewitsch, the "nucleus of the posterior longitudinal fasciculus," and the nucleus of the prosterior commissure. Whether any of these nuclei is the same as the interstitial nucleus of Cajal, or the nucleus of van (jehuchten in fishes, is uncertain. By the nucleus of the medial longitudinal fasciculus is here meant the interstitial nucleus of C'ajal. 
of the posterior or medial longitudinal fasciculus. It is uncrossed and lies near the floor of the brain cavity and next the median line. In the cord it occupies a similar position near the ventral sulcus. Its fibres terminate in the ventral horn. Some fibres have been traced into the lumbar cord. (Figs. 3 I 8 and 350 .)

IV. The Rubro-spinal Tract (von Monakow's Tract).-This consists of axones of the red nucleus (nucleus ruber) located in the tegmentum of the midbrain. These axones cross and descend to the fa rd, being joined by axones of the other cells in the reticular formaalo $\mathrm{n}$ in the region of the pons. In the cord the tract lies mingled with mis ce]

un.

ar $n$ tral to the lateral pyramidal tract. Its fibres terminate in the rt of the ventral horn. This tract is a lower link in a threeכath from cerebellum to cord composed as follows: $(a)$ $\therefore$ cells in cerebellar cortex to nucleus dentatus in cerebel, axones of cells in nucleus dentatus via superior cerebellar ...ile to the nucleus ruber; $(c)$ axones of nucleus ruber as the . upro-spinal tract to $(d)$ efferent peripheral neurones of cord. (Figs. 318, 323 and 337.)

V. The Deitero-spinal Tract (Vestibulo-spinal Tract).-This tract originates from Deiters' nucleus which lies in the medulla and receives fibres from the vestibular division of the acoustic nerve and also from the cerebellum (see below). It occupies the ventral and mesial periphery of the cord. The more lateral fibres are uncrossed, those near the ventral sulcus come from the nuclei of both sides. Descending with these fibres are probably axones of other vestibular terminal nuclei, and of other nuclei in the gray reticular formation (reticulo-spinal fibres) of the medulla. These fibres all terminate in the ventral horn. Some fibres have been traced to the sacral cord. Deiters' nucleus receives fibres fron the cerebellum, and thus this tract is a segment of a second efferent cerebellar pathway: (a) Axones of cells in cerebellar cortex to nucleus fastigii in cerebellum; (b) Axones of cel's of nucleus fastigii as the fastigiobulbar tract to Deiters' nucleus; (c) axones of cells of Deiters' nucleus as the Deitero-spinal tract to $(d)$ efferent peripheral neurones of cord. According to some authorities some fibres proceed from cerebellum to cord without interruption in Deiters' nucleus. (Figs. 3 I8 and 323.)

All the fibres of $\mathrm{V}$ are sometimes collectively called the anterolateral descending tract or marginal bundle of Löwenthal.

Tract III and the mesial part of $\mathrm{V}$ constitute the major portion of 
the descending fibres of an important bundle in the segmental brain known as the medial longitudinal fasciculus.

VI. The Fasciculus of Thomas.-Besides the reticulo-spinal fibres already mentioned are fibres in the lateral column which originate in the reticular formation of the medulla and terminate in the gray of the cervical cord. These are known as the tract of Thomas. (Fig. 3IS.)

VII. Helweg's tract is a small triangular bundle of fibres lying along the ventro-lateral margin of the cord, and is traceable upward as far as the olives (Fig. 3IS). The origin and destination of its fibres are not definitely known. Some of its fibres appear to originate in the cord and terminate in the inferior olivary nucleus in the medulla (spino-olivary fibres).

VIII. The Septo-marginal Tract.-This is a small bundle of fibres lying next the posterior septum. It appears to change its location in different levels, e.g., in the sacral cord it occupies a small dorso-median triangle, in the lumbar region it forms an oval bundle (of Flechsig) at the midclle of the septum and a superficial bundle, in the thoracic and cervical cord its fibres are more scattered. It is probably composed of descending axones of cells in the cord. (Fig. 3IS.)

IX. The so-called "comma" tract of Schultze is a small comma-shaped bundle of descending fibres lying about the middle of the posterior column (Fig. 3I8). It is most prominent in the dorsal cord. Its fibres are believed by some to be descending branches of spinal ganglion cells, by others to be descending axones from cells situated in the gray matter of the cord (column cells).

In general the descending tracts fall into two categories: (I) Tracts which are descending or efferent suprasegmental paths, or are parts of such paths. These comprisc $(a)$ the efferent pallial path (tract I), (b) the efferent midbrain paths (tracts II and III) and (c) the efferent cerebellar paths (tracts IV and V). (2) Descending intersegmental tracts. Of the latter some originate in nuclei lying in the segmental brain (nucleus of the medial longitudinal fasciculus, nucleus ruber, nucleus of Deiters) which receive efferent suprasegmental fibres, and thus form links of descending suprasegmental paths. Other reticulo-spinal fibres come from other cells in the gray reticular formation. Other still shorter tracts (spino-spinal), from cells in the cord, form the descending fibres in the ground bundles (see below). Tracts VIII and possibly IX are also in this category.

The descending path from the pallium which terminates atround the bodies of the splanchnic efferent neurones in the cord is stated to consist of (wo neurones, a fallio-bulbar neurone to the medulla and a bulb(o-spinal neurone to the efferent peripheral neurone in the cord. The axones of the latter pass out of the cord ats preganglionic fibers to the sympathetic ganglion cells. In the cord the fibres of this path 
probably lie in the ventro-lateral columns, though some authorities place them in the dorsal columns.

This pallio-bulbo-spino-sympathetic path is the path by which various psychic states (emotions, perceptions, memories) affect the splanchnic effectors, as seen in blushing, perspiring, erection, pupillary changes, etc.

Many tracts contain fibres proceeding in a direction opposite to that of most of the fibres.

Fundamental Columns or Ground Bundles (Shorter InterSegmental Tracts of Cord or Spino-spinal Tracts)

The ascending and descending tracts above described are known as the long-fibre tracts of the cord. If the area which these tracts occupy be subtracted from the total area of white matter, it is seen that a considerable area still remains unaccounted for. This area is especially large in the antero-lateral region, and extends up along

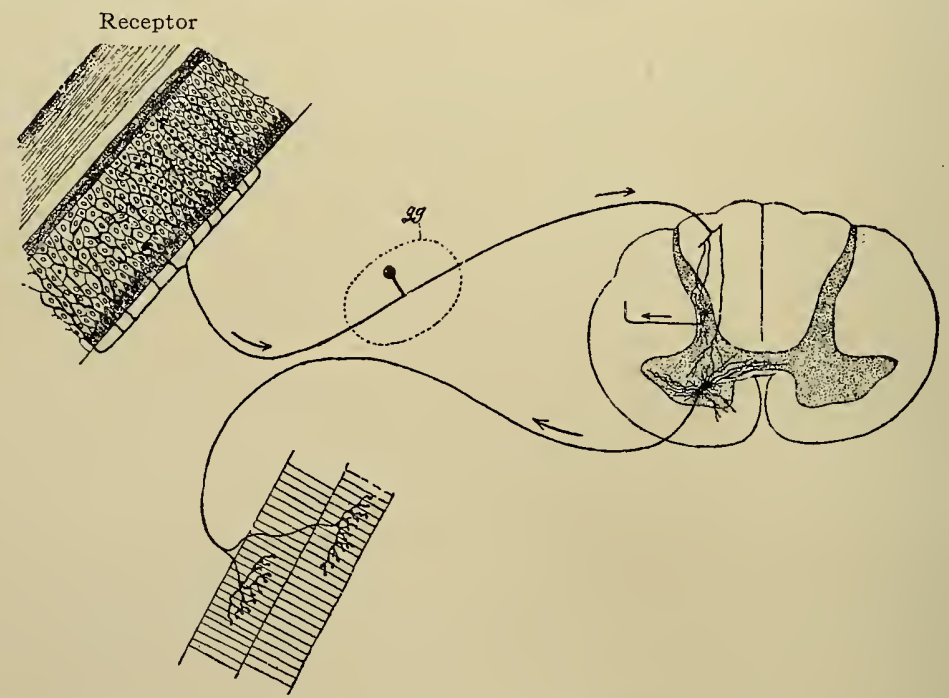

FIG. 320.-Diagram illustrating a Two-neurone Spinal Reflex Arc. Groups of neurones are represented by one neurone. $g g$, Spinal ganglion. (Van Gehuchten.)

the lateral side of the posterior horn between the latter and the crossed pyramidal tract (Figs. $3 \mathrm{II}$ and 3I8). A small area in the posterior column just dorsal to the posterior commissure, and extending up a short distance along the medial aspect of the horn, should also be included. These areas are occupied by the fundamental columns or short-fibre (spino-spinal or proprio-spinal) systems of the cord. The 
fibres serve as longitudinal commissural fibres to bring the different segments of the cord into communication (Fig. 32I). The shorter fibres lie nearest the gray matter and link together adjacent segments. The longer fibres lie farther from the gray matter and continue through several segments. The origin of these fibres as axones of cells of the gray matter, and the manner in which they re-enter the gray matter as terminals and collaterals have been considered (pp. 444 and 445).

The fact, alluded to above, that the shorter fibres lie nearest, or mingled with the gray is, in a general way, true throughout the central nervous system. A result of this in the cord is the superficial position of many of the longest tracts.

Attention has already been called to the concept of neural arcs which may traverse the cord or both cord and suprasegmental structures (page $42 \mathrm{I}$ ).

It must be kept in mind that there are probably no isolated neural arcs and that every neural reaction involving any given arc always influences and is influenced by other parts of the nervous system.

From the neurones thus far studied and the tracts which their axones form, the following neural arcs may be constructed:

(1) A Two-neurone Spinal Reflex Arc (Fig. 320).-(a) Peripheral afferent neu-

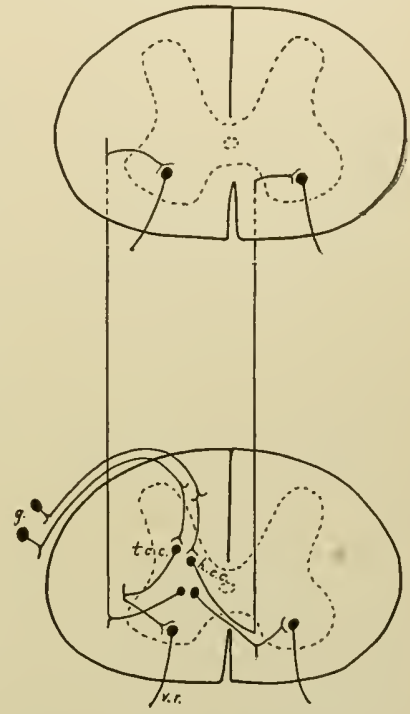

FIG. 32I.-Diagram illustrating Three-neurone Spinal Reflex Arcs of one segment and more than one segment. Groups of neurones are represented by one neurone. $g$, Spinal ganglion cells; h.c.c., heteromeric column cell; t.c.c., tautomeric column cell; v.r., ventral root. rones; their peripheral processes and receptors, the spinal ganglion cells, their central processes with collaterals terminating around motor cells of anterior horn; (b) peripheral efferent neurones, i.e., motor cells of anterior horn with axones passing to effectors. Such a two-neurone reflex arc is chiefly uncrossed and in most cases involves only one segment or closely adjacent segments. As it involves only one synapsis (see chapter VI) (in the ventral gray) it is sometimes termed a monosynaptic arc.

(2) A Three-neurone Spinal Reflex Arc (Fig. 32 I).-(a) Peripheral afferent neurones as in ( 1 ), but terminating around column cells of the cord. (b) Cord neurones (column cells) - axones in the fundamental 
columns with collaterals and terminals to anterior horn cells of different levels. (c) Peripheral efferent neurones as in the two-neurone reflex. Such a three-neurone or disynaptic reflex arc may involve segments above or below the segment of entrance of the stimulus and is uncrossed or crossed according as the cord neurones are tautomeric or heteromeric.

The independence of the cord as a reflex mechanism is much diminished in man.

(3) A Cerebellar Arc may be constituted as follows: (a) Peripheral afferent neurones to $(b)$ column cells in cord (e.g., Clarke's column) via spino-cerebellar tracts to cerebellar cortex; $(c)$ various associative cortical cerebellar neurones; $(d)$ axones of cortical cells to $(e)$ dentate nucleus the axones of which (superior peduncle) pass to $(f)$ nucleus ruber via rubro-spinal tract to $(g)$ efferent peripheral neurones in cord. Another arc would consist of $(a),(b)$ and $(c)$ the same, $(d)$ cerebellar cortex to nucleus fastigii in cerebellum to $(e)$ nucleus of Deiters to $(f)$ efferent peripheral neurones to effectors (Figs. 323 and $337)$.

FIG. 322.-Diagram showing the Most Important Direct Paths which an Impulse follows in passing from a Receptor $(S)$ to the Cerebral Cortex and from the latter back to an Effector $(M)$ (e.g., muscle), also some of the cranial-nerve connections with the cerebral cortex. Groups of neurones are represented by one or several individual neurones. $A$, Sensory cortex; $B$, motor cortex; $C$, level of third nerve nucleus; $D$, level of sixth and seventh nerve nuclei; $E$, level of fillet or sensory decussation; $F$, level of pyramidal or motor decussation; $G$, spinal cord.

\section{From Periphery to Cortex.}

Neurone No. I.-The Peripheral afferent Neurone: r, Spinal, cell bodies in spinal ganglia; receptor, $S$, peripheral arm of spinal ganglion cell; central arm of spinal ganglion cell as fibre of dorsal root to column of Goll or of Burdach, thence to nucleus of one of these columns in the medulla. $V_{1}$, Cranial (example, fifth cranial nerve, trigeminus; cell bodies in Gasserian ganglion); receptor; peripheral arm of Gasserian ganglion cell; central arm of Gasserian ganglion cell to medulla as afferent root of fifth nerve, thence to terminal nuclei in medulla.

Neurone No. 2.-2, Spinal connection-Cell body in nucleus of Goll or of Burdach; axone passing as fibre of fillet to thalamus. $V_{2}$, Cranial nerve connection (trigeminal), cell body in one of trigeminal nuclei in medulla, axone as fibre of secondary trigeminal tract to thalamus.

Neurone No. 3.-3, Cell body in thalamus, axone passing through internal capsule to terminaticn in cortex. (Various association neurones in cortex omitted.)

\section{From Cortex to Periphery}

Neurone No. 4.-4, Cell body in motor cerebral cortex; axone through internal capsule and pes to $(a)$ motor nuclei of cranial nerves $(b)$ by means of pyramidal tracts to ventral gray of spinal cord.

Neurone No. 5.-5, Spinal, cell body in ventral gray of cord; axone as motor fibre of ventral root through mixed spinal nerve to effector (muscle).

Neurone No. 5.-Cranial $-V_{5}$, Cell body in motor nucleus of trigeminus; axone passing to muscle as motor fibre of fifth nerve.

$I I I_{5}$, Peripheral efferent neurone of third nerve-oculomotor. $V I_{5}$, Peripheral efferent neurone of sixth nerve-abducens. $V I I_{5}$, Peripheral efferent neurone of seventh nerve-facial. XII glossal. 
(4) A Cerebral or Pallial Arc: (a) Peripheral afferent neurones, via long ascending arms to $(b)$ nucleus gracilis or cuneatus, thence as medial lemniscus to $(c)$ thalamus to cortex pallii; (d) associative neurones of cortex; $(e)$ cortical precentral neurones via pyramid to $(f)$ efferent peripheral neurones to effectors. Another arc would involve the spino-thalamic tract instead of the lemniscus. (Figs. 322 and 323.)

Similar arcs may include efferent sympathetic neurones. (See pp. 465 and 436 .)

\section{TECHNIC}

(I) Carefully remove the cord (human if possible; if not, that of a large dog) with its membranes, cut into two or three pieces if necessary, and lay on sheei cork. Slit the dura along one side of the cord, lay the folds back, and pin the clura to the cork. Care must be taken to leave the dura very loose, otherwise it will flatten the cord as it shrinks in hardening. With a sharp razor now cut the corrl, but not the dura, into segments alsout $x \mathrm{~cm}$. thick. frix in Orth's fluirl (p. 7). bieces of the cord may be cut out as wanterl and $\mathrm{em}$ berdferl in celloidin. Scetions should be cut atorout $15^{\mu}$ in thickness.

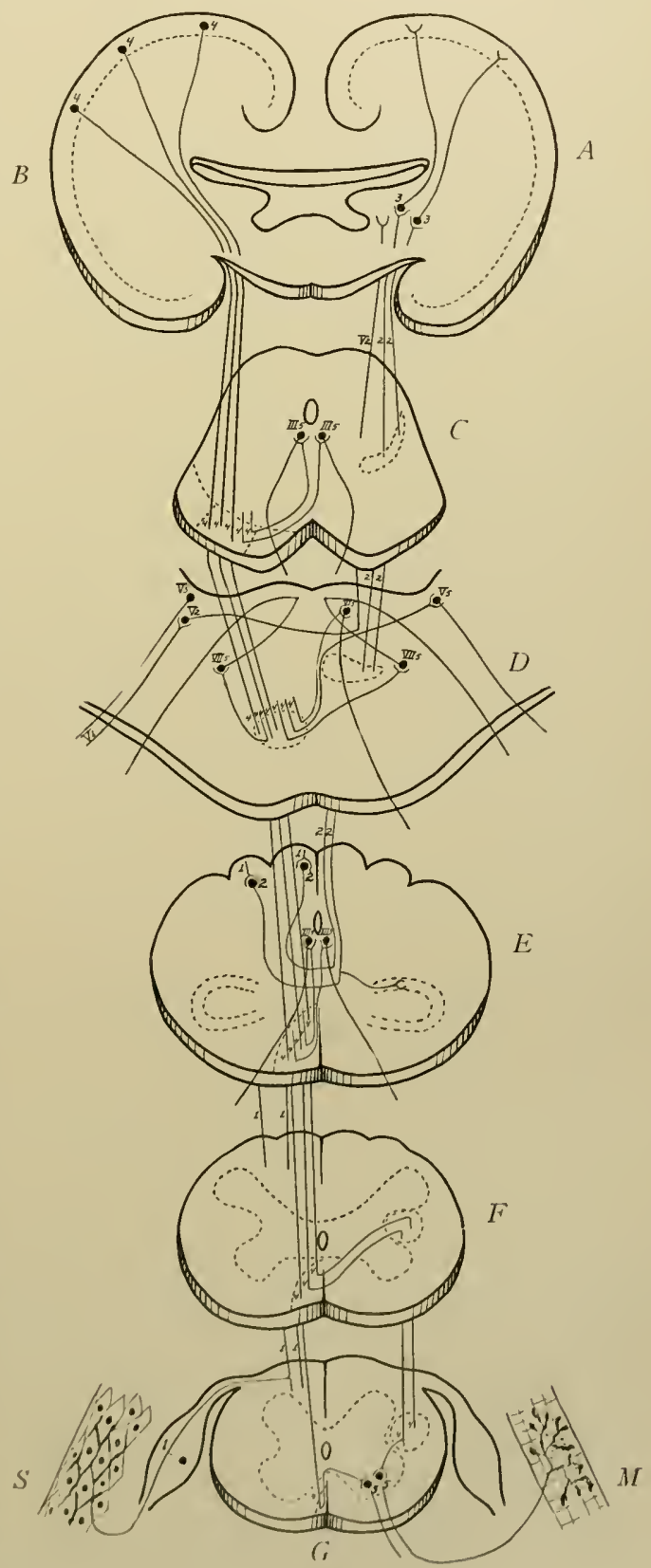

Fiss. 322 . 
(2) For the study of the general internal structure of the cord, stain a section through the lumbar enlargement of a cord prepared according to the preceding technic (I) in hæmatoxylin-picro-acid-fuchsin (technic 3, p. 2I) and another section through the same level in Weigert's hæmatoxylin (technic p. 32). Mount both in balsam. For Weigert staining, material fixed in formalin or in Orth's fluid should be further hardened in Müller's fluid for at least a month, changing the fluid frequently at first to remove the formalin. Mallory's glia stain should also be used with material fixed in Zenker's fluid (technic, p. 29). The silver method of Cajal (alcohol-fixation) should also be used (technic, p. 37) and that of Nissl.

(3) From a cord prepared according to technic I, remove small segments from each of the following levels: (I) the twelfth dorsal, (2) the mid-dorsal, and (3) the cervical enlargement. The segments are embedded in celloidin, sections cut $I_{5}$ to $20 \mu$ thick, stained by Weigert's method (page 32 ), and mounted in balsam. Medullated sheaths alone are stained by this method and appear dark blue or black.

(4) A human cord from a case in which death has occurred some time after fracture of the vertebræ with resulting crushing of the cord, furnishes valuable but of course rarely available material. If death occur within a few weeks after the injury, the method of Marchi should be used; if after several weeks, the method of Weigert (page 32). The picture in the cord is dependent upon the fact that axones cut off from their cells of origin degenerate and disappear. After a complete transverse lesion of the cord, therefore, all ascending tracts are found degenerated above the lesion, all descending tracts below the lesion. The method of Marchi gives a positive picture of osmic-acid-stained degenerated myelin in the affected tracts. The method of Weigert gives a negative picture, the neuroglia tissue which has replaced the degenerated tracts being unstained in contrast with the normal tracts, the myelin sheaths of whose fibres stain, as usual, dark blue or black.

(5) Human cords from cases which have lived some time after the destruction of the motor cortex, or after interruption of the motor tract in any part of its course, may also be used for studying the descending fibre tracts.

(6) The cord of an animal may be cut or crushed, the animal kept alive for from two weeks to several months, and the cord then treated as in technic (4). The most satisfactory animal material may be obtained from a large dog by cutting the cord half-way across, the danger of too early death from shock or complications being much less than after complete section.

(7) The cord of a human fœtus from the sixth month to term furnishes good. material for the study of the anterior and posterior root fibres, the plexus of fine fibres in the gray matter, the groupings of the anterior horn cells, etc. The pyramidal tracts are at this age non-medullated and are consequently unstained. in Weigert preparations. The cord of an infant of about one month is also excellent. Here the majority of the pallio-spinal fibres are medullated but very thinly so that they are easily distinguishable by their lighter stain. The Weigert-Pal method gives the best results.

(8) For the study of the course of the posterior root fibres within the cord, cut any desired number of posterior roots between the ganglia and the cord 
and treat material by the Marchi or the Weigert method, according to the time elapsed between the operation and the death of the animal.

\section{BRAIN}

\section{General Structure}

The principal peculiarities of the brain as distinguished from the cord depend upon two factors; certain peculiarities of the receptors and effectors of the head and the development of higher coördinating apparatus in the central nervous system of the head.

Besides the receptors of the general senses (p. 436), there are in the head the highly specialized receptors of smell, sight, hearing and position (semicircular canals), which are respectively concentrated into certain localities and form, together with certain accessory structures, the organs known as the nose, eye and ear. Each of these groups of receptors has its own special connection with the brain (nerves I, II, and VIII) and its own paths within the latter (see below). The special receptors of taste show a less degree of aggregation into an organ and, together with other visceral receptors, are innervated by afferent portions of a group of nerves (VII, IX and $\mathrm{X}$ ) which have a common continuation within the brain. The remaining somatic receptors of the general senses, scattered over the anterior part of the head, are innervated by one nerve (V) having its own central continuations. It has already been stated that all the afferent peripheral neurones which innervate these receptors (except the mesencephalic V) follow the general law of having their bodies located outside the neural tube. The central processes (root fibres) usually split on entering the tube, but the descending arms are the longer. Nerves I and II present certain special peculiarities.

The splanchnic effectors of the head include the usual splanchnic effectors - smooth muscle and glands-and also the branchial striated voluntary muscles. The somatic effectors are the remaining (myotomic) striated voluntary muscles. The striated voluntary muscles of the head fall into three groups; those of the eye (somatic); of the jaw, face, pharynx and larynx (splanchnic, modified branchial musculature); and of the tongue (somatic). The peripheral path to the smooth muscles and glands follows the same general law as in the body, i.e., neurone bodies in the central nervous system send preganglionic root fibres to sympathetic ganglion cells, which in turn send their axones to the effectors (p. 439). The peripheral path to 
the splanchnic (branchial) striated voluntary muscles, on the other hand, follows the same law as obtains for the somatic striated voluntary muscles, i.e., neurone bodies in the central nervous system send their efferent root fibres uninterrupted to the muscle. These distinctions are shown centrally by differences in grouping of the neurone bodies which supply respectively the somatic muscles, the splanchnic voluntary muscles, and the smooth muscles and glands via the sympathetic ganglia

The higher coördinating apparatus' or suprasegmental structures (p. 420) of the brain are essentially expansions of the dorsal walls of parts of the brain, each expansion having manifold afferent and efferent connections with the rest of the nervous system and having the endings and beginnings in it of its afferent and efferent paths complexly interrelated by enormous numbers of association neurones. The presence of these latter has probably necessitated the extensive layers of externally placed neurone bodies (cortex) characteristic of suprasegmental structures.

The structure of the basal part of the brain, connected with the cranial nerves (segmental brain, p. 420), is affected by both the peculiarities of peripheral structures mentioned above and by the presence of bundles of fibres and masses of gray forming portions of paths to and from suprasegmental structures (see below).

The following summary embodies the resulting general structural features of the brain:

\section{Segmental Brain and Nerves}

Afferent Peripheral (Segmental) Neurones.--(I) Splanchnic Group comprising nerves VII (geniculate ganglion), IX (superior and petrosal ganglia) and $\mathrm{X}$ (jugular and nodose ganglia). The nerves of taste probably belong entirely to this group. The peripheral arms of these ganglia innervate visceral receptors and the central arms form a descending tract in the medulla, the fasciculus solitarius, which has its terminal nucleus giving rise to secondary tracts.

(2) General Somatic Group (common or general sensibility).Semilunar ganglion of $\mathrm{V}$. The peripheral arms of the semilunar ganglion cells pass to the skin of the anterior part of the head, to the mouth and meninges. The central arms of the ganglion cells form a descending tract in the medulla, the radix spinalis $\mathrm{V}$. The terminal nucleus of this tract is the continuation in the medulla of the dorsal 
horn. The axones of the terminal nucleus form a secondary tract to the thalamus and thence a tertiary thalamo-cortical tract passes to the central cortex cerebri. Axones of the mesencephalic nucleus of nerve $\mathrm{V}$ innervate the muscle-tendon receptors of the jaw muscles and nerves III, IV and VI probably contain fibres to the muscletendon receptors of the eye muscles.

(3) Vestibulo-Semicircular Canal Group.-Ganglion of Scarpa of VIII. The peripheral arms pass to vestibule and semicircular canals. The central arms constitute the vestibular portion of VIII, forming descending tracts in medulla and terminating in several vestibular terminal nuclei (including Deiters' nucleus).

(4) Acoustic Group.-Ganglion spirale of VIII. The peripheral arms terminate in the organ of Corti of the cochlea. The central arms form the cochlear part of VIII and terminate in the medulla in various nuclei which originate the secondary tract (lateral fillet) to the midbrain, and medial geniculate body in thalamus. A third (or fourth?) system of thalamo-cortical neurones passes to the temporal cortex cerebri (also p. 582).

(5) Visual Group.-Ganglion in retina. The second neurone system begins in the retina and forms the secondary tract (optic "nerve") to the lateral geniculate body in thalamus. The third thalamo-cortical neurone system passes to the calcarine cortex cerebri (also p. 56I).

(6) Olfactory Group.- "Ganglion" cells in olfactory mucous membrane form the olfactory nerve (fila olfactoria) which terminates in the olfactory buli. Secondary tracts from the olfactory bulb (and tertiary tracts) proceed to diencephalon and hippocampus (also P. $5^{8} 5$ ).

Efferent Peripheral (Segmental) Neurones.-(i) Splanchnic. (a) Lateral nuclei in the gray matter of the hindbrain. Their axones pass to the striated voluntary muscles of the jaw (V), face (VII), pharynx and larynx (IX and X). (b) Nuclei more deeply placed in the gray of midl-and hindbrain. Their axones pass as preganglionic fibres to sympathetic ganglia of hearl and body. (c) Sympathetic. Their borlies compose the sympathetic ganglia of the head (ciliary, sphenopalatine, submaxillary and otic). 'These ganglia receive the above preganglionic fibres and are thus connected with cranial nerves III, V, VII, IX, and X.

(2) Somatic.- Medial nuclei located near the median line in the gray matter of hindbrain (XII and VI), and midbrain (IV and III), to 
muscles of tongue (XII) and eye (VI, IV and III). Nerve III also contains splanchnic neurones whose axones pass to sympathetic ganglia (ciliary). Nerves III, IV and VI probably also contain afferent nerve fibres (p. $474,(2)$ ).

InTrasegmental and Intersegmental Neurones.-These are represented principally by the gray reticular formation of the hindbrain and midbrain and long descending tracts external to it. The gray reticular formation is composed of neurone bodies and short intersegmental tracts intermingled. Among the former are certain well differentiated nuclei (e.g., nucleus ruber, nucleus of Deiters, and nucleus of the medial longitudinal fasciculus) the axones of which form long, principally descending, intersegmental tracts external to the gray reticular formation. These may also be links in efferent suprasegmental paths. (See also p. $477 \mathrm{~B}, \mathrm{XIV}, \mathrm{XVI}$, XVII and XVIII.) Other cells in the gray reticular formation form the shorter tracts within it. The reticular formation may also contain motor nuclei of the cranial nerves and is traversed by various fibres passing to tracts and by terminals from tracts.

\section{Afferent and Efferent Suprasegmental Paths}

These paths are paths from receptors of body and head to cerebellum, midbrain roof (colliculi) and pallium and paths from cerebellum, midbrain roof and pallium to effectors of head and body. There are also paths connecting pallium, mid-brain roof and cerebellum.

\section{Afferent Suprasegmental Paths}

\section{A. Afferent Pallial}

I. General Somatic Sensation: Spinal and trigeminal ganglionic + spinothalamic and bulbo-thalamic (medial fillet) crossed + thalamo-pallial (to postcentral cortex) neurones. (Fig. 322.)

II. Visceral Sensation, including Taste. This important path is not well known. The gustatory path enters by ganglionic neurones of nerves $\mathrm{V}$ (?), IX and $\mathrm{X}$ (fasciculus solitarius). Its secondary tract may lie partly in the medial fillet.

III. Hearing (cochlear): Spiral ganglionic + lateral fillet (crossed) and brachium of inferior colliculus + geniculo-pallial (to temporal cortex) neurones (Fig. 330).

IV. Vestibular: A somewhat doubtful path except perhaps to the pallium through the cerebellum (paths $\mathrm{X}+\mathrm{VII}$ ). 
V. Sight: Retinal bipolar ganglionic + retino-geniculate (optic nerve and tract, crossed and uncrossed in chiasma) + geniculo-pallial (to calcarine cortex) neurones. (Fig. 350.)

VI. Smell: Olfactory ganglionic + bulbo-rhinencephalic-pallial (crossed and uncrossed to hippocampal cortex) neurones. (Fig. 35I.)

VII. Cerebello-pallial: Cerebellar cortical + dentato-rubral and dentatothalamic (superior cerebellar peduncle, crossed) + rubro-thalamo-pallial neurones. (Fig. 337.)

\section{B. Afferent Mesencephalic}

VIII. A comparatively unimportant path composed of spinal ganglionic + spino-collicular (crossed) and bulbo-collicular (? medial fillet, crossed) neurones.

\section{Afferent Cerebellar}

IX. The spinal ganglionic (innervating proprioceptors of body) + spinocerebellar path to vermis of cerebellum. The dorsal spino-cerebellar and ventral spino-cerebellar tracts of this path pursue somewhat different routes. (Figs. 323 and 337.) This path may receive accessions from the columns of Goll and Burdach and their nuclei in the bulb.

$\mathrm{X}$. The vestibular ganglionic (Scarpa) + Deitero-cerebellar path. The vestibular ganglionic neurones may send axones to the cerebellum without interruption. (Figs. 323, 33I and 337.) Paths via other cranial nerves, especially nerves $\mathrm{V}$ and II, may also pass to the cerebellum.

Olivo-cerebellar neurones form part of another, not well known, afferent cerebellar path.

Efferent pallial path XIII is obviously also an afferent cerebellar path.

\section{Eif erent Suprasegmental Paths}

\section{A. Efferent Pallial}

XI. Voluntary Motor: Pallio (precentral cortex) - spinal (crossed) + spinal peripheral somatic motor neurones, also pallio-tegmental and pallio-bulbar (crossed and uncrossed) + cranial peripheral somatic motor and branchiomotor neurones. A short intersegmental system probably is intercalated between the pallial and peripheral neurone systems. (Figs. 322, 323.)

Xil. Splanchnic Efferent (except branchiomotor): This is the pallio-bulbospino-sympathetic path mentioned on page 467 and comprises also descending pallial fibres which act upon neurones in the brain whose axones pass out as cranial preganglionic fibres.

Xill. Pallio-cerelbellar: Pallio-pontile + ponto-cercbellar (misldle cerebellar fecluncle, crosserl) neurones. (Figs. 323,337 .)

XIV. Pallio-rubral + rubro-spinal (crossed) + peripheral motor neurones

(p. 5r2). This path may partly supplement, physiologically, path XI.

Other less important paths are mentioned on p. 523 . 


\section{B. Efferent Mesencephalic}

XV. Colliculo-bulbar and colliculo-spinal (crossed) + cranial (especially VII for squint reflex) and spinal peripheral motor neurones. (Fig. 350.)

XVI. Colliculo-medial longitudinal fasciculus: This path probabiy consists of collicular neurones which pass to the nucleus of the medial longitudinal fasciculus + the latter nucleus and its descending axones in the medial longitudinal fasciculus + peripheral motor neurones of brain (especially oculomotor) and cord. (Fig. 350.)

Inasmuch as the superior colliculus receives optic fibres, paths XV and XVI are probably largely concerned in optic reflexes.

\section{Efferent Cerebellar}

XVII. Cerebellar cortico (cortex of cerebellar hemispheres) - dentate + dentato-rubral (superior cerebellar peduncle, crossed) + rubro-spinal (crossed) + peripheral motor neurones. (Figs. 323,337.)

XVIII. Cerebellar cortico (cortex of vermis) - fastigial + fastigio-Deiters (mesial part of inferior cerebellar peduncle) + Deitero-bulbar and Deitero-spinal (crossed and uncrossed) + peripheral oculomotor and spinal motor neurones. (Figs. 323, 33r.)

Inasmuch as Deiters' nucleus also receives directly vestibular nerve fibres, there exists the important vestibulo-Deiters + Deitero-bulbar and Deitero-spinal + peripheral motor neurones reflex path whereby the vestibulo-semicircular canal receptors directly influence the position of eyes and body.

Afferent pallial path VII is obviously also an efferent cerebellar path. (Figs. 323,337 .)

The large efferent pallial paths XI and XIII markedly affect the configuration of the brain. These two paths are added ventrally to the segmental and intersegmental apparatus and form the pes pedunculi or crusta (added ventrally to the tegmentum of the midbrain), the pons Varolii (added ventrally to the tegmentum of midbrain, isthmus and hindbrain) and the pyramids (added ventrally to the hindbrain). Arising from the neopallium (p. 532), they are to be regarded as largely more recent acquisitions by the vertebrate nervous system.

\section{Suprasegmental Structures}

These are the pallium or cerebral hemispheres, the inferior and superior colliculi or corpora quadrigemina and the cerebellum. They consist essentially of the endings and beginnings of their respective afferent and efferent paths and of their own association neurones, the bodies of which lie in their respective cortices.

The corpora quadrigemina are relatively of much less importance in the human brain.

In accordance with the above there are usually to be distinguished in transverse sections of the brain at various levels the following: 
A. Peripheral (segmental) neurones. (I) Efferent ("motor" nuclei and root fibres).

(2) Central continuations of afferent neurones (afferent roots). (a) Those entering at and therefore belonging to the segment involved, (b) Those entering above or below the segment and represented in the segment by descending or ascending (overlapping) tracts.

B. Terminal muclei of (2) and the secondary tracts originating from them. These may fall under category $\mathrm{C}$ or D (below).

C. Intrasegmental and Intersegmental muclei and tracts of the segmental brain, consisting principally of the gray reticular formation and long descending tracts (arrangement much modified in forebrain).

D. Nuclei and tracts forming parts of afferent and efferent suprasegmental paths. The afferent paths include some of the nuclei and tracts under $\mathrm{B}$, and their continuations, and the efferent include some of the longer systems under $\mathrm{C}$, together with efferent suprasegmental tracts to them.

E. Suprasegmental structures (not present in many transections).

The general histology of the brain is similar to that of the cord. Cells of the motor cranial nuclei have an arrangement of chromophilic substance similar to their analogs in the cord, while cells of afferent cranial ganglia present a chromophilic picture similar to their cord analogs. Certain cells whose axones act directly upon motor cells in cord and brain (e.g., cells in motor cortex and certain cells in reticular formation) resemble motor cells, in arrangement of chromophilic substance, while certain cells in close connection with afferent peripheral neurones resemble the latter. The neuroglia cells and fibres also present the same general characteristics as those in the cord, with variations peculiar to certain localities (e.g., parts of the cerebellum).

\section{Hindbrain or Rhombencephalon}

This includes the medulla, cerebellum and part of the tegmentum and pons. Its peripheral nerves are the V, VI, VII, VIII, IX, X, and XII. ${ }^{1}$

The Mfidula Oblongata or Bulb is the continuation upward of the spinal cord and extends from the lower limit of the pyramidal decussation below to the lower margin of the pons above. ${ }^{2}$ the X.

'It is better probably to reckon the so-called medullary or bull ar part of the XI with

2 It weuld be better to include in the term medulla oblongata what here falls under frontile tegmentum of the hindbrain. 


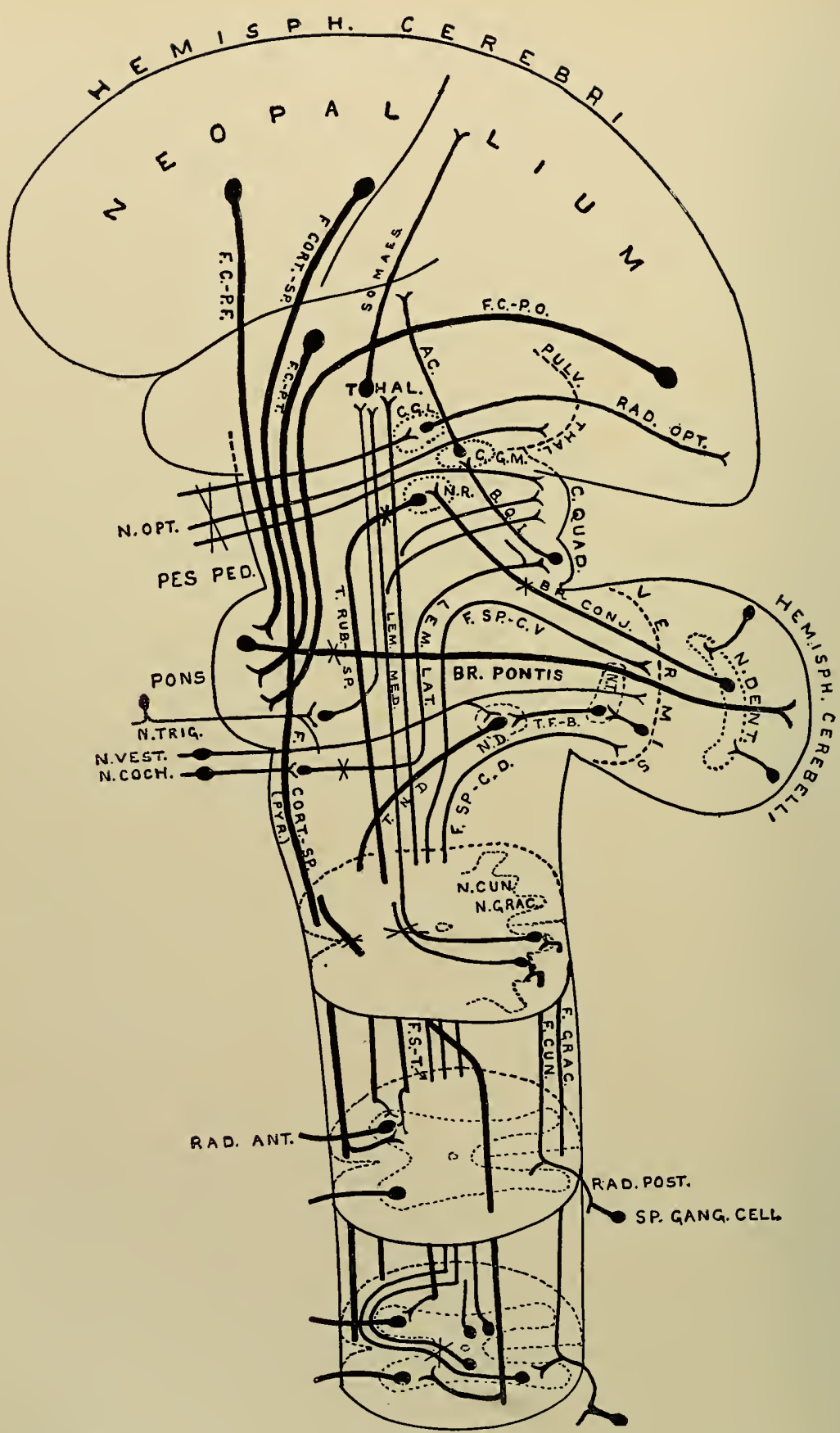

Fig. 323. 
Externally, the medulla shows the continuation upward of the anterior fissure and posterior septum of the cord. On either side of the anterior fissure is a prominence caused by the anterior pyramid, and to the outer side of the pyramid the bulging of the olivary body may be seen. The antero-lateral surface of the medulla is also marked by the exit of the fifth to the twelfth (inclusive) cranial nerves. The VI and XII (somatic motor) emerge near the midventral line, the others, including the splanchnomotor portions of the $\mathrm{V}$, VII, IX and $\mathbf{X}$, emerge more laterally. The posterior surface shows two prominences on either side. The more median of these, known as the clava, is caused by the nucleus gracilis, or nucleus of the column of Goll; the other, the cuneus, lying just to the outer side of the clava, is due to the nucleus cuneatus or nucleus of the column of Burdach. Lateral to this is a third eminence, the tuberculum cinereum, due in part to the descending root of the $\mathrm{V}$ and its underlying terminal nucleus, the continuation of the dorsal gray column of the cord. This eminence merges anteriorly with the eminence of the restiform body. 'The central canal of the cord continues into the medulla, where it gradually approaches the dorsal surface and opens into the cavity of the fourth ventricle. The floor of the fourth ventricle exhibits a medial eminence (trigonum hypoglossi) occupied caudally by the nucleus hypoglossi. Lateral to this is a triangular area, the ala cinerea (trigonum vagi), surrounded by furrows. This is partly occupied by nuclei of the vagus. Cephalad and laterally a broader

FIG. 323.-Principal afferent and efferent suprasegmental pathways (excepting the rhinopallial connections, the efferent connections of the midbrain roof and the olivocerebellar connections). Efferent peripheral neurones of cranial nerves are omitted. Each neurone group (nucleus and fasciculus) is indicated by one or several individual neurones. Decussations of tracts are indicated by an $\mathrm{X}$. ac, Acoustic radiation, from medial geniculate body to temporal lobe; $b r$. conj, brachium conjunctivum (superior cerebellar peduncle); $b r$. pontis, brachium pontis, from pons to cercbellum; b.q.i, brachium quadrigeminum inferius; c.g.l, lateral or external geniculate body; c.g.m, medial or internal geniculate body; c.quad, corpora quadrigemina; f.cort.-sp, pallio-spinal fasciculus (pyramidal tract); f.c.-p.f, frontal pallio-pontile fasciculus (irom frontal lobe); f.c.-p.l, temporal pallio-pontile fasciculus (from temporal lobe); f.c.-p.o, occipital pallio-pontile fasciculus (from occipital lobe); f.cun, fasciculus cuneatus (column of Burdach); l.f.-b, fastigio-bulbar tract; $f$. grac, fasciculus gracilis (column of Goll); f.s.-l, spino-thalamic fasciculus; $f . s p .-c . d$, dorsal spino-cerebellar fasciculus (tract of Jlechsig); f.sp.-c.v, ventral spino-cerebellar fasciculus; lem. lat, lateral lemniscus or lateral fillet; lem. med, merlial lemniscus or fillet; $n$. coch, cochlear nerve; $n . c u n$, (terminal) nucleus of the column of Bur(atch; $n . d$, nucleus of Deiters; $n . d e n l$, nucleus dentatus; $n$.grac, nucleus of the column of Goll; $n . o p l$, optic nerve; $n . r$, nucleusuber; $n . l$, nucleus tecti (or fastigii); n.lrig, trigeminal nerve; n.vest, vestibular nerve; pes.ped, pes perlunculi (crusta); pulv thal, pulvinar thalami; pyr, pyramid; rad. ant, ventral spinal root; rad. post, dorsal spinal $r(x$, ; rad. opl, optic radiation (from latcral geniculate borly to calcarine region); som oss. bundles from thalamus to posteentril region of neopollium; sp. gang, spinal ganglion; l.f.-b., tractus fastigio-bulbaris; thal, thalamus; $t . n . d$, tract from the nucleus of Deiters; 6. rub.-sp, rubıo-spinal tract (von .llonakow). (Litteral view of brain.) 
triangular area with an angle directed into the lateral recess marks the area occupied by the nuclei of the acoustic nerve (area acustica). Still further cephalad near the median line are eminences indicating the positions of the nucleus abducentis and genu facialis. The roof of the fourth ventricle is formed by the thin plexus chorioideus and the cerebellum. (Fig. 324.)

The Pons is a mass of fibres and gray matter extending across the ventral surface of portions of mid- and hindbrain. The term is often

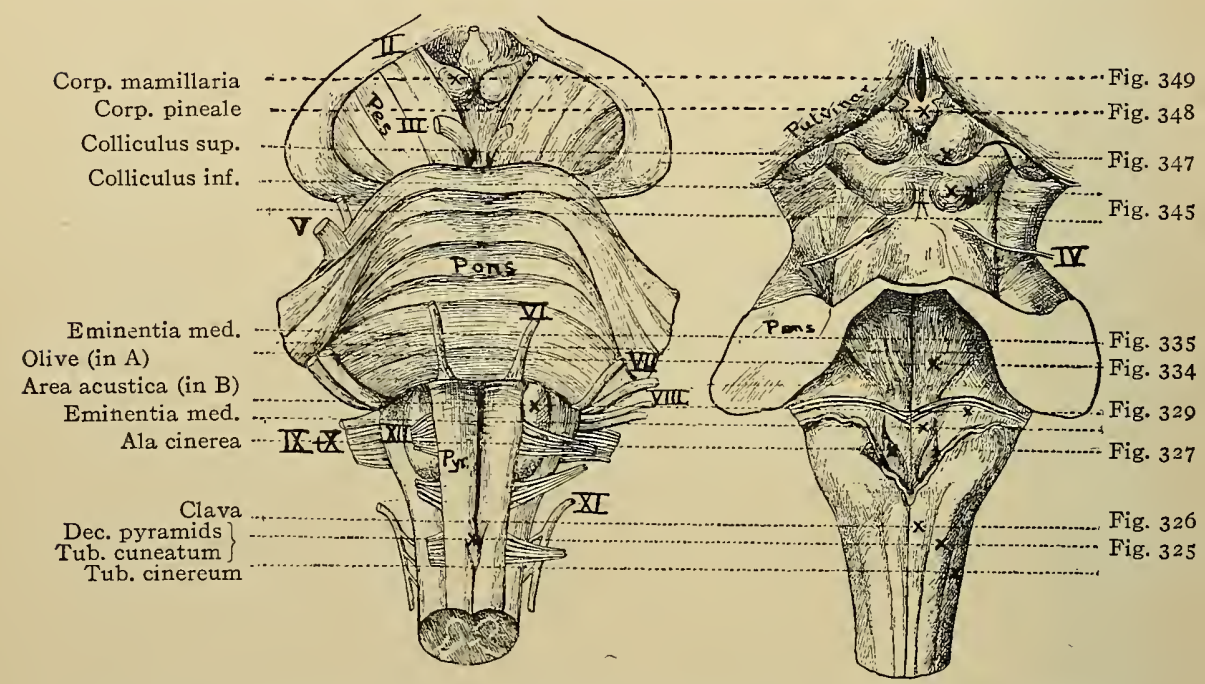

FIG. 324.-Ventral (A) and dorsal (B) views of part of Brain Stem (cerebellum removed). Structures named at the left are indicated by their reference lines running to an $\mathrm{X}$. On the right are named the figures showing transverse sections through the brain at the levels indicated by the reference lines. The level for Fig. 349 is not quite accurately indicated.

used to include the whole of the basal part of the brain thus covered by the pons. It is better, however, to restrict it to the pons itself. The part of the hindbrain dorsal to the pons, which is the continuation forward of the medulla, may be included in the term tegmentum of the hindbrain.

The Cerebellum is described on p. 507 .

\section{TECHNIC}

The technic of the medulla (and the rest of the segmental brain) is the same as that of the cord (page 47r). Transverse sections should be cut through the following typical levels, stained by Weigert's method (page 32 ), and mounted in balsam: 
I. Through the pyramidal decussation.

2. Through the sensory decussation.

3. Through the lower part of the olivary nucleus.

4. Through the middle of the olivary nucleus.

5. Through the entrance of the cochlear nerve.

6. Through the entrance of the vestibular nerve.

7. Through the roots of the sixth and seventh cranial nerves.

8 . Through the roots of the fifth cranial nerve.

The methods of Nissl, Cajal and glia stains should also be used when practicable.

\section{PRACTICAL STUDY}

\section{Transverse Section of the Medulla through the Decussation of the Pyram- idal Tracts (Motor Decussation) (Figs. 324 and 325)}

The most conspicuous features of this section are the decussation of the pyramids, the larger size of the dorsal horn and the beginning of the gray reticular formation. Surrounding the centra' canal is the central gray.

Efferent Peripheral Neurones.-Nuclei of first cervical spinal nerve in ventral gray, and root fibers passing out to emerge on ventral aspect. Nuclei of $X I$, in mesial position in central gray, or in ventral gray. Axones pass out laterally from latter and emerge on the lateral surface. The mesial or deep nuclei are best reckoned with nerve X.

Afferent Roots, their Terminal Nuclei and Secondary Tracts.--Some afferent fibres of the first cervical spinal nerve are still entering at this level.

Ascending afferent roots: The dorsal funiculus comprising the fasciculus cuneatus and fasciculus gracilis remain as in the cord. Collaterals and terminals from them can be seen entering the subjacent gray.

Descending afferent roots: Some of the fine fibres between the enlarged dorsal horn and the periphery, occupying the position of the zone of Lissauer in the cord, are descending afferent root fibres of the $\mathrm{V}$-cranial nerve or tractus spinalis trigemini (spinal $V$ ). Collaterals and terminals from these fibres terminate in the gelatinous substance of Rolando and also traverse it to form a plexus of medullated fibres in its inner side very similar to the cord. 'The axones of the dorsal horn cells (or terminal nucleus of the $V$ ) form the secondary tracts of the $\mathrm{V}$ which cannot be distinguished (p. 506 and Fig. 336).

Secondary tracts, forming parts of afferent suprasegmental paths: These form a mass of fibres along the lateral periphery of the merlulla which consists of (a) the dorsal spino-cerebellar (b) the ventral spino-cerelsellar and (c) the spinothalamic tracts.

Intersegmental Neurones. - The neurone bodies are, as in the cord, scattered throughout the gray. The continuation of the ventro-lateral intersegmental tracts of the cord (and the colliculo-spinal tract) is the U-shaped mass of fibres around the ventral gray. 'This mass consists of the long descending, the shorter ascending and descending intersegmental tracts, and the colliculo-spinal; i.e., (a) rubro-spinal (in lateral arm of $U$ ), (b) Deitero-spinal (lateral and mesial), 


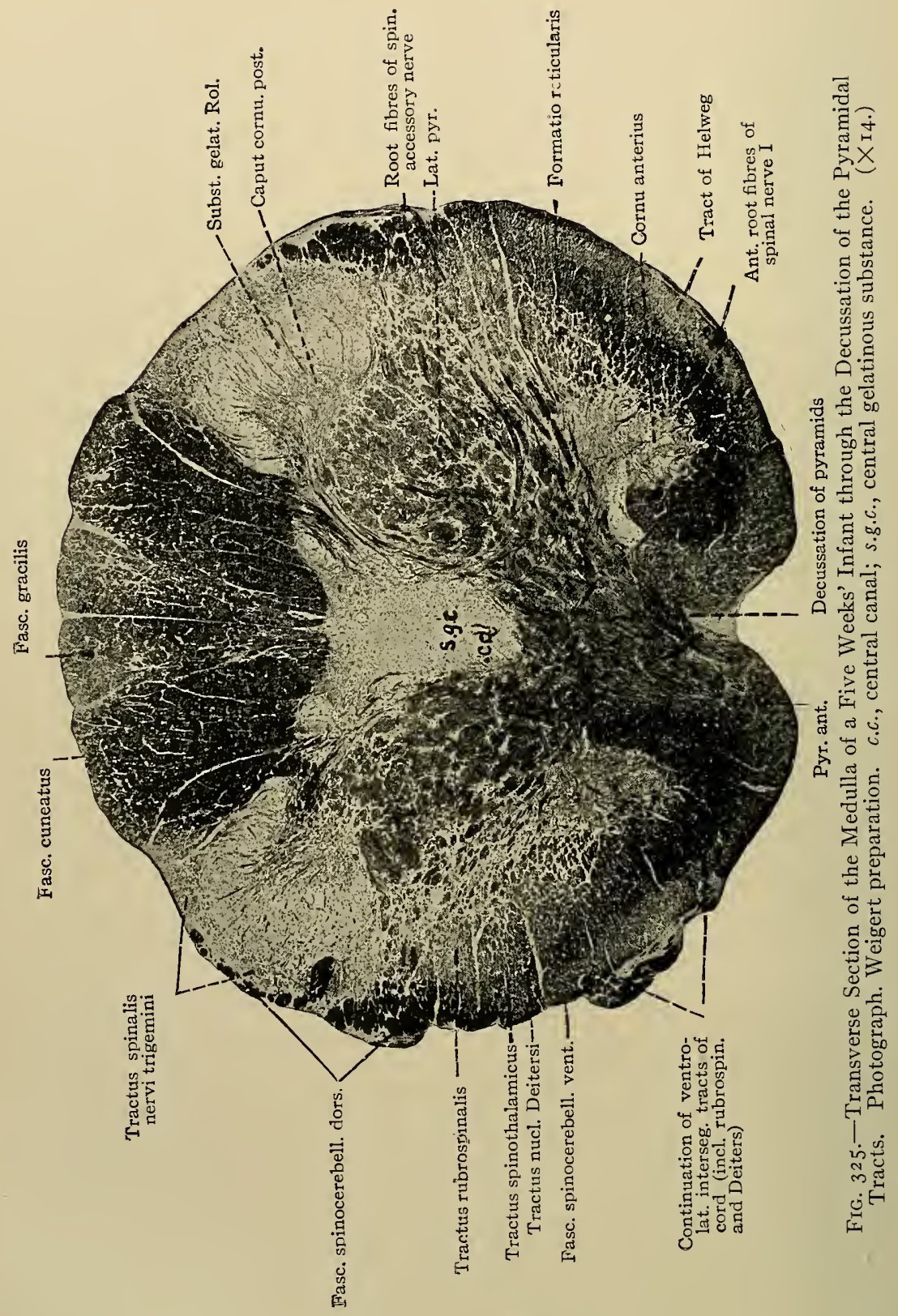


(c) tract from nucleus of medial longitudinal fasciculus (mesial), (d) colliculospinal (mesial), (e) shorter descending and ascending tracts which may be regarded as the equivalent of the ground bundles of the cord comprising shorter reticulo-spinal and spino-reticular fibres. The shortest of these fibers, which in the cord were next the lateral gray, are now mingled with the gray, the combination constituting the gray reticular formation. Other short intra- and intersegmental tracts lie in and adjoining the dorsal horn, as in the cord.

Descending suprasegmental paths include certain of the above long descending intersegmental tracts as previously explained. Besides these there are the effercnt suprasegmental neurones known as the pallio-spinal or pyramidal tracts and the colliculo-spinal tracts. Bundles of fibres are seen crossing (pyramidal dccussation) from the anterior pyramid of one side to the opposite dorso-lateral column, where they turn downward as the crossed pyramidal tract. In their passage through the gray matter, they cut off the ventral horn from the rest of the gray matter. These fibres, as already noted in the cord, are descending axones from motor cells situated in the precentral cerebral cortex. In the pyramidal decussation most of these fibres cross to the opposite dorso-lateral region to pass down the cord as the crossed pyramidal tract (p. 464 , and Fig. $3 \mathrm{r} 8$; Fig. $322, \mathrm{~F}$ ). The remaining fibres stay in their original anterior position and continue down the cord as the direct pyramidal tract (p. $46_{5}$, and Fig. 3 I 8 ; Fig. $322, \mathrm{~F}$ ). A few pass to the ventral tract in the same side, thus becoming uncrossed fibres in the lateral tract. The bundles of fibres do not cross in a transverse plane, but take a downward direction at the same time. For this reason transverse sections show these fibres cut rather obliquely. Because of the fact that the fibres cross in alternate bundles, the number of decussating fibres seen in any one section is greater on one side than on the other (Fig. 324).

\section{Transverse Section of the Medulla through the Decussation of the Fillet or Lemniscus (Sensory Decussation) (Figs. 324 and 326 )}

The most conspicuous features are the appearance of the nuclei cuneatus and gracilis, the decussation and formation of the medial lemniscus or fillet, and the increase of the gray reticular formation.

Peripheral Efferent Neurones.-In the lateral part of the central gray is the dorsal nucleus of the $\mathrm{X}$ (nucleus alce cinerea). In the ventral part of the central gray is the nucleus hypoglossi and, passing ventrally and emerging lateral to the j)yramids, may be seen the axones of its cells-the root fibres of the XII.

In the nucleus XII can be distinguished (Weigert stain) coarse fibres which are the root filses, and fine fibres which are terminals of other fibres ending in the nucleus. Among these have been distinguished collaterals from secondary vagoglossopharyngeal and trigeminal tracts (three-neurone reflex; and from various parts of the reticular formation. Whether pyramidal fibres reach the nucleus directly or via intercalated neurones is uncertain.

Afferent Roots, their Terminal Nuclei and Secondary Tracts.-Entering afferent root filores are usually not present.

The funiculi or fasciculi cuncatus and gracilis have diminished, and internal to them have appeared large masses of gray. These are the nuclei of the columns, 


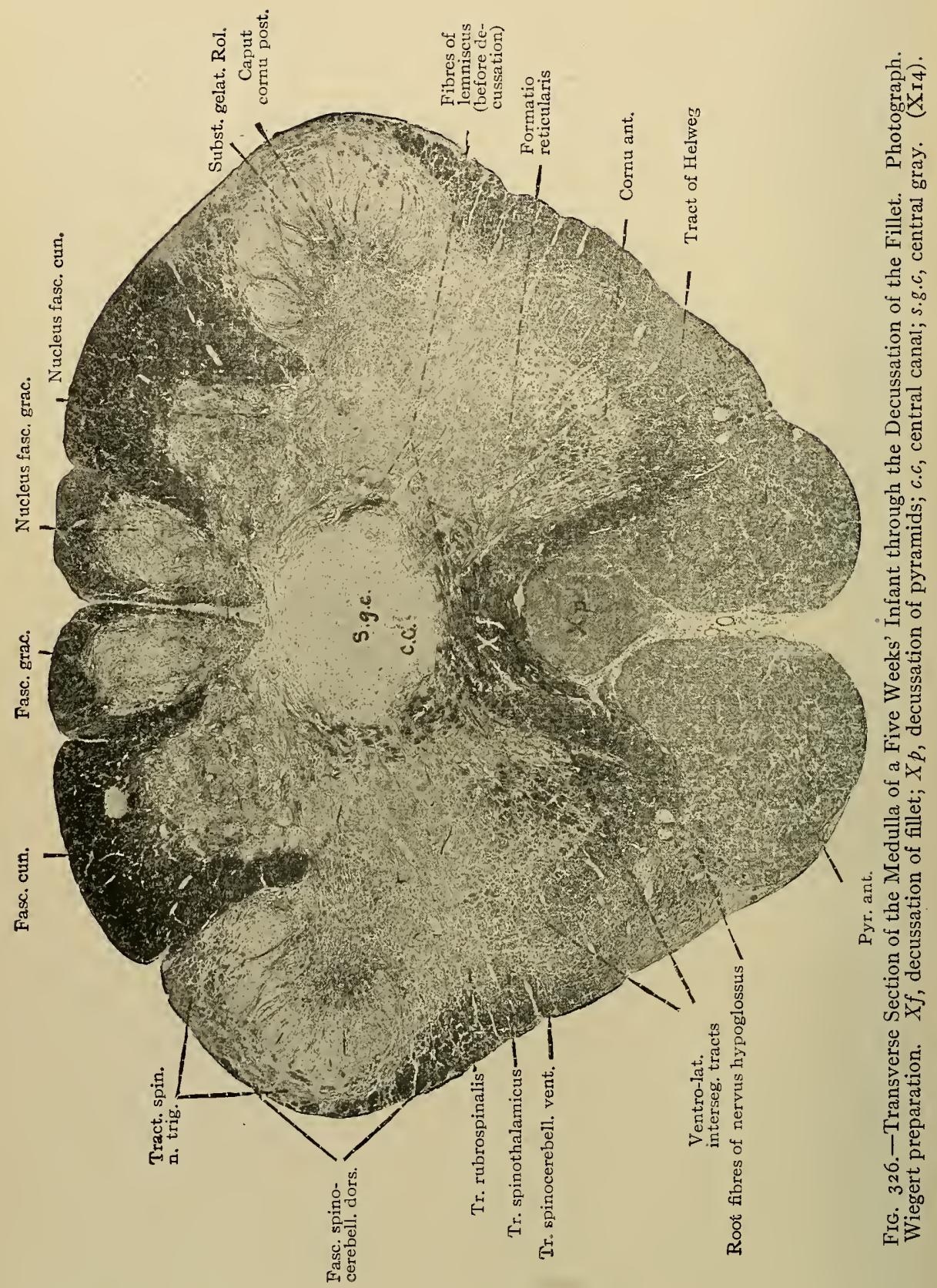


and are known, respectively, as the nucleus of the column of Goll or the nucleus gracilis, and the mucleus of the column of Burdach or the nucleus cuneatus. In the higher sensory decussation levels thcre is usually an accessory cuneate nucleus.

These nuclei serve as nuclei of termination for the fibres of the posterior funiculi. Their termination in these nuclei is the ending of that system of fibres which has been traced upward from their origin in the cells of the spinal ganglia; the completion of the course of the spinal peripheral afferent neurones. As the fibres of the posterior columns are constantly terminating in these nuclei, there is, in passing from below upward, a constant increase in the size of the nuclei and a corresponding decrease in the size of the posterior columns, until, just below the olive, the whole of the column of Goll and most of the column of Burdach are replaced by their respective nucl $\leqslant$ i. (Pp. $46 \mathrm{I}, 462$.

Study the plexus of fine fibres in these nuclei, formed by the terminals of the column fibres, also the coarser fibres (axones of the cells of the nuclei) gathered in the ventral part of the nuclei, whence they emerge and curve ${ }^{1}$ around the central gray, cross to the opposite side ventral to it and dorsal to the pyramids, and then turn brainward forming the bundle of fibres known as the medial lemniscus or medial fillet.

The spinal $\mathrm{V}$ has increased and also its terminal nucleus, the dorsal horn. The spino-cerebellar and spino-thalamic tracts occupy about the same positions.

In the central gray dorsal to the central canal is a nucleus representing a union of the caudal ends of the terminal nuclei of the fasciculi solitarii (see next section)-the mucleus commissuralis. Fibres of the fasciculi solitarii also decussate here.

Intersegmental Neurones. - The rubro-spinal and Deitero-spinal tracts and the tract from the nucleus of the medial longitudinal fasciculus occupy about the same positions. The reticular formation has increased, the whole of the ventral horn and intermediate gray containing bundles of longitudinal fibres. The formation is also traversed by transverse fibres, representing the beginnings or terminations of various longitudinal fibres.

Efferent Suprasegmerital Neurones. - The decussation of the pyramids has now nearly or entirely ceased. The lateral pyramidal tracts are no longer in the lateral columns but are parts of the anterior pyramidal tracts which form two large masses of fibres one on each side of the ventral sulcus. The colliculospinal tract occupies the same position.

\section{Transverse Section of the Medulla through the Lower Part of the Inferior Olivary Nucleus (Figs. 324 and 327 )}

The central canal has opened into the fourth ventricle, the central gray (including the central gelatinous substance) now being spread out on its floor. The roof of the ventricle is formed by its chorioid plexus. The most conspicuous new feature is the olive.

Efferent Peripheral Neurones - The nucleus of the XII is large and occupies

'Fibres having a transverse curverl or arched course are in general termed arcuate fibres. If they are decply located, they are incrnal arcuale fibres, if near the periphery, they are superficiat or external arcuale fibres. Obviously the same fibre may be, in different parts of its course, internal arcuatc, external arcuatc, and longitudinal. 


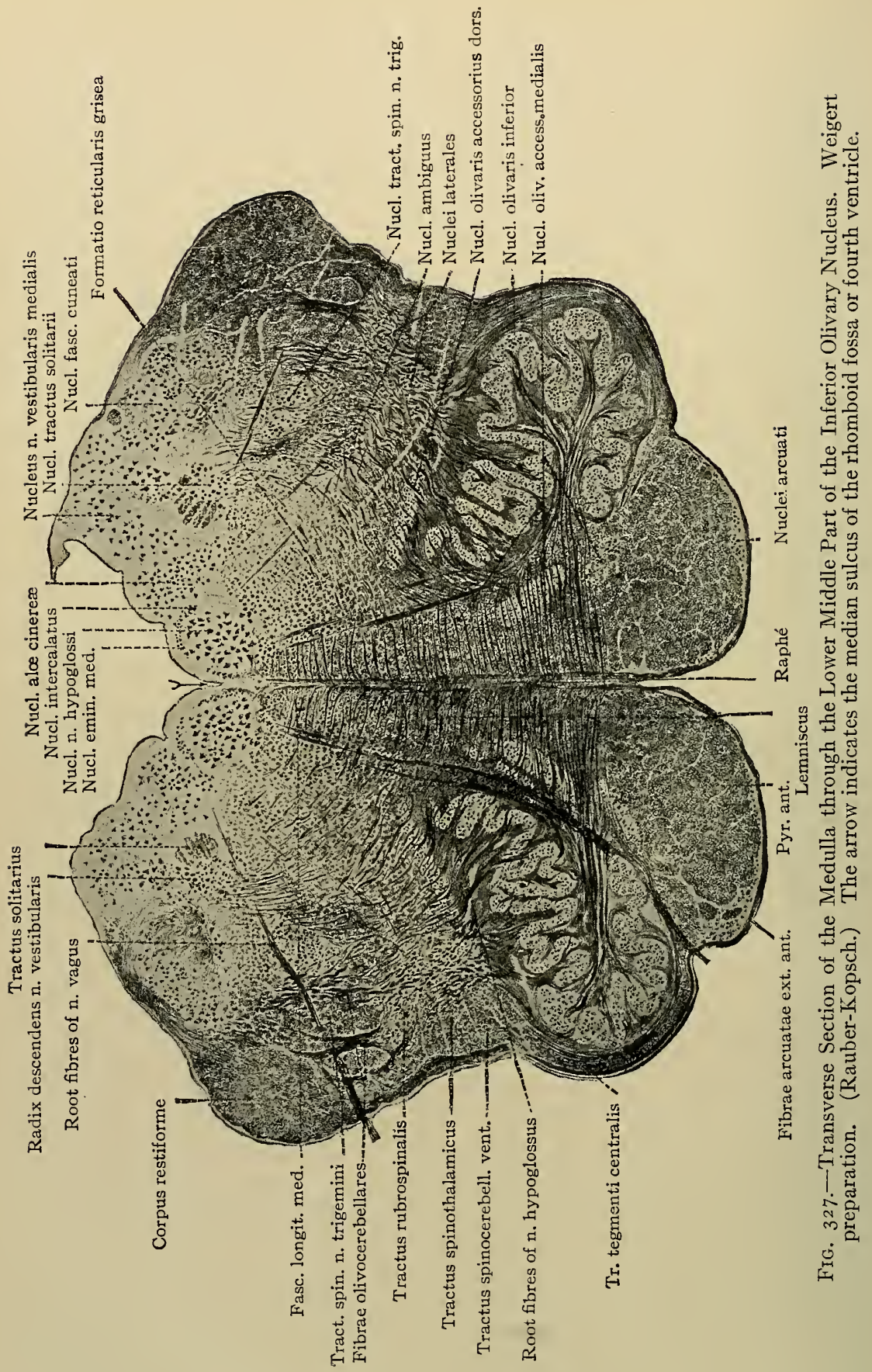


the swelling in the floor of the ventricle each side of the median line known as the eminentia or trigonum hypoglossi. The root fibres of the XII pass lateral to the medial lemniscus, between the olive and pyramid, and then emerge at the groove between olive and pyramid.

The dorsal nucleus of the $\mathbf{X}$ occupies a swelling lateral to the preceding and known as the ala cinerea. Some of the root fibres of the $\mathrm{X}$ are axones from this nucleus. They probably innervate (via sympathetic neurones) some, at least, of the smooth muscles, heart (and glands ?), innervated by the vagus (X). The dorsal nucleus appears to be relatively deficient in terminals. What it does receive appears to come from the secondary vago-glossopharyngeal and trigeminal tracts.

The bodies of another group of peripheral efferent neurones form the nucleus ambiguus, often difficult to distinguish, in the reticular formation. Their axones pass obliquely dorsally and mesially, join the other root fibres of the $\mathrm{X}$, and then bending abruptly, pass with them to the lateral surface of the medulla. Some pass across the median line and leave by the root of the opposite side. They probably innervate the striated muscles of the pharynx, larynx (and œsophagus ?). The nucleus receives various terminals, some at least appearing to come from the secondary trigeminal tracts and from the lateral part of the retic-

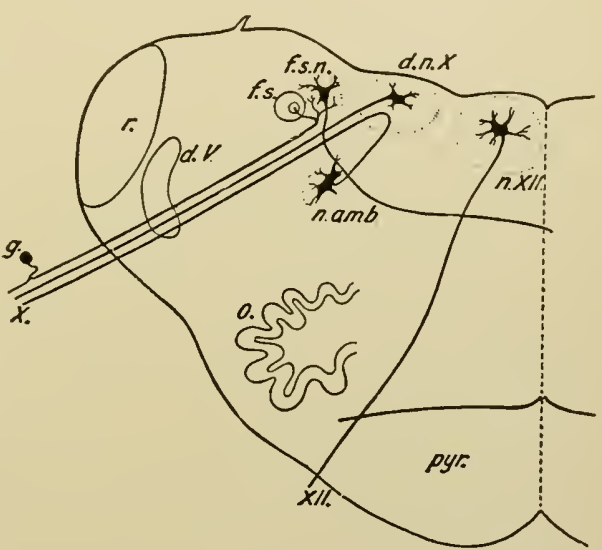

FIG. 328.-Diagram of Origin of Cranial Nerves $\mathrm{X}$ and XII. (Schäfer.) pyr, Pyramid; $o$, olivary nucleus; $r$, restiform body; $d . V$, spinal root of fifth nerve; $n . X I I$, nucleus of hypoglossal; XII, hypoglossal nerve; d.n.X.XI, dorsal nucleus of vagus; n.amb, nucleus ambiguus; f.s., solitary fasciculus (descending root of vagus and glosso-pharyngeal); f.s.n, nucleus of solitary fasciculus; $X$, motor fibre of vagus from nucleus ambiguus; $g$, ganglion cell of sensory root of vagus sending central arm into solitary fasciculus (f.s.) and collateral to its nucleus (f.s.n.); f.s.n, cell of nucleus of solitary fasciculus sending axone as internal arcuate fibre to opposite side of cord (secondary vagus and glossopharyngeal tract.) This course of the secondary tract is doubtful. ular formation. (Fig. 328.)

\section{Afferent Peripheral Roots, their Terminal Nuclei and Secondary Tracts.-} Other root fibres are the afferent fibres of the $\mathrm{X}$ which form a common root with the preceding. Sometimes they can be seen joining the fasciculus solitarius of which they form a part. Some filbres or collaterals may enter the adjacent gray (tcrminal muclcus of the $\mathrm{X}$ ) (see Fig. 328).

If the roots of the $X$ do not show well in the section, defer their study until the following section where the IX shows similar relations.

The spinal $\mathrm{V}$ is partly pierced and partly covered by transverse fibres, principally olivo-cerchellar fibres (sec below). Its terminal nucleus is less conspicuous. Two new bundles of descending root fibres have appeared; one is the fasciculus solitarius comprosed of the afferent root filores of the $X, I X$ (including gustatory 
fibres), and higher up, of the VII. A small mass of gray of a gelatinous appearance near it is its terminal nucleus. The course of the secondary tract cannot be made out and is not accurately known. The other bundle is the descending vestibular root. It lies lateral to the fasciculus solitarius. Accompanying it are cells which constitute its terminal nucleus. Occupying the floor of the ventricle lateral to the dorsal nucleus of the $\mathrm{X}$ is another terminal nucleus of the vestibular nerve, the nucleus medialis (triangular or chief nucleus.) Internal arcuate fibres emerging from these regions may represent secondary tracts (probably reflex) from these nuclei. (P. 493; Fig. 331.)

The nucleus gracilis has disappeared. The nucleus cuneatus may be present, much diminished, and give rise to some internal arcuate fibres to the medial lemniscus. The lemniscus is now a tract which has become built up on each side of the median line. This latter is known as the raphé (i.e., "seam," stitched by the decussating fibres).

The ventral spino-cerebellar tract and spino-thalamic tract are in about the same lateral position, but the dorsal spino-cerebellar tract has moved dorsally and together with olivo-cerebellar fibres (see below) begins to form the restiform body (see below).

In the lateral part of the reticular formation, between spinal $\mathrm{V}$ and olive are seen the nuclei laterales. In these nuclei some of the spino-cerebellar fibres end. The axones of these nuclei partly enter the restiform body on the same side and partly cross to the opposite restiform body (p. 507; Fig. 337). They form some of the ventral external arcuate fibres seen in the section. The lateral nuclei are thus partial interruptions in the spino-cerebellar path. The nuclei arcuati are well marked.

Other Afferent Cerebellar Neurones.-A new and important convoluted mass of gray is the inferior olivary nucleus, forming the bulge of the lateral surface of the medulla known as the olive. Near it are the dorsal and medial accessory olives. The axones of the olivary cells are the olivo-cerebellar fibres. They cross through the fillets, pass through or around the opposite olivary nucleus, thence proceed dorso-laterally, being gathered into more compact bundles, traverse or surround the spinal $\mathrm{V}$ and dorsal to it bend longitudinally, forming a great part of the restiform body. The latter produces an eminence on the dorso-lateral surface of the medulla. The restiform body, thus formed by these spino-cerebellar and olivo-cerebellar fibres, together with certain others, passes into the cerebellum higher up, forming the major part of the inferior cerebellar arm or peduncle. According to many authorities fibres from the columns and nuclei of Goll and Burdach of the same side (dorsal external arcuate fibres) and opposite side (ventral external arcuate fibres) may join the restiform body. (Comp. p. 507 .)

Fibres appearing on the external surface of the olivary nucleus are the termination of a large tract descending to the olivary nucleus, the central tegmental tract. Its origin in higher levels is not accurately known.

Intersegmental Neurones.-The reticular formation is now still more extensive.

The original U-shaped mass of intersegmental tracts (and the colliculo-spinal tract) has now become widely separated into two parts. The lateral part, con- 
sisting principally of the rubro-spinal tract and uncrossed Deitero-spinal fibres, lies mesial to, or partly mingled with, the spino-thalamic and ventral spino-cerebellar tracts. The mesial part of the $\mathrm{U}$, consisting principally of crossed and uncrossed Deitero-spinal fibres and fibres from other nuclei in the reticular formation, and of fibres from the nucleus of the medial longitudinal fasciculus, now forms the medial longitudinal fusciculus dorsal to the fillet. Near this bundle, or united with it, is the colliculo-spinal tract (predorsal tract). When these tracts have passed down to below the formation of the fillet and the olives, they assume the positions noted in the lower levels of the medulla.

Efferent Suprasegmental Neurones.-The pyramids are the same. Small bundles of more lightly stained fibres present in the fillet here and in higher levels (Weigert stain, not indicated in the figures) are efferent pallial fibres detached from pes or pyramids. They are aberrant fibres which rejoin the pyramids or are fibres innervating motor cranial nuclei. The colliculo-spinal tract (see above).

\section{Transverse Section of the Medulla through the Middle of the Olivary Nucleus}

Such a section is so similar to 3 and 5 that its detailed description may be omitted. The nucleus cuneatus has disappeared; the fillet increased somewhat; fasciculus solitarius and descending vestibular root have increased; also their terminal nuclei. The olivary nucleus, olivo-cerebellar fibres, and the restiform body have greatly increased. The formatio reticularis has increased in extent.

\section{Transverse Section of the Medulla through the Entrance of the Cochlear Root of Nerve VIII (Figs. 324 and 329)}

Efferent Peripheral Neurones.-The dorsal vagus nucleus is not present, but the nucleus ambiguus is usually present and probably sends some axones to nerve IX, passing out with the afferent fibres (see below). The nucleus XII has disappeared and also its root fibres.

Afferent Roots, their Terminal Nuclei and Secondary Tracts.-Usually the afferent root fibres of ncrve IX are present. They enter on the lateral aspect of the medulla ventral to the restiform body, traverse the spinal $\mathrm{V}$, and pass to the fasciculus solitarius or its terminal nucleus. The fasciculus solitarius is smaller, and just above the entrance of the IX consists of only a comparatively few descending afferent root filbres of the VII.

The filures of the cochlear nerve enter the extreme lateral angle of the medulla, where many or, according to some, all of them terminate in two masses of cells enveloping externally the restiform borly and known as the ventral (or accessory) and dorsal (or lateral) cochlear nuclei. Most of the axones of the dorsal nucleus pass across in the floor of the ventricle (strice medullares) to form a part of the opposite secondary tract (laleral lemniscus) of the cochlear nerve. The axones of the ventral nucleus also decussate, but by a more ventral route, (trapezius), and also form a part of the lateral lemniscus. This latter decussation takes place at at higher level (sec next section). 


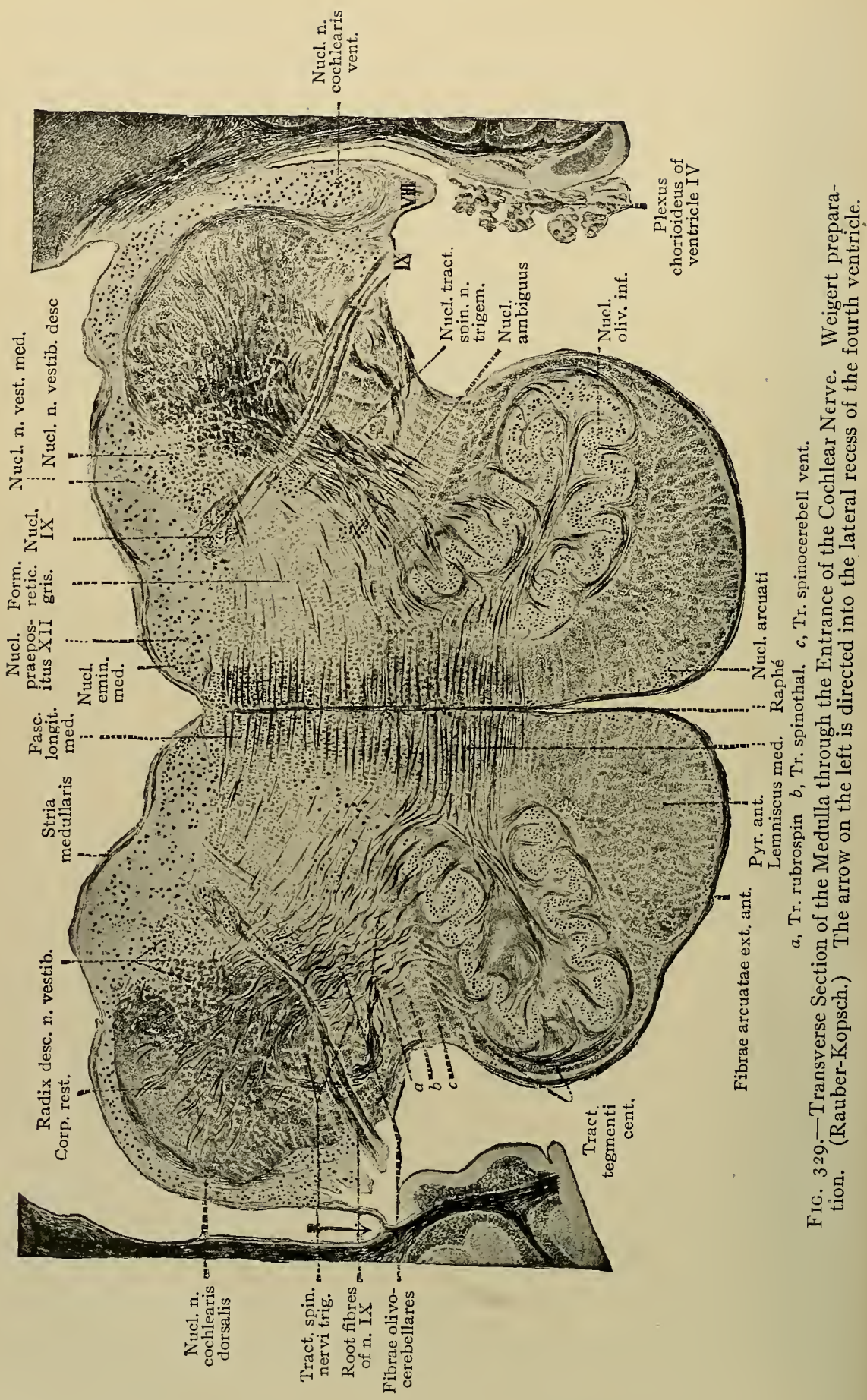


The auditory nerve is divided into two parts: the cochlear nerve (ganglion spirale) and the vestibular nerve (ganglion of Scarpa). The fibres of the cochlear root enter at a lower level than those of the vestibular. Some of them enter the ventral cochlear nucleus; the remainder pass dorsalward to the dorsal cochlear nucleus, or nucleus of the acoustic tubercle. According to some authorities, some root fibres pass to the superior olivary and trapezoid nuclei. The axones of the cells of the ventral and dorsal nuclei form the secondary cochlear tract (lateral fillet). These fibres decussate and send collaterals to, or are partially interrupted in, the nucleus olivaris superior, trapezoideus, nucleus of lateral fillet and inferior colliculus (posterior corpus quadrigeminum). According to some authorities all the fibres of the lateral lemniscus terminate in the inferior colliculus. From the inferior colliculus the path is formed by the arm or brachium of the latter to the medial geniculate body and thence to the temporal cortex cerebri. It is thus not possible to state definitely how many neurone systems are involved, but the principal ones are: (I) ganglion spirale, (2) dorsal and ventral nuclei and (decussation) lateral lemniscus, (3) posterior corpus quadrigeminum and its brachium, (4) medial geniculate body of the thalamus and geniculo-cortical fibres. If the lateral lemniscus fibres be regarded as simply passing by the posterior corpus quadrigeminum, giving collaterals to it (Cajal), the path would in part consist of three neurone systems analogous to those of the paths from the cord, trigeminus and eye. (Figs. 323, 330.)

The fibres of the vestibular root enter higher and mesial to those of the cochlear root, passing dorsally along the inner side of the restiform body to four terminal nuclei, which cannot all be clearly seen in any one section; (a) Deiters' nucleus (lateral vestibular nucleus) situated at the end of the main bundle of root fibres, just internal to the restiform body; $(b)$ von Bechterew's nucleus (superior vestibular nucleus) situated somewhat dorsal to Deiters' nucleus in the lateral wall of the fourth ventricle; $(c)$ the median or principal nucleus of the vestibular division-a large triangular nucleus, occupying a considerable part of the floor of the fourth ventricle; $(d)$ the descending vestibular nucleus which accompanies the descending fibres of the vestibular root (spinal eighth). Fibres also pass to the cercbellum. The axones of the cells of the terminal vestibular nuclei form the secondary vestibular tracts, some axones going to (a) the cerebellum (?),(b) the midbrain, especially to the nuclei of nerves III and IV (via Deiters and von Bechterew, the former by the medial longitudinal fasciculus), (c) the medulla and cord, probably to various motor nuclei, via the medial longitudinal fasciculus, lateral tract from I)eiters' nucleus and other tracts in the reticular formation. (Figs. 323, 331 and 337.)

The descending vestitular root is large, as is also its terminal nucleus and the merlial terminal vestibular nucleus, in the present section.

The spinal $V$ is unchanged, its terminal nucleus being rather indistinct. Secondary trigeminal i racts cannot be distinguished-such fibres probal,ly either join the merlial lemniscus or form an independent ascending tract in the reticular formation. 'The fillet is about the same. The vent ral spino-cerclellat and spinothalamic tracts are in the same positions.

Other Afferent Cerebellar Neurones. - The olives are still larger and send many bundles of olivo-cerclucllar fibres to the opposite restiform borly which has 

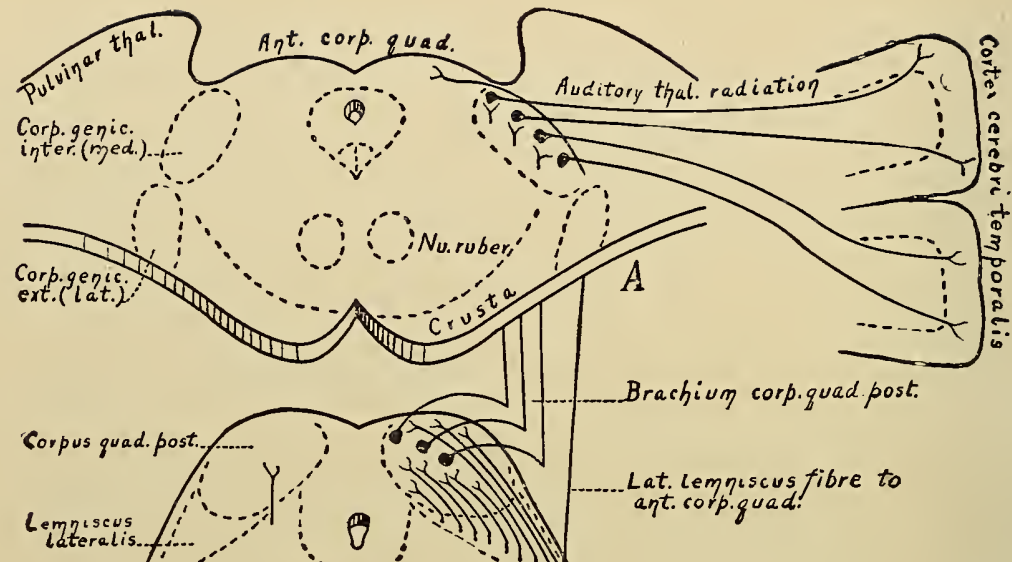
a.t. corp.guad!
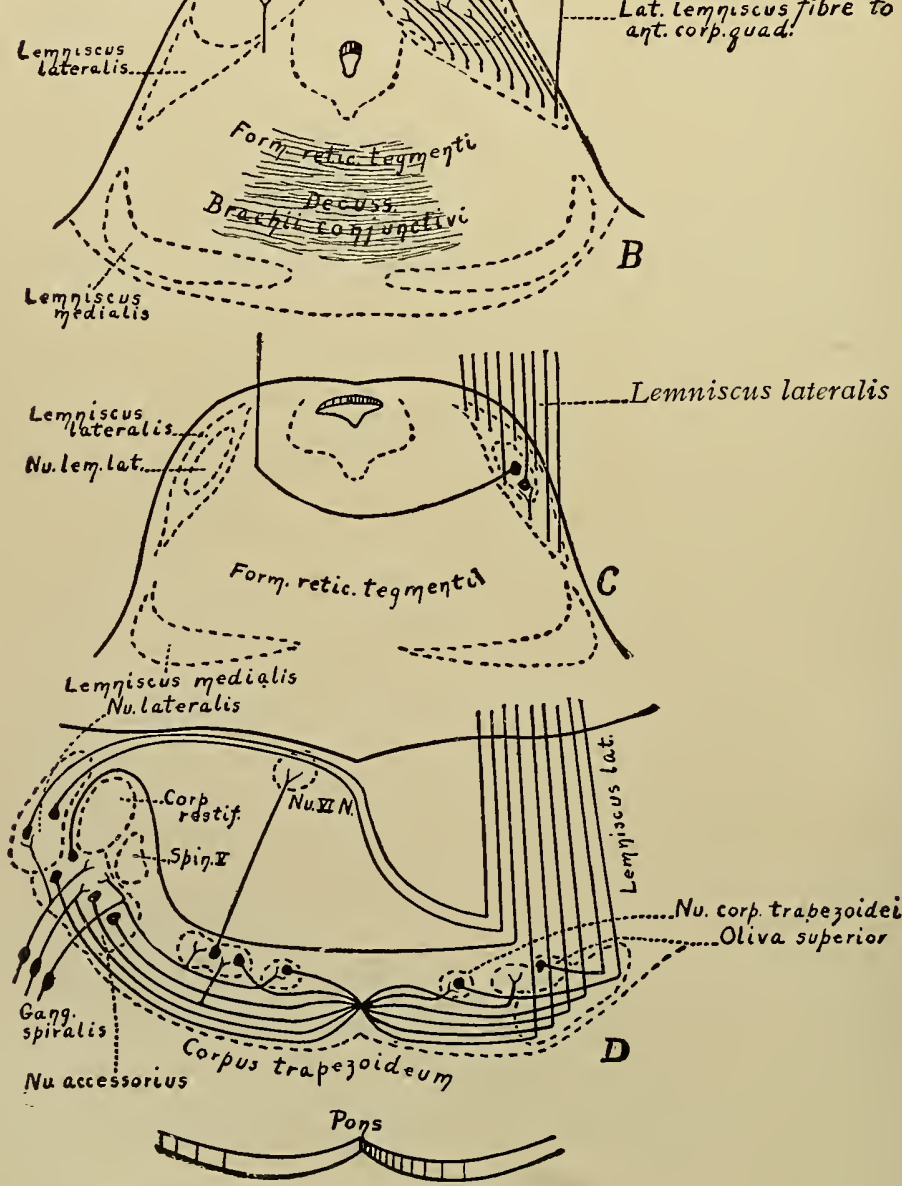

FIG. 330 


\section{EXPLAN.ITION OF FIG. $33^{\circ}$}

Fig. 330.-Diagram showing Connections of the Cochlear Portion of the Auditory (VIII) Nerve. A, Section at level of superior colliculi (Ant. corp. quad.) and red nucleus; $B$, through level of inferior colliculi (Corpus. quad. post.); $C$, through level of nucleus of lateral lemniscus $(N u$. lem. lat.); $D$, through pons at level of VIII nerve. Spacing between the different levels is not proportionate. In $B$ and $C$ the basis pedunculi is omitted. Each neurone group is indicated by one or several individual neurones.

Neurone No. I.-Cell bodies in spiral ganglion (gang. spiralis); peripheral processes end in organ of Corti; central processes terminate principally in ventral or accessory nucleus ( $N u$. accessorius) and lateral nucleus ( $N u$. lateralis) or tuberculum acusticum; some also terminate in superior olives (Oliva superior), and nuclei of trapezoid body ( $\mathrm{N} u$. corp. trapezoidei) of same and opposite sides.

Veurone No. 2. (and 3?).-Axones of cells in accessory nucleus, in superior olives, and in nuclei of trapezoid body, constitute a ventral path in the lower border of the tegmentum, and form the lateral part of the lateral lemniscus (Lemniscus lateralis) or lateral fillet on the opposite side. Axones of cells in the lateral nucleus traverse the floor of the fourth ventricle as the striæ medullares, forming a dorsal pathway, decussate and then turn ventrally to a point dorsal to the superior olive and join the lateral lemniscus as its mesial part. Some axones also of cells in the accessory and lateral nuclei pass dorsally, looping around the restiform body, and then proceed ventrally (bundle of Held) to join the opposite lemniscus. The lateral lemniscus passes upward to the inferior colliculus, some of the axones terminating en ronte in the nucleus of the lateral lemniscus. From cells in this nucleus some axones again join the lateral lemniscus, and a few decussate and then pass upward to the inferior colliculius. The axones of the lateral lemniscus terminate in the inferior colliculus, or pass on to terminate in the internal geniculate body, merely giving off collaterals to the superior colliculus. Some fibres of the lateral lemniscus probably go to the superior colliculus.

Neurone No.3 (and 4?). - Axones of cells in the gray matter of the inferior colliculus form its brachium (Brachium corp. quad. post.) and ascend to terminate in the internal or medial geniculate body (Corp. genic. inter.).

Neurone No.4 (and 5?).-Axones of cells in the internal geniculate body pass as a part of the thalamic radiation via the posterior part of the internal capsule to the cortex of the temporal lobe of the cerebrum.

The axones which constitute the ventral path (NTeurones I and 2) form a bundle of fibres known as the trapezoid body (Corpus trapezoideum) or trapezius. The decussation of these is peculiar in that the dorsal axones of the bundle on one side become the ventral ones on the opposite side; this accounts for the convergence of the axones at the median raphé.

Axones of cells in the superior olive pass to the nucleus of VI nerve (reflex). There is possibly also a descending path from the lateral nucleus to the spinal cord (not indicaterl). 


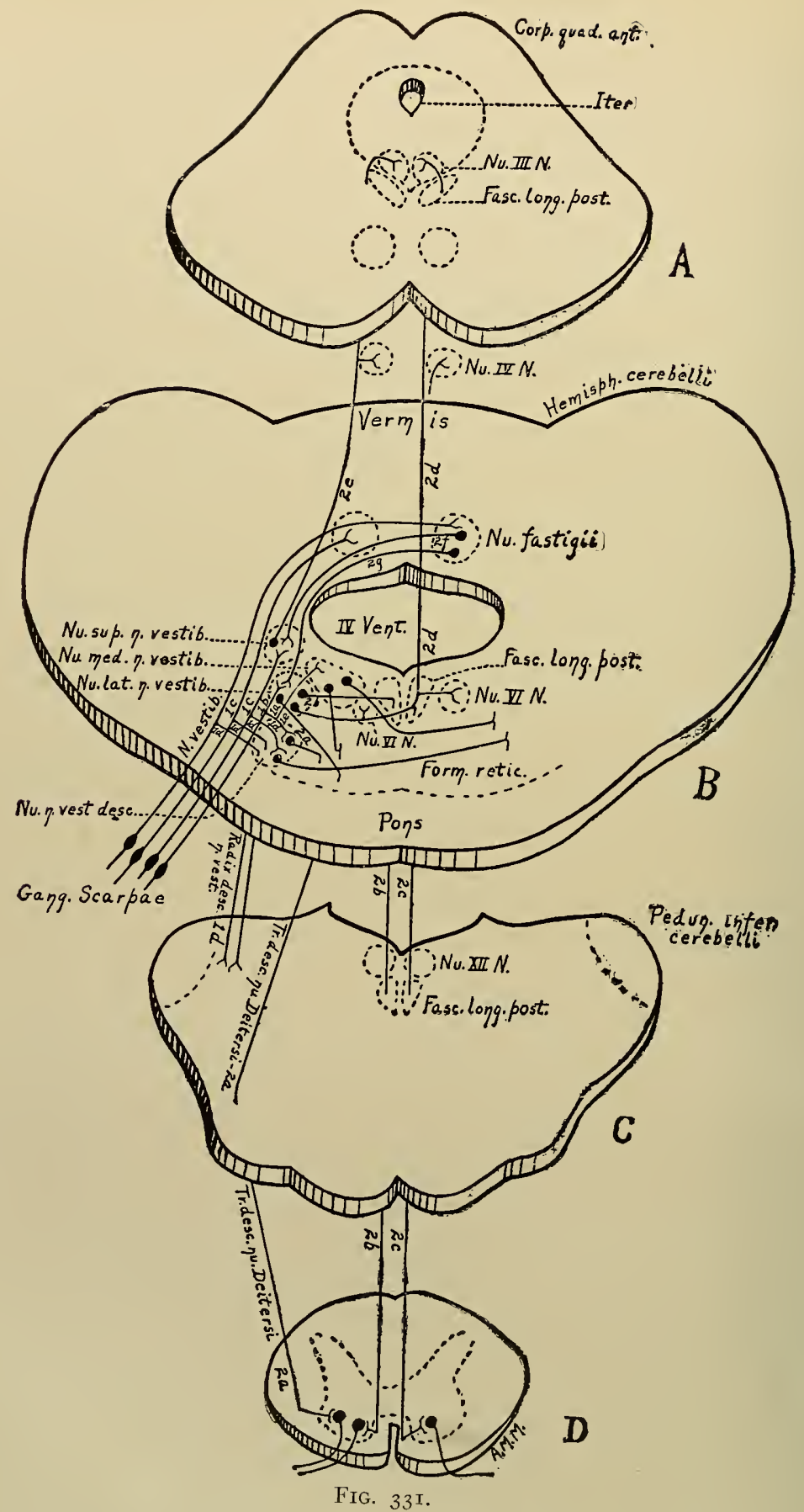


Fig. 33I.-I'rincipal Connections of the Vestibular Portion of the Auditory (VIII) Terve. $A$, Section at level of oculomotor (III) nerve; $B$, section through pons and cerebellum; $C$, through inferior olives; $D$, through spinal cord. Each neurone group is indicated by one or several individual neurones.

Teurone . To. I. - Cell bodies in ganglion of Scarpa; peripheral processes end in semicircular canals; central processes bifurcate, and ascending arms go to Deiters' nucleus

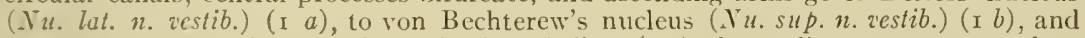
to nuclei fastigii and cortex of vermis of cerebellum (i $c$ ); descending arms go to nucleus of descending root ( $N u, n$. vestib. desc.) ( $\mathrm{f} d)$ and (collaterals?) to principal or median nucleus ( $(\mathrm{T} u$. med. n. restib.) (I $e$ ).

Veurone Wo. 2.- Axones of some cells in Deiters' nucleus descend (Tr. desc. $n u$. Deitersi) uncrossed to antero-lateral column of the cord, axones of other cells enter the posterior longitudinal fasciculus (Fasc. long. post., 2 b) of same side and descend to anterior column of the cord, others pass to the medial longitudinal fasciculus of opposite side whence some $(2 c)$ descend to anterior column of the cord, occupying a position near the anterior median fissure, while some $(2 d)$ ascend in the medial longitudinal fasciculus and terminate principally in the nuclei of VI, IV, and III nerves. Axones of cells in von Bechterew's nucleus ascend $(2 \ell)$, joining lateral part of medial longitudinal fasciculus of same side, and terminate in nuclei of IV and III nerves. Axones of cells in the nucleus of the descending root probably pass in part to lateral part of reticular formation of same and opposite sides, ascending and descending (to other motor nuclei?). Axones of cells in the median nucleus probably pass largely into the reticular formation, possibly also to the medial longitudinal fasciculus (not indicated). Axones of cells in the nuclei fastigii of the cerebellum pass to von Bechterew's nucleus $(2 f)$ and to Deiters' nucleus $(2 g)$. The cerebellar associations intercalated between these $(2 f, 2 \mathrm{~g})$ and the vestibular fibres to the cerebellum ( $\mathrm{I} C$ ) are not known. (It is evident that impulses other than vestibular ones entering the cerebellum may also by $2 f$ and $2 g$ act indirectly upon the motor nuclei innervated by axones of the cells in Deiters' and ron Bechterew's nuclei. Compare Ïigs. 323 and 337.) 


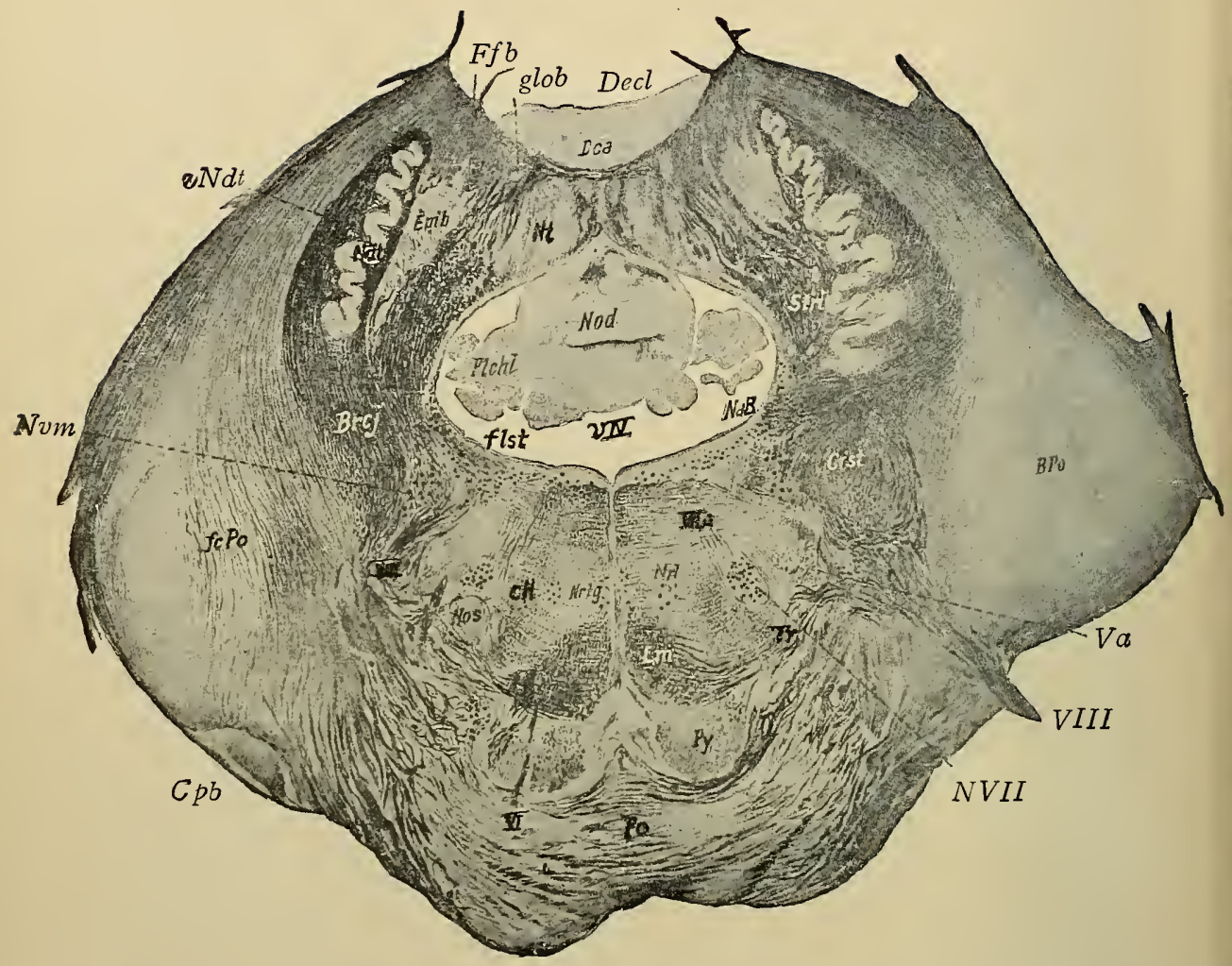

FIG. 332.-Section through the Hindbrain at the Level of the Junction of Pons and Cerebellum and the Entrance of the Vestibular Part of the Eighth Nerve. Weigert preparation. (Marburg.) Va, Radix spinalis trigemini (spinal root of the fifth); VI, nervus abducens (external eye muscle nerve); VIIa, pars nuclearis nervi facialis (pars prima, crus of origin or ascending facial root); VIII, nervus acusticus (eighth, vestibular part); $B P o$, brachium pontis (middle cerebellar peduncle); $B r c j$, brachium conjunctivum (superior cerebellar peduncle); $c H$, fasciculus tegmenti centralis; $C p b$, corpus pontobulbare (Essick); Crst, corpus restiforme; Dca, decussatio cerebelli anterior; Decl, declive; $E m b$, embolus (nucleus emboliformis); $f c$ Po, fibræ pontocerebellares; Ffb, fasciculus fastigiobulbaris (uncinate bundle of Russell); flst, pedunculus flocculi; glob, nucleus globosus; $\mathrm{Lm}$, Lemniscus medialis (mesial fillet tract); NVII, nucleus facialis (motor nucleus of facial nerve); $N a B$, nucleus angularis, or superior, vestibularis (Bechterew); $N d t$, nucleus dentatus cerebelli; Nod, nodulus cerebelli; Nos, nucleus olivaris superior; $N r l$, nucleus reticularis lateralis (nucleus of the lateral column); Nrtg, nucleus reticularis tegmenti; $N$, nucleus tecti (nucleus fastigii); $N v m$, nucleus vestibularis magnocellularis or lateralis (Deiters) (large-celled nucleus of vestibular nerve); Plchil, plexus chorioideus lateralis; $P o$, pons; $P$ y, pyramid; Stri, stratum intermedium (pedunculi); $T r$, corpus trapezoides; $v I V$, ventriculus quartus; $v N d t$, vellus nuclei dentati cerebelli (fleece of the cerebellar olive). 
still further increased in size. External arcuate fibres may be present and probably contain fibres to the restiform body and possibly fibres from the cerebellum, which end in the reticular formation. The arcuate nuclei are present. The central tegmental tract is larger.

Intersegmental Neurones.-The reticular formation is very extended. Its composition (longitudinal and transverse fibres, and cells) should be examined carefully. The rubro-spinal tract is in the same position, but the lateral Deiterospinal tract is now more internally located. Its fibres cannot usually be distinguished, but are bending inward and toward its nucleus of origin (located somewhat higher). The medial longitudinal fasciculus may be partially separated from the medial lemniscus. It is a complex bundle and contains at various levels $(a)$ descending and ascending fibres from Deiters' nucleus and other cells scattered in the reticular formation, $(b)$ descending fibres from the nucleus of the medial longitudinal fasciculus in the tegmentum of the midbrain. The fibres of this fasciculus probably terminate in many nuclei, especially those of eye-muscle nerves (III, IV and VI) (comp. Figs. 33I, 337 and 350).

Efferent Suprasegmental Neurones.-The pyramids and colliculo-spinal tracts are in the same positions. The aberrant efferent pallial fibres already noted (p. 49I) may be seen in the lemniscus.

\section{Section through the Hindbrain at Level of Junction of Pons and Cerebellum and Entrance of Vestibular Nerve (Figs. 324 and 332)}

The most conspicuous features are the nuclei and fibres of the pons, added ventrally to the preceding structures, which are now collectively known as the tegmentum; the cerebellum, enclosing dorsally the fourth ventricle; and the connections (inferior and middle peduncles, arms, or brachia) of the cerebellum with the rest of the brain. The greater part (restiform body) of the inferior peduncle represents ascending ccrebellar connections from all parts below this level, the middle peduncle (from the pons) is the second link in the descending connection from the pallium to the cerebellum (pallio-cerebellar path). The superior peduncle (efferent cerebellar) is not fully formed at this level. (Comp. p. 508.)

Efferent Peripheral Neurones.-In this level and that of the next section are present the nuclei and root fibres of nerves VII and VI.

The rucleus of nerve VII, or mucleus facialis is scen occupying a lateral position in the reticular formation similar to that of the nucleus ambiguus. In it may be made out the usual plexus of fine terminals and the coarser root fibres which proceed dorso-mesially to the floor of the ventricle, where they partially envelop the nucleus of the VI (usually not present in this level). They then turn cephalarl, forming a compact longitudinal bundic (next section) and finally turn ventro-laterally and caudally to emerge on the lateral aspect at the catudal border of the pons. 'This latter part is the second part as distinguished from the first part of the connection. The bend is known as the genu facialis. Iccording to some authorities, some of the filbres cross and pass out in the root of the opprosite side.

Four groups of cells composing the nucleus facialis have been distinguished: three ventral groups which, passing from the most mesial to the inost lateral group, innervate respectively the muscles of tympanum, of pinna and of mouth 
and face. The dorsal group (to superior branch of facial) innervates the frontalis, corrugator supercilii and orbicularis palpebrarum. Among the terminals in the nucleus have been distinguished collaterals (reflex) from the lateral part of the reticular formation, from the secondary acoustic and trigeminal tracts and other adjacent fibres; also terminals of the colliculo-spinal tract. Whether the nucleus receives direct terminals from the pyramids or whether fibres of the latter are only connected with it via intercalated neurones is uncertain.

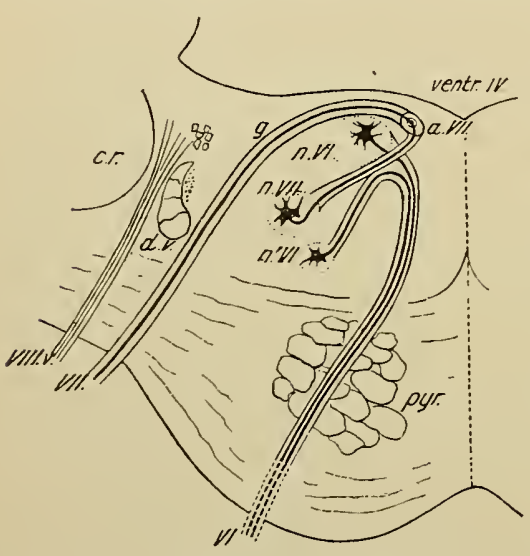

FIG. 333.-Diagram of Origin of Sixth and Seventh Cranial Nerves. (Schäfer.) pyr, Pyramid; $c r$, restiform body; $d V$, spinal root of fifth nerve; Ventr. $I V$, fourth ventricle; VIII.v, vestibular root of eighth nerve; $n . V I$, chief nucleus of sixth nerve; $n^{\prime} V I$, accessory nucleus of sixth nerve; $V I$, sixth nerve; $n . V I I$, nucleus of seventh nerve, from which the axones pass dorsomesially to the floor of the ventricle, where they turn brainward, appearing as a bundle of transversely cut fibres, $a V I I$, and ascend to the "genu." $g$, where they turn and pass ventro-laterally and somewhat caudally to the surface as the seventh nerve, VII.

Some of the root fibres of the VI are usually seen in the ventral border of the tegmentum. For nucleus see next section.

Afferent Roots, their Terminal Nuclei and Secondary Tracts.-The afferent vestibular root fibres enter at the caudal border of the pons and pass lateral to the spinal $\mathrm{V}$, mesial to the ventral cochlear nucleus and restiform body, and enter the field previously occupied by the descending vestibular root, the fibres of which are a continuation of the root. Scattered large cells in this region form the nucleus of Deiters. Dorso-mesial to this is still the medial vestibular terminal nucleus and dorsal to Deiters' at the external angle of the fourth ventricle is the superior restibular terminal nucleus (von Bechterew). Fibres seen passing from the vestibular region to the cerebellum and lying near the ventricle are partly vestibular root fibres to the cerebellum (especially to the nucleus tecti, or fastigii, see below), and partly descending fibres from cerebellar nuclei (especially from the nuclei fastigii, forming fastigio-bulbar fibres) to Deiters' nucleus, other vestibular nuclei, and other cells in the reticular formation. It is thus evident that such nuclei as Deiters' may act as parts of vestibular bulbo-spinal reflex arcs and also as parts of efferent and possibly afferent cerebellar paths. Internal arcuate fibres from the vestibular area are probably principally fibres (secondary tracts) from the various vestibular nuclei to the medial longitudinal fasciculus and other tracts in the reticular formation. (Comp. pp. 493, 501, 507, Figs. 331, 337.)

The nucleus olivaris superior lies ventral to the nucleus facialis and lateral to the central tegmental tract. This nucleus together with several other small nuclei in its immediate vicinity (preolivary nucleus, semilunar nucleus, trapezoid nucleus) is one of the nuclei intercalated in the cochlear path (Fig. 330) which provides reflex connections (e.g., with the VI and VII motor nuclei). Lateral 
to it is seen a mass of fibres which pass by it toward the median line through the medial lemniscus, and decussate, finally turning longitudinally dorso-lateral to the opposite superior olive. These are fibres of the trapezius, and together with the more dorsal secondary cochlear fibres (p. +93) form the lateral lemniscus (See Fig. 330 and page 493). The lateral lemniscus is thus one of the links in the cochlear or auditory pathway. Fibres pass from superior olive to nucleus of nerve VI (reflex). In some cases the slender afferent root of VII (portio intermedia or nerve of Irisberg) from the ganglion geniculi may be seen entering between the vestibular and main facial roots. Its fibres proceed to a gray mass which may be regarded as a continuation of the nucleus fasciculi solitarii and there probably partly terminate and partly send descending arms to join the fasciculus solitarius.

The spinal $\mathrm{V}$ occupies the same position though separated from the surface by the pontile fibres; internal to it is its terminal nucleus. Note the change in the shape of the lemniscus. The ventral spino-cerebellar and spino-thalamic tracts are in the same position though here separated from the surface by the added pontile fibres.

Other Afferent Cerebellar Neurones.-The inferior olives are not present, and the olivo-cerebellar fibres are here entering the cerebellum as a part of the restiform body. The central tegmental tract (to the olives) occupies the ventral part of the reticular formation. The restiform body is entering the white matter of the cerebellum. It has been seen to be composed of the dorsal spino-cerebellar tract, olivo-cerebellar fibres and fibres from the lateral and possibly other nuclei in the reticular formation. The dorsal spino-cerebellar tract terminates in the cortex of the vermis or middle lobe of the cerebellum, the olivo-cerebellar fibres terminate in all parts of the cerebellar cortex. The fibres mesial to the restiform body, consisting of ascending vestibular fibres to the cerebellum and descending fibres to vestibular and other nuclei (see cerebellum), are sometimes called the $i n$ ternal or juxta-restiform body. This and the restiform body proper constitute the inferior cerebellar peduncle. The pons consists of gray matter-the pontile nuclei -and of transverse and longitudinal fibres. The longitudinal fibres include the pyramids which pass through to the medulla and cord, and other fibres from the pallium (pallio-pontile or cerebro-pontile) which terminate in the pontile nuclei. The axones of the latter form the transverse pontile fibres (ponto-cerebellar fibres) which cross and pass to the cortex of the opposite cerebellar hemisphere. They constitute the middle cerebellar peduncle or brachium pontis. (Comp. p. 508 .)

The pallio-pontile and ponto-cerebellar neurones constitute the pallio-pontocercbellar path connecting one cerebral with the opposite cerebellar hemisplere (1). 477: path XIII). There are probally also transverse fibres in the pons connecting cerebellum and reticular formation. Fibres passing vertically in the rajpe from pons to reticular formation (perpendicular fibres of pons) may be in part continuations of these and in gart efferent pallial fibres from pes to tegmentum. The latter are cither aberrant fibres or fibres innervating directly or indircetly motor cranial nuclei.

Intersegmental Neurones. - The reticular formation is extensive. In it there may be distinguished, besides the nucki alrearly mentioned, various more 
or less well-defined reticular nuclei (see Fig. 332). The fibres of the lateral Deitero-spinal tract (not distinguishable) are here emerging from Deiters' nucleus. The medial longitudinal fasciculus occupies the same position, but is here well separated from the fillet. Some of the internal arcuate fibres in the dorsal part of the reticular formation may be fibers from Deiters' nucleus to the medial longitudinal fasciculus. They may be crossed or uncrossed, and may descend in it as already mentioned (pp. 466 and 499; or ascend (see Fig. 33I). Other internal arcuate fibres here, as elsewhere, pass from the various terminal nuclei to form secondary tracts. Other transverse fibres are axones of cells of reticular nuclei or collaterals and terminals ending in them.

Efferent Suprasegmental Neurones.-The colliculo-spinal tract lies ventral to the medial longitudinal fasciculus. The pyramids are in the same position, but are partly surrounded by pontile fibres and nuclei. (See also pons, above.)

The Cerebellum.- The gray matter consists of the external gray or cortex, and internal muclei forming interruptions or relays in paths from the cortex. The white matter consists of the fibres of various afferent and efferent cerebellar paths and possibly association fibres of the cerebellum. The cortex is studied elsewhere. The internal nuclei can usually be distinguished. They are the nucleus dentatus cerebelli (corpus dentatum), a convoluted mass of gray resembling the inferior olives (and sometimes called the cerebellar olives), and mesial to this the nucleus globosus, nucleus emboliformis and the nucleus tecti or fastigii. The nucleus fastigii receives fibres from various parts of the cerebellar cortex and also vestibular root fibres (p. 497). Its axones, in part at least, pass to Deiters' nucleus and other nuclei in the reticular formation (fastigio-bulbar tract). This forms a link of the cortico-fastigio-Deitero-spinal path (p. 478, XVIII). The nucleus dentatus, nucleus globosus, and nucleus emboliformis also receive fibres from the cerebellar cortex. Their axones form the superior cerebellar peduncle. (bruchium conjunctivum), cross and pass to the red nucleus, reticular formation, nucleus of nerve III, and thalamus. At the level of the section the superior peduncle is not yet fully formed. This forms links in both the cortico-dentato-rubrospinal and the cerebello-pallial paths (p. 478 , XVII and VII. See also p. 507.)

Note where possible the structure of the plexus chorioideus of the fourth ventricle. It consists of a layer of cuboidal epithelial cells next the ventricle which are ectodermic, and an outer mesodermic part consisting of connective tissue and blood-vessels.

\section{Transverse Section of the Hindbrain through the Roots of Nerves VI (Ab- ducens and VII (Facial) (Figs. 324 and 334)}

Efferent Peripheral Neurones.-The nucleus facialis is usually not present, but various portions of the root fibres may be present (see preceding section), especially the longitudinal part.

The nucleus of the VI or nucleus abducentis is present in about the middle of the floor of the ventricle and just beneath the central gray or partly within it. Its fibres, the root fibres of the abducens, are seen passing ventrally. The nucleus receives collaterals from the axones of Deiters' nucleus (secondary vestibular) on the same and opposite sides and collaterals or terminals from the superior 


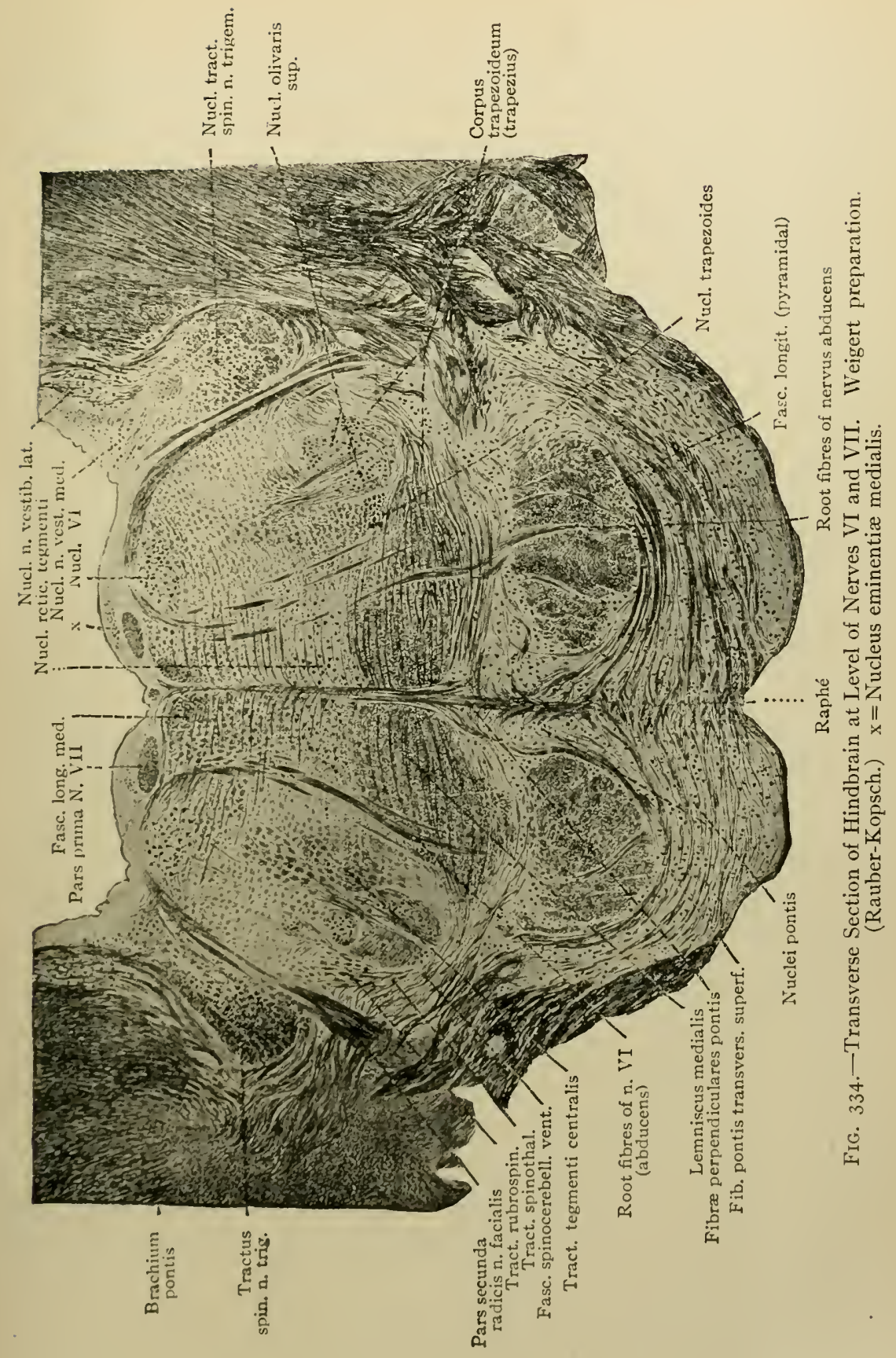


olive (secondary cochlear). The exact mode of connection with the pyramids is not well known.

Afferent Roots, Their Terminal Nuclei and Secondary Tracts.-The lateral (Deiters') and medial vestibular nuclei are usually still present, also possibly fastigio-bulbar fibres. The ventral cochlear nucleus has disappeared, but other cochlear nuclei (superior olivary and trapezoid) are usually present. Often fibres can be seen passing from the superior olive to the nucleus VI.

Fibres of the secondary cochlear tract (corpus trapezoideum) are still traversing the medial lemniscus, and decussating. The tract they are forming (lateral lemniscus) is not yet very distinct.

The spinal $\mathrm{V}$ is in the same position, but it and its terminal nucleus tend to separate into groups of fibres and cells, and to change their relative positions. The medial lemniscus is more flattened in cross section, extending transversely instead of dorso-ventrally. The ventral spino-cerebellar tract and spinothalamic tract are in the same positions in the external part of the tegmentum ventral to the spinal $\mathrm{V}$ and external to the superior olive.

Other Afferent Cerebellar Connections.-The restiform body has now merged with the white matter of the cerebellum. The nuclei and transverse fibres of the pons (ponto-cerebellar neurones) have increased. The longitudinal fibres in the pons at this level are principally the pyramids, but some are palliopontile fibres which terminate in the nuclei pontis. Perpendicular fibres are present.

Intersegmental Neurones.- The reticular formation is practically unchanged. One of its nuclei (nucleus reticularis tegmenit) can be seen as a lighter area (Weigert) in the medial part, dorsal to the medial lemniscus. The rubro-spinal tract is in the same position near or mingled with the spino-thalamic and ventral spino-cerebellar tracts. These fibres are not easily distinguished among the various fibres of the cochlear tract which cross them. The medial longitudinal fasciculus is now a well-marked tract occupying the same position. From now on, it contains ascending fibres from Deiters' nucleus and perhaps other reticular nuclei besides the descending fibres from the nucleus of the medial longitudinal fasciculus.

Efferent Suprasegmental Neurones.-The pyramids and colliculo-spinal tract (predorsal fasciculus) occupy the same positions. The fastigio-bulbar fibres have been mentioned. The superior cerebellar peduncle is now more distinct as it is being formed by fibres from the dentate nucleus. It lies near the dorso-lateral part of the ventricle.

\section{Transverse Section of the Hindbrain Through the Roots of Nerve V (Trigem- inus) (Figs. 324 and 335 )}

Efferent Perip" eral Neurones.-Motor nucleus of $V$. This is mesial to the terminal nucleus of the $\mathrm{V}$ and its coarse efferent root fibres may be seen, in favorable levels, passing out just internal and somewhat cephalad to the entering afferent fibres. It is probable some of the fibres cross and pass out in the opposite motor root. Some of the finer terminal fibres present in the nucleus are afferent root fibres of the $V$ (two-neurone arc) and collaterals of secondary 


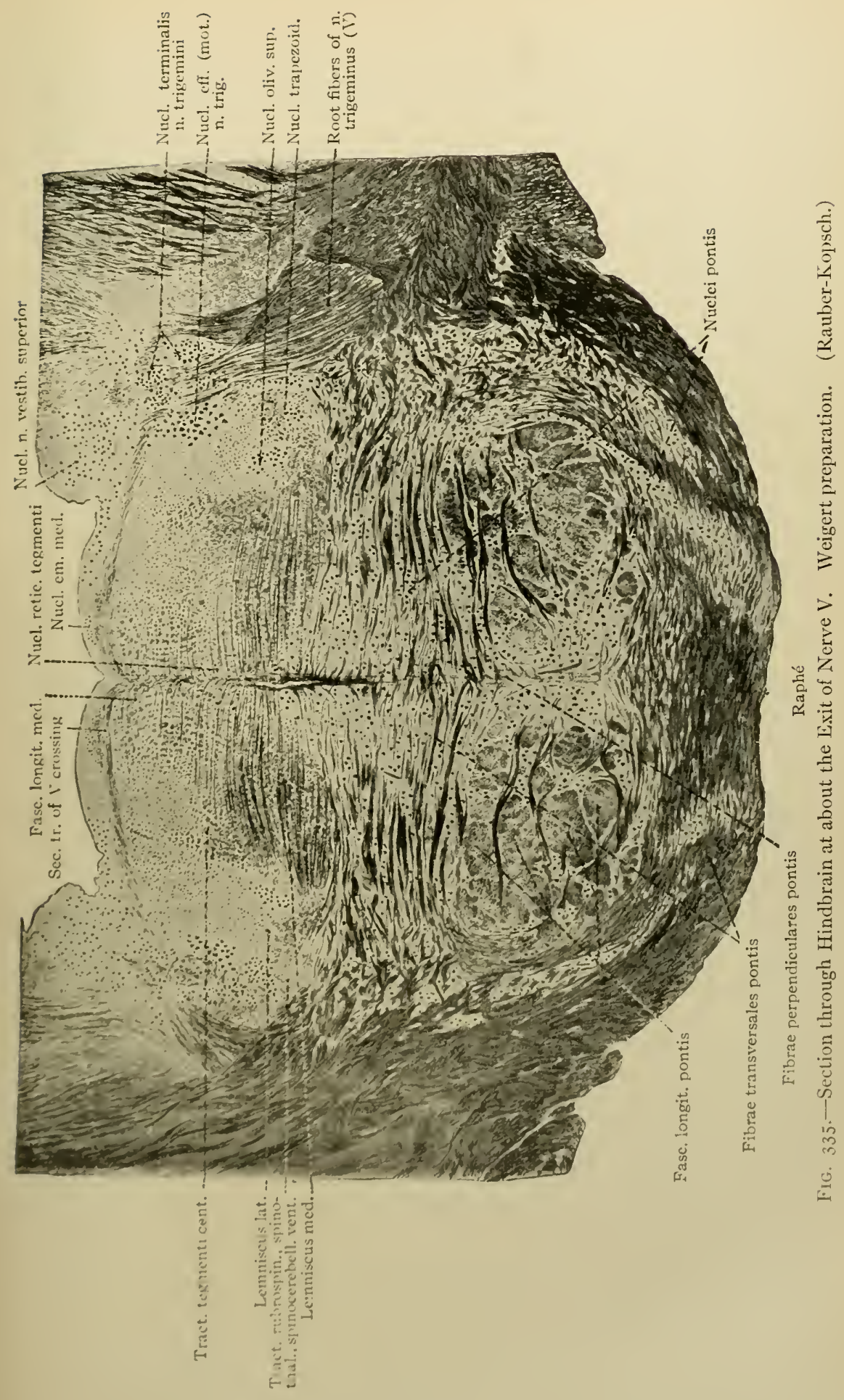


tracts of $\mathrm{V}$ (three-neurone arc). The nature of its connections with efferent pallial fibres is not known. Many collaterals are also received from the mesencephalic root. (Fig. 336.)

Afferent Roots, their Terminal Nuclei and Secondary Tracts.-The afferent fibres of the $\mathrm{V}$ pass through the pons and enter the tegmentum where they divide into short ascending and long descending arms. The former, together with collaterals, terminate in the cephalic end of the terminal nucleus of the V. This is broken up into groups of cells which lie dorso-lateral to the entering fibres and is sometimes known as the "principal sensory" nucleus of the V. The long descending arms pass down to the cord as the spinal V, giving off collaterals and terminals to the nucleus en route. (Fig. 336.) A third source of

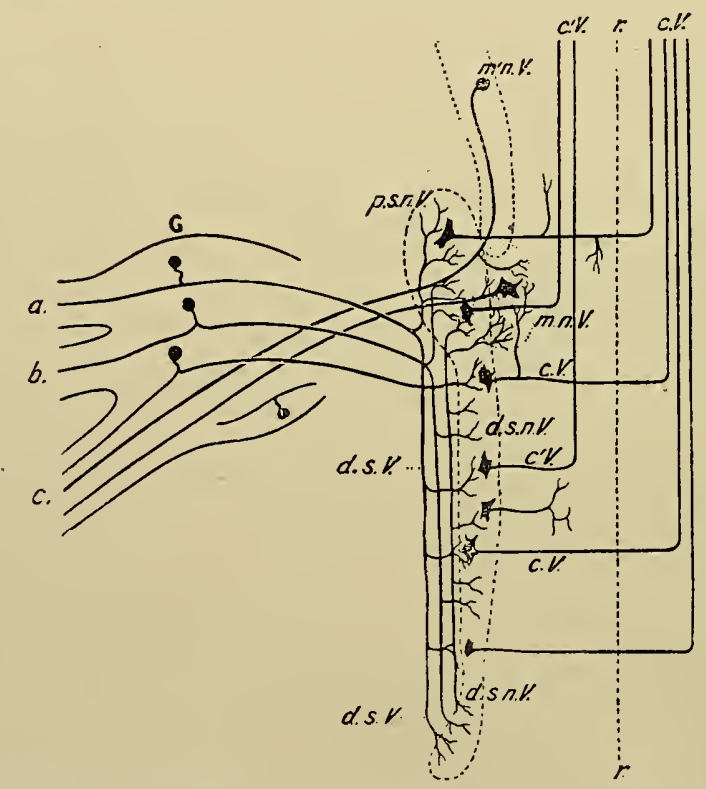

Fig. 336.-Diagram of Origin of Fifth Cranial Nerve. (Schäfer.). G, Gasserian ganglion; $a, b, c$, the three divisions of the nerve; m.n.V, principal motor nucleus; p.s.n.V, principal terminal "sensory" nucleus; d.s.n.V terminal nucleus of spinal root; d.s. $V$, descending or spinal root; $c . V$ and $c^{\prime} . V$, secondary trigeminal tracts (axones of cells in terminal nuclei); $r$, median raphé; $m^{\prime} . n . V$, mesencephalic nucleus.

fibres of the $\mathrm{V}$ is a series of cells extending upward into the roof of the mesencephalon. The axones of these cells form the mesencephalic root of the V. There is reason to suppose, from their peculiar location and for other reasons, that these are afferent peripheral neurones which have remained within the neural tube. From the region of the terminal nucleus of the V, a transverse bundle passes to the opposite side in the floor of the fourth ventricle. This is considered a secondary decussating trigeminal tract which forms an ascending tract in the dorsal part of the reticular formation. Fibres of secondary tracts give off collaterals to various efferent nuclei and probably axones of some cells of the terminal nuclei become intersegmental fibres in the reticular formation. Second- 
ary tracts to the thalamus (via fillet and also in reticular formation?) form part of the trigeminal afferent pallial path.

The superior terminal vestibular nucleus (of von Bechterew) may still be present. The superior olivary and trapezoid nuclei may be present. The secondary (and tertiary (?), see Fig. 330) cochlear tract or lateral lemniscus is now well formed and may be seen lying dorso-lateral to the superior olivary and trapezoid nuclei.

The medial lemniscus is still more flattened. The spino-thalamic and ventral spino-cerebellar tracts occupy the same positions.

Otter Afferent Cerebellar Neurones.-The transverse pons fibres are the same, but the longitudinal fibres have increased owing to the presence of more pallio-pontile fibres. The perpendicular pontile fibres are seen passing dorsally in the raphe into the tegmentum.

The central tegmental tract is in nearly the same position.

Intersegmental Neurones. - The reticular formation is somewhat diminished. In it is the nucleus reticularis tegmenti. The rubro-spinal tract is in the same position, mingled with the spino-thalamic and ventral spino-cerebellar tracts. The medial longitudinil fasciculus is unchanged.

Efferent Suprasegmental Neurones.-The pyramids now occupy the central part of the pons, and are broken up into a number of bundles. In the dcrsal part of the pons fibres pass obliquely dorsally. These are probably efferent pallial fibres which act directly or indirectly on some of the efferent nuclei of cranial nerves (motor path to cranial nerves). The pallio-pontile fibres have been mentioned.

The superior peduncle is now a large bundle of fibres flattened against the ventricular surface of the dorso-lateral brain wall.

The colliculo-spinal tract is in the same position.

\section{Cerebellum}

The cerebellum, connected with the rest of the brain by its three peduncles, consists of two lateral lobes or hemispheres connected by a median lobe, the vermis. These are divided into various lobules, the surfaces of which are marked by parallel transverse folds or laminæ. When these are cut across it is seen that they give off secondary or tertiary laminæ, the whole producing the appearance known as the arbor vite. The surface of the cerebellum is composed of gray matter, the cortex, enveloping the white matter. Besides this there are masses of gray within, the internal nuclei of the cerebellum (dentatus, globosus emboliformis, and fastigii), embedded in its white matter. Fibres entering the cortex are the terminations of the fibres of the restiform body (dorsal spino-cerebellar tract to the cortex of the vermis, olivo-cerebellar fibres to the whole cortex, fibres from the lateral nucleus and possibly other nuclei in the reticular formation, also per- 
haps some fibres from the columns and nuclei of Goll and Burdach of the same and opposite sides), vestibular root fibres to the vermis, the ventral spino-cerebellar tract to the vermis, and the pontile fibres to the cortex of the hemispheres. The cortical cells do not send axones outside the cerebellum, all efferent fibres being interrupted in the internal nuclei. The dentate nucleus receives fibres from the cortex of the hemispheres; the globose and emboliform nuclei receive fibres from the cortex of the vermis; and the nucleus fastigii receives fibres from various parts. The axones of the first three form the superior

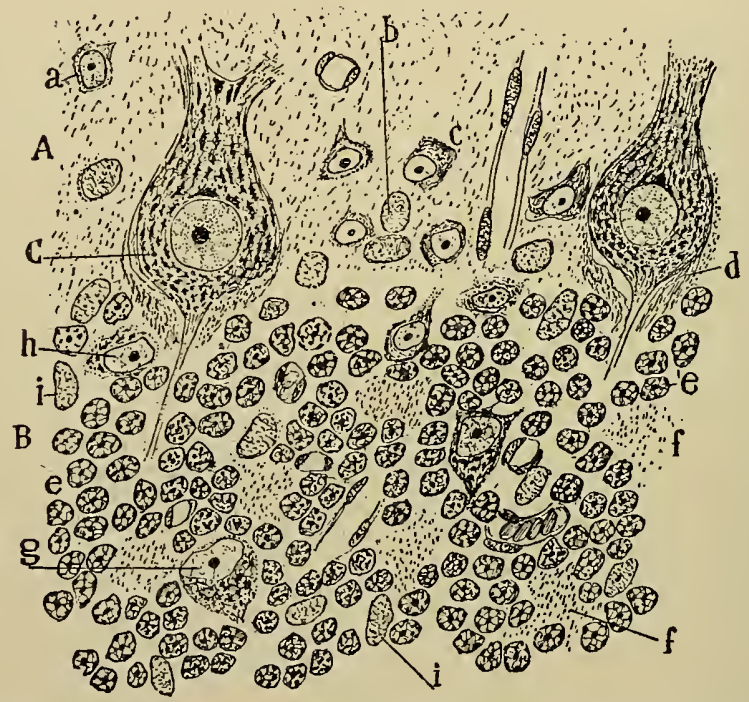

Fig. 338.-Part of a Vertical Section through the Adult Human Cerebellar Cortex. Nissl Method. (Cajal.) $A$, Inner portion of the molecular layer; $B$, granular layer; $C$, body of a Purkinje cell; $a$, stellate cell of the molecular layer; $b$, nuclei of the epitheliallike neuroglia cells (cells of the fibres of Bergmann); $c$, stellate cell with marginal chromophilic substance; $d$, fibrillar mass corresponding to the baskets; $e$, nuclei of the granule cells; $f$, islands or glomeruli in the granular layer; $g, h$, Golgi cells in the granular layer; $i$, nuclei of neuroglia cells.

peduncle; the axones of the nucleus fastigii are fastigio-bulbar fibres, principally crossed, to vestibular nuclei and possibly other reticular formation nuclei. There may be some efferent fibres in the middle peduncle to reticular formation nuclei, but the major part, at least, of this peduncle consists of the ponto-cerebellar fibres already described. The inferior and middle peduncles are thus largely afferent and the superior peduncle is efferent, to red nucleus, thalamus and nucleus of nerve III. (Fig. 323, p. 546 and Fig. 337.) See also pp. 475 and 476 . 
In the cortex can be distinguished an outer or molecular layer with few cells and few medullated fibres, an inner, granular or nuclear layer. and between the two a single row of large flask-shaped cells, the cells of Purkinje (Figs. 338,339 ). These latter give off several main dendrites, which enter the molecular layer and form a remarkably rich arborization extending to the surface. The Golgi method shows the larger and medium branches smooth, but the terminal branches thickly beset with "gemmules." The dendritic arborization is flattened, extending at right angles to the laminæ. The axone is

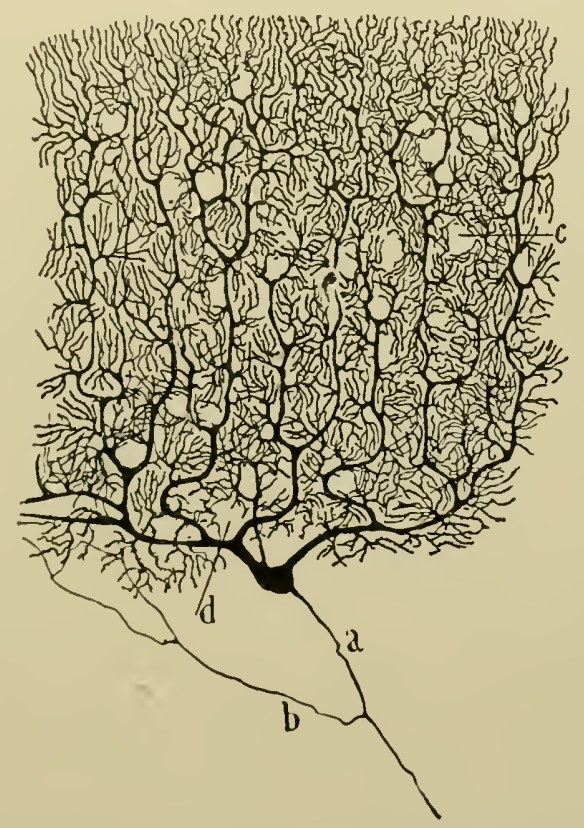

F'Ic. 339.-P'urkinje Cell of Arult Human Cerebellum. Golgi preparation. (Cajal.) $a$, Ixone; $b$, recurrent collateral; $d$, spaces occupied by basket cells; $c$, spaces occupied by blocerl-ressels.

given off from the end opposite to the dendrites and passes through the granular layer into the white matter, either to one of the internal cerchellar nuclei where it terminates, or to some other part of the cortex. The Purkinje cells are almost the only cells the axones of which enter the white matter. It is evident that all intracortical connections must ultimately converge on these cells to reach the efferent cerebellar patlis. The axones of the Purkinje cells give off collaterals not far from their origin, which pass into the molecular layer and appear to terminate there in end "buttons" upon the 
bodies of adjacent Purkinje cells. The medullated axones form the coarser fibres traversing the granular layer. The cell body is fairly well filled with small chromophilic bodies of uniform size, often showing a slightly concentric arrangement (Fig. 338).

The cells in the molecular layer (stellate cells) are either superficial stellate cells with irregular branching dendrites and a short axone or deep stellate (basket) cells. These latter are cells the axones of which (apparently non-medullated) have a narrow neck and unusual thickening beyond the neck. They extend at right angles to the laminæ

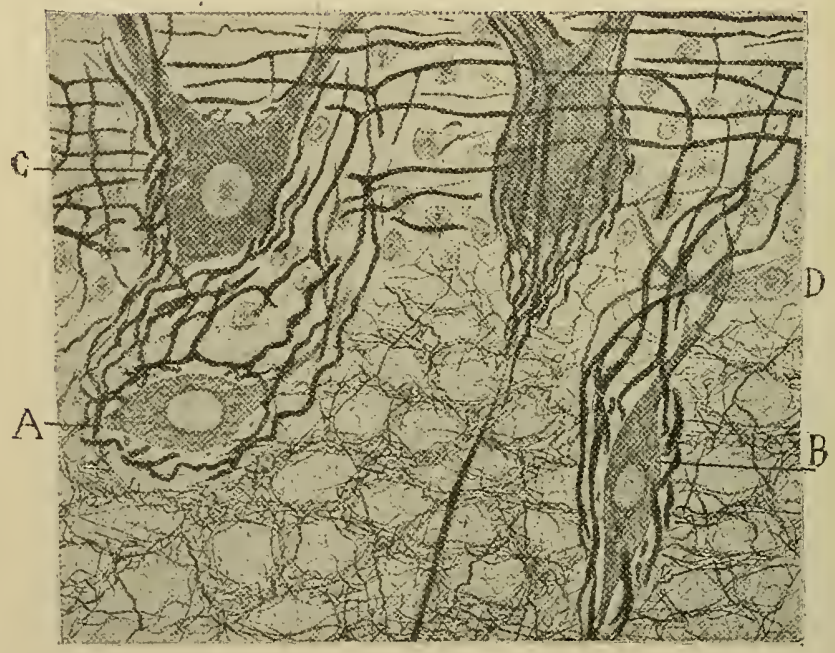

Frg. 340.-Section of Adult Human Cerebellum. Silver Method of Cajal. (Cajal.) $A, B$, Cells in the granular layer enveloped by basket fibres; $C$, cell of Purkinje. The axone of one of the cells of Purkinje is shown.

for a distance of several Purkinje cells, giving off to each Purkinje cell one or more collaterals which pass toward the granular layer and envelop, with their terminal arborizations, the body and proximal, non-medullated portion of the axone of the Purkinje cell. Collaterals of other basket cell axones may terminate around the same Purkinje cell, forming the "basket" (Fig. 340). The dendrites of the basket cells ramify throughout the molecular layer. Besides the cells contained in it, and the dendritic arborizations of the Purkinje cells, the molecular layer contains the axones of the granule cells and the terminations of the climbing fibres (see below). In ordinary stains it presents a general punctate appearance, with the scattered nuclei of the 


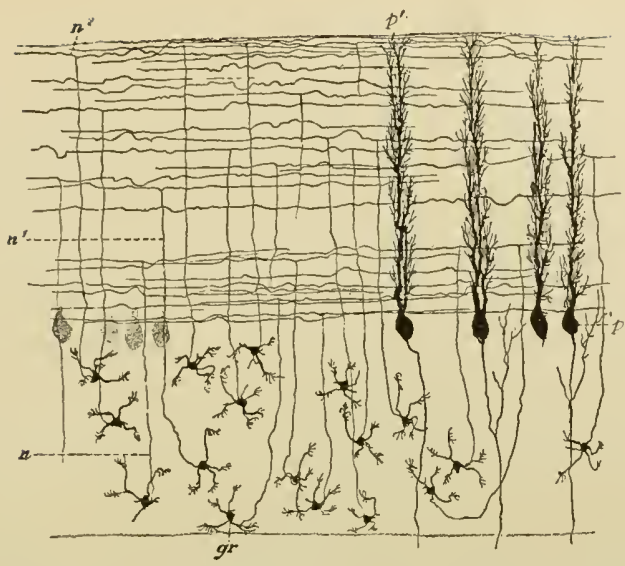

FIG. 34r.-Diagram of Longitudinal Section of Cerebellar Lamin ${ }^{2}$. Golgi method. (Kölliker.) gr, Cell of the granular layer; $n$, axone of granule cell; $n^{\prime}$, the same in molecular layer where it branches and runs in long axis of lamina; $p$, Purkinje cell showing how much less extensively its dendrites $\left(p^{\prime}\right)$ branch in long axis of lamina. (Compare Fig. 343.)

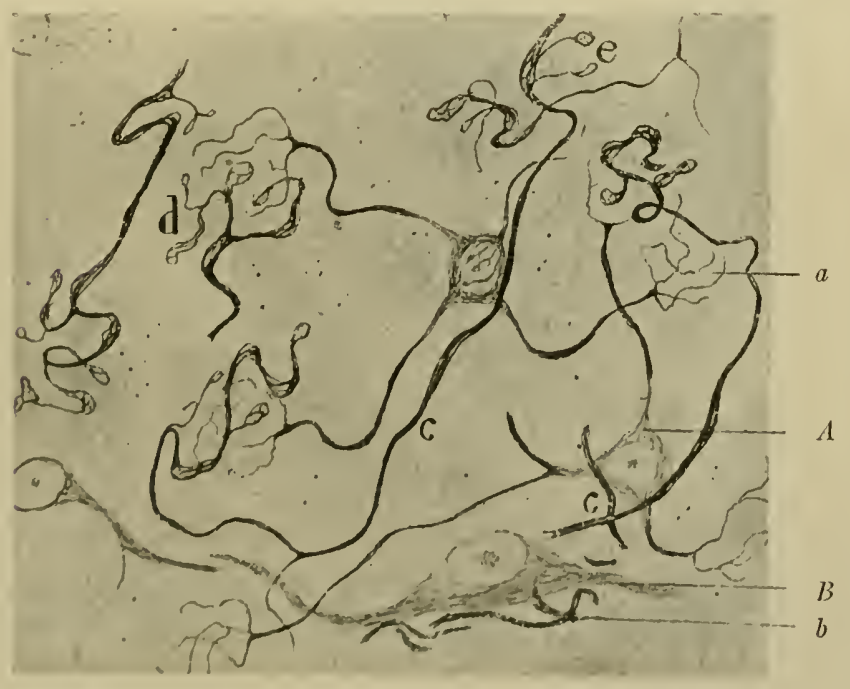

Frs. 342.- (iranule C'clls and Mossy Filores in the ('crelocllum of Arlult Cat. Silver methrod of (ajal. (Cajal.) $A$, (iranule cell; $B$, (Golgi cell; $a$, dendritic arlorization of granule coll; $l$, mossy fibres pássing l,y Ciolgi cell; $c$, mossy fibre; $d$, termination of a mossy"fibre; $e$, terminal processes given off from a thickening in at mossy fibre. 
short axone and basket cells, and the coarser dendrites of the Purkinje cells distinguishable (Fig. 338).

The granular layer with ordinary stains presents the appearance of closely packed nuclei with clear spaces here and there ("islands" or "glomeruli") and also a few larger cells (Fig. 338). Most of these nuclei belong to the gramule cells, which are caryochrome cells. The granule cells are small and possess three to six dendrites which are

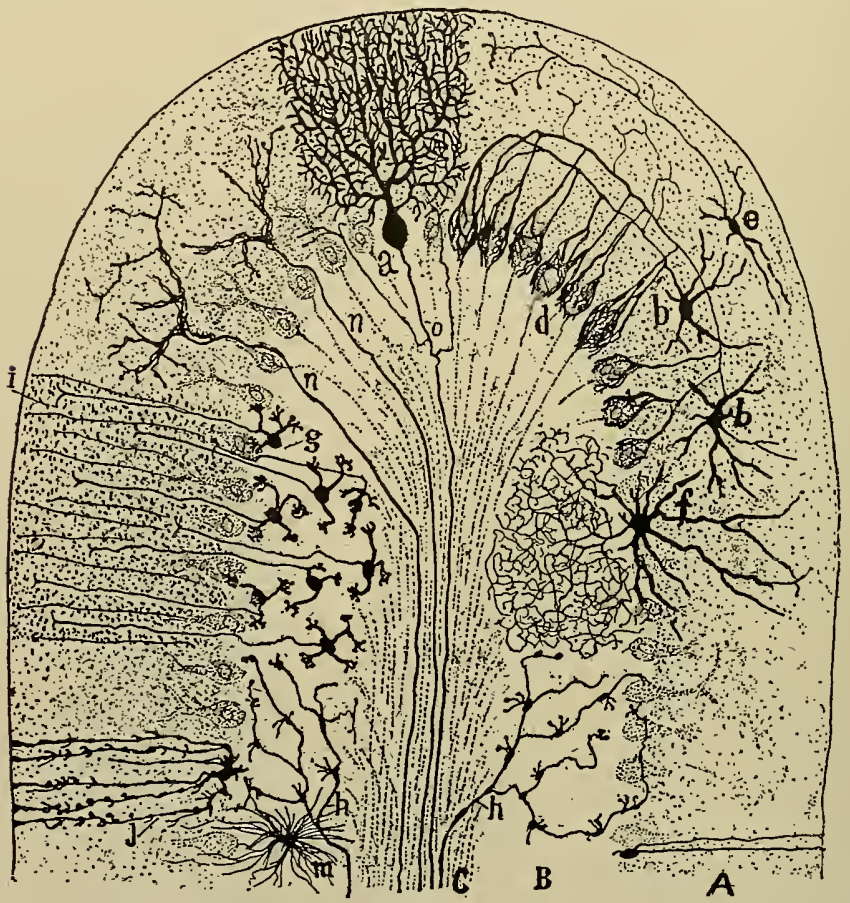

FIG. 343.- Semi-diagrammatic transverse Section of a Cerebellar Lamina of a Mammal, as shown by the Golgi Method. (Cajal.) $A$, Molecular layer; $B$, granular layer; $C$, white matter; $a$, Purkinje cell, seen flat; $b$, basket cells of the molecular layer; $d$, their terminal arborizations which envelop the bodies of the Purkinje cells; $e$, superficial stellate cells; $f$, Golgi cell; $g$, granule cells with their axis-cylinders ascending and bifurcating at $i ; h$, mossy fibres; $j$, neuroglia cell; $m$, neuroglia cell in granular layer; $n$, climbing fibres.

comparatively short and terminate in the glomeruli with a compact arborization, each branch of which ends in a small varicosity. The axones of the granule cells, which are non-medullated, ascend into the molecular layer where each divides into two branches running longitudinally along the laminæ and terminating in varicosities (Figs. 34I, 343). These are the parallel fibres of the molecular layer. They thus run at right angles to and through the dendritic expansions of 
the Purkinje cells and their cross sections together with the terminal dendritic arborizations of the Purkinje cells give the molecular layer its punctate appearance. The scattered larger cells in the granular layer are principally short axone or Golgi cells, whose main dendrites usually penetrate and branch within the molecular layer. Their axones often form very extensive and complicated arborizations in the granular layer, the terminations of which are concentrated in the glomeruli (Fig. $343, f$ ). Dislocated cells of this type may have their cell bodies in the molecular layer.

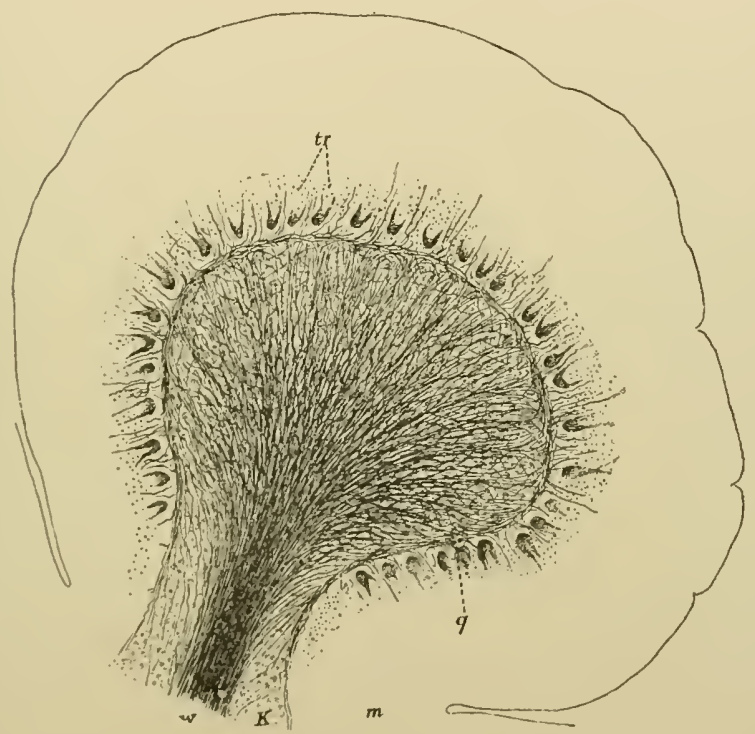

FIG. 344--Cross Section of a Cerebellar Convolution Stained by WVeigert's Method. (Köliker.) $m$, Molecular layer; $K$, granular layer; $w$, white matter; $q$, fine fibres passing from white matter into the molecular layer; $t r$, dots represent longitudinal fibres of molecular layer among bodies of P'urkinje cells.

In the cortex there are also the terminations of the afferent cercbellar fibres already mentionerl (p. 507). These are of two types, mossy fibres and climbing fibres. The mossy fibres, so called from the appearance of their terminations in embryos, are the coarsest fibres of the white matter. While in the latter they bifurcate, branches going to different laminæ. These main branches give off secondary branches which enter the granular layer and there arborize. Juring their course, and also at their terminations, these branches are thickenert in places and there give off short, thick, terminal 
branches which end in varicosities. These terminal branches are located within the glomeruli. The glomeruli thus contain the dendritic terminations of the granule cells, the axonal terminations of the Golgi cells, and the terminations of the mossy fibres.

(Figs. 343 and 342.)

The climbing fibres pass from the white matter, through the granular layer to the cells of Purkinje. Passing by the bodies of the latter they arborize into terminals which envelop the smooth dendritic branches of the Purkinje cells; i.e., all but the terminal dendritic arborizations. (Fig. 343,n). Three kinds of fibres thus terminate around the Purkinje cells; the granule axones, probably in contact with its terminal dendritic arborizations; the climbing fibres around its coarser dendritic branches; and the basket fibres around its body. The respective sources of the mossy and climbing fibres are unknown. There is some evidence that the climbing fibres are from the pons.

It is evident from the above that all of the cells of the cerebellar cortex except the Purkinje cells are association cells of the cortex.

The medullated fibres of the cerebellum (Fig. 344) pass from the white matter into the granular layer and ramify throughout the latter, forming quite a dense plexus separating groups of granule cells. Sometimes straight fibres can be seen passing through toward the molecular layer which are probably either the climbing fibres or axones of the Purkinje cells. Beneath and between the bodies of the Purkinje cells is a plexus of fibres extending into the deeper part of the molecular layer, the remainder of this layer containing few or no medullated fibres. These fibres in the vicinity of the Purkinje cells are probably principally formed by the recurrent collaterals of the Purkinje cells already mentioned. The remaining fibres of the granular plexus would apparently consist of the arborizations of mossy fibres and of the Golgi cells. Whether the former are medullated is, however, somewhat uncertain.

Most of the neuroglia cells in the cerebellum are of the same general type as seen elsewhere, but in the Purkinje cell layer are apparently epithelial-like cells which send vertical processes to the periphery. Some of these processes, as seen in the Golgi method, are rough and branched, others are smooth. In ordinary stains these processes are sometimes visible and are known as the fibres of Bergmann. (Figs. 338, 343.) 


\title{
Isthmus
}

\section{PRACTICAL STUDY}

9. Transverse Section through the Isthmus at the Exit of Nerve IV (Trochlearis)

\author{
(Figs. 324 and 345)
}

In this there are to be distinguished three parts, the thin roof (superior medullary velum), the tegmentum and, ventral to the latter, the pons. The tegmentum consists essentially of the reticular formation, efferent cerebellar and midbrain connections, and externally the afferent pallial connections. The pons contains the efferent pallial paths to the cerebellum and to parts of the nervous system caudal to it. The cavity is the itcr or aquaductus Sylvii. Next to this is the central gray of the brain wall.

Efferent Peripheral Neurones.-The root fibres of the $I V$ are seen in the roof. They originate from nuclei lying further forward in the ventral part of the central gray. The fibres pass from the nuclei dorsally and caudally in the outer part of the central gray and finally decussate in the roof and emerge. It is only the latter part of this course which is seen in this level.

Afferent Roots, their Terminal Nuclei and Secondary Tracts.-The mesencephalic root of the $\mathrm{V}$ lies in the lateral part of the central gray. Mingled with its fibres may be seen the rounded cells, the axones of which form these fibres.

The lateral lemniscus occupies part of the lateral swelling on the surface of the tegmentum, forming the major part of the external structure known as the trigonum lemnisci. Groups of cells among its fibres constitute the dorsal mucleus of the lateral lemniscus. The medial lemniscus is now still more flattened. The spino-thalamic tract is in about the same position, between the two lemnisci. Thus at this level, the principal afferent suprasegmental paths form an L-shaped mass, enveloping the rest of the tegmentum and representing general bodily scnsation and hearing. There are also cranial nerve ascending paths lying probably within the reticular formation and fillet (secondary vago-glossopharyngeal and trigeminal tracts, representing visceral, taste, and general head sensation). These cannot be distinguished in the section.

The ventral spino-cerebellar tract is on the surface, and now comes to lie external to the superior cerebellar peduncle. At about this point it turns caudally, and passes back into the cerebellum, accompanying the superior peduncle.

Otl er Afferent Cerebellar Connections. - The central tegmental tract occupies the same position. (For the pallio-cercbellar connection see "Efferent Suprasegmental Neurones" below.)

Intersegmental Neurones.- I'he reticular formation is diminished in extent. One of its nuclei, the nucleus centralis superior, lies near the raphé. The rubrospinal tract has moved somewhat mesially. It is dorsal to the medial lemniscus. The medial Jongitudinal fasciculus is in the same position, and is a well-marked bundle lying at the boundary between the ventral part of the central gray and the reticular formation.

Efferent Suprasegmental Neurones. -The pyramids are now broken up into bundles which may show a tendency to gather in the ventral part of the pons 


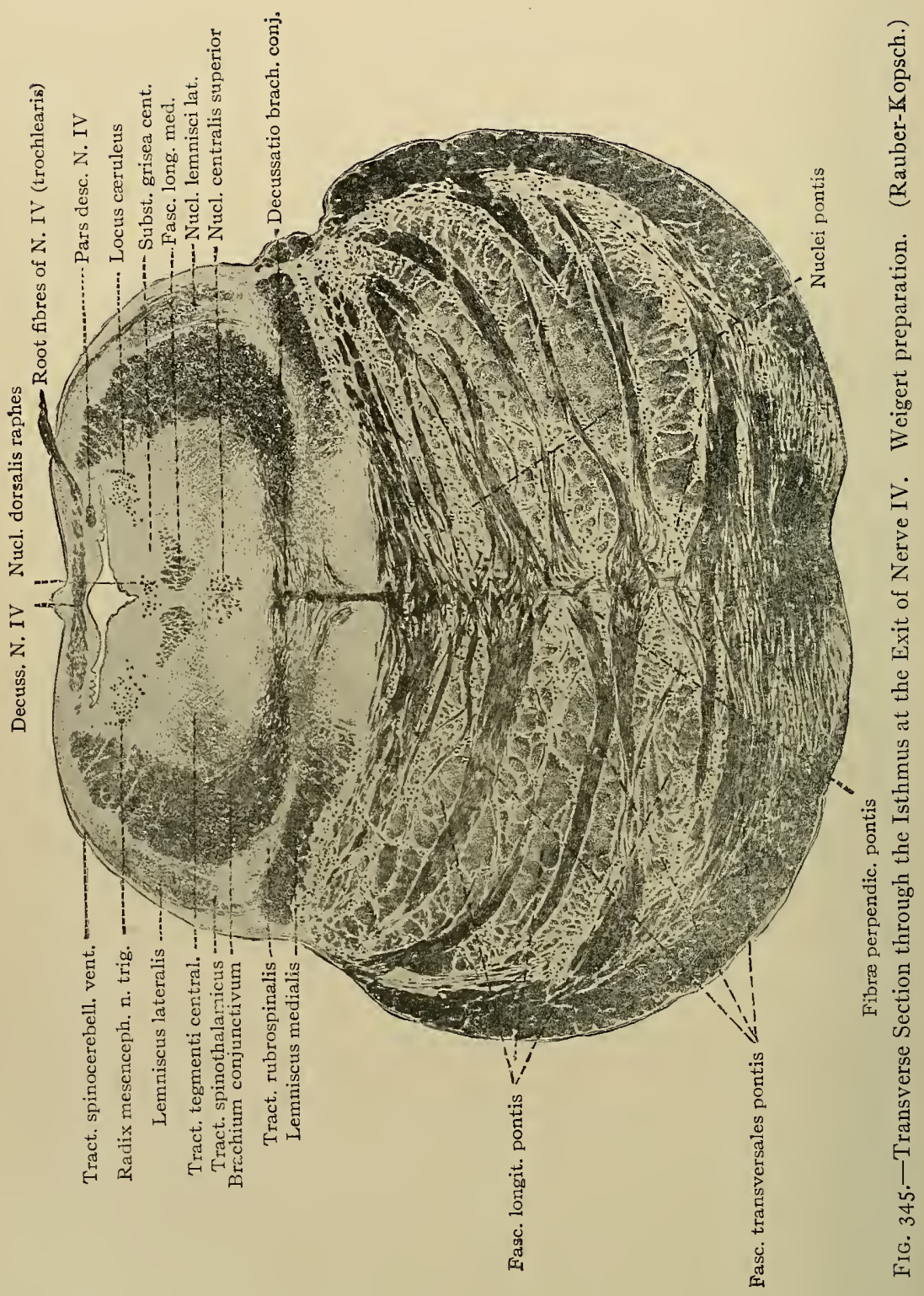


(distinguishable in one-month infant, where they are medullated while the palliopontile system is not). Bundles apparently forming lateral and mesial portions of the medial lemniscus (not indicated in the figure) are aberrant efferent pallial fibres. Such bundles have been seen passing from pons to tegmentum and also imbedded in the medial lemniscus in lower levels (pp. 50r, 49I). Some of these fibres are possibly fibres acting directly or indirectly on the efferent peripheral neurones or motor nuclei of the cranial nerves (see p. 52I).

The pallio-pontile fibres are still more numerous. The gray matter in the pons (nuclei pontis) is very extensive. The transverse fibres of the pons no longer pass at this level into the cerebellum, but are collected at the sides of the pons to pass backward to the cerebellum (compare with an external view of the brain).

The superior cerebellar peduncles or brachia conjunctiva are two large crescentric bundles of fibres in the lateral part of the reticular formation. Some of their fibres have begun to decussate in the ventral part of the reticular formation.

The colliculo-spinal tract or predorsal fasciculus lies ventral to the medial longitudinal fasciculus.

\section{Midbrain or Mesencephalon}

The dorsal surface of the midbrain presents four rounded prominences, the two inferior and two superior colliculi (posterior and anterior corpora quadrigemina). Ventrally are seen two diverging masses of longitudinal fibres, the pes pedunculi, separated by a deep groove or sulcus. In the midbrain are to be distinguished, $(a)$ the expanded roof, the colliculi or corpora quadrigemina, (b) the tegmentum containing the segmental (cranial nerves IV and III) and intersegmental apparatus and the afferent suprasegmental paths, and $(c)$ the basis pedunculi, ventral to the tegmentum and comprising the principal efferent pallial paths (pes pedunculi) and the substantia nigra.

The cavity of the midbrain is the aqueduclus Sylvii or iler.

\section{PRACTICAL STUDY}

I0. Transverse Section through Midbrain at Level of Superior Colliculi (Anterior Corpora Quadrigemina) and Exit of Nerve III (Oculomotor) (Figs.

$$
324 \text { and 347) }
$$

(omparerl with the preceling section, the following are the most conspicuous changes: The roof has now enlargerl into the superior colliculi; the tegmentum now contains the nuclei and roots of nerve 111 and the red nucleus; instead of the pons, the ventral part of the brain is now composed of the basis perlunculi, consisting of a mass of efferent pallial fibres and the substantia nigra. The 
term crura cerebri or cerebral peduncles is loosely used to include all except the roof of the brain at this level, i.e., tegmentum and basis pedunculi.

Efferent Peripl eral Neurones. -The nucleus of nerve III or oculomotor nucleus is located in the ventral part of the central gray in a $\mathrm{V}$-shaped trough formed by the fibres of the medial longitudinal fasciculus. The nucleus is divided into large and small-celled groups. The large-celled groups are two lateral groups subdivided

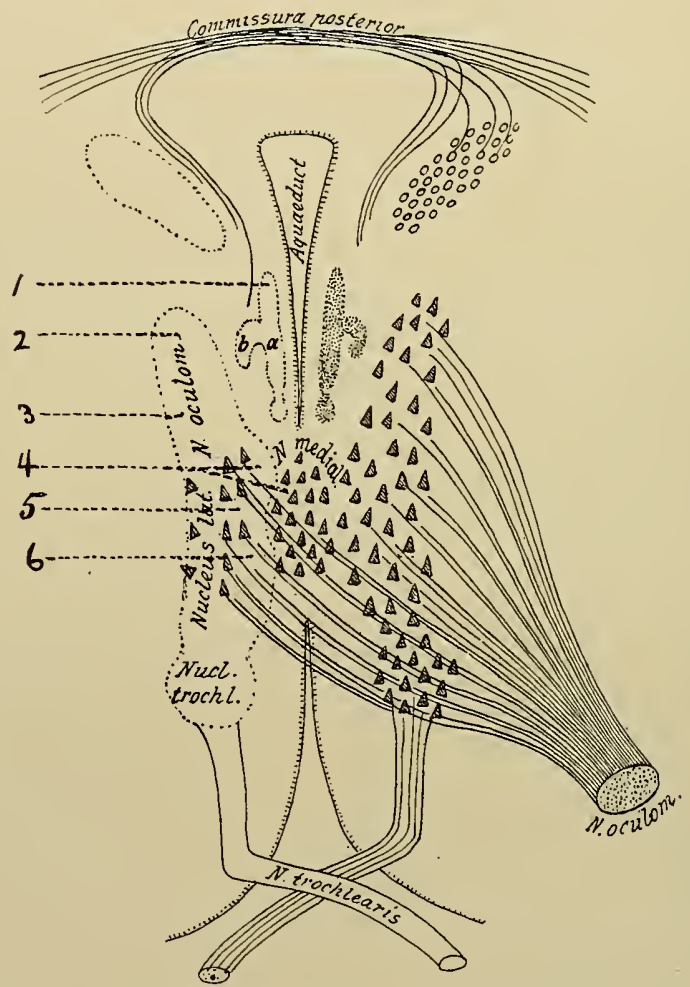

Fig. 346.-The region of the aquæductus Sylvii seen from above. Schema showing the position of the nuclei of nerves III and IV and their subdivisions. (Edinger.) I. The small-celled nucleus (here represented as one on each side) $a$, its ciliary, $b$, its pupillary portion. 2, The portion of the large-celled nucleus sending uncrossed fibres to M. levator palpebræ; 3 , portion sending uncrossed fibres to M. rectus superior; 4 and 5, portions sending crossed and uncrossed fibres to $\mathrm{Mm}$. rectus internus and obliquus inferior; 6 , portion sending crossed fibres to $M$. rectus inferior. The nucleus trochlearis sends crossed fibres to M. obliquus superior.

into anterior and posterior dorso-lateral and anterior and posterior ventro-mesial, and a central or median group-nine in all. Between the cephalic or anterior groups are on each side a small-celled group known as the Edinger-Westphal nucleus, and still further forward are two small-celled anterior median nuclei. The connections of these groups with the extrinsic muscles of the eye innervated by nerve III (internal, superior and inferior recti, and inferior oblique) and the levator palpebræ superioris and intrinsic eye muscles (ciliary and sphincter 


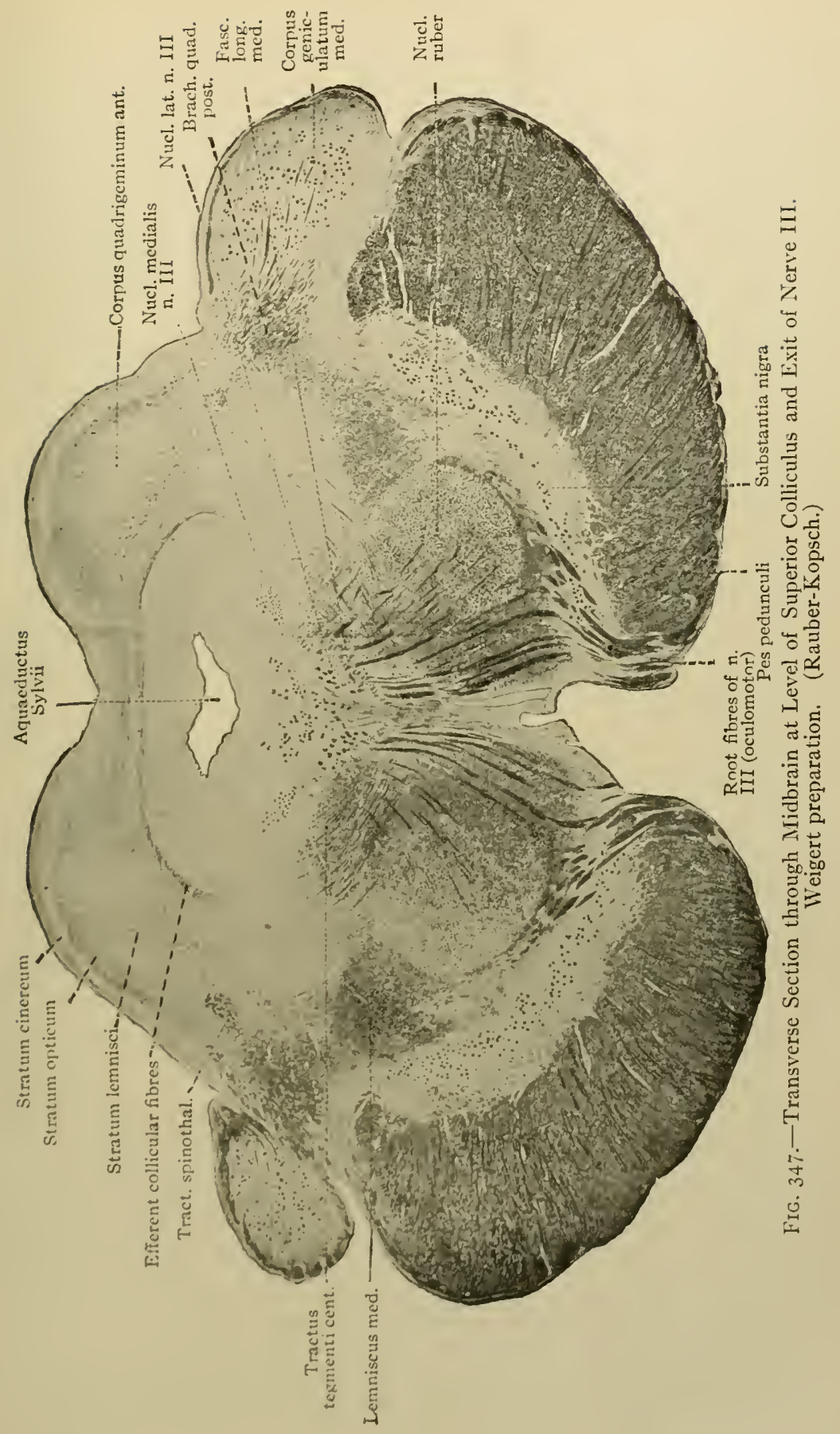


pupillæ, via ciliary sympathetic ganglion) are uncertain. From $a$ priori grounds, the innervation of the intrinsic muscles by the small-celled groups and the other muscles by the large-celled groups would seem probable. Some of the fibres, usually stated to be from the posterior dorsal-lateral group, decussate. Recent observations (Cajal) would indicate that the decussating fibres come from the ventro-mesial lateral groups. What is perhaps the prevailing view as to these relations is shown in Fig. 346. The various fibres pass ventrally in a number of bundles, some passing mesial to, some traversing, and some passing lateral to the superior cerebellar peduncle and red nucleus. Ventral to these the root fibres come together and emerge on the ventral aspect of the midbrain (Figs. 347 and 348.)

The nucleus of nerve III receives terminals and collaterals from the medial longitudinal fasciculus, i.e., from the ascending axones from Deiters' nucleus and the descending axones from the nucleus of the medial longitudinal fasciculus, possibly collaterals from the colliculo-spinal tract, collaterals or terminals from the superior cerebellar peduncle and from the reticular formation. Its exact relation to efferent pallial fibres is not known. Fibres in the medial longitudinal fasciculus connect the nuclei of III, IV and VI (synergic movements of eye muscles).

Immediately caudal to the nucleus of nerve III (not in the plane of the section) is the nucleus of the nerve IV occupying a position similar to the lateral groups of the nerve III nucleus. The course of the root fibres of the IV has been mentioned (preceding section). It receives terminals similar to those received by III.

Afferent Roots, their Terminal Nuclei and Secondary Tracts.-The mesencephalic trigeminal root is sometimes distinguishable on the lateral border of the central gray.

The lateral lemniscus has partly or wholly terminated in the inferior colliculus at a lower level. Fibres from the latter form its arm or brachium, representing another link of the cochlear path. In the present section the fibres of the brachium of the inferior colliculus are seen entering the medial geniculate body (which is a part of the thalamus). Axones of the cells of this body constitute the last relay of the cochlear path (III, p. 476) to the temporal cortex cerebri (not present in this section). As already stated, there is doubt as to how far this path is interrupted in various nuclei along its course such as the superior olives, trapezoid, lateral lemniscus nucleus and inferior colliculus. It may be reducible to three principal neurone groups: (I) the spinal ganglion and its cochlear nerve, (2) the lateral lemniscus and (3) the geniculo-temporal system.

The medial lemniscus is now a laterally placed, curved bundle, displaced laterally by the red nucleus. According to some authorities, some of its fibres terminate in the superior colliculus. The spino-thalamic tract is plainly distinguishable as a bundle dorsal to the dorsal edge of the medial lemniscus. At this level, then, the afferent paths from cord to pallium have practically united.

In the superior colliculus are terminations of the optic tract (the continuation past the optic chiasma of the so-called optic nerve) (see below).

The central tegmental tract is displaced dorsally by the red nucleus.

Intersegmental Neurones.-The reticular formation is smaller. The rubro- 
spinal tract (not distinguishable) is emerging from its nucleus of origin, the nucleus ruber. Just below this level its fibres decussate (ventral decussation of Forel) and pass to the location they have been seen to occupy in preceding levels. The nucleus ruber or red nucleus is very conspicuous, occupying a large part of the reticular formation. It consists of a large-celled part which gives rise to the rubro-spinal tract and to rubro-bulbar fibres, and a smaller-celled portion. The latter sends fibres to the thalamus and thence to the pallium. The nucleus ruber, probably the first named part, also receives fibres from the pallium. According to this it will be seen that the nucleus ruber is a part of the descending cerebello-rubro-bulbar and spinal path (XVII, p. 478), of the cerebellopallial path (VII, p. 477), and of the pallio-rubro-bulbar and spinal path (XIV, p. 477). The red nucleus probably also receives collaterals from the colliculospinal tract.

The medial longitudinal fasciculus is diminished, some of its descending fibres having been formed from reticular formation nuclei below this level. Many of its fibres, both ascending and descending, send collaterals and terminals to the cells of the oculomotor nucleus.

Efferent Suprasegmental Neurones.-The ventral part of the brain is composed of a mass of efferent pallial fibres, the pes pedunculi. The pyramidal fibres from the precentral areas of the cerebral hemisphere (pallio-spinal and some pallio-bulbar fibres) occupy about the middle three-fifths, but are also scattered through other parts of the pes, especially the mesial part. The leg fibres are probably more numerous laterally, the arm fibres in the middle, and the face fibres mesially. In the lateral part of the pes are pallio-pontile fibres from the occipital and temporal lobes of the cerebral hemispheres (occipito-temporal pallio-pontile fibres). In the mesial part are efferent pallial fibres from the frontal lobe, in part to the pons nuclei (frontal pallio-pontile) and in part possibly from the lower frontal region to the motor nuclei of cranial nerves VII and XII. Besides the above pallio-pontile and pallio-spinal fibres in the pes there are two other aberrant peduncular bundles (p. 517) which probably contain efferent pallial fibres which pass to the motor nuclei of nerves $V$, VII and XII. These two bundles, which may be termed the mesial and lateral pedunculo-tegmental bundles ("medial accessory fillet" and "lateral peduncular fillet") detach themselves from the pes higher up, and below this level come to lie in the vicinity of the medial lemniscus.

Dorsal to the pes and constituting the remainder of the basis pedunculi is a mass of gray matter which, on account of the pigmentation of its cells, is known as the substantia nigra. The substantia nigra receives collaterals from the adjoining pes fibres. These are probably fibres from the motor cortex but according to some maty lie from the corpus striatum or corpus subthalamicum. The axones of the cells of the substantia nigra enter the tegmentum. Their destination is unknown.

The superior cerebellar peeluncle has completed its decussation below this level and its fibers are secen surrounding or within the nucleus ruber which is one of their terminal nuclei. Other fibres of the superior cerebellat peduncle icrminate in the thalamus and some are stated to terminate in the nucleus of nerve III. 
Internal arcuate fibres from the gray matter of the superior colliculus pass through the reticular formation, and form an oblique decussation. This decussation is the dorsal, or fountain-like decussation of Meynert. The fibres originate from cells in the superior colliculus (tectum opticum), and after decussation form the descending colliculo-bulbar and spinal tract (tecto-spinal or predorsal tract) (see also below).

The Anterior Corpus Quadrigeminum or Superior Colliculus.--In this four principal layers may be distinguished besides the usual covering of neuroglia cells and fibres: (I) An outer white layer, stratum zonale. This consists of fine nerve fibres coming from the superior brachium, possibly fibres from the optic tract and cerebral cortex. Among them are small nerve cells, mostly horizontal and with tangential or centrally directed axones. (2) A gray layer, the stratum cinereum. This consists of radially arranged nerve cells with their larger dendrites proceeding outward, and their axones inward. The largest cells lie deepest. In this layer the optic fibers principally terminate. (3) The stratum opticum consists principally of optic fibres which send their terminals mostly into the preceding layer, but also into the deeper layers. It also contains cells whose axones pass into the next layer. (4) Deep gray-white layer, or stratum lemnisci, because it is stated to contain fibres from the medial lemniscus which terminate in the superior colliculus (denied by some). This layer contains large and medium stellate cells whose axones, together with axones from cells in the more superficial layers, either pass across to the opposite colliculus or sweep ventrally around the central gray, decussate in the raphe and proceed caudally as the colliculo-bulbar and spinal tract. The above relations have been principally ascertained by the Golgi method. The superior colliculus also possibly receives fibres from the lateral lemniscus and spino-thalamic tract. It also receives fibres from the occipital and temporal cortex cerebri (palliocollicular fibres).

Belonging to the midbrain is the posterior commissure (not in the section) the fibres of which cross in the roof just anterior to the superior colliculus. Its fibres originate from collicular cells (in turn receiving optic fibres), decussate and terminate in the nucleus of the medial longitudinal fasciculus and other nuclei in the reticular formation.

The colliculus thus consists essentially of $(a)$ afferent fibres from the retina (optic tract), the pallium and possibly other parts of the nervous system, and (b) efferent neurones to other parts of the brain and cord brought into various relations with each other in the colliculus either directly or by $(c)$ the association cells of the colliculus, the axones of which do not leave the latter.

\section{Forebrain or Prosencephalon}

\section{INTERBRAIN (DIENCEPHALON OR THALAMENCEPHALON)}

In the interbrain or diencephalon, three parts may be distinguished; the thalamus, epithalamus, and hypothalamus. The epithalamus consists principally of the pineal body, the habenulæ, and striæ thalami. The hypothalamus consists mainly of the structures in 
the ventral expansion of the interbrain, such as the corpora mamillaria, tuber cinereum, infundibulum and posterior lobe of the hypophysis. The epithalamus and hypothalamus are principally connected with olfactory paths (see p. 530 and Fig. 35I). Certain extensions forward of the tegmentum are also termed subthalamic (e.g. corpus subthalamicum or corpus Luysii).

The thalamus comprises the great bulk of the interbrain. It consists of a number of nuclei forming links in afferent and efferent pallial paths and of other nuclei connected with the corpora striata. There is much difference of opinion both as to the number of the nuclei and their connections. According to some authorities the thalamus may be regarded as divided into internal and external segments (usually separated by the lamina medullaris medialis). The internal segment consists of an anterior nucleus, median nucleus, the "median center" or nucleus of Luys, and a nucleus arcuatus. The external segment consists of a dorso-lateral, an external ventrolateral, an internal ventro-lateral, and a ventral nucleus. To the external segment should be added the pulvinar and lateral and medial geniculate bodies (metathalamus). The various nuclei of this external segment receive the fibres of the afferent pallial paths and complete the paths by sending fibres to the cortex pallii. These paths are (I) the medial lemniscus, spino-thalamic, and secondary trigeminal tracts (general sensory from body and face) to the ventrolateral nuclei and thence to the cortex of the central region of the pallium; (2) the lateral fillet or brachium of inferior colliculus (hearing) to the medial geniculate body, and thence to the temporal region of the pallium; (3) the optic tract to the lateral geniculate body and thence to the occipital pallial cortex; (4) part of the superior cerebellar peduncle (also said to be distributed to nuclei of inner segment). The visceral (including gustatory) and vestibular paths to the pallium are not definitely known. (See also p. 475.) As the olfactory nerve belongs $(0)$ the endbrain, its path to the pallium does not traverse the thalamus. Besirles giving rise to the above thalamo-cortical fibres, the external thalamic segment in all probability receives many descending fibres from the cortex pallii. The various fibres connecting thalamus and cortex constitute the thalamic radialions. In general the anterior parts of the cortex are connecterl with the anterior part of the external thatamic segment, the midclle with the midclle, and the posterior with the pesterior. It is also probable that the thalamocortical fibres from the various lateral nuclei are arranged dorso- 
ventrally, so that the fibres from the ventral parts pass to the ventral part of the central region of the pallium, dorsal to dorsal, etc. (E. Sachs.)

The nuclei of the internal segment of the thalamus do not appear, according to some recent researches, to have direct connections with the cortex pallii. The anterior nucleus receives the bundle of Vicq d' Azyr (mamillo-thalamic tract) and probably sends fibres to the nucleus caudatus (see p. 533). It thus belongs to the olfactory apparatus. The median nucleus is also probably connected with the nucleus caudatus. The median center of Luys and the nucleus arcuatus send fibres to adjoining thalamic nuclei, especially the lateral nuclei, and appear to be thus association nuclei. Other authorities affirm that ascending tracts are received by some of these internal nuclei and that they have direct cortical connections. Descending tracts coming directly from the thalamus have not been definitely demonstrated.

\section{PRACTICAL STUDY}

Ir. Transverse Section through the Junction of Midbrain and Thalamus. (Figs. 324 and 348 .)

The most conspicuous change from the last section is the appearance, or increase, of the geniculate bodies and pulvinar, and the thalamic radiations.

Efferent Peripheral Neurones.-The nuclei and root fibres of the III nerve are still present.

Afferent Roots, their Terminal Nuclei, Secondary Tracts, and Tertiary Neurones.-The fibres of the optic tract (optic "nerve") are seen entering the ventral surface of one of their terminal nuclei, the lateral or external geniculate body. Other optic fibres (not entirely traceable) enter the pulvinar thalami and the superior colliculus (Stro) (see also preceding section). On the dorso-lateral surface of the lateral geniculate body, a bundle of fibres accumulates which represents the beginning of the geniculo-calcarine tract to the occipital cortex, thereby completing the visual path.

Internal to the lateral geniculate body is the medial (internal) geniculate body which now contains the terminals of the brachium of the inferior colliculus. Fibres from its cells gather on its lateral surface. These represent the beginning of the geniculo-temporal tract to the temporal cortex, thereby completing the auditory path.

Internal and ventral to the medial geniculate body are the medial lemniscus (bulbo-thalamic) and spino-thalamic tracts about to terminate in the ventrolateral thalamic nuclei whose axones complete the general sensory path by passing to the central cortex.

The central tegmental tract can hardly be distinguished.

Intersegmental Neurones. - The nucleus ruber is still large, but the remainder 


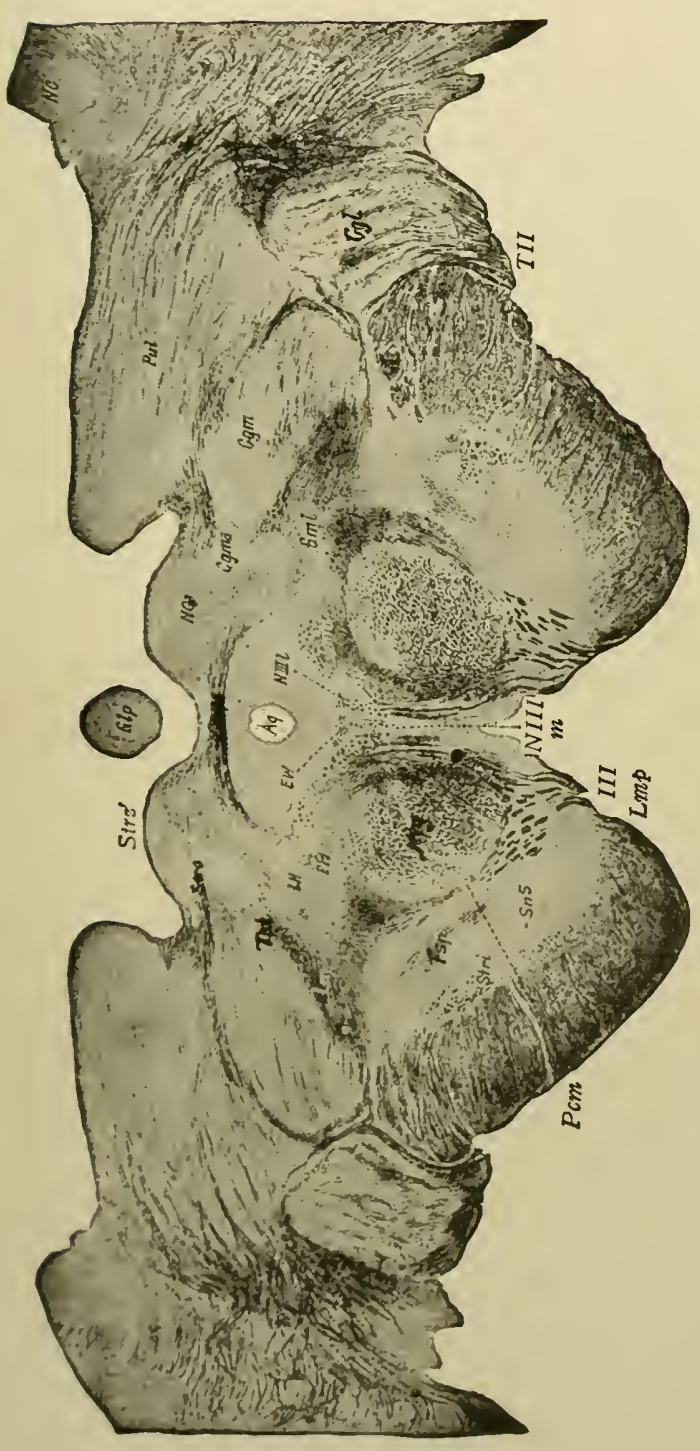

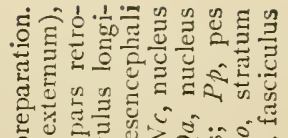

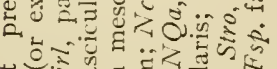

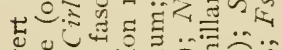

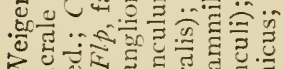

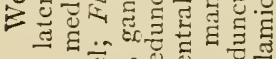

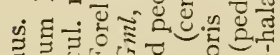

菏.

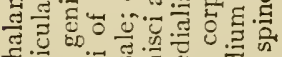

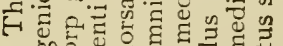

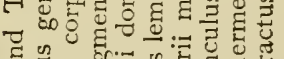

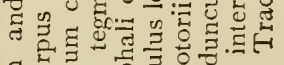

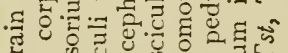

कि

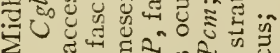

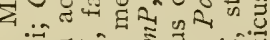

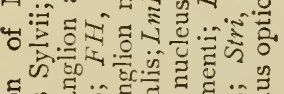

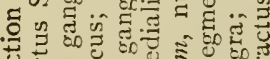

莙苟

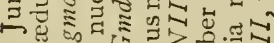

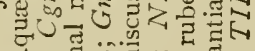

Ð

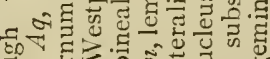

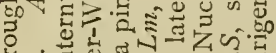

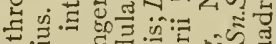

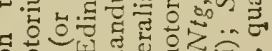

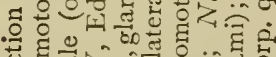

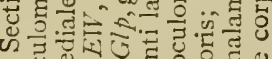

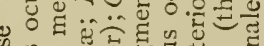

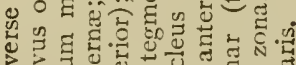

की

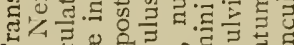

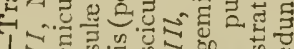

1. $\begin{aligned} & 4 \\ & \text { on }\end{aligned}$

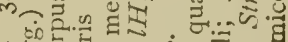

0

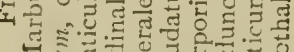

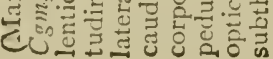


of the reticular formation has nearly disappeared. The medial longitudinal fasciculus is much diminished as its ascending fibres terminate in the nucleus of nerve III and many of its descending fibres originate from cells below this level.

Efferent Suprasegmental Neurones.-The pes pedunculi occupies the same position, and dorsal to it is the diminished substantia nigra. Along the ventromesial border of the pes a bundle of fibres can sometimes be distinguished (Fig. 348, Lmp), which at lower levels comes to lie mesial to the medial lemniscus (aberrant peduncular fibres, comp. p. 52 I). Descending pallial fibres (not distinguishable) also probably form part of the thalamic radiations (pp. 523, 530).

Fibres of the superior cerebellar peduncle may be seen within and around the nucleus ruber. Some of these terminate in the latter, some pass further forward to end in the thalamus (compare pp. 507, 52I).

The Superior Colliculus is somewhat diminished. The posterior commissure passes across in the roof dorsal to the central gray (see preceding section).

The Thalamus (can hardly be included under the preceding structures).The corpora geniculata have been mentioned. The pulvinar thalami is a large gray mass dorsal to the medial geniculate body. Fibres passing laterally from it contribute to the retrolenticular portion of the internal capsule (Cirl). These fibres are a part of the thalamic radiations.

The mucleus caudatus (a portion of the corpus striatum of the endbrain) is present.

I2. Section through the Interbrain at the Level of the Optic Chiasma. (Figs. 324 and 349.)

Efferent Peripheral Neurones.-None present.

Afferent Roots, their Terminal Nuclei, Secondary Tracts, and Tertiary Neurones.-Fibres of the optic nerve are seen decussating and forming the optic chiasma. The further continuation of the optic fibres to their termination is called the optic tract. Both nerve and tract constitute the secondary optic tract, the optic chiasma being analogous to the decussation of the medial lemniscus and of the lateral lemniscus (trapezius). (For further description of optic paths see Fig. 350.)

The medial lemniscus, spino-thalamic, and secondary trigeminal tracts are

Fig. 349.- Section through the Interbrain at the Level of the Optic Chiasma. (The chorioid plexus of the third ventricle has been removed.) Weigert preparation. (Marburg) $C e$, capsula externa; $C e x$, capsula extrema; $C h I I$, chiasma nervorum opticorum (or optic chiasma); $C i$, capsula interna; $C l$, claustrum; $C m l$, ganglion laterale corp. mammillaris; $\mathrm{Cmm}$, ganglion mediale corp. mammill.; Coa, commissura anterior; Cospm, commissura supramammillaris; $C$ sth, corpus subthalamicum; $e$, nucleus externus gangl. med. corpor. mammillaris; $F m p$, fasciculus mammillaris princeps; $F_{0}$, fornix; $F p$, fibræ perforantes (pedunculi); frlf, fasciculus retroflexus (Meynert); Fsp, fasciculus subthalamico-peduncularis; $F u$, fasciculus uncinatus; $G h b$, ganglion habenulæ; $g l p$, globus pallidus; $H$, area tegmenti Forel; $H I$, pars dorsalis areæ tegmenti; $H I I$, pars ventralis areæ tegmenti; $I$, insula Reillii; $i$, nucleus internus gangl. medial. corp. mammillaris; $L m l$, lamina medullaris lateralis; Narc, nucleus arcuatus thalami; $N c$, nucleus caudatus; $N L$, nucleus Luysii (nucleus centralis, or median centre, thalami;) $N l$, nucleus lateralis thalami; $N l v$, nucleus lateralis ventralis thalami; $N t g$, nucleus ruber tegmenti; $P p$, pes pedunculi; $P u$, putamen; $S n S$, substantia nigra; $S t$, stria cornea; Strz, stratum zonale thalami; $T I I$, tractus opticus; $T b c$, tuber cinereum; $T t$, tænia thalami; VIII, ventriculus tertius; $Z i$, zona incerta. 


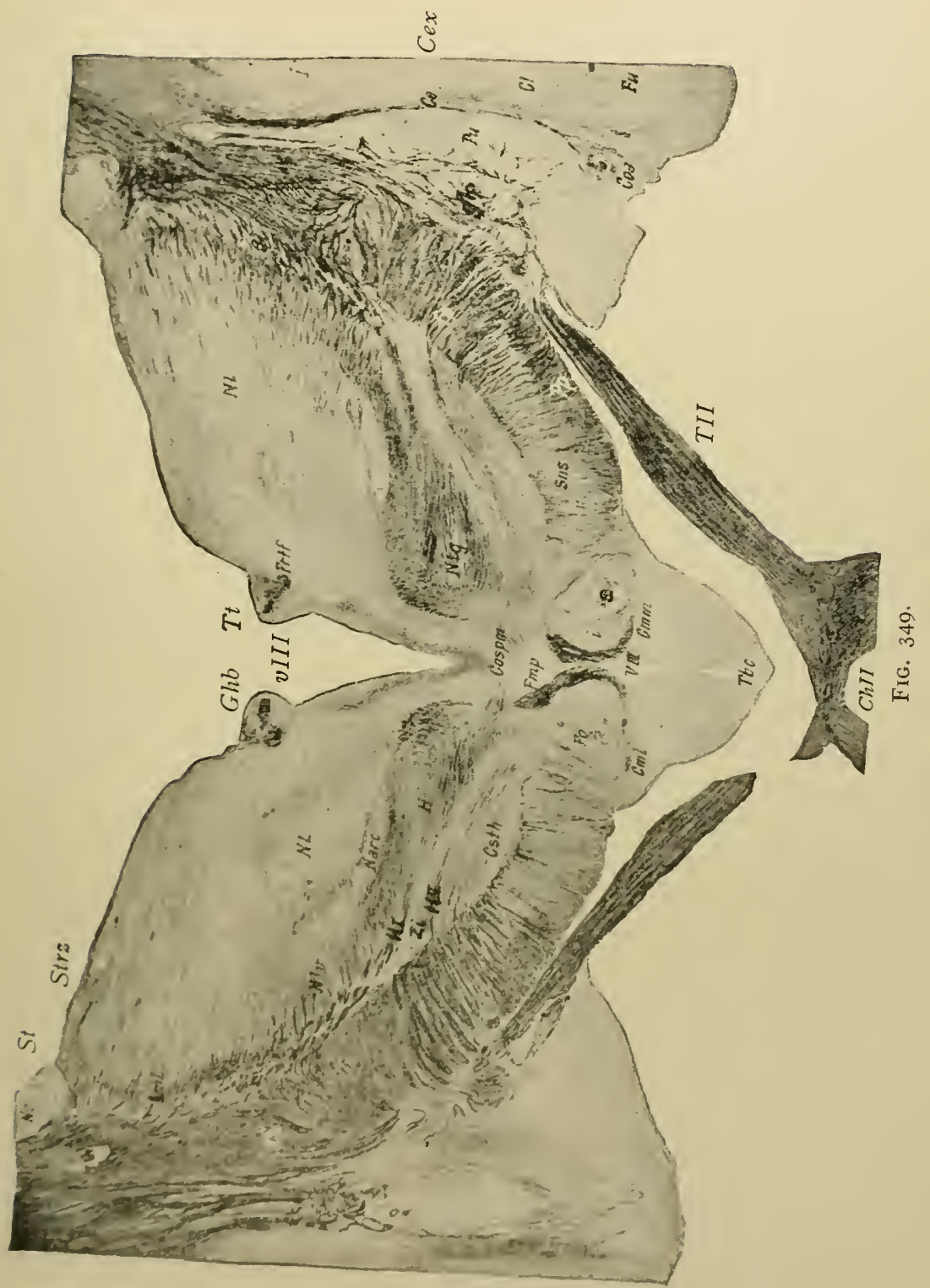




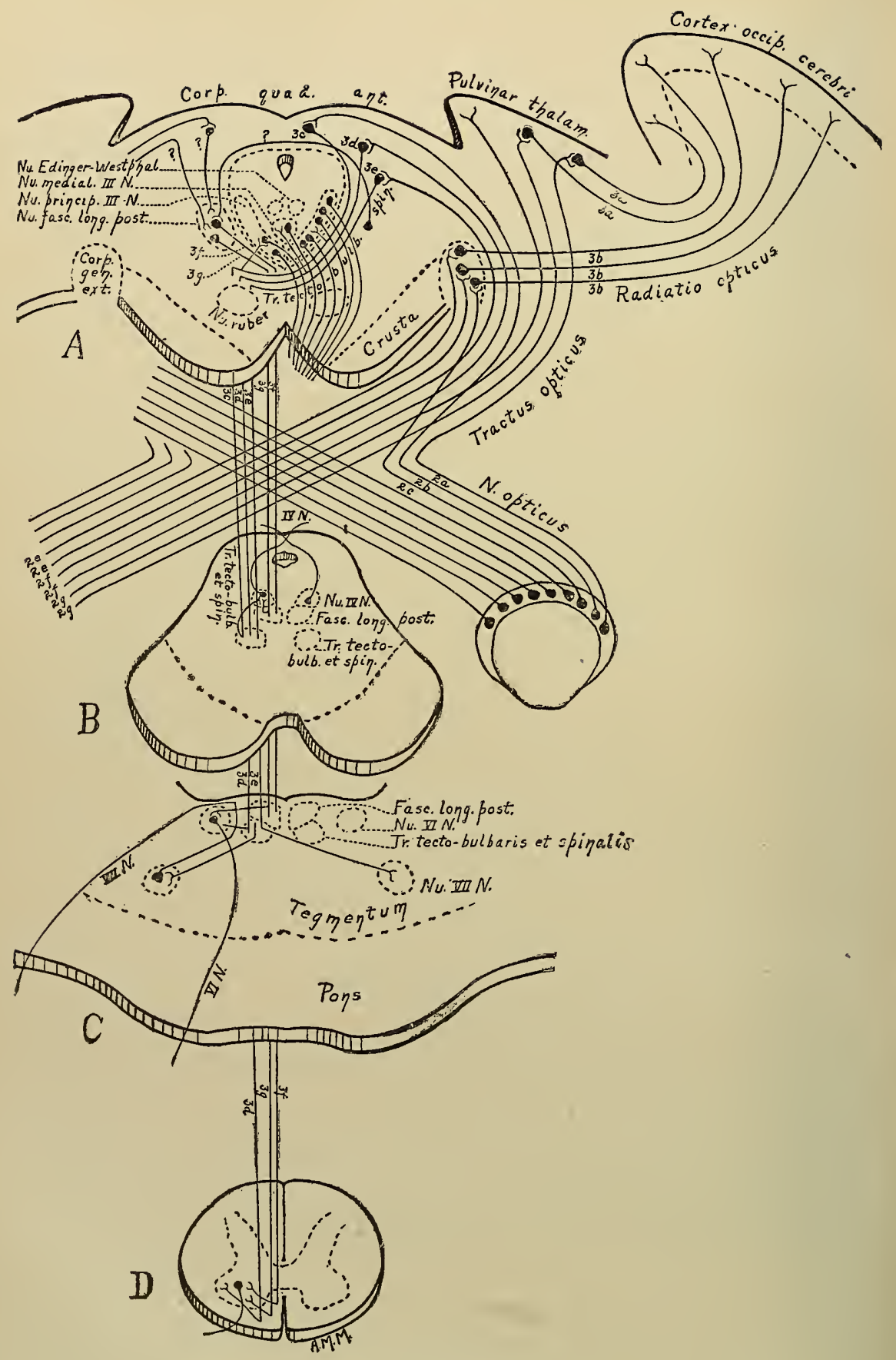

FIG. 350 . 
EXPLANATION OF FIG. $35^{\circ}$.

FIG. 350.-Diagram of the Optic (II) Nerve and some of its Principal Connections. $A$, Level of nerves II and III; $B$, level of nerve IV; $C$, level of nerves VI and VII; $D$, spinal cord. Neurone groups are represented by one or several individual neurones.

The rods and cones (receptors) and the bipolar cells (=Neurone No. I) of the retina are not indicated.

Veurone No. 2. - 2a, Axones of ganglion cells in temporal part of retina pass to pulvinar of thalamus of same side; $2 b$, axones of ganglion cells in temporal retina pass to superior colliculus of same side; $2 c$, axones of ganglion cells in temporal retina pass to external geniculate body of same side; $2 e$, axones of ganglion cells in nasal side of retina cross in optic chiasma and pass to external geniculate body of opposite side; $2 f$, axones of ganglion cells in nasal side of retina cross in optic chiasma and pass to superior colliculus of opposite side; $2 \mathrm{~g}$, axones of ganglion cells in nasal side of retina cross in optic chiasma and pass to pulvinar of thalamus of opposite side. Macular fibres are partly crossed and partly uncrossed.

Veurone No.3.- $3 a$, Axones of cells in pulvinar to cortex of occipital lobe of cerebrum (this connection is disputed) 33 , axones of cells in external geniculate body to cortex of occipital lobe of cerebrum; $3 a$ and $3 b$ constitute the primary optic radiation; $3 c, 3 d$ and $3 e$, axones of cells in deep (fourth) layer of superior colliculus (see p. 522) decussate ventral to medial longitudinal fasciculus (dorsal tegmental decussation or decussation of Meynert) and form the tractus colliculo-bulbaris et spinalis or predorsal bundle. (Tr. tectobulb. et spin.) to bulb (medulla) and anterior column of cord, innervating by collaterals and terminals, directly or indirectly, chiefly the nuclei of III, IV, VI, and VII cranial nerves and motor nuclei of spinal nerves. $3 f$ and $3 g$ (possibly another neurone intercalated between these and optic terminals), axones of cells in interstitial nucleus of Cajal ( $N u$. fasc. long. post.) form part of medial longitudinal fasciculus and descend on same side to anterior column of cord next to anterior median fissurc, innervating nuclei of III, IV, and VI cranial nerves and motor nuclei of spinal nerves.

Veurone No. 4.-Axones of cells in above-mentioned motor nuclei. Axones from cells in median nucleus of nerve III $(N u$. med. $N . I I I)$ and possibly in Edinger-Westphal nucleus, probably innervaté the intrinsic muscle of eyeball (ciliary and pupillary reflex path).

Pallio-tectal fibres, by means of which the collicular reflex centre is brought under the control of the cerebral cortex, are not inclicated.

It is evident from the diagram that the cerebral pathway of the optic nerve is via the external geniculate body (and pulvinar of thalamus?), and the reflex pathway is via the superior colliculus. 
now lost in the ventro-lateral thalamic nuclei, cells of which constitute the tertiary neurones of the various afferent pallial paths (see pp. 523 and 524).

Intersegmental Neurones. - The cephalic end of the nucleus ruber is still present on the right $(\mathrm{Ntg})$. On the left are seen some fibres in its place, lateral to which are two transverse bundles enclosing a strip of gray matter. These are known as the area tegmenti or field of Forel and represent a subthalamic forward extension of the tegmentum. The gray or zona incerta may be regarded as representing a continuation of part of the reticular formation. The ventral bundle of fibres $(H I I)$ are probably fibres from the nucleus lenticularis $(g l p$ and $P u$, right) passing through the pes (or rather through the posterior part of its continuation - the internal capsule) as perforating fibres ( $f p$ on right) to the corpus subthalamicum (Csth, left) and other subthalamic regions, possibly also to the nucleus ruber. Some of these fibres are collaterals of pes fibres and may consequently come directly from the pallium. The dorsal bundle $(H I)$ probably contains fibres connecting red nucleus and pallium. Other fibres in this region are probably fibres of the superior cerebellar peduncle which have passed by the nucleus ruber to the lateral nucleus and median center of the thalamus (see also p. 523) and possibly also fibres of the secondary trigeminal tract. The nucleus of the medial longitudinal fasciculus falls in the level between this and the preceding section. In general it seems probable that portions of the corpus striatum, the corpus subthalamicum and certain other subthalamic nuclei, the substantia nigra and part of the nucleus ruber represent certain phylogenetically old, rather obscure, efferent forebrain paths.

Efferent Suprasegmental Neurones.-The pes pedunculi now lies partly between the thalamus and nucleus lenticularis (see p. 533) constituting the greater part of the internal capsule. The parts of the internal capsule as shown in horizontal sections of the hemispheres are shown in Fig. 352. The most dorsal part is here passing into the corona radiata (p. 533) of the cerebral hemispheres (not included in the section). The part present in this level is the most posterior part of the capsule (occipito-temporal pallio-pontile fibres (see p. 533).

Dorsal to the mesial part of the pes is the corpus subthalamicum which has replaced the substantia nigra. It receives collaterals from the pes and is said to contribute fibres to the latter. It also appears to be connected by fibres with the nucleus lenticularis (see above). Superior cerebellar peduncle (see Intersegmental Neurones above).

Thalamus. - At this level the ventro-lateral nucleus, the nucleus arcuatus, and the median center of Luys can usually be distinguished. At the outer border of the thalamus, fibres accumulate forming the lateral medullary lamina. These fibres continue outward as thalamic radiations, entering the internal capsule which they may follow a distance, or cross obliquely and enter the corona radiata.

Epithalamic and Hypothalamic Structures and their Connections.-The ganglia habenula are two small masses of gray matter occupying eminences on the mesial walls of the thalamus. A bundle of fibres near each is the stria medullaris (near the tania thalami) consisting of fibres from the olfactory bulb and trigonum and representing afferent olfactory connections (p. 532). The ganglion habenulæ contains a mesial small-celled and a lateral large-celled nucleus. Their 
axones form the fasciculus retroflexus of Meynert to the interpeduncular ganglion, situated more caudally (Fig. 35I). There is also a commissura habenularis connecting the two ganglia. The stria terminalis (stria cornea), another olfactory connection, lies in the groove between the ventricular surfaces of nucleus caudatus and thalamus. The tuber cinereum is seen projecting ventrally. Dorsal to this are seen the corpora mammillaria containing lateral and mesial nuclei. The mammillary body receives some fibres of the fornix (from the rhinopallial cortex, see below) and also fibres from the medial fillet and other

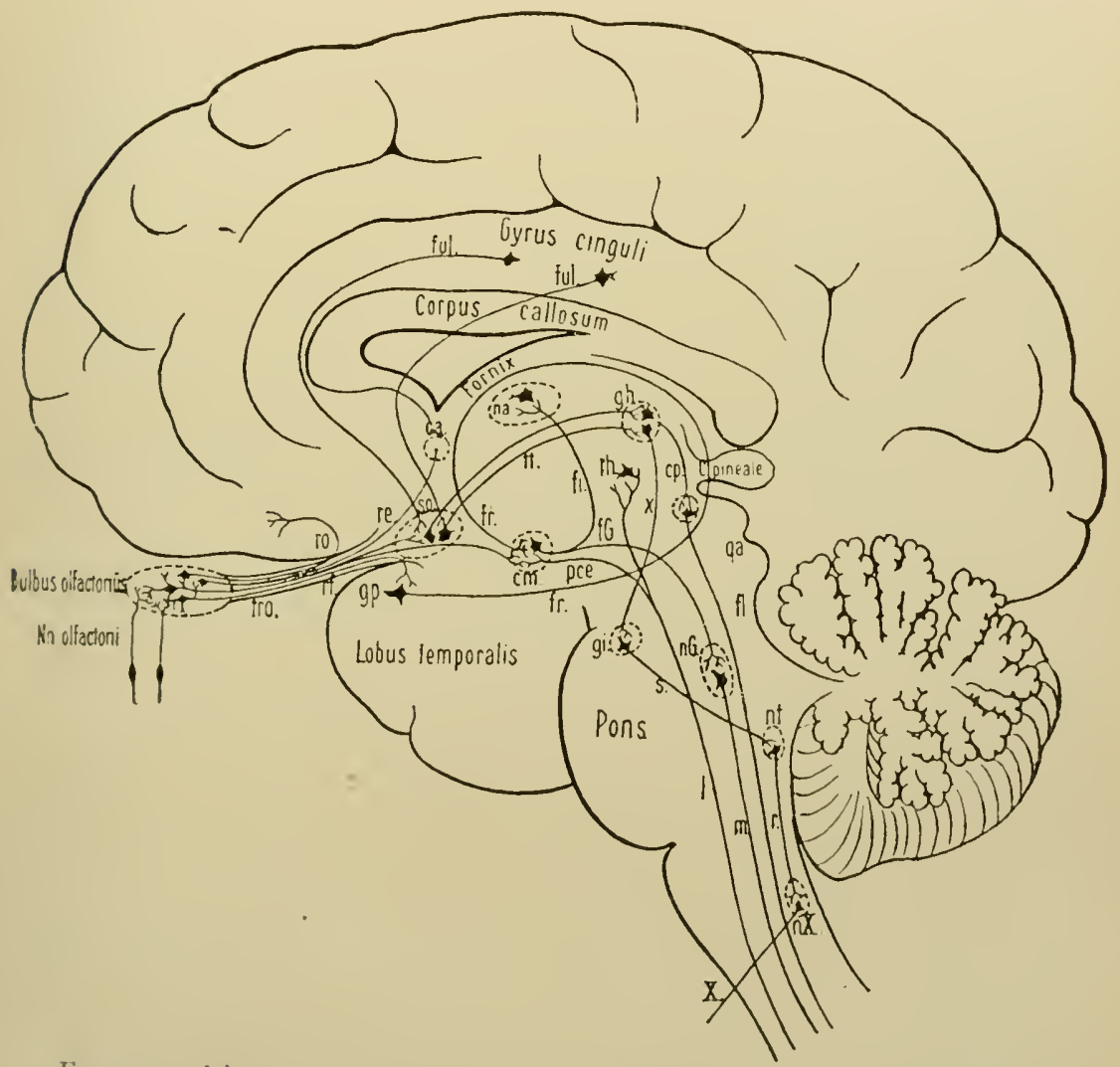

Fig. $35 \mathrm{r}$-D - Diagram of Olfactory Paths (von Bechterew.) X, Root fibres of vagus; $C a$, commissurat anterior; $c m$, corpus mammillare; $c p$, fibres from nucleus habenulic to posterior commissure; $f G$, tritet from corpus mammillare to Gudrlen's nucleus; fi, fasciculus mammillo-thalamicus; $f$, fasciculus longitudinalis medialis; $f r$, fornix; ful, fibres of $l$, lemniscus medialic; na, nucleus anterior the filses from (iudden's nucleus to substantiat reticularis grisea; $n X$, nucleus mulurius nuri, na, Cidden's nucleus; $n$ l, nucleus legmenti (v. Gudken); corpera cquadrigemin nerri vagi; peli, peelunculus corporis mammillaris from fillet; qu, nerves, re radix lateralis frues from nucleus tegmenti ( $v$. Ciudden) to nuclei of cranial nerves; re, radix lateralis tractus olfactorii; $r f$ fibres of tractus olfactorius to trigonum olfactorum; ro, radix medialis tractus olfactorii; s, filses from ganglion interpedunculare torius; $\|$, tacniat hat , so, area of trigonum olfactorium; th, thalamus; tro, tractus olfac- 
ascending tracts in the reticular formation. It gives rise to the bundle of Vicq d'Azyr (mammillo-thalamic tract) to the dorsal nucleus of the thalamus, and mammillo-tegmental fibres to the nucleus of Gudden (Fig. 35I) and red nucleus. The fibres entering the mammillary body from the fillet and other ascending tracts, together with the mammillo-tegmental tract, constitute its peduncle. It is not improbable that the mammillary body is a link in the afferent gustatory path (p. 476, II). (See also Endbrain.)

On the right is seen the posterior part of the mucleus lenticularis of the corpus striatum.

\section{The Endbrain or Telencephalon.}

The endbrain consists of pallium (dorsal expanded part), corpus striatum, and rhinencephalon. Two principal parts of the pallium may be distinguished; the olfactory pallium or rhinopallium (archipallium), including principally the cornu ammonis and gyrus dentatus; and the neopallium including the greater part of the cerebral hemispheres.

The rhinencephalon ${ }^{1}$ includes the olfactory nerves and bulb, the trigonum olfactorium, the tuberculum olfactorium or anterior perforated space, and the gyrus hippocampi, in part at least (pyriform lobe). The olfactory nerve is composed of axones of cells in the olfactory mucous membrane which terminate in the olfactory bulb. They there form synapses with the dendrites of the mitral cells, the axones of which constitute the secondary tract, part of which decussates in the pars olfactoria of the anterior cerebral commissure. A secondary tract ("lateral root") proceeds, with tertiary tracts, to the cortex of the gyrus hippocampi and thence to the cornu ammonis. Efferent axones of cornu ammonis cells are collected in the fimbria and descend by the fornix to the mammillary body, the further caudal connections of which have been described (p. 53I). Fibres of the fimbria also cross, forming the commissure of the fornix (olfactory pallial commissure). Secondary olfactory tracts also pass to the trigonum, whence tertiary neurones pass as the stria medullaris to the ganglion habenulæ (see p. 530 and Fig. 35r). The principal commissure of the rhinencephalon is the anterior cerebral commissure.

The corpus striatum consists of the nucleus caudatus and nucleus lenticularis, the connections and significance of which are obscure. They receive collaterals from the descending pallial fibres which pass by them and also apparently send out fibres to join the latter. They also have connections with rhinencephalon and thalamus.

${ }^{1}$ The term rhinencephalon is often used to include also the olfactory pallium. 
The pallium consists of an extensive external convoluted sheet of gray matter (cortex pallii or cortex cerebri) and of white matter underlying the gray. In the white matter may be distinguished the corona radiata composed of the afferent and efferent pallial fibres connecting the pallium with other parts of the brain (projection fibres). The remaining fibres of the white matter are association fibres of the pallium and are either crossed or commissural, connecting the two hemispheres (corpus callosum and fornix commissure), or are uncrossed. The term association fibres is often restricted to the uncrossed fibres.

The afferent connections of the neopallium (p. 523) and rhinopallium (p. 532) have been summarized and also the efferent connections of the rhinopallium (p. 532).

The following are the principal descending or efferent connections of the neopallium: (I) The pyramidal or pallio-spinal tract. This is composed of the axones of the giant cells (of Betz) of the arm, body, and leg precentral motor areas. They descend in the corona radiata, the posterior limb of the internal capsule, middle part of the pes, and thence through pons and medulla to the cord. Their decussation and further course has been described. (2) The descending tracts to the motor nuclei of the cranial nerves originate from precentral cells of the various areas controlling the muscles in question and pass down in the vicinity of the genu of the internal capsule. Their path is not so well known but they apparently do not pass down in the pes through(o) their course (p). 524, 521 , etc.).

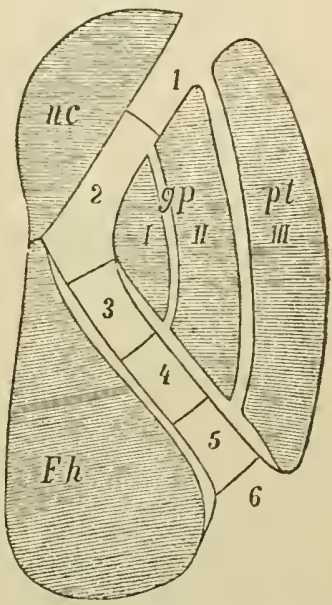

FIG. 352.-Scheme of General Arrangement of fibres in Internal Capsule. (von Bechterew.) I, II, $I I I$, The three parts of the lenticular nucleus; $n c$, nucleus caudatus; $F$, thalamus; $g p$, globus pallidus; $p t$, putamen; $I$, fibres of anterior thalamic peduncle; 2, fibres of medial (frontal) pons system (irontal pallio-pontile fibres); 3 , efferent pallial fibres to motor nuclei of cranial nerves; 4 , pyramidal fibres (efferent pallial fibres to motor nuclci of spinal nerves); 5 , pyramidal fibres mingled with those of the afferent (sensory) path; 6, libres of the lateral pons system (occipitio-temporal pallio-pontile fibres). The various systems are not sharply marked of as inclicated, but are more or less intermingled.

The pallio-pontile system to the pons (continuation to opposite cercbellar hemisphere). This originates in various parts of the cortex. The filores from the occipital (?) and temporal regions pass down in the extreme posterior part of the internal capsule and lateral 


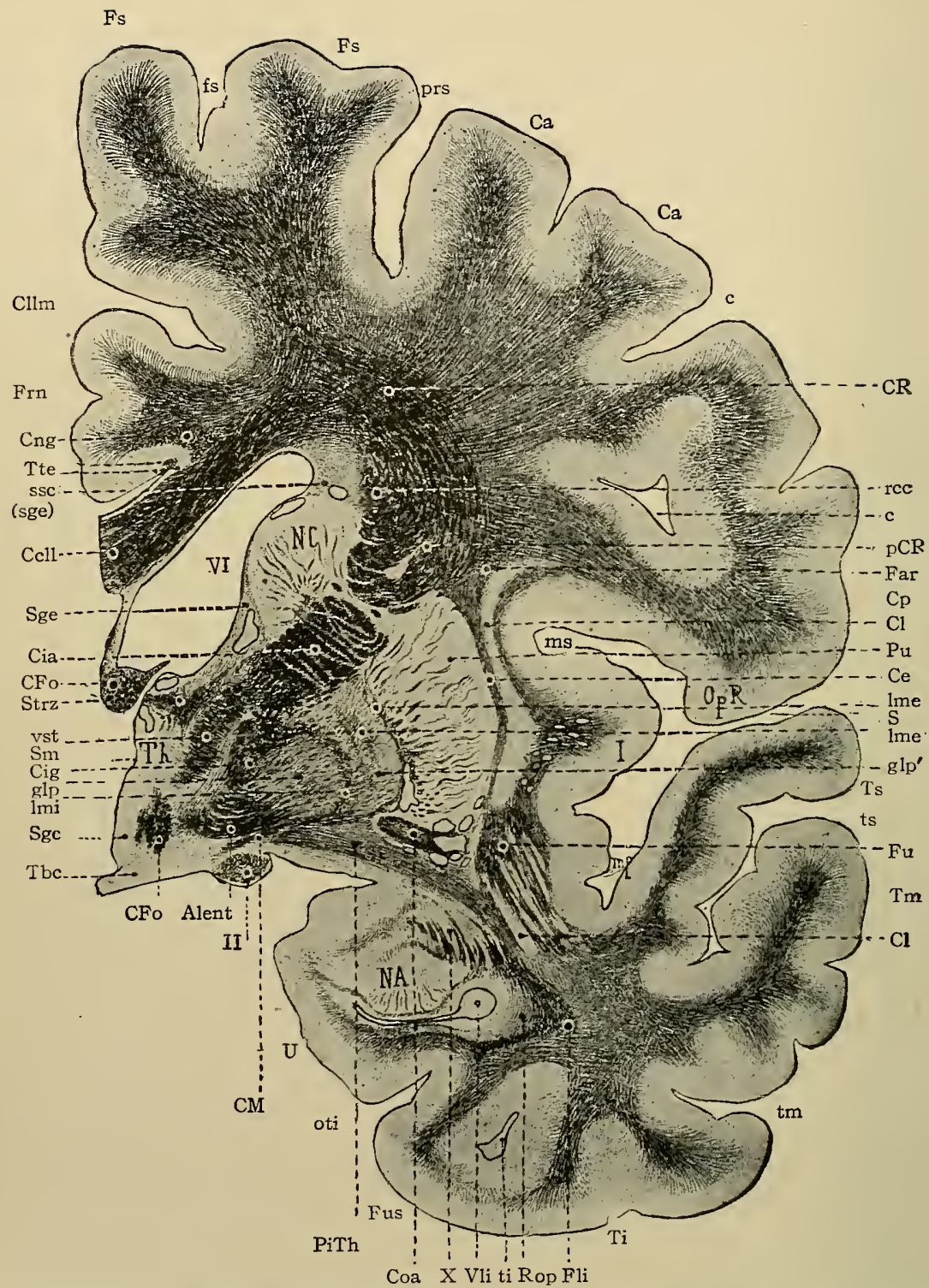

FIG. 353.- Transverse Section through the Cerebral Hemispheres, Corpora Striata and Thalamus. Weigert preparation. (Dejerine.) II, Tractus opticus; Alent, ansa lenticularis; $c$, sulcus centralis (Rolandicus); $C a$, gyrus centralis anterior; $C c l l$, corpus callosum; $C e$, capsula externa; $C F_{0}$, columna fornicis; $C i a$, crus anterior capsul. int.; $\mathrm{Cig}$, genu capsul. int.; $\mathrm{Cl}$, claustrum; $\mathrm{cllm}$, sulcus callosomarginalis; $\mathrm{Cng}$, cingulum; Coa, commissura anterior; $C p$, gyrus centralis posterior (ascending parietal convolution); $C R$, corona radiata; Far, fasciculus arcuatus; $F l i$, fasciculus longitudinalis inferior; $F r n$, gyrus fornicatus; $f s$, sulcus frontalis superior; $F s$, gyrus frontalis superior; $F u$, fasciculus uncinatus; Fus, gyrus fusiformis; $g l p$, globus pallidus (inner segment); $g l p^{\prime}$, globus pallidus (outer segment); $I$, insula; lme, lamina medullaris externa nuclei lenticularis; lme', supplementary lamina of the outer segment of the globus pallidus; $l m i$, 
part of the pes, those from the frontal region pass down in the anterior limb of the internal capsule and mesial part of the pes. (4) Palliotectal fibres to the midbrain roof. (5) Fibres to the substantia nigra and corpus subthalamicum. (6) Fibres to the red nucleus. (7) Pallio-thalamic fibres (see p. 523). (8) Fibres, or collaterals, to the corpora striata. (Fig. 352.)

The crossed association fibres of the neopallium (corpus callosum) connect principally corresponding parts of the hemispheres. The long uncrossed association fibres (furthest from the gray matter) form certain more or less well-defined bundles among which are the following: (I) The cingulum, a longitudinal bundle near the corpus callosum; also contains projection fibres and belongs to the olfactory part of the brain as well as to the neopallium. (2) The superior longitudinal fasciculus or fasciculus arcuatus; connects frontal with occipital and part of temporal lobes. (3) The inferior longitudinal bundle connecting temporal and occipital lobes. It may, however, be a projection bundle. (4) The uncinate fasciculus connecting frontal and temporal lobes. (5) The perpendicular fasciculus of Wernicke connecting inferior parietal and fusiform lobules. Projection fibres may form portions of these bundles.

Besides the above long association fibres there are shorter association fibres nearer the gray matter which connect adjoining convolutions (fibræ proprix of Meynert).

\section{PRACTICAL STUDY}

\section{Transverse Section through the Cerebral Hemispheres, Corpora Striata and Thalamus (Fig. 353)}

First distinguish in general (I) the pallium, its cortex and white matter, (2) the corpus striatum and its divisions, i.e., the caudate nucleus and the lenticular nucleus, the latter being subdivided into the putamen and globus pallidus and (3) the thalamus and other structures of the interbrain.

lamina medullaris interna nuclei lenticularis; $m p$ and $m s$, sulcus circularis (Reili); $N A$, nucleus amygdaliformis; $N c$, nucleus caudatus; $O p R$, operculum; ot $i$, sulcus occipitotemporalis inferior; $p C R$, pes corone rarliate; JiTh, pedunculus inferior thalami; prs, sulcus precentralis; P' $u$, putamen; $r c c$, stratum reticulum coronæ radiat $x ; R o p$, racliatio optica; $S$, fissura Sylvii (posterior branch); Sgc, substantia grisea centralis; $s M$, sulcus Monroi; Sge, sulsstantia grisca sul,ependymalis; ssc, stratum subcallosum: Strz, stratum zonale thalami; Tbc, tuber cinercum; Th, thalamus opticus; $t i$, sulcus temporalis inferior; $T i$, gyrus temporalis inferior; $l m$, sulcus temporalis merlius; Tm, gyrus temporalis medius; $t s$, sulcus temporalis superior; $T$ 's, gyrus temporalis superior; Tte, tenia tecta; I', uncus; l'l, ventriculus lateralis; Vli, ventriculus lateralis (cornu inferius); vsl, perlunculus anterior thaliami; $X$, pedunculus putaminis; C $I I$, Commissure of Meynert. 
Afferent Roots, their Terminal Nuclei, Secondary Tracts, and Tertiary Neurones.- The optic tract here forms a part of the ventral surface of the brain. The geniculo-cortical portion of the optic path forming a part of the optic radiation may be seen. Other afferent pallial connections are hardly distinguishable among the fibres connecting thalamus and pallium.

Efferent Suprasegmental Neurones.--A great part of the pes has now entered the corona radiata. The part now about to enter the corona is the anterior limb of the internal capsule (Fig. 352). The ansa lenticularis is, according to some authorities, composed of fibres or collaterals from the pes to the lenticular nucleus. The fibres of the anterior peduncle of the thalamus are evident. They are considered by some as composed of cortico-thalamic fibres. The anterior pillars of the fornix (efferent rhinopallial) are shown cut through twice, the upper section shows its earlier course emerging from the fimbria, the lower section is near its termination in the mammillary body.

For other structures of thalamus, epithalamus, and hypothalamus see Fig. 353.

The Pallium. - In the white matter distinguish as far as possible the corona radiata, the corpus callosum, and the long association bundles (inferior longitudinal fasciculus, fasciculus uncinatus, and fasciculus arcuatus) (see p. 535). Note the nucleus amygdaliformis, the anterior perforated space and the anterior commissure, belonging to the rhinencephalon.

Other details shown in Fig. 353 should be studied.

The Cerebral Cortex.-The following types of cells are found in the cerebral cortex: (I) Pyramidal cells. This is the prevailing type and is characterized by a long apical dendrite usually directed toward the surface of the brain. This dendrite gives off branches, and usually reaches the outer cortical layer, there to break up into a number of branches. From the cell body are also given off a number of basal dendrites. By the Golgi and Ehrlich methods, gemmules can be demonstrated on the dendrites. The axone proceeds from the base of the cell (opposite to the apical dendrite) and usually passes into the white matter. It gives off several collaterals on its way to the white matter. (2) Stellate cells. These have dendrites passing in various directions. Many, especially the smaller (granules), may have short axones (Golgi's second type). (3) Polymorphous cells of a triangular or spindle shape are usually found in the deepest layers of the cortex and send their axones into the white matter. (4) Horizontal cells (of Cajal), found in the outer layer, with long horizontal dendrites and axones confined to the outer layer. (5) Inverted pyramidal cells (of Martinotti) with axones directed toward the surface. (Fig. 355.)

The largest cells of the cortex (giant cells of Betz) are very rich in chromophilic substance arranged similarly to that in the efferent 
root cells of cord and brain. The medium and small cells have fewer chromophilic bodies which are often in the shape of irregular masses, either near the periphery of the cell or around the nucleus. The neurofibrils vary in their arrangement according to the shape of the cell.
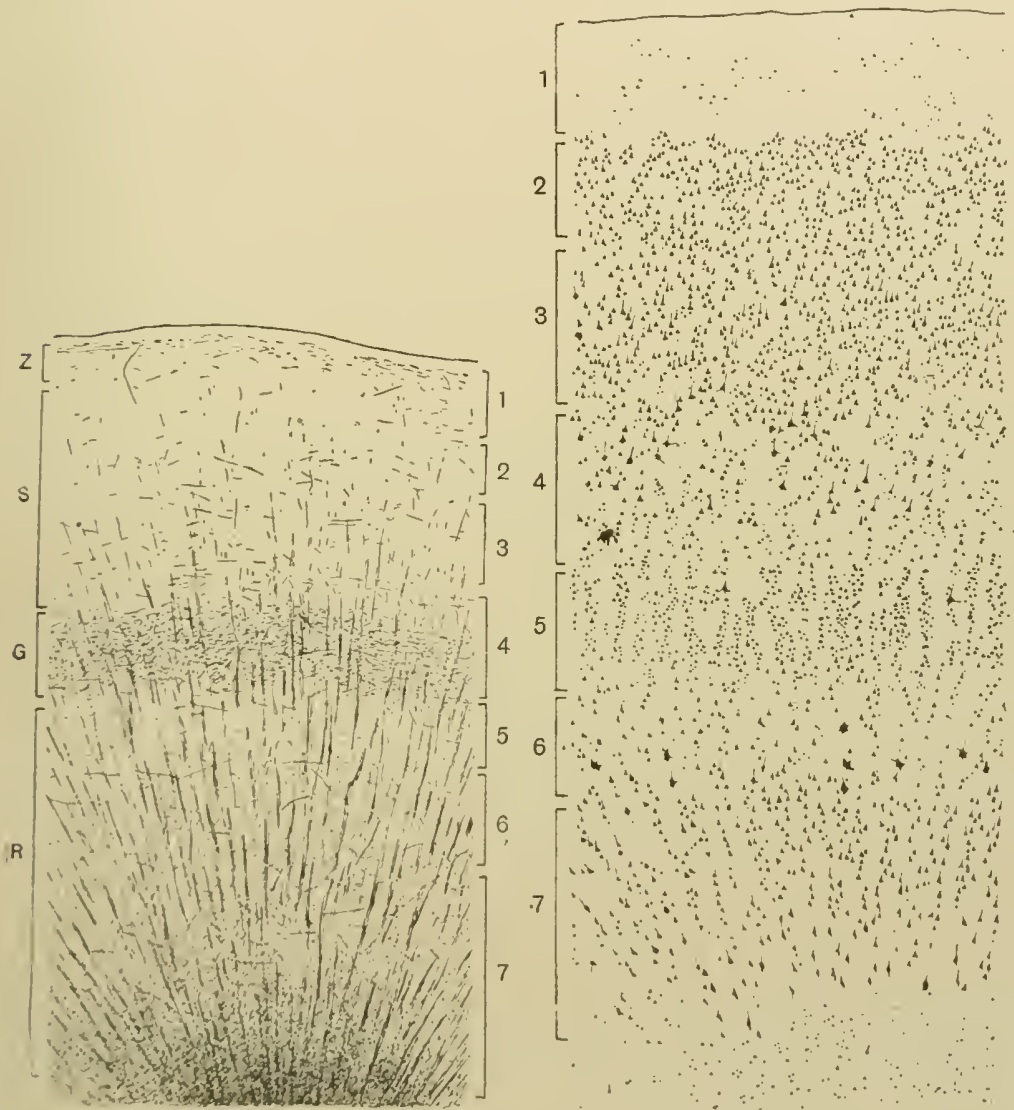

Fis: 354-Vertical Sections of Calcarine Area of Adult Human Cortex. Lefl, Weigert preparation showing filore arrangement. Right, Arrangement of Cells. (Camplell.) $G$, Line of (iennari; $K$, radiary layer; $S$, supraradiary layer; $Z$, Layer of superfirial tangential filores in molecular layer; 1 , molecular layer; 2, external granular (small pyramid) layer; 3, loyramid layer; 4, (large granules) and 5 (small granules), internal grinular layer; 6, ganglionic layer (containing solitary cells of Meynert); 7, multiform layer.

'The neuroglia cells and fibres are in general similar to those in other parts of the nervous system.

The cells of the cortex are arranged in layers which liave the same gencral character throughout, but in various regions exhibit variations 

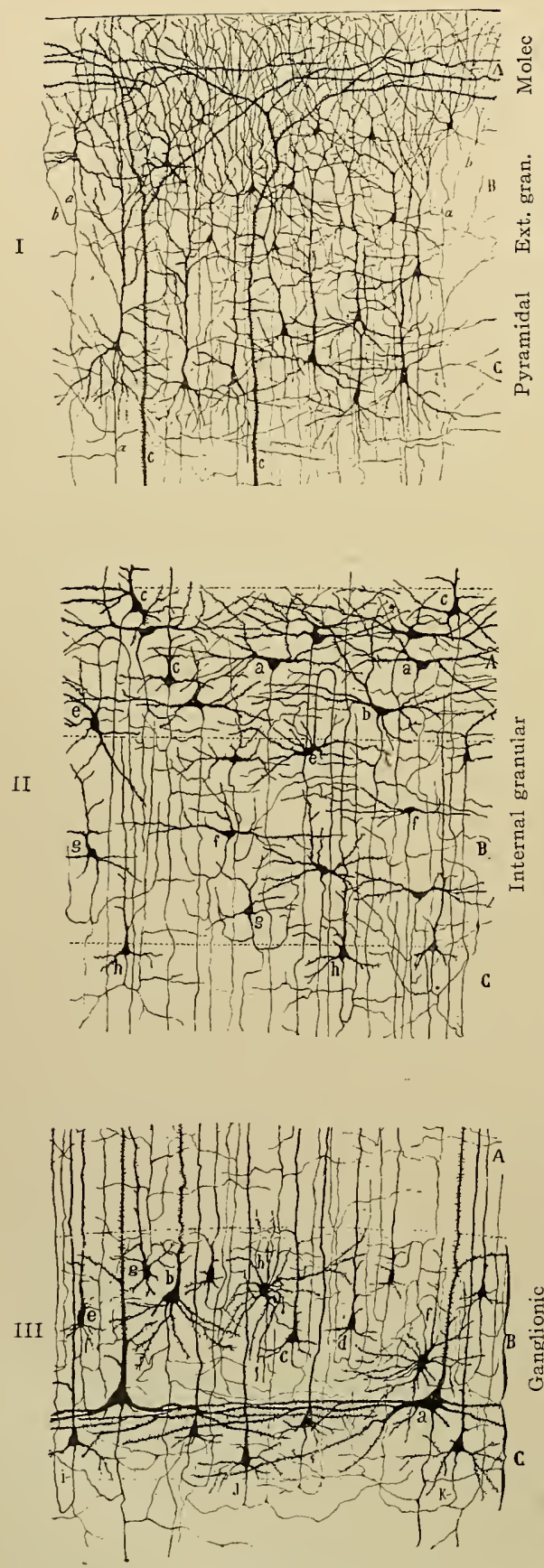

Fig. 355 . such as suppression, diminution, enlargement, or subdivision of certain layers. The cell layers of the cortex are: (I) Molecular layer (zonal layer, plexiform layer of Cajal). This contains the horizontal cells, other cells with short axones, and also receives the axones of the Martinotti cells. Besides this, it contains the terminal branches of the apical den-

FIG. 355.-Vertical section of Calcarine Area of Cortex of an Infant I $^{-20}$ days old. (Cajal, combined from three, $I, I I$ and $I I I$, somewhat overlapping figures. The multiform layer is not included). Golgi's method.

I. $-A$, Molecular layer; $B$, external granular layer (of small pyramids); $C$, pyramidal layer (of medium pyramids); $a$, descending axones; $b$, ascending collaterals; $c$, apical dendrites of large pyramidal cells in ganglionic layer.

II. - A, Sublayer of large stellate cells; $B$, sublayer of small stellate cells; $C$, outer part of ganglionic layer; $a$, crescentic stellate cells; $b$, and $f$, horizontal, spindle-shaped stellate cells; $c$, medium-sized pyramidal cells; $e$, stellate cells with arched axones; $g$, triangular stellate cells with stout, arched collaterals; $h$, pyramidal cells with arched axones.

III.- $A$, Part of internal granular layer; $B$, sublayer of small pyramidal cells with arched ascending axones; $C$, sublayer of large pyramids; $a$, large pyramidal cell; $b$, medium-sized pyramidal cell with long descending axone; $c$, small pyramidal cell with arched ascending axone; $d$, pyramidal cell with axone split into two arched ascending branches; $e$, pyramidal cells whose axone sends out various ascending branches; $f, g, h$, stellate cells with ascending axones which branch in $B$ and in the sublayer of small stellate cells; $i, j, k$, pyramidal cells with arched ascending axones which send branches into the ganglionic layer. 
drites of the pyramidal cells. (2) External granular layer, very often termed the layer of small pyramids. The dendrites of the cells of this layer mostly enter the first layer, their axones pass downward into the white matter. (3) Pyramidal layer, often called the layer of superficial medium and large pyramids. This is composed principally of typical pyramids sending dendritic branches into the first layer and axones into the white matter. The larger cells are in the deeper part (sublayer of large pyramids). This layer also contains many granule cells with short axones and cells of Martinotti, (4) Internal gramular layer. Here the predominating elements are stellate cells, the larger usually sending their axones into the white matter. Among these are many short axone granules, the axones of which end in the same layer or in more superficial layers. (5) Ganglionic layer or deep layer of large and medium sized pyramids. These send their axones into the white matter. Mingled with them are short axone and Martinotti cells. (6) Multiform layer or layer of polymorphous cells. These usually send their axones into the white matter. Mingled with them are short axone and Martinotti cells. (Figs. 354, 355 and 356.)

The cells of the cortex obviously fall into two classes: efferent projection cells and association cells. Which cells are projection cells is not definitely known, except in the case of the precentral motor cortex where it has been established that these cells are the cells of Betz, the axones of which form the pyramidal tract and the fibres to motor cranial nerve nuclei. An examination of this area shows that the association cells must enormously outnumber the efferent projection cells in the cortex. The association cells comprise the short axone cells and cells the fibres of which enter the white matter, but terminate in some other part of the cortex, forming the association fibres of the white matter. (Compare p. 533.)

It is thus evident that every part of the cortex contains terminations of association fibres. The areas containing the terminations of afferent projection fibres are those which receive the thalamocortical continuations of the afferent pallial paths and the continuations of the olfactory paths. From observations made with the Golgi method it seems probable that the afferent projection fibres are coarse fibres which may ramify throughout the greater part of the thickness of the cortex, but are confined mainly to the third and fourth layers. The principal areas of the cortex receiving the afferent projection fibres are the hippocampal area (olfactory), the cal- 


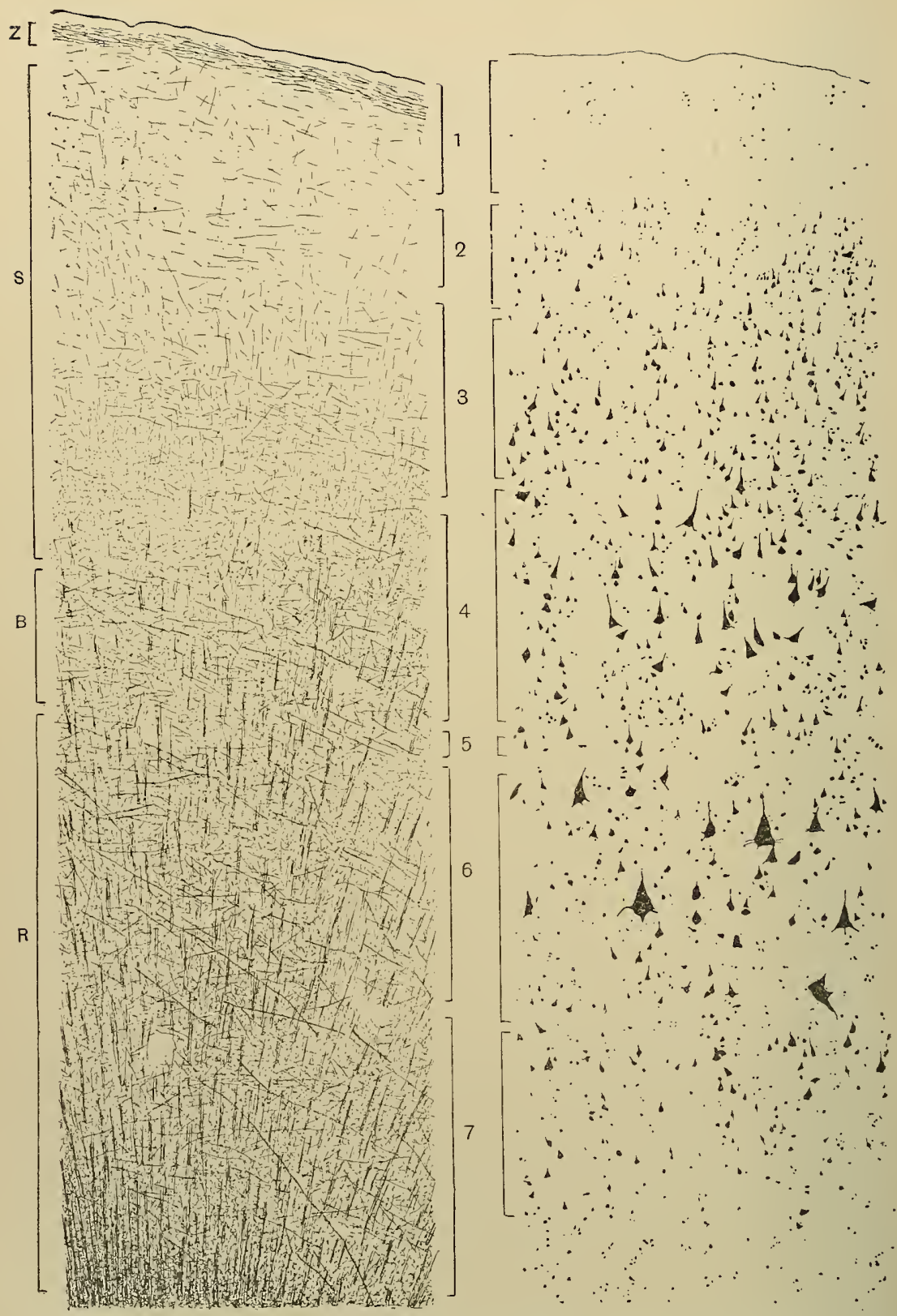

Fig. 356.--Vertical Sections of Precentral or Motor Area of Adult Human Cortex. Left, Weigert preparation showing fibre arrangement. Right, Arrangement of cells. (Campbell.) $B$, Position of line of Baillarger, its position obscured by surrounding wealth of fibres; $R$, radiary layer; $S$, supraradiary layer; $Z$, layer of superficial tangential fibres in molecular layer, dense and well defined; $x$, molecular layer; 2 , external granular layer (small pyramids), 3 (medium-sized) and 4 (large), pyramid layer; 5 , internal granular layer, indistinct and with scattered granule or stellate cells; 6 , ganglionic layer (large deep pyramids); 7 , multiform layer. 
carine area (visual, fibres from lateral geniculate body), the transverse temporal gyri of Heschl (auditory, fibres from medial geniculate body), and the pre- and postcentral areas (postcentral only, according to some authorities, area of general sensation from body and head, fibres from ventrolateral thalamic nuclei).

(Fig. 357.)

The medullated fibres of the cortex consist of radially, obliquely, and tangentially running fibres. The radial fibres enter the cortex from the white matter in bundles known as the radiations of Meynert which extend a variable distance toward the periphery, diminishing until they end usually in the third layer. They consist mainly of the axones of the adjoining cells passing to the white matter. Their fibres are of varying calibre, the coarsest originating from the largest cells. The oblique fibres form a dense plexus of coarse and fine fibres between the radial fibres, the interradiary plexus. Toward the surface (in the second and third cell layers) they form a delicate plexus of fine fibres. This latter plexus lies principally superficial to the radiations of Meynert and is the supraradiary plexus. A denser aggregation of irregular fibres constitute the line or stria of Baillarger located in the layer of superficial large pyramids. It is probable that this represents a layer especially rich in terminals of fibres from the white matter. Other striæ are also described. Besides representing the terminals of fibres from the white matter the oblique fibres in general are also composed of medullated collaterals of axones of pyramids and possibly arborizations of short axone cells. A few coarser fibres ascending to the molecular layer are ascending fibres from Martinotti cells. The deep tangential fibres, most marked on the sides of the convolutions and in the sulci, are considered short association fibres belonging to the fibre proprix of Meynert. In the molecular layer are the superficial tangential fibres consisting of the axones of the horizontal cells and the terminals of the axones of Martinotti cells. (Figs. 354 and 356.)

'The cortex is divided into various areas by various investigators, the areas being distinguished $(a)$ by the time of medullation (myelogenetic methorl of Flechsig), (b) by the number and arrangement of the merlullated fibres (myeloarchitecture), especially the number, thickness, and distinctness of the strice, such as that of Baillarger, formed by them, and (c) by the number and arrangement of the cells (cytoarchitecture). Many such areas have been thus distinguished by different investigators with results agrecing in many respects but differing in others. The areas most clearly definerl and concerning 


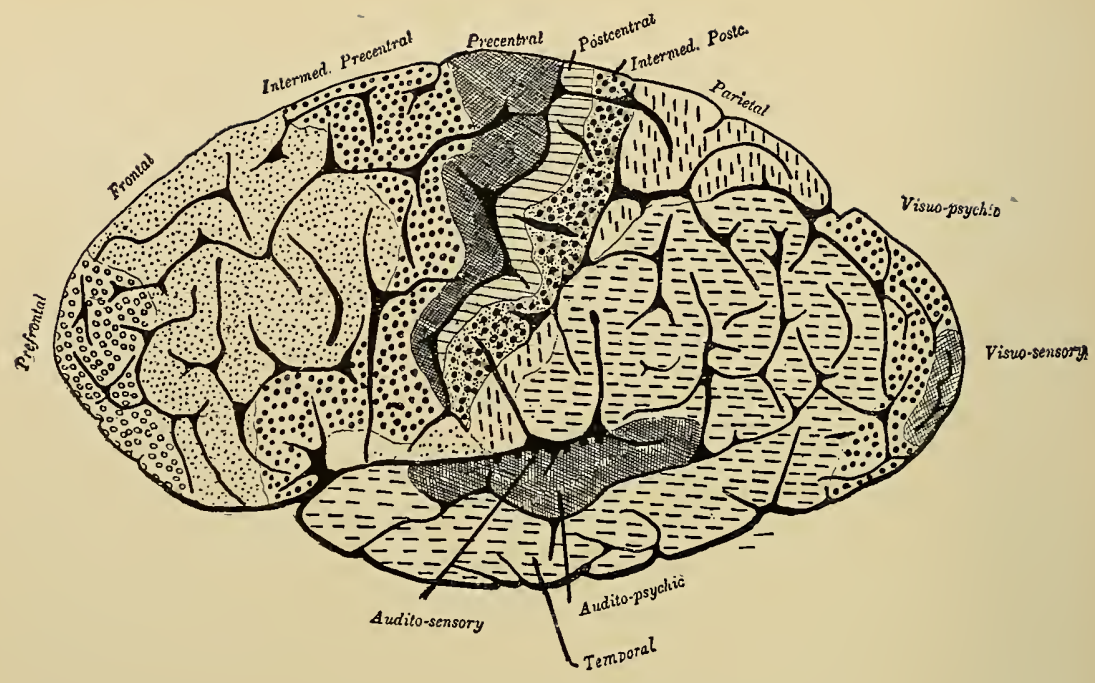

I

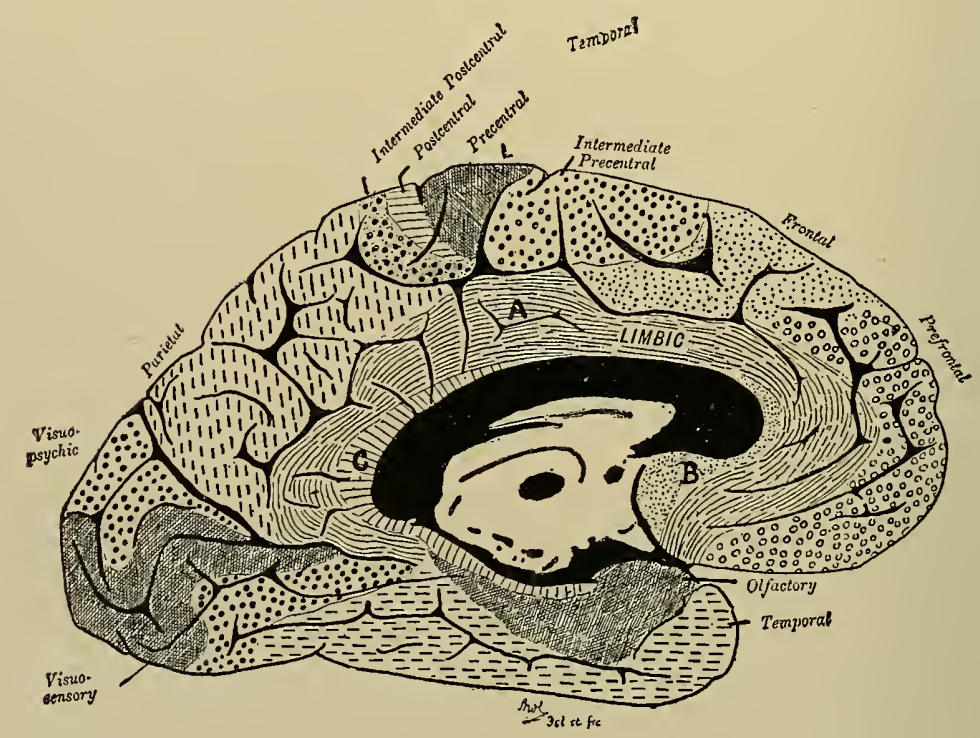

II

FIG. 357.-Diagram (orthogonal) showing Cortical Areas as determined by the Arrangement and Distribution of Fibres and Cells (A. W. Campbell). Large portions of important areas are concealed within fissures, e.g., the calcarine or visual (visuo-sensory, within the calcarine fissure) precentral (motor) and postcentral (within the fissure of Rolando) and especially the acoustic (audito-sensory) which is almost completely hidden within the Sylvian fissure. $A, B$ and $C$, parts of the limbic lobe. 
which there is perhaps the most general agreement are the various sensory (afferent projection) areas already enumerated (p. 539) and the motor (efferent projection) precentral area (Fig. 357). These areas myelinate first (at or soon after birth), next areas adjacent to them, and last areas occupying a considerable portion of the human pallium but much less extensive in other mammals. There is much difference of opinion as to the extent to which these last myelinating areas are supplied with projection fibres. According to some authorities the areas myelinating last have no projection fibres and are consequently entirely composed of association cells and fibres. Perhaps the two best-marked of the various areas are the motor and visual areas the structure of which is shown in figures 354,355 and 356 . The motor precentral cortex is characterized by the presence of the giant cells of Betz, by an almost complete absence of an internal granular layer and by a great wealth of fibres. The calcarine or visual area is characterized by a strongly marked line of Gennari (=Baillarger) and by a double or triple internal granular layer containing large granules in place, apparently, of the superficial large pyramids. The line of Gennari is probably partly composed of the terminals of the fibres of the optic path (geniculo-calcarine fibres).

\section{TECHNIC}

(I) The general structure of the cerebellum is well brought out by staining sections of formalin-Müller's fluid-fixed material with hæmatoxylin-picro-aciclfuchsin (tcchnic 3, p. $2 \mathrm{I}$ ), and mounting in balsam.

(2) The arrangement of the cell layers of both cerebellum and cerebrum as well as certain details of internal structure of the cells, can be studied in sections of alcohol-or formalin-fixed material stained by the method of Nissl (technic, p. 38).

(3) The distribution of the medullated nerve fibres of either the cerebellar or cerebral cortex is best demonstrated by fixing material in Müller's fluid (technic 4, p. 6) or in formalin-Müller's fluid, and staining rather thick sections by the Weigert or Weigert-Pal method (technic, p. 33).

(4) The external morphology of the cerebellar and cerebral neurones and the relations of cell and fibre can be thoroughly understood only by means of sections stained by one of the (jolgi methorls (technic, pp. 35 and 36). Especially in the case of the cercbellum, sections should be made both at right angles, and Jongiturlinal to the long axis of the convolution. Golgi preparations from cmbryonic material and from the brains of lower animals furnish instructive pictures.

(5) The silver method of Cajal should be userl especially with alcohol fixit- 
tion (technic p. 38 , No. 2), both for the neurofibrils and for the external morphology of the neurones. It is especially successful with the cerebellum.

(6) Neuroglia stains should also be used.

\section{The Pituitary Body}

(See page 407.)

\section{The Pineal Body}

The pineal body originates as a fold of the wall of the primary brain vesicle. It lies upon the dorsal surface of the inter- and midbrain, being connected with the former. The pineal body is apparently of the nature of a rudimentary sense organ, being sometimes referred to as the median or pineal eye. In man it is surrounded by a firm connective-tissue capsule, which is a continuation of the pia mater. This sends trabeculæ into the organ, which anastomose and divide it into many small chambers. The latter contain tubules or alveoli lined with cuboidal epithelium. This may be simple or stratified, and frequently almost completely fills the tubules. Within the tubules are often found calcareous deposits known as "brain sand."

\section{TECHNIC}

The general structure of the pituitary body and of the pineal body can be studied by fixing material in formalin-Müller's fluid (technic 5, p. 7 ) and staining sections with hæmatoxylin-eosin (technic I, p. 20).

\section{General References for Further Study}

Bailey and Miller: A Text-book of Embryology, New York, I909. Chaps. XVII and XVIII. I 899 .

Barker: The Nervous System and its Constituent Neurones, New York,

Dejerine: Anatomie des centres nerveux, Paris, 1895 .

Edinger L.: Vorlesungen über den Bau der nervösen Zentralorgane des Menschen und der Tiere, Leipsig, I908 and I9II. I906.

Van Gehuchten: Anatomie du système nerveux de l'homme, Louvaine,

Golgi: Untersuchungen über den feineren Bau des centralen und peripherischen Nervensystems, Jena, 1894.

Johnston, J. B.: The Nervous System of Veterbrates, I906.

Kölliker: Handbuch der Gewebelehre des Menschen, Leipsic, 1896.

Von Lenhossék: Der feinere Bau des Nervensystems im Lichte neuester Forschungen, Berlin, I 895 . 


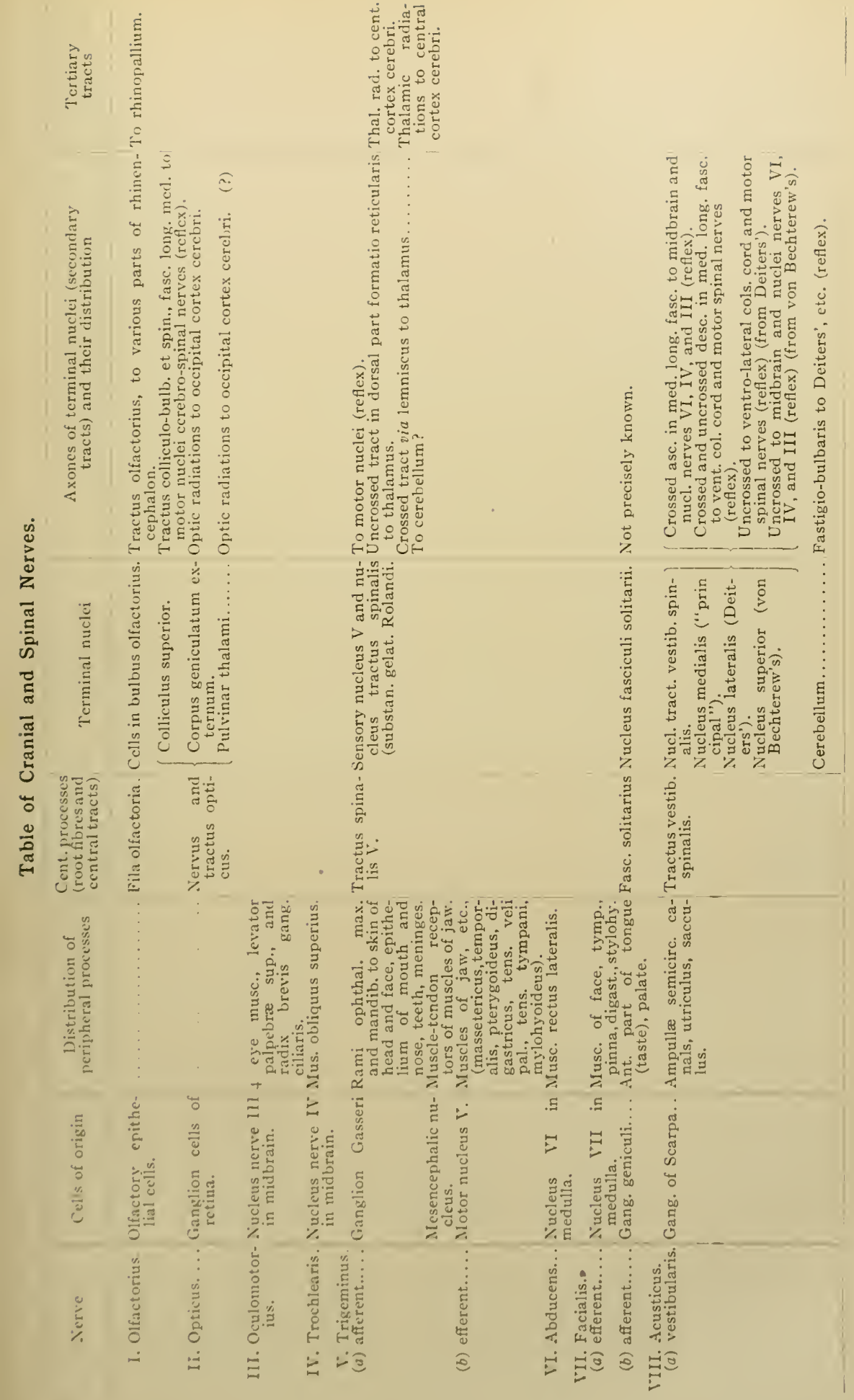




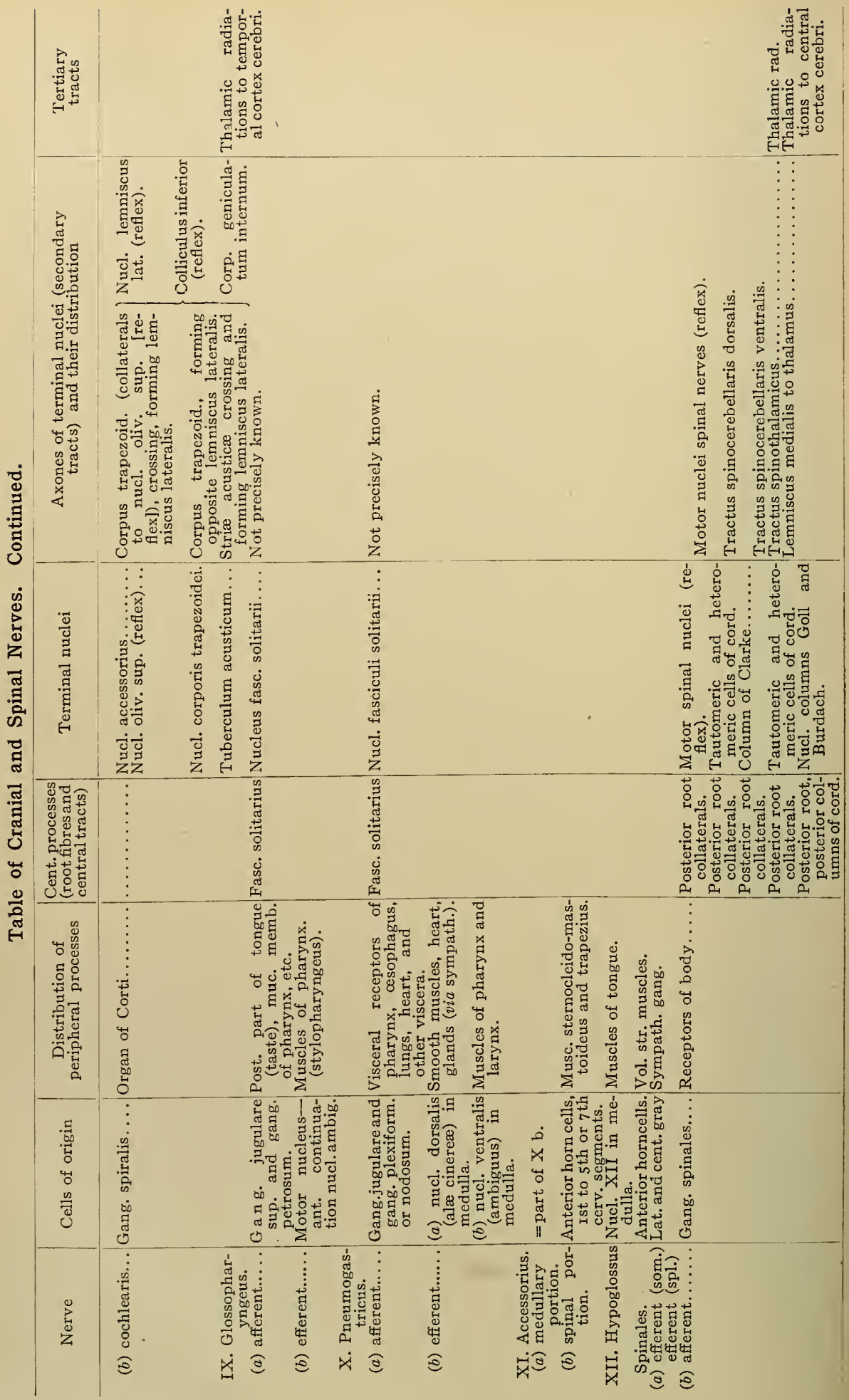



I9IO.

Marburg: Atlas des menschlichen Centralnervensystems, Leipzig and Wein,

Meyer, Adolf: Critical Review of the Data and General Methods and Deductions of Modern Neurology. Journ. of Comp. Neurol., Vol. VIII, Nos. 3 and $4, \mathrm{I} S 9 \mathrm{~S}$.

Obersteiner: Anleitung beim Studieren des Baues der nervösen Centralorgane, Leipsic.

Quain's Elements of Anatomy, Vol. III, Neurology, Parts I and 2, I908.

Ramón y Cajal: Beitrag zum Studium der Medulla Oblongata, etc., Leipsic, I 896 . - Les nouvelles idées sur la structure du système nerveux chez l'homme et chez les vertébrés, Paris, I894. - Histologie du système nerveux de l' homme et des vértebrés, trad. par L. Azoulay, Tomes I and II, Paris, I909.

Studien über die Hirnrinde des Menschen, Leipsig, I900.

Spalteholz, W.: Handatlas of Human Anatomy (trans. by L. F. Barker) Vol. III, Igc3. 


\section{CḦAPTER X́III}

\section{THE ORGANS OF SPECIAL SENSE}

\section{The Organ of Vision}

THE eyeball and optic nerve constitute the organ of vision. To be described in connection with them are the eyelid and the lacrymal apparatus.

The Eyeball or Bulbus Oculi.-This is almost spherical, although slightly flattened antero-posteriorly. It consists of a wall enclosing a cavity filled with fluid.

The wall of the eyeball consists of three coats: (a) An external fibrous coat - the sclera and cornea; $(b)$ a middle vascular-the chorioid; and (c) an internal nervous - the retina (Fig. $35^{8}$ ).

The Sclera (Figs. 358 and 359).-This consists of dense fibrous tissue with some elastic fibres. The fibres run both meridionally and equatorially, the tendons of the straight muscles of the eyeball being continuous with the meridional fibres, those of the oblique muscles with the equatorial fibres. The few cells of the sclera lie in distinct, very irregular cell spaces, and frequently contain pigment granules. Pigmented cells in considerable numbers are regularly present near the corneal junction, at the entrance of the optic nerve, and on the inner surface of the sclera. Where the optic nerve pierces the sclera, the continuity of the latter is broken by the entering nerve fibres, forming the lamina cribosa (Fig. ${ }^{6}{ }_{7}$ ). The pigmented layer of the sclera next the chorioid is known as the lamina fusca, and is lined internally by a single layer of flat non-pigmented endothelium. Anteriorly a loose connective tissue attaches the sclera to the scleral conjunctiva.

The CoRnea (Figs. 360 and 363).-This is the anterior continuation of the sclera so modified as readily to allow the light to pass through it. It is about $\mathrm{I} \mathrm{mm}$. thick and consists of five layers, which from before backward are as follows (Fig. 360):

(I) Anterior epithelium.

(2) Anterior elastic membrane or membrane of Bowman.

(3) Substantia propria corneæ. 
(4) Posterior elastic membrane or membrane of Descemet.

(5) Posterior endothelium or endothelium of Descemet.

(I) The anlcrior cpilhclium. (Fig. $360, \mathrm{I}$ ) is of the stratified squamous type and consists of from four to eight layers of cells. The deepest cells are columnar and rest upon the anterior elastic membrane. The middle cells are polygonal and are connected by short

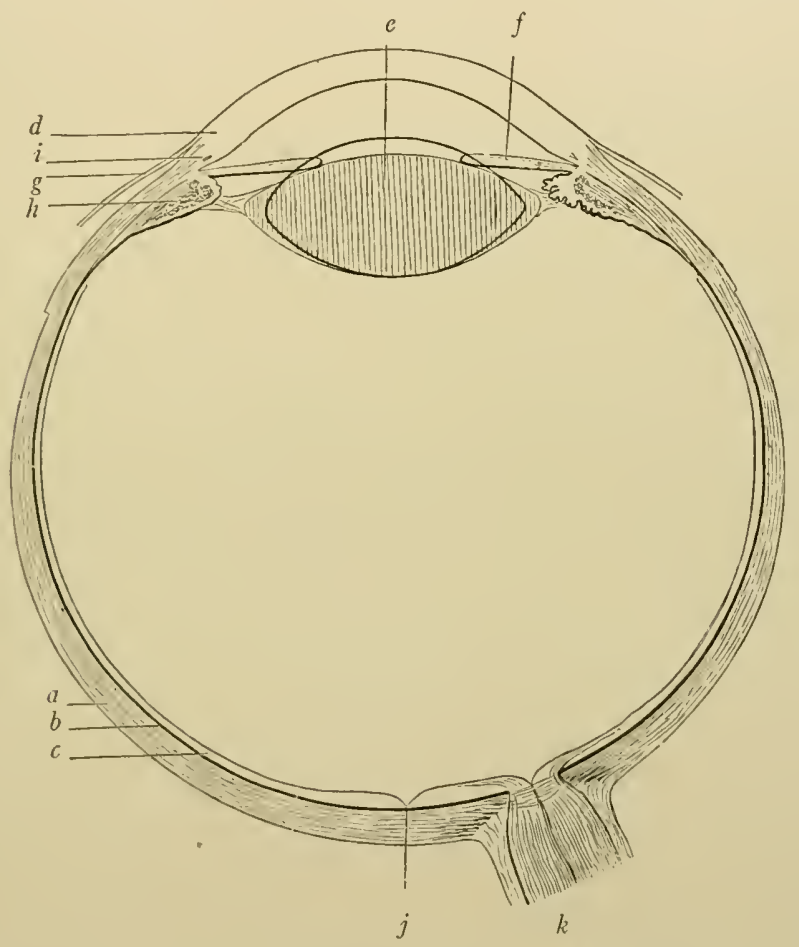

Fic 358.-I)iagram of Eyeball showing Coats. (Merkel-Henle.) $a$, Sclera; $b$, chorioid; $c$, retina; $d$, cornea; $e$, lens; $f$, iris; $g$, conjunctiva; $h$, ciliary body; $i$, sclero-corneal junction and canal of Schlemm; $j$, fovea centralis; $k$, optic nerve.

intercellular bridges. The surface cells are flat. Along the margin of the cornea the epithelium is continuous with that of the conjunctiva (Fig. 363).

(2) The anlerior claslic membrane (Fig. 360,2 ) is a highly developed basement membrane, its anterior surface being pitted to receive the bases of the decpest epithelial cells. It is apparently homogeneous, and while called an elastic membrane, does not conform rhemically to either fibrous or clastic tissue. By means of special technic, at fibrillar structure has been demonstrated. 
(3) The substantia propria (Fig. 360,3 ) constitutes the main bulk of the cornea. It consists of connective tissue the fibrils of which are doubly refracting and are cemented together to form bundles and lamellæ. In the human cornea the lamellæ are about sixty in number. The lamellæ are parallel to one another and to the surface of the cornea, but the fibres of adjacent lamellæ cross one another at an angle of about twelve degrees. The lamellæ are united by cement substance. Fibres running obliquely through the lamellæ from posterior to anterior elastic membranes hold the lamellæ firmly together. They are known as perforating or arcuate fibres.

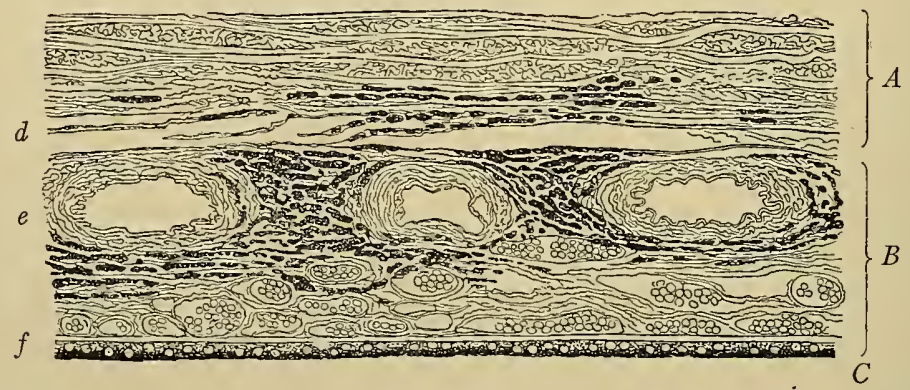

Fig. 359.-Vertical Section through Sclera, Chorioid, and Pigment Layer of Retina. (Merkel-Henle.) $A$, Sclera; $B$, chorioid; $C$, pigment layer of retina; $d$, lamina suprachorioidea; $e$, Haller's layer of straight vessels; $f$, choriocapillaris; $g$, vitreous membrane.

Between the lamellæ are irregular flat cell spaces which communicate with one another and with the lymph spaces at the margin of the cornea by means of canaliculi. Seen in secticns vertical to the surface of the cornea, these spaces appear fusiform. In the spaces are the connective-tissue cells of the cornea or corneal corpuscles. These are flat cells corresponding in shape to the spaces and sending out processes into the canaliculi (Figs. 361 and 362 ).

(4) The posterior elastic membrane or membrane of Descemet (Fig. 360, 4) resembles the anterior, but is much thinner. Like the anterior, it does not give the chemical reaction of elastic tissue.

(5) The posterior endothelium or endothelium of Descemet (Fig. $360,5)$ consists of a single layer of flat hexagonal cells, the nuclei of which frequently project slightly above the surface.

The cornea contains no blood-vessels.

THE CHORIOID.--This is made up of four layers. which from without inward are as follows (Fig. 359): 
(I) The lamina suprachorioidea.

(2) The layer of straight vessels-Haller's layer.

(3) The capillary layer-choriocapillaris.

(4) The vitreous membrane-lamina citrea-membrane of Bruch.

(I) The lamina suprachorioidea (Fig. $359, d$ ) is intimately connected with the lamina fusca of the sclera and consists of loosely arranged bundles of fibrous and elastic tissue among which are scattered pigmented and non-pigmented connective-tissue cells. Numerous lymph spaces are found between the bundles of connective tissue and between the lamina suprachorioidea and lamina fusca. The latter are known as the perichorioidal lymph spaces (Fig. 363).

(2) The layer of straight vessels (Fig. $359, e)$ consists of fibro-elastic tissue containing numerous pigmented and non-pigmented cells, supporting the large blood-vessels of the layer. The latter can be seen with the naked eye, and, as they are straight and parallel, give to the layer a striated appearance. The arteries lie to the inner side. The veins-vene vorlicos $Q$ - are larger than the arteries and converge toward four points one in each quadrant of the eyeball.

A narrow boundary zone, rich in

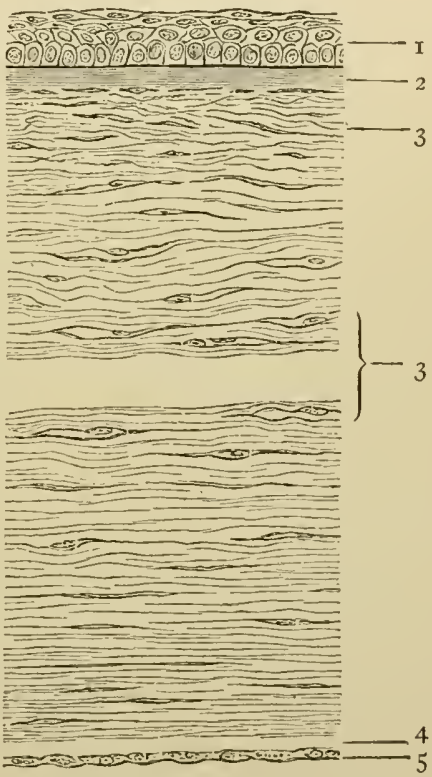

FIG. 360.-Vertical Section of Cornea. (Merkel-Henle.) I, Anterior epithelium; 2, anterior elastic membrane; 3, substantia propria corneæ; 4, posterior elastic membrane; 5 , posterior endothelium. elastic fibres and free from pigment, limits this layer internally. It is much more highly developed in some of the lower animals than in man. Formed of connectivetissue bundles in ruminants and horses, it is known as the tapetum fibrosum, while in the carnivora its structure-several layers of flat colls-gives it the name of the tapehum cellulosum.

(3) The choriocapillaris (Fig. 359, f) consists of connective tissue supporting a dense network of capillaries, which is most dense in the region of the macula lutea. 'This layer is usually described as free from pigment, although it not infrequently contains some pigmented cells. 
(4) The vitreous membrane (Fig. 359, g) is a clear, apparently structureless membrane about two microns thick. Its outer surface is grooved by the capillaries of the choriocapillaris, while its inner surface is pitted by the retinal epithelium.

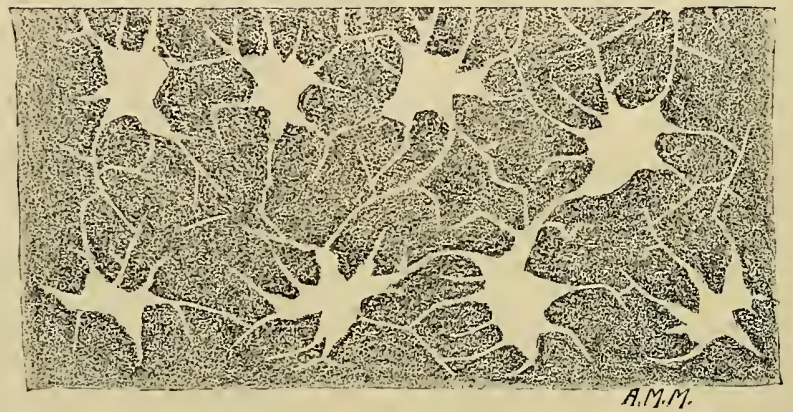

Fig. 36r.-Section of Human Cornea cut Tangential to Surface- $\times 350$ (technic 9, p. 97)-showing corneal cell spaces (lacunæ) and anastomosing canaliculi.

The Ciliary Body.-This is the anterior extension of the chorioid and consists of the ciliary processes and the ciliary muscle (Fig. 363 ). It extends from the ora serrata (a wavy edge which marks the anterior

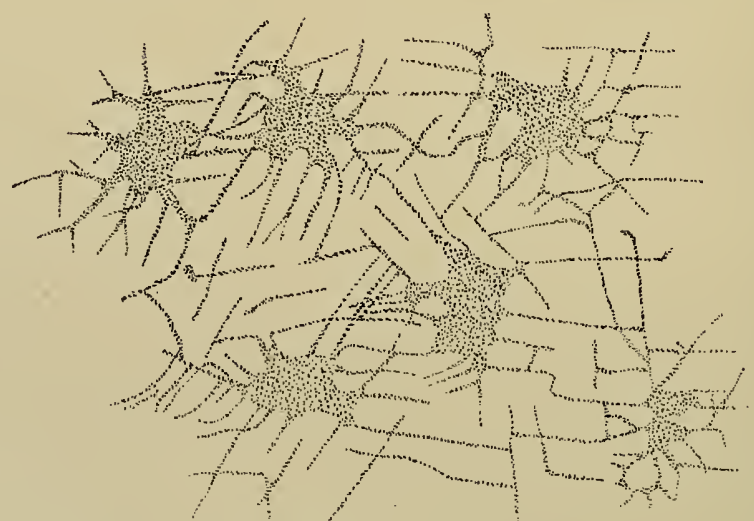

FIG. 362.-Section of Human Cornea cut Tangential to Surface $-\times_{350}$ (technic 8, p. 97) - showing corneal cells and their anastomosing processes.

limit of the nervous elements of the retina-see Retina) to the margin of the iris (see below).

The ciliary processes (Fig. 363), from seventy to eighty in number, 
are meridionally-running folds of the chorioid from which are given off numerous irregular secondary folds. The processes begin low at the ora serrata, gradually increase in height to about I mm., and end abruptly at the margin of the iris. The ciliary processes consist of connective tissue containing many pigmented cells and supporting numerous blood-vessels. Invaginations lined with clear columnar epithelium have been described as ciliary glands. The ciliary folds

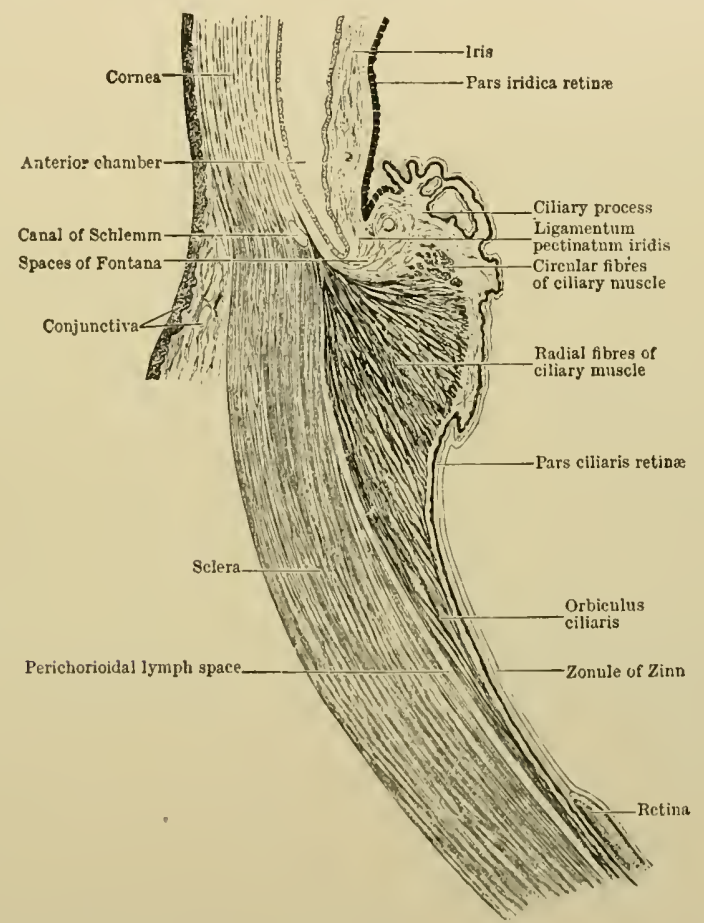

FIG. 363.- Vertical Section through Human Sclero-corneal Junction. (Cunningham.)

are covered by the vitrcous membrane, and internal to the latter is a continuation forward of non-nervous elements of the retina-pars ciliaris relince (Fig. 363 ). 'This consists of two layers of cclumnar epithelial cells, the outer layer being pigmented, the inner nonpigmented.

The ciliary muscle (Fig. $36_{3}$ ) is a band of smooth muscle which encircles the iris. It lies in the outer anterior part of the ciliary body, and on cross section has a generally triangalar shape. It is divisible into three groups of muscle cells: (a) An inner circular group near the base of the iris-circular muscle of Müller; (b) an 
outer meridional group lying next to the sclera and known as the tensor choricideæ, and $(c)$ a middle radial group. The meridional and radial groups both take origin in the posterior elastic lamina of the cornea, the former passing backward along the margin of the sclera to its insertion in the ciliary body near the ora serrata, the latter radiating fan-like to a broad inserticn in the ciliary body and processes.

The ciliary body is closely attached to the sclero-corneal junction by the ligamentum pectinatum (Fig. 363), a continuation of the

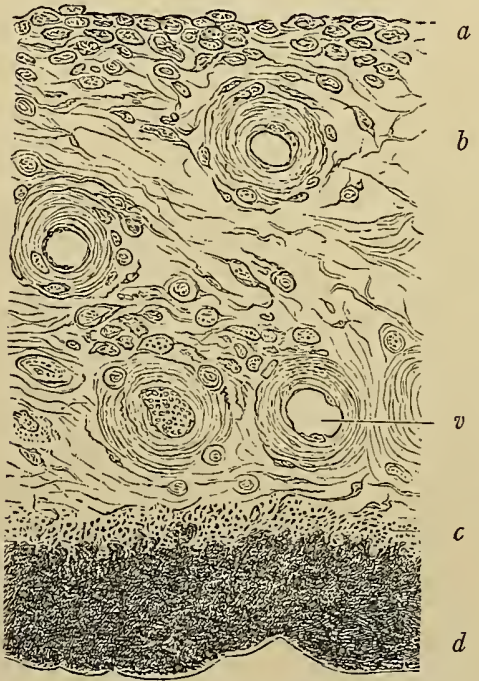

FIG. 364.--Vertical Section through Iris. (Merkel-Henle.) a, Anterior endothelium; $b$, stroma or substantia propria; $c$, vitreous membrane; $d$, pigment layer; v, blood-vessel. posterior elastic lamina of the cornea. Within the ligament are spaces (spaces of Fontana) lined with endothelium. These are apparently lymph spaces, and communicate with each other, with similar spaces around the canal of Schlemm, and with the anterior chamber. The canal of Schlemm (Fig. $3_{3}$ ) is a venous canal which encircles the cornea, lying in the sclera close to the corneal margin. Instead of a single canal there may be several canals.

THE IRIS (Fig. 364).-This represents a further continuation forward of the chorioid. Its base is attached to the ciliary body and ligamentum pectinatum. From this point it extends forward as a diaphragm in front of the lens, its centre being perforated to form the pupillary opening. It is deeply pigmented, and to its pigment the color of the eye is due. Four layers may be distinguished, which from before backward are as follows:

(I) The anterior endothelium.

(2) The stroma.

(3) The vitreous membrane.

(4) The pigmented epithelium.

(I) The anterior endothelium is a single layer of pigmented cells continuous with the posterior endothelium of the cornea (Fig. $364, a)$. 
(2) The stroma is divisible into two layers: an anterior reticular layer, containing many cells, scme of which are pigmented, and a vascular layer, the ressels of which are peculiar in that their walls contain almost no muscle, but have thick connective-tissue sheaths. In the posterior part of the stroma are bundles of smooth muscle. Those nearest the pupillary margin encircle the pupil, forming its sphincter muscle, while external are scattered radiating bundles forming the dilator muscle.

(3) The vitreous membrane is continuous with, and has the same structure as the membrane of Bruch.

(4) The pigmented epithelium (Fig. $364, d$ ) consists of several layers of cells and is continuous with the pas ciliaris retinæ. Except in albinos, both layers are pigmented.

The Retina.-The retina is the nervous tunic of the eye. It lines the entire eyeball, ending only at the pupillary margin of the iris. Its nerrous elements, however, extend only to the ora serrata, which marks the outer limit of the ciliary body (Fig. 363). The nervous part of the retina is known as the pars optica retine, the non-nervous extension over the ciliary processes as the pars ciliaris retina, its further continuation over the iris as the pars iridica retina. Modifications of the optic portion of the retina are found in the region of the macula lutea and of the optic nerve entrance.

The Pars Optica Retina.-This is the only part of the retina directly concerned in the reception of impulses, and may be regarded as the extremely complex sensory end-organ of the optic nerve. It is divisible into ten layers, which from without inward are as follows (Fig. $36_{5}$ ):

(I) Layer of pigmented epithelium.

(2) Layer of rods and cones.

(3) Outer limiting membrane.

(4) Outer nuclear layer.

(5) Outer molecular layer.

(6) Inner nuclear layer.

(7) Inner molecular layer.

(8) Layer of nerve cells.

(9) Layer of nerve fibres.

(Io) Inner limiting membrane.

I.ayer of neuro-epithelium.

'The layer of pigmented epithelium (Fig. $36_{5}, B, \mathrm{I}$ ) consists of a single layer of regular hexagonal cells (Fig. 25, p. 76). The nuclei 
lie in the outer part of the cell, while from the inner side thread-like projecticns extend down between the rods and cones of the layer next internal. The pigment has the form of rod-shaped granules. Its distribution seems to depend upon the amount of light being admitted to the retina. When little or no light is being admitted, the pigment is found in the body of the cell, the processes being wholly or almost

$A$

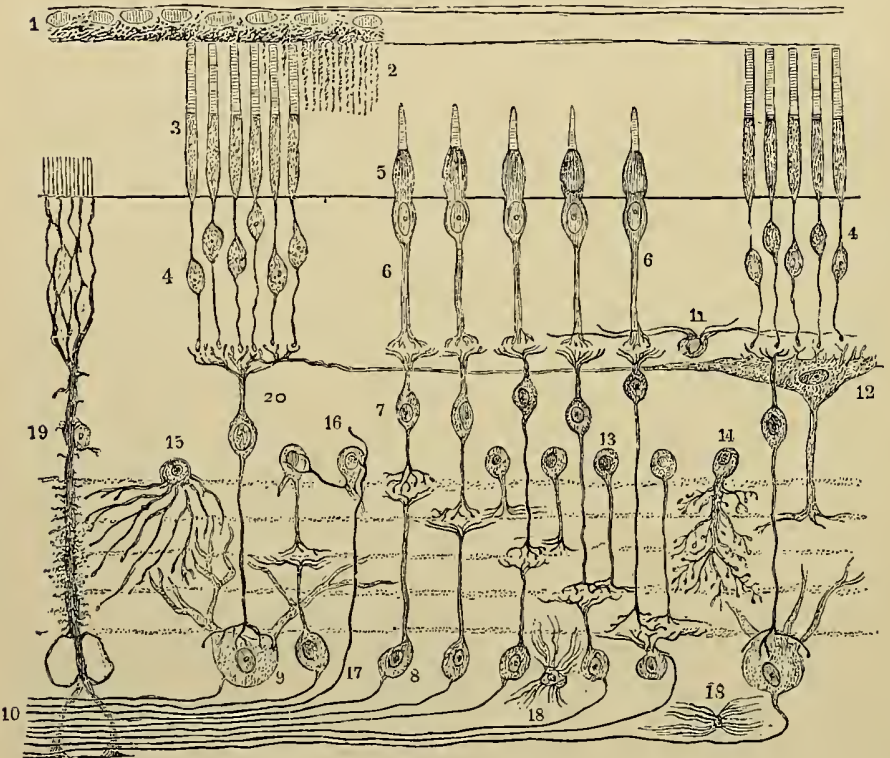

$B$

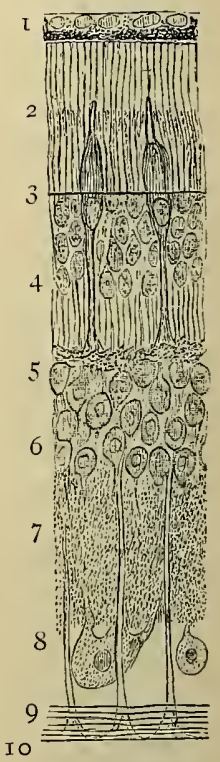

FIG. $365 .-A$, Scheme of retina as shown by the Golgi method. $B$, Vertical section of retina to show layers as demonstrated by the hæmatoxylin-eosin stain. (MerkelHenle.) B.-I, Layer of pigmented epithelium; 2, layer of rods and cones; 3 , outer limiting layer; 4, outer nuclear layer; 5 , outer molecular layer; 6 , inner nuclear layer; 7 , inner molecular layer; 8, layer of nerve cells; 9 , layer of nerve fibres; 10, inner limiting layer. A.- I, Pigment layer; 2, processes of pigmented epithelial cells extending down between rods and cones; 3 , rods; 4 , rod-cell nuclei and rod fibres; 5 , cones; 6 , cone fibres; 7 , bipolar cells of inner nuclear layer; 8 , ganglion cells of nerve-cell layer; 9 , larger ganglion cells of nerve-cell layer; Io, fibres of optic nerve forming layer of nerve fibres; I I and I 2 , types of horizontal cells; I3, I4, I5, and I6, types of cells the bodies of which lie in the inner nuclear layer; I7, efferent optic-nerve fibre ending around cell of inner nuclear layer; I8, neuroglia cells; I9, Müller's fibre; 20 , rod-bipolar cell of inner nuclear layer.

free from pigment; when the retina is exposed to a bright light, some of the pigment granules pass down into the processes so that the pigment becomes more evenly distributed throughout the cell.

The layer of rods and cones and the outer nuclear layer (Fig. 365, $B, 2,4)$ are best considered as subdivisions of a single layer, the neuroepithelial layer. This consists essentially of two forms of neuro- 
epithelial elements, rod visual cells and cone visual cells. These, with supporting connective tissue, constitute the layer of rods and cones and the outer nuclear layer, the separation into sub-layers being due to the sharp demarcation between the nucleated and non-nucleated parts of the cells, and the separation of the two parts by the perforated outer limiling membrane.

The rod visual cell (Fig. $365, A, 4$ ) consists of rod, rod-fibre, and nucleus. The rod (Fig. $365, A, 3$ ) is a cylinder from 30 to $40 \%$ in length and about $2 \mu$ in diameter. It is divisible into an outer clear portion, which contains the so-called "visual purple" and an inner granular portion. At the outer end of the latter is a fibrillated ellipsoidal body, much more distinct in some of the lower animals, the ellipsoid of Krouse. At its inner end the rod tapers down to a fine fibre, the rod fibre, which passes through a perforation in the outer limiting membrane into the outer nuclear layer, where it expands and contains the nucleus of the rcd visual cell. These nuclei are situated at various levels in the fibre and constitute the most conspicuous element of the ouler muclear layer (Fig. $365, B, 4$ ).

The cone visual cell (Fig. $365, A, 5,6$ ) consists of cone, cone-fibre, and nucleus. The cone (Fig. $365, A, 5$ ) is shorter and broader than the rod, and like the latter is divisible into two parts. The outer part is short, clear, and tapering, the inner part broad and granular, and like the rod contains a fibrillated ellipsoid body. The cone fibre (Fig. $36_{5}, A, 6$ ) is much broader than the rod fibre, passes completely through the outer nuclear layer and ends in an expansion at the margin of the outer molecular layer. The nucleus of the cone cell usually lies just beneath the outer limiting membrane.

The remaining layers of the retina must be considered in relation on the one hand to the neuro-epithelium, on the other to the optic nerve. The inner muclear layer (Fig. $3_{5}, B, 6$ ) and the layer of nerve cells (Fig. ${ }_{3} 6_{5}, B, 8$ ) are composed largely of nerve-cell bodies, while the two molecular layers (Fig. $365, B, 5,7$ ) are formed mainly of the ramifications of the processes of these cells. In the inner nuclear layer are two kinds of nerve elements, rod bipolar cells and cone bipolar cells. The bodies of these cells with their large nuclei form the bulk of this layer. From the rod bipolars (Fig. 365, A, 20) processes (dendriles) pass outward to ramify in the outer molecular layer arcund the terminations of the rod fibres. From the cone bipolars (Jig. $365, A, 7$ ) similar processes (dendrites) extend into the outer molecular layer where they ramify around the terminations of the 
cone cells. Two other forms of nerve cells occur in the inner nuclear layer. One is known as the horizontal cell (Fig. $365, A$, I2). Its processes ramify almost wholly in the outer molecular layer. The other lies along the inner margin of the inner nuclear layer and sends its dendrites into the inner molecular layer (Fig. 365, A, 13, I4, I5, and I6). Many of these latter cells appear to have no axone and are consequently known as amacrine cells.

The outer molecular layer is thus seen to be formed mainly of terminations of the rod and cone visual cells, of the dendrites of the rod and cone bipolars, and of the processes of the horizontal cells.

From the cone bipolar a process (axone) extends inward to ramify in the inner molecular layer, while from the rod bipolar a process (axone) passes inward through the inner molecular layer to terminate around the cells of the nerve-cell layer.

The layer of nerve cells (Fig. $36_{5}, B, 8$ ) consists for the most part of large ganglion cells whose dendrites ramify in the inner molecular layer, and whose axones pass into the layer of nerve fibres and thence into the optic nerve. Some small ganglion cells are also found in this layer, especially in the region of the macula lutea (see page 559).

The inner molecular layer is thus seen to be composed mainly of the processes (axones) of the rod and cone bipolars and of the dendrites of the ganglion cells of the nerve-cell layer.

The layer of nerve fibres (Fig. $36_{5}, B, 9$ ) consists mainly of the axones of the just-described ganglion cells, although a few centrifugal axcnes of brain cells (Fig. $365, A$, I 7 ) are probably intermingled.

The outer and inner limiting layers or membranes (Fig. $365, B, 3, \mathrm{IO}$ ) are parts of the sustentacular apparatus of the retina, being connected with the cells or fibres of Müller (Fig. 365, A,19 and Fig. 366). The latter form the most conspicuous elements of the supportive tissue of the retina. They are like the nerve elements proper, of ectodermic origin and are elongated cells which extend through all the retinal layers, excepting the layer of rods and cones and the pigment layer. The inner ends of the cells, which are conical and fibrillated, unite to form the inner limiting membrane (Fig. 366, Io). Through the inner molecular layer the cell takes the form of a narrow stalk with numerous fringe-like side fibrils (Fig. 366, 7). This widens in the inner nuclear layer, where cup-like depressions in the sides of the Müller's cell are caused by the pressure of the surrounding nerve cells (Fig. $366, b)$. This wide portion of the cell in the inner nuclear layer contains the nucleus (Fig. 366,a). In the outer molecular layer the 
cell again becomes narrow (Fig. 366,5 ) and in the outer nuclear layer broadens out into a sponge-like reticulum (Fig. 366, 4), which supports the rod and cone bipolars. At the inner margin of the layer of rods and cones the protoplasm of the Müller's cells spreads out and unites to form the so-called outer limiting membrane (Fig. 366, 3), from which delicate fibrils (fibre baskets) pass outward between the

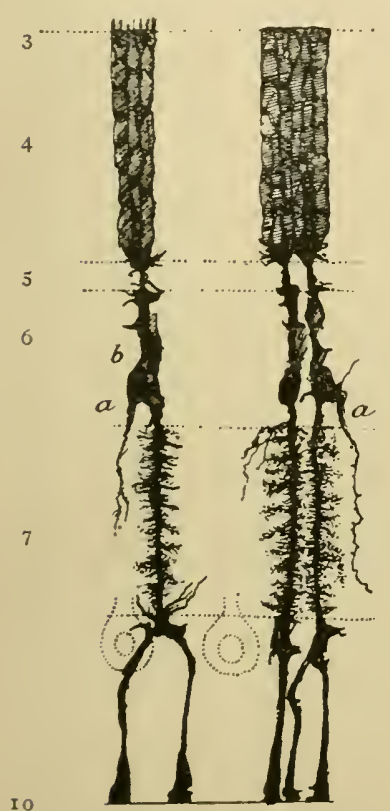

Fic. 366.-Two Müller's Fibres from Retina of $\mathrm{Gx}$ showing Relation to Layers of Retina. (Ramón y Cajal.) 3, Outer limiting layer; 4, outer nuclear layer; 5 , outer molecular layer; 6 , inner nuclear layer; 7 , inner molecular layer; 8, layer of nerve cells; 9 , layer of nerve fibres; 10 , inner limiting layer; $a$, nucleus; $b$, cup-like depression caused by pressure from surrounding cells.

The Optic Nerve.-The optic nerve (Fig. $367, d$ ) is enclosed by two connective-tissue sheaths, both of which are extensions of the brain membranes. The outer or dural shealh, (Fig. $367, a$ ) is continuous with the dura mater of the brain posteriorly, while anteriorly it blends with the sclera. The inner or pial sheath, (Fig. $367, b$ ) is an extension of the pia mater and is separated from the outer sheath by ler's cells, which are neuroglia elements, spider cells also occur in the retina (Fig. $365, A$, I 8 ).

The retina of the macula lutea presents certain peculiarities. Its name is derived from the yellow pigment which is distributed diffusely through the inner layers, extending as far out as the outer molecular layer. The ganglion-cell layer and the inner nuclear layer are thicker than in other parts of the retina. In the layer of rods and cones there is a gradual reduction in the number of rods, while the number of cones is correspondingly increased.

In the centre of the macula is a depression, the fovea centralis. As the retina approaches this area it becomes greatly thinned, little remaining but the layer of cone cells and the somewhat thickened layer of pigmented epithelium.

At the ora serrata the nervous elements of the retina cease. The non-nervous retinal extension over the ciliary body (pars ciliaris retince) and over the iris (pars iridica retina) have been described in connection with the ciliary body and iris. rods and cones. In addition to the Mül- 
the subdural space (Fig. $367, c)$. The pial sheath is divisible into two sub-layers: an outer fibrous layer (the so-called arachnoid), and an inner vascular layer. These two layers are separated by a narrow space, the subarachnoid space. The optic nerve fibres, in passing through the sclera and chorioid, separate the connective-tissue

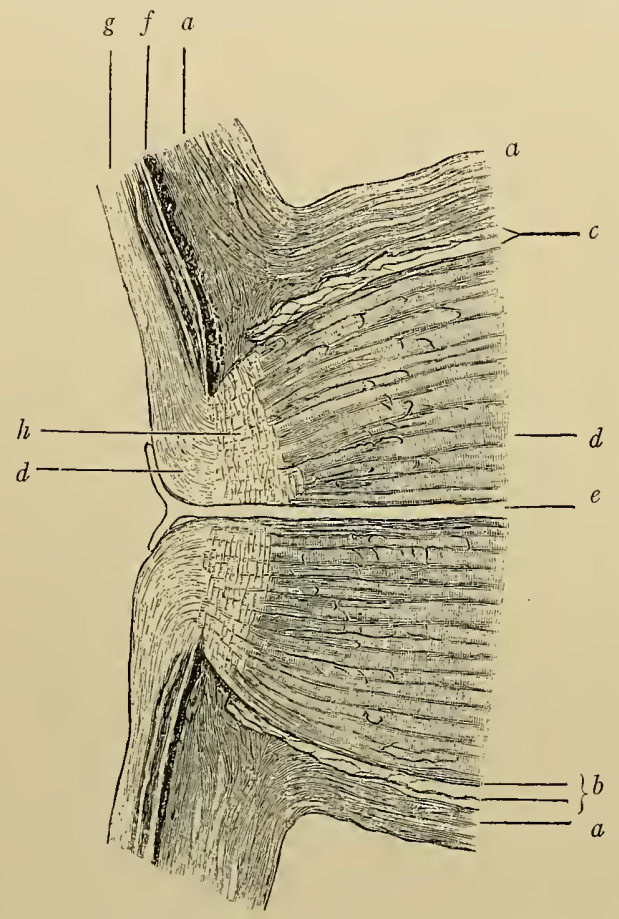

Frg. 367.-Section through Entrance of Optic Nerve into Eyeball. (Merkel-Henle.) a, Dural sheath; $b$, pial sheath, inner and outer layers; $c$, space between inner and outer layers of pia mater; $d$, optic nerve; $e$, central artery of retina; $a^{\prime}$, sclera; $f$, chorioid; , retina; $h$, lamina cribrosa. bundles so that they form a lattice-work, the already mentioned lamina cribrosa (Fig. $367, h$ ). The optic nerve fibres are medullated, but have no neurilemma. As they pass through the lamina cribrosa the medullary sheaths are lost, the fibres reaching the retina as naked axones.

\section{Relations of Optic Nerve to Retina AND BRAIN}

The rod and cone visual cells are the neuro-epithelial beginnings of the visual tract (Fig. 365, A, 3, 4, 5, and 6). By their expanded bases in the outer molecular layer, the rod and cone cells communicate with the neurone system No: I. of the optic tract. This comprises (a) rod neurones, (b) cone. neurones, $(c)$ horizontal neurones.

Neurone System No. I.-(a) Rod neurones. The cell bodies of these neurones (Fig. $365, A, 20$ ) lie in the inner nuclear layer. Their dendrites enter the outer molecular layer where they form networks around the expanded bases of the rod cells. Their axones pass through the inner molecular layer and end in arborizations around the bodies and dendrites of cells of the nerve-cell layer (neurone system No. II.). (b) Cone neurones (Fig. $365, A, 7$ ). These have their cell bodies in the inner nuclear layer. Their dendrites pass to the outer molecular layer where they form networks around the expanded bases 
of the cone cells. Their axones pass only into the inner molecular layer where they end in arborizations around the dendrites of neurones whose cell bodies are in the layer of nerve cells (neurone system No. II.). (c) Horizonlal neurones (Fig. $365, A$, I I and I2). These serve as association neurones between the visual cells and may be divided into rod association neurones and cone association neurones. The cone association neurones are the smaller and more superficial, and both dendrites and axones end in the outer molecular layer around the terminal expansions of the cone visual cells (Fig. $365, A$, I I). The rod association neurones are larger, more deeply seated, and behave in a similar manner toward the rod visual cells (Fig. $365, A$, I 2$)$. Some of these cells send processes to the inner molecular layer.

Neurone System No. II.This has been already partly described in connection with the axone terminations of neurone system No. I. The cell bodies of the second neurone system (Fig. $365, A, 8,9)$ are in the layer of nerve cells and are, as above noted, associated either directly or by means of their dendrites with the axones of the

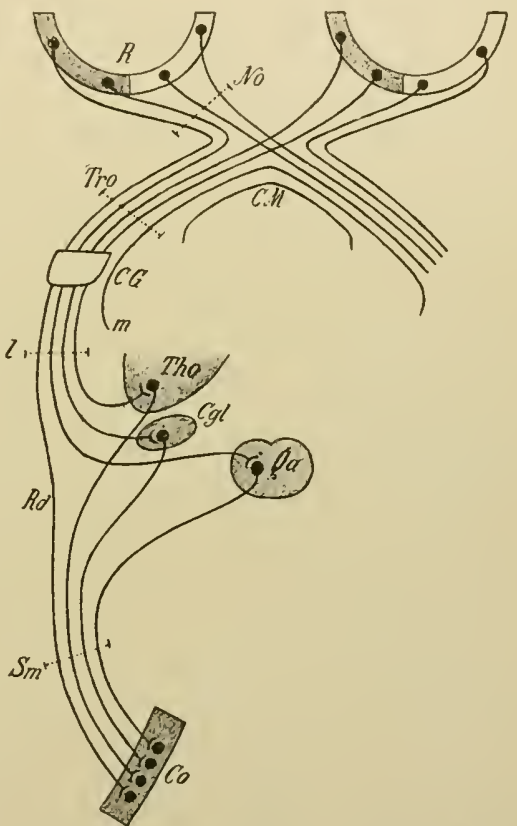

Fic. 368.-Diagram showing Main Relations of Optic Tract. (Testut.) $R$, Retina; $N o$, optic nerve; $C M$, optic decussation or chiasma; Tro, optic tract; Tho, thalamus; $\mathrm{Cgl}$, lateral geniculate body; $Q a$, anterior corpus quadrigeminum; $R d$, fibre of optic tract passing directly to cortex; Sm, third neurone system of optic tract (excepting $R d$ ) connecting thalamus, lateral geniculate body, and anterior corpus quadrigemnium with the cortex, Co. first neurone system. Their axones pass into the layer of nerve fibres and ultimately become fibres of the optic nerve (Fig. $365, \Lambda$, Io).

The optic nerves (Fig. $368, \mathrm{No}$ ) unite at the base of the brain to form the optic decussation or chiasmu (Fig. 368, CM). Here the axones from the mesial part of the retina cross to the optic tract of the opposite side, while those from the lateral part of the retina remain in the optic tract of the same side. The axones of the optic tract 


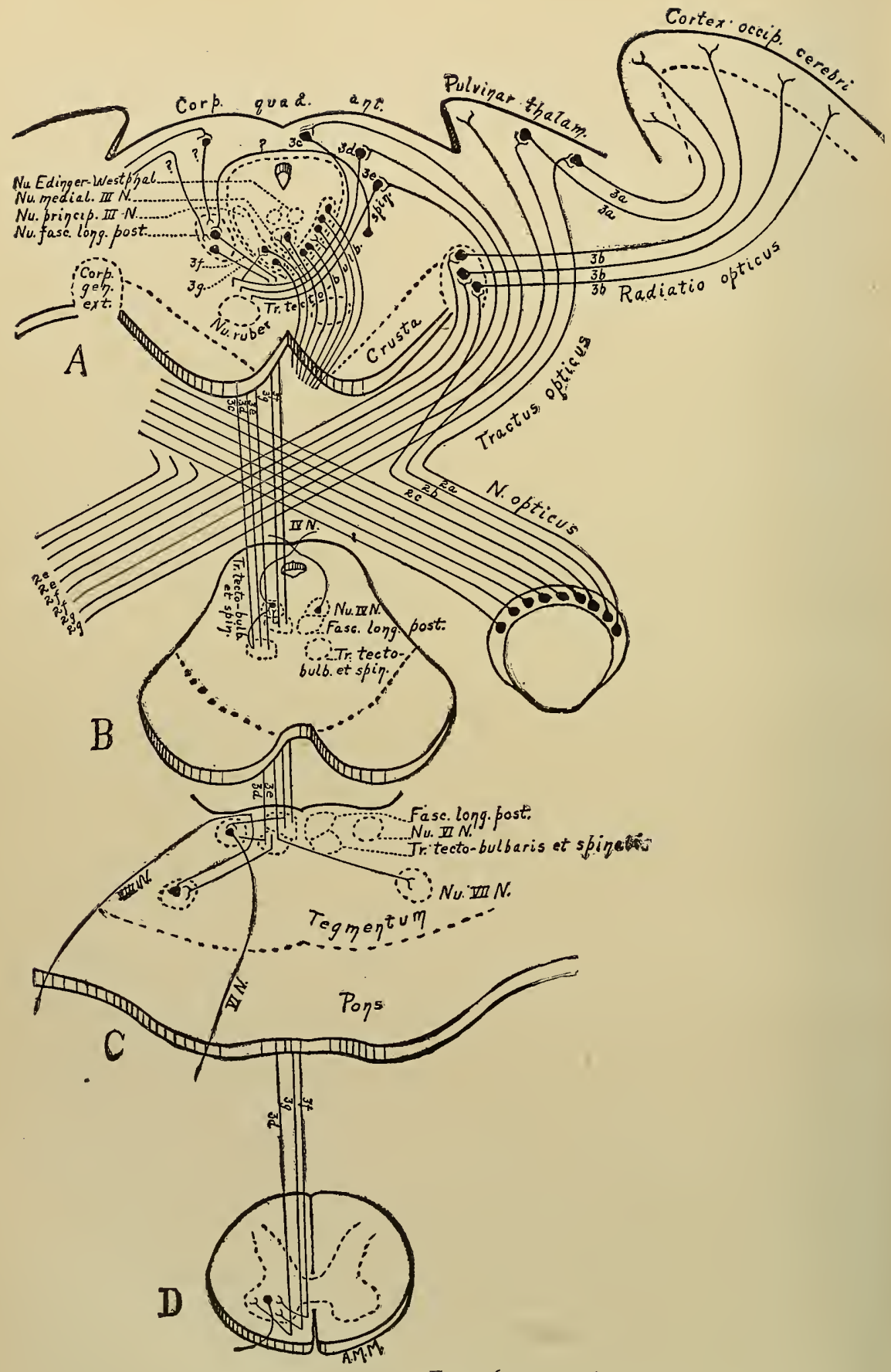

FIG. 369 . 


\section{EXPLANATION OF FIG. 369.}

FIG. 369.-Diagram of the Optic Nerve (II) and some of its Principal Connections. $A$, Level of nerves II and III; $B$, level of nerve IV; $C$, level of nerves VI and VII; $D$, spinal cord. The rods and cones (sensory cells) and the bipolar cells (=Neurone No. I) of the retina are not indicated.

Neurone No. 2.-2 a, Axones of ganglion cells in temporal part of retina pass to pulvinar of thalamus of same side; $2 b$, axones of ganglion cells in retina pass to anterior corpus quadrigeminum of same side; $2 c$, axones of ganglion cells in retina pass to external geniculate body (Corp. gen. ext.) of same side; $2 e$, axones of ganglion cells in nasal side of retina cross in optic chiasma and pass to external geniculate body of opposite side; $2 f$, axones of ganglion cells in nasal side of retina cross in optic chiasma and pass to anterior corpus quadrigeminum of opposite side; $2 g$, axones of ganglion cells in nasal side of retina cross in optic chiasma and pass to pulvinar of thalamus of opposite side.

Neurone No. 3.-3a, Axones of cells in pulvinar to cortex of occipital lobe of cerebrum (this connection is disputed); $3 b$, axones of cells in external geniculate body to cortex of occipital lobe of cerebrum; $3 a$ and $3 b$ constitute the primary optic radiation; $3 c, 3 d$ and $3 c$, axones of cells in middle layer of tectum (roof) of anterior corpus quadrigeminum decussate ventral to posterior longitudinal fasciculus (dorsal tegmental decussation or decussation of Meynert) and form the tractus tecto-bulbarlis et spinalis (Tr. tecto-bulb. et spin.) to bulb (medulla) and anterior column of cord, innervating by collaterals and terminals, directly or indirectly, chiefly the nuclei of III, IV, VI, and VII cranial nerves and motor nuclei of spinal nerves. $3 f$ and $3 g$ (possibly another neurone intercalated between these and optic terminals), axones of cells in nucleus of posterior longitudinal fasciculus (Nu. fasc. long. post.) (Nucleus of Darkschewitsch) form part of posterior longitudinal fasciculus and descend on same side to anterior column of cord next to anterior median fissure, innervating nuclei of III, IV, and VI cranial nerves and motor nuclei of spinal nerves.

Neurone No. 4.-Axones of cells in above-mentioned motor nuclei. Axones from cells in median nucleus of III nerve ( $\mathrm{Nu}$. med. III N. ), and possibly in Edinger-Westphal nucleus, probably innervate the intrinsic muscles of eyeball (ciliary and pupillary reflex path).

It is evident from the diagram that the cerebral pathway of the optic nerve is via the external geniculate body (and pulvinar of thalamus), and the reflex pathway in via the anterior colliculus (anterior corpus quadrigeminum). 
(Fig. 368, Tro) terminate in the thalamus, in the lateral geniculate body, and in the anterior corpus quadrigeminum (Fig. 368).

Neurone System No. III.-The neurones of this system have their cell bodies in the thalamus, lateral geniculate body, and anterior corpus quadrigeminum (Fig. 368). The axones of the two former terminate in the cortical visual centers in the occipital lobe (Fig. 368, Co). The axones of the latter form descending reflex paths (see Anterior Corpora Quadrigemina, p. 522).

Neurone system No. I is the analog of the primary afferent neu-

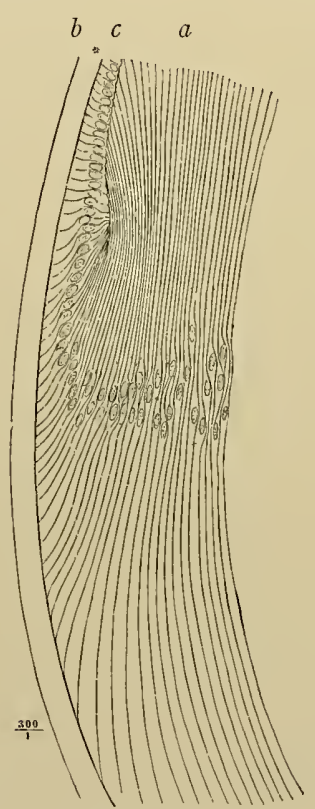

Fig. 370 . rones (the cerebro-spinal ganglion cells). Neurone system No. II is the analog of the secondary afferent neurone system which receives the afferent root fibres and "originates the secondary decussating tracts

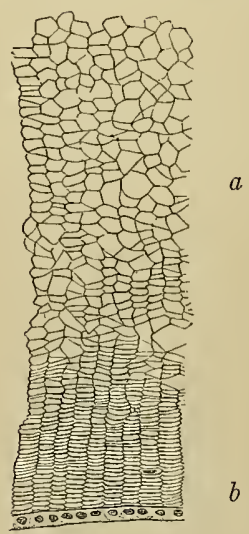

Fig. 37 I.

FIG. 370.-From Longitudinal Section through Margin of Crystalline Lens, showing longitudinal sections of lens fibres and transition from epithelium of capsule to lens fibres. (Merkel-Henle.) $a$, Lens fibres; $b$, capsule; $c$, epithelium.

Fig. 37x.-From Cross Section of Crystalline Lens, showing transverse sections of lens fibres and surface epithelium. (Merkel-Henle.) $a$, Lens fibres; $b$, epithelium.

to the thalamus, such as the medial and lateral fillets. Neurone system No. III is the tertiary afferent neurone system or thalamo-cortical system. It will thus be seen that the optic chiasma is the analog of the decussations of the medial and lateral fillets. (See also pp. 475,476 A.)

The Lens.-The lens is composed of lens fibres which are laid down in layers (Fig. 370, a). The lens fibre is a long hexagonal, flattened prism with serrated edges. Most of the lens fibres are nucle- 
ated, the nucleus lying at about the centre of the fibre near the axis of the lens. The most central of the lens fibres are usually nonnucleated. The fibres extend meridionally from before backward through the entire thickness of the lens. They are united by a small amount of cement substance. The lens is surrounded by the lens capsule (Fig. 370, b), a clear homogeneous membrane which is about I $2 \mu$ thick over the anterior surface of the lens, about half as thick over the posterior surface. Between the capsule and the anterior and lateral surfaces of the lens is a single layer of cuboidal epithelial cells (Fig. 370, c), the lens epithclium. At tached to the capsule of the lens anteriorly and posteriorly are membrane-like structures which constitute the suspensory ligament of the lens. These pass outward and unite to form a delicate membrane, the zomula ciliaris or zonule of Zinn (Fig. 363). This bridges over the inequalities of the ciliary processes and, continuing as the hyaloid membrane, forms a lining for the vitreous cavity of the eye. The triangular space between the two layers of the suspensory ligament and the lens is known as the canal of Petit.

The vitreous body is a semifluid substance containing fibres which run in all directions and a small number of connective-tissue cells and leucocytes. Traversing the vitreous in an antero-posterior direction is the so-called hyaloid or Cloquet's canal, the remains of the embryonic hyaloid artery (page 570).

Blood-vessels.-The blood-vessels of the eyeball are divisible into two groups, one group being branches of the central artery of the retina, the other being branches of the ciliary artery.

'The central artery of the retina enters the eyeball through the centre of the optic nerve. Within the eyeball it divides into two branches, a superior and an inferior. These pass anteriorly in the nerve-fibre layer, giving off branches, which in turn give rise to capillaries which supply the retina, passing outward as far as the neuroepithelial layer and anteriorly as far as the ora serrata. The smaller branches of the retinal arteries do not anastomose. In the embryo a third vessel exists, the hyaloid artery. This is a branch of the central retinal artery and traverses the vitreous to the posterior surface of the lens, supplying these structures. The hyaloid canal, or canal of Cloquet, of the adult vitreous, represents the remains of the degenerate hyaloid artery (page 570 ). The veins of the retina accompany the arteries.

The ciliary arteries are divisible into long ciliary arteries, short 
ciliary arteries, and anterior ciliary arteries. The long ciliary arteries are two in number and pass one on each side between the chorioid and sclera to the ciliary body, where each divides into two branches, which diverge and run along the ciliary margin of the iris. Here the anastomosis of the two long ciliary arteries forms the greater arierial circle of the iris. This gives rise to small branches which pass inward supplying the surrounding tissues and unite near the margin of the pupil to form the lesser arterial circle of the iris. The branches of the short ciliary arteries pierce the sclera near the optic nerve entrance, supply the posterior part of the sclera, and terminate in the chorio-capillaris of the chorioid. The anterior ciliary arteries enter the sclera near the corneal margin and communicate with the chorio-capillaris and with the greater arterial circle of the iris. The anterior ciliary arteries also supply the ciliary and recti muscles and partly supply the sclera and conjunctiva. Small veins accompany the ciliary arteries; the larger veins of this area are peculiar, however, in that they do not accompany the arteries, but as venæ vorticosæ converge toward four centres, one in each quadrant of the eyeball. At the sclero-corneal junction is a venous channel, the canal of Schlemm, which completely encircles the cornea (Fig. ${ }_{3} 6_{3}$ ).

Lymphatics.-The eyeball has no distinct lymph-vessel system. The lymph, however, follows certain definite directions which have been designated by Schwalbe "lymph paths." He divides them into anterior lymph paths and posterior lymph paths.' The anterior lymph paths comprise $(a)$ the anterior chamber which communicates by means of a narrow cleft between iris and lens with the posterior chamber; $(b)$ the posterior chamber; $(c)$ the lymph canaliculi of the sclera and cornea and the canal of Petit. The posterior lymph paths include (a) the hyaloid canal (see above); (b) the subdural and intra-pial spaces, including the capsule of Tenon; $(c)$ the perichorioidal space, and $(d)$ the perivascular and pericellular lymph spaces of the retina.

Nerves.-The nerves which supply the eyeball pass through the sclera with the optic nerve and around the eyeball in the suprachorioid layer. From these nerves, branches are given off as follows:

(I) To the chorioid, where they are intermingled with ganglion cells.

(2) To the ciliary body, where they are mingled with ganglion cells to form the ciliary plexus. The latter gives off branches to the ciliary body itself, to the iris, and to the cornea. Those to the 
cornea first form a plexus in the sclera - the plexus annularis - which encircles the cornea. From this, branches pierce the substantia propria of the cornea, where they form four corneal plexuses, one in the posterior part of the substantia propria, a second just beneath the anterior elastic membrane, a third sub-epithelial, and a fourth intra-epithelial. The fibres of the last named are extremely delicate and terminate freely between the epithelial cells. Krause describes end-bulbs as occurring in the substantia propria near the margin of the sclera, while according to Dogiel some of the fibres are connected with end-plates.

\section{The Lacrymal Apparatus}

The lacrymal apparatus of each eye consists of the gland, its excretcry ducts, the lacrymal canal, the lacrymal sac, and the nasal duct.

The lacrymal gland is a compound tubular gland consisting of two main lobes. Its structure corresponds in general to that of a serous gland. The excretory ducts are lined with a two-layered columnar epithelium which becomes simple columnar in the smaller ducts. The alveoli are lined with irregularly cuboidal serous cells, which rest upen a basement membrane beneath which is a richly elastic interstitial tissue.

The lacrymal canals have a stratified squamous epithelial lining. This rests upon a basement membrane beneath which is the stroma containing many elastic fibres. External to the connective tissue are some longitudinal muscle fibres.

The lacrymal sac is lined with a two-layered stratified or pseudostratified columnar epithelium resting upon a basement membrane. The stroma contains much diffuse lymphatic tissue.

The nasal duct has walls similar in structure to those of the lacrymal sac. In the case of both sac and duct the walls abut against periosteum, a dense vascular plexus being interposed.

The blood-vessels, lymphatics, and nerves of the lacrymal gland have a distribution similar to those of other serous glands.

\section{The Eyelid}

The eyelid consists of an outer skin layer, an inner conjunctival layer, and a middle connective-tissue layer.

The epidermis is thin and the papilla of the derma are low. Small sebaceous glands, sweat glands, and fine hairs are present. 
The conjunctiva (Fig. 372, d) is a mucous membrane consisting of a lining epithelium and a stroma. The epithelium is stratified columnar consisting of two or three layers of cells. Among these

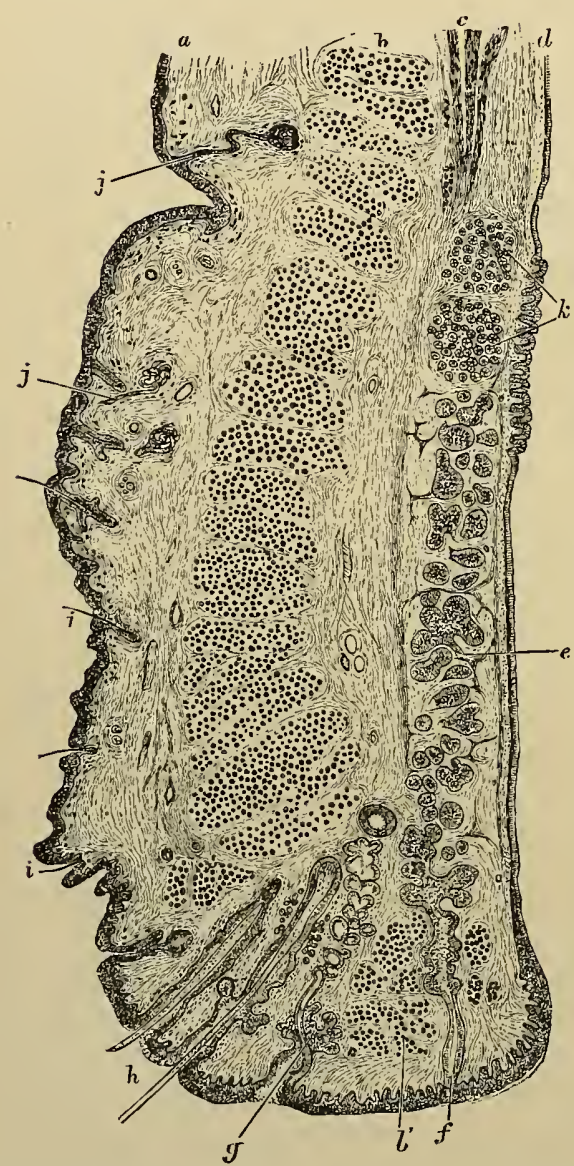

FIG. 372.--Vertical Section through Upper Eyelid. (Waldeyer.) $a$, Skin; $b$, orbicularis muscle; $b^{\prime}$, ciliary bundle of muscle; $c$, involuntary muscle of eyelid; $d$, conjunctiva; $e$, tarsus contaning Meibomian glands; $f$, duct of Meibomian gland; $g$, sebaceous gland with duct lying near eyelashes; $h$, eyelashes; $i$, small hairs in outer skin; $j$, sweat glands; $k$, posterior tarsal glands.

height of the lid. It contains the tarsal or Meibomian glands (Fig. $372, e)$. These are from thirty to forty in number, each consisting of a long duct which opens externally on the margin of the lid behind the lashes (Fig. 372,f), and internally into a number of branched let cells. Although not always upon the surface, they are believed to be mucous cells, probably analogous to the so-called Leydig's cells found in the larvæ of amphibians and fishes. Diffuse lymphoid tissue is regularly present in the stroma, while lymph nodules are of rare occurrence. Small glands, similar to the lacrymal glands in structure, are usually present (Fig. 372, $k$ ).

At the margin of the eyelid where skin joins mucous membrane are several rows of large hairs, the eyelashes (Fig. $372, h)$. Connected with their follicles are the usual sebaceous glands (Fig. 372, g) and the glands of Mall, the latter probably representing modified sweat glands.

The middle layer contains the tarsus (Fig. $3 \uparrow^{2}, e$ ) and the muscular structure of the eyelid (Fig. 372, b). The tarsus is a plate of dense fibrcus tissue which lies just beneath the conjunctiva and extends about two-thirds the cells are cells resembling gob- 
tubules. The duct is lined with stratified squamous epithelium. The tubules resemble those of the sebaceous glands. Between the tarsus and the skin are the muscular structures of the eyelid in which both smooth and striated muscle are found.

Blood-vessels. - Two main arteries pass to the eyelid, one at each angle, and unite to form an arch, the tarsal arch, along the margin of the lid. A second arch, the external tarsal arch, is formed along the upper margin of the tarsus. From these arches are given off capillary networks which supply the structures of the lid.

Lymphatics.-These form two anastomosing plexuses, one anterior, the other posterior to the tarsus.

Nerves.-The nerves form plexuses in the substance of the lid. From these, terminal fibrils pass to the various structures of the lid. Many of the fibres end freely in fine networks around the tarsal glands, upon the blood-vessels, and in the epithelium of the conjunctiva. Other fibres terminate in end-bulbs which are especially numerous at the margin of the lid.

\section{Development of the Eye}

The eyes begin their development very early in embryonic life. As optic depressions they are visible even before the closure of the medullary groove.

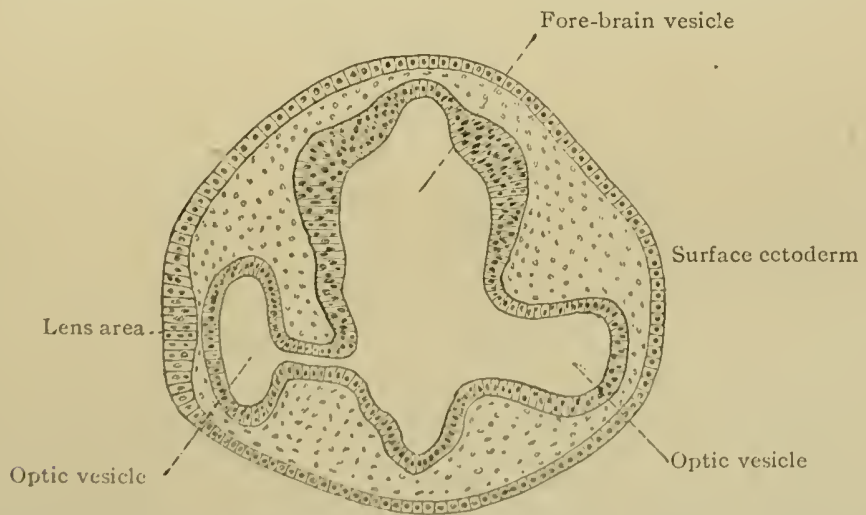

Fro. 373.- Section through hear of chick of two days' incubation. (1)uval.) The formation of the optic vesicle and stalk appears to be somewhat more advanced on the left than on the right.

As a result of the closure of this groove, the optic depressions are transformed into the oplic resicles. The connection between vesirle and brain now becomes narrowed so that the two are connecterl only by the thin optic stalk. The surface of the optic vesicle becomes firmly adherent to the epiclermis and as a 
result of proliferation of ectodermic cells at this point is pushed inward (invaginated), forming the optic cup. The invagination of the optic vesicle extends also to the stalk, the sulcus in the latter being known as the chorioid

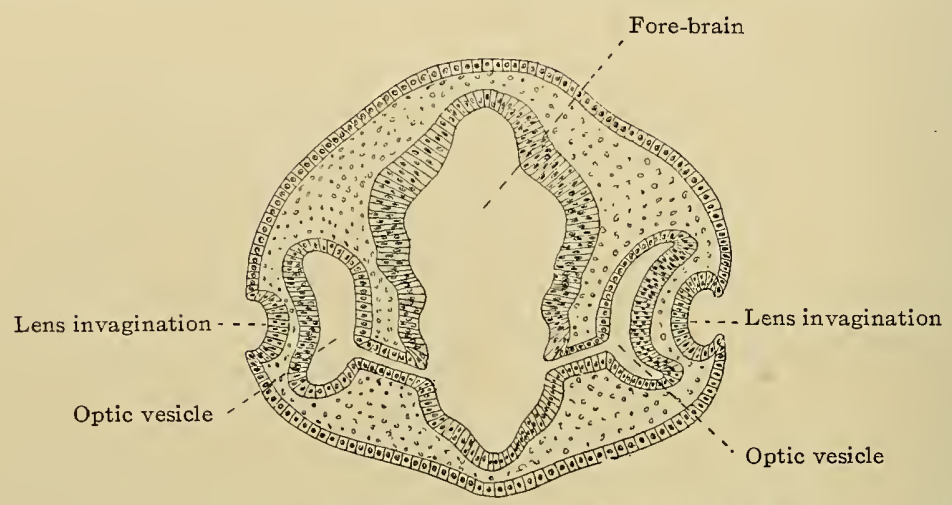

FIG. 374.-Section through head of chick of three days' incubation. (Duval.)

fissure. The latter serves for the introduction of mesenchyme, and the development of the hyaloid retinal artery. Three distinct parts may now be distinguished in the developing eye, which at this stage is known as the secondary

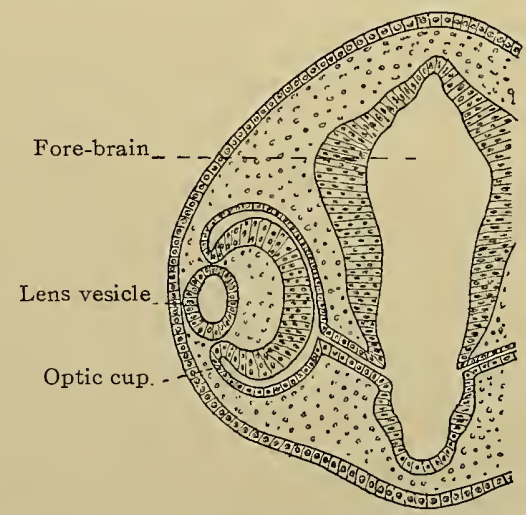

FIG. 375.- Showing somewhat later stage in development of optic cup and lens than is shown in Fig. 374. (Duval.)

optic vesicle: (a) The proliferating epidermis which is to form the lens; (b) the more superficial of the invaginated layers which is to become the retina; and (c) the surrounding mesodermic tissue from which the outer coats of the eye are to develop.

\section{TECHNIC}

(I) For the study of the general structures of the eyeball the eye of some large animal, such as an ox, is most suitable. Fix the eye for about a week in ten- 
per-cent. formalin. Then wash in water and bisect the eye in such a manner that the knife passes through the optic-nerve entrance and the centre of the cornea. The half eye should now be placed in a dish of water and the structures shown in Fig. $35^{\mathrm{S}}$ identified with the naked eye or dissecting lens. On removing the vitreous and retina, the pigmented epithelium of the latter usually remains attached to the chorioid from which it may be scraped and examined in water or mounted in glycerin. In removing the lens note the lens capsule and the suspensory ligament. The lens may be picked to pieces with the forceps, and a small piece, after further teasing with needles, examined in water or mounted in glycerin or eosin-glycerin. The retinal surface of the chorioid shows the iridescent membrane of Bruch. By placing a piece of the chorioid, membrane-ofBruch-side down, over the tip of the finger and gently scraping with a knife in

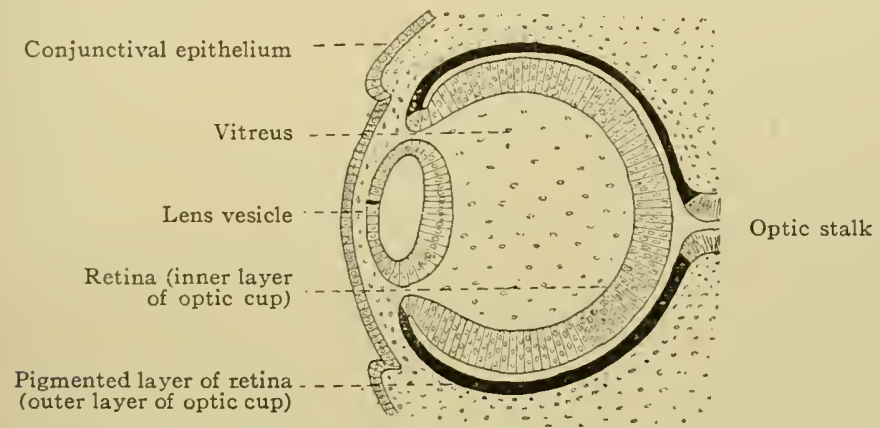

FIG. 376.-Diagram of developing lens and optic cup. (Duval.) The cells of the inner wall of the lens vesicle have begun to elongate to form lens fibres. The epithelium over the lens is the anlage of the corneal epithelium. The mesodermal tissue between the latter and the anterior wall of the lens vesicle is the anlage of the substantia propria corneæ.

the direction of the larger vessels, the latter may be distinctly seen. By now staining the piece lightly with hæmatoxylin and strongly with eosin, clearing in oil or origanum and mounting in balsam, the choriocapillaris and the layer of straight vessels become distinctly visible with the low-power lens. In removing the chorioid note the close attachment of the latter to the sclera, this being due to the intimate association of the fibres of the lamina suprachorioidea and of the lamina fusca. If the brown shreds attached to the inner side of the sclera be examined, the pigmented connective-tissue cells of the sclera can be seen.

(2) For the study of the finer structure of the coats of the eyc, a human eye if it is possible to obtain one, if not, an eye from one of the lower animals, should be fixed in formalin-Müller's fluid (technic 5, p. 7) and hardened in alcohol. ( $\Lambda$ few drops of strong formalin injected by means of a hypodermic needle directly into the vitreous often improves the fixation.) The eye should next be divided into quadrants by first carrying the knife through the middle of the cornea and of the optic-nerve entrance, and then dividing each half into an anterior and a postcrior half. Block in celloidin, cut the following sections, and stain with hxmatoxylin-cosin (tcchnic I, p. 20).

(a) Section through the sclero-corneal junction, including the ora serrata, 
ciliary body, iris, and lens. Before attempting to cut this section almost all of the lens should be picked out of the block, leaving only a thin anterior and lateral rim attached to the capsule and suspensory ligament. The block should then be so clamped to the microtome that the lens is the last part of the block to be cut. The above precautions are necessary on account of the density of the lens, making it difficult to cut.

(b) Section through the postero-lateral portion of the eyeball to show structure of sclera, chorioid, and retina. This section should be as thin as possible and perpendicular to the surface.

(c) Section through the entrance of the optic nerve. Hæmatoxylin-picroacid-fuchsin also makes a good stain for this section. It is instructive in cutting the eye to cut a small segment from the optic nerve and to block it with the opticnerve entrance material in such a manner that it is cut transversely. In this way both longitudinal and transverse sections of the optic nerve appear in the same section.

(d) For the study of the neurone relations of the retina, material must be treated by one of the Golgi methods (page 35).

(4) The connective-tissue cells and cell spaces of the cornea may be demonstrated by means of technics 8 and 9, page 97 .

(5) The different parts of the lacrymal apparatus may be studied by fixing material in formalin-Müller's fluid and staining sections in hæmatoxylin-eosin.

(6) The Eyelid. An upper eyelid, human if possible, should be carefully pinned out on cork, skin side down, and fixed in formalin-Müller's fluid. Vertical sections should be stained with hæmatoxylin-eosin or with hæmatoxylin-picroacid-fuchsin.

\section{The Organ of Hearing}

The organ of hearing comprises the external ear, the middle ear, and the internal ear.

\section{The External Ear}

The external ear consists of the pinna or auricle, the external auditory canal, and the outer surface of the tympanic membrane.

The pinna consists of a framework of elastic cartilage embedded in connective tissue and covered by skin. The latter is thin and contains hairs, sebaceous glands, and sweat glands.

The external auditory canal consists of an outer cartilaginous portion and an inner bony portion. Both are lined with skin continuous with that of the surface of the pinna. In the cartilaginous portion of the canal the skin is thick and the papillæ are small. Hair, sebaceous glands, and large coiled glands (ceruminous glands) are present. The last named resemble the glands of Mall (page 568) and are probably modified sweat glands. Their cells contain numerous fat droplets and 
pigment granules. They have long narrow ducts lined with a twolayered epithelium. In children these ducts open into the hair follicles; in the adult they open on the surface near the hair follicles. The secretion of these glands plus desquamated epithelium constitutes the ear wax. In the bony portion of the canal the skin is thin, free from glands and hair, and firmly adherent to the periosteum.

The tympanic membrane (ear drum) separates the external ear from the middle ear. It consists of three layers: a middle layer or substantia propria, an outer layer continuous with the skin of the external ear, and an inner layer continuous with the mucous membrane of the middle ear.

The substantia propria consists of closely woven connective-tissue fibres, the outer fibres having a radial direction from the head of the malleus, the inner fibres having a concentric arrangement and being best developed near the periphery.

The outer layer of the tympanic membrane is skin, consisting of epidermis and of a thin non-papillated corium, excepting over the manubrium of the malleus, where the skin is thicker and papillated.

The inner layer is mucous membrane and consists of a stroma of fibro-elastic tissue covered with a single layer of low epithelial cells.

Blood-vessels.-Blood is supplied to the tympanic membrane by two sets of vessels, an external set derived from the vessels of the external auditory meatus and an internal set from the vessels of the middle ear. These give rise to capillary networks in the skin and mucous membrane, respectively, and anastomose by means of perforating branches at the periphery of the membrane. From the capillaries the blood passes into two sets of small veins, one extending around the periphery of the membrane, the other following the handle of the malleus.

Lymphatics. - These follow in general the course of the bloodvessels. They are most numerous in the outer layer.

Nerves.- The larger nerves run in the substantia propria. From these, branches pass to the skin and mucous membrane, beneath the surfaces of which they form plexuses of fine fibres.

\section{THE MHDLE İAR}

The middle ear or tympanum is a small chamber separated from the external car by the tympanic membrane and communicating with the pharynx by means of the Eustachian tube. Its walls are formed 
by the surrounding bony structures covered by periosteum. It is lined with mucous membrane and contains the ear ossicles and their ligamentous and muscular attachments. The epithelium is of the simple low cuboidal type. In places it may be ciliated and not infrequently assumes a pseudostratified character with two layers of nuclei. Beneath the epithelium is a thin stroma which contains some diffuse lymphoid tissue and blends with the dense underlying periosteum. Small tubular glands are usually present, especially near the opening of the Eustachian tube.

The fenestra rotunda is corered by the secondary tympanic membrane. This consists of a central lamina of connective tissue covered on its tympanic side by part of the mucous membrane of that chamber, on the opposite side by a single layer of endothelium.

The ossicles are composed of bone tissue arranged in the usual systems of lamellæ. The stapes alone contains a marrow cavity. Over their articular surfaces the ossicles are corered by hyaline cartilage.

The Eustachian Tube.-This is a partly bony, partly cartilaginous canal lined with mucous membrane. The epithelium of the latter is of the stratified columnar ciliated variety consisting of two layers of cells. In the bony portion of the tube the stroma is small in amount and intimately connected with the periosteum. In the cartilaginous portion the stroma is thicker and contains, especially near the pharyngeal opening, lymphoid tissue and simple tubular mucous glands.

\section{The Internal Ear}

The internal ear consists of a complex series of connected bony walled chambers and passages containing a similar-shaped series of membranous sacs and tubules. These are known, respectively, as the osseous labyrinth and the membranous labyrinth. Between the two is a lymph space, which contains the so-called perilymph, while within the membranous labyrinth is a similar fluid, the endolymph.

The bony labyrinth consists of a central chamber, the iestibule, from which are given off the three semicircular canals and the cochlea. The vestibule is separated from the middle ear by a plate of bone in which are two openings, the fenestra ovalis and the fenestra rotunda. Just after leaving the vestibule each canal presents a dilatation, the ampulla. As each canal has a return opening into the vestibule and as the anterior and posterior canals have a common return opening 
(the canalis communis), there are five openings from the restibule into the semicircular canals (Fig. 377). The bony labyrinth is lined with periosteum, covered by a single layer of endothelial cells.

The Vestibule and the Semicircular Canals.-In the vestibule the membranous labyrinth is subdivided into two chambers, the saccule and the utricle, which are connected by the utriculo-saccular duct. From the latter is given off the endolymphatic duct which communicates, through the aqueduct of the vestibule, with a subdural lymph space, the endolymphatic sac. The saccule opens by means of the ductus reuniens into the cochlea, while the utricle opens into the ampullx of the semicircular canals. The saccule and utricle only partly fill the vestibule, the remaining space, crossed by fibrous bands and lined with endothelium, constituting the perilymphatic space.

SACCULE AND UTRICLE.--The walls of the saccule and of the utricle consist of fine fibroelastic tissue supporting a thin

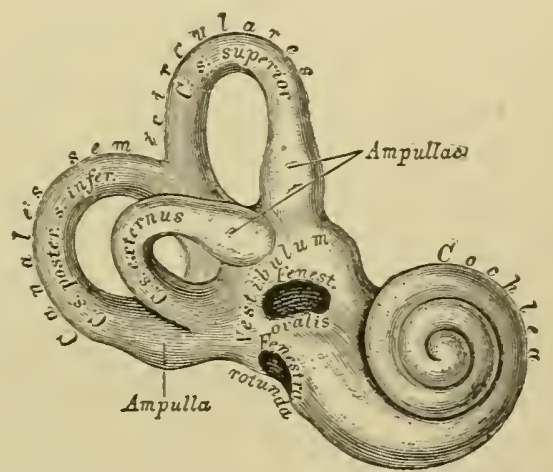

FIG. 377.-The Bony Labyrinth. $X_{3}$. (Heitzmann.) basement membrane, upon

which rests a single layer of low epithelial cells. In the wall of each chamber is an area of special nerve distribution, the macula acustica. .Here the epithelium changes to high columnar and consists of two kinds of cells, sustentacular and neuro-cpithelial. The sustentacular cells are long, irregular, nucleated cylinders, narrow in the middle, widened at each end, the outer end being frequently split and resting upon the basement membrane. The neuro-epithelial cells or "hair cells" are short cylinders which extend only about halfway through the epithelium. The basal end of the cell is the larger and contains the oval nucleus. The surface of the cell is provided with a cuticular margin from which project several long hair-like processes, the auditory hairs. Small crystats of calcium carbonate are found on the surfaces of the hair cells. These are known as otoliths and are embedded in a soft substance, the otolithic membrane. The hair cells are the neuro-epithelial end-organs of the vestibular division of the auditory nerve and are, therefore, closely associated with the nerve frbres. The latter on piercing the basement membrane 
lose their medullary sheaths and split up into several small branches, which form a horizontal plexus between the basement membrane and the bases of the hair cells. From this plexus are given off fibrils which end freely between the hair cells.

Semictrcular Canals.- The walls of the semicircular canals are similar in structure to the walls of the saccule and utricle; they also bear a similar relation to the walls of the bony canal. Along the concavity of each canal the epithelium is somewhat higher, forming the

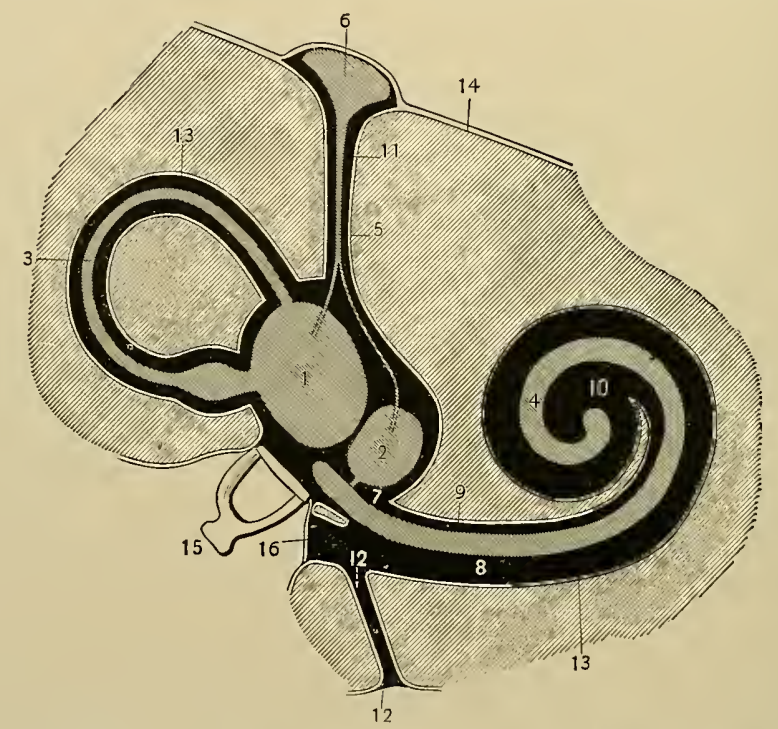

FIG. 378.-Diagram of the Perilymphatic and Endolymphatic Spaces of the Inner Ear. (Testut.) Endolymphatic spaces in gray; perilymphatic spaces in black. I, Utricle; 2, saccule; 3, semicircular canals; 4, cochlear canal; 5, endolymphatic duct; 6, subdural endolymphatic sac; 7 , canalis reuniens; 8 , scala tympani; 9, scala vestibuli; ro, their union at the helicotrema; $1 x$, aqueduct of the vestibule; 12 , aqueduct of the cochlea; $I_{3}$, periosteum; I4, dura mater; $x_{5}$, stapes in fenestra ovalis; 16 , fenestra rotunda and secondary tympanic membrane.

raphé. In each ampulla is a crista acustica, the structure of which is similar to that of the maculæ of the saccule and utricle. With the adjoining high columnar cells, this forms the so-called semilunar fold. As in the case of the maculæ the hair cells have otoliths upon their surfaces, the otolithic membrane here forming a sort of dome over the hair cells known as the cupula.

The Cochlea.-The bony cochlea consists of a conical axis, the modiolus, around which winds a spiral bony canal. This canal in man makes about two and one-half turns, ending at the rounded tip of the cochlea or cupola. Projecting from the modiolus partly across 
the bony canal of the cochlea is a plate of bone, the bony spiral lamina (Fig. $3 S \mathrm{I}, x$ ). This follows the spiral turns of the cochlea, ending at the cupola in a hook-shaped process, the hamulus. Along the outer

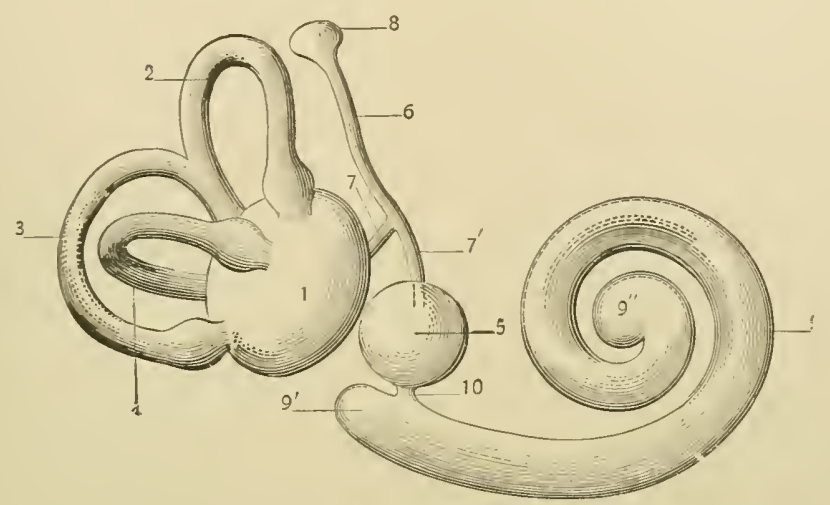

FIG. 379.-Diagram of the Right Membranous Labyrinth. (Testut.) I, Utricle; 2 superior semicircular canal; 3, posterior semicircular canal; 4, external semicircular canal; 5 , saccule; 6 , endolymphatic duct; 7 and $7^{\prime}$, canals connecting utricle and saccule respectively with the endolymphatic duct; 8 , endolymphatic sac; 9 , cochlear duct; $9^{\prime}$, its vestibular cul-de-sac; $9^{\prime \prime}$, its terminal cul-de-sac; 10 , canalis reuniens.

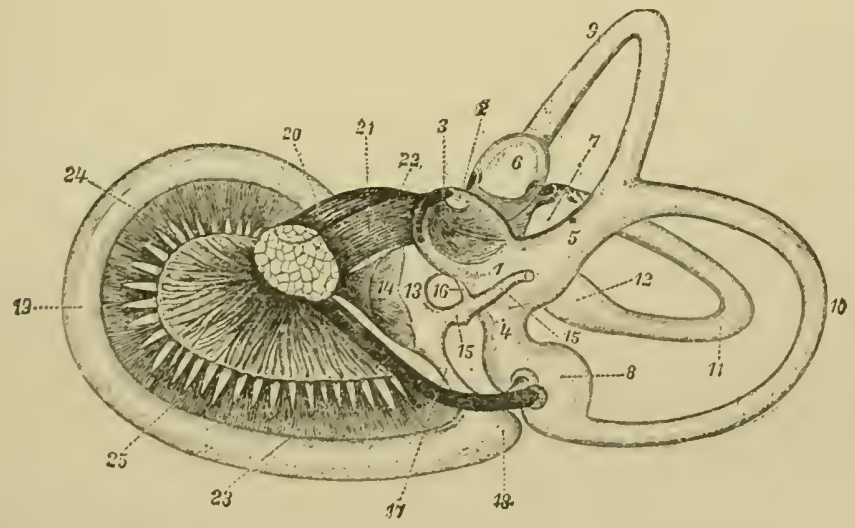

Fic. 380. - The Membranous Labyrinth from the Right Internal Ear of a Human Embryo at the Fifth Month; seen from the Medial Side. (After Retzius, from Barker.) I-5, Utriclc; 2, utricular recess; 3 , macula acustica of utricle; 4, postcrior sinus; 5 , superior sinus; $6,7,8$, superior, lateral, and posterior ampullæ; 9, 10, 11 , supcrior, posterior, and lateral semicircular canals; 12, widened mouth of lateral semicircular canal opening in to the utricle; I3, saccule; I4, macula acustica of the saccule; 15, cndolymphatic duct; 16 , utriculo-saccular duct; 17 , ductus reuniens; 18 , vestibular cul-de-sac of cochlear duct; 19 , cochlear duct; 20 , facial nerve; $21-24$, auditory nerve; 21 , its vestibular branch; 22, saccular branch; 23 , branch to inferior ampulla; 24 , cochlcatr branch; 25 , distrilution of cochlcar branch within the bony spiral lamina.

side of the canal, opposite the bony spiral lamina, is a projection of thickened periosteum, the spiral ligament (Fig. $38 \mathrm{I}, h$ ). A connective tissue membrane, the membranous spiral lamina (Fig. 38 I, s), 
crosses the space intervening between the spiral ligament and the bony spiral lamina, thus completely dividing the bony canal of the cochlea into two parts, an upper, scala vestibuli (Fig. $38 \mathrm{r}, l$ ) and a lower, scala tympani (Fig. $38 \mathrm{r}, k$ ). These are perilymphatic spaces, the scala vestibuli communicating with the perilymph space of the vestibule, the scala tympani communicating with the perivascular

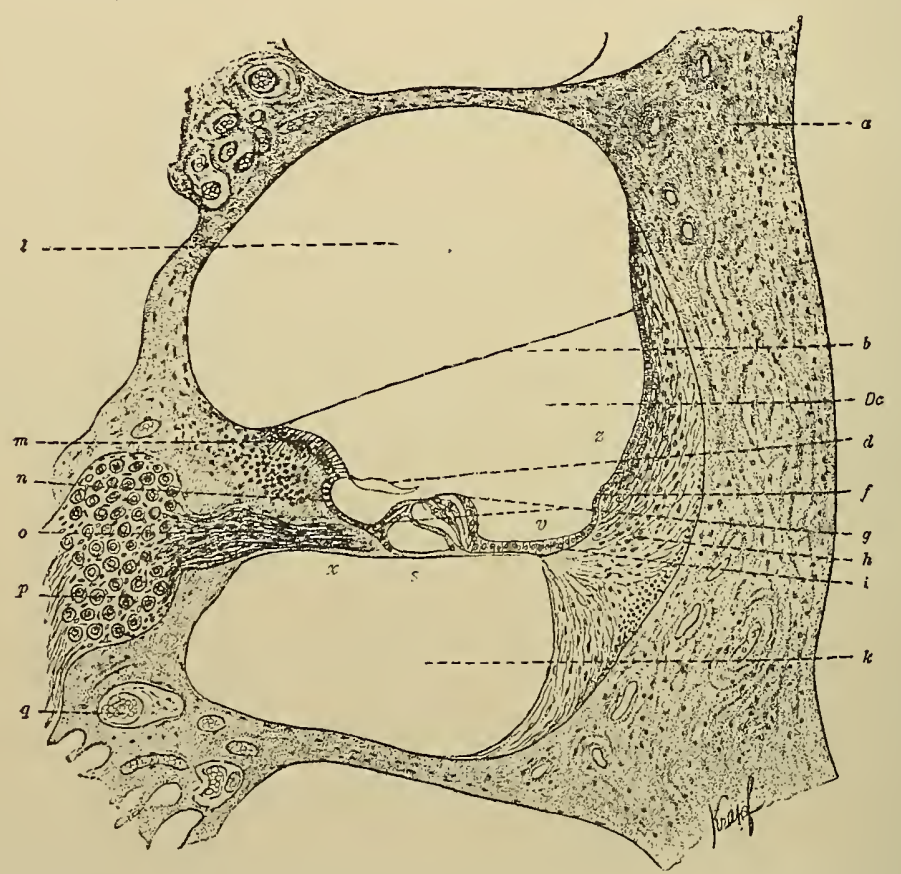

FIg. 381.--Section through a Single Turn of the Cochlea of a Guinea-pig. (Böhm and von Davidoff.) $a$, Bone of cochlea; $l$, scala vestibuli, $D c$, scala media or cochlear duct; $k$, scala tympani; $b$, membrane of Reissner; $d$,membrana tectoria or membrane of Corti; $f$, spiral prominence; $g$, organ of Corti; $h$. spiral ligament; $i$, basilar membrane (outer portion-zona pectinata-covered by cells of Claudius); $z$, stria vascularis; $v$, external spiral sulcus; $r$, crista basilaris; $s$, membranous spiral lamina; $x$, bony spiral lamina; $m$, spiral limbus; $n$, internal spiral sulcus; $o$, medullated peripheral processes (dendrites) of cells of spiral ganglion passing to the organ of Corti; $p$, spiral ganglion; $q$, blood-vessel.

lymph spaces of the veins of the cochlear duct. The scala vestibuli and the scala tympani communicate with each other in the cupola by means of a minute canal, the helicotrema.

The Cochlear Duct (Membranous Cochlea or Scala Media).This is a narrow, membranous tube lying near the middle of the bony cochlear canal and following its spiral turns from the vestibule, where it is connected with the saccule through the canalis reuniens, 
to its blind ending in the cupola. It is triangular in shape on transverse section, thus allowing a division of its walls into upper, outer, and lower (Fig. $38 \mathrm{r}, D c$ ).

The upper or vestibular wall is formed by the thin membrane of Reissner (Fig. ${ }_{3} S_{1}, b$ ) which separates the cochlear duct from the scala vestibuli. The membrane consists of a thin central lamina of connective tissue covered on its vestibular side by the vestibular endothelium, on its cochlear side by the epithelium of the cochlea.

The cuter wall of the cochlear duct is formed by the spiral ligament, which is a thickening of the periosteum. The outer part of the spiral ligament consists of dense fibrous tissue, its projecting part of more loosely arranged tissue. From it, two folds project slightly into the duct. One, the crista basilaris (Fig. $3 S_{\mathrm{I}}, r$ ), serves for the attachment of the membranous spiral lamina; the other, the spiral prominence (Fig. $38 \mathrm{r}, f$ ), contains several small veins. Between the two projections is a depression, the external spiral sulcus (Fig. $3 S_{\text {I }}, v$ ). That part of the spiral ligament between the spiral prominence and the attachment of Reissner's membrane is known as the siria vascularis (Fig. $38 \mathrm{r}, \approx$ ). It is lined with granular cuboidal epithelial cells, which, cwing to the absence of a basement membrane, are not sharply separated from the underlying connective tissue. For this reason the capillaries extend somewhat between the epithelial cells, giving the unusual appearance of a vascular epithelium.

The lower or tympanic wall of the cochlear duct has an extremely complex structure. Its base is formed by the already mentioned bony and membranous division-wall between the scala media and the scala tympani (bony spiral lamina and membranous spiral lamina).

The bony spiral lamina has been described (page 577 ).

The membranous spiral lamina consists of a substantia propria or basilar membrane, its tympanic covering, and its cochlear covering.

The basilar membrane (Fig. $3 \mathrm{SI}_{\mathrm{I}}$ ) is a connective-tissue membrane composed of fine straight fibres which extend from the bony spiral lamina to the spiral ligament. Among the fibres are a few connectivetissue cells. On either side of the fibre layer is a thin, apparently structureless membrane.

The tympanic covering of the basilar membrane consists of a thin layer of connective tissue - an extension cf the periosteum of the spiral lamina- covered over by a single layer of flat endothelial cells.

The cochleur covering of the basilar membrane is epithelial. Owing to the marked difference in the character of the epithelium, the basilar 
membrane is divided into an outer portion, the zona pectinata (Fig. $38 \mathrm{I}, i)$ and an inner portion, the zona tecta (Fig. $38 \mathrm{I}, s$ ). The epithelium of the former is of the ordinary columnar type; that of the latter is the highly differentiated neuro-epithelium of Corti's organ.

The Organ of Corti.-The spiral organ or the organ of Corti (Fig. $38 \mathrm{I}, g$, and Fig. 382 ) is a neuro-epithelial structure running the entire length of the cochlear canal with the exception of a short distance at either end. It rests upon the membranous portion of the spiral lamina, and consists of a complex arrangement of four different kinds

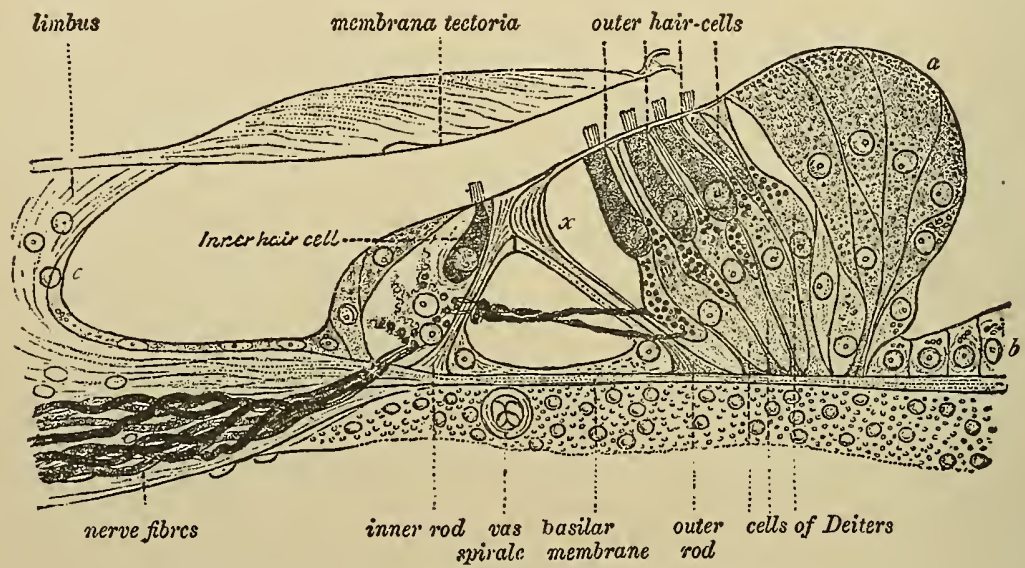

FIG. 382.--Semidiagrammatic Representation of the Organ of Corti and Adjacent Structures (Merkel-Henle.) $a$, Cells of Hensen; $b$, cells of Claudius; $c$, internal spiral sulcus; $x$, Nuel's space. The nerve fibres (dendrites of cells of the spiral ganglion) are seen passing to Corti's organ through openings (foramina nervosa) in the bony spiral lamina. The black dots represent longitudinally-running branches, one bundle lying to the inner side of the inner pillar, a second just to the outer side of the inner pillar within Corti's tunnel, the third beneath the outer hair cells.

of epithelial cells. These are known as: (x) pillar cells, (2) hair cells, (3) Deiter's cells, and (4) Hensen's cells (Fig. 352).

(1) The pillar cells are divided into outer pillar cells and inner pillar cells. They are sustentacular in character. Each cell consists of a broad curved protoplasmic base which contains the nucleus, and of a long-drawn-out shaft or pillar which probably represents a highly specialized cuticular formation. The end of the pillar away from the base is known as the head. The head of the outer pillar presents a convexity on its inner side, which fits into a corresponding concavity on the head of the inner pillar, the heads of opposite pillars thus "articulating" with each other. From their articulation the pillars diverge, so that their bases which rest upon the basilar membrane are 
widely separated. There are thus formed by the pillars a series of arches known as Corti's arches, enclosing a triangular canal, Corti's innnel. This canal is filled with a gelatinous substance and crossed by delicate nerve fibrils. As the outer pillar cells are the larger, they are fewer in number, the estimated number in the human cochlea being forty-five hundred of the outer cells and about six thousand of the inner cells.

(2) The hair cells or auditory cells lie on either side of the arches of Corti, and are thus divided into inner hair cells and outer hair cells. Both inner and outer hair cells are short, cylindrical elements which do not extend to the basilar membrane. Each cell ends below in a point, while from its free surface are given off a number of fine stiff hairs.

The inner hair cells lie in a single layer against the inner side of the inner pillar cells, one hair cell resting upon about every two pillars.

The outer hair cells lie in three or fcur layers to the outer side of the outer pillar cells, being separated from one another by sustentacular cells, the cells of Deiter, so that no two hair cells come in contact.

(3) Deiter's Cells (Fig. 382).-These like the pillar cells are sustentacular. Their bases rest upon the basilar membrane, where they form a continuous layer. Toward the surface they become separated from one another by the hair cells. The long slender portions of the Deiter's cells, which pass in between the hair cells, are known as phalangeal processes. Between the innermost of the outer hair cells and the outer pillar is a space known as Nuel's space (Fig. $382, x$ ).

(4) Hensen's Cells (Fig. $382, a$ ).-These are sustentacular cells, which form about eight rows to the outer side of the outermost Deiter's cells. These cells form the outer crest of Corti's organ and consequently have a somewhat radial disposition, their free surfaces being broad, their basal ends narrow. They decrease in height from within outward, and at the end of Ccrti's organ become continuous with the cells of Claudius (Fig. 382 , b), the name given to the cochlear epithelium covering the basal membrane to the outer side of Corti's organ.

'The phalangeal processes of the 1)eiter's cells are cemented together and to the superficial parts of the outer pillars in such a manner as to form a sort of cuticular membrane, the lamina reticularis, through which the heads of the outer hair cells project. This mem- 
brane also extends out as a cuticula over the cells of Hensen and of Claudius.

The Membrana Tectoria.-This is a peculiar membranous structure attached to a projection of the bony spiral lamina known as the spiral limbus (Fig. 382), the concavity beneath its attachment being the internal spiral sulcus (Fig. $382, c$ ). The membrane is non-nucleated and shows fine radial striations. It bridges over the internal spiral sulcus and ends in a thin margin, which rests upon Corti's organ just at the outer limit of the outer hair cells.

Blood-vessels. - The arteries consist of two small branches of the auditory-one to the bony labyrinth, the other to the membranous labyrinth. The latter divides into two branches - a vestibular and a cochlear. The vestibular artery accompanies the branches of the auditory nerve to the utricle, saccule, and semicircular canals. It supplies these parts, giving rise to a capillary network, which is coarse meshed except in the cristæ and maculæ, where the meshes are fine. The cochlear artery also starts out in company with the auditory nerve, but accompanies it only to the first turn of the cochlea. Here it enters the modiolus where it gives off several much coiled branches, the glomerular arteries of the cochlea. Branches from these pierce the vestibular part of the osseous spiral lamina and supply the various structures of the cochlear duct. The veins accompany the arteries, but reach the axis of the modiolus through foramina in the tympanic part of the bony spiral lamina.

Lymphatics. - The scala media contains endolymph and is in communication with the subdural lymph spaces by means of the endolymphatic duct, the endolymphatic sac, and minute lymph channels connecting the latter with the subdural spaces. The perilymph spaces - scala tympani and scala vestibuli-are connected with the pial lymph spaces by means of the perilymphatic duct. Lymph spaces also surround the vessels and nerves. These empty into the pial lymphatics.

Nerves. - The vestibular branch of the auditory nerve divides into branches which supply the saccule, utricle, and semicircular canals, where they end in the maculæ and cristæ as described on page 575. The ganglion of the vestibular branch is situated in the internal auditory meatus. The cochlear branch of the auditory nerve enters the axis of the modiolus, where it divides into a number of branches which pass up through its central axis. From these, 
numerous fibres radiate to the bony spiral laminx, in the bases of which they enter the spiral ganglia (Fig. $3^{8}$ I, p).

The cells of the spiral ganglia are peculiar, in that while of the same general type as the spinal ganglion cell they maintain their embryonic bipolar condition (see page 4Ig) throughout life. Their axones follow the already described course through the modiolus and thence through the internal auditory meatus to their terminal nuclei in the medulla (see page 493). Their dendrites become medullated like the dendrites of the spinal ganglion cells and pass outward in bundles in the bony spiral lamina (Fig. $38 \mathrm{I}, o$, and Fig. 382). From these are given off branches which enter the tympanic portion of the lamina, where they lose their medullary sheaths and pass through the foramina nervosa (minute canals in the tympanic part of the spiral lamina) to their terminations in the organ of Corti. In the latter the fibres run in three bundles parallel to Corti's tunnel. One bundle lies just inside the inner pillar beneath the inner row of hair cells (Fig. 382 ). A second bundle runs in the tunnel to the outer side of the inner pillar (Fig. 382). The third bundle crosses the tunnel (tunnel-fibres) and turns at right angles to run between the cells of Deiter beneath the outer hair cells (Fig. 382). From all of these bundles of fibres are given off delicate terminals which end on the hair cells. (For details of acoustic tract see pp. 475, 476, and 493.)

\section{Development of the Ear}

The essential auditory part of the organ of hearing, the membranous labyrinth, is of ectodermic origin. This first appears as a thickening followed by an invagination of the surface ectoderm in the region of the posterior cerebral vesicle. This is known as the auditory pit. By closure of the lips of this pit and growth of the surrounding mesodermic tissue is formed the otic vesicle or olocyst, which is completely separated from the surface ectorlerm. Diverticula soon appear passing off from the otic vesicle. These are three in number and correspond respectively to the future endolymphatic duct, the cochlear duct and the membranous semicircular canals. Within the saccule, utricle, and ampullic special differentiations of the lining epithelium give rise to the maculex and cristac acustica. Of the cochlear duct the upper and lateral walls become thinnerl to form Reissner's membrane and the epithelium of the outer wall, while the lower wall becomes the basilar membrane, its cpithclium undergoing an claborate specialization to form the organ Corti.

Of the cochlea, only the membranous cochlear duct develops from the otic vesicle; the scala vestibuli, scata tympani, and bony cochlea developing from the surrounding mesorlerm. The mesodermic connective tissue at first com- 
pletely fills in the space between the cochlear duct and the bony canal. Absorption of this tissue takes place, resulting in formation of the scala tympani and scala vestibuli.

During the differentiation of the above parts a constriction appears in the body of the primitive otic vesicle. This results in the incomplete septum which divides the utricle from the saccule.

The middle ear is formed from the upper segment of the pharyngeal groove, the lower segment giving rise to the Eustachian tube.

The external ear is developed from the ectoderm of the first branchial cleft and adjacent branchial arches. The tympanic membrane is formed from the mesoderm of the first branchial arch, its outer covering being of ectodermic, its inner of entodermic origin.

\section{TECHNIC}

(I) For the study of the general structure of the pinna and walls of the external auditory meatus, material may be fixed in formalin-Müller's fluid (technic 5, p. 7) and sections stained with hæmatoxylin-eosin (technic r, p. 20). In sections of the wall of the cartilaginous meatus the ceruminous glands may be studied, material from children and from new-born infants furnishing the best demonstrations of these glands.

(2) For the study of the inner ear the guinea-pig is most satisfactory on account of the ease with which the parts may be removed. Remove the cochlea of a guinea-pig with as much as possible of the vestibule and semicircular canals and fix in Flemming's fluid (technic 7, p. 7). A small opening should be made in the first turn of the cochlea in order to allow the fixative to enter the canal. After forty-eight hours the cochlea is removed from the fixative and hardened in graded alcohols (page 8). The bone is next decalcified, either by one of the methods mentioned on page Io or in saturated alcoholic solution of picric acid. If one of the aqueous decalcifying fluids is used, care must be taken to carry the material through graded alcohols. Embed in celloidin or paraffin, cut sections through the long axis of the modiolus, through the utricle and saccule, and through the semicircular canals. Stain with hæmatoxylin-eosin and mount in balsam.

(3) The neurone relations of the cristæ, maculæ, and cochlear duct can be demonstrated only by means of the Golgi method. The ear of a new-born mouse or guinea-pig furnishes good material. The cochlea together with some of the base of the skull should be removed and treated by the Golgi rapid method (page 35). Sections should be thick and must of course be cut through undecalcified bone. Good results are difficult to obtain.

\section{The Organ of Smell}

The olfactory organ consists of the olfactory portion of the nasal mucosa. In this connection it is, however, convenient to describe briefly the olfactory bulb and the olfactory tract.

The Olfactory Mucosa.-This has been described (page 299). The 
peculiar olfactory cells there described are not neuro-epithelium but are analogs of the spinal ganglion cell, being the only example in man of the peripherally placed ganglion cell found in certain lower animals. Each cell sends to the surface a short dendrite which ends in several short, stiff, hair-like processes. From its opposite end each cell gives off a longer centrally directed process (axone), which as a fibre of one of the olfactory nerves passes through the cribriform plate of the ethmoid (Fig. ${ }_{3} s_{3}, \mathrm{ethm}$ ) to its terminal nucleus in the olfactory bulb (Fig. $3 \delta_{3}$ ).

The Olfactory Bulb.- This is a somewhat rudimentary structure analogous to the much more prominent olfactory brain lobe of some

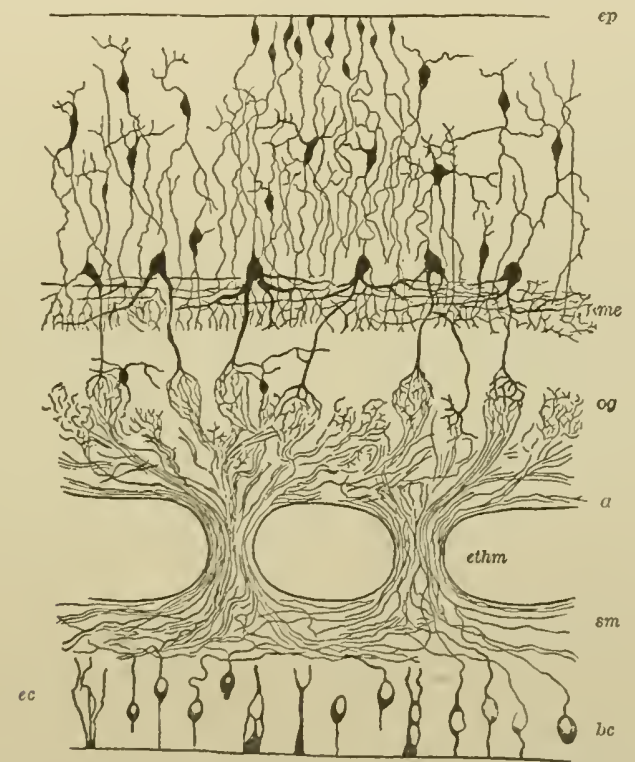

FIG. 383.-Diagram of Structure of Olfactory Mucosa and Olf actory Bull). (Ramón y Cajal.) bc, Bipolar cells of olfactory mucosa; sm, submucosa; $c t h m$, cribriform plate of ethmoid; $a$, layer of olfactory fibres; og, olfactory glomeruli; me, mitral cells; $e p$, epithelium of olfactory ventricle.

of the lower animals. It consists of both gray matter and white matter arranged in six fairly distinct layers. These from below upward are as follows: (a) The layer of olfactory fibres; $(b)$ the layer of glomeruli; $(c)$ the molecular layer; $(d)$ the layer of mitral cells; $(e)$ the granule layer; $(f)$ the layer of longitudinal fibre bundles. Through the centre of the last-named layer runs a band of neuroglia which represents the obliterated lumen of the embryonal lobe. The relations of these layers to the olfactory neurone system are as fellows: 
The layer of clfactory fibres (Fig. $3^{8} 3, a$ ) ccnsists of a dense plexiform arrangement of the axones of the above-described olfactory cells. From this layer the axcnes pass into the layer of olfactory glomeruli where their terminal ramifications mingle with the dendritic terminals of cells lying in the more dorsal layers, to form distinctlyoutlined spheroidal or oval nerve-fibre nests, the olfactory glomeruli (Fig. 383 , $o g$ ). The latter mark the ending of neurone system No. I. of the olfactory conduction path. (For olfactory tract see pp. $475,477, a$.)

The molecular layer contains both small nerve cells and large nerve cells. These send their dendrites into the olfactory glomeruli. The smaller cells belong to Golgi Type II. (see page I32) and appear to be association neurones between adjacent glomeruli. The axones of the larger cells, the so-called brush cells, become fibres of the olfactory tract.

Of the mitral cells (Fig. $383, m e$ ), the main dendrites end in the olfactory glomeruli, while their axones, like those of the brush cells, become fibres of the olfactory tract.

In addition to the fibres which pass through it (axones of mitral and of brush cells), the granular layer contains numerous nerve cells. Many of these are small and apparently have no axones (amacrine cells). Their longer dendrites pass toward the periphery, their shorter dendrites toward the olfactory tract. Larger multipolar cells, whose axones end in the molecilar layer, also occur in the granular layer.

The layer of longitudinal fibre bundles consists mainly of the centrally directed axones of the mitral and brush cells. These fibres run in distinct bundles separated by neuroglia. Leaving the bulb they form the olfactory tract by means of which they pass to their cerebral terminations.

The brush cells and mitral cells with their processes thus constitute neurone system No. II. of the olfactory conduction path.

\section{TECHNIC}

(I) Carefully remove the olfactory portion of the nasal mucosa (if human material is not available, material from a rabbit is quite satisfactory). This may be recognized by its distinctly brown color. Fix in Flemming's fluid (technic 7 , p. 7), or in Zenker's (technic 9, p. 8). Stain thin vertical sections with hæmatoxylin-eosin (technic I, p. 20) and mount in balsam.

(2) For the study of the nerve relations of the olfactory cells material should be treated by the rapid Golgi method (page 35 ). 


\section{The Organ of Taste}

The organ of taste consists cf the so-called taste buds of the lingual mucosa. These have been mentioned in connection with the papillie of the tongue (page 226) and under sensory end-organs (Fig. 297).

The taste buds are found in the side walls of the circumvallate papilla (page 226). of some few of the fungiform papilla, in the mucosa of the posterior surface of the epiglottis, and especially in folds (foliate papilla) which occur along the postero-lateral margin of the tongue.

The taste bud ( $\mathrm{Fig} .38_{4}$ ) is an ovoid epithelial structure embedded in the epithelium and connected with the surface by means of a minute canal, the gustatory canal (Fig. $\left.3 s_{4}, a\right)$, the outer and inner ends of which are known, respectively, as the outer and inner taste pores.

Each taste bud consists of two kinds of cells, neuro-epithelial cells or gustatory cells and sustentacular cells (Fig. 384). The gustatory cells are long, delicate, spindle-shaped cells which occupy the centre of the taste bud, each ending externally in a ciliumlike process, which usually projects through the inner pore. The inner end of the cell tapers down to a fine process, which may be single or branched. The sustentacular cells are long, slender

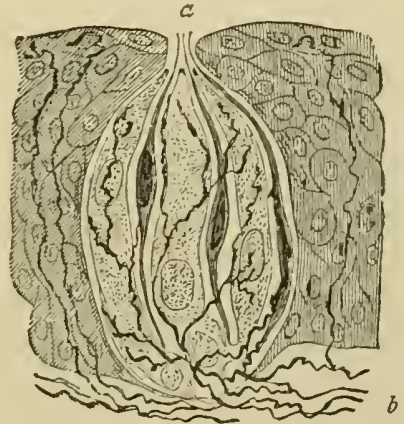

Fig. 384.-Taste-bud from Side IVall of Circumvallate Papilla. (Merkel-Henle.) $a$,'Tastepore; $b$, nerve fibres, some of which enter the taste-bud-intrageminal fibres; while others end frecly in the surrounding epithelium-intergeminal fibres. cells which form a shell several cells thick around the gustatory cells. Sensory terminals of the glosso-pharyngeal nerves (Fig. $384, b)$ end within the taste buds in a network of varicose fibresintrageminal fibres. Other sensory terminals of the same nerve end frecly in the epithelium between the taste buds. These are finer and smoother than the intrageminal fibres and are known as intergeminal fibres (Fig. 384 ).

\section{TECHNIC}

(I) The general structure of the taste buds is shown in the sections of tongue (technic, p. 227).

(2) For the sturly of the nerve terminals the method of Golgi should be userl (page 35). 


\section{General References for Further Study}

Kölliker: Handbuch der Gewebelehre des Menschen. McMurrich: The Development of the Human Body. Ramón y Cajal: La rétine des vertébrés. La Cellule, ix., I893. Schwalbe: Lehrbuch der Anatomie der Sinnesorgane: I887. 


\section{INDEX}

ABDUCENS (nerve VI) $475,499,502$

Absorption, 273

of fat, 273

Accessorius (nerve $\mathrm{XI}$ ) $48_{3}$

Accessory nasal sinuses, 299

olivary nucleus, 490

Achromatic element of intranuclear network, 48

spindle, 54

Acid aniline dyes, 20

cells, $245,249,272$

Acidophile granules, rc6

Acini, 217

Acoustic group of segmental neurones, 475

Acromegaly, 400

Acrosome, 343

Acusticus (nerve VIII), 475, 48 I, 49 I, 493

Adelomorphous cells, 249

Adenoids, r8o

Adipose tissue, 87

Adrenal gland, $4 \mathrm{I} 2$

blood-ressels of, 4 I4

chromaffin granules of, 414

development of, 4 is

lipoid granules of, 4 I 2

lymphatics of, 4 I 4

nerves of, 4 I 4

phxochrome granules, $4 \mathrm{I} 4$

phacochromoblasts, 415

sympathoblasts, 4 rs

Adventitia of arterics, $15^{6}$

of lymph vessels, 165

of veins, 158

Afferent ccrcbellar neurones, $490,493,501$, 504,507

peripheral nerves, 4 I 9

segmental neurones, 476

path.s, $47^{6}$

pallial, 46 $\mathrm{r}, 462,464,474,476,523$

roots, their terminal nuclei and seconrlary tracts, $48_{3}, 485,489,491$, $500,504,506,515,520,524,526$, $53^{6}$

suprascgmental neurones, 421
Agminated follicles, 260

Air cells, 3 ro

passages, 3 Io

sacs, 3 Io

vesicles, 3 I 0

Ala cinerea, $48 \mathrm{r},+89$

Alcohol, as a fixative, 6 dilute, as a fixative, 6 for hardening, $S$

Ranvier's, 4 strong, as a fixative, 6

Alcohol-ether celloidin, I I

Alimentary tract, 219

development of, 296

endgut, 263

foregut, 243

headgut, 220

midgut, 255

Altmann's granule theory of protoplasmic structure, 44

Alum-carmine, rg for staining in bulk, 2I

Alveolar ducts, 3 10

glands, 2 I $5,2 \mathrm{I} 7$

passages, $3 \mathrm{rr}$

sacs, 310

Alveoli, 2 I 7, 2S2, 300

Amacrine cells, $55^{8}, 596$

Amitosis, 53

Amoeboid movement, 5 I technic for, 63

Amphiaster, 54

Amphicytes, 427

$\Lambda$ mphipyrenin, 48

A mpulla, 574

Amygdaliform nucleus, $53^{6}$

Analsolism, 50

Anaphase, $5^{6}$

Aniline dyes, acid, 22 basic, 19

Anistropic line, I I5 sulsstance, in 5

Annular tcrminations, 434

Annuli fibrosi, If, 2

Ansa lenticulisis, 536 
Anterior corpus quadrigemini, 5 I 7 , 522

cerebral commissure, 532,536

horns, 444,448

root or motor cells of, 44I, 444

perforated space, 532, 536

pyramids, 464

white commissure, 536

Antero-lateral funiculus, 448 white column, 448

Antrum, 355

Anus, 267

Aorta, I 56

Apathy, concerning cilia, 76

Apical body, 343

Aponeurosis, 92

Appendix epididymidis, 342

testis, 342

vermiformis, 265

Aqueductus Sylvii, 4I 7, 5I5, 5I 7

Arachnoid membrane, 422, 560

Arbor vitæ, 507

Arborescent terminations, 434

Arborizations, terminal, I3I, 429

Arc, cerebellar, 470

cerebral, 470

pallial, $47 \mathrm{I}$

spinal reflex, 469

three-neurone, 469 , 506

two-neurone, 469,504

Archipallium, 532

Archoplasm, 49

Arciform nucleus, 499, 530

Arcuate fasciculus, 535

fibres, $487,49 \mathrm{I}, 499$

external, $487,490,499$

internal, $487,500,502,522$

Area, acustica, 482

hippocampal, 539

tegmenti, 530

Areolar (loose) connective tissue, 87

Arrector pili muscle, 39 I

Arrectores pilorum, 394

Arteriæ arciformes, 326

rectæ, 328

Arteries, 153

adventitia of, ${ }_{5} 6$

anterior spinal, 454

aorta and other large, ${ }_{5} 6$

arcuate, 326

arteriole, 154

bronchial, 3 I 3
Arteries, carotid, 4 I I

ciliary, 565

coats of, 153

coronary, I6 I

development of, $\mathbf{1}_{3} 3$

greater arterial circle of iris, 566

hepatic, 289

interlobar, 326

interlobular, 326

intima of, 154

large, like the aorta, ${ }_{5} 6$

lesser arterial circle of the iris, 566

lymph channels of, I 59

media of, I55

medium-sized, I 54

nerves of, I59

phrenic, 328

posterior spinal, 454

precapillary, $\mathrm{I}_{54}$

pulmonary, 3 I 3

recurrent, $\cdot 328$

renal, 3 I 8,325

small, I53

structural peculiarities of some, 157

sulco-commissural, 454

technic of, $\mathrm{I} 60$

vasa vasorum, 159

Arteriole, I 54

Articular cartilages, 204

Articulations, 204; see Joints

diarthrosis, 204

synarthrosis, 204

synchondrosis, 204

syndesmosis, 204

technic of, 205

Arytenoid cartilages, $30 \mathrm{I}$

Ascending degeneration, 459

fibre tracts of spinal cord, 445, 459

direct cerebellar, $46_{3}$

Gowers', 464

long arms of dorsal root fibres, $46 \mathrm{r}$ posterior columns, 443,448

funiculi, $448,46 \mathrm{I}$

spino-tectal, 465

-thalamic, 462

tract of Flechsig, 463

tracts forming parts of afferent pal-

lial paths, $46 \mathrm{I}$

to cerebellum, 463

tractus spino-cerebellaris dorsalis, 463

ventralis, 463

uncrossed cerebellar, 463 
Asters, 58

Atresia of follicle, 363

Atrophy method of determining fibre tracts of the cord, 459

Attraction sphere, 49

Auditory canal, 572

cells, $58 \mathrm{I}$

hairs, 575

nerve, 49 I, 493

cochlear branch of, 49 I, 493

restibular branch of, 475, 49I, 493

path, $475,49 \mathrm{I}, 492,493,524$

pit, $5^{8} 3$

Auerbach, end-buttons of, 38

end-feet of, 38

plexus of, 264, 270

Auricle, 573

muscle of, I6I

Auriculo-ventricular ring, I6r

Axis cylinder, I 26, I32; see also Axone

Axolemma, I34

and neurilemma, relation of, ${ }_{13} 6$

Axonal degeneration, I42, 459

Axone, the, 126, 132, 418

Bethe's views of, 136

Cajal's views of, 137

collaterals of, 132

degenerative changes in, I39

development of, 4 is

fibres of Remak, I33

medullary sheath of, 132

medullated, 133

naked axone, I 32

non-medullated, 132

terminal arborizations of, 132

Axone-hill, 132

BAILLARGER, line of, $54 \mathrm{I}$

I3alsam, Canada, for mounting, 23

liarker, concerning the neurone, 127,130

Jartholin, glands of, 376

duct of, 277

Basal filament, 2 I3

granule, 76

Basic aniline dyes, 19

l'asket cells, 222, 510

Basophile granules, 106

Bellini, duct of, 321,324

Berkley, concerning pituitary body, 409

Bertini, columns of, 320

Bethe, concerning continuity of axolemma and neurilemma, ${ }_{13} 6$
Betz, cells of, $533,536,539$

Bioblasts, 44

Bipolar nerve cells, I 27, 428

Bladder, urinary, 330

Blastoderm, 62

Blastomeres, 62

Blocking, I 2

Blood, IO3

corpuscles, 103

crenation of red cells, 104

development of, Io8

diapedesis, 107

dust, IcS

erythrocytes of, 103

granules, elementary, rc 8

granules of Ehrlich, 107

hæmatin, 104

hæmatokonia, ros

hrmoglobin of, 104

hæmolysis, 104

Jenner's stain for, 3 I

leucocy tes of, 105

macrocytes, 103

microcytes, 103

phagocy tosis, 107

plasma of, Iо3

platelets, roS

red cells of, 103

crenation of, 104

technic for, 63

smears, technic of, Iro

specific gravity of, IO3

stroma of, 104

technic of, 110

thrombocytes, ros

vascular unit, 3 I 3

white cells of, 105

Blood-islands, 108, 163

Blood-sinuses of hamoly $\mathrm{mph}$ nodes, 173

Jloorl smear, 6

Jlood-vascular unit, 313

Blood-vessel system, I5 I

arteries, 153

capillaries, $15 \mathrm{I}$

development of , $\mathrm{I}_{3}$

heart, 161

lining of, $15 \mathrm{r}$

technic of, 160,163

valsat vasorum, 150

vcins, 158

J3lood-vessels, 15 I

lymph channcls of, 159 
Blood-vessels, nerves of, I59

technic of, 160

Body cavities, 144

Bone, breakers, 199

cells, 190,200

decalcification of, Iо

formers, 198

tissue, Ioo

calcination of, IOI

cells of, IOI

cementum, 233

corpuscles of, Ior

decalcification of, 3,9 , IOI

intercellular substance of, roI

lacunæ and canaliculi of, IOI

lamellæ of, IOI

technic of, IO2

Bone-marrow, I $92^{2}$

blood-vessels of, 196

development of, 203

gelatinous, I95

marrow spaces, I88, I89, I98, 200

red, I94

cells of, I9o

erythroblasts of, $\mathrm{I} 92$

fat cells of, I93

giant cells, I93

leucocytes, I93

marrow cells, I92

mast cells of, I 93

megakaryocytes, I93

myelocytes of, 192

myeloplaxes of, 193

non-nucleated red blood cells of, I93

normoblasts, I92

nucleated red blood cells of, I02

osteoclasts, I93

plasma cells of, I93

polykaryocytes of, I9.3

technic of, I97

yellow, I95

Bones, I88

blood-vessels of, I 95

canaliculi of, IgI

cancellous or spongy, I88

cells of, I9O, 200

origin of, 198

circumferential lamellæ of, I 9 I

development of, 197

intracartilaginous, 199

intramembranous, 197
Bones, subperichondral development of, $20 I$

subperiosteal, $20 \mathrm{I}$

growth of, 203

hard or compact, I 88

Haversian canals of, 189

lamellæ, I9o

spaces, 202

Howship's lacunæ, I99

intermediate lamellæ, I9 I

interstitial lamellæ, I9I

lymphatics of, I96

marrow, I92

red, 194

yellow, I95

nerves of, 196

nutrient canal, 196

foramen, 196

vessels, 196

osteoblasts, $\mathbf{1} 98$

osteoclasts, i 99

osteogenetic tissue, 197

perforating fibres, I92

perichondrium, I99

pericranium, 198

periosteal buds, 200

periosteum of, I9I, I99

Sharpey's fibres, I92

technic of, 196

developing bone, 203

Volkmann's canals, I9I, I96

Bony cochlea, 576

spiral lamina, 577

Borax-carmine, alcoholic solution, 22

Born's theory of corpus luteum, 362

Bowman, capsule of, 320,322

glands of, 300

membrane of, 548

sarcous elements of, Ir 6

Brachia conjunctiva, 5c2, $5 \mathrm{I} 7$

Brachium of posterior corpus quadrigeminum, 520

pontis, 499

Brain, the, 473

cerebral cortex of, 536

contrasted with spinal cord, 473

development of, 4 I 6

effectors, 473

endbrain (telencephalon), 4r7, 532

corpus striatum, 532

pallium, 533

rhinencephalon, 532 
Brain, forebrain (prosencephalon), 416, 522 diencephalon (thalamencephalon), 417,522

epithalamus, 523

hypothalamus, 523

thalamus, 523

interbrain, 522

general histology of, 479

structure of, 473

hemispheres of, 478

higher coördinating apparatus of, 473

hindbrain (rhombencephalon), 4I7, 479

cerebellum, 499

isthmus, 5 . 5

medulla oblongata (bulb), 479

nerves of, 479

pons, 4 I $7,482,499$

tegmentum, 479, 499

membranes of, 422

arachnoid, 422

blood-vessels of, 422

cerebral dura, 422

dura mater, 422

pia mater, 422

cerebralis, 424

Pacchionian bodies of, 424

relation of optic nerve to, 560

technic of, 424

midbrain (mesencephalon), $4 \mathrm{I} 6$

aqueductus Sylvii, 5 I 7

basis pedunculi, 5 I 7

corpora quadrizemina, 478,522

iter, 5 I 7

pes pedunculi, $52 \mathrm{I}$

posterior commissure, 522

substantia nigra, 52 I

tegmentum, 5 I 7

pallium, 478

pineal eye, $5+4$

pituitary body, 407

receptors, 473

relation of, to optic nerve, 560

sand, 547

segmental brain and nerves, 420,474

afferent peripheral neurones, 474

efferent peripheral neurones, 475

semicircular canals, 473

suprasegmental structures, 478

technic of, 482,543

ventricles, 416,417

vesicles, 410
Branca, concerning the centrosome, $5^{8}$

Bridges, intercellular, 3,70 , II 2

Bronchi, 304

alveolar, 309

blood-vessels of, 3 I 3

cartilages of, 306

development of, 3 I 5

lobular, 309

lymphatics, 3 I 5

nerves of, 3 I 5

primary, 304

respiratory, 310

structure of walls of, 304

technic of, 317

terminal, 310

Bruch, membrane of, 55 I

Brunner's glands, 25 2, 262, 272

Bulb, 479; see Medulla

Bulbus oculi, 548; see Eyeball.

Bundle of Löwenthal, 466 of Vicq d'Azyr, 524, 532

Burdach, column of, 459,462

nucleus of, $4 \delta 2,4 \delta I$

Bursæ, 208

Busch-Marchi staining method, 35

Bütschli's theory of protoplasm structure, 44

diagram of, 45

Cachexia strumipriva, 403

Cajal, cells of, 536

concerning the neurone, $131, \mathbf{1} 35$, 138

interstitial nucleus of, $465,485,49 \mathrm{I}$, $5 \mathrm{CO}, 504$

methods for staining neurofibrils in nerve cells, 37

Cajeput oil for clcaring sections, 23

Calcarine area, $5+3$

Calcification, 82

centre, 197,200

zonc, 202

Calcination, 539

Canala balsam, 23

Canal, gastro-intestinal, 2.45

gustatory, $5^{8} 7$

lacrymal, $5^{\circ} 7$

of Cloquet, $5^{(1)} 5$

of l'etit, $5{ }^{6} 5$

of Sclilemm, $55+$

portal, 290

Volkmann's, 191, 196 
Canaliculi of bone, IOI, I9I of connective tissue, 83

Canalis communis, 575

Canalized fibrin, 373

Cancellous bone, I88, 198

Capillaries, I5 I

chyle, 270

development of, 163

technic of, 160

Capillary endothelium, I5 I network, I 52

Capsule of Bowman, 320,322

internal, 530

of ganglion cells, 419

of Glisson, 287

of Tenon, 566

Carbol-xylol for clearing specimens, 23

Carboxyhæmoglobin, I07

Carmine alum, I9, 2I

borax, 22

gelatin, 25

neutral, 20

picro-, 2 I

Carotid gland, 409

Cartilage, 97

arytenoid, $30 \mathrm{I}$

cells, 98

chondrin, 98

classification of, 98

cricoid, 301

development of, $8 \mathrm{I}$, 100

elastic, 99

embryonal, 99

epiphyseal, 203

fibrous, 99

hyaline, 98

intercellular matrix of, roo

intermediate, 199

laryngeal, 540

of developing bone, 199

perichondrium of, 99

Santorini's, 30I

technic of, I00

thyreoid, 30I

tracheal, 302

Wrisburg's, 301

Cartilages, articular, 204

costal, 204

skeletal, 204

Caryochromes, I29, 5 I 2

Caudate nucleus, 526, 532, 535

Cavernous sinuses, 350
Cavity of embryonic vesicle, 6 I

Cedarwood oil as solvent, I3

Cell, the, 43

amitosis, 53

body of, $44, \mathbf{I} 26$

centrosome of, 49

crusta of, 46

cuticula of, 47

cytoplasm of, 45

cytoreticulum of, 45

deutoplasm granules of, 46

division, direct, 53

indirect, 53

endoplasm of, 46

exoplasm of, 46

function of, 5 I

hyaloplasm of, 44

intranuclear network of, 48

irritability of, 5 I

karyoplasm of, 46

linin of, 48

membrane of, 47

metabolism of, 50, 2I3

metaplasm granules of, 46

microsomes, 44

mitosis, 53

motion of, 5 I

nuclear membrane of, 48

nuclein of, 48

nucleolus of, 48

nucleoreticulum, 48

nucleus, 47

origin of word, 47

patches, 373

paraplasm granules of, 46

plastids, 46

plastin, 44

protoplasm of, 43

Altmann's granule theory of, 44

Bütschli's foam or emulsion theory of, 44

fibrillar theory of, 45

reproduction of, 52

space (lacuna), IoI

spongioplasm of, 44

technic for study of, 44

trophospongium, 46

typical, 43

diagram of, 43

structure of, 43

vital properties of, 5O, $2 \mathrm{I} 3$

function of, 285 
Cell, function of, Opie's theory of, 286 origin of, $2 \delta_{5}$ structure of, $28_{5}$ technic of, 287

Cell-islands of Langerhans, $2 \delta_{5}$

Celloidin, alcohol-ether, I I

clove-oil, I 2

embedding, II

sections, I.t clearing of, 23 mounting of, 23

Cells, acid, $245 \cdot 249,272$ active, 2 I 2

adelomorphous, 249

air, 310

amacrine, 558,586

amceboid, I4t

auditory, $5 \mathrm{~S}_{\mathrm{I}}$

basal, 300

basket, 222

blood, IO3

bone, IOI, I90, 2c0, 233

brush, $5 S_{5}$

capsule, 4 IS

cartilage, $9 S, 201$

cementoblasts, 235

central, 249

centro-acini, of Langerhans, $2 S_{3}$

centro-tubular, $28_{3}$

chief, $245,249,272,405,407$

chromaffin, 4 Io

chromophile, 407

ciliated, 75,304

clasmocytes, 85

clasmatocytes, 85

clear, 405

colloid, 403, 405

compound tactile, $43 \mathrm{I}$

connective-tissue, $8_{3}$

corneum, 383

crescents of Gianuzzi, 222, 278, 302

daughter, 57

decirlual, 370

Deiter's, 580

dctomorphous, 2.49

demilunes of Heidenhain, 222

cmuty, 212

endothelial, 15 I

eosinophile, 106, 174

equithelial, 70

crythroblasts, 192

crythrocytes, 10.3
Cells, extrinsic, 443

fat, $S_{7,1} 93$

fibroblasts, $S_{2}$

fixed, $\mathrm{S}_{3}$

fotal, 3 I 2, 3I6

ganglia, 4 I 9

giant, I93

gland, 212

goblet, 75, 21 2, 257, 272

Golgi, Type I., I30, I32

Type II., I32, 446

granule, 538

gustatory, $5^{8} 7$

hair, $575,5 S I$

hecatomeric, 445

Hensen's, $5^{\text {so }}$

heteromeric, 445

interstitial, $33^{\circ}$

intrinsic, 443

Kupffer's, 292

I.angerhans', 283

leucocytes, I03, I 93

Leydig's, 568

liver, 290

loaded, 2 I 2

lutein, 359

lymphoid, 170

macrocytes, 103

marrow, 192

mast, $83,174,192$

megakaryocyte, I93

megalocyte, $\mathrm{IS}_{4}$

microcyte, 103

migratory leucocytes, 259

mitral, 586

mononuclear, i 84

mossy, I +3

mucous, 21 2, 221, 257, 272

multinuclear, I $\delta_{4}$

muscle, II I

myelocytes, 192

myeloplaxes, 193

nerve, IIr; for classification see Nerve cells

neurilemma, 144,419

ncuroblasts, I 26, 142, 418

neur()-epithelial, 575

neuroglia, $143,418,453,514$

normoblasts, 102

nucleaterl red blood, 108, 102

ofintoblasts, 228,241

of Claturlius, $58 \mathrm{~g}$ 
Cells of oral glands, 22 I, 222

olfactory, 300

osteoblasts, r98, 235

osteoclasts, 193, I99, 235

ovum, $52,355,356$

oxyntic, 249

oxyphile, 405

Paneth's, 260, 273

parietal, 249

peptic, 249, 272

phæochromoblasts, 4I 2, 4I 5

phagocytes, I07, I74

pigmented, 46, 76, I3 I, 384, 388, 554

pillar, 580

plasma, 85, I93

polykaryocytes, I93

prickle, 383,387

primitive ova, 354,378

Purkinje, 509

red blood, I03, I 84

replacing, 73, 252, 259

reserve, 403

respiratory, 3 I I

resting, 54, 2I 2, 403

secreting, 2 I 3

serous, $22 \mathrm{I}$

Sertoli's, 335,378

sex, 378

signet-ring, 88

simple tactile, $43 \mathrm{I}$

single primitive, 52

smooth muscle, II 2, 38 I

spermatids, 337,344

spermatoblasts, 345

spermatocytes, 337,344

spermatogenic, 335

spermatogones, 336,344

spider, $\mathrm{r}_{43}$

spleen, I 84

supporting, 335

sustentacular, 284, 3co, 335, 575

sympathoblasts, 4 I 5

tactile, 433

tautomeric, 445

tendon, 92

thrombocytes, I08

wandering, 86, 259

white blood, ro5, 184

Cementing glycerin mounts, 22

Cementoblasts, 235

Cementum, 233

development of, 242
Central canal, 4r6, 449

cells, 249

chromatolysis, $\mathrm{r}_{42}$

gelatinous substance, 449

gray, $483,485,487,502$

nervous system, $4 \mathrm{I} 6$

neurones, 4 I8

spindle, 54

tegmental tract, 490 , 50r, 507, 5I5, 518,526

vein, 289

Centriole, 49

Centro-acinar cells of Langerhans, 283

Centrosome, 49, 62

archoplasm, 49

attraction sphere, 49

centriole, 49

daughter, 53

of fertilization, 62

Centro-tubular cells of Langerhans, 283

Cerebellar arc, 470

connections, $463,464,468,499,506$

cortex, $507,5 \times 2$

peduncles, 502

Cerebello-olivary fibres, 490, 49 I, 499

Cerebellum, 4I 7, 502, 507

arbor vitæ, 507

ascending paths to the, 462

association cells of, $5 \mathrm{I} 4$

basket cells of, 5 Io

cells of, 509,5 ro, 5 I I

climbing fibres of, 5 10

cortex of, 509, 5I4

dentate nucleus of, 500, 502, 507

descending tracts from, 464

development of, $4 \mathrm{x} 7$

fibres of, 5 ro

climbing, $5 \mathrm{IO}, 5 \mathrm{I} 3$

mossy, $5 \mathrm{x} 3$

of Bergmann, $5^{r_{4}}$

parallel, 5I 2

general histology, 507

glomeruli of (islands), 5 I 2

granular layer, $5 \circ 7$

gray matter of, 507

hemispheres, of, 502, 507

internal nuclei of, 502, 507

laminæ of, 507

middle peduncle of, 508

molecular layer, $5 \circ 9$

neuroglia of, $5^{\mathrm{r}} 4$

nuclear layer, 509 
Cerebellum, nucleus dentatus, 507

emboliformis, 507

globosus, 502, 507

tecti or fastigii, $502,507,53 \mathrm{~S}$

peduncles of, 508

Purkinje cells of, 509

technic of, 543

vermis, 507

Cerebral arc, $47 \mathrm{I}$

cortex, 48 I

dura, 422

hemispheres, 417

development of, 4 I 7

membranes, 422

peduncles, 5 IS

vesicle, 4 r 6

Cerebro-spinal ganglia, $\mathrm{x} 26,4 \mathrm{r} 8,426$

central processes of, 436

Dogiel's classification of, 427

peripheral processes of, 429

technic of, $44 \mathrm{r}$

nervous system, 4I 7

neurones, efferent peripheral, 441

Ceruminous glands, 572

Cervical enlargement of cord, 443

segments of cord, 443

Cervix, 367

epithelium of, 368

external os, 368

ovula Nabothi, 368

plicæ palmatæ, 368

technic of, 379

Chain ganglia, 436

Cheeks, mucous membrane of, 220

Chemotaxis, 5 I

Chiasma, optic, $526,5^{61}$

Chief cells, 245, 249, 272, 405, 407

Chloride of gold for staining connectivetissue cells, 28

Chloroform as solvent, $\mathrm{r}_{3}$

Choledochus ductus, 295

Choriocapillaris, 55 I

Chorion, $37 \mathrm{I}$

Chorionic villi, $37 \mathrm{r}$

Chorioid, the, $55^{\circ}$

choriocapillaris of, 55 I

fissure, 570

Haller's layer of, 558

lamina citrea, $55 \mathrm{I}$

suprachorioidea, $55 \mathrm{I}$

perichorioidal lymph spaces of, $55 \mathrm{I}$

plexus, 417, 482, 487, 502
Chorioid, tapetum cellulosum of, $55 \mathrm{r}$

fibrosum of, $55^{\mathrm{I}}$

venæ vorticosæ of, $55^{\mathrm{x}}$

vitreous membrane of, 552

Chroma ${ }_{\star}$ affin cells, 4 ro

granules, 4I 2

organs, 4 Io

Rose, concerning chromaffin cells, 4 ro

Chromatic element of intranuclear network, 48

Chromatin, 48

Chromatolysis, $\mathrm{r}_{42}$

Chromatolytic changes, 363

Chrome-silver method of Golgi, 29

Chromophilic bodies, 129 significance of, $130, \mathrm{r}_{3} 8$

Chromosomes, 55

daughter, 55

Chyle vessels, 270

Cilia, 52, 7 r, 76, 340

Ciliary artery, 565

body, $55^{2}$

blood-vessels of, 565

canal of Schlemm, 554

ligamentum pectinatum, 554

muscles of, $55^{2}$

processes of, 552

spaces of Fontana, 554

vitreous membrane of, 553

ganglion, 436

movement, 52

technic for, 570

muscle, 553

plexus, 566

processes, 552

Cingulum, 535

Circulatory system, $\mathrm{r}^{\mathrm{I}}$

blood-vessel system, I.5 I

carotid gland, 4 ro

coccygeal gland, $4 \mathrm{rr}$

development of, $\mathrm{r}_{3}$

lymph-vessel system, I66

Circumferential lamellæ, i 9

Circumvallate papillix, 225

Cirl, concerning fibres of internal capsule, 526

Clarke's columns, 450, 455, 463

Clasmatocyles, 85

Clasmocytes, 85

Claude, concerning devclopment of pancrcas, 297 
Claudius, cells of, $58 \mathrm{I}$

Clava, the, $48 \mathrm{I}$

Clearing specimens before mounting, 23

Clefts of Schmidt-Lantermann, 135

Climbing fibres, 510 , 513

Clitoris, 376

Cloquet's canal, 565

Closed skein (spireme), 54

Clove-oil celloidin, 2

Coagulum sheath, $\mathrm{I}_{37}$

Coccygeal glands, $4 \mathrm{II}$ segments of spinal cord, 455,488

Cochlea, 576

bony spiral lamina of, 577

cupola of, 576

cupula of, 576

hamulus cf, 577

helicotrema, 578

membranous spiral ligament of, 577

modiolus of, 576

scala tympani, 578

vestibuli, 578

spiral ligament of, 577

Cochlear duct, 578

basilar membrane of, 579

crista basilaris, 579

external spiral sulcus, 579

membrane of Reissner, 579

organ of Corti, 580

spiral prominence of, 579

stria vascularis, 579

zona pectinata, 580

tecta, 580

ncrve, $475,489,49 \mathrm{r}, 493$

tracts, 49I, 500, 504

Colom, I6 5

Cohnheim's field, II6

Collagenous fibres, $8 \mathrm{I}$

Collaterals, r 32,447

Colliculi, 41 7, 51 7, 522

Colloid, 40:2, 405, 408

Colostrum corpuscles, 400

Column cells, 444

hecateromeric, 444

heteromeric, 444

tautomeric, 444

technic of, 445

of Burdach, 459, 462, 48r, 487

of Goll, 459, 462, 48I, 4.87

Columnæ rectales, 266

Columins of Bertini, 320

of Sertoli, 335 .
Comma tract of Schultze, 467

Commercial formalin, 4

Commissura habenularis, 53 I

Commissural fibres, 532

Common dental germ, 238 senses, 436

Compact bone, i 88

Compound tactile cells, $43 \mathrm{I}$

Conduction path, 42I, $46 \mathrm{I}$

afferent pallial, $46 \mathrm{I}, 476$

afferent and efferent suprasegmental, 476

auditory, 493

descending suprasegmental, 483

efferent cerebellar, 499

pallial, 476

pallio-cerebellar, 499

pallio-spino-peripheral efferent, 465

to cerebellum, 462

trigeminal afferent pallial, 507

Cone association neurones, 56I

fibres, 557

neurones, 560

-visual cell, 557

Cones, layer of rods and, 556

Conjunctiva, 568

end bulbs of, 433,569

Connective tissue, 80,82

adipose or fat, 87

aponeurotic, 92

areolar, 87

bcne, Ioo

cartilage, 97

cells of, 83

characteristics of, so

chlorid-of-gold method for demonstrating cells of, 28

classification of, 8I

dense fibrous, 87

elastic, 92

elastin of, 87,95

embryonal, 8I, 82, 197, 203

fat, 87

fibres of, 86

elastic, 86

fibrillated, 86

reticular, 94

white, 86

yellow, 86

fibrillar, 82

fibroblasts, 82

fixed cells of, 83 
Connective tissue, formed, $9 \mathrm{r}$

gelatin of, 86

gelatinous, $\delta_{2}$

histogenesis of, So

intralobular, $9 \mathrm{r}, 2 \mathrm{r} \overrightarrow{5}$

interalveolar, $3 \mathrm{r} 3$

intercellular substance of, 86

interlobar, 215

interlobular, 89,2 I 5

intrafascicular, 208, 425

ligaments, 91

loose, 87

Mallory's stain for, 29, 30

mast cells of, 83

mucous, 82

neuroglia, $142,418,453,514$

pigmented cells of, $8_{7}$

plasma cells of, 83

reticular, 94

retinaculæ cutis, $3 S_{2}$

staining cells of, 29

technic for, 95, ro2

theories of development of fibres of, $8 \mathrm{r}$

wandering cells of, 83

Constrictions of Ranvier, $\mathrm{r} 34$

Contact theory of neurones, ${ }_{3} 3$

Continuity theory of neurones, 139

Convoluted tubules, $320,322,323$

Cord, spinal, 4 16; see Spinal Cord

Corium, 380; see Derma

Cornea, the, 548

anterior elastic membrane of, 549

epithelium of, 549

corpuscles of, $55^{\circ}$

endothelium of Descemet of, 550

membrane of Bowman of, 548

of Descemet of, $55^{\circ}$

posterior elastic membrane of, 550

substantia propria of, 550

Corneal corpuscles, $55^{\circ}$

Cornu ammonis, 532

Cornua of cord, 448

Corona radiata of ovum, 356

of pallium, $533,536,537$

Coronary arteries, 162

Corpora amylacea, 348

cavernosa, 349

lutea of pregnancy, 360

spuria, 36o

vera, 360

mammillaria, 531
Corpora, quadrigemina, $417,493,5 x$

anterior, 517,522

development, of, 417

posterior, 493, 517

striata, 4 r 7

Corpus albicans, 360

callosum, 536

dentatum, 502

hæmorrhagicum, 359

Highmori, or mediastinum testis, 333

luteum, 359

theory of, 362

Luysii, 523

quadrigeminum, anterior, $5^{17} 7,5^{22}$

spongiosum, 349

striatum, 532,535

candate nucleus, 532

lenticular nucleus, 532

putamen, 535

subthalamicum, 521, 530

trapezoideum, 500, 504

Corpuscles, blood, 103

bone, ror

colostrum, 400

corneal, 550

crescentic, 348

Golgi-Mazzoni, 394, 434

Grandry's, 43I

Hassall's, I 77

Meissner's, 350, 394, 432

Merkel's, $43 \mathrm{I}$

Pacinian, 196, 350, 433

renal, 320

Ruffini's, 394, 434

salivary, $18 \mathrm{c}$

splenic, I 82

tactile, 432

Vater-Pacinian, 394

Wagner, 394

Cortex cerebelli, 509, 514; see also Cerebellum

ccrebri, 533,536

arcas of, $54 \mathrm{I}$

association filores of, 543

barren or molecular layer of, 5.38

cells of, 536, 539

of Bet $\%, 536$

of Cajal, 537

of Ciclgi, Type II., 536

of Martinotti, 536,539

pyramidal, 536 
Cortex cerebri, commissural fibres, 533 corona radiata of, 533,536 deep tangential fibres of, $54 \mathrm{x}$ external granular layer, 539 ganglionic layer, 539 horizontal cells of Cajal, 536 internal granular layer, 539 interradiary plexus, $54 \mathrm{I}$ inverted pyramidal cells of Martinotti, 536

layer of polymorphous cells, 536 of pyramidal cells of, 539

line of Baillarger, 54 I of Gennari, 543

molecular layer, 538

multiform layer, 539

plexiform layer of Cajal, 538

polymorphous cells of, 536

projection fibres, 543

radiations of Meynert, 54I

stellate cells of, 536

superficial, tangential fibres of, $54 \mathrm{x}$

supraradiary plexus of, 54I

Cortical labyrinths, 320

pyramids, 320 ; see also Kidney

Corti's arches, $58 \mathrm{r}$

organ, 580; see also Organ of Corti tunnel, 580

Cotyledons, 37 I

Cowper's glands, 348

technic of, 349

Cox-Golgi method of staining nerve tissue, 36

Cranial nerves, $474,545,546$; see also Nerves, cranial

Crenation of red blood cells, IO4

Crescentic corpuscles, 348

Crescents of Gianuzzi, 222, 278, 302

Cretinism, 403

Cricoid cartilage, 3 or

Crista acustica, 576

basilaris, 579

Crossed pyramidal tracts, 464,485

Crura cerebri, 5 I 8

Crusta (exoplasm), 46

Crypt of Lieberkühn, 252,260

Cumulus ovigerus, $35^{6}$

Cuneus, $48 \mathrm{t}, 487$

Cupola, 576

Cupula, 576

Cutaneous sensation, 436
Cuticle, 382 ; see Epidermis

Cuticula, 47, 7 I dentis, 233

Cuticular membrane, 7I, 240, 255

Cystic duct, 294, 297

Cytoarchitecture, 54I

Cytoplasnı, 46 of nerve cells, I 28

Cytoreticulum, 45

Cytosomes, 2 I 3

DARKSCHEWITSCH, nucleus of, 465

Daughter cells, 57 centrosomies, 53

chromosomes, 55 stars, 56

Decalcification, 3,9

Decalcifying, ,o fluids, ro

Decidua basalis, 370 capsularis, 370 graviditatis, $\dot{3} 70$ menstrualis, 369 placentalis subchorialias, 374 reflexa, 370 serotina, 370 subchorionic-placental, 374 vera, 370

Decidual cells, 370

Decolorizing fluid for Weigert's hæmatoxylin, 33

Decussation of fillet, 485

motor, 483

optic, $56 \mathrm{I}$

of Forel, 522

of Meynert, 522

of pyramids, $464,485,487$

sensory, 435,485

Deep sensation, 436

Degenerating nerves, Marchi's method for staining, 34,140

Degeneration of neurones, 140

Wallerian law of, I4I

Dehiscent glands, $2 \mathrm{I} 7$

Deiter's cells, $58 \mathrm{I}$

nucleus, $466,476,493,499,502$

descending tract from, 466,483 , $487,490,502$

Delafield's hæmatoxylin, I7

Delomorphous cells, 249

Demilunes of Heidenhain, 222

Dendrites, the, I $26, \mathrm{I} 3 \mathrm{I}, 4 \mathrm{I} 8$ 
Dental germ, 23s

groove, $23 \mathrm{~S}$

papilla, $23 \mathrm{~S}$

periosteum, 237

pulp, 230

layer of Weil of, $22 \mathrm{~S}$

odontoblasts, $22 \mathrm{~S}$

ridgë, 239

sac, 239

sheath, Neumann's, 232

shelf, $23 \mathrm{~S}$

Dentate nucleus, $458,500,502,507$

Dentinal canals, 230

development of, $24 \mathrm{I}$

fibres of, 230

interglobular spaces of, 232

lines of Schreger, 232

nerves of, 236

Neumann's dental sheath, 232

Tomes' granular layer, 232

Dentine, 228

chemical composition of, 228

secondary, 232

Derma, or corium, 380

corpuscles of Meissner, 350, 394, 432

muscle cells of, $38 \mathrm{r}$

papillæ, compound, $38 \mathrm{I}$

nerve, $38 \mathrm{r}$

simple, $38 \mathrm{r}$

vascular, $38 \mathrm{r}$

pars papillaris, $38 \mathbf{r}$

reticularis, 380

pigmentation of, 384

subcutaneous tissue of, $38 \mathrm{r}$

Descemet, endothelium of, $55^{\circ}$

membrane of, $55^{\circ}$

Descending degeneration, 459

fibre tracts of the spinal cord, 464 ; sec Fibre tracts of spinal cord (descending)

Deutoplasm, 46, 357

Diapedesis, 107

Diaphysis of bone, 203

Diarthrosis, 204

articular cartilages, 20.7

glenoid ligaments, 20.5

interarticular cartilages, 205

joint capsule, 2of,

Diaster, 56, 60

Diencephalon, 417,522

Digestive system, 219
Digestive system, alimentary tract of, 219

development of, 238,296

endgut, 263

foregut, 243

gall-bladder, 295

gastro-intestinal canal, 245

headgut, 220

large intestine, $26_{3}$

larger glands of, 275

liver, $2 S_{7}$

mesentery, 268

midgut, 255

mouth, 220

œsophagus, 243

omentum, 269

pancreas, $2 \mathrm{SI}$

peritoneum, 267

pharynx, 242

rectum, 266

salivary glands, 276

small intestine, 255

stomach, 247

teeth, 227

tongue, 223

vermiform appendix, 265

Direct cerebellar tract, 463

pyramidal tract, 464

Discus proligerus, 356

Dissociation of tissue elements, 4

Disynaptic arc, 470

Dogiel's end plates, $35^{\circ}, 5^{67}$

theory of structure of spinal ganglion, 427

Dorsal accessory olivary nucleus, 490

decussation of Meynert, 522

gray commissure, 449

root fibres of white matter, $45^{\circ}$

spino-cerebellar tract, 463

white commissure, 450

Duct systems of glands, $2 \mathrm{I}+$

Ducts, aberrans Halleri, 342

alveolar, 3 ro

Bartholini's, 277

Bcllini's, 321, 324

bilc, 290

choledocluus, 295

cochlear, 578

common, 294

cystic, 294, 297

endolymphatic, 575

ejaculatory, 342 
Ducts, excretory, $214,276,282,329,364$

Fallopian tube, 364

Gärtner's, 363

hepatic, 289

mesonephric, 377

Müllerian, 348

nasal, 567

of Müller (embryonal), 342

of sweat glands, 384

oviduct, 364

pancreatic, $28 \mathrm{I}$

pronephric, 377

reuniens, 575

Santorini's, 282

secondary pancreatic, $28 \mathrm{I}$

seminal, 339

Stenoni's, 277

thoracic, 164

thyreo-glossal, 403

utriculo-saccular, 575

vas deferens, 340

epididymis, 339

vas efferentia, 339

Wharton's, 278

Wirsung's, 28 I

Wolffian, 377

Ductus aberrans Halleri, 342 reuniens, 575

Duodenum, 262

Brunner's glands, 262 technic of, 275

Dura mater, 422

blood-vessels of, 424

cerebral, 422

spinal, 422

technic of, 424

Dyes, acid aniline, 20

basic aniline, $\mathbf{I} 9$

nuclear, I7

plasma, I9

Dynamic centre of cell, 57

EAR, 572; see also Organ of Hearing

blood-vessels of, 582

development of, 583

drum, 573

external, 572

auditory canal, 572

auricle, 572

blood-vessels of, 573

ceruminous glands of, 572

ear drum, 573
Ear, external, auditory canal, 572 lymphatics of, 573

nerves of, 573

pinna, 572

tympanic membrane, 573

internal, 574

ampulla, 574

blood-vessels of, $58 \mathrm{I}$

canalis communis, 575

cochlea, 576

ducts of, 578

ductus reuniens, 575

endolymph of, 574

endolymphatic duct, $5^{82}$

sac, 582

fenestra ovalis, 574

rotunda, 574

lymphatics of, 582

membrana tectoria, 582

membranous labyrinth, 574

nerves of, 582

crgan of Corti, 580

osseous labyrinth of, 574

perilymph of, $5^{82}$

saccule, 575

scala media, 578

semicircular canals, 575

utricle, 575

utriculo-saccular duct, 575

vestibule, 574

middle, or tympanum, 573

fenestra rotunda of, 574

ossicles of, 574

stapes, 574

wax, 573

Ebner's glands, 226

hydrochloric salt solution, ro

Ectoderm, 62

derivations from, $67, \mathrm{I} 26, \mathrm{I} 42,395$

Edinger-Westphal nucleus, $5 \mathrm{I} 8$

Effectors, 4I8, 424, 426

Efferent pallial paths, $46 \mathrm{I}, 462,464,475$, 477

peripheral neurones, 4I $8,44 \mathrm{I}, 483$, $485,487,49 \mathrm{I}, 499,502,504,5 \mathrm{I} 5$, $5 \mathrm{I} 8,524$

root fibres, $4 \mathrm{I} 8$

suprasegmental neurones, 499, 502,

$504,507,5 \mathrm{I} 5,52 \mathrm{I}, 526,530,536$

Egg cords, Pflüger's, 354

technic of, 366

nests, 354 
Ehrlich, granules of, 10-

Ejaculatory ducts, 34I

Elastic cartilage, 99

fibres, 86

tissue, 92

Verhœeff's differential stain for, $2 S$

Weigert's stain for, 28

Elastin, $\delta_{7}, 95$

Eleidin, 383

Ellipsoid of Krause, 5.57

Embedding, I I celloidin, I I paraffin, $I_{3}$

Emboliform nucleus, 507

Embryonal tissue, 82 fat tissue, 88

Eminentia hypoglossi, $48 I, 489$ medialis, 48 I

Emulsion theory of protoplasmic structure, 44

Enamel, 233

cells, 237,239

chemical composition of, 233

cuticula dentis of, 233 membrane of, 240

development of, 238

fibres, 233

organ, 239

prisms, 233

lines of Retzius of, 233

Endbrain (telencephalon), 417, 532

corpus striatum, 532

pallium, 533

neopallium, 532

olfactory pallium, 532

rhinencephalon, 532

anterior perforated space, 532

gyrus hippocampi, 532

olfactory bulb, 532, 595

nerve, 532

pyriform lobe, 532

trigonum olfactorium, 532

tuberculum olfactorium, 532

End-buttons, 509

of Aucrbach, 38

-feet of Auerbach, 38

-bults, 433

of Krause, 227, 350, 394

Endgut, $26_{3}$

large intestinc, $26 / 3$

mesentery, 267

omentum, 267
Endgut, peritoneum, 207

rectum, 266

vermiform appendix, 265

Endocardium, I6r primitive, 164

Endochondral ossification, Iog

Endolymph, 574

Endolymphatic duct, 575

sac, 575

Endomysium, 208

Endoneurium, I33, 425

Endoplasm, 46

Endosteum, 195

Endcthelial tube, I64

Endothelium, 7० of Descemet, $55^{\circ}$

Engelmann, showing ciliated epithelial cell, 76

Entoderm, 62 tissue derivations from, $67,296,315$

Eosin, 19 -glycerin, 22

-hrematoxylin stain, 20

Eosinophile granules, I06, I 74

Epiblast, 62

Epicardium, I6I

Epicranium, 198

Epidermis (or cuticle), 382

eleidin, 383

keratin, $38_{3}$

keratohyaline granules, $38_{3}$

mitosis of cells of, $3^{8} 3$

pareleidin, 384

pigmentation of, $3^{8} 4$

prickle cells of, 383

stratum corneum of, 383

cylindricum $\mathrm{cf}, 383$

germinativum of, $38_{2}$

granulosum of, 383

lucidum of, 383

Malpighii of, 382

mucosum of, $38_{2}$

spinosum of, 383

Epididymis, 339

cells of, 339

vas deferens, $34^{\circ}$

vas epididymis of, $34^{\circ}$

vasa efferentia of, 339

Epidural space, 422

Epiglottis, 301

Epimysium, 208

Eipincurium, 425 
Epiphyseal cartilage, 203

Epiphysis of bone, 203

Epithalamus, 522, 530

Epithelium, 70

basal membrané of, 70

cells of, 70

ciliated, 75

classification of, $7 \mathrm{I}$

cuboidal, 72

cuticular membrane of, 7 I

endothelium, 77

follicular, 355

general characteristics of, 70

germinal, 354,378

glandular, 77, 21 2

histogenesis of, 70

intercellular bridges of, 70

lens, 564

membrana propria of, 70

mesothelium, 77

neuro, 77

pigmented, 76

pseudo-stratified, 73

replacing cells of, 73

respiratory, 3I I

simple, $7 \mathrm{r}$

columnar, $7 \mathrm{I}$

pseudo-stratified, 73

squamous, 7 I

stratified, 73

columnar, 74

squamous, 73

transitional, 74

surface, of mucous membranes, 2 I 8

syncytium, 373

tactile cells of, $43 \mathrm{I}$

technic of, 78

transitional, 74

Eponychium, 387

Epoöphoron, 363

Erectile tissue, 349, 376

Ergastoplasm, 213, 272

Erythroblasts, 192

Erythrocytes, I03

Erythrosin, 20

Eustachian tube, 574

Excretory ducts, 214, 276, 282, 329, 364

substances in cells, $46,2 \mathrm{I} 3$

Exoplasm; 46, 82

External arcuate fibres, 490, 499 ear, 572 ; see Ear, external geniculate bodies, 493, 520
External os, 368 spiral sulcus, 579

Extero-ceptors, 436

Extracellular network, 139

Eye, the, 548; see Organ of vision

blood-vessels of, $5^{6} 5$

development of, 569

eyeball or bulbous oculi, 548

eyelid, 567

lacrymal apparatus, 567

lens, 564

lymphatics, 566

nerves of, 566

neurone systems of, 560

optic nerve, 559

technic of, 570

Eyeball (or bulbus oculi), 548

blood-vessels of, 565

chorioid of, $55^{\circ}$

ciliary body of, $55^{2}$

cornea of, 548

development of, 569

iris of, 554

lens, 564

lymphatics of, 566

nerves of, $555,559,565$

retina of, 555

sclera of, 548

technic of, 570

Eyelashes, 567

Eyelid, the, $5^{6} 7$

blood-vessels of, 569

conjunctiva of, 568

epidermis of, 567

glands of, 568

of Mall, 568

lymphatics of, 569

Meibomian glands of, 568

muscles of, 568

nerves of, 569

tarsus of, 568

technic of, 569

FACIALIS (nerve VII), 474, 475, 544

Fallopian tube, 364; see Oviduct

ampulla of, 364

blood-vessels of, 365

coats of, 364

development of, 376

fimbriated extremity of, 364

isthmus of, 364

lymphatics of, 365 
Fallopian tube, nerves of, 365 ovarian extremity, 364 technic of, 366

False corpora lutea, 360

Fascicles of muscle, 207 of nerves, 421,425

Fasciculus arcuatus, 535, 536 of Thomas, 467 inferior longitudinal, 535 medial longitudinal, $467,485,49 \mathrm{I}$, $500,504,507,5$ I 5,5 I 8

perpendicular of W'ernicke, 535 predorsal, 504

retroflexus of Meynert, 53 I

solitarius, 487,489

superior longitudinal, 535 uncinate, 535,536

Fastigio-bulbar tract, 500, 502

Fat, absorption of, 273

technic of, 275

blood supply of, 9I

development of, 90 technic of, 9 I

globules, 237

osmic-acid stain for, 31

subcutaneous, $38_{2}$

tissue, 87

histogenesis of, 87

technic of, 97

Fat-droplets in cells, 46,90

Fat-lobules, 88

Fauces, mucous membrane of, 220

Feltwork of fibres, 440

Female genital organs, 352 pronucleus, 59

Fenestra ovalis, 574 rotunda, 574

Fenestrated membrane, 94

Ferrein, pyramids of, 320

Fertilization of the ovum, 58, $35^{8}$

Fibre proprix of Meyncrt, 535,

$54 \mathrm{I}$

Fibre baskets, 559

systemis, $42 \mathrm{I}$

efferent, 424

main motor, 424

short, 445,468

proprio-spinal, 468

spino-spinal, 46,8

tracts of cord, 459

methods of determining, 459

(ascending), $46 \mathbf{r}$
Fibre tracts of cord, ascending, direct cerebellar, 463

Gowers', 464

long ascending, 445

arms of dorsal root fibres, 46 I

of spinal cord, 459

posterior columns, $443,44 \mathrm{~S}$

spino-collicular, 462

spino-tectal, 465

spino-thalamic, $462,48_{3}, 501$, $504,515,526$

tract of Flechsig, $4 \sqrt{3}_{3}$

tractus spino-cerebellaris dorsalis, $463,483,487,490,507$

ventralis, $463,483,487,501,515$

uncrossed cerebellar, 463,487 , 490,507

(descending), 464

anterior marginal bundle of Löwenthal, 466

anterior pyramids, $464,483,487$, 504,507

antero-lateral, 466

cerebro-spinalis, 464

comma tract of Schultze, 467

crossed pyramidal, 464

descending tract from Deiter's nucleus, 466

direct pyramidal, 465

fasciculus of Thomas, 467

from the interstitial nucleus of Cajal, 465

fundamental, 445, 468

Helweg's, 467

marginal bundles of Löwenthal, 466

origin of tracts, 443

oval bundle of Flechsig, 463

pallio-spinalis, 464,533

pyramidal, 464, 533

rubro-spinal, $465,466,483,487$, $490,504,5$ I 5

septo-marginal, 466,467

short, $445,462,468$

tecto-spinal tract, 465

tractus cortico-spinalis, 464

vestibulo-spinal, 466

Von Munakow's tract, 466

Fibres, afferent nerve, 419 ; see also Nerve fibres

arcuate, 487,489

association of pallium, $42 \mathbf{I}, 533,535$ 
Fibres, calcified, I97

cartilage, 99

commissural, 533

cone, 557

connective-tissue, 82

development of, 82

cortical, 388

dentinal, 228

efferent root, $4 \mathrm{I} 8$

enamel, 233

external arcuate, 490,499

genioglossal, 224

heart miscle, I I 9

hyoglossal, 224

intergeminal, $5^{87}$

internal arcuate, $487,500,502,522$

interzonal, 56

intrageminal, $5^{87}$

involuntary striated (heart) muscle, II9

lens, 5 Jo, 564

Mallory's method of staining connective-tissue, 29,30

mantle, 54

Müller's, 558

nerve, I3I; see also Nerve fibres medullated, 133

non-miedullated, I 32

neuroglia, $\mathbf{1} 43$

of areolar tissue, 87

of bone, IOI

of developing muscle, I 24

of formed connective tissue, 92

of Remak, I33

of Sharpey, 192, 234

olfactory, layer of, 584

perforating or arcuate, of cornea, $55^{\circ}$

projection, $533,535,539,543$

radiate, 292

reticulo-spinal, 467

rod, 557

styloglossal, 224

superficial arcuate, $487,490,499$

tendon, 9 I

tunnel, 583

voluntary muscle, II 4 , I I 7

Weigert's method for staining elastic, 28

method for staining nerve, 3 I

white or fibrillated, 86

yellow or elastic, 86

Fibrillar connective tissue, 86
Fibrillar theory of protoplasmic structure, 44

Fibroblasts, 82

Fibrous cartilage, 99

Field of Forel, 530

Fila olfactoria, 475

Filar mass, 45

Filiform papillæ, 225

Fillet (or medial lemniscus), $462,485,487$, $49 \mathrm{I}, 493,504$

Filum terminale, 442

Fimbria, 533

Fissure, anterior median, 448 chorioid, 570

Fixation, 5

by injection, 6

in toto, 6

Fixatives, 6, 7, 8

Fixed cells, 55I

Flechsig, oval bundle of, 467

myelogenetic method of, 459, 54I

tract of, 463

Flemming concerning cell-division, 53

Flemming's fluid, 7

Foam theory of protoplasm structure, 44

Fœtal cells, 3 I 2, 3 I 6 structures, appendix epididymidis, 342

of genital system, 342,363

testis, 342

ductus, aberrans Halleri, 342

organ of Giraldès, 342

paradidymis, 342

Foliate papillæ, 587

Follicle, Graafian, 354; see also Graafian follicle

Fcllicles, agminated, 260 solitary, 253

Follicular cavity or antrum, 355

Folliculi linguales, 180 ; see Tonsils

Fontana, spaces of, 554

Foramen cæcum lingui, I 80

Forebrain (prosencephalon), 4I 7, 522 diencephalon (thalamencephalon), 4r 7, 522

epithalamus, 523

hypothalamus, 523

thalamus, 523

interbrain, 522

section through junction of midbrain and thalamus, 524 
Foregut, the, 243

general structure of walls of the gas-

tro-intestinal canal, 245

œsophagus, 243

stomach, $24 i$

Forel, decussation of, $52 \mathrm{I}$ field of, 530

Formaldehyde, as a fixative, 6 for macerating, 4 -bichromate method, 36

Formalin, commercial, 4

Formalin-Müller's fluid (Orth's), 7

Formed connective tissue, 9I

Fornix, 53I

anterior pillars of, 536

commissure, 533

Fossa navicularis, 352

Fountain-like decussation of Mleynert, 522

Fourth ventricle, $481,487,493$

Fovea centralis, 559

Fraenkel's theory of corpus luteum, 362

Free endings of sympathetic ncrves, $44 \mathrm{I}$

Frozen sections, 15

Fuchsin, I0

Function of cells, 5I

Fundamental columns of spinal cord, 445

Fundus, 248

$$
\text { glands, } 249
$$

Fungiform papillx, 225

Funiculus cuneatus, 462,485

gracilis, 462,485

posterior, $46 \mathrm{I}$

Fusiform lobules, 535

GAGE, showing muscle fibres, i 19

Gage's hrmatoxylin, I 7

Galea capiris, $3+3$

Gall-bladder, 295

Galvanotaxis, 5 I

Ganglia, 418, 419

amphicytes, 427

cerebral, 426

ccrebro-spinal, 418,426 ,

chain, 436

ciliary, 436

Gasscrian, 506

habenularis, 530

of Corti, 583

of Scarpa of VIII., 475, 493

otic, $43^{6}$

peripheral, $43^{5}$

satcllite cells, 427
Ganglia, sphenopalatine, 436

spinal, 426

spiral, $58_{3}$

spirale of V'III., 475,493

structure of, 426

sympathetic, 436

submaxillary, 436

technic for, $4+4$

terminal, 436

vertebral, 436

Ganglion cells, 4 I9

capsule of, 419

cardiac, I6 2

development of, $4 \mathrm{I} S$

Gärtner's canal, 377

duct, 363

Gasserian ganglion, 506

Gastric crypts, 248

glands, 248,249

pits, 248

Gastro-hepatic omentum, 268

Gastro-intestinal canal, general structure of the walls of, $2+5$

Gelatin, 86

carmine for injecting, 25

Prussian blue, for injecting, 25

Gelatinous marrow, 195

substance of Rolando, 449

Gemmules, $5 \circ 9$

Geniculatc body, 493, 520, 524

ganglion of, VII., 474

Genio-glossal fibres, 224

Genital gland, 378

organs, fcmale, $35^{2}$

male, 333

ridge, 378

systenı, 333; sec also Reproduclive system

development of, 376

rudimentary structures connected with development of, 342,363

Genito-urinary system; sec Urinary sys$1 \mathrm{em}, 31 \mathrm{~S}$, and Reprotuclive syslcm, 333

Genuari, line of, 543

(ientian violet, 19

(icnu-facialis, 49)

Germ hill, 356

latyers, 612

tissues deriverl from, 67

primitive, 6$)_{2}$

Gcrminal epithelium, 354,378 
Germinal spot, 357

vesicle, 59,357

Giant cells of Betz, 533, 536

Gianuzzi, crescents of, 222, 278, 302

Giraldés, organ of , 342,377

Gland cells, 2I 2, 2 I 3

Glands, 2I 2

accessory thyreoid, 403

acini of, 2 I 7

adrenal, 4I 2

alveolar saccular, 2I4

compound, 2I 7

simple, 2I 5, 2I 7

alveoli of, $2 \mathrm{I} 7$

Bartholin's, 376

Bowman's, 300

Brunner's, 252, 272

cardiac, 252

carotid, 4 ro

cells of, 2I 2, 2 I 3

ceruminous, 572

classification of, $2 \mathrm{I} 2$

ccccygeal, 4I 1

compound, 2 I 4

corpus luteum, 359

Cowper's, 348

dehiscent, 217

development of, 2I 5

duct, 2 I 5

ductless, 2I 4,2 I 7

Ebner's, 226

epithelium of, 2 I 3

excretory ducts of, 2 I4

fundus, 249

gall-bladder, 295

gastric, 249

general structure of, 2 I 2

genital, 378

giant, 192

hæmolymph, I73

internal secreting, 255,217

interstitial tissue of, 2 I 5

intraepithelial, 340

kidney, 3 I 8

lacrymal, 567

large, of digestive system, 275

Lieberkühn's, 252, 260

lingual, 222

Littré's, 35I, 352

liver, 287

lobes of, $2 \mathrm{I} 4$

lobules, $2 \mathrm{I} 4$
Glands, lymph, I67

Mall's, 568

mammary, 395

Meibomian, 2I 7, 568

mixed, $22 \mathrm{I}$

mucous, $22 \mathrm{I}$

membranes of, 2 I 8

of internal secretion, 2I 5, 2I 7

of the oral mucosa, 220

ovary, $35^{2}$

pancreas, $28 \mathrm{I}$

parathyreoids, 404

parenchyma of, 2 I 5,277

parotid, 277

peptic, 249

pineal, 544

prehyoid, 403

prostate, 347

pyloric, 249

racemose, 2 I 5

reticular, 2 I 7

saccular, 2 I 7

compound, $2 I 7$

simple, 2 I 5

salivary, 276

sebaceous, 35 I, 384,39 I, 568,572

secreting portions of, $2 \mathrm{I} 3$

serous, $22 \mathrm{I}$

simple, 2 I 4

spleen, I $8 \mathrm{I}$

sublingual, 277

submaxillary, 278

sudoriferous, $2 \mathrm{r} 4$

suprahyoid, 403

sweat, 384,567

tarsal, 568

thymus, I 75

thyreoid, 402

accessory, 403

tonsils, 178

tubular, 2I4, 2 I 5

compound, $2 \mathrm{I} 6$

simple branched, 2 I 6

simple coiled, 2 I 6

simple straight, 2 I 5

tubulo-alveolar, 2 I 4

tympanic, 409

Tyson's, 35I

uterine, 367

Glandulæ sudoriparæ, 384

vestibulares majores, 376

minores, 376 
Glandular epithelium, 77, 21 2

Glans penis, $35^{\circ}$

Glenoid ligamients, 205

Glisson, capsule of, $2 S_{7}$

Gliosis, 144

Globus major, 334

minor, 334

pallidus, 535

Glomerulus cf kidney, 320

blood-ressels of, 325

olfactory, $5^{8} 5$

Glosso-pharyngeal (IX. nerve), $474,49 \mathrm{r}$, 496

Glycerin for mounting specimens, 22 jelly, 22

Glycogen granules, 290

Goblet cells, 75, 21 2, 257, 272

Gold chlorid for staining connective tissue cells, 28

Gold-size for glycerin mounts, 22

Golgi cell, Type I., 130, 132

cell, Type II., 13 I , 132, 446, 536

method, bichlorid, 36

chrome-silver, 29

Cox modification, 36

formalin bichromate, 36

mixed, 35

rapid, 35

silver, for nerve tissue, 35

slow, for nerve tissue, 35

muscle-tendon organs of, 434

net, 139

organs of, 434

Golgi-Mazzoni corpuscles, 394, 434

Goll, column of, 459,462

nucleus of, $462,481,487$

Gowers' tract, 464

Graafian follicles, 354

antrum of, 355

corona radiata, $35^{6}$

cumulus ovigerus, $35^{6}$

development of, $35^{6}, 376$

discus proligerus, $35^{6}$

egg nest, 354

epithelium of, 354

follicular cavity of, 355

germ hill of, $35^{6}$

liquor folliculi, 355

nerves of, 363

orum of, 355

I'füger's eggr cords, 354

primitive, 355

39
Graafian follicles, prinitive ova, 354

rupture of, $35^{8}$

stratum granulosum, 355

technic cf, 305

theca folliculi, 356

tunica fibrosa, 356

vasculosa, 356

Graded alcohols, 7

Grandry, corpuscles of, 43 I

Granule theory of protoplasmic structure, 40

Gray matter, 420, 449

rami communicantes, 437

reticular formation, 476,485

Greater omentum, 235

Ground bundles of spinal cord, 445, 468

Grübler's methylene bluc, 3 I, 38

water-soluble cosin, 3 I

Gums, mucous membrane of, 220

Gustatory canal, 587

Gyrus dentatus, 532

hippocampi, 532

of Heschl, 54 I

HABENULA, 530

Hæmalum, Mayer's, I8

Hæmatcin, I 7, 104

Hæmatoidin, crystals of, 360

Hæmatokonia, 108

Hæmatoxylin, 7

and eosin, for staining double, 20

and picro-acid fuchsin, 2I

Delafield's, 17

Gage's, 17

Heidenhain's, I 8

Mallory's stain, 29

Weigert's, 19, 32

Hxmoglobin, 104

Hæmolymph nodes, $x 73$

blood sinuses of, 173

blood-vessels of, 175

cells of, 174

cosinophiles, 177

mast cells, 174

pliagocytes, 174

development of, 175

function of, I 75

hilum of, 173

marrow-lympli, 174

relation of, to lymphatic system, 175

spleno-lymph, 174

technic of, 175 
Hæmolysis, 104

Hair, 387

arrector pili muscle of the, 39 I blood-vessels of, 394

bulb, 387

-cells, $575,58 \mathrm{I}$

cells of the, 389

connective-tissue follicle of, 389

cortex of, 388

cortical fibres of, 388

cuticle of, 388

development of the, 358,395

excretory duct of, 39I

eyelashes, 567

follicle, 387,388

germ, 392

growth of the, 392

hyaline membrane, 389

inner root sheath, 388

cuticle of, 389

Henle's layer of, 389

Huxley's layer of, 389

lanugo, the, 388

lymphatics, 394

medulla of, 387

nerves of, $394,430,43 \mathrm{I}, 440$

outer root sheath, 388

papilla of, 387

prickle cells, 389

root of the, 387

root sheath, 388

sebaceous glands of the, $39 \mathrm{I}$

sebum of the, 392

shaft of, 387

shedding of the, 392

stratum cylindricum, 389

technic of the, 393

vitreous membrane, 389

Halleri, ductus aberrans, 342

Haller's layer, $55^{\mathrm{I}}$

Hamulus, 577

Hardening, 8

Hassal's corpuscles, I 77

Haversian canals, I 89

development of, 202

fringes, 205

lamellæ, I90

spaces, 202

systems, I9I

development of, 202

Hayem's fluid, 63

Head, sympathetic ganglia of, 436
Headgut, 220

mouth, 220

pharynx, 242

teeth, 227

tongue, 223

Hearing, organ of, $57^{2}$; see Ear

Heart, I6I

annuli fibrosi, 162

auricular muscle, $\mathbf{6} 6 \mathbf{I}$

auriculo-ventricular ring, I6I

blood-vessels of, I62

coronary arteries of, $\mathrm{I} 6 \mathrm{I}$

development of, I 64

endocardium of, I6I

epicardium of, $\mathbf{I} 6 \mathrm{I}$

lymphatics of, $\mathrm{I} 62$

muscles of, I6I

myocardium of, 140

nerves of, I 42,440

technic of, $\mathrm{I}_{42}$

valves of, I4I

-muscle, I I9, I6I; see also Involuntary striated muscle

Hecateromeres, 445

Heidenhain, concerning voluntary striated muscle, i I 7

demilunes of, 222

Heidenhain's hæmatoxylin, I8

Heisterian valve, 295

Helicotrema, 578

Heller's plexus, 268

Helweg, tract of, 467

Hemispheres of cerebellum, 500, 507

Hendrickson, concerning coats of liver ducts, 295

Henle, concerning ovum, 363

Henle's layer, 389

loop, 320, 322, 333

sheath, I36, 426

Hensen's cells, 58 I

line, II 5

Hepatic artery, 289

cells, 291

cords, $29 \mathrm{I}$

cylinders, 297

duct, 289

Hermann, showing centrosome, 49

Heschl, transverse temporal gyri of, 54I

Heteromeres, 445

Hilum of liver, 287

of kidney, 3I 8

Hindbrain, 417, 479 
Hindbrain (rhombencephalon), bulb, 479 cerebellum, 4I \&, 499

medulla oblongata, $4 \mathrm{r} \delta, 479$

section of, through, at level of junction of pons and cerebellum, and entrance VIII, vestibubular, 499 through roots of VI, abducens, and VII facial nerves, 500

through roots of $\mathrm{V}$, trigeminus nerve, 502

His, marginal veil of, $4 \mathrm{I} 7$

myclospongium of, $4 \mathrm{I} 7$

spongioblasts of, $4 \mathrm{I} 7$

Histogenesis, 67

Holmgren, showing trophospongium, 46

Horizontal cells, $538,55^{8}$

Howship's lacunæ, 199

Huxley's layer, 389

Hyaline cartilage, 98

Hyaloid canal, 565 membrane, $5^{6} 5$

Hyaloplasm, 44, I 24

Hydatid of Morgagni, 342 stalked, 342

Hydrochloric acid for decalcifying, Io

Hyoglossal fibres, 224

Hypoblast, 62

Hypoglossal (XII nerve), 48I, 485, 487, 497

Hyponychium, 387

Hypophysis cerebri, 407; see also Pituitury body

Hypothalamus, 522, 533

INCISURES of Schmidt-Latncrmann, $\mathbf{1 3 5}$

Incremental lines of Schreger, 232

Indirect cell division, 53; sec Milosis

Inferior brachium quadrigeminum, 520 cerebellar pedunclc; sce Restijorm body

colliculi, 417

Infundibula, 3 10

Injection, 25

apparatus, 25

double, 26

separatc organs, 26,

whole animals. 26

Innervation of muscles, 434, 44 I

Inokomma, I 7

Interalveolar connective tissue, 313

Intcrarticular cartilages, 205

Interbrain, 416,522
Interbrain, epithalamus, 523

hypothalamus, 523

thalamus, 523

Intercallated discs, I 2 I

Intercellular bridges of epithclium, 70, 73

bridges of muscle tissue, 112

substance, 68

of connective tissue, 86

silver-nitrate method of staining, 28

Interfilar mass, 45

Intermcdiate cartilage, 199

lamellæ, I9 I

neurones, $4 \mathrm{I} S$

Internal arcuate fibres, $487,500,502,522$ capsule, 530

ear, 574 ; see also Ear, internal

nuclci of cerebellum, 502, 507

Internode, I 34

Intero-ceptors, 436

Interradiary plexus, $54 \mathrm{I}$

Intersegmental neurones, $421,48_{3}, 487$, $490,499,501,504,507,515,520$, $524,530,536$

tracts, 468

Interstitial lamellæ, r9 I

nucleus of Cajal, 465, 485, 491, 500, 504

Intestine; see Small intestine, 255; Large inlesline, 263

Intestines, devclopment of, 296

Intima, 154

of arteries, 154

of lymph vessels, 165

of veins, 158

Intracartilaginous ossification, 199

Intracellular canals, 46

Intrafascicular connective tissue, 208

Intramembranous ossification, 197

Intranuclcar network of typical cell, 48

Inverse fernicnt, 273

Invertin, 273

Involuntary striated muscle (heart), i I 9 Cohnhcim's ficld, 120

development of, $\mathrm{I} 24$

intercallated discs of, $12 \mathrm{r}$

McCallum's vicws, 120

membrane of Krause, 120

muscle columus of Kölliker, 120

nerves of, 440

sarcoplasm of, 120

technic of, 125 
Involuntary smcoth muscle, I I intercellular bridges of, I 12 nerves of, 440

Iodine, to remove mercury, 9

Iris, the, 554

greater arterial circle, 566

layers of the, 554

lesser arterial circle, 566

muscles of the, 555

pigmentation of, 554

Irritability of cells, 5 I

Islands, blood, ro8, I6 3 of Langerhans, 285

Isolated smooth muscle cells, I I 3 technic of, 124

Isotropic line, I I 5 substance, I I 5

Isthmus, 4I 7, 5 I 5 section through, at exit of $I V$, trochlearis, nerve, $5^{\mathrm{I}} \mathrm{5}$

Iter, 4I $7,464,5$ I 5,5 I 7

JENNER's blood stain, 3I

Joint capsule, 205

stratum fibrosum, 205

synoviale, 205

synovial membrane, 205

Joints, 204; see Articulations

Jugular ganglion of $\mathrm{X}, 474$

Juxta-restiform body, 5CI

KARYOLYSIS, 383

Karyoplasm, 46

Karyosomes, 48

Katabolism, 50

Keratin, 383

Keratohyaline granules, 383,387

Kidney, the, 3 I 8

arteriæ arciformes, 326

rectæ, 328

blood-vessels of, 325

Bowman's capsule of, 320

capillaries of, 328

columns of Bertini, 320

convoluted tubules of, 320

cortex of, $3 \mathrm{I} 8$

cortical pyramids of, 320

development of, 321,376

duct of Bellini, $32 \mathrm{I}$

epithelium of, 324

glomerulus of, 320

Henle's loop, 322, 323
Kidney, interlobar arteries of, 326

hilum, of, 3 I 8

labyrinths of, 320

lobulated, 318

location of tubules in, 324

lymphatics $\mathrm{cf}, 328$

main excretcry duct of, 329

Malpighian body, 320

pyramid, 320

medulla of, 3 I 8

medullary (or Malpighian) pyramid, 320

rays, 320

nerves of, 328

papillæ of, $32 \mathrm{I}$

pelvis of, 3 I 9

pyramids of Ferrein, 320

renal artery; 318

corpuscle, 320

vein, $3 \mathrm{I} 8$

renculi or lobes of, 318

septa renis, 320

single lobe of, 3 i 8

stellate veins of Verheyn, 328

technic of, $33 \mathrm{I}$

ureter, 3I8, 329

uriniferous tubule, 320 ; see also $U r i$ niferous tubule

Kidney-pelvis, 329

calyces of, 329

development of, 376

technic of, $33 \mathrm{I}$

Kölliker, muscle columins of, I 16

concerning bronchi, 304

showing Golgi cell type II, I3 I spleen cells, 184

Krause, ellipsoid, 557

end-bulbs, $227,350,394,567$

line of, I 5

membrane of, II 7 , I 20

Kupffer, cells of, 292

LABIA minora, sebaceous glands of, 384

Labyrinth, membranous, 574

osseous, 574

Lacrymal apparatus, 567

canal, ${ }_{567}^{67}$

gland, 567

blood-vessels of, 567

lymphatics of, $5^{67}$

nerves of, 567

technic for, $57^{\circ}$ 
Lacrymal apparatus, nasal duct of, 567 sac, 567

Lacteals, 240

Lacunx, IoI

origin of, $\mathrm{I} 9 \mathrm{~S}$

Laguesse, concerning derelopment of pancreas, 297

concerning lung lobules, 310

I.amellæ, circumferential, I9I

ground, rgi

Haversian, rgc

intermediate, I9I

interstitial, $\mathrm{I} 9 \mathrm{I}$

of bone tissue, ror

Lamina, bony spiral, 577

citrea, $55 \mathrm{I}$

cribrosa, 548,560

fusca, 548

membranous spiral, 577

reticularis, $58 \mathrm{I}$

suprachorioidea, $55 \mathrm{I}$

Laminxe of cerebellum, 507

Langerhans, cell islands of, 285

centro-acinar cells of. 283

centro-tubular cells of, 283

Langley and Sewell ccncerning sccretion, 272

Lanugo hairs, 387

Large intestine, 263

Auerbach's plexus, 264, 270

blood-vessels, 268

coats of, $26_{3} 3$

development of, 296

gland tubules, 263

Heller's plexus of, 268

linex coli, 264

lymphatics, 270

nerves, 270

plexus of Meissner, 263, $27 \mathrm{I}$

myentericus, 270

technic of, 275

Larynx, the, 3 or

bloorl-vessels of, 303

cartilages of, $30 \mathrm{r}$

arytenoid, 3 or

cricuirl, 301

epiglottis, 301

Santorini's, 30I

thyroid, $30 \mathrm{I}$

W'risburg's, 3 or

cells of, 3 or

develop, ment of, 315
Larynx, epithelium of, 30 I

lymphatics, 304

nerves, 304

perichondrium, $30 \mathrm{I}$

technic, 304

vocal cords, $30 \mathrm{I}$

Law of Wallerian degencration, I+I

Layer of Weil, 228

Lecithin, 4I 3

Lemniscus, bulbo-thalamic, 524

lateral, 49 I, 493, 499, 501, 504, 5 I5

medial, $462,485,487,49 \mathrm{I}, 493,504$

Lenhossék, concerning ciliated epithelium,

Lens, 565

epithelium, 565

fibres, 565 .

hyaloid membrane, 56,5

invaginaticn, 570

suspensory ligament; $5^{65}$

vesicle, $57 \mathrm{I}$

zonula ciliaris, 565

zonule, of Zinn, $5^{6} 5$

Lenticular capsule, $56_{5}$

nucleus, 532, 535

Leopold, concerning pregnant uterus, 370

Leucocytes, I05

acidophile, ro6

basophile, 106

granular, ro6

lymphocytes, 105

migratory, 259

mononuclear, 105

neutrophile, 106

of milk, 400

polymorphonuclear, to 5

polynuclear, 105

transitional, 105

Lewis, concerning shitpe of blocd-rells, $\mathrm{IO}_{3}$

Lieberkühn, crypts of, 252, 260

glands of, 252

Ligament, glenoid, 205

spiral, 577

structure of, $9 \mathrm{I}$

suspensory, 564

Ligamentum nuchir, () 3

pectinatum, 554

I inex coli, 264

I ine of Gennari, 543

of Batillarger, 5.4

Lines of Retrius, 2.3 .3

Lingual glinds, 222

tonsils, 180 
Lingualis, genic-glossus fibres of, 224 hypoglossus fibres of, 224 longitudinal fibres of, 224 styloglossus fibres of, 224

Linin, 48 transverse fibres of, 224

Lipoid granules, 4 I 2

Liquor ferri sesquichlorati, 28 folliculi, 355

Lissauer, zone of, 443,450

Littré, glands of, 35I, 352

Liver, the, 287

bile duct of, 290

blood supply of, 288

capillary network, 289

capsule of Glisson, 287

cells of, $29 \circ$

of Kupffer, 292

central vein of, 289

compared with other compound tubular glands, 293

connective tissue, 287

cords of liver cells, $29 \mathrm{r}$

development of, 297

ducts, 289

common, 294

cystic, 294

hepatic, 289, 294

glycogen granules, 290

Heisterian valve, 295

hepatic artery, 289

cords, $29 \mathrm{I}$

duct, 289

hilum, 287

intralobular secreting tubules, 290

lobes of, 287

lobules, 288

lymphatics, 294

main ducts, 289,294

nerves, 294

portal canal, 290

vein, 289

radiate fibres, 292

reticulum, 292

septa, $2 S_{7}$

sublobular vein, 289

technic of, 297

tubules of, 293

Lobulated kidney, 3 I 8

Löwenthal, anterior marginal bundle of, 466

Longitudinal cleavage, 55
Longitudinal fasciculus, 49I.500, 504, 5 I 5,526

Loops of Henle, 320, 322, 323

Loose (areolar) connective tissue, 87

Lugol's solution, 28

Lumbar enlargement of spinal cord, 443 segments of cord, 443

Lungs, the, 308 air cells, 3 ro sacs, 3 Io vesicles, 3 Io

alveolar bronchi, 309 ducts, 3 Io

passage, 3 II

sacs, 309

alveoli of, 3 I I

blood-vessels of, $3 \mathrm{I} 3$

bronchial artery, $3 \times 3$

system, 3 I 3

capsule of. 308

cells of, 3 I I

development of, $3 \times 5$

epithelium of, 3 ro

fœtal cells of, 3 II

infundibula of, 3 Io

interalveolar connective tissue of, 3 I 3

lobes of, 308

lobules of, 308

lymphatics of, 3 I 5

nerves of, $3 \mathrm{I} 5$

parietal pleura, 308

pulmonary artery, 308

Iobule, 308

pleura of, 308

respiratory bronchi, 310

cells, 3 I I

epithelium, 3 I I

septa of, 308

technic of, $3 \mathrm{I} 7$

terminal bronchi of, 3 to

Lunula, 387

Lutein cells, 359

granules, 359

Luteum, corpus, 359

Luys, nucleus of, 523

Lymph, capillaries, I $_{5}, 270$

glands, I67; see Lymph nodes

nodes, 167

blood-vessels of, r $7 \mathrm{c}$

capsule of, 167

chains of, 167

connective tissue of, $\mathrm{r} 68$ 
Lymph nodes, cords of, I 69

cortex of, 169

development of, I 7 I

germinal centre of, I69

lymphatics of, $r 7 \mathrm{r}$

medulla of, $x 69$

nerves of, I7 I

nodules of, I 69

reticular connective tissue of, I 70

sinuses of, 169

technic of, 172

nodule, 95, I69, 242, 265

germinal centre of, I 69

paths of the eye, 566

spaces, I 65

pericellular, 165

perivascular, 159

vessel system, I 64

capillaries of, 165

development of, $\mathrm{x} 66$

lymph capillaries, 165

spaces, I 65

relation of, to hæmolymph node,

I 75

stomata of, 165

technic of, 165

vessels, coats of, I 65

structure of, I 64

Lymphatic organs, 167

development of, $171,175,177,180$, I 86

hæmolymph nodes, 173

lymph nodes, 95, 167

spleen, $\mathrm{r} 8 \mathrm{r}$

technic of, I 72, I75, I78, I 81, I 87

thymus, 175

tonsils, 178

tissue, 167,168

Lymphocytes, 105

Lymphoid cells, r 70

tissue, 170

Macerating fluids, 4

Maceration, 4

Macrocytes, 103

Macro-nucleus, 57

Macula acustica, 575

lutea, 557

fovea centralis, 559

Male genital organs, 333

pronucicus, 54

Mall, glands of, 568
Mall, concerning development of fibrillar connective tissue, $\mathrm{S}_{2}$

Mallory's aniline blue stain for connective tissue, 30

phosphomolybdic acid hæmatoxylin stain for connective tissue, 29

phosphotungstic acid hæmatoxylin stain for connective tissue, 30

Malpighian bodies, $\mathrm{I} \mathrm{S}_{2}$

body of kidney, 320

development of, 321, 376

pyramid, 320 ; see Kidney

Mamillo-thalamic tract, 524

Mammary gland, 395

active, 398

alveoli of active, $39 \mathrm{~S}$

ampulla of, 397

blood-vessels of, 400

cells of, 398

colostrum corpuscles, 400

development of, $40 \mathrm{r}$

ducts of, 397

of nipple, 397

inactive, 397

interlobar septa of, 397

interlobular septa of, 397

lobular ducts of, 397

lymphatics of, 400

milk, 399

nerves of, 400

secretion of, 399

structure of, 395

technic of, 4 or

Mantle fibres, 54

Marchi's method for staining degenerating nerves, 34

Busch's modification of, 35

Maresh's modification of Bielschowsky's stain for connective tissue, 3 I

Marginal bundle of Löwenthal, 460 veil of His, 4r 7

Marrow, r92; see Bone marrow lymp̨h nodes, $x 74$

Martinotti, cells of, $53^{6}, 539$

Mast cells, 85, 174, 192, 193

Matrix of nail, 385

Maturation, $5^{8}$ of ovuni, $5^{8}, 35^{8}$

of spermatozoïn, $5^{8}$

Mayer's hicmalum, 18

McCallum, concerning heart muscle, 120

Media of arteries, 155 
Media of lymph vessels, ${ }^{6}{ }_{5}$ of veins, 158

Medial eminence, 48I, 489 fillet, $462,485,487$

Median center of Luys, 524, 530 lemniscus, $462,485,487,526$ raphé, $488,490,507$ septum, posterior, 448

Mediastinum testis, 333

Medulla oblongata, 4I 7, 479 accessory olivary nucleus, 490 olives, 490

afferent cerebellar neurones, 474, 477, $490,493,501,504,507$

roots, $483,485,489$ secondary tracts of, 483,485 , $489,49 \mathrm{I}$

terminal nuclei of, $483,485,489$, 49 I

ala cinerea, $48 \mathrm{I}, 489$

anterior fissure, $48 \mathrm{I}$

ground bundles, $483,487,489$, 499

pyramid, 48I, 499

arciform (arcuate) mucleus, 490

arcuate fibres, 487,489

area acustica, 482

auditory nerve, 493

central canal, $48 \mathrm{I}$

gelatinous substance, 487

gray matter, $483,486,489$

tegmental tract, 490,499

cerebellar peduncles, 499

cerebello-olivary fibres, 490

chorioid plexus, 487

clava, 48I

cochlear nerve, 475, 489, 49 I, 493 nuclei, 493

column of Burdach, 459, 462, 48 I, 487 of Goll, 459, 462, 48I, 487

compared with spinal cord, $48 \mathrm{I}$

corpus restiforme, $48 \mathrm{I}, 485,489,490$, 499, 5 ०, 504

crossed pyramidal tract, 464,483

cranial nerves of, $479,480,495$

cuneus, $48 \mathrm{I}$

decussation of fillet, 485

of pyramids, $48 \mathrm{r}$

Deiter's nucleus, 493, 499

tract, 487

descending root of fifth nerve, 483 , 487
Medulla oblongata, descending or spinal root of vestibular portion of - eighth nerve, 493 suprasegmental paths, 485

tract from Deiter's nucleus, 483 , 49I, 499

from the vestibular núclei, 490

development of, 4 I 6

direct cerebellar tract, 483

pyramidal tract, 483

funiculus, 483

horns, dorsal, $48 \mathrm{I}$

nucleus of ninth cranial nerve, $49 \mathrm{I}$

of tenth nerve, $48 \mathrm{I}, 485,489$

dorsal external arcuate fibres, 490

spino-cerebellar tract, 483,487 , 490

efferent peripheral neurones, 483 , 485,487

suprasegmental neurones, 485,489 , 499

eminentia hypoglossi, 489

external arcuate fibres, $487,490,499$

fasciculus cuneatus, $48_{3}$

gracilis, 483

solitarius, 489 , 49 I

fillet or medial lemniscus, 462,485 , 487

formatio reticularis, $485,489,49 \mathrm{I}$

fourth ventricle, $48 \mathrm{I}, 487,493$

funiculus cuneatus, 485

gracilis, 485

gelatinous substance of Rolando, 483

general structure of, 479

genu facialis, $48 \mathrm{I}$

Gowers' tract, 483

gray reticular formation, 485,487

internal arcuate fibres of, 487,500 , $5 \circ 2,522$

intersegmental neurones, 483,487 , 489,499

lateral fillet, 49 I, 493

lemniscus, 49 I, 493

longitudinal fasciculus, 49I, 500 median lemniscus, $462,485,487,489$, 49 r, 493

longitudinal fasciculus, 49I, 499

raphé, 488,490

nuclei arcuati, 490

of the floor of the ventricle, $48 \mathrm{I}$

laterales, 490 
Medulla oblongata, nuclei of posterior columns, $48 \mathrm{I}, 495$

nucleus, abducentis, 482

accessory cuneate, 487

alæ cinereæ, 485

ambiguus, 489

arcuatus, 490

commissuralis, 487

cuncatus, $48 \mathrm{I}, 4 \delta_{5}, 487$

gracilis, $48 \mathrm{I}, 487$

hypoglossi, 48r, 485

of acoustic nerve, $4 \delta_{2}, 489,493$

of the column of Burdach, 462,48 I, 487

of the column of Goll, $48_{1}, 48_{7}$

of the fifth spinal nerve, $48_{3}, 4 \delta_{5}$, 489

of origin of eleventh cranial (spinalaccessorv) nerve, $48_{3}$

of origin of twelfth cranial (hypog!ossal) nerve, $4 \delta$ I, 485,487

of vagus nerve, 482,489

olives, 489

clivary nucleus, $487,490,49 \mathrm{I}$

olivo-rerebellar fibres, $49 c, 493$

pallio-spinal tract, $48_{5}$

peduncles of, 499

peripheral neurones of, 479

plexus chorioideus, 487

pons Varolii, 482, 499

posterior columns of, 487

longitudinal fasciculus, $49 \mathrm{I}$

septum, 48 r

predorsal tract, 49 I

pyramidal decussation, 485,487

tracts, 485,487

raphé, 488,490

restiform body, $481,485,489,490$

reticular formation, $485,487,489$, 490,491

root fibres of spinal $\mathrm{V}, 4^{8} 3$

and nuclcus of origin of sixth

(abducens) cranial nerve, 499

of seventh (fucial) cranial nerve, $49 \mathrm{I}$

and nuclei of eightle (auditory) cranial nerve, 49) 3

of ninth (glosso-phuryngeal) and tenth (vagus) cranial nerves, 49) I

of cleventh (spinal-accessury) crianial norve, $4^{8} 3$
Medulla oblongata, root of twelfth (hypoglossal) cranial nerve. 485

rubro-spinal tract, $48_{3}, 48_{7}, 490$

secondary cochlear tract, 493

vestibular tract, 493

sensory tract of fifth nerve, $48_{3}$, 487

section through decussation of fillet, 485

entrance of cochlear branch of eighth, 49 I

lower part of inferior olivary nucleus, 487

middle of olivary nucleus, 49 I

pyramidal decussation, 483

sensory decussation, 485

roots and motor nuclei of the fifth nerve, 499, 500, 502, 506

solitary fasciculus, 489 , $49 \mathrm{I}$

spinal (descending) root of fifth cranial nerve, 483,487

$\mathrm{V}, 475,48_{3}, 48_{5}, 489,493,501,504$, 506

spino-cercbellar tract, 483,487 , 489

-tectal tract, $48.3,490$

-thalamic tract, 489

stria medullares, 49 I

technic of, 482

tecto-spinal tract, 483,487

tegmentum, 499

terminal nucleus of the descending (sensory) root fibres of the fifth nerve, $48_{3}$

tract of Gowers, 483

from interstitial nucleus of Cajal, 465

of Helweg, 460,407

of Iöwenthal, 4606

tractus spinalis trigemini, 483

trapezius, 491

trigonum lyypoglossi, 481,489

vagi, 481

tuberculum cincreum, .181

ventral external arcuitt: fibres, 490

horn, 485,487

spino cerebellar tract, $4^{8} 3$

vestibular nerves, 175

vestibulo-spinal trat,$+8_{3}$

Medullary laminit, 530

lyramid (Malpighian), 320 
Medullary rays, 320 sheath, 134

Medullated axones, $\mathrm{r} 33$ nerve fibres, Weigerts' stain for, 32

Megalocytes, I 84

Meibomian glands, 217,568

Meissner, corpuscles of, $350,394,432$ plexus of, $254,262,263,271,436$

Melanin, I3I

Membrana chorii, 37 I elastica externa, ${ }_{5} 6$ interna, 154

limitans olfactoria, 300

preformativa, $24 \mathrm{I}$

propria, 70

tectoria, 582

Membrane, basal, 70 cuticular, 7 r, 240 mucous, 218 of Bowman, 548 of Descemet, 550 of Krause, II 7, I 20 of Reissner, 579 peridental, 234 serous, 165 synovial, 205

Membranes of brain and cord, 422

Membranous cochlea, 578

labyrinth, 574

spiral lamina, 577

ligament, 577

Meninges, 422

Meniscus, tactile, 43 I

Menopause, 40I

Menstrualis, decidua, 369

Menstruating uterus, 368

Menstruation, 369

Mercuric chlorid as a fixative, 8

Merkel's corpuscles, 43 I

Mesencephalic root of fifth (trigeminus) cranial nerve, 475

Mesencephalon, 4I7

Mesenchyme, 77

Mesentery, 268

Mesoappendix, 265

Mesoblast, 62

Mesoderm, 62 tissue derivations from, 68, 1 22, 296, 315,377

Mesonephros, 364 derivations from, 342,364

Mesothelium, 70, 77
Metabolism of cells, 50, 2I3

Metanephroi, 377

Metaphase, 55

Metaplasm, 46

Metathalamus, 523

Methods for studying fibre tracts of the cord, 459

atrophy, 459

axonal degeneration, 459

comparative anatomy, 459

myelogenetic, 459

physiology, 46r

secondary degeneration, 459

von Guddens, 459

Methyl blue, 19

green, 19

violet, $\mathrm{rg}$

Methylene blue, 38

Meynert, decussation of, 522

fasciculus retroflexus of, $53 \mathrm{I}$

fibræ propriæ of, 535

radiations of, $54 \mathrm{I}$

Micron (micromillimeter), I 5

Microsomes, 44

Microtome, I4

Midbrain, 4 I6, 5I 7

anterior corpora quadrigemina of, $5^{17}, 5^{22}$

aqueductus Sylvii, $5^{\mathrm{I}} 7$

basis pedunculi, 5 I 7

brachia conjunctiva, 520

cerebral peduncles, $5 \mathrm{I} 7,52 \mathrm{I}$

colliculi, 4I 7, 5 I 7

corpora quadrigemina, $4 \mathrm{I} 7,522$

cranial nerves III. and IV., 520

crura cerebri, 5 I 8

decussation of Forel, $52 \mathrm{I}$

of Meynert, 522

Edinger-Westphal nucleus, 518

fourth cranial nerve, 520

geniculate bodies of, 520

inferior brachium quadrigeminum, 520

colliculi, 520

internal arcuate fibres, 522

iter, $5 \mathrm{I} 7$

lateral peduncular fillet, $52 \mathrm{I}$

lemniscus, 462,520

medial accessory fillet, 52 I

mesencephalic root of fifth nerve, 475

520

optic nerve, 475 
Midbrain, pes pedunculi, 521

posterior commissure, 522

corpora quadrigemina, 517

longitudinal fasciculus, $52 \mathrm{I}$

red nucleus of, $5^{1} \overline{7}, 520,52 \mathrm{I}$

reticular formation, 520

root fibres and nucleus of origin of third (oculomotor) cranial nerve, $5 \mathrm{I} 7$

section through exit of third (oculomotor) cranial nerve, $5 \mathrm{r} 7$

spino-tectal tract, 522

substantia nigra, 517,521

superior cerebeliar peduncles of, 521 colliculi, 520,522

tegmentum, 4r6, 5 I8

Middle ear, 573; see Ear, middle

Midgut, 255

small intestine, 255

Migratory leucocytes, 259

Milk, 399

cells of, 399

colostrum corpuscles of, 400

teeth, 239, 24 I

Minot, concerning endothelium and mesothelium, 77

concerning the pregnant uterus, 370

Mitochondria, 213

Miton, 45

Mitosis, 53

anaphase, $5^{6}$

metaphase, 55

method of demonstrating by Flemming's fluid, 8

prophase, 53

technic for, 63

telophase, 56

Mitotic figure, 55

Mitral cells, 584

Mixed spinal nerve, 424

Modiolus, 576

Mollier, concerning splenic pulp), 185

Monaster, 54, 55

Mononuclear cclls, 184

leucocytes, ro5

Monophyletic theory of blood cells, 109

Monosynaptic are, 460

Mordanting, 32

Morgagni, hydatirl of, 342

Mossy cills, 143

Motion of cells, 5 I

Motor cells of anterior horns, 444
Motor decussation, $4 \mathrm{~S}_{3}$

end plate, 442

nuclei, $4+\mathrm{I}$

peripheral nerves, 44I

precentral area, $5+3$

Mounting, 22

celloidin specimens, 23

in balsam, 23

in glycerin, 22

paraffin sections, 23

Mouth, the, 220

blood-vessel of, 222

end bulbs in mucous membrane, 433

glands of, 220

lymphatics of, 222

mucous membrane of, 220

nerves of, 222, 226, 432, 587

technic of, 223

Mucin, 82, 22 I

Mucous glands, 22 I

membranes, 2 I 8

end bulbs in, 433

general structure of, 218

of alimentary tract, 219

tactile cells of, 43 I

corpuscles of, 432

tissue, 82

Mucus, 213,22I

Müller, cells of, $55^{8}$

circular muscle of, 553

fibres of, $55^{8}$

Müller's fluid, 7

Müllerian ducts, 348

Multinuclear cells, 184

Multipolar nerve cells, I27, 444

Muscle, arrector pili, $39 \mathrm{r}$

auricular, $16 \mathrm{r}$

cclls, in

ciliary, 553

circular, of Müller, 553

columns of Kölliker, Ir6, Ir 7

discs, II 5

fibrilla, I I 4

nuclei, I I 2

of sweat glands, 395

spindles, or neuro-muscular bundles,

434

tendon junction, 208, 434

organs of Golgi in, 434

peripheral nerve terminations in, 434

tissuc, I I I 
Muscle tissue, classification of, I I I

development of, I 22

heart, II9

histogenesis, I I 9

intercellular bridges of, I I 2

involuntary smooth, II I

striated, II 9

technic cf, I 24

voluntary striated, I 14

anisotropic substance, II 5

Cohnheim's fields, i 6

cross fibre nets of, I 7

end bulbs of, 434

ergastoplasm, I 14

Heidenheim's scheme of structure of, II 7

Hensen's line, I I 5

inokomma, II 7

isotropic substance, I I 5

Krause's line, I 15

mesophragma, I I 7

muscle columns of Kolliker, $\mathrm{x}$ \%6, II7

discs, II 5

spindles, 434

nerves, terminations in, 434

Pacinian corpuscles of, 434

Ruffini's theory of nerve terminations in, 434

sarcolemma, II 4

sarcoplasm, I I 5

sarcous element of Bowman, i 16

technic of, I 24

telophragma, I 7

ultimate fibrillæ, I I 5

white and red fibres, II 7

Muscles, voluntary, 207

capsule of, 207

endomysium, 208

epimysium, 207

fascicles of, 207

growth of, 209

intrafascicular connective tissue of, 208

perifascicular sheath, 208

perimysium, 208

Muscular system, 207

blood-vessels of, 2 ro

lymphatics of, 2 IO

nerves of, 2 I I

technic of, 2 I I

tendons of, $9 \mathbf{I}, 208$
Muscular system, voluntary muscle, 207

Muscularis mucosæ of mucous membranes, 2 I 8

Musculature of intestine, I13, 263, 264

Myelin, I34

Myeloarchitecture, 54 I

Myelocytes, I92

Myelogenetic method for determining fibre tracts of cord, $459,54 \mathrm{I}$

Myeloplaxes, I93

Myelospongium of His, $4 \mathrm{I} 7$

Myentericus, plexus, 270

Myoblast, I 22, 2 IC

Myocardium, I6I

primitive, $\mathrm{I} 64$

Myofibril, i 7

Myotome, 122

Myxœdema, 40.3

NАвотні, ovula, 368

Nails, the, 385

cells of the, 387

development of, 396

eponychium of, 387

growth of, 387

hyponychium, 387

keratohyalin of, 387

lunula of, 387

matrix of, 385

prickle cells of, 387

structure of, 385

technic of, 387

Nail-bed, 385

root, 385

groove, 385

wall, 385

Nares, 299

accessory nasal sinuses, 299

cells of, 300

basal, 300

olfactory, 300

sustentacular, 300

development of, 3 I 5

glands of Bowman, 300

membrana limitans olfactoria, 300

structure of, 299

olfactory region, 299

respiratory region, 299

vestibular region, 299

technic of, 304

zone of oval nuclei, 300

of round nuclei, 300 
Nasal duct, 567

Nemileff, showing amitosis, 53 scheme of medullated nerve fibre, 137

Necpallium, 532, 533, 535 association fibres of, 535 fibræ of Meynert, 535

Nerve cells, I26; see also Neurone amacrine, 558,586 amphicytes, 427 anterior horn, $4+4$ association, 514, 539

basket, 222, 510

Betz', 533, 536, 539

bipolar, 127, 428

brush, 585

Cajal's, 539

caryochromes, I29, 5 I 2

cerebro-spinal ganglia, 486

column, 444, 45r, 469

cone-bipolar, 557

cone-visual, 557

efferent projecticn, 539

cpendymal, 48

extrinsic, 443

ganglion, $418,426,443,539$

giant, of Betz, 533, 536, 539

glia, I44

Golgi, Type I, I30, I 32

Type II, I3 I, I32, 446, 536

hecateromeric, 444,445

heteromeric, 445

horizontal, 538,558

in gray matter of cord, 444

intrinsic, 443

inverted pyramidal, 536

large granule cells, 5 I 2

marginal, $45^{\circ}$

Martinotti's, 536

mitral, 584

mossy, 143

motor, of the anterior horn. I 29, 444

Müller's, $55^{8}$

multipolar, 127,444

neurilemma, 4 I?

neuroblasts, $126,142,418$

neuroglia, $143,418,453,514$

nuclcus of, 127

of motor arca of cerebral cortex, 539

outside the spinal corcl, 444

peripheral motor, $44 \mathrm{I}$

scensory, 426

jigment in, 131
Nerve celis, polymorphous, 536,539

Purkinje, $5 \times 9$

pyramidal. 536

inverted, 536

rod-bipolar, 557

rod-visual, 557

root, $441,444,45 \mathrm{I}$

satellite, 427

small granule, $5^{\mathrm{I}} 2$

somatochromes, I 29

spider, I 4.3

spinal ganglion, 426, 443

spongioblasts, I $43,4 \mathrm{I} 7$

stellate, 5 Io, 5.36

sympathetic ganglion, 436

tautomeric, 445,463

unipolar, I 27,428

endings, 429; see Peripheral nerve terminalions

fibres, I 3 I

afferent, 4 I9

root, 420

association, $421,533,535$

climbing, 510,513

commissural, 533

cone, 557

deep tangential, 54 $\mathrm{I}$

efferent root, 4 I 8

fastigio-bulbar, 500, 504

felt works of, $44^{\circ}$

layer of, of retinit, $55^{8}$

lens, 564

medullated, I 33

of cercbellum, 5 I I

mossy, 5 I 3

motor end plates of, 442

neuroglia, 143

non-medullated, I 32

of Bergmann, 5I4

of Rinlak, 133

of trapezius, 5or

origin of, of white malter of corrl,

443

pallial, 533

pallio-pontile, 501, 504

pallio-tectill, 535

pallis thalamic, 535

parillel, of the cercbellum, 512

perpendicular pontile, $499,501,507$

prestganglionic, 437

pregranglionic, 437

projection, 533 
Nerve fibres, rod and cone, 557 superficial tangential, 54I

terminations, 430

motor, 440

sensory, 430

tissue, I 26

Golgi methods of staining, 35

neuroglia, I4 2

neurone, $\mathbf{I} 26$

axone, 132

cell body, I 26

dendrites, $13 \mathrm{I}$

protoplasmic processes, I 3 I

technic for, 38, I 44

Nerves, cranial, table of, 545, 546

I (olfactorius), 475, 532

II (opticus), 473, 520, 524, 559

optic decussation of, $526,5^{61}$

chiasma, 526, 56 I

tract, $520,526,536$

motor and sensory nuclei of, $42 \mathrm{I}$

III (oculomotor), 475, 5I 7

oculomotor nucleus, $5 \mathrm{I} 7$

root fibres and nucleus of origin of third, $502,5 \mathrm{I} 7,5 \mathrm{I} 8$

IV (trochlearis), $475,5 \mathrm{I} 5,5 \mathrm{I} 8,520$

root fibres and nucleus of origin of fourth, $5 \mathrm{I} 5,5 \mathrm{I} 8,520$

$\mathrm{V}$ (trigeminus), 475, 485, 489, 493, 5०r, 5०4, $5 \circ 6$

mesencephalic root of fifth, 475 , $506,5^{15}, 5^{20}$

motor nucleus of fifth, 504

"principal sensory" nucleus, 506

semilunar ganglion of fifth, 474

sensory nucleus of fifth, 506

and motor root fibres of fifth, 506

spinal root of fifth, 483,487

terminal nucleus of, 483

VII (abducens), 475

nucleus of origin of sixth, 499, 500, 502

abducentis, 499, 502

root fibres of sixth, $499,500,502$

VII (facial), 474, 475, 544

ganglion geniculate, 474

nucleus facialis, 499,502

of origin of seventh, 499

root fibres of seventh, 502

VIII (auditory), 475, 48I, 49 I, 493, cochlear branch of eighth, 475,489 , 49I, 493
Nerves, ganglion of Scarpa, 475, 493

spirale, 474, 475, 493

nuclei of eighth, 49I, 504

Deiter's, 500, 50I

von Bechterew's, 493, 507

root fibres of eighth, 493

vestibular branch of eighth, 475 , 49I, 493

IX (glosso-pharyngeal), 474, 49I

descending or sensory root fibres of the ninth, 489

dorsal nucleus of ninth, 49 I

ganglia of, 474

motor nucleus of ninth, $49 \mathrm{I}$

root fibres of ninth, $49 \mathrm{I}$

$\mathrm{X}$ (vagus), 474,489

descending or sensory root fibres of tenth, 489

dorsal nucleus of tenth, $48 \mathrm{I}, 485$, 489

ganglion jugular, 474

nodose, 474

motor nucleus of tenth, 489

root fibres of tenth, 489

XI (spinal accessory), 483

nucleus of origin of eleventh, 483

root fibres of eleventh, 483

XII (hypoglossal), 475, 48I

nucleus of origin of $\mathrm{twelfth}, 48 \mathrm{I}$, 485,487

root fibres of twelfth, 487,489

mixed spinal, 424

olfactory, 532

peripheral, 424

spinal, anterior, motor or efferent roots of, 485

sensory, or afferent portions, 426

Nervous system, the, $4 \mathrm{I} 6$

cerebro-spinal, $4 \mathrm{I} 6$

connective tissue of, I42

development of, 4 I 6

general structure of, 4 I 6

sympathetic, $4 \mathrm{I} 6$

cerebro-spinal, 4I 6

afferent peripheral neurones, 424

brain, 473

cerebro-spinal ganglia, 424

cranial nerves, $473,546,547$

development of, $4 \mathrm{I} 6$

efferent peripheral cerebrospinal

neurones, 44I

general structure of, $4 \mathrm{I} 6$ 
Nervous system, histological development of, $4 \mathrm{I} 6$

membranes of brain and cord, 422 segmental part, 420,474

spinal cord, 442

nerves, 424, 546, 547

suprasegmental part, 420,478

sympathetic, $4 \mathrm{I} 6$

development of, $4 \mathrm{IS}$

ganglia, 4I 6, 436

Neumann's dental sheath, 232

Neural arc, 42 I, 469

cerebellar, 470

cerebral, 470

disynaptic, 470

mono-synaptic, 469

pallial, 470

three-neurone, spinal, 469,485

two-neurone, spinal, 469, 504

canal, I 26

fold, 4 I 6

groove, 416

plate, 4 I 6

tube, $4 \mathrm{I} 6$

Neuraxone, I 26

Neurilemma, 132, I35

and axolemma, relation of, 136

-cells, 4I 9

Neurite, I 26

Neuroblasts, I26, I48, 4 I 8

Neuro-epithelium, 77

cone bipolar cells, 557

visual cells, 557

rod bipolar cells, 557

visual cells, 557

Neurofibrils, 128

Cajal's method of staining, 37

importance of, in neurone, 138

Neuroglia, I 42

mossy cells of, I 43

Müller's cells of, $55^{8}$

neuroblasts, I26, I 42,4 I 8

of cerebellum, $5 \mathbf{1} 4$

spider cells, 143

spongioblasts of, $\mathrm{I} 43$

technic for, 144

Neurokeratin network, 135

Neurological staining methorls, 32

Neuromuscular bundles, 434

Neurone, the, 12 ,

axone of, 132

canals in, 131
Neurone, caryochromes, I29

cell body, I 26

chromophilic bodies, I 29

contact theory of, ${ }_{3} 8$

continuity theory of, I3 $S$

cytoplasm of, 128

degenerative changes in, I 39

dendrites of, $\mathrm{I} 3 \mathrm{I}$

development of, I 26

extracellular network of, 139

functional centre of, 137

genetic centre of, I 37

Golgi net, I 39

long axone neurone, 132

neurofibrils of, 127

Nissl, special method of technic for, 38

nucleolus, I 28

nucleus, 127

nutritive centre of, 137

pericellular network of, 139

perifibrillar substance, $\mathrm{I} 28$

physiological significance of, 137

pigment in, $13 \mathrm{I}$

protoplasmic processes, I $3 \mathbf{I}$

retraction theory of, ${ }_{1} 38$

short axone neurone, 132

somatochromes, I 29

synapsis of, 138

technic for, 38 , 144

theory, 138

trophic centre of, 137

Neurones, afferent peripheral, 4I9, 474

segmental, 42I, 474

suprasegmental, 42 I

associative, 470

central, 418, 420

cone association, 56 $\mathrm{I}$

cord, 469

cortical precentral, 469

efferent periplieral, segmental, 4 18, $44 \mathrm{I}, 474$

suprasegmental, 42I, 485

peripheral segmental, 42 I, 474

intermecliate, 418,420

intersegmental, $42 \mathrm{I}, 476$

intrascymental, $42 \mathrm{I}, 47^{6}$

pallio-pontile, 5or

peripheral, 419

afferent, 420, 426, 469, 474

efferent, 418, 420,4

ponto-cercbellar, 501, 504 
Neurones, rod-association, $56 \mathrm{I}$

somatic (peripheral), 4I9 474, 475

splanchnic (peripheral), 4I9, 474, 475

suprasegmental associative, 422

thalamo-cortical $4^{6}{ }_{2}$

visceral (peripheral), 4I9

Neuroplasm, I34

Neutral carmine, 20

Neutrophile granules, Io6

Nipple, 397

Nissl method for staining nerve cells, 38 pathological value of, I 30 concerning chromophilic bodies, I30

Nitric acid for decalcifying, ro for dissociating muscle tissue, 5

Nodes, lymph, I67 of Ranvier, I 34

Nodose ganglion of $\mathrm{X}$ nerve, 474

Non-medullated axones, $\mathrm{I}_{32}$

Normoblasts, I 92

Notochord, anlage of, 62

Nuclear dyes, I 7

alum carmine, I9

basic anilin, I 9

carmine, $\mathrm{I} 7$

combination of Gage's and Mayer's formulas, I8

Delafield's hæmatoxylin, I 7

Gage's hæmatoxylin, I7

hæmatoxylin, I7

Heidenhain's hæmatoxylin, I8

Mayer's hæmalum, I8

Weigert's hæmatoxylin, 19

eccentricity, $\mathrm{I}_{42}$

fluid, 49

groups, 373

membrane, 48

sap, 49

structures, method of demonstrating

Nuclein, 48 by Flemming's fluid, 7

Nucleolus of typical cell, 48

false, 48

Nucleoplasm, 49

Nucleoreticulum, 48

Nucleus, the, 47

abducentis, 502

accessory olivary, 490

alæ cinereæ, 485

ambiguous, 489

amygdaliformis, 536

arciform, 499, 530
Nucleus, arcuatus, 499, 530

caudatus, 526, 532, 535

centralis superior, $5^{\mathrm{I}} 5$

chromatin of, 48

commissuralis, 487

cuneatus, 487

Deiter's, $466,476,483,500,502$

dentate, $466,500,502,507$

dorsal cochlear, 493

Edinger-Westphal, 5 I 8

emboliformis, 502, 507

facialis, 499

fastigii, $466,500,502,507$

function of, 47

funiculi cuneati, 462,485

gracilis, 462,485

globosus, 502, 507

hypoglossi, 485

interstitial, of Cajal, 465, 485, 49r, 500,504

karyoplasm of, 49

lenticularis, 532, 535

linin of, 48

medialis, 490

membrane of, 48

network of, 48

nuclein of, 48

nucleoreticulum of, 48

nucleolus of, 48

oculomotor, $5 \mathrm{r} 8$

of acoustic tubercle, 493

of a typical cell, 47

of column of Burdach, 462, 48 $\mathrm{r}, 487$

of Goll, 462, 48r, 487

of Darkschewitsch, 465

of Luys, 523

of origin, $42 \mathrm{I}$

oculomotor, $5 \mathrm{I} 8$

olivary, $487,490,49$ I, 500, 507

pontis, 504

preolivary, 500

pulposus, 204

red, $436,466,476,5$ I 7,524

resting, 57

reticularis tegmenti, 504

ruber, $436,466,476,517,524$

semilunar, 500

tecti, 466, 500, 502

terminal, $42 \mathrm{~T}, 490$

trapezoid, 49r, 500, 507

triangular, 490

ventro-lateral, 530 
Nucleus, vestibular, 490, 493, 500 von Bechterew's, 500, 507

Nuel's space, 58 I

Nutrient canal, ro6

foramen, 196

vessels, of bone, 196

Nutritive center of neurone, 137

Occloyotor III nerve, 5 I 7,5 IS nucleus, $5 \mathrm{r} 7,5 \mathrm{r} 8$

Odontoblasts, 228, 24 I

Esophagus, the, 243

technic of, 215

Oil of origanum Cretici for clearing specimens, 23

Olfactorius (I nerve), 47.5, 532

Olfactory bulb, 532,585

granule layer, 586 of longitudinal fibre bundles, 586 of mitral cells, 586

of olfactory fibres, 586

molecular layer, $5^{86}$

olfactory glomeruli of, 586

path, $475,532,533,539$

pallial commissure, 533

group of segmental neurones, 475

nerve, 475,532

organ, $5^{8} 4$

pallium, 532

tract, $475,539,586$

Olivary nucleus, $48 \mathrm{r}, 487,493,500,507$

Olives, $487,489,49 \mathrm{r}, 493$, 50 I

Omentum, 268

gastro-hepatic, 268

greater, 268

Opie, concerning the pancreas, 286

concerning the cell-islands of Langerhans, 286

Oppel's method of staining intralobular connective tissue of liver, 296

Optic chiasma, 526, 56 I

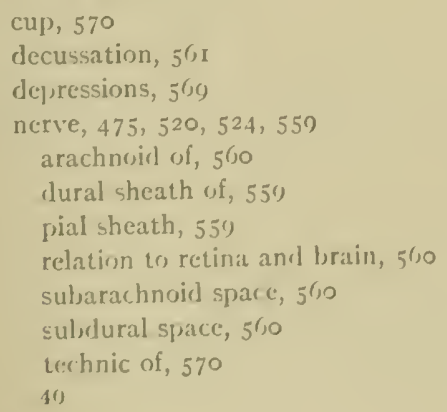

Optic stalk, 569

tract, $520,524,536$

vesicle, 569,570

Opticus (II nerve), 473

Ora serıata, 552, 555

Oral glands, cells of, 220

crescents of Gianuzzi, 222

demilunes of Heidenhain, 222

mixed glands, 221

mucous glands, 22 I

serous glands, 221

technic of, 223

Orange $\mathrm{G}, 20$

Organ of Corti, 580

acoustic neurones to, 475

cells of Claudius of, $5^{8} \mathrm{I}$

Corti's arches, $58 \mathrm{I}$

$$
\text { tunnel, } 58 \mathrm{r}
$$

Deiter's cells, $5^{8 \mathrm{r}}$

hair or auditory cells, $58 \mathrm{I}$

Hensen's cells, 58 I

lamina reticularis of, $58 \mathrm{I}$

Nuel's space of, 58 I

phalangeal processes, $58 \mathrm{r}$

pillar cells, $5^{80}$

of Giraldès (paradidymis), 342, 377

of hearing, 572; see also Ear

blood-vessels of, $5^{82}$

development of, $5^{8} 3$

ear, external, 572

internal, 574

middle, 573

lymphatics, 582

nerves, $5^{82}$

technic of, $5^{84}$

of smell, 584; see Olfactory organ

olfactory bulb, -85

mucosa, 584

tract, 586

technic of, 586

of taste, $5^{87}$

cclls of, $5^{87}$

foliate papillw, $5^{87}$

gustatory canial, $5^{87}$

intergeminal filses of, $5^{87}$

intrageminal fibres of, $5^{87}$

taste burls, 226, 227, 4.32, 433, 587

technic of, 587

of vision, 548

bloxl vesscls of, $5^{6 / 5}$

development of, 5 (\%)

cycball or bubus oculi, 5.48 
Organ of vision, eyelid, 567

lacrymal apparatus, $5^{6} 7$

lens, 564

lymphatics of, 566

nerves of, 566

neurone systems of, 560

optic nerve, $473,520,524,559$

technic of, 570

of Zuckerkandl, 41 2

Organs, the, I49

circulatory system, I 5 I

digestive system, 219

acoustic neurones to, 475

glands, duct, 2 I 2

ductless, 402

lymphatic organs, 167

muscular system, 207

nervous system, $4 \mathrm{x} 6$

reproductive system, 333

respiratory system, 299

skeletal system, I88

skin and its appendages, 380

special sense organs, 548

urinary organs, 318

of special sense, 548

organ of hearing, 572

of smell, 584

of taste, 587

of vision, 548

Organs of Golgi, peripheral nerve terminations in, 434

Orth's fluid (formalin-Müller's), 7

Osmic acid as a fixative, 8

action on fat, 8

on myelin, 8

stain for fat, $3 \mathrm{I}$

Osseous labyrinth, 574

Ossicles of middle ear, 574

Ossification, 82

centres, 197

endochondral, I99

intracartilaginous, 199

intramembrancus, 199

subperichondral, I99

subperiosteal, I99

Osteoblasts, I98, 235

Osteoclasts, I99, 235

Osteogenetic tissue, 197

Otic ganglion, 436

vesicle, 583

Otocyst, $5_{8} 3$

Otolithic membrane, 575
Otoliths, 575

Oval bundle of Flechsig, 467

Ovary, the, 352

blood-vessels of, 363

corpora lutea, of pregnancy, 360 spuria, 360

vera, 360

corpus albicans, 360

hæmorrhagicum, 359

luteum, 359

cortex of, 353

development of, 376

egg nest, 354

epoöphoron, $3^{6} 3$

Fallopian tube, 353, 364

germinal epithelium of, 354

Graafian follicles, 354

hæmatoidin crystals, 360

hilum of, 353

lutein cells, 359

lymphatics of, 363

medulla of, 353

nerves of, 363

ovarian stroma, 353

oviduct, 353

ovum, 352,355

paroöphoron, 363

Pflüger's egg tubes or cords, 354

primitive ova, 354,378

rudimentary structures connected with, 363

secretion of, 352

structure of, 353

technic of, 365

tunica albuginea, 353

zona vasculosa, 353

Oviduct, the, 364 ; see Fallopian tubes

Ovula Nabothi, 368

Ovum, the, $52,355,356$

atresia of follicle, 363

cells of, 357

deutoplasm granules, 357

development of, 356

fertilization of, $58,35^{8}$

germinal spot, 357

maturation of, 357

perivitelline space, 357

primitive, 378

segmentation of, 62

yolk granules of, 357

zona pellucida of, 357

Oxyhæmoglobin, ro7 
Oxyntic cells, 249

Oxyphile cells, 405

Pacchioniax bodies, 424

Pacinian bodies, 196,433

corpuscles, $350,+34$

Palate, mucous membrane of, 220

Palatine tonsils, $\mathrm{I} 78$; see Tonsils

Pallial connections, 46r, 462, 464, 469, 470, $+77,522$

Pallio-pontile fibres, 50I, 504, 5 I 7

Pallio-ponto-cerebellar path, 5 or

Pallio-spino-peripheral efferent conduction path, 465

Pallio-thalamic fibres, 523, 535

Pallio-tectal fibres, 535

Pallium, 41 7, 532, 536

cortical areas of, 536

fibres of, 533

Pancreas, the, 28I

blood-vessels of, 286

cell-islands of Langerhans, 285

centro-acinar cells of Langerhans, 283

development of, 295

duct of Santorini, 282

of Wirsung, $28 \mathrm{I}$

ductus choledochus, 295

excretory ducts, 282

intracellular secretory tubules of, 285

lobules of, $28 \mathrm{I}$

lymphatics of, 286

nerves of, 286

Opie, concerning cell-islands, 286

secondary excretory duct of, 286

secretion of, 285

sustentacular cells of, 284

technic of, 287

terminal tubules of, 282

zymogen granules of, 282

Pancth, cells of, 260, 273

Panniculus adiposus, 383

Papillac, circumvallate, 225, 587

compound, $38 \mathbf{I}$

filiform, 225

foliate, 587

fungiform, 225, $5^{87}$

nerve, $38 \mathrm{r}$

of mouth, 220

pharynx, 242

simple, $38 \mathrm{I}$

vascular, $3^{8}{ }_{3}$

Paradidymis, or organ of Giralelès, 342,377
Paraffin embedding, $\mathrm{I}_{3}$

apparatus for, 14

oven, I 3

section-cutting, I5

sections, staining and mounting of, 23

Paraganglia, to9

carotid gland, 4 ro

chromafin organs, 410

coccygeal gland, $4 \mathrm{I}$ I

organ of Zuckerkandl, $4 \mathrm{I} 2$

tympanic gland, 4 I 2

Paralinin, 48

Paramiton, 45

Paranuclein, 48

Paranucleus, 213

Paraplasm, 46

Parathyreoids, 404

chief or clear cells of, 405

development of, 406

function of, 406

oxyphile cells, 405

Pool's theory of, 406

technic of, 406

Pareleidin, $38_{4}$

Parenchyma of glands, 215,277

Parietal cells, 249

peritoneum, 267

pleura, 308

Paroöphoron, 303

Parotid gland, 277

development of, 297

intercalated tubule of, 267

nerves of, 280

Stenoni's duct of, 277

technic of, 281

Pars ciliaris retinx, 555,559

iridica retinac, 555, 559

optica retince, 555

papillaris, 38 I

reticularis, 380

Javlow, concerning secretion, 273

Peduncle, inferior, 499, 500 middle, 499 superior, 499, 500, 502, 507, 517

Pellicula, 47

Penicillus, 183

Penis, 349

arterices of, 350

cavernous sinuees, 350

corporat catwernosit of, $3.4 \%$

corpus sprongiosum of, 340

erectile tissue, $34 \%$ 
Penis, glans, $35^{\circ}$

glands of Tyson of, 35 I

lymphatics, $35^{\circ}$

nerve endings of, 350

prepuce of, $35^{\mathrm{I}}$

sebaceous glands of, $35 \mathrm{I}$

technic of, $35^{2}$

tunica albuginea of, 349

Pepsinogen granules, 272

Peptic cells, 249, 272 glands, 249

Perforated space, anterior, 536

Perforating fibres, I 92

of cornea, $55^{\circ}$

of Sharpey, I 92

Perforatorium, 343

Periaxial sheath, I 34

Pericardial cavity, 165

Pericellular network, I39

Perichondrium of bone, 199 of cartilage, 99

Perichorioidal lymph spaces, $55 \mathrm{I}$

Pericranium, I98

Peridental membrane, 234

Perifascicular sheath, 208, 425

Perifibrillar substance, I 28

Perilymph, 574

Perimysium, 208

Perineurium, 425

Periosteal buds, 200

Periosteum, I9I, I99

Peripheral afferent neurones, 4I9, 426, 474

cerebro-spinal ganglia, 426

symphathetic ganglia, 436

efferent neurones, 42O, 44I, 475

motor neurone system, $44 \mathrm{I}$

nerves, 4I 9,424

afferent part of, 424

cranial, 424

efferent part of, 424

endoneurium of, 425

epineurium of, 425

fascicles of, 425

intrafascicular connective tissue of, 425

medullated fibres of, $\mathrm{I} 33$

motor or efferent, 424

motor nerve terminations, 44I

non-medullated fibres of, 132

perifascicular sheath of, 425

perineurium of, 425

sensory or afferent, 424
Peripheral nerves, sensory nerve terminations, 429

sheath of Henle, 426

spinal, 424

structure of, 424

technic of, 426

nerve terminations, 429

annular, 434

diffuse, 430

end-bulbs, 43 I, 433

free endings, 430

in penis, 350

golgi Mazzoni corpuscles, 394, 434

grandry, corpuscles, 43 I

in mucous membrane of mouth and conjunctiva, 432

in muscle-tendon junctions, 434

in skin, $394,43^{\circ}$

in smooth muscle, 430

in voluntary muscle, 434

Krause's end-bulbs in penis, $35^{\circ}$

Meissner's corpuscles in papillæ of penis, $35^{\circ}$

muscle spindles, 434

muscle-tendon organs of Golgi, 434

neuromuscular bundles, 434

Pacinian bodies, 196, 433

corpuscles of penis, $35^{\circ}$

nerve terminations, Ruffini's theory of, 434

spiral terminations, 434

tactile cells, 43 I

corpuscles, 432

meniscus, $43 \mathrm{I}$

taste buds, 432

Peripheral nervous system, 4I 6

spinal nerves, 424

Peritoneal cavity, I65

Peritoneum, 267

parietal, 267

subserous tissue of, 268

visceral, 267

Perivitelline space, 357

Permanent teeth, 239, 242

Perpendicular fasciculus of Wernicke, 535

pontile fibres, 499, 50I, 507

Pes pedunculi, 4I 7, 5 I 7, 52 I, 526, 530, 536

Petit, canal of, 565

Petrosal ganglion of IX, 474

Peyer's patches, 260

Pflüger's egg tubes or cords, 354

Phæochrome granules, 4I 2

Phæochromoblasts, 4 I 5 
Phagocytes, 10;, Iit

Phagocytosis, Io 7

Phalangeal processes, $j \delta \mathrm{I}$

Pharyngeal tonsils, I So; sec Tonsils

Pharyn. the, 243

blood-ressels of, 243

lymphatics of $2+3$

nerves of. 243

structure of, 242

technic of, $2+3$

Phloroglucin, ro

Pia mater, 422

arachnoid, 422

blood-ressels of, +24

cerebralis, 422

Pacchionian bodies, 424

spinalis, 422

technic of, 424

Picric acid as a fixative, $S$

as plasma dye, 20

Picro-acid-fuchsin, 20

Picro-carmine, 2 I

Pigment granules in cells, $46,3_{4}$

in connective tissue, 87

in epithelium, $; 6$

hair, $38 s$

in iris, 554

in nerve cells, I $3 \mathrm{I}$

Pillar cells, $58 \mathrm{I}$

Pineal body, $5+4$

brain sand of, 547

technic of, $5+7$

Pineal cyce, $5+4$

Pinna, $57^{2}$

Pituitary body, 40 7

Berkley; concerning posterior lobe, 408

function of, 409

relation of, to p,regnancy, 409

technic of, 408

Placenta, 37 I

blood-vessels of, 374,375

canalized fibrin, 373

cell patches, 373

chorionic villi, $37 \mathrm{I}$

cotylerdons, 371

fat 4 ning villi, 37 I

fortalif 371

free so lloating villi of, $37 \mathrm{t}$

lymphatics of, 375

memlerana rlourii of, 37 I

nerves of, 375
Placenta, nuclear groups, 373

septa of, $3 i+t$

subchorionic placental decidua, 374

syncytium of, 373

techuic of, 379

uterina, 373

villi of, $37 \mathrm{r}$

Plasma cells, I 193

l'lasma dyes, $\mathrm{I} 7, \mathrm{I} 9$

acid aniline, 20

eosin, 19

neutral carmine, 20

picric acid, 20

Plasmosome, +8

Plastids, $4^{6}$

Plastin, 44

Platelets, blood, IoS

Pleura, parictal, $3 c 8$

pulmonary, 308

Pleural carity, I $\sigma_{5}$

I'leuroperitoneal cleft, $\mathrm{I}_{5}$

Plexiform layer of Cajal, ${ }_{53} \mathrm{~S}$

Plexus annularis, 567

Auerbach's $264,270,436$

ciliary, 566

chorioideus, $417,482,487,502$

IIeller's 268

interradiary, $54 \mathrm{I}$

Meissner's, 25t, 262, 263, 271, 436

myentericus, 270

prevertebral, +36

supraradiary, 54 t

Plicx palmatx, $36 \mathrm{~S}$

Pncumogastricus (vagus nerve) 474,489

Polar bodies, $59,35^{\mathrm{S}}$

globules, $6 \mathbf{I}$

rays, 54

Polykaryocytes, 193

Polymorphonuclear leucocytes, ro5

Polymorphous cells, 105

Polynuclear leucocytes, ro5

I'ons Varolii, 17, 482, 499, 501, 515

longiturlinal libres of, 499, 501

perpemelic ular filores, 499, 50r, 507

pontile nuclei of, sor

pyramid of, $50 \mathrm{r}$

transverse filtres of, 50 r

I'onl, I: H., concerning parathyreoid gland, son

P'orsipphoron, 3'3

Portal cattial, 2yo

$v\left(\mathrm{cin}, 2 \mathrm{~K}_{0}\right.$ ) 
Posterior columns of spinal cord, 443,448 origin of fibres of, 443

column of Burdach, 459, 462, 485 of Goll, 459, 462, 485

commissure, 522,526

corpus quadrigemium, 493, 5I 7

distribution of fibres of, 452

funiculus, 448,46 I

horns, 445,448

longitudinal fasciculus, $49 \mathrm{I}$

median septum, 448

nerve root, $45^{\circ}$

root fibres, 450

nucleus funiculi cuneati, 462,485

funiculi gracilis, 462,485

of the column of Burdach, 462,485

of the column of Goll, 462,485

origin of, 443

tract, or terminal zone of Lissauer, 443,450

zone of Lissauer, 443

Potassium hydrate, as a macerating fluid, 4

Precapillary artery, I 54

Predorsal fasciculus, 504

Prepuce, 35I

Preparation of sections, 5

Preserving, 9

Prevertebral plexuses, 436

Prickle cells, 383,387

Principal sensory nucleus of nerve V, 506

Projection fibres, 533

Pronephric or Wolffian ducts, 377

Pronephroi, 376

Pronucleus, female, 59 male, 59

Prophase, 53

Proprio-ceptors, 436

Proprio-spinal tract of cord, 468

Prosencephalon, 416, 522

Prostate gland, 347

blood-vessels of, 348

capsule of, 347

corpora amylacea of, 348

crescentic corpuscles of, 348

epithelium of, 3I 7

lymphatics of, 348

Müllerian duct, 348

nerves of, 348

technic of, 349

trabeculæ, 347

uterus masculinus, 348
Prostate gland, utriculus prostaticus, 348 vesicula prostatica, 348

Protargol, for staining intercellular substance, 28

Protoplasm, 43, 45

streaming of, 52

theories of structure of, 44

Protoplasmic movement, 52 processes, I3 I

Proximal convoluted tubule, 3I9

Prussian blue gelatin as an injecting fluid, 25

Pseudochromosomes, 2 I 3

Pseudopodia, 50

Pulvinar radiations, 524

thalami, 524

Pulmonary artery, 3 I 3

lobule, 308, 3I3

pleura, 308

Pulp cavity, 227

cord, I84

splenic, I84

Purkinje cells, 509

Putamen, 535

Pyloric glands, 252

Pylorus, 252

Pyramid, cortical, 320

of Ferrein, 320

Malpighian, 320

Pyramidal cells, 536

decussation, 464,485

tracts, 464 anterior pyramids, $464,483,487$, 504,507

crossed pyramidal, 464

direct pyramidal, 465

pyramidal decussation, 464

Pyramids, 4I 7, 504, 507, 5I 5

Pyrenin, 48

Pyriform lobe, 532

RACEMOSE glands, 2 I 5

Radiations of Meynert, 54I

Radix spinalis, V, 483,487

Rami communicantes, gray, 437

white, 425,436

Ranvier's alcohol as a macerating fluid, 4

nodes or constrictions of, I34

showing muscle fibres, II 5

Raphé, of semicircular canals, 576

median, $488,490,507$ 
Receptors, +19, +24, +25, 429 ,

$$
436
$$

extero-ceptors, 436

intero-ceptors, 436

proprio-ceptors, 436

Sherrington's classification of, +36

Rectum, 266

anus, 267

columnæ rectales, 266

technic of, 275

Red blood cells, ro3; see also Blood nucleated, 109,192

nucleus, 436, 466, 476, 5 I 7

Reduction of chromosomes, 55, $3+4$

Reflex arc, disynaptic, 470

monosynaptic, 469

three-neuronc spinal, 469

two-neurone spinal, 469

Reissner, mcmbrane of, 579

Remak, fibres of, I33

Renal corpuscle, 320

development of, 320,377

Renculus, 318

Replacing cells, 73,252

Reproduction cells of, 52

Reproductive system, 333

development of, 376

rudimentary structures connected with the, 342,363

female organs, 352

clitoris, 376

Fallopian tube, 364

ovary, $35^{2}$

oviduct, 364

placenta, 37 I

urethra, $35 \mathrm{I}$

uterus, 366

vagina, 375

vestibule, 376

male organs, 333

Cowper's glands, 348

ejaculatory ducts, 34 I

penis, 349

prostate gland, 347

seminal ducts, 339

vesicle, $34 \mathrm{I}$

seminifcrous tubule, 334

spermatozoa, 343

testis, 333

urethra, 351

Respiratory bronchus, 310

cells, 311
Respiratory cpithelium, 3 II

system, 299

bronchi, 304

development of, 3 I 5

larymx, 3 or

lungs, $30 \mathrm{~S}$

nares, 299

technic of, 304, 31 7

trachea, $30 \mathrm{I}$

Restiform body, $48 \mathrm{I}, 485,489,490,499$, 501,504

Rete testis, tubules of, 334, 339

vasa efferentia, 339

Reticular formation, $476,485,487,489$, $490,491,501,504,507$

glands, 2 I 7

process, 448,455

tissue, 170

Reticulin, 95

Retina, 555

blood-vessels of, 565

ellipsoid of Krause, 557

fibre-basket of, 559

fovea centralis, 559

ganglionic layer of, 555

horizontal cells of, $55^{8}$

inner limiting membrane of, $55^{8}$

molecular layer, 557, 558

nuclear layer, 557

layer of nerve cclls, $55^{8}$

of nerve fibres, $55^{8}$

of neuro-epithelium, 555,556

of pigmented cpithelium, 555

of rods and cones, $55^{6}$

macula lutea, 559

Müller's cells and fibres, $55^{8}$

ora scrrata, 555

outer limiting membrane, 558

molecular layer, $55^{8}$

nuclear layer, 556

pars ciliaris retinte, 555,550

iridica retina, $55.5,559$

optica retinac, 555

relation to optic nerve, 560

rod and conc cells of, 557

visual purple of, 557

Retinaculac cutis, 38 ,

Retraction hypothesis, 138

Retrolenticular portion of internal cap)sulc (C' $\mathrm{irl}$ ), 526

Retzius, lines of, 233 
Rhinencephalon, 4I 7,532

Rhinopallium, 532

Rhombencephalon, 41 7, 479

Ribboning paraffin sections, I 5

Rod association neurones, 561

fibres, 557

neurones, 560

Rod-visual cells, 557

Rods, layer of rods and cones, 557

Rolando, gelatinous substance of, 449

Root canal, 228 cells, 44I, 444, 45 I

Roots, afferent, 4I 9

Rose concerning chromaffin cells, 4 ro

Ruffini, corpuscles of, 394

theory of nerve terminations, 434

Rugæ, 246, 248, 295

Rühle, concerning the uriniferous tubule, 325

SAccular glands, 2 I 5,2 I 7

Saccule, and utricle, 575

auditory hairs of, 575

macula acustica, 575

neuro-epithelial cells of, 575

otolithic membrane of, 575

otoliths of, 575

sustentacular cells of, 575

Sachs, E., concerning thalamus, 523

Sacral segments of spinal cord, 458

Safranin, I9

Salivary corpuscles, I80 glands, 276

blood-vessels of, 278

development of, 297

lymphatics of, 280

minute structure of, 22 I

nerves of, 280

parenchyma of, 277

parotid, the, 277

sublingual, the, 277

submaxillary, the, 278

technic of, $28 \mathrm{I}$

Santorini, cartilage of, $30 \mathrm{I}$

duct of, 282

Sarcolemma, I 14

Sarcoplasm, II4

Sarcostyles, 209

Sarcous elements of Bowman, I 6

Satellite cells, 427

Scala media, 578

tympani, 578
Scala vestibuli, 578

Scarpa's ganglion, 475, 493

Schaper, concerning placenta, $37 \mathrm{I}$

Schlemm, canal of, 554

Schmidt-Lantermann, clefts of, 135 incisures of, I35 segments of, 135

Schreger, lines of, 232

Schultze, comma tract of, 467

Schwalbe, lymph paths of, 566

Schwann, sheath of, 132, I35

Sclera, the, 548

Scrotum, skin of, 333

Sebaceous glands, $384,39 \mathrm{I}$

development of, 395

of glans penis, $35 \mathrm{I}, 384$

of labia minora, 384

of margin of lips, 384

of prepuce, 384

Sebum, 392

Secondary cochlear tract, 493, 504, 507 trigeminal tract, 483

vestibular tract, 493

Secretion, 42, 213, 27 I

Secretory tubules, 276

Golgi method of demonstrating, 29

of parietal cells of stomach, $25 \mathrm{I}$

Section cutting, I4

celloidin specimens, I4

frozen sections, I 5

paraffin specimens, $\mathrm{I} 4$

staining, 17,20

Segmental brain, 420,474

nerves, 420,474

paths, 476

Segmentation cavity, 6 I

of ovum, 62

Segments of Schmidt-Lantermann, $\Upsilon_{35}$ of spinal cord, 448,455

Semen, 343

Semicircular canals, 473,576 crista acustica of, 576 cupula of, 576

raphé of, 576 semilunar, fold of, 576

Seminal ducts, 339 vas epididymis, 340 vas deferens, 340 vasa efferentia, 339 vesicles, $34 \mathrm{I}$

Seminiferous tubules, 334

cells of, 335 
Scminiferous tubules, columns of Sertoli, 335

convoluted portion of, 334

development of, 344

glandular cells of, 335

rete testis, 334,339

spermatids, 337

spermatocytes, 337

spermatogenic cells, 335

spermatogones, 336

spermatozoa, 337

straight portion of, 338

supporting cells of, 335

sustentacular cells of, 335

Semilunar fold, 576

ganglion of $\mathrm{Y}, 474$

Senses, common, 436

general, 436

special, 436

Sensory decussation, 485

path, general, 441, 461, 462, 474, 491, 524

peripheral nerves, 426

Septa renis, 320 ; see Kidney

Septo-marginal tract, 467

Septum linguac, 224

Serial sections, 15

Serous cells, $22 \mathrm{I}$

membranes, 165

Sertoli, cells of, 335,378

columns of, 335

Sewell and Langley concerning secretion, 272

Sex cells, 378

Sharpey, showing bone lamella, 19 I

Sharpey's fibres, 192, 234

Sheath of Henle, r.36, 426

medullary, 132,134

Neumann's dentil sheath, 232

of Schwann, I 32

perifascicular, 208, +25

myelin, 134

Sherrington concerning receptors, 436

Short fibre tracts, 445

Signet-ring cell, 88

Silver nitrate methorl, (iolgi, 35

of staining interc cllular substance, 28

thein, cloged, 54

thelletal gystem, 188

articulations, 204

bone-marrow, 192

lones, 188
Skeletal sy'stem, cartilages, 204

Skin and its appendages, 380

blood-1-essels of, 399

color of, $38_{4}$

corium, 380

corpuscles of Grandry; 43 I

of Meissner, 394,432

of Ruffini, 394, 434

of Wagner, 394

cuticle, $3^{8}{ }_{2}$

derma, 3 So

development of, 395

elcidin of, $3^{8} 3$

cnd-bulbs in, 430

epidermis of, $3 \delta_{2}$

glands of, 384

glandulæ sudoripar:e, $38_{4}$

Golgi-Mazzoni corpuscles of, 394,434

hair follicles of, 387

junction of, with mucous membrane of mouth, 220, $38_{4}$

keratohyalin granules, $38_{3}, 387$

Krause's end-bulbs, 39.4

lymphatics of, 394

mammary gland, 395

Merkel's corpuscles of, 431

mitosis of cells of, 383

nails, $38_{5}$

nerves of, 394,430

of scrotum, $38 \mathrm{I}$

Pacinian bodies of, 434

panniculus adiposus of, $3_{3}$

papillic of, ${ }_{3} \delta_{1}$

parcleidin, $38_{4}$

pars papillaris, 38 i

pars reticularis, 380

peripheral nerve ter minations in, 304 . 430

prickle cells of, 383,387

retinaculie cutis, $3 \mathrm{Si}_{2}$

sebace ous glands of $33_{4}, 30 \mathrm{r}$

subcutancous tissue of 381

sweat glands (glandula suboriparar),

$$
38.4
$$

jores of $33^{84}$

tartile cells of, 43 I

rorpuseles, 4.33

ter hunic: of, 38.4

for blocel vessely of, 30)5

Vatcr-Pacinian corpuscles of, 39.4

imall intestines, 255

agminated follides, 260 
Small intestines, Auerbach's plexus, 263

blood-vessels of, 268

Brunner's glands of, 262, 273

cells of, 257

chyle capillaries of, 270

coats of, 262

crypts of Lieberkühn, 260

development of, 296

lacteals of, 275

lymphatics, 270

Meissner's plexus, 262

migratory leucocytes, 257

muscle of, 262

nerves of, 270

Paneth's cells, 260, 273

Peyer's patches of, 260

plexus myentericus, 270

replacing cells, 259

secreting cells, 257,272

solitary follicles, 260

technic of, 275

valvulæ conniventes of, 255

villi of, 256

Smell, organ of, $5^{84}$

Smooth muscle, $\mathrm{rrg}$; see Involuntary muscle

Sodium hydrate as a macerating fluid, 4

Solitary fasciculus, 487,489

follicles, 253

Somatic receptors, 473

(peripheral) neurones, 420,473

Somatochromes, 129

Spaces of Fontana, 554

Special dental germs, 238

senses, 436

Spermatids, 337,344

Spermatoblast, 345

Spermatocytes, 337,344

Spermatogenesis, 58 , 344

technic of, 346

Spermatogones, 336

Spermatozoa, 58, 337, 343

acrosome, 337

apical body, 337

axial thread, 344

development of, 58,344

diagram of, 343

galea capitis, 343

perforatorium, 343

structure of, 58,343

technic of, 346

Sphenopalatine ganglion, 436
Spider cells, $x_{43}$

Spinal accessory nerve, 483

Spinal cord, 4 I 6

anterior columns of, 448

funiculus, 448

horns of, 444,448

marginal bundle of Löwenthal, 466

median fissure, 448

nerve roots of, $45^{\circ}, 459$

pyramids, 465

white commissure of, $45^{\circ}$

antero-lateral columns of, 448

funiculus, 448

white column, 448

ascending tract, $463,483,487$

descending tract, 466

arachnoid membrane of, 422

arrangement of fibres of, $45^{2}$

arteries of, 454

ascending tracts of, $46 \mathrm{I}$

blood-vessels of, 454

cell-groupings of, $45^{\circ}$

cells of dorsal horn, 450

cells of the intermediate gray matter, $45^{\circ}$

of Golgi, Type II, 446

of ventral horn, $45^{x}$

cells outside cord, with axones to white matter of cord, 444

central canal of, 4I6, 449

gelatinous substance, 449

cervical enlargement of, 446, 455

segmients of, 446

Clarke's column of, $450,455 \cdot 463$

coccygeal segments of, 446

collaterals of, 447

column of Burdach, 459, 46 I

column of Goll, 459,462

cells, $444,46 \mathrm{r}$

hecateromeric, 444

heteromeric, 444

tautomeric, 444

comma tract of Schultze, 467

conduction paths of, $42 x, 46 r$

cornua of, 448

crossed pyramidal tract, 464

descending paths from higher centres, 464

tract from Deiter's nucleus, 466

from vestibular nuclei, 466

diagram showing tracts of, 460 
Spinal cord, direct ascending paths to higher centres, $46 \mathrm{I}$ cerebellar tract, 463 pyramidal tract. $4^{6} 4$ reflex collaterals, 452

dorsal gray columns, 448

commissure, 449

spino-cerebellar tract, $4^{6} 3$

white columins, 448

dura mater of, 422

ependyma of, 453

fasciculus, medial longitudinal, 467 of Thomas, 467

fibre tracts of, 459

methods of determining, 459

filum terminale of, 442

finer tructure of, 4.53

fundamental columns of, 445,468

ganglion cells of, 443

gelatinous substance of Rolando, 449

general topography of, 448

Gowers' tract, $463,464,465$

gray matter of, 420,449

ground bundles of, $445,4^{6 / 8}$

Helweg's tract, 464

interchange of fibres, 452

intermediate gray matter, 450

intermedio-lateral column, $45^{\circ}$

lateral horn of, 450

long ascending arms of dorsal root fibres, 461

long ascending fibre tracts, 445

longitudinal section of six days' chick embryo, 447

lumbar enlargement of, 443,448 segments of, 443

main motor fibre sy'stems of, 418,441 marginal bundle of Löwenthal, 400

marginal zone, 449

medial fillet (lemniscus), $4^{6 / 2}$

medullated fibres of, 406,443

membranes of, 422

arachnoirl 422

bloorl-vessels of, +2.4

dura mater, 422

pia mater, 422

spinal dura, 422

technic of, 42.4

mixerl spinal nerve, 424

motor cells of anterior horu, $\$ 1.1$

multipular gangliosn cells of, 112, 127 $439,4.4$
Spinal cord, neuroglia cells, 453

fibres, 453

tissue, 450

neurone systems of, 421

nucleus, Darkschewitsch's, $4^{6} 5$

Deiter's, 466

funiculi cuneati, 462

gracilis, 462

origin of fibres of white matter, +43

of posterior columns of, 443

oval bundle of Flechsig, $46_{3}$

peripheral motor or efierent neurone system, $418,4+$ I

sensory or afferent neurone system, 419,426

pia mater, spinalis, 422,448

plexus of fine fibres, 452

posterior columns, 443,448

funiculus, 448,46 I

horns, 445,448

median septum, $+48^{\circ}$

nerve roots, 450

root fibres, 450

postero-lateral groures, +48

sulci, 448

pyramidal decussation, $4^{6} 4$

tracts, 464

reflex arcs, 469

reticular process, 448,455

root cells, $44 \mathrm{I}, 444,45 \mathrm{I}$

rubro-spinal tract, 466

sacral segments of, $4+40$

scheme of neurone relations of, 460

section through cervical enlarge-

ment of, 405

through lumbar enlargement, 4.48

through micl-thoracic region, 455

through six-lay click (mbryo, +17

through twelfth thoracic segment, 455

segments of, 4.4 .3

septo-marginal tract, $4^{67}$

shape of, 443

short libre systems of, $4.15,162,468$

shorter intersegmental tracls, 460

size of, 14.3

spino tectal tracl, 465

thalamic tract, f(t) 2

leclesplinal Iried, fb5

lachnic of, $4.46,471$

thoracir segments of, 143 
Spinal cord, tract from interstitial nucleus of Cajal, 465

tractus cerebro-spinalis, 464

cortico-spinalis, 464

pallio-spinalis, 464

reticulo-spinalis, 467

spino-cerebellaris dorsalis, $46_{3}$

cerebellaris ventralis, 463

spinalis, 468

thalamis, $4 \mathrm{I} 4$

variations in structure at different

levels, 455

veins of, 454

ventral gray columns, 448

commissure, 449

spinal cerebellar tract, 463

white columns, 448

vestibulo-spinal tract, 466

von Monakow's tract, 466

white commissure, $45^{\circ}$

matter, $420,446,45^{\circ}$

zona spongiosa, 449

terminalis, $45^{\circ}$

zone of Lissauer, 443

Spinal ganglia, 426

amphicytes, 427 .

capsule of, 426

development of, 4 I 8

technic of, 446

ganglion cells, 436,443

central processes of, 436

classification of, 427

collaterals from, 47

descending arms from central processes of, 436

development of, $4 \mathrm{I} 8$

Dogiel's classification, 427

ectodermic origin, 416

modes of termination of peripheral processes of, 429

peripheral processes of, 429

Ruffini's classification of terminations in muscle spindles,

$$
434
$$

satellite cells, 427

structure of, 426

technic of, 44I, 446

Spinal nerves, 424

Spindle, achromatic, 54

Spino-cerebellar tract (dorsal), 463

(ventral), 426, 463

Spino-collicular tract, 462
Spino-peripheral motor neurone system, $44^{2}$

Spino-spinal tract, 468

Spino-tectal tract, $465,483,487$, 490

Spino-thalamic tract, $462,483,501,504$, 515,526

Spiral ganglion, 475,493

lamina, 577

ligament, 577

limbus, 582

organ, 579

prominence, 579

sulcus, external, 579

terminations, 434

Spireme, closed, 54

open, 55

Spireme-thread, 54

Splanchnic effectors, 473

Splanchnic (peripheral) neurones, 4I9

Spleen, I8I

ampullæ, 183

blood-vessels, 182

cavernous veins, 183

cells of, 184

central arteries of, $\mathrm{I} 83$

connective tissue framework, 18 I

cords of, I 84

corpuscles of, 182

development of, I 86

function of, 186

development of, 186

germinal centres of, 182

lymphatics of, 186

Malpighian bodies, $\mathrm{I} 82$

Mollier, concerning splenic pulp, I85

nerves of, 187

Spleen, penicillus, 183

pulp of, 184

cords of, 184

spindles of, 183

techñic of, 187

Spleen-sinus, 183

Splenic corpuscles, 182

pulp, I84

Spheno-lymph nodes, 174

Spongioblasts, I42, 4I3

of His, 4I 7

Spongioplasm, 44

Spongy bone (cancellous), I 88

primary, 202 
Staining, I 7

differential. 3

double with hiematoxylin-eosin, 20 in bulk, 2I

methods, special. 2S

chloride of gold, is

Golgi's chrome silver for secretory tubules, 20

Jenner's, for blood, $3 \mathrm{I}$

Mallory's aniline blue for connective tissue, 30

phosphomolybdic acid hæmatoxillin stain for connective tissue, 29

phosphotungstic acid hrmatoxylin stain for connective tissuc, 30

Maresh's modification of Bielschow'sky's stain for fine connective tissue fibrils, 3 I

osmic acid, for fat, 3 I

silver nitrate, for intercellular substance, 28

Verhoefi's differential stain for clastic tissue, 28

IV'cigert's clastic-tissue stain, $2 \mathrm{~S}$ parafin sections, 23

sections, 20

double with hæmatoxylin-eosin, 20

triple with hrematoxylin-picro-acidfuchsin, 2 I

with picro-acid-fuchsin, 20

with picro-carmine, 2 I

selective, 3

special neurological methods, $32^{\circ}$

Cajal's methods for ncurofibrils in nerve cells, 37

Cox-golgi method, 36

Golgi lichlorid method, 36 silver method, 35

Marchi's, for degencrating nerves, 34

Nissl's methorl, $3^{8}$

Weigert's, for medullaterl nerve fithres, 32

I'eigert-I'al mothorl, 33

Stains, nuclear dyes, 17

plisma rlyes, if

Stalkerl hyrlaticl, 342

Stapes, 57.4

Stellate cells, $510,53^{6}$
Stenoni, duct of, $2 \pi$ i

Stöhr, concerning muscle fibres Iro scheme of spleen, $\mathrm{r}_{3}$

Stomach, $2+7$

acid cells of, $2+5,249$

adelomorphous cells of, $2+9$

Auerbach's plexus, 270

blood-1 essels of, $26 \mathrm{~S}$

cardiac gland of, 252

chief cells of, 2.49

delomorphous cells of, $2+0$

development of, 296

epithelium of, $24 \mathrm{~S}$

funclus glands of, $2+9$

gastric glands of, $2+9$

pits of, 248

IIcller's plexus, $26 \mathrm{~S}$

lymphatics of, 270

M eissner's plexus, 270

mucous membrane of $24 \mathrm{~S}$

muscular coat of, 254

nerves of, 270

oxyntic cells, 249

parietal cells of, 249

peptic cells of, 249

glands of, 249

plexus myentericus, 270

pyloric glands of, 252

replacing cells, 252

rugie of, 248

secretion of, 272

solitary follicles of, 253

stroma of, 252

technic of, $25+$

tuluules of, 250

Stomata, 165

Stratum cincreum, 522

corneum, 38,3

cylindricum, $38_{2}$

filorosum, 205

germinativum $3 \% 2$

granulosum, 3.55

lemnisci, 522

lucidum, 38.3

Nalpiglbii, 382

muccosum, $38_{2}$

ग)tirum, 522

spinesum, 38.3

submuscosum, 36r,

suprisvasculare 3 tor,

vaturulare 36,6

zoniale, 522 
Stratum cinercum, synoviale, 205

Streaming of protoplasm, 52

Stria cornea, $53 \mathrm{I}$

medullaris, 530

of Baillarger, 54I

terminalis, $53 \mathrm{I}$

vascularis, 579

Strix thalami, 522

Styloglossal fibres, 224

Subarachnoid space, 422

Subchorionic placental decidua, 374

Subcutaneous tissue, 318

Subdural space, 422

Sublingual gland, 277

crescents of Gianuzzi of, 278

development of, of, 297

duct of Bartholin of, 278

nerves of, 280

technic of, $28 \mathrm{I}$

Sublingualis minor, 278

Submaxillary ganglion, 436

gland, 278

development of, 297

duct of Wharton of, 278

nerves of, 280

technic of, 278

Submucosa, 2I8

Subperichondrial ossification, $20 \mathrm{I}$

Subperiosteal ossification, 201

Substantia alba, 420

grisea, 420

nigra, I3 $1,5 \mathrm{I} 7,526$

propria corneæ, $55^{\circ}$

Sulcus, external spiral, 582

Superficial sensation, 436

Superior cerebellar peduncles, 499, 500, $502,507,517$

colliculus, $4 \mathrm{I} 7,5^{17} 7,5^{22}$

ganglion of IX, 474

longitudinal fasciculus, 49I, 500, 504 515526

olive $493,500,5 \circ 2,5 \circ 4,5 \circ 7$

Suprachorioidea $55 \mathrm{I}$

Supraradiary plexus $54 \mathrm{I}$

Suprasegmental arc $42 \mathrm{I}$

brain 478

cerebral hemispheres 420,478

connections (afferent and efferent) 476

corpora quadrigemina, 420,478

intersegmental nuclei and tracts of segmental brain, 479
Suprasegmental nuclei and tracts forming suprasegmental paths, 479 pallium, 420, 478

paths, 476

afferent, 476

efferent, 477

peripheral (segmental) neurones,

479

structures, 478

terminal nuclei, 479

neurones, $42 \mathrm{I}$

afferent, $42 \mathrm{I}$

associative, $42 \mathrm{I}$

efferent, 42 I

Suspensory ligament, 565

Sustentacular cells, $284,300,335,575$

Sweat glands, 384

development of, 395

ducts of, 384

muscle tissue of, 395

pore, 384

Sympathetic ganglia, 4I8, 436

cells of, 439

chain ganglia, 436

development of, $4 \mathrm{I} 8$

in Auerbach's plexus, 436

in Meissner's plexus, 436

pigmentation of cells of, 439

prevertebral plexuses, 436

structure of, 436

technic of, 446

termination of nerves, 440

vertebral ganglia, 436

nervous system, $4 \mathrm{I} 6$

Synapsis of neurones, 138

Synarthrosis, 204

Synchondrosis, 204

Syncytial cells, $4 \mathrm{I} 7$

Syncytium, 68, 373

Syndesmosis, 204

Synovial membrane, 205

villi, 205

System, a, 68

Szymonowizc, showing intercellular bridges, 74

showing medullated nerve fibre, $\mathbf{I} 36$

TACTILE cells, 43 I

corpuscles, 223, 432

of Meissner, 432

of Wagner, 394 :

meniscus, 432 
Trenia thalami, 530

Tapetum cellulosum, $55 \mathrm{I}$

fibrosum. 55 I

Tarsal glands, $56 \mathrm{~S}$

Tarsus, 568

Taste buds, $226,227,+32,+33,587$ organ of, 587 ; see Organ of Tuste

Tautomeles, 445,463

Teasing. 4

Technic, general, 3

Tecto-spinal tract, $465,483,487,490$

Teeth, 227

apical foramina, $22 \mathrm{~S}$

blood-resscls of, 235

cementum of, 227,233

crown of, 227

cuticula dentis, 233

dental germ, 238

groove, $23 \mathrm{~S}$

papilla, ${ }_{23} 8$

periosteum, 234

pulp, 228

sac, 239

dentinal canals, 230

fibres, 228

dentine of, $227,22 \mathrm{~S}$

development of , 238

common dental germ, 238

cuticular memurane, 240

dental papilla, 238

enamel organ, 239

special dental germ, 238

technic of, 242

Tomes' process, 440

enamel of, 227,233

cells, 237

fibres, 233

organ, 239

prisms, 233

fang of, 227

interglobular spaces, 232

layer of Weil, 228

lines of Ketzius, 233

of Schreger, 232

lymphatics of, 230 ,

milk, 239, 2\$1

nerves of, $23^{\prime}, 430$

Neumann's dental sheath, 232

roflontoblasts of, 228

peridental membrane, 234

permanent, 23\%, 242

pulfs cavity, 227
Teeth, root of, 227

root-canal of, 228

special dental germs, $23 \mathrm{~S}$

technic of, $2+2$

Tomes' granular layer, 232

process, 240

true molars, $2+2$

Tegmentum, 4 I $7,478,4 S_{3}, 499,515,5 \mathrm{I} 7$

central tegmental tract, $490,+99$

development of, 4 I 7

fillet, 462

fourth cranial nerve, $5 \mathrm{I} 5,5 \mathrm{I} 7$

lateral lemniscus, 49I, 493, 499, 50I, 504,515

nucleus ruber, $436,466,476,517,524$

posterior longitudinal fasciculus, $49 \mathrm{r}$, $500,504,515,526$

reticular formation, $476,4 \delta_{5}, 4 \delta_{7}, 48_{9}$, $490,491,501,504,507$

superior colliculi, 417, 517,522

peduncles, $499,500,502,507,517$

Telencephalon, 417, 532

Telophragma, I I 7

Telophase, 56

Tendon, structure of, 9I sheaths, 208

Tendon-muscle junction, 208

organs of Golgi in, 434

peripheral-nerve terminations in, 434

Tenon, capsule of, 566

Tensor chorioidex, 554

Terminal arborizations, 131, 429

bronchus, 3 ro

nucleus, $42 \mathrm{I}, 490$

Terminations, nerve, 429

annular, 434

arborescent, 434

Ruffini's theory of, 4.34

spiral, 434

Testis, 333

blood-vessels of, 342

corpus Ifighmori, 333

development of, 376

ducts of, $334,339,342$

e jaculatory, 34 r

cpidirlymis of, 3.33

lobules of, 3.3 .3

lymphatics of, 342

mediastinum, 3.33

nerves, 3.42

rete, 339

secretion of, 34.3 
Testis, semen, 343

seminal ducts of, 339

vesicles, 34 I

seminiferous tubules of, 334

spermatozoa, 337,343

technic of, 346

tunica albuginea of, 333

vaginalis, 333

vasculosa, 333

vas deferens, 334

Thalamencephalon, 522

Thalamo-cortical neurones, 462,523

Thalamus, 4I $7,523,526,530$

anterior peduncle of, 536

bundle of Vicq d'Azyr, 524

external segment of 523

geniculate bodies, 520

internal segment of, 523

mamillo-thalamic tract, 524

metathalamus, 523

nuclei of, 523

nucleus of Luys, 523

pulvinar, 523,524

Sachs, E., concerning the, 523 , 524

thalamic radiations, 523,526

Theca folliculi, $35^{6}$

Theoharra and Bensley concerning secretion, 272

Thermostat, 13

Thermotaxis, $5 \mathrm{I}$

Thionin, I9

Thomas, fasciculus of, 467

Thoracic duct, I6 5

technic for, $\mathrm{I} 66$

Three-neurone afferent suprasegmental conduction path, $42 \mathrm{I}$

spinal reflex arc, 469, 506

Thrombocytes, ios

Thymus, I75

blood-vessels of, 177

development of, I 77

Hassall's corpuscles, I7 6

lymphatics of, $\mathrm{x} 77$

nerves of, $\mathrm{I} 77$

technic, $\mathrm{I} 78$

Thyreoid, 402

absence of, 403

blood-vessels of, 403

cartilage, $30 \mathrm{I}$

cells of, 403

colloid of, 402
Thyreoid, development of, 403

isthmus of, 402

lymphatics of, 403

nerves of, 403

Timofeew, concerning nerve fibres in prostate gland, 348

Tissue, 68

-elements, dissociation of, 4

Tissues, 67

adipose, 87

areolar, 87

blood, ro3

bone, roo

cartilage, 97

classification of, 69

connective 80,82

derivatives from ectoderm entoderm, mesoderm, $67,68^{\circ}$

dissociation of, 4

elastic, 92

embryonal, 82

endothelium, 77

epithelial, 70

erectile, 349,376

examination of fresh, 4

histogenesis of, 67

lymphatic, I 64

lymphoid, I 70

mesothelium, 77

muscle, I I I

nerve, $\mathbf{1} 26$

osseous, 100

osteogenetic, I 97

reticular, 94, I70

subcutaneous, $38 \mathrm{I}$

subserous, 268

white fibrous, 82

Toluidin blue, I 9

Toluol, as solvent, I3

Tomes' granular layer, $23 \mathrm{I}$

process, 240

Tongue, 223

blood-vessels of, 226

circumvallate papillæ, 225

connective tissue of, 224

Ebner's glands, 226

end-bulbs of Krause, 227

fibres of, 224

filiform papillæ, 225

fungiform papillæ, 225

glands of, 222, 226

longitudinal fibres of, 224 
Tongue, lymph follicles of, 226

spaces of, 227

muscles of, 223

nerves of, 227

papillic of. 225

seprum lingux. 224

taste buds, $220,227,432,5 s_{7}$

technic of, 227

transiverse fibres of, 224

vertical fibres of, 224

Tonsils, $1 ; \mathrm{S}$

blood-1-ssels of, I80

crypts of, 179

development of, ISo

germ centre of, $1 ; 9$

lingual; felliculic linguales, iso

foramen cacum lingui of, iso

lymphatics of. ISo

lymphoid infiltration of epithelium, 179

nerves of, I 80

nodule of, 179

palatine or true, i $7 \mathrm{~S}$

pharyngeal, iso

adenoids of, $\mathrm{I}$ So

salivary corpuscles of, I 80

technic of, $\mathrm{I} 8 \mathrm{I}$

Trachea, 301

blood-icssels of, 303

cartilages of, 302

glands of, 302

lymphatics of, 304

nerves of, 304

technic of, 304

Tract, a, 42 s

antero-lateral ascending-ventral spino-cercbellar, $4^{(\mathrm{s}} 3$

antero-lateral descending, 466

Burlach's, 459, 4r,2

central tegmental, 490, 50r, 507

cerebro spinalis, $4^{5} 4$

cochlear, 49I, 500, 504

comma, of Schultze, 407

cortico pinali, $4^{r} \cdot 4$

croocel pyramidal, 4/4, 485

Deitero ypinal tract, 466, 483,487, $4 \mathrm{r}^{\circ}, 502$

decencline from pallium (6) motor nucli $i, \leqslant 33$

from inter titial nucleu of C a jal, $4^{65}$

$$
\text { if } 5,487
$$

Tract from nucleus of posterior longitudinal fasciculus, 465

from vestibular nuclei, 460

direct cerebellar, $46_{3}$

pyramidal, 465

dorsal spino-ccrebellar, $46_{3}, 483,487$, 490,507

dorso-lateral ascending-dorsal spinoccrcbellar, 463

fasciculus of Thomas, 467

fastigio-bulbar, 500, 502

fundamental or ground bundles, 445 , $46 \mathrm{~S}$

Flechsig's, 463

Goll's, $459,+62$

Gower's, 464

Helweg's, 467

intersegmental (shorter) $46 \$$

Lissauer's, $443,45^{\circ}$

long ascending arms of dorsal root fibres, 46 I

manillo-thalamic, 524, 532

marginal bundle of Löwenthal, 466

medial lemniscus, $462,485,487,489$ $491,493,517$

optic, 524

oval bundle of Flechsig, 463,467

pallio-spinalis, 464,533

-tectal, 535

posterior, 462

funiculi, 46I

predorsal, $49 \mathrm{I}$

fasciculus, 517

pyramidal, 464, 533

anterior, 465

ruh)ro-spinal, $466,483,487,490,504$, 515

secondary cochlear, 493, 504, 507

vago-glossopharyugeat and trigeminal, 515,526

vestibular, 493

scpto-marginal, 467

short fibre, $4.45,462,468$

spinalis trigemini, 485,500

spine-rereluellar, ventral, 463, 483, $4^{8} 7,508$

-cullimlo, f1.5, 483,487,490,502, $504,507,517$

-spinal, 468.8

lectal, $46,5,4^{8} 3,487,400$ 
Tract, spinothalamic, $462,483,501,504$, 515,526

tecto-spinal, $465,483,487,490$

uncrossed cerebellar, 463

ventral spino-cerebellar, $463,50 \mathrm{O}$, $5 \circ 4,5$ I 5

vestibulo-spinal, 466

Von Monakow's, 466

Tractus, see Tract

Transitional leucocytes, I05

Transverse temporal gyri of Heschl, 54I

Trapezoid nucleus, 493

Trapezium, 493

Trigeminus (V nerve), 475, 485, 489, 493, $5 \circ 1,5 \circ 4,5 \circ 6$

Trigonum hypoglossi, 48I, 489

olfactorium, 532

vagi, 48 I

Trochlearis (IV nerve), 475, 5I 5, 5I 8,520

Trophic centre of neurone, 137

Trophospongium, 46

True corpora lutea, 360

tonsils, $\mathrm{r} 78$

vocal cords, $30 \mathrm{I}$

Tuberculum cinereum, 48I, 53I olfactorium, 532

Tubular glands, $2 \mathbf{I}_{5}$

Tubules arched, 320,323

collecting $32 \mathrm{I}, 324$

distal, 320,323

first or proximal, $32 \mathrm{C}, 322$

intercalated, 276

intermediate, 277

junctional, 377

salivary, 276

second or distal, 320,323

secreting, 276

seminiferous, 334

serous, 222

straight, 32 I, 324

uriniferous, 320

Tubulo-alveolar gland, 282

Tunica albuginea, of ovary, 353

of penis, 349

of testis, 333

dartos, 38I

fibrosa, 356

vaginalis, 33.3

vasculosa, 333,356

Two-neurone spinal reflex arc, 469, 504

Tympanic gland, 4I 2

membrane, 573
Tympanum, 573; see also Ear, middle

Tyson, glands of, $35^{\mathrm{I}}$

Ultimate fibrillæ, I 5

Uncinate fasciculus, 535

Unipolar nerve cells, I2 7, 428

Ureter, 329

development of, 376

technic of, 33 I

Urethra, female, 35 I

glands of Littré of, $35 \mathrm{I}$

male, $35 \mathrm{I}$

fossa navicularis, $35^{2}$

glands of Littrè of, $35^{2}$

technic of, 352

Urinary bladder, 330

blood-vessels of, $33 \mathrm{I}$

development of, 376

system, 3 I 8

development of, 376

te chnic of, 33 I

kidney, 3 I 8

-pelvis, 329

ureter, 329

urinary bladder, $33^{\circ}$

technic of, 33 I

Uriniferous tubule, 320

arched tubule of, $32 \mathrm{I}, 323$

ascending arm of Henle's loop, 320 , 323

blood-vessels of, 325

Bowman's capsule, 320,322

descending arm of Henle's loop, 320,322

development of, 376

duct of Bellini, 32 r, 324

epithelium of, 324

first or proximal convoluted, 320,322

foramina papillaria, $32 \mathrm{I}$

glomerulus, 320

Henle's loop, 320,323

location in kidney of, 324

Malpighian body, 32 I

membrana propria of, 32 I

neck of, 322

renal corpuscle, 320

Rühle, concerning, 325

second or distal convoluted, 320,323

straight or collecting, 32 I, 324

Uterus, 366

blood-vessels of, 375 
Uterus, cervix, 367

coats of, 366

decidua basalis, 370

capsularis, 370

graviditatus, $37^{\circ}$

menstrualis, 369

reflexa, $37^{\circ}$

serotina, 370

subchorionic placental, 374

vera, 370

decidual cells of, 370

development of, 376 ; see also $R e$ productive system, devclopment of lymphatics of, 375

masculinus, $24 \mathrm{~S}$

mucosa of menstruating, 368

of pregnant, $37^{\circ}$

of resting, 367

stratum submucosum, 366

supravasculare, 366

vasculare, 366

nerves of, 375

placenta, 371

plicx palmatx, 368

pregnant, 370

theories concerning, 369

stage of menstruation proper, 369

of preparation, 368

of reparation, 369

technic of, 379

with placenta in situ, technic of, 379

Utricle, 575; see Saccule and utricle

Utericulo-saccular duct, 575

Utriculus prostaticus, 348

Uvula, mucous membrane of, 220

VAGINA, 375

blood-vessels of, 376

coats of, 375

lymphatics of, 376

nerves of, 376

rugae of, 376

technic of, 376

Vagus (X) ncrve, 474, 489

Valve, Hcisterian, 295

Valves of heart, $\mathrm{I}_{3}$

of veins, 158

Valvulac conniventes, 24 6), 255

Vas deferens, $34^{\circ}$

technic of, 34 6

eprididymis, $34^{\circ}$
Vasa efferentia, 339

vasorum, 159

Vascular papillæ, ${ }_{3} S \mathrm{I}$

sy.stem, I5I; sce also Circulatory system

Vater-Pacinian corpuscles, 394

Veins, ${ }_{5} \mathrm{~S}$

adventitia of, 158

arcuate, 328

central, $2 S 9$

coats of, ${ }_{5} S$

development of, ${ }^{6} 63$

intima of ${ }^{5} S$

lymph channels of, I59

media of, 158

musculature of, 158

nerves of, 158

perivascular lymph spaces of, I 59

portal, 289

renal, 3 I 8

splenic, 182

stellate, of Verheyn, 328

sublobular, 289

technic of, 160

valves of, 158

vasa vasorum, I 59

ven $x$ vorticos $x, 55$ I

Venæ vorticosæ, $55 \mathrm{I}$

Ventral spino-cerebellar tract, 463

Ventricles of brain, $4 \mathrm{r} 6,4 \mathrm{I} 7,4 \mathrm{Sr}, 487,499$ of heart, I6I

Verheyn, stellate veins of, 328

Verhorf's differential elastic-tissue stain, 28

Vermiform appendix, 265

lymph nodules of, 266

mesoappendix, 265

technic of, 275

Vermis of cerelsellum, 4I $5,449,455$

Vertebral ganglia, 436

Vesicle, air, 310

brain. 416

germinal, 59

optic, $5^{60}$

otic, $5^{8} 3$

seminal, 342

Vesicula prostatica, 348

Vestibular ganglion, 425

nerve, $425,44 \mathrm{I}$

nuclei, 44 !

descending tract from, 4.41

Vestibule, 575 
Vestibule, ductus reuniens of, 575

endolymphatic duct, 575

sac, 575

saccule of, 575

utricle of, 575

utriculo-saccular duct of, 575

Vestibulo-semicircular canal group of segmental neurones, 475

Vicq d'Azyr bundle of, 524, 532

Villi, 256

development of, 297

fastening, $37 \mathrm{I}$

floating, $37 \mathrm{I}$

free, $37 \mathrm{I}$

lacteals of, 275

synovial, 205

terminal, $37 \mathrm{I}$

Visceral neurones, 419, 475

peritoneum, 267

Visual area, 488

group of segmental neurones, 475

path, 475,524

purple, 557

Vision, organ of, 548 ; see Organ of Vision

Vital properties of cells, $50,2 \mathrm{I} 3$

function, $5 \mathrm{I}$

irritability, $5^{\mathrm{I}}$

metabolism, 50, 2 I 3

motion, 5 I

reproduction, 52

Vitreous body of the eye, 565

Cloquet's canal, 565

hyaloid canal of, 565

membrane of chorioid, 553

of iris, 553

Vocal cords, $30 \mathrm{I}$

Volkmann's canal, I9I, I96

Voluntary striated muscle, I I4; see $M u s$ cle, striated, voluntary

Von Bechterew's nucleus, 500, 507

Von Bibra, concerning chemical composition of dentine, 228

of enamel, 233

Von Gudden, concerning method of determining fibre tracts of cord, 459

Von Monakow's bundle, 466

WAGNER, corpuscles of, 394

Wallerian degeneration, law of, I4I
Wandering cells, 83

Washing after fixation, 9

Weigert's elastic-tissue stain, 28

hæmatoxylin, I9

method of staining medullated nerve fibres, 32

Weigert-Pal method, 33

Weil, layer of, 228

Wernicke, perpendicular fasciculus of, 535

Wharton's duct, 278

Wheeler, showing amitosis, 52

White blood cells (leucocytes), I05

or fibrillated fibres, 86

fibrous tissue, 82

matter, $420,45^{\circ}$

rami communicantes, 425,436

Wilson, E. B., diagrams showing mitosis, 54,56

Wirsung, duct of, $28 \mathrm{I}$

Wolffian body, 338,377

IVrisburg, cartilage of, $30 \mathrm{I}$ nerve of, $50 \mathrm{I}$

XYLoL and cajeput oil for clearing, 23

-balsam, 23

damar, 30

Xylol-paraffin for embedding, I 3

Yellow or elastic fibres, 86

Yolk granules, 357

ZENKER's fluid for decalcifying, Io for fixation, 8

Zinn, zonule of, 565

Zona incerta, 530

pectinata, 580

pellucida, 6r, 357

spongiosa, 449, 450

tecta, $5^{80}$

Zone of Lissauer, 443

of oval nuclei, 300

of 1 ound nuclei, 300

Zonula ciliaris, 565

Zonule of Zinn, $5^{6} 5$

Zuckerkandl, organ of, 4I 2

Zymogen granules, 282

technic of, 287 

This book is due on the expiration of a definite pe iod fter the date fibrary or by special aras provided by the Libu riar in charge.

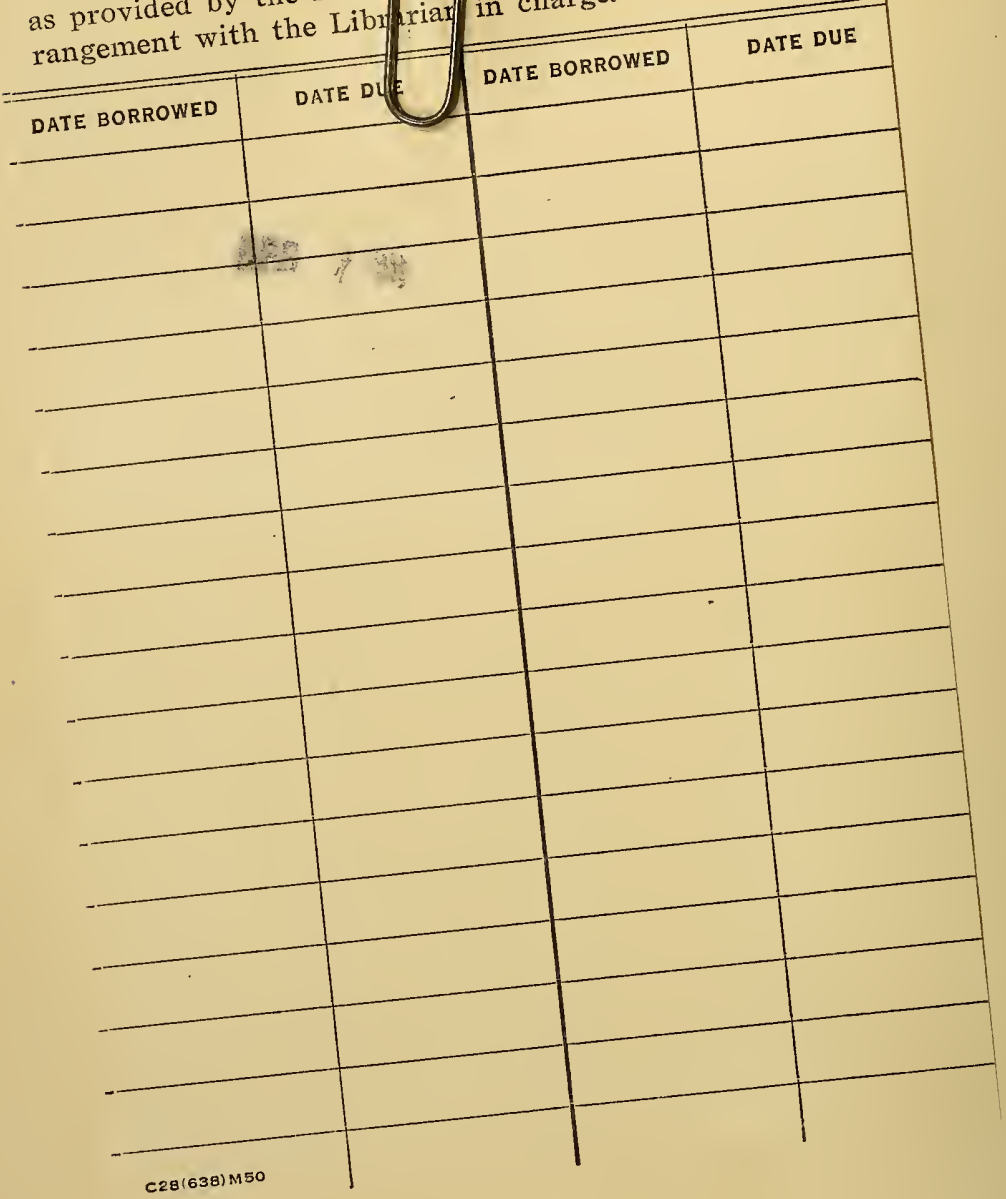




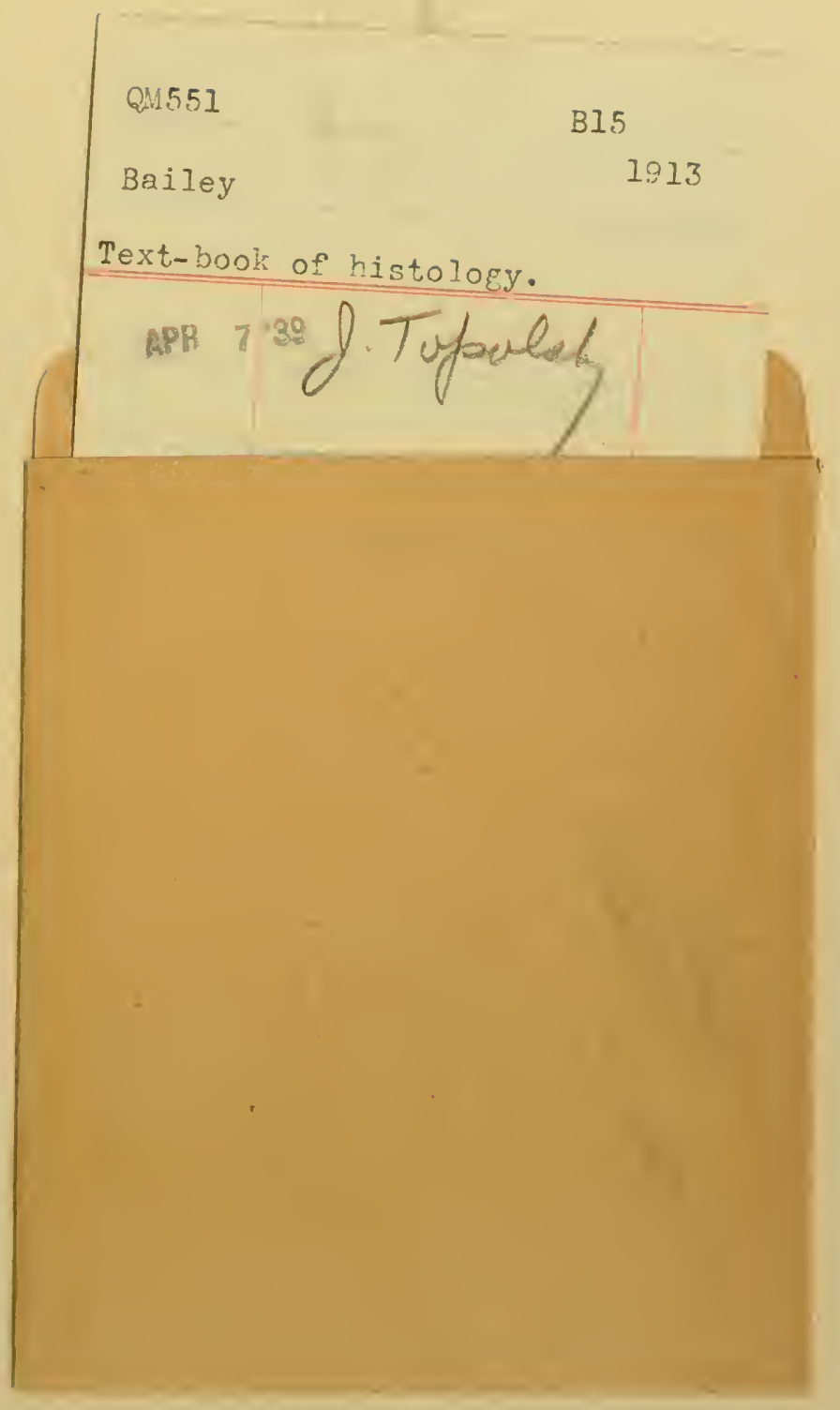


